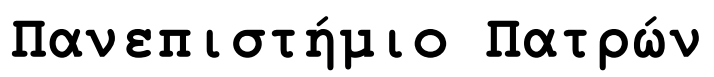

Tиท́

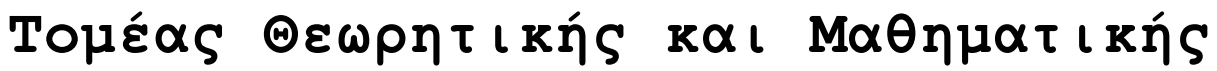

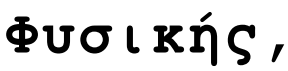

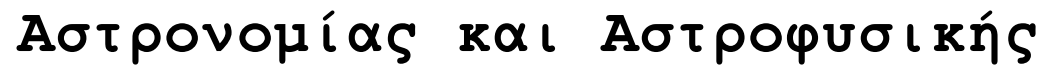

$\Theta \varepsilon \dot{\varepsilon} \mu$ :

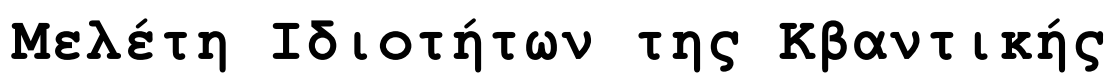

$\Pi \lambda \eta \rho о \varphi о \rho i \alpha s$

$\sigma \varepsilon \quad K \beta \alpha \nu \tau$ เ Kó $\Sigma$ vง $\sigma \tilde{\eta} \mu \alpha \tau \alpha$

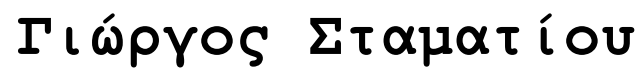

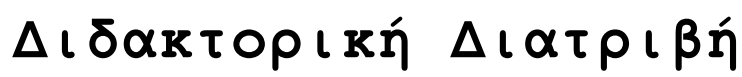

I.X.NI.KA.

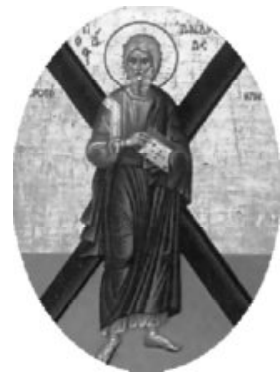

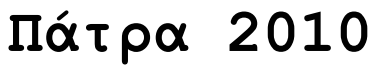



$\Sigma \tau \eta \nu \operatorname{M\eta \tau \varepsilon ́\rho ~\mu ov~}$ 



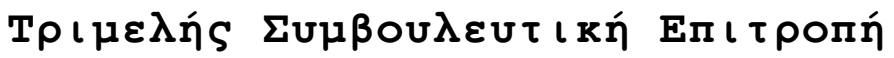

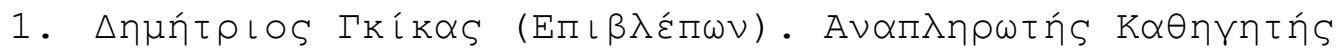

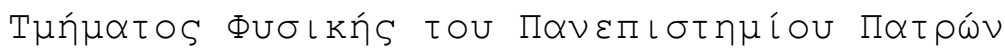

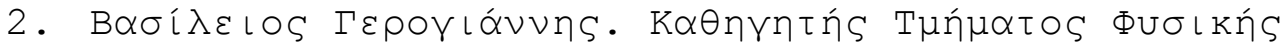

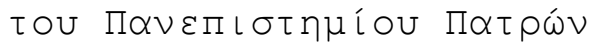

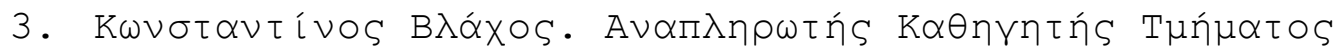

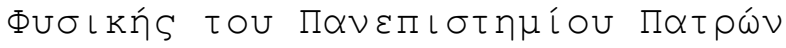

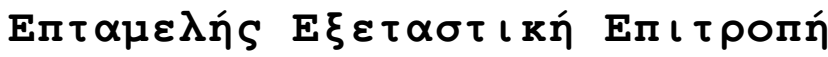

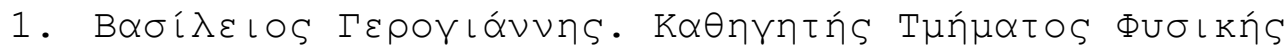

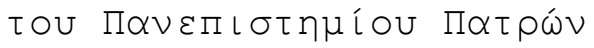

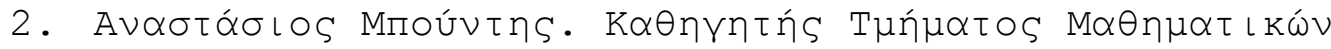

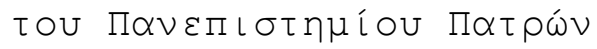

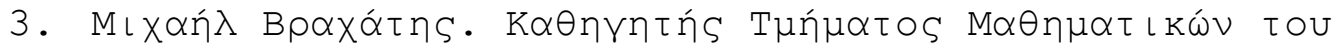

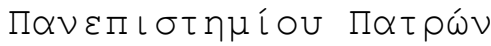

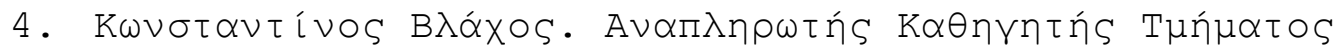

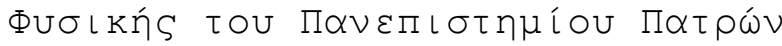

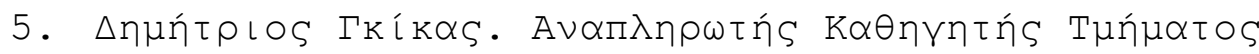

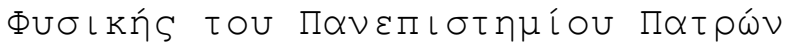

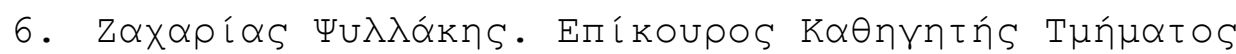

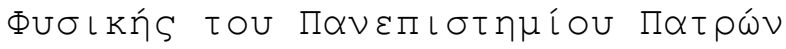

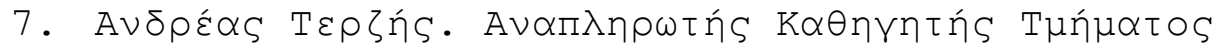

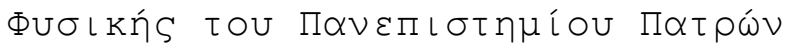





\section{Eux $\alpha \rho\llcorner\sigma \tau i \varepsilon S$}

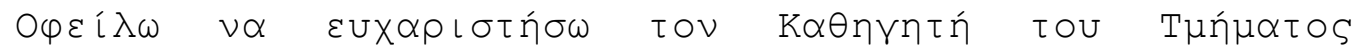

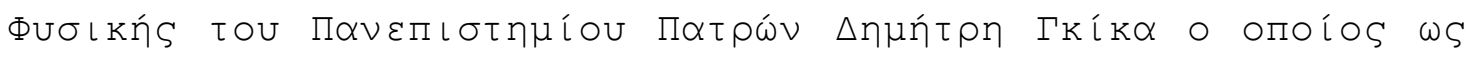

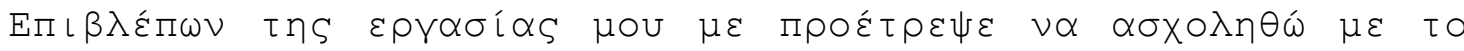

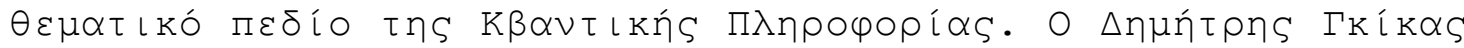

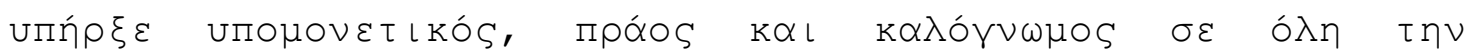

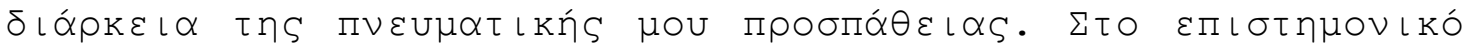

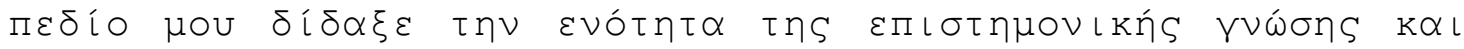

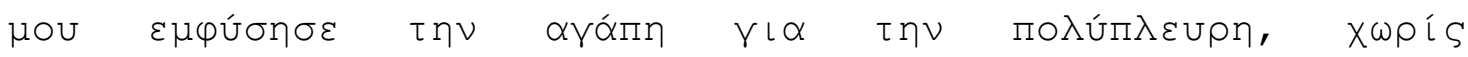

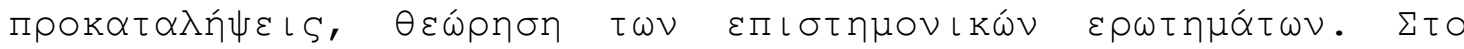

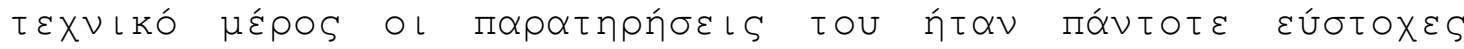

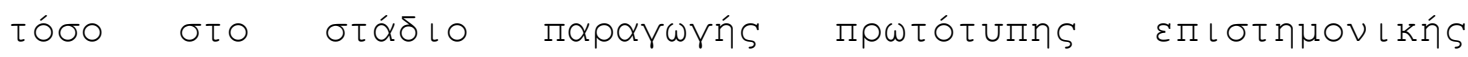

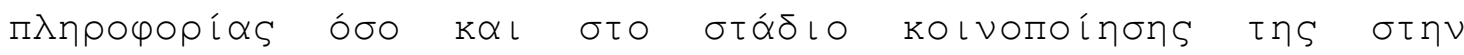

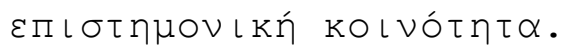

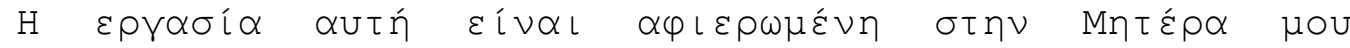

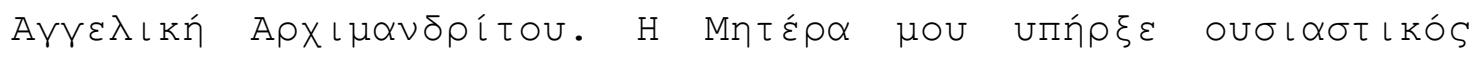

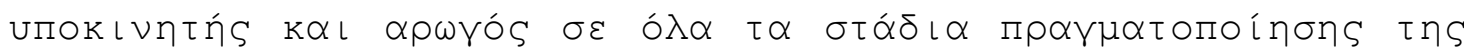

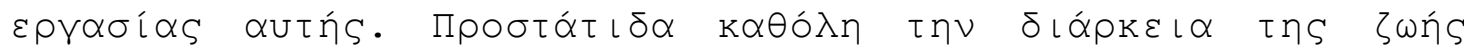

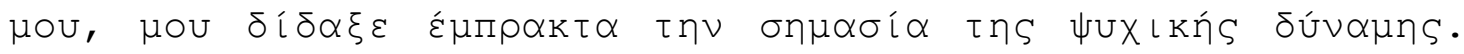

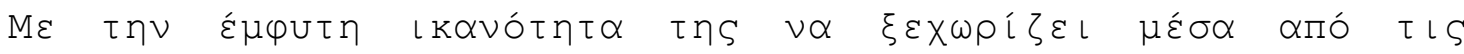

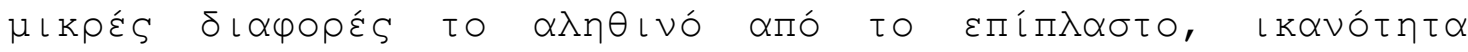

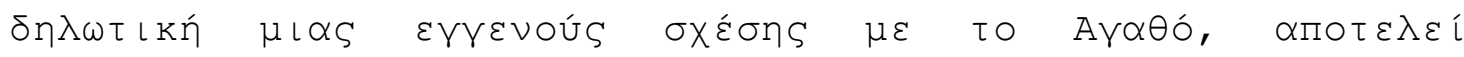

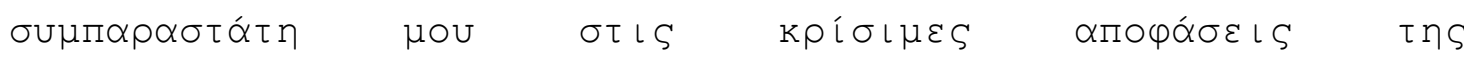

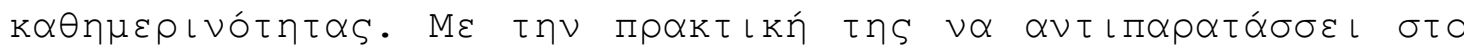

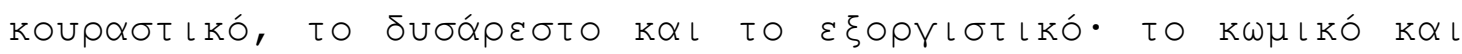

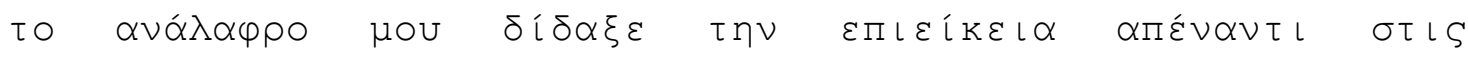

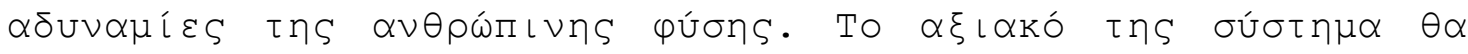

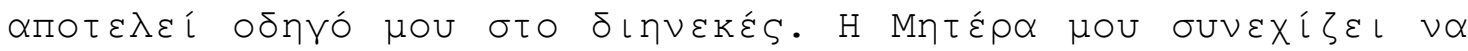

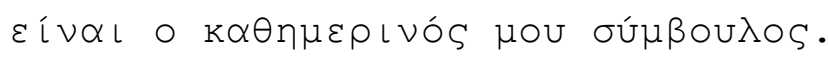

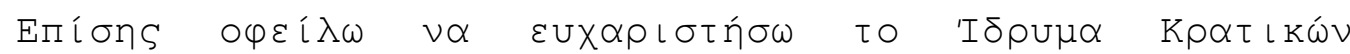

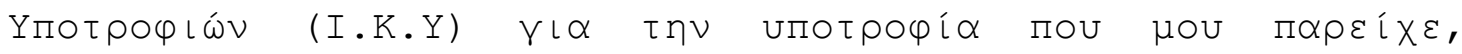

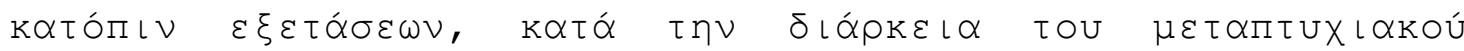

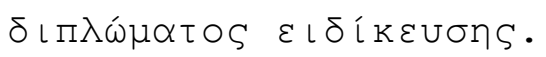





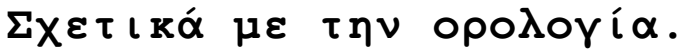

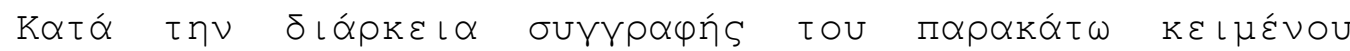

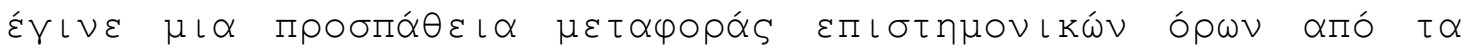

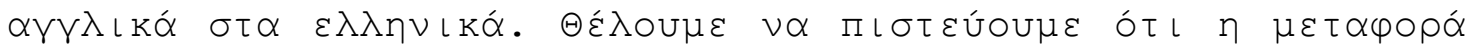

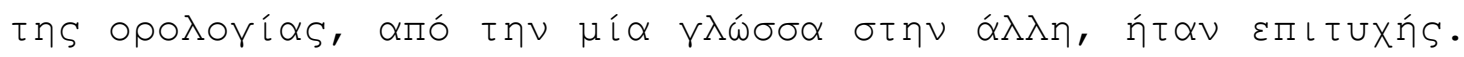

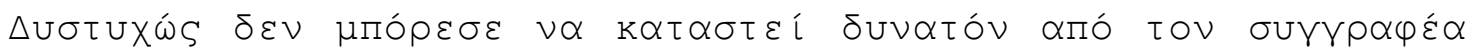

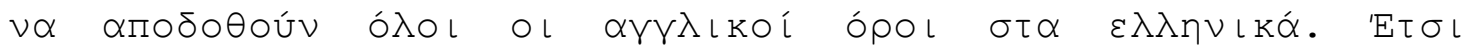

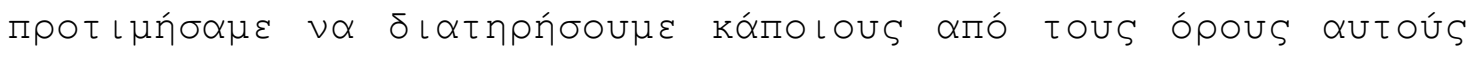

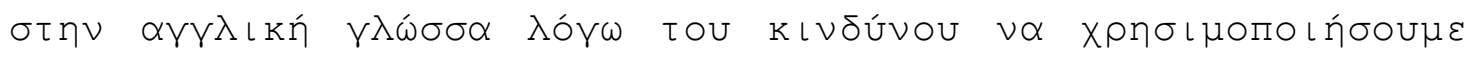

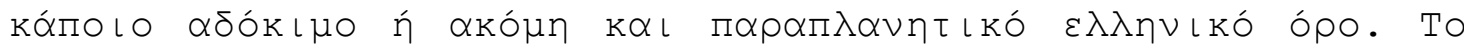

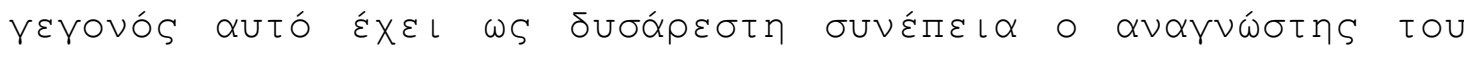

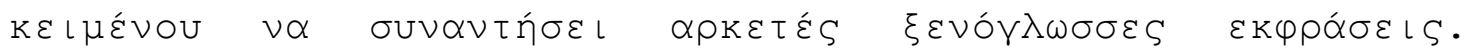

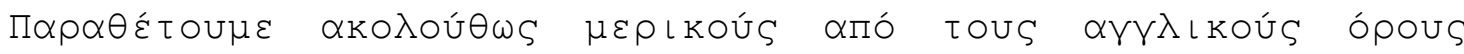

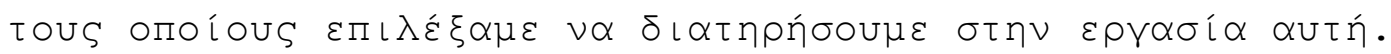

Entanglement, Entangled, Disentangled, Unentagled, Quantum Kicked Rotor, Quantum Kicked Top, Majorization, Majorized, Distillable Entanglement, Entanglement Cost, Entanglement of Formation, Cross Norm, Robustness, Squashed Entanglement, Bit, Qubit, Qutrit, Ancilla, Saturation Index, Entangling Power.

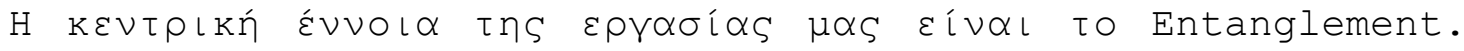

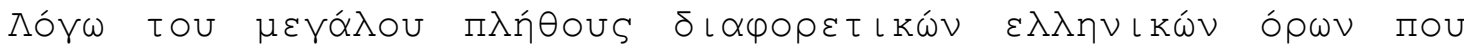

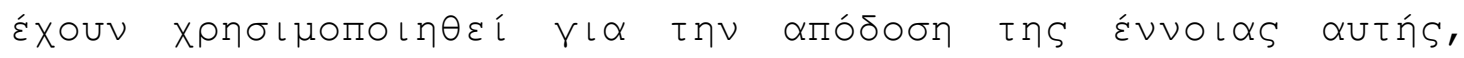

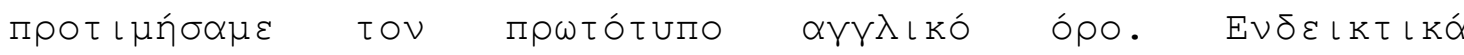

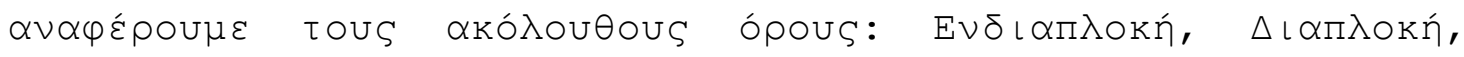

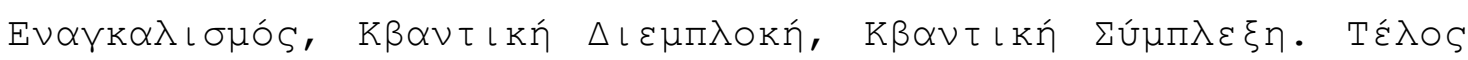

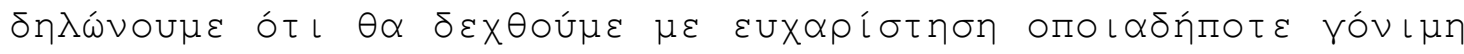

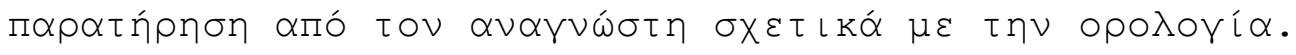





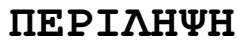

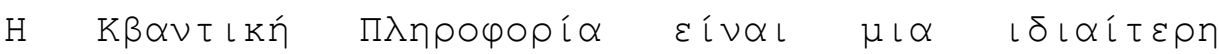

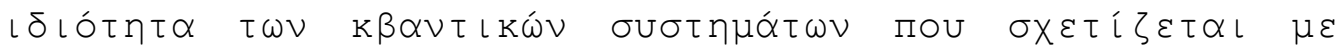

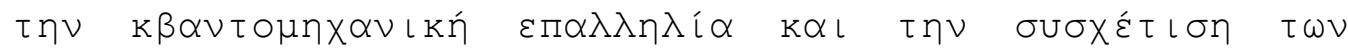

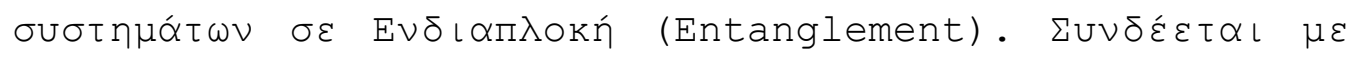

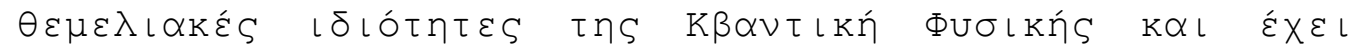

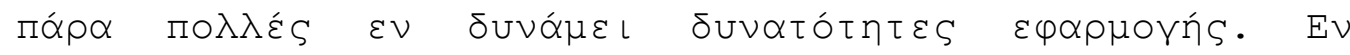

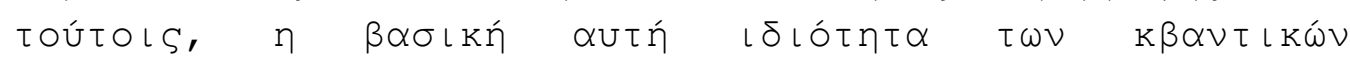

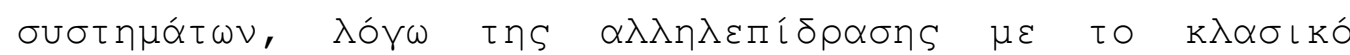

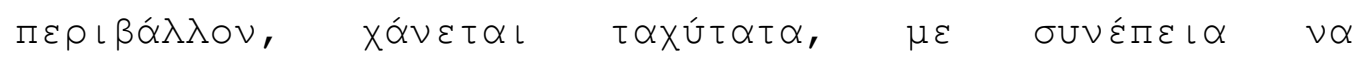

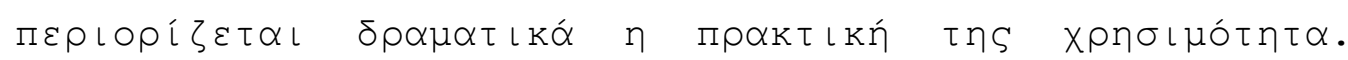

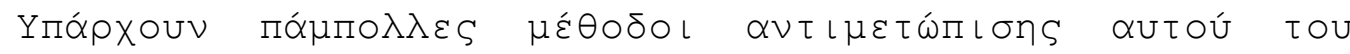

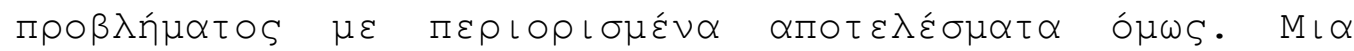

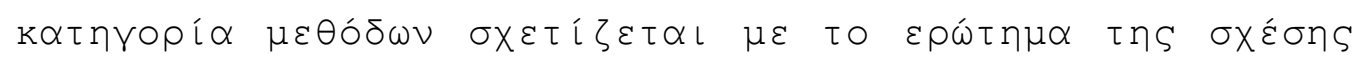

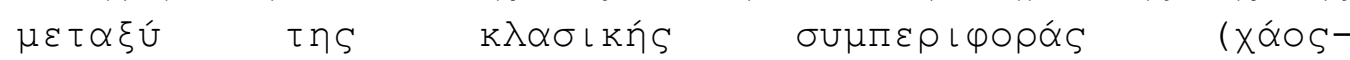

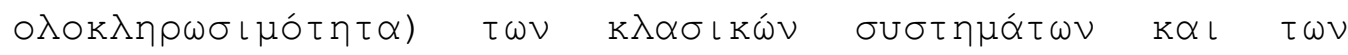

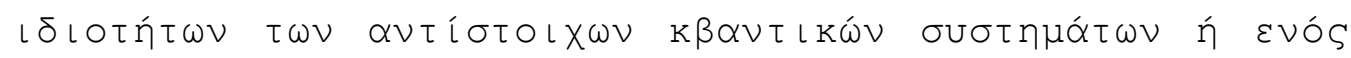

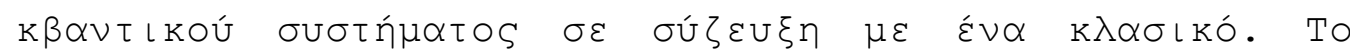

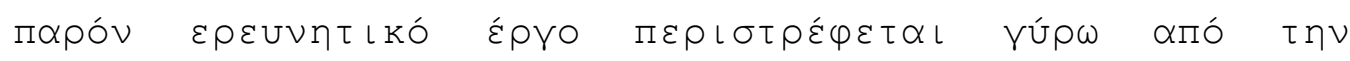

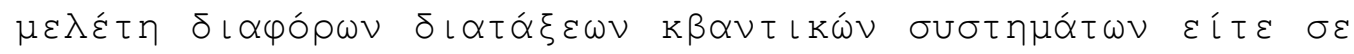

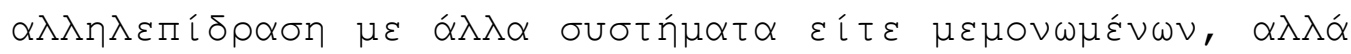

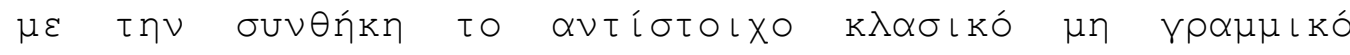

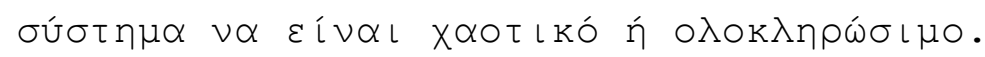

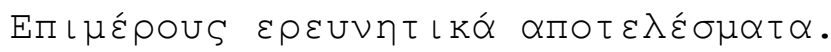

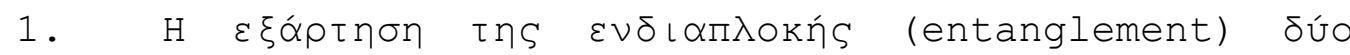

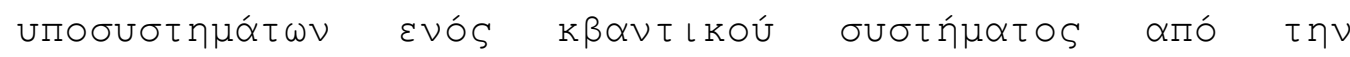

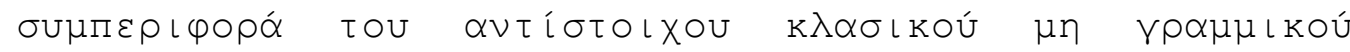

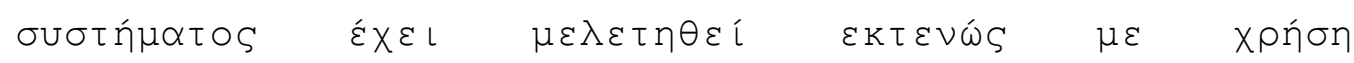

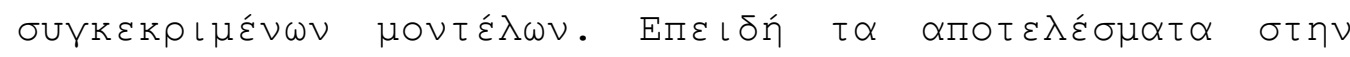

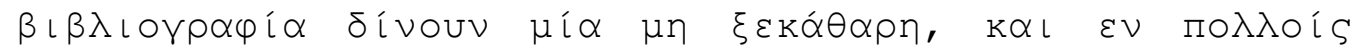

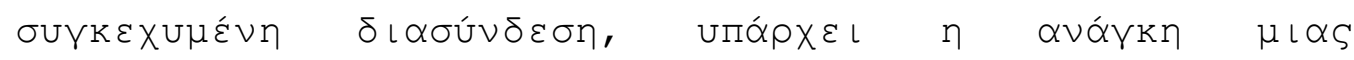

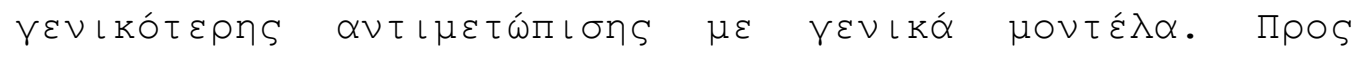

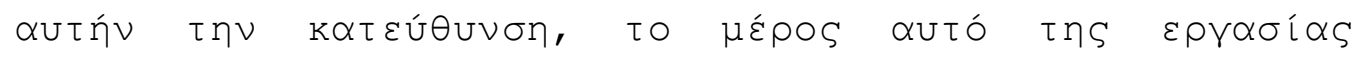

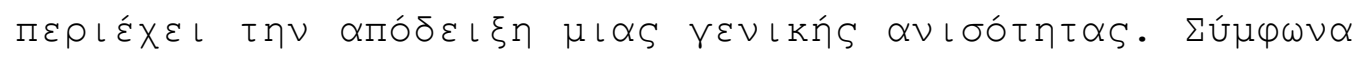

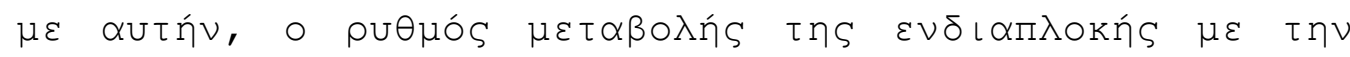

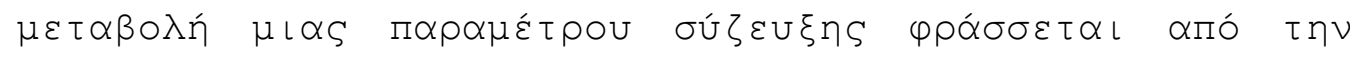

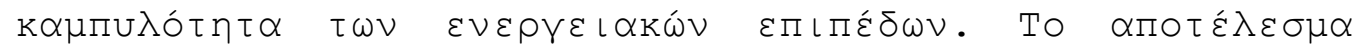

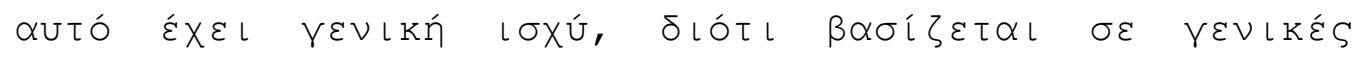

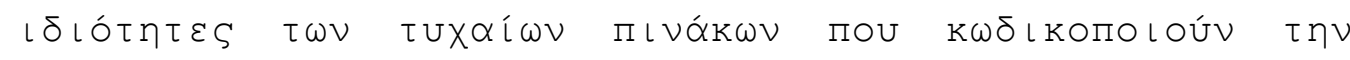




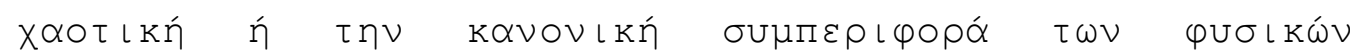
$\sigma U \sigma \tau \eta \mu \alpha ́ \tau \omega \nu$.

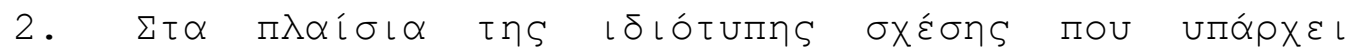

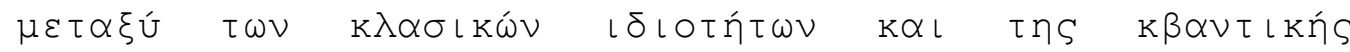

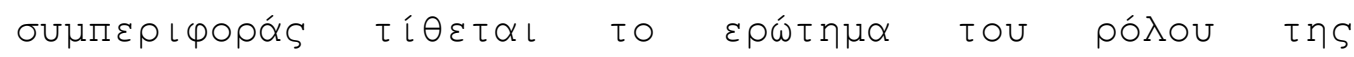

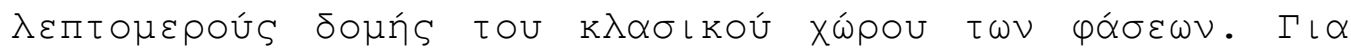

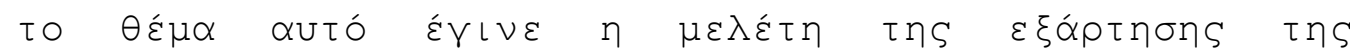

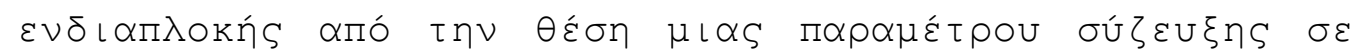

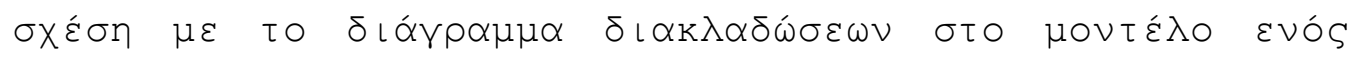

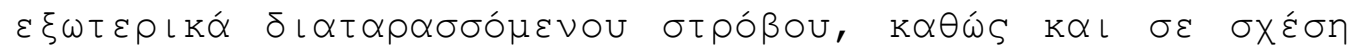

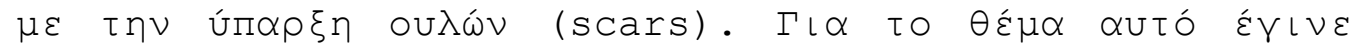
$\varepsilon \kappa \tau \varepsilon \vee \eta ́ S ~ \alpha \vee \alpha ́ \lambda U \sigma \eta$.

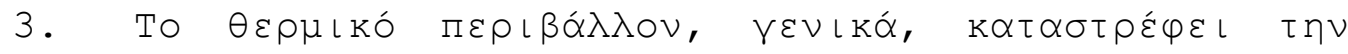

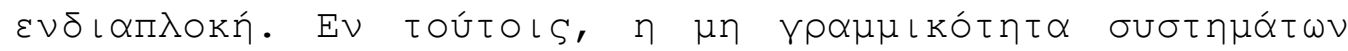

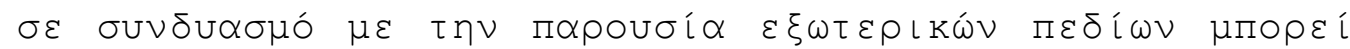

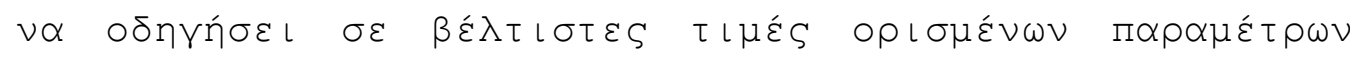

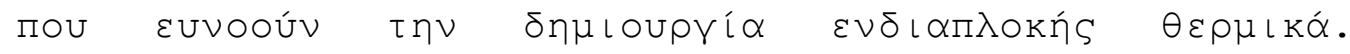

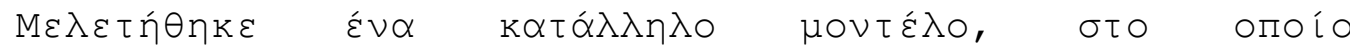

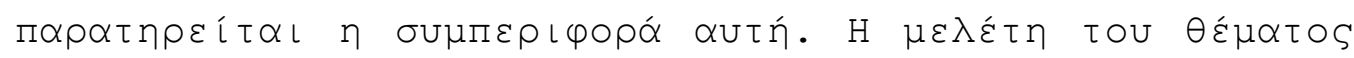

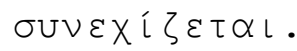

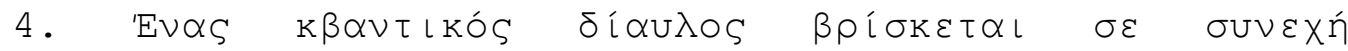

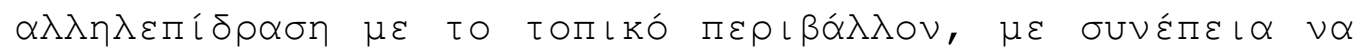

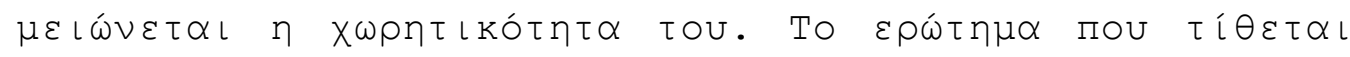

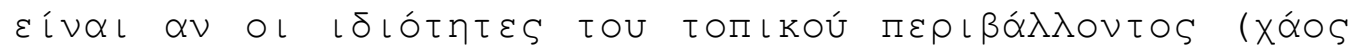

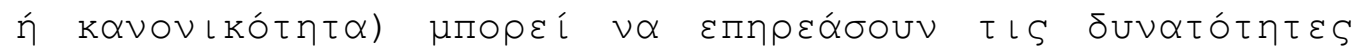

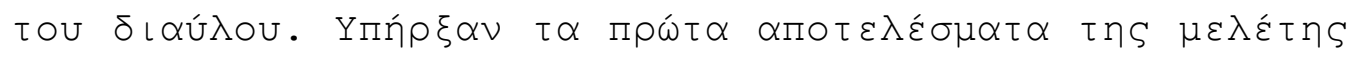

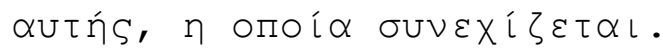

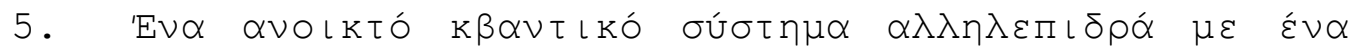

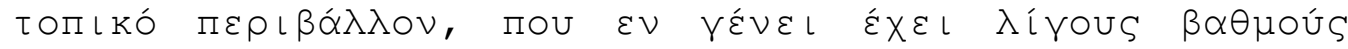

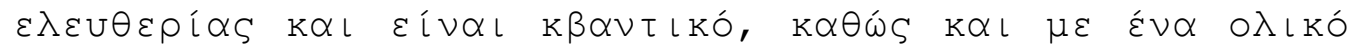

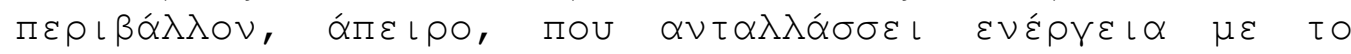

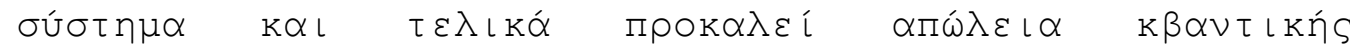

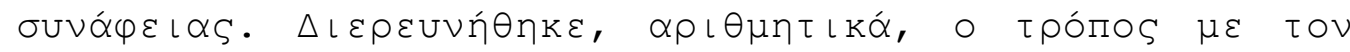

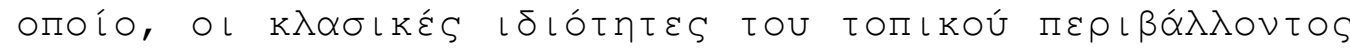

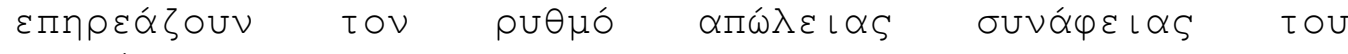

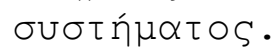




\section{$\Delta \eta \mu о \sigma \iota \varepsilon \tilde{u} \sigma \varepsilon \iota \varsigma:$}

1. Demetris P.K. Ghikas, Georgios Stamatiou: A curvature dependent bound for entanglement change in classically chaotic systems, International Journal of Quantum Information, Vol. 5 (2007) 685-704.

2. Georgios Stamatiou, Demetris P.K. Ghikas: Quantum entanglement dependence on bifurcations and scars in non-autonomous systems. The case of quantum kicked top, Physics Letters A 368 (2007) 206-214. 



\section{ABSTRACT}

Quantum Information is a particular property of quantum systems which is associated with the quantum mechanical superposition principle and the correlation of the systems in the entangled states. It is related to fundamental properties of Quantum Physics and it has very many potential applications. Nevertheless, due to the interaction with the classical environment, this basic property of quantum systems is lost very quickly, with the result, its practical usefulness to be dramatically reduced. There are many methods of dealing with this problem, but with partial success. One category of methods, not extensively investigated, is associated with the question of the relation between the behavior of the classical systems (chaos-integrability) and the properties of the corresponding quantum systems, or of a quantum system interacting with a classical one. The present Thesis is concerned with the study of various arrangements of quantum systems either in interaction with other systems, or isolated, but with the condition that the corresponding classical non linear system is chaotic or integrable.

Results presented in the thesis.

1. The dependence of entanglement of two subsystems of a quantum system on the behavior of the corresponding classical non linear system has been studied extensively with the use of concrete models. Because the results in the literature do not give a clear, and in many cases give a contradicting picture, a more general approach is needed with generic models. To this purpose, in this part of the thesis the proof of a general inequality is presented. According to this, the rate of change of entanglement with respect to the change of an interaction parameter is bounded by the curvature of the energy levels. This result has a general validity, because it is based on general properties of random matrices, which may encode the regular or chaotic behavior of physical systems.

2. In the framework of the particular relation that exists between classical properties and quantum 
behavior, a question is posed about the role of the detailed structure of the classical phase space. For this matter, the dependence of entanglement on the position of a parameter of interaction in connection to the bifurcation diagram in the model of quantum kicked top was studied. Also an analysis was carried out for a possible impact of the existence of scars on the behavior of entanglement. This problem was extensively analyzed numerically.

3. In general, a hot environment destroys entanglement. Nevertheless, the non linearity of systems combined with the presence of external fields may lead to optimal values of certain parameters which favor the thermal creation of entanglement. A particular model was studied in which this behavior is observed.

4. A quantum channel is in constant interaction with its local environment, and as a result its capacity is reduced. The question posed, is whether the properties of the local environment (chaos or integrability) may influence the capabilities of the channel. The first results of this study are presented.

5. An open quantum system interacts with a quantum local environment, which in general has few degrees of freedom, and a global infinite one, which exchanges energy with the system and leads finally to the loss of quantum coherence. It was numerically investigated how the classical properties of the local environment influence the decoherence rate of the system

Publications:

\begin{tabular}{|c|c|}
\hline $\begin{array}{l}\text { 1.Demetris P.K. Ghikas, Georgios } \\
\text { dependent bound for entanglement } \\
\text { chaotic systems, } \\
\text { Information, Vol. } 5 \text { (2007) 685-704. }\end{array}$ & $\begin{array}{crr}\text { Stamatiou: } & \text { A curvature } \\
\text { change in classically } \\
\text { Journal } & \text { of } & \text { Quantum }\end{array}$ \\
\hline $\begin{array}{l}\text { - Georgios Stamatiou, } \\
\text { ntanglement dependence on bifurca }\end{array}$ & $\begin{array}{lll}\text { P.K. Ghikas: } & \text { Qua } \\
\text { ions and scars in }\end{array}$ \\
\hline
\end{tabular}




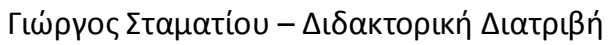

\section{MEPIEXOMENA}

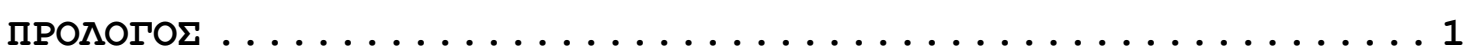

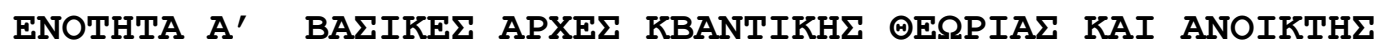

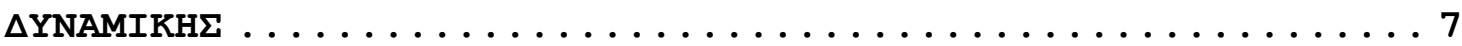

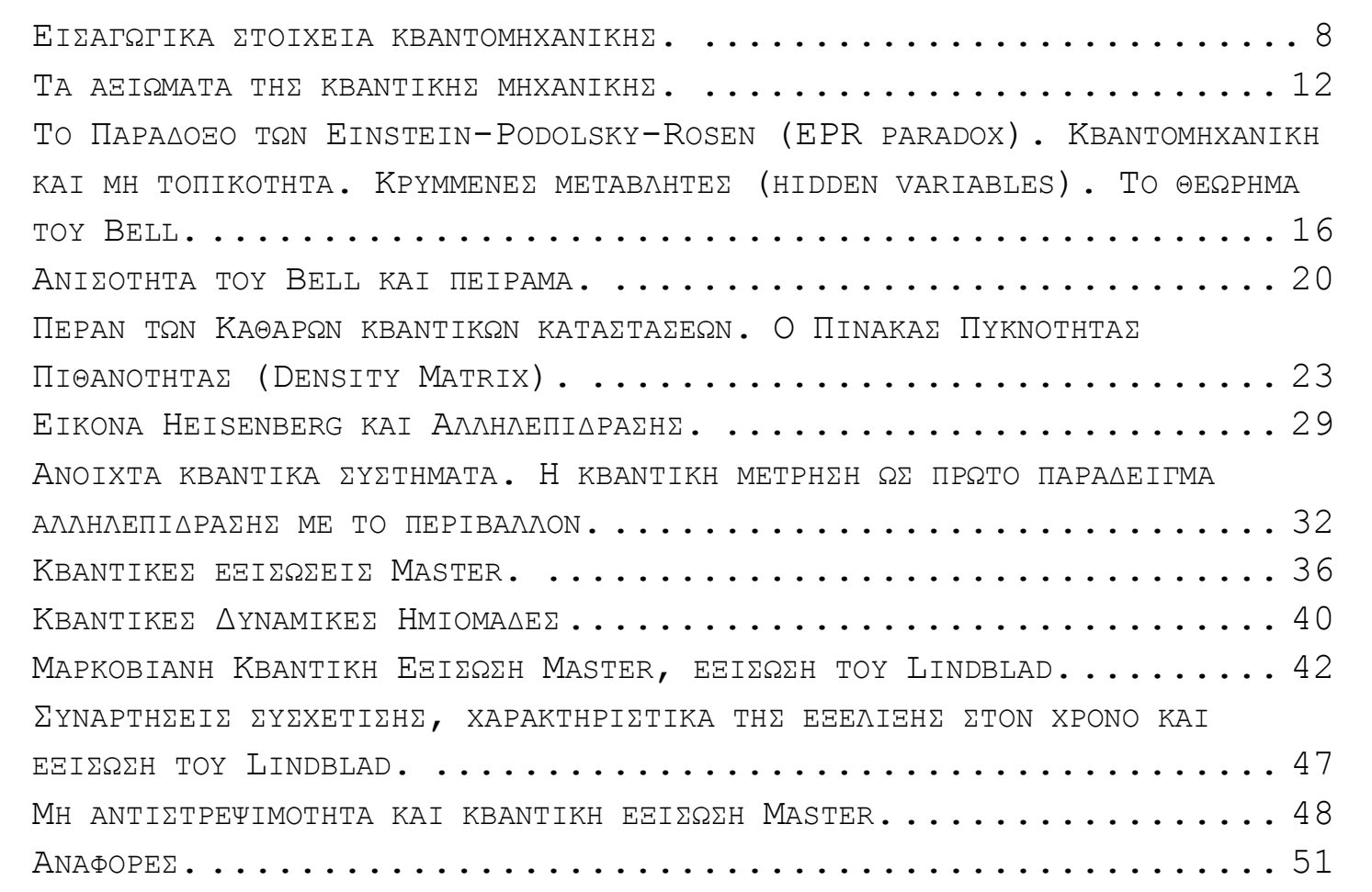

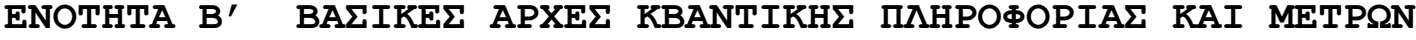
TOY ENTANGLEMENT $\ldots \ldots \ldots \ldots \ldots \ldots \ldots \ldots \ldots \ldots \ldots$

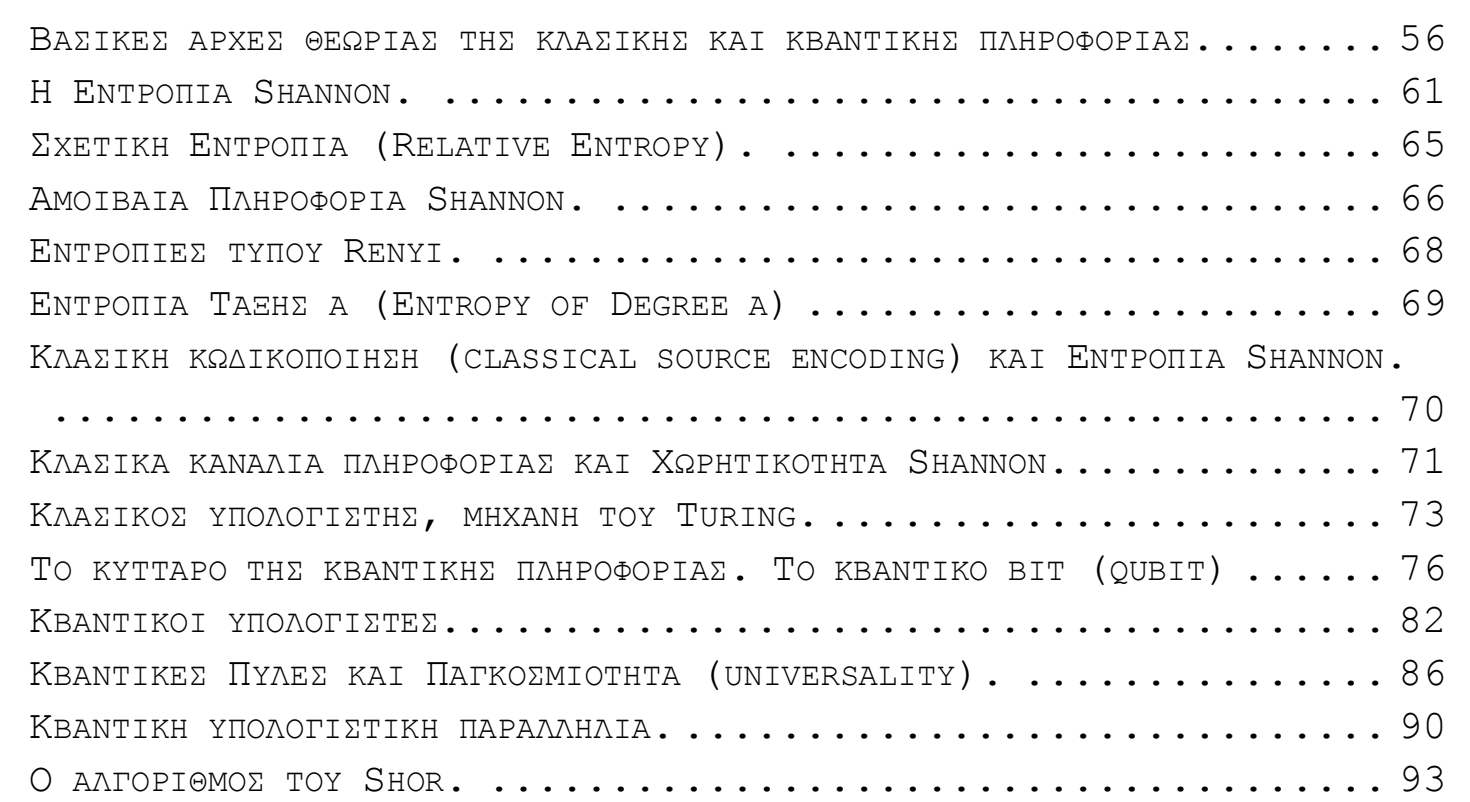




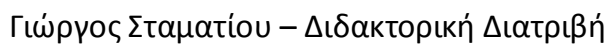

\begin{tabular}{|c|}
\hline BANTIKE $E$ ENTPOHIE $\ldots \ldots \ldots \ldots \ldots \ldots \ldots \ldots \ldots \ldots$ \\
\hline 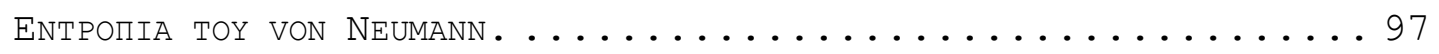 \\
\hline ГPAMMIKH ENTPOMIA $($ LINEAR ENTROPY $) \ldots \ldots \ldots \ldots \ldots \ldots \ldots \ldots$ \\
\hline 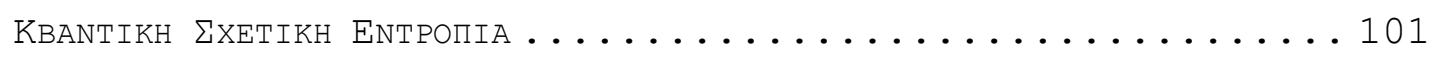 \\
\hline 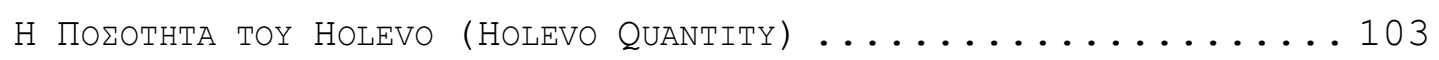 \\
\hline 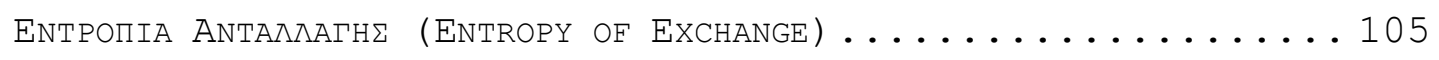 \\
\hline 'Aд \\
\hline 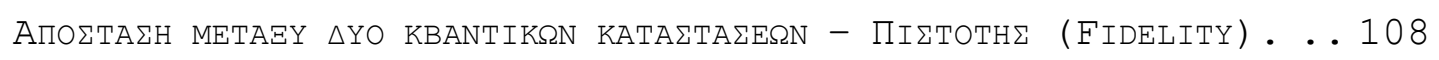 \\
\hline 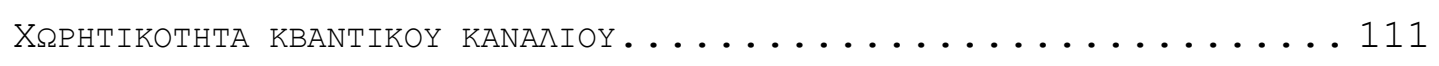 \\
\hline MH TOПIKH KBANTIKH $\Sigma Y \Sigma X E T I \Sigma H-E N T A N G L E M E N T ~ \ldots \ldots \ldots \ldots \ldots \ldots \ldots \ldots . \ldots 112$ \\
\hline 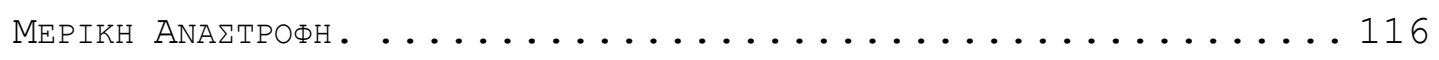 \\
\hline 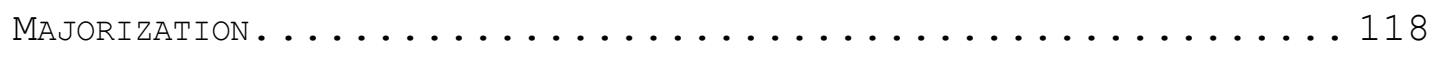 \\
\hline 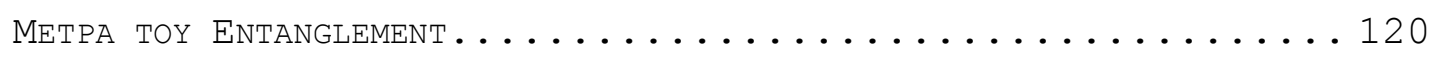 \\
\hline ENTANGLEMENT OF FORMATION $\ldots \ldots \ldots \ldots \ldots \ldots \ldots \ldots \ldots \ldots$ \\
\hline 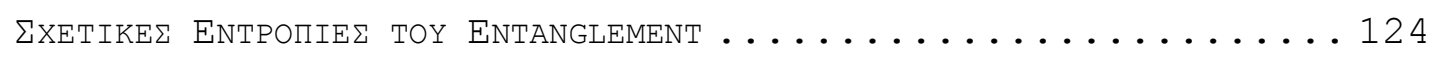 \\
\hline О \\
\hline MONOTONA TOY ENTANGLEMENT BAIIEMENA $\Sigma$ E NOPMA.............127 \\
\hline 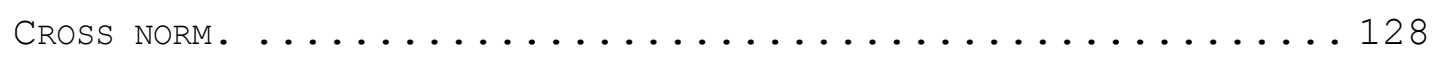 \\
\hline \begin{tabular}{|ll} 
TO SQUASHED ENTANGLEMENT . \\
\end{tabular} \\
\hline$\bullet \cdots$ \\
\hline
\end{tabular}

ENOTHTA $\Gamma^{\prime} \quad \Sigma T O I X E I A \quad$ KBANTIKOY XAOY $\ldots \ldots \ldots \ldots \ldots$

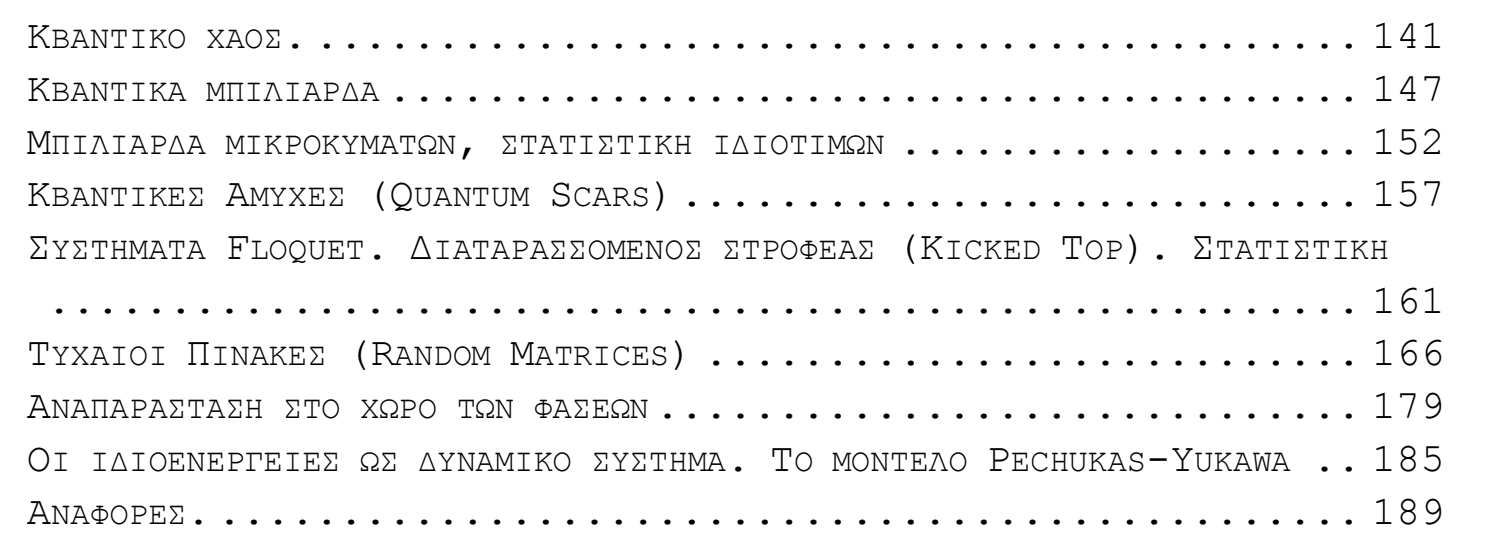

ENOTHTA $\Delta^{\prime}$ EEAPTH $\mathrm{TH}$ TOY ENTANGLEMENT AПO THN KYPTOTHTA

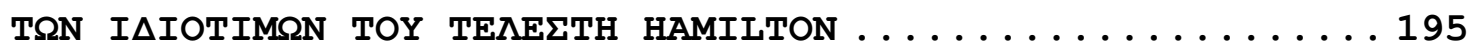

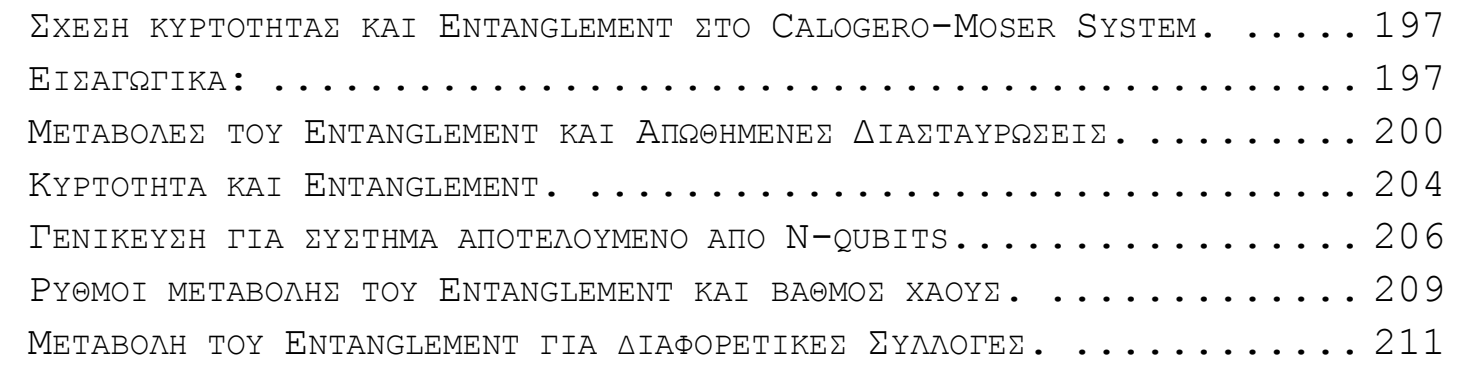




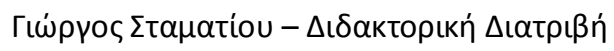

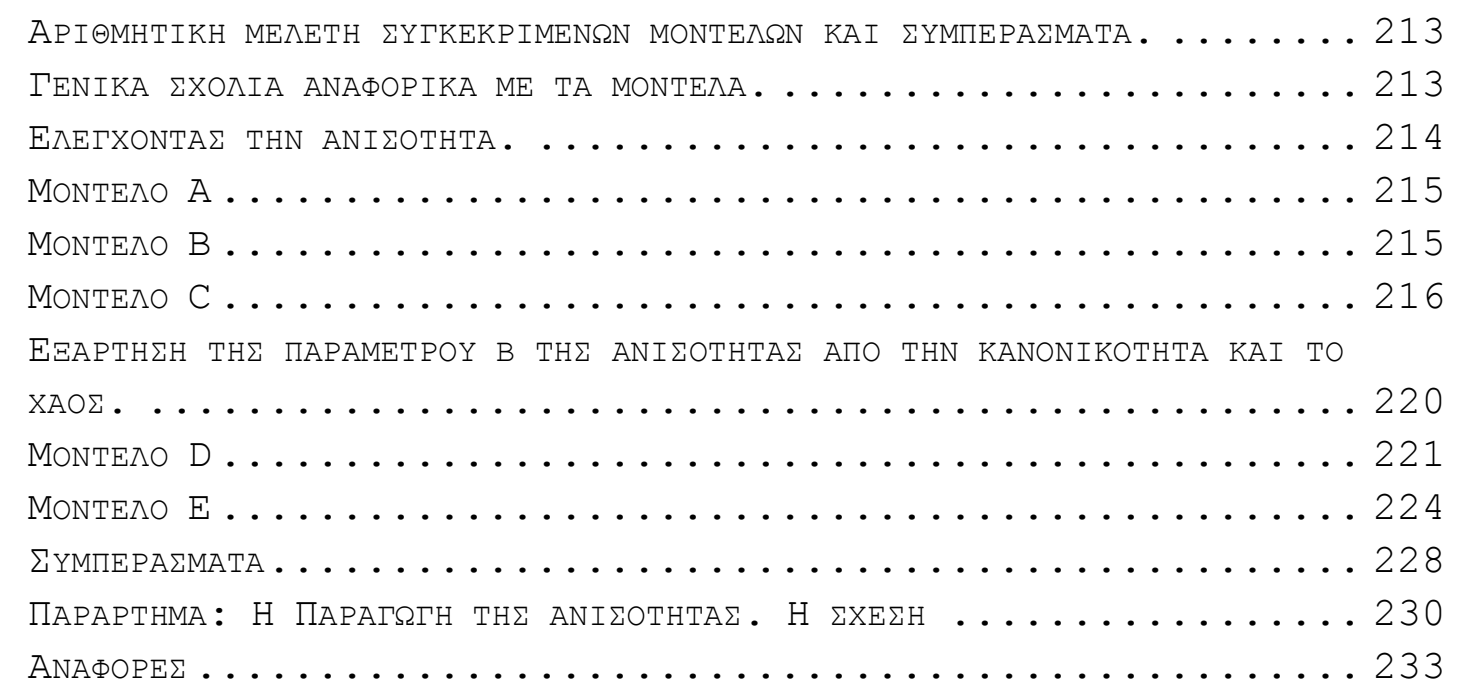

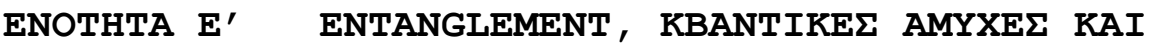

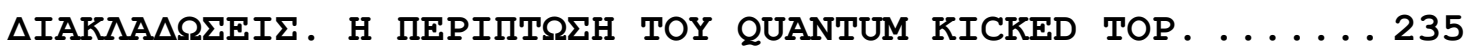

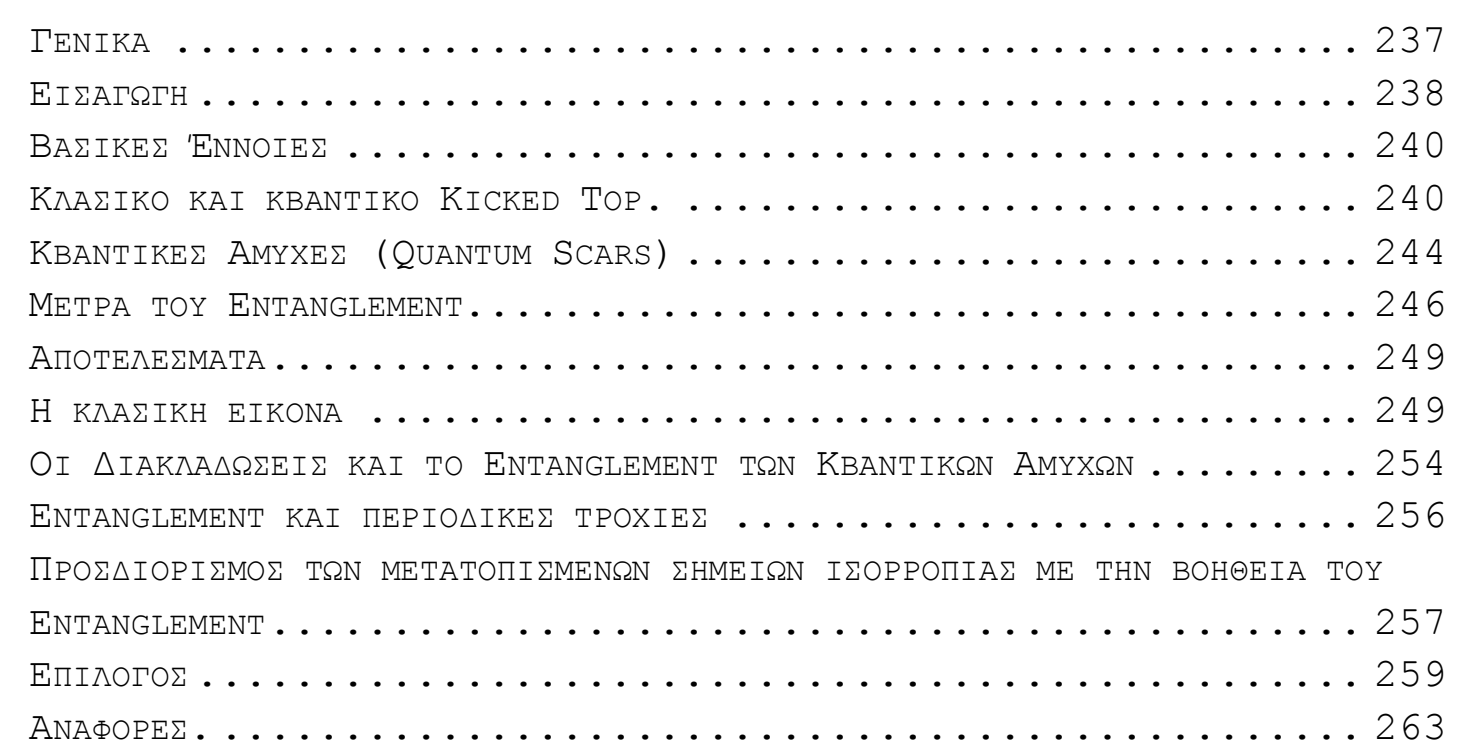

ENOTHTA $\Sigma T^{\prime}$ @EPMIKO ENTANGLEMENT $\Sigma$ E TETPATSNIKE

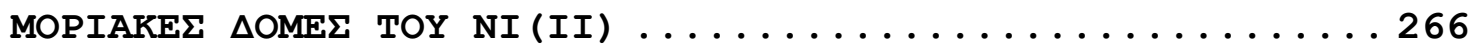

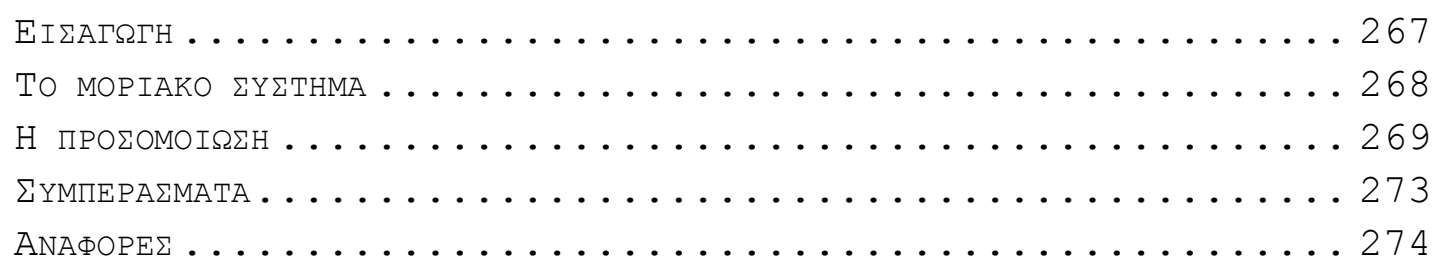




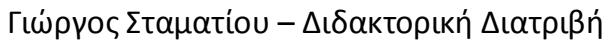

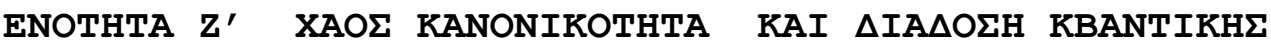

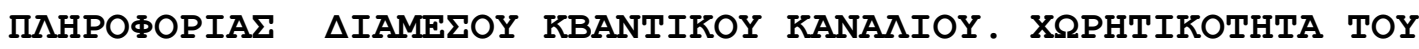

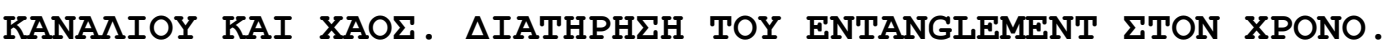

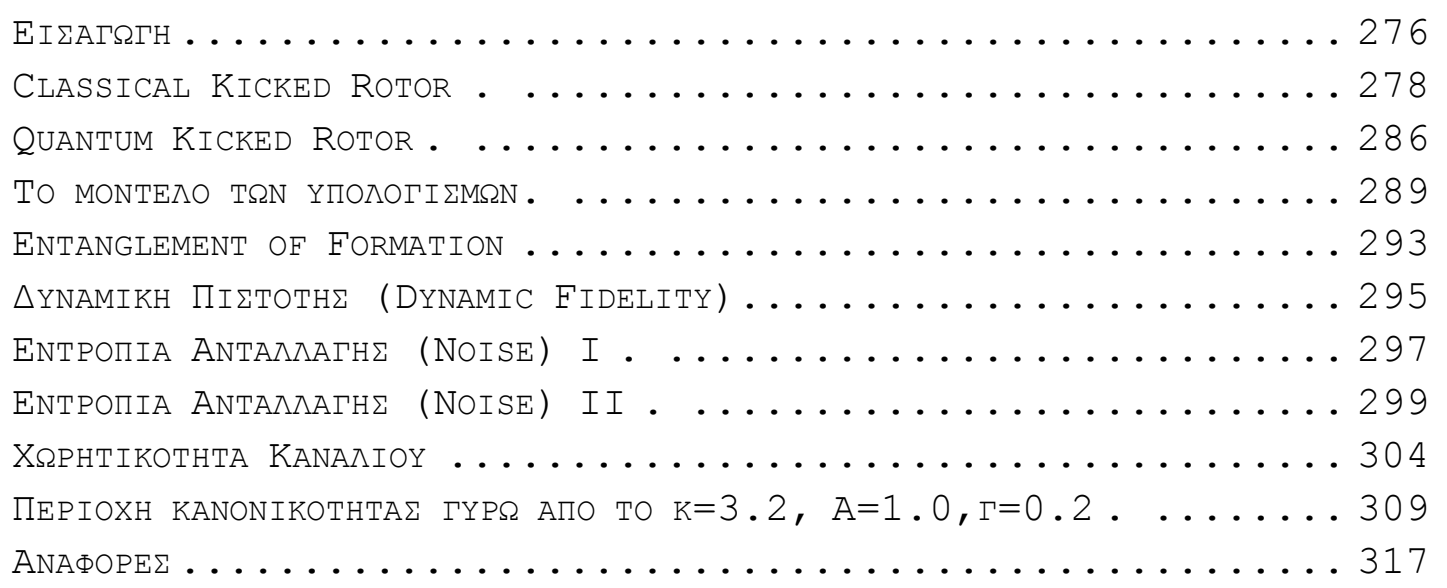

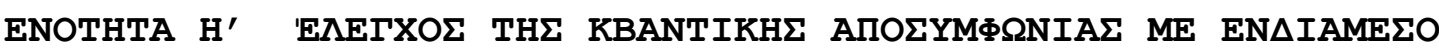

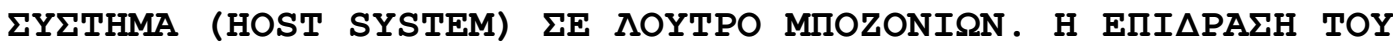

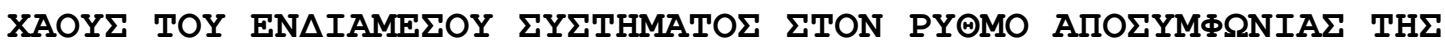

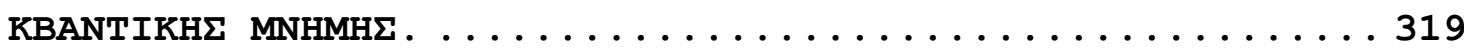

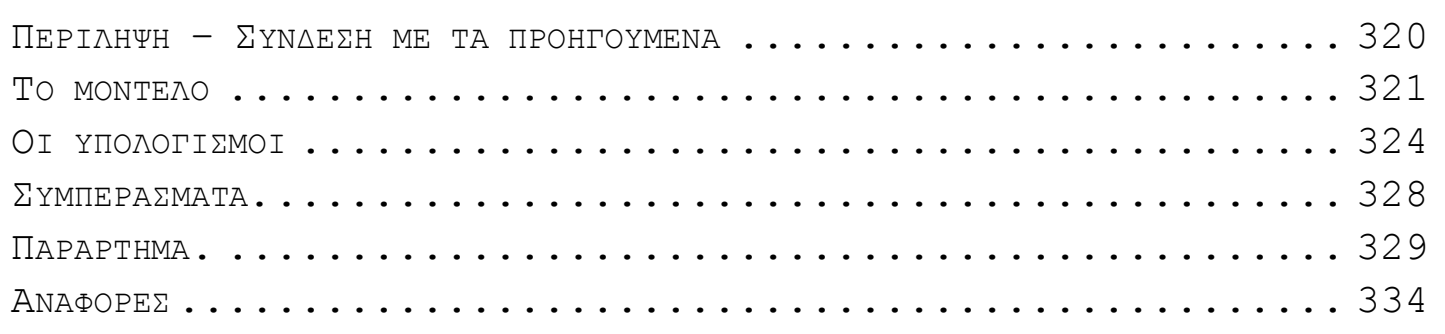

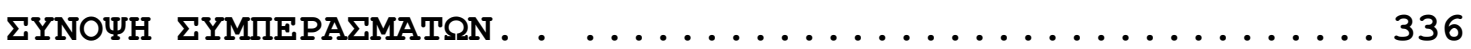

ПАРАРТНМА: ФЕМАТІКН ВІВАIОГРАФІА..............П1-П6 


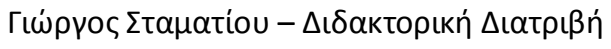

\section{ПРОЛОГОГ}





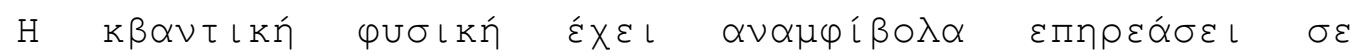

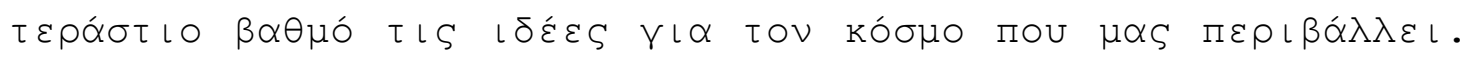

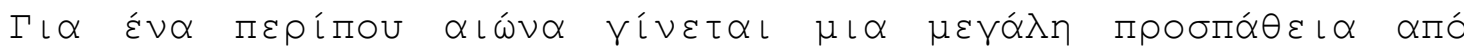

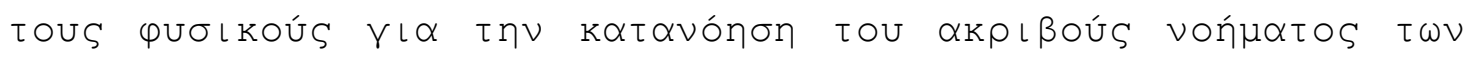

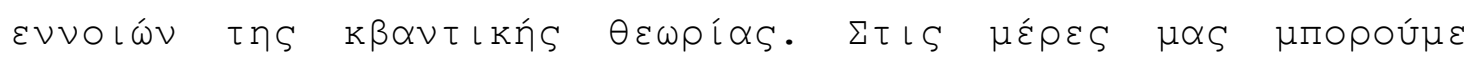

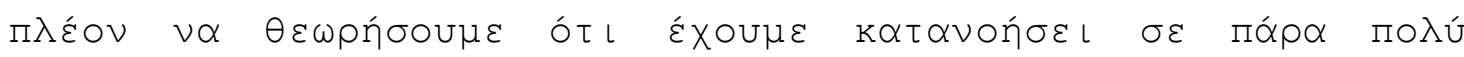

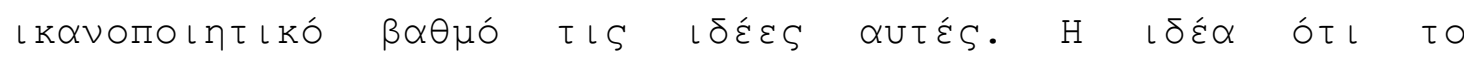

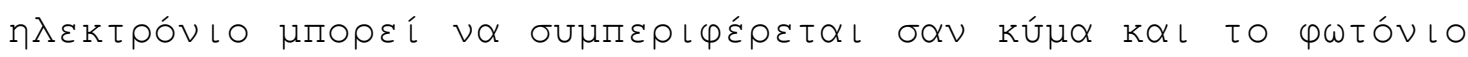

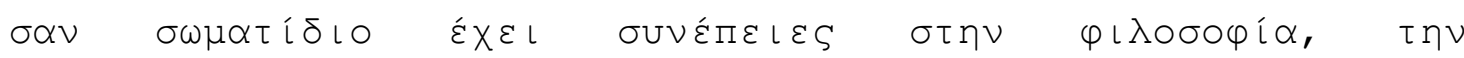

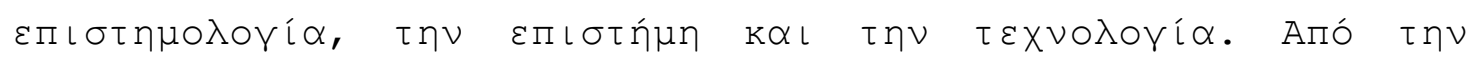

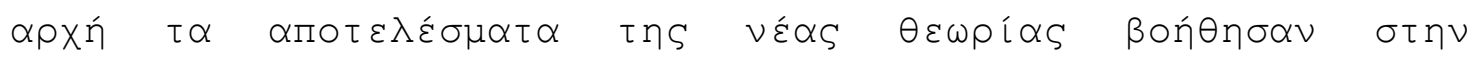

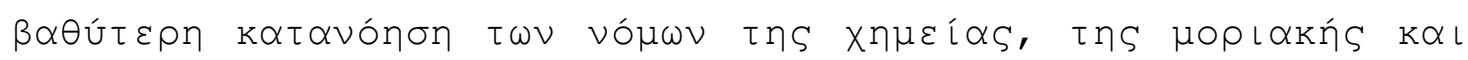

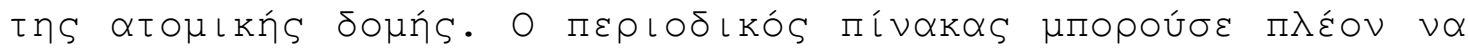

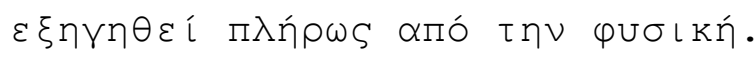

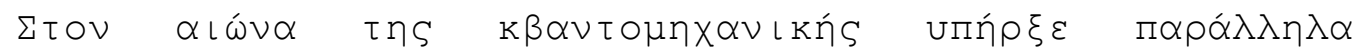

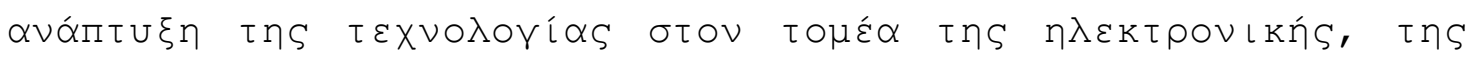

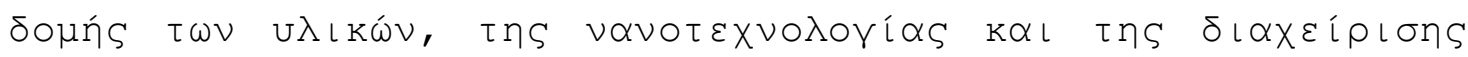

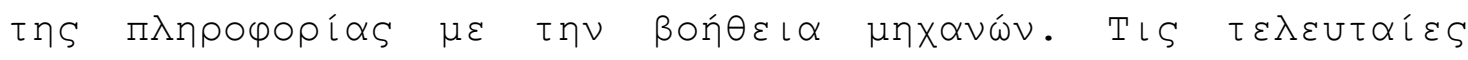

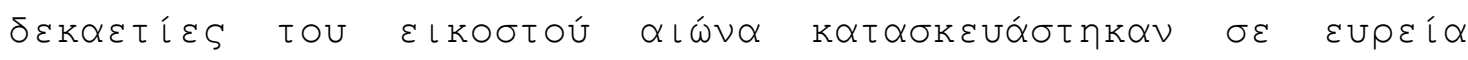

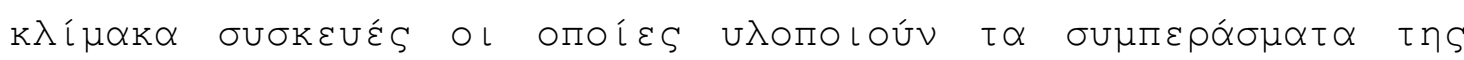

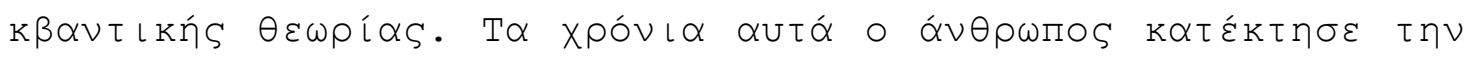

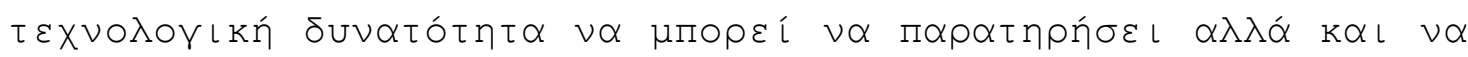

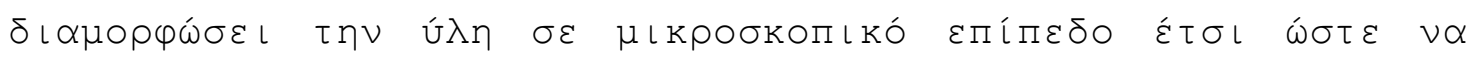

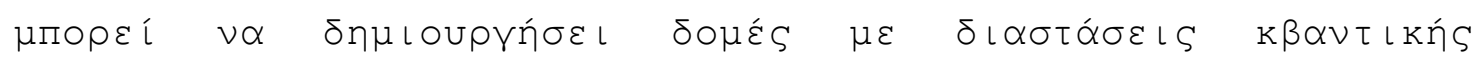

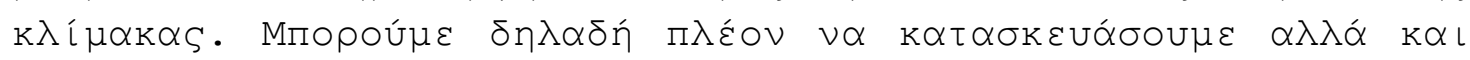

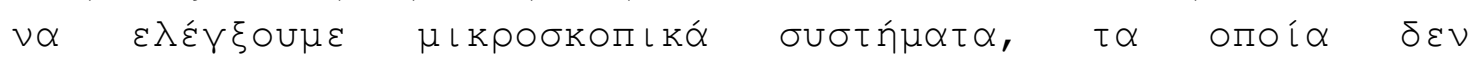

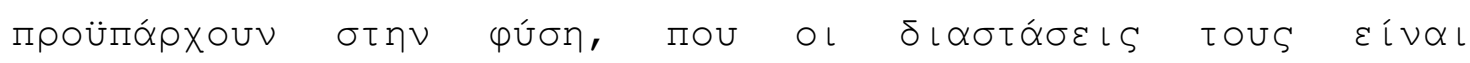

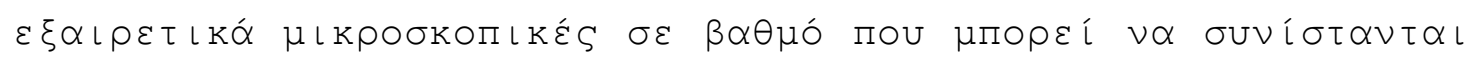

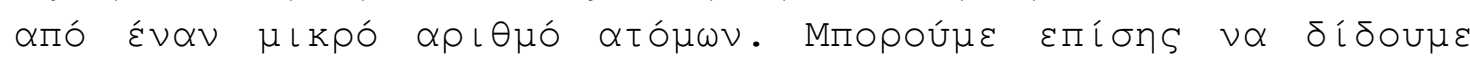

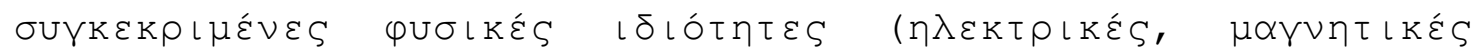

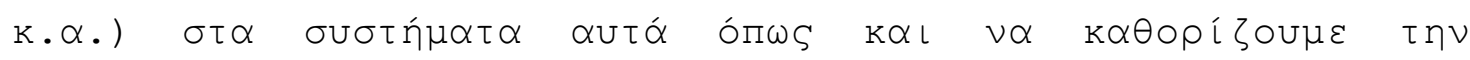

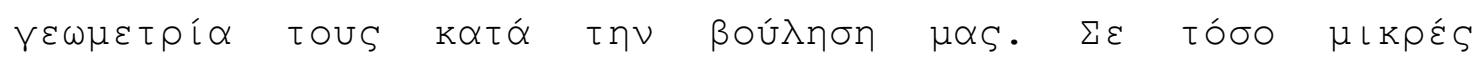

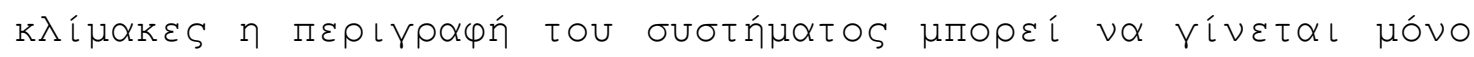

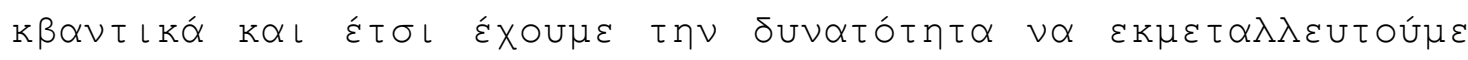

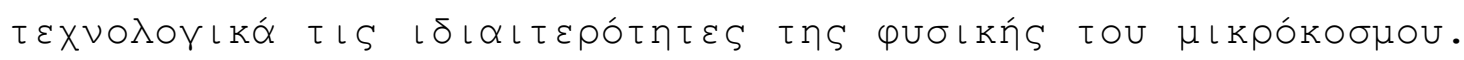

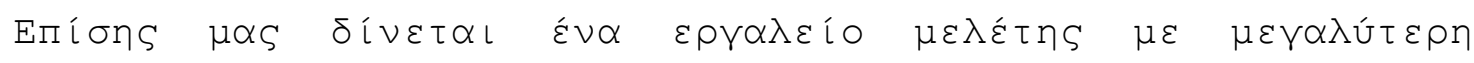

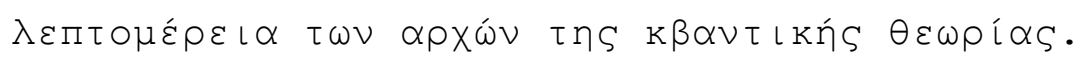

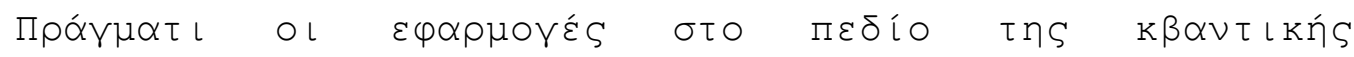

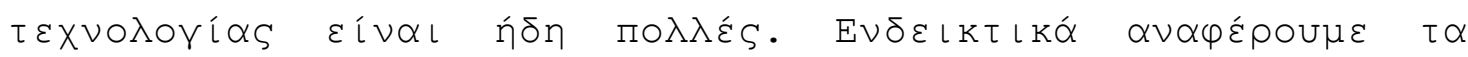

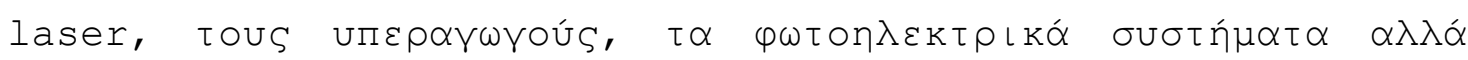




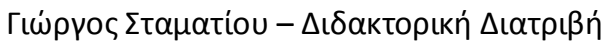

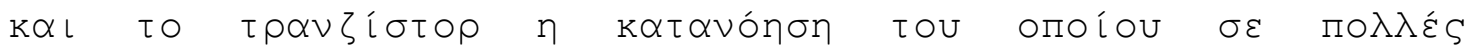

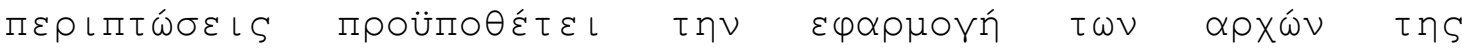

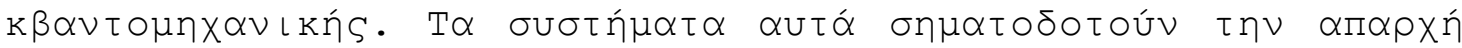

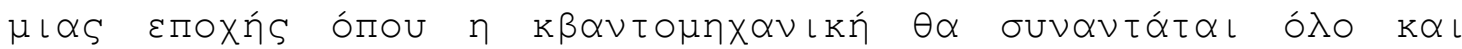

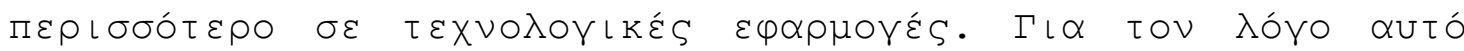

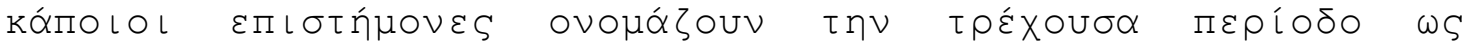

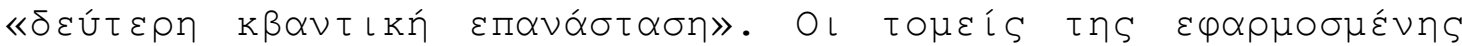

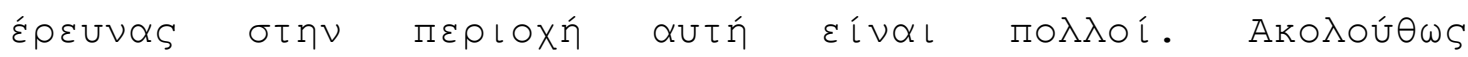

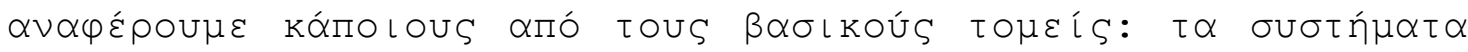

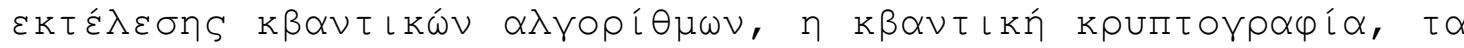

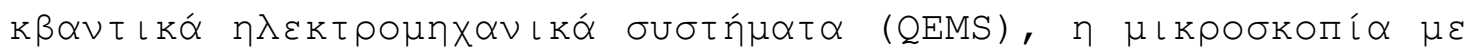

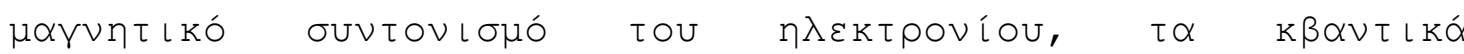

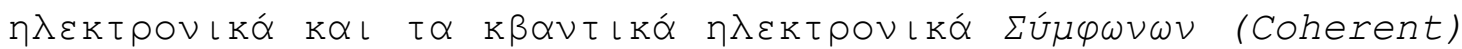

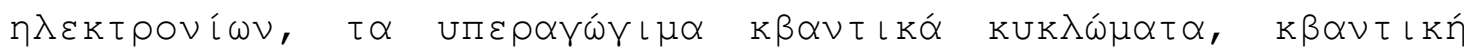

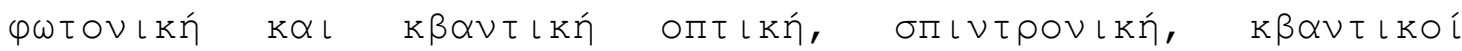

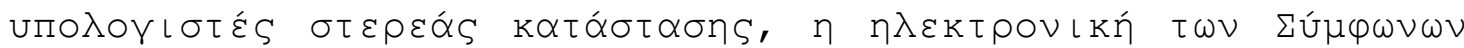

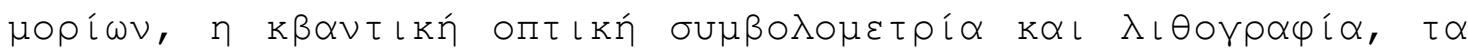

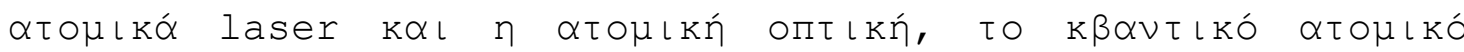
$\beta \alpha \rho u \tau o ́ \mu \varepsilon \tau \rho o$.

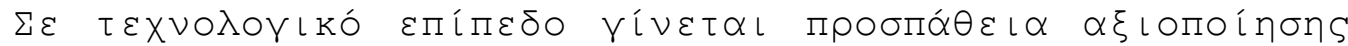

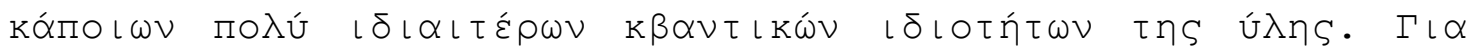

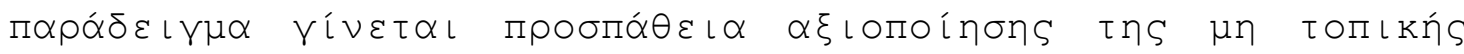

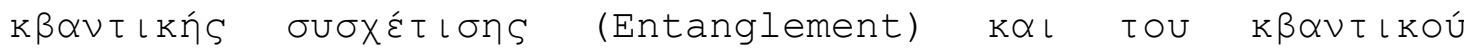

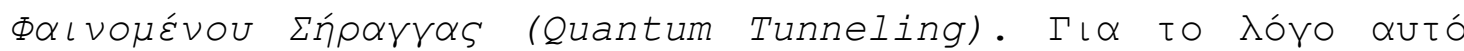

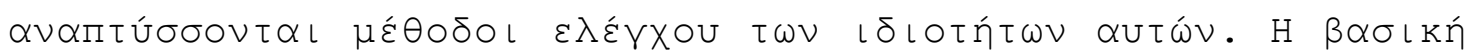

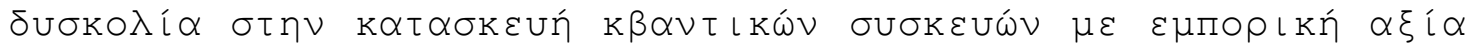

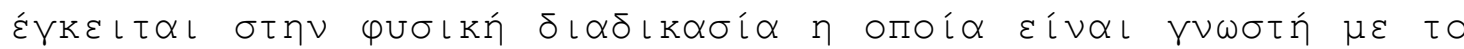

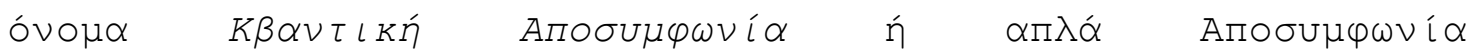

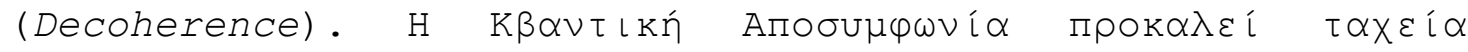

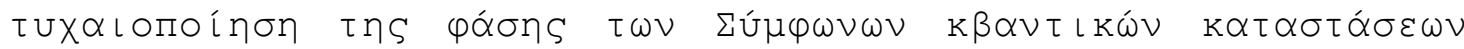

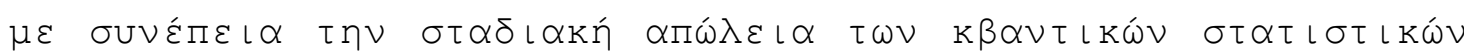

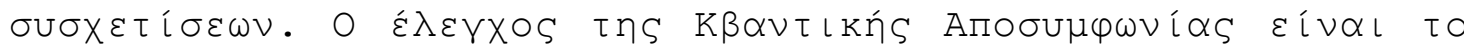

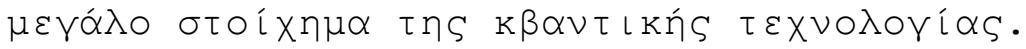

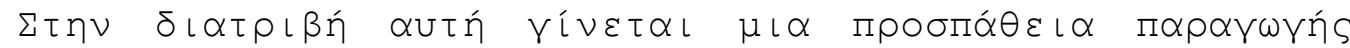

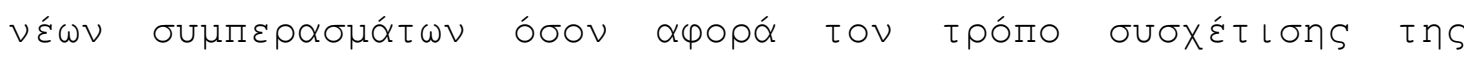

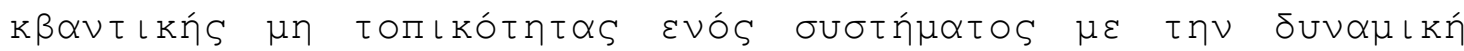

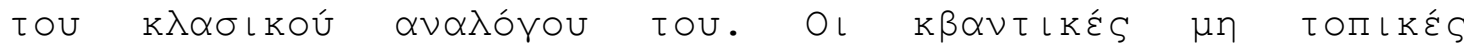

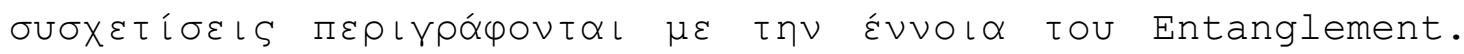

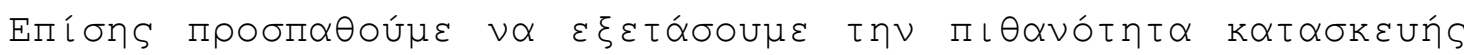

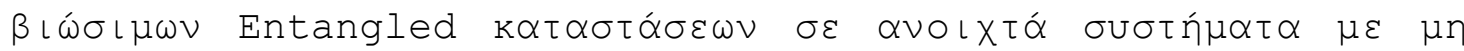




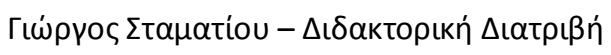

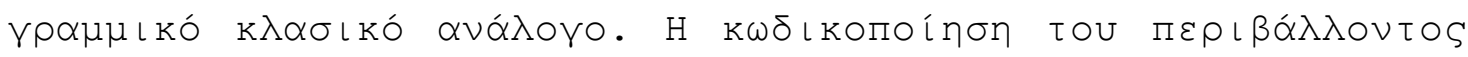

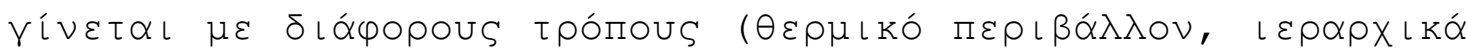

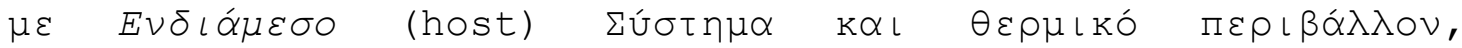

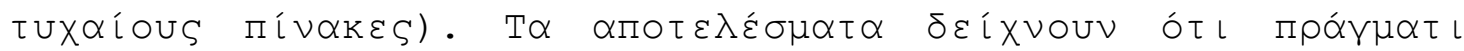

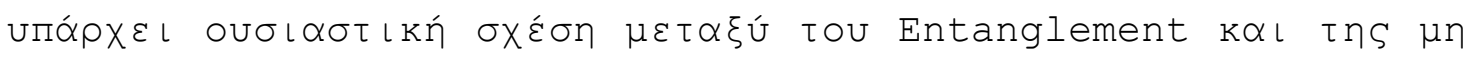

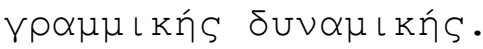

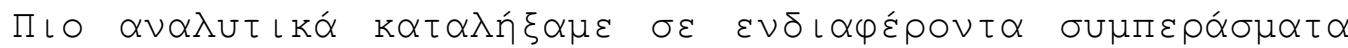
$\sigma \tau \iota \varsigma \quad \varepsilon \xi \bar{\zeta} \varsigma \quad \Pi \varepsilon \rho \iota \Pi \tau \omega ́ \sigma \varepsilon\llcorner\varsigma:$

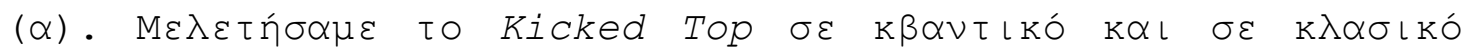

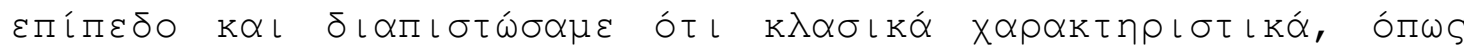

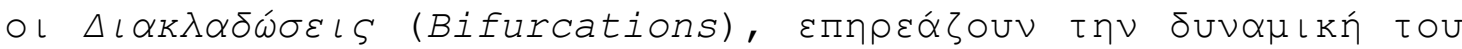
Entanglement.

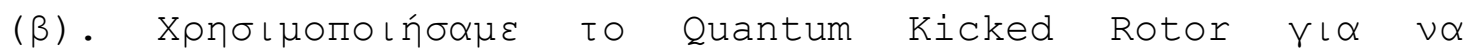

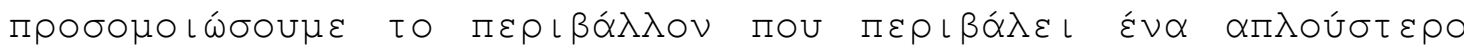

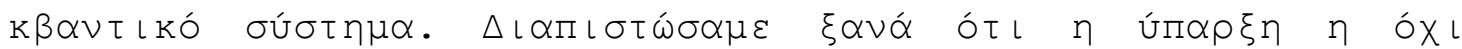

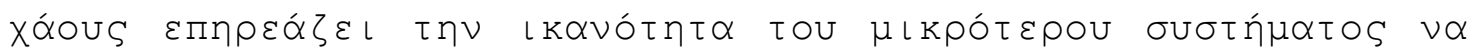

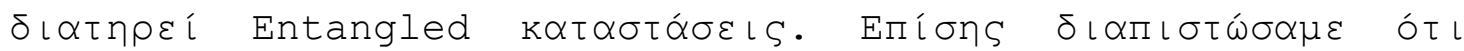

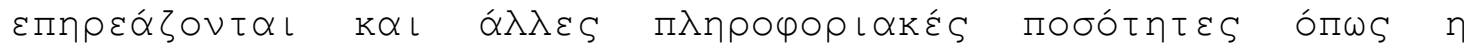

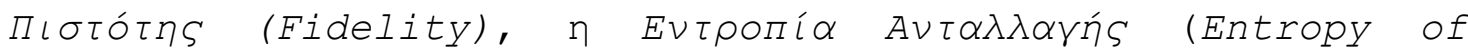

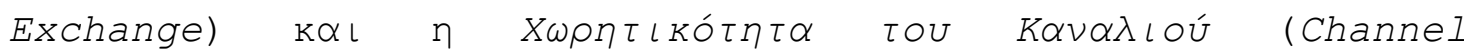
Capacity) .

(

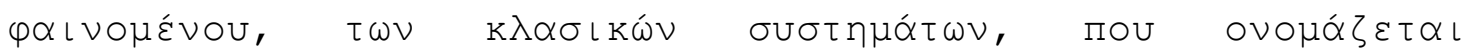

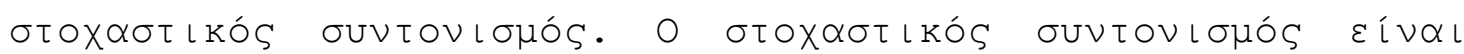

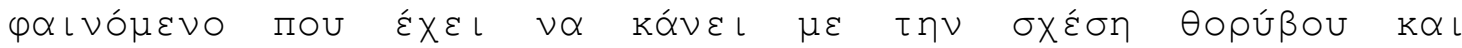

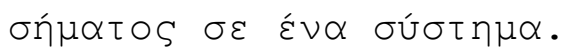

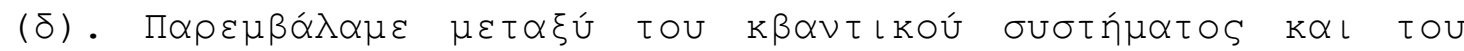

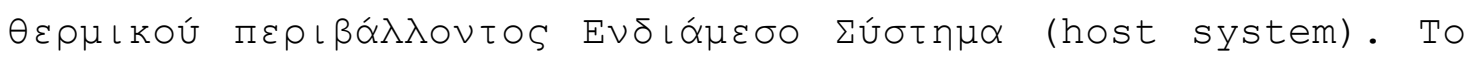

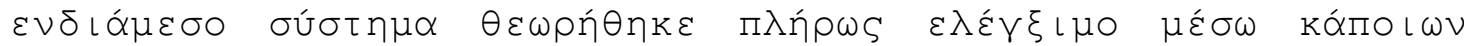

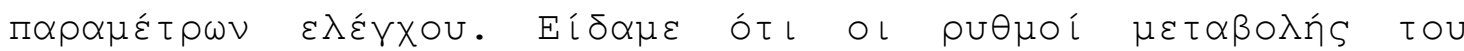

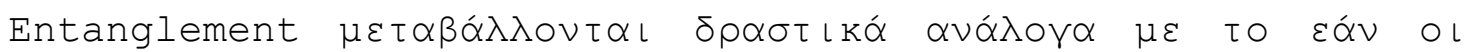

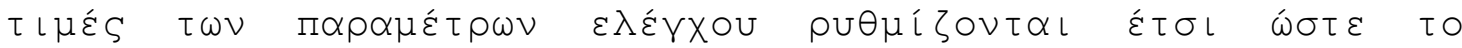

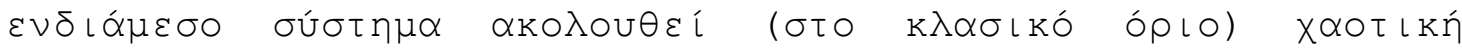

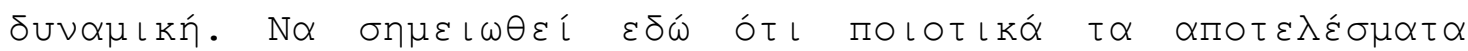

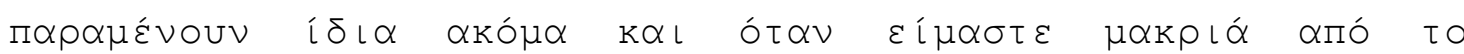

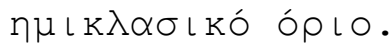




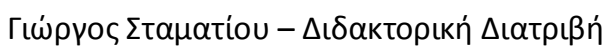

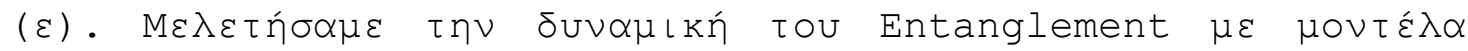

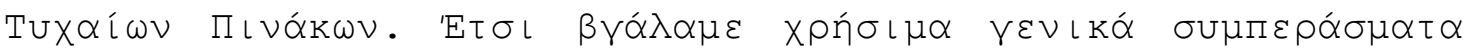

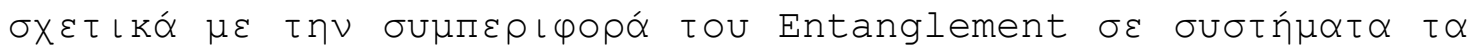

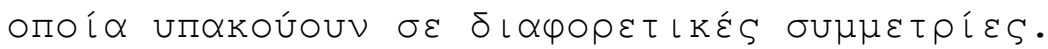

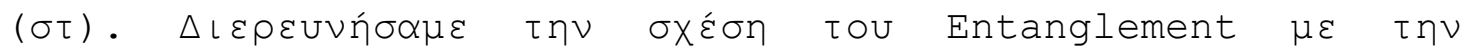

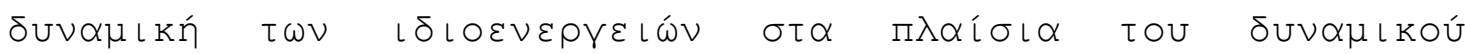

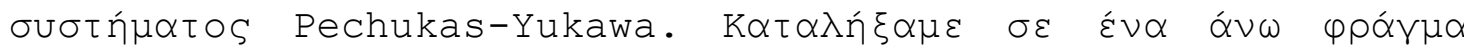

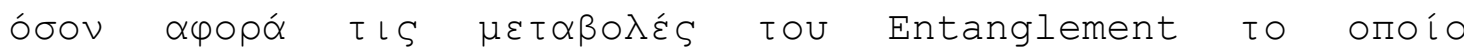

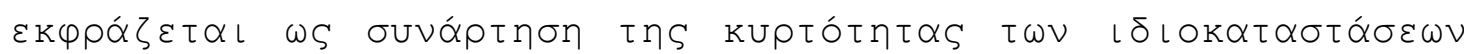

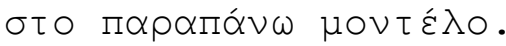

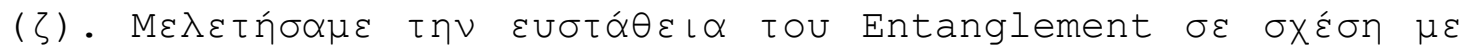

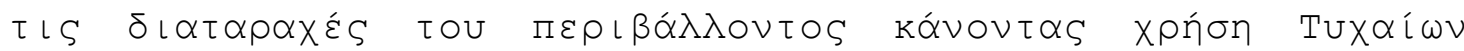

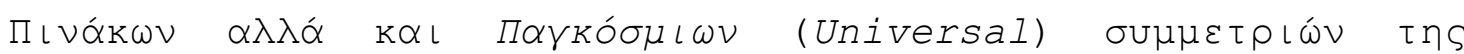

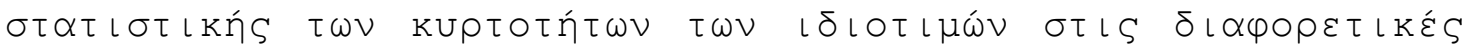

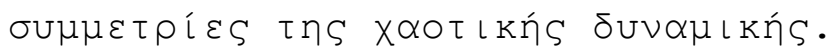

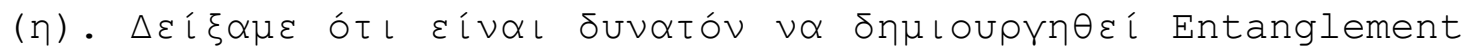

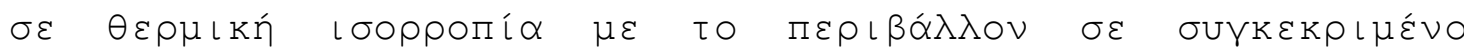

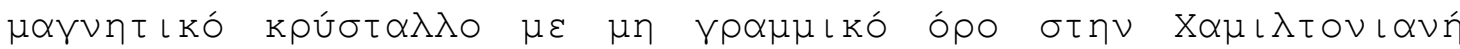

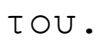

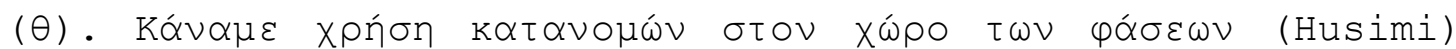

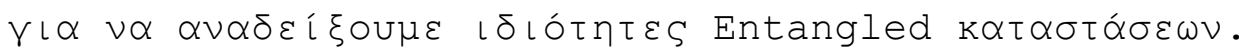

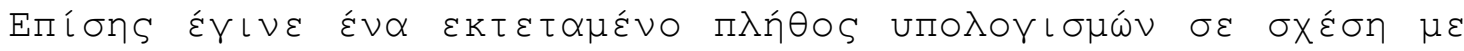

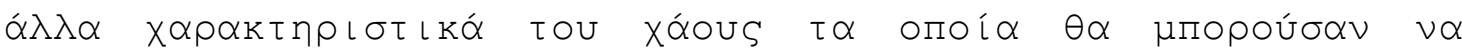

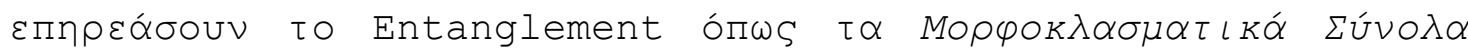

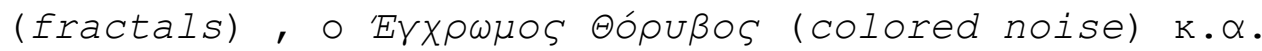

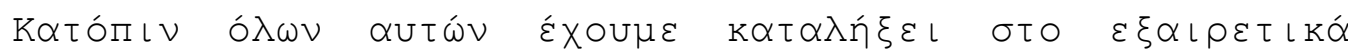

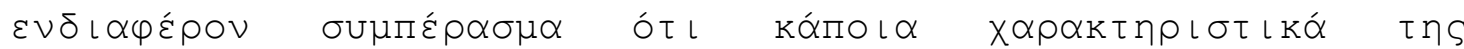

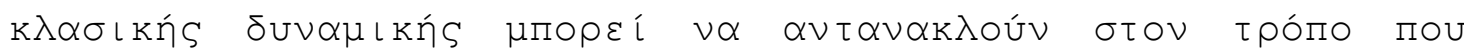

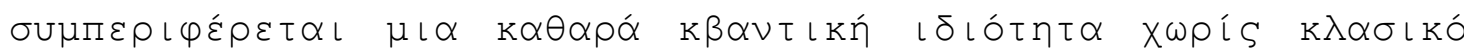

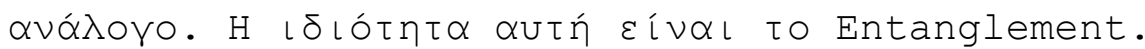

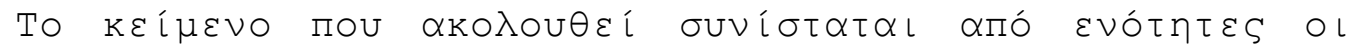

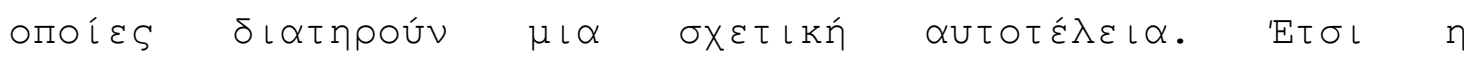

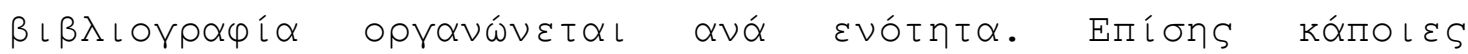

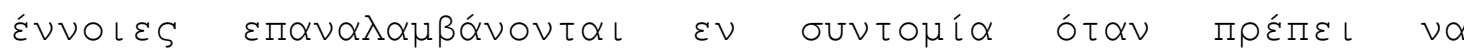

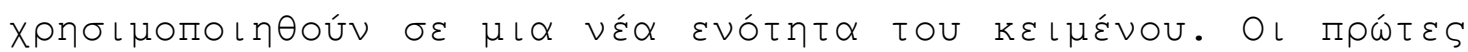

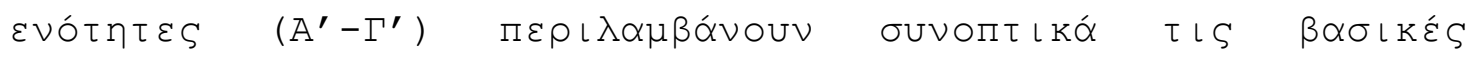




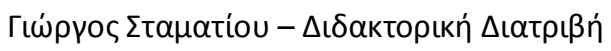

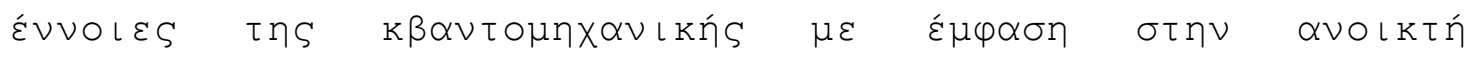

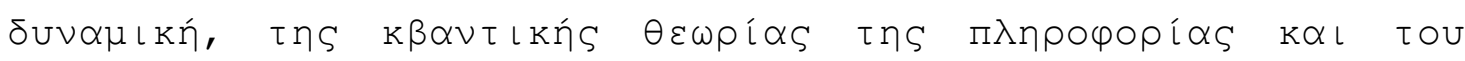

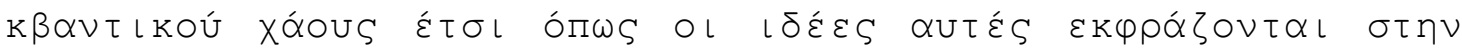

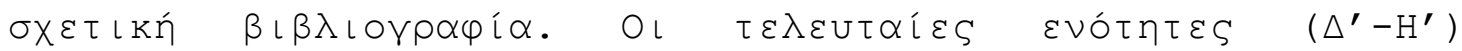

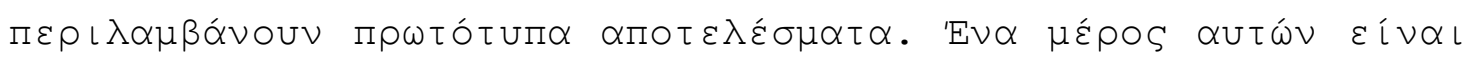

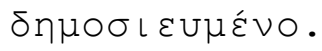





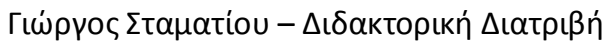

ENOTHTA $A^{\prime}$

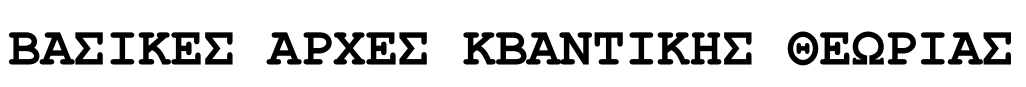

KAI

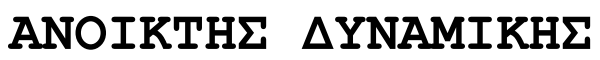





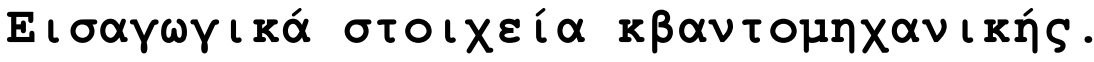

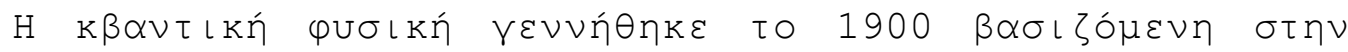

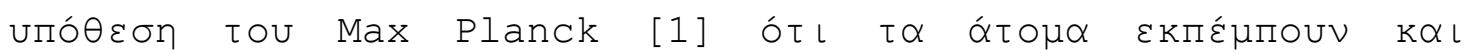

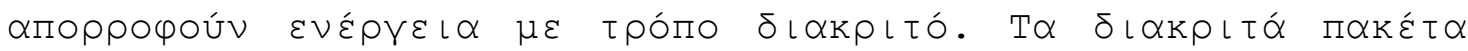

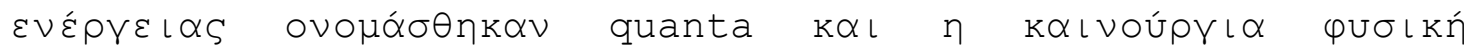

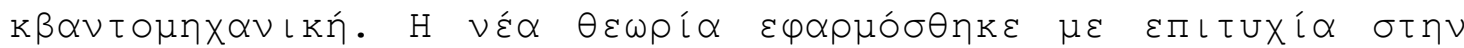

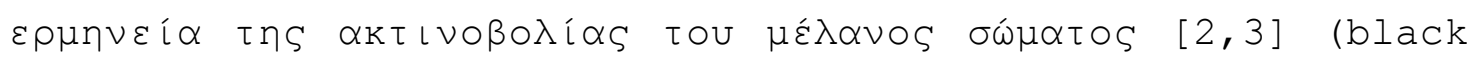

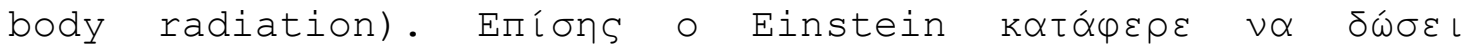

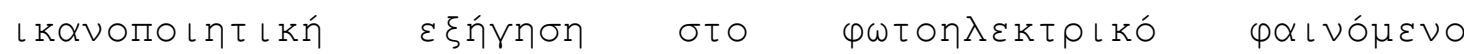

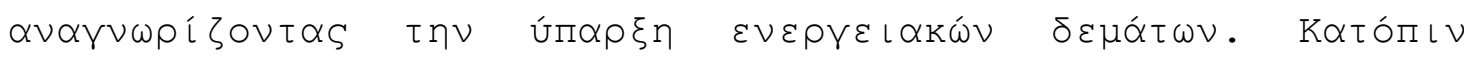

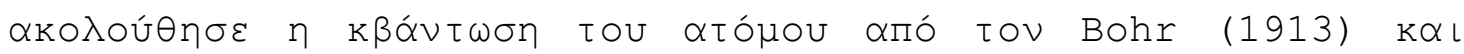

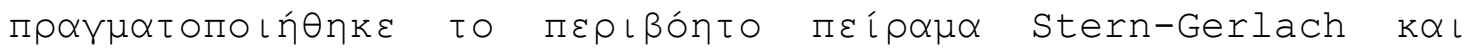

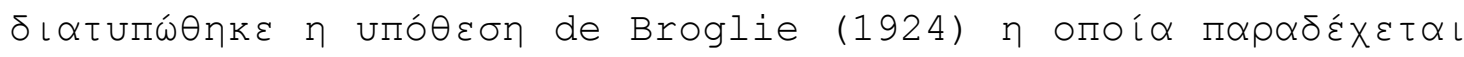

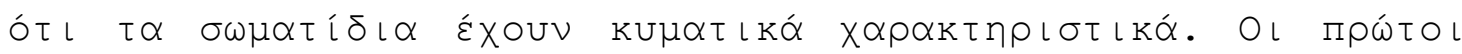

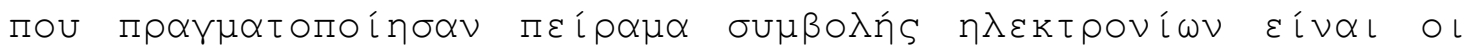

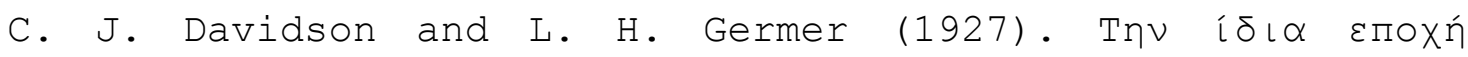

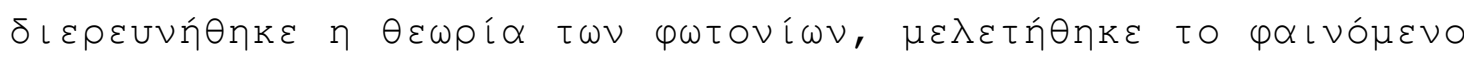

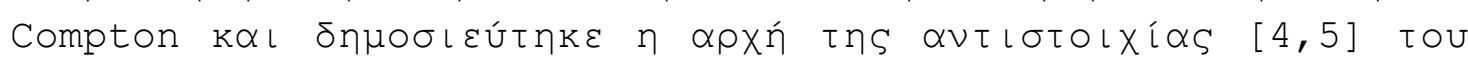

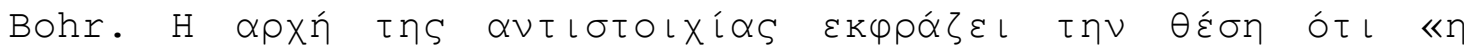

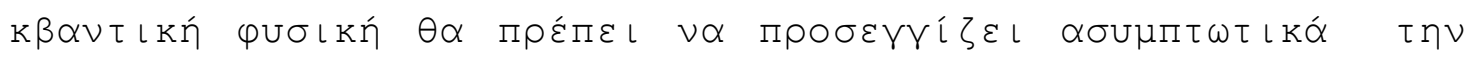

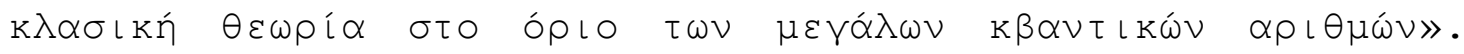

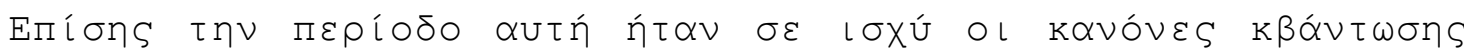

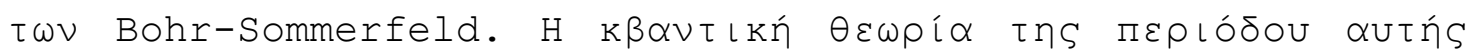

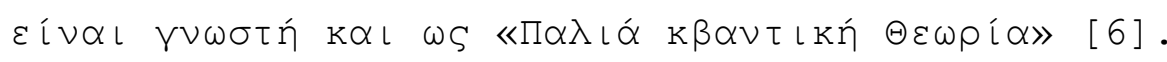

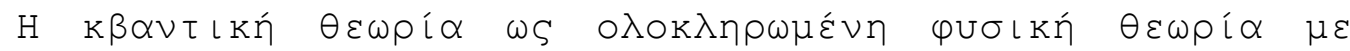

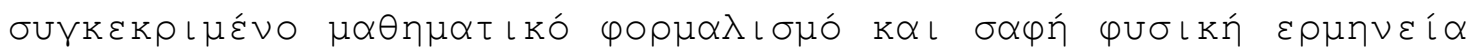

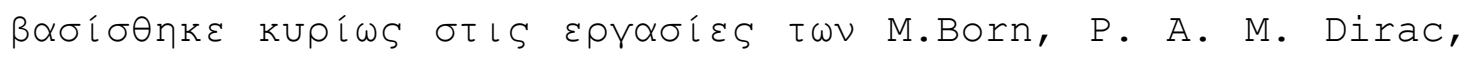
P. Jordan, W. Pauli, E. Schrodinger $k \alpha l$ W. Heisenberg. Ol

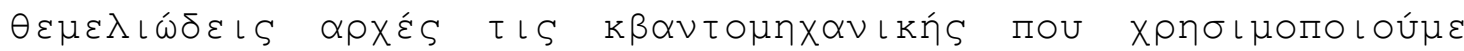

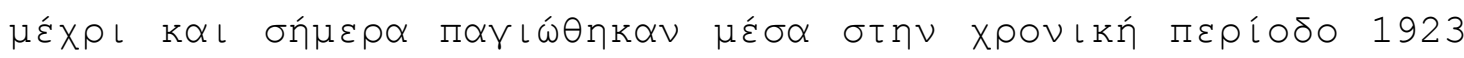

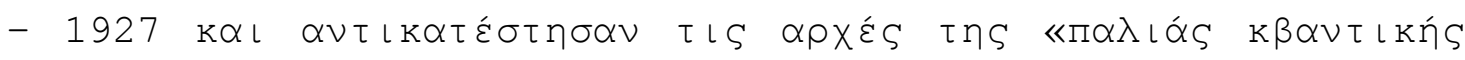

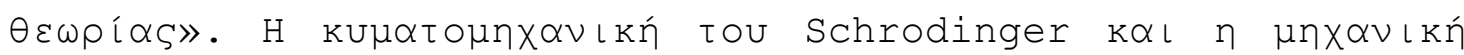

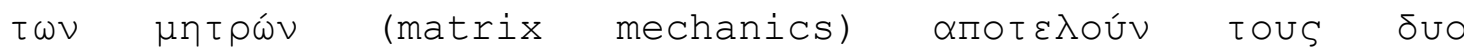

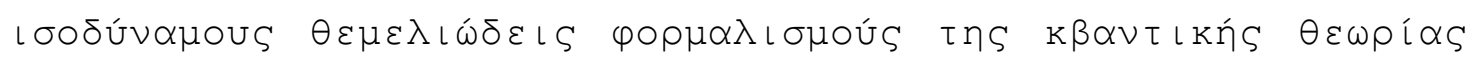

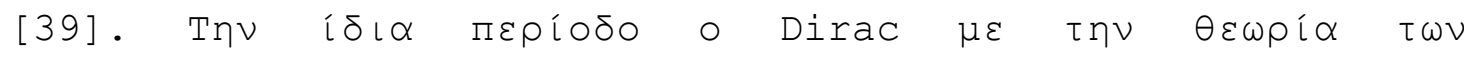

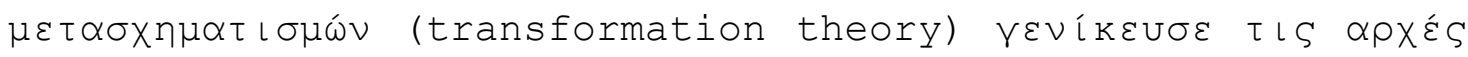

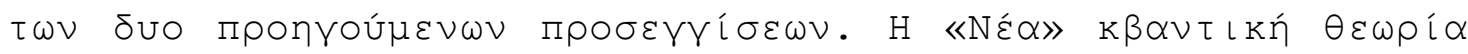

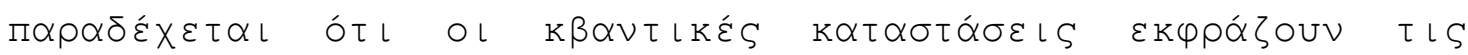

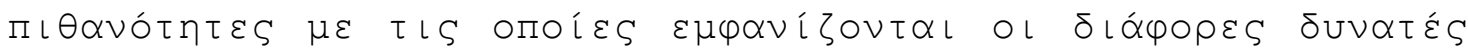




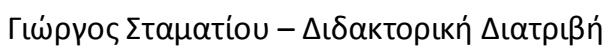

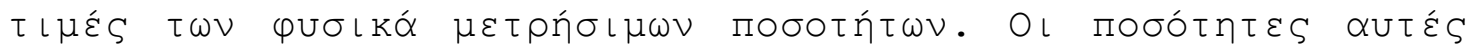

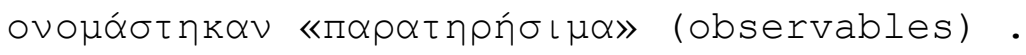

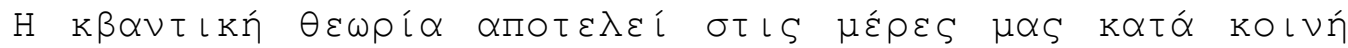

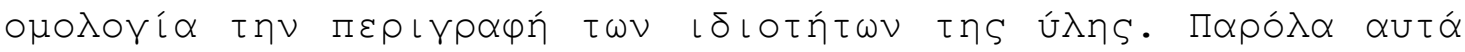

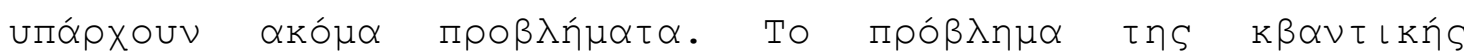

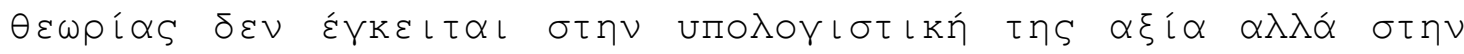

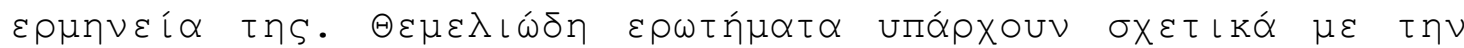

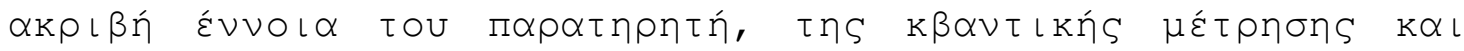

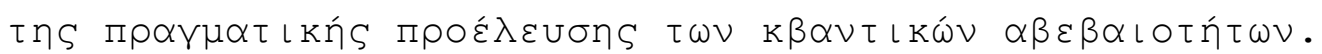

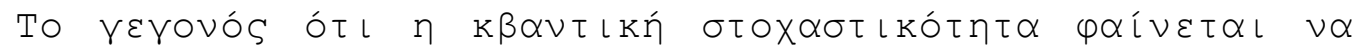

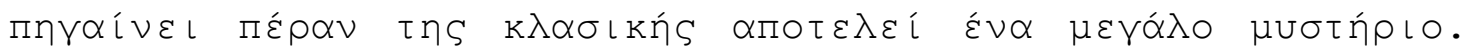

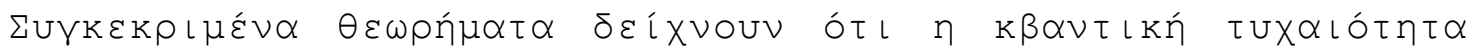

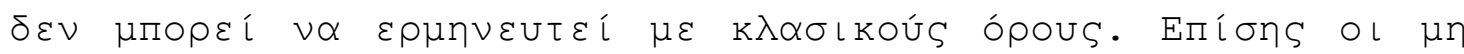

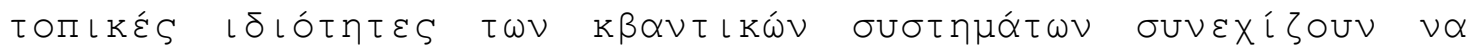

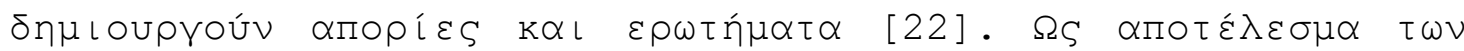

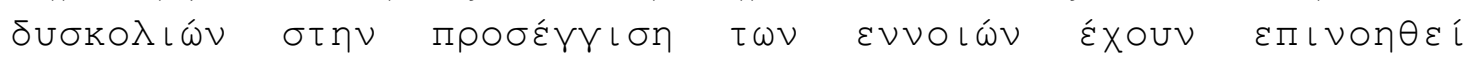

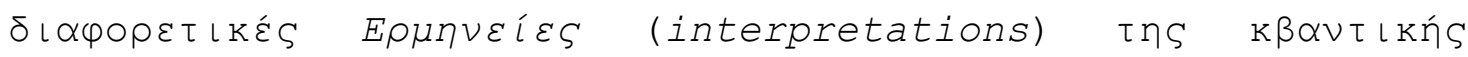

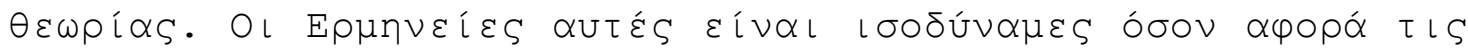

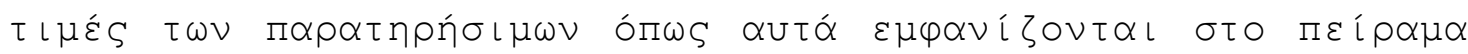

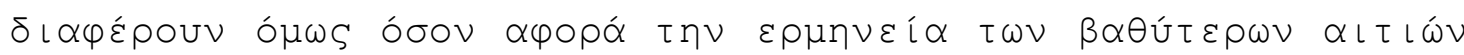

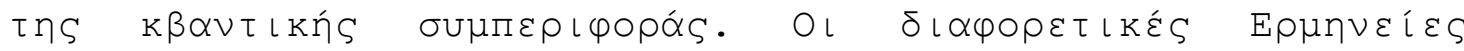

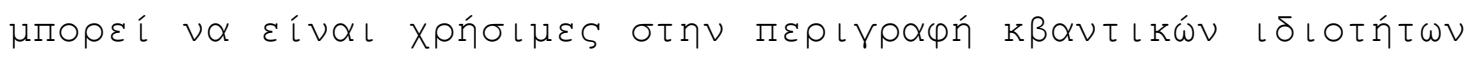

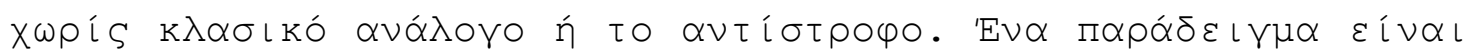

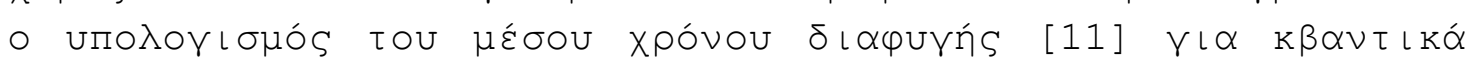

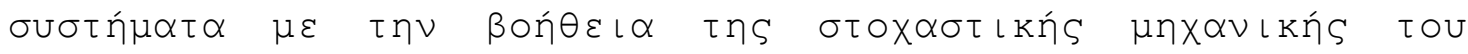

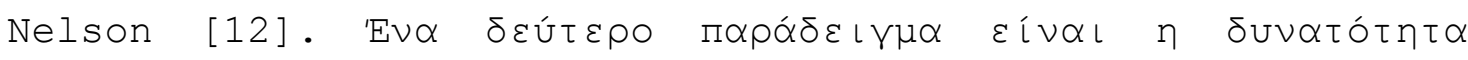

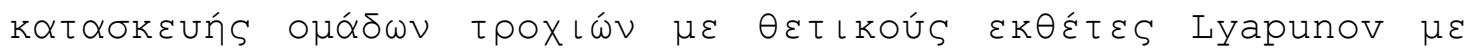

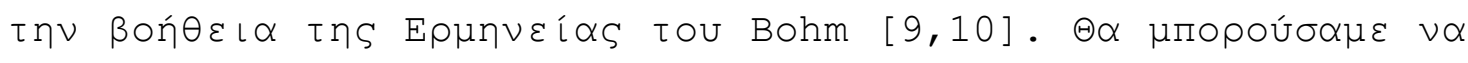

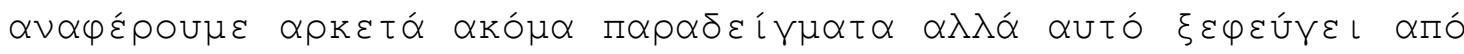

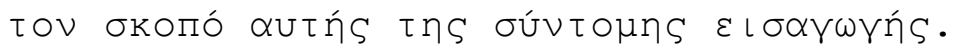

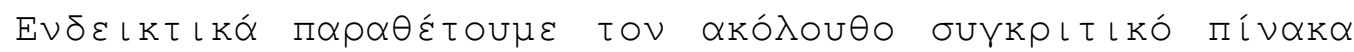

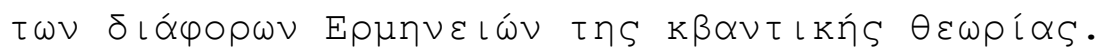




\begin{tabular}{|c|c|c|c|c|c|c|}
\hline Interpretation & Deterministic? & $\begin{array}{l}\text { Waveform } \\
\text { Real? }\end{array}$ & $\begin{array}{l}\text { Unique } \\
\text { History? }\end{array}$ & $\begin{array}{l}\text { Hidden } \\
\text { Variables? }\end{array}$ & $\begin{array}{l}\text { Collapsing } \\
\text { Wavefunctions? }\end{array}$ & $\begin{array}{l}\text { Observer } \\
\text { role? }\end{array}$ \\
\hline $\begin{array}{l}\text { Copenhagen } \\
\text { interpretation } \\
\text { (Waveform not } \\
\text { real) }\end{array}$ & No & No & Yes & No & NA & NA \\
\hline $\begin{array}{l}\text { Ensemble } \\
\text { interpretation } \\
\text { (Waveform not } \\
\text { real) }\end{array}$ & No & No & Yes & Agnostic & No & None \\
\hline $\begin{array}{l}\text { Copenhagen } \\
\text { interpretation } \\
\text { (Waveform real) } \\
\text { Objective } \\
\text { collapse theories }\end{array}$ & No & Yes & Yes & No & Yes & None \\
\hline $\begin{array}{l}\text { Consistent } \\
\text { histories } \\
\text { (Decoherent } \\
\text { approach) }\end{array}$ & Agnostic $^{1}$ & Agnostic $^{1}$ & No & No & No & $\mid$ Interpretational $^{2}$ \\
\hline Quantum logic & Agnostic & Agnostic & Yes $^{3}$ & No & No & Interpretational $^{2}$ \\
\hline $\begin{array}{l}\text { Many-worlds } \\
\text { interpretation } \\
\text { (Decoherent } \\
\text { approach) }\end{array}$ & Yes & Yes & No & No & No & |None \\
\hline $\begin{array}{l}\text { Stochastic } \\
\text { mechanics }\end{array}$ & No & No & Yes & No & No & None \\
\hline $\begin{array}{l}\text { Many-minds } \\
\text { interpretation }\end{array}$ & |Yes & Yes & No & No & No & Interpretational ${ }^{4}$ \\
\hline $\begin{array}{l}\text { Bohm-de Broglie } \\
\text { interpretation } \\
\text { ("Pilot-wave" } \\
\text { approach) }\end{array}$ & Yes & Yes $^{5}$ & Yes $^{6}$ & Yes & No & None \\
\hline $\begin{array}{l}\text { Transactional } \\
\text { interpretation }\end{array}$ & No & Yes & Yes & No & Yes $^{7}$ & None \\
\hline $\begin{array}{l}\text { Consciousness } \\
\text { causes collapse }\end{array}$ & No & Yes & Yes & No & Yes & |Causal \\
\hline $\begin{array}{l}\text { Relational } \\
\text { Quantum } \\
\text { Mechanics }\end{array}$ & No & Yes & Agnostic $^{8}$ & No & Yes $^{9}$ & None \\
\hline
\end{tabular}




\begin{tabular}{|l|l|l|l|l|l|l|}
\hline $\begin{array}{l}\text { Incomplete } \\
\text { measurements }\end{array}$ & No & No $^{10}$ & Yes & Yes & \\
\hline
\end{tabular}

\footnotetext{
${ }^{1}$ If wavefunction is real then this becomes the many-worlds interpretation. If wavefunction less than real, but more than just information, then Zurek calls this the "existential interpretation".

${ }^{2}$ Quantum mechanics is regarded as a way of predicting observations, or a theory of measurement..

${ }^{3}$ But quantum logic is more limited in applicability than Coherent Histories.

${ }^{4}$ Observers separate the universal wavefunction into orthogonal sets of experiences.

${ }^{5}$ Both particle AND guiding wavefunction are real.

${ }^{6}$ Unique particle history, but multiple wave histories.

${ }^{7}$ In the TI the collapse of the state vector is interpreted as the completion of the transaction between emitter and absorber.

${ }^{8}$ Comparing histories between systems in this interpretation has no well-defined meaning.

${ }^{9}$ Any physical interaction is treated as a collapse event relative to the systems involved, not just macroscopic or conscious observers.

${ }^{10}$ The nature and collapse of the wave function are derived, not axiomatic.
}

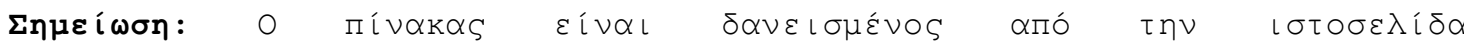
http://en.wikipedia.org/wiki/Interpretation_of_quantum_mechanics. $\Gamma\llcorner\alpha$

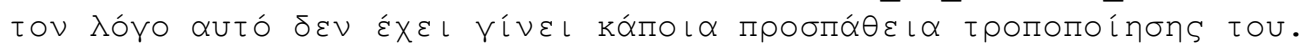

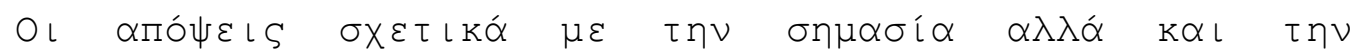

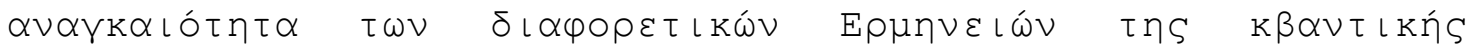

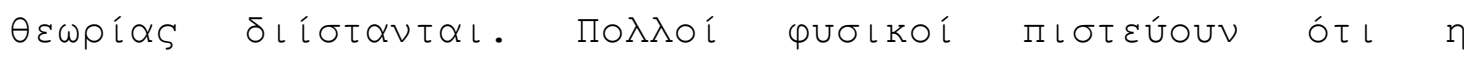

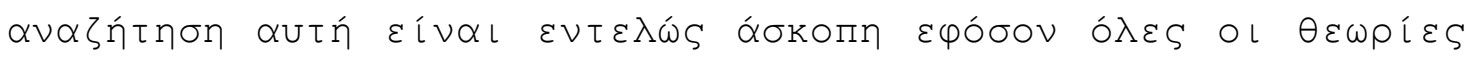

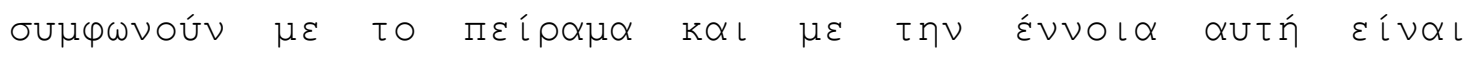

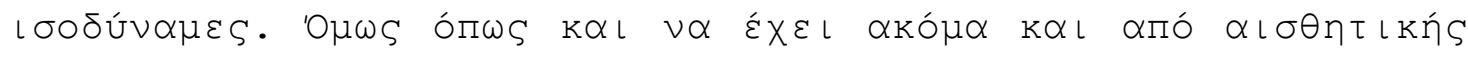

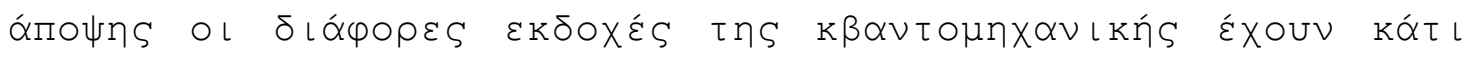

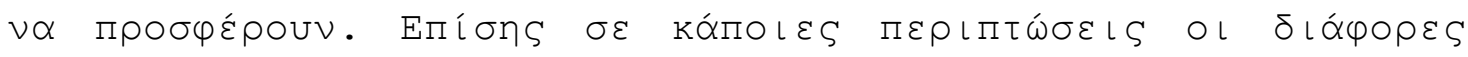

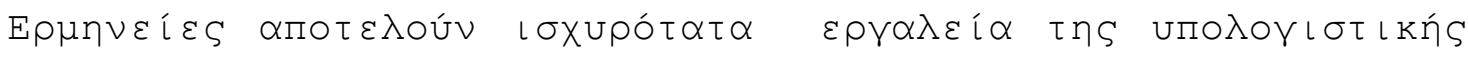

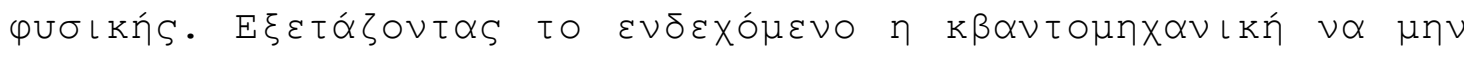

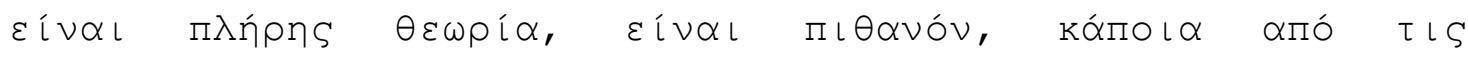

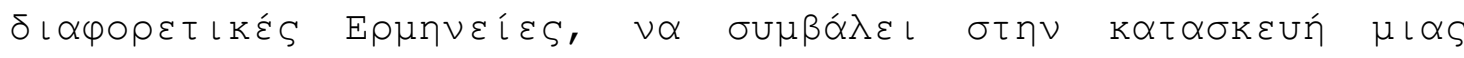

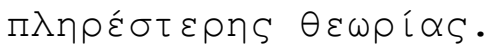




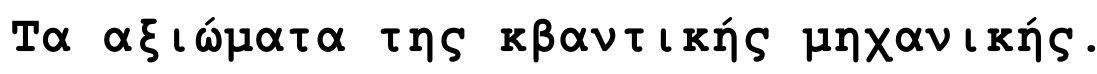

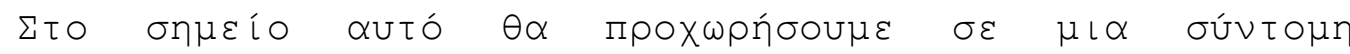

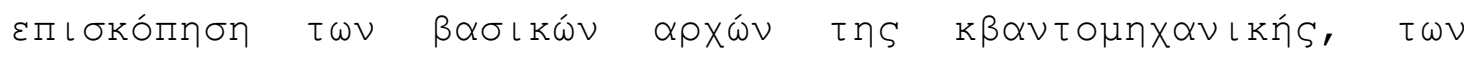

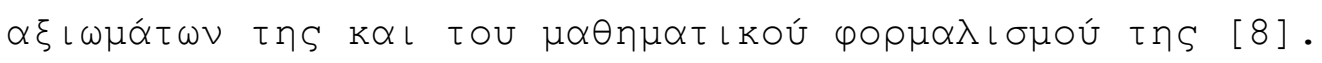

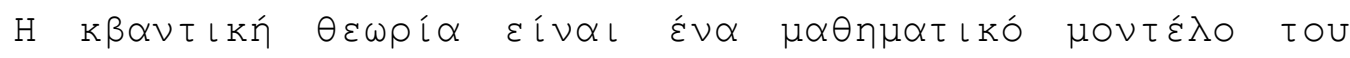

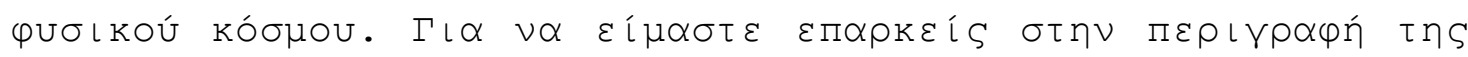

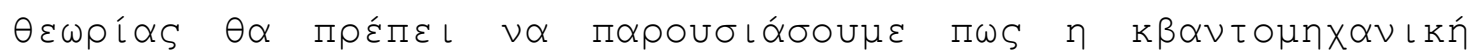

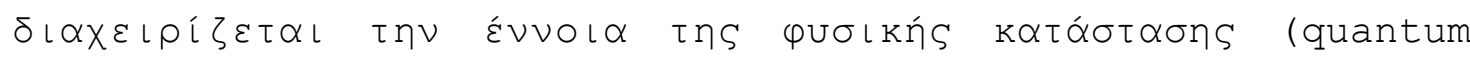

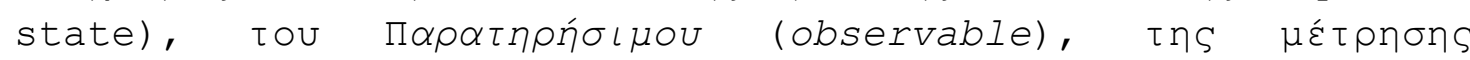

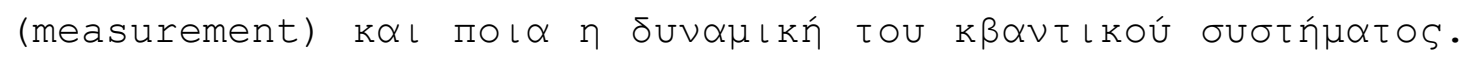

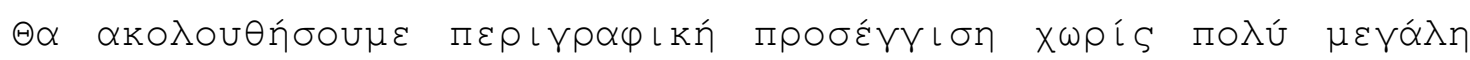

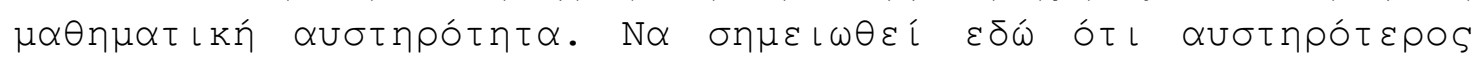

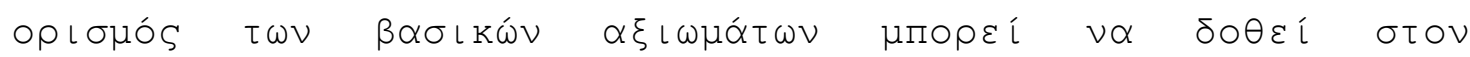

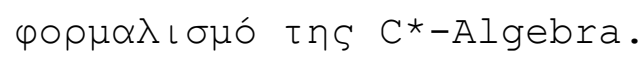

\section{A $\xi i \omega \mu \alpha$ 1:}

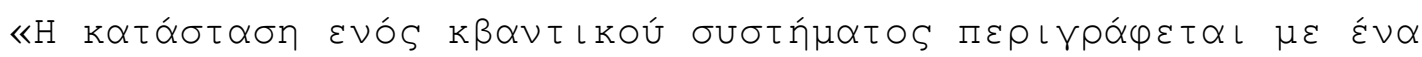

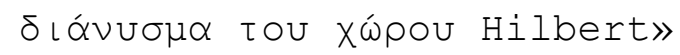

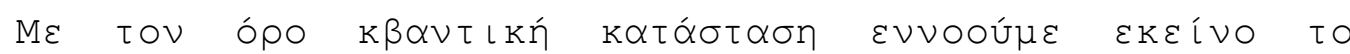

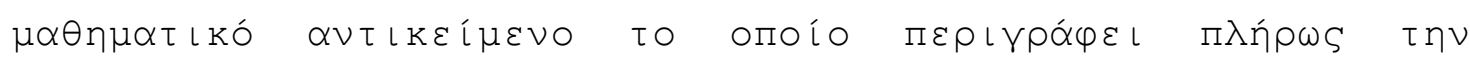

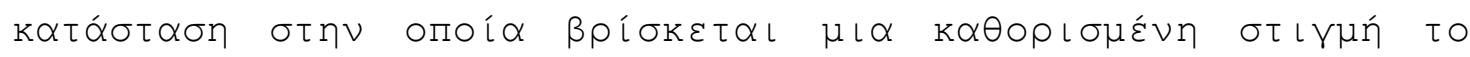

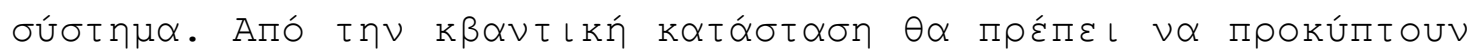

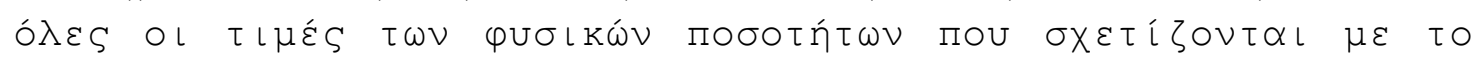

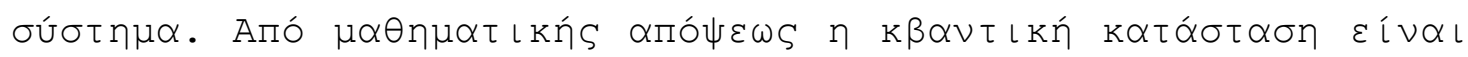

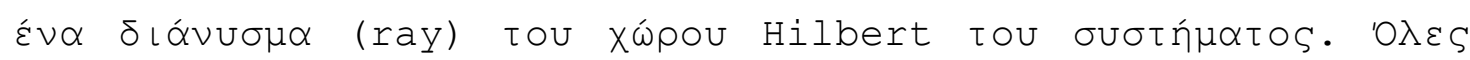
O

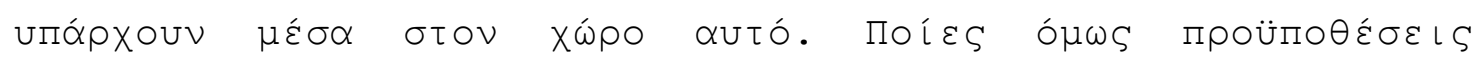

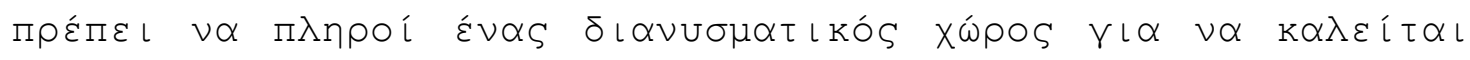

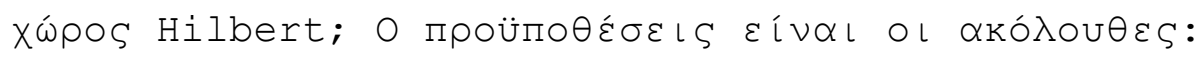

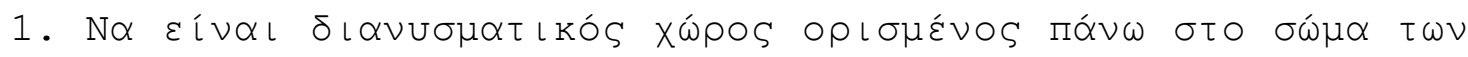

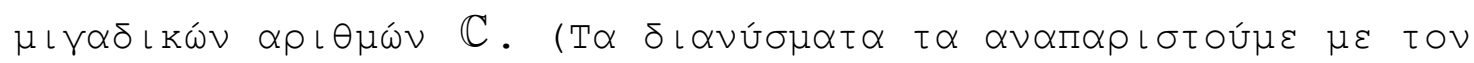

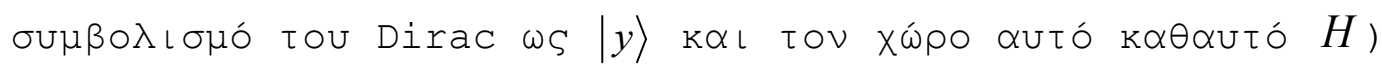

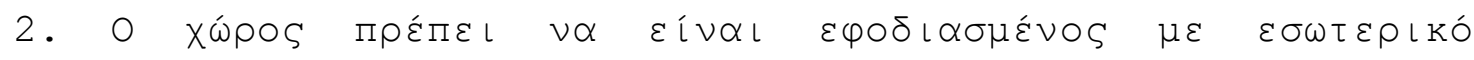

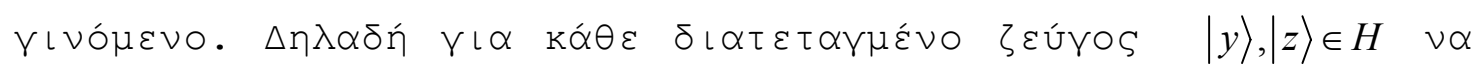




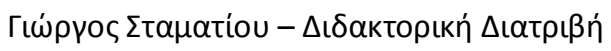

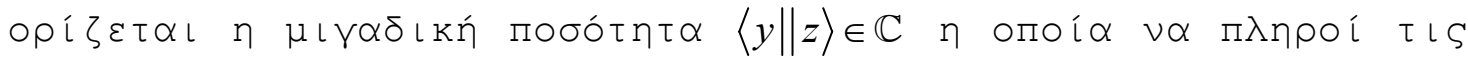

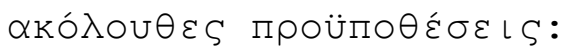

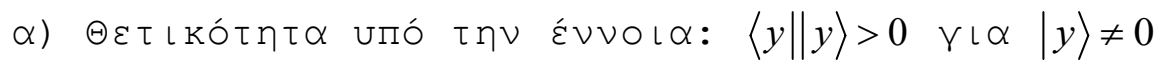

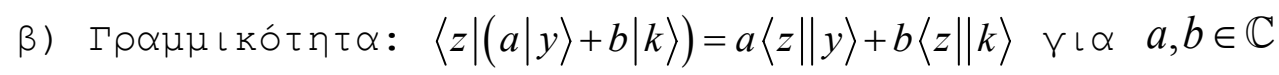

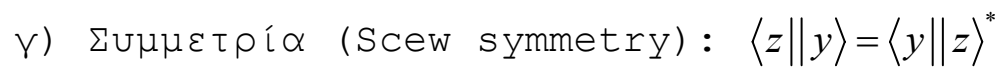

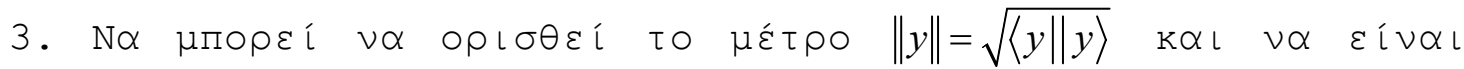
$\Pi \varepsilon \Pi \varepsilon \rho \propto \sigma \mu \varepsilon \dot{v} \vee \circ$.

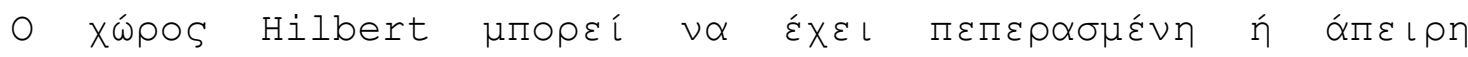

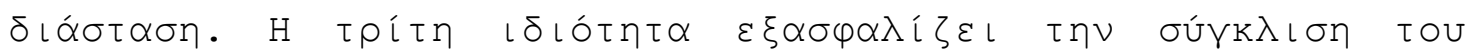

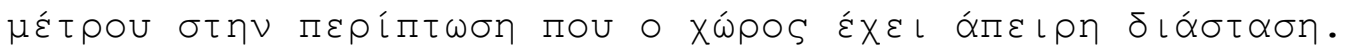

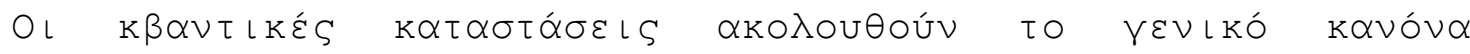

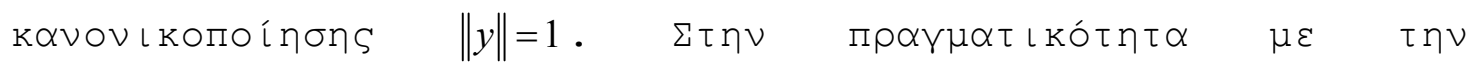

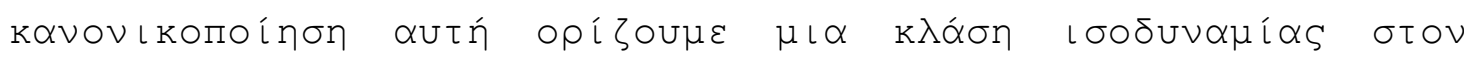

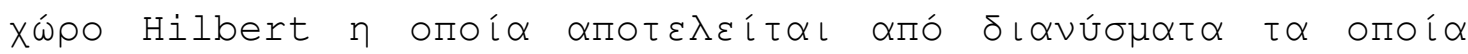

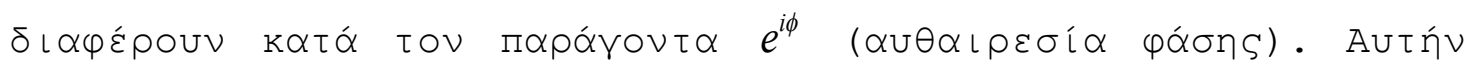

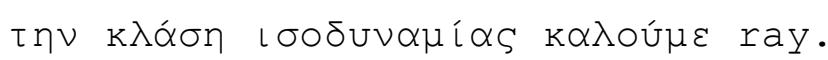

\section{A $\xi i \omega \mu \alpha 2$ :}

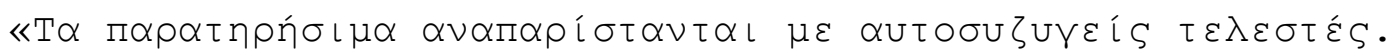

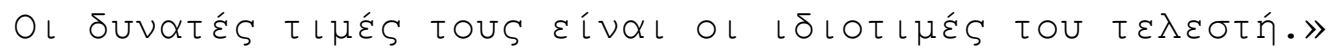

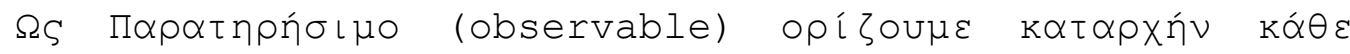

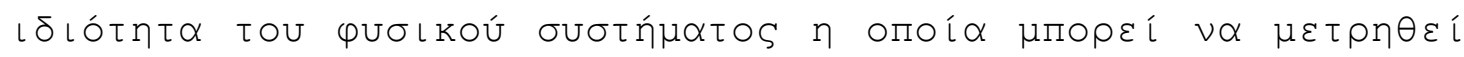

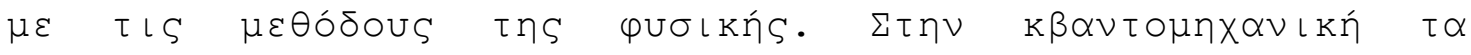

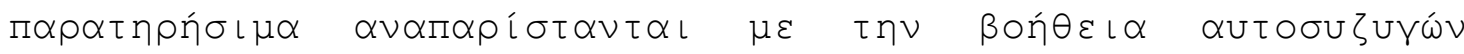

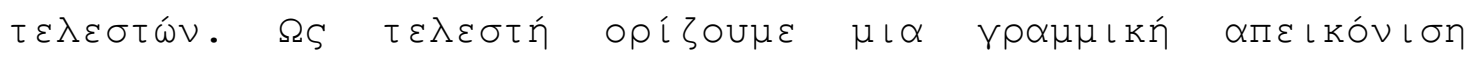
$T: H \rightarrow H . \Delta \eta \lambda \alpha \delta n$

$$
\begin{gathered}
|y\rangle \in H \Rightarrow T|y\rangle=|z\rangle \in H \\
|z\rangle,|y\rangle \in H a, b \in \mathbb{C} \Rightarrow T(a|y\rangle+b|z\rangle)=a T|y\rangle+b T|z\rangle
\end{gathered}
$$




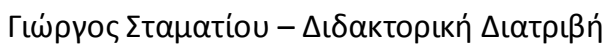

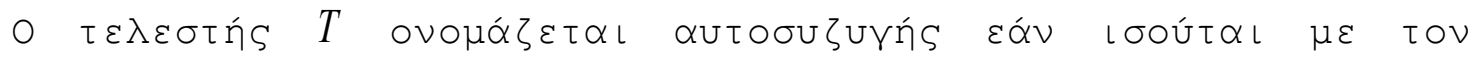

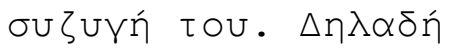

$$
T=T^{+}
$$

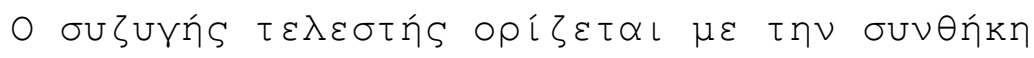

$$
\langle z|| T y\rangle=\left\langle T^{+} z|| y\right\rangle \quad \forall|y\rangle,|z\rangle \in H
$$

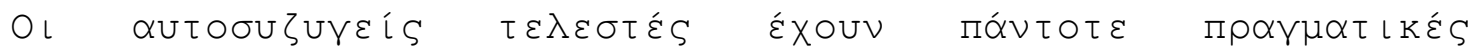

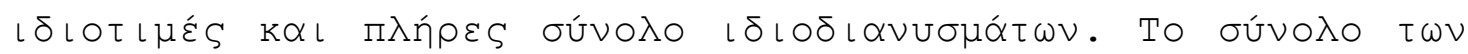

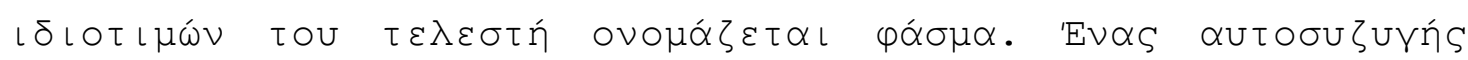

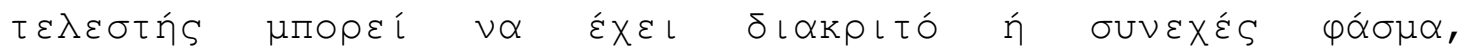

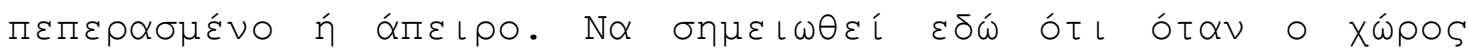
घí

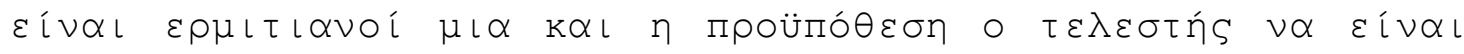

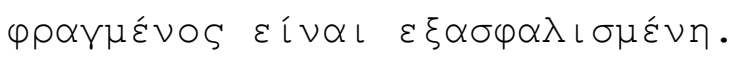

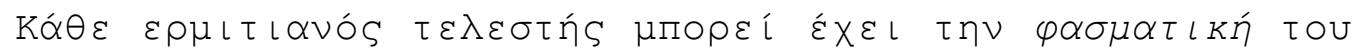
$\alpha \nu \alpha \Pi \alpha \rho \alpha ́ \sigma \tau \alpha \sigma \eta$ (spectral decomposition).

$$
T=\sum_{n} t_{n} P_{n}
$$

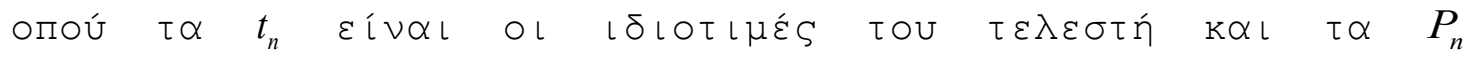

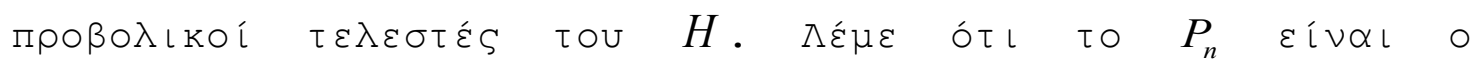

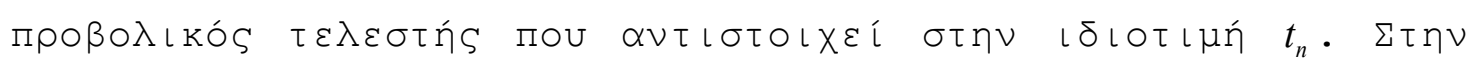

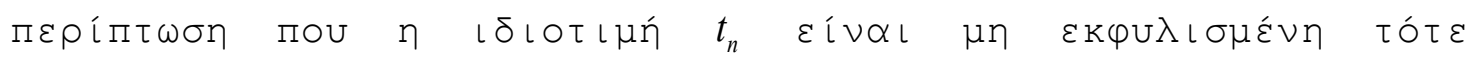
$P_{n}=|n\rangle\langle n|$ о́поч $T|n\rangle=t_{n}|n\rangle$.

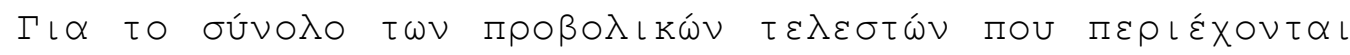

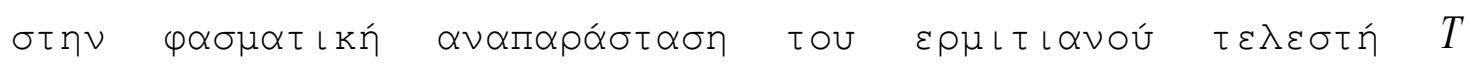

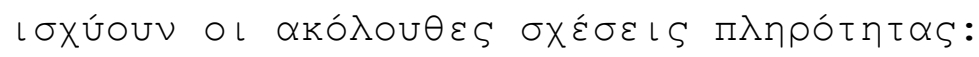

$$
\begin{gathered}
P_{n} P_{m}=\delta_{n m} P_{m} \\
P_{n}^{+}=P_{n}
\end{gathered}
$$

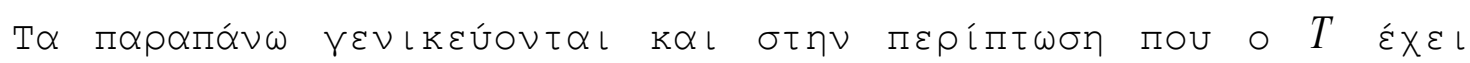

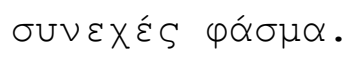




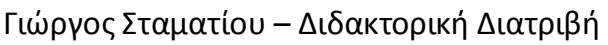

\section{A $\xi i \omega \mu \alpha$ 3:}

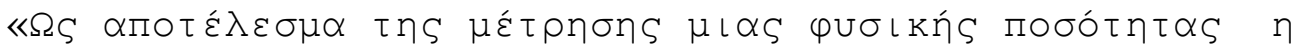

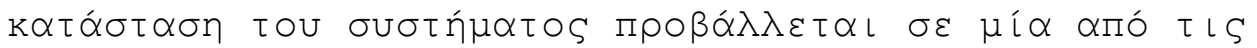

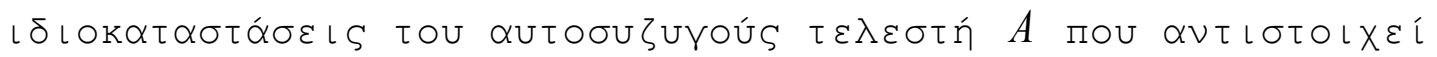

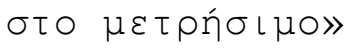

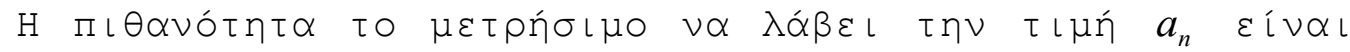

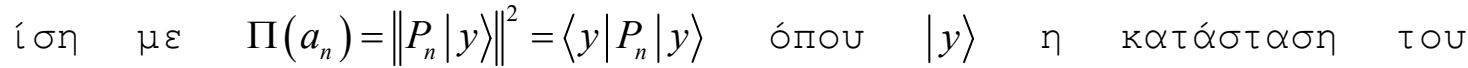

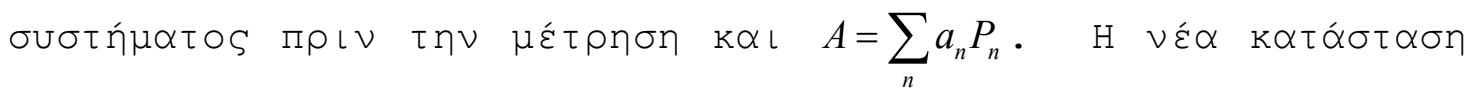

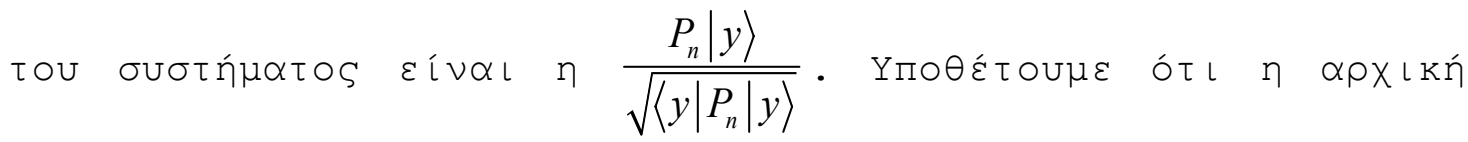

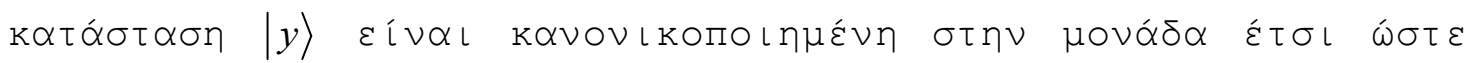

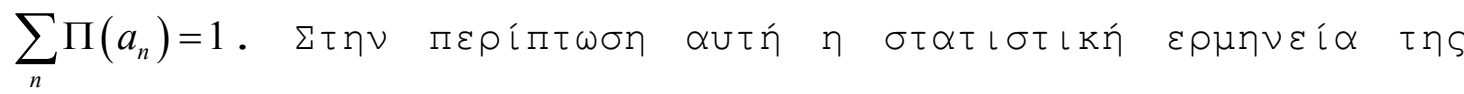

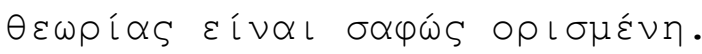

\section{Ał $i \omega \mu \alpha$ 4:}

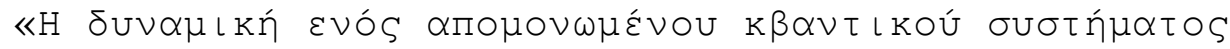

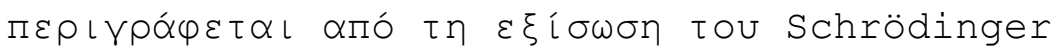

$$
i \hbar \frac{\partial}{\partial t}|y(t)\rangle=H|y(t)\rangle
$$

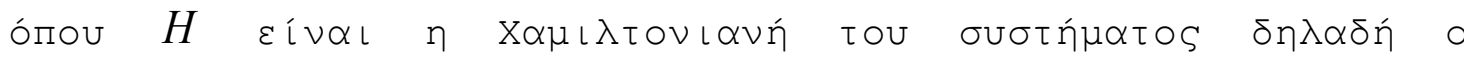

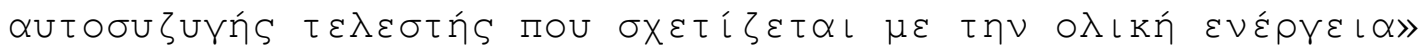

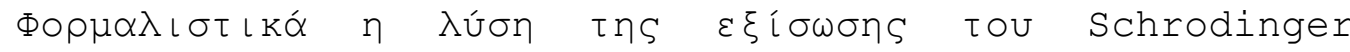

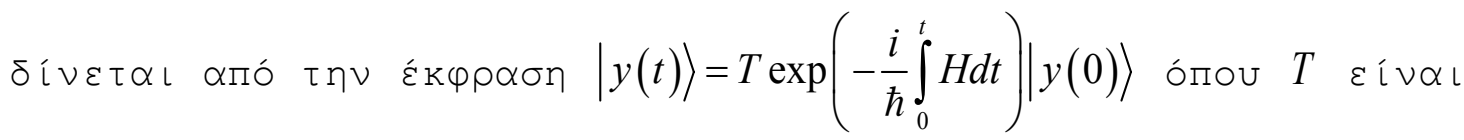

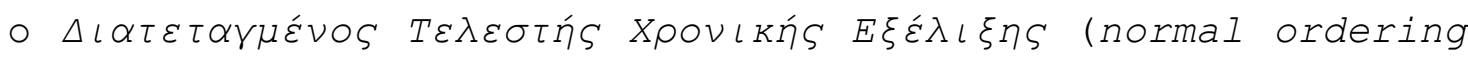

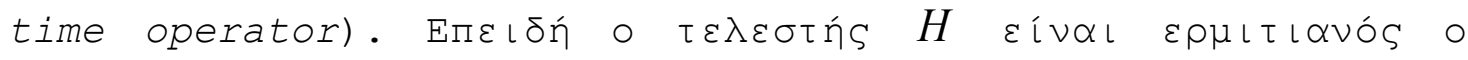

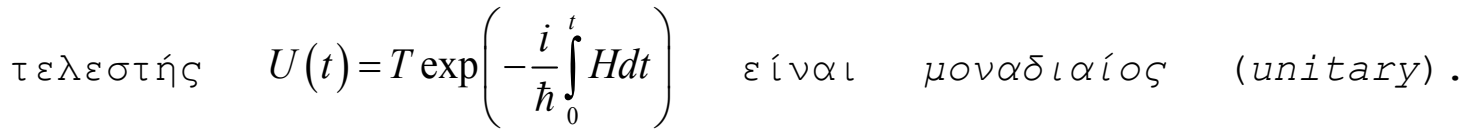

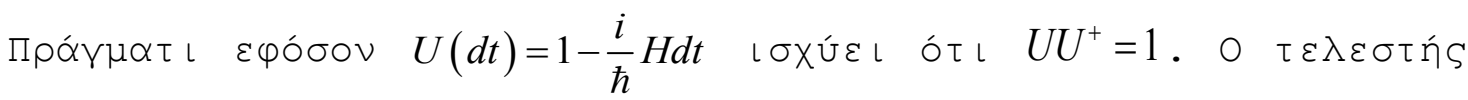




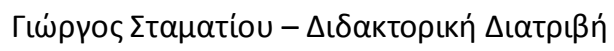

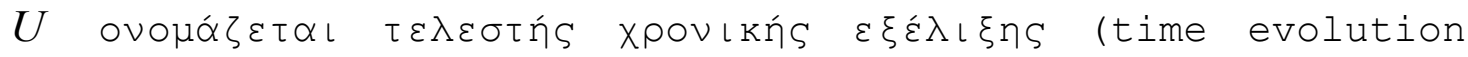

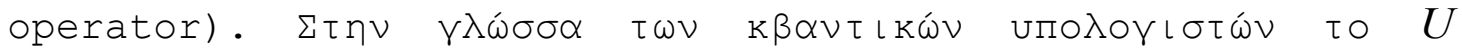

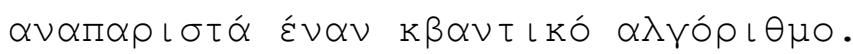

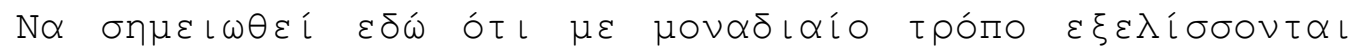

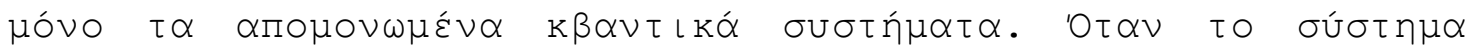

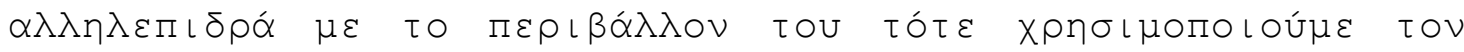

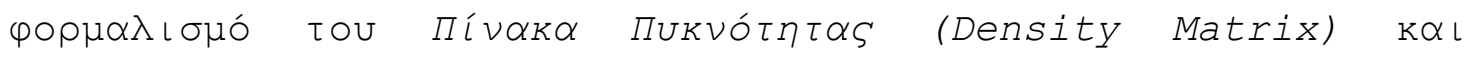

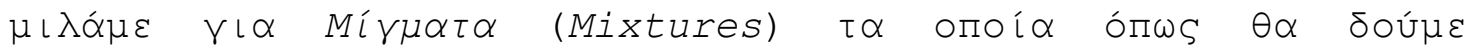

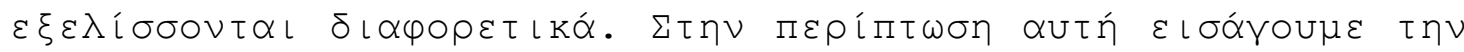

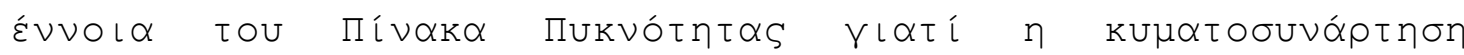

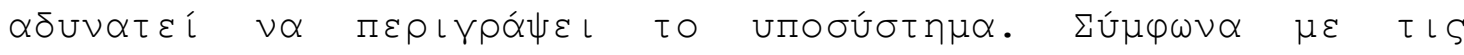

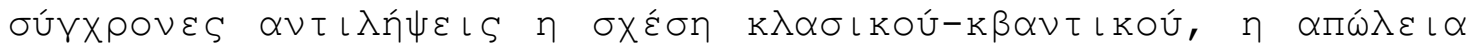

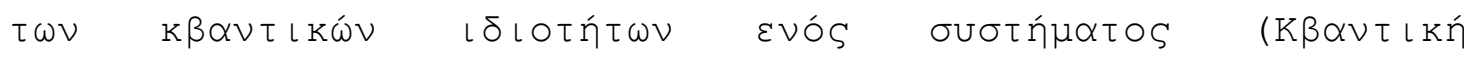

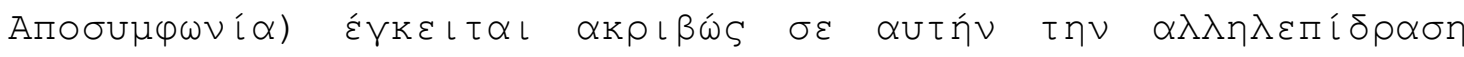

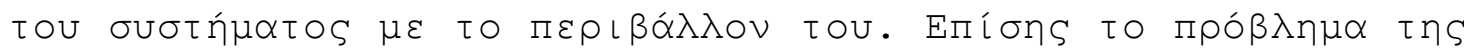

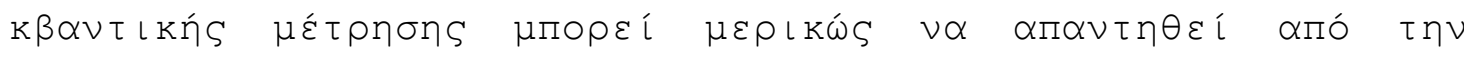

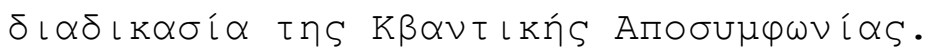

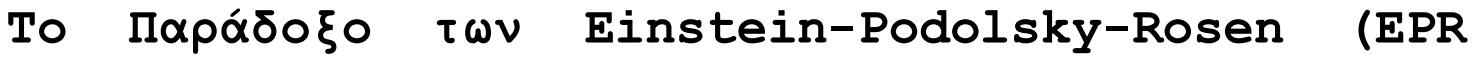

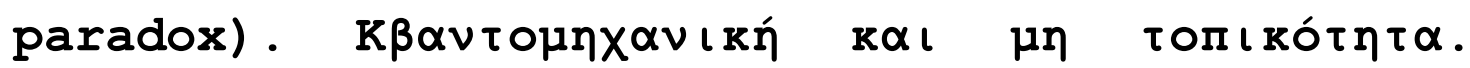

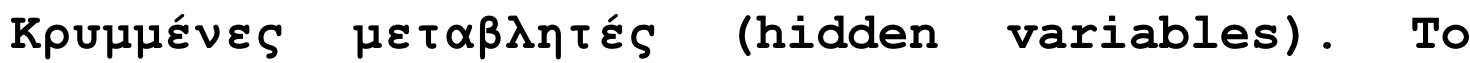

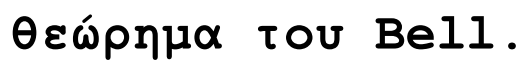

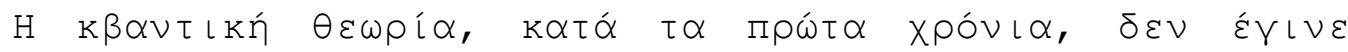

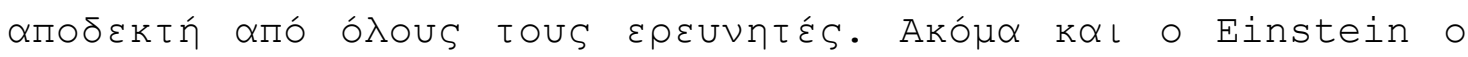

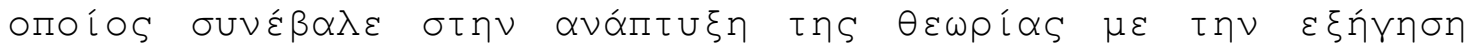

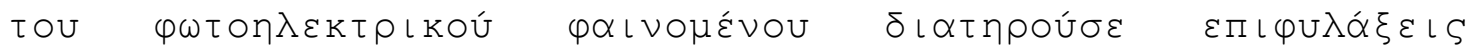

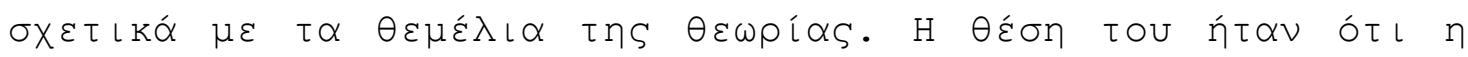

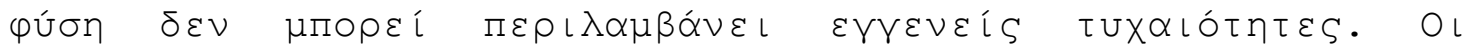

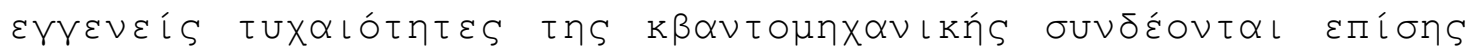

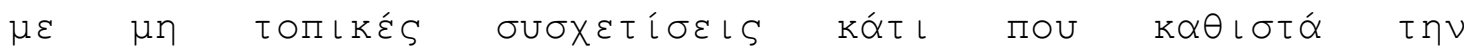

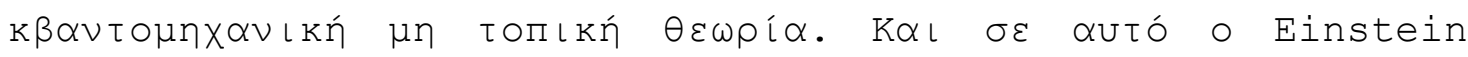

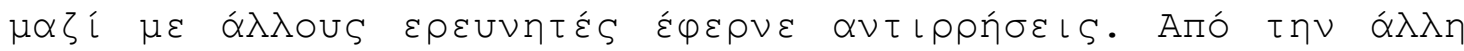

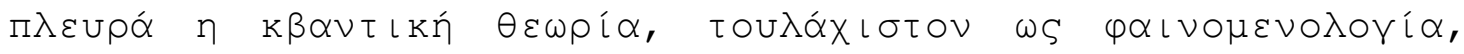

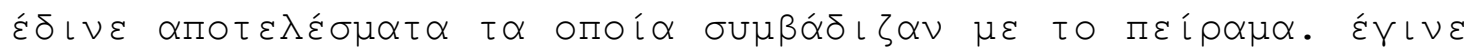

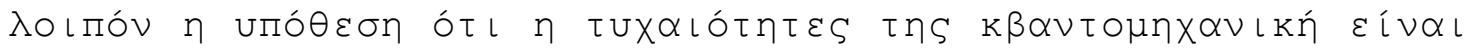

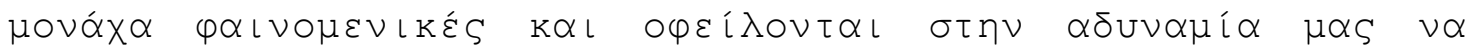

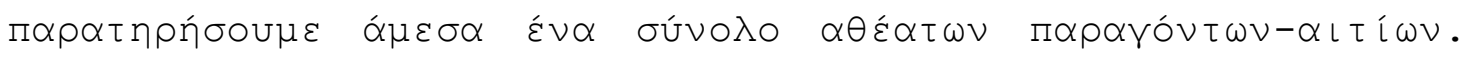




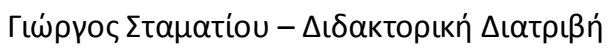

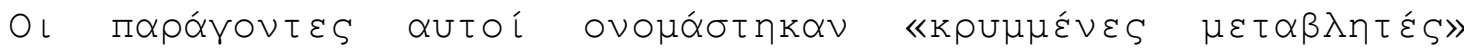

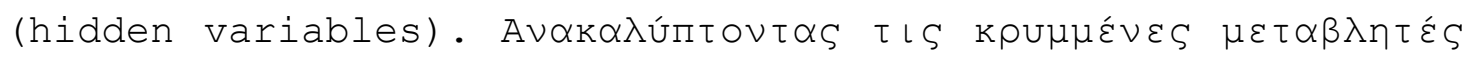

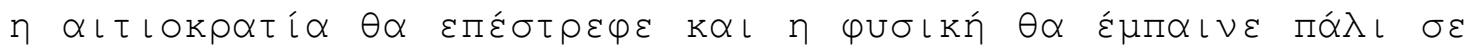

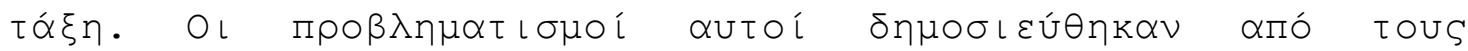

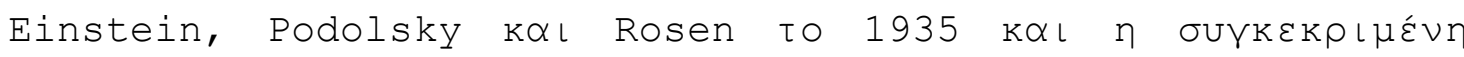

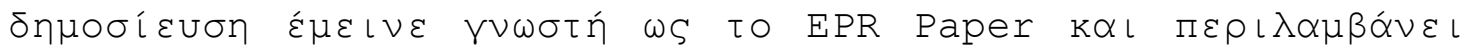

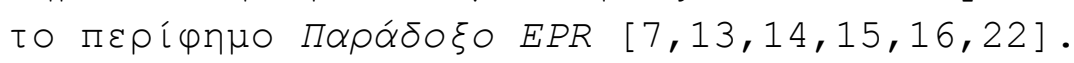

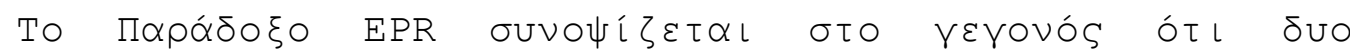

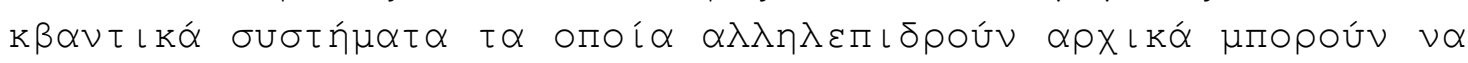

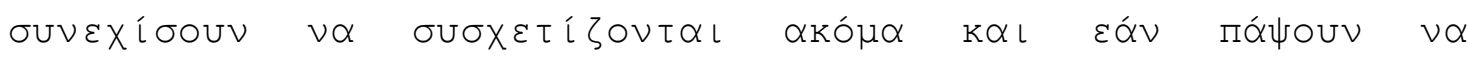

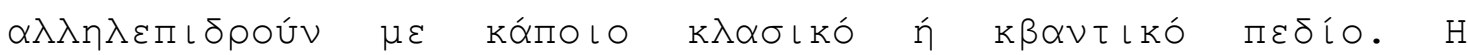

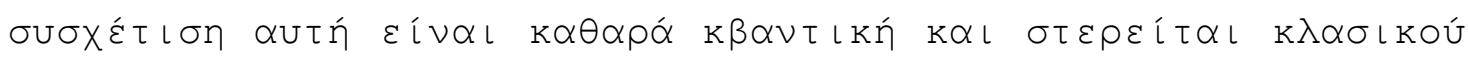

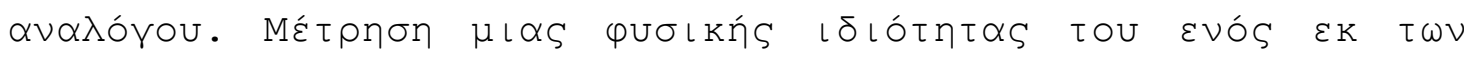

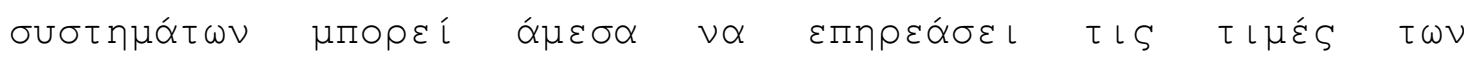

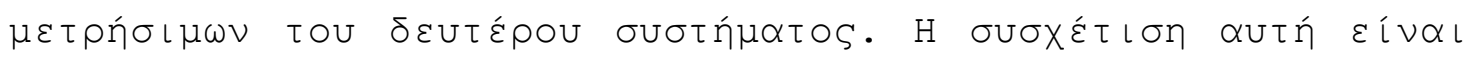

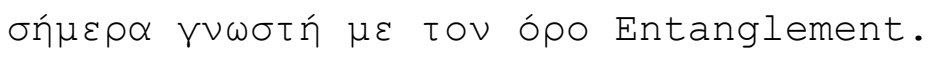

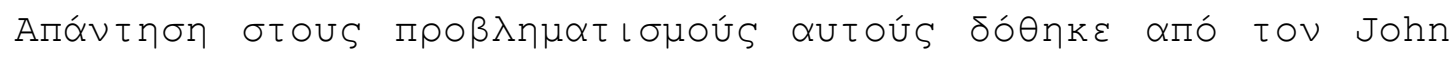

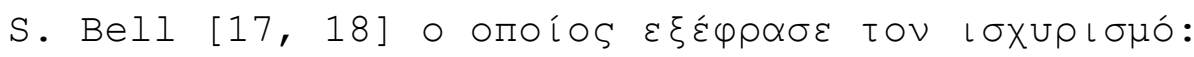

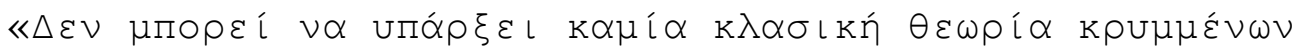

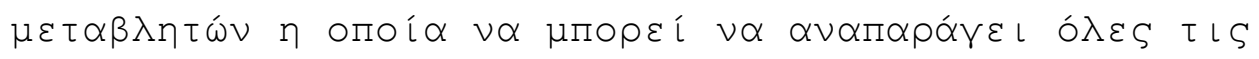

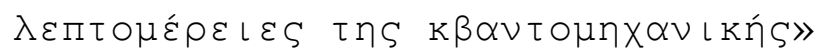

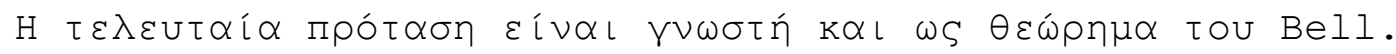

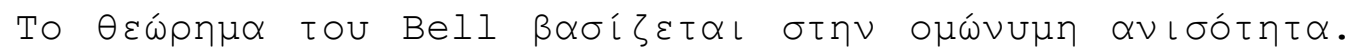

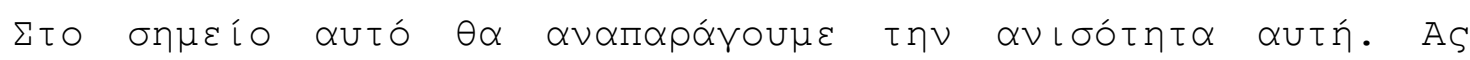

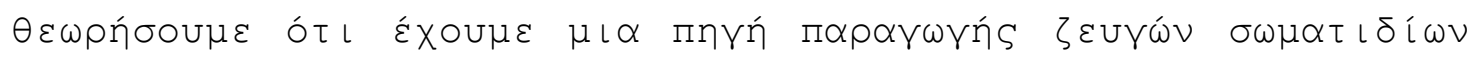

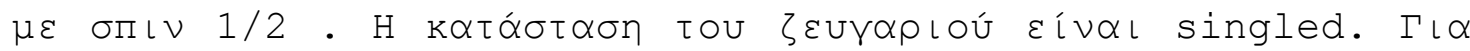

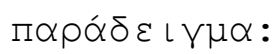

$$
|y\rangle=\frac{1}{\sqrt{2}}(|01\rangle-|10\rangle)
$$

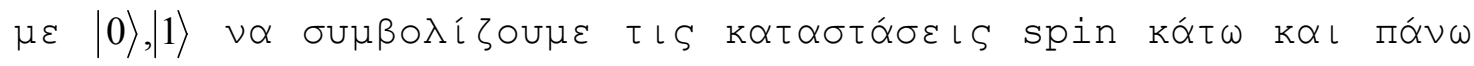
$\alpha \nu \tau i \sigma \tau \circ\llcorner\chi \alpha$. 


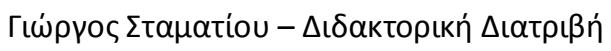

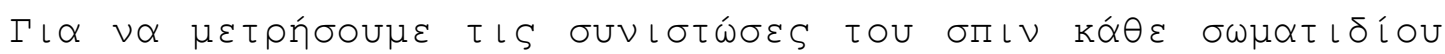

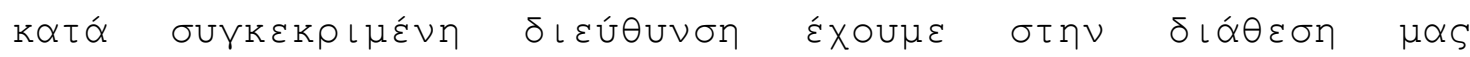

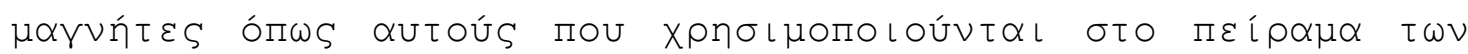

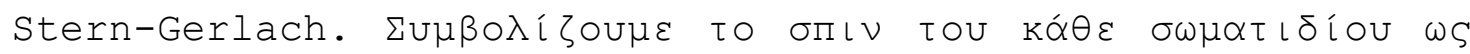

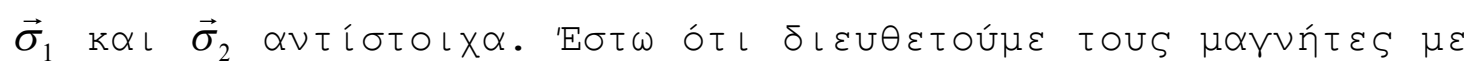

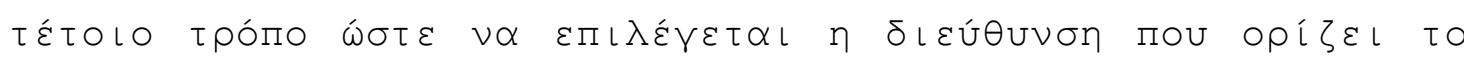

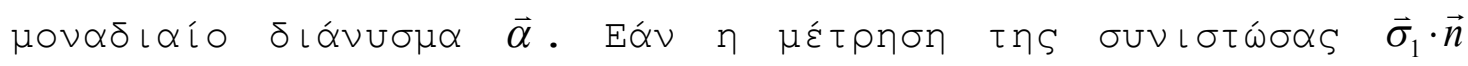

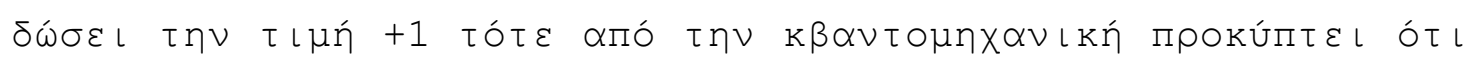

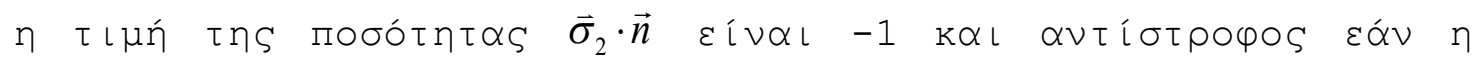

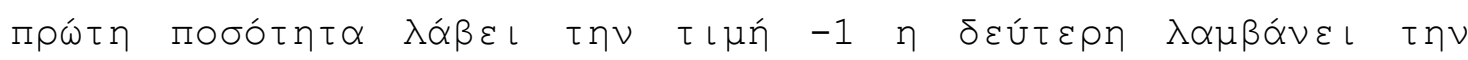

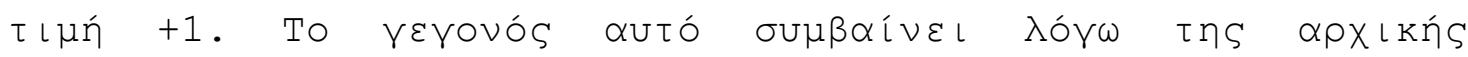

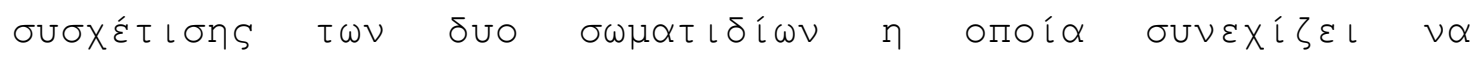

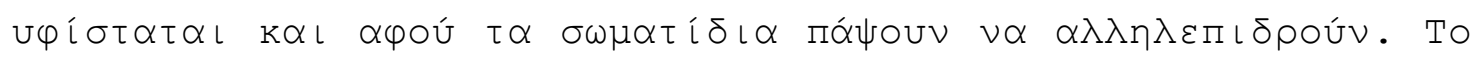

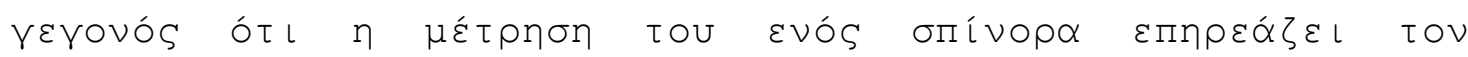

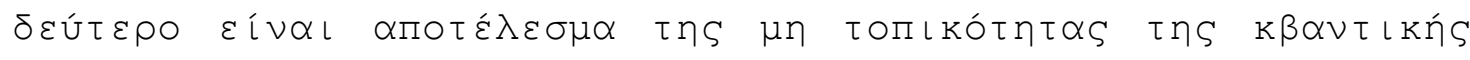
$\theta \varepsilon \omega \rho i \alpha_{S}$.

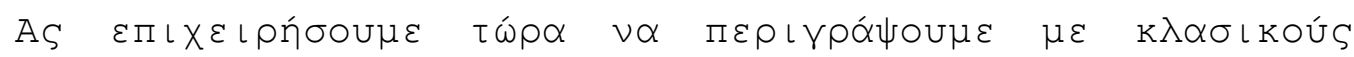

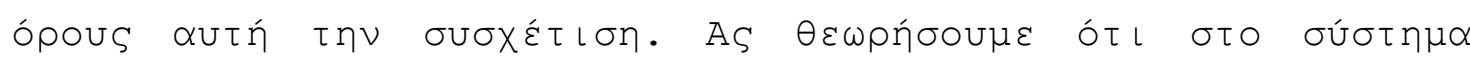

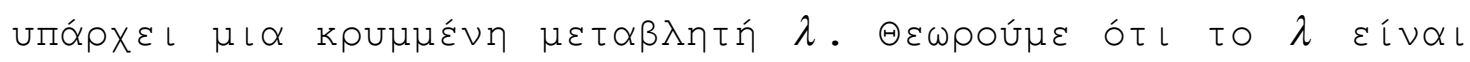

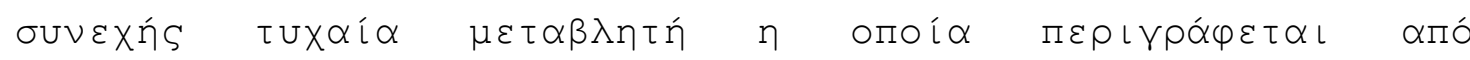

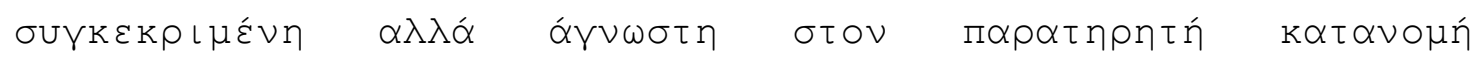

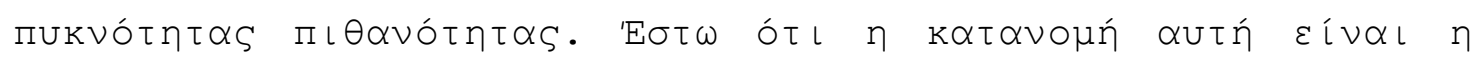

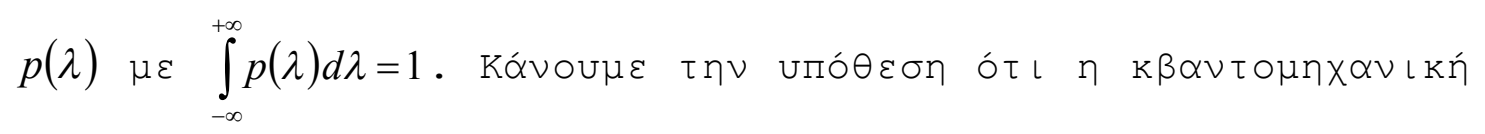

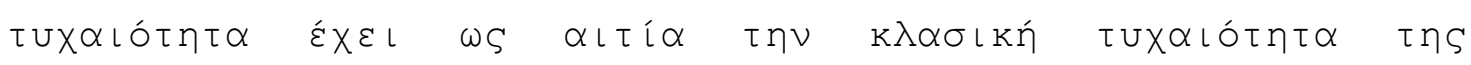

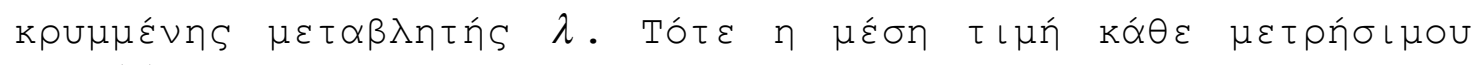
$T \equiv T(\lambda) \quad \iota \sigma \circ u ́ \tau \alpha \iota \mu \varepsilon$

$$
\langle T\rangle=\int_{-\infty}^{+\infty} T(\lambda) p(\lambda) d \lambda
$$

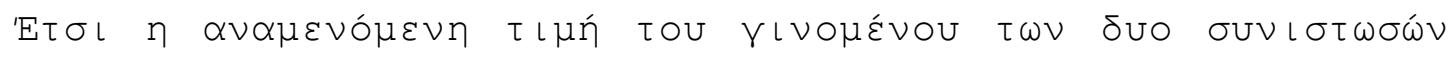
$\vec{\sigma}_{1} \vec{a} \quad \mathrm{k} \alpha \iota \quad \vec{\sigma}_{2} \vec{b} \quad \iota \sigma \circ u ́ \tau \alpha \iota \quad \mu \varepsilon$

$$
P(\vec{a}, \vec{b})=\int d \lambda p(\lambda) A(\vec{a}, \lambda) B(\vec{b}, \lambda)
$$




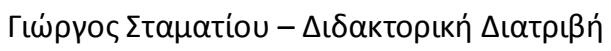

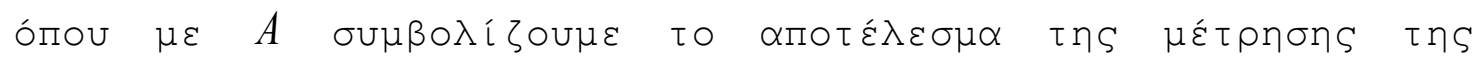

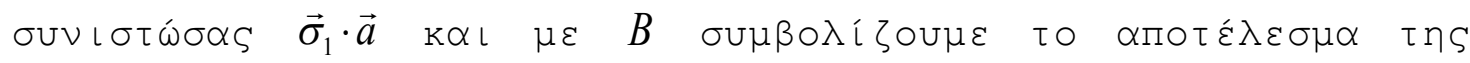

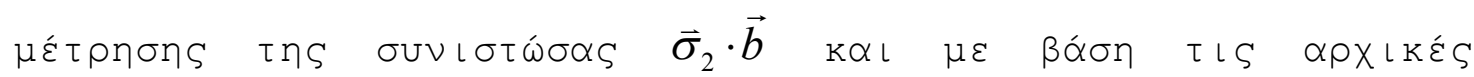

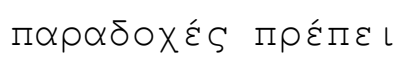

$$
A(\vec{a}, \lambda)= \pm 1 \quad \mathrm{k} \alpha \iota \quad B(\vec{b}, \lambda)=\mp 1
$$

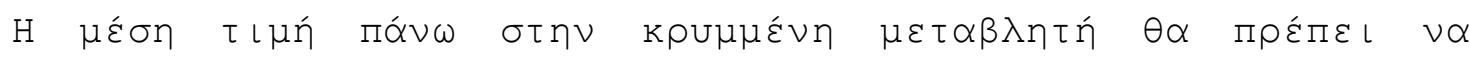

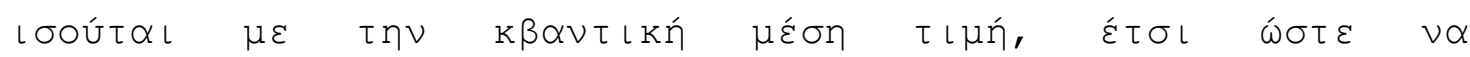

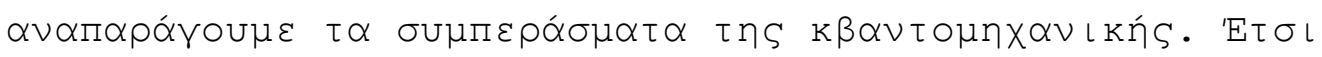

$$
\left\langle\vec{\sigma}_{1} \vec{a} \sigma_{2} \vec{b}\right\rangle=-\vec{\alpha} \cdot \vec{b}
$$

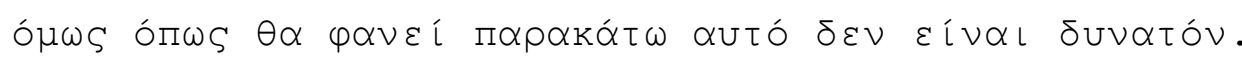

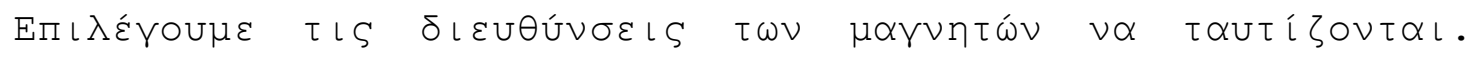
'E七 $\sigma \iota \quad \vec{a}=\vec{b} \quad \mathrm{k} \alpha \iota$

$$
A(\vec{a}, \lambda)=-B(\vec{a}, \lambda)
$$

бUレยாஸ́S

$$
P(\vec{a}, \vec{b})=-\int d \lambda p(\lambda) A(\vec{a}, \lambda) A(\vec{b}, \lambda)
$$

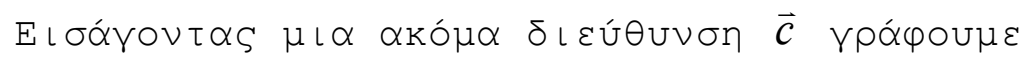

$$
\begin{gathered}
P(\vec{a}, \vec{b})-P(\vec{a}, \vec{c})=-\int d \lambda P(\lambda)[A(\vec{a}, \lambda) A(\vec{b}, \lambda)-A(\vec{a}, \lambda) A(\vec{c}, \lambda)] \\
\quad=\int d \lambda P(\lambda) A(\vec{a}, \lambda) A(\vec{b}, \lambda)[A(\vec{b}, \lambda) A(\vec{c}, \lambda)-1]
\end{gathered}
$$

бUขยாஸ́ร

$$
|P(\vec{a}, \vec{b})-P(\vec{a}, \vec{c})| \leq \int d \lambda P(\lambda)[1-A(\vec{b}, \lambda) A(\vec{c}, \lambda)]
$$

ó

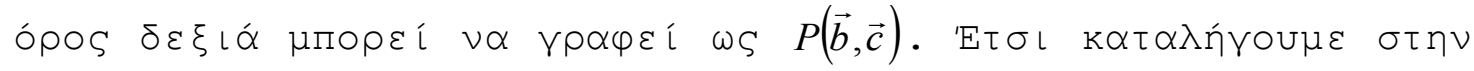
$\alpha \vee\llcorner\sigma o ́ \tau \eta \tau \alpha:$

$$
1+P(\vec{b}, \vec{c}) \geq|P(\vec{a}, \vec{b})-P(\vec{a}, \vec{c})| \quad(\text { A } \vee \text { เó́ ๆฺ } \alpha \text { Bell })
$$




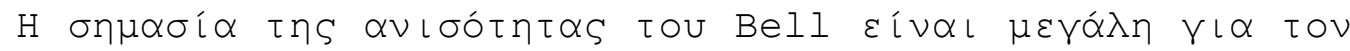

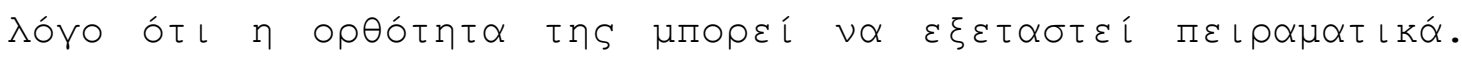

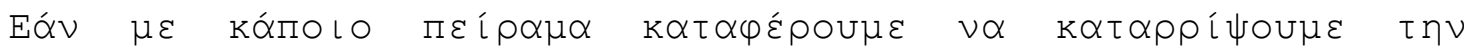

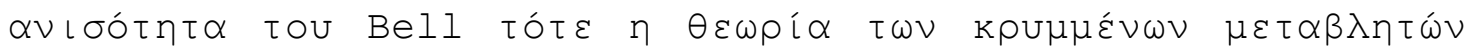

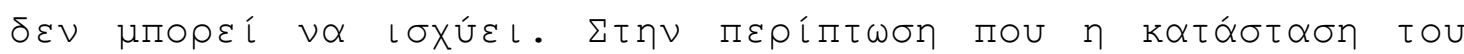

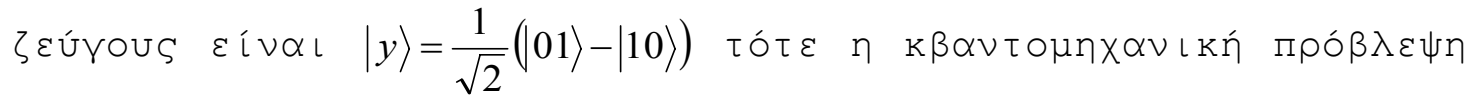

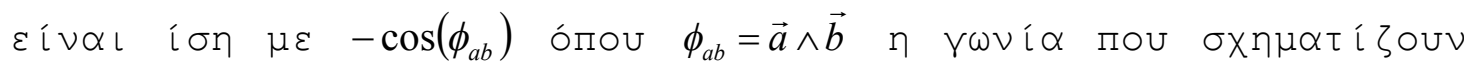

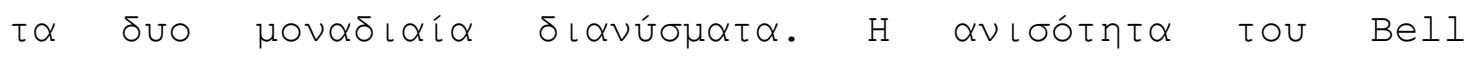

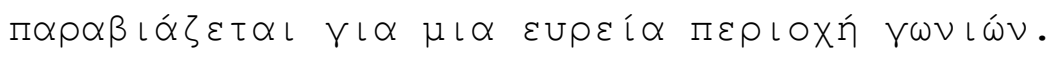

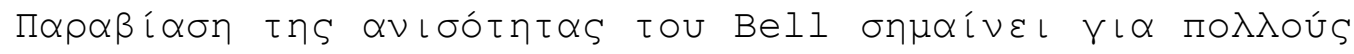

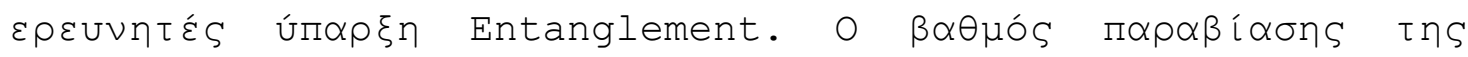

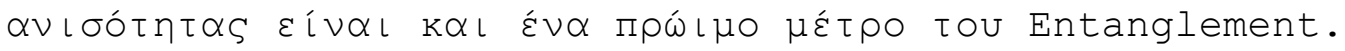

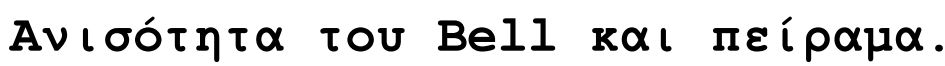

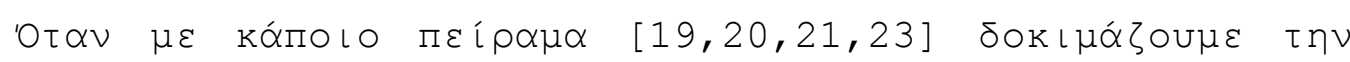

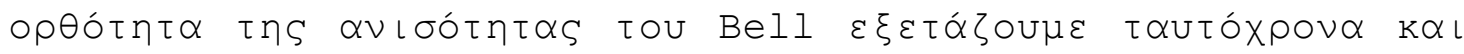

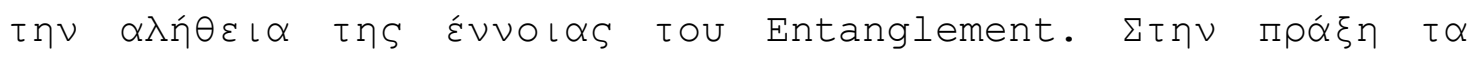

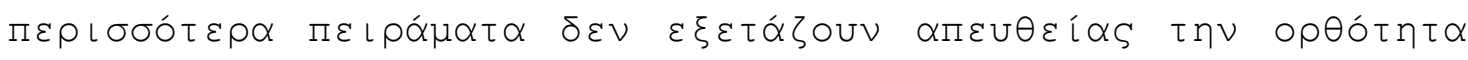

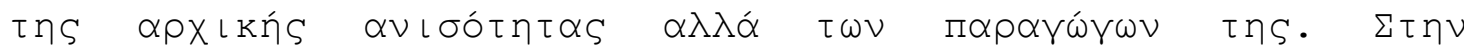

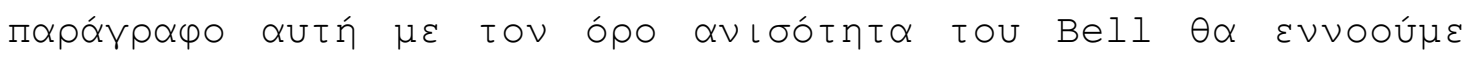

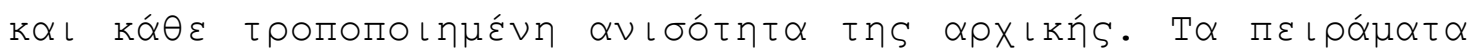

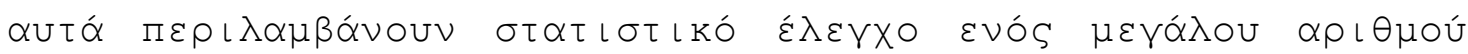

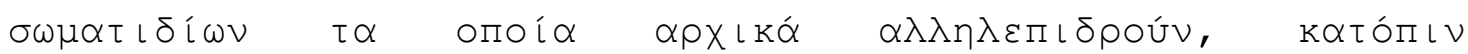

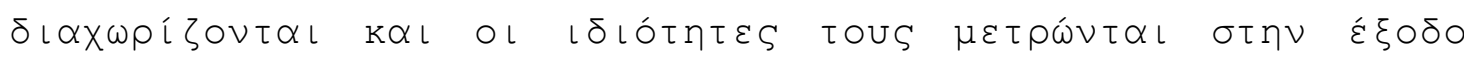

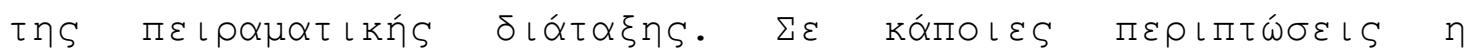

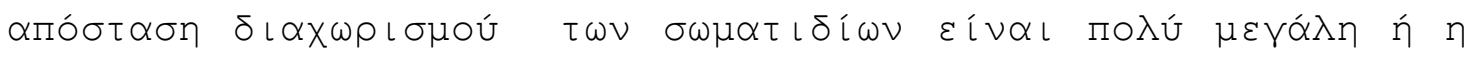

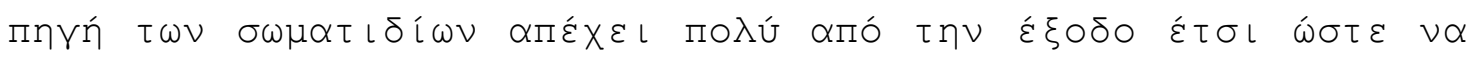

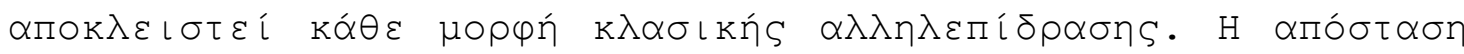

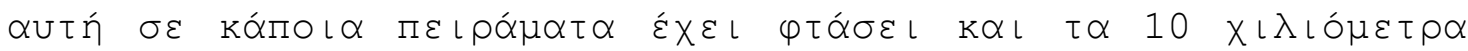
$[19]$ !

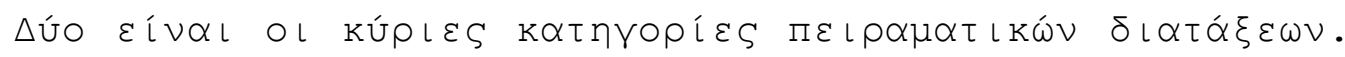

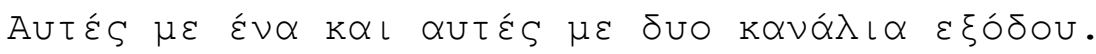

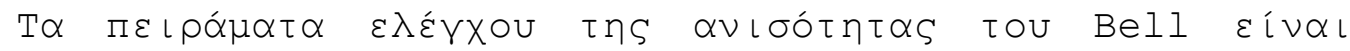

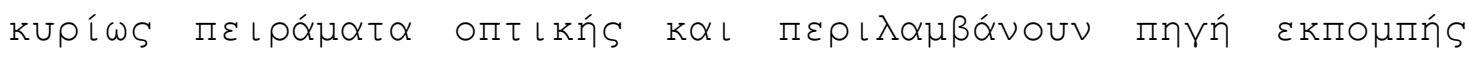




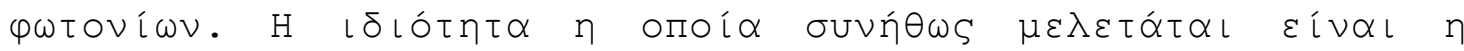

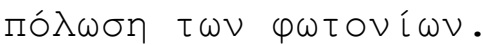

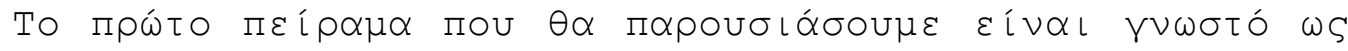

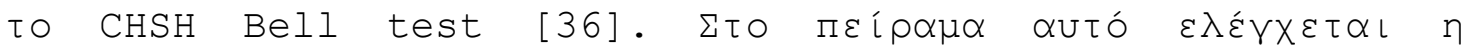

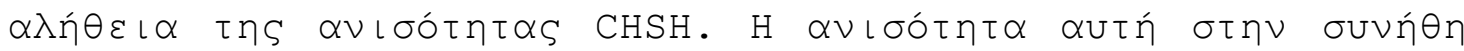

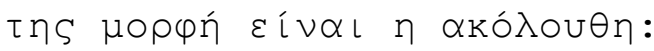

$$
-2 \leq S \leq+2
$$

о́по

$$
S=E(a, b)-E\left(a, b^{\prime}\right)+E\left(a^{\prime}, b\right)+E\left(a^{\prime}, b^{\prime}\right)
$$

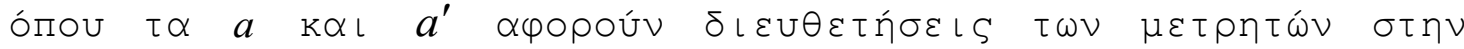

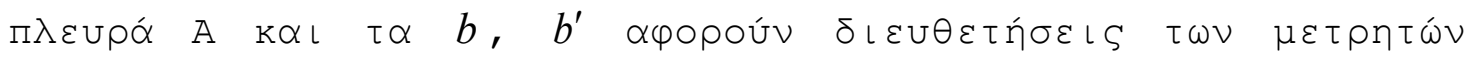

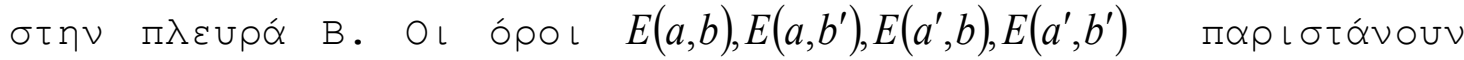

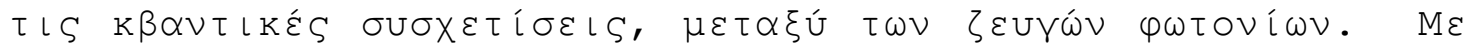

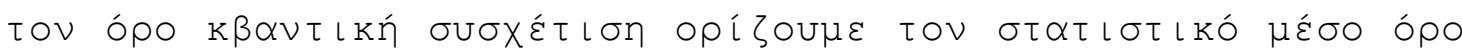

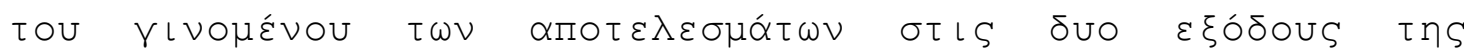

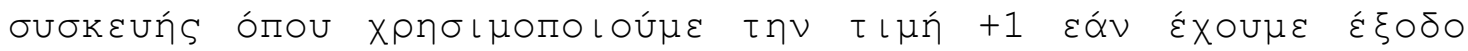

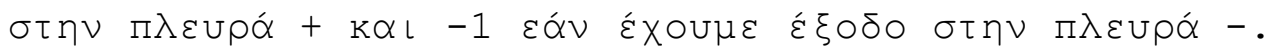

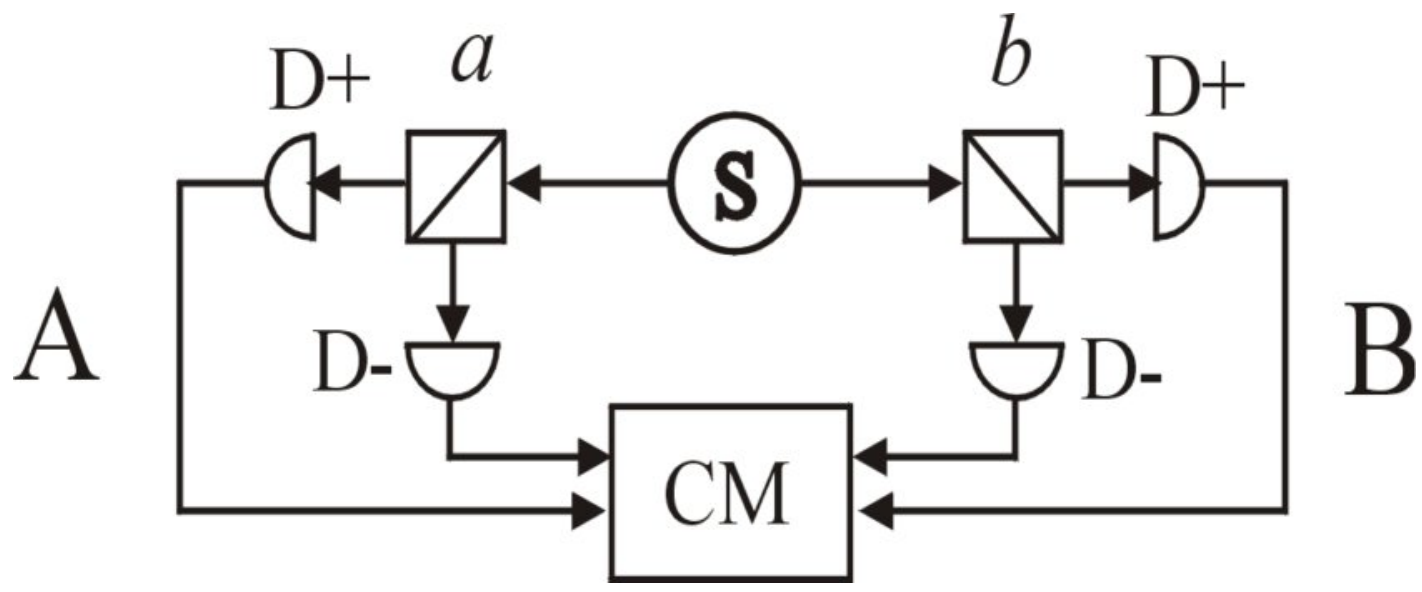

[http://commons.wikimedia.org/wiki/File:Two_channel.png]

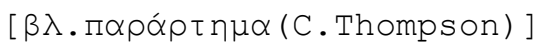

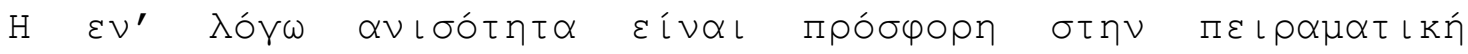

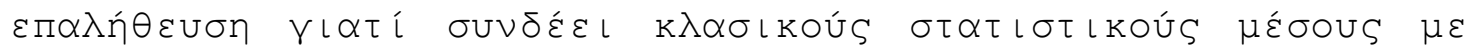

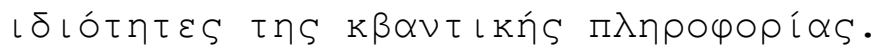




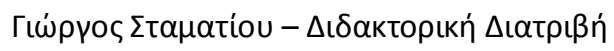

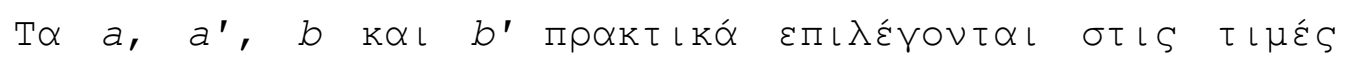

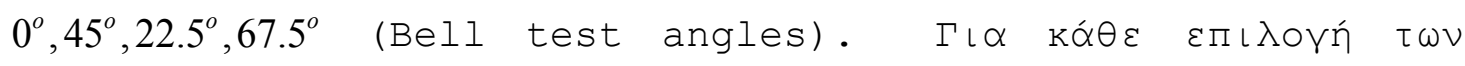

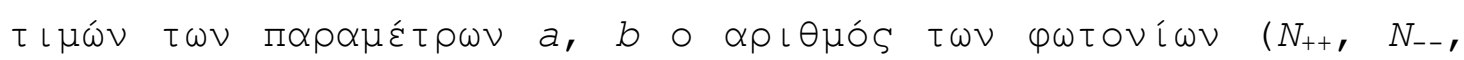

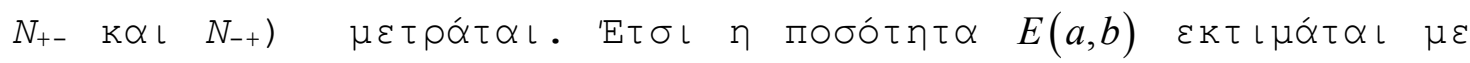

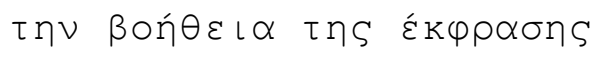

$$
E=\left(N_{++}+N_{--}-N_{+-}-N_{-+}\right) /\left(N_{++}+N_{--}+N_{+-}+N_{-+}\right)
$$

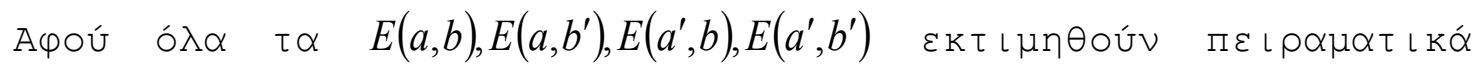

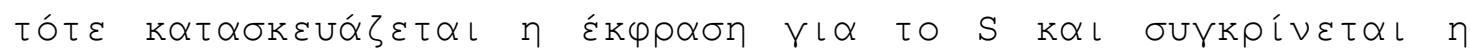

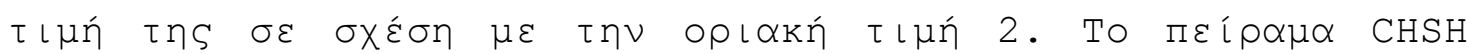

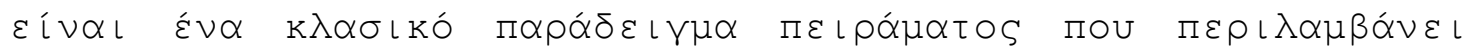

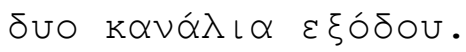

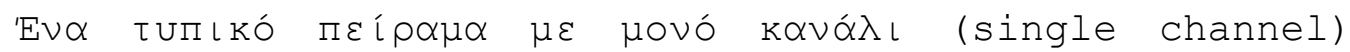

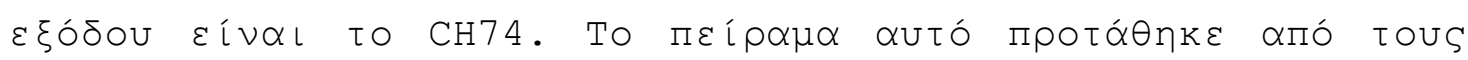

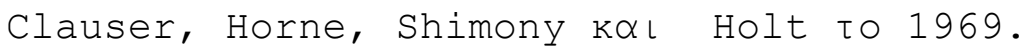

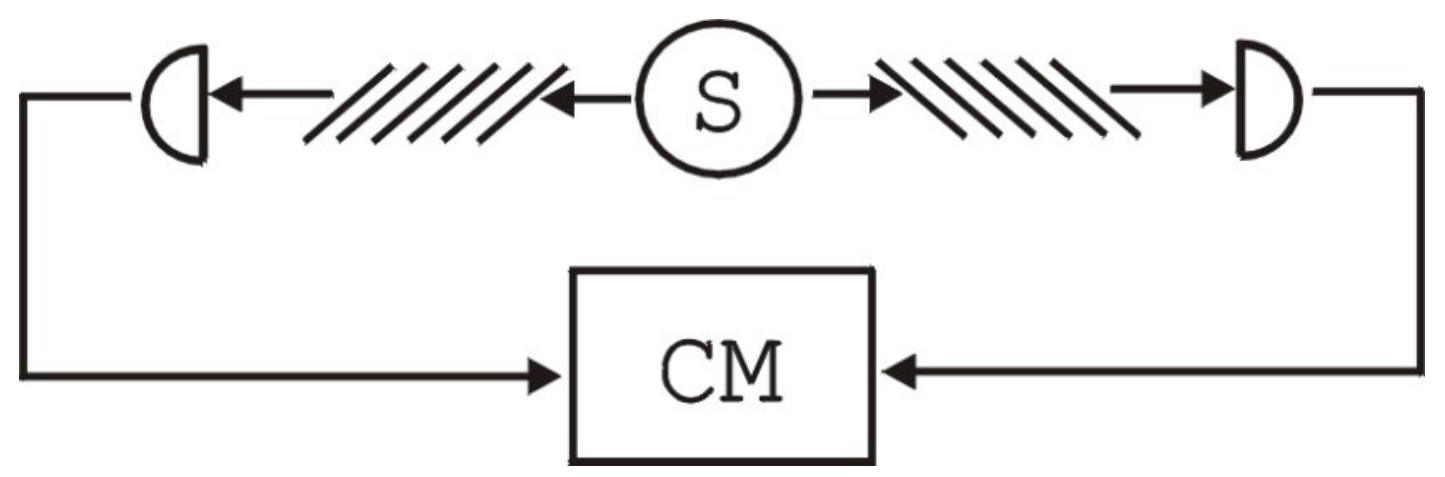

[http://en.wikipedia.org/wiki/File:Single-channel_Bell_test.png]

$[\beta \lambda . п \alpha \rho \alpha ́ \alpha \tau \eta \mu \alpha($ C.Thompson) ]

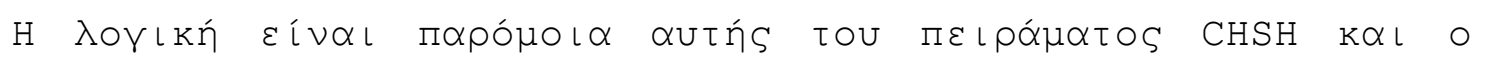

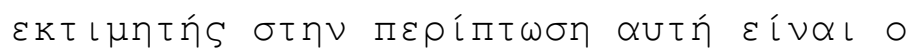

$$
\begin{gathered}
S=\left(N(a, b)-N\left(a, b^{\prime}\right)+N\left(a^{\prime}, b\right)+N\left(a^{\prime}, b^{\prime}\right)-N\left(a^{\prime}, \infty\right)-\right. \\
N(\infty, b)) / N(\infty, \infty)
\end{gathered}
$$

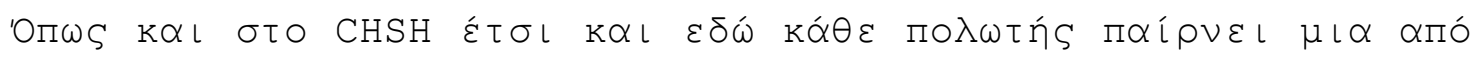

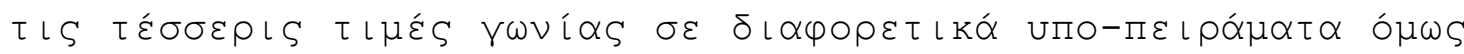

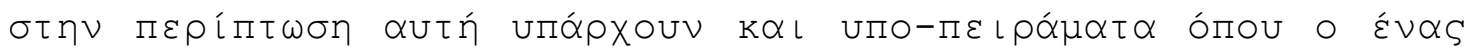

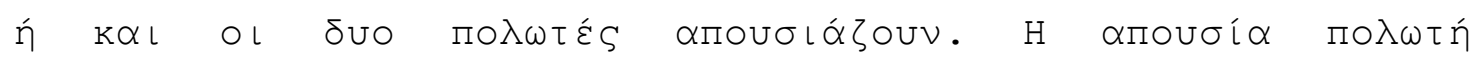




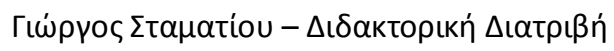

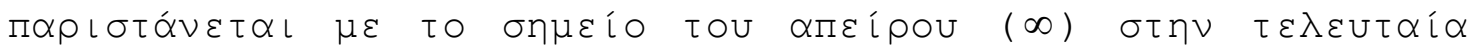

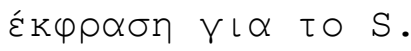

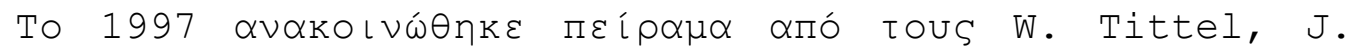
Brendel, B. Gisin, T. Herzog, H. Zbinden, N. Gisin [19] ol

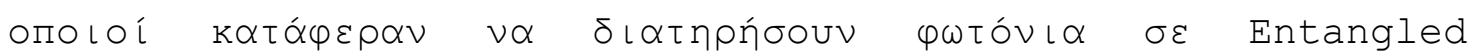

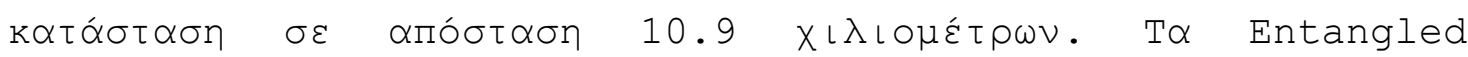

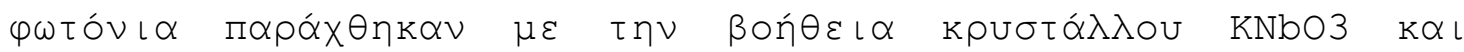

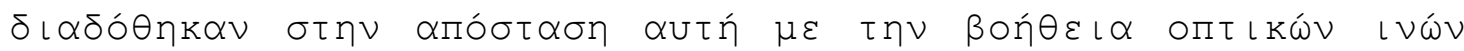

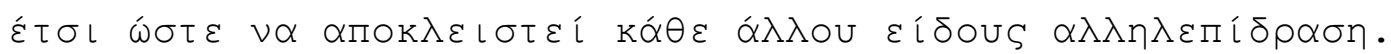

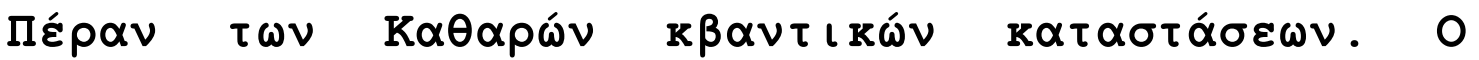

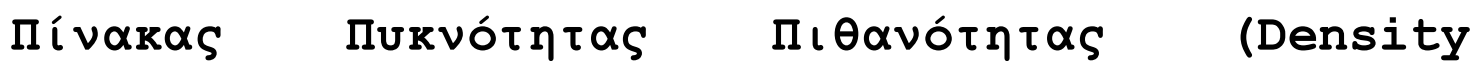 Matrix) .
}

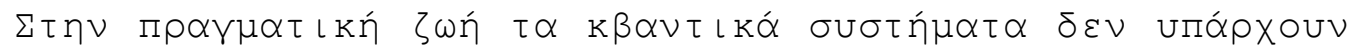

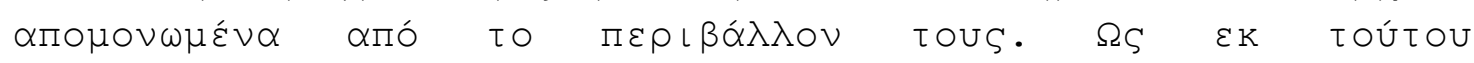

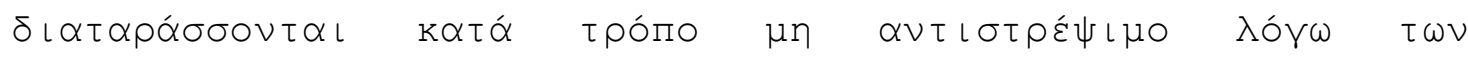

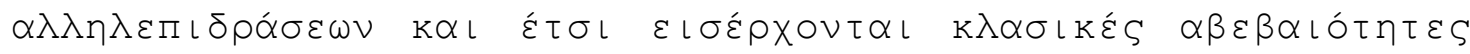

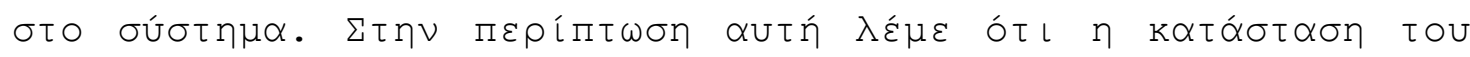

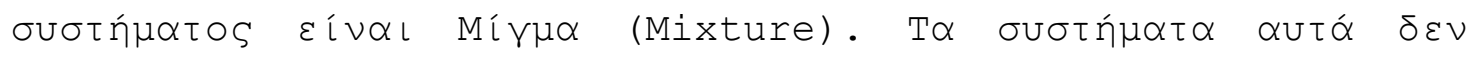

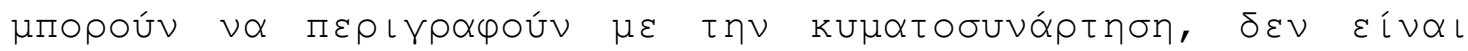

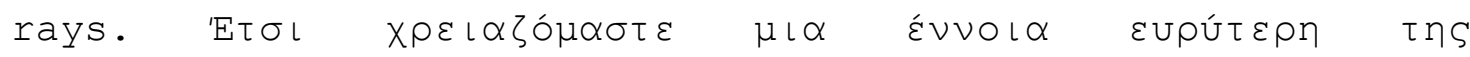

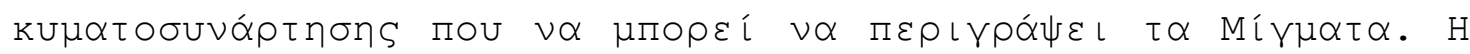

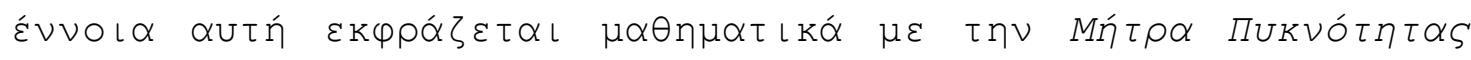

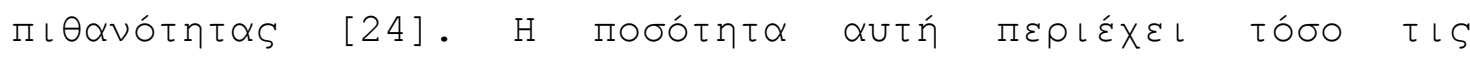

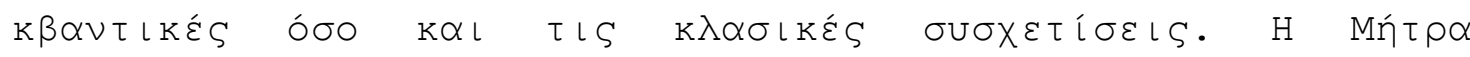

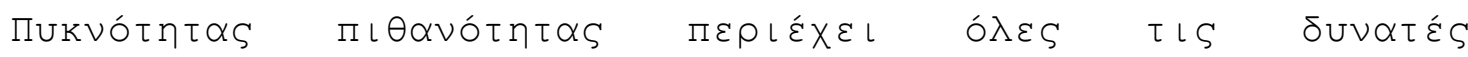

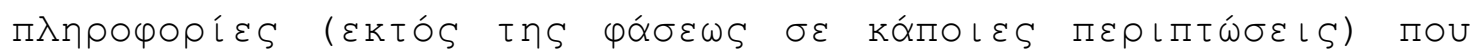

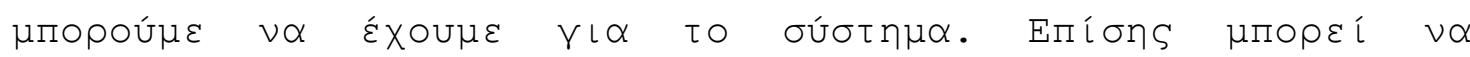

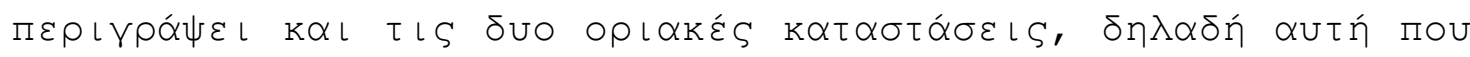

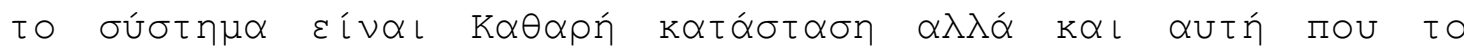

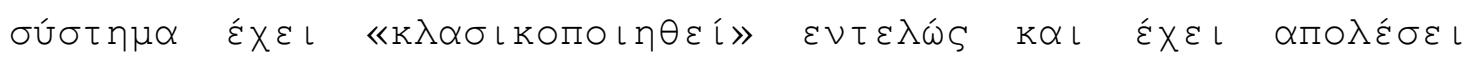

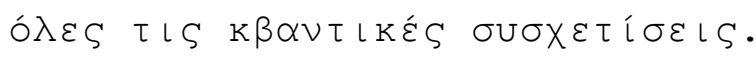




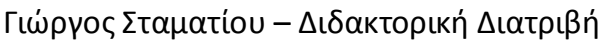

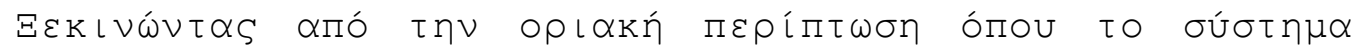

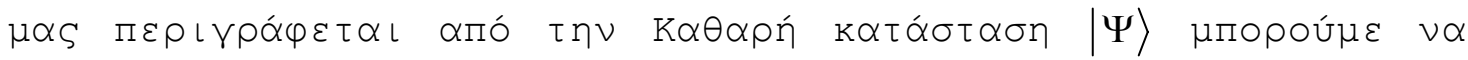

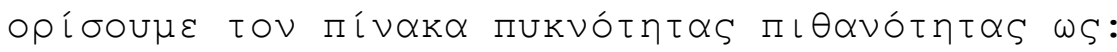

$$
\rho=|\Psi\rangle\langle\Psi|
$$

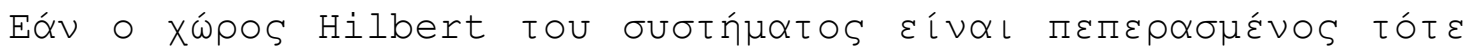

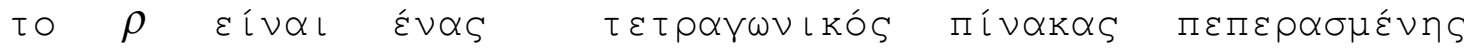

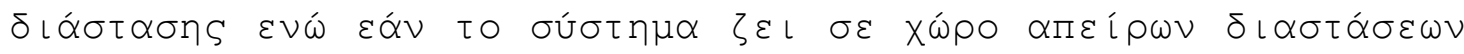

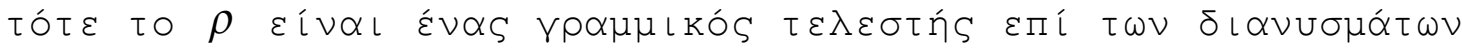

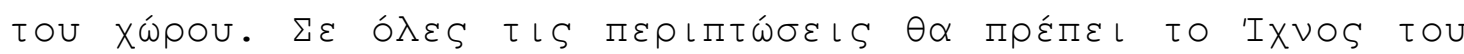

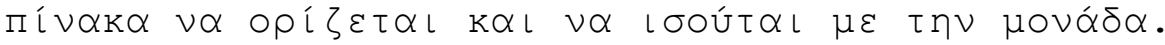

$$
\operatorname{tr}(\rho)=1
$$

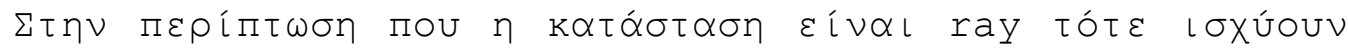

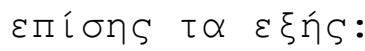

$$
\rho^{2}=\rho
$$

$K \propto \iota$

$$
\operatorname{tr}\left(\rho^{2}\right)=1
$$

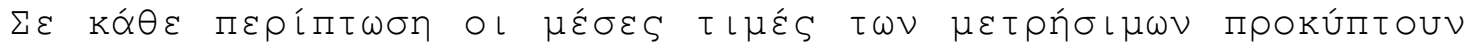

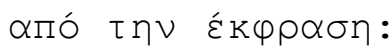

$$
\langle A\rangle=\operatorname{tr}(\rho A)
$$

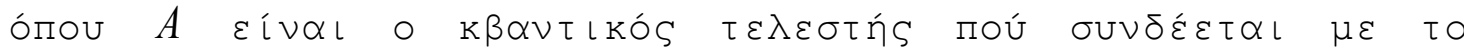

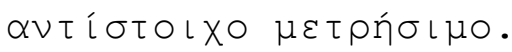

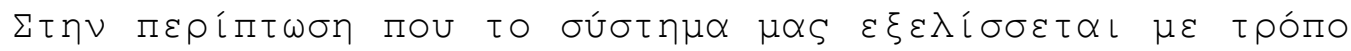

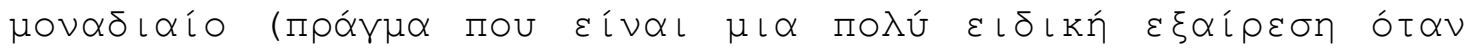

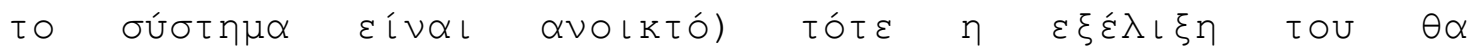

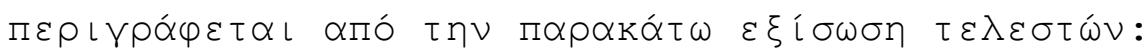

$$
\dot{\rho}=-\frac{i}{\hbar}[H, \rho]
$$




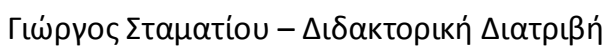

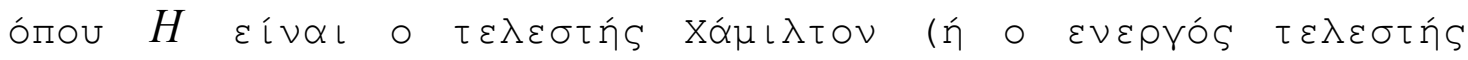

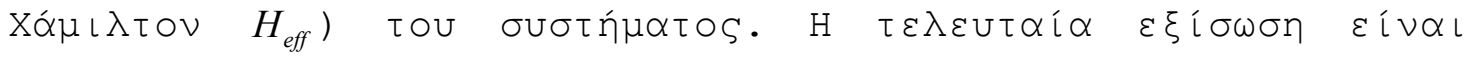

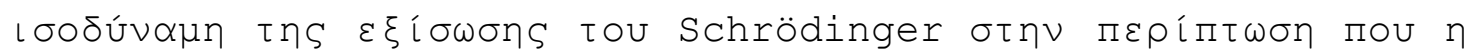

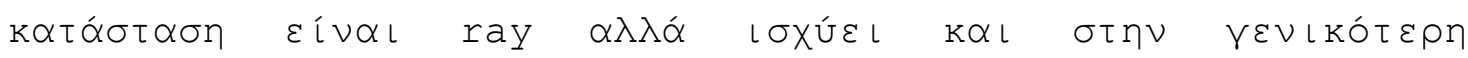

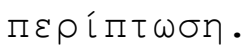

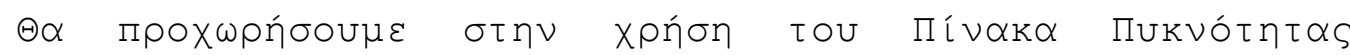

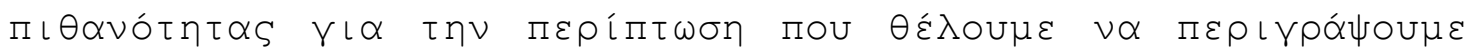

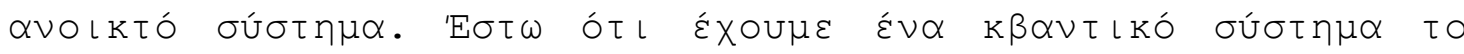

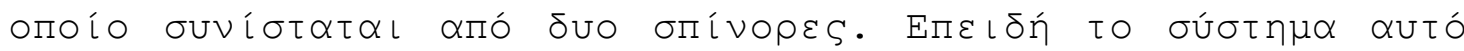

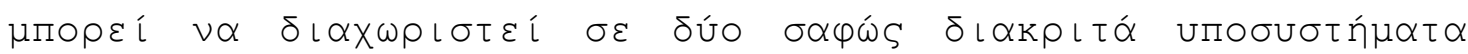

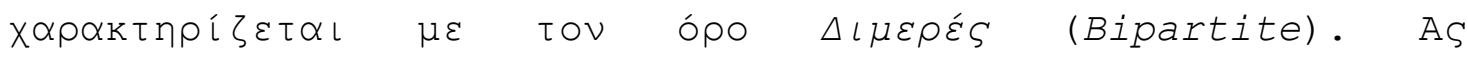

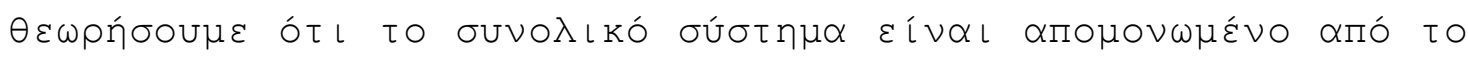

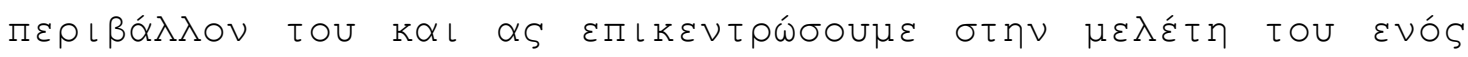

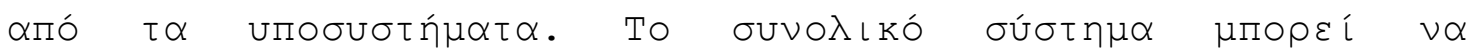

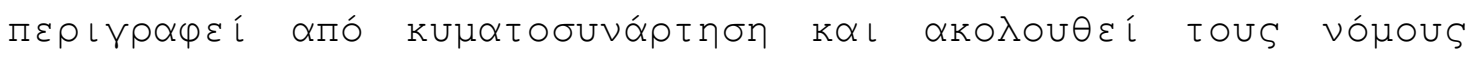

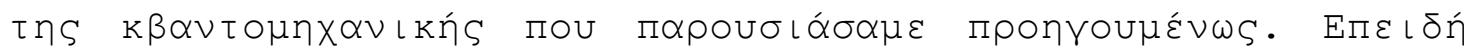

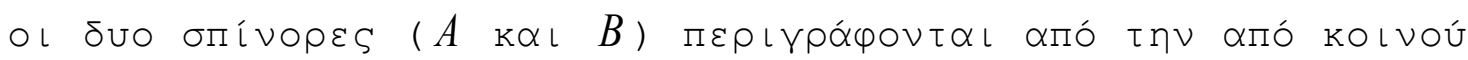

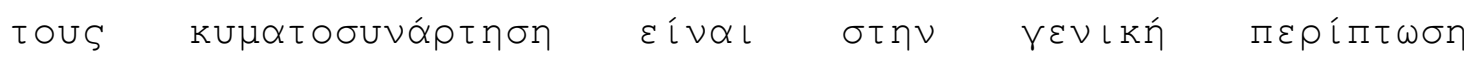

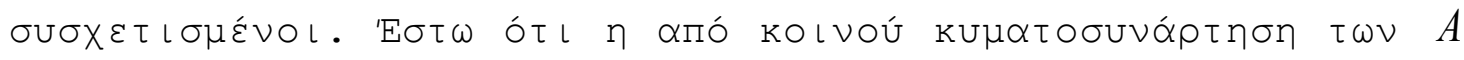

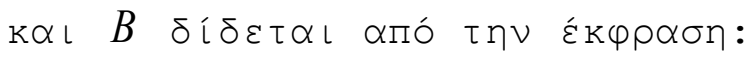

$$
|\Psi\rangle_{A B}=\alpha|00\rangle+\beta|11\rangle
$$

ń $\alpha \lambda \lambda \iota \omega ́ S$

$$
|\Psi\rangle_{A B}=\alpha|0\rangle_{A} \otimes|0\rangle_{B}+\beta|1\rangle_{A} \otimes|1\rangle_{B}
$$

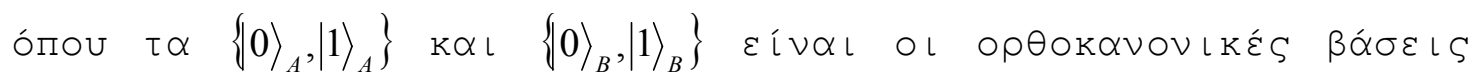

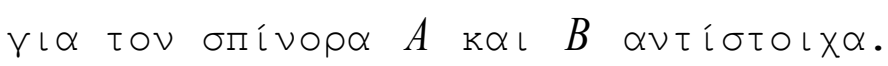

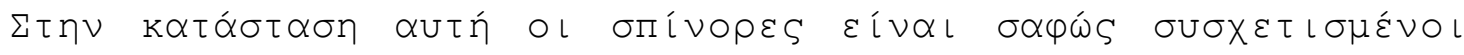

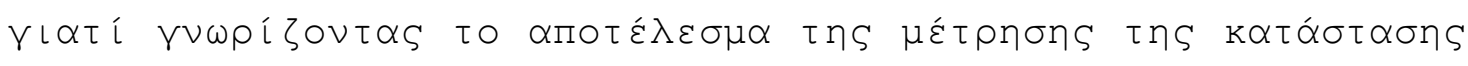

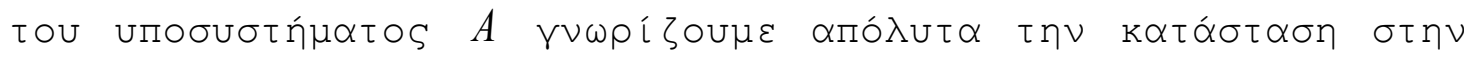

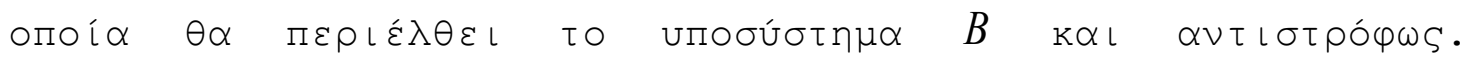

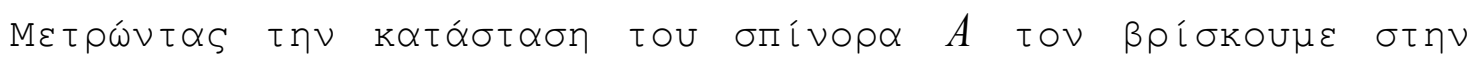

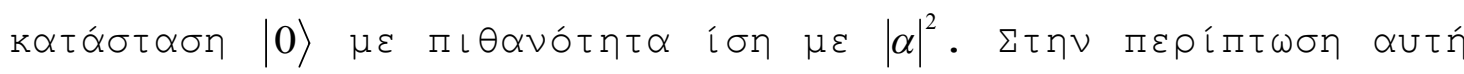

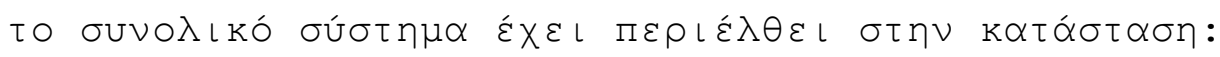




$$
|0\rangle_{A} \otimes|0\rangle_{B}
$$

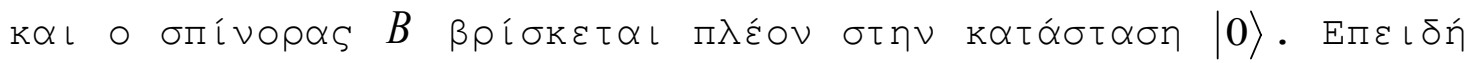

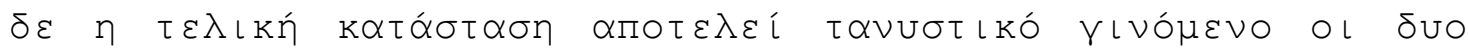

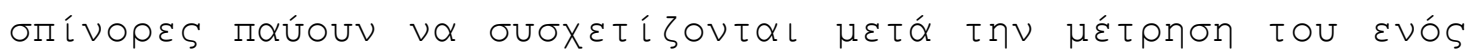

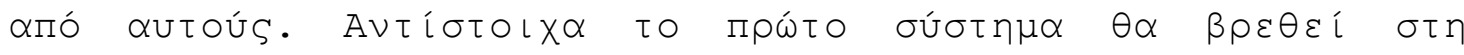

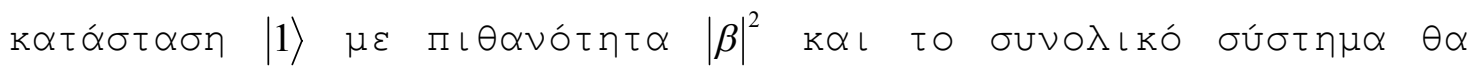

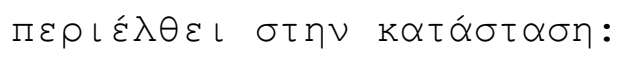

$$
|1\rangle_{A} \otimes|1\rangle_{B}
$$

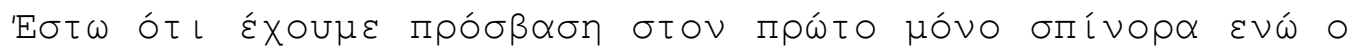

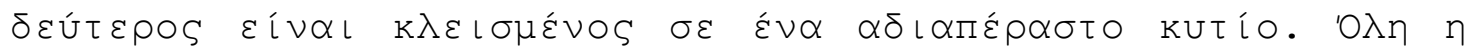

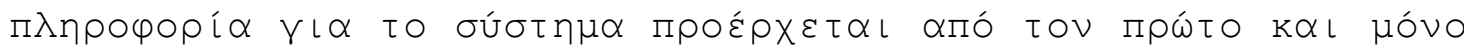
бாі́

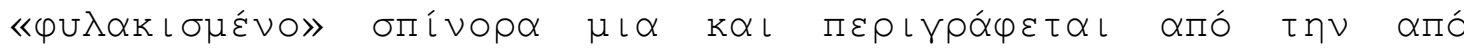

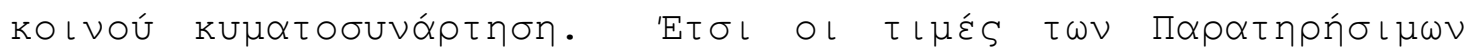

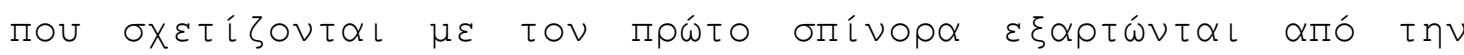

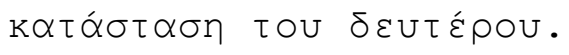

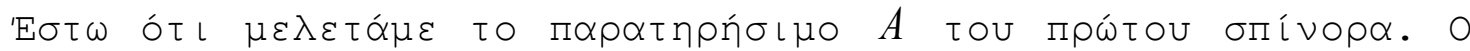

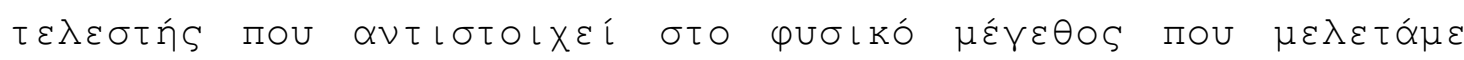

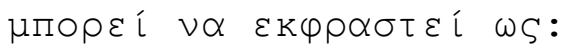

$$
A_{1} \otimes 1_{2}
$$




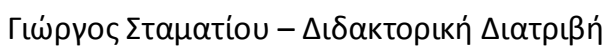

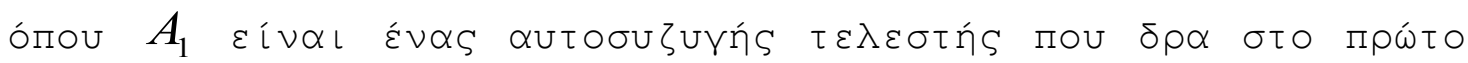

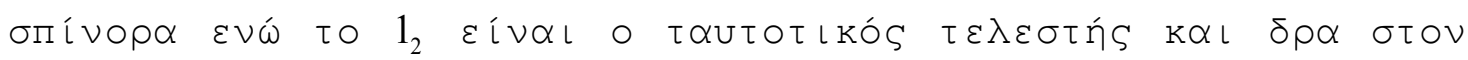

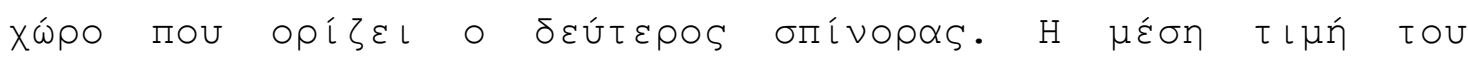

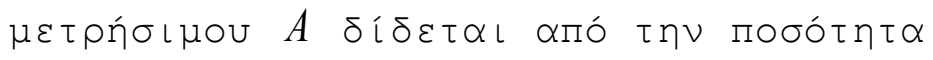

$$
\begin{gathered}
\left\langle\Psi\left|A_{1} \otimes 1_{2}\right| \Psi\right\rangle= \\
=\left[\left(\alpha^{*}\left\langle 00\left|+\beta^{*}\langle 11|\right) A_{1} \otimes 1_{2}(\alpha|00\rangle+\beta|11\rangle)\right]=\right.\right. \\
=|\alpha|^{2}\left\langle 0\left|A_{1}\right| 0\right\rangle+|\beta|^{2}\left\langle 1\left|A_{1}\right| 1\right\rangle
\end{gathered}
$$

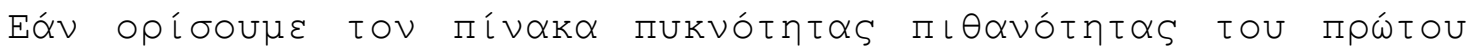

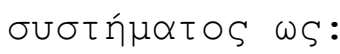

$$
\rho_{1}=|a|^{2}|0\rangle\left\langle\left. 0|+| \beta\right|^{2} \mid 1\right\rangle\langle 1|
$$

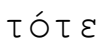

$$
\langle A\rangle=\operatorname{tr}\left(\rho_{1} A_{1}\right)
$$

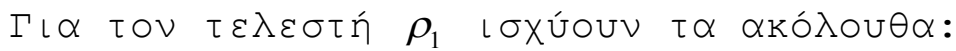

$$
\begin{aligned}
& \operatorname{tr}\left(\rho_{1}\right)=1
\end{aligned}
$$

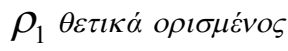

$$
\begin{aligned}
& \rho_{1}=\rho_{1}^{+} \varepsilon \rho \mu \imath \tau \iota \alpha \nu o ́ \varsigma
\end{aligned}
$$

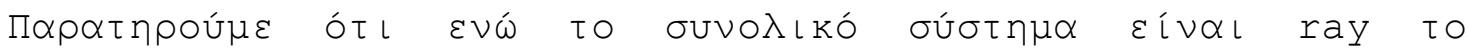

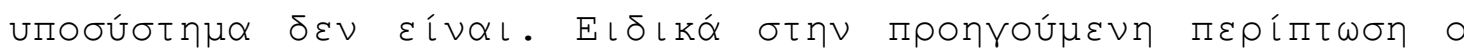

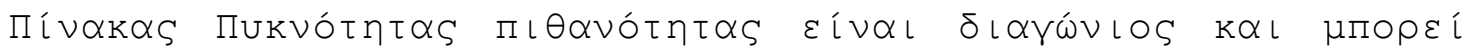

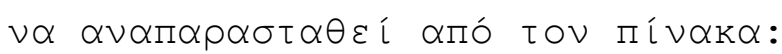

$$
\rho_{1}=\left(\begin{array}{cc}
|a|^{2} & 0 \\
0 & |\beta|^{2}
\end{array}\right)
$$

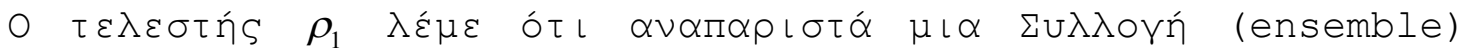

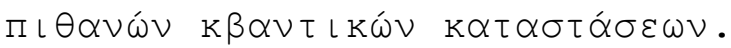




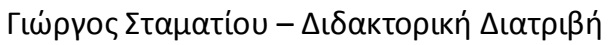

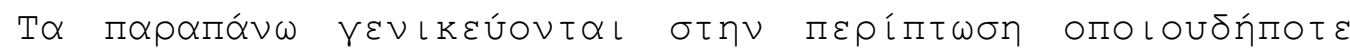

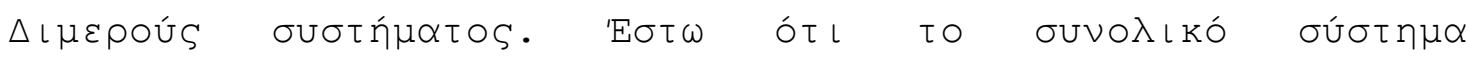

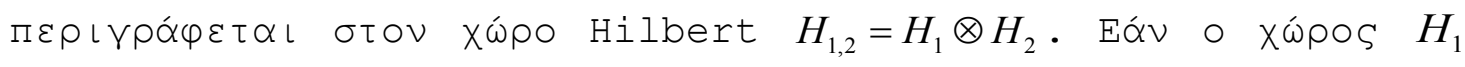

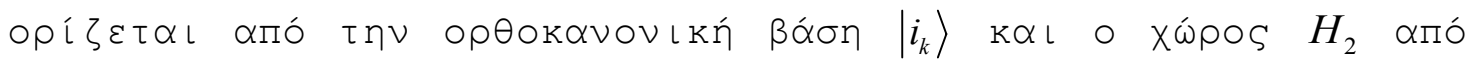

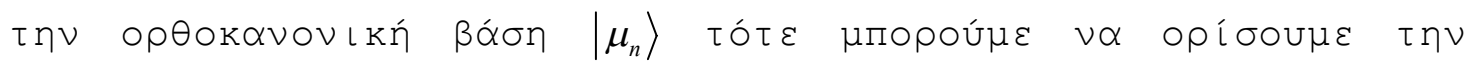

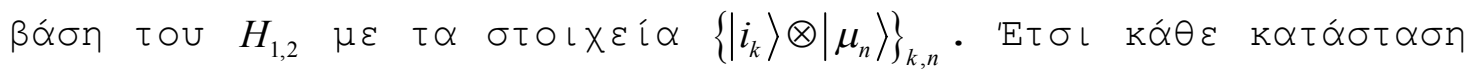

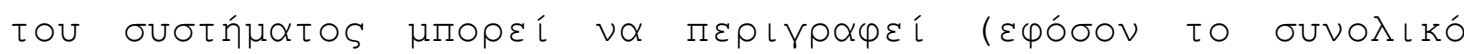

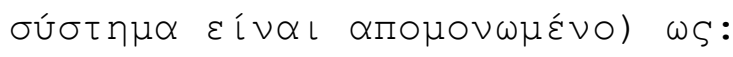

$$
|\Psi\rangle=\sum_{k=1}^{K} \sum_{n=1}^{N} c_{k, n}\left|i_{k}\right\rangle \otimes\left|\mu_{n}\right\rangle
$$

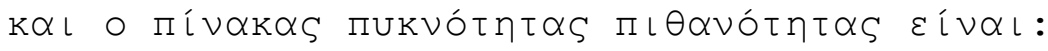

$$
\rho=|\Psi\rangle\langle\Psi|
$$

ń

$$
\rho=\sum_{k, k^{\prime}, n, n^{\prime}} c_{k^{\prime}, n^{\prime}}^{*} c_{k, n}\left|l_{k} \mu_{n}\right\rangle\left\langle l_{k^{\prime}} \mu_{n^{\prime}}\right|
$$

opíbovtas ws Mepıkó 'IXvos (partial trace) tou $\rho$ ws npos tov

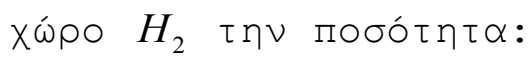

$$
\begin{gathered}
\rho_{1}=t r_{2}(\rho) \\
=\sum_{n=1}^{N}\left\langle\mu_{n}|\rho| \mu_{n}\right\rangle
\end{gathered}
$$

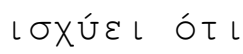

$$
\rho_{1}=\sum_{k, k^{\prime}, n} c_{k^{\prime}, n}^{*} c_{k, n}\left|\iota_{k}\right\rangle\left\langle u_{k^{\prime}}\right|
$$

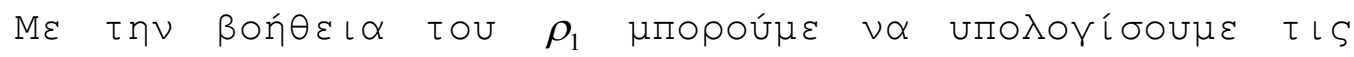

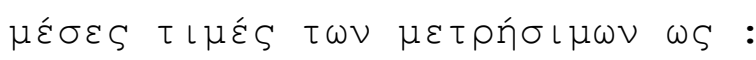

$$
\langle A\rangle=\operatorname{tr}\left(\rho_{1} A_{1}\right)
$$




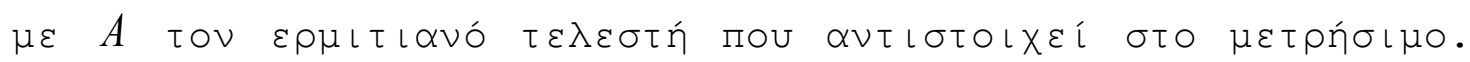

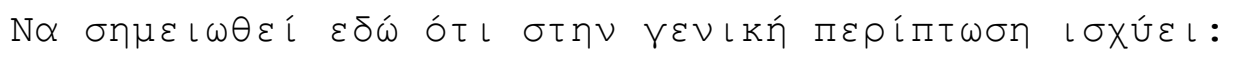

$$
\operatorname{tr}\left(\rho_{1}^{2}\right) \leq 1
$$

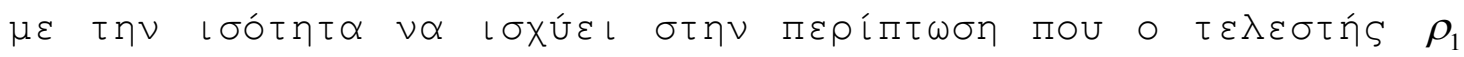

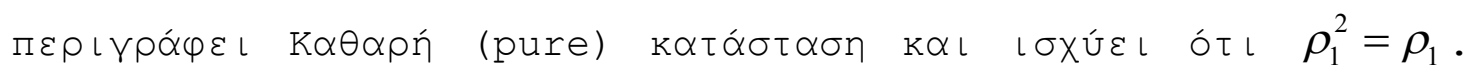

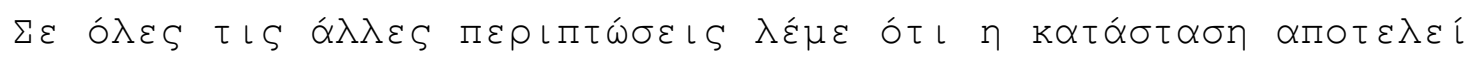
Mi $ү \mu \alpha$.

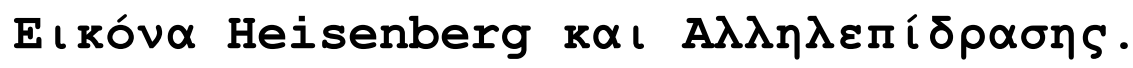

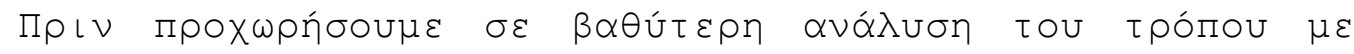

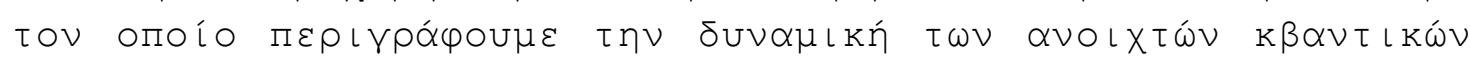

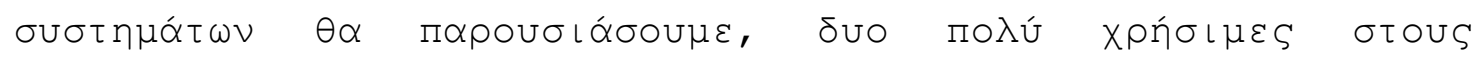

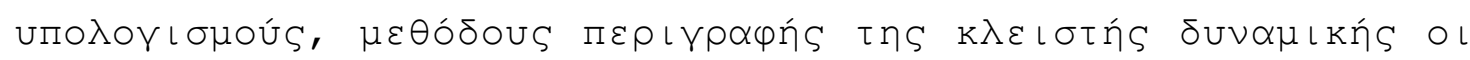

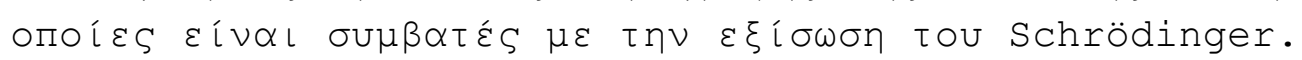

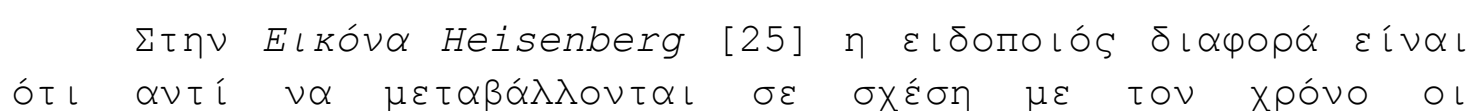

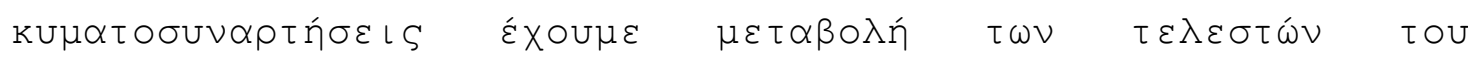

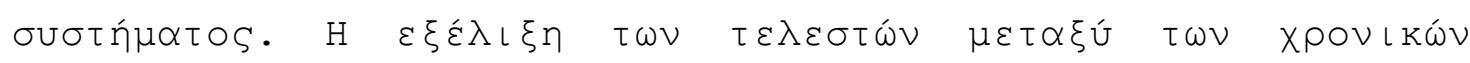

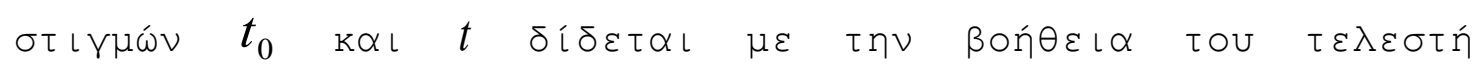

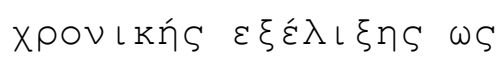

$$
A_{H}(t)=U^{+}\left(t, t_{0}\right) A(t) U\left(t, t_{0}\right)
$$

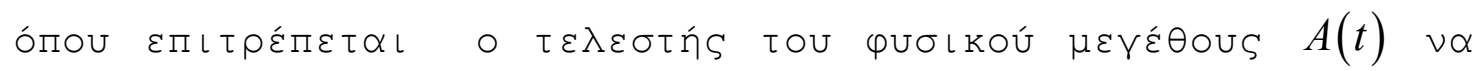

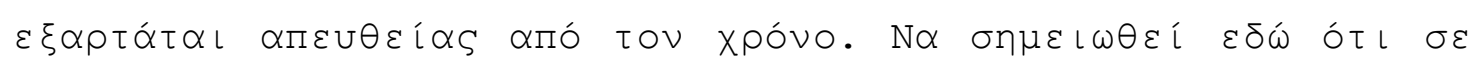

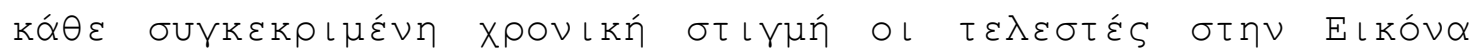

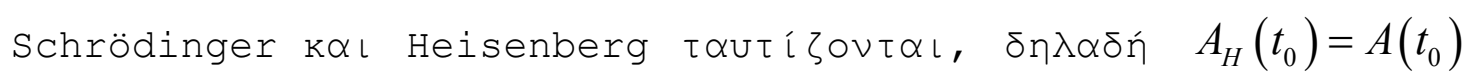

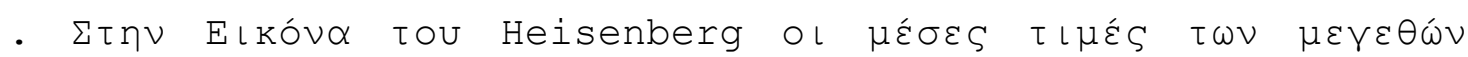

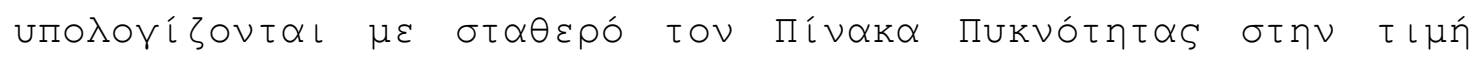
$\rho_{H}\left(t_{0}\right)$. 


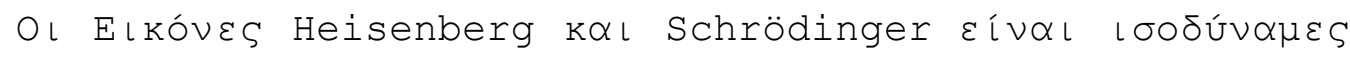

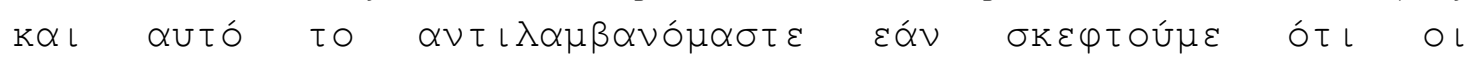

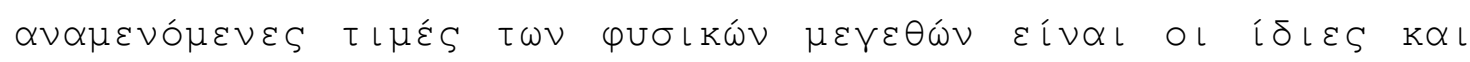

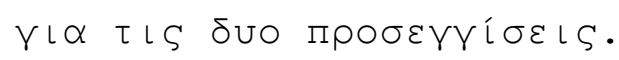

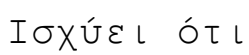

$$
\langle A(t)\rangle=\operatorname{tr}[A(t) \rho(t)]=\operatorname{tr}\left[A_{H}(t) \rho_{H}\left(t_{0}\right)\right]
$$

Eпi ons

$$
\frac{d A_{H}}{d t}=i\left[H_{H}(t), A_{H}(t)\right]+\frac{\partial A_{H}}{\partial t}
$$

$\mu \varepsilon$

$$
H_{H}(t)=U^{+}\left(t, t_{0}\right) H(t) U\left(t, t_{0}\right)
$$

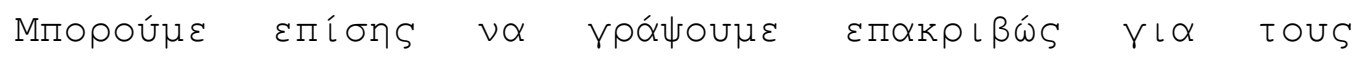

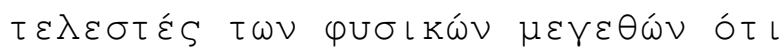

$$
\frac{\partial A_{H}(t)}{\partial t}=U^{+}\left(t, t_{0}\right) \frac{\partial A(t)}{\partial t} U\left(t, t_{0}\right)
$$

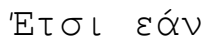

$$
\frac{d A_{H}(t)}{d t}=0
$$

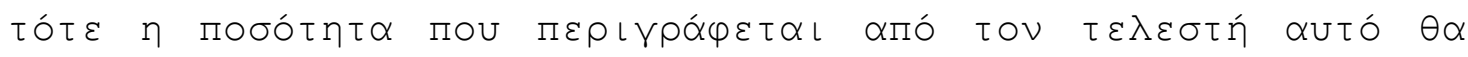

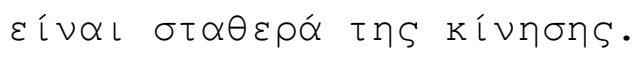

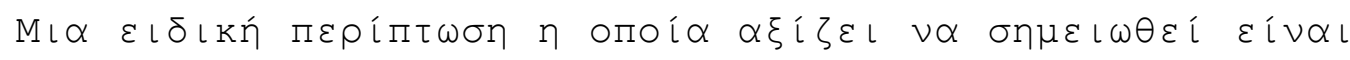

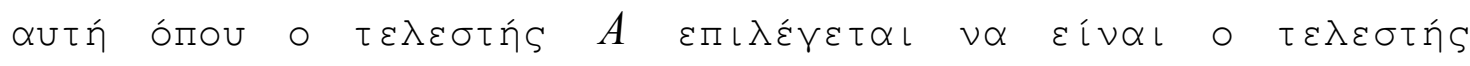

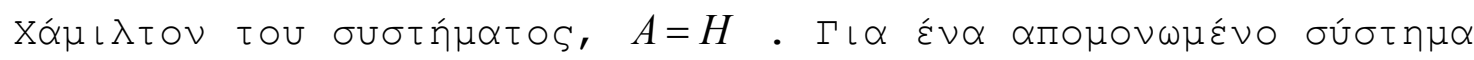

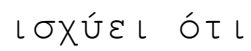

$$
\frac{\partial H}{\partial t}=0
$$




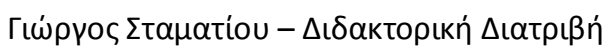

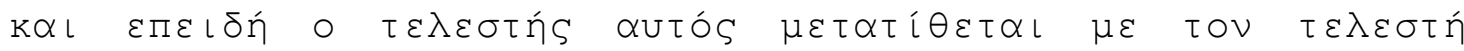

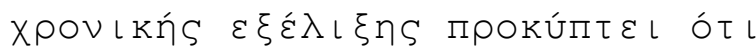

$$
\frac{d H_{H}}{d t}=0
$$

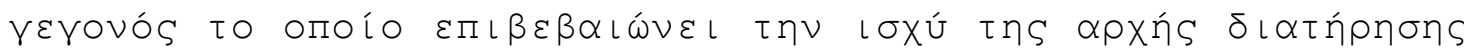

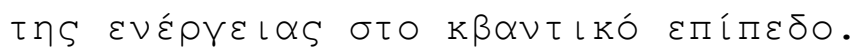

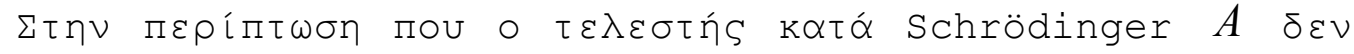

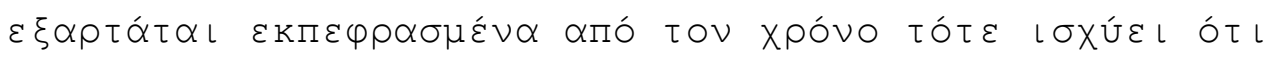

$$
\frac{d A_{H}(t)}{d t}=i\left[H, A_{H}(t)\right]
$$

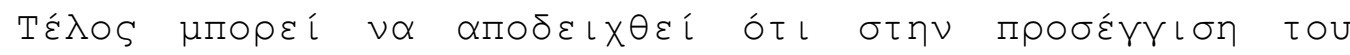

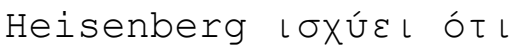

$$
\begin{gathered}
\frac{d\langle A(t)\rangle}{d t}=\left\langle\frac{d A_{H}(t)}{d t}\right\rangle= \\
\left.=\operatorname{tr}\left\{i\left[H_{H}(t), A_{H}(t)\right]+\frac{\partial A_{H}(t)}{\partial t}\right) \rho_{H}\left(t_{0}\right)\right\}
\end{gathered}
$$

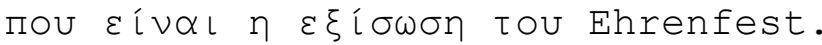

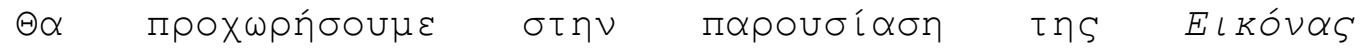

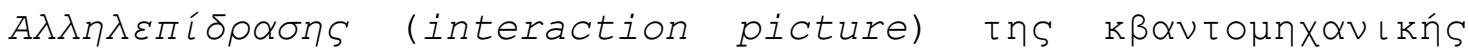

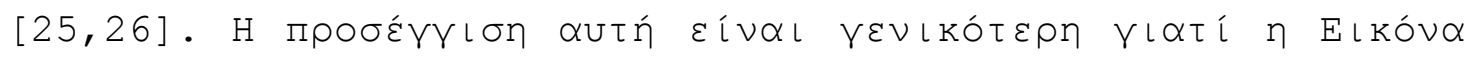

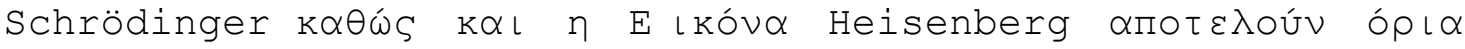

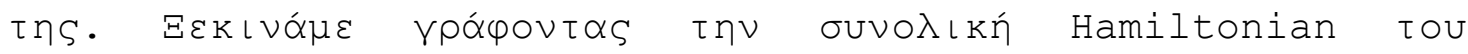

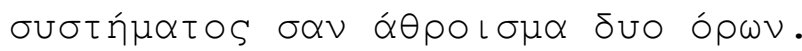

$$
H(t)=H_{0}+\hat{H}_{I}(t)
$$

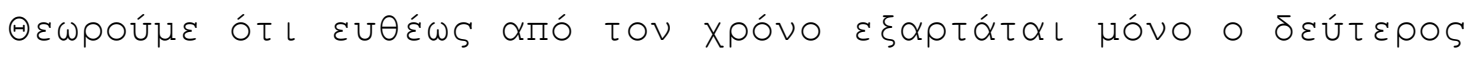

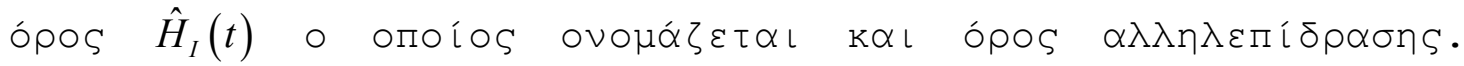




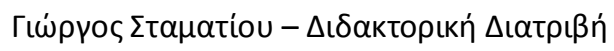

X X

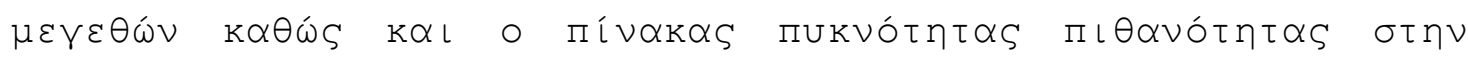

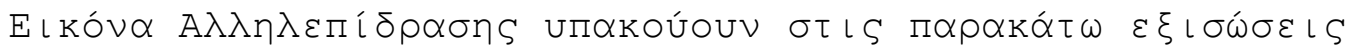

$$
A_{I}(t) \equiv U_{0}^{+}\left(t, t_{0}\right) A(t) U_{0}\left(t, t_{0}\right)
$$

$K \propto L$

$$
\rho_{I}(t) \equiv U_{I}\left(t, t_{0}\right) \rho\left(t_{0}\right) U_{I}^{+}\left(t, t_{0}\right)
$$

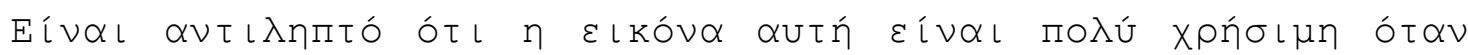

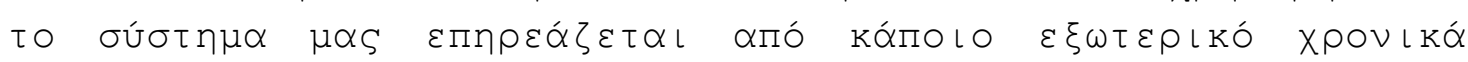

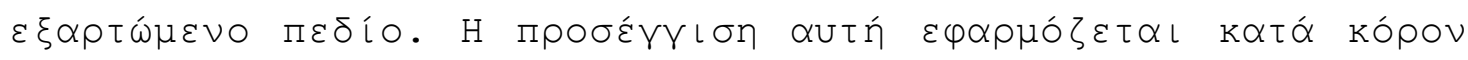

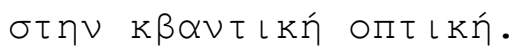

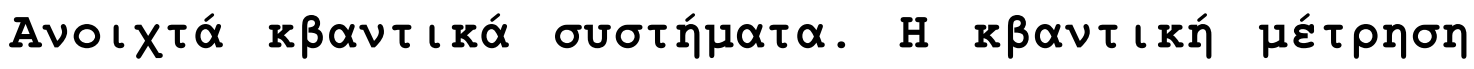

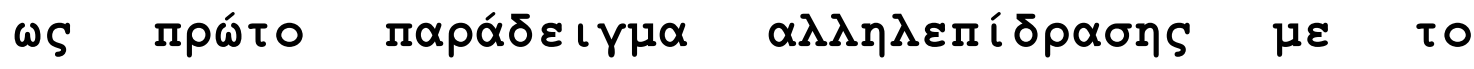

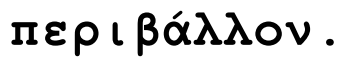

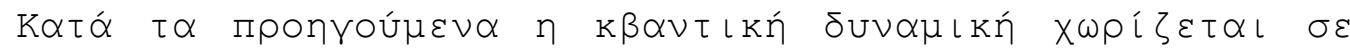

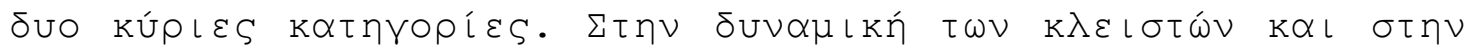

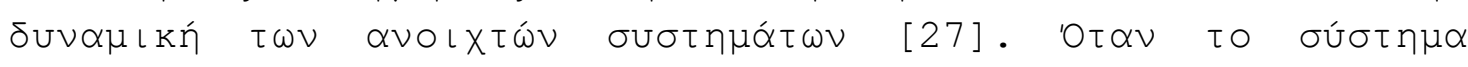

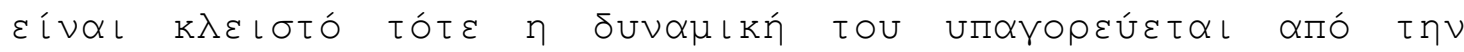

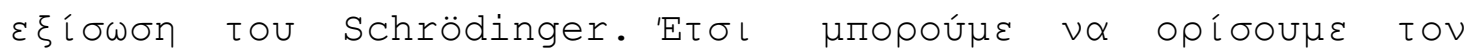

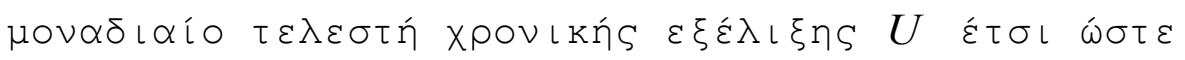

$$
i \hbar \frac{\partial U}{\partial t}=H U
$$

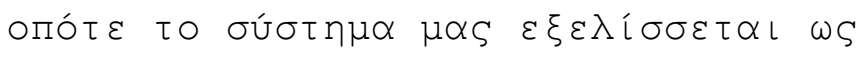

$$
|\Psi(t)\rangle=U|\Psi(0)\rangle
$$

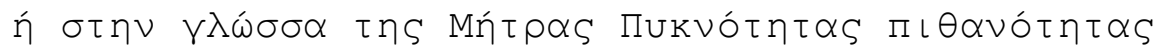

$$
\rho(t)=U \rho(0) U^{+}
$$

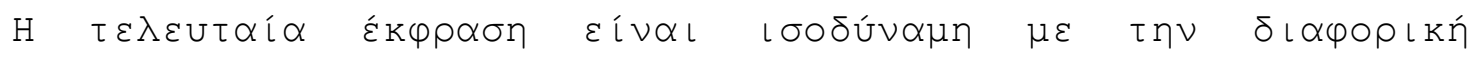
$\varepsilon \xi i \sigma \omega \sigma \eta \Pi \iota \nu \alpha ́ k \omega \nu$ 


$$
\dot{\rho}=-\frac{i}{\hbar}[H, \rho]
$$

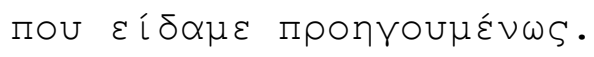

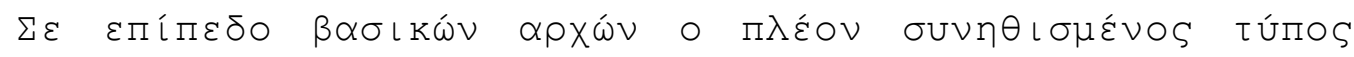

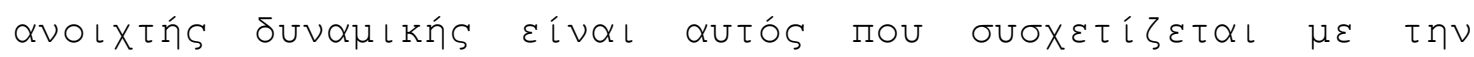

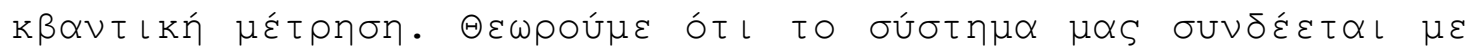

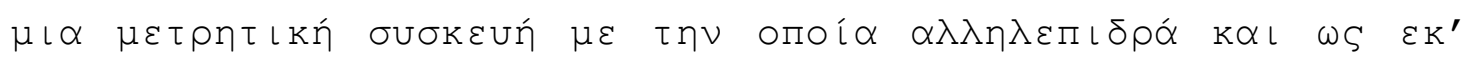

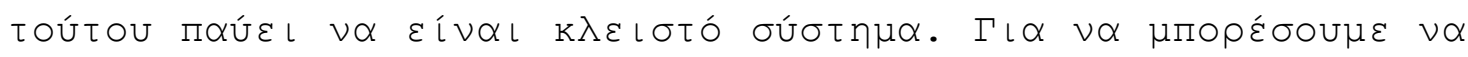

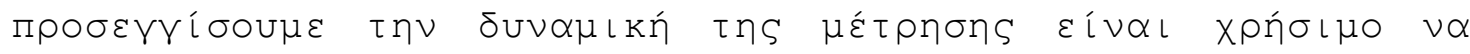

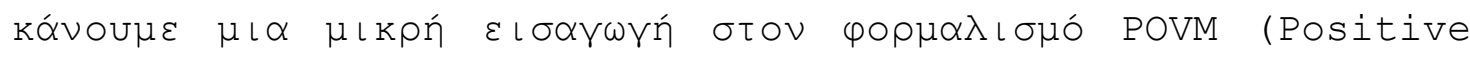

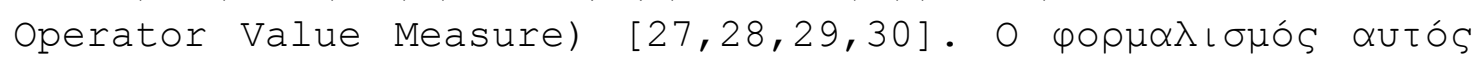

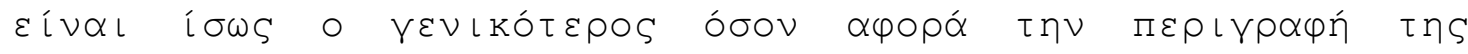

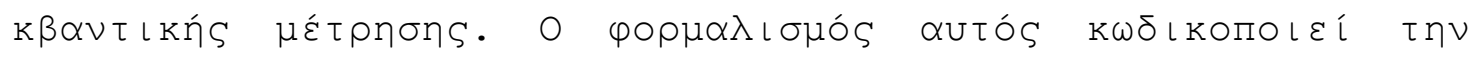

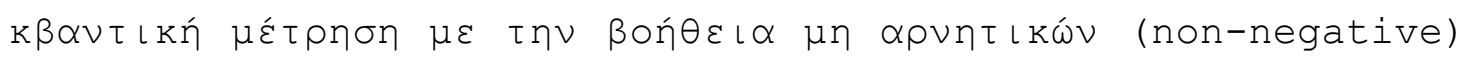

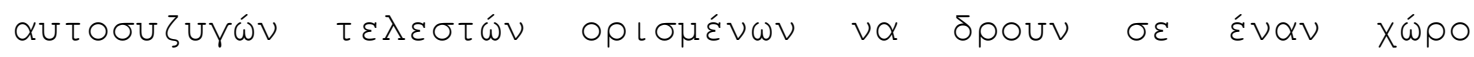

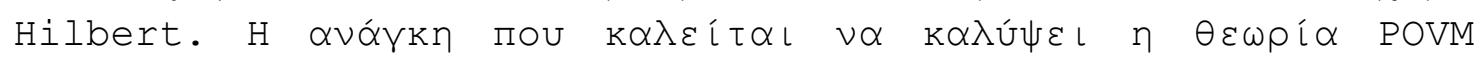

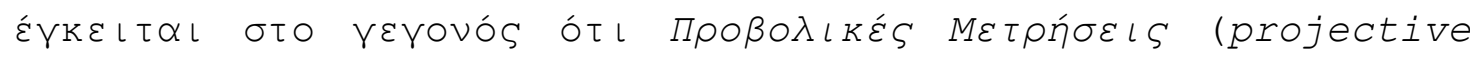

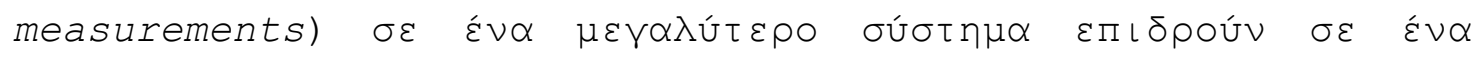

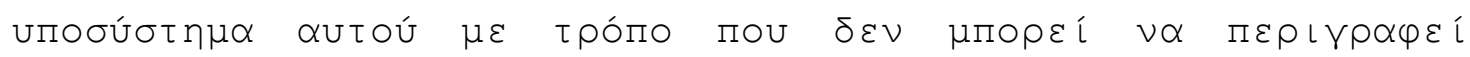

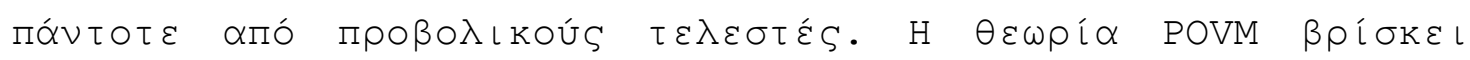

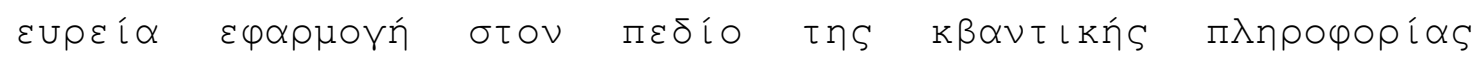

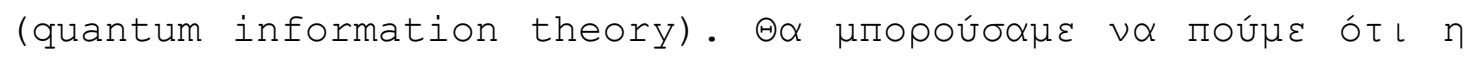

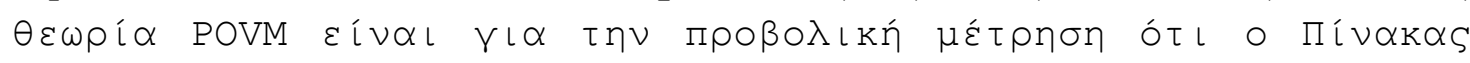

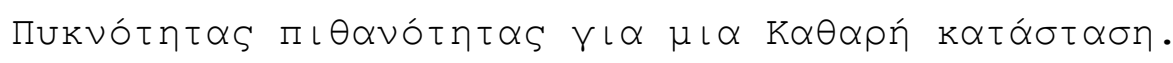

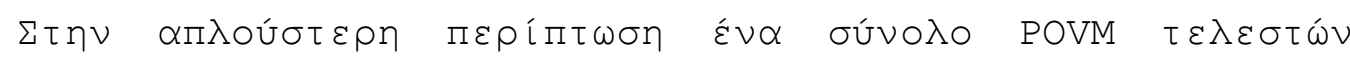

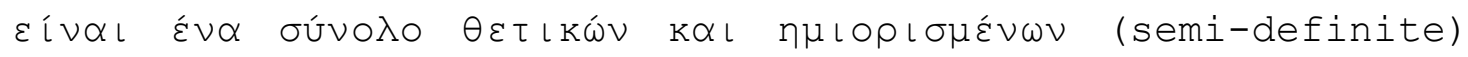

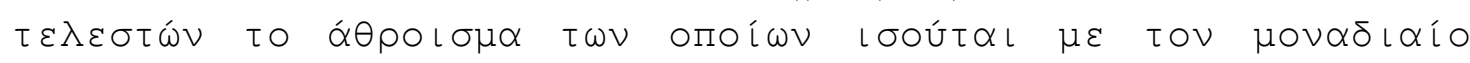
$\tau \varepsilon \lambda \varepsilon \sigma \tau \dot{n}$.

$$
\begin{gathered}
\sum_{i=1}^{M} F_{i}=1 \\
F_{i}>0
\end{gathered}
$$

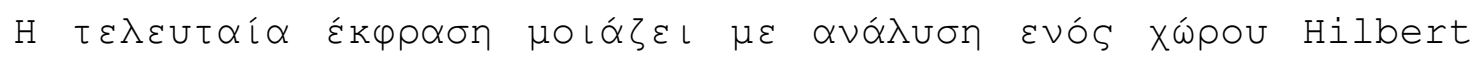

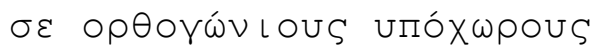




$$
\sum_{i=1}^{N} E_{i}=1
$$

$\mu \varepsilon$

$$
E_{i} E_{j}=\delta_{i j} E_{i}
$$

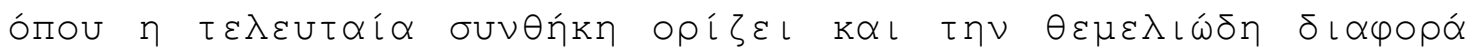

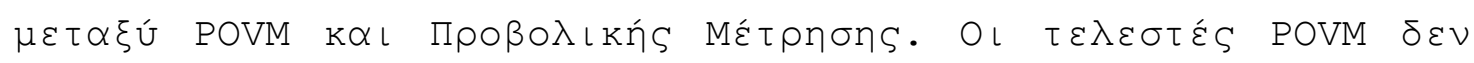

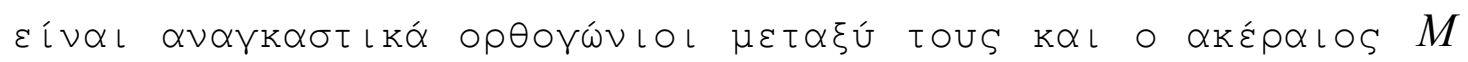

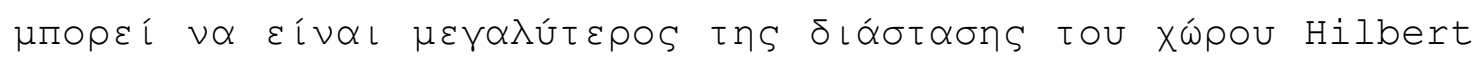

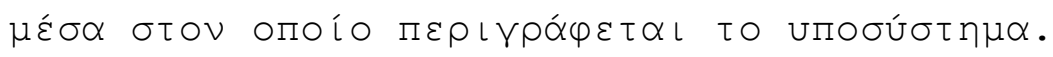

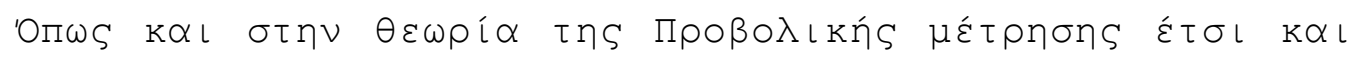

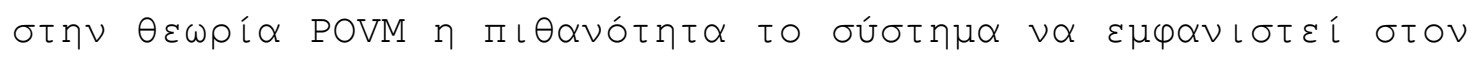

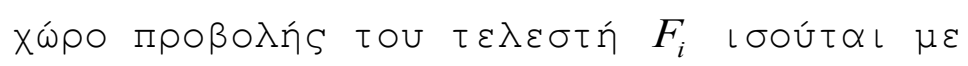

$$
P_{i}=\operatorname{tr}\left(F_{i} \rho\right)
$$

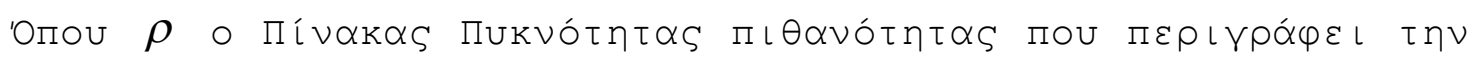

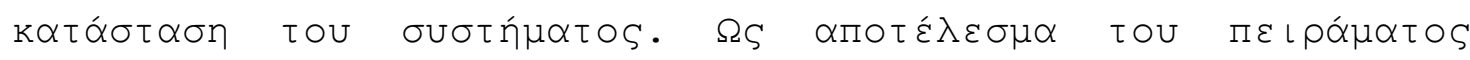

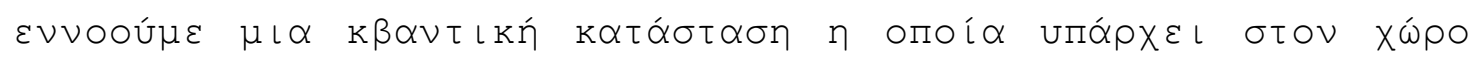

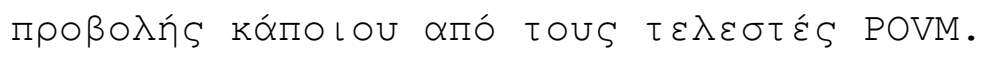

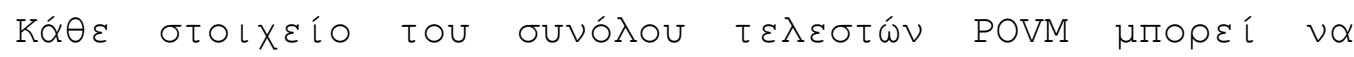

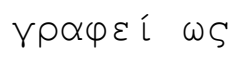

$$
F_{i}=M_{i}^{+} M_{i}
$$

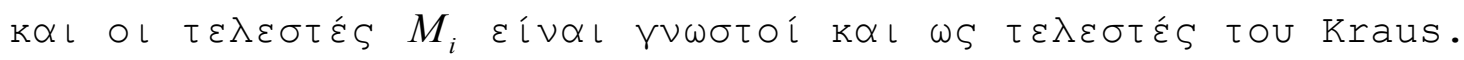

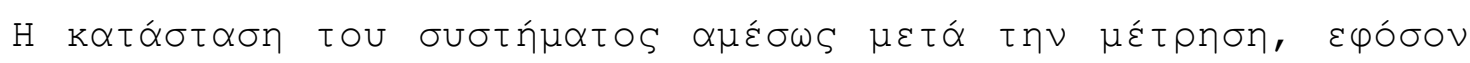

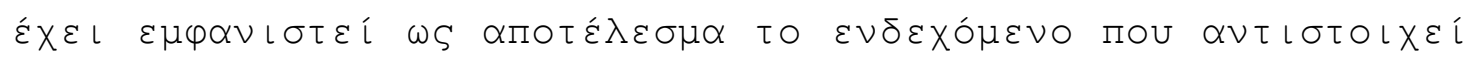

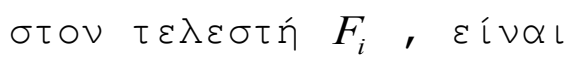

$$
\rho_{\text {After }}=\frac{M_{i}^{+} \rho M_{i}}{\operatorname{tr}\left(M_{i}^{+} \rho M_{i}\right)}
$$

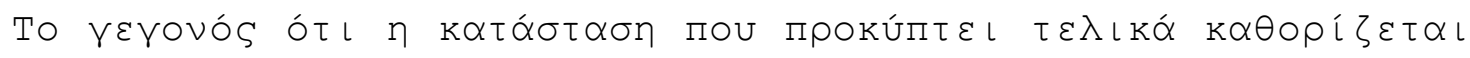

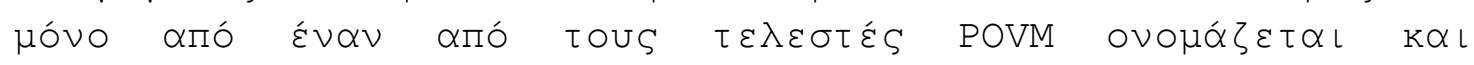




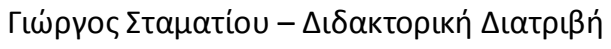

Eпı

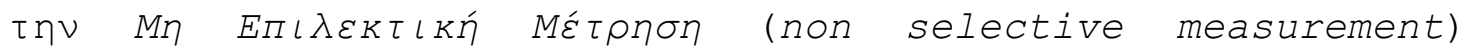

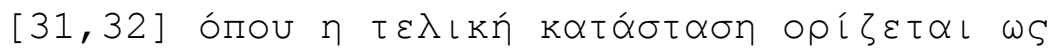

$$
\rho_{\text {After }}=\sum_{i=1}^{M} M_{i}^{+} \rho M_{i}
$$

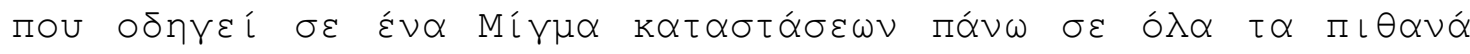

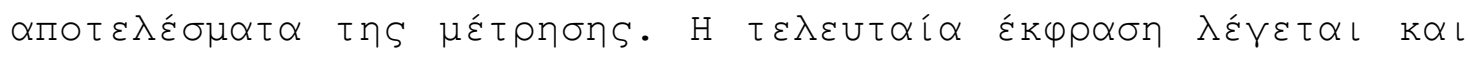

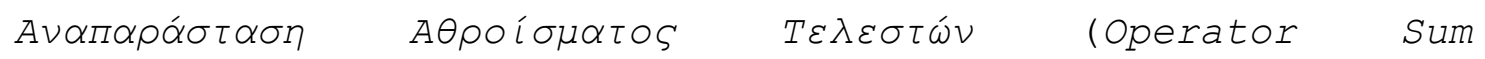

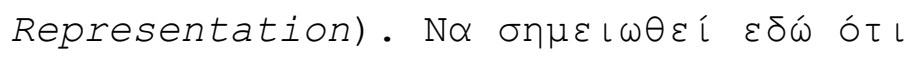

$$
\begin{gathered}
\operatorname{tr}\left(\sum_{i=1}^{M} M_{i}^{+} \rho M_{i}\right)= \\
=\operatorname{tr}\left(F_{i} \rho\right)= \\
=\sum_{i=1}^{M} P_{i}=1
\end{gathered}
$$

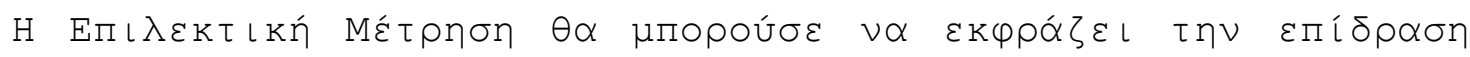

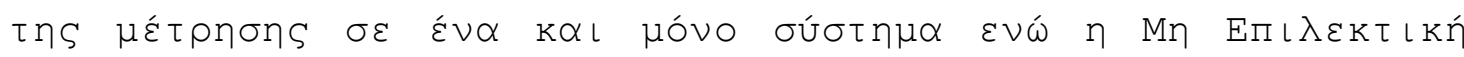

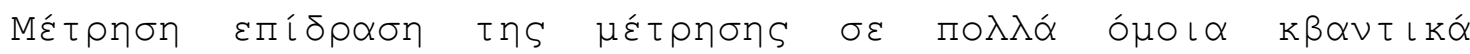

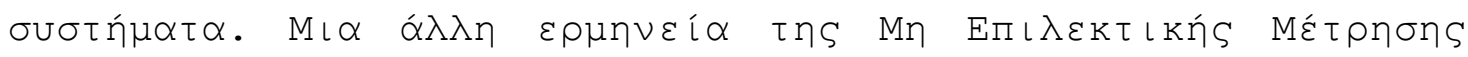

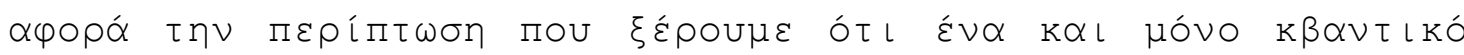

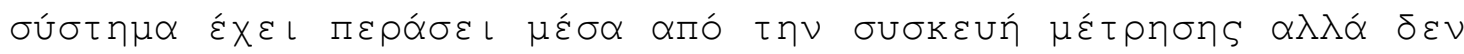

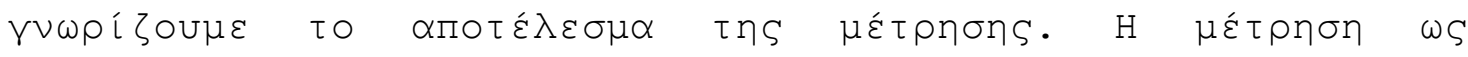

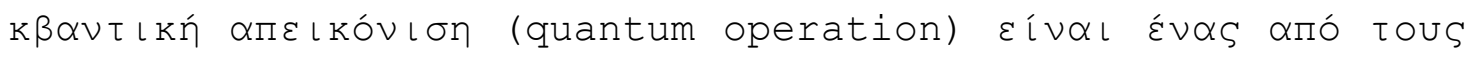

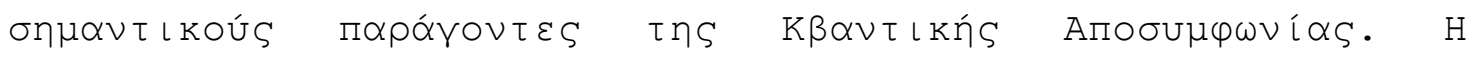

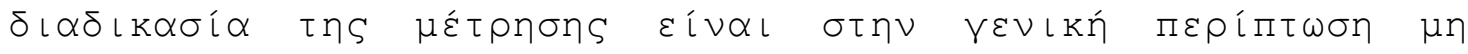

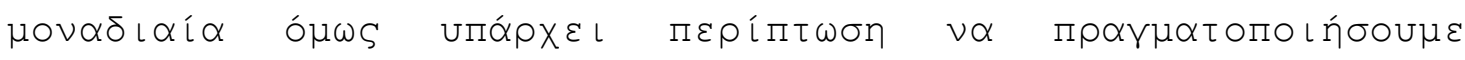
пвір

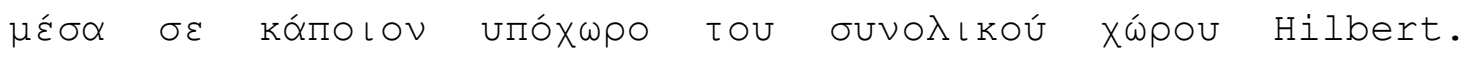

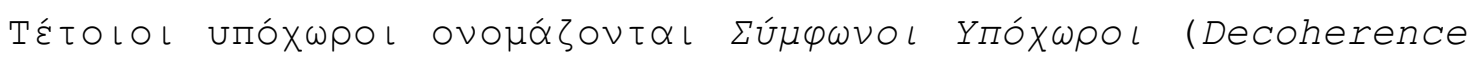
Free Subspaces). 


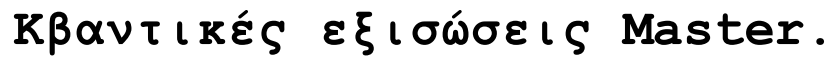

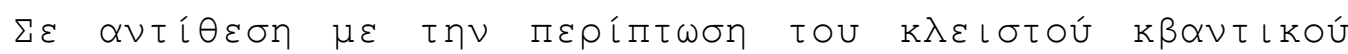

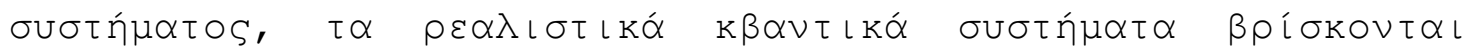

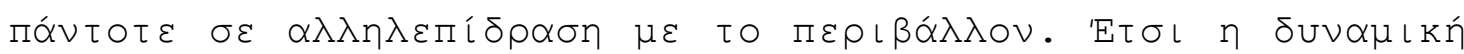

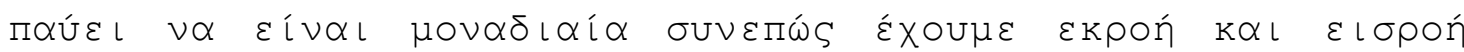

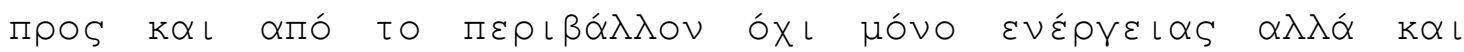

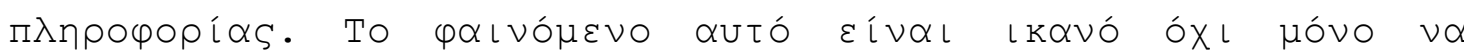

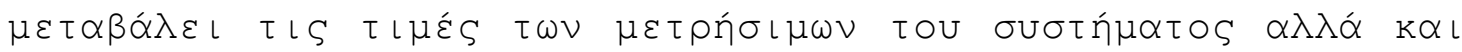

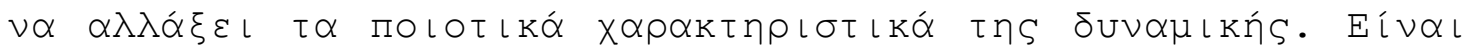

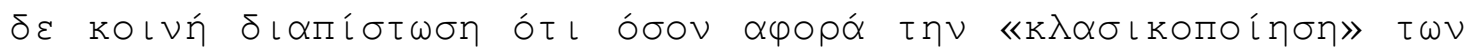

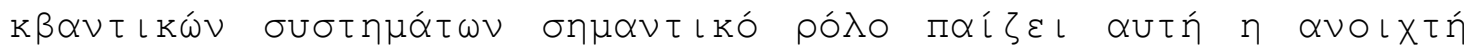

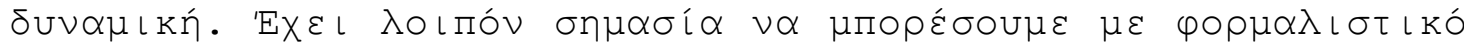

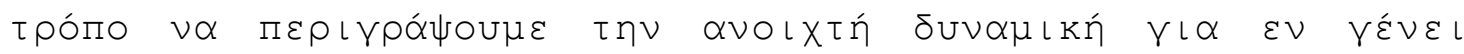

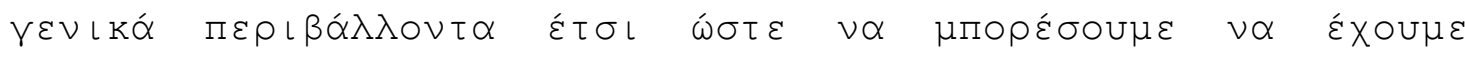

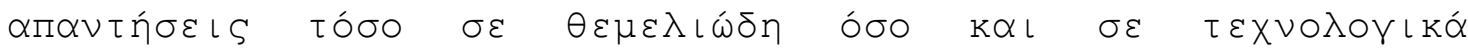

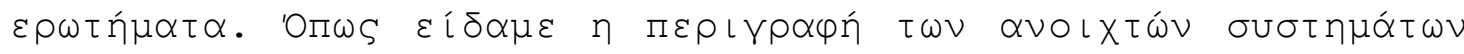

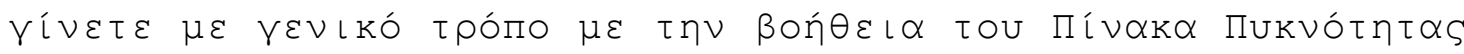

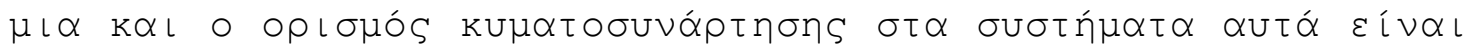

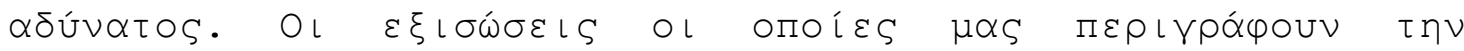

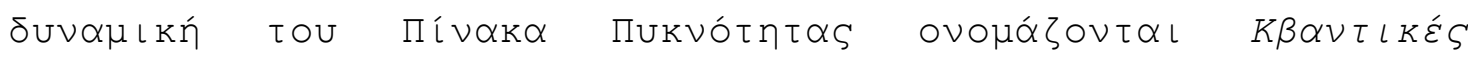

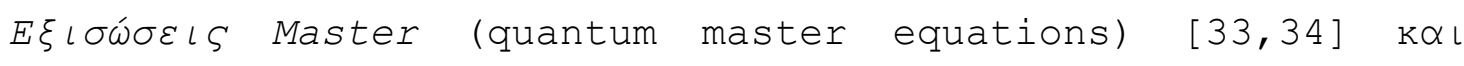

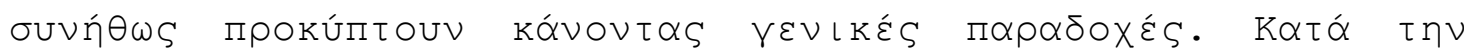

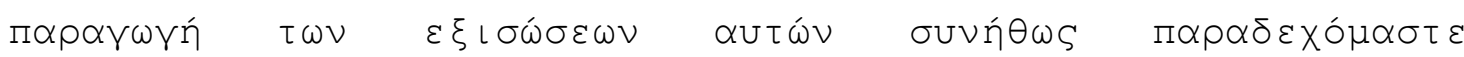

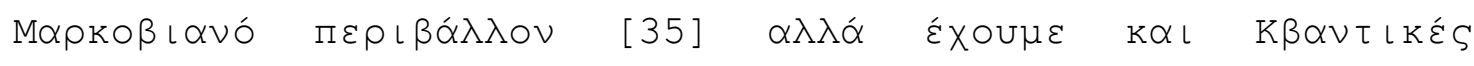

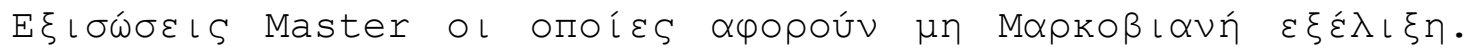

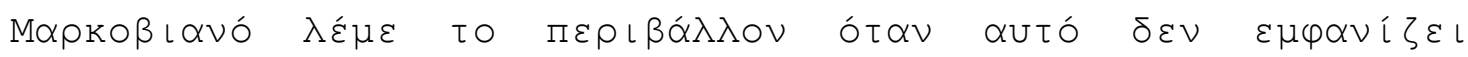

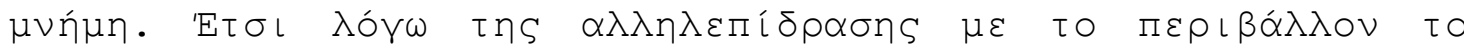

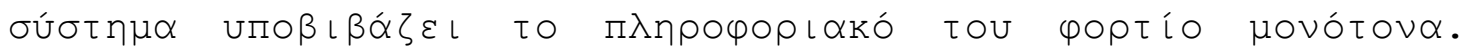

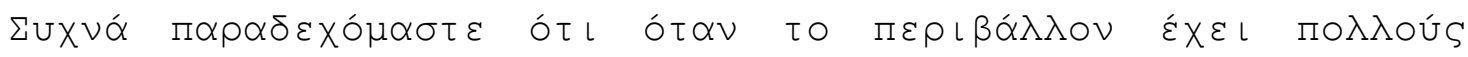

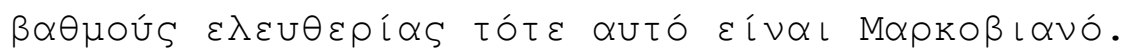

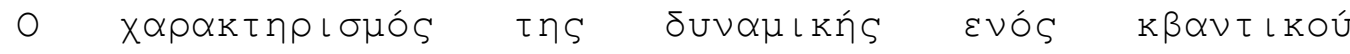

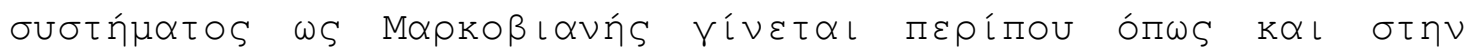

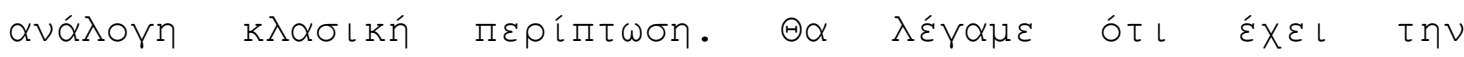

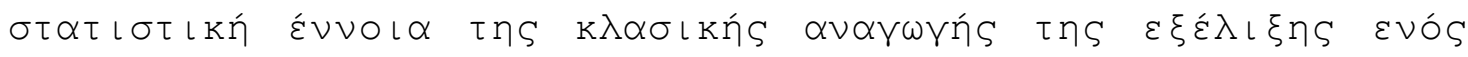

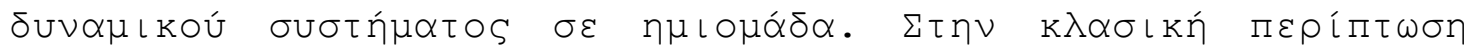

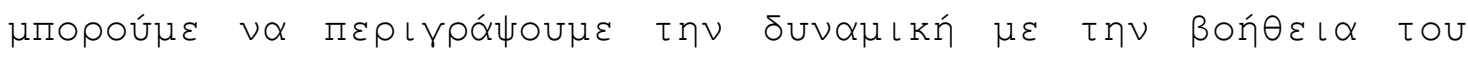

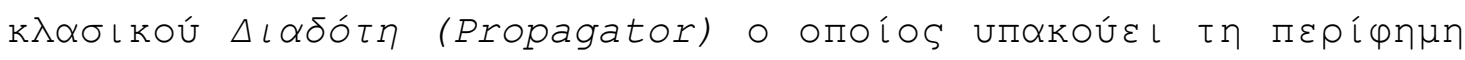
$\varepsilon \xi i \sigma \omega \sigma \eta \tau \omega \nu$ Chapman - Kolmogorov. 


$$
T\left(x_{3}, t_{3} \mid x_{1}, t_{1}\right)=\int d x_{2} T\left(x_{3}, t_{3} \mid x_{2}, t_{2}\right) T\left(x_{2}, t_{2} \mid x_{1}, t_{1}\right)
$$

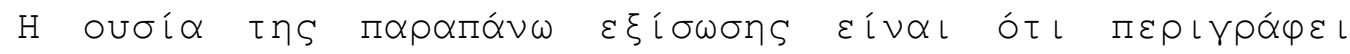

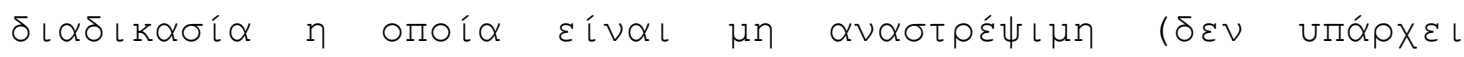

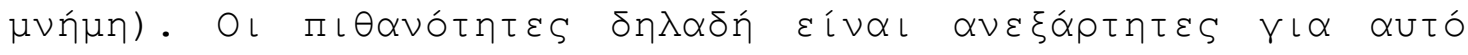

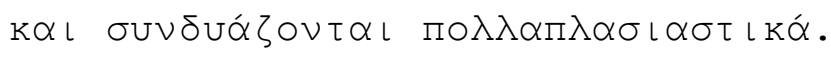

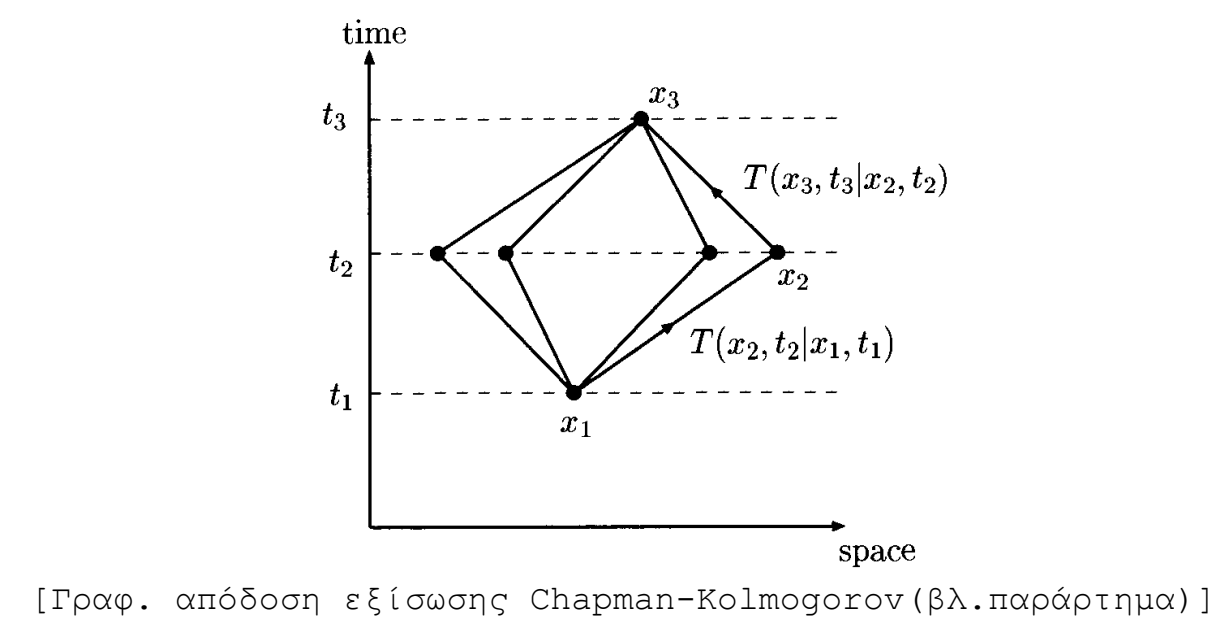

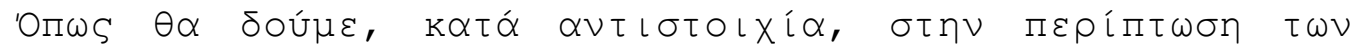

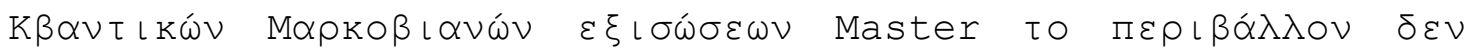

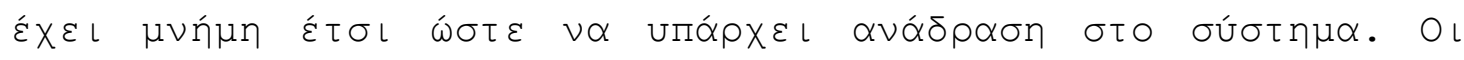

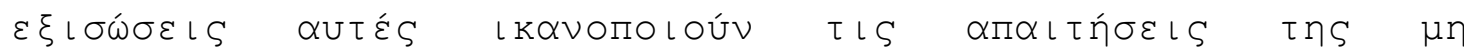

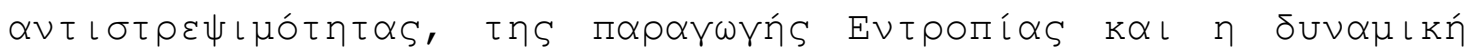

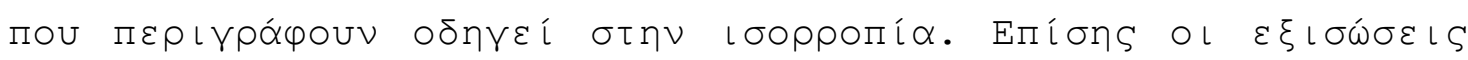

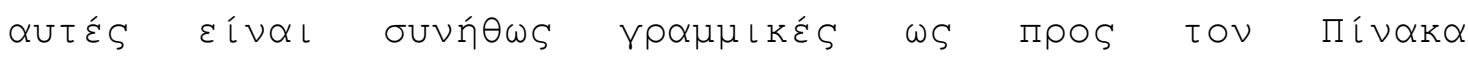

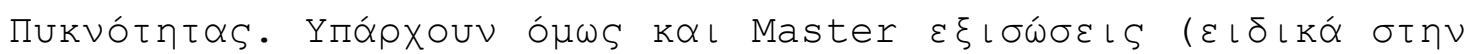

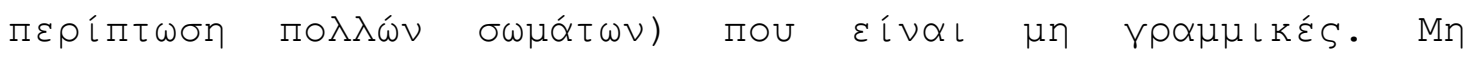

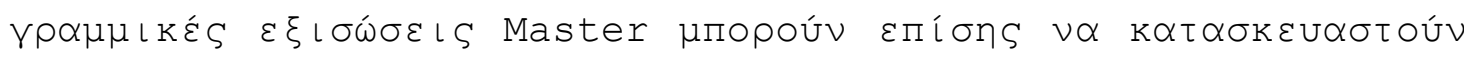

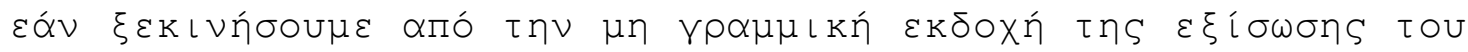

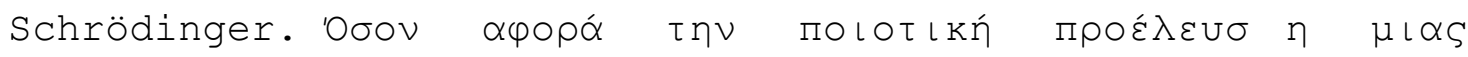

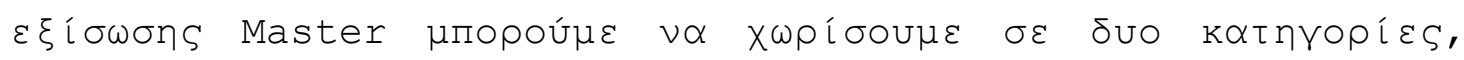

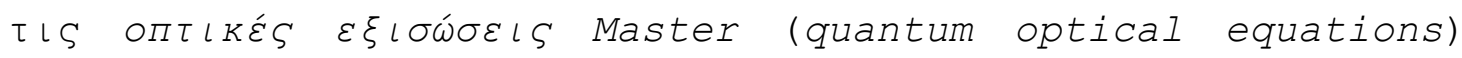

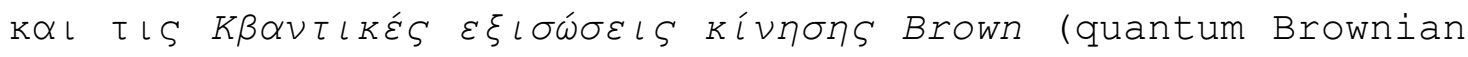

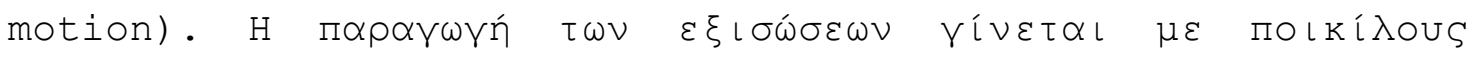

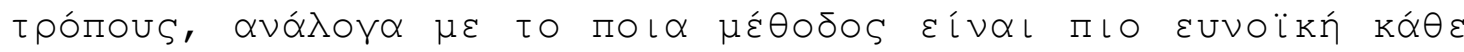

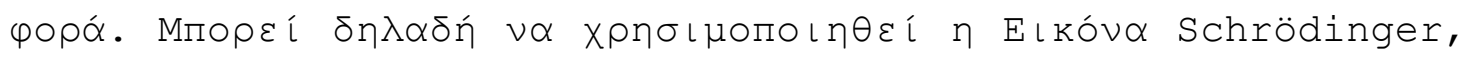

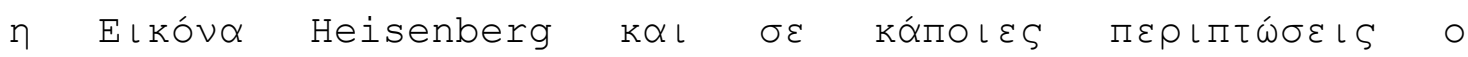




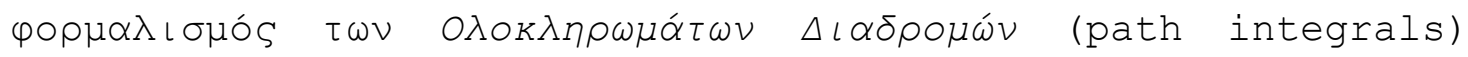

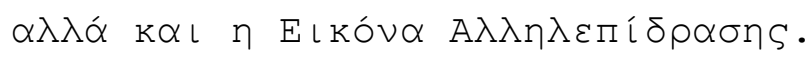

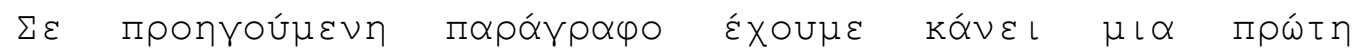

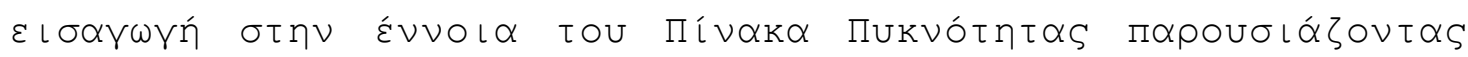

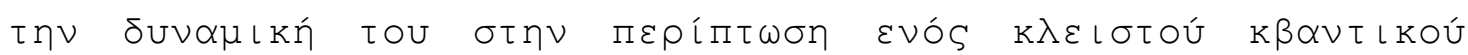

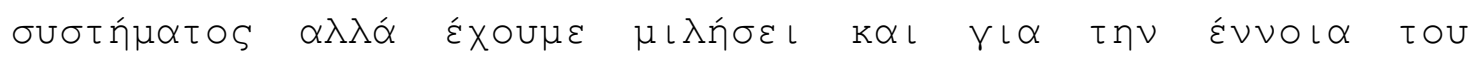

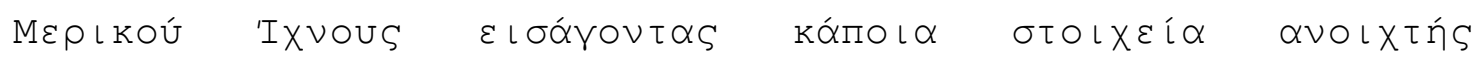

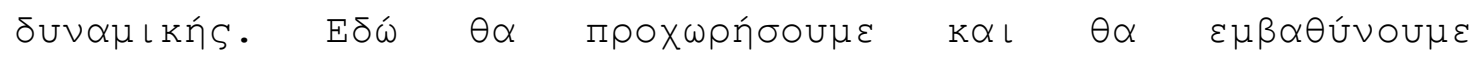

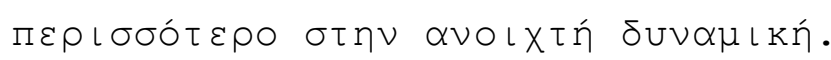

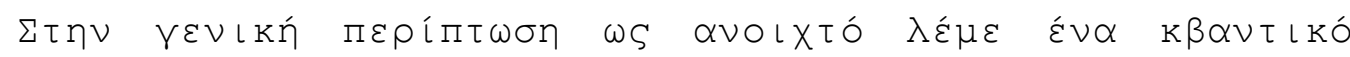

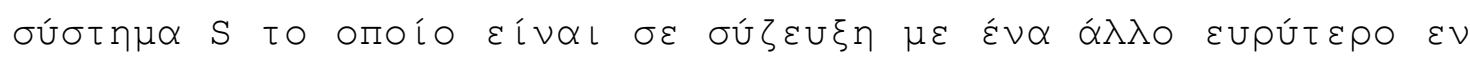

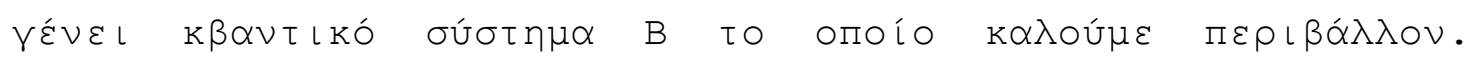

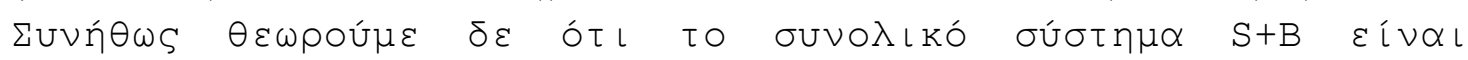

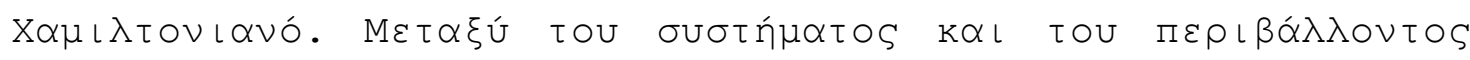

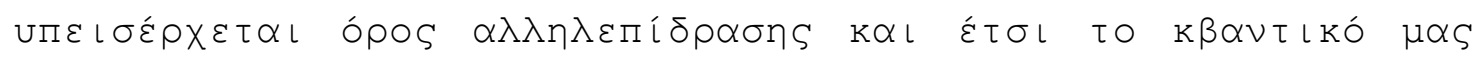

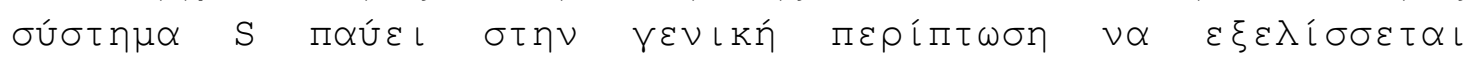
$\mu \circ v \alpha \delta\llcorner\alpha i \alpha$.

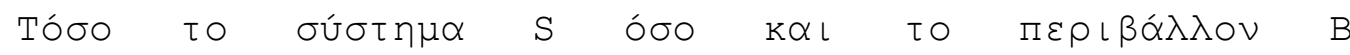

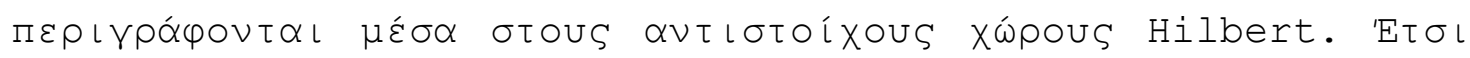

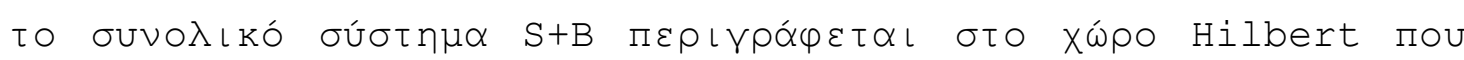

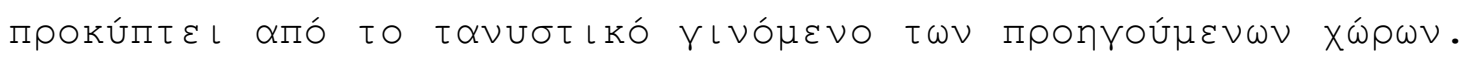

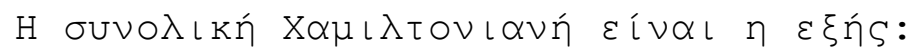

$$
H(t)=H_{S} \otimes I_{B}+I_{S} \otimes H_{B}+\hat{H}_{I}(t)
$$

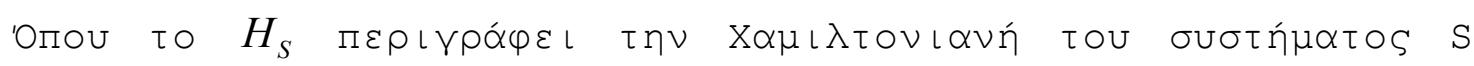
(

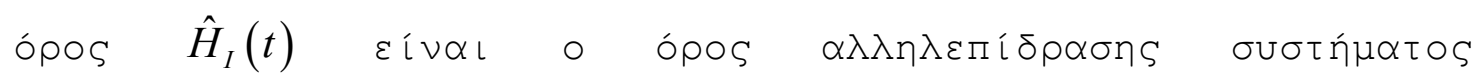

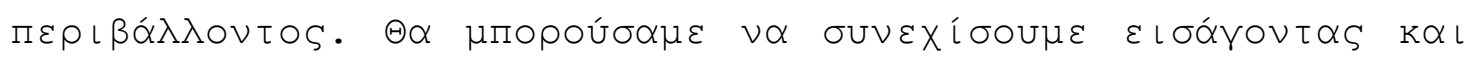

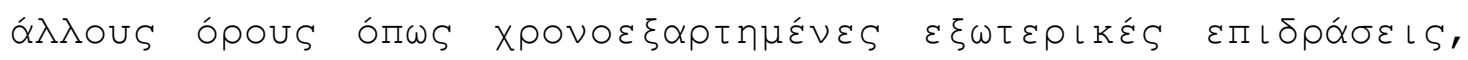

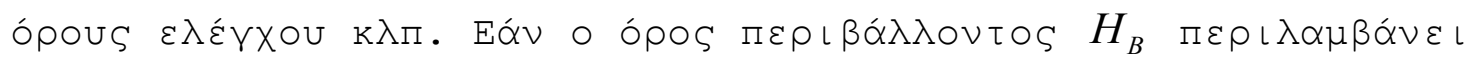

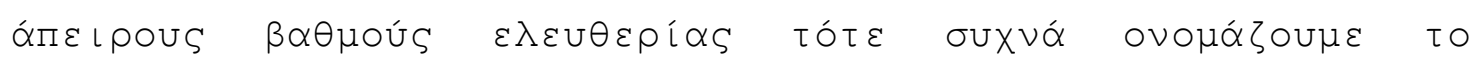

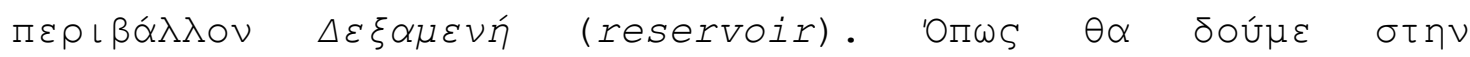

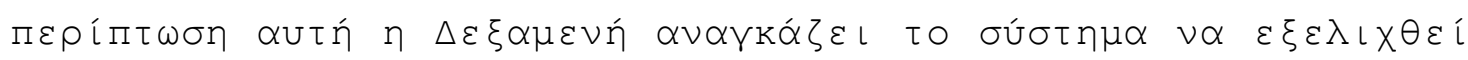

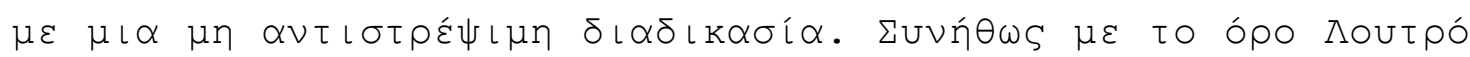

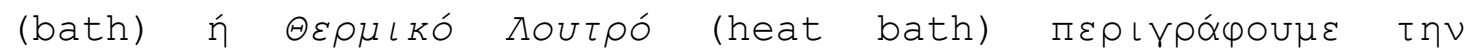

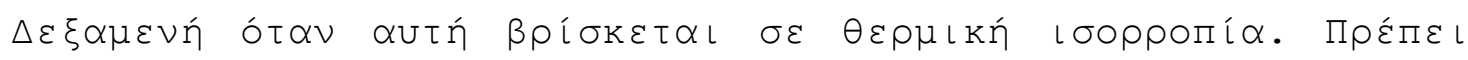




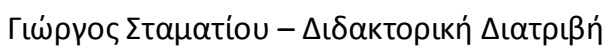

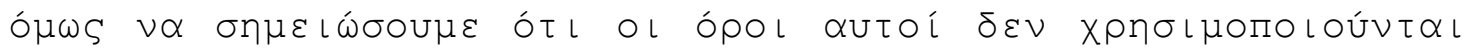

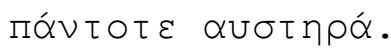

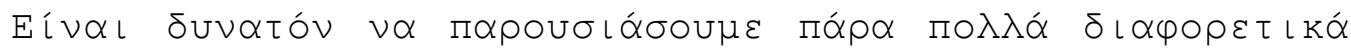

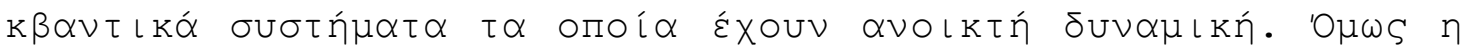

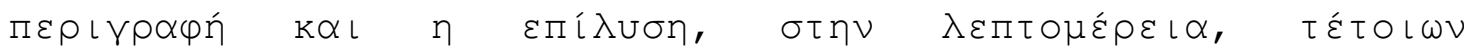

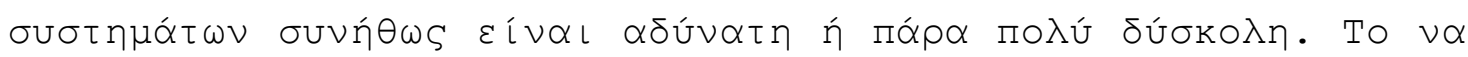

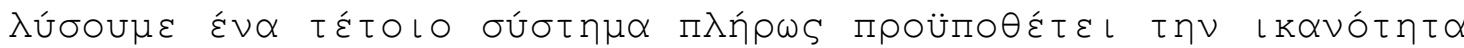

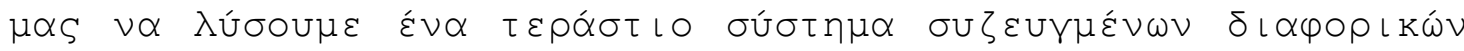

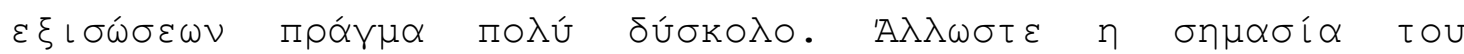

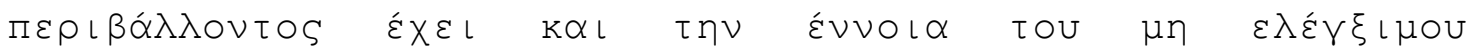

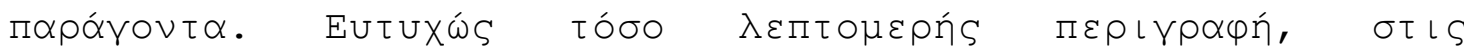

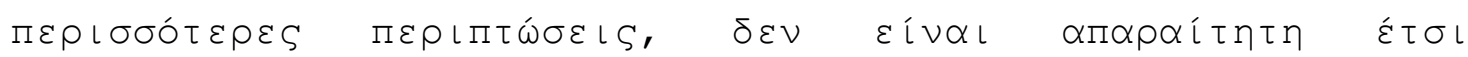

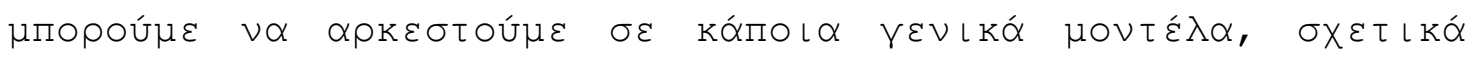

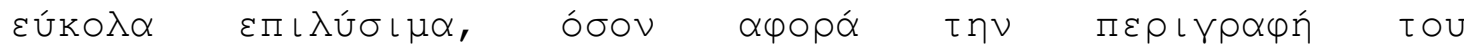

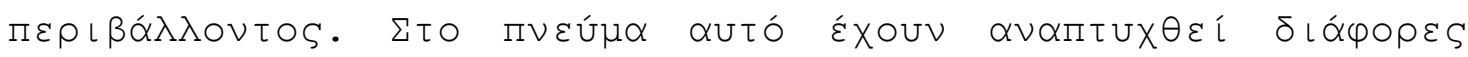

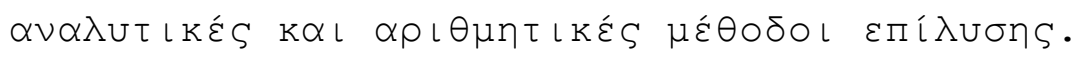

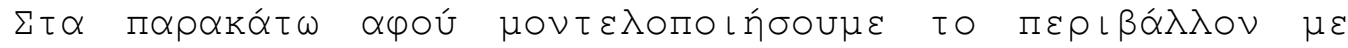

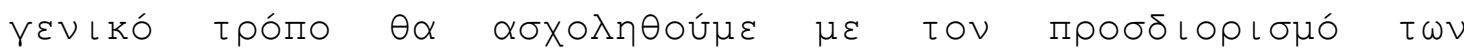

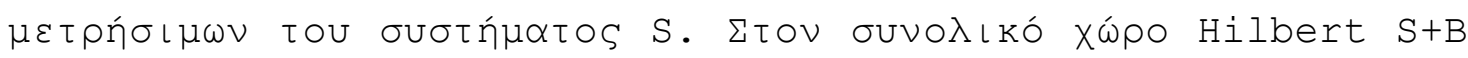

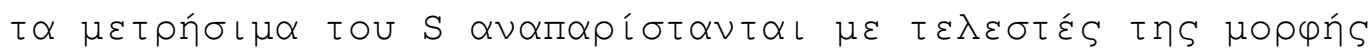

\section{$A \otimes I_{B}$}

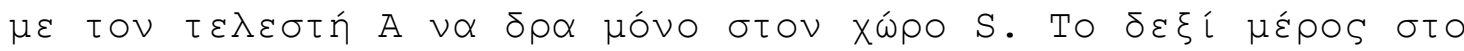

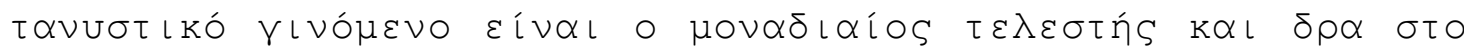

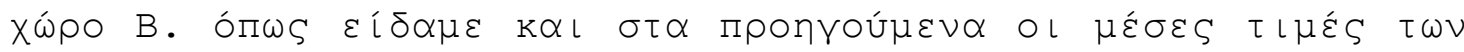

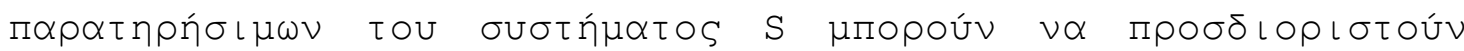

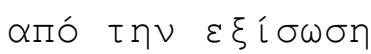

$$
\langle A\rangle=\operatorname{tr}_{S}\left(A \rho_{S}\right)
$$

$\mu \varepsilon$

$$
\rho_{S}=\operatorname{tr}_{B}(\rho)
$$

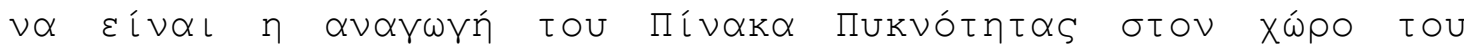

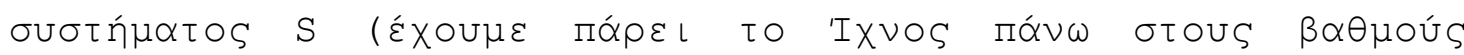

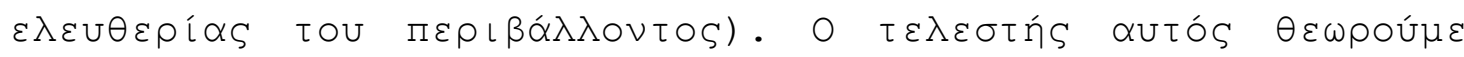

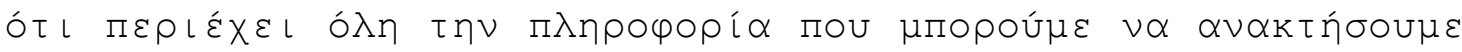




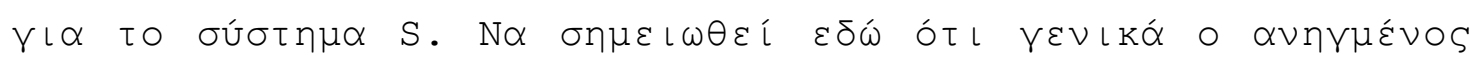

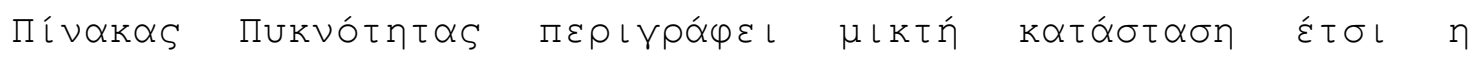

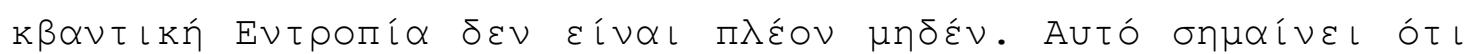

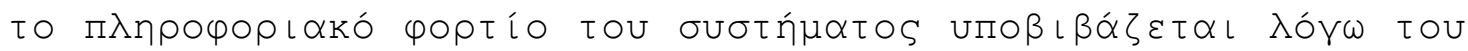

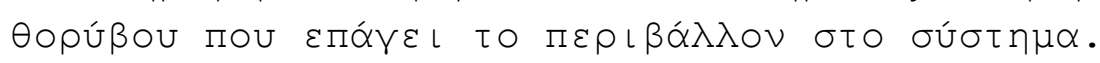

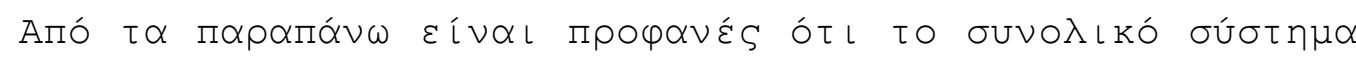

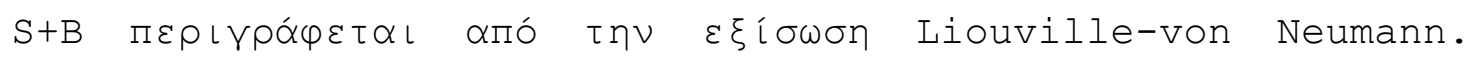

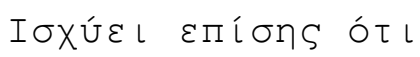

$$
\frac{d}{d t} \rho_{S}(t)=-i t r_{B}[H(t), \rho(t)]
$$

$\mu \varepsilon \quad \hbar=1$.

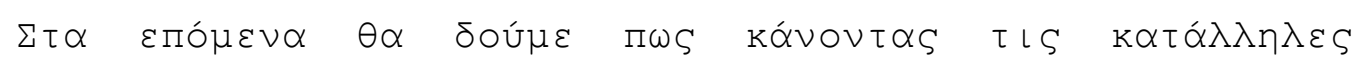

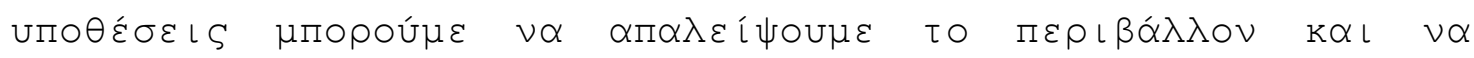

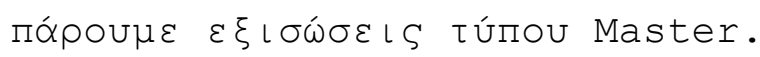

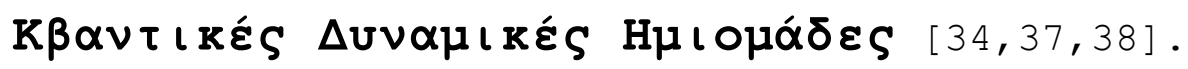

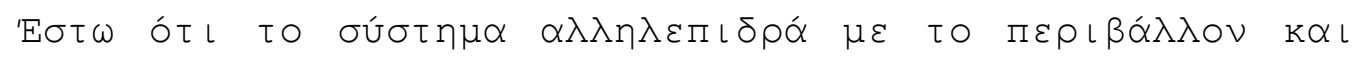

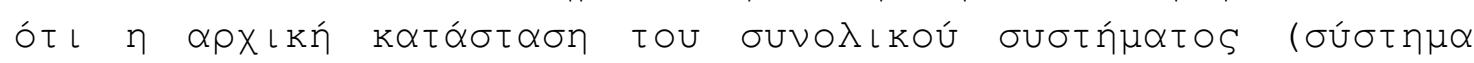

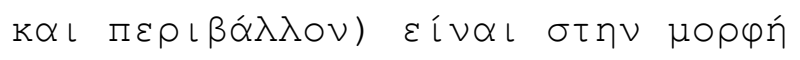

$$
\rho(0)=\rho_{S}(0) \otimes \rho_{B}
$$

Мпороú

$$
\rho_{S}(t)=V(t) \rho_{S}(0) \equiv \operatorname{tr}_{B}\left\{U(t, 0)\left[\rho_{S}(0) \otimes \rho_{B}\right] U^{+}(t, 0)\right\}
$$

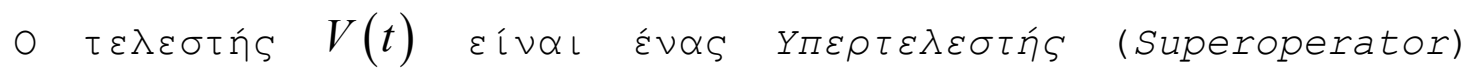

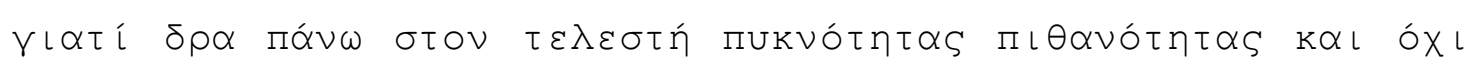

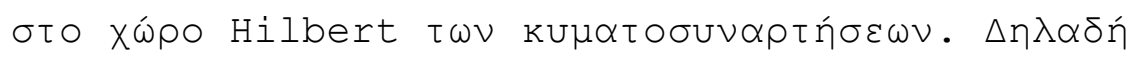

$$
V(t): S\left(\mathcal{H}_{S}\right) \rightarrow S\left(\mathcal{H}_{S}\right)
$$




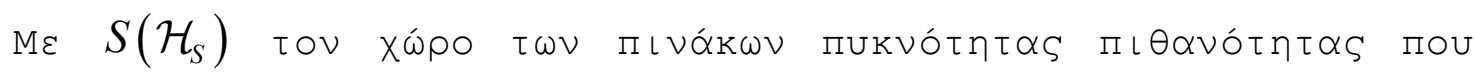

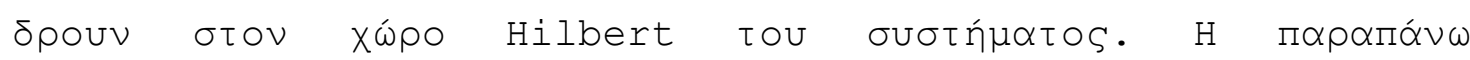

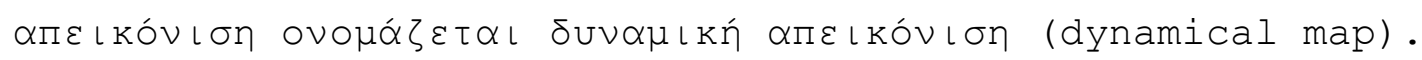

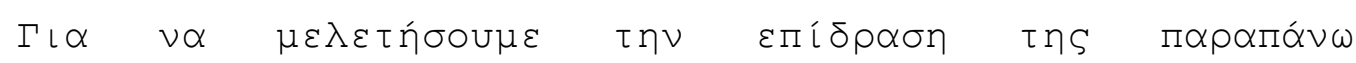

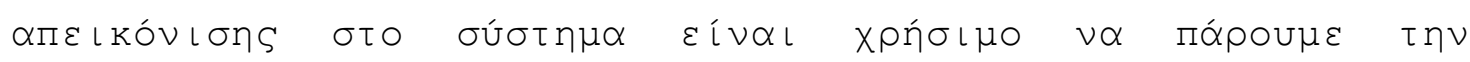

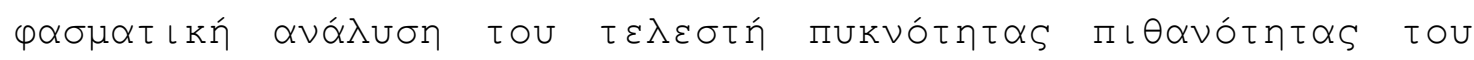

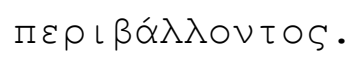

$$
\rho_{B}=\sum_{a} \lambda_{a}\left|\varphi_{a}\right\rangle\left\langle\varphi_{a}\right|
$$

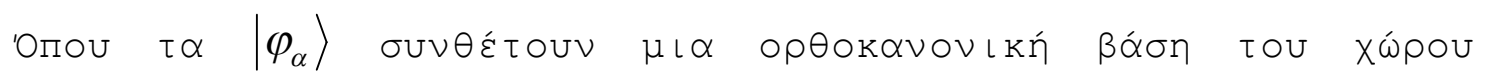

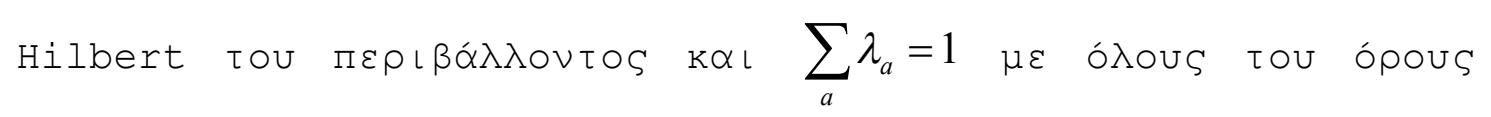
$\theta \varepsilon \tau$ เ KOÚS.

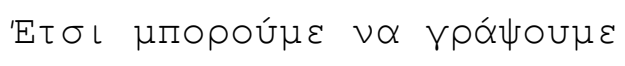

$$
V(t) \rho_{S}=\sum_{\alpha, \beta} W_{\alpha \beta}(t) \rho_{S} W_{\alpha \beta}^{+}(t)
$$

$\mu \varepsilon$

$$
W_{\alpha \beta}(t)=\sqrt{\lambda_{\beta}}\left\langle\varphi_{\alpha}|U(t, 0)| \varphi_{\beta}\right\rangle
$$

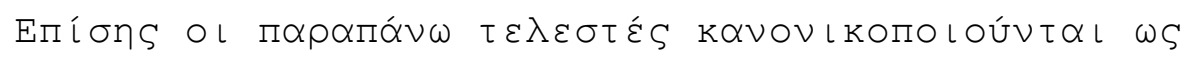

$$
\sum_{\alpha, \beta} W_{\alpha \beta}^{+}(t) W_{\alpha \beta}(t)=I_{S}
$$

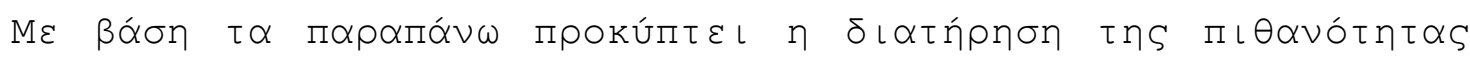

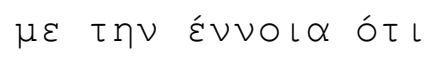

$$
\operatorname{tr}_{S}\left[V(t) \rho_{S}\right]=t r_{S} \rho_{S}=1
$$

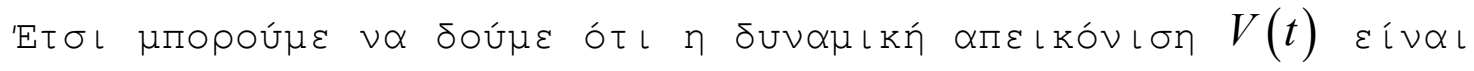

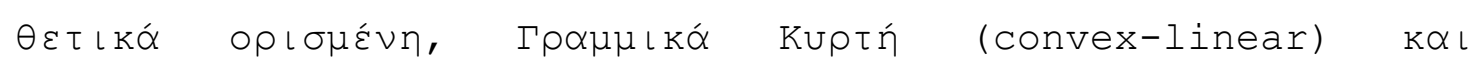

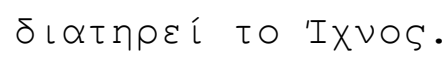




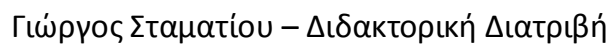

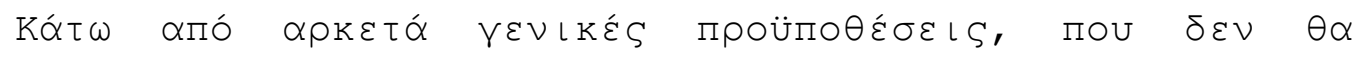

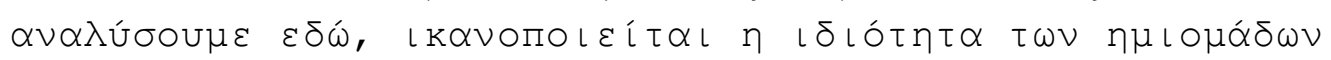

$$
V\left(t_{1}\right) V\left(t_{2}\right)=V\left(t_{1}+t_{2}\right), \quad t_{1}, t_{2} \geq 0
$$

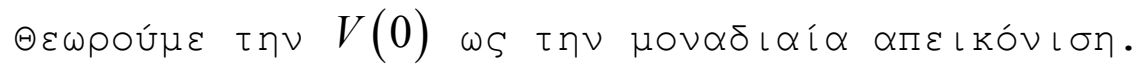

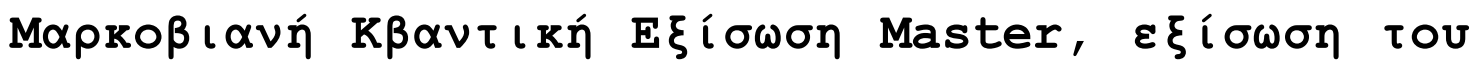
Lindblad.

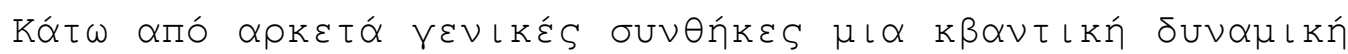

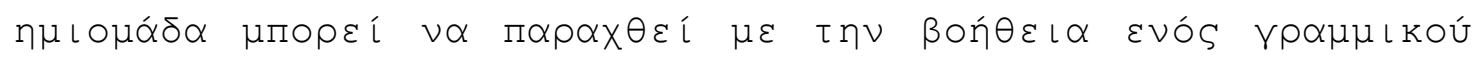

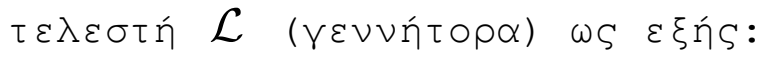

$$
V(t)=e^{t \mathcal{L}}
$$

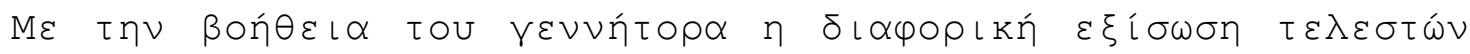

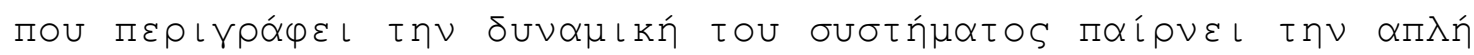

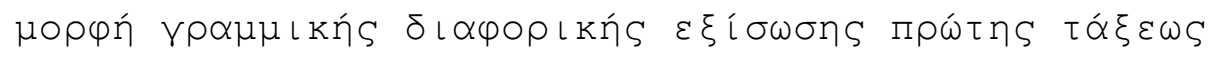

$$
\dot{\rho_{S}}(t)=\mathcal{L} \rho_{S}(t)
$$

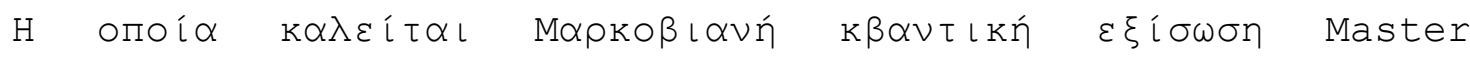
(Markovian quantum master equation).

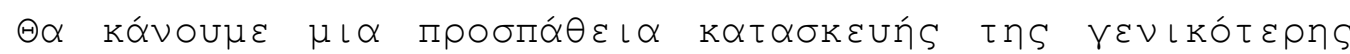

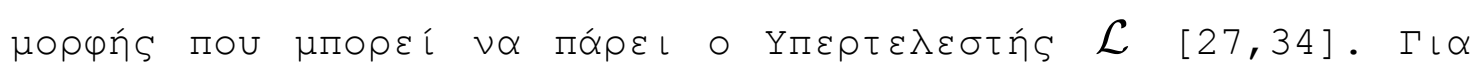

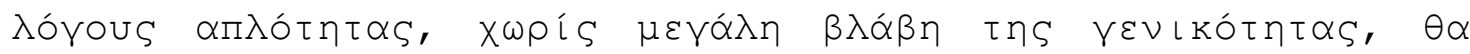

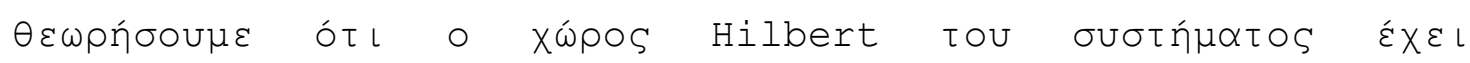

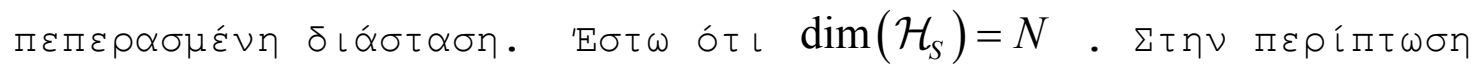

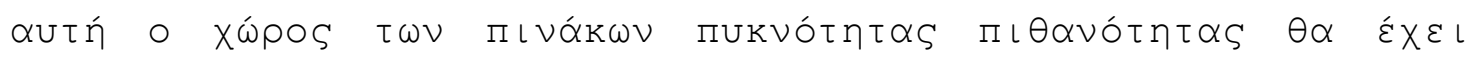

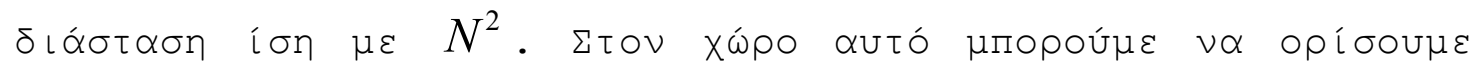

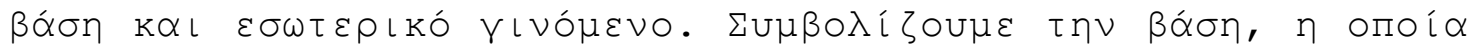

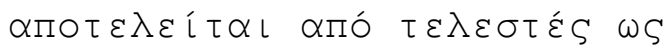

$$
F_{i}, i=1,2, \ldots, N^{2}
$$




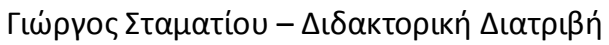

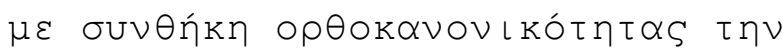

$$
\left(F_{i}, F_{j}\right) \equiv \operatorname{tr}_{S}\left(F_{i}^{+} F_{j}\right)=\delta_{i j}
$$

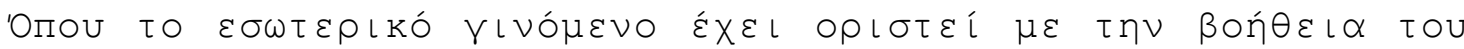
'IXVOUS.

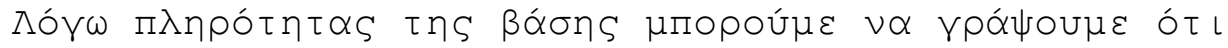

$$
W_{\alpha \beta}(t)=\sum_{i=1}^{N^{2}} F_{i}\left(F_{i}, W_{\alpha \beta}(t)\right)
$$

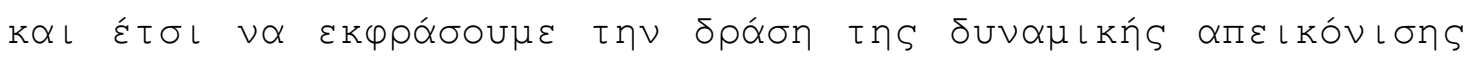
$\omega S$

$$
V(t) \rho_{S}=\sum_{i, j=1}^{N^{2}} c_{i j}(t) F_{i} \rho_{S} F_{j}^{+}
$$

$\mu \varepsilon$

$$
c_{i j}(t)=\sum_{\alpha \beta}\left(F_{i}, W_{\alpha \beta}(t)\right)\left(F_{j}, W_{\alpha \beta}(t)\right)^{*}
$$

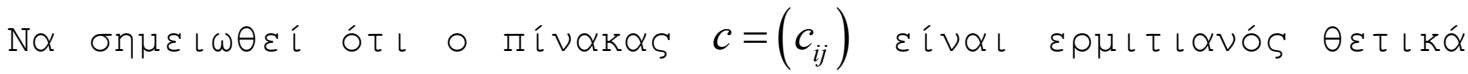

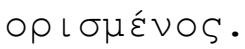

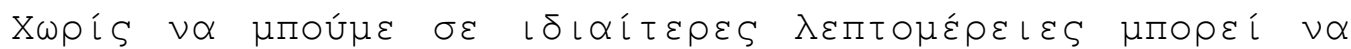
$\alpha \Pi \circ \delta \varepsilon\llcorner X \theta \varepsilon$ ót ı

$$
\mathcal{L} \rho_{S}=-i\left[H, \rho_{S}\right]+\sum_{i, j=1}^{N^{2}-1} \alpha_{i j}\left(F_{i} \rho_{S} F_{j}^{+}-\frac{1}{2}\left\{F_{j}^{+} F_{i}, \rho_{S}\right\}\right)
$$

'Опоч

$$
\alpha_{i j}=\lim _{\tau \rightarrow 0}\left[\frac{c_{i j}(\tau)}{\tau}\right], \quad i, j=1,2, \ldots, N^{2}-1
$$




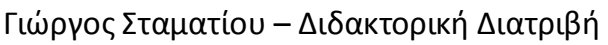

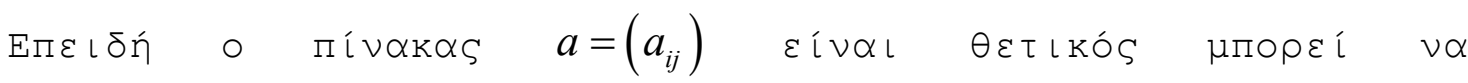

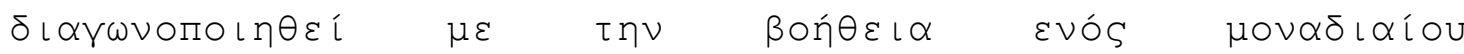
$\mu \varepsilon \tau \alpha \sigma \times \eta \mu \alpha \tau\llcorner\sigma \mu \circ u ́ u$

$$
u \alpha u^{+}=\left[\begin{array}{cccc}
\gamma_{1} & 0 & \cdots & 0 \\
0 & \gamma_{2} & \cdots & 0 \\
0 & 0 & \ddots & 0 \\
0 & 0 & 0 & \gamma_{N^{2}-1}
\end{array}\right]
$$

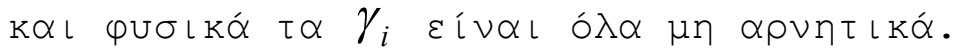

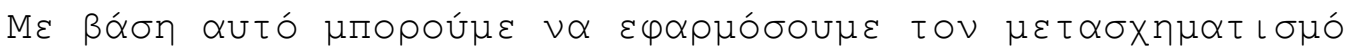

$$
F_{i}=\sum_{k=1}^{N^{2}-1} u_{k i} A_{k}
$$

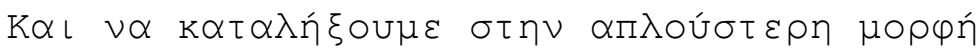

$$
\mathcal{L} \rho_{S}=-i\left[H, \rho_{S}\right]+\sum_{k=1}^{N^{2}-1} \gamma_{k}\left(A_{k} \rho_{S} A_{k}^{+}-\frac{1}{2} A_{k}^{+} A_{k} \rho_{S}-\frac{1}{2} \rho_{S} A_{k}^{+} A_{k}\right)
$$

ท́

$$
\dot{\rho}_{S}(t)=-i\left[H, \rho_{S}(t)\right]+\mathcal{D}\left[\rho_{S}(t)\right]
$$

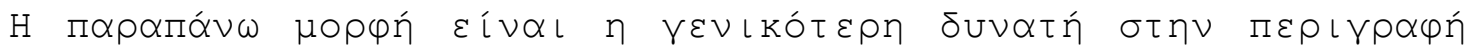

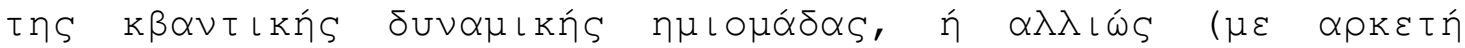

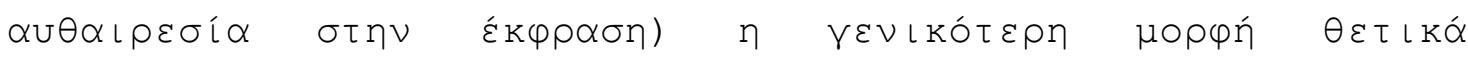

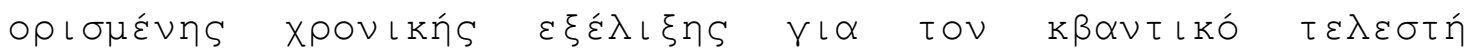

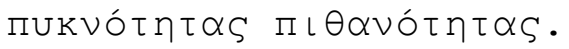

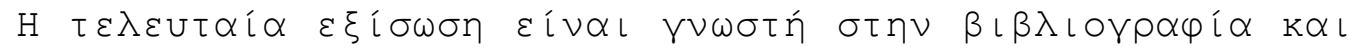

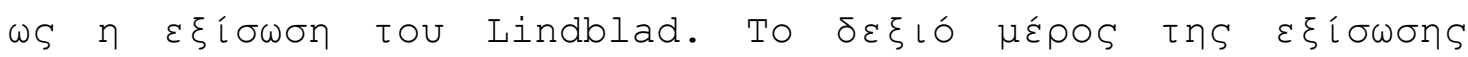

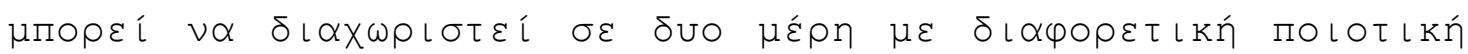

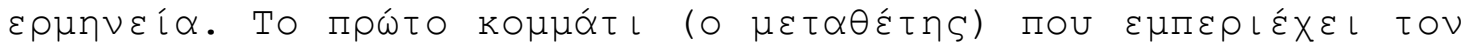




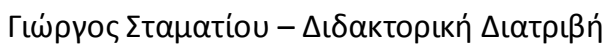

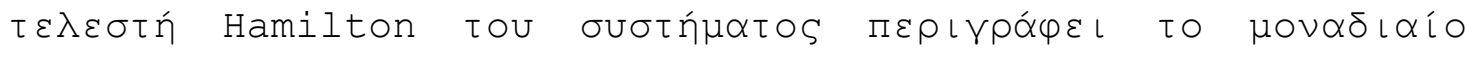

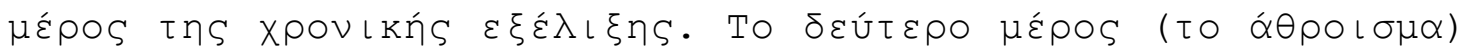

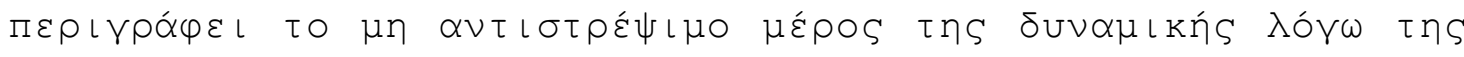

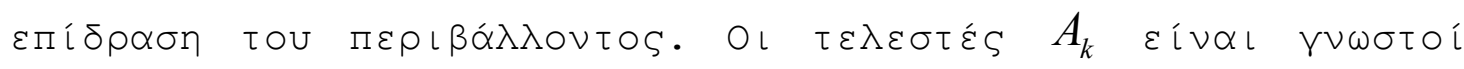

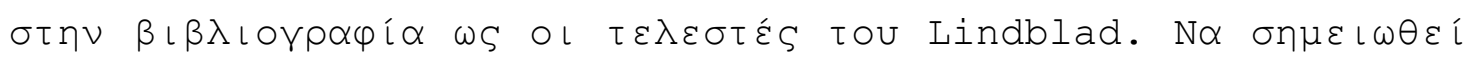

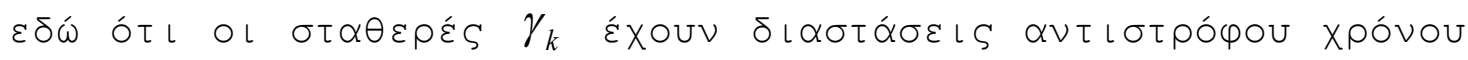

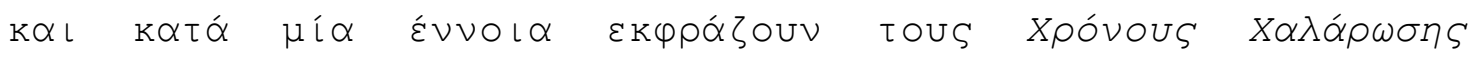

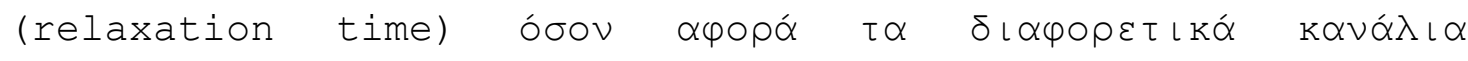

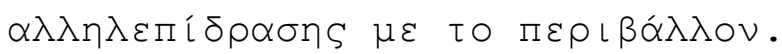

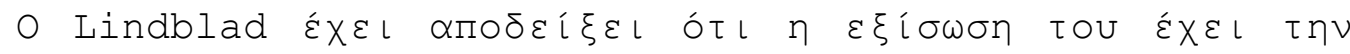

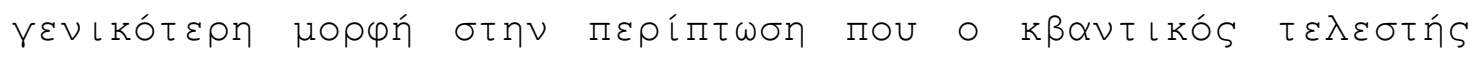

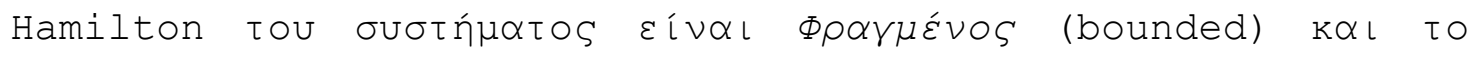

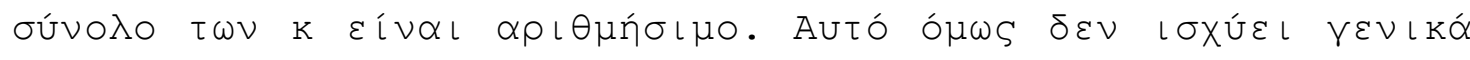

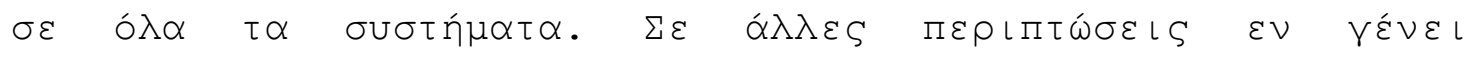

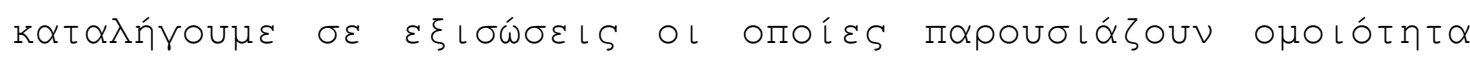

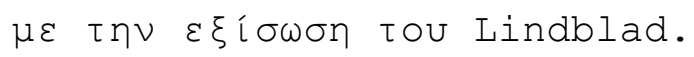

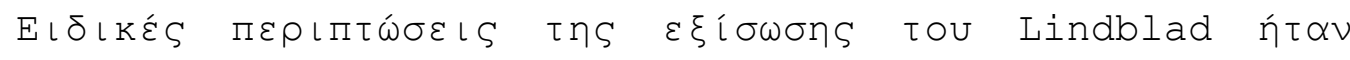

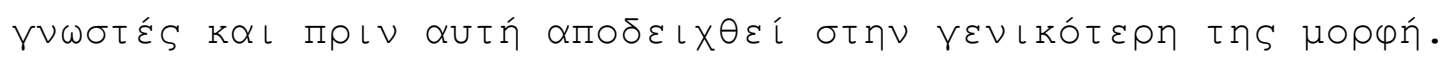

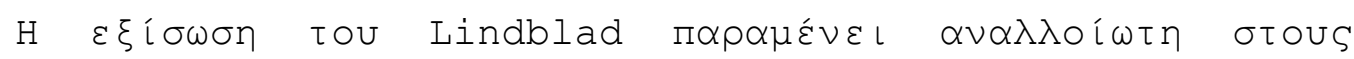

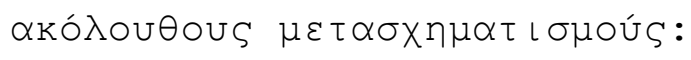

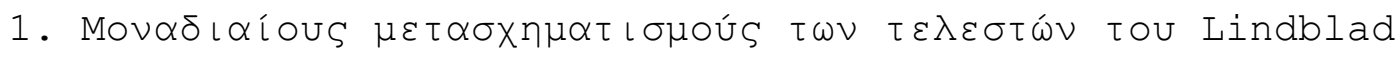

$$
\sqrt{\gamma_{i}} A_{i} \rightarrow \sqrt{\gamma_{i}^{\prime}} A_{i}^{\prime}=\sum_{j} u_{i j} \sqrt{\gamma_{j}} A_{j}
$$

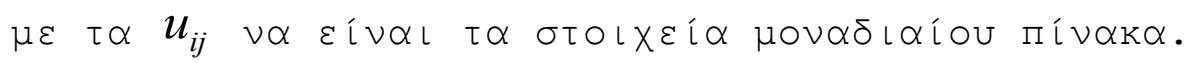

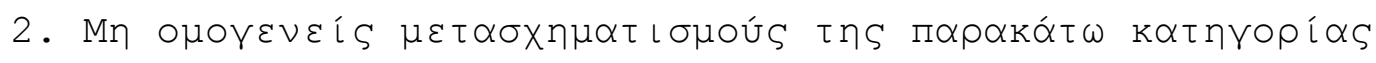

$$
A_{i} \rightarrow A_{i}^{\prime}=A_{i}+\alpha_{i}
$$




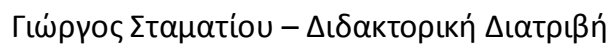

$$
H \rightarrow H^{\prime}=H+\frac{1}{2 i} \sum_{j} \gamma_{j}\left(a_{j}^{*} A_{j}-a_{j} A_{j}^{+}\right)+b
$$

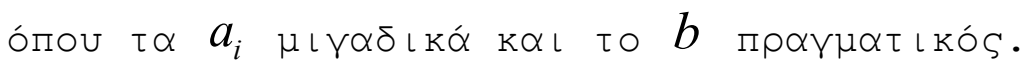

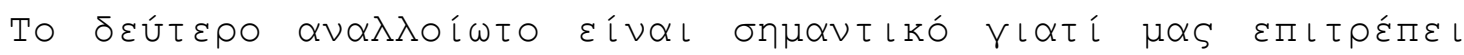

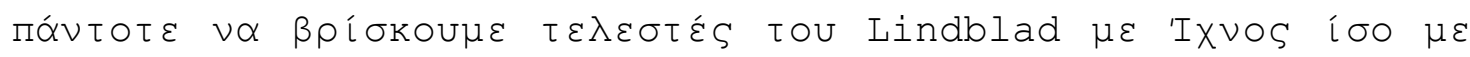

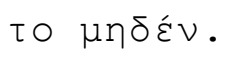

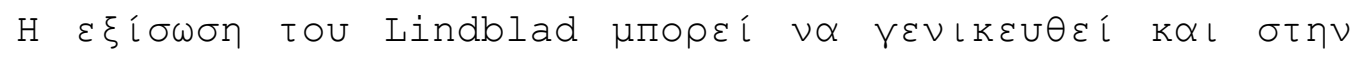

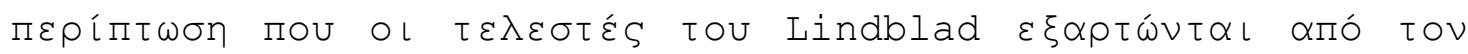
xpóvo.

$$
\dot{\rho}_{S}(t)=\mathcal{L}(t) \rho_{S}(t)
$$

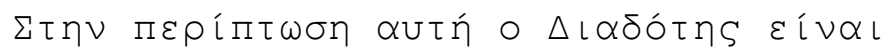

$$
V\left(t, t_{0}\right)=T \exp \left[\int_{t_{0}}^{t} d \omega \mathcal{L}(\omega)\right], \quad T \rightarrow \text { time ordering operator }
$$

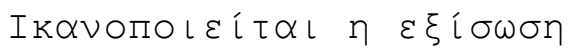

$$
\frac{\partial V\left(t, t_{0}\right)}{\partial t}=\mathcal{L}(t) V\left(t, t_{0}\right)
$$

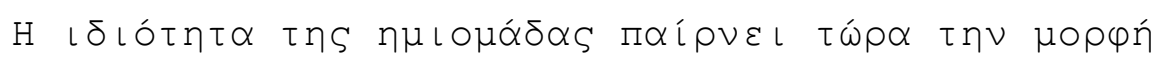

$$
V\left(t, t_{1}\right) V\left(t_{1}, t_{0}\right)=V\left(t, t_{0}\right)
$$

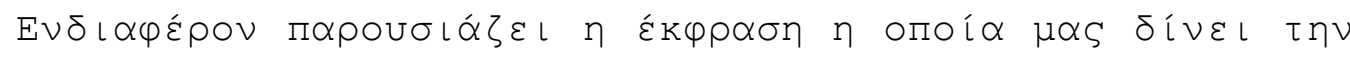

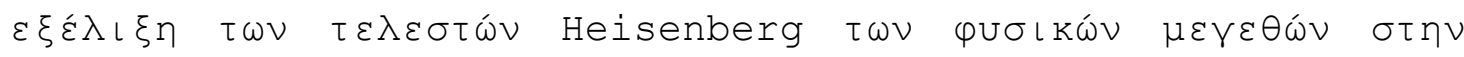

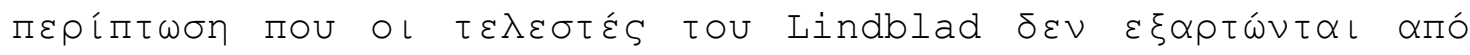
tov xpóvo. 


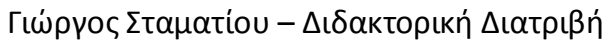

$$
\begin{gathered}
\frac{d A_{H}(t)}{d t}=\mathcal{L}^{+} A_{H}(t) \\
=i\left[H, A_{H}(t)\right]+\sum_{k} \gamma_{k}\left[A_{k}^{+} A_{H}(t) A_{k}-\frac{1}{2} A_{H}(t) A_{k}^{+} A_{k}-\frac{1}{2} A_{k}^{+} A_{k} A_{H}(t)\right]
\end{gathered}
$$

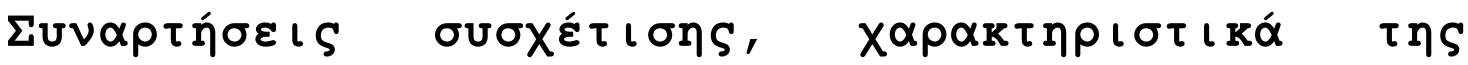

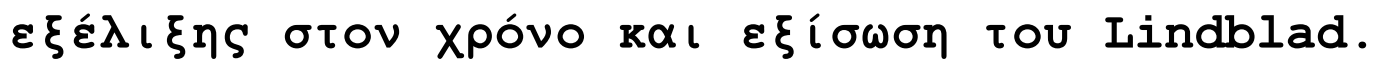

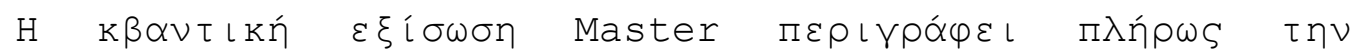

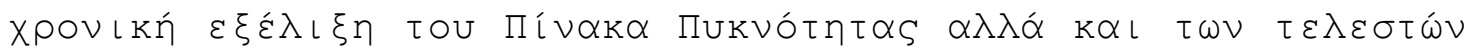

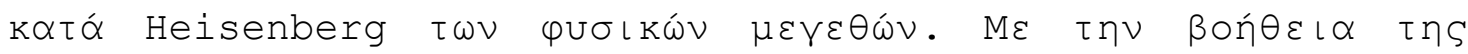

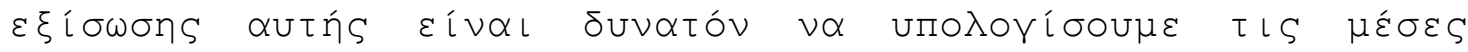

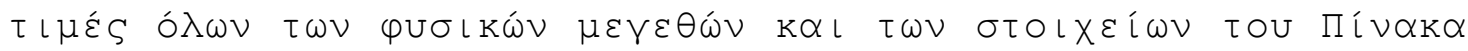

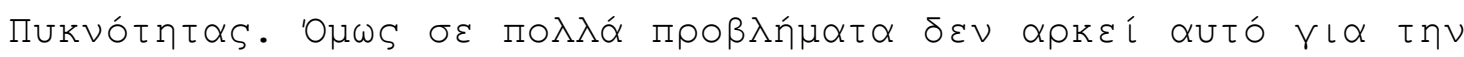

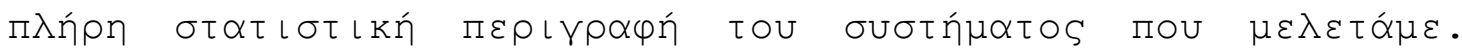

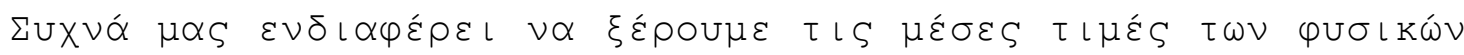

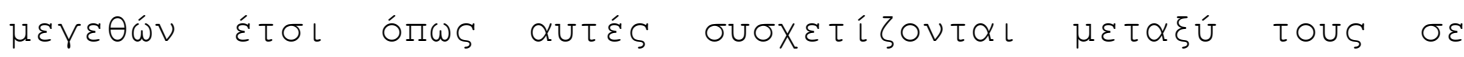

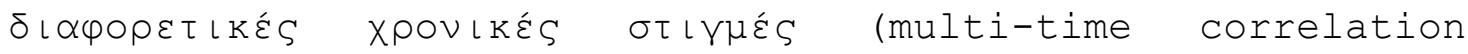
functions). $\Sigma \varepsilon$ kó

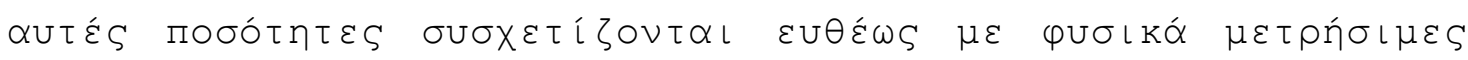

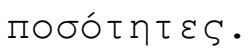

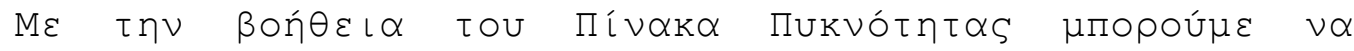

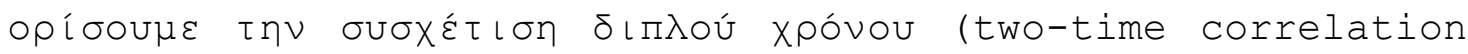

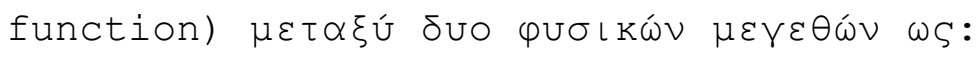

$$
\sigma(t, s) \equiv\langle B(t) C(s)\rangle=\operatorname{tr}_{S}\left[B(t) C(s) \rho_{S}(0)\right]
$$

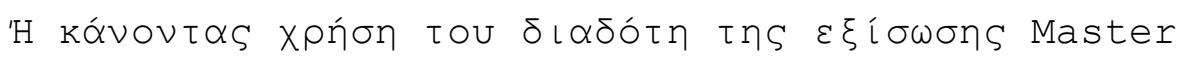

$$
\sigma(t, s)=\langle B V(t, s) C V(s, 0)\rangle=\operatorname{tr}_{S}\left[B V(t, s) C V(s, 0) \rho_{S}(0)\right]
$$

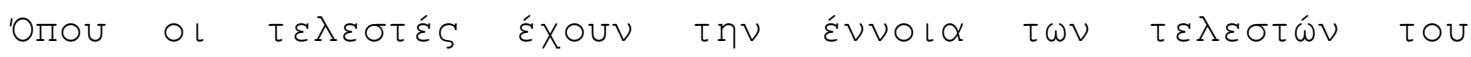

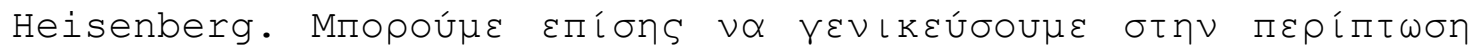

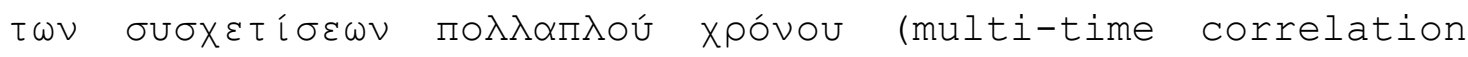
functions) ws: 


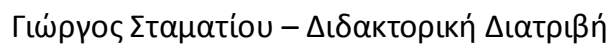

$$
\begin{gathered}
\sigma\left(t_{1}, t_{2}, \ldots, t_{n} ; s_{1}, s_{2}, \ldots, s_{m}\right) \equiv\left\langle B_{1}\left(s_{1}\right) B_{2}\left(s_{2}\right) \ldots B_{m}\left(s_{m}\right) C_{n}\left(t_{n}\right) \ldots C_{2}\left(t_{2}\right) C_{1}\left(t_{1}\right)\right\rangle \\
=\operatorname{tr}_{s}\left[C_{n}\left(t_{n}\right) \ldots C_{2}\left(t_{2}\right) C_{1}\left(t_{1}\right) \rho_{S}(0) B_{1}\left(s_{1}\right) B_{2}\left(s_{2}\right) \ldots B_{m}\left(s_{m}\right)\right]
\end{gathered}
$$

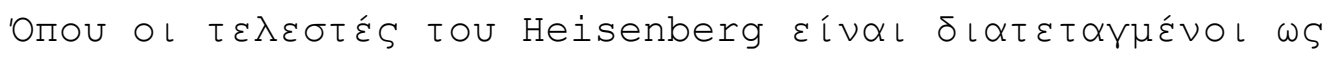

$$
s_{m}>s_{m-1}>\ldots>s_{1} \geq 0, t_{m}>t_{m-1}>\ldots>t_{1} \geq 0
$$

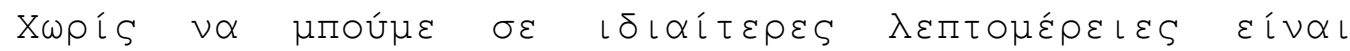

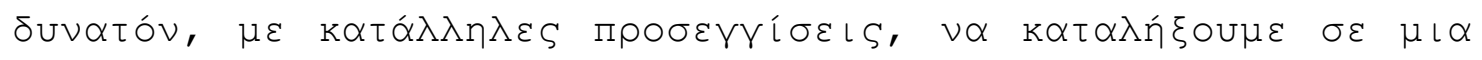

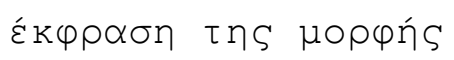

$$
\begin{gathered}
\frac{d}{d \tau}\left\langle B_{i}(t+\tau) B_{l}(\tau)\right\rangle=\operatorname{tr}_{s}\left[\left(\mathcal{L}^{+} B_{i}\right) V(t+\tau, t) B_{l} \rho_{S}(t)\right] \\
=\sum_{j} G_{i j}\left\langle B_{j}(t+\tau) B_{l}(t)\right\rangle
\end{gathered}
$$

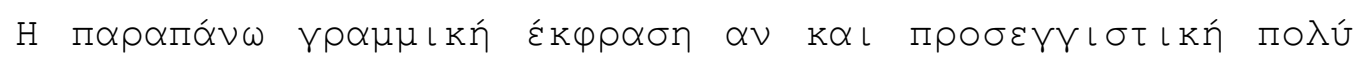

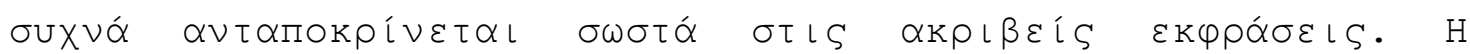

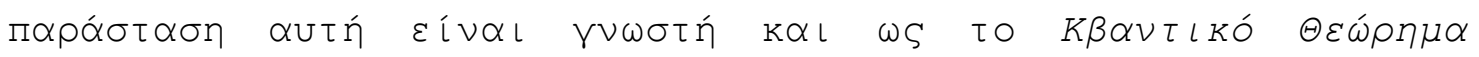

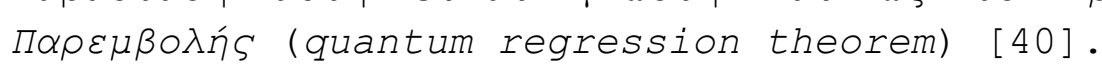

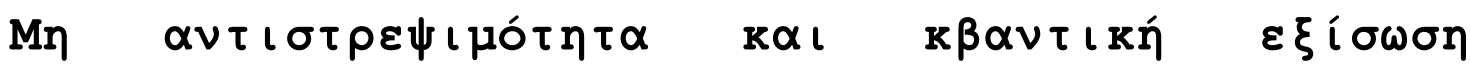 Master.}

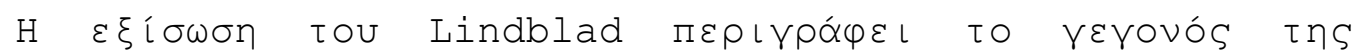

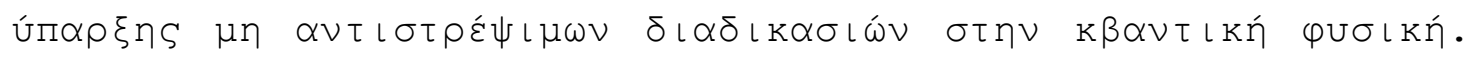

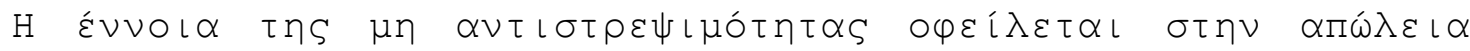

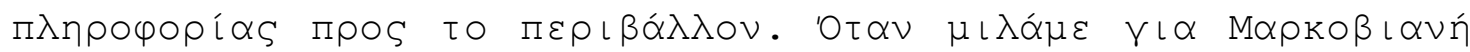

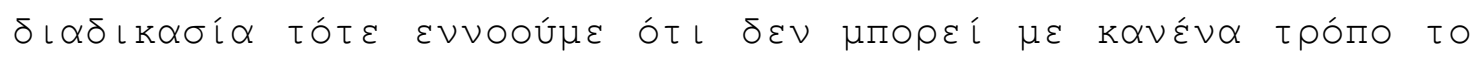

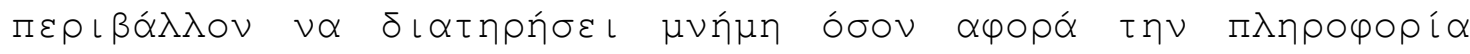

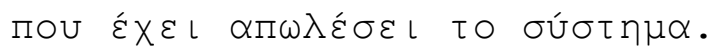

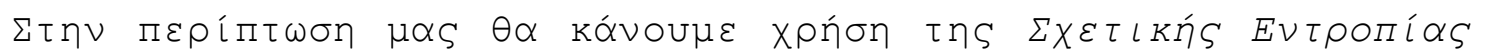

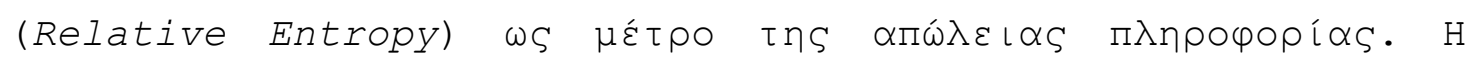

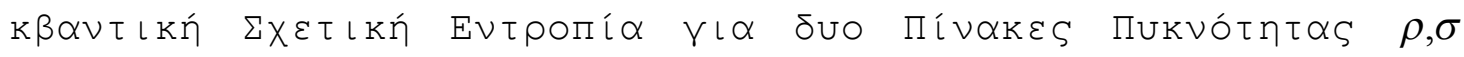




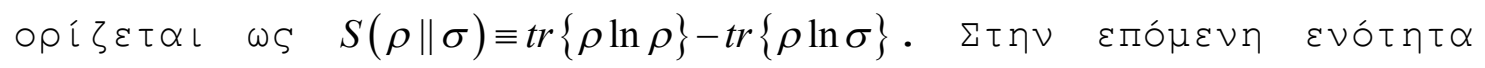

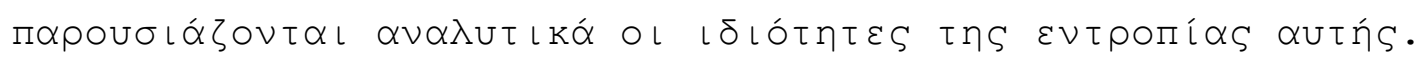

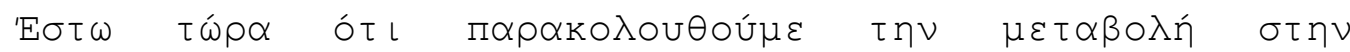

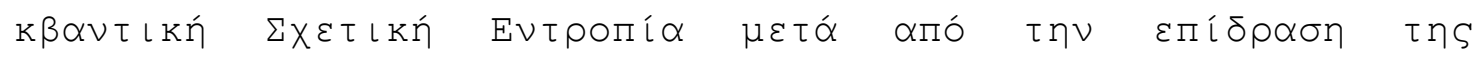

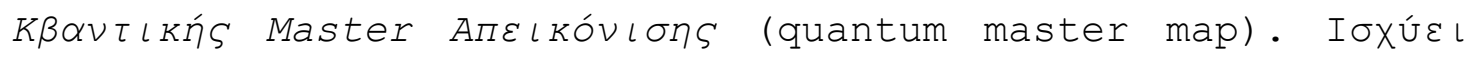
ót l

$$
\begin{gathered}
S\left(V(t) \rho \| V(t) \rho_{0}\right)= \\
=S\left\{t r\left[U(t, 0) \rho \otimes \rho_{B} U^{+}(t, 0)\right] \| \operatorname{tr}\left[U(t, 0) \rho_{0} \otimes \rho_{B} U^{+}(t, 0)\right]\right\} \\
\leq S\left[U(t, 0) \rho \otimes \rho_{B} U^{+}(t, 0) \| U(t, 0) \rho_{0} \otimes \rho_{B} U^{+}(t, 0)\right] \\
=S\left(\rho \otimes \rho_{B} \| \rho_{0} \otimes \rho_{B}\right) \\
=S\left(\rho \| \rho_{0}\right)
\end{gathered}
$$

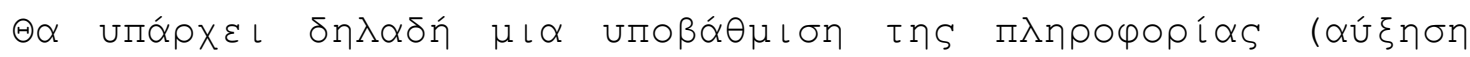

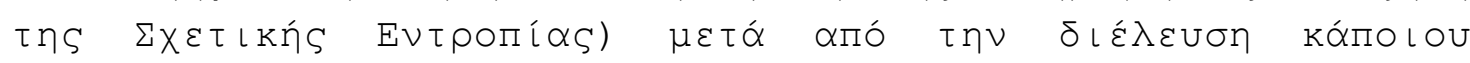

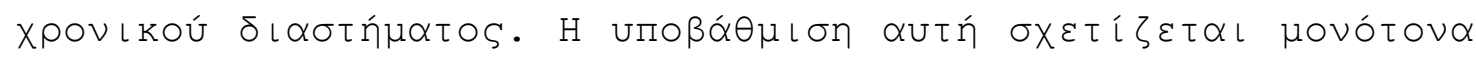
$\mu \varepsilon$ tov Xpóvo.

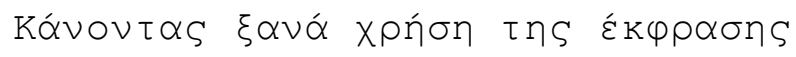

$$
V(t)=e^{\mathcal{L} t}
$$

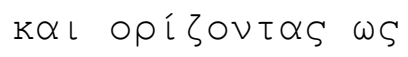

$$
\sigma[\rho(t)] \equiv-\frac{d}{d t} S\left[\rho(t) \| \rho_{0}\right] \geq 0
$$

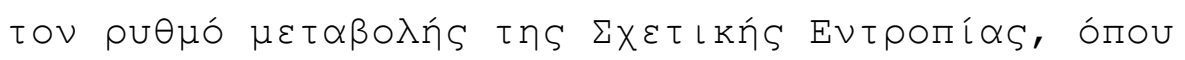

$$
\rho(t)=V(t) \rho(0)
$$




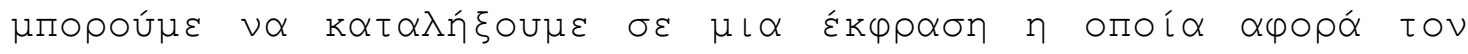

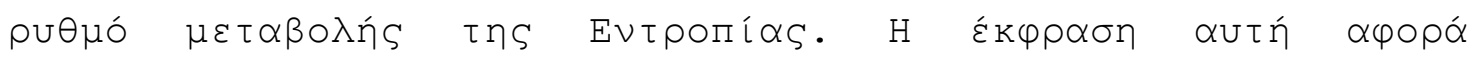

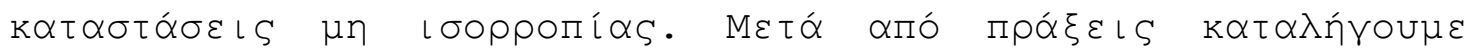

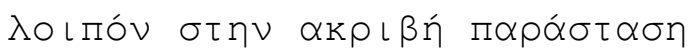

$$
\sigma(\rho)=-k_{\beta} \operatorname{tr}[\mathcal{L}(\rho) \ln \rho]+k_{\beta} \operatorname{tr}\left[\mathcal{L}(\rho) \ln \rho_{0}\right] \geq 0
$$

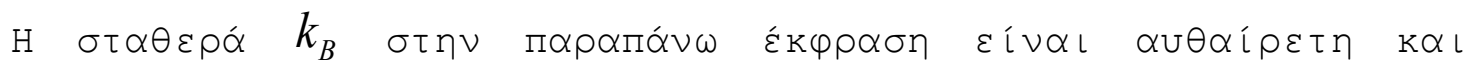

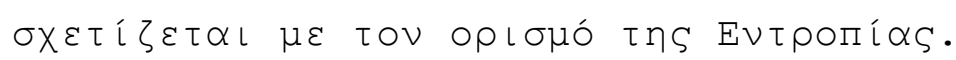

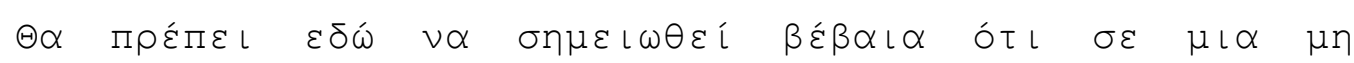

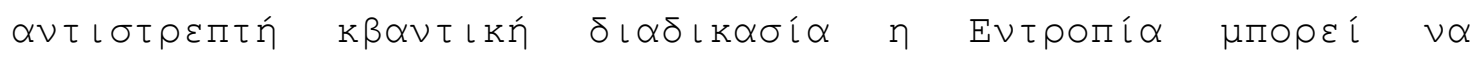

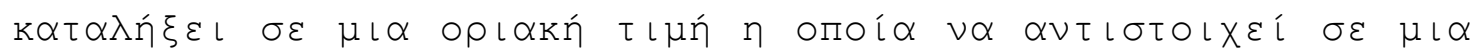

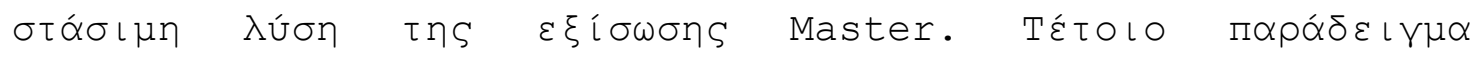

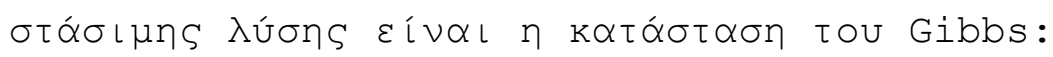

$$
\rho_{\text {thermal }}=\frac{1}{Z} e^{-\beta H}
$$

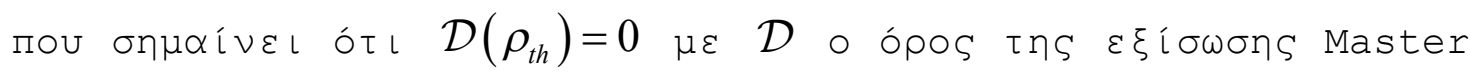

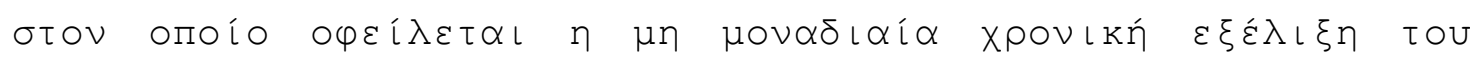

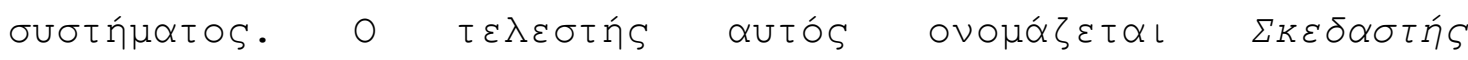
(dissipator) tnS $\varepsilon \xi i ́ \sigma \omega \sigma n S$ Master. 



\section{Aข $\alpha \varphi \circ \rho \varepsilon ́ \varsigma$}

[1]. A short history of atomic physics in the twentieth century, D Kleppner - Reviews of Modern Physics, 1999, vol.71, issue 2, S78-S84

[2]. Planck's Law and the light quantum hypothesis, S Bose - Journal of Astrophysics and Astronomy, 1994, vol.15, no. $1, \quad 3-11$

[3]. Black-body theory and the quantum discontinuity, 18941912, TS Kuhn, University of Chicago Press, 1987

[4]. Decoherence, chaos, and the correspondence principle, S Habib, K Shizume, WH Zurek - Physical Review Letters, 1998, vol 80, issue 20, 4321-4365

[5]. Bohr's correspondence principle: the cases for which it is exact, AJ Makowski, KJ Gorska - Physical Review A, $2002,66,062103$

[6]. Understanding quantum mechanics, $R$ Omnès, Princeton University Press, 1999

[7]. On the problem of hidden variables in quantum mechanics, JS Bell - Reviews of Modern Physics, 1966, vol.38, issue 3, 447-452

[8]. The principles of quantum mechanics, PAM Dirac, Oxford University Press, 1981

[9]. Regular and chaotic causal trajectories for the Bohm potential in a restricted space, S Konkel, AJ Makowski Physics Letters A, 1998, vol.238, issue 2-3, 95-100

[10]. Quantum chaos in terms of Bohm trajectories, H Wu, DWL Sprung - Physics Letters A, 1999, vol.261, issue 3-4, $150-157$

[11]. Classical Hamiltonian systems and stochastic mechanics: A possible new ..., DPK Ghikas - Physics Letters A, 1989, vol.137, issues 4-5, 183-187 


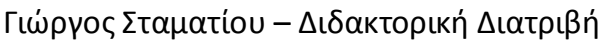

[12]. Derivation of the Schrödinger equation from Newtonian mechanics, E Nelson - Physical Review, 1966, vol. 150, issue 4, 1079-1085

[13]. Realization of the Einstein-Podolsky-Rosen paradox for continuous variables, ZY Ou, SF Pereira, HJ Kimble, KC Peng - Physical review letters, 1992, vol.68, issue 25, $3663-3666$

[14]. Bell's theorem and the EPR paradox, D Home, F Selleri - La Rivista del Nuovo Cimento (1978-1999), 1991 Springer, vol.14, no. 9, 1-95

[15]. An experimental test of the EPR paradox, G Faraci, D Gutkowski, S Notarrigo, AR ... - Lettere Al Nuovo Cimento ..., 1974 - Springer, vol.9, no. 15, 607-611

[16]. Two paradoxes in quantum mechanics, HP Krips Philosophy of Science, 1969, vol. 36, no.2, 145

[17]. Hidden variables and the two theorems of John Bell, ND Mermin - Reviews of Modern Physics, 1993, vol. 65, issue 3, 803-815

[18]. On the einstein-podolsky-rosen paradox, JS Bell... Physics, 1964, vol. 1, no. 3, 195-200

[19]. Violation of Bell inequalities by photons more than $10 \mathrm{~km}$ apart, W Tittel, J Brendel, $\mathrm{H}$ Zbinden, $\mathrm{N}$ Gisin Physical Review Letters, 1998, vol. 81, issue 17, 3563-3566

[20]. Experimental test of Bell's inequalities using timevarying analyzers, A Aspect, J Dalibard, G Roger - Physical Review Letters, 1982, vol. 49, issue 25, 1804-1807

[21]. Proposed experiment to test local hidden-variable theories, JF Clauser, MA Horne, A Shimony, RA Holt Physical Review Letters, 1969, vol. 23, issue 15, 880-884

[22]. Can quantum-mechanical description of physical reality be considered complete?, A Einstein, B Podolsky, N Rosen... - Physical review, 1935, vol. 47, issue 10, 777-780 


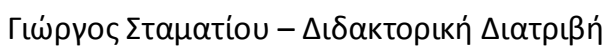

[23]. Experimental test of local hidden-variable theories, SJ Freedman, JF Clauser - Physical Review Letters, 1972, vol. 28, issue 14, 938-941

[24]. Density matrix theory and applications, K Blum, Springer, 1996

[25]. Quantum Mechanics, C Cohen-Tannoudji, B Diu, F Laloë, S Hemley, Wiley Interscience, 1977

[26]. A Modern Approach to Quantum Mechanics, Townsend, John S. 2nd ed.. Sausalito, CA: University Science Books, 2000

[27] . Quantum theory of open systems, EB Davies, Academic Press , 1976

[28]. States, Effects, and Operations, K.Kraus, Lecture Notes in Physics 190, springer, 1983.

[29]. Quantum Theory: Concepts and Methods, A. Peres, Kluwer Academic Publishers, 1993

[30]. Quantum Computation and Quantum Information, M. Nielsen and I. Chuang, Cambridge University Press, 2000, ISBN : $0-521-63503-9$

[31]. Quantum-mechanical model for continuous position measurements, CM Caves, GJ Milburn - Physical Review A, 1987, vol. 36, issue 12, 5543-5555

[32]. Operations and measurements. II, KE Hellwig, K Kraus - Communications in mathematical physics, 1970, vol.16, no. $2, \quad 142-147$

[33]. Handbook of stochastic methods, CW Gardiner, Springer, 1985

[34]. On the generators of quantum dynamical semigroups, G Lindblad, Communications in Mathematical Physics, 1976, vol. 48, no. 2, 119-130 


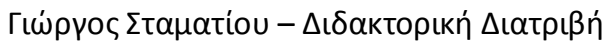

[35]. Markovian master equations, EB Davies Communications in mathematical Physics, 1974 - Springer, vol. 39, no. 2, 91-110

[36]. Experimental violation of a Bell's inequality with efficient detection, MA Rowe, D Kielpinski, V Meyer, CA Sackett, WM ... - Nature, 2001, 409 (6822):791-794

[37]. Quantum groups and quantum semigroups, T Hayashi Journal of Algebra, 1998, vol. 204, issue 1, 225-254

[38]. Quantum dynamical semigroups and applications, R Alicki, K Lendi -Springer, Lec. Notes Phys. 717, 2007

[39]. Mathematical foundations of quantum mechanics, J Von Neumann, Princeton University Press , 1996

[40]. Formal Theory of Quantum Fluctuations from a Driven State, Melvin Lax, Phys. Rev.,1963, vol. 129, issue 5, $2342-2348$ 


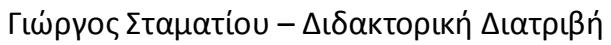

ENOTHTA B'

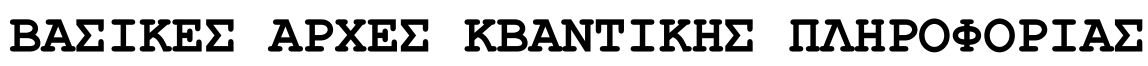
KAI

METPSN TOY ENTANGLEMENT 



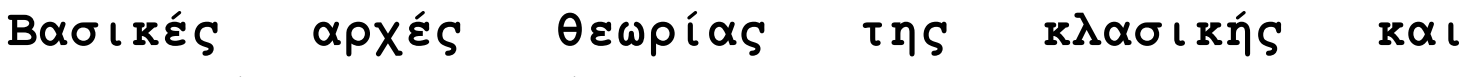

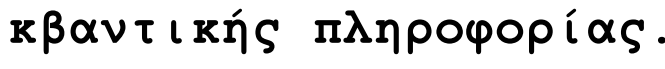

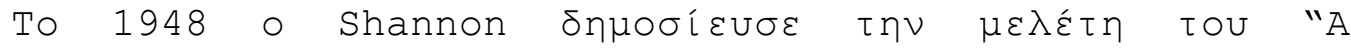
Mathematical Theory of Communication" [1]. H $\mu \varepsilon \lambda \varepsilon \dot{\tau} \eta$ autń

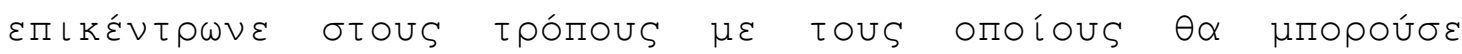

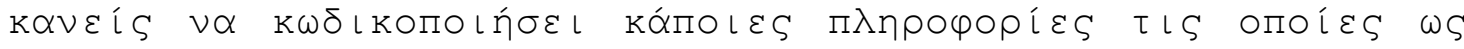

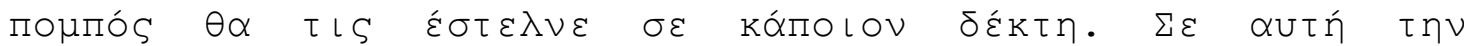

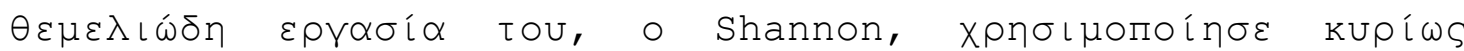

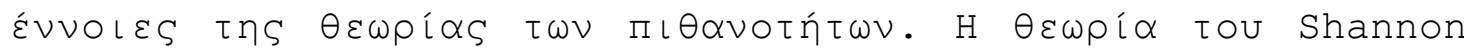

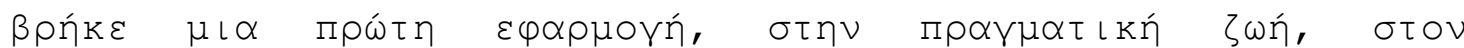

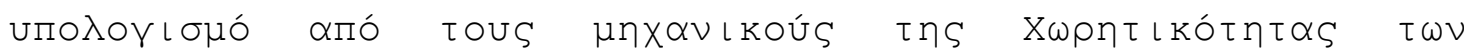

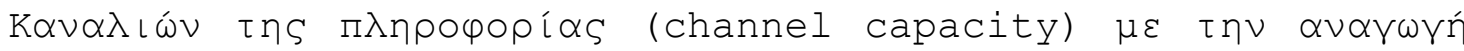

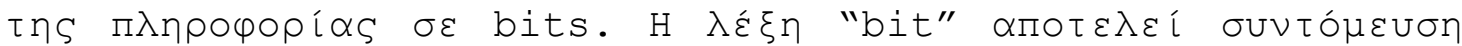

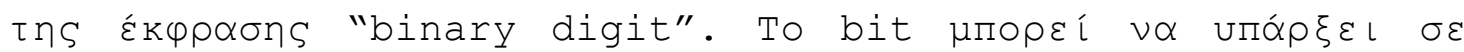

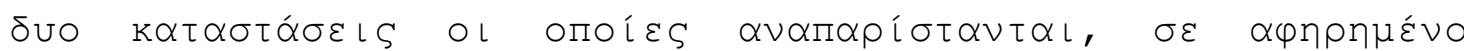

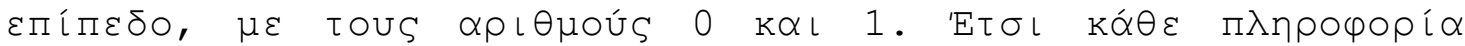

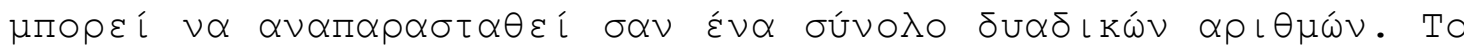

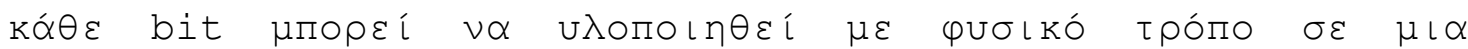

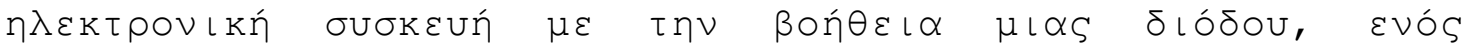

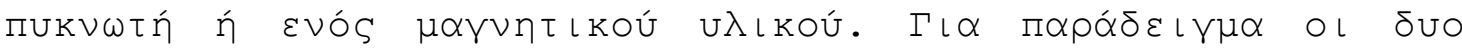

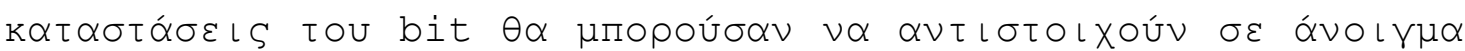

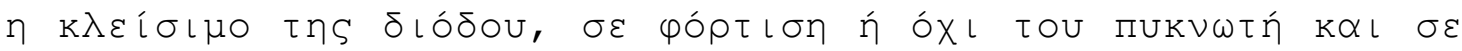

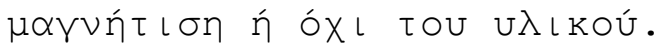

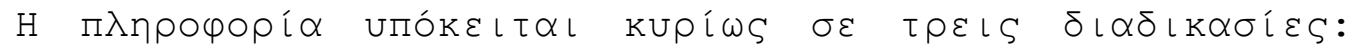

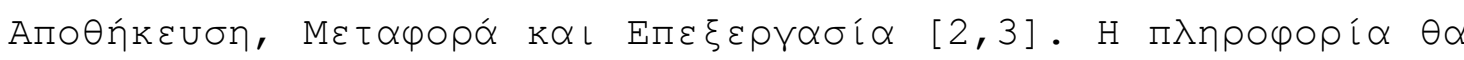

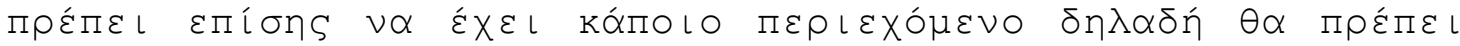

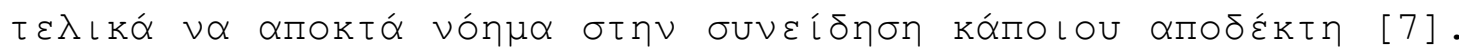

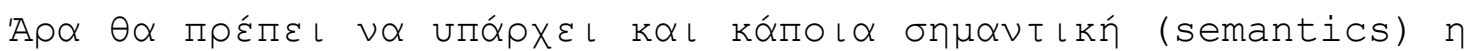

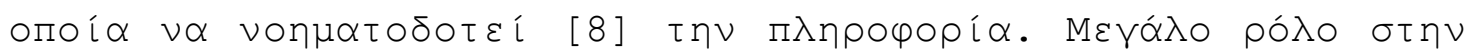

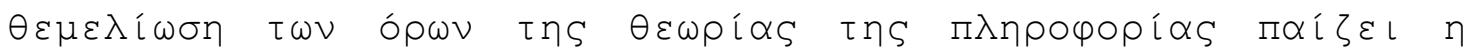

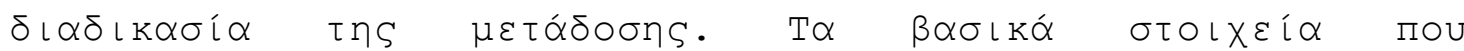

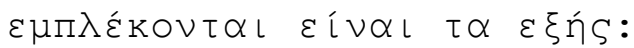

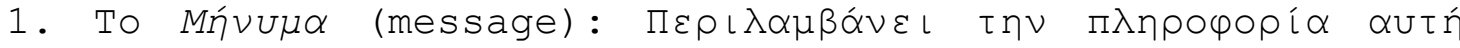
к $\alpha \theta \alpha u \tau \dot{n}$.

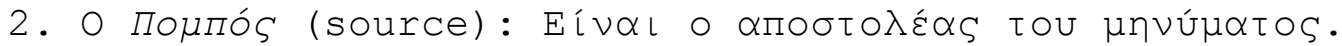




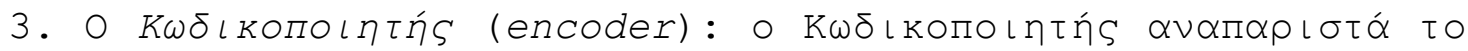

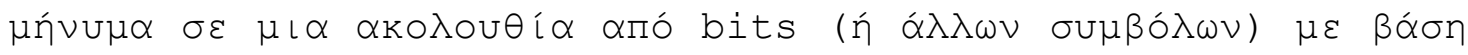

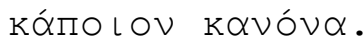

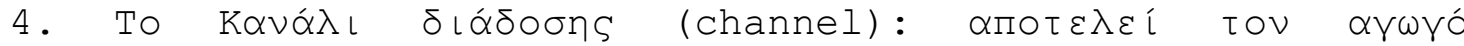

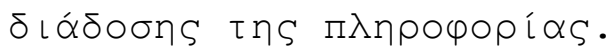

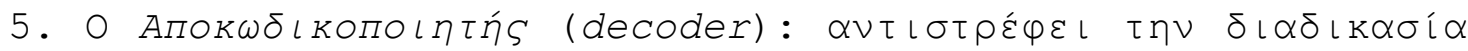

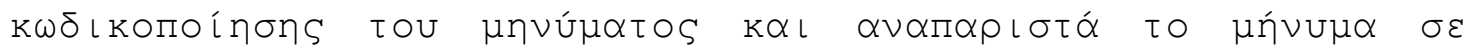

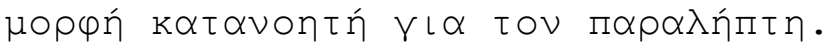

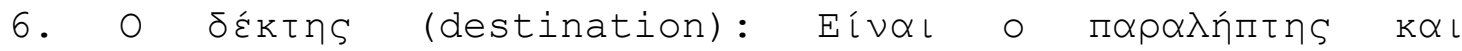

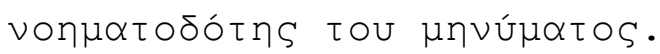

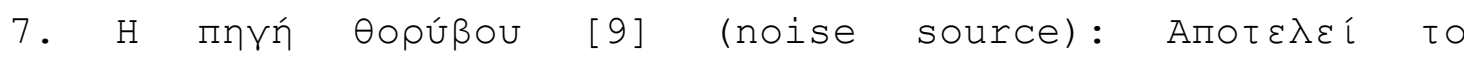

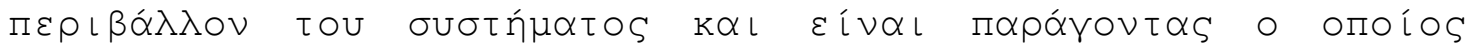

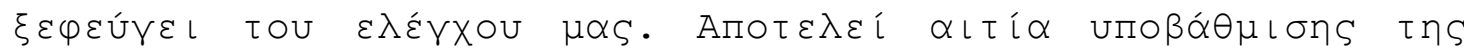

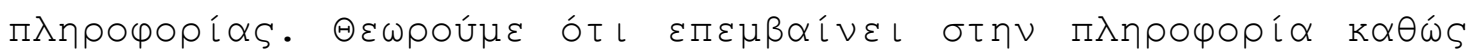

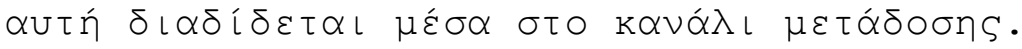

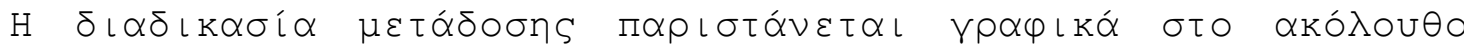
$\sigma \times \eta ́ n \alpha:$

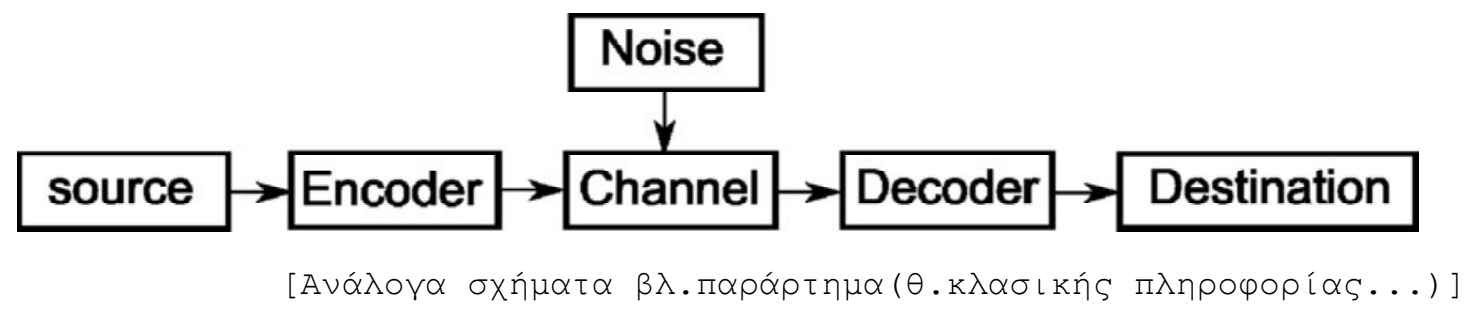

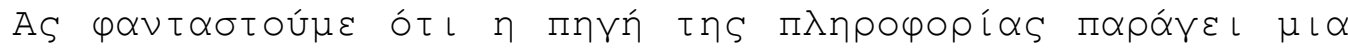

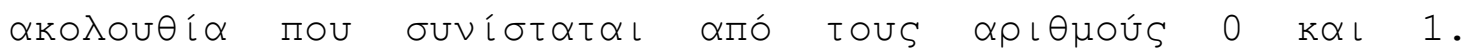

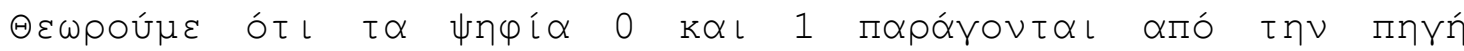

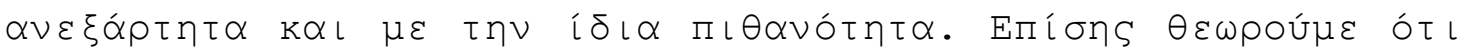

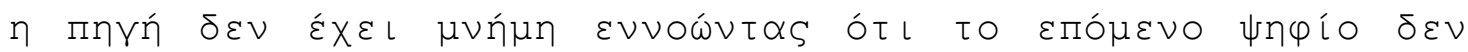

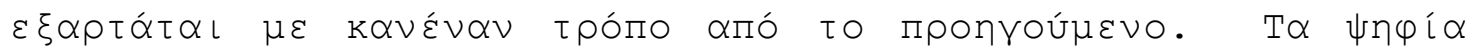

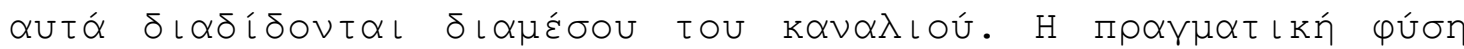

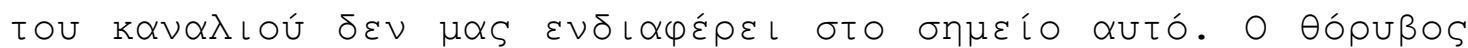

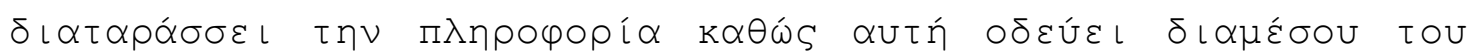

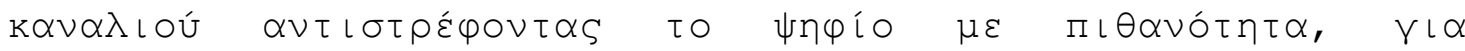




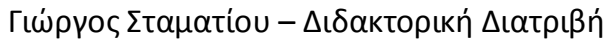

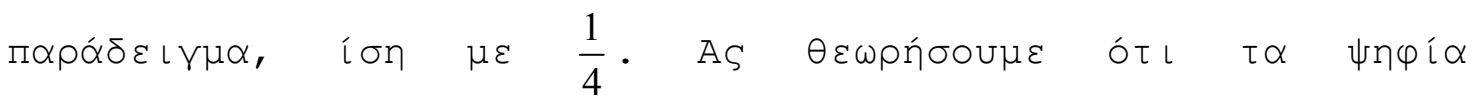

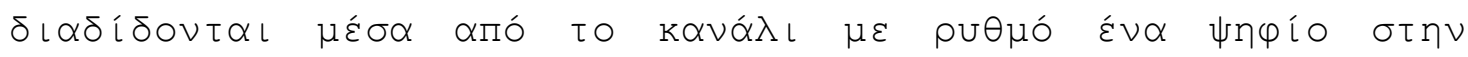

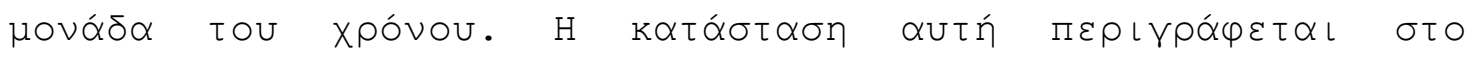

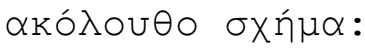

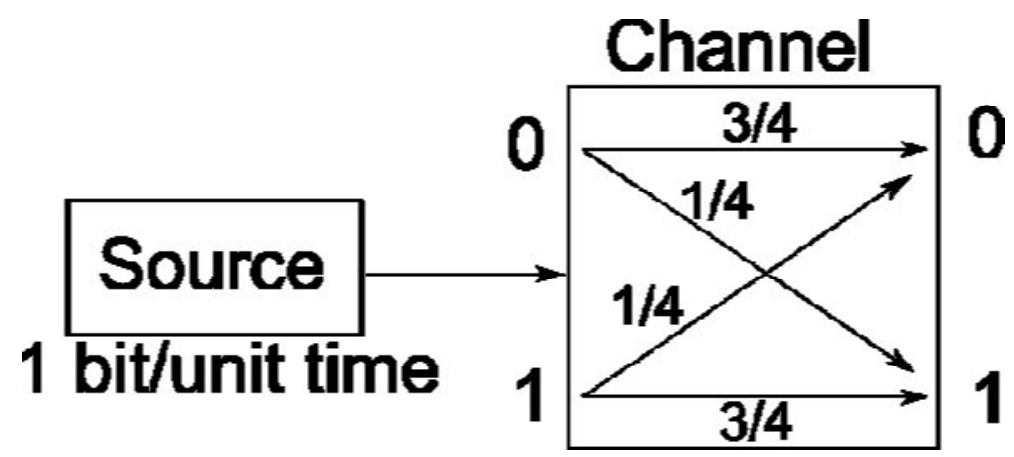

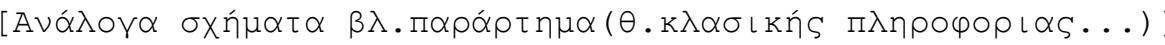

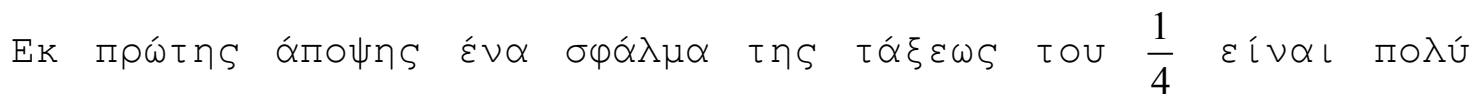

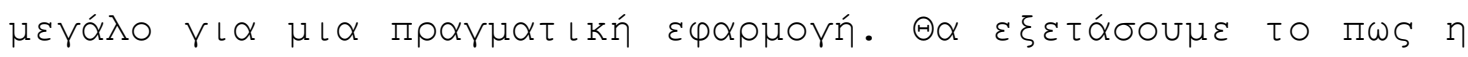

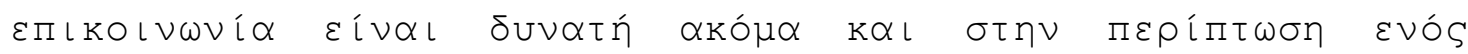

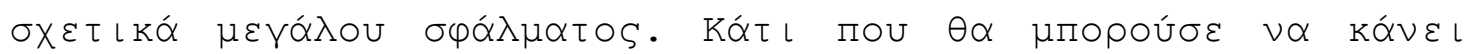

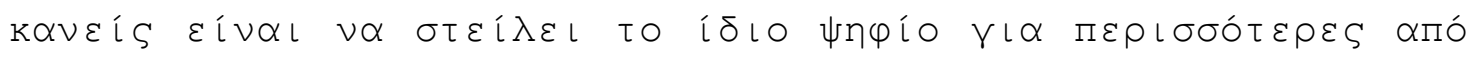

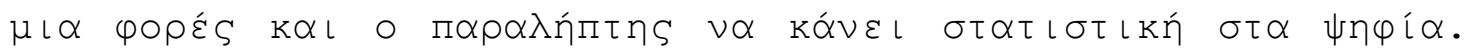

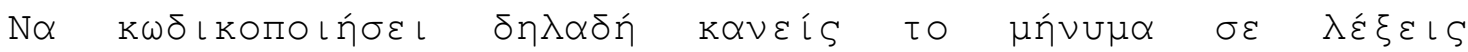

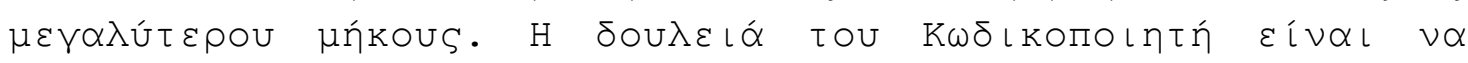

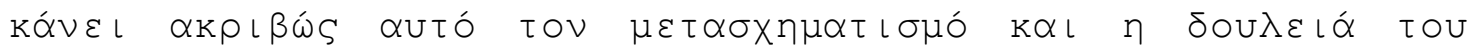

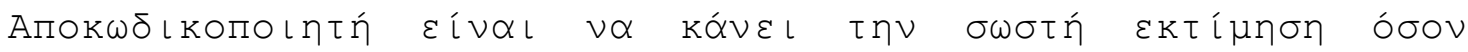

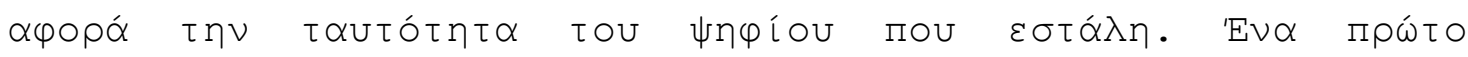

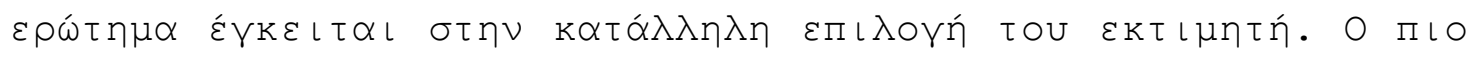

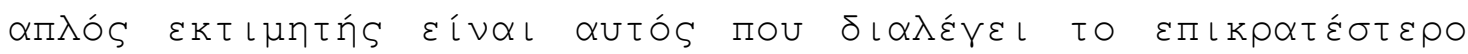

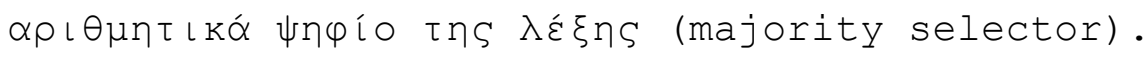

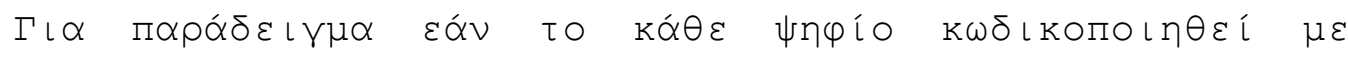

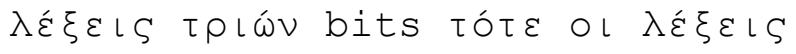

$$
100,010,001,000
$$

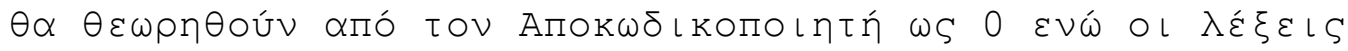

$$
\text { 110, 011, 101, } 111
$$




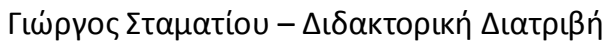

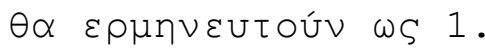

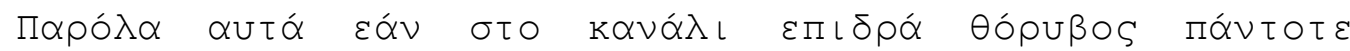

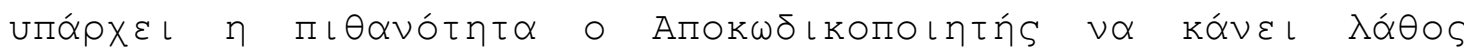

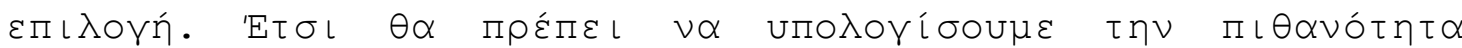

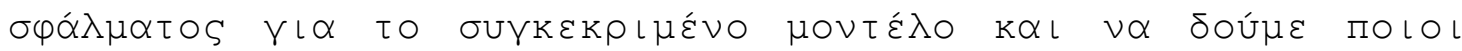

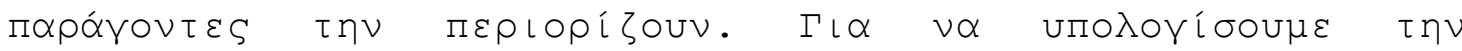

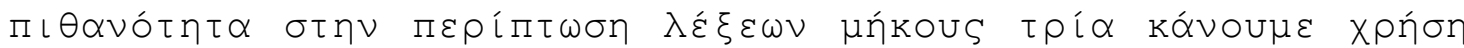

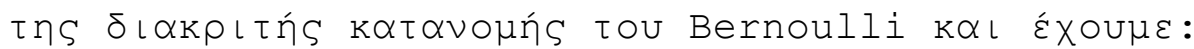

$$
\left(\begin{array}{l}
3 \\
2
\end{array}\right)\left(\frac{1}{4}\right)^{2} \frac{3}{4}+\left(\frac{1}{4}\right)^{3}=\frac{10}{64}<\frac{1}{4}
$$

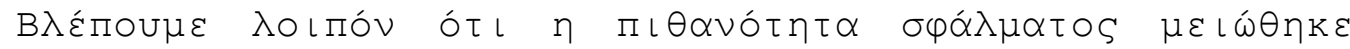

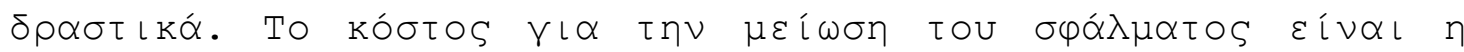

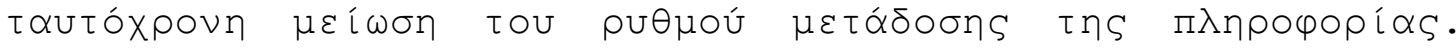

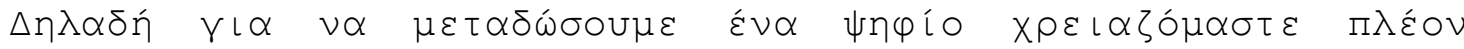

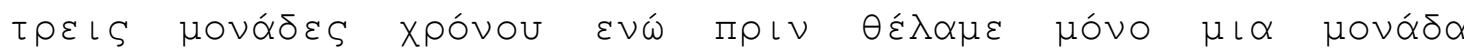
xpóvou.

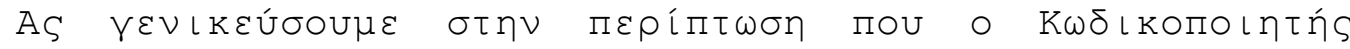

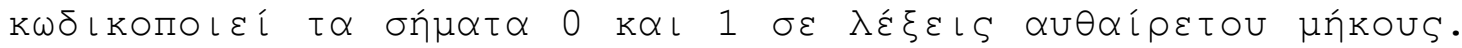

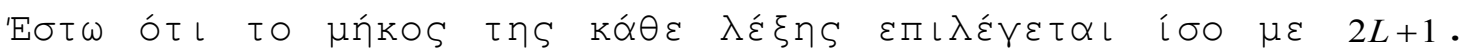

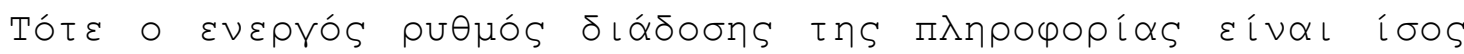
$\mu \varepsilon$

$$
\text { rate }=\frac{1}{2 L+1}
$$

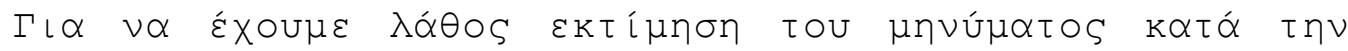

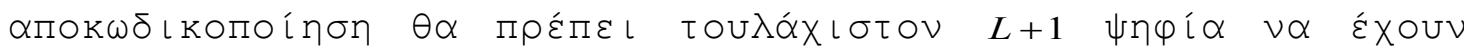

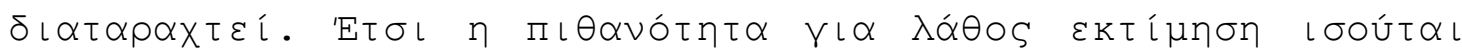
$\mu \varepsilon:$

$$
P_{\text {error }}=\sum_{k=L+1}^{2 L+1}\left(\begin{array}{c}
2 L+1 \\
k
\end{array}\right) \lambda^{k}(1-\lambda)^{2 L+1-k}
$$




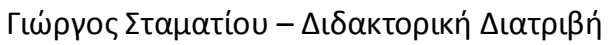

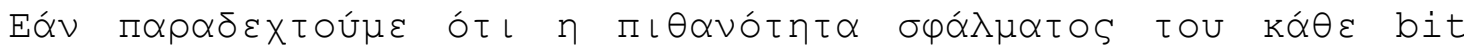

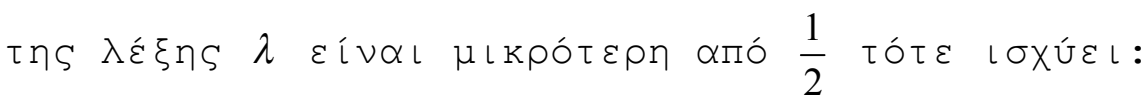

$$
(2 L+1) \lambda<L+1
$$

$\varepsilon \varphi o ́ \sigma \circ \nu$

$$
\lambda<\frac{1}{2}
$$

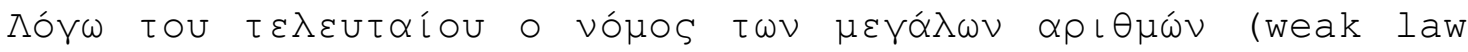

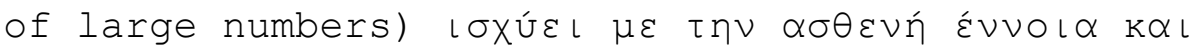

$$
\lim _{L \rightarrow \infty} P_{\text {error }}=0
$$

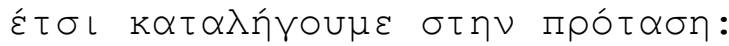

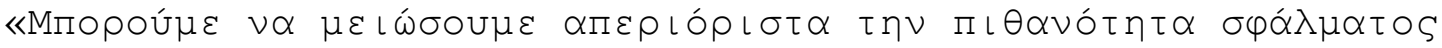

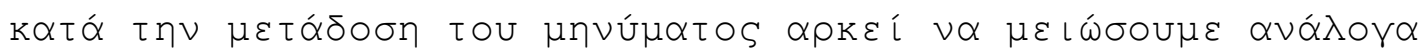

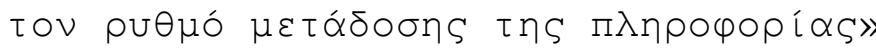

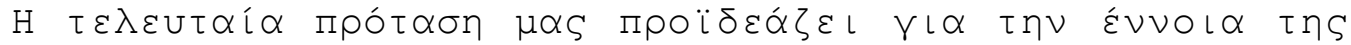

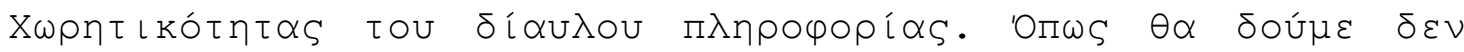

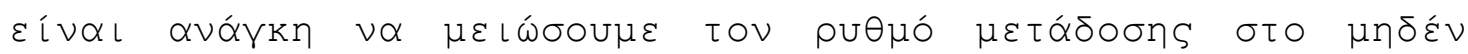

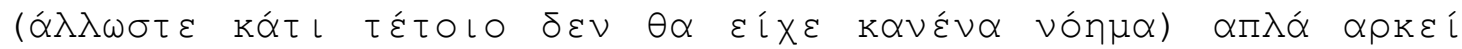

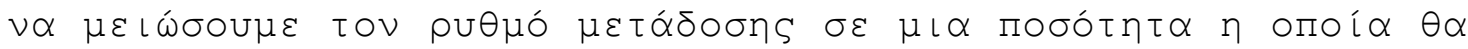

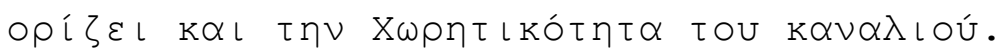

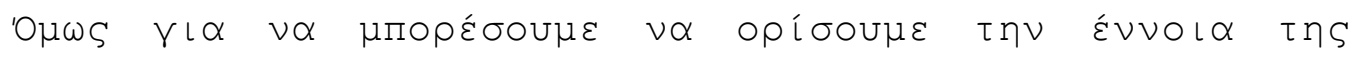

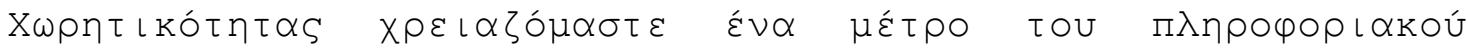

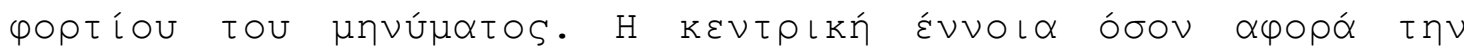

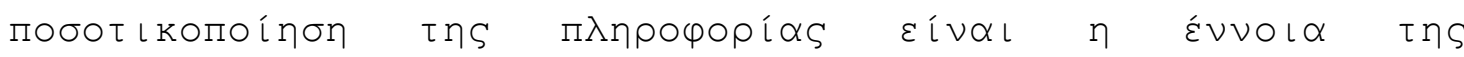

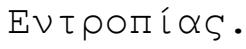




\section{H Eviponía Shannon.}

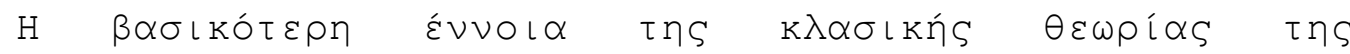

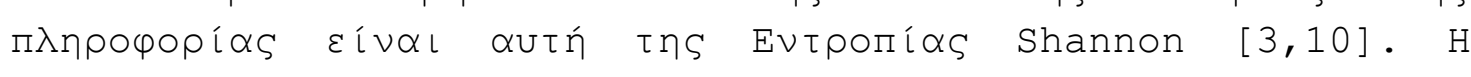

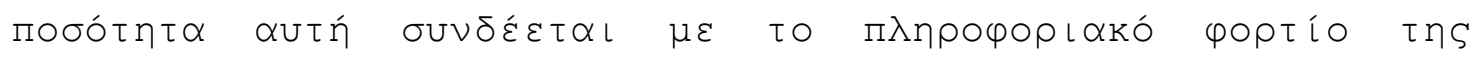

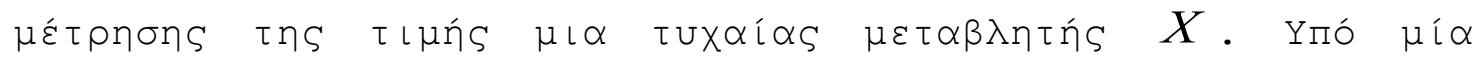

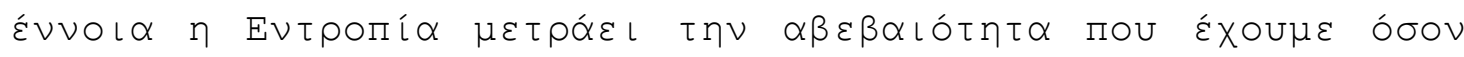

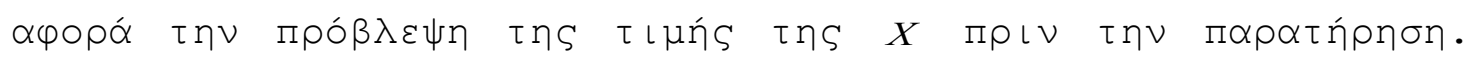

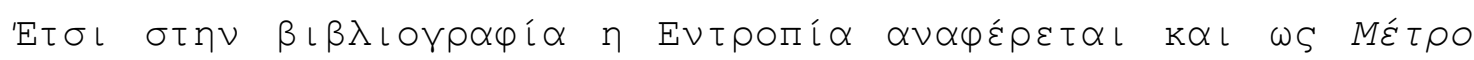
A $\beta \varepsilon \beta \alpha \iota$ ótntas (uncertainty measure).

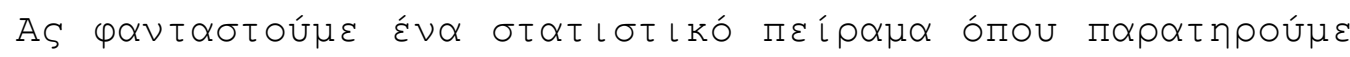

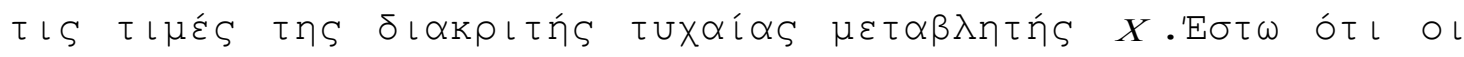

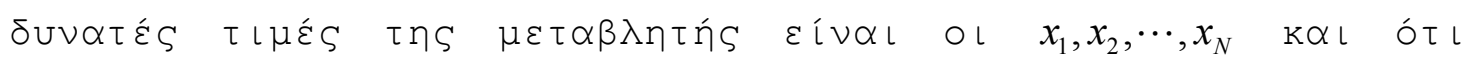

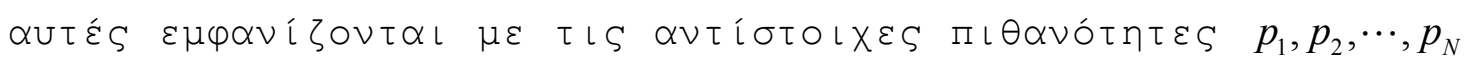

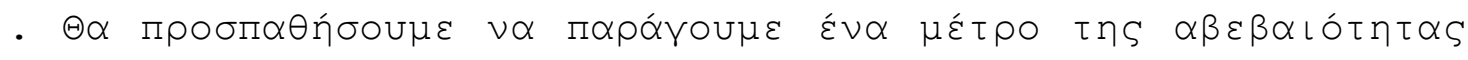

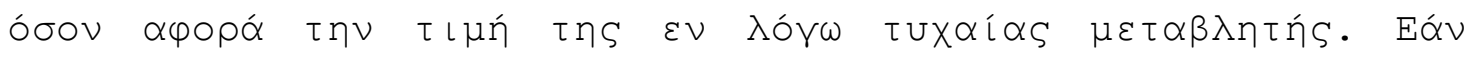

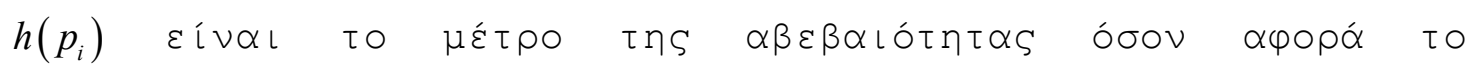

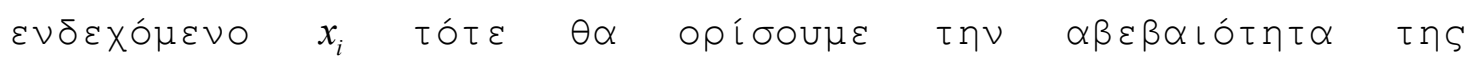

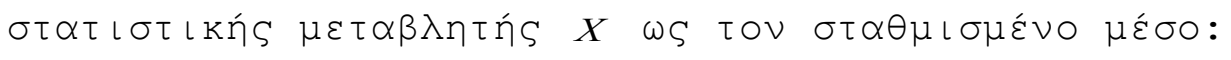

$$
H\left(p_{1}, p_{2}, \cdots, p_{N}\right)=\sum_{i=1}^{N} p_{i} h\left(p_{i}\right)
$$

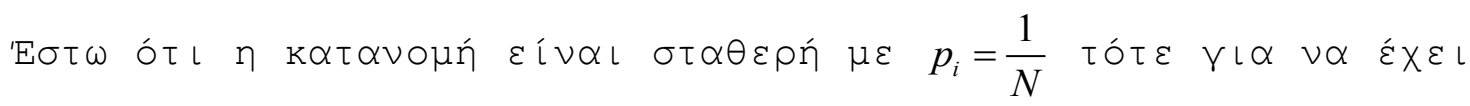

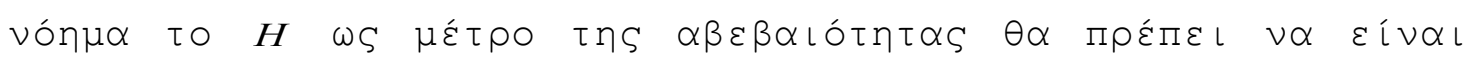

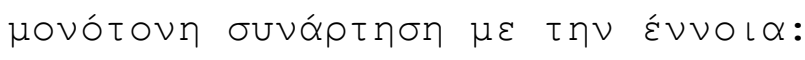

$$
H(N)<H\left(N^{\prime}\right)
$$

$\varepsilon \alpha ́ v$

$$
N<N^{\prime}
$$




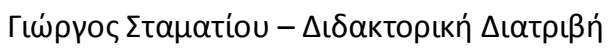

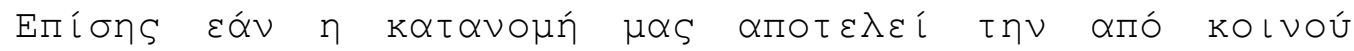

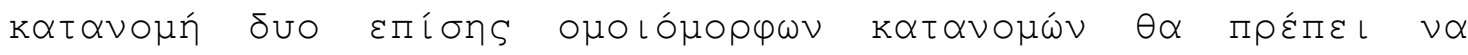
เ $\sigma \times \dot{\varepsilon} \varepsilon$ :

$$
H(N L)=H(N)+H(L)
$$

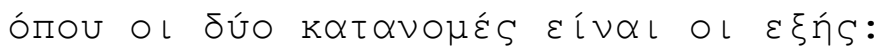

$$
x_{1}, x_{2}, \cdots, x_{N} \quad \mu \varepsilon \quad p_{i}=\frac{1}{N}
$$

$K \alpha \iota$

$$
x_{1}, x_{2}, \cdots, x_{L} \quad \mu \varepsilon \quad p_{i}^{\prime}=\frac{1}{L}
$$

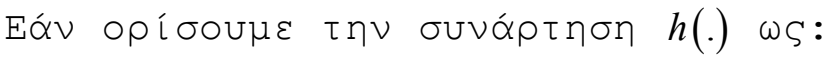

$$
h(x)=-K \log (x)
$$

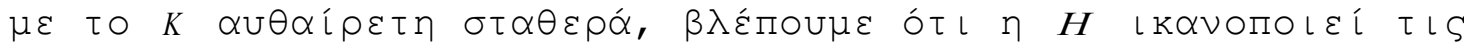

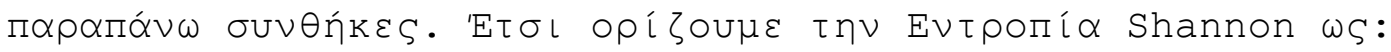

$$
H\left(p_{1}, p_{2}, \cdots, p_{N}\right)=-\sum_{i=1}^{N} p_{i} \log \left(p_{i}\right)
$$

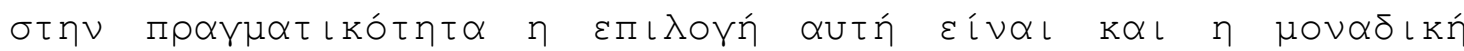

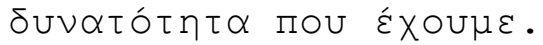

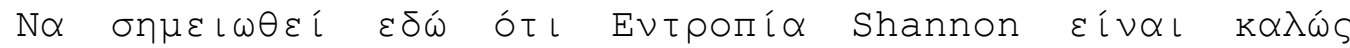

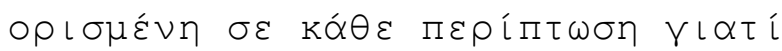

$$
\lim _{x \rightarrow 0}(x \log x)=0
$$

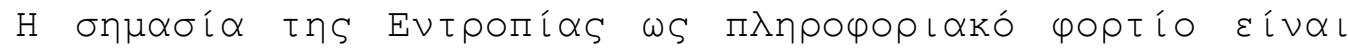

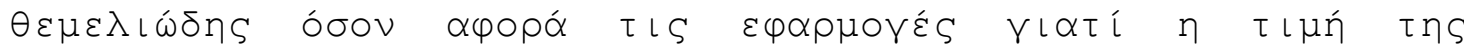

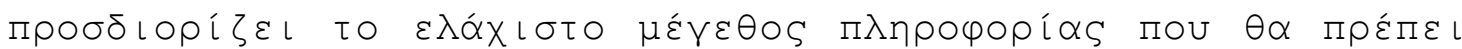

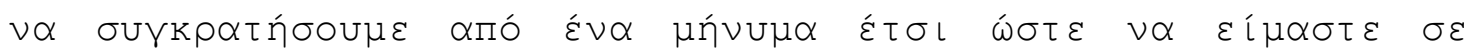

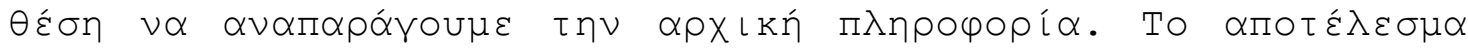

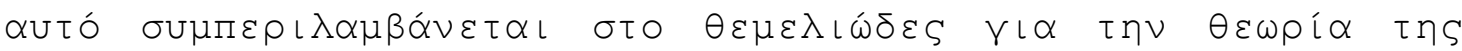




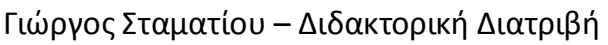

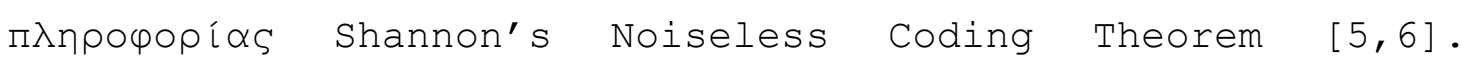

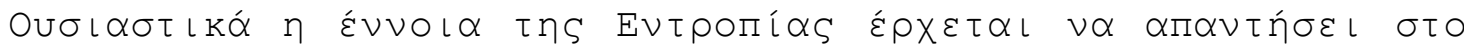

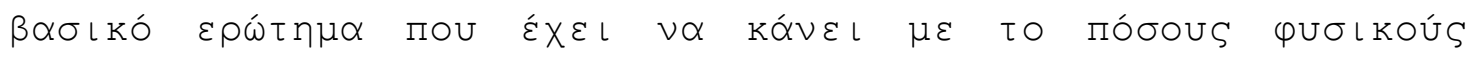

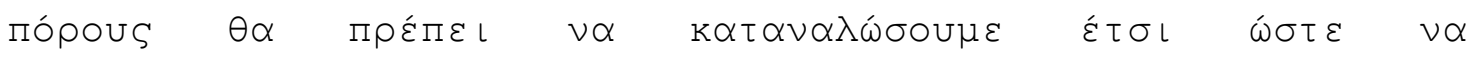

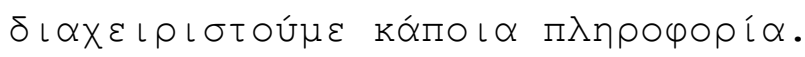

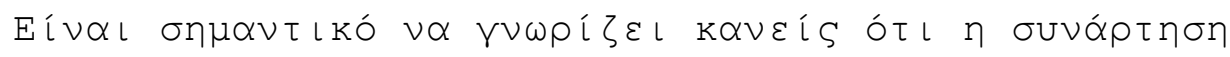

$$
H\left(p_{1}, p_{2}, \ldots, p_{M}\right)=-C \sum_{i=1}^{M} p_{i} \log p_{i}
$$

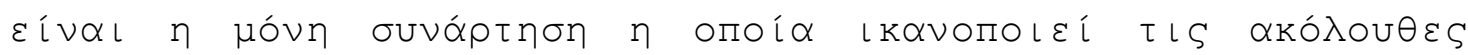

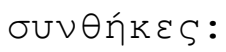

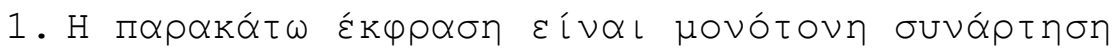

$$
H\left(\frac{1}{L}, \frac{1}{L}, \ldots, \frac{1}{L}\right)=f(L)
$$

2. ıoXúkl ótı

$$
f(M L)=f(M)+f(L), \quad M, L=1,2, \ldots
$$

3. (Grouping Axiom)

$$
\begin{gathered}
H\left(p_{1}, \ldots, p_{M}\right)= \\
=H\left(p_{1}+\ldots+p_{k}, p_{k+1}+\ldots+p_{M}\right)+\left(p_{1}+\ldots+p_{k}\right) H\left(\frac{p_{1}}{\sum_{i=1}^{k} p_{i}}, \ldots, \frac{p_{k}}{\sum_{i=1}^{k} p_{i}}\right) \\
+\left(p_{k+1}+\ldots+p_{M}\right) H\left(\frac{p_{k+1}}{\sum_{i=k+1}^{M} p_{i}}, \ldots, \frac{p_{M}}{\sum_{i=k+1}^{M} p_{i}}\right) \\
k=1,2, \ldots, M-1
\end{gathered}
$$




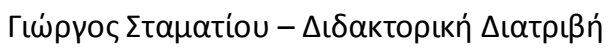

4. H ouvóptnon

$$
H(p, 1-p)
$$

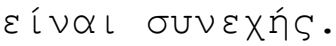

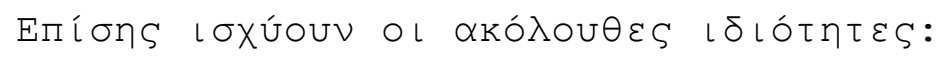

1.

$$
H\left(p_{1}, p_{2}, \ldots, p_{M}\right) \leq \log M
$$

2 .

$$
H\left(X_{1}, X_{2}, \ldots, X_{M}\right) \leq \sum_{i=1}^{M} H\left(X_{i}\right)
$$

ónоu $\tau \alpha$

$$
X_{1}, X_{2}, \ldots, X_{M}
$$

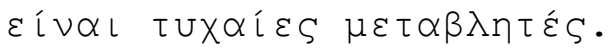

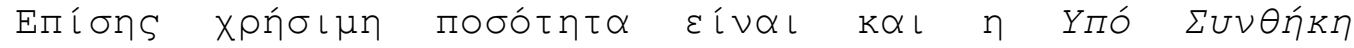
Evтропi (Conditional Entropy)

$$
H(X \mid Y) \equiv-\sum_{y \in Y} p(y) \sum_{x \in X} p(x \mid y) \log p(x \mid y)
$$

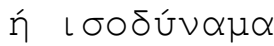

$$
H(X \mid Y) \equiv-\sum_{y \in Y} \sum_{x \in X} p(x, y) \log p(x \mid y)
$$

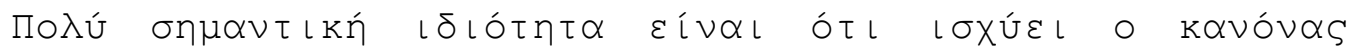
$\alpha \lambda v \sigma i \delta \alpha s$ (chain rule)

$$
\begin{gathered}
H(X, Y)=H(X)+H(Y \mid X) \\
H(X \mid Y) \leq H(X)
\end{gathered}
$$




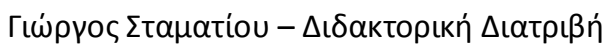

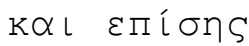

$$
\begin{gathered}
H(X, Y, Z)=H(X, Y)+H(Z \mid X, Y) \\
H(Z \mid X, Y) \leq H(Z \mid Y)
\end{gathered}
$$

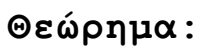

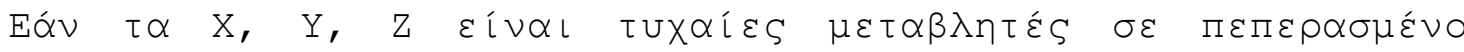

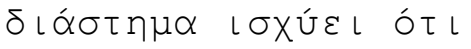

$$
H(X, Y, Z) \leq H(X, Y)+H(Y, Z)-H(Y)
$$

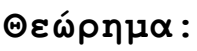

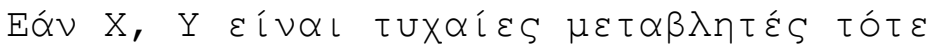

$$
H(X \mid Y) \leq p \log (d-1)+H(p, 1-p)
$$

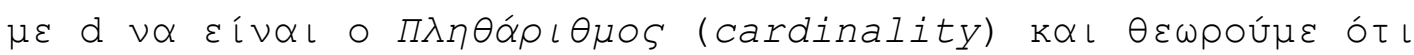

$$
p \equiv \operatorname{prob}(X \neq Y)
$$

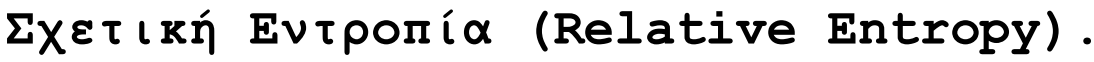

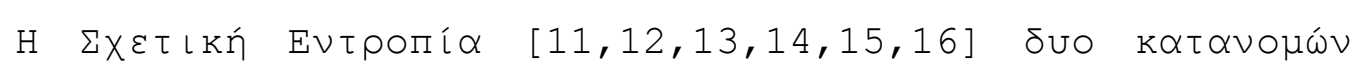

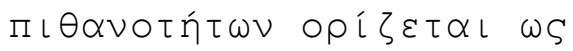

$$
D\left(p_{1} \| p_{2}\right) \equiv \sum_{x} p_{1}(x)\left[\log p_{1}(x)-\log p_{2}(x)\right]
$$

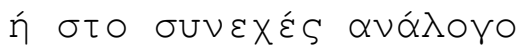

$$
D\left(p_{1} \| p_{2}\right)=\int_{-\infty}^{+\infty} p_{1}(x) \log \frac{p_{1}(x)}{p_{2}(x)} d x
$$




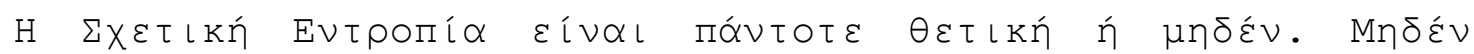

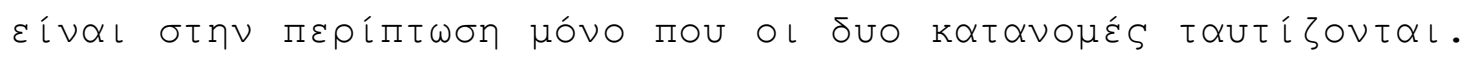

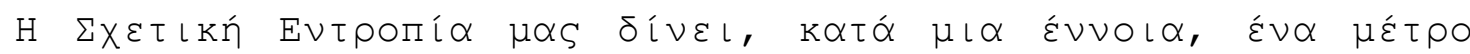

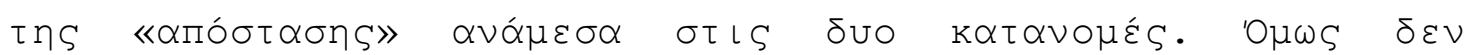

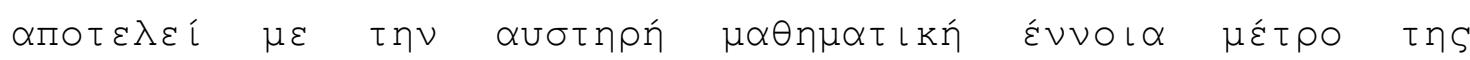

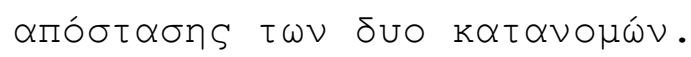

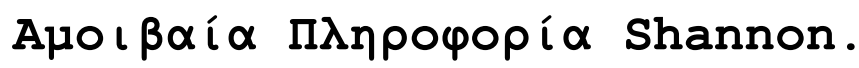

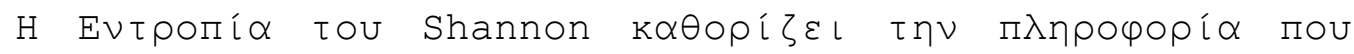

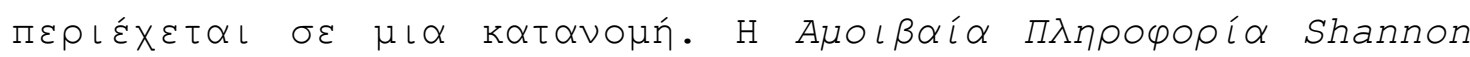

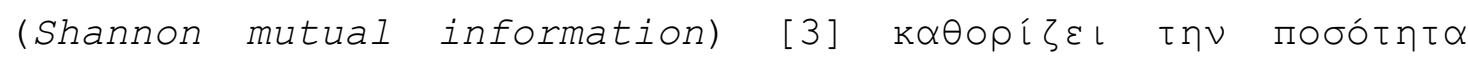

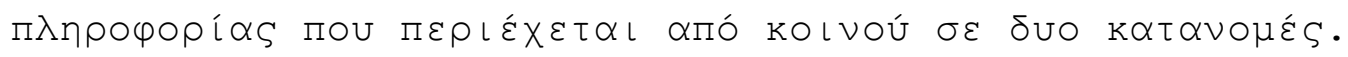

$$
I(X \wedge Y) \equiv \sum_{x, y} p(x, y) \log \left[\frac{p(x, y)}{p(x) q(y)}\right]
$$

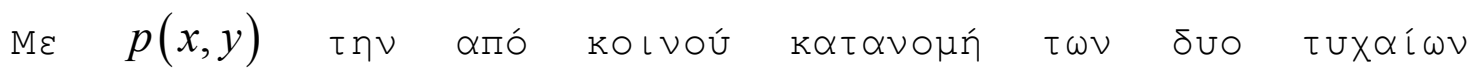

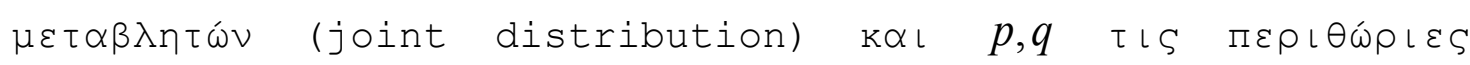

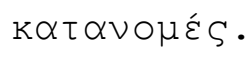

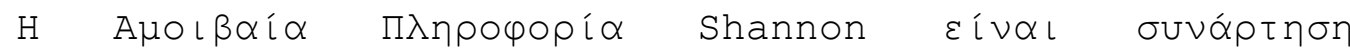

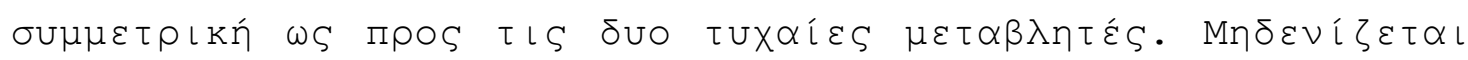

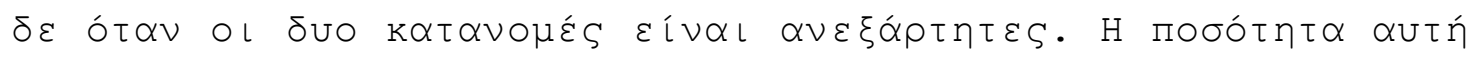

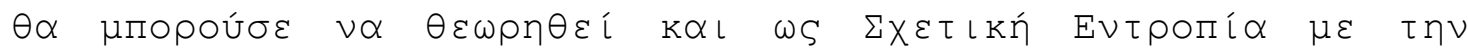

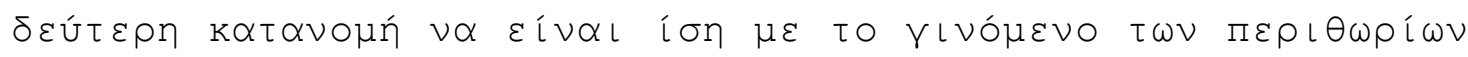

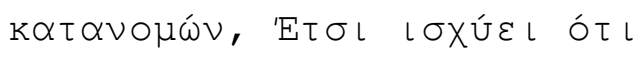

$$
I(X \wedge Y) \geq 0
$$

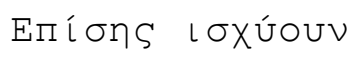

$$
I(X \wedge Y) \leq H(X)
$$

$K \propto l$

$$
I(X \wedge Y) \leq H(Y)
$$




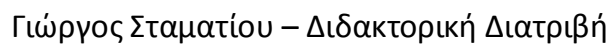

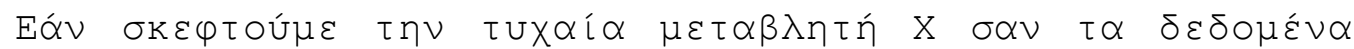

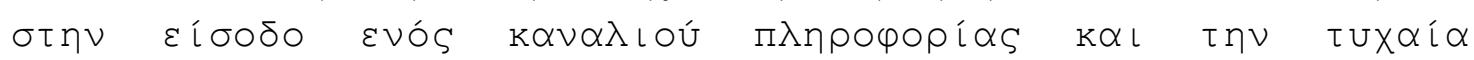

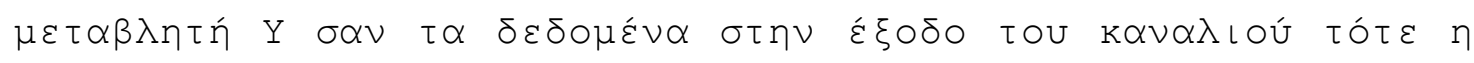

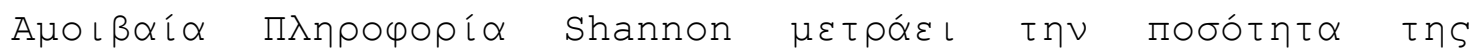

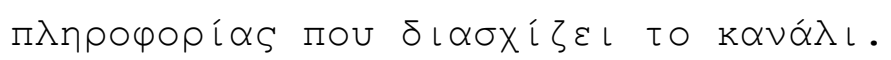

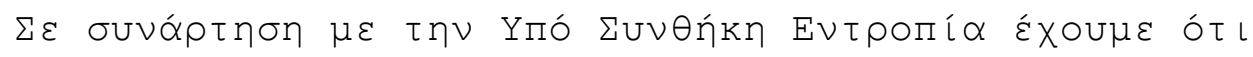

$$
I(X \wedge Y)=H(X)-H(X \mid Y)
$$

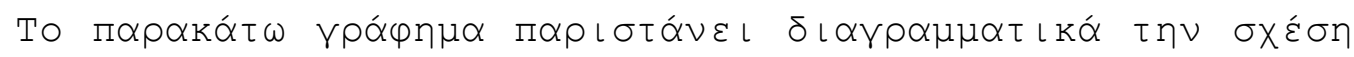

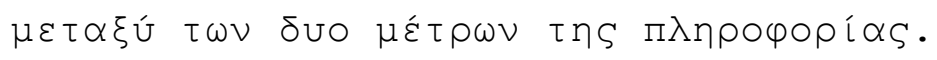

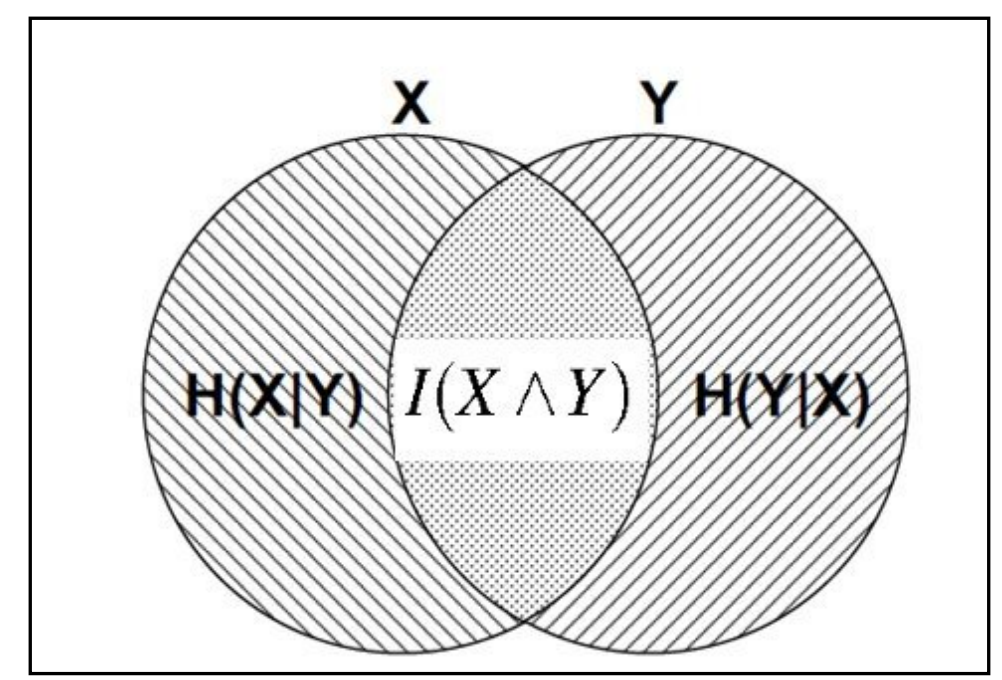

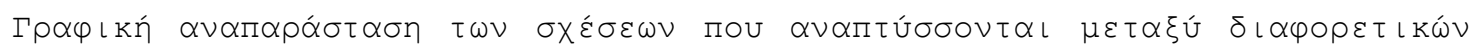

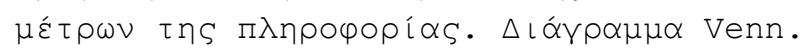

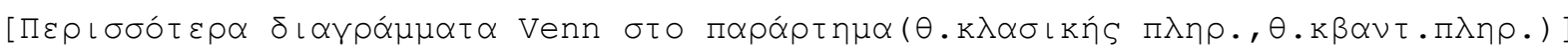




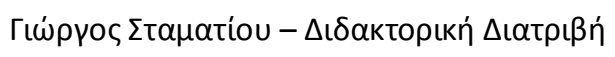

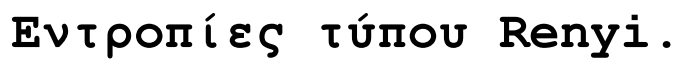

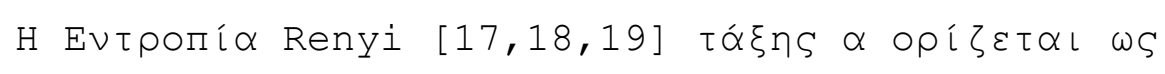

$$
H_{a}\left(p_{1}, p_{2}, \ldots, p_{M}\right) \equiv \frac{1}{1-a} \log \sum_{k=1}^{M} p_{k}^{a}
$$

$\mu \varepsilon$

$$
a \neq 1
$$

$K \alpha \iota$

$$
\left(p_{1}, p_{2}, \ldots, p_{M}\right)
$$

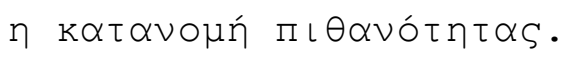

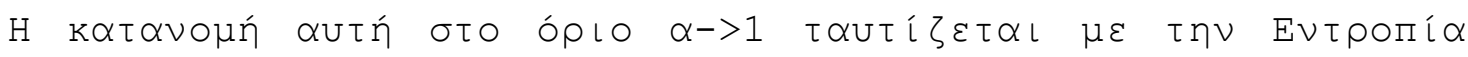
tou Shannon.

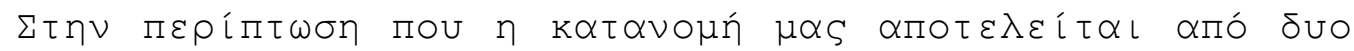

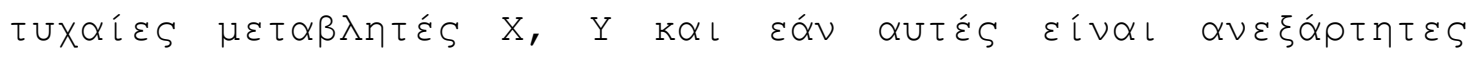

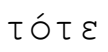

$$
H_{a}(X, Y)=H_{a}(X)+H_{a}(Y)
$$

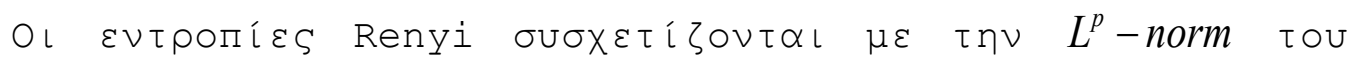

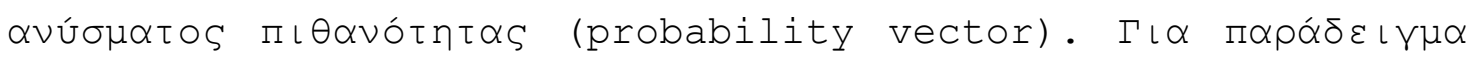

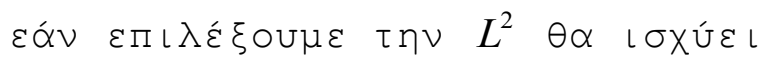

$$
H_{2}(p)=-2 \log \|p\|_{2}
$$

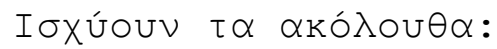

$$
\begin{gathered}
H_{0}(X)=\log M=\log |X| \\
H_{\infty}(X)=-\log \left(\sup _{i=1 . . . M}\left\{p_{i}\right\}\right)
\end{gathered}
$$




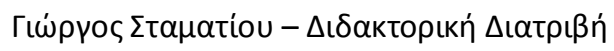

$$
\begin{gathered}
H_{2}<2 H_{\infty} \\
H_{\infty}<H_{2} \\
H_{1}(X) \geq H_{2}(X)
\end{gathered}
$$

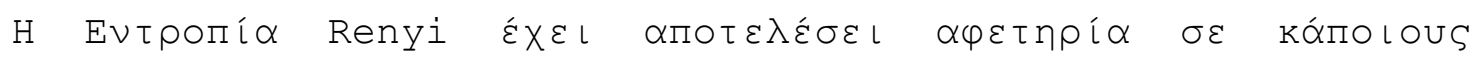

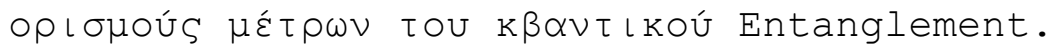

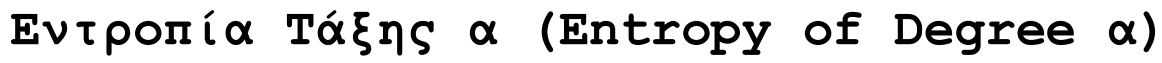

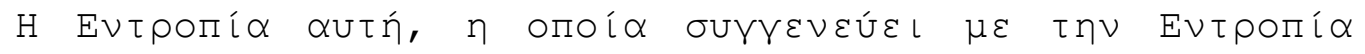

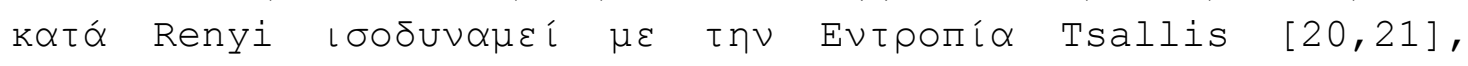

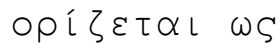

$$
H^{a}\left(p_{1}, p_{2}, \ldots, p_{M}\right) \equiv \frac{1}{2^{1-a}-1}\left(\sum_{k=1}^{M} p_{k}^{a}-1\right)
$$

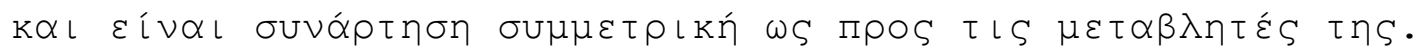

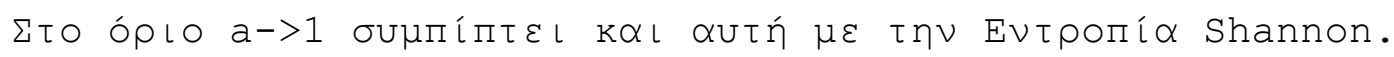

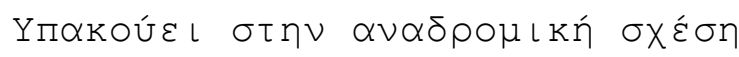

$$
\begin{gathered}
H^{a}\left(p_{1}, p_{2}, \ldots, p_{M}\right)=H^{a}\left(p_{1}+p_{2}, p_{3}, \ldots, p_{M}\right) \\
\quad+\left(p_{1}+p_{2}\right)^{a} H^{a}\left(\frac{p_{1}}{p_{1}+p_{2}}, \frac{p_{2}}{p_{1}+p_{2}}\right)
\end{gathered}
$$

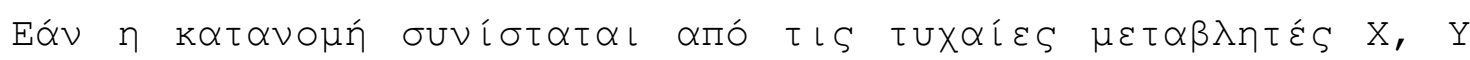

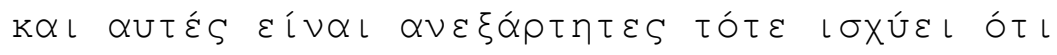

$$
H^{a}(X, Y)=H^{a}(X)+H^{a}(Y)+\left(2^{1-a}-1\right) H^{a}(X) H^{a}(Y)
$$




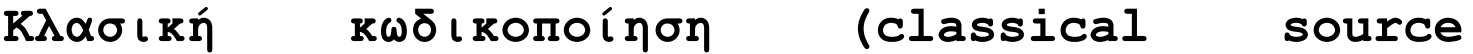 encoding) $k \alpha \iota$ Eviponí $\alpha$ Shannon.}

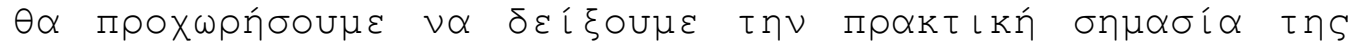

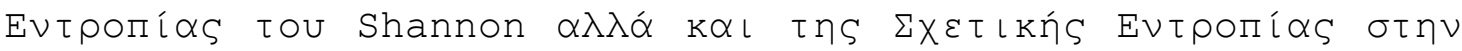

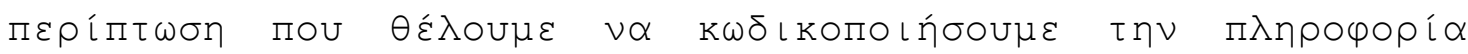

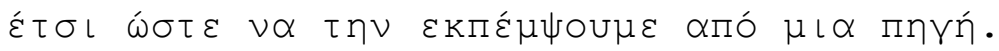

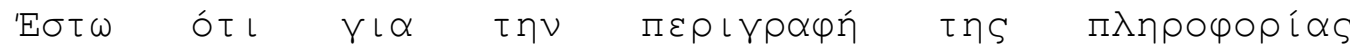

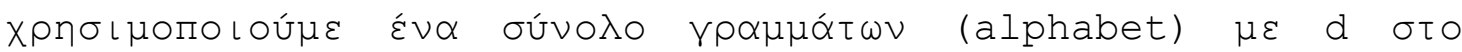
$\Pi \lambda \dot{\theta} \theta$ ○ $\sigma \tau \circ\llcorner\chi \varepsilon \dot{\imath} \alpha$

$$
\{0,1,2, \ldots, d-1\}
$$

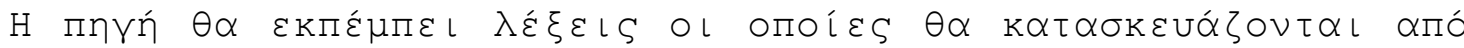

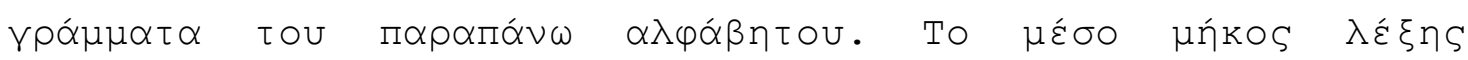
(codeword) $\theta \alpha$ \&iv $\alpha \iota$

$$
L(C) \equiv \sum_{x} p(x) \ell(x)
$$

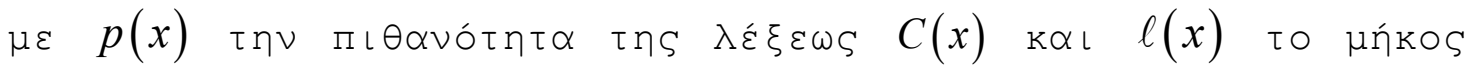

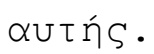

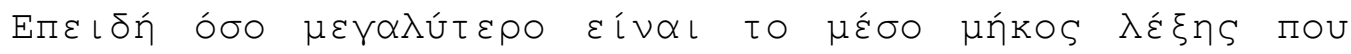

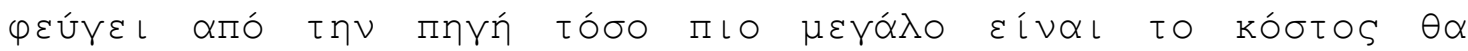

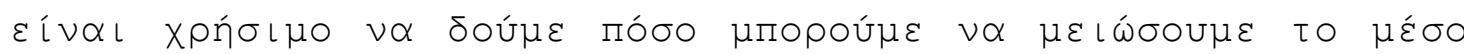

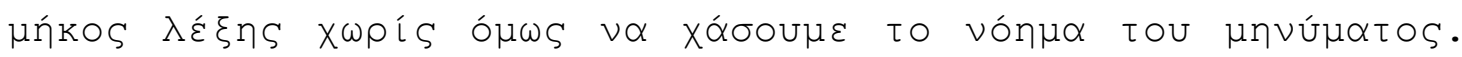

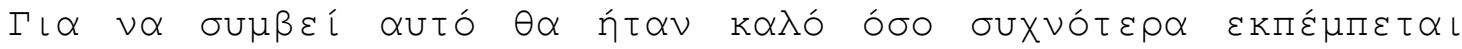

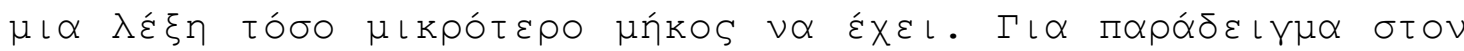

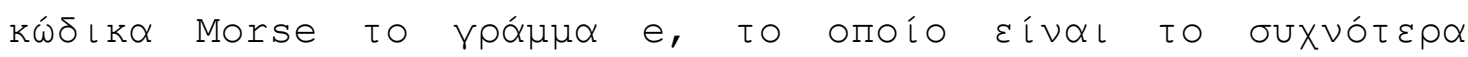

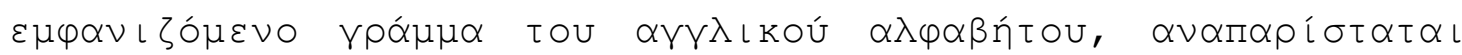
$\mu \varepsilon \quad \mu \iota \alpha \quad \alpha \Pi \lambda \dot{n} \tau \varepsilon \lambda \varepsilon i \alpha$.

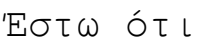

$$
\begin{array}{r}
\bar{n}=\sum_{i=1}^{M} p_{i} n_{i} \\
\mu \varepsilon \quad\left(\begin{array}{l}
p_{i}=p\left(x_{i}\right) \\
n_{i}=\ell\left(x_{i}\right)
\end{array}\right)
\end{array}
$$




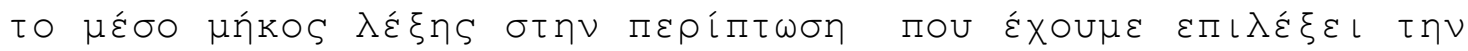

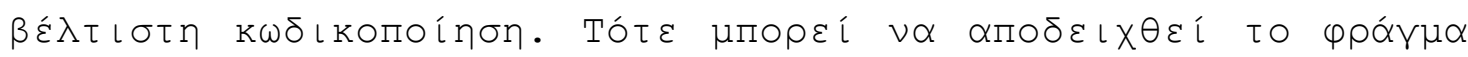
(Noiseless Coding Theorem)

$$
\bar{n} \geq \frac{H(x)}{\log d}
$$

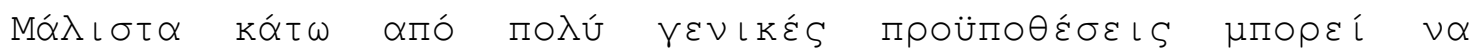

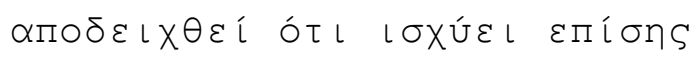

$$
\bar{n}<\frac{H(x)}{\log d}+1
$$

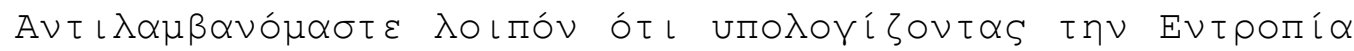

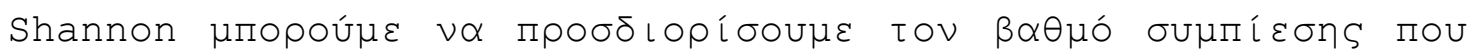

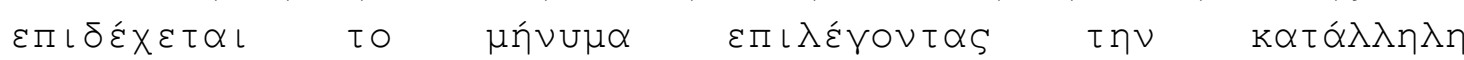

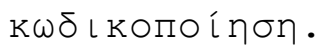

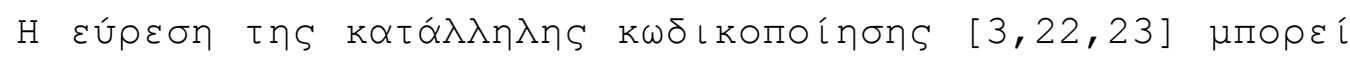

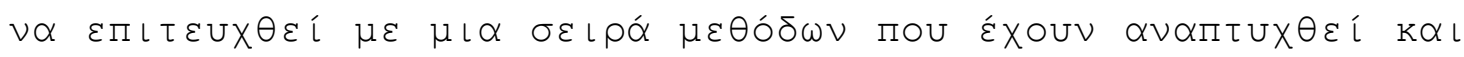

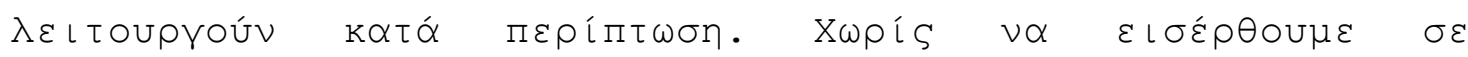

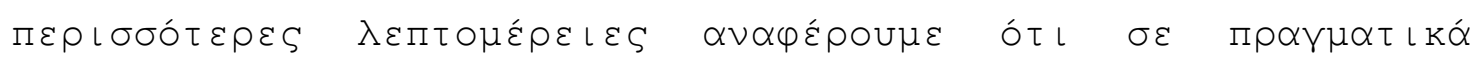

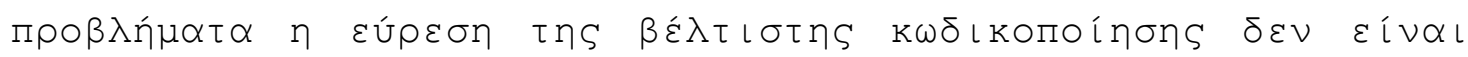

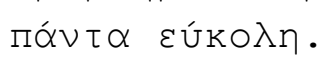

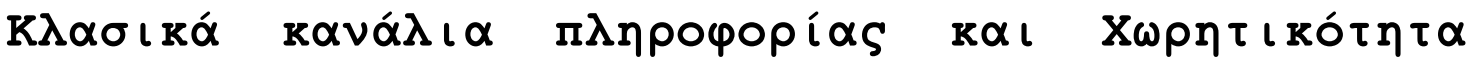 Shannon.}

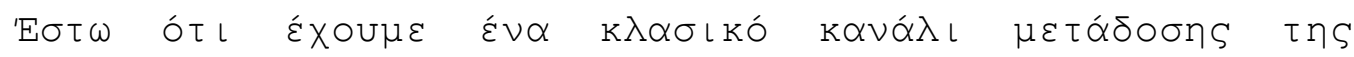

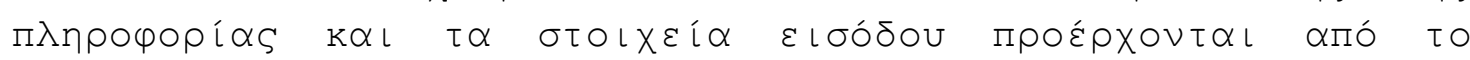

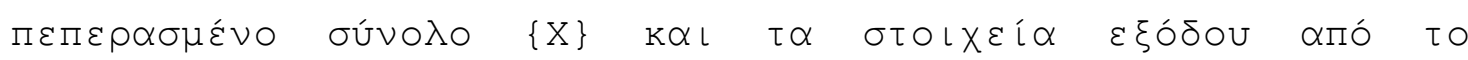

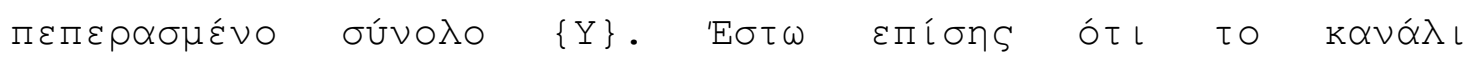

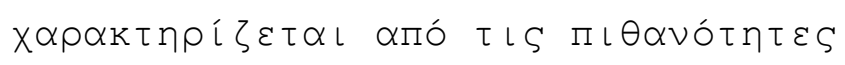

$$
T_{y x} \equiv \operatorname{prob}(Y=y \mid X=x)
$$




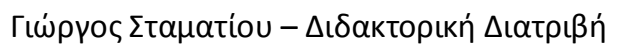

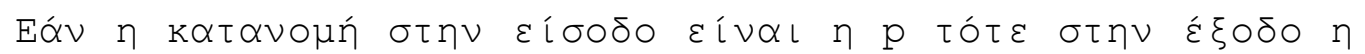

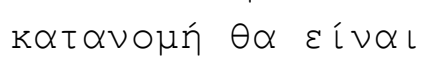

$$
q(y)=\sum_{x} T_{y x} p(x)
$$

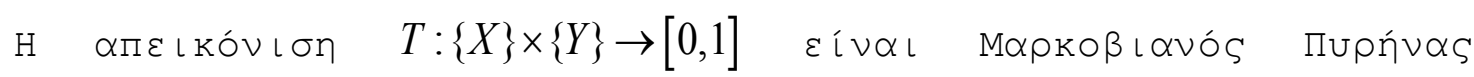
(Markov Kernel).

$$
\sum_{y} T_{y x}=1
$$

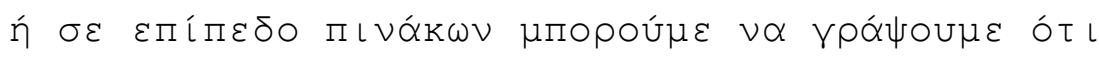

$$
q=T p
$$

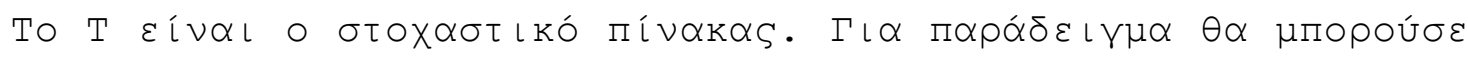

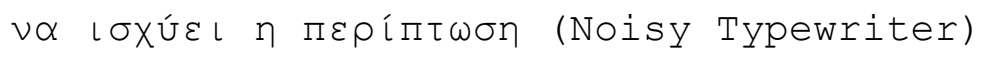

$$
T_{y x}=\left\{\begin{array}{cc}
1 / 2 & y=x \eta^{\prime} y=x+1 \\
0 & \alpha \lambda \lambda o v
\end{array}\right.
$$

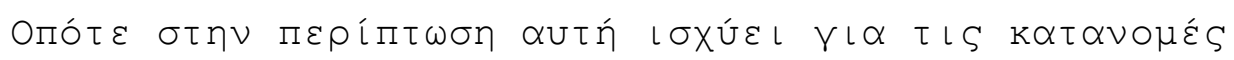

$$
p\left(x, y \phi=\frac{p(x)}{2} y \quad x \tau \alpha \dot{m} \quad y=x \quad=+1\right.
$$

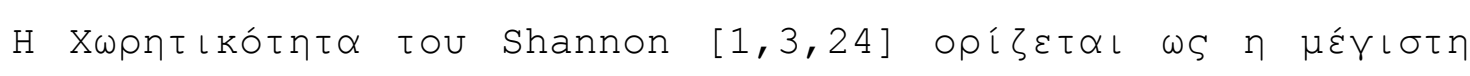

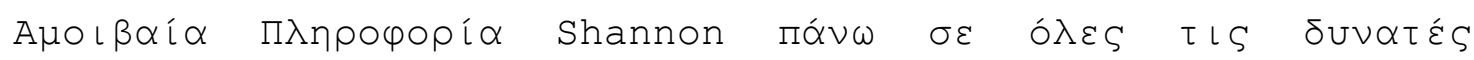

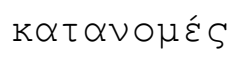

$$
C=\sup _{p}[I(X \wedge Y)]
$$

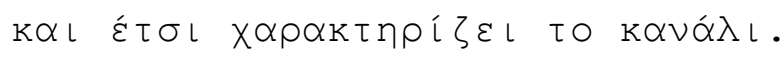

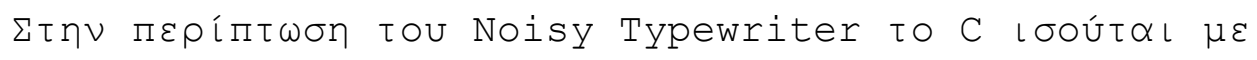

$$
-\sum_{x} \frac{p(x)+p(x-1)}{2} \log \left[\frac{p(x)+p(x-1)}{2}\right]-1
$$




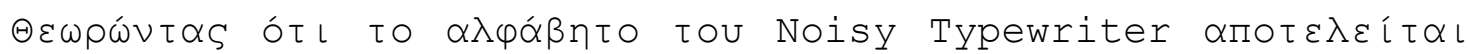

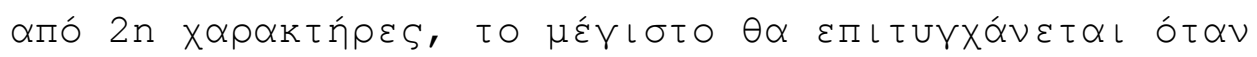

$$
\frac{p(x)+p(x-1)}{2}=\frac{1}{2 n}
$$

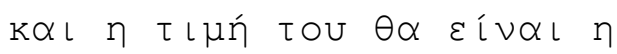

$$
\log (2 n)-1=\log n
$$

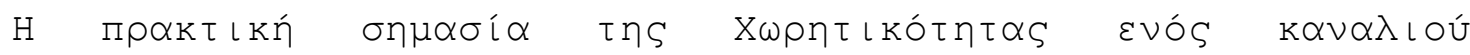

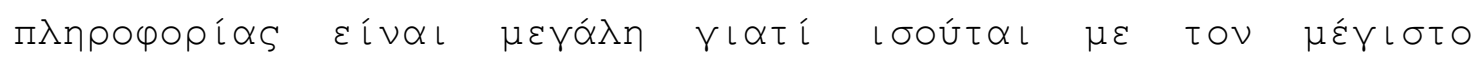

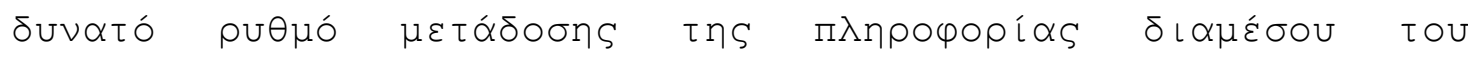

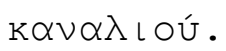

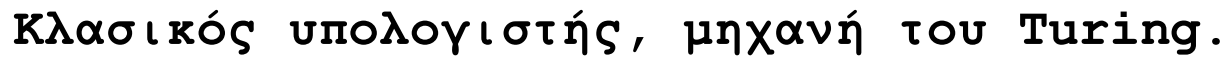

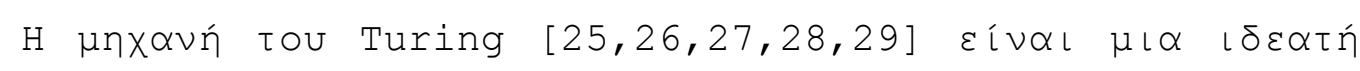

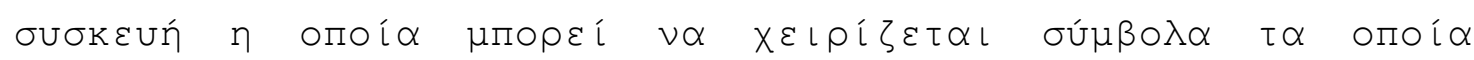

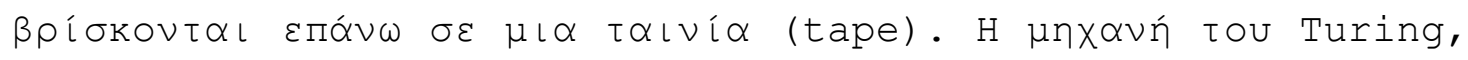

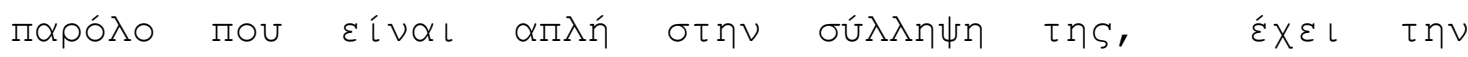

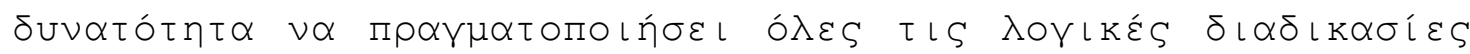

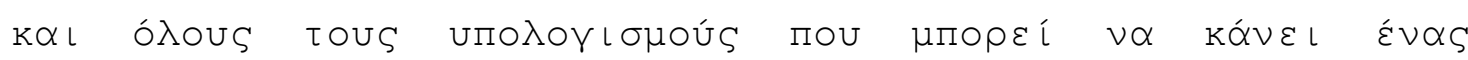

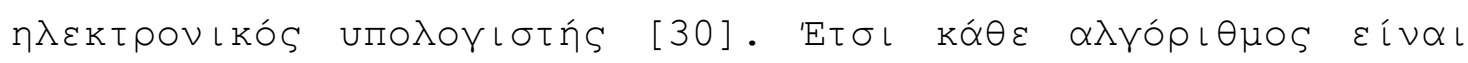

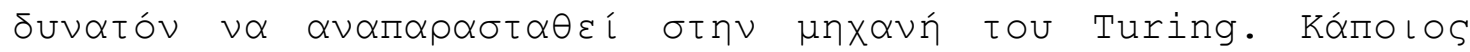

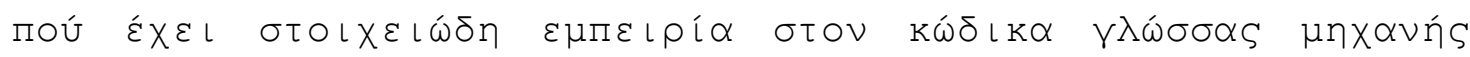

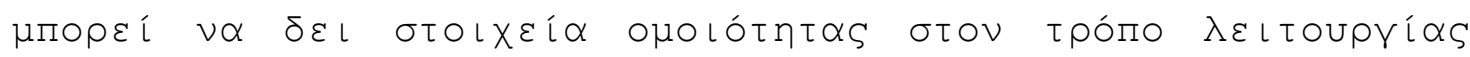

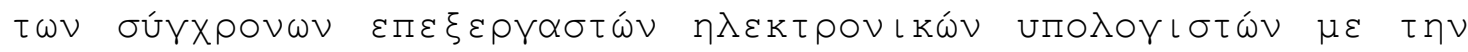

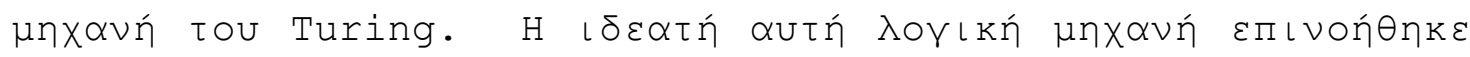

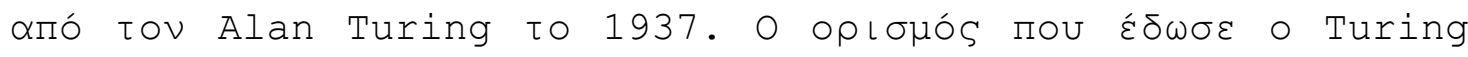

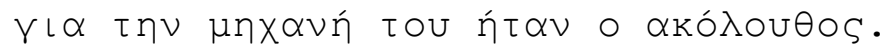

..."an infinite memory capacity obtained in the form of an infinite tape marked out into squares on each of which a symbol could be printed. At any moment there is one symbol in the machine; it is called the scanned symbol. The machine can alter the scanned symbol and its behavior is in part determined by that symbol, but the symbols on the tape elsewhere do not affect the behavior of the machine. 


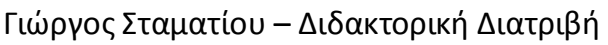

However, the tape can be moved back and forth through the machine, this being one of the elementary operations of the machine. Any symbol on the tape may therefore eventually have an innings" (Turing 1948)

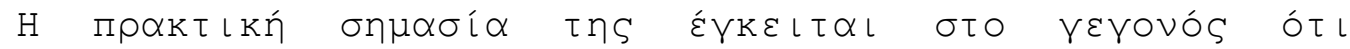

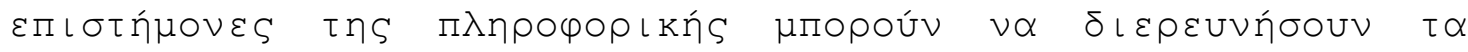

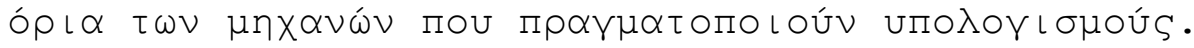

[http://en.wikipedia.org/wiki/File:Turing_machine_2a.svg]

[http://commons.wikimedia.org/wiki/File:Turing_machine_2b.svg]
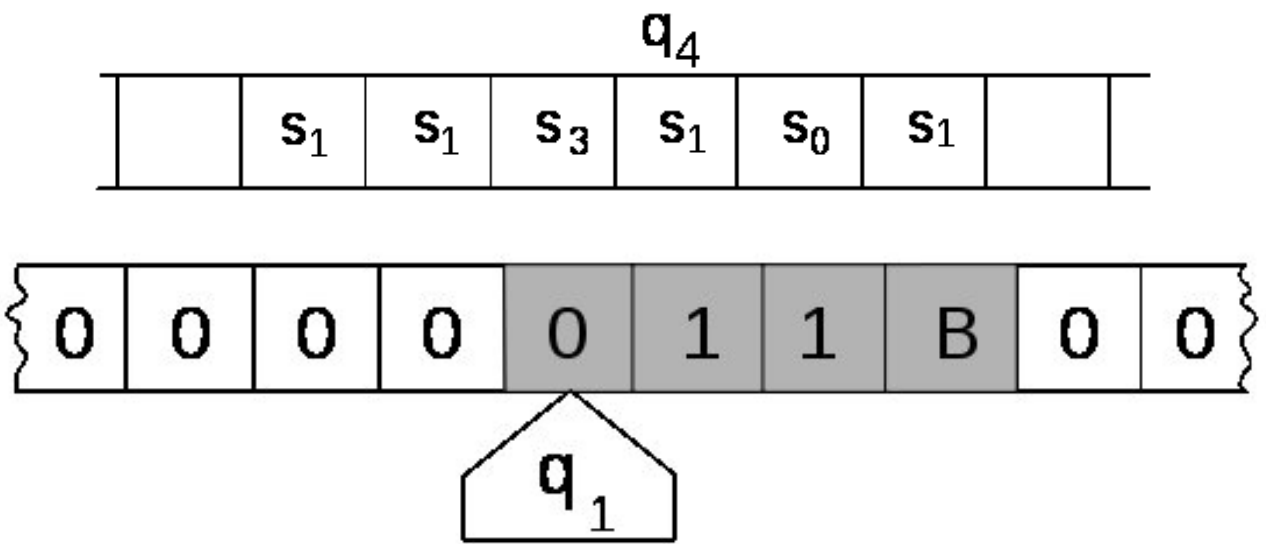

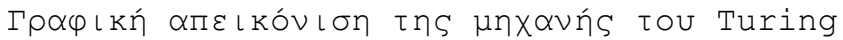

$[\beta \lambda . п \alpha \rho \alpha ́ \rho \tau \eta \mu \alpha]$

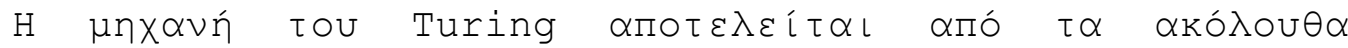
$\sigma \tau O\llcorner X \varepsilon i ́ \alpha:$

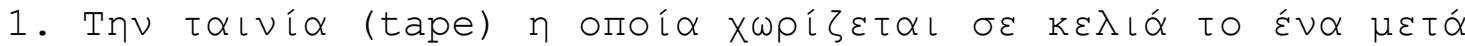

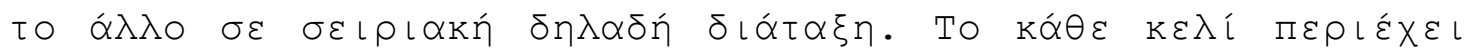

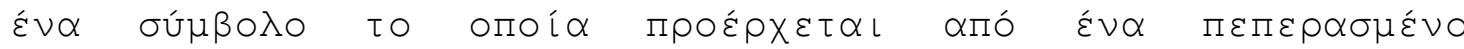

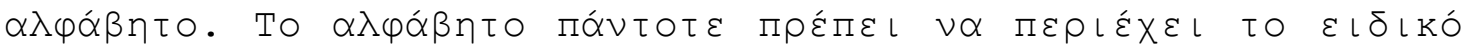

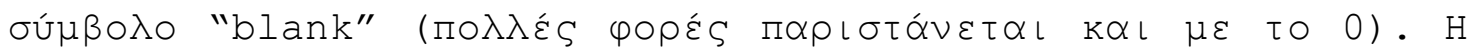

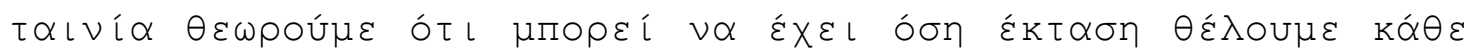

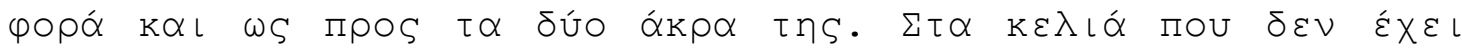

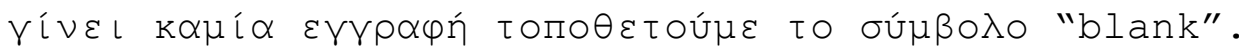

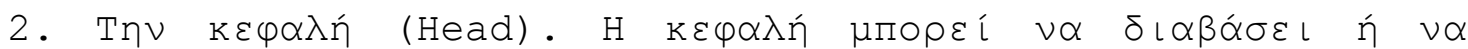

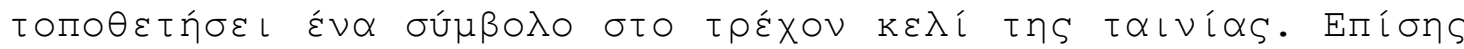

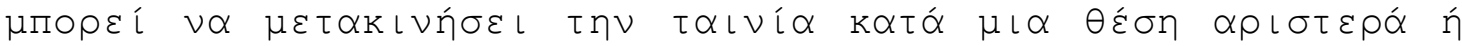




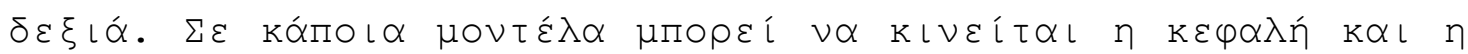

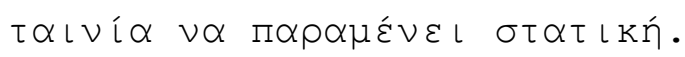

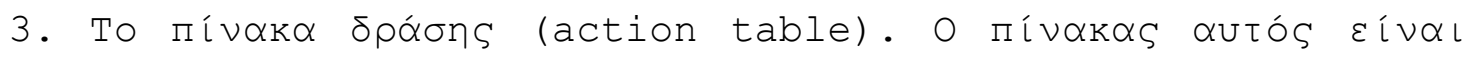

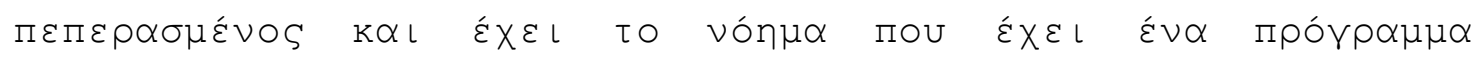

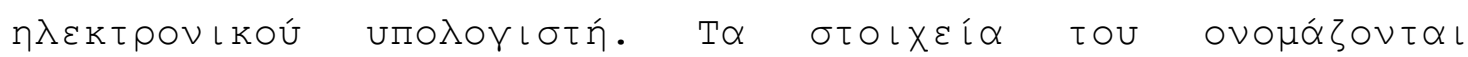

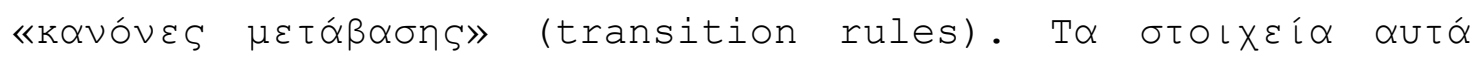

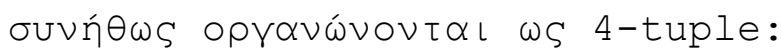

\section{$\left\langle\right.$ State ${ }_{0}, S_{y m b o l}$, State next $_{\text {, Action }\rangle}$}

"if the machine is in state state $_{0}$ and the current cell contains symbol then move into state state $_{\text {next }}$ taking $\boldsymbol{A c t i o n}^{\prime \prime}$

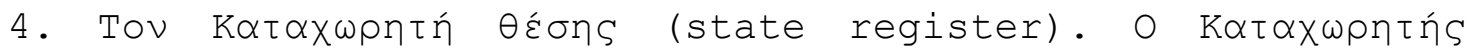

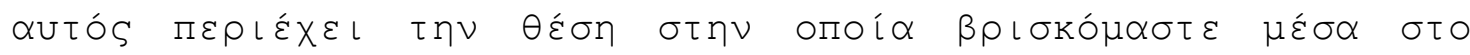
action table.

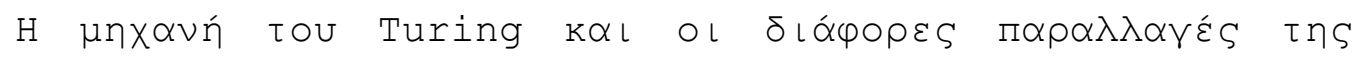

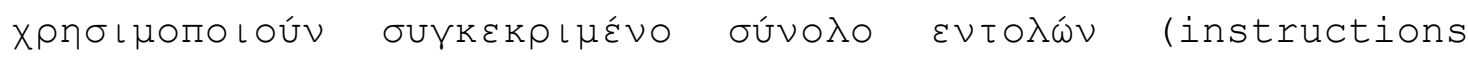

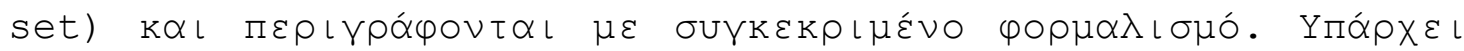

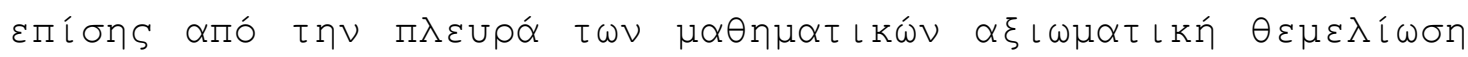

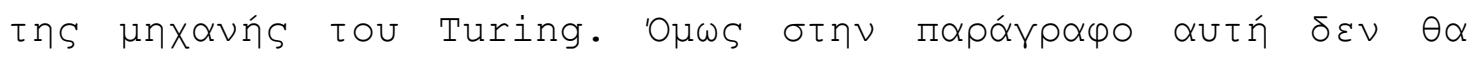

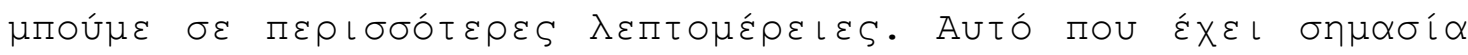

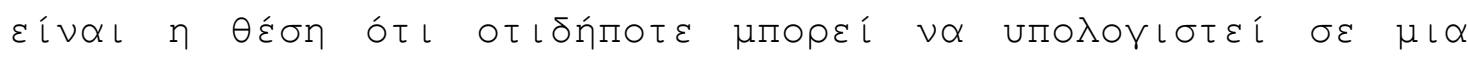

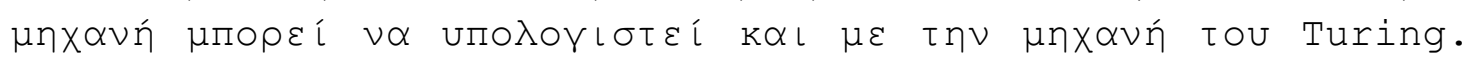

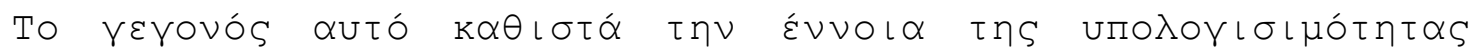

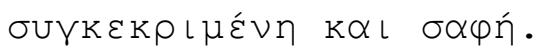

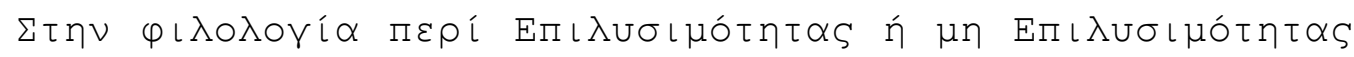

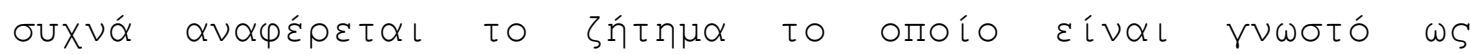

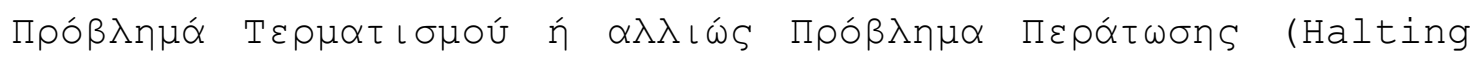

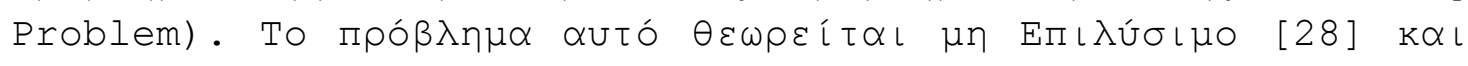

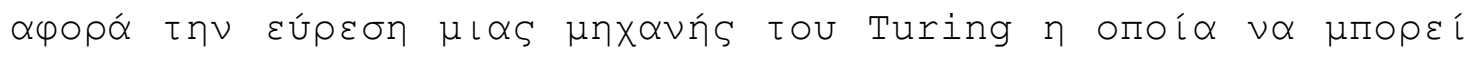

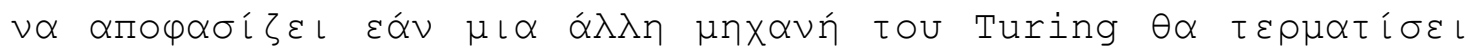

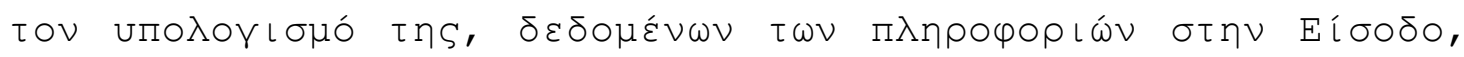

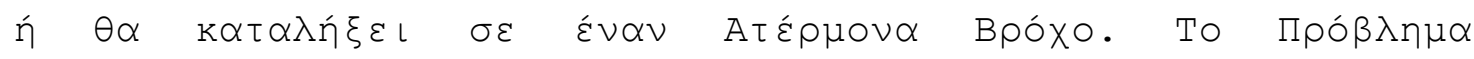

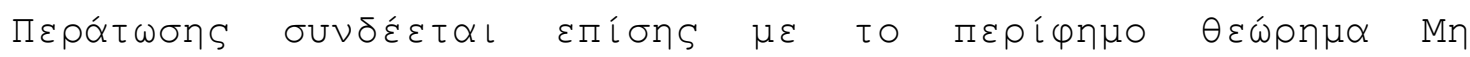

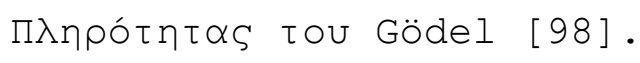




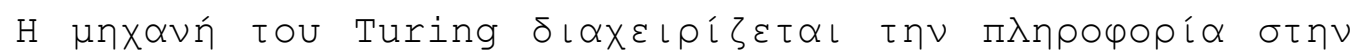

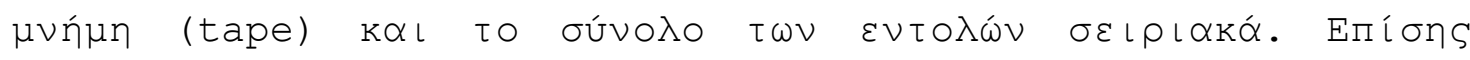

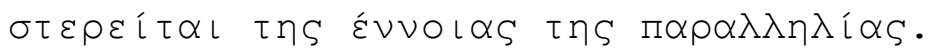

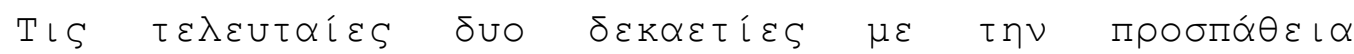

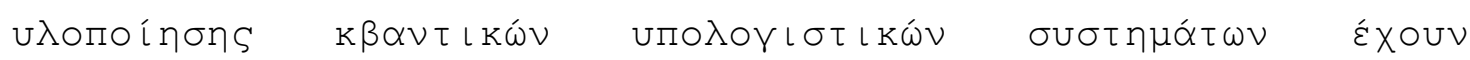

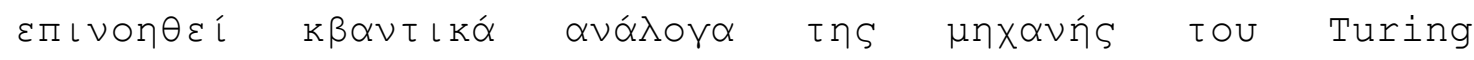

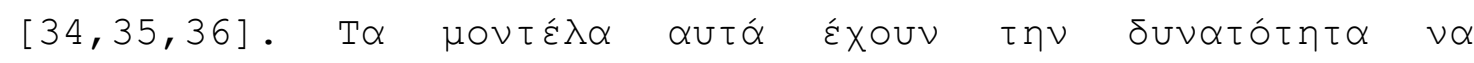

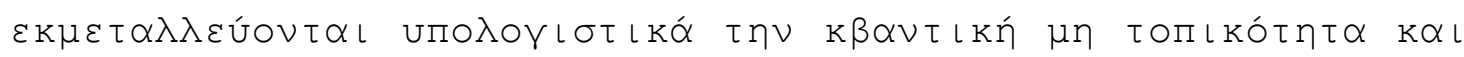
$\pi \alpha \rho \alpha \lambda \lambda \eta \lambda i \alpha$.

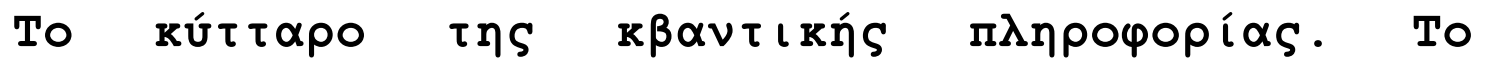

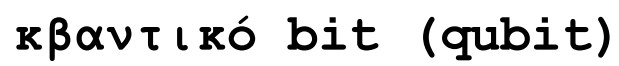

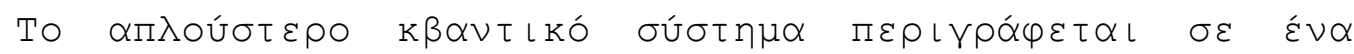

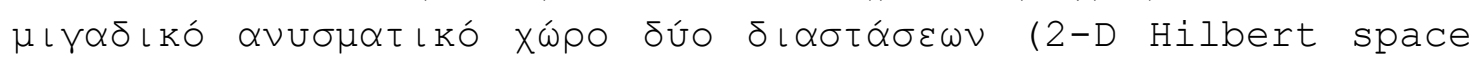

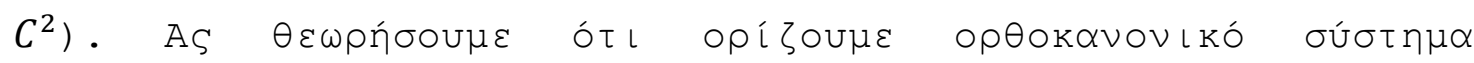

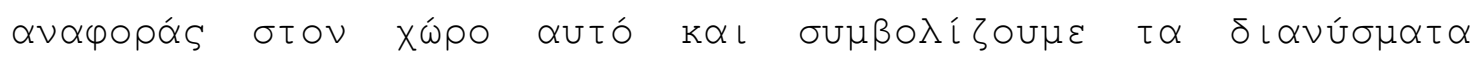

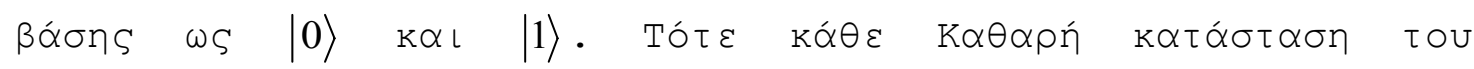

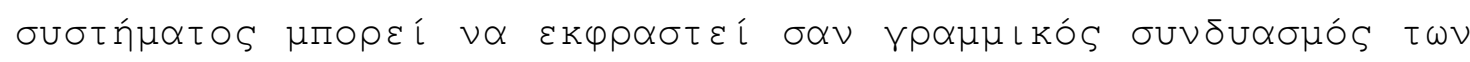

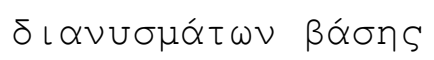

$$
|\psi\rangle=\alpha|0\rangle+\beta|1\rangle
$$

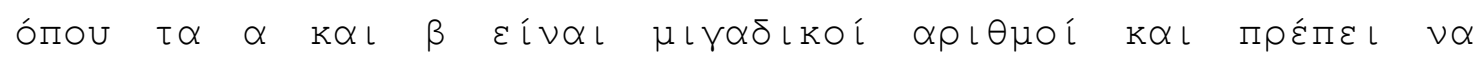

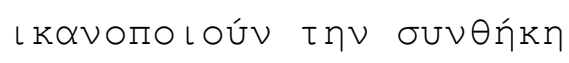

$$
|a|^{2}+|\beta|^{2}=1
$$

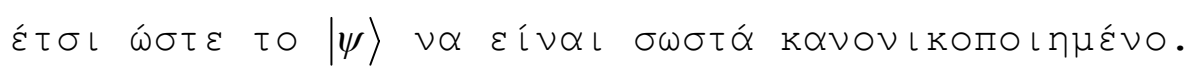

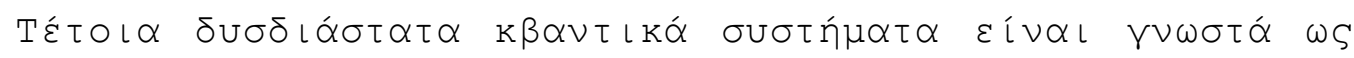

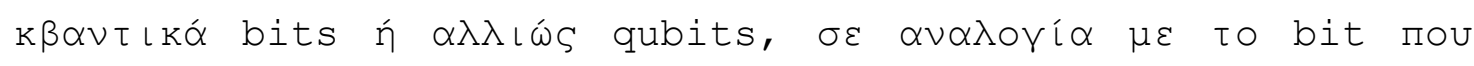

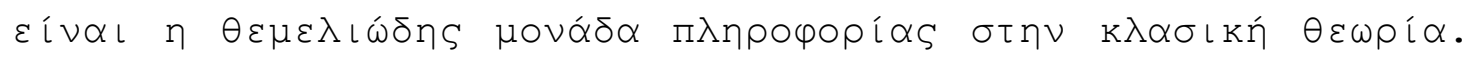

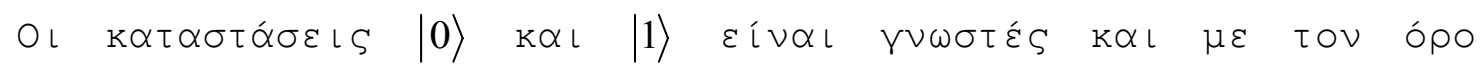

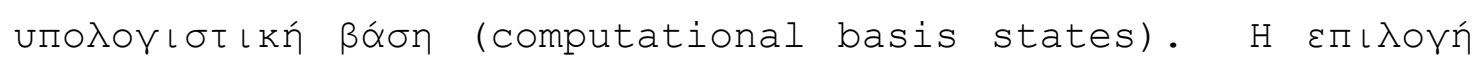

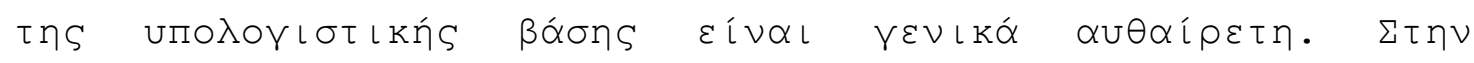

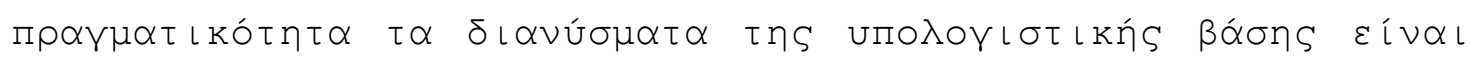

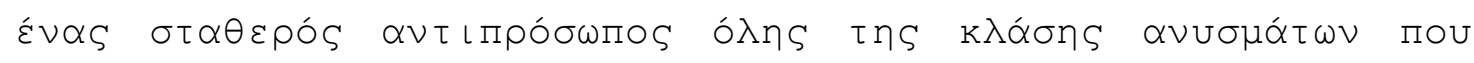




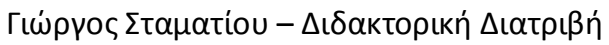

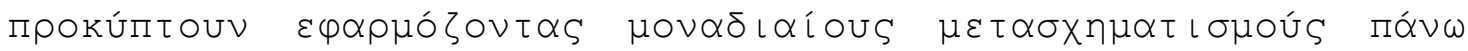

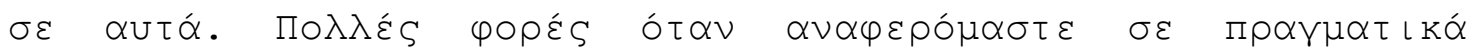

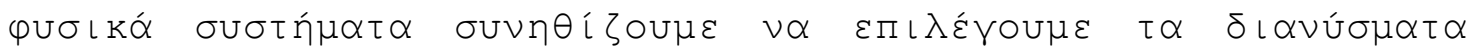

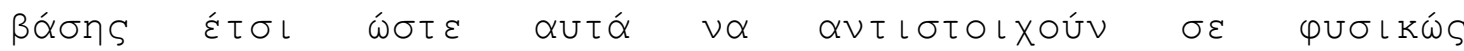

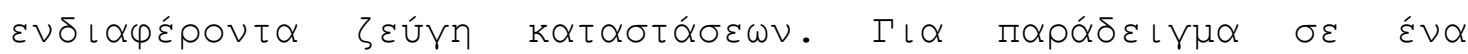

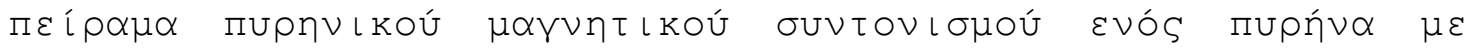

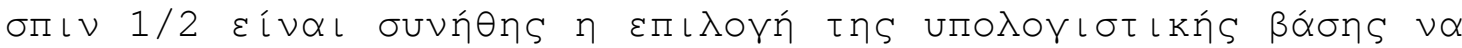

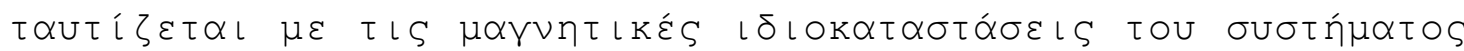

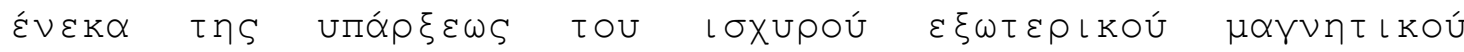
$\Pi \varepsilon \delta i ́ \mathrm{ou}$.

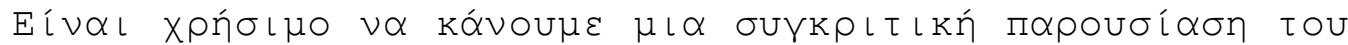

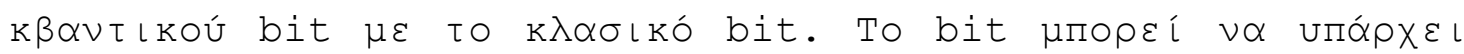

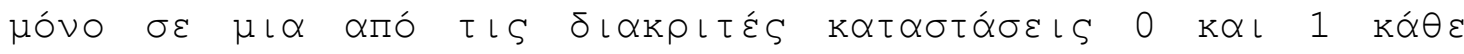

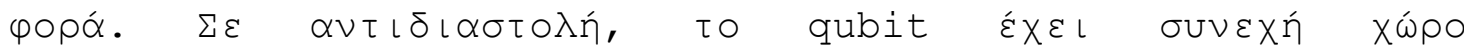

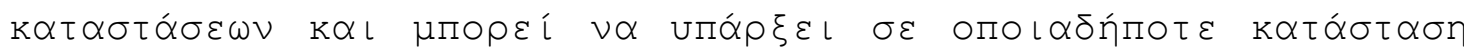

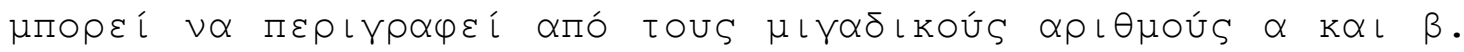

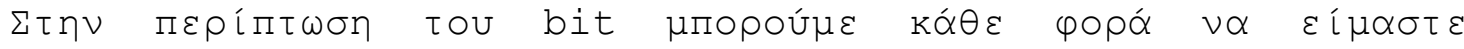

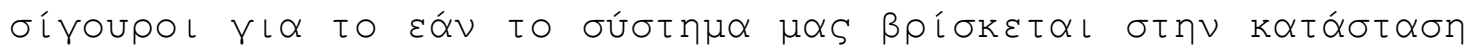

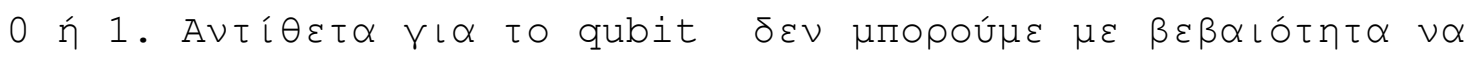

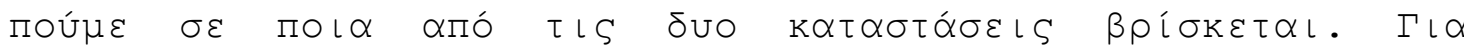

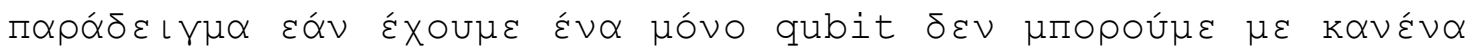

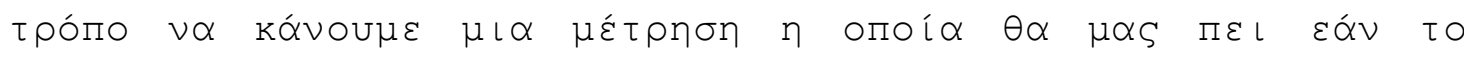

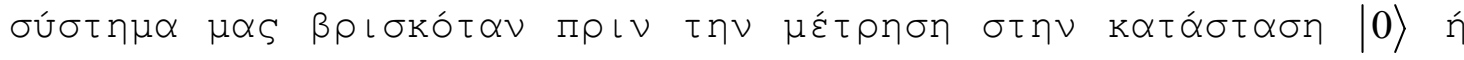

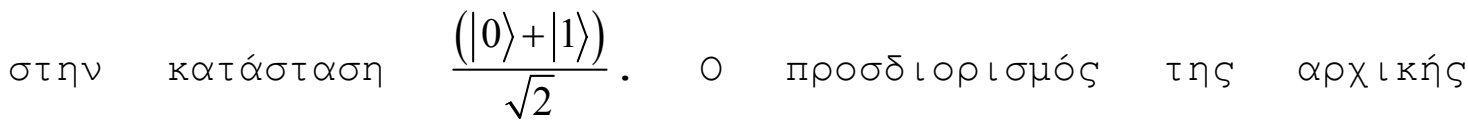

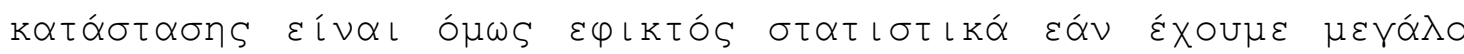

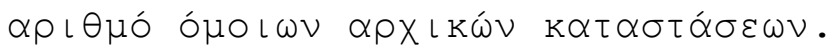

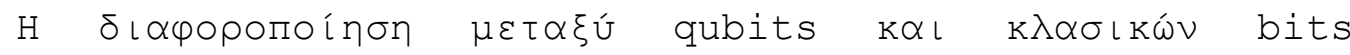

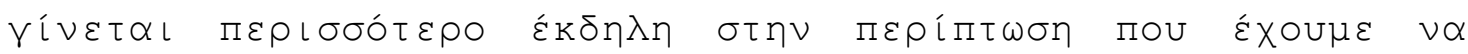

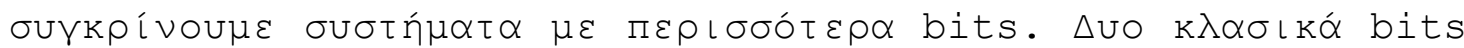

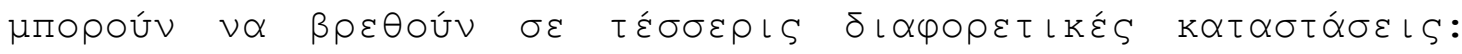

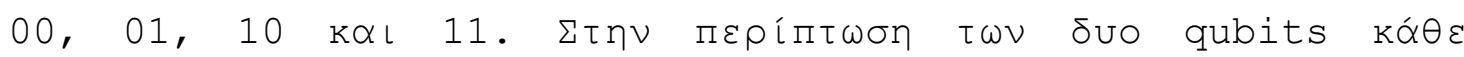

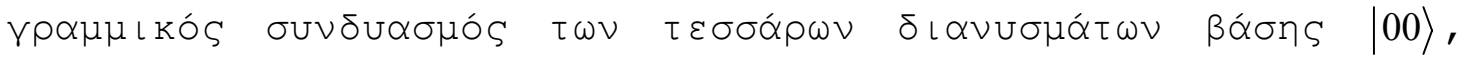

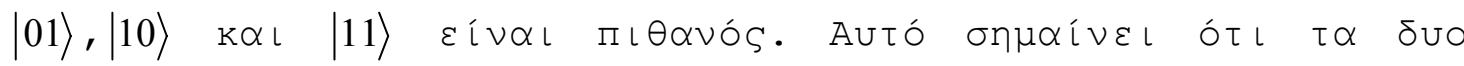

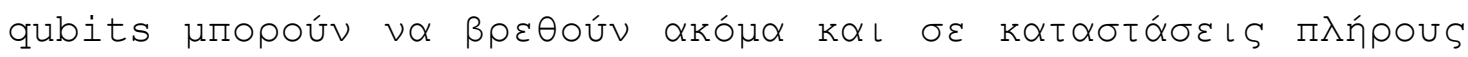

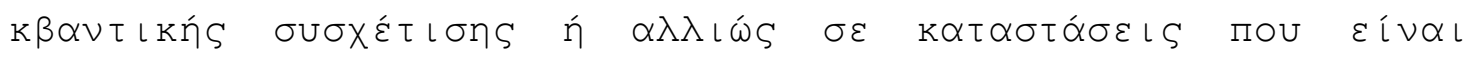

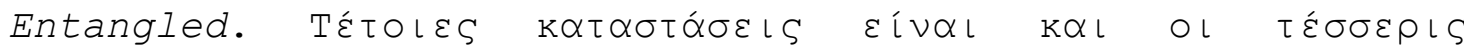

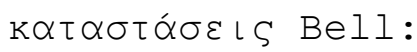




$$
\begin{array}{cc}
\left|\Phi_{+}\right\rangle=\frac{(|00\rangle+|11\rangle)}{\sqrt{2}} & \left|\Phi_{-}\right\rangle=\frac{(|00\rangle-|11\rangle)}{\sqrt{2}} \\
\left|\Psi_{+}\right\rangle=\frac{(|01\rangle+|10\rangle)}{\sqrt{2}} & \left|\Psi_{-}\right\rangle=\frac{(|01\rangle-|10\rangle)}{\sqrt{2}}
\end{array}
$$

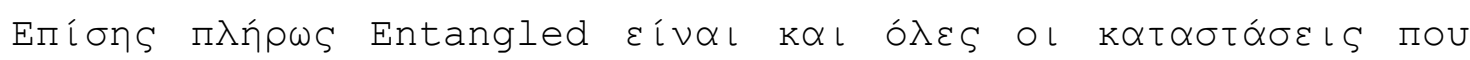

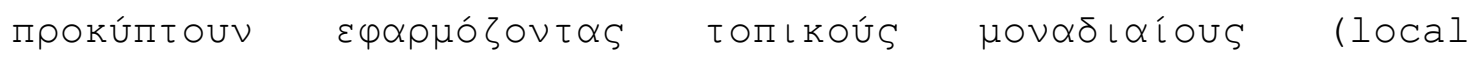

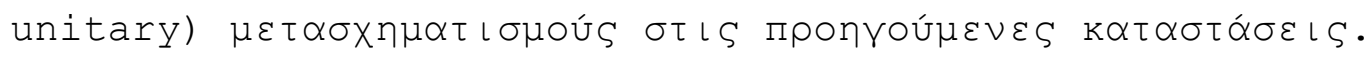

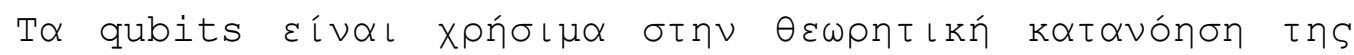

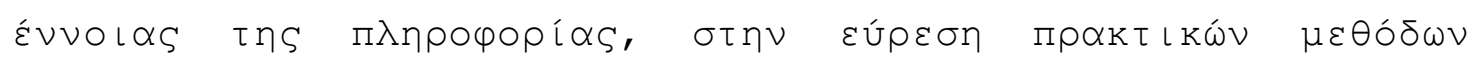

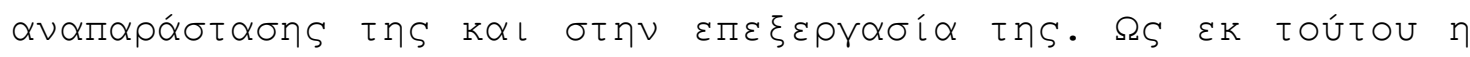

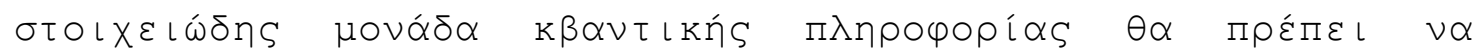

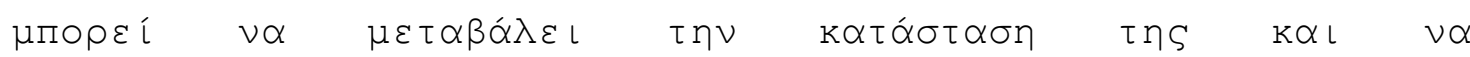

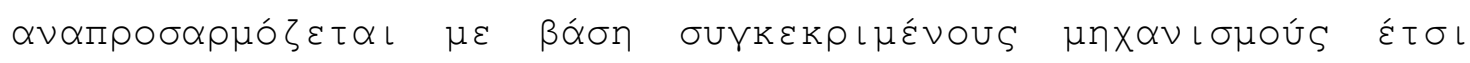

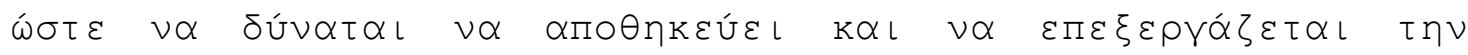

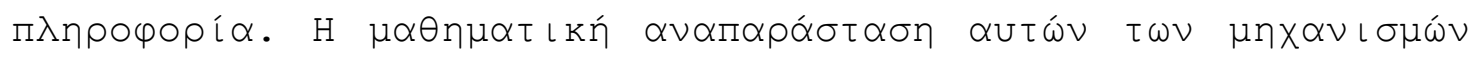

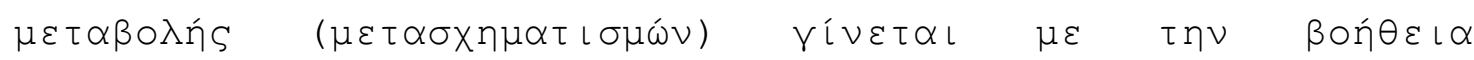

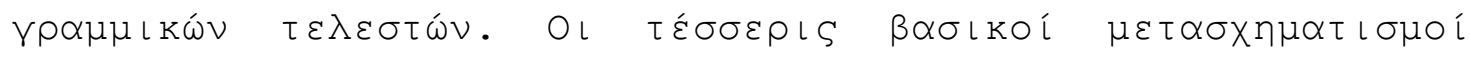

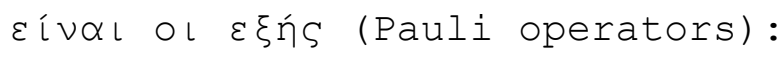

$$
\begin{array}{ccc}
I=\left[\begin{array}{ll}
1 & 0 \\
0 & 1
\end{array}\right] & X=\left[\begin{array}{ll}
0 & 1 \\
1 & 0
\end{array}\right] \\
Y=\left[\begin{array}{cc}
0 & -i \\
i & 0
\end{array}\right] & Z=\left[\begin{array}{cc}
1 & 0 \\
0 & -1
\end{array}\right]
\end{array}
$$

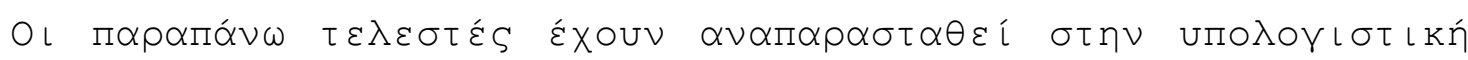

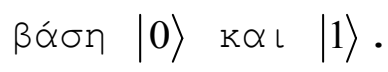

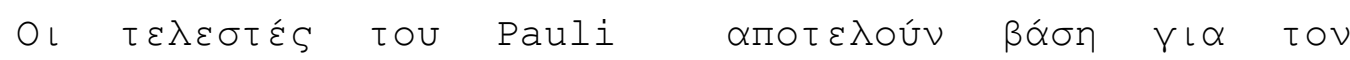

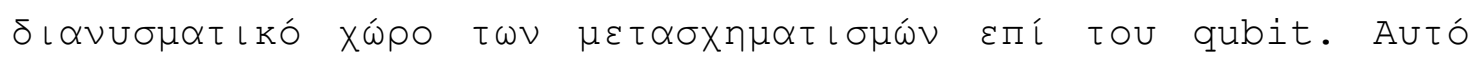

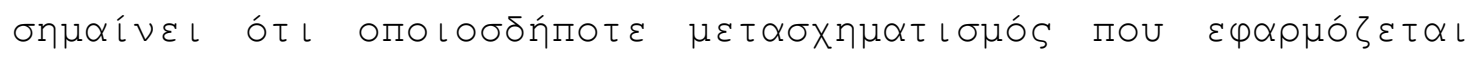

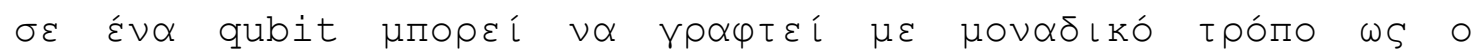

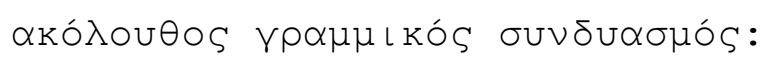

$$
P=a \cdot I+b \cdot X+c \cdot Y+d \cdot Z
$$




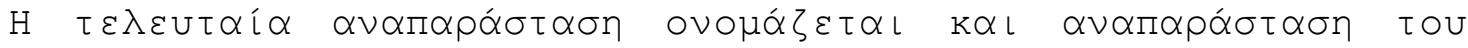
Bloch (Bloch representation).

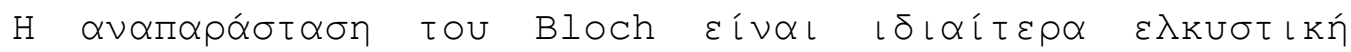

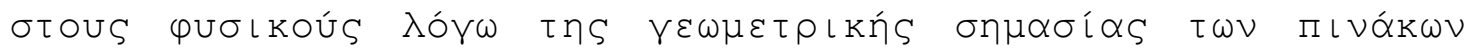

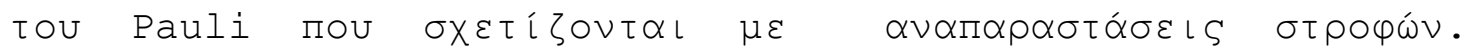

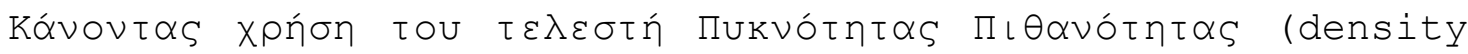

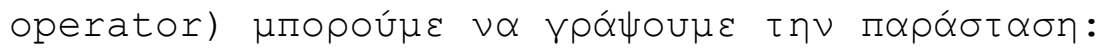

$$
\rho=\frac{I+\vec{\gamma} \cdot \vec{\Omega}}{2}
$$

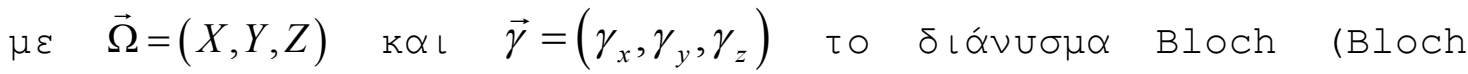

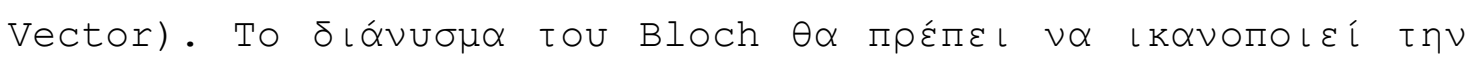

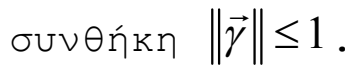

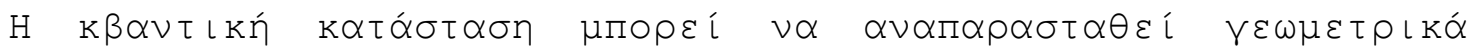

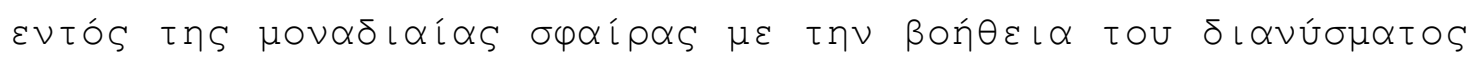

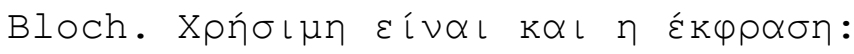

$$
\rho=\frac{I}{2}+\frac{1}{2}\|\vec{\gamma}\|\left[\begin{array}{cc}
\cos \theta & \sin \theta e^{-i \phi} \\
\sin \theta e^{i \phi} & -\cos \theta
\end{array}\right]
$$

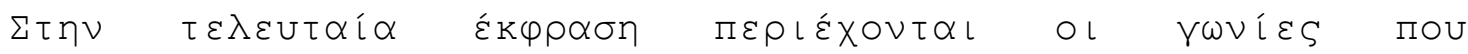

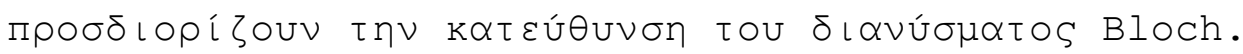

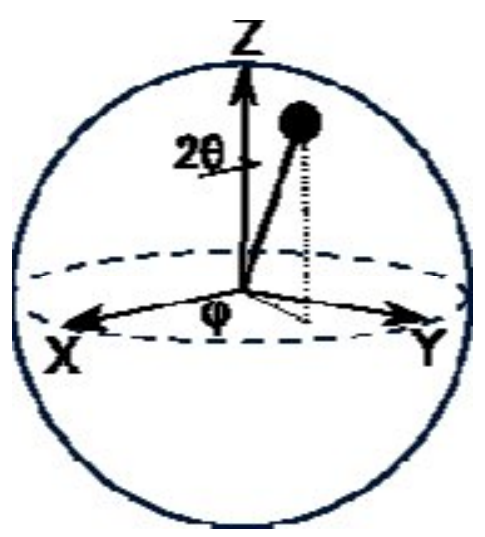

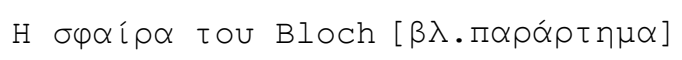




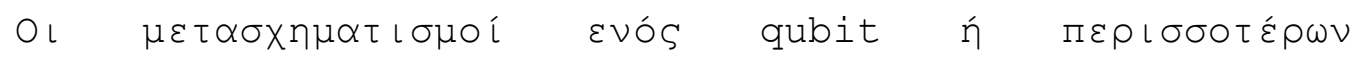

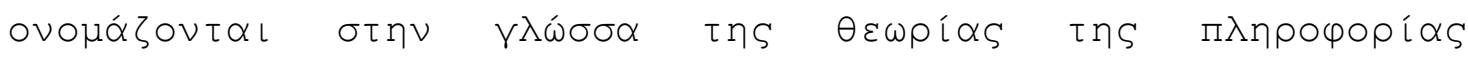

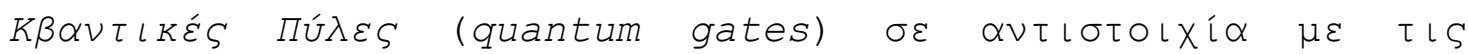

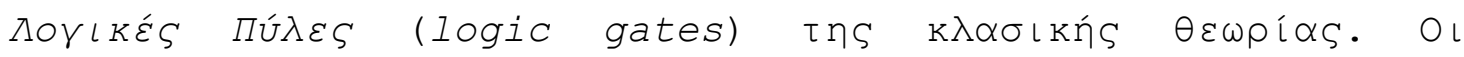

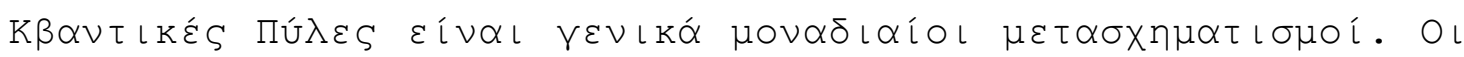

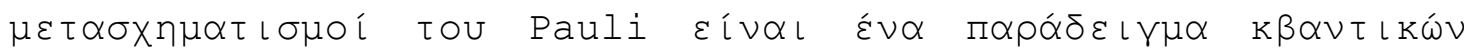

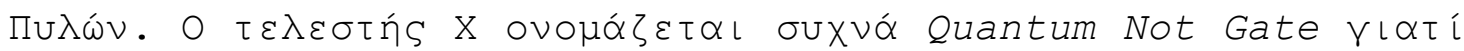

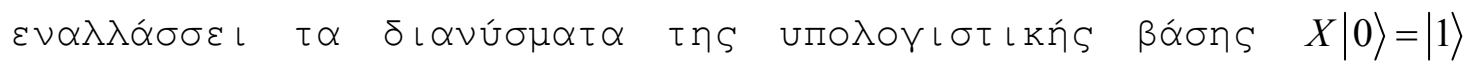

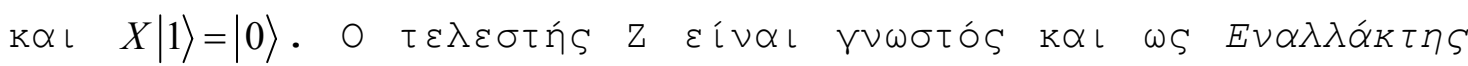

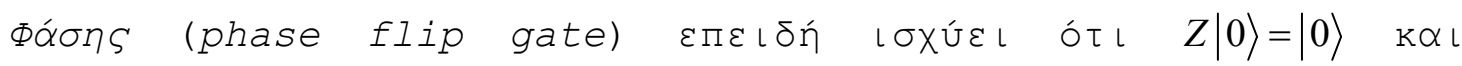
$Z|1\rangle=-|1\rangle$.

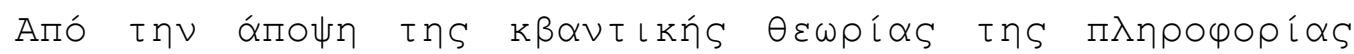

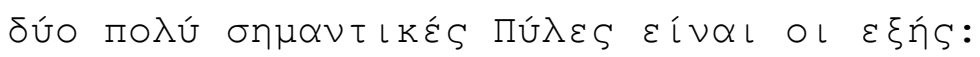

$$
\begin{aligned}
& H=\frac{1}{\sqrt{2}}\left[\begin{array}{cc}
1 & 1 \\
1 & -1
\end{array}\right] \quad \text { Hadamard Gate } \\
& S=\left[\begin{array}{cc}
1 & 0 \\
0 & e^{i \pi / 4}
\end{array}\right] \quad \text { Phase Shift Gate }
\end{aligned}
$$

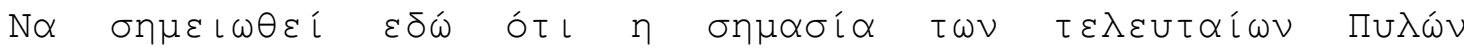

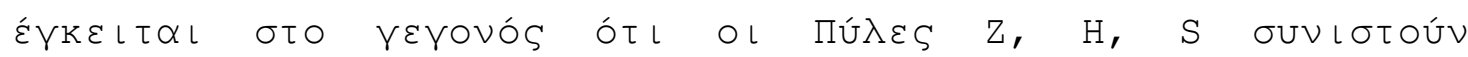

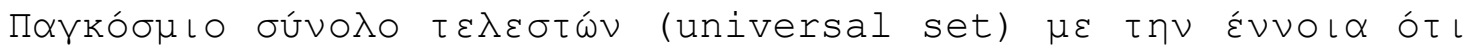

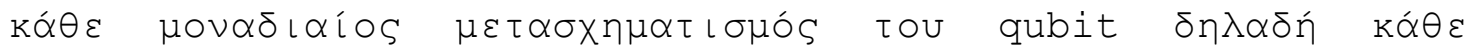

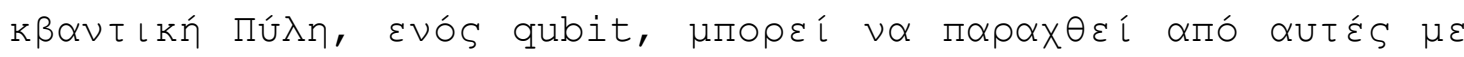
$\alpha \Pi \varepsilon \rho\llcorner o ́ \rho \iota \sigma \tau \eta ~ \alpha k \rho i ́ \varepsilon\llcorner\alpha$.

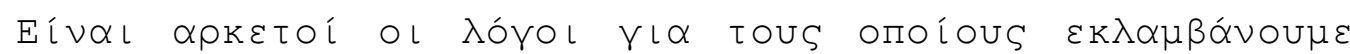

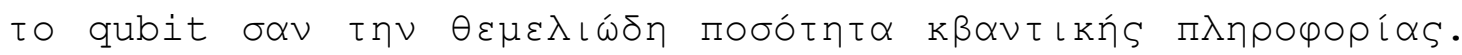

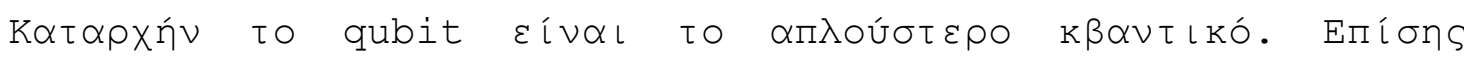

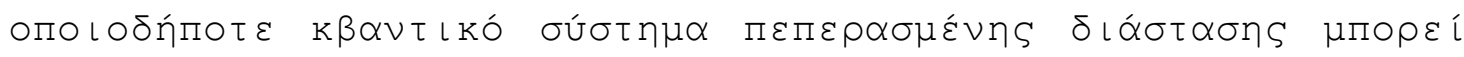

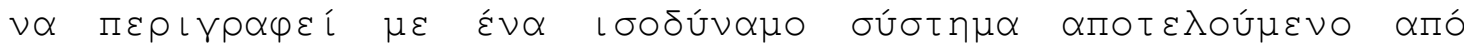

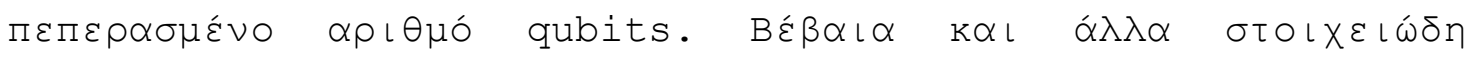

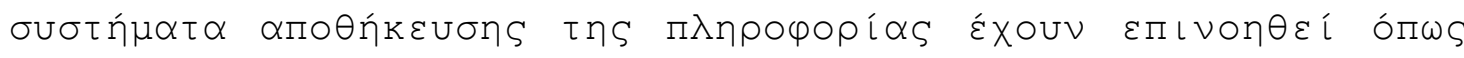

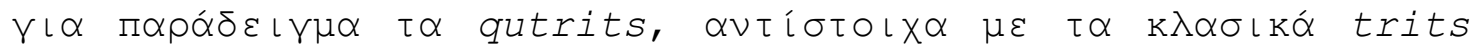

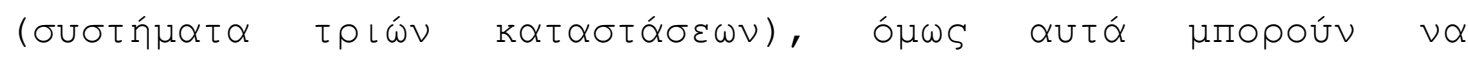




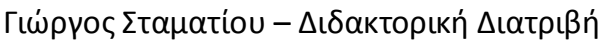

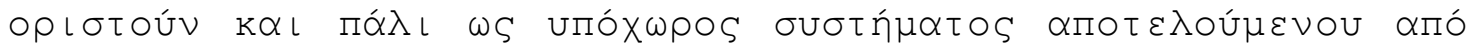

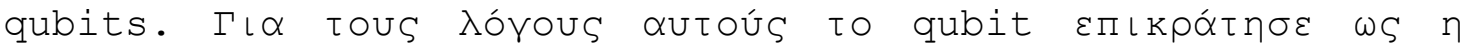

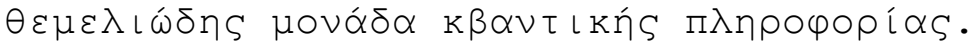

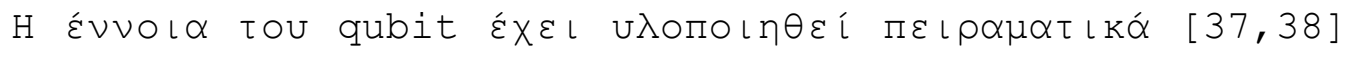

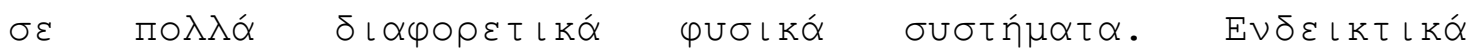

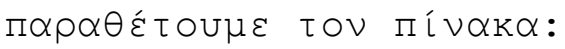

\begin{tabular}{|c|c|c|c|c|}
\hline Physical support & Name & $\begin{array}{l}\text { Information } \\
\text { support }\end{array}$ & $|0\rangle$ & $|1\rangle$ \\
\hline \multirow[t]{3}{*}{\begin{tabular}{|l|} 
Single photon \\
(Fock states)
\end{tabular}} & Polarization encoding & \begin{tabular}{|l|}
$\begin{array}{l}\text { Polarization of } \\
\text { light }\end{array}$ \\
\end{tabular} & Horizontal & Vertical \\
\hline & Photon number & Photon number & Vacuum & Single photon state \\
\hline & Time-bin encoding & Time of arrival & Early & Late \\
\hline \begin{tabular}{|l|} 
Coherent state of \\
light
\end{tabular} & Squeezed light & Quadrature & $\begin{array}{l}\text { Amplitude-squeezed } \\
\text { state }\end{array}$ & Phase-squeezed state \\
\hline \multirow[t]{2}{*}{ Electrons } & Electronic spin & Spin & Up & Down \\
\hline & Electron number & Charge & No electron & One electron \\
\hline Nucleus & \begin{tabular}{|l|} 
Nuclear spin addressed \\
through NMR
\end{tabular} & Spin & Up & Down \\
\hline Optical lattices & Atomic spin & Spin & Up & Down \\
\hline \multirow[t]{3}{*}{$\begin{array}{l}\text { Josephson } \\
\text { junction }\end{array}$} & $\begin{array}{l}\text { Superconducting } \\
\text { charge qubit }\end{array}$ & Charge & \begin{tabular}{|l|} 
Uncharged \\
superconducting island \\
$(Q=0)$
\end{tabular} & $\begin{array}{l}\text { Charged superconducting island } \\
(Q=2 e \text {, one extra Cooper pair })\end{array}$ \\
\hline & \begin{tabular}{|l|} 
Superconducting flux \\
qubit
\end{tabular} & Current & Clockwise current & Counterclockwise current \\
\hline & \begin{tabular}{|l|} 
Superconducting phase \\
qubit
\end{tabular} & Energy & Ground state & First excited state \\
\hline $\begin{array}{l}\text { Singly-charged } \\
\text { quantum dot pair }\end{array}$ & Electron localization & Charge & Electron on left dot & Electron on right dot \\
\hline Quantum dot & Dot spin & Spin & Down & Up \\
\hline 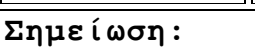 & $\overline{\Pi \Pi \imath \vee \alpha k \alpha S}$ & $\overline{\varepsilon i \iota \alpha \iota l}$ & 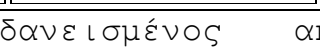 & 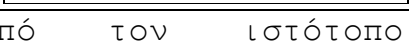 \\
\hline http://en.wi] & kipedia.org/wik & i/Qubit. & 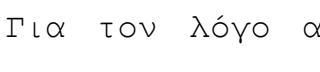 & 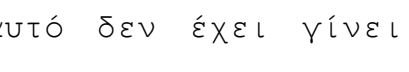 \\
\hline
\end{tabular}




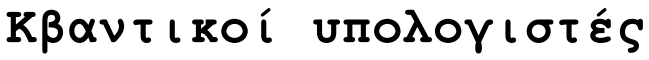

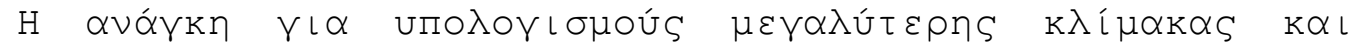

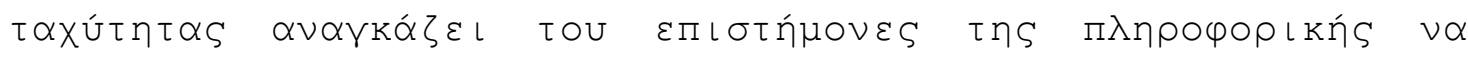

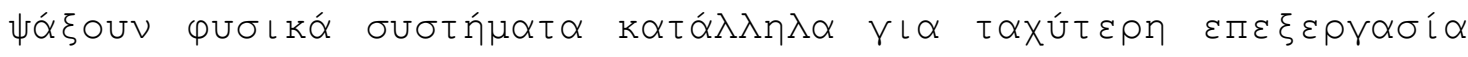

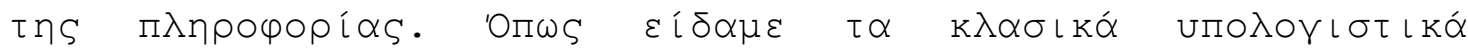

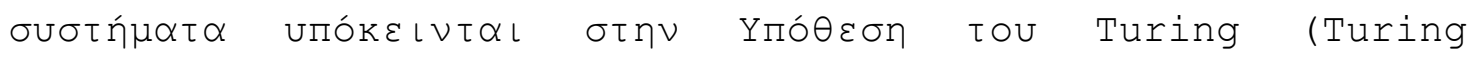

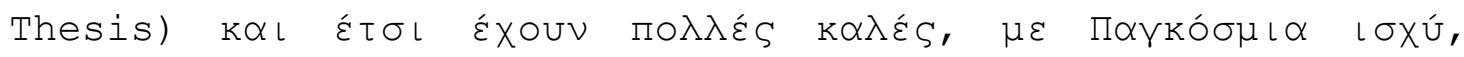

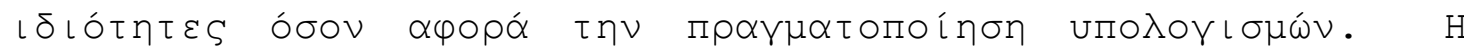

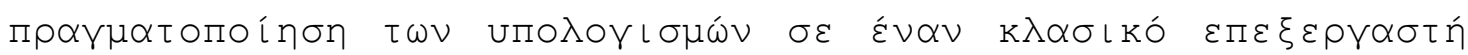

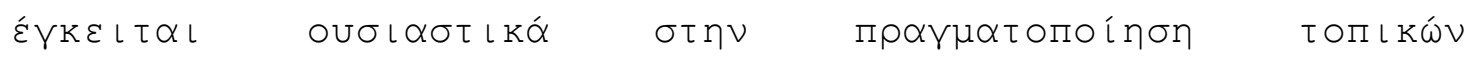

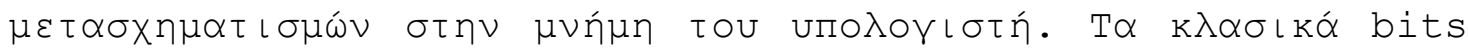

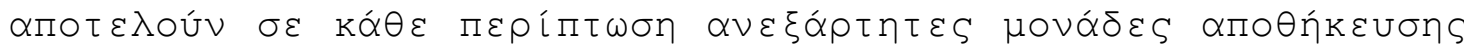

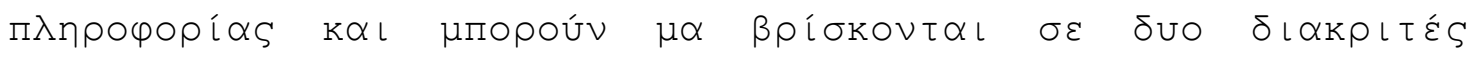

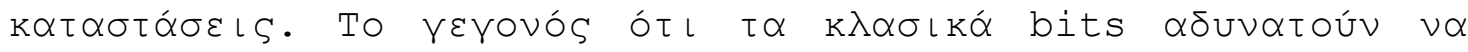

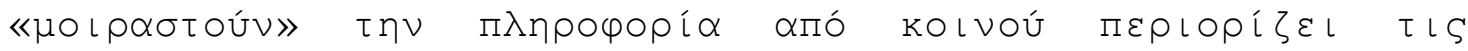

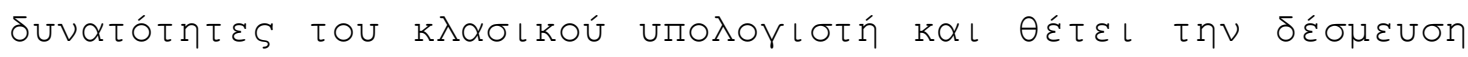

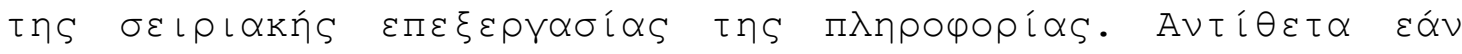

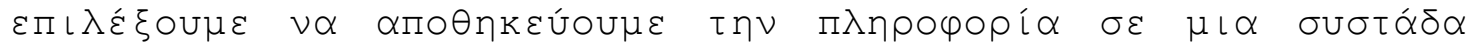

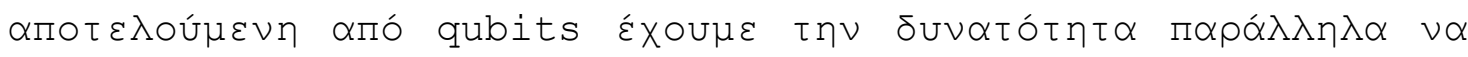

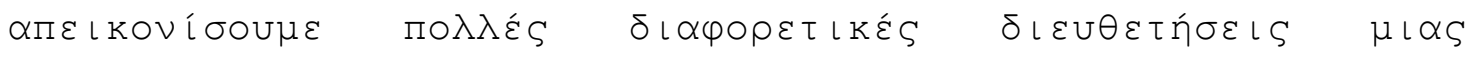

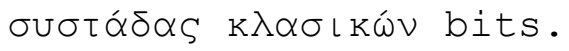

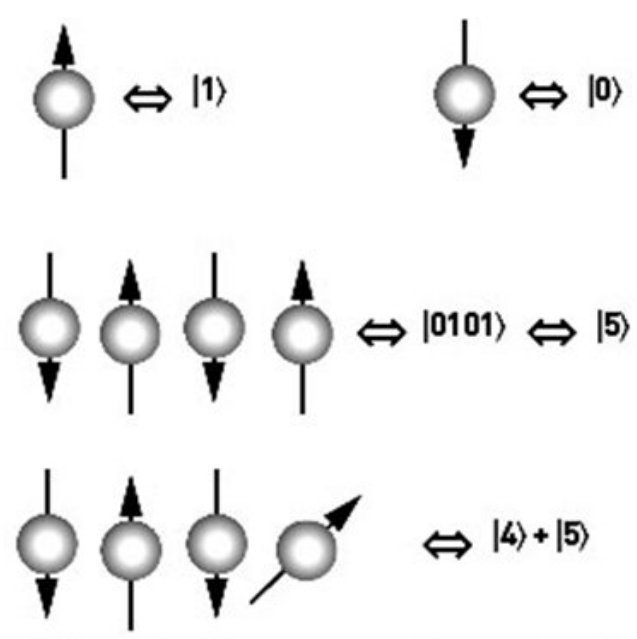

[http://commons.wikimedia.org/wiki/File:Quantum_computer.svg]

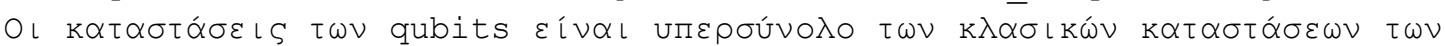
bits

$[\beta \lambda . п \alpha \rho \alpha ́ \rho \tau \eta \mu \alpha]$ 


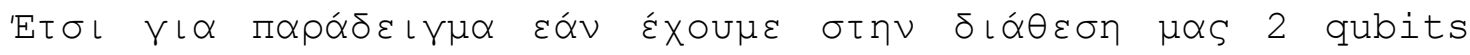

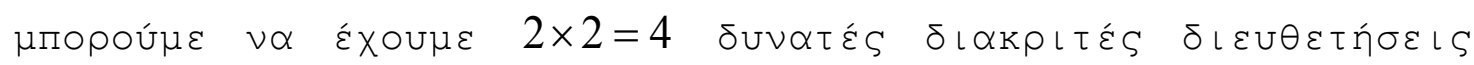

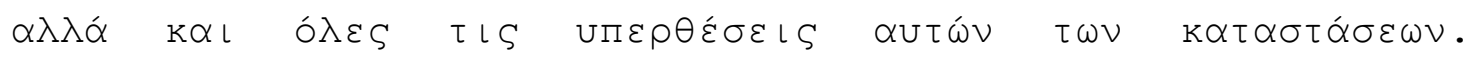

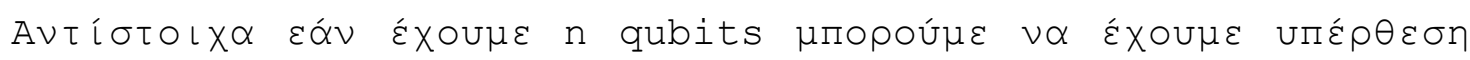

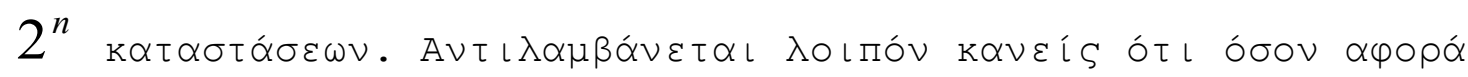

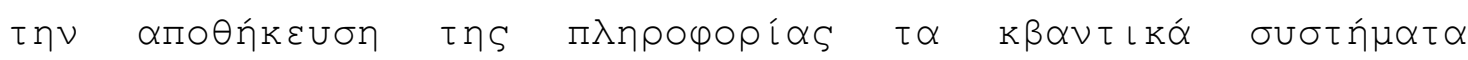

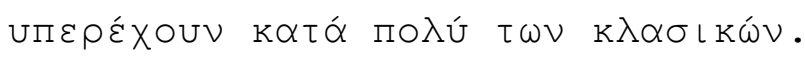

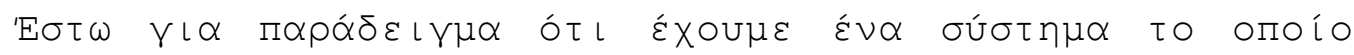

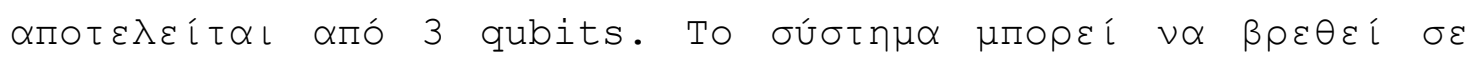

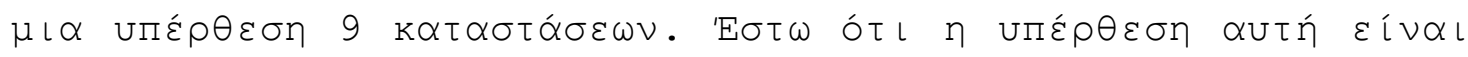
$\eta \varepsilon \xi \tilde{n} s:$

$$
a|000\rangle+b|001\rangle+c|010\rangle+d|011\rangle+e|100\rangle+f|101\rangle+g|110\rangle+h|111\rangle
$$

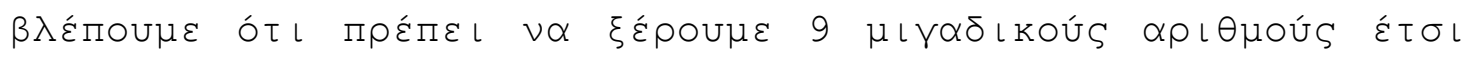

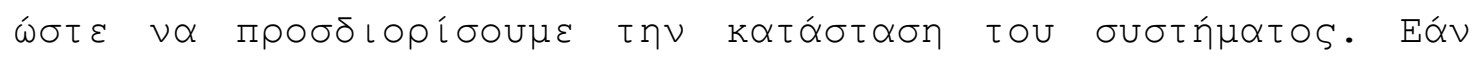

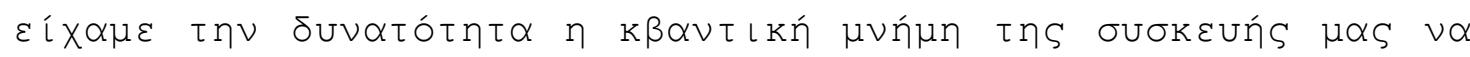

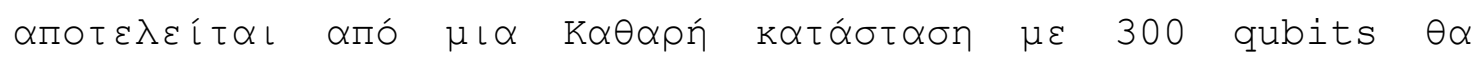

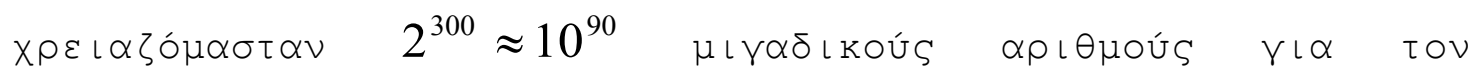

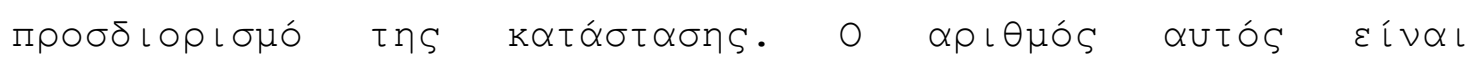

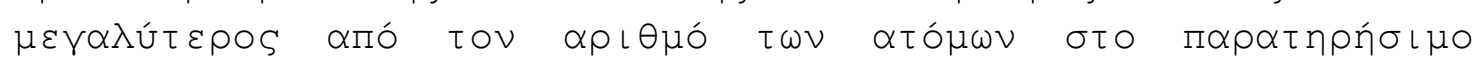

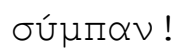

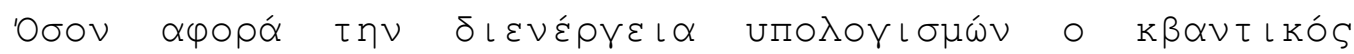

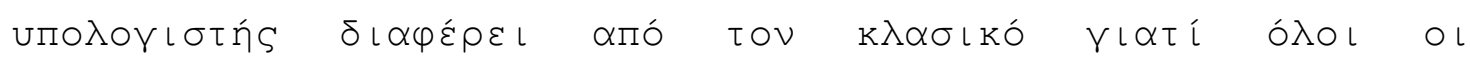

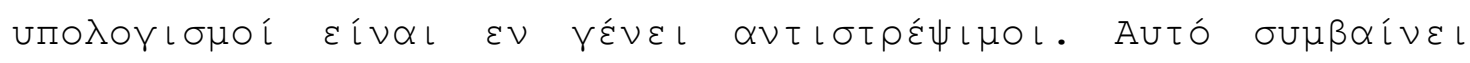

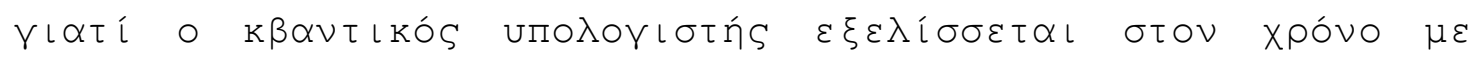

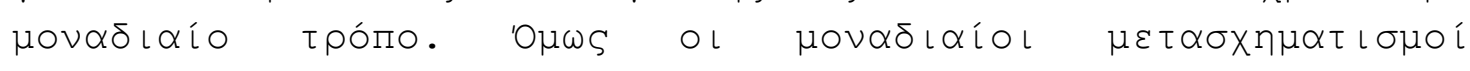

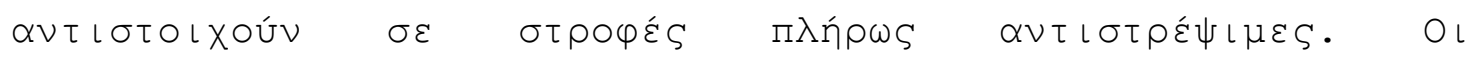

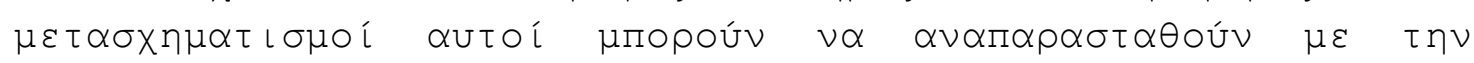

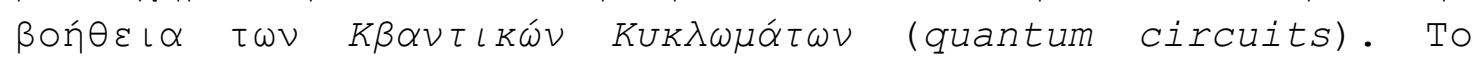

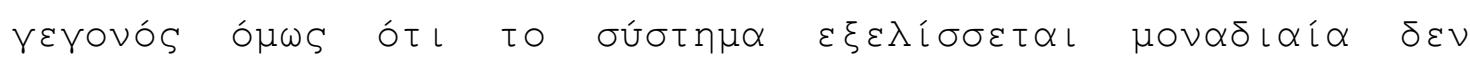

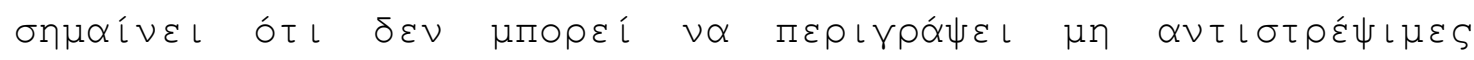

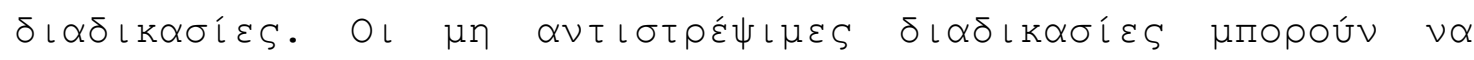

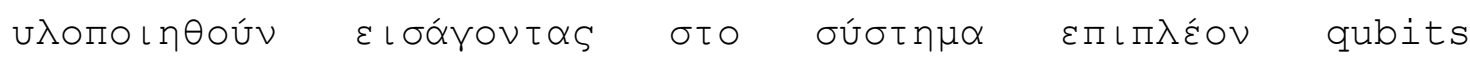

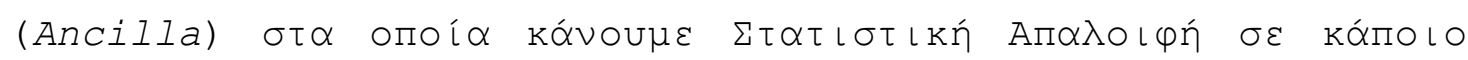

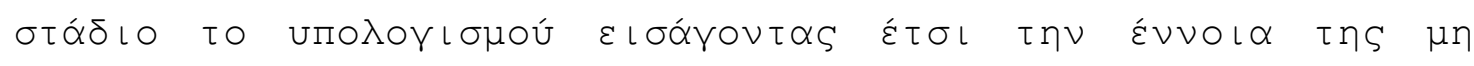




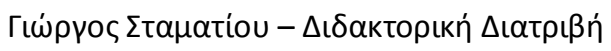

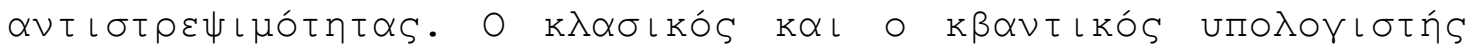

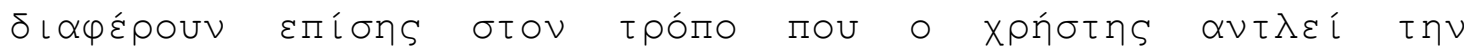

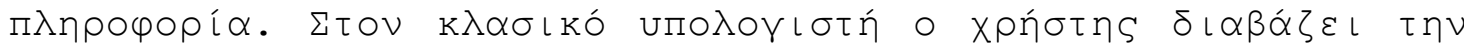

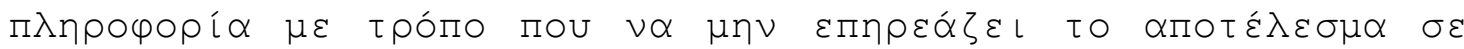

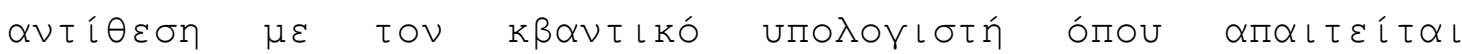

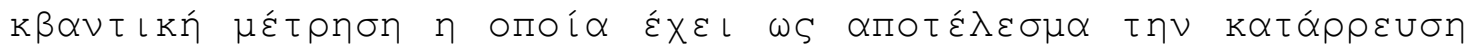

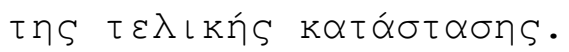

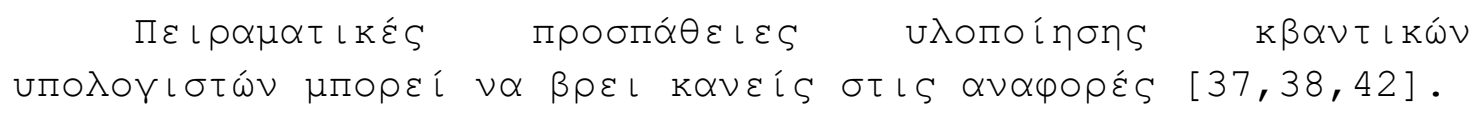

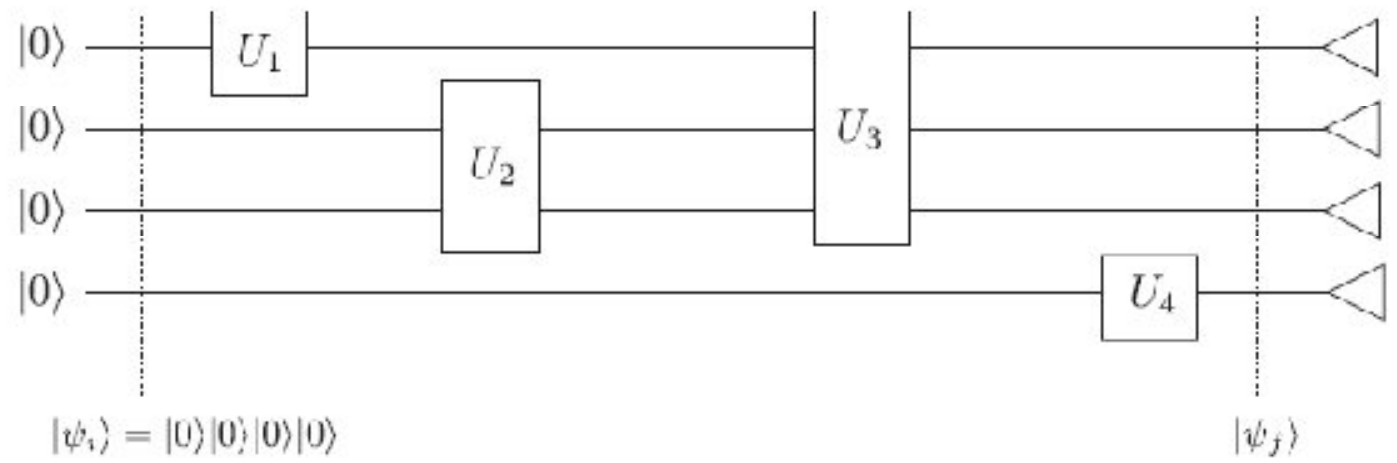

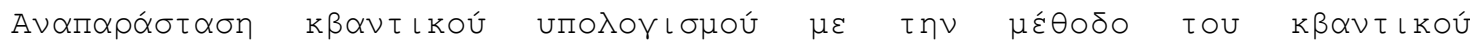

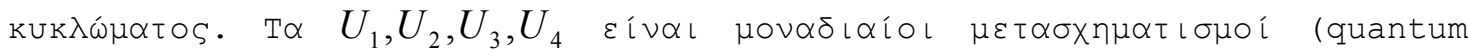

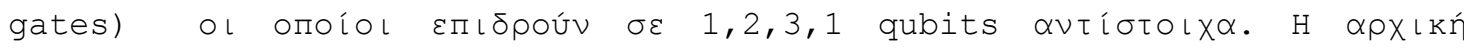

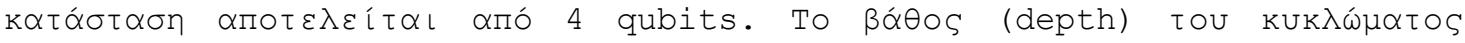

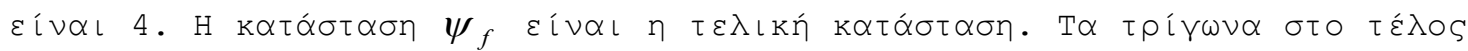

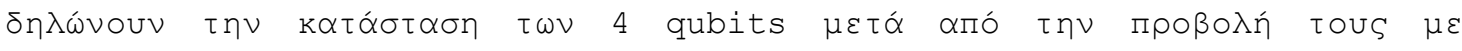

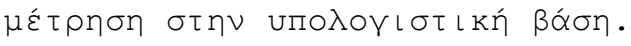

$[\beta \lambda . п \alpha \rho \alpha ́ \rho \tau \eta \mu \alpha($ P.Kaye,... ) ] 


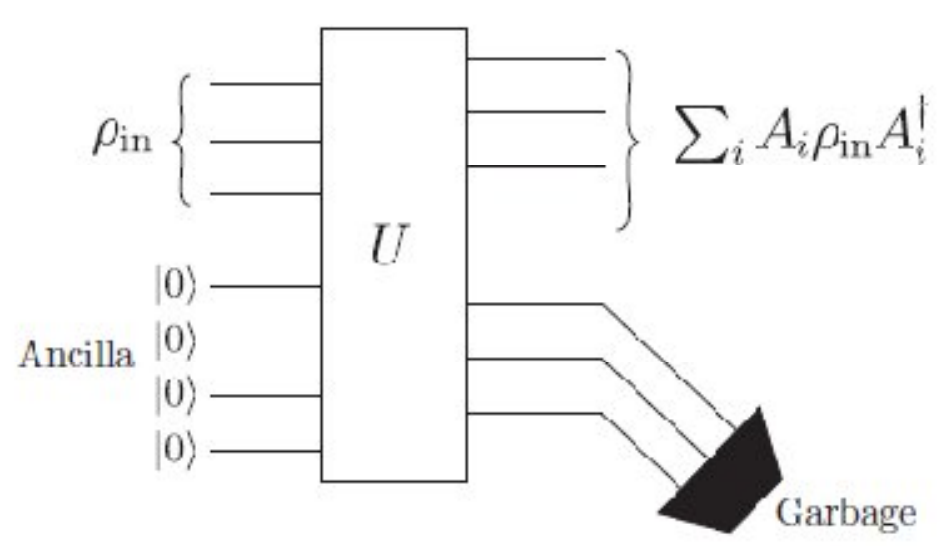

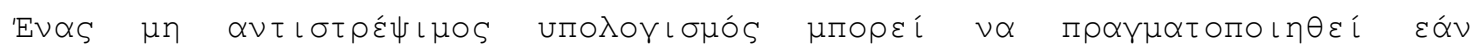

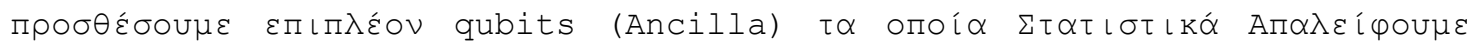

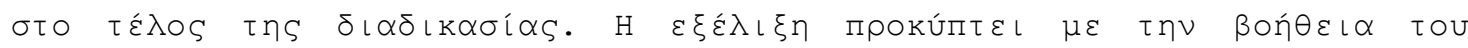

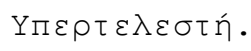
$[\beta \lambda . \pi \alpha \rho \alpha ́ \rho \tau \eta \mu \alpha($ P.Kaye, .. ) ]

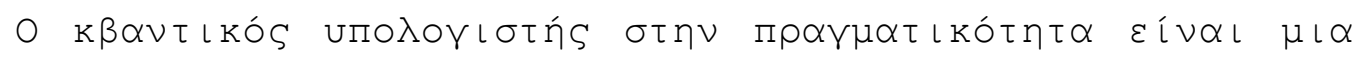

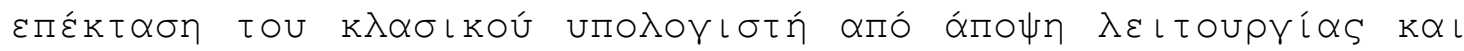

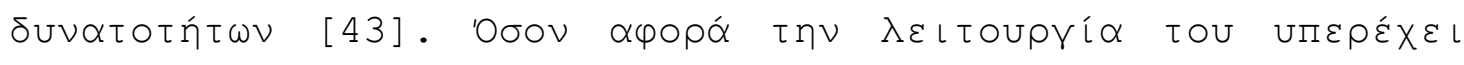

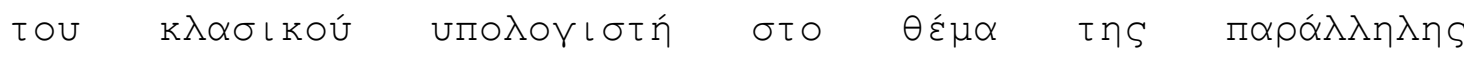

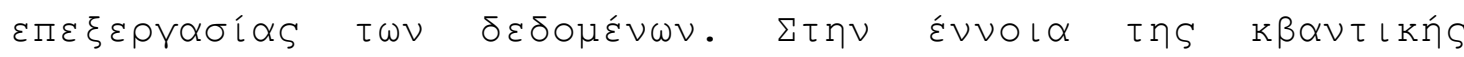

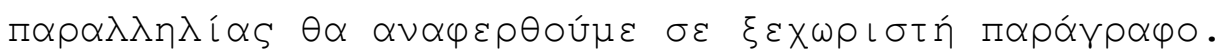

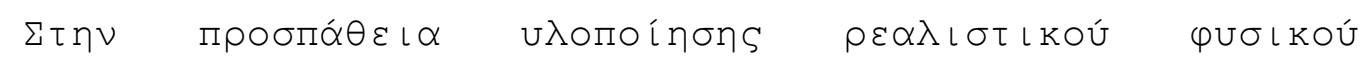

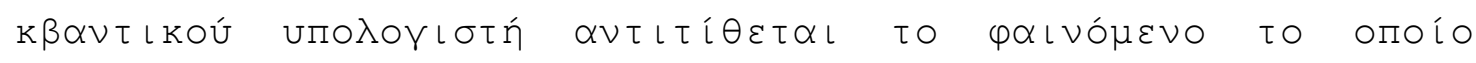

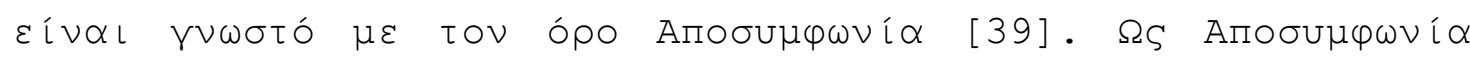

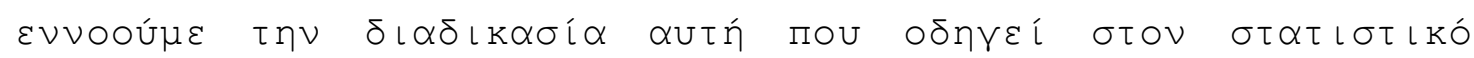

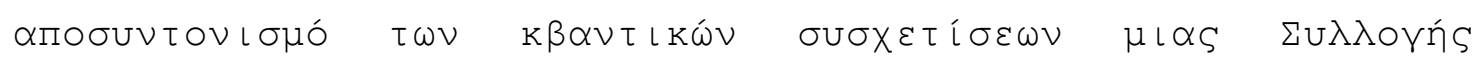

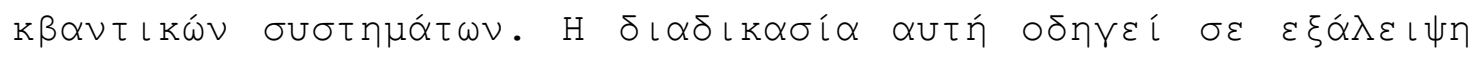

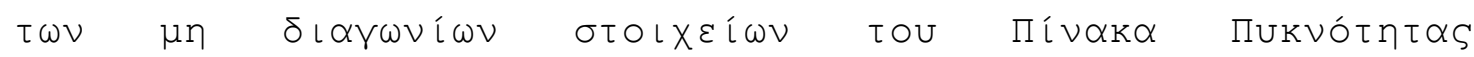

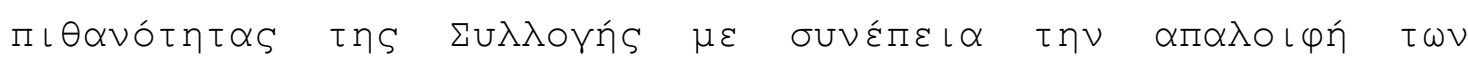

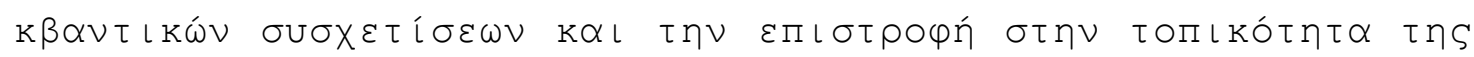

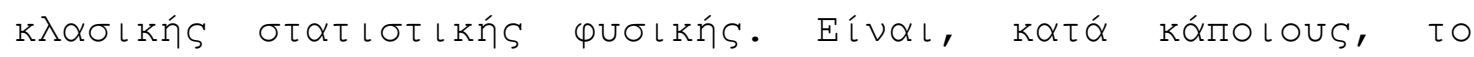

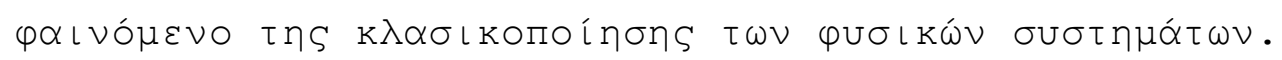




\section{$\mathrm{K} \beta \alpha \nu \tau$ เ Kर́ $S$ \\ Пú $\lambda \varepsilon \varsigma \quad \mathrm{k} \alpha \iota$

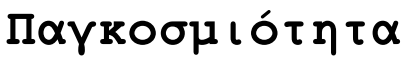 (universality).}

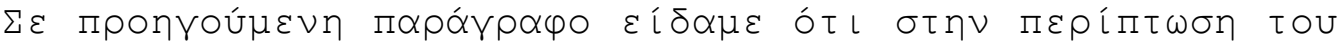

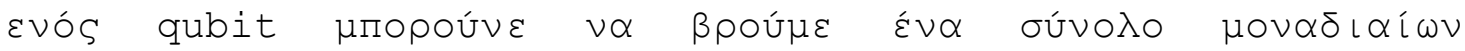

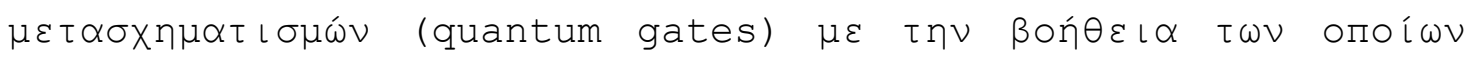

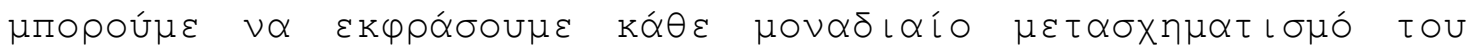

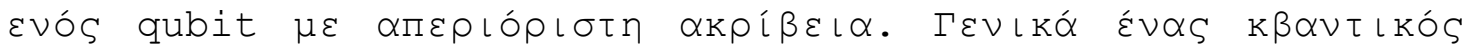

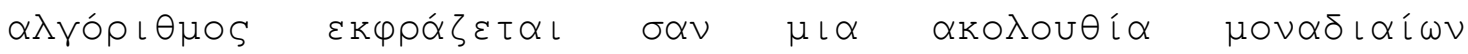

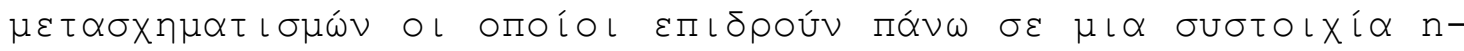

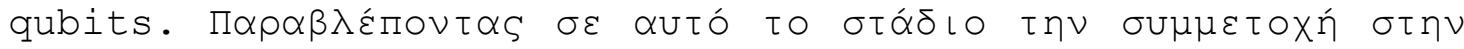

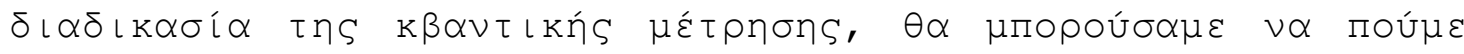
ót ı́ ó

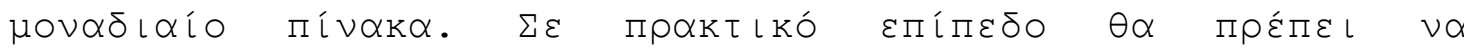

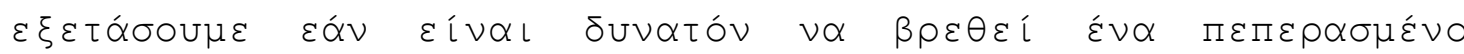

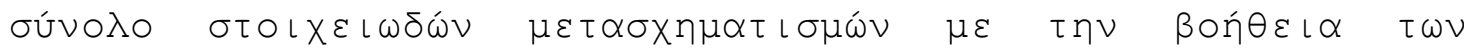

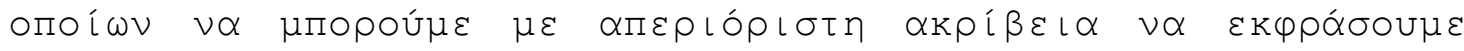

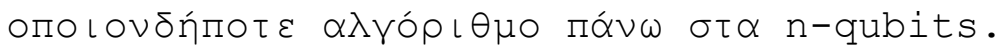

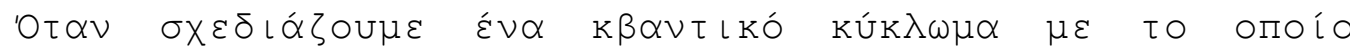

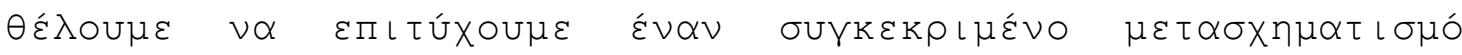

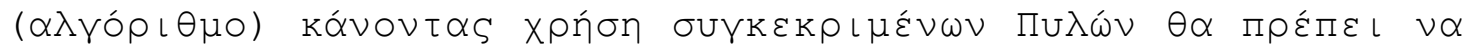

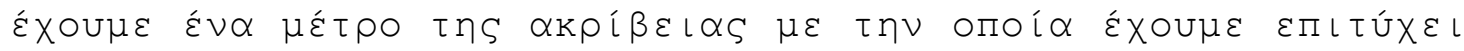

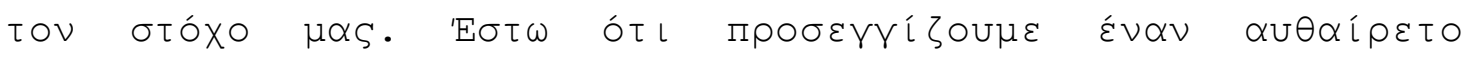

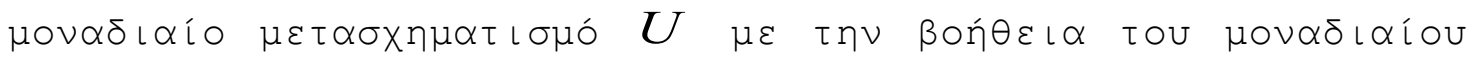

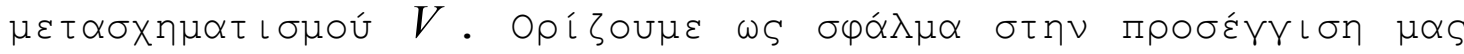

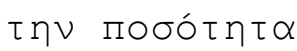

$$
E(U, V) \equiv \max _{|\psi\rangle} \|(U-V)|\psi\rangle \|
$$

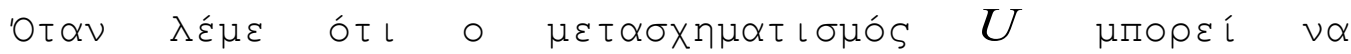

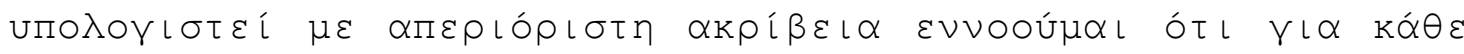

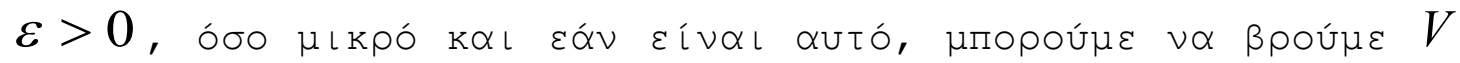
$\tau \varepsilon \dot{\varepsilon} \tau\llcorner O \omega ́ \omega \tau \varepsilon$

$$
E(U, V)<\varepsilon
$$

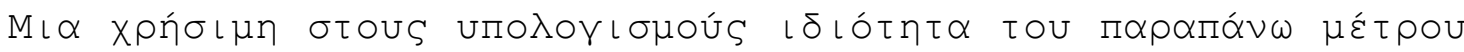

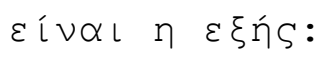




$$
E\left(U_{n} U_{n-1} \ldots U_{1}, V_{n} V_{n-1} \ldots V_{1}\right) \leq E\left(U_{n}, V_{n}\right)+E\left(U_{n-1}, V_{n-1}\right)+\cdots+E\left(U_{1}, V_{1}\right)
$$

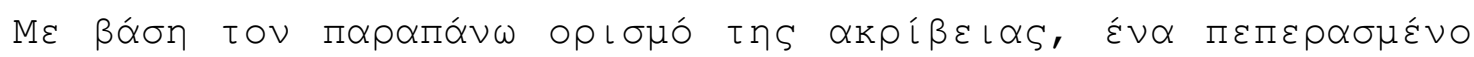

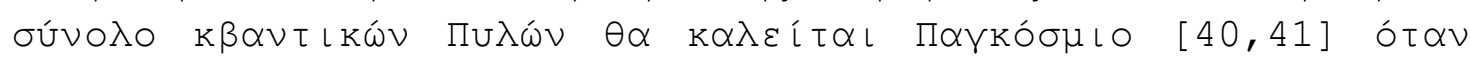

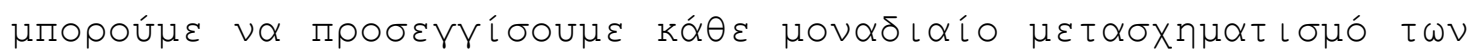

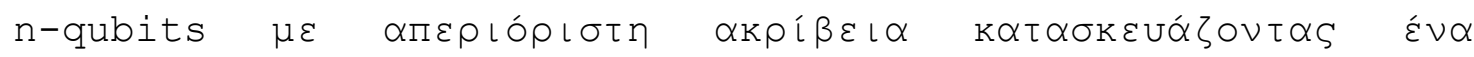

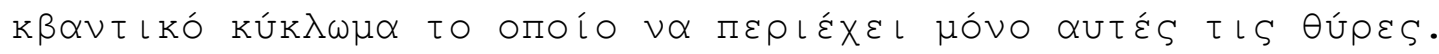

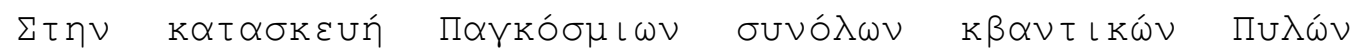

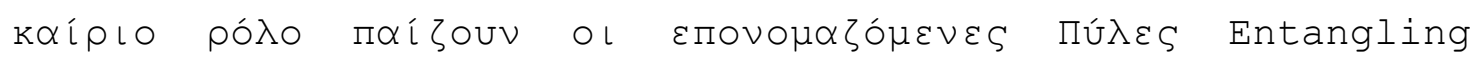

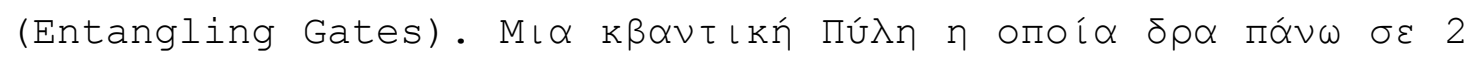

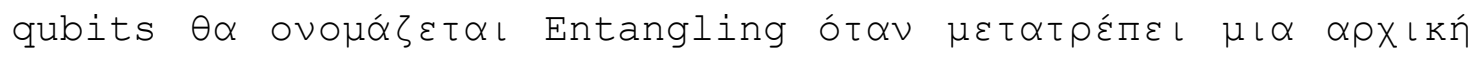

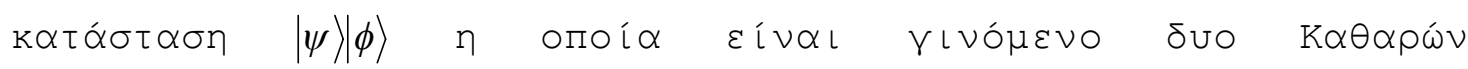

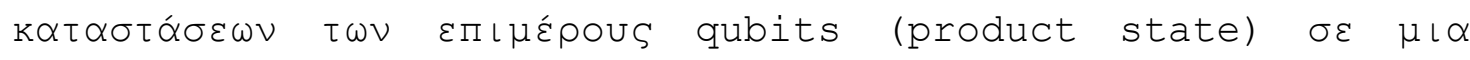

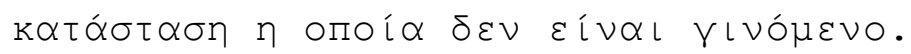

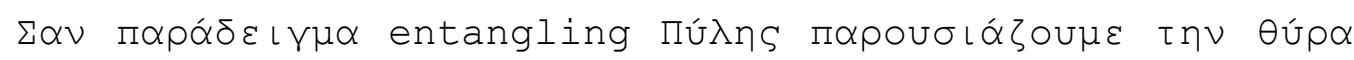

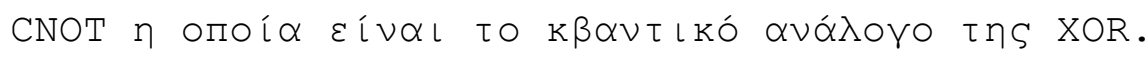

$$
\text { CNOT }=\left[\begin{array}{llll}
1 & 0 & 0 & 0 \\
0 & 1 & 0 & 0 \\
0 & 0 & 0 & 1 \\
0 & 0 & 1 & 0
\end{array}\right]
$$

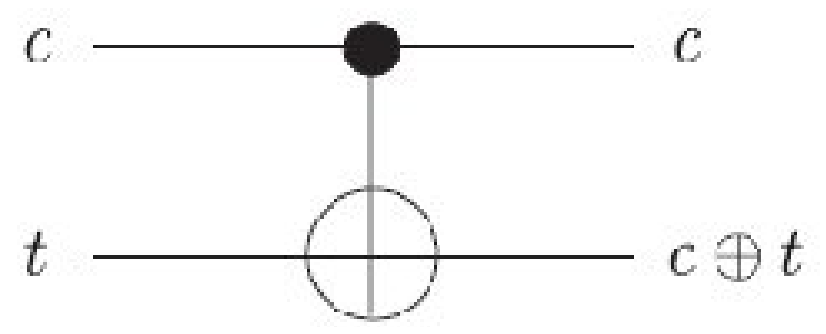

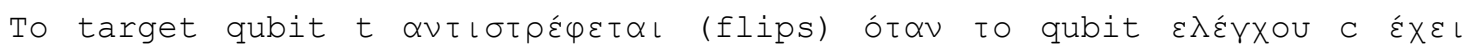

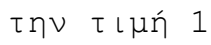




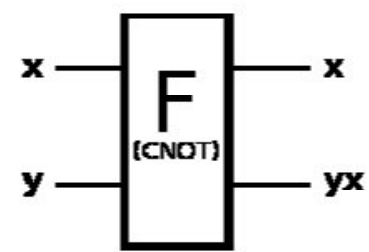

input output

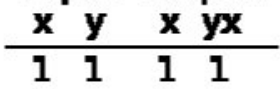

$\begin{array}{llll}1 & -1 & 1 & -1\end{array}$

$\begin{array}{llll}-1 & 1 & -1 & -1\end{array}$

$\begin{array}{llll}-1 & -1 & -1 & 1\end{array}$
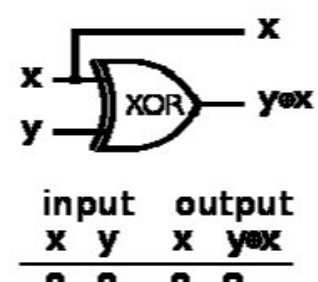

00000

$0 \begin{array}{llll}0 & 1 & 0 & 1\end{array}$

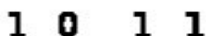

$\begin{array}{llll}1 & 1 & 1 & 0\end{array}$

$$
\begin{array}{r}
1-0 \\
-1-1
\end{array}
$$

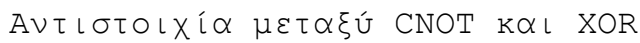

$$
[\beta \lambda . \sqcap \alpha \rho \alpha ́ \alpha \tau \eta \mu \alpha(\text { k. пÚ } \lambda \varepsilon \varsigma)]
$$

[http://en.wikipedia.org/wiki/File:Cnot-compared-to-xor.svg]

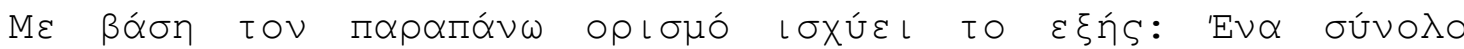

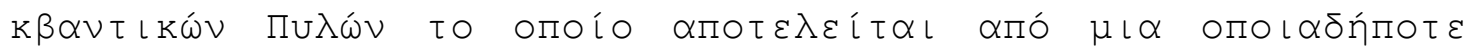

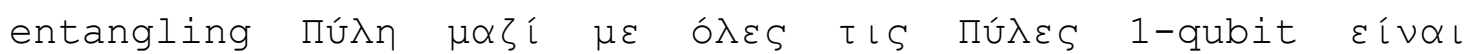

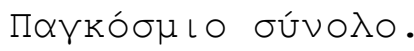

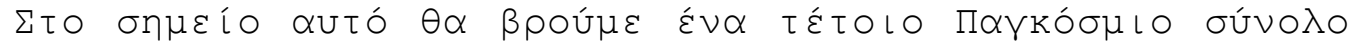

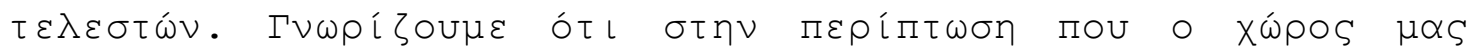

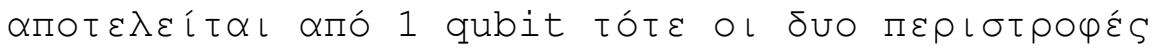

$$
G=\left\{R_{l}(\beta), R_{m}(\gamma)\right\}
$$

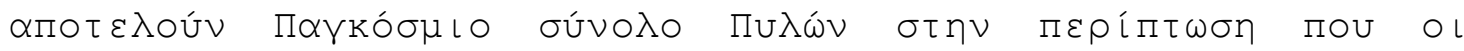

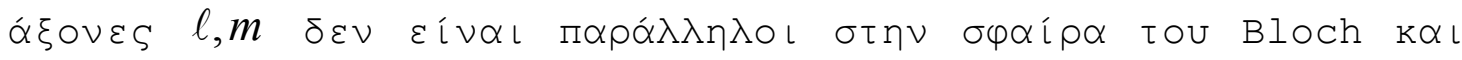

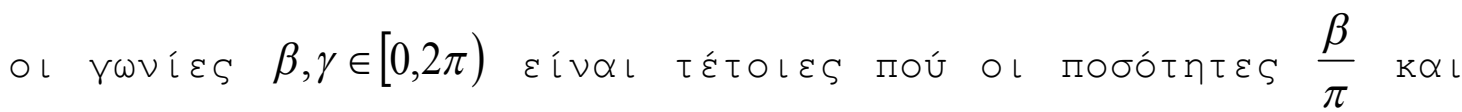

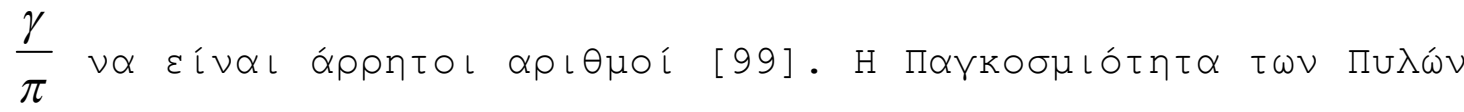

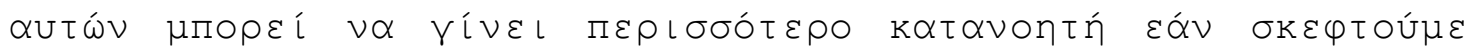

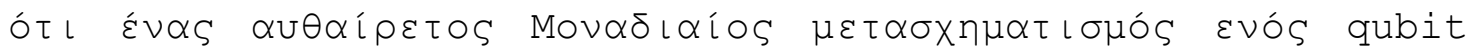

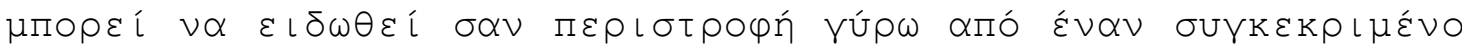

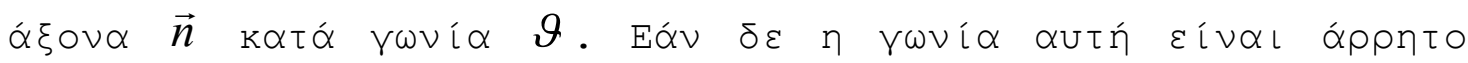




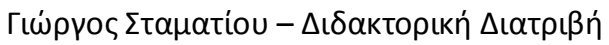

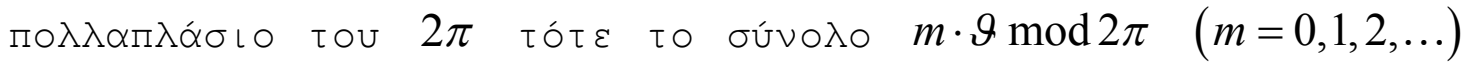

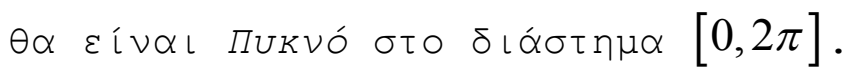

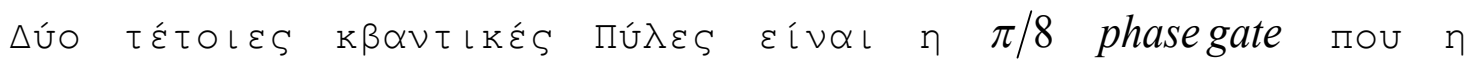

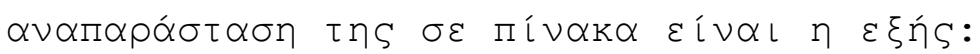

$$
T=\left[\begin{array}{cc}
1 & 0 \\
0 & \exp \left(i \frac{\pi}{4}\right)
\end{array}\right]
$$

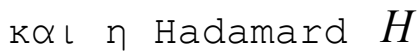

$$
H=\frac{1}{\sqrt{2}}\left[\begin{array}{cc}
1 & 1 \\
1 & -1
\end{array}\right]
$$

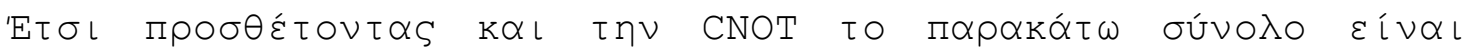
Паүкóouı

\section{$\{C N O T, H, T\}$}

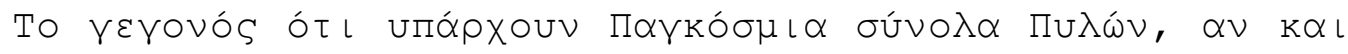

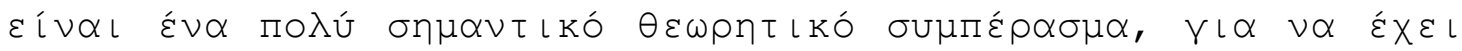

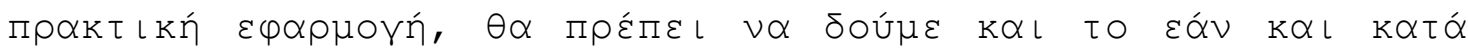

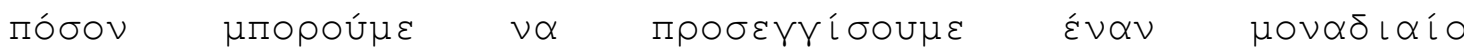

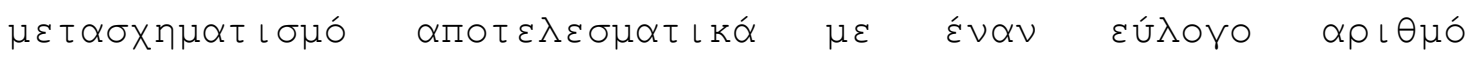

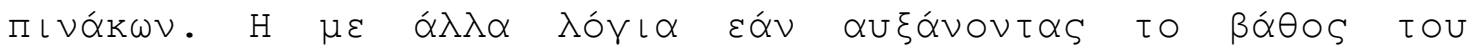

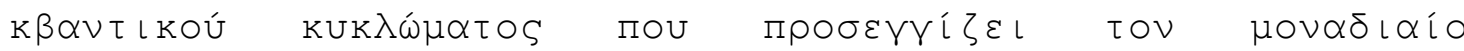

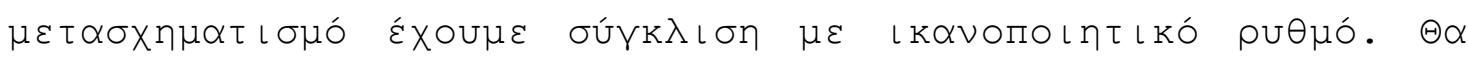

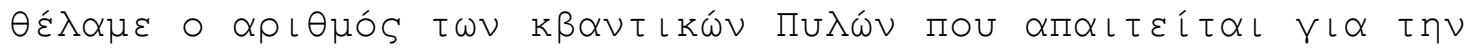

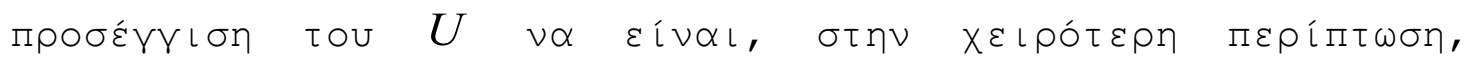

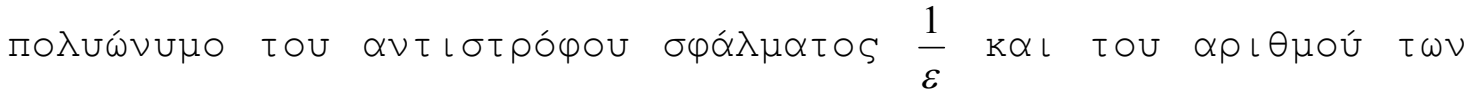

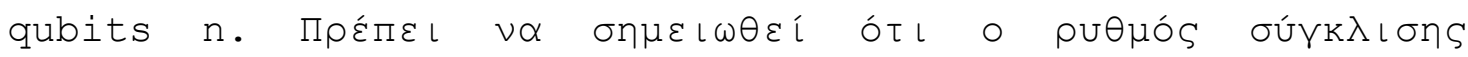

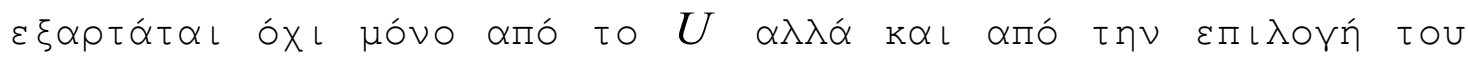

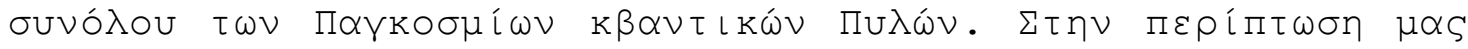




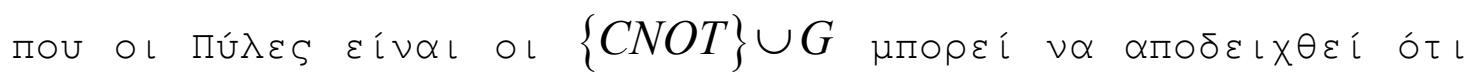

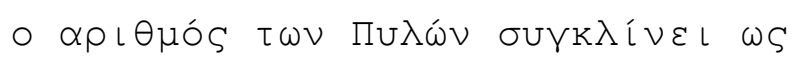

$$
O\left[m \log ^{c}\left(\frac{m}{\varepsilon}\right)\right]
$$

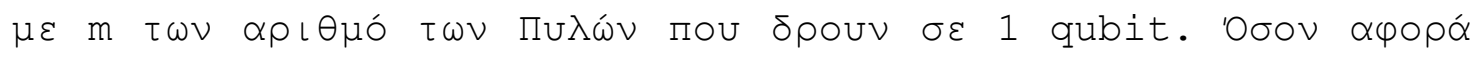

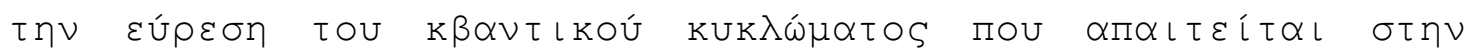

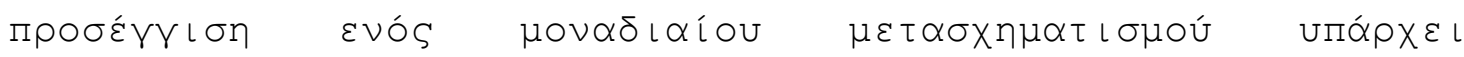

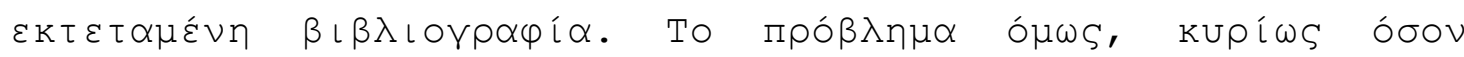

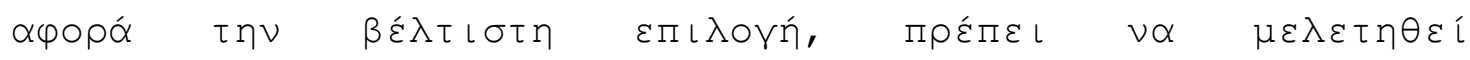

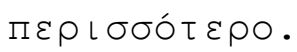

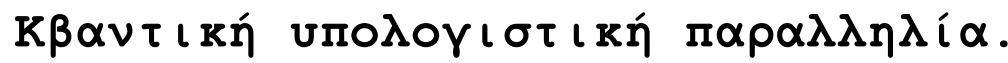

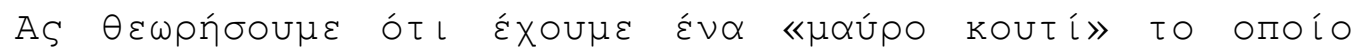

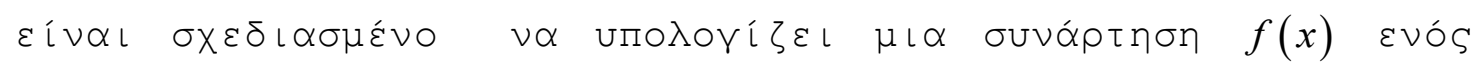

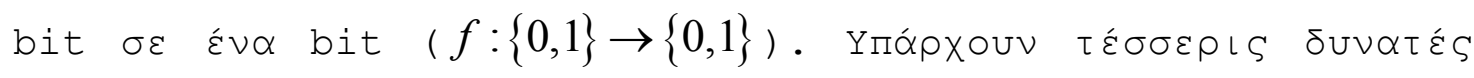

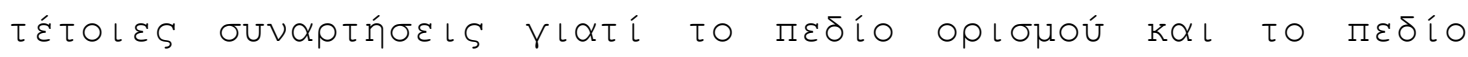

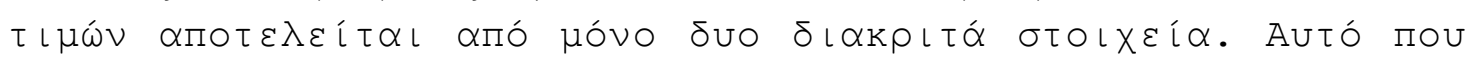

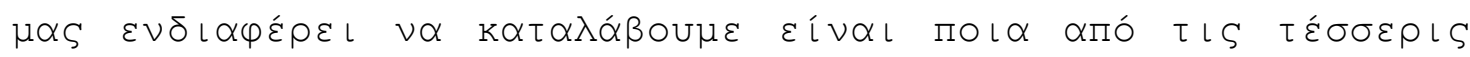

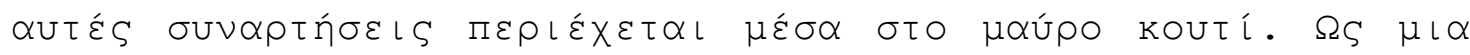

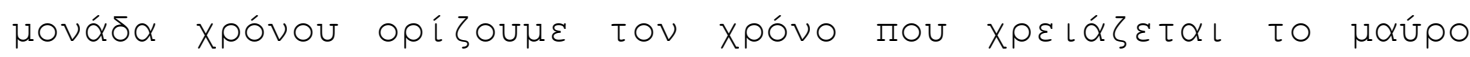

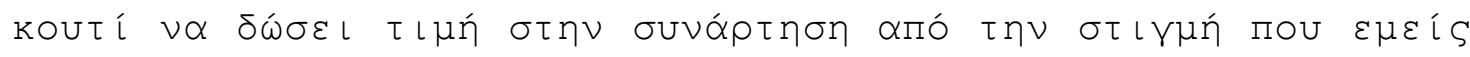

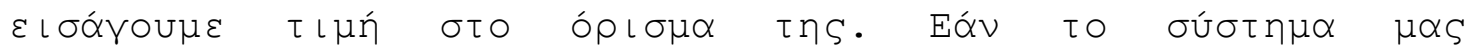

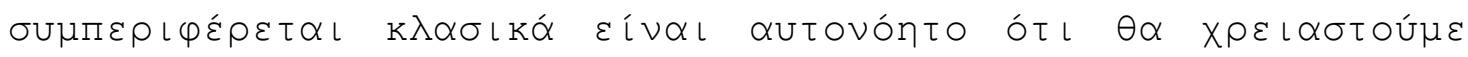

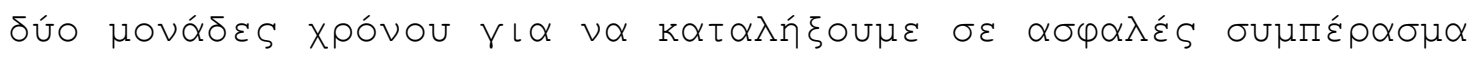

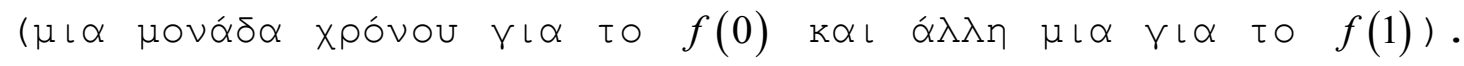

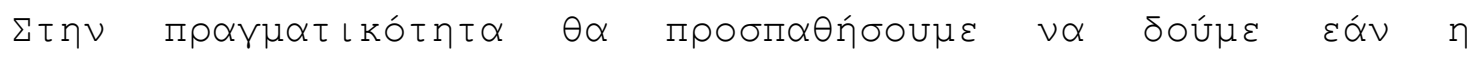

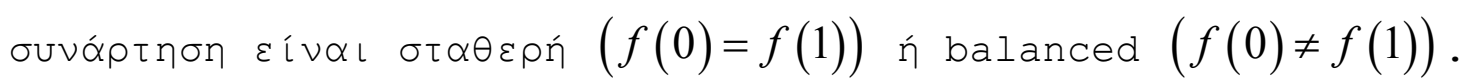

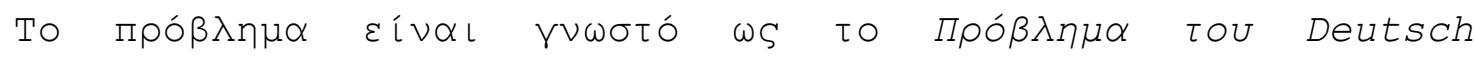
(Deutsch's problem) [44,45].

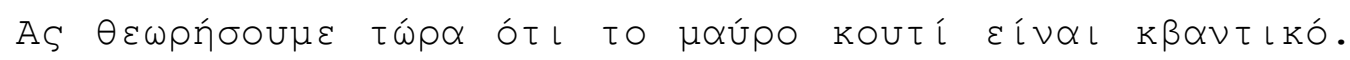

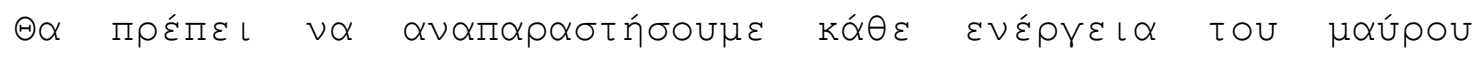

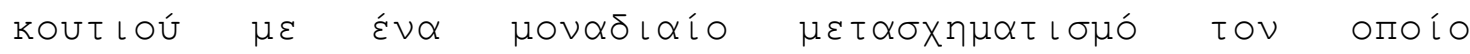




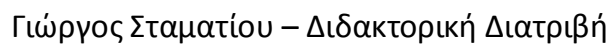

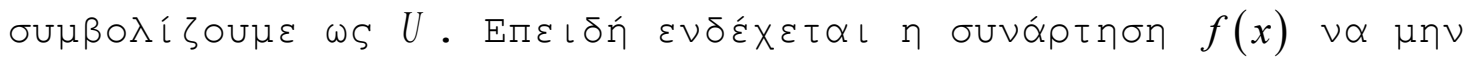

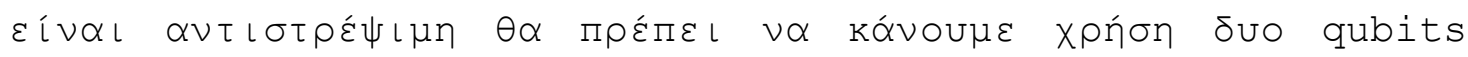

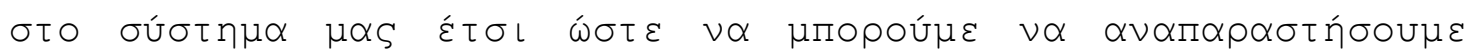

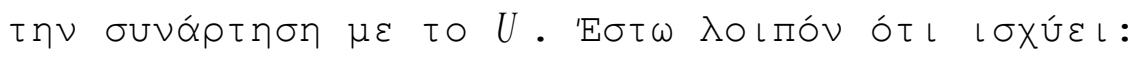

$$
U|x\rangle|y\rangle=|x\rangle|y \oplus f(x)\rangle
$$

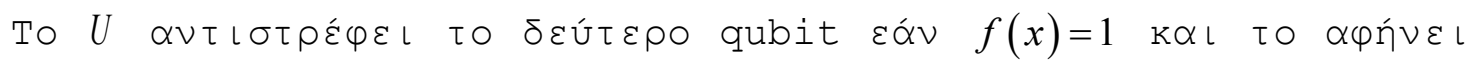
$\alpha \nu \alpha \lambda \lambda \circ i \omega \tau \circ \varepsilon \alpha \dot{\alpha} v f(x)=0$.

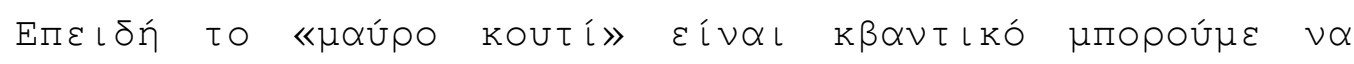

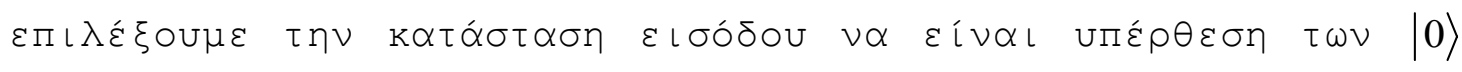

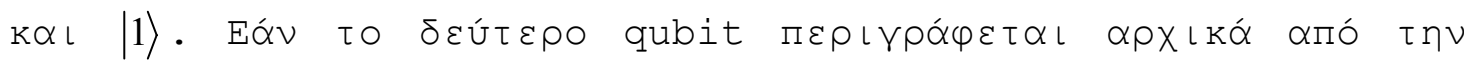

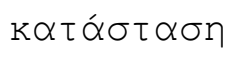

$$
\frac{|0\rangle-|1\rangle}{\sqrt{2}}
$$

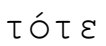

$$
\begin{gathered}
U\left[|x\rangle \frac{|0\rangle-|1\rangle}{\sqrt{2}}\right]= \\
|x\rangle \frac{|f(x)\rangle-|1 \oplus f(x)\rangle}{\sqrt{2}}= \\
|x\rangle(-1)^{f(x)} \frac{|0\rangle-|1\rangle}{\sqrt{2}}
\end{gathered}
$$

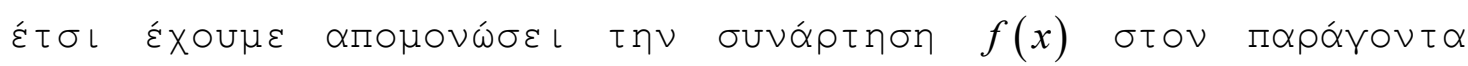

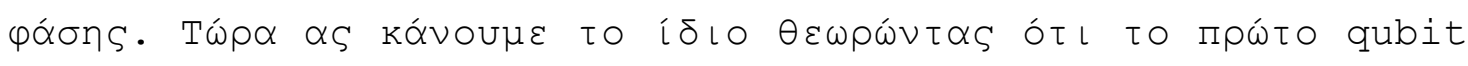

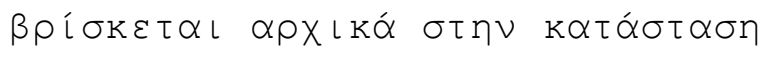

$$
\frac{|0\rangle+|1\rangle}{\sqrt{2}}
$$




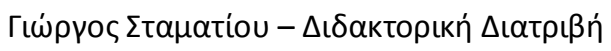

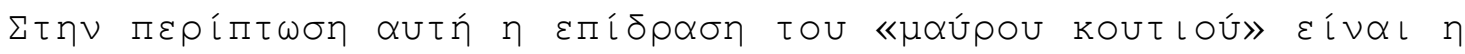

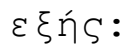

$$
\begin{gathered}
U\left[\left(\frac{|0\rangle+|1\rangle}{\sqrt{2}}\right)\left(\frac{|0\rangle-|1\rangle}{\sqrt{2}}\right)\right]= \\
\frac{1}{\sqrt{2}}\left[(-1)^{f(0)}|0\rangle+(-1)^{f(1)}|1\rangle\right] \frac{1}{\sqrt{2}}(|0\rangle-|1\rangle)
\end{gathered}
$$

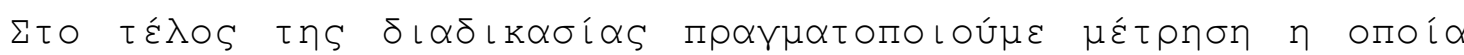

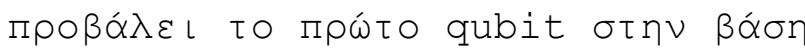

$$
| \pm\rangle=\frac{1}{\sqrt{2}}(|0\rangle \pm|1\rangle)
$$

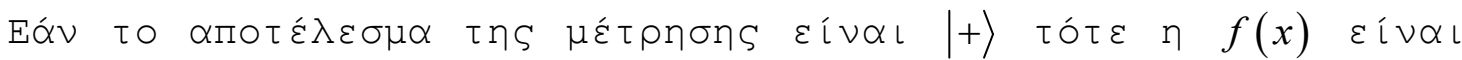

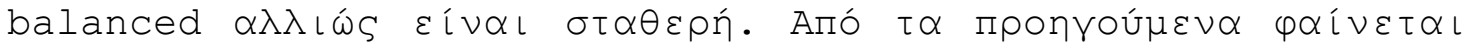

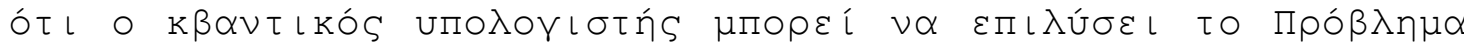

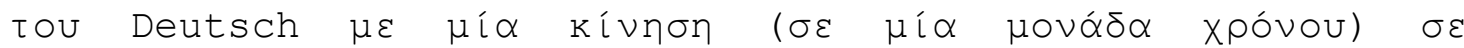

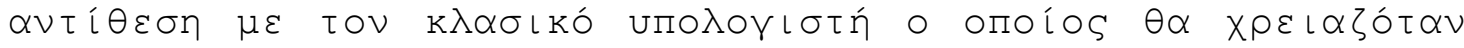

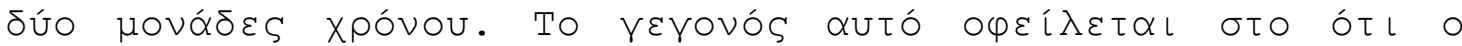

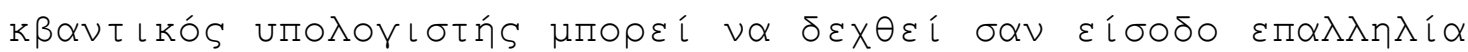

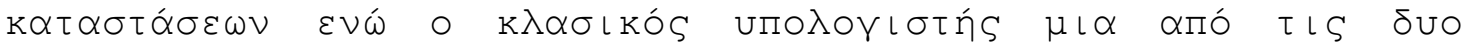

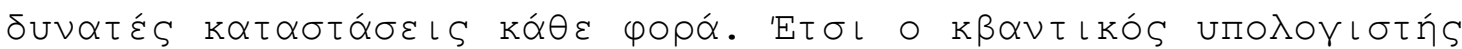

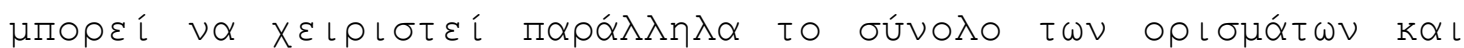

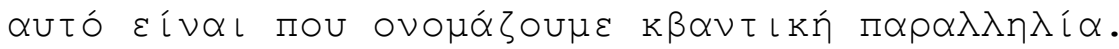

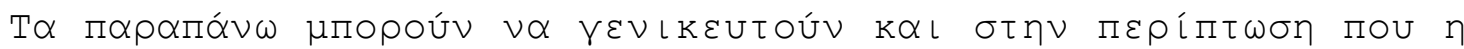

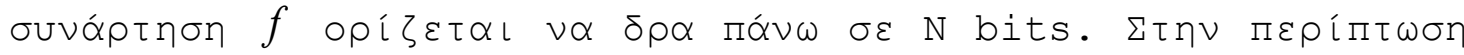

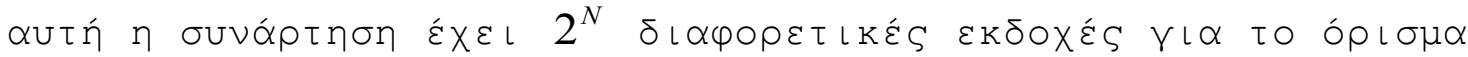

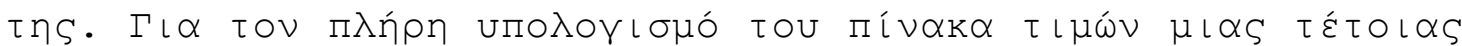

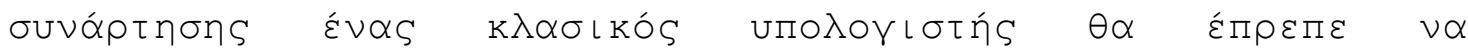

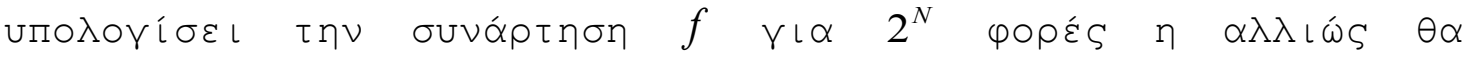

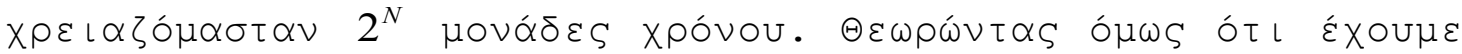

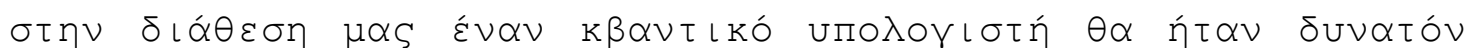

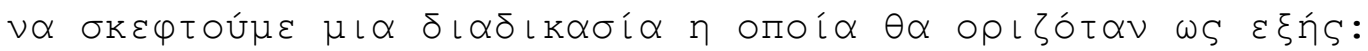

$$
U|x\rangle|0\rangle=|x\rangle|f(x)\rangle
$$




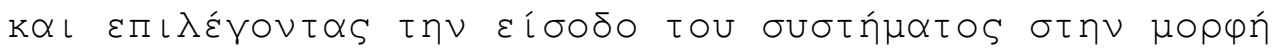

$$
\bigotimes_{N}\left(\frac{|0\rangle+|1\rangle}{\sqrt{2}}\right)=\frac{1}{2^{\frac{N}{2}}} \sum_{n=0}^{2^{N}-1}\left|x_{n}\right\rangle
$$

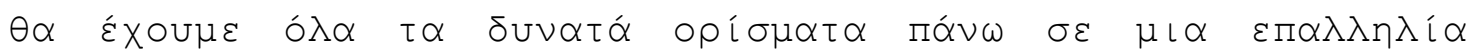

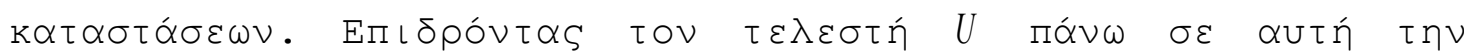

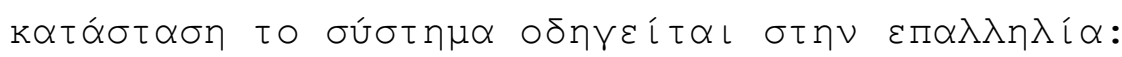

$$
\frac{1}{2^{\frac{N}{2}}} \sum_{n=0}^{2^{N}-1}\left|x_{n}\right\rangle\left|f\left(x_{n}\right)\right\rangle
$$

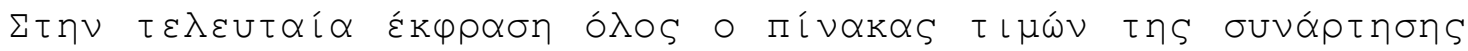

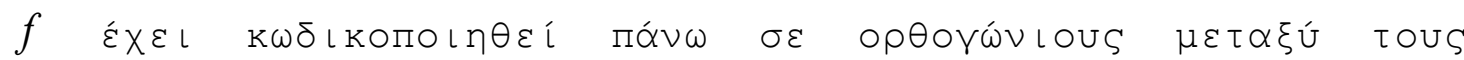

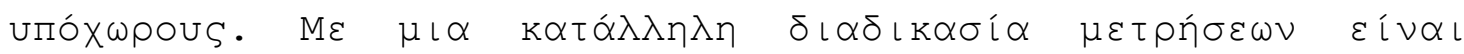

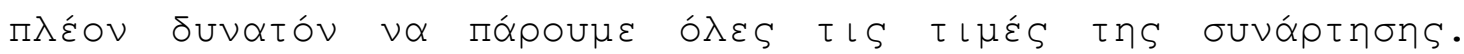

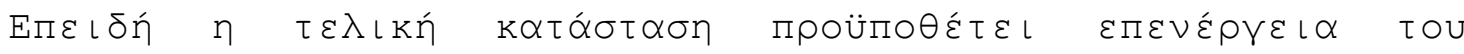

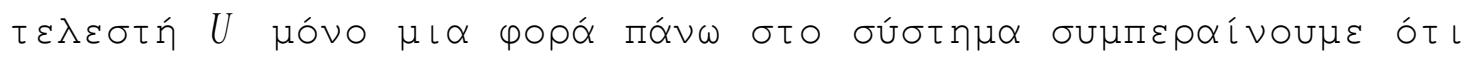

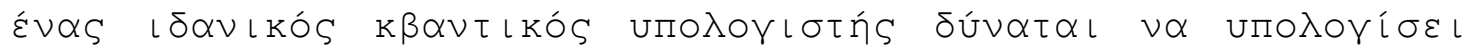

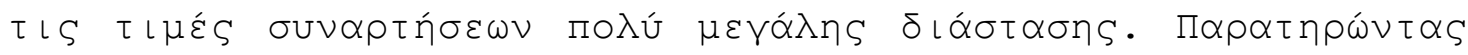

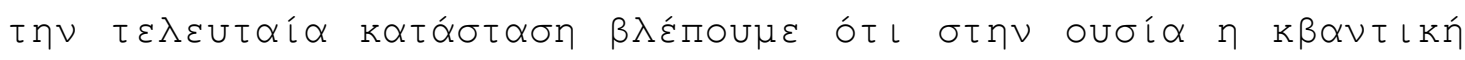

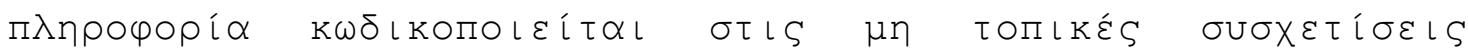

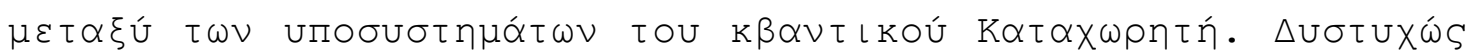

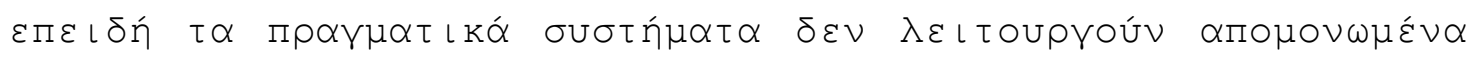

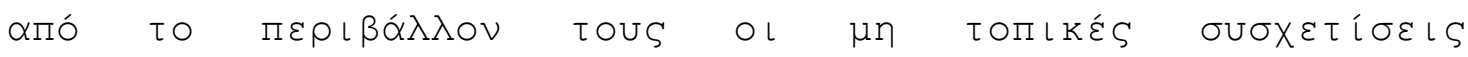

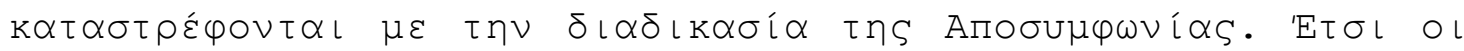

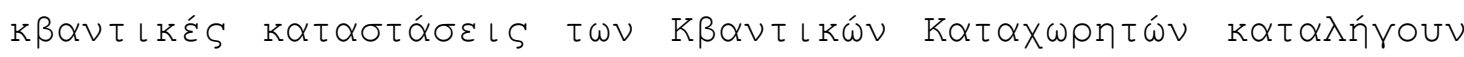

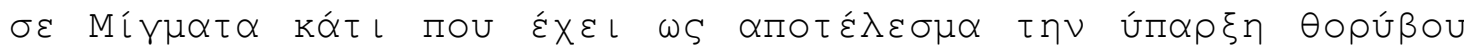
$\sigma \tau \alpha$ 更

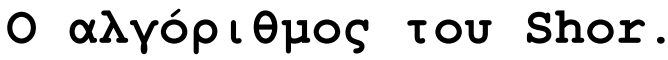

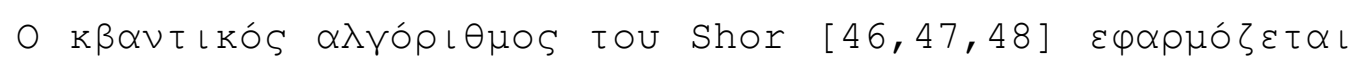

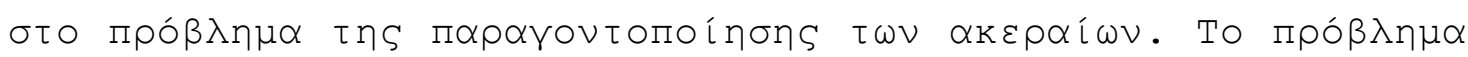

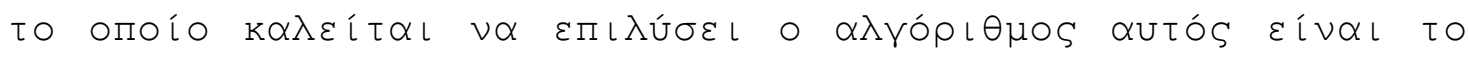
$\varepsilon \xi \dot{n} S:$ 


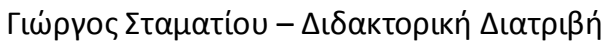

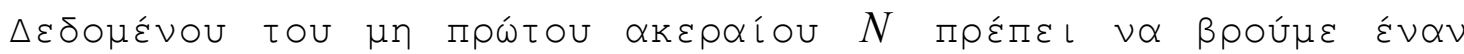

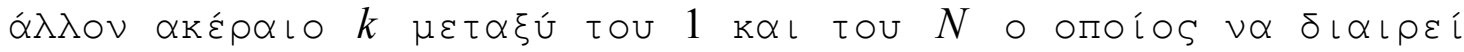

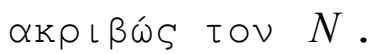

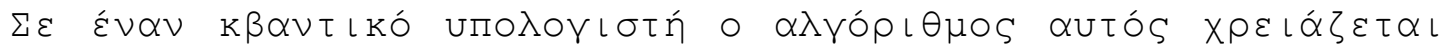

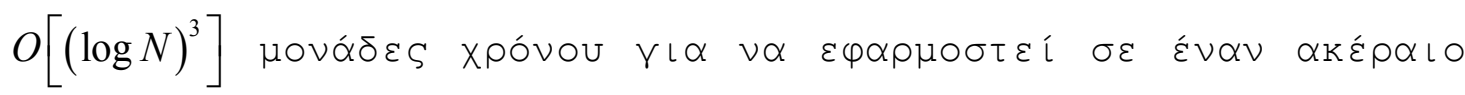

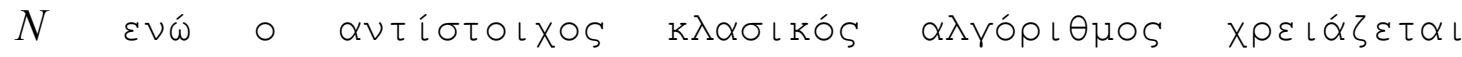

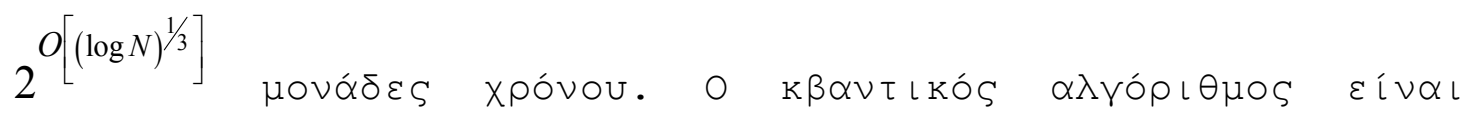
$\varepsilon$

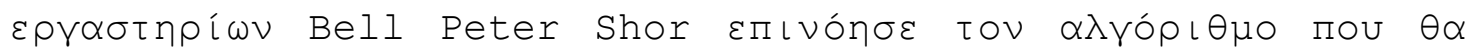

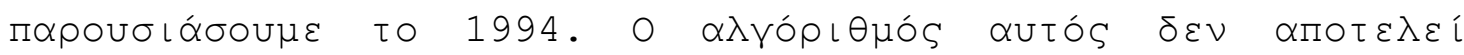

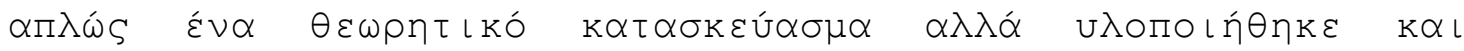

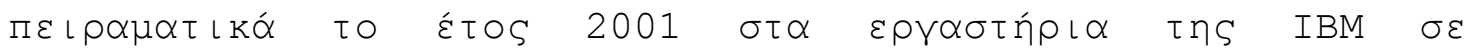

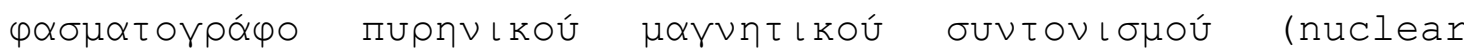

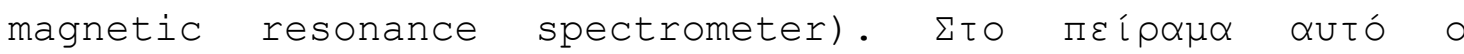

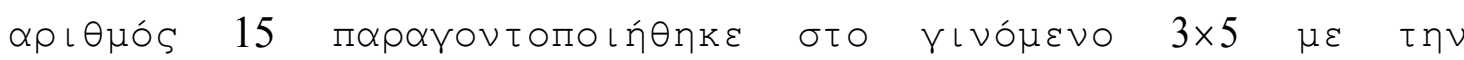

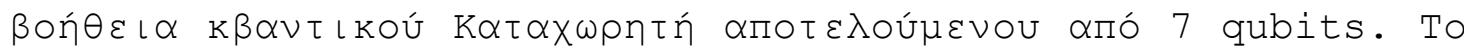

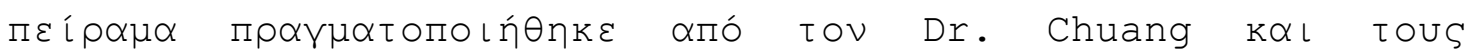
ouveprótes tou Dr. Lieven M. K. Vandersypen, Matthias

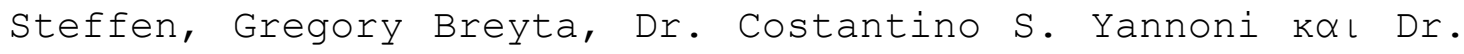

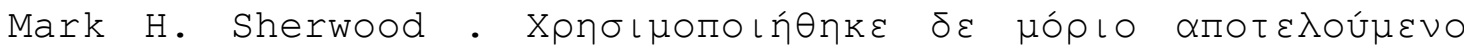

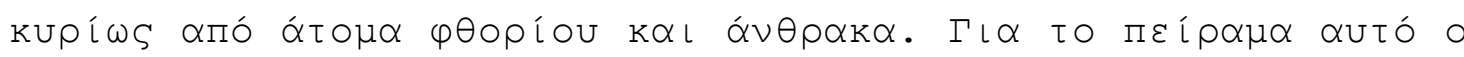
Dr. Chuang $\delta\llcorner\alpha \Pi \imath \sigma \tau \omega \sigma \varepsilon$ ót ı:

«We have to move far beyond this, but at least we have demonstrated that it is possible».

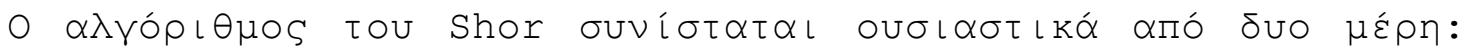

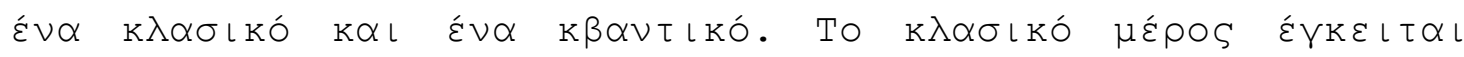

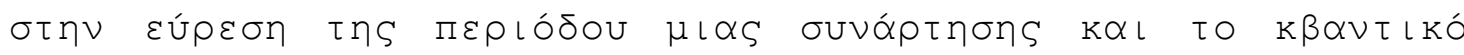

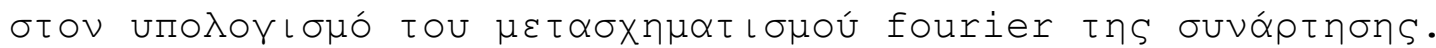

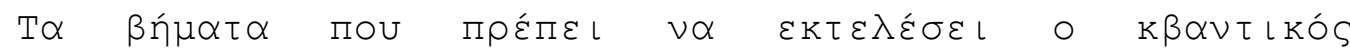

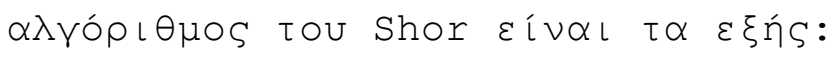

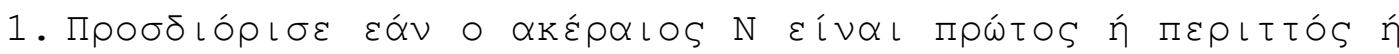

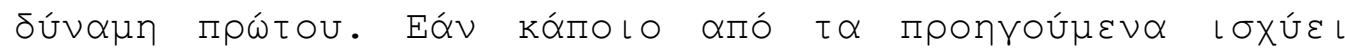

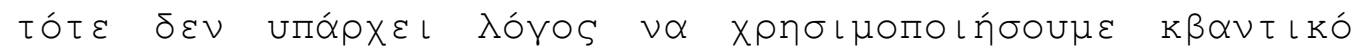

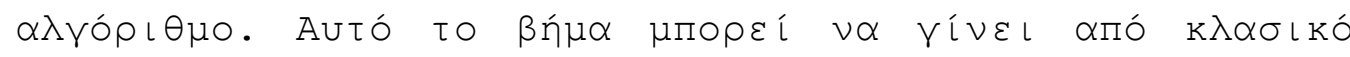

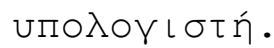




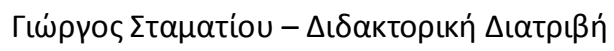

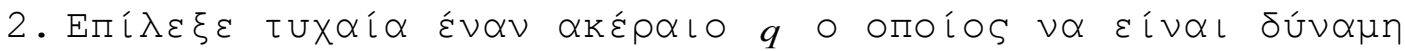

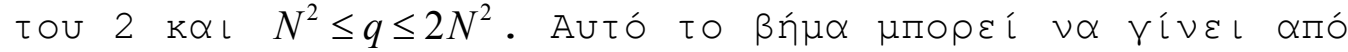

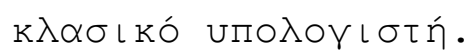

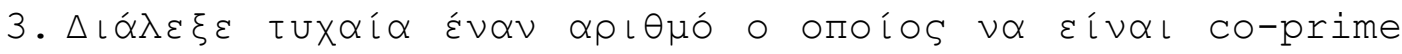

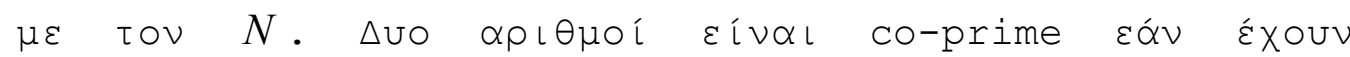

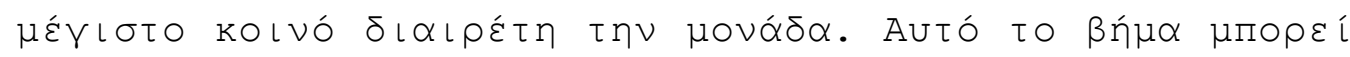

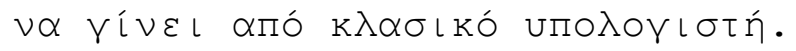

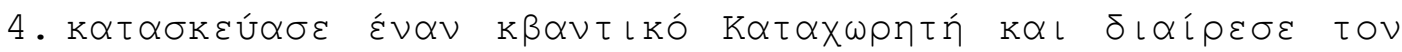

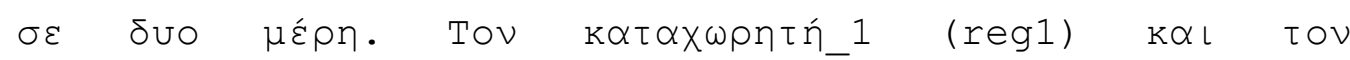

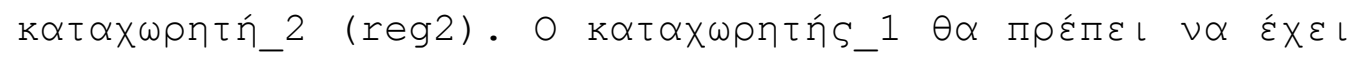

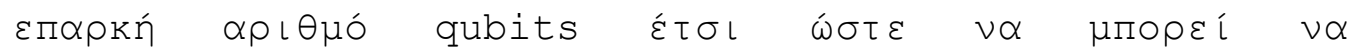

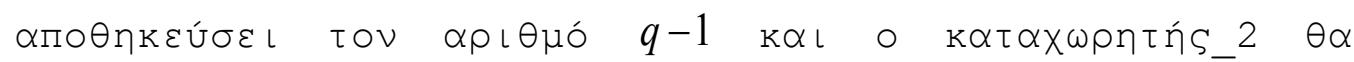

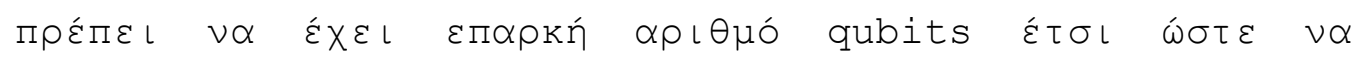

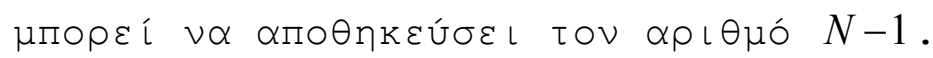

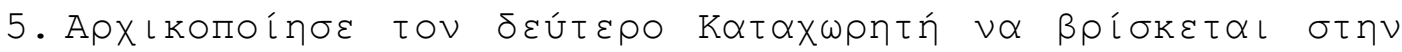

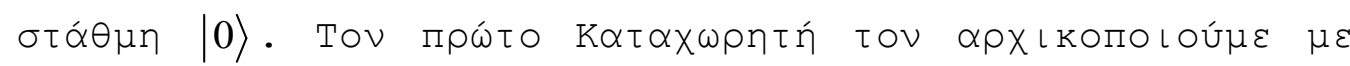

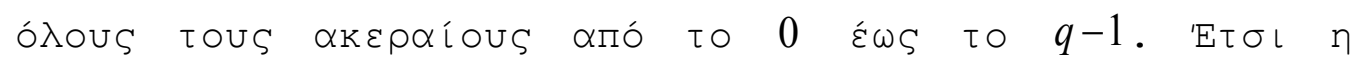

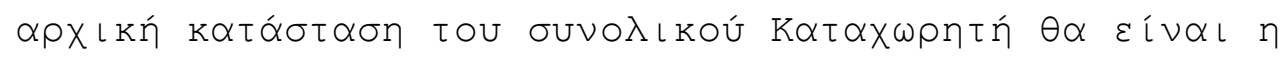

6.

$$
\frac{1}{\sqrt{q}} \sum_{c=0}^{q-1}|c, 0\rangle
$$

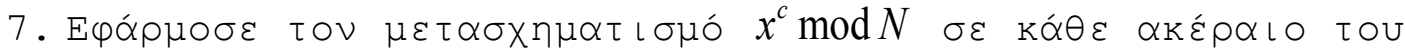

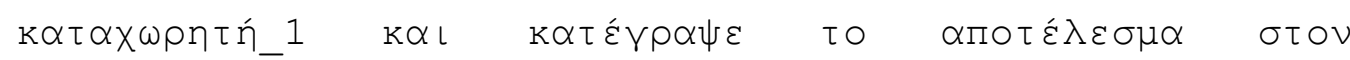

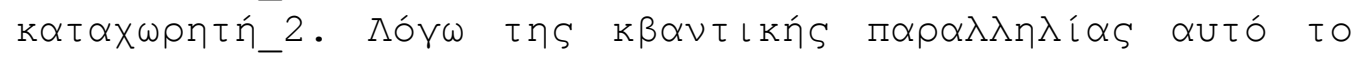

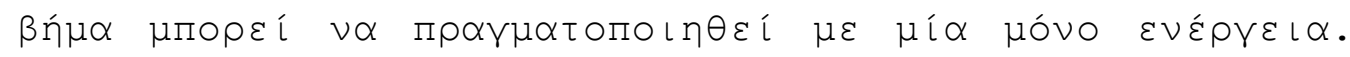

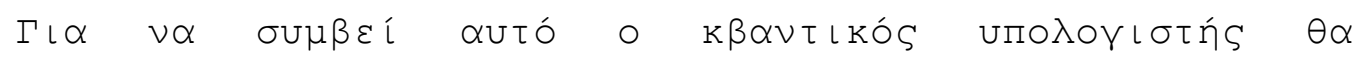

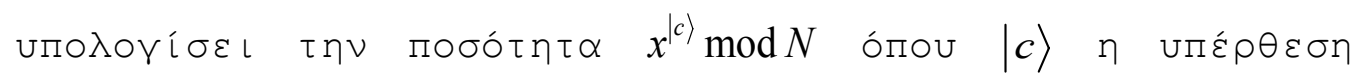

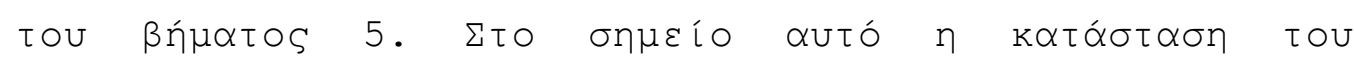

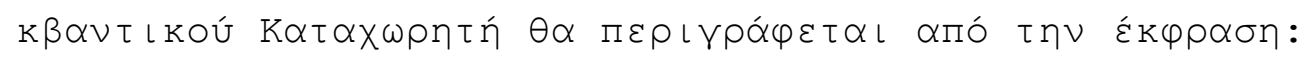

8. 


$$
\frac{1}{\sqrt{q}} \sum_{c=0}^{q-1}\left|c, x^{c} \bmod N\right\rangle
$$

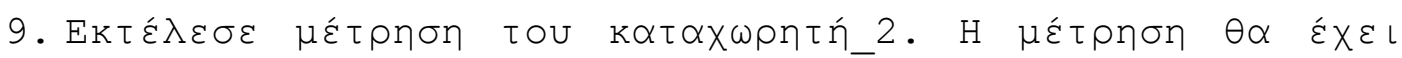

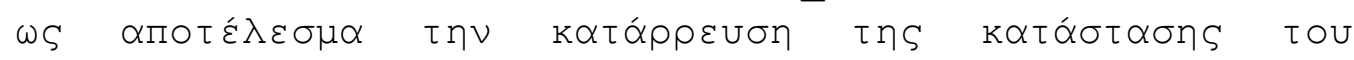

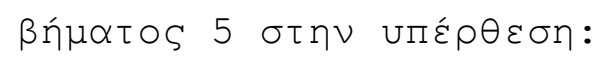

$$
\frac{1}{\sqrt{\|A\|}} \sum_{c^{\prime} \in A}\left|c^{\prime}, k\right\rangle
$$

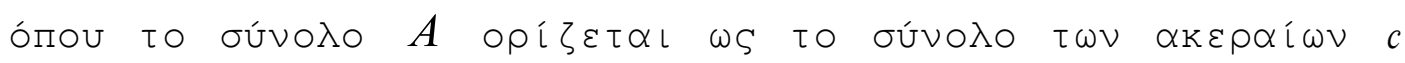

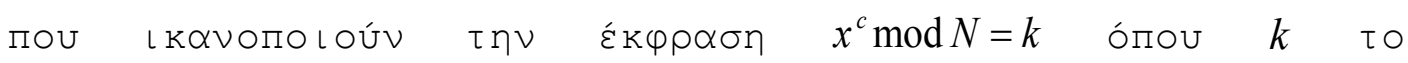

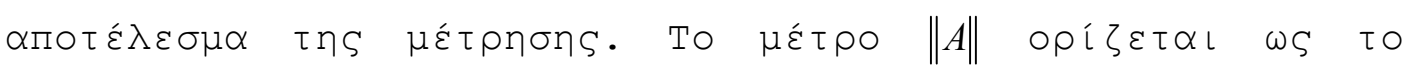

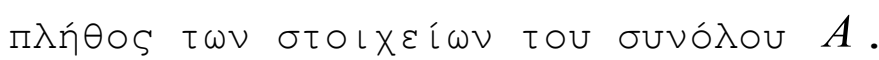

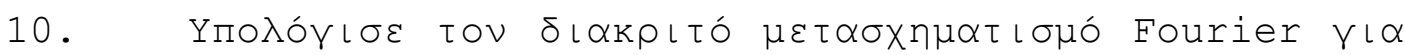

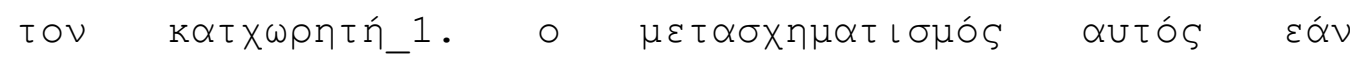

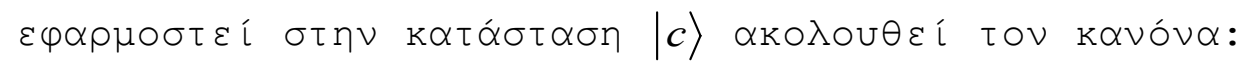

$$
|c\rangle=\frac{1}{\sqrt{q}} \sum_{a=1}^{q-1}|a\rangle e^{\frac{2 \pi i c a}{q}}
$$

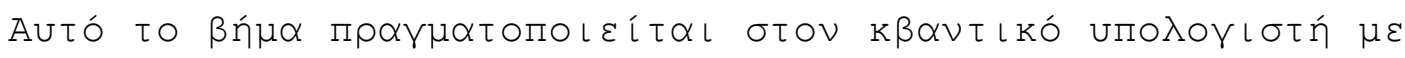

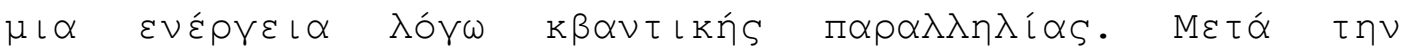

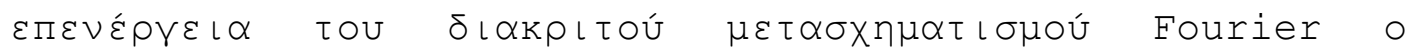

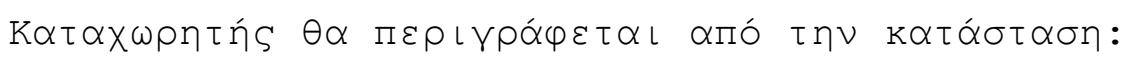

$$
\frac{1}{\sqrt{\|A\|}} \sum_{c^{\prime} \in A} \frac{1}{\sqrt{q}} \sum_{c=0}^{q-1}|c, k\rangle e^{2 \pi i c^{\prime} a / q}
$$

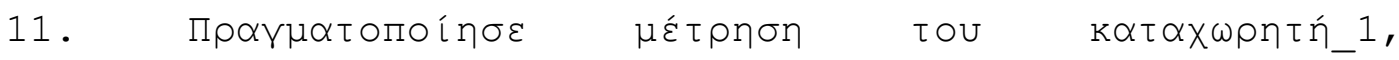

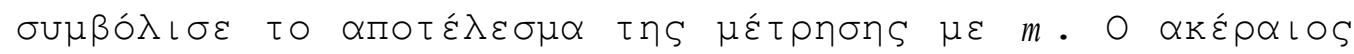

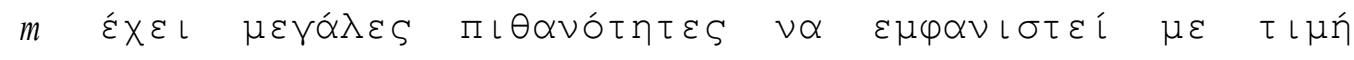

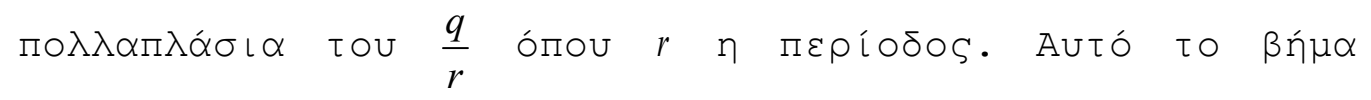

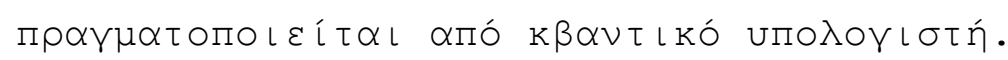




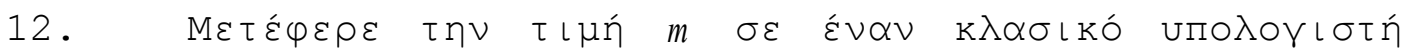

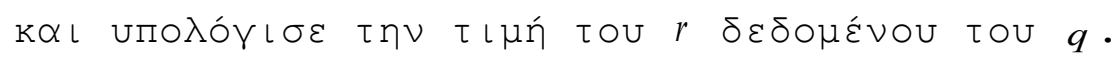

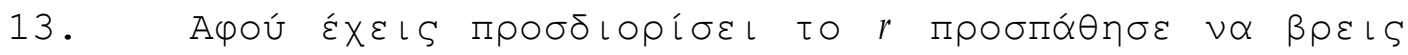

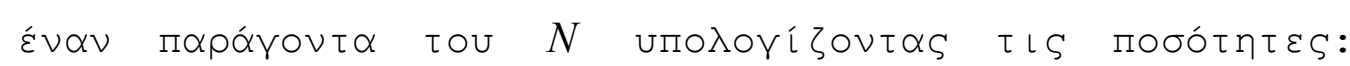

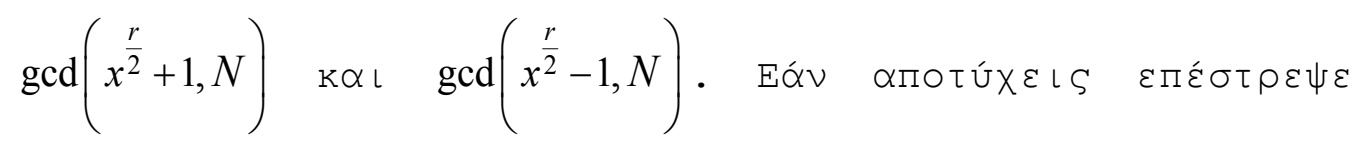

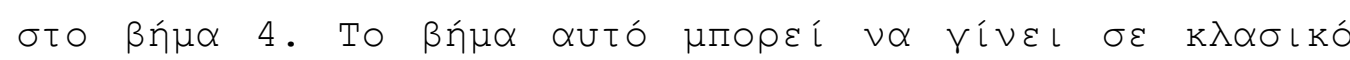
uпо入оүьбт́.

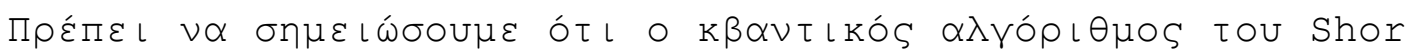

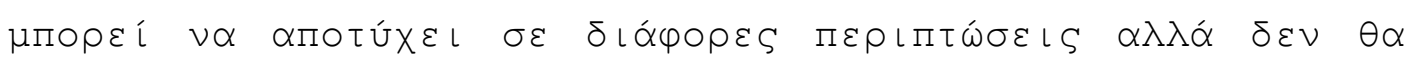

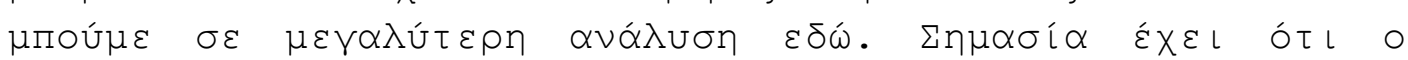

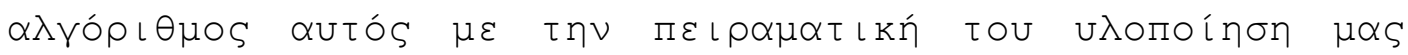

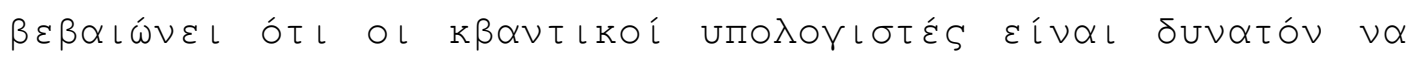

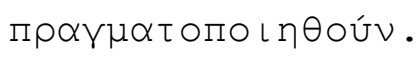

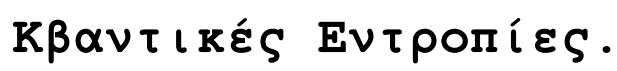

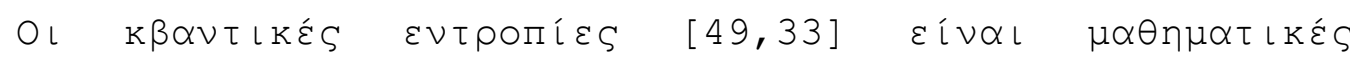

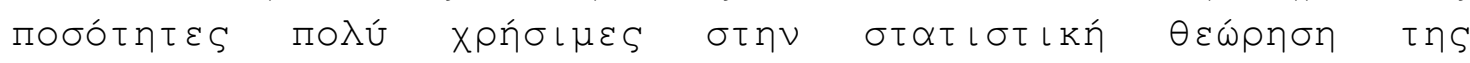

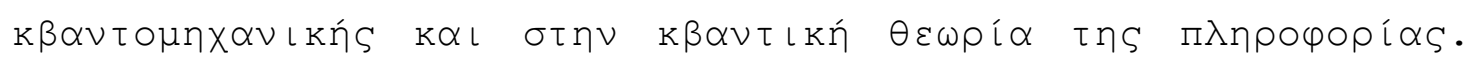

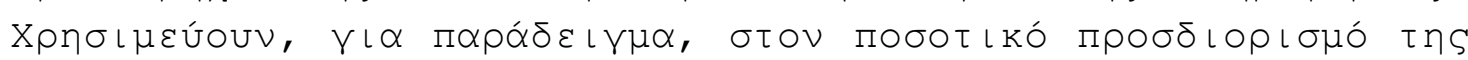

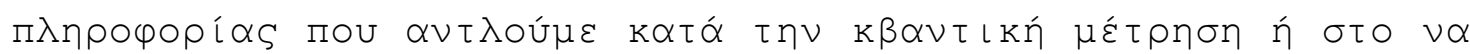

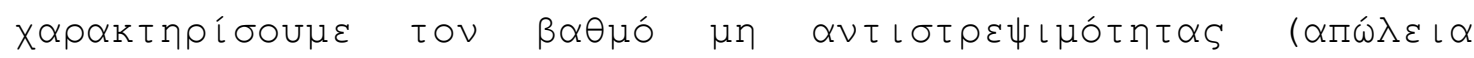

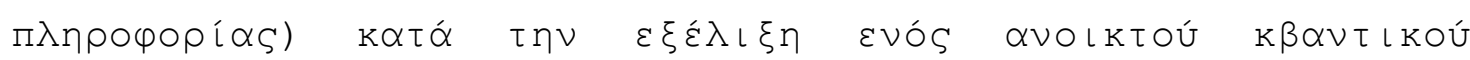

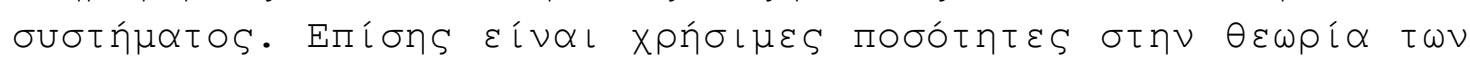

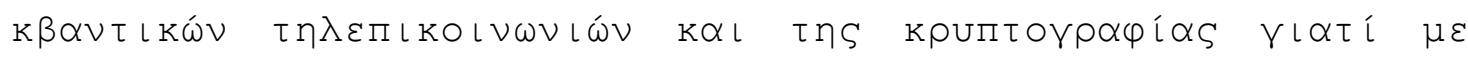

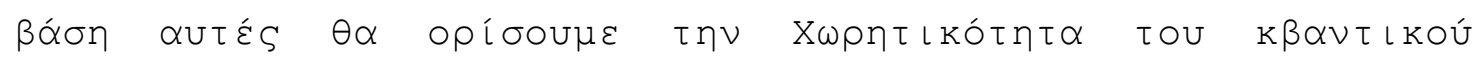

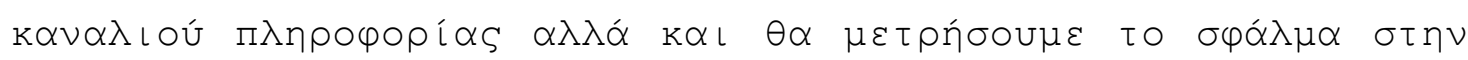

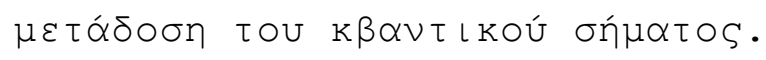

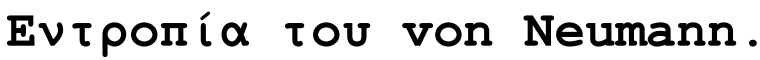

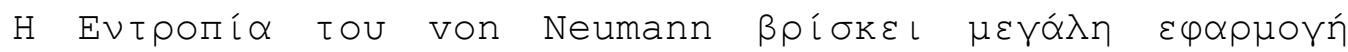

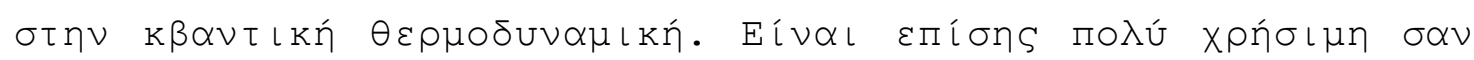

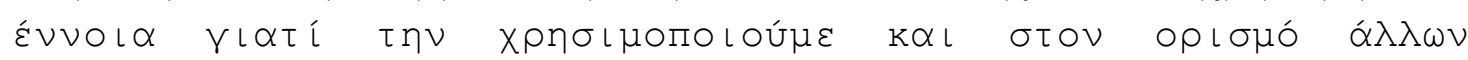

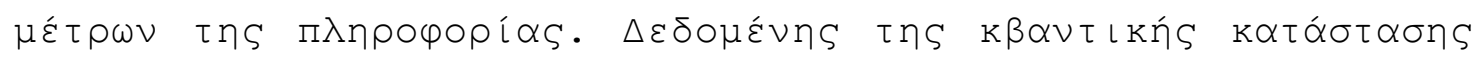




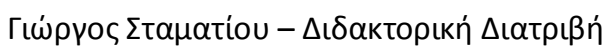

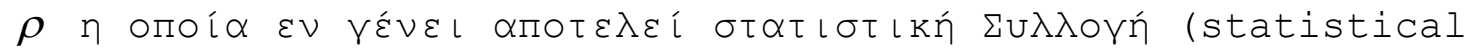

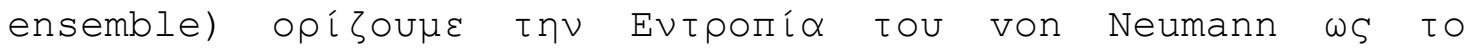

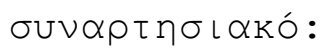

$$
S(\rho) \equiv-\operatorname{tr}(\rho \ln \rho)
$$

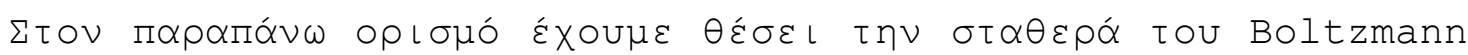

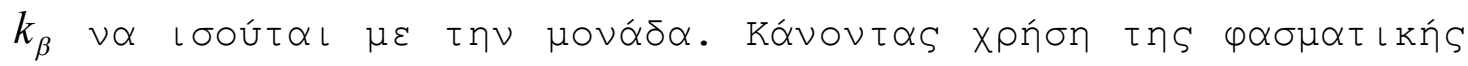

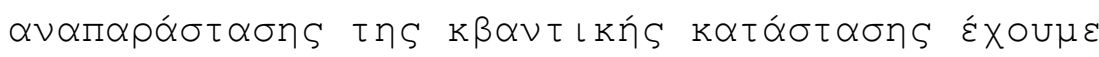

$$
\begin{gathered}
\rho=\sum_{i} p_{i}\left|\varphi_{i}\right\rangle\left\langle\varphi_{i}\right|, \quad p_{i} \geq 0 \\
\sum_{i} p_{i}=1
\end{gathered}
$$

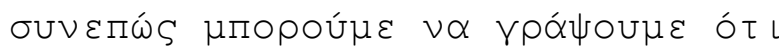

$$
\begin{gathered}
S(\rho)=-\sum_{i} p_{i} \ln p_{i} \quad\left(=H\left(p_{1}, p_{2}, \ldots\right)\right) \\
0 \cdot \ln 0 \equiv 0
\end{gathered}
$$

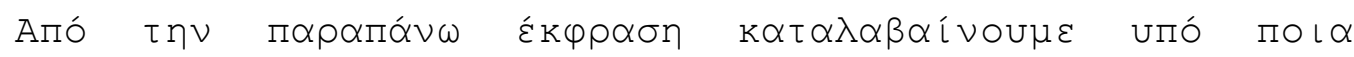

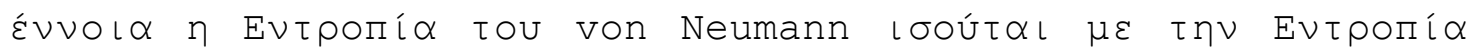

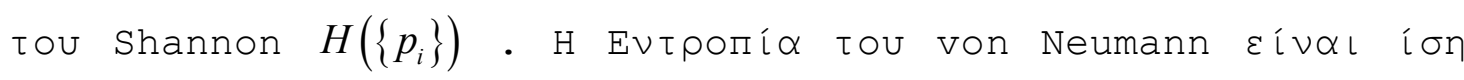

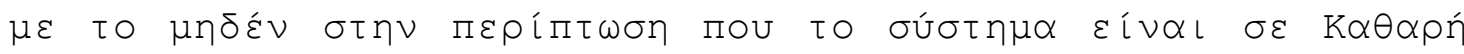

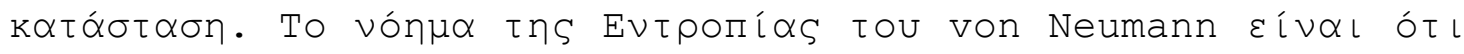

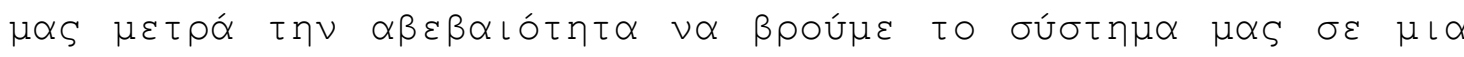

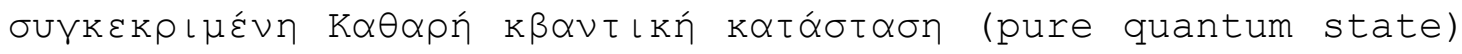

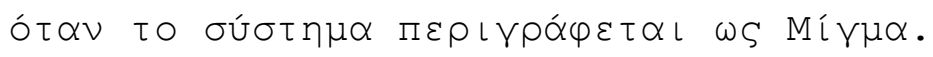

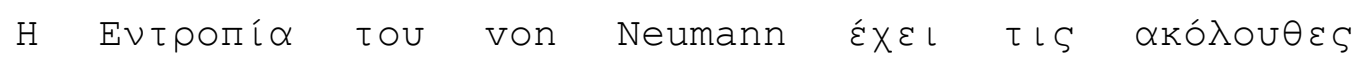
ıঠı́tntes [33, 49,50,51].

1. $E \vee \curlyvee \varepsilon ́ \vee \varepsilon\llcorner$

$$
S(\rho) \geq 0
$$

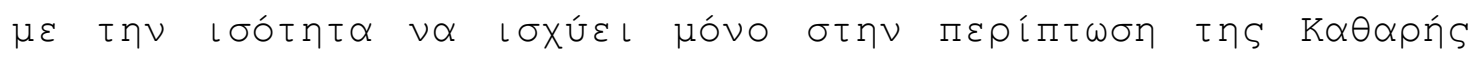

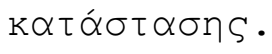




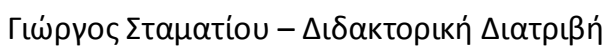

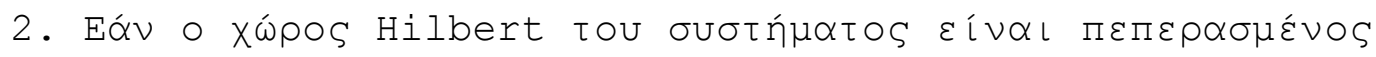

$$
\operatorname{dim} \mathcal{H}=D<\infty
$$

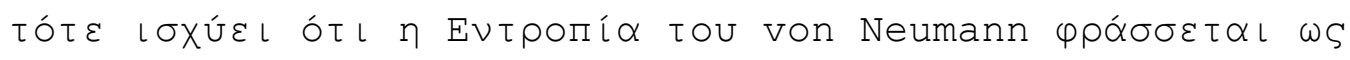

$$
S(\rho) \leq \ln D
$$

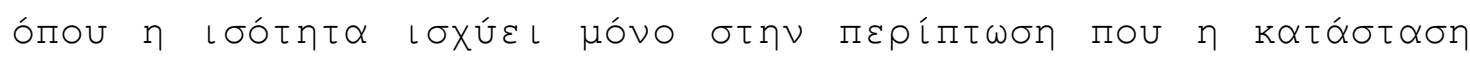

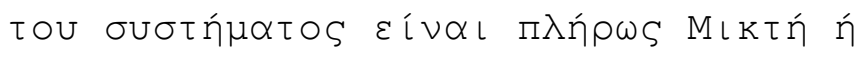

$$
\rho=\frac{I}{D}
$$

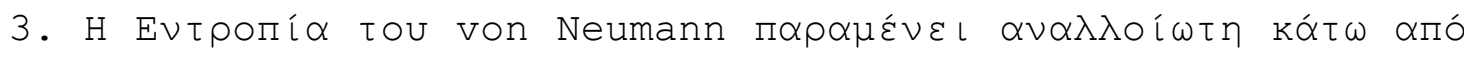

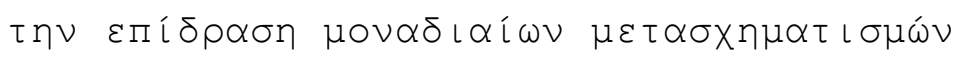

$$
S\left(U \rho U^{+}\right)=S(\rho)
$$

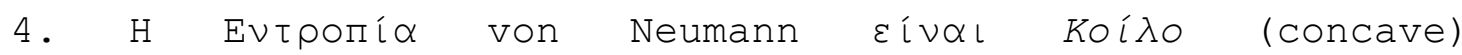

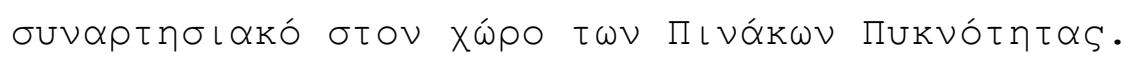

$$
\begin{gathered}
S\left(\sum_{i} \lambda_{i} \rho_{i}\right) \geq \sum_{i} \lambda_{i} S\left(\rho_{i}\right), \quad \lambda_{i} \geq 0 \\
\sum_{i} \lambda_{i}=1
\end{gathered}
$$

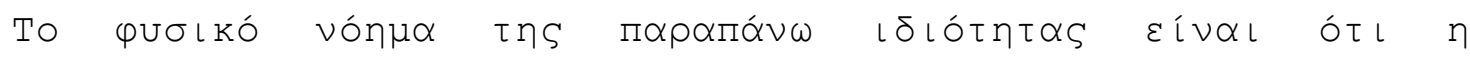

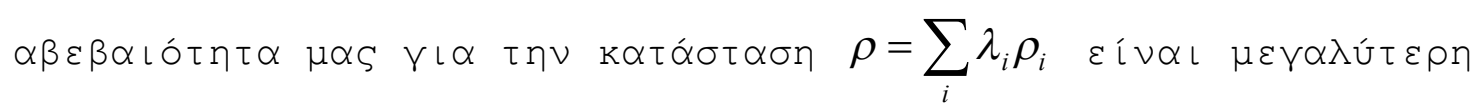

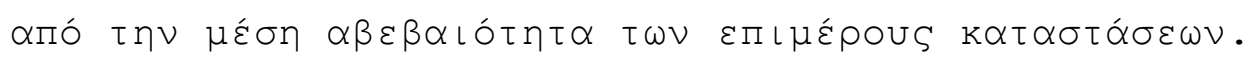

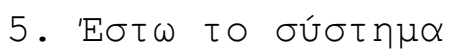

$$
\mathcal{H}=\mathcal{H}^{(1)} \otimes \mathcal{H}^{(2)}
$$

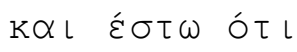

$$
\rho^{(1)}=t r_{2}(\rho)
$$




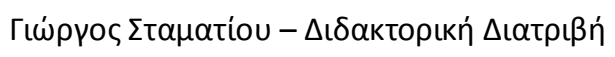

$$
\rho^{(2)}=\operatorname{tr}_{1}(\rho)
$$

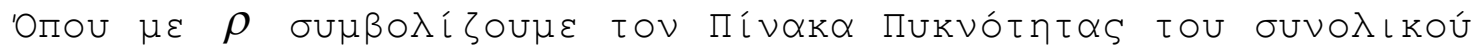

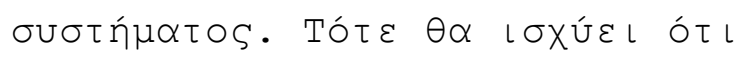

$$
S(\rho) \leq S\left(\rho^{(1)}\right)+S\left(\rho^{(2)}\right)
$$

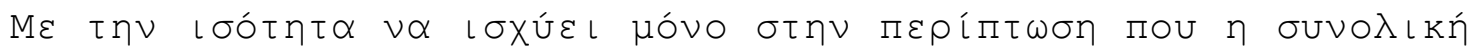

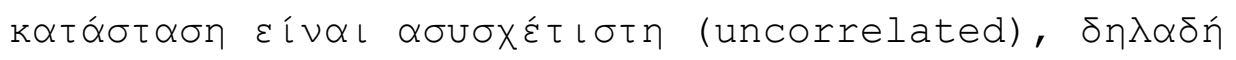

$$
\rho=\rho^{(1)} \otimes \rho^{(2)}
$$

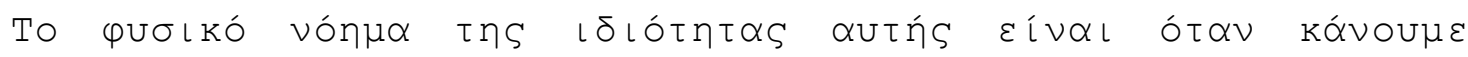

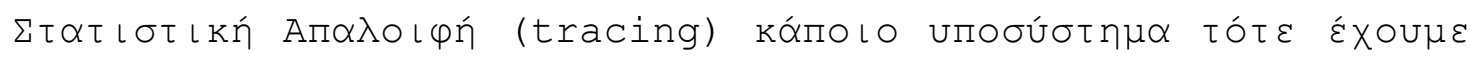

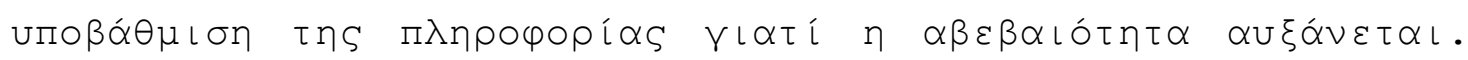

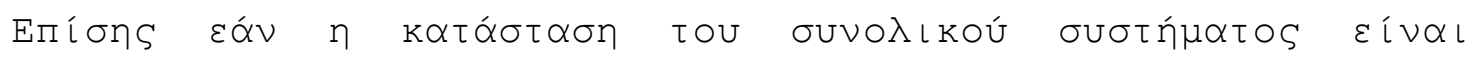

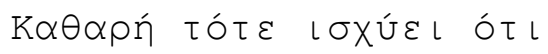

$$
S\left(\rho^{(1)}\right)=S\left(\rho^{(2)}\right)
$$

Enions [51] (strong subadditivity)

$$
S\left(\rho_{A B C}\right)+S\left(\rho_{B}\right) \leq S\left(\rho_{A B}\right)+S\left(\rho_{B C}\right)
$$

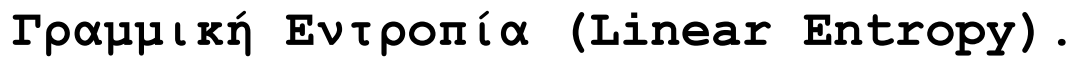

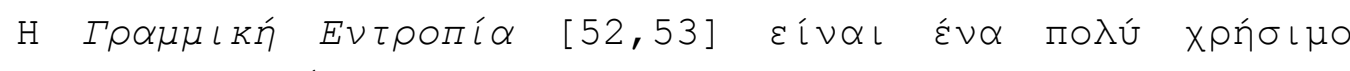

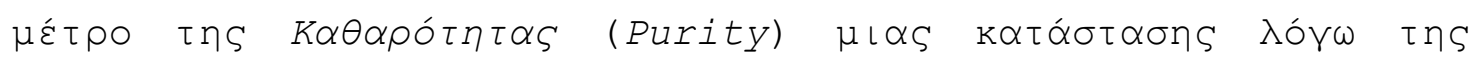

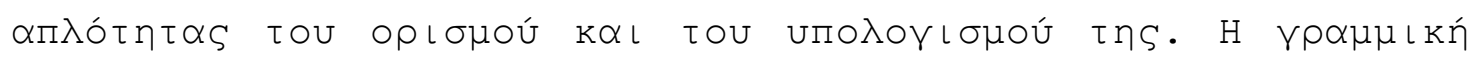

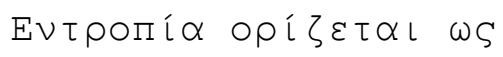

$$
S_{l} \equiv 1-\operatorname{tr}\left(\rho^{2}\right)
$$

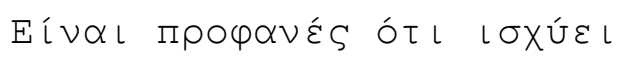

$$
0 \leq S_{l}(\rho) \leq 1
$$




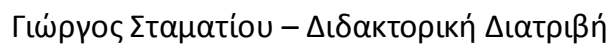

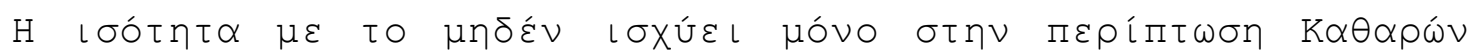
(pure) $\quad k \alpha \tau \alpha \sigma \tau \alpha ́ \sigma \varepsilon \omega \nu$.

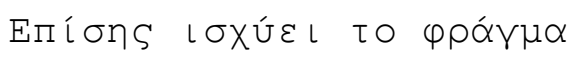

$$
S_{l}(\rho) \leq 1-\frac{1}{D}
$$

$\mu \varepsilon$

$$
\operatorname{dim} \mathcal{H}=D
$$

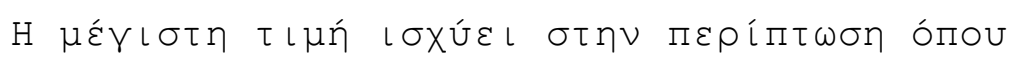

$$
\rho=I / D
$$

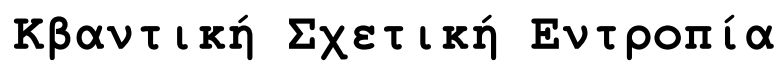

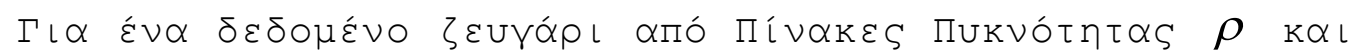

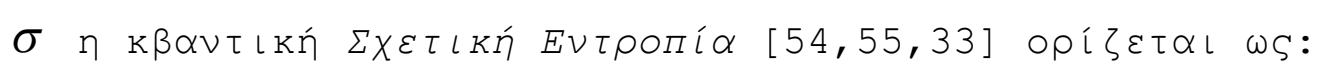

$$
S\left(\rho_{1} \| \rho_{2}\right) \equiv \operatorname{tr}\left(\rho_{1} \ln \rho_{1}\right)-\operatorname{tr}\left(\rho_{1} \ln \rho_{2}\right)
$$

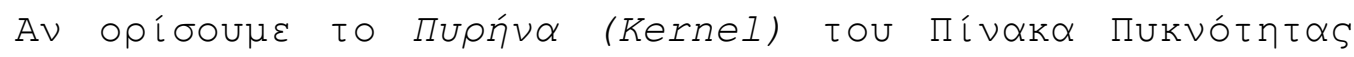

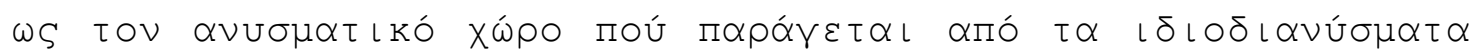

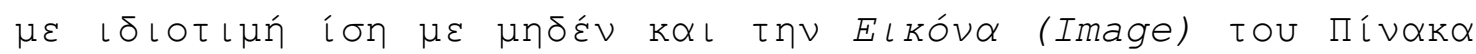

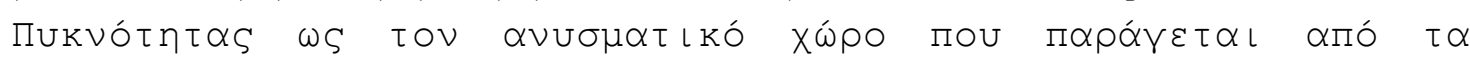

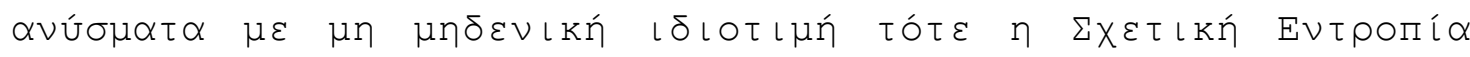

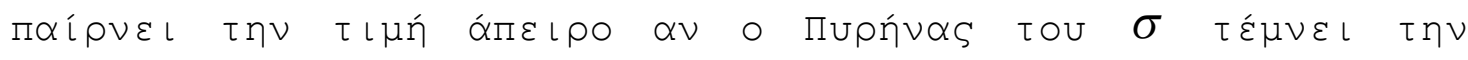

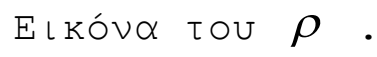

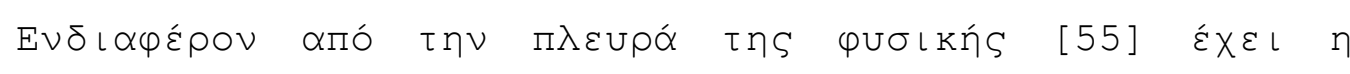

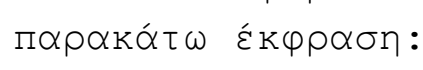

$$
S\left(\rho \| \rho^{(1)} \otimes \rho^{(2)}\right)=S\left(\rho^{(1)}\right)+S\left(\rho^{(2)}\right)-S(\rho)
$$

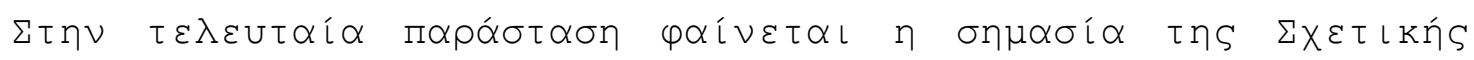

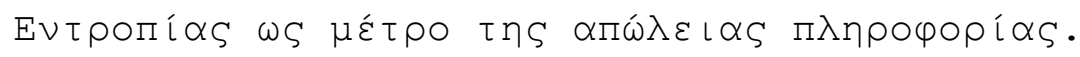




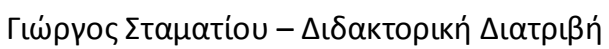

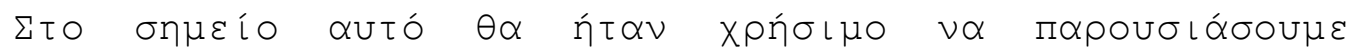

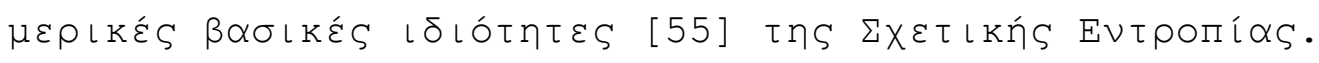

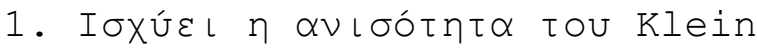

$$
S(\rho \| \sigma) \geq 0
$$

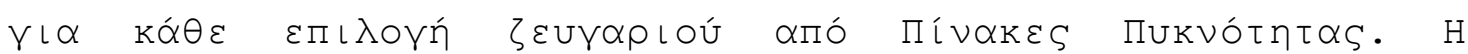

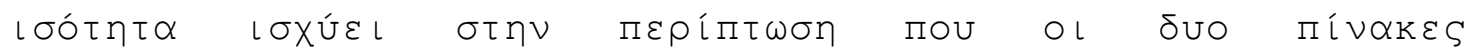
$\tau \alpha u \tau i \zeta$ ¿ $\tau \alpha \iota$.

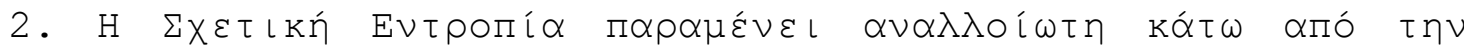

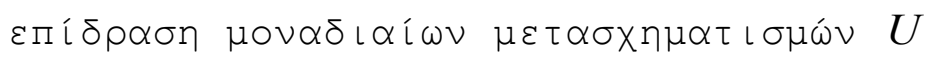

$$
S\left[\left(U \rho U^{+}\right) \|\left(U \sigma U^{+}\right)\right]=S(\rho \| \sigma)
$$

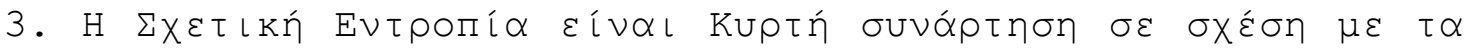

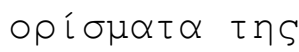

$$
S(\rho \| \sigma) \leq \lambda S\left(\rho_{1} \| \sigma_{1}\right)+(1-\lambda) S\left(\rho_{2} \| \sigma_{2}\right)
$$

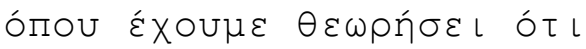

$$
\begin{gathered}
\rho=\lambda \rho_{1}+(1-\lambda) \rho_{2} \\
\sigma=\lambda \sigma_{1}+(1-\lambda) \sigma_{2} \\
0 \leq \lambda \leq 1
\end{gathered}
$$

4. E⿺́र $\theta \varepsilon ́ \sigma o v \mu \varepsilon$

$$
\begin{gathered}
\rho^{(1)}=t r^{(2)} \rho \\
\sigma^{(1)}=t r^{(2)} \sigma
\end{gathered}
$$

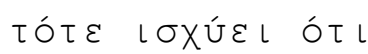




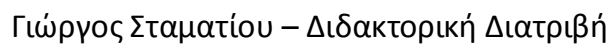

$$
S\left(\rho^{(1)} \| \sigma^{(1)}\right) \leq S(\rho \| \sigma)
$$

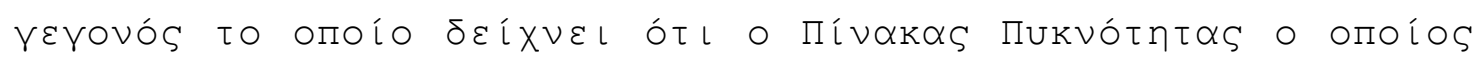

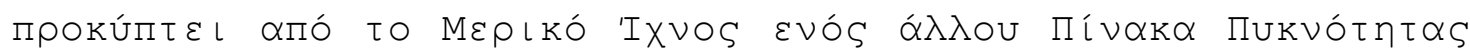

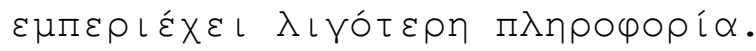

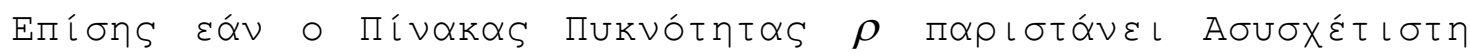

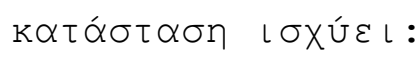

$$
S\left(\rho^{(1)} \| \sigma^{(1)}\right)=S\left[\left(\rho^{(1)} \otimes \rho^{(2)}\right) \|\left(\sigma^{(1)} \otimes \rho^{(2)}\right)\right]
$$

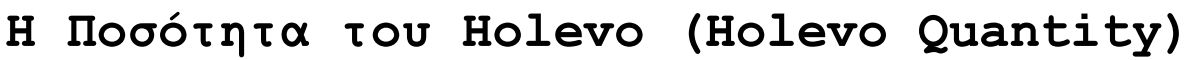

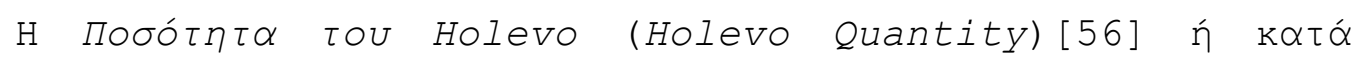

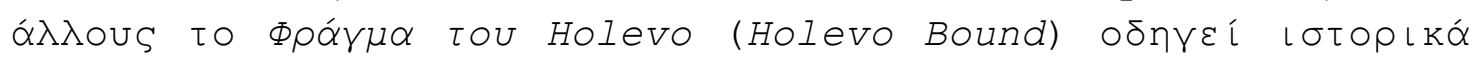

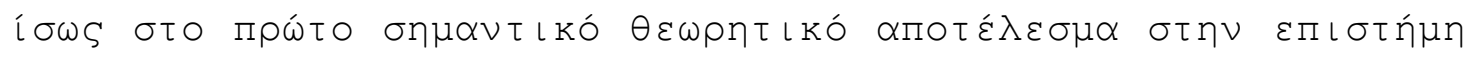

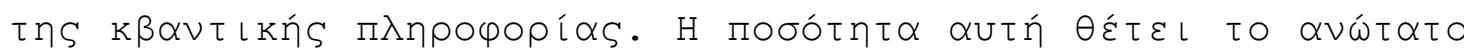

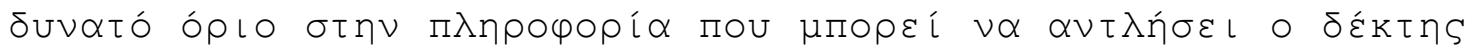

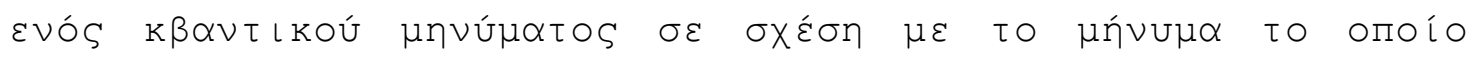

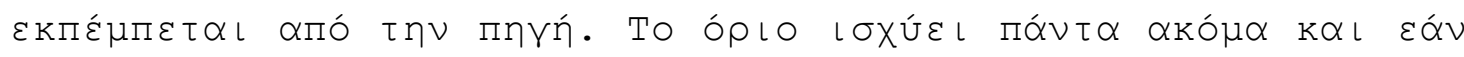

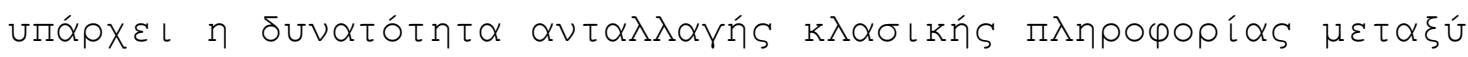

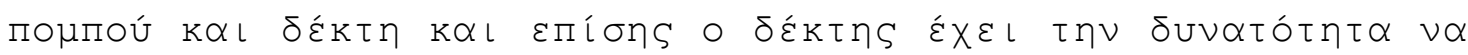

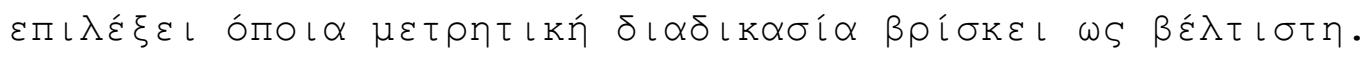

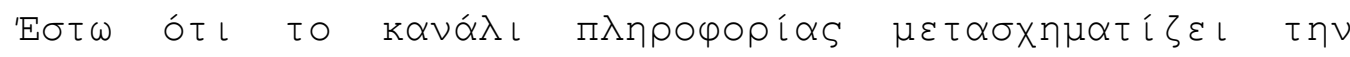
$\varepsilon\llcorner\sigma \varepsilon \rho x o ́ \mu \varepsilon \vee \eta ~ \Pi \lambda \eta \rho о \varphi о \rho i ́ \alpha \omega S$

$$
\mathcal{E}: B(\mathcal{M}) \rightarrow B(\mathcal{K})
$$

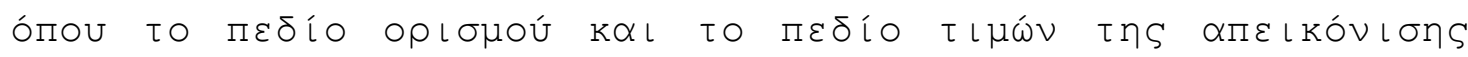

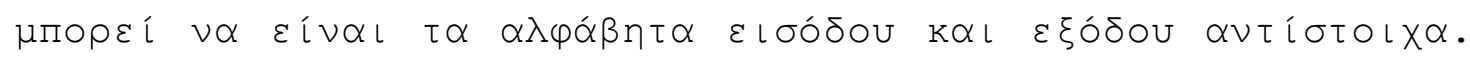

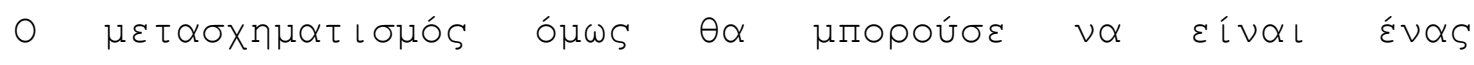

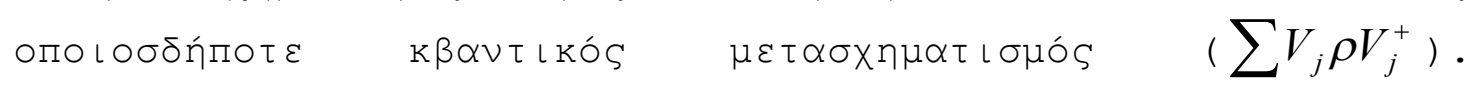

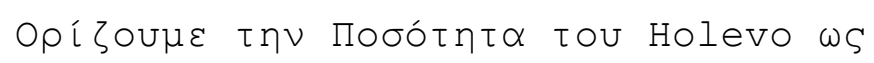

$$
I\left[\left(p_{x}, \rho_{x}\right), \mathcal{E}\right] \equiv S[\mathcal{E}(\rho)]-\sum_{x} p_{x} S\left[\mathcal{E}\left(\rho_{x}\right)\right]
$$


$\mu \varepsilon \quad \rho=\sum_{x} p_{x} \rho_{x}$

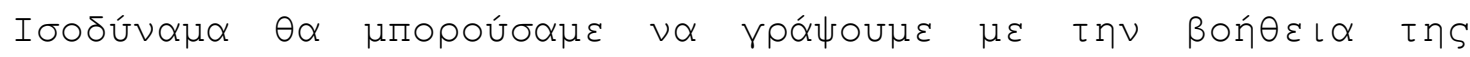

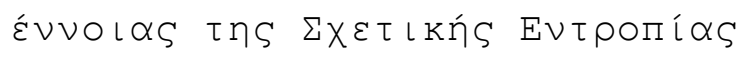

$$
I\left[\left(p_{x}, \rho_{x}\right), \mathcal{E}\right]=\sum_{x} p_{x} S\left[\mathcal{E}\left(\rho_{x}\right) \| \mathcal{E}(\rho)\right]
$$

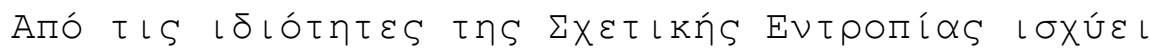

$$
I\left[\left(p_{x}, \rho_{x}\right), \mathcal{E}\right] \geq I\left[\left(p_{x}, \rho_{x}\right), \mathcal{E}^{\prime} \circ \mathcal{E}\right]
$$

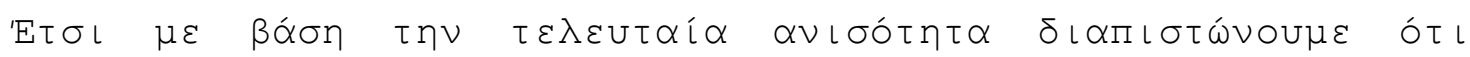

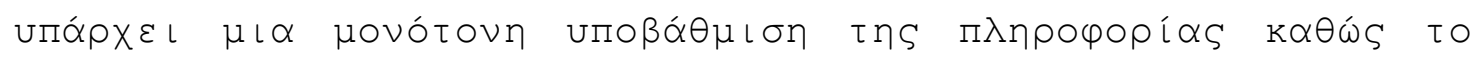

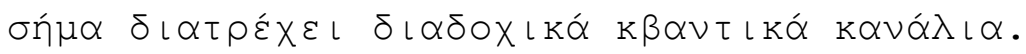

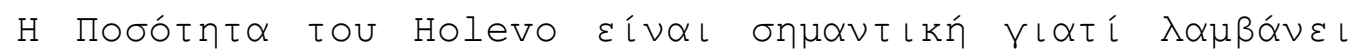

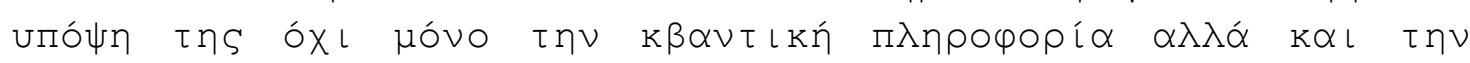

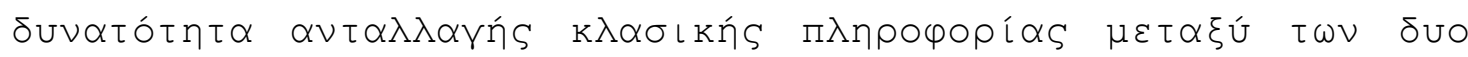

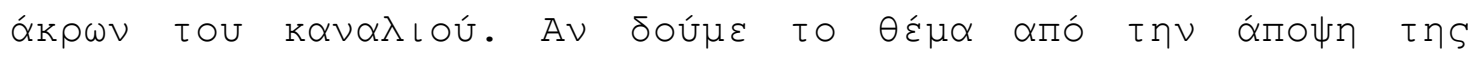

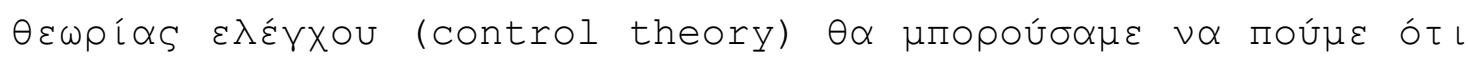

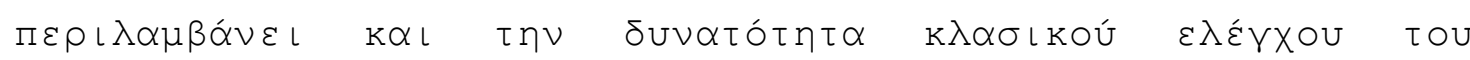

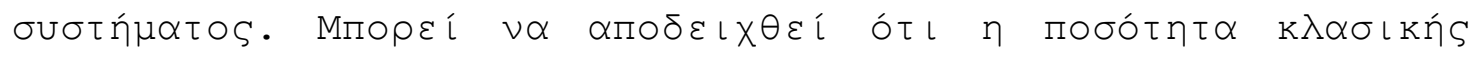

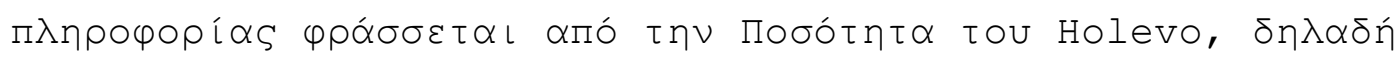

$$
I(X \wedge Y) \leq I\left[\left(p_{x}, \rho_{x}\right), \mathcal{E}\right]
$$

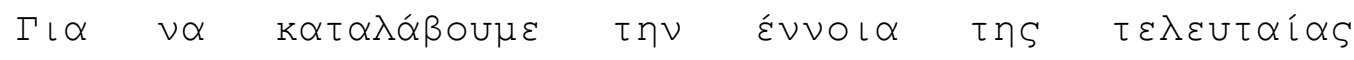

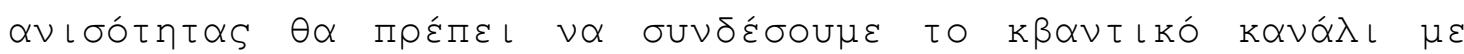

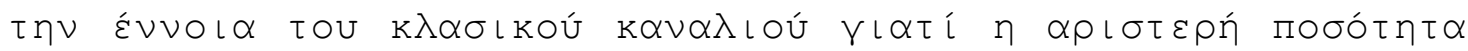

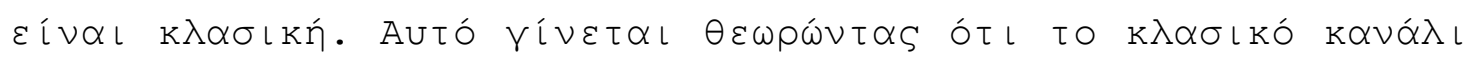

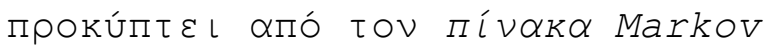

$$
T_{y x}=\operatorname{Tr}\left(\rho_{x} V_{y}\right)
$$

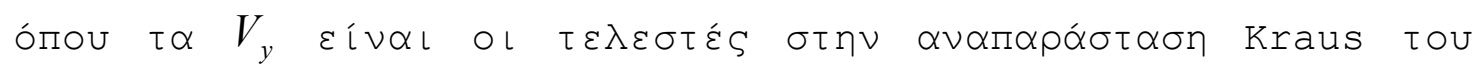

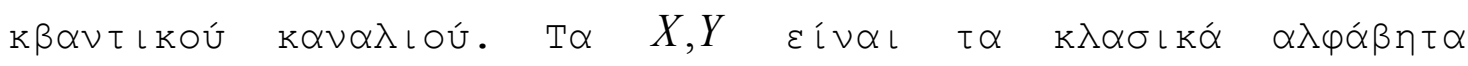

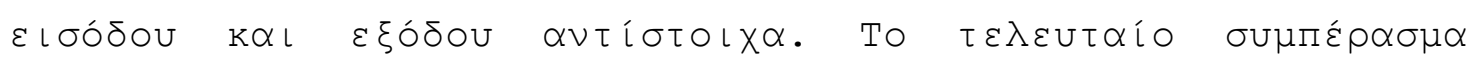

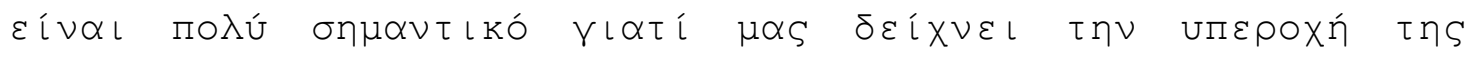




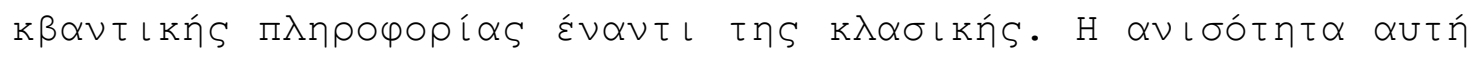

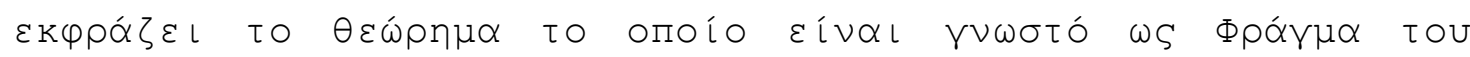
Holevo (Holevo bound) [56,57].

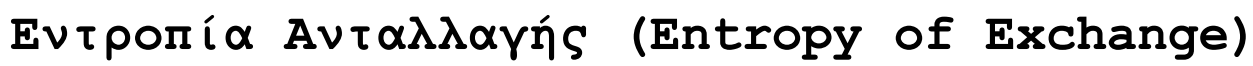

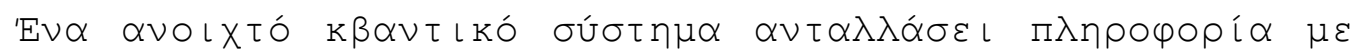

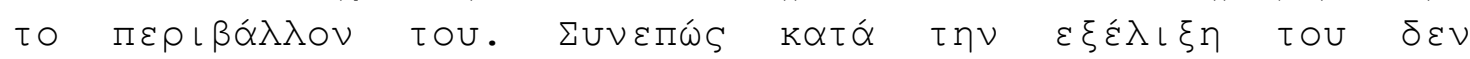

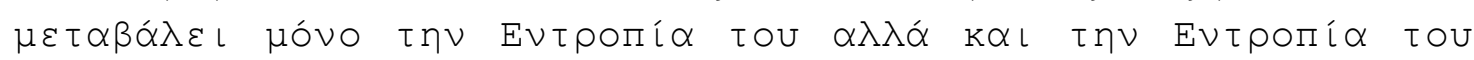

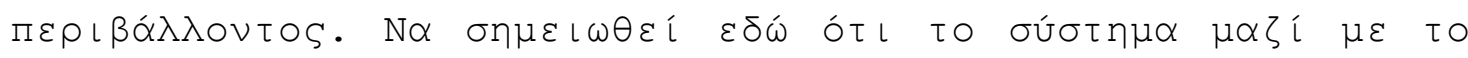

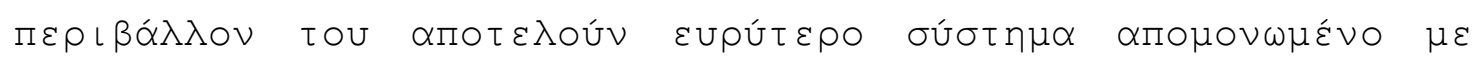

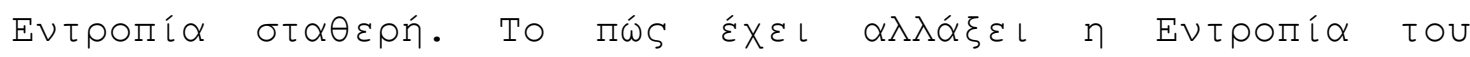

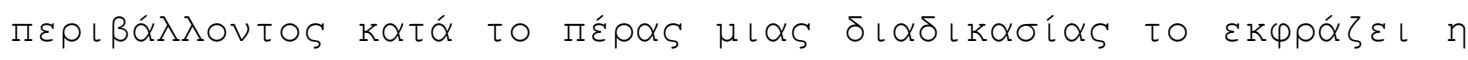

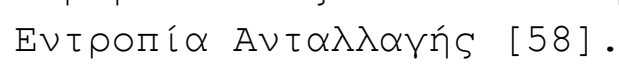

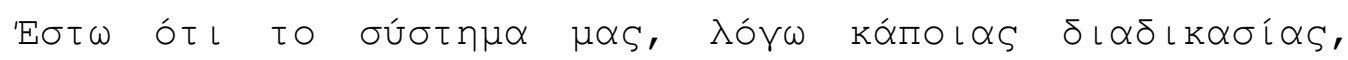
$\mu \varepsilon \tau \alpha \sigma \times \eta \mu \alpha \tau i \zeta \varepsilon \tau \alpha \iota \quad \omega S$

$$
\begin{gathered}
\mathcal{E}: B(\mathcal{M}) \rightarrow B(\mathcal{K}) \\
\mathcal{E}(D)=\sum_{i} V_{i} D V_{i}^{*} \quad V_{i}: \mathcal{M} \rightarrow \mathcal{K}
\end{gathered}
$$

Av

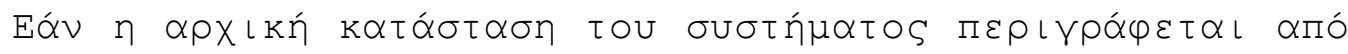

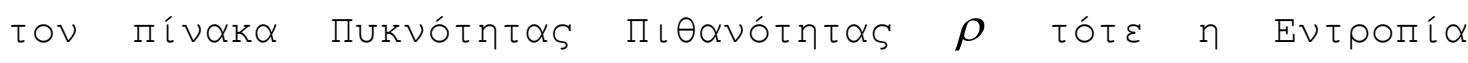

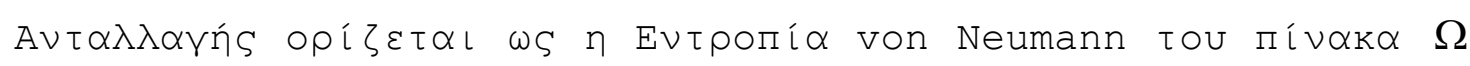

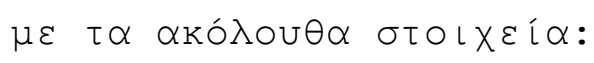

$$
\omega_{i j}=\operatorname{Tr}\left(V_{i} \rho V_{j}\right) \quad i, j=1 . . N
$$

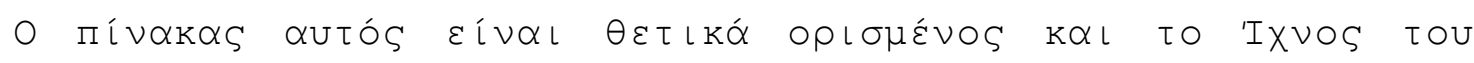

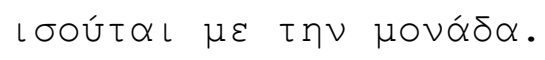

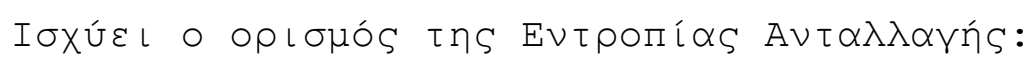

$$
S(\rho, \mathcal{E}) \equiv S(\Omega)
$$




$$
\omega_{i j}=\operatorname{Tr}\left(V_{i} \rho V_{j}\right) \quad i, j=1 . . N
$$

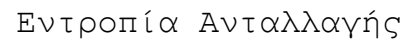

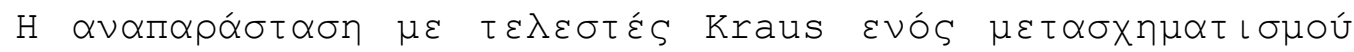

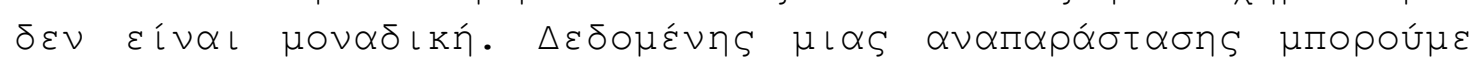

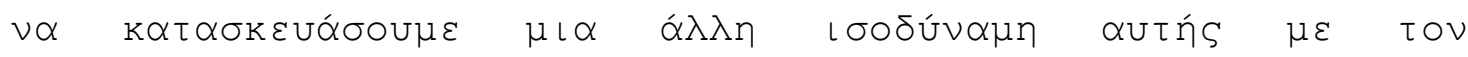
$\mu \varepsilon \tau \alpha \sigma \chi \eta \mu \alpha \tau \iota \sigma \mu o ́$

$$
V_{i}=\sum_{j} c_{i j} W_{j}
$$

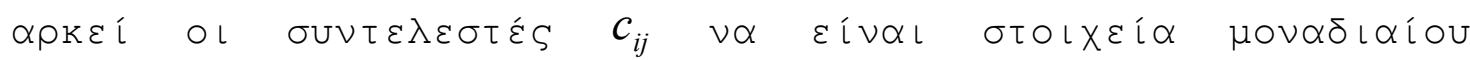

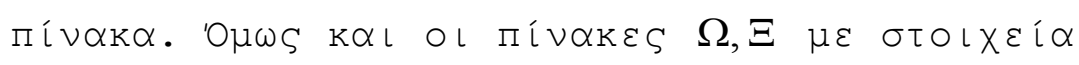

$$
\omega_{i j}=\operatorname{Tr}\left(V_{i} \rho V_{j}\right) \quad \xi_{i j}=\operatorname{Tr}\left(W_{i} \rho W_{j}\right) \quad i, j=1 . . N
$$

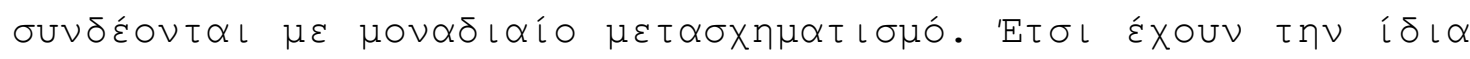

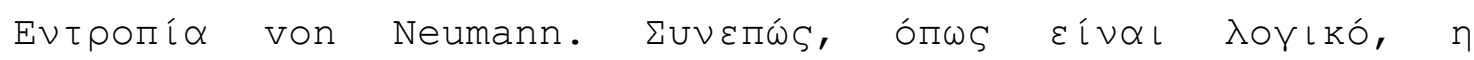

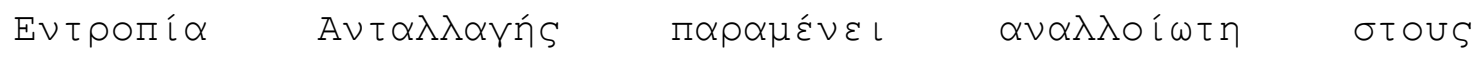

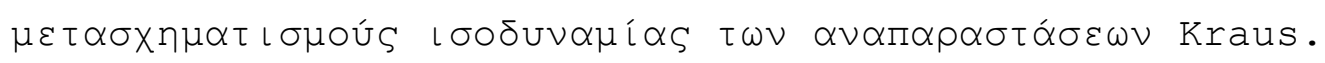

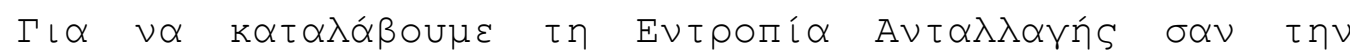

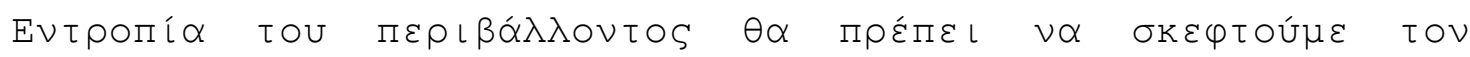

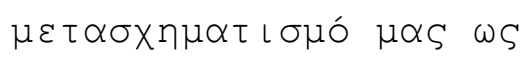

$$
\mathcal{E}(\rho)=\operatorname{Tr}_{e}\left[U\left(\rho_{e} \otimes \rho\right) U^{*}\right]
$$

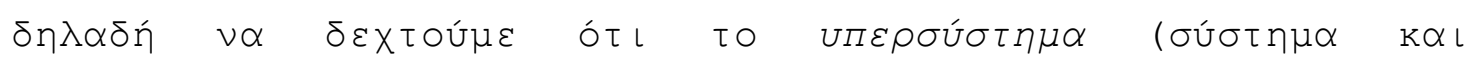

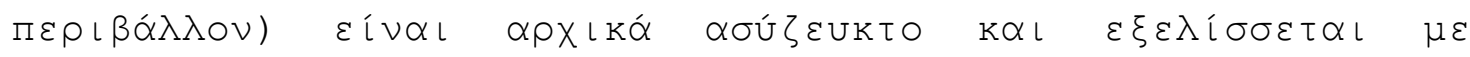

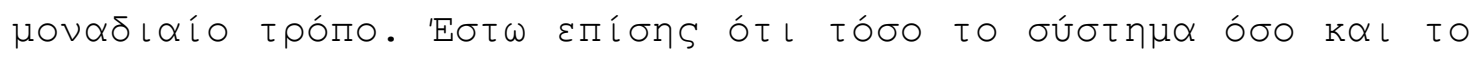

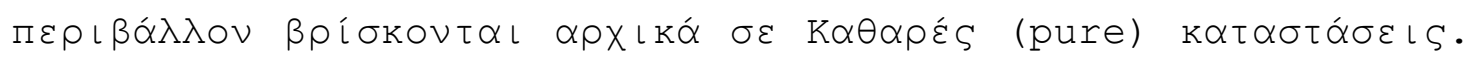

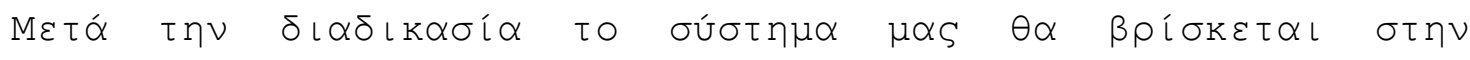

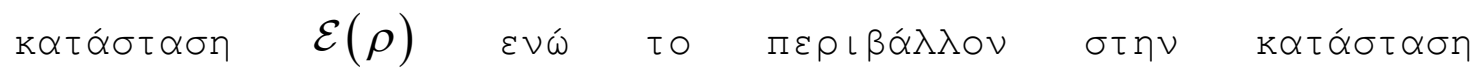
$\Omega, \quad \omega_{i j}=\operatorname{Tr}\left(V_{i} \rho V_{j}\right)$. 


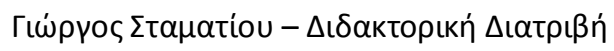

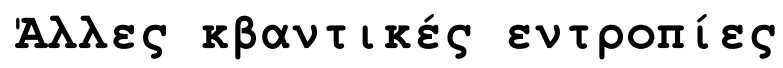

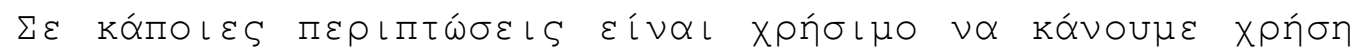

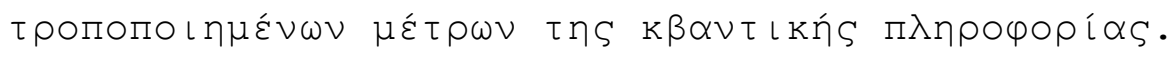

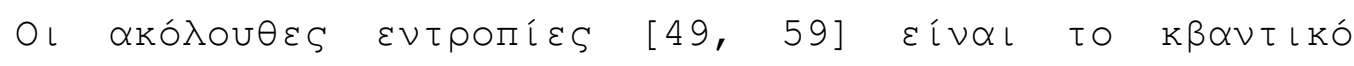

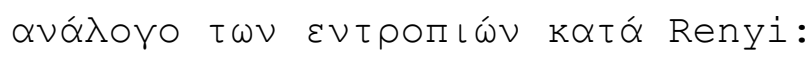

$$
\begin{gathered}
S^{a}(\rho) \equiv \frac{1}{2^{(1-a)}-1}\left[\operatorname{tr}\left(\rho^{a}\right)-1\right] \\
S_{a}(\rho) \equiv \frac{1}{1-a} \log \left[\operatorname{tr}\left(\rho^{a}\right)\right] \\
S_{a}\left(\rho_{1} \| \rho_{2}\right) \equiv \frac{1}{a(1-a)} \operatorname{tr}\left[\left(I-\rho_{2}^{a} \rho_{1}^{-a}\right) \rho_{1}\right]
\end{gathered}
$$

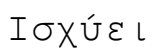

$$
S_{a}\left(\rho_{1} \| \rho_{2}\right) \geq S_{a}\left[\mathcal{E}\left(\rho_{1}\right) \| \mathcal{E}\left(\rho_{2}\right)\right]
$$

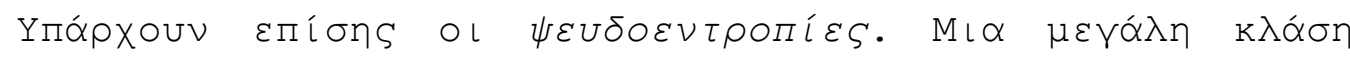

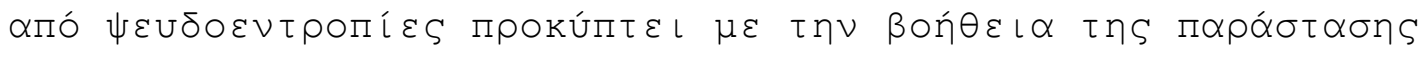

$$
S_{f}^{K}\left(\rho_{1} \| \rho_{2}\right)=\left\langle K \rho_{1}^{1 / 2}, f\left[\Delta\left(\frac{\rho_{2}}{\rho_{1}}\right) K \rho_{1}^{1 / 2}\right]\right\rangle
$$

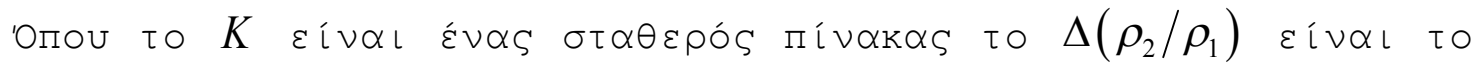

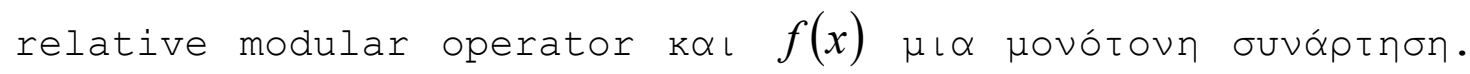

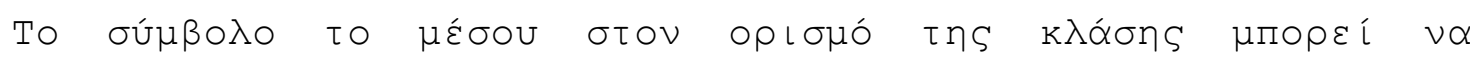

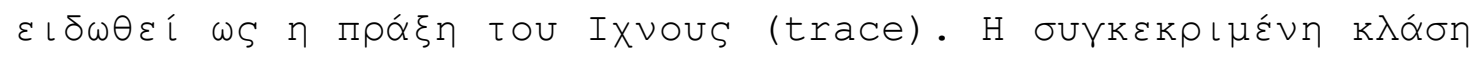

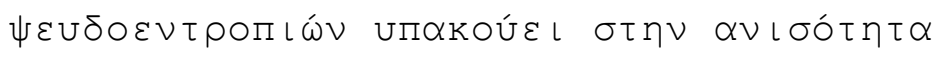

$$
S_{f}^{\mathcal{E}^{*}(K)}\left(\rho_{1} \| \rho_{2}\right) \leq S_{f}^{K}\left(\mathcal{E}\left(\rho_{1}\right) \| \mathcal{E}\left(\rho_{2}\right)\right)
$$




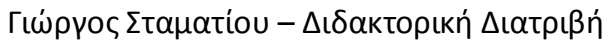

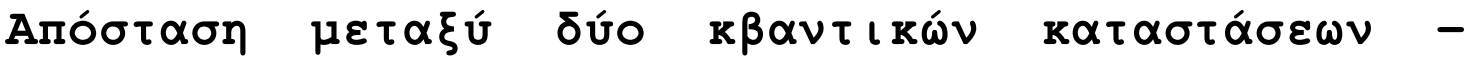

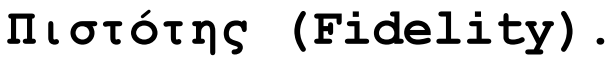

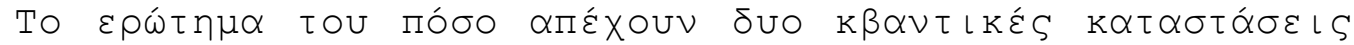

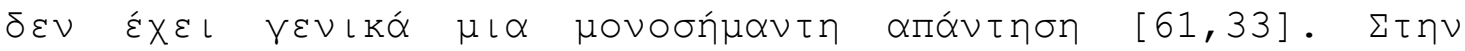

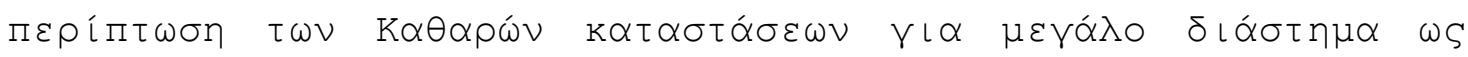

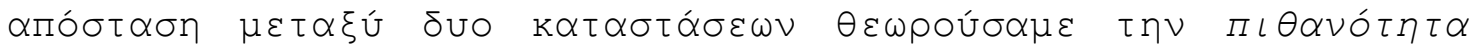

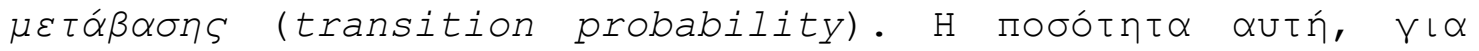

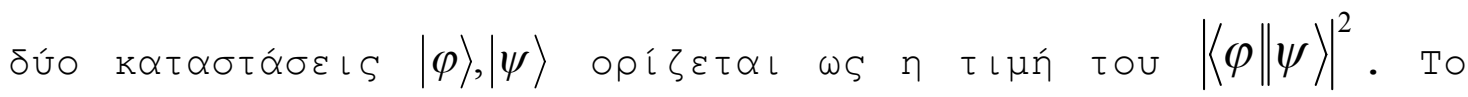

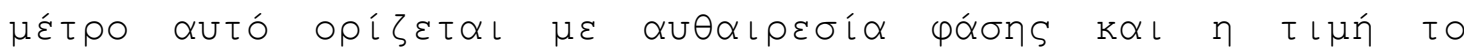

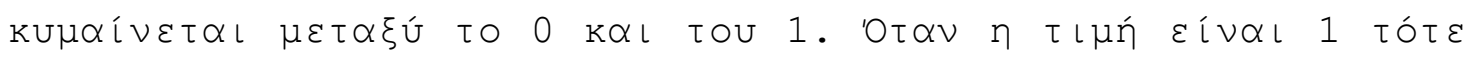

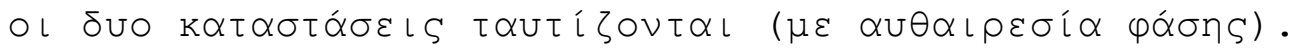

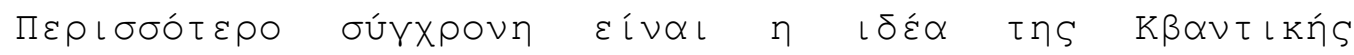

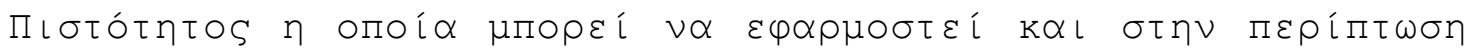

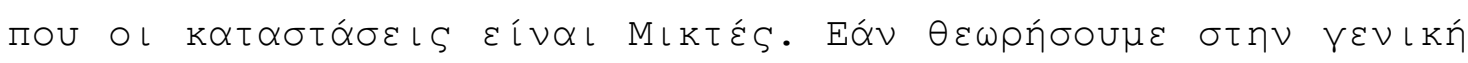

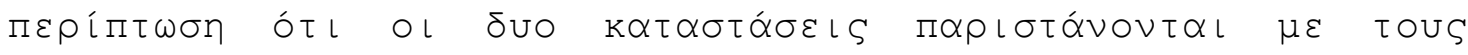

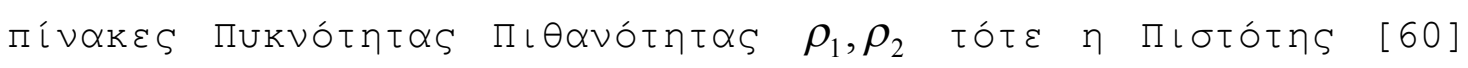

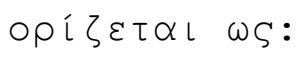

$$
F\left(\rho_{1}, \rho_{2}\right)=\operatorname{tr} \sqrt{\sqrt{\rho_{1}} \rho_{2} \sqrt{\rho_{1}}}
$$

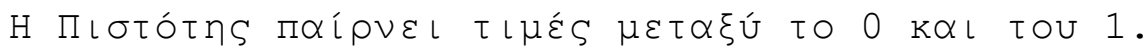

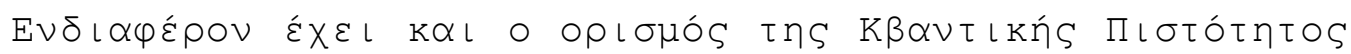

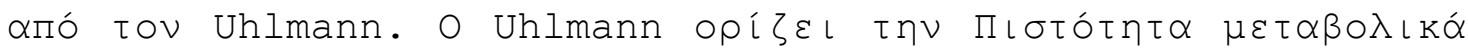
$\omega S$

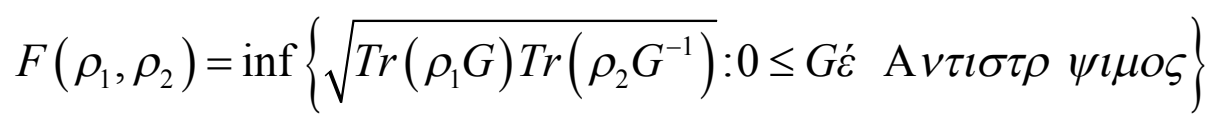

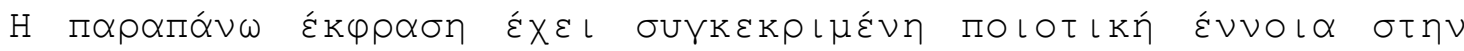

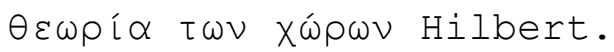

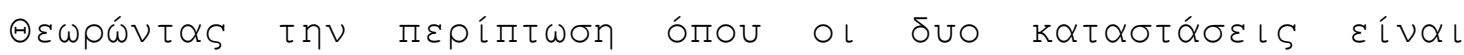

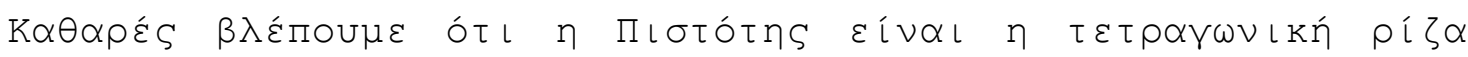

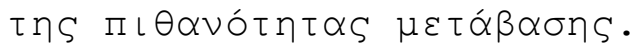

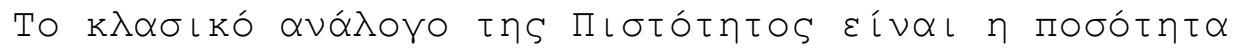




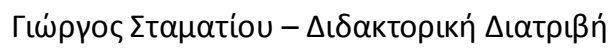

$$
F(p, q)=\sum_{i} \sqrt{p_{i} q_{i}}
$$

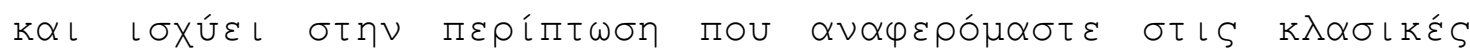

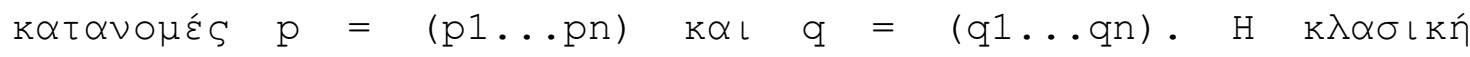

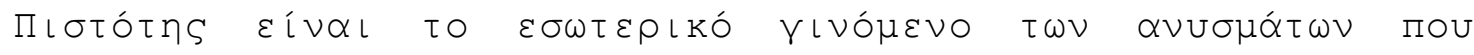

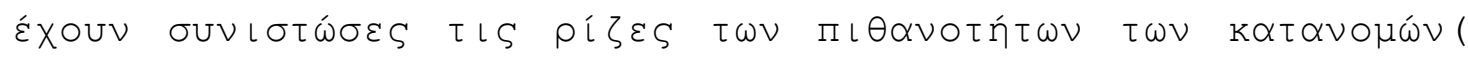
$\left(\sqrt{p_{1}}, \ldots, \sqrt{p_{n}}\right)$ к $\left.८ \quad\left(\sqrt{q_{1}}, \ldots, \sqrt{q_{n}}\right)\right)$.

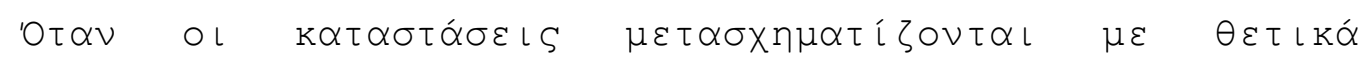

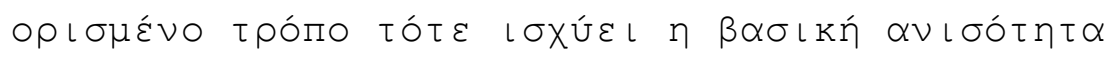

$$
F\left[\mathcal{E}\left(\rho_{1}\right), \mathcal{E}\left(\rho_{2}\right)\right] \geq F\left(\rho_{1}, \rho_{2}\right)
$$

Eníons ıoxúouv $\tau \alpha$ ópı $\alpha$

$$
1-F\left(\rho_{1}, \rho_{2}\right) \leq \frac{1}{2} \operatorname{Tr}\left|\rho_{1}-\rho_{2}\right| \leq \sqrt{1-F\left(\rho_{1}, \rho_{2}\right)}
$$

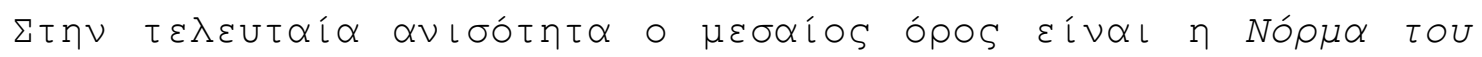

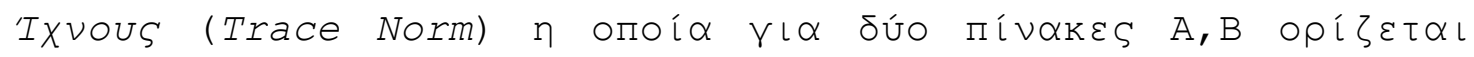
$\varepsilon \delta \omega ́ \omega$

$$
D(A, B)=\frac{1}{2}\|A-B\|_{t r}
$$

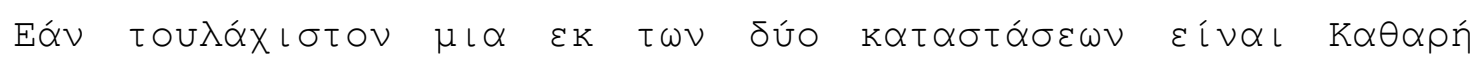
ı $\sigma \times \dot{\varepsilon} \varepsilon\llcorner$

$$
1-F(\psi, \rho)^{2} \leq D(\psi, \rho)
$$

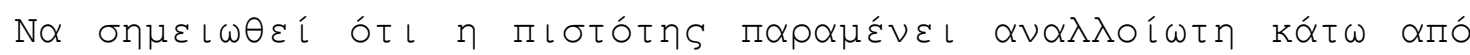

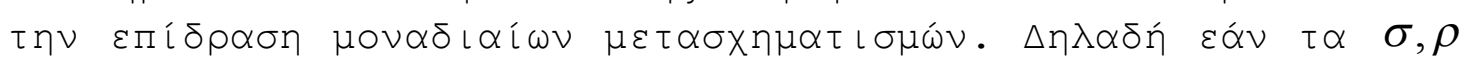

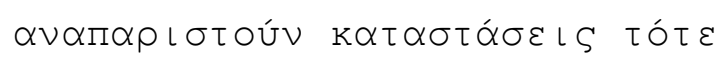

$$
F(\rho, \sigma)=F\left(U \rho U^{*}, U \sigma U^{*}\right)
$$

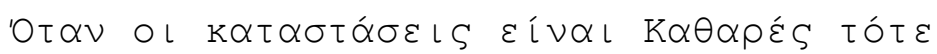




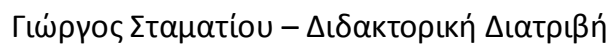

$$
F(\rho, \sigma)=\operatorname{tr}(\rho \sigma \rho)^{1 / 2}=\operatorname{tr}(|\phi\rangle\langle\phi \mid \psi\rangle\langle\psi \mid \phi\rangle\langle\phi|)^{1 / 2}=|\langle\phi \mid \psi\rangle|
$$

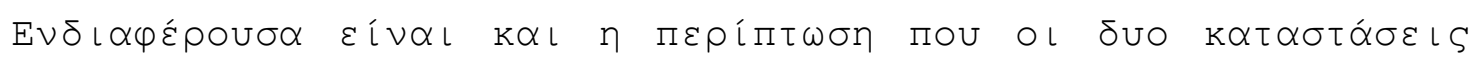

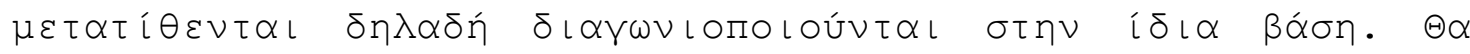

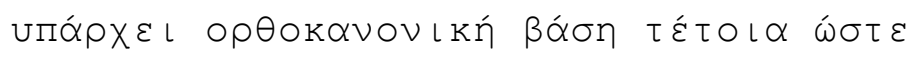

$$
\rho=\sum_{i} p_{i}|i\rangle\langle i| \quad \text { к } \propto \iota \quad \sigma=\sum_{i} q_{i}|i\rangle\langle i|
$$

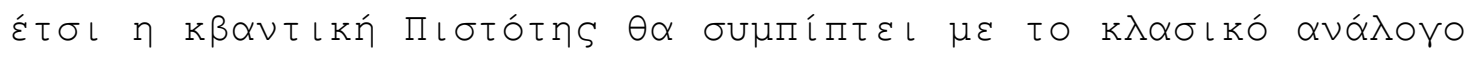

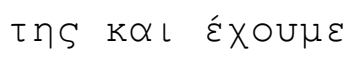

$$
F(\rho, \sigma)=\sum_{i} \sqrt{p_{i} q_{i}}
$$

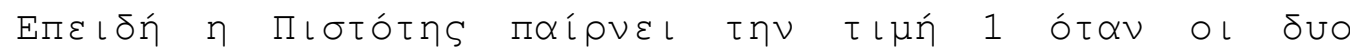

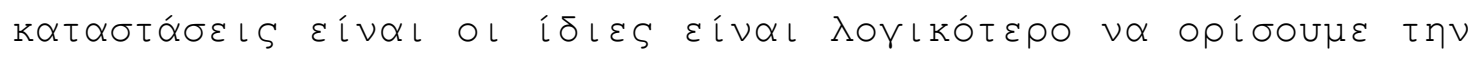

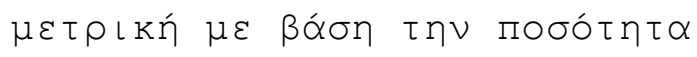

$$
\tilde{E}(\rho, \sigma) \equiv 1-F(\rho, \sigma)
$$

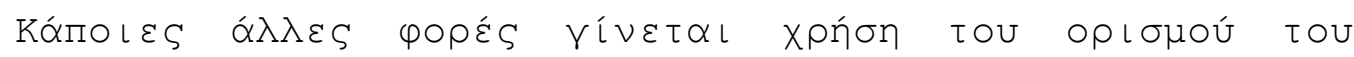

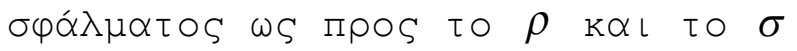

$$
E(\rho, \sigma) \equiv 1-F(\rho, \sigma)^{2}
$$

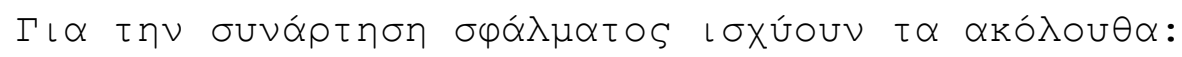

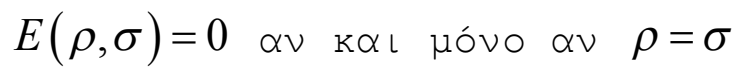

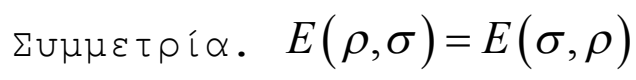

$$
\begin{aligned}
& E[\mathcal{E}(\rho), \mathcal{E}(\sigma)] \leq E(\rho, \sigma)
\end{aligned}
$$

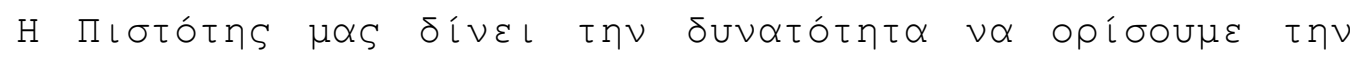

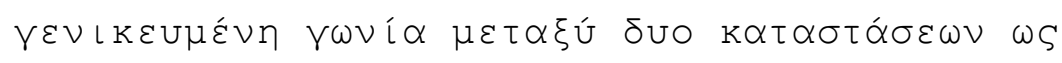




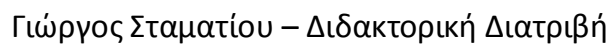

$$
A(\rho, \sigma) \equiv \operatorname{Arccos} F(\rho, \sigma)
$$

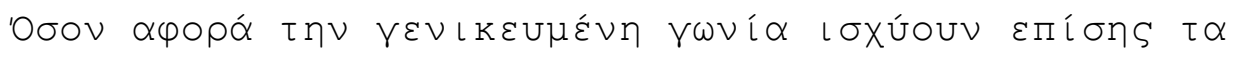

$$
\begin{aligned}
& A(\rho, \sigma)=0 \Leftrightarrow \rho=\sigma
\end{aligned}
$$

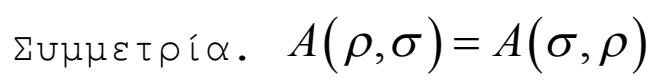

$$
\begin{aligned}
& A[\mathcal{E}(\rho), \mathcal{E}(\sigma)] \leq A(\rho, \sigma)
\end{aligned}
$$

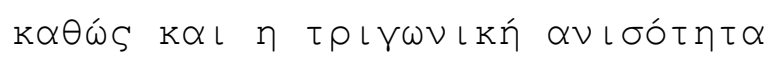

$$
A(\rho, \sigma) \leq A(\rho, g)+A(g, \sigma)
$$

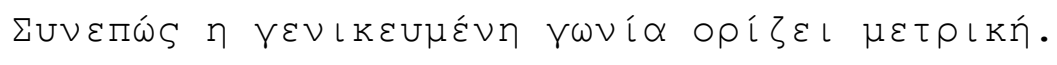

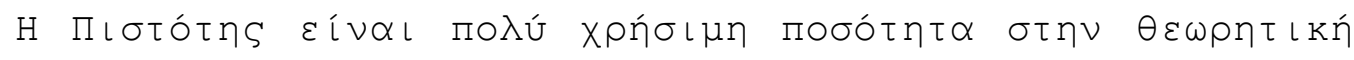

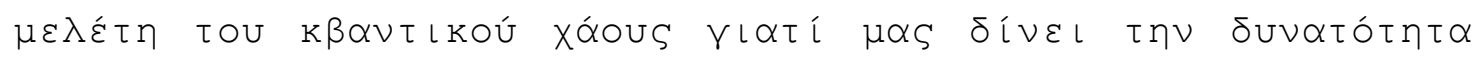

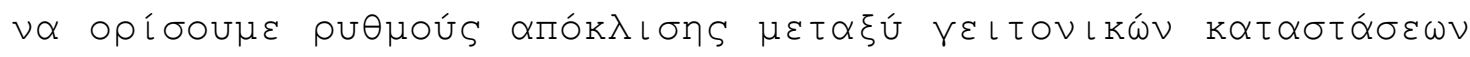

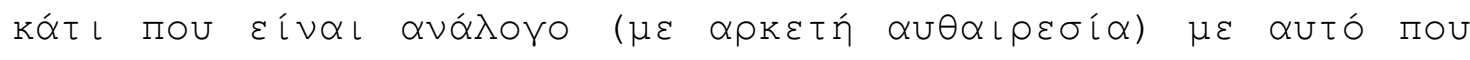

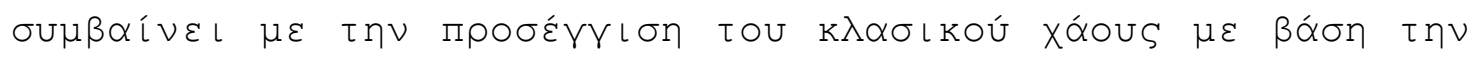

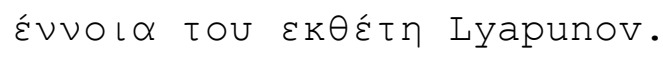

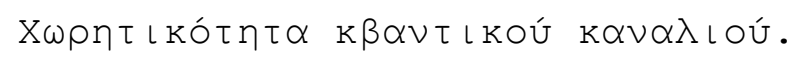

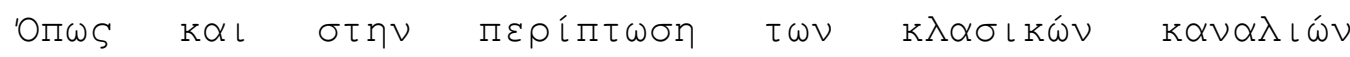

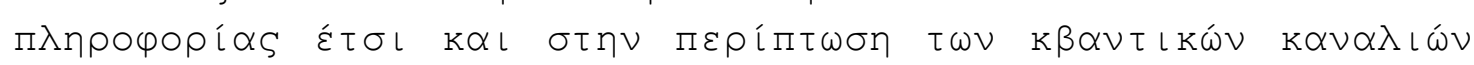

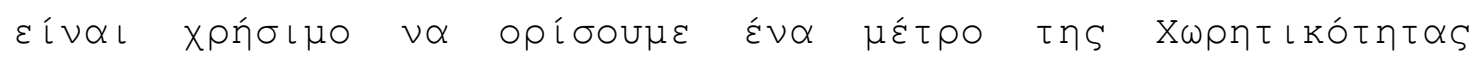

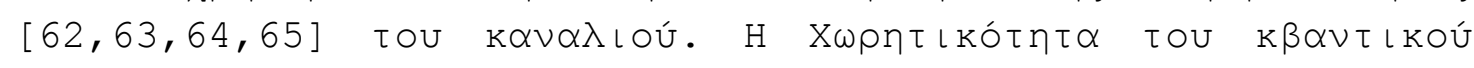

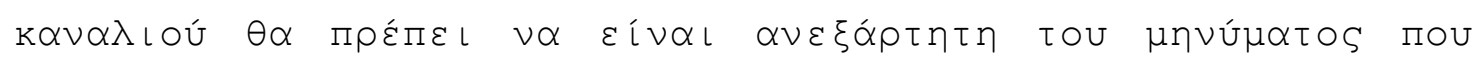

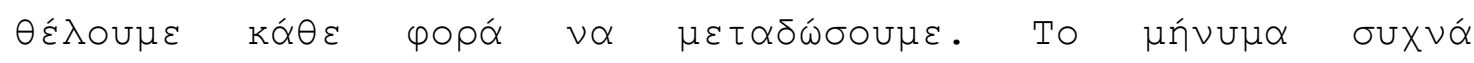

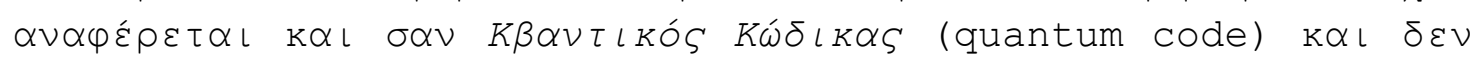

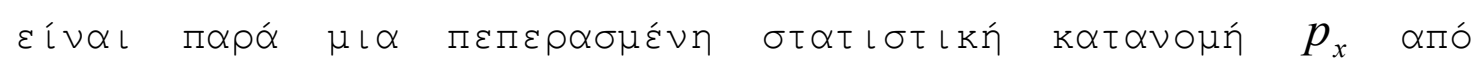

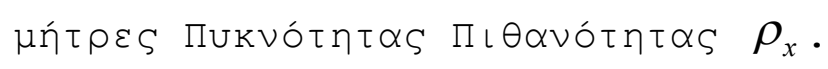

$$
\left(p_{x}, \rho_{x}\right)
$$

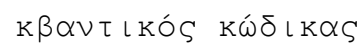




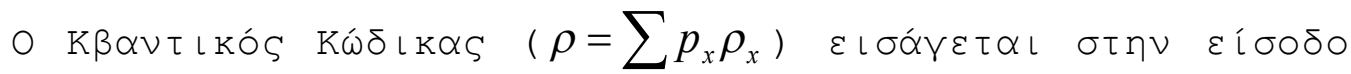

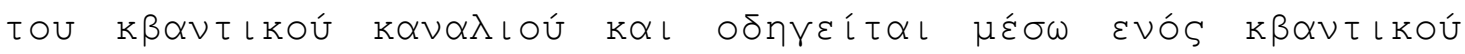

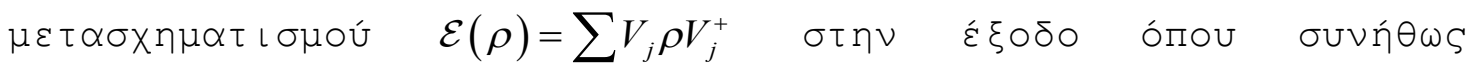

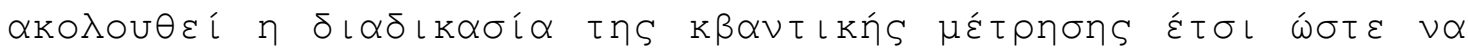

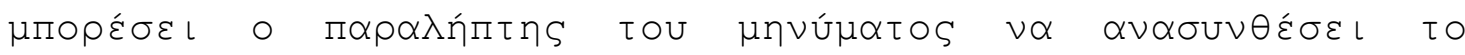

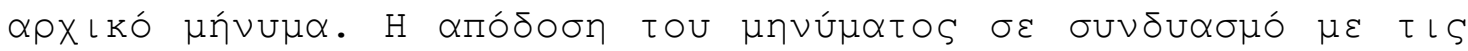

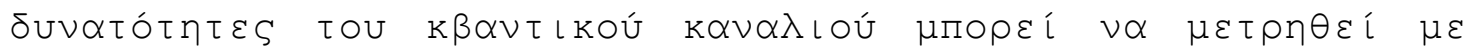

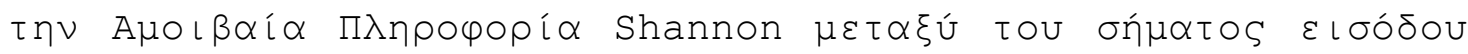

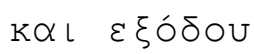

$$
\chi\left(p_{x}, \rho_{x} ; \mathcal{E}\right)=\sum_{x} p_{x} S\left(\mathcal{E}\left(\rho_{x}\right) \| \mathcal{E}(\rho)\right)=S(\mathcal{E}(\rho))-\sum_{x} p_{x} S\left(\mathcal{E}\left(\rho_{x}\right)\right)
$$

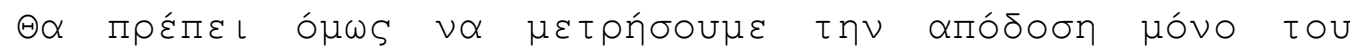

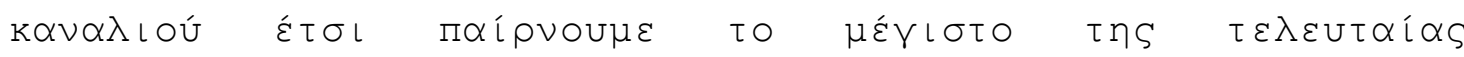

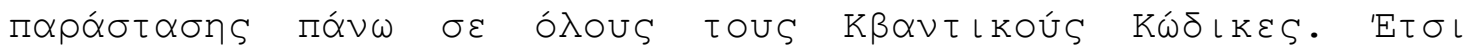

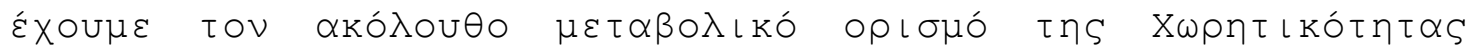

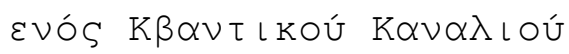

$$
C_{H o}(\mathcal{E}) \equiv \sup \left\{\chi\left(p_{x}, \rho_{x} ; \mathcal{E}\right): \tau \alpha\left(p_{x}^{\prime}, \rho_{x}\right) \varepsilon v \alpha \iota \kappa \beta \alpha v \tau \alpha^{\prime} \kappa \varsigma \kappa \delta \imath \kappa \alpha \varsigma\right\}
$$

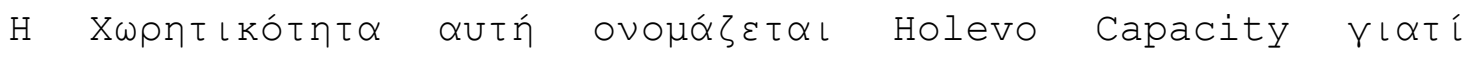

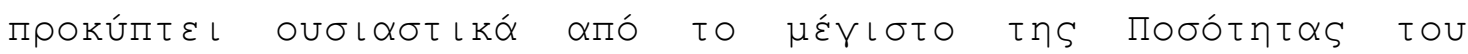

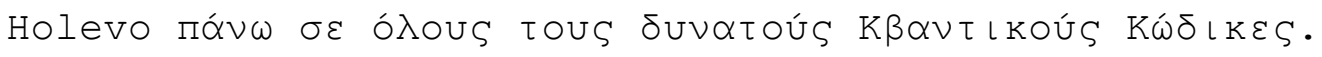

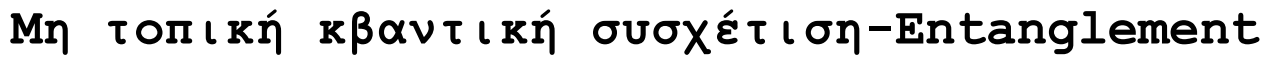

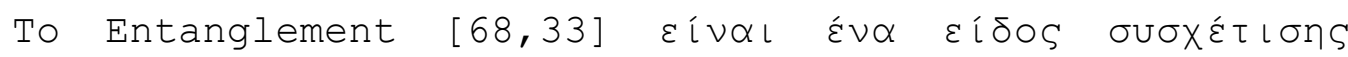

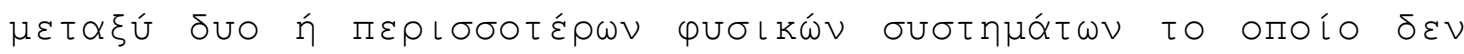

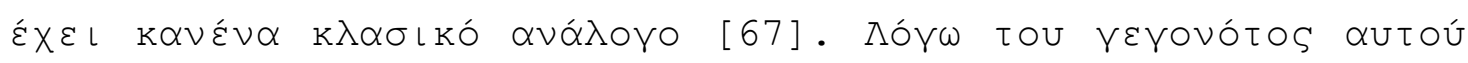

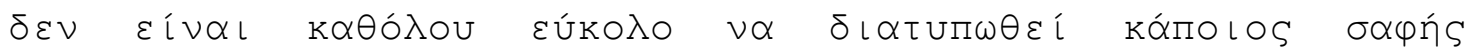

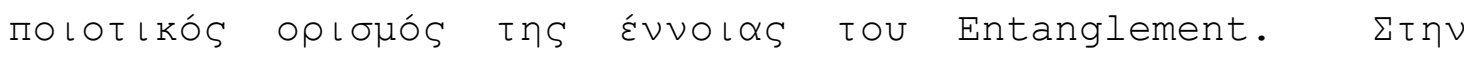

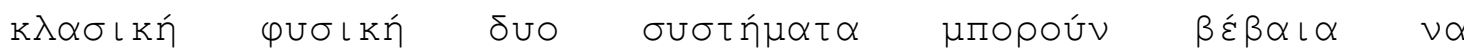

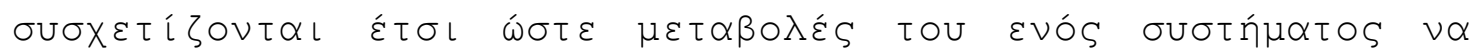

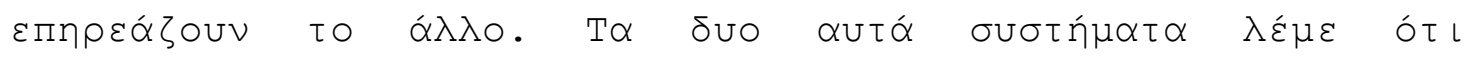

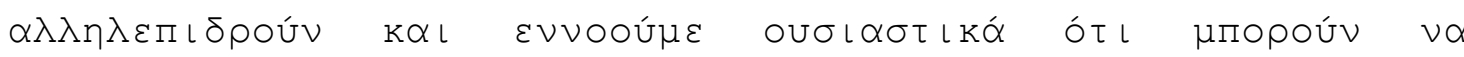

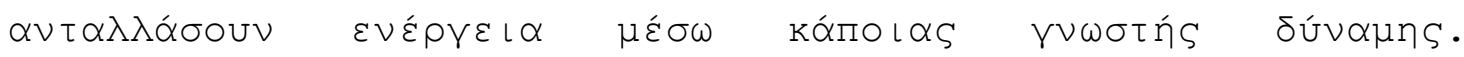

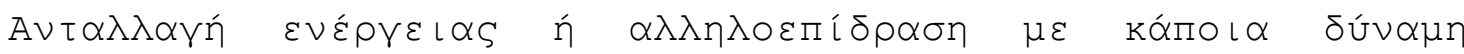




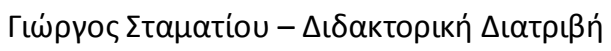

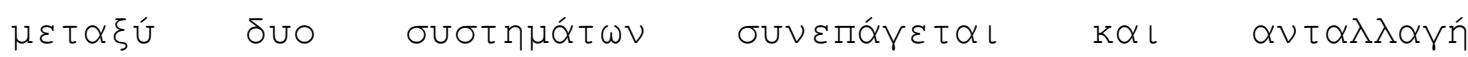

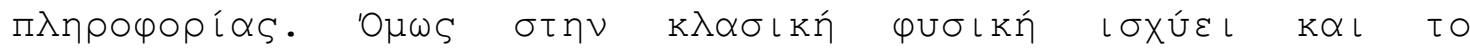

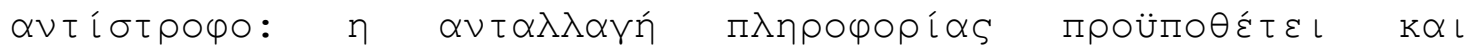

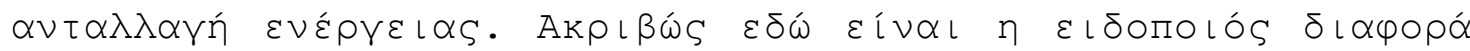

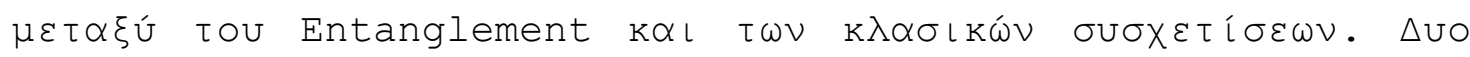

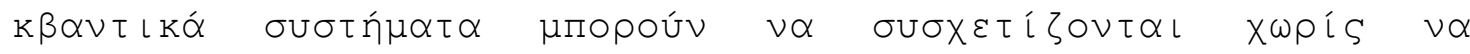

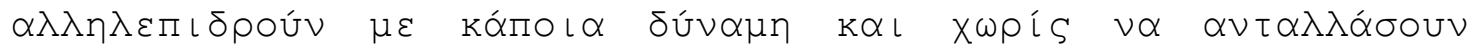

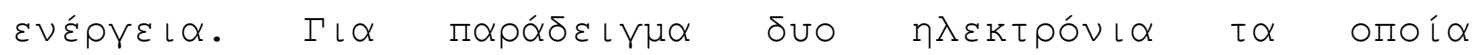

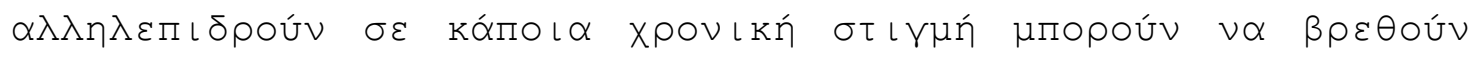

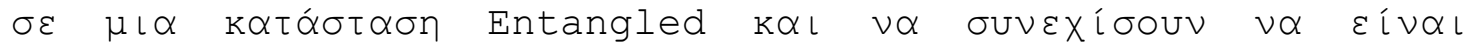

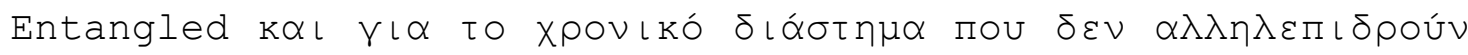

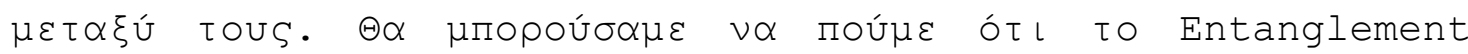

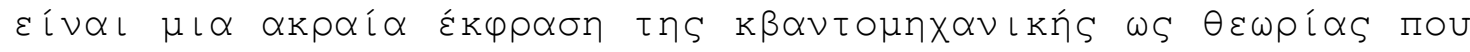

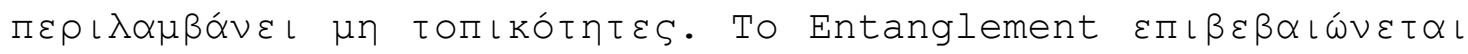

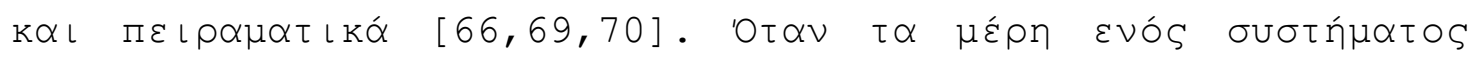

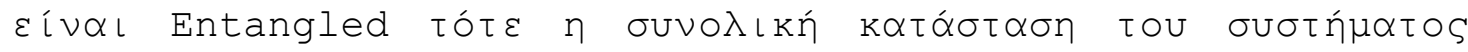

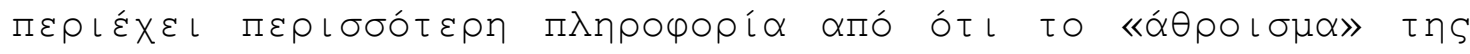

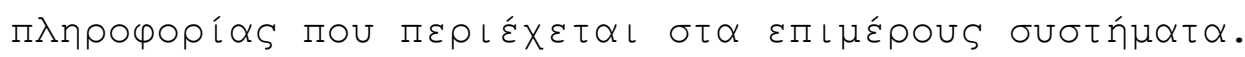

"Best possible knowledge of a whole does not include best possible knowledge of its parts."

(Schrödinger)

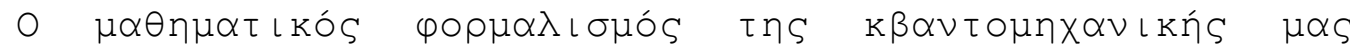

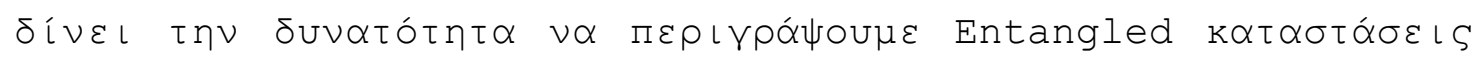

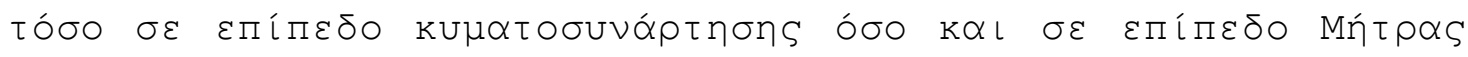

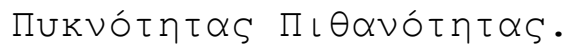




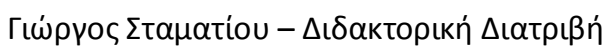

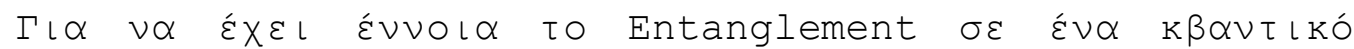

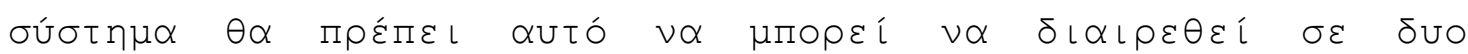

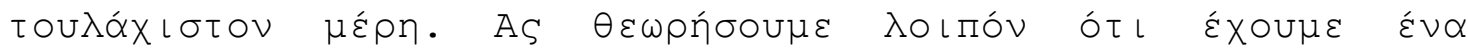

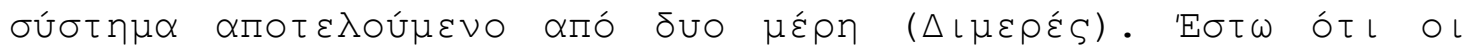

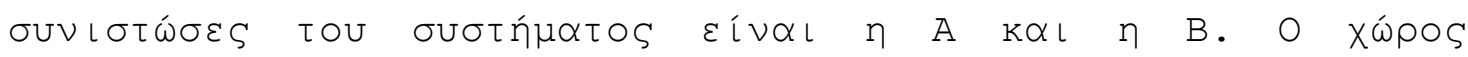

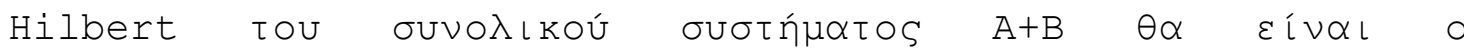

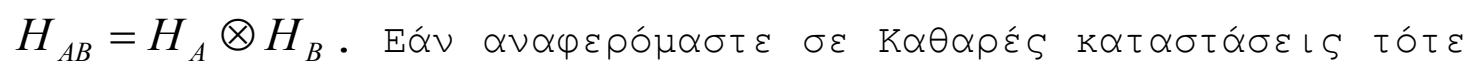

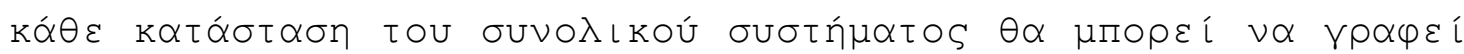
$\omega S$

$$
\Psi=\sum_{i j} c_{i j} a_{i} \otimes b_{j}
$$

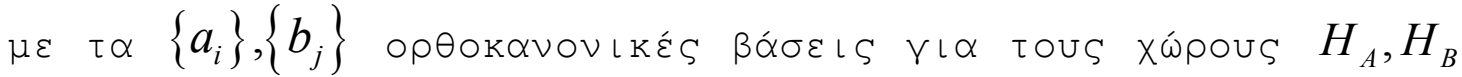
$\alpha \vee \tau i \sigma \tau \circ\llcorner\chi \alpha$.

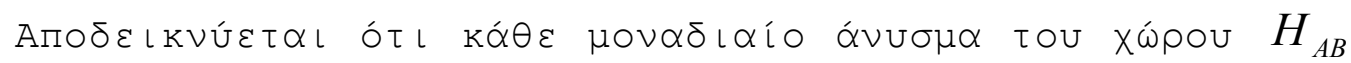

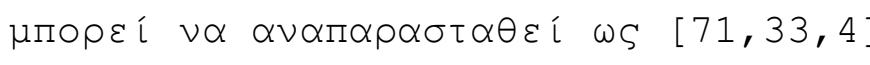

$$
\Psi=\sum_{k} \sqrt{p_{k}} g_{k} \otimes h_{k}
$$

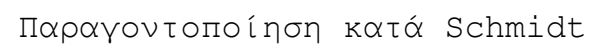

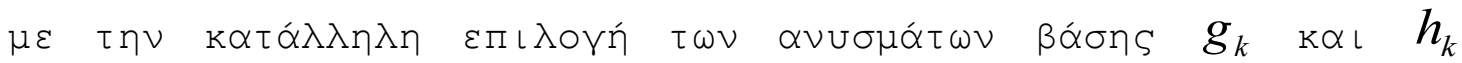

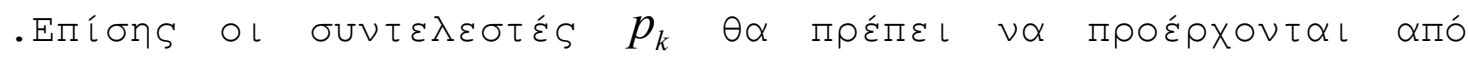

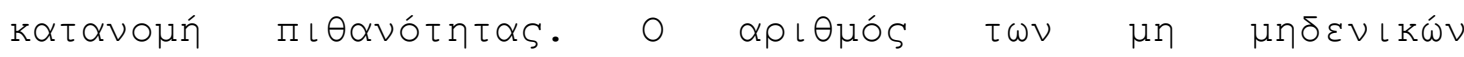

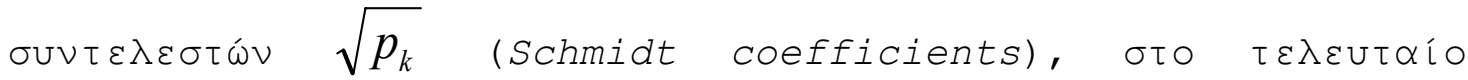

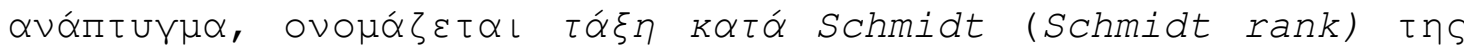

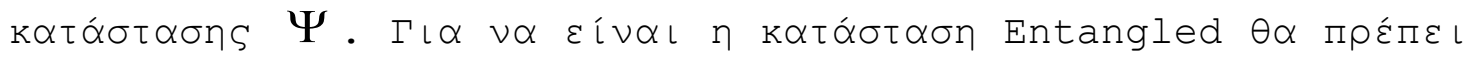

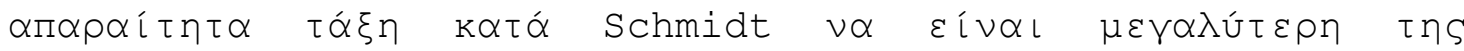

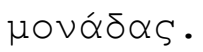

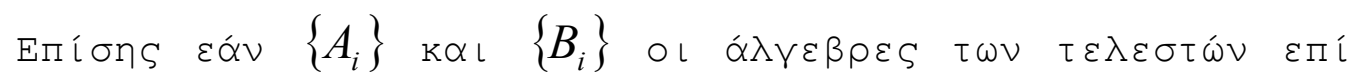

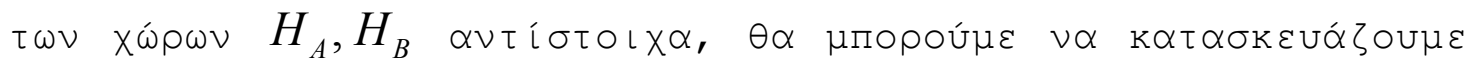

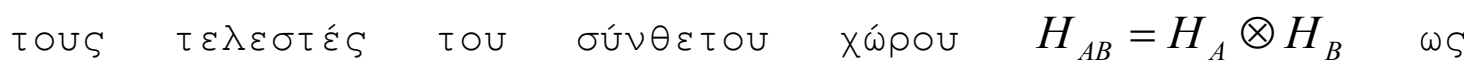

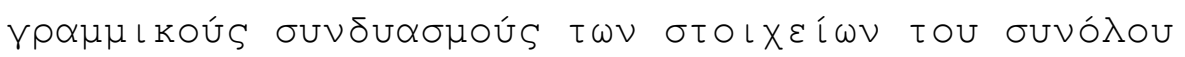




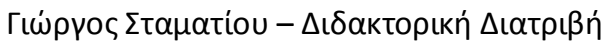

$$
\left\{A_{i} \otimes B_{j}\right\}
$$

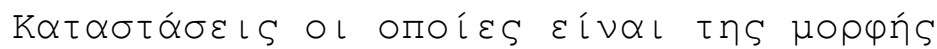

$$
\Psi=\psi_{A} \otimes \psi_{B}
$$

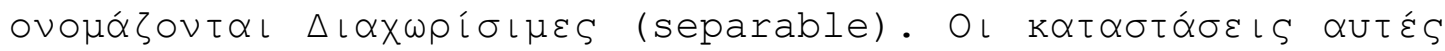

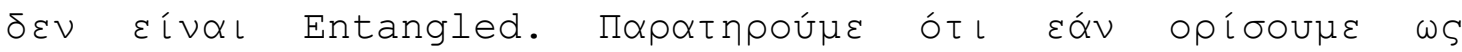

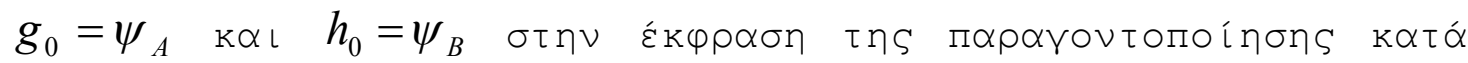

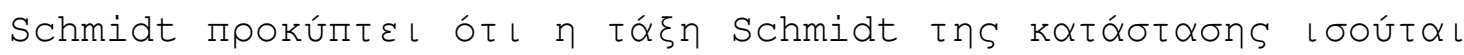

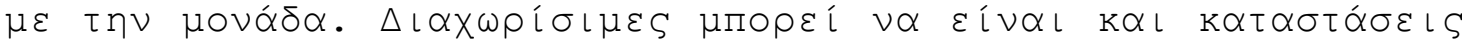

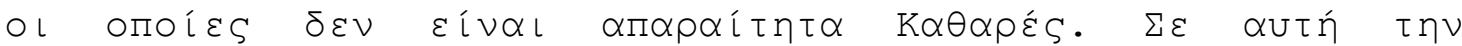

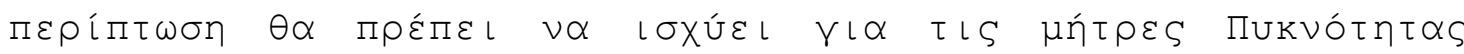

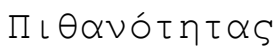

$$
\rho=\rho_{A} \otimes \rho_{B}
$$

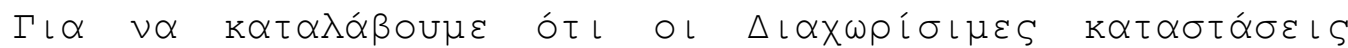

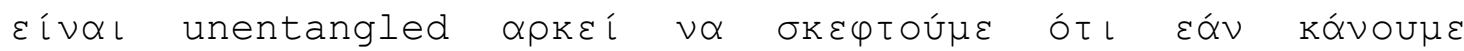

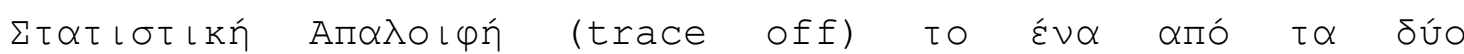

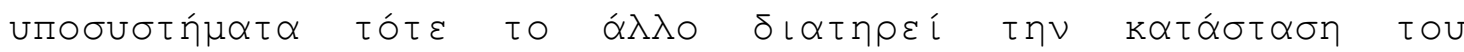
$\alpha \vee \alpha \lambda \lambda \circ i \omega \tau \eta . \Delta \eta \lambda \alpha \delta n$

$$
\begin{aligned}
& \operatorname{Tr}_{A}(\rho)=\operatorname{Tr}_{A}\left(\rho_{A} \otimes \rho_{B}\right)=\rho_{B} \\
& \operatorname{Tr}_{B}(\rho)=\operatorname{Tr}_{B}\left(\rho_{A} \otimes \rho_{B}\right)=\rho_{A}
\end{aligned}
$$

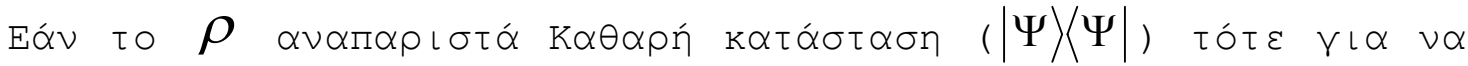

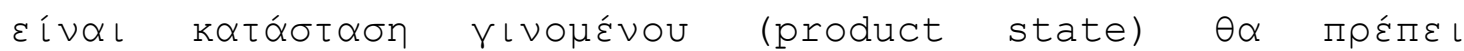

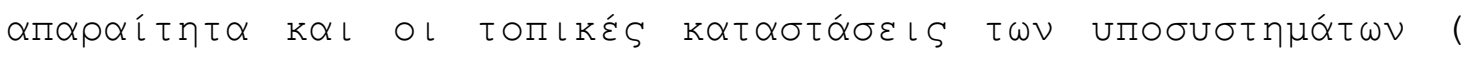

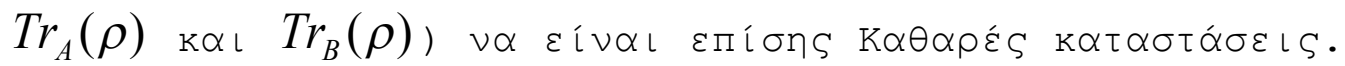

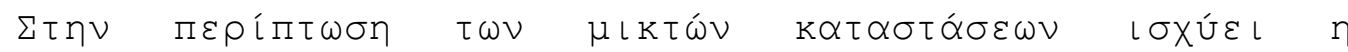

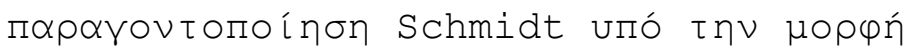

$$
\rho_{A B}=\sum_{i}^{L} \lambda_{i} G_{i}^{A} \otimes G_{i}^{B}
$$




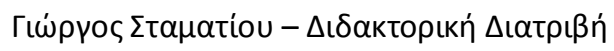

$$
L=\max \left(\operatorname{dim}\left(\mathcal{H}_{A}\right)^{2}, \operatorname{dim}\left(\mathcal{H}_{B}\right)^{2}\right)
$$

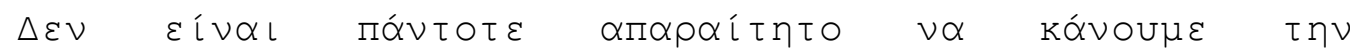

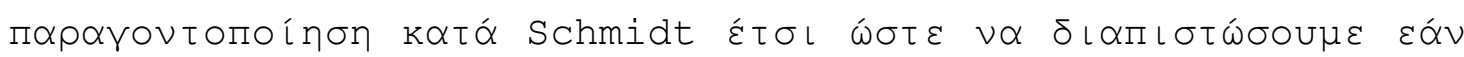

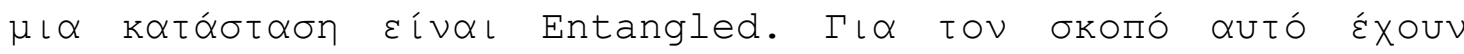

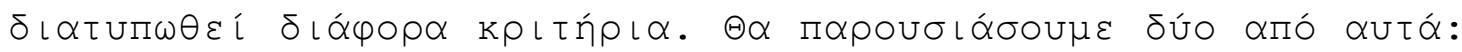

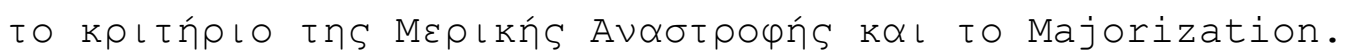

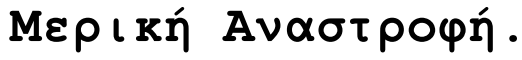

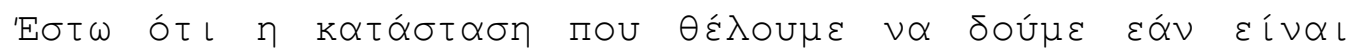
Entangled $\varepsilon i v \alpha \iota n$

$$
\begin{gathered}
\rho_{A B}=\sum_{i, j=1}^{N_{A}} \sum_{\mu, v=1}^{N_{B}} a_{i j}^{\mu \nu}(|i\rangle\langle j|)_{A} \otimes(|\mu\rangle\langle v|)_{B} \\
\{|i\rangle\} \quad i=1,2, \ldots, N_{A} ; N_{A} \leq \operatorname{dim}\left(\mathcal{H}_{A}\right) \\
\{|\mu\rangle\} \quad i=1,2, \ldots, N_{B} ; N_{B} \leq \operatorname{dim}\left(\mathcal{H}_{B}\right)
\end{gathered}
$$

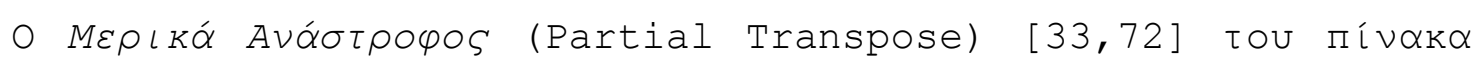

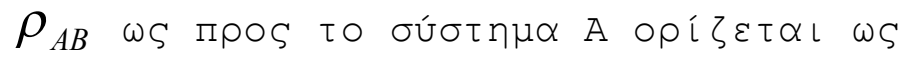

$$
\rho_{A B}^{T_{A}}=\sum_{i, j=1}^{N_{A}} \sum_{\mu, v=1}^{N_{B}} a_{i j}^{\mu v}(|j\rangle\langle i|)_{A} \otimes(|\mu\rangle\langle v|)_{B}
$$

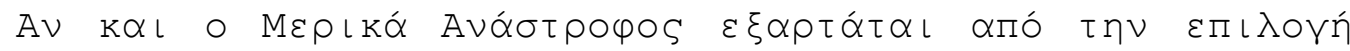

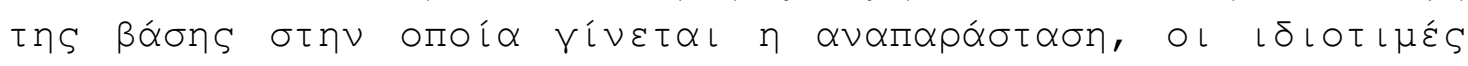

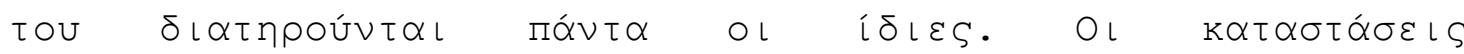

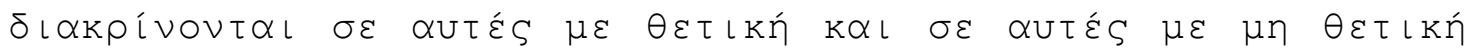

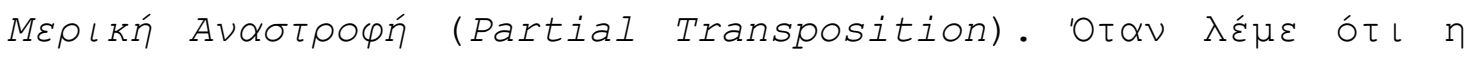

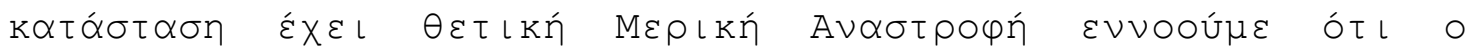




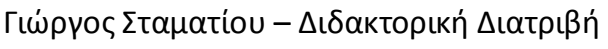

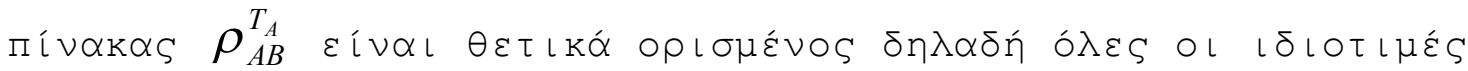

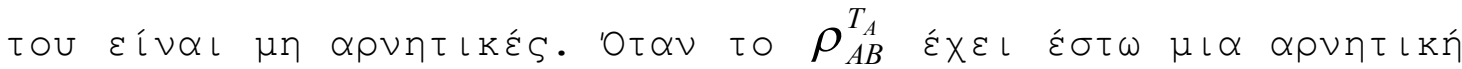

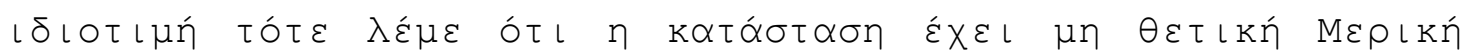
Av $\propto \sigma \rho \circ \varphi n$.

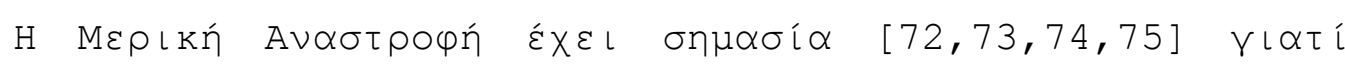

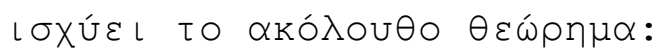

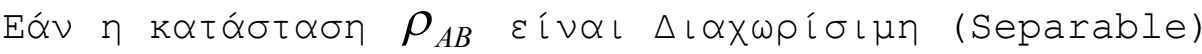

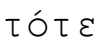

$$
\begin{aligned}
& \rho_{A B}^{T_{A}} \geq 0 \quad \text { к } \alpha\left\llcorner\quad \rho_{A B}^{T_{B}}=\left(\rho_{A B}^{T_{A}}\right)^{T} \geq 0\right.
\end{aligned}
$$

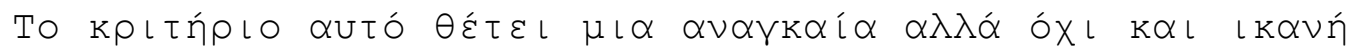

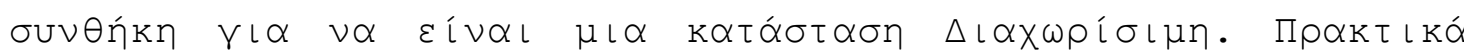

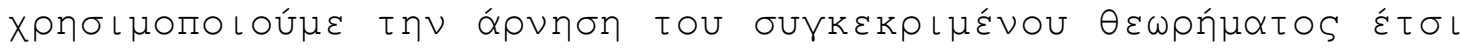

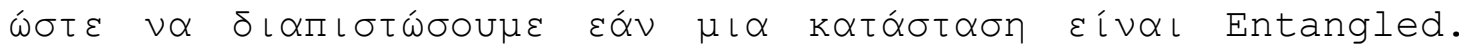

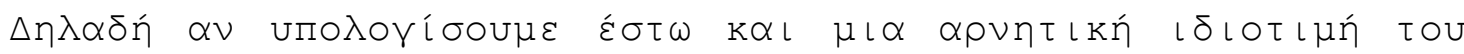

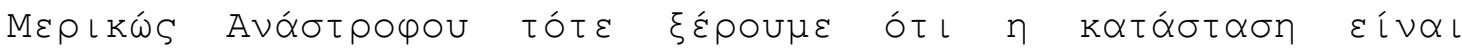

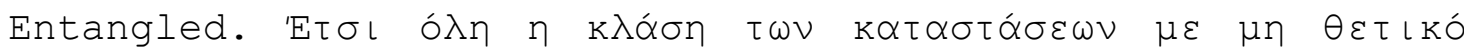

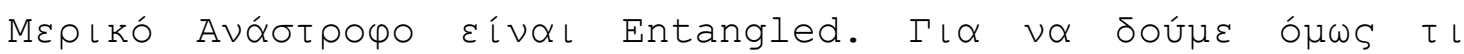

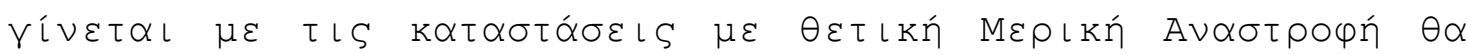

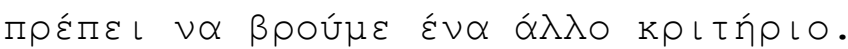

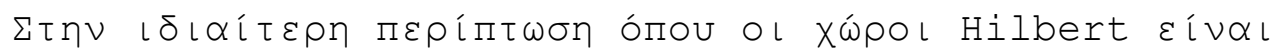

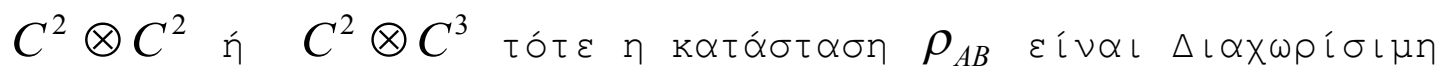

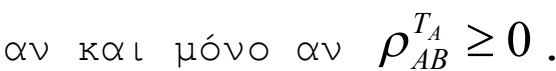

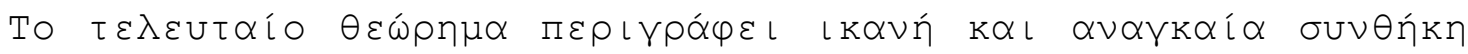

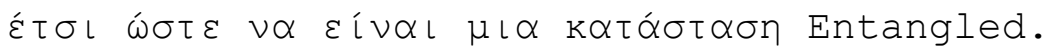




\section{Majorization.}

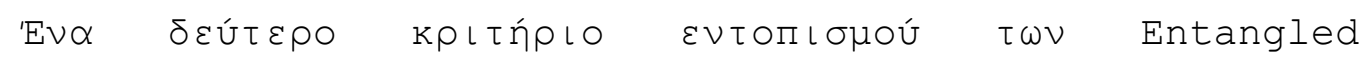

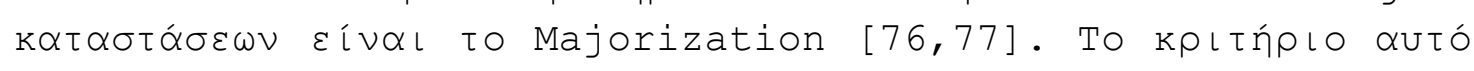

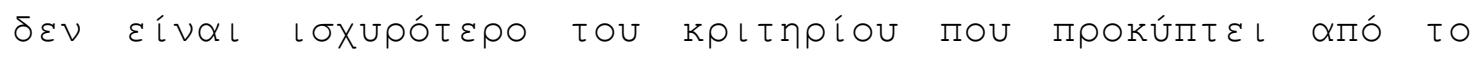

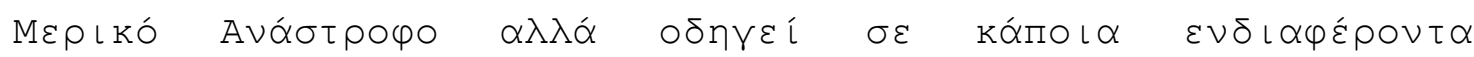

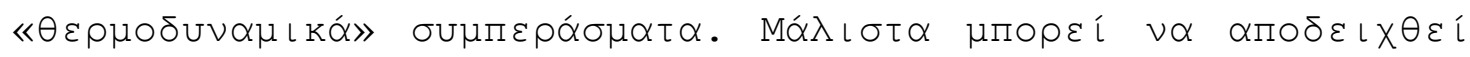

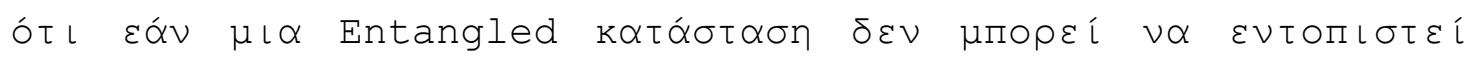

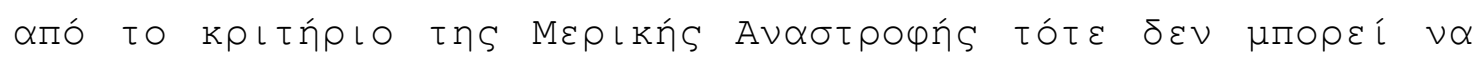

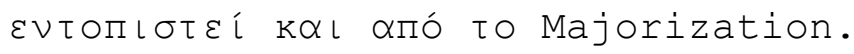

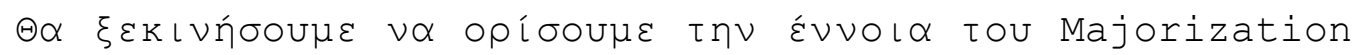

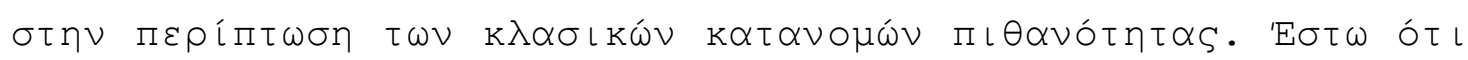

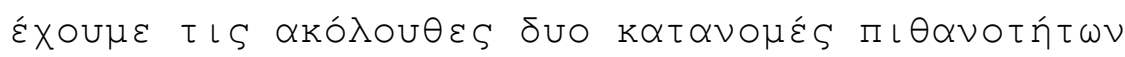

$$
x=\left(x_{1}, x_{2}, \ldots x_{d}\right) \text { ка } y=\left(y_{1}, y_{2}, \ldots y_{d}\right)
$$

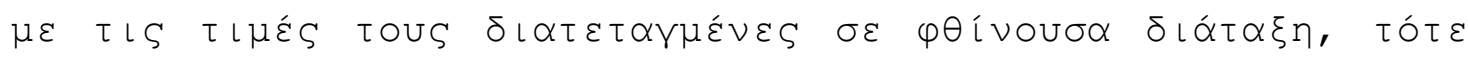

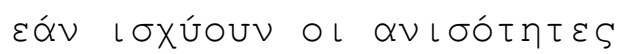

$$
\sum_{i=1}^{l} x_{i} \leq \sum_{i=1}^{l} y_{i}
$$

$\mu \varepsilon$

$$
l=1,2 \ldots d-1
$$

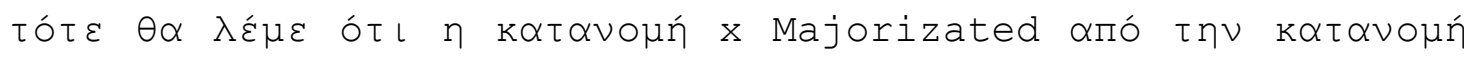

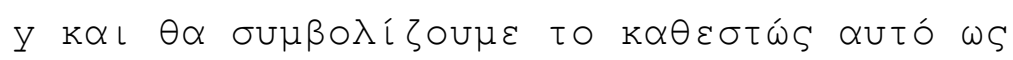

$$
x \prec y
$$

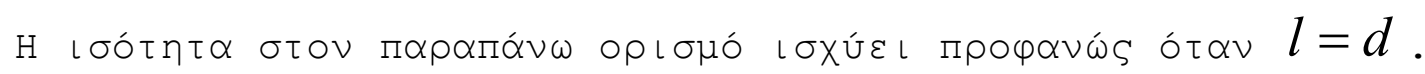

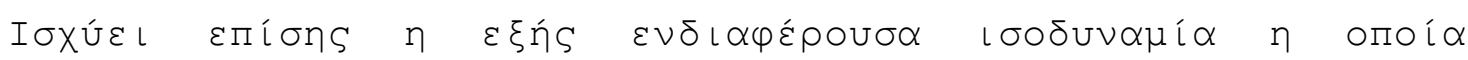

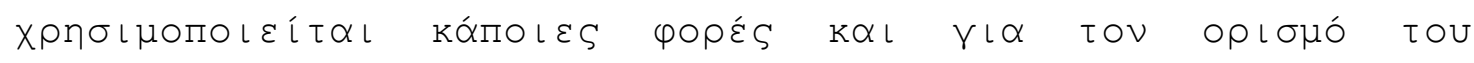
Majorization. 


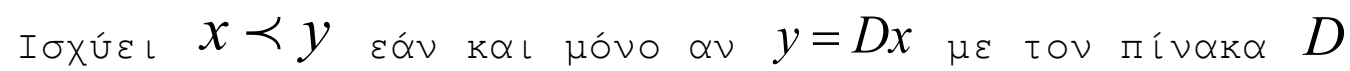

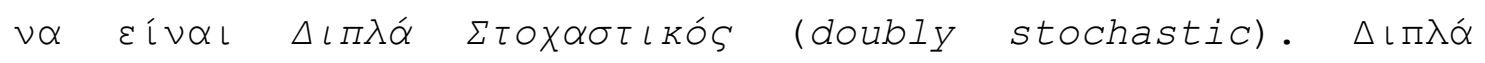

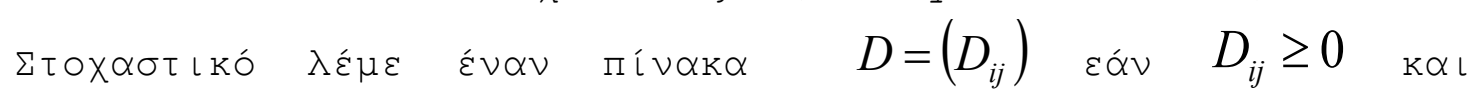
$\sum_{i} D_{i j}=\sum_{j} D_{i j}=1$.

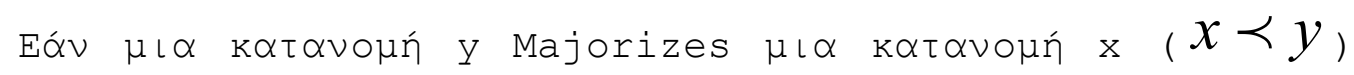

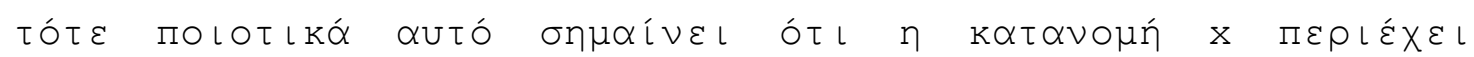

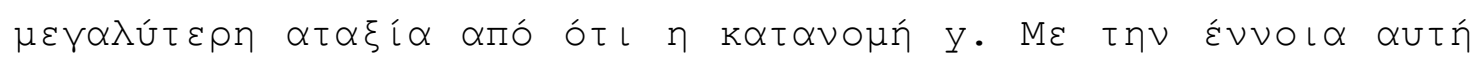

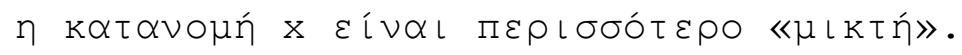

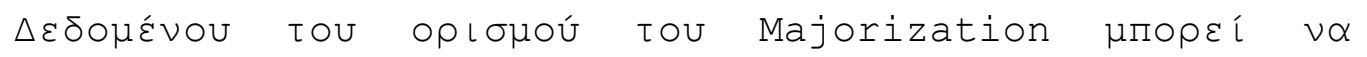

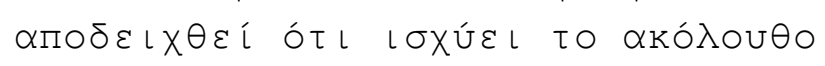

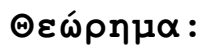

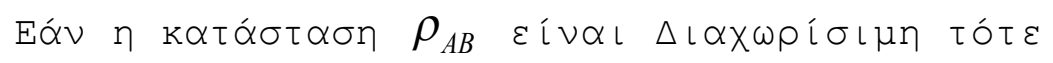

$$
\lambda\left(\rho_{A B}\right) \prec \lambda\left(\rho_{A}\right) \quad \text { к } \alpha \iota \quad \lambda\left(\rho_{A B}\right) \prec \lambda\left(\rho_{B}\right)
$$

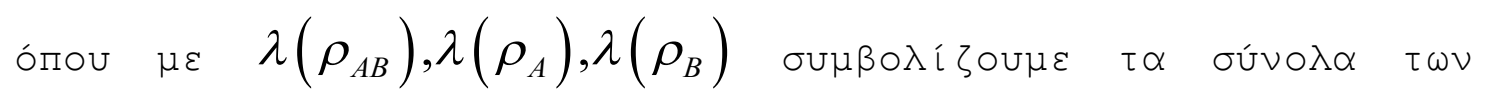

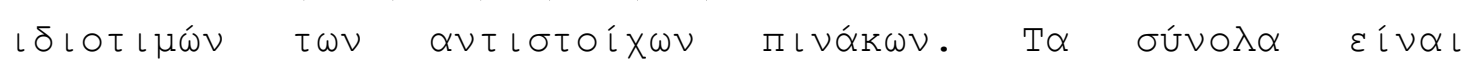

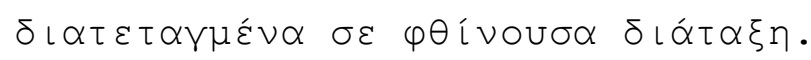

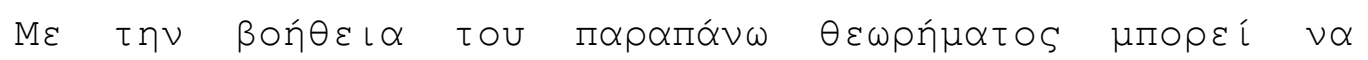

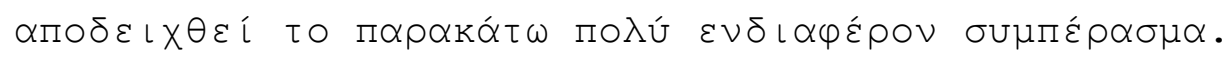

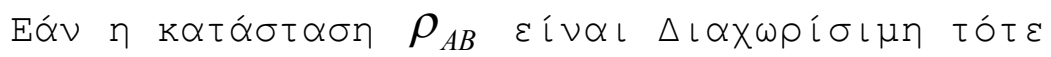

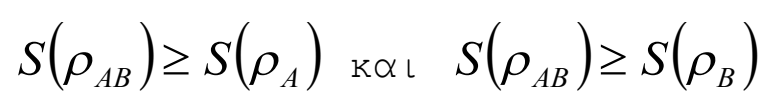

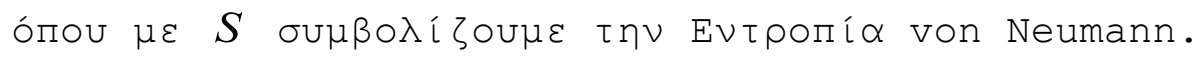

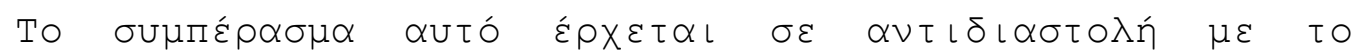

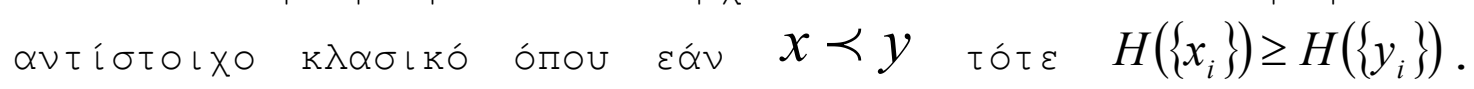

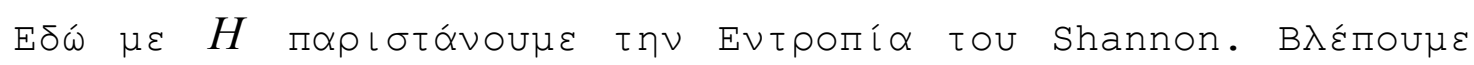




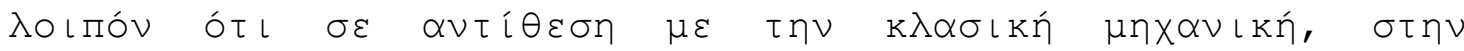

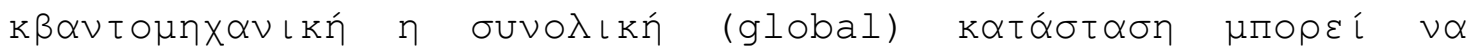

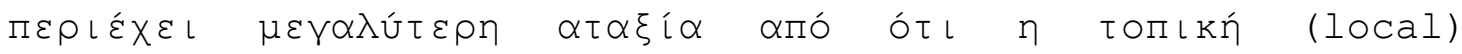

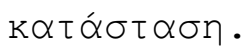

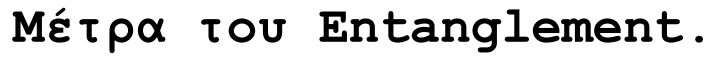

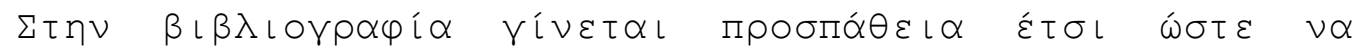

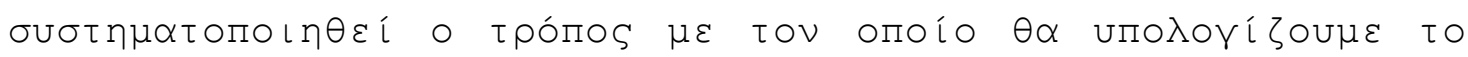

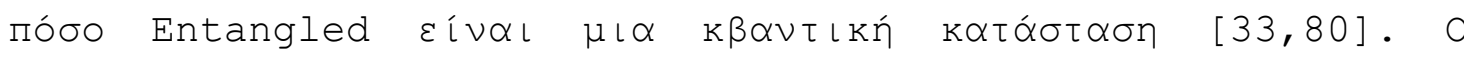

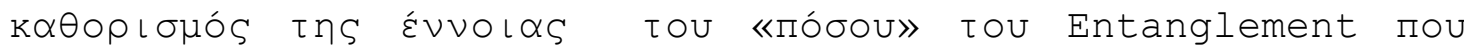

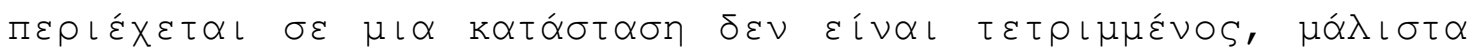

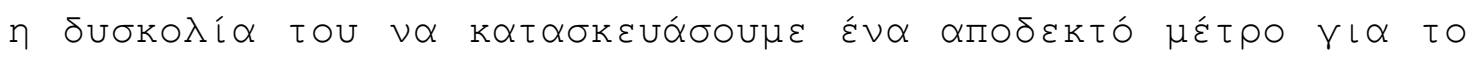

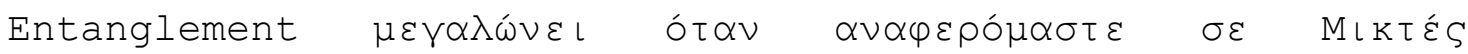

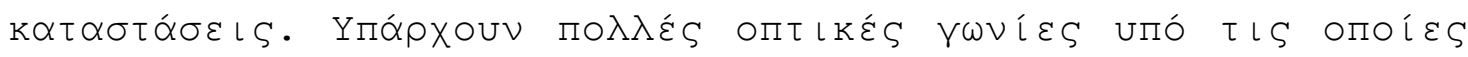

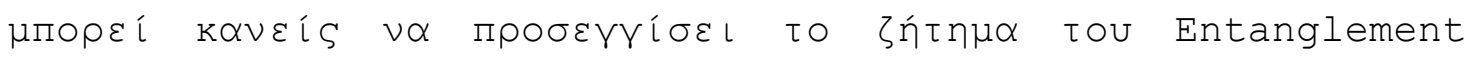

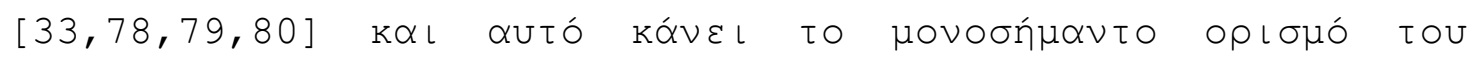

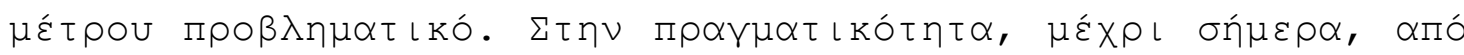

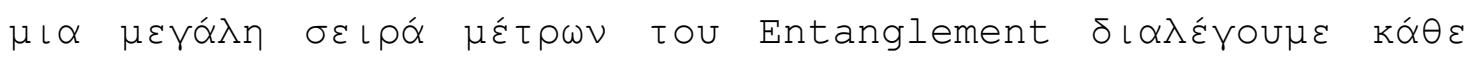

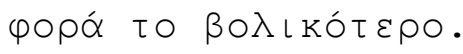

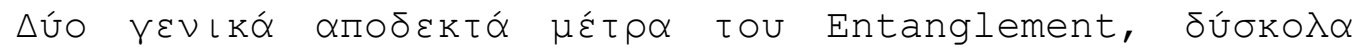

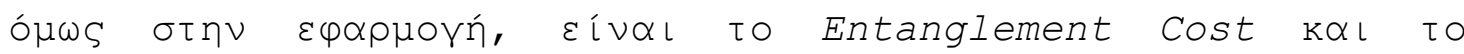
Distillable Entanglement.

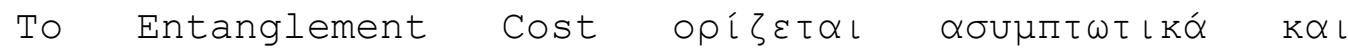

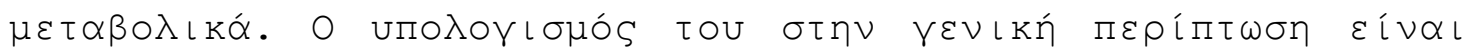

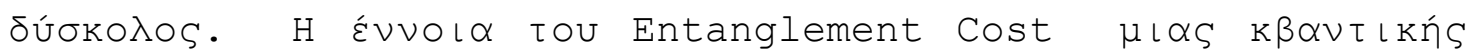

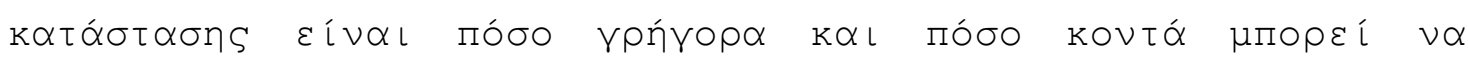

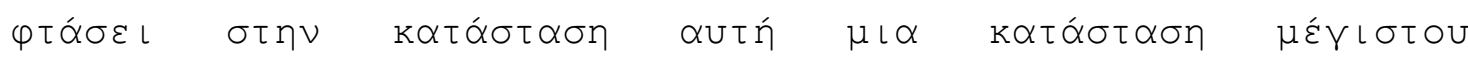

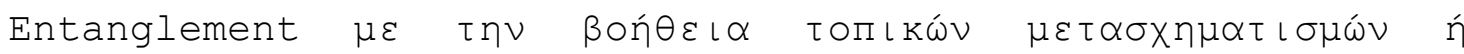

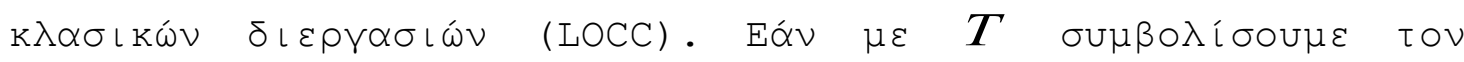

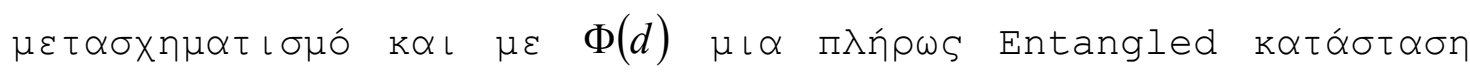

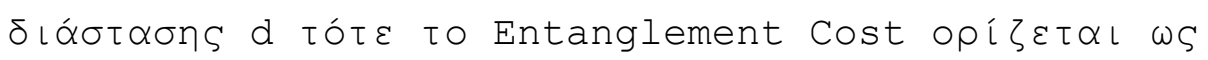

$$
E_{C}(\rho) \equiv \inf \left\{r: \lim _{n \rightarrow \infty}\left[\inf _{T} D\left(\rho^{\otimes n}, T\left(\Phi\left(2^{r n}\right)\right)\right)\right]=0\right\}
$$




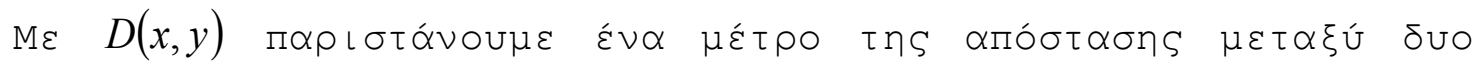

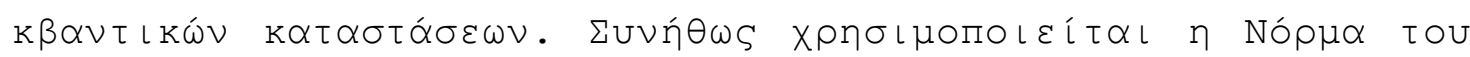
'IXVOUS.

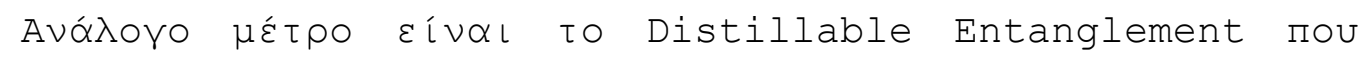

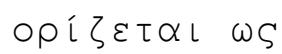

$$
D(\rho) \equiv \sup \left\{r: \lim _{n \rightarrow \infty}\left[\sup _{T} \operatorname{tr}\left|T\left(\rho^{\otimes n}\right)-\Phi\left(2^{r n}\right)\right|\right]=0\right\}
$$

\section{Distillable Entanglement}

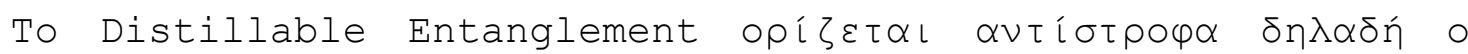

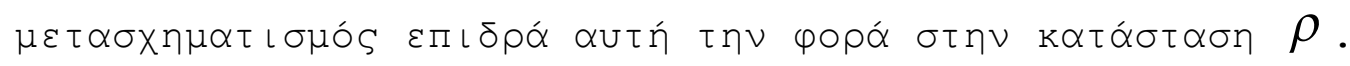

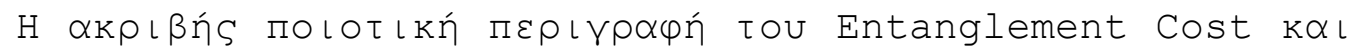

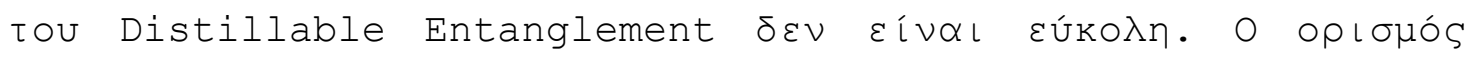

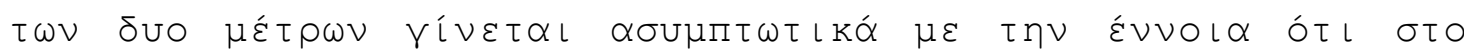

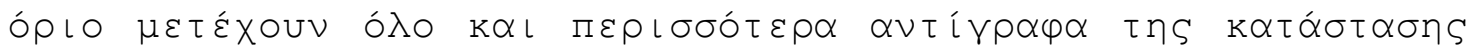

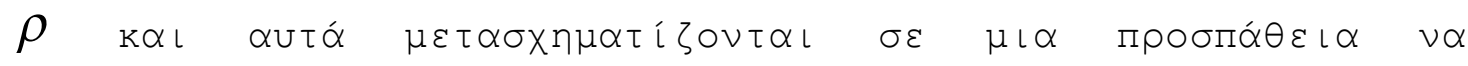

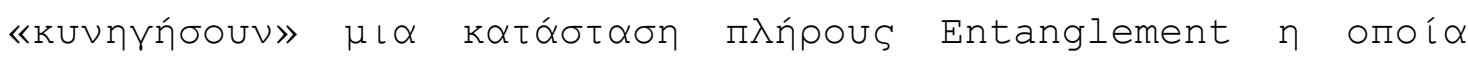

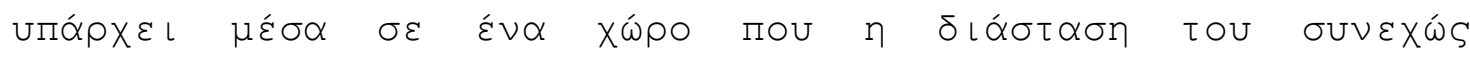

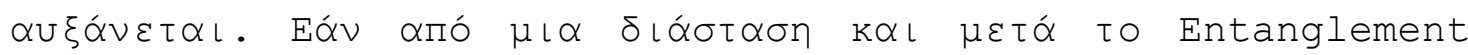

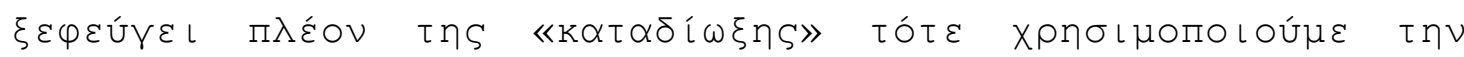

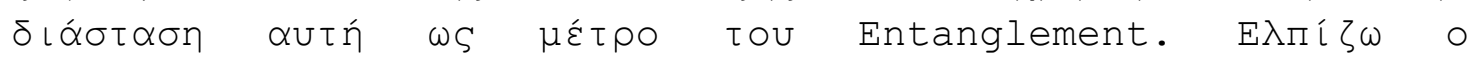

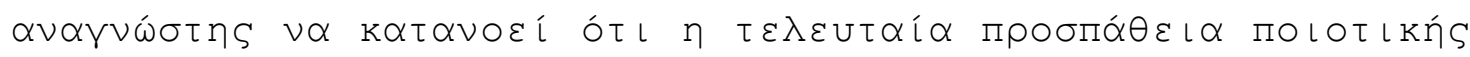

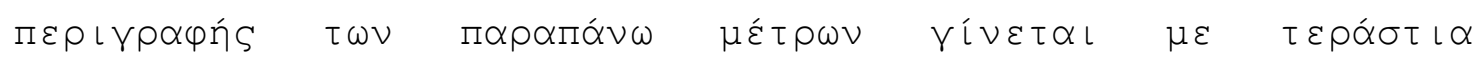

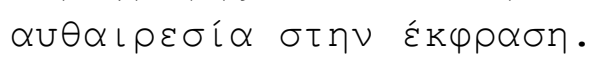

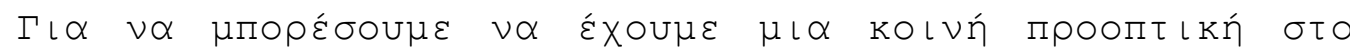

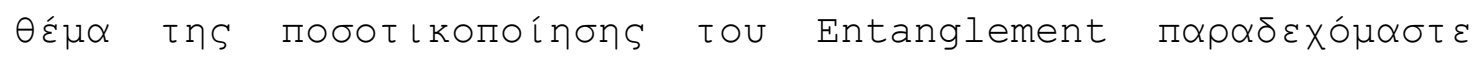

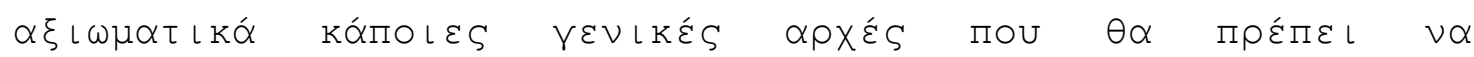

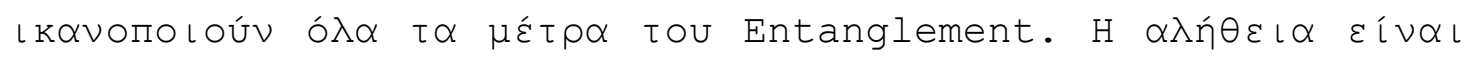

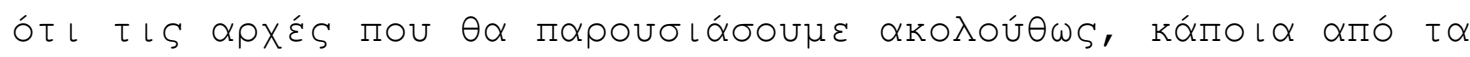

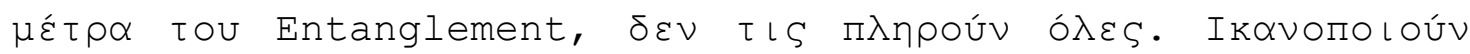

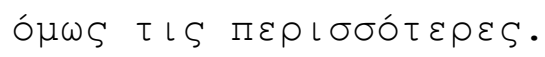

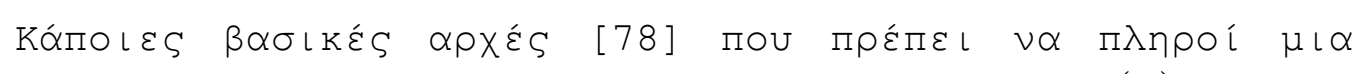

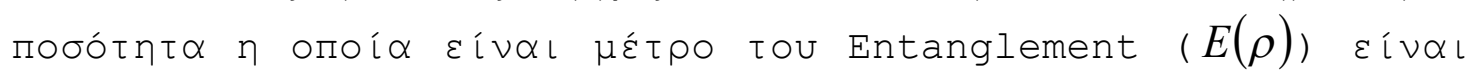

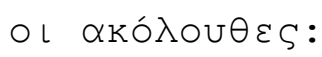




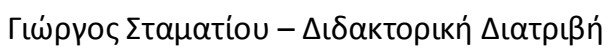

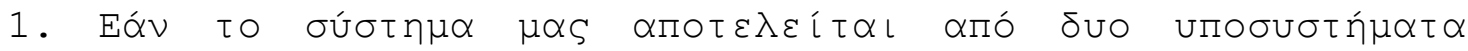

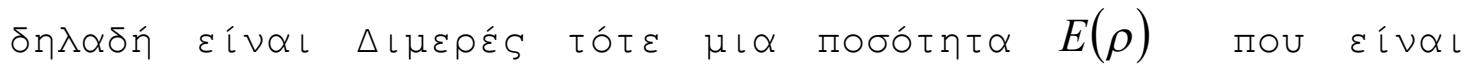

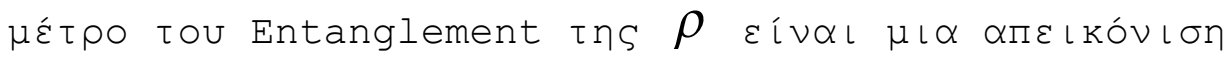

$$
\rho \rightarrow E(\rho) \in R^{+}
$$

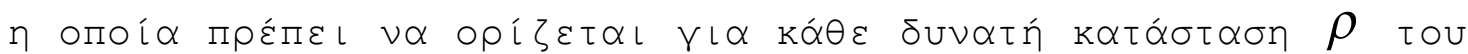

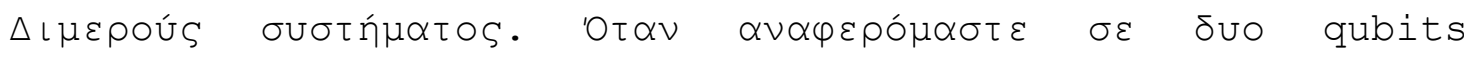

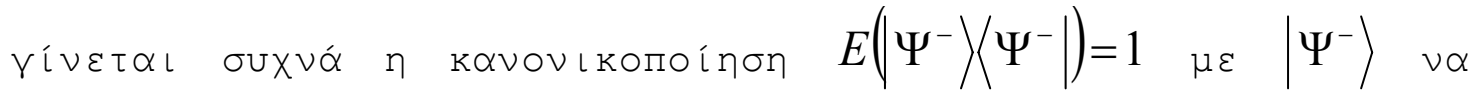

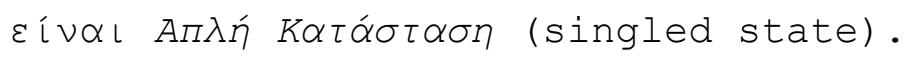

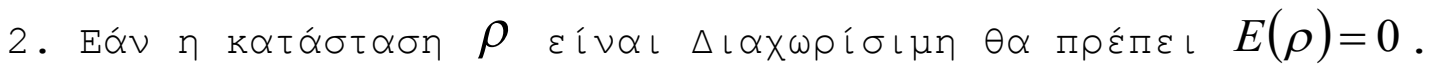

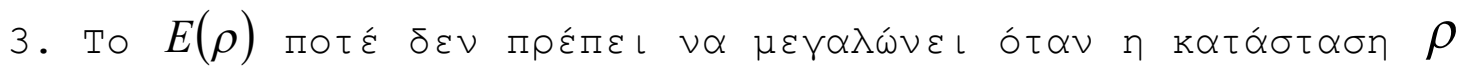

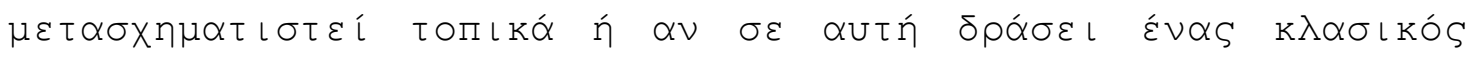
$\mu \varepsilon \tau \alpha \sigma \times \eta \mu \alpha \tau \iota \sigma \mu o ́ s \quad$ (LOCC).

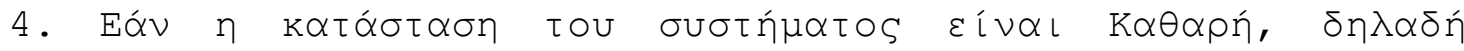

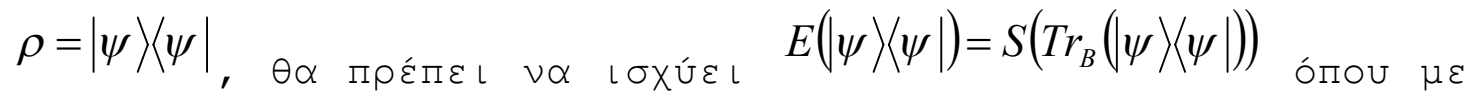

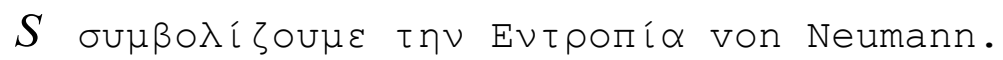

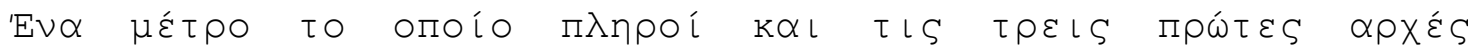

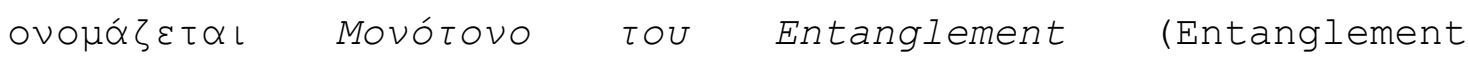
Monotone). $\Omega S$ MÉtpo tou Entanglement (Entanglement Measure)

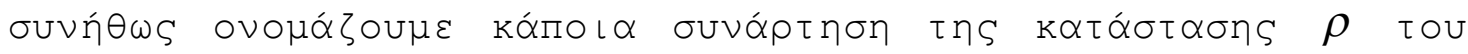

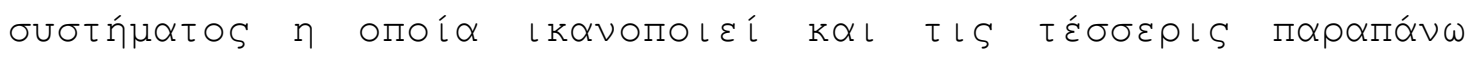

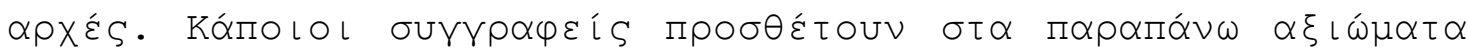

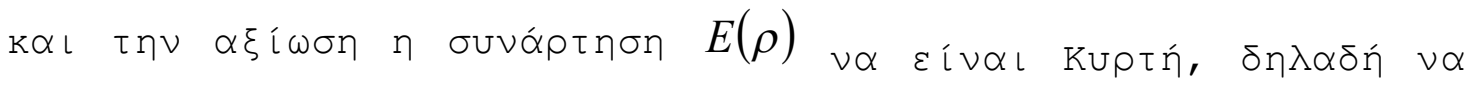

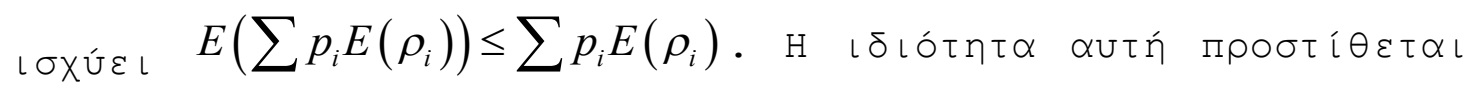

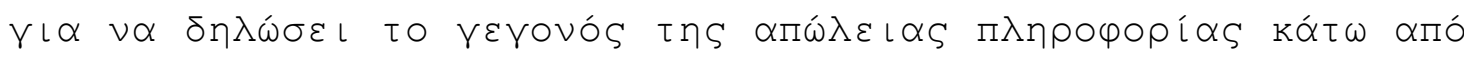

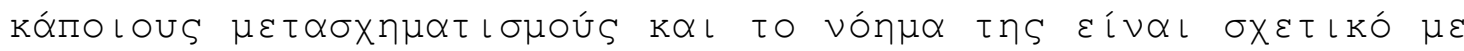

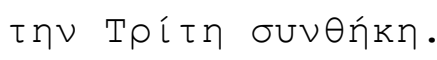




\section{Entanglement of Formation.}

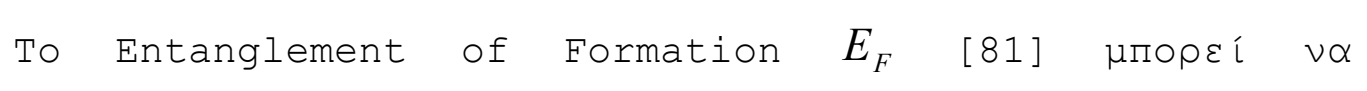

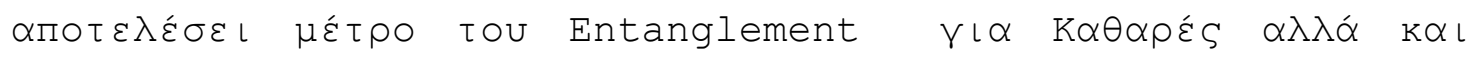

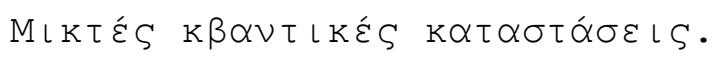

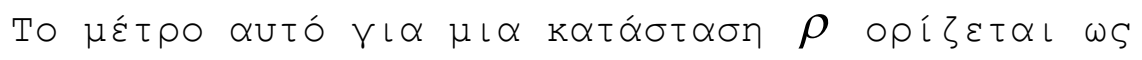

$$
\left.E_{F}(\rho) \equiv \inf \left\{\sum_{i} p_{i} E\left(\left|\psi_{i}\right\rangle\left\langle\psi_{i}\right|\right)\left|\rho=\sum_{i} p_{i}\right| \psi_{i}\right\rangle\left\langle\psi_{i}\right|\right\}
$$

Entanglement of Formation

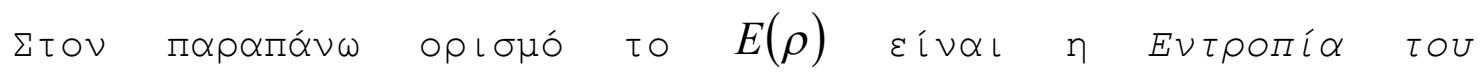

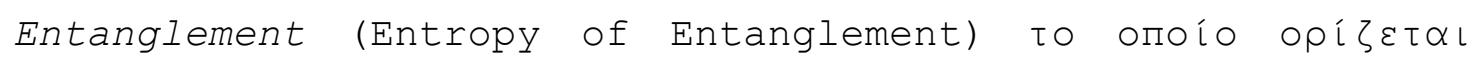

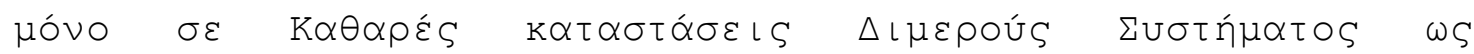
$E\left(\rho_{A B}\right)=S\left(\rho_{A}\right)=S\left(\rho_{B}\right) \quad \Delta \eta \lambda \alpha \delta \dot{n}$ to Entanglement of Formation

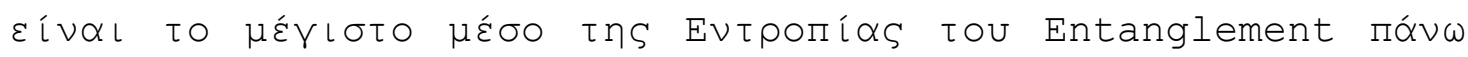

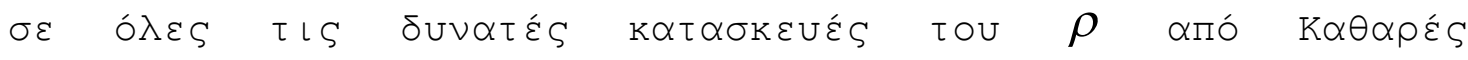

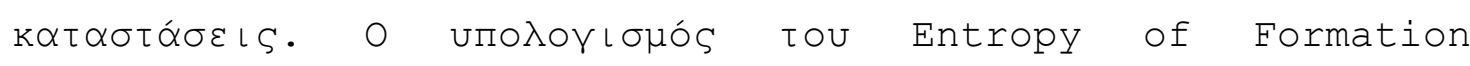

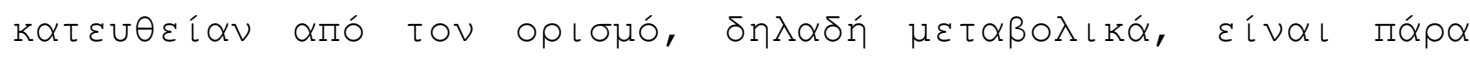

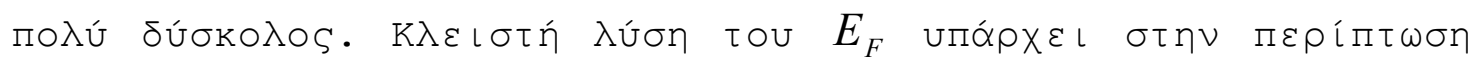

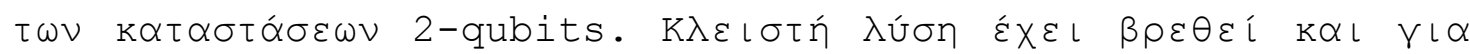

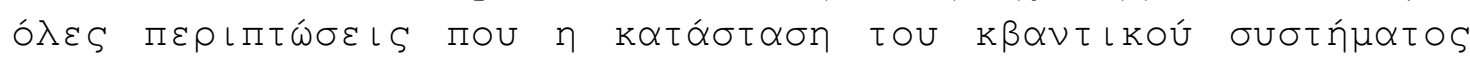

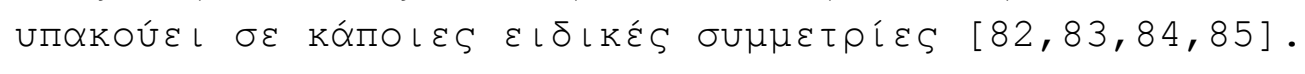

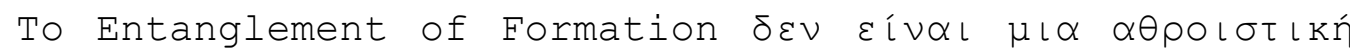

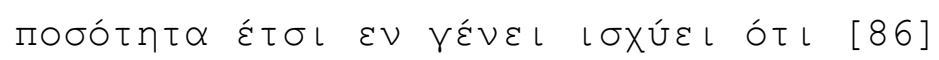

$$
E_{F}\left(\rho^{A B} \otimes \sigma^{A B}\right) \neq E_{F}\left(\rho^{A B}\right)+E_{F}\left(\sigma^{A B}\right)
$$

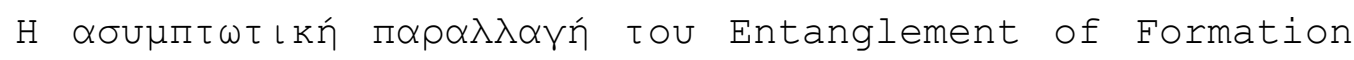

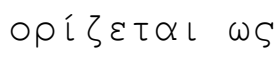

$$
E_{F}^{\infty}(\rho) \equiv \lim _{n \rightarrow \infty} \frac{E_{F}\left(\rho^{\otimes n}\right)}{n}
$$




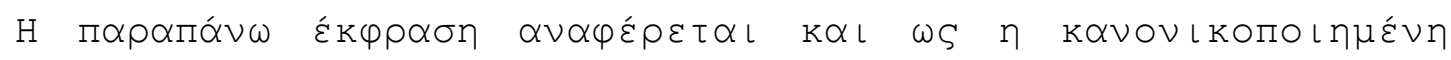

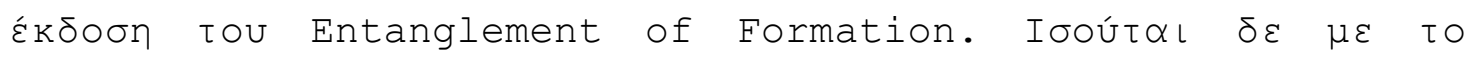
Entanglement Cost $\delta \eta \lambda \alpha \delta n$

$$
E_{F}^{\infty}(\rho)=E_{C}(\rho)
$$

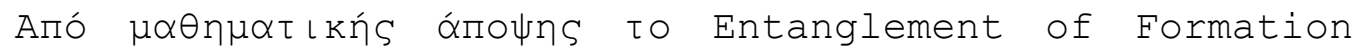

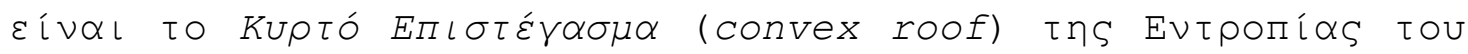

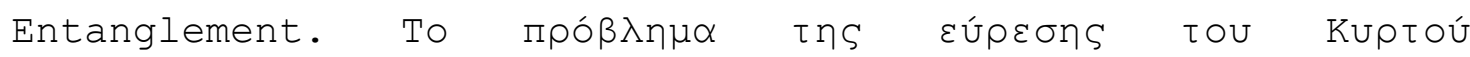

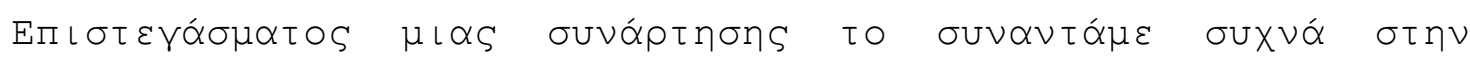

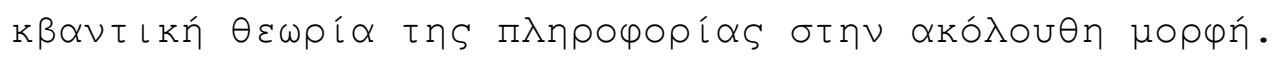

$$
\begin{gathered}
M(\rho)=\inf _{\left\{p_{i},\left|\psi_{i}\right\rangle\right\} \in \mathcal{D}(\rho)}\left[\sum_{i} p_{i} m\left(\left|\psi_{i}\right\rangle\right)\right] \\
\mathcal{D}(\rho)=\left\{\left\{p_{i},\left|\psi_{i}\right\rangle\right\}_{i=1}^{s}, s œ \tau \xi \eta \rho \mid\left\{\left|\psi_{i}\right\rangle\right\}_{i=1}^{s} \subset \mathcal{H},\right. \\
\left.p_{i} \geq 0, \sum_{i=1}^{s} p_{i}=1, \rho=\sum_{i=1}^{s} p_{i}\left|\psi_{i}\right\rangle\left\langle\psi_{i}\right|\right\}
\end{gathered}
$$

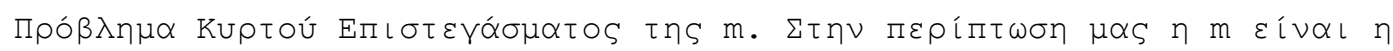

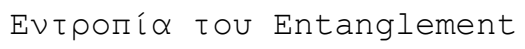

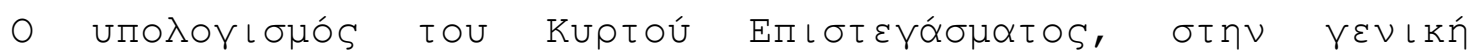

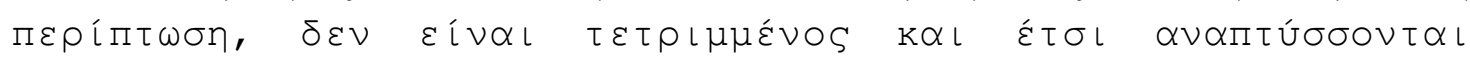

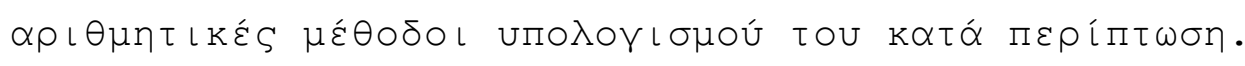

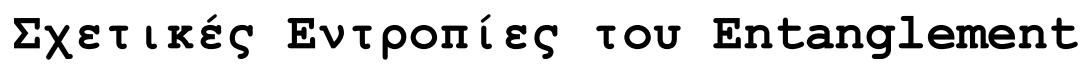

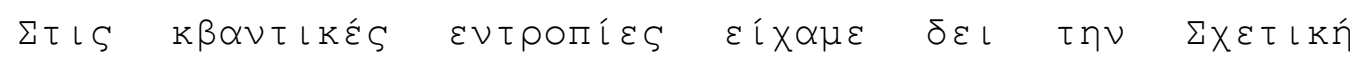

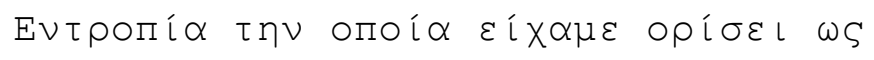

$$
\begin{aligned}
& S(\rho \mid \sigma) \equiv-S(\rho)-\operatorname{tr}(\rho \log \sigma)
\end{aligned}
$$

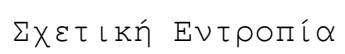




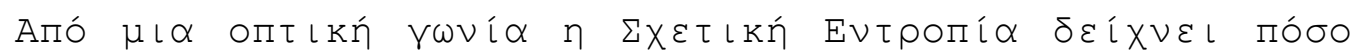

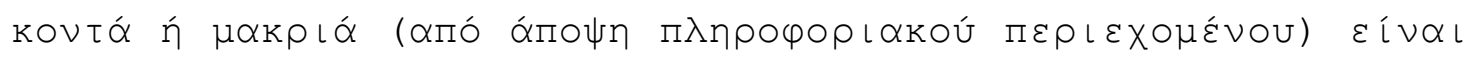

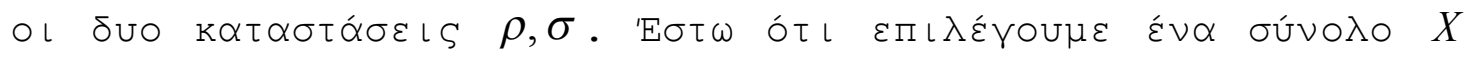

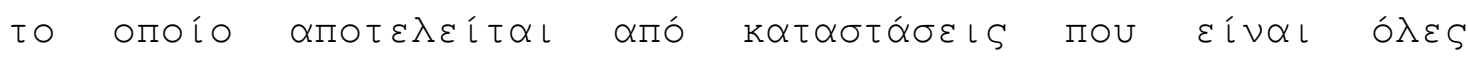

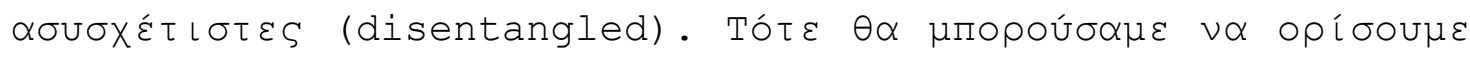

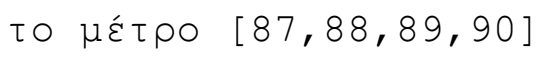

$$
E_{R}^{X}(\rho \mid \sigma) \equiv \inf _{\sigma \in X}[S(\rho \mid \sigma)]
$$

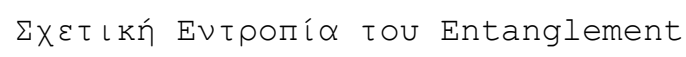

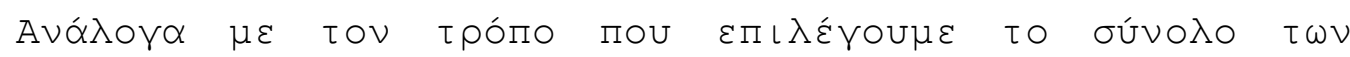

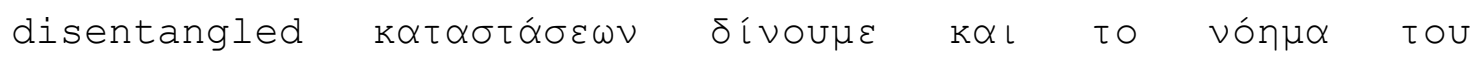

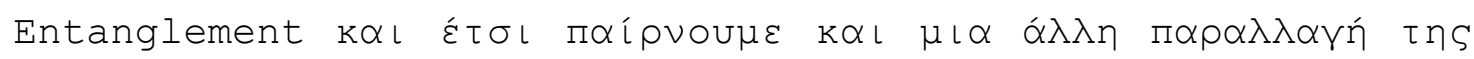

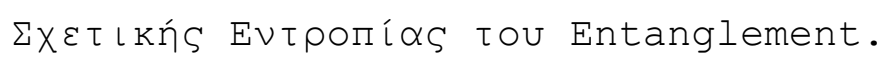

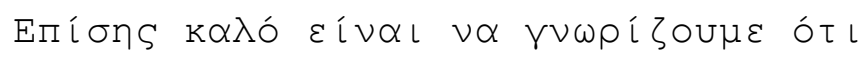

$$
E_{R}^{X}\left(\rho^{\otimes n}\right) \neq n E_{R}^{X}(\rho)
$$

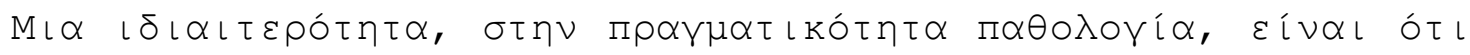

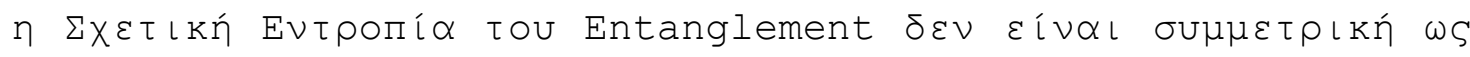

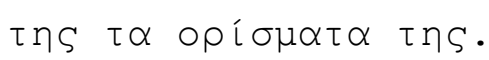

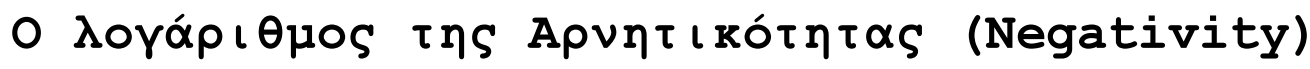

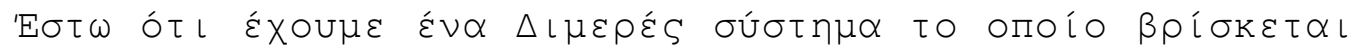

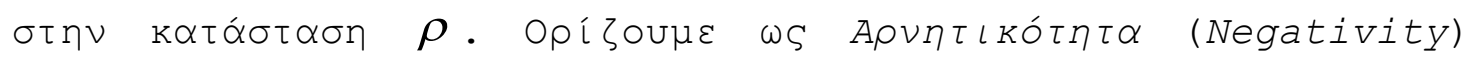

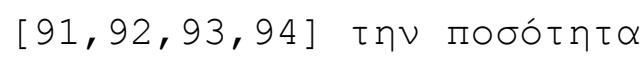

$$
N(\rho) \equiv\left\|\rho^{T_{B}}\right\|
$$

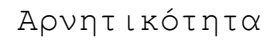

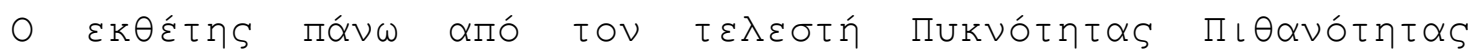

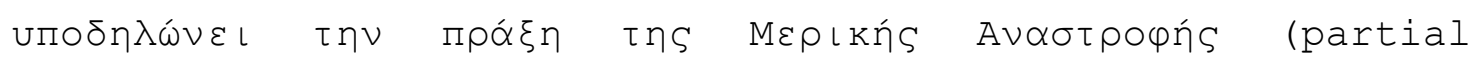

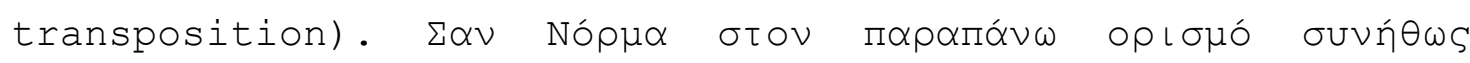

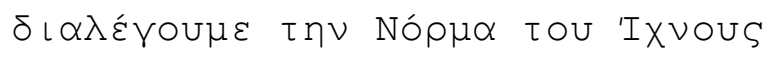




$$
\begin{aligned}
& \|X\| \equiv \operatorname{tr}\left(\sqrt{X^{+} X}\right) \\
& \text { H Nóppu tou 'Ixvous }
\end{aligned}
$$

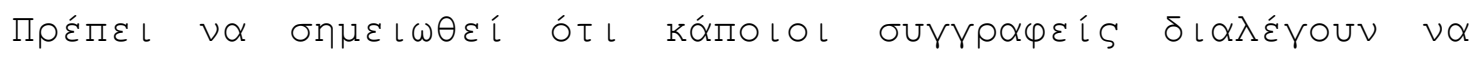

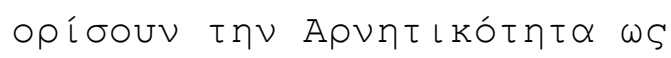

$$
\frac{\left(\left\|\rho^{T_{B}}\right\|-1\right)}{2}
$$

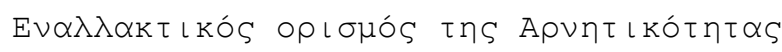

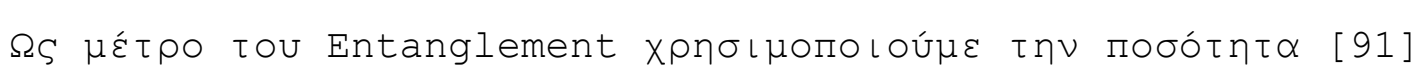

$$
E_{N}(\rho) \equiv \log \left\|\rho^{T_{B}}\right\|=\log [N(\rho)]
$$

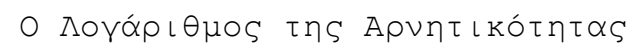

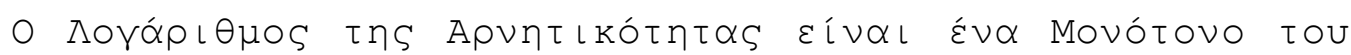

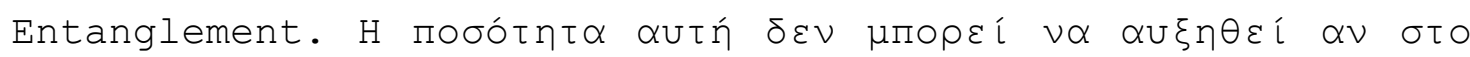

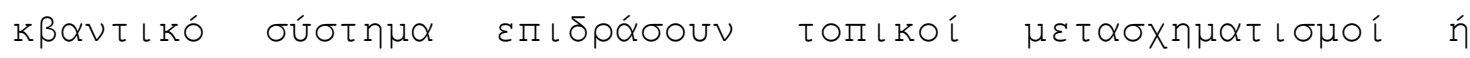

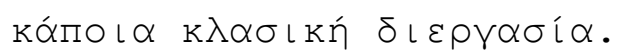

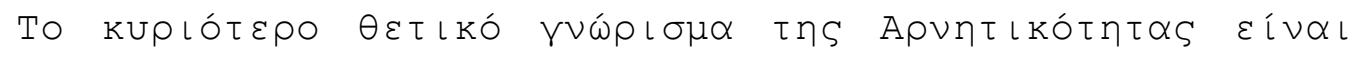

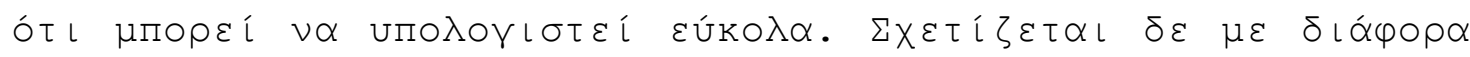

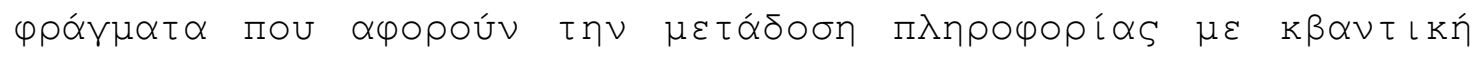

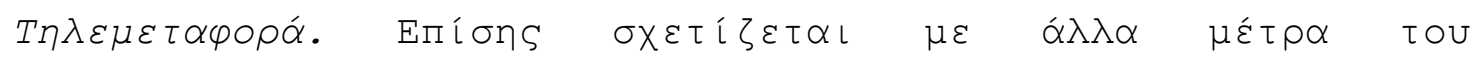

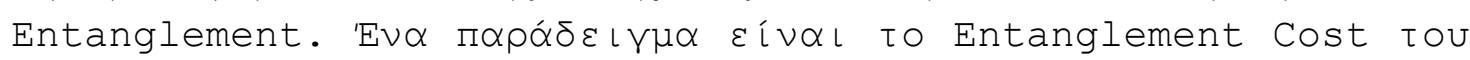

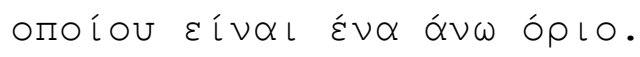

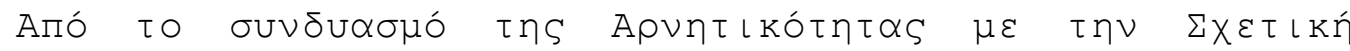

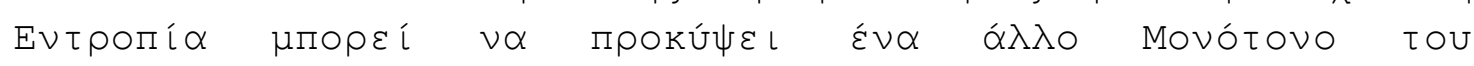

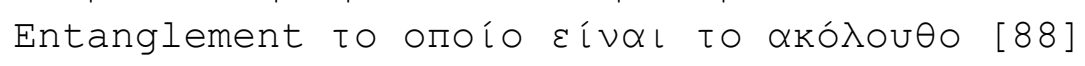

$$
B(\rho) \equiv \min _{o \lambda \alpha \tau \alpha \sigma}\left[S(\rho \mid \sigma)+E_{N}(\sigma)\right]
$$

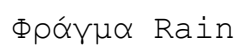

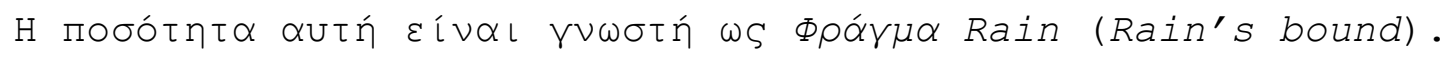




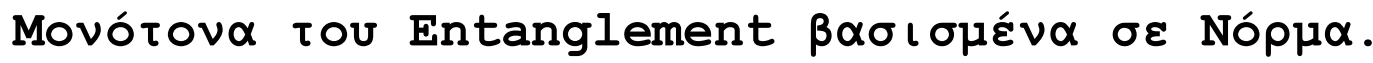

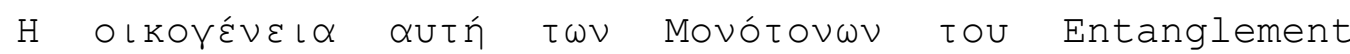

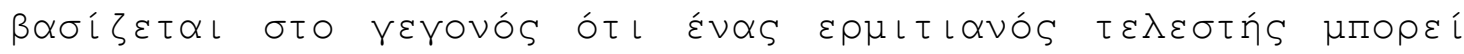
$\nu \alpha \varepsilon \kappa \varphi \rho \alpha \sigma \tau \varepsilon i$ wS

$$
h=a \Omega-b \Delta \quad a, b \geq 0
$$

$$
\Omega \in X, \Delta \in Y
$$

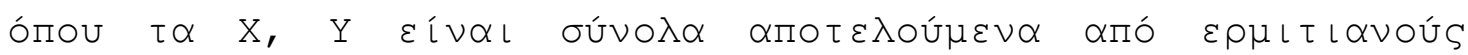

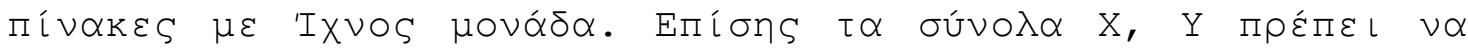

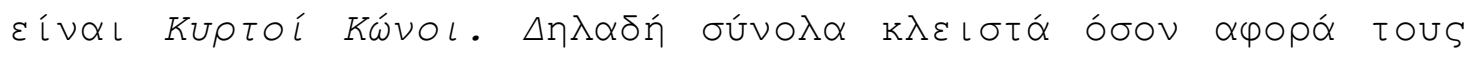

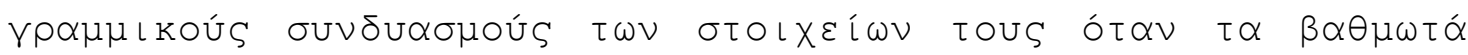

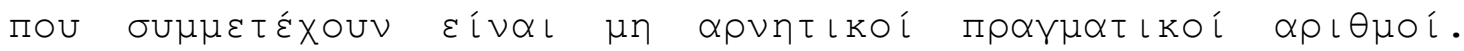

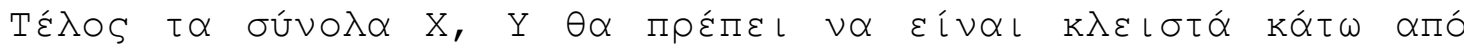

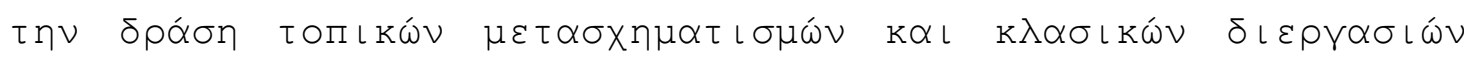
$(\mathrm{LOCC})$.

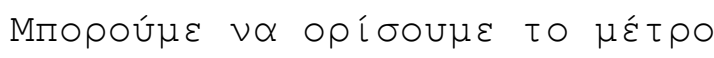

$$
\|h\|_{X, Y} \equiv \inf _{\Omega \in X, \Delta \in Y}\{a+b \mid h=a \Omega-b \Delta, a, b \geq 0\}
$$

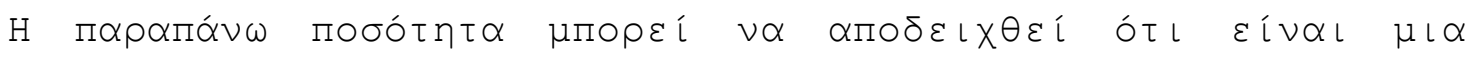
Nóp $\mu \alpha$.

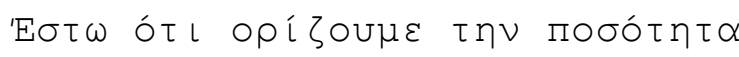

$$
R_{X, Y}(h) \equiv \inf _{\Omega \in X, \Delta \in Y}\{b \mid h=a \Omega-b \Delta, a, b \geq 0\}
$$

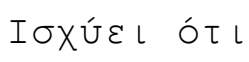

$$
R_{X, Y}(\rho) \equiv \inf \left\{b \mid b \geq 0, \exists \Delta \in Y, \Omega \in X_{\varepsilon}^{\prime} \tau \sigma \omega^{\prime} \sigma \tau \varepsilon \frac{\rho+b \Delta}{1+b}=\Omega\right\}
$$




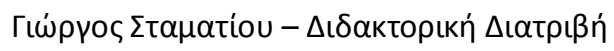

$$
=\frac{\|h\|_{X, Y}-1}{2}
$$

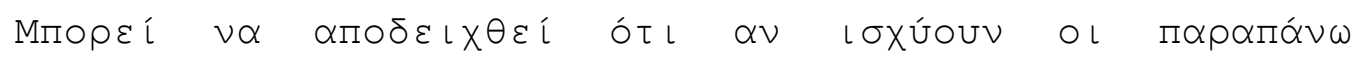

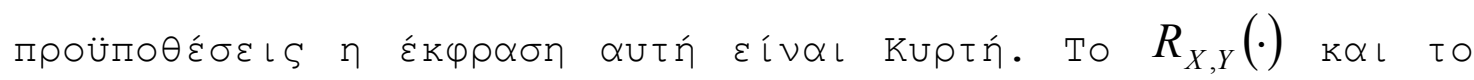
$\|h\|_{X, Y}$ Eíval LOCC Movótovo.

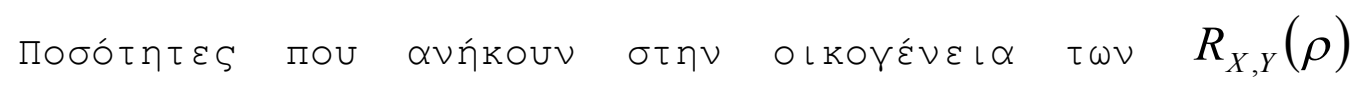

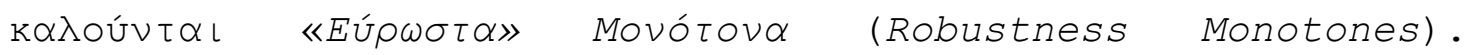

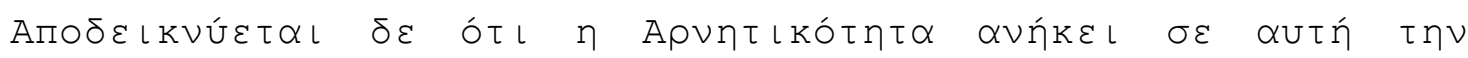

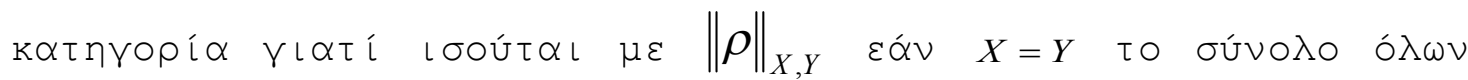

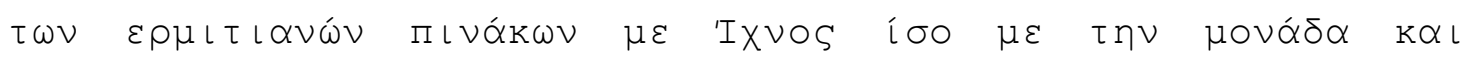

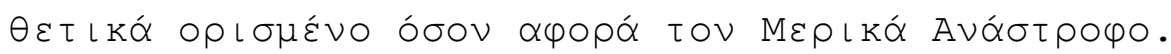

\section{Cross norm.}

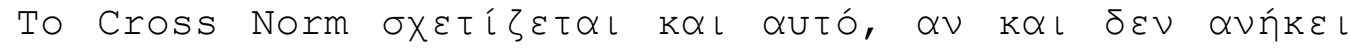

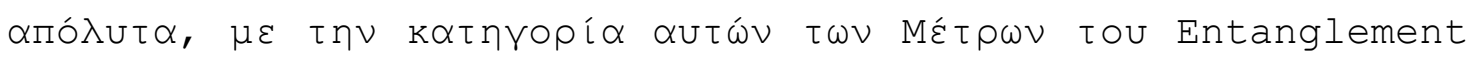

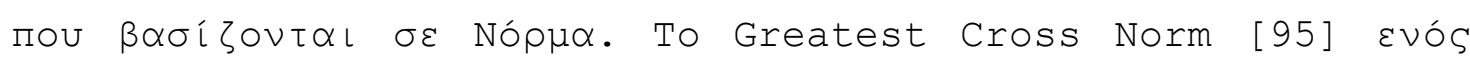

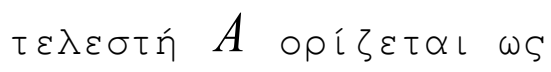

$$
\begin{gathered}
\|A\|_{\gamma} \equiv \inf \left[\sum_{i=1}^{n}\left\|u_{i}\right\|_{1}\left\|_{i}\right\|_{1}: A=\sum_{i} u_{i} \otimes v_{i}\right] \\
\text { Greatest Cross norm }
\end{gathered}
$$

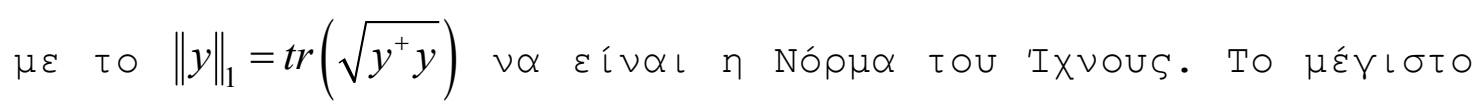

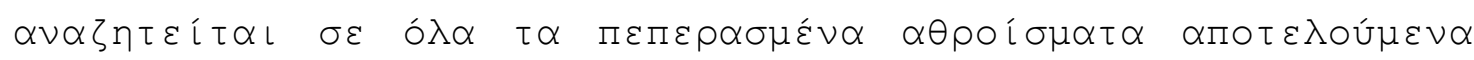

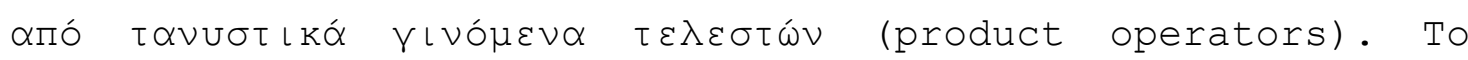

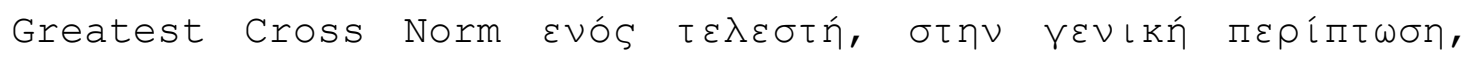

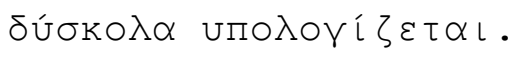




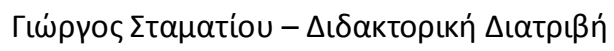

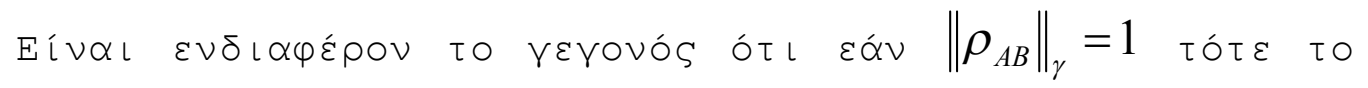

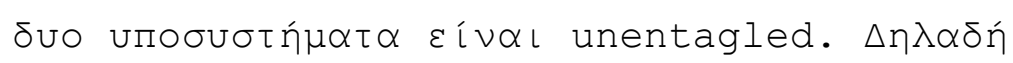

$$
\left\|\rho_{A B}\right\|_{\gamma}=1 \Rightarrow \rho_{A B}=\rho_{A} \otimes \rho_{B}
$$

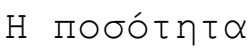

$$
E_{\gamma}(\rho) \equiv\|\rho\|_{\gamma}-1
$$

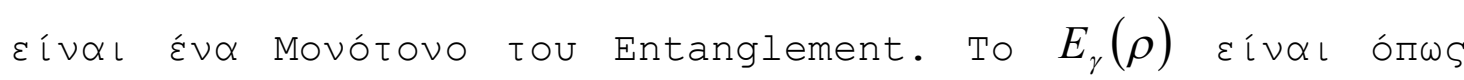

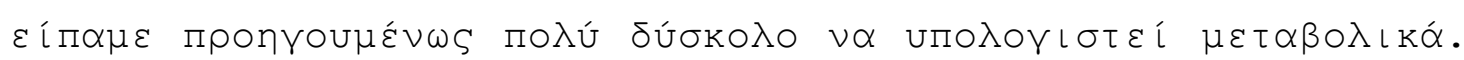

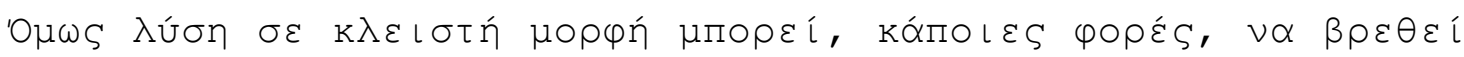

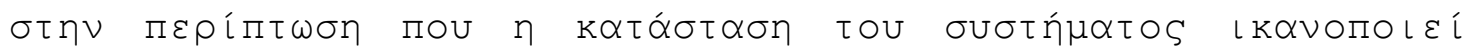

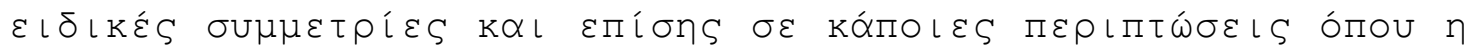

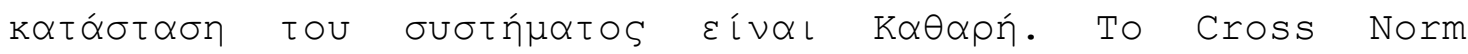

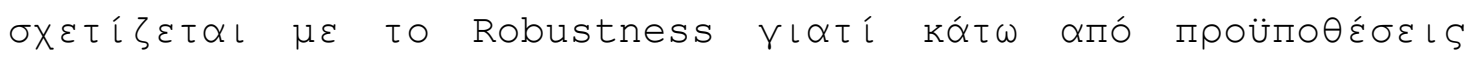

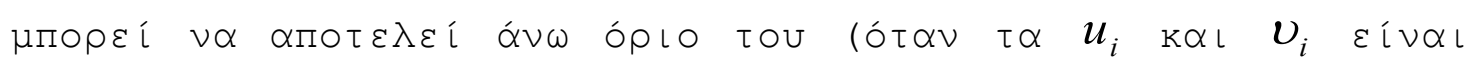

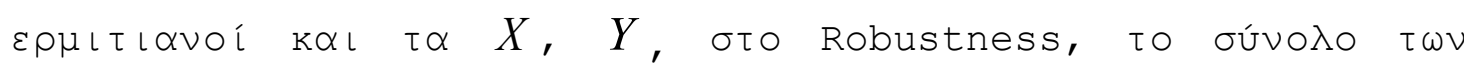
unentangled $k \alpha \tau \alpha \sigma \tau \alpha ́ \sigma \varepsilon \omega \nu)$.

\section{To Squashed Entanglement}

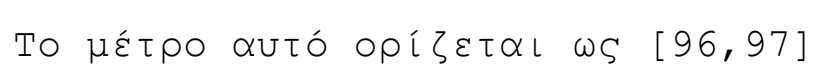

$$
E_{s q} \equiv \inf \left[\frac{1}{2} I\left(\rho_{A B E}\right) \mid t r_{E}\left(\rho_{A B E}\right)=\rho_{A B}\right]
$$

Squashed Entanglement

$\mathrm{K} \propto \mathrm{L}$

$$
I\left(\rho_{A B E}\right) \equiv S(A E)+S(B E)-S(A B E)-S(E)
$$

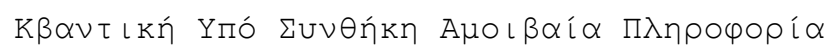




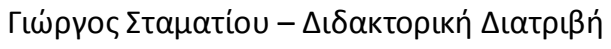

To Squashed Entanglement Eíval Kuptó Movótovo tou

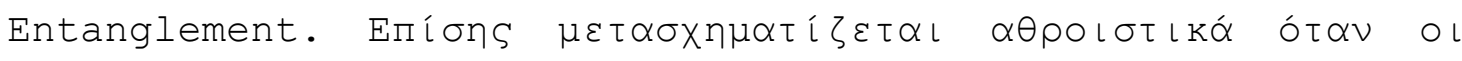

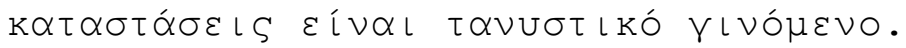

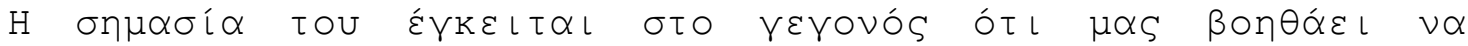

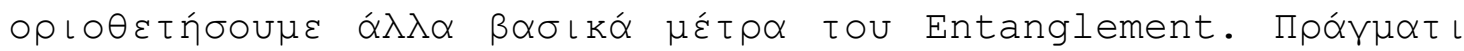

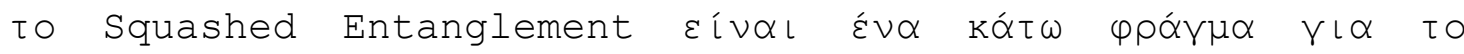

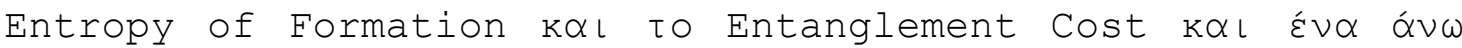

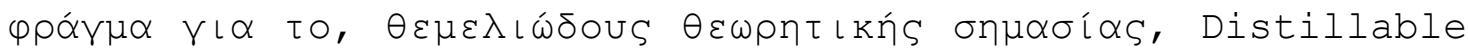

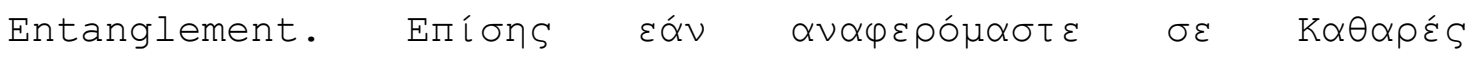

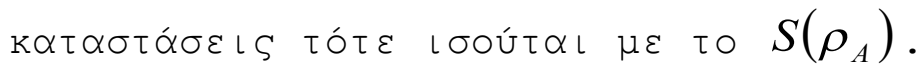





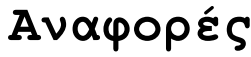

[1]. A mathematical theory of communication, 1948, CE Shannon, W Weaver - Bell Syst. Tech. J

[2]. Information theory and reliable communication, RG Gallager - 1968, John Wiley \& Sons, Inc. New York, NY, USA

[3]. Elements of information theory, TM Cover, JA Thomas 2006 - Wiley

[4]. Quantum information theory, CH Bennett, PW Shor - IEEE Transactions on Information Theory, 1998, vol. 44, no. 6, $2724-2742$

[5]. Recent results in the Shannon theory, A Wyner - IEEE Transactions on Information Theory, 1974, vol. IT20, no. 1, $2-10$

[6]. Coding and information theory, S Roman - 1992, Springer

[7]. The concept of information, R Capurro, B Hjørland Annual review of information science ..., 2003, vol. 37, 343411

[8]. Cybernetics or Control and Communication in the Animal and the ..., N Wiener - 1965, MIT Press

[9]. An introduction to information theory: symbols, signals \& noise, JR Pierce - 1980, Dover

[10] . Entropy and information theory, RM Gray - 1990, http://ee.stanford.edu/ gray/it.pdf

[11]. Information Theory and Statistics. S. Kullback. Dover, New York, 1968.

[12]. Notions generalisees d'incertitude, d'entropie et d'information du point de vue de la theorie des martingales. A. Perez. In Transactions First Prague Conf. on Information Theory, Stat. Decision Functions, and Random Processes,. Czech. Acad. Sci. Publishing House, 1957, pages $183-208$ 


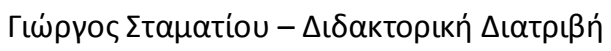

[13]. Sur la theorie de l'information dans le cas d'un alphabet abstrait. A. Perez. In Transactions First Prague Conf. on Information Theory, Stat. Decision Functions, Random Processes. Czech. Acad. Sci. Publishing House, 1957, pages 209-244

[14]. Sur la convergence des incertitudes, entropies et informations echantillon vers leurs valeurs vraies. A. Perez. In Transactions First Prague Conf. on Information Theory, Stat. Decision Functions, and Random Processes. Czech. Acad. Sci. Publishing House, 1957, pages 245-252

[15]. Dynamical systems with completely positive or zero entropy. M. S. Pinsker. Soviet Math. Dokl., 1:937-938, 1960

[16]. A general formulation of the fundamental Shannon theorem in information theory. R. L. Dobrushin. Uspehi Mat. Akad. Nauk. SSSR, 14:3-104, 1959.

[17]. On measures of information and entropy. A. Rényi (1961). Proceedings of the 4th Berkeley Symposium on Mathematics, Statistics and Probability 1960. pp. 547-561.

[18]. Alpha-divergences for Classification, Indexing and Retrieval. A. O. Hero, O.Michael and J. Gorman (2002), http://www. eecs.umich.edu/ hero/Preprints/cspl-328.pdf

[19]. On the dimension and entropy of probability distributions, A Renyi - Acta Mathematica Hungarica, 1959, vol. 10, no. 1-2, 193-215

[20]. Tsallis Entropy and Jaynes' Information Theory Formalism, A Plastino , Brazilian Journal of Physics, March, 1999, vol. 29, no. 1, 50-60

[21]. C. Tsallis, J. Stat. Phys., 1988, 52, 479

[22]. Introduction to the Theory of Error-Correcting Codes, Vera Pless (1982), John Wiley \& Sons, Inc.

[23]. Algebraic Coding Theory, Elwyn R. Berlekamp (1984), Aegean Park Press (revised edition) 
[24]. Channel capacity. Saleem Bhatti. Lecture notes for M.Sc. Data Communication Networks and Distributed Systems D51 -- Basic Communications and Networks.

[25]. Church 1934:90 footnote in Davis 1952

[26]. Turing 1936-7 in Davis 1952:149

[27]. Computability and $\lambda$-Definability, Turing, The Journal of Symbolic Logic, Dec. 1937, vol. 2, issue 4, 153-163

[28]. On computable numbers, with an application to the Entscheidungs problem, Turing, Proceedings of the London Mathematical Society, Ser. 2, 1937, vol. 42, 230-265

[29]. On Computable Numbers, with an Application to the Entscheidungs problem. A correction. Turing, Proceedings of the London Mathematical Society, 1937, Ser. 2, Vol. 43, $544-546$

[30]. Computation: finite and infinite machines, ML Minsky - 1967, Prentice-Hall, Inc, ISBN:0-13-165563-9.

[31]. After the Transistor, the Qubit?, Robert W. Keyes, Computing in Science and Engineering, Jan./Feb. 2005, vol. 7, no. 1, pp. 36-41

[32]. The Fight For The Best Quantum Bit (Qubit), Henrik Ingerslev Jørgensen, University of Copenhagen, 2008, June 27, http://www.sciencedaily.com

[33]. Quantum computation and quantum information, MA Nielsen, I Chuang, LK Grover - American Journal of Physics, 2002, vol. 70, issue 5, 558-559 and ref[30],pg.53 in thesis

[34]. Quantum mechanical Hamiltonian models of Turing machines, P Benioff - Journal of Statistical Physics, 1982 - Springer, vol. 29, no. 9,515-546

[35]. Quantum theory, the Church-Turing principle and the universal quantum computer, D Deutsch - Proceedings of the Royal Society of London. Series A, ..., 1985, vol. 400, 97117 


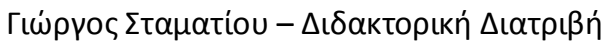

[36]. Quantum complexity theory, E Bernstein, U Vazirani Proceedings of the twenty-fifth annual ..., 1993, 11-20

[37]. Experimental Aspects of Quantum Computing, Henry 0 . Everitt (Editor), Springer, April 1, 2005, ISBN-10: 0387230459

[38]. Experimental Quantum Computation and Information, F. De Martini and C. Monroe (editors), International School of Physics Enrico Fermi, vol.148, 165-196.

[39]. Decoherence, einselection, and the quantum origins of the classical, WH Zurek - Reviews of Modern Physics, 2003, vol.75, issue 3, 715-775

[40]. Two-bit gates are universal for quantum computation, DP DiVincenzo - Physical Review A, 1995, vol.51, issue 2, 1015-1022

[41]. Elementary gates for quantum computation, A Barenco, $\mathrm{CH}$ Bennett, R Cleve, DP DiVincenzo, $\mathrm{N}_{\text {... - Physical Review }}$ A, 1995, vol. 52, issue 5, 3457-3467

[42]. A silicon-based nuclear spin quantum computer, BE Kane - Nature, 1998, vol. 393, 133-137

[43]. On the power of quantum computation, DR Simon - SIAM Journal on Computing, 1997, 26(5), 1474-1483

[44]. Experimental realization of Deutsch's algorithm in a one-way quantum computer, MS Tame, R Prevedel, M Paternostro, P Böhi, MS ... - Physical review letters, 2007, vol. 98, issue 14, 14501

[45]. Simple quantum computer, IL Chuang, Y Yamamoto Physical Review A, 1995, vol. 52, issue 5, 3489-3496

[46]. Quantum computation and Shor's factoring algorithm, A Ekert, R Jozsa - Reviews of Modern Physics, 1996, vol. 68, issue 3, 733-753

[47]. Algorithms for quantum computation: Discrete logarithms and factoring, PW Shor - ANNUAL SYMPOSIUM ON FOUNDATIONS OF ..., 1994, 124-134 
[48]. Polynomial-Time Algorithms for Prime Factorization and Discrete Logarithms on a Quantum Computer, PW Shor SIAM Review, 1999, vol. 41, no. 2, 303-332

[49]. Quantum entropy and its use, M Ohya, D Petz Springer, 2004, ISBN 3-540-20806-2

[50]. Inequalities for quantum entropy: A review with conditions for equality, MB Ruskai - Journal of Mathematical Physics, 2002, vol. 43, issue 9, 4358

[51]. A fundamental property of quantum-mechanical entropy, EH Lieb, MB Ruskai - Physical Review Letters, 1973, vol. 30 , issue $10,434-436$

[52]. Linear entropy as an Entanglement measure in twofermion systems, F Buscemi, P Bordone, A Bertoni - Physical Review A, 2007, vol. 75, issue 3, 032301

[53]. Decoherence and linear entropy increase in the quantum baker's map, AN Soklakov, R Schack - Physical Review E, 2002, vol.66, issue 3, 036212

[54]. The proper formula for relative entropy and its asymptotics in quantum probability, F Hiai, D Petz Communications in Mathematical Physics, 1991, vol. 143, no. $1, \quad 99-114$

[55]. The role of relative entropy in quantum information theory, V Vedral - Reviews of Modern Physics, 2002, vol. 74, issue 1, 197-234

[56]. Bounds for the quantity of information transmitted by a quantum communication ..., AS Holevo - Problemy Peredachi Informatsii, 1973, vol. 9, issue 3, 197-234

[57]. The capacity of the quantum depolarizing channel, C King - IEEE Transactions on Information Theory, 2003, vol. 49 , no. 1, 221-229

[58]. Entropy exchange and Entanglement in the JaynesCummings model, E Boukobza, DJ Tannor - Physical Review A, 2005, vol. 71, issue 6, 063821 


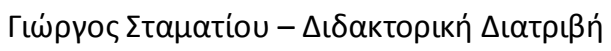

[59]. Conditional entropies and their relation to Entanglement criteria, KGH Vollbrecht, MM Wolf - Journal of Mathematical Physics, 2002, vol. 43, issue 9, 4299

[60]. Fidelity for mixed quantum states, R Jozsa - Journal of Modern Optics, 1994, vol. 41, issue 12, 2315-2323

[61]. Distance measures to compare real and ideal quantum processes, A Gilchrist, NK Langford, MA Nielsen - Physical Review A, 2005, vol. 71, issue 6, 062310

[62]. The capacity of the quantum channel with general signal states, AS Holevo - IEEE Transactions on Information Theory, 1998, vol. 44, no. 1, 269-273

[63]. Tema con variazioni: quantum channel capacity, D Kretschmann, RF Werner - New Journal of Physics, 2004 , vol. 6,26

[64]. Classical information capacity of a quantum channel, P Hausladen, R Jozsa, B Schumacher, M ... - Physical Review A, 1996, vol. 54, issue 3, 1869-1876

[65]. On capacity of a quantum communications channel, AS Holevo - Problemy Peredachi Informatsii, 1979, vol. 15, issue $4,3-11$

[66]. Experimental Entanglement of four particles, CA Sackett, D Kielpinski, BE King, C Langer, $V$ Meyer, ... Nature, 2000, vol. 404, 256-259

[67]. Quantum states with Einstein-Podolsky-Rosen correlations admitting a hidden-variable model, R.F. Werner, Phys. Rev. A 40, 4277 (1989).

[68]. Quantum Entanglement, $R$ Horodecki, P Horodecki, M Horodecki, K ... - Reviews of Modern ..., 2009, vol. 81, issue $2, \quad 865-942$

[69]. Experimental long-lived Entanglement of two macroscopic objects, B Julsgaard, A Kozhekin, ES Polzik Nature, 2001, vol. 413, 400-403 
[70]. Experimental demonstration of five-photon Entanglement and open-destination ..., $Z$ Zhao, YA Chen, AN Zhang, T Yang, HJ Briegel, JW ... - Nature, 2004, vol. 430, $54-58$

[71]. Entangled quantum systems and the Schmidt decomposition, A Ekert, PL Knight - American Journal of Physics, 1995, vol. 63, issue 5, 415-423

[72]. Separability criterion for density matrices, A Peres - Physical Review Letters, 1996, vol. 77, issue 8, 14131415

[73]. Distinguishing separable and entangled states, AC Doherty, PA Parrilo, FM Spedalieri - Physical Review Letters, 2002, vol. 88, issue 18, 187904

[74]. Separability of mixed states: necessary and sufficient Conditions, M Horodecki, $\mathrm{P}$ Horodecki, $\mathrm{R}$ Horodecki - Physics Letters A, 1996, vol. 223, issue 1-2, $1-8$

[75]. Inseparability criteria for continuous bipartite quantum states, E Shchukin, W Vogel - Physical review letters, 2005, vol. 95, issue 23, 230502

[76]. Majorization criterion for distillability of a bipartite quantum state, T Hiroshima - Physical review letters, 2003, vol. 91, issue 5, 057902

[77]. Majorization and the interconversion of bipartite states, MA Nielsen, G Vidal - Quantum information and computation, 2001, vol. 1, no. 1, 76-93

[78]. Entanglement measures and purification procedures, V Vedral, MB Plenio - Physical Review A, 1998, vol. 57, issue $3, \quad 1619-1633$

[79]. Quantifying Entanglement, $\mathrm{V}$ Vedral, MB Plenio, MA Rippin, PL Knight - Physical Review Letters, 1997, vol. 78, issue $12,2275-2279$ 


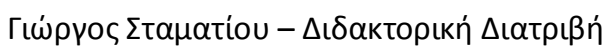

[80]. An introduction to Entanglement measures, MB Plenio, S Virmani - Quantum information \& computation, 2007, 7,1, http://arxiv.org/abs/quant-ph/0504163

[81]. ENTANGLEMENT OF FORMATION, WK Wootters - Quantum communication, computing and ..., 2000 - Springer, part I, 69-73, ISBN: 978-0-306-46307-5

[82]. Entanglement of formation of an arbitrary state of two qubits, WK Wootters - Physical Review Letters, 1998, vol. 80, issue 10, 2245-2248

[83]. Entanglement of formation for symmetric Gaussian states, G Giedke, MM Wolf, O Krüger, RF Werner, JI Cirac Physical review letters, 2003, vol. 91, issue 10, 107901

[84]. Entanglement of formation for isotropic states, BM Terhal, KGH Vollbrecht - Physical Review Letters, 2000, vol. 85, issue 12, 2625-2628

[85]. Entanglement of a pair of quantum bits, S Hill, WK Wootters - Physical Review Letters, 1997, vol. 78, issue $26, \quad 5022-5025$

[86]. On strong superadditivity of the Entanglement of formation, KMR Audenaert, SL Braunstein - Communications in Mathematical ..., 2004 - Springer, vol. 246, no. 3, 443-452

[87]. Information, relative entropy of Entanglement, and irreversibility, L Henderson, V Vedral - Physical Review Letters, 2000, vol. 84, issue 10, 2263-2266

[88]. Asymptotic relative entropy of Entanglement, $K$ Audenaert, $J$ Eisert, $E$ Jane, MB Plenio, S Virmani, ... Physical Review Letters, 2001, vol. 87, issue 21, 217902

[89]. Bounds on relative entropy of Entanglement for multiparty systems, MB Plenio, V Vedral - Journal of Physics A: Mathematical and General, 2001, vol. 34, no. 35, 6997

[90]. Calculating the relative entropy of Entanglement, S Wu, Y Zhang 1997, http://arxiv.org/abs/quant-ph/0004018 
[91]. Logarithmic negativity: a full Entanglement monotone that is not convex, MB Plenio - Physical review letters, 2005, vol. 95, issue 9, 090503

[92]. Comparison of the relative entropy of Entanglement and negativity, A Miranowicz, S Ishizaka, B Horst, A Grudka - Physical Review A, 2008, vol. 78, issue 5, 052308

[93]. Negativity, Entanglement witness and quantum phase transition, $X$ Wang, SJ Gu - Journal of Physics A: Mathematical and Theoretical, 2007, vol. 40, no. 35, 10759

[94]. A comparison of the Entanglement measures negativity and concurrence, F Verstraete, K Audenaert, J Dehaene, B De ... - Journal of Physics A: ..., 2001, vol. 34, no. 47, 10327

[95]. A new class of Entanglement measures, O Rudolph Journal of Mathematical Physics, 2001, vol. 42, issue 11, 5306

[96]. "Squashed Entanglement": An additive Entanglement measure, M Christandl, A Winter - Journal of Mathematical Physics, 2004, vol. 45, issue 3, 829

[97]. Squashed Entanglement for multipartite states and Entanglement measures based ..., D Yang, K Horodecki, M Horodecki, P ... - Arxiv preprint , 2007, http: //arxiv.org/abs/arXiv:0704.2236

[98]. Mathematical Logic, Stephen Cole, Kleene - 1967, Dover Publications Inc., ISBN: 0486425339

[99]. On Universal and Fault-Tolerant Quantum Computing, Boykin P. Oscar, Mor Tal, Pulver Matthew, RoychowdhuryVwani, Vatan Farrokh, 1999, eprint arXiv:quantph/9906054 



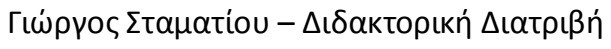

ENOTHTA $\Gamma^{\prime}$

¿TOIXEIA

KBANTIKOY XAOY 



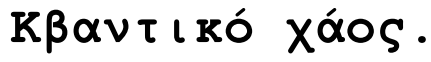

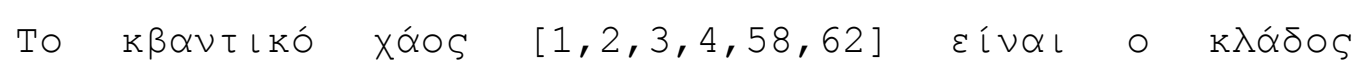

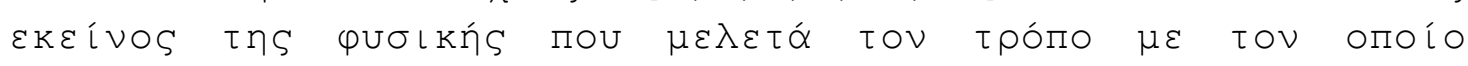

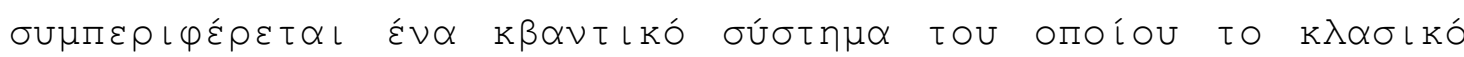

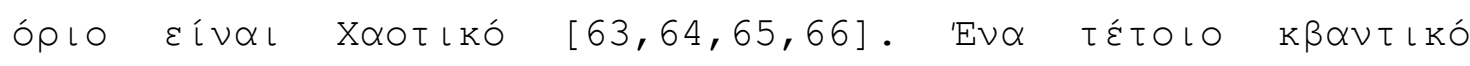

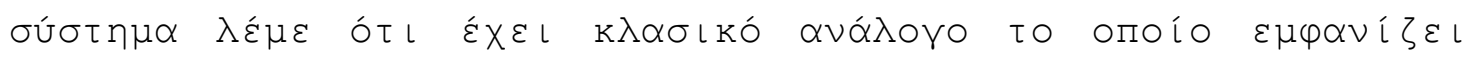

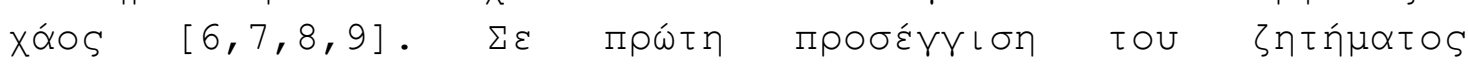

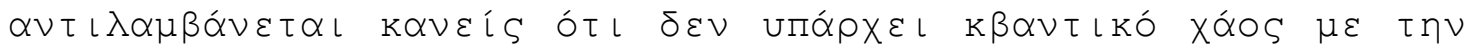

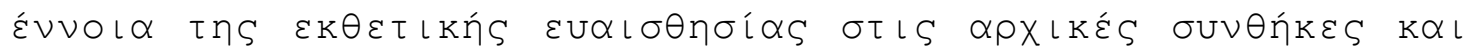

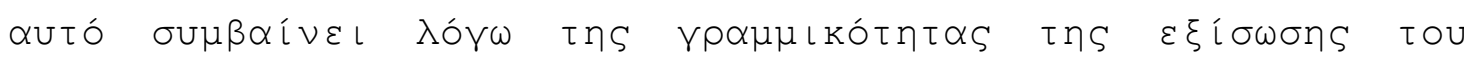

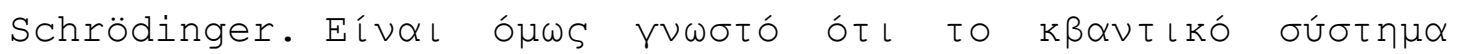

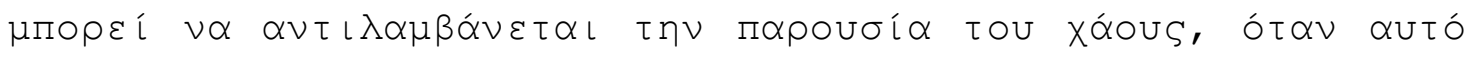

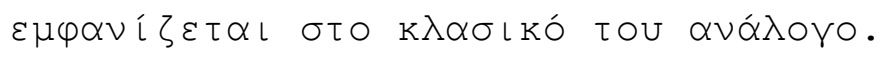

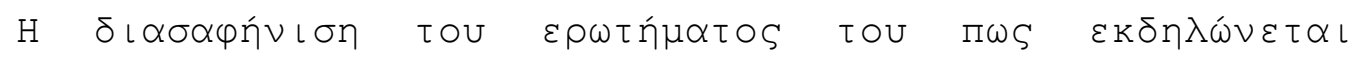

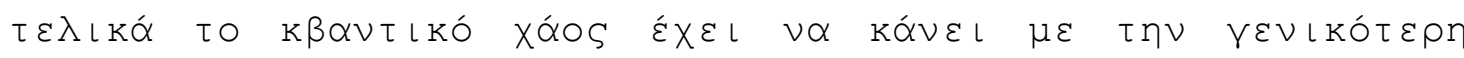

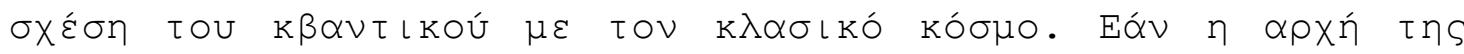

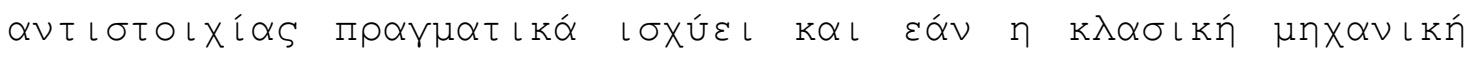

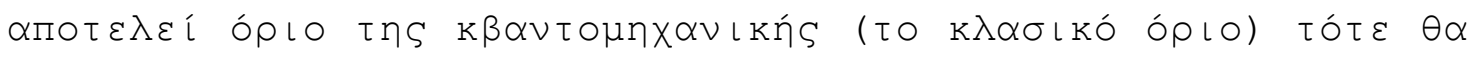

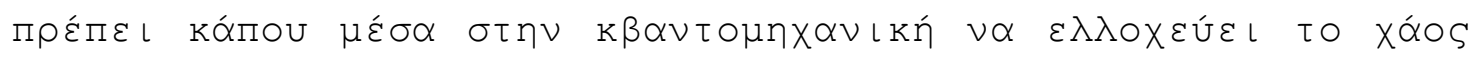

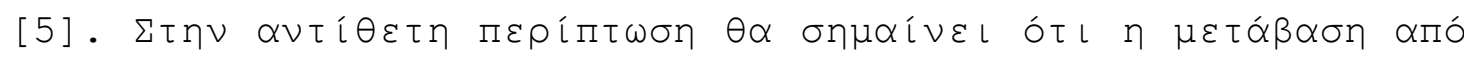

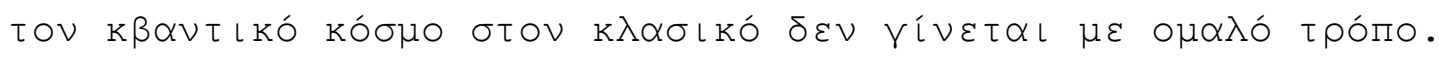

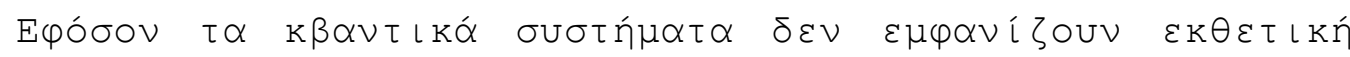

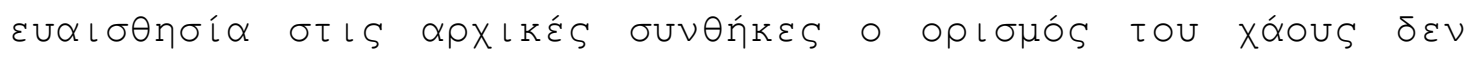

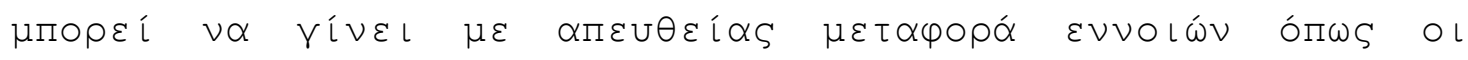

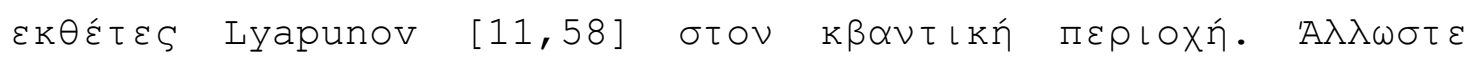

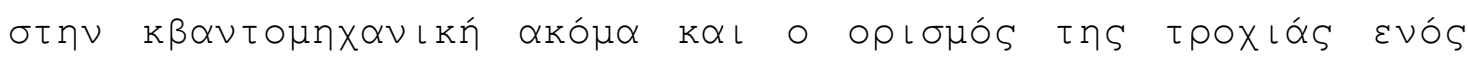

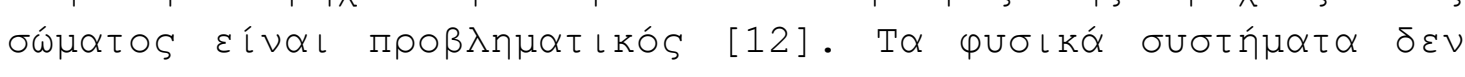

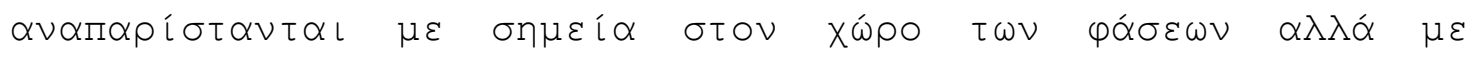

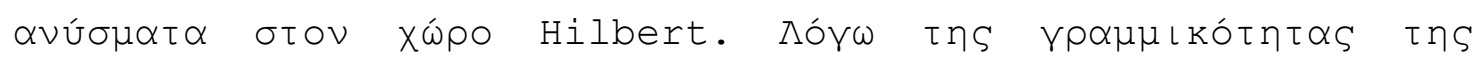

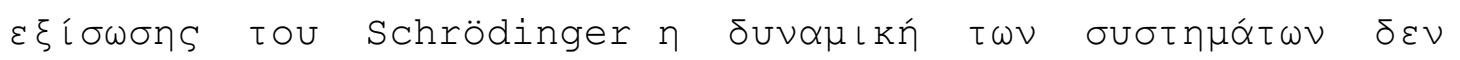

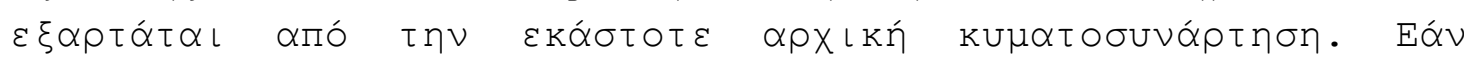

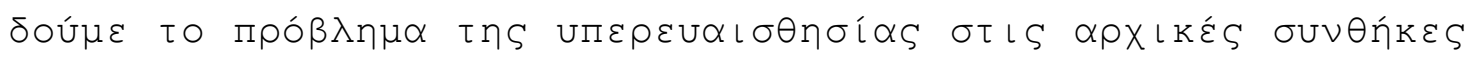

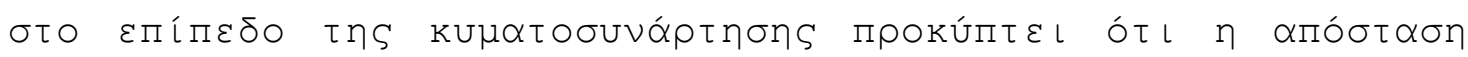

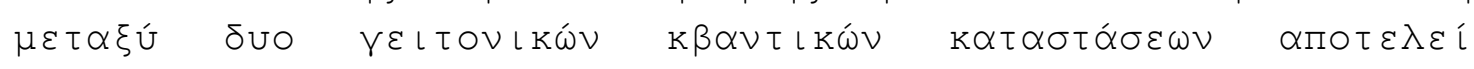

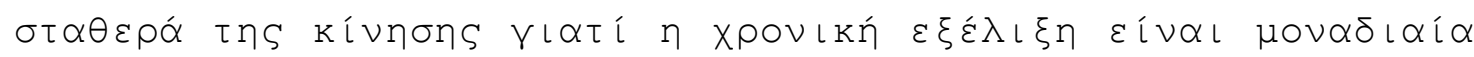

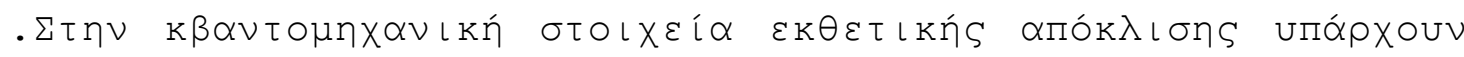

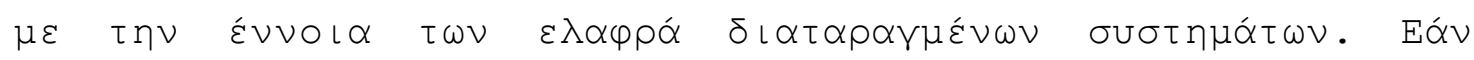

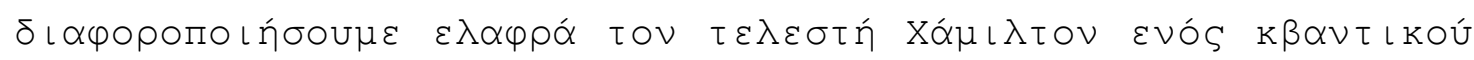

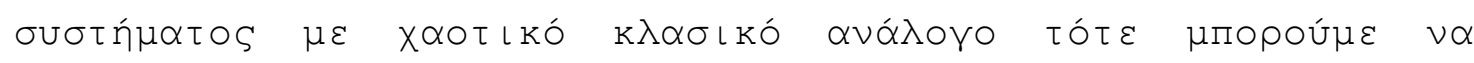




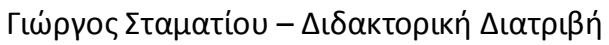

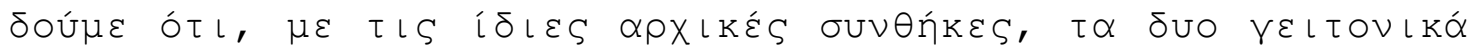

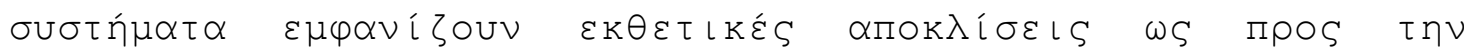

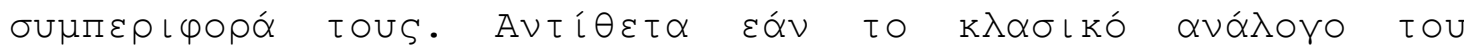

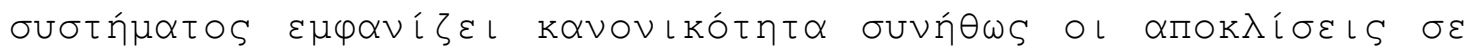

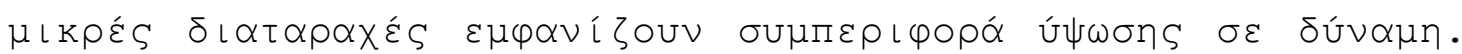

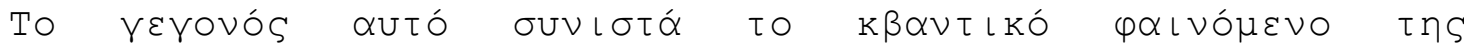
$\Pi \varepsilon \tau \alpha \lambda \circ u ́ \delta \alpha \varsigma$.

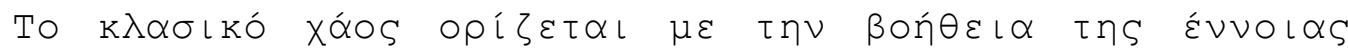

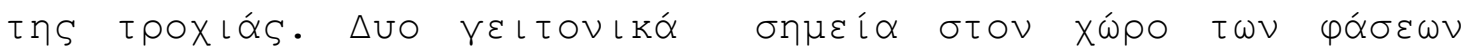

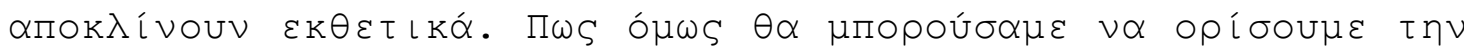

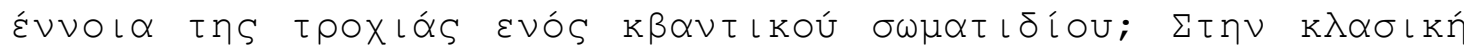

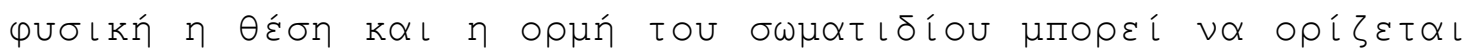

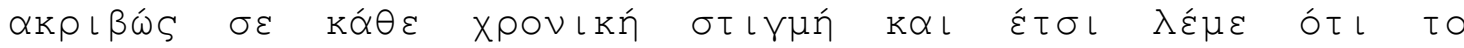

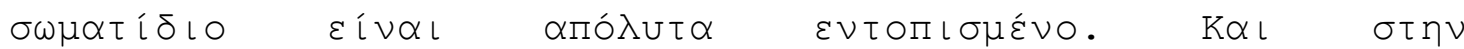

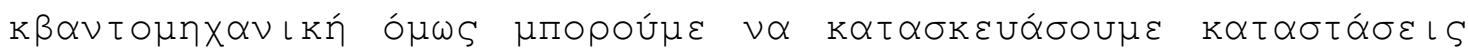

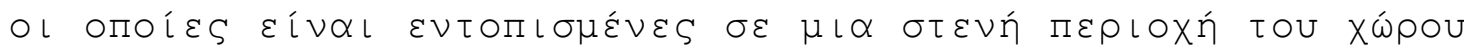

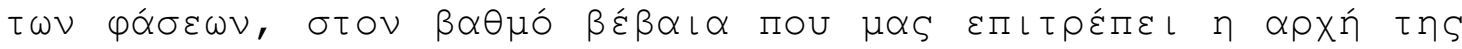

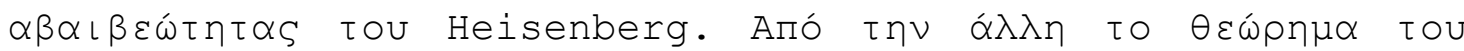

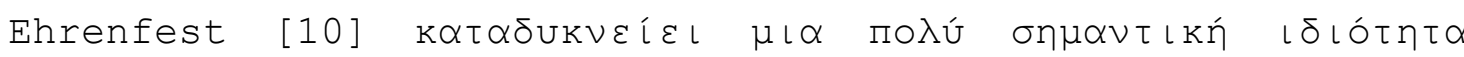

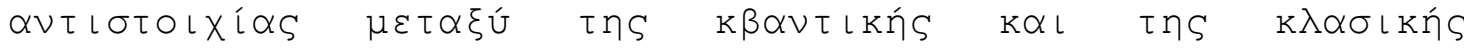

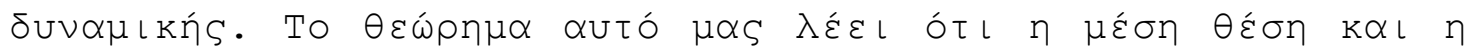

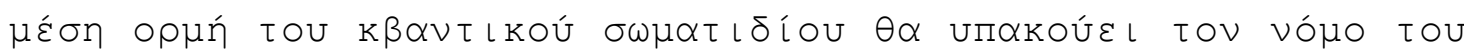

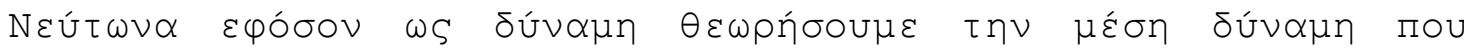

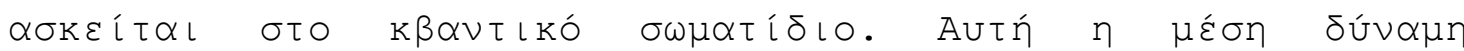
(

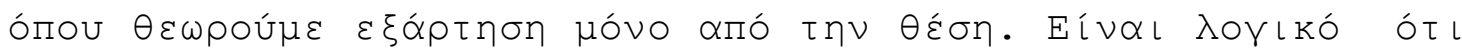

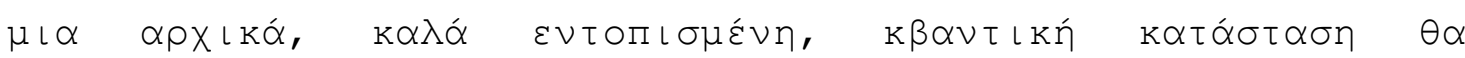

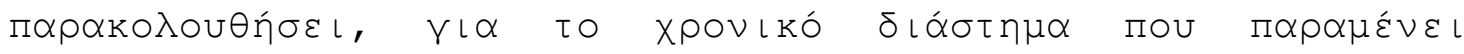

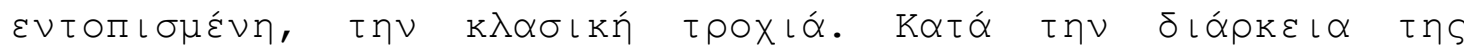

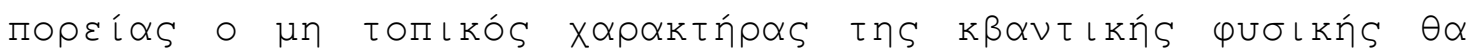

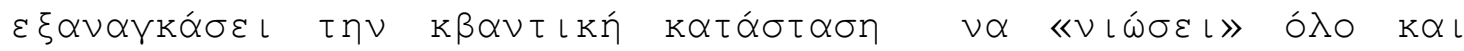

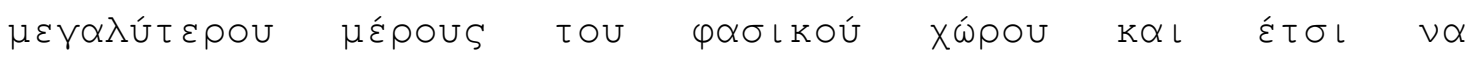

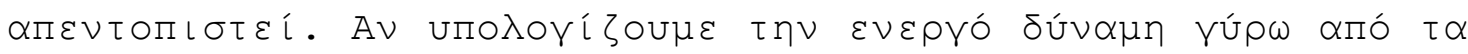

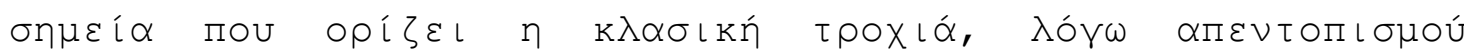

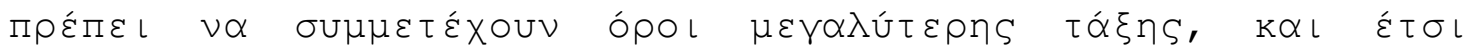

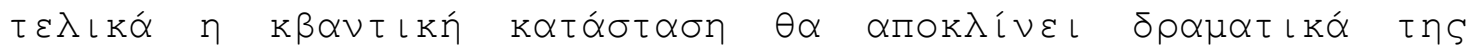

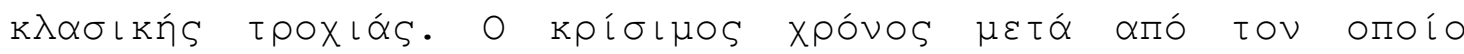

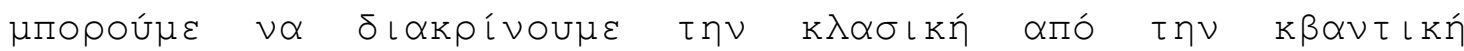

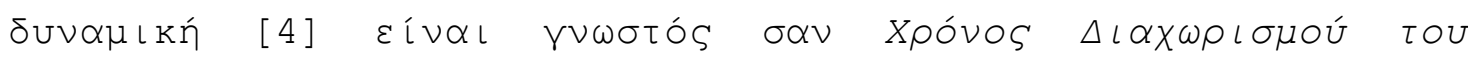

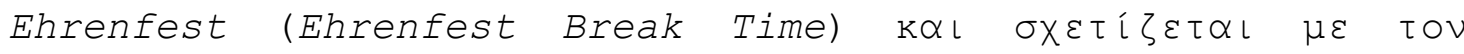




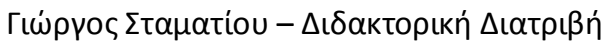

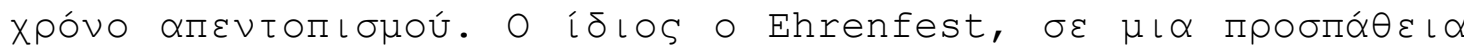

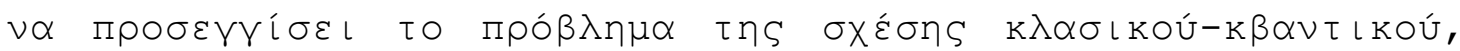

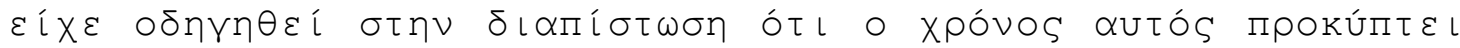

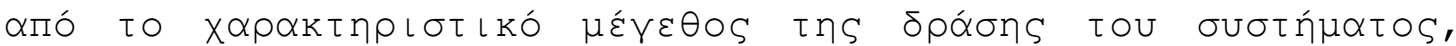

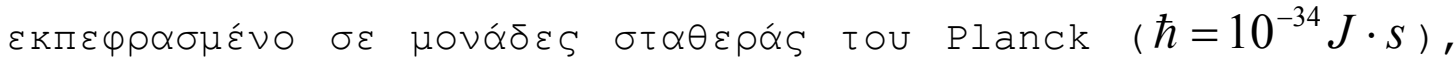

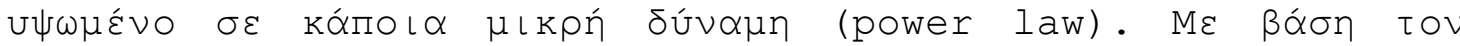

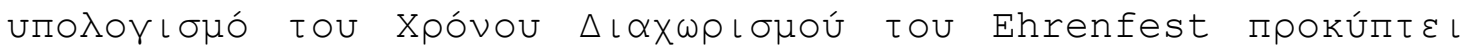

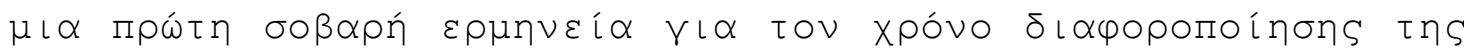

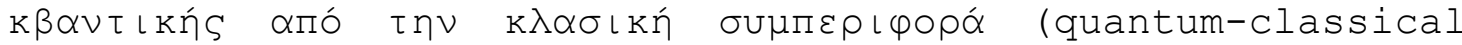

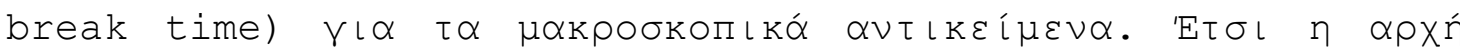

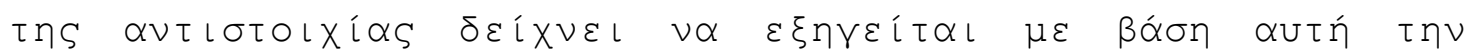

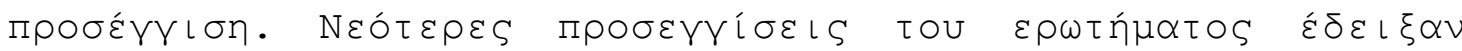

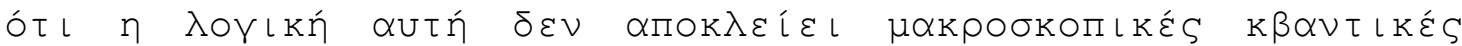

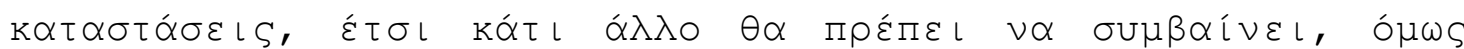

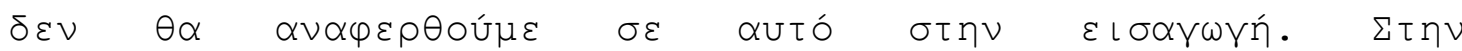

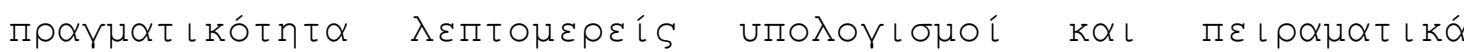

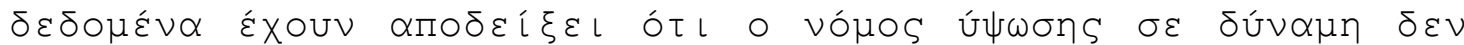

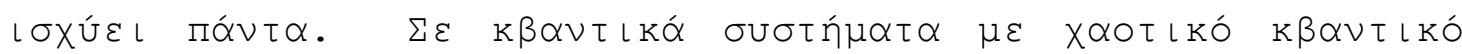

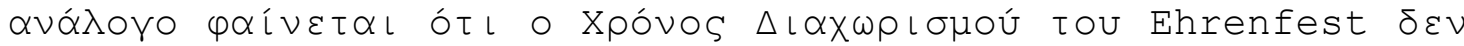

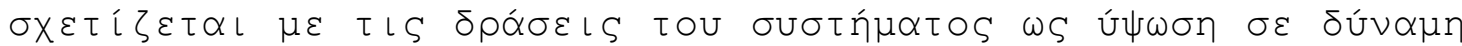

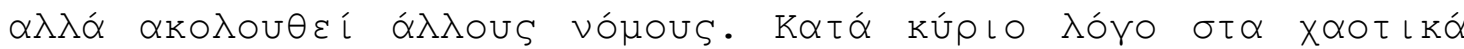

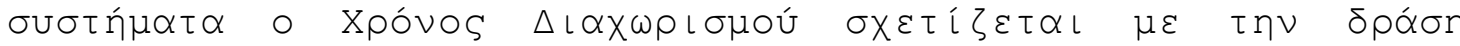

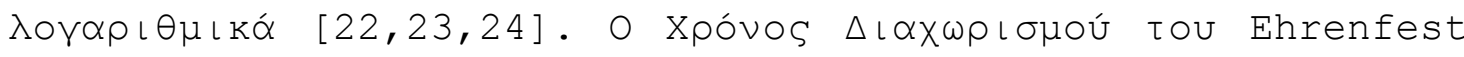

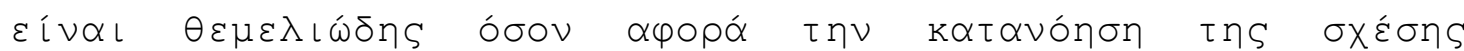

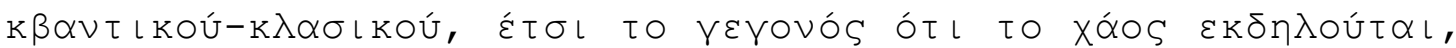

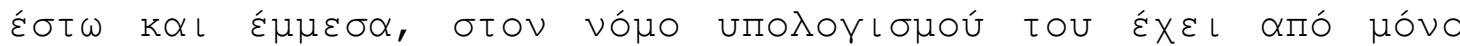

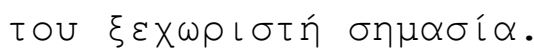

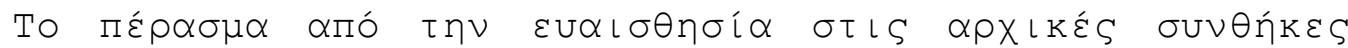

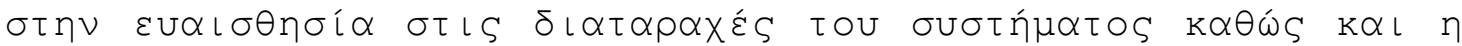

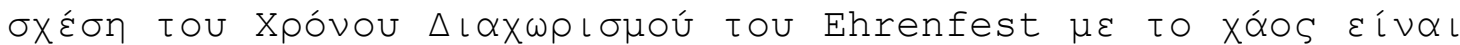
ov

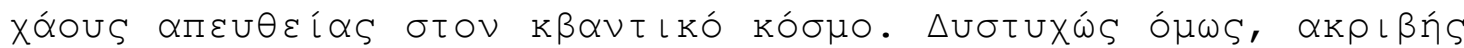

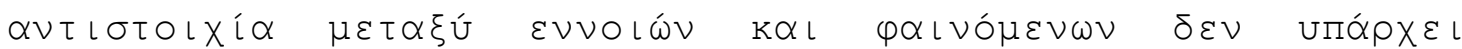

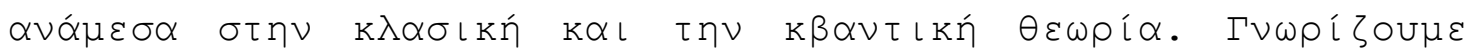

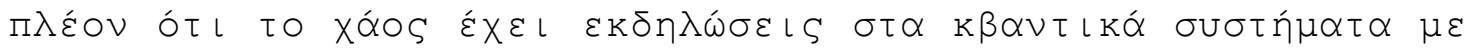

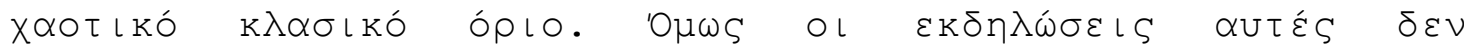

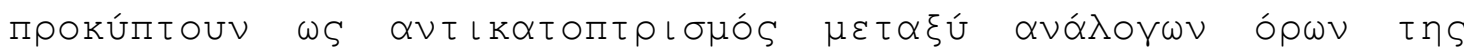

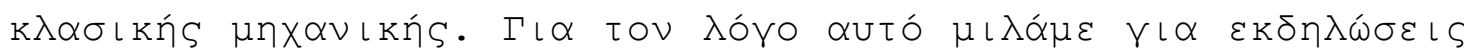

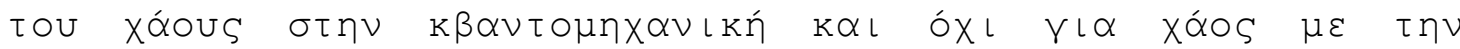

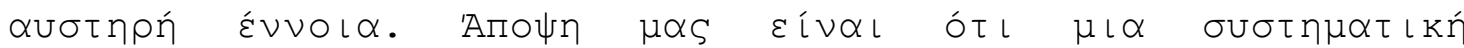




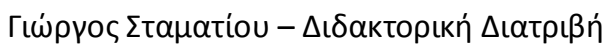

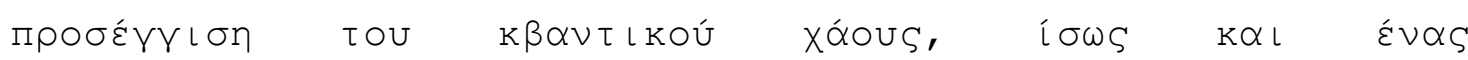

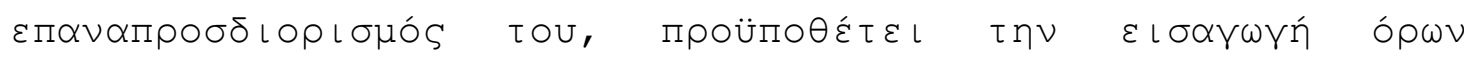

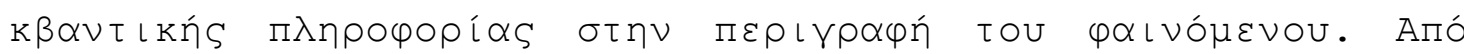

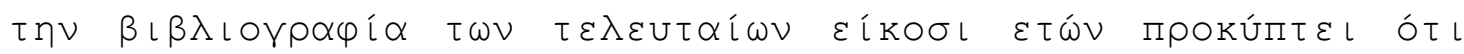

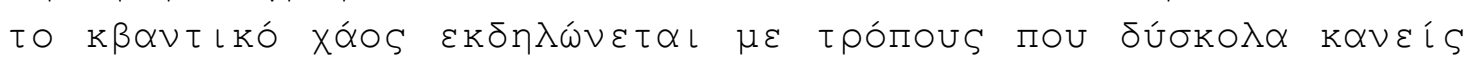

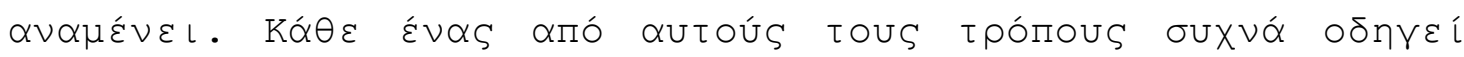

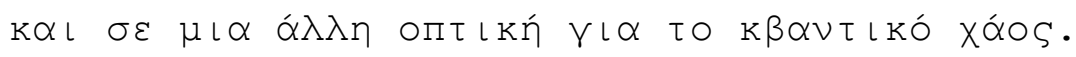

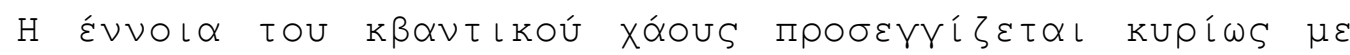

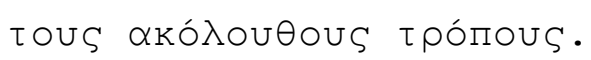

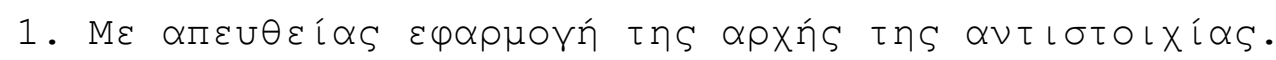

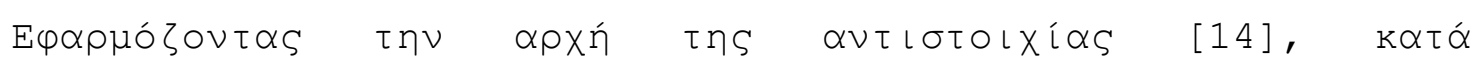

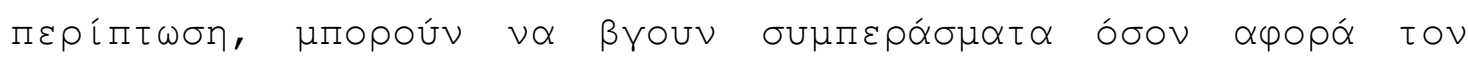

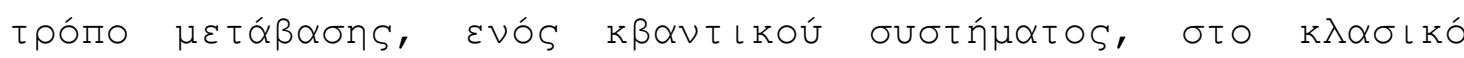
Xóos.

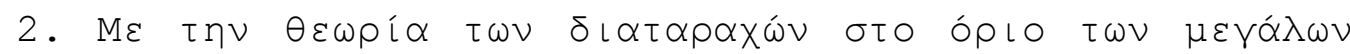

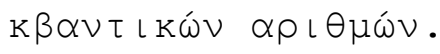

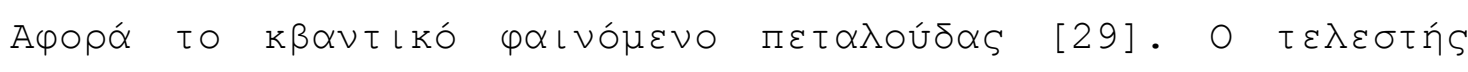

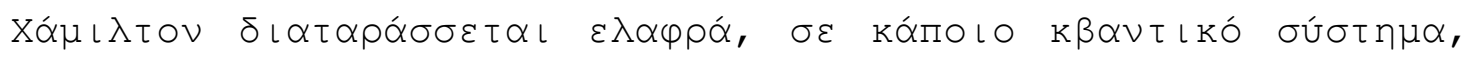

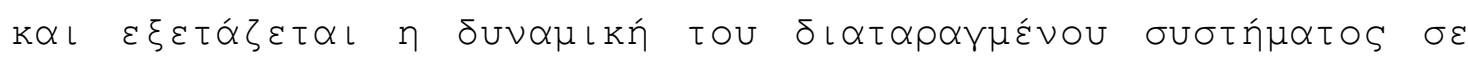

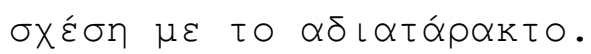

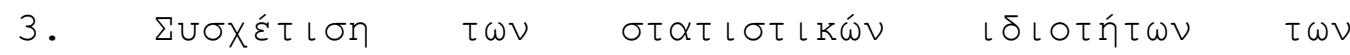

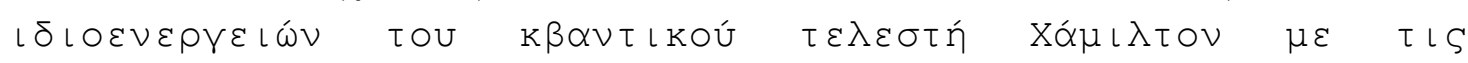

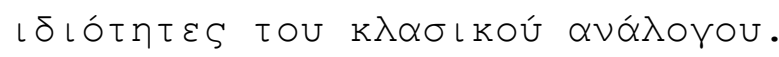

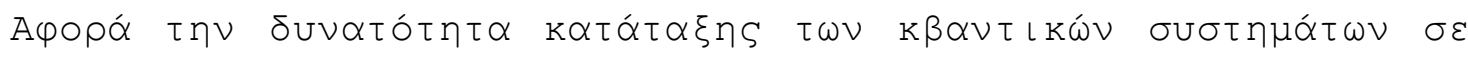

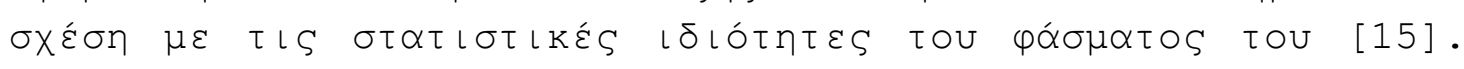
'EX

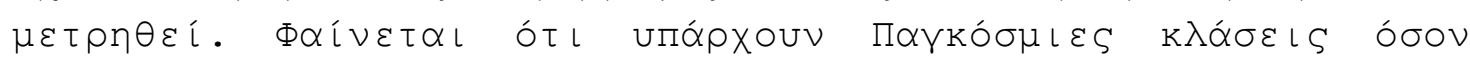

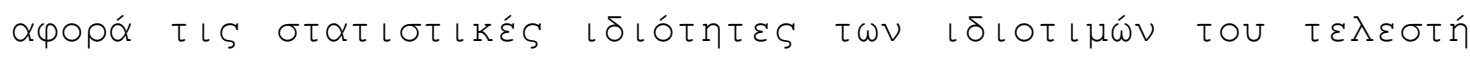

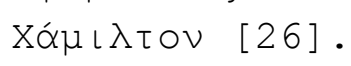

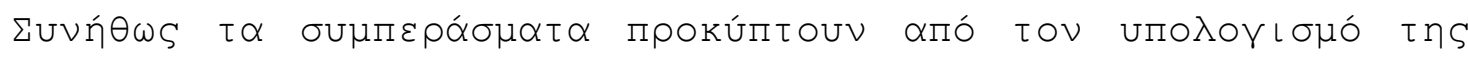

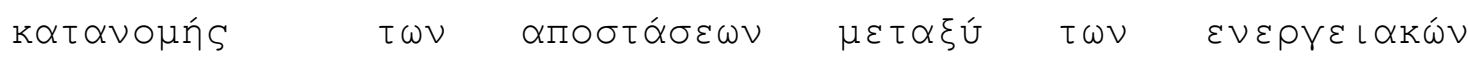

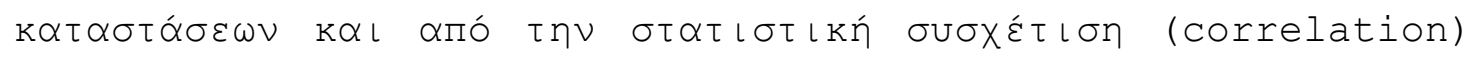

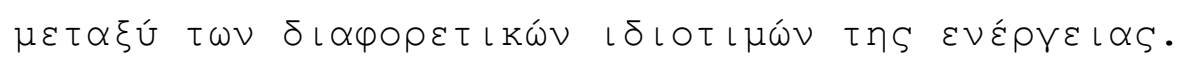




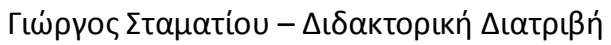

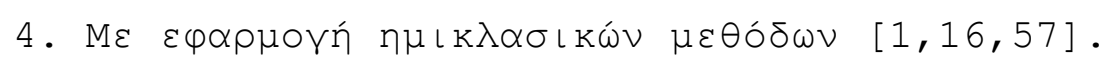

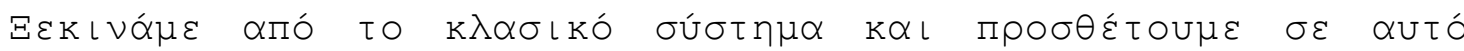

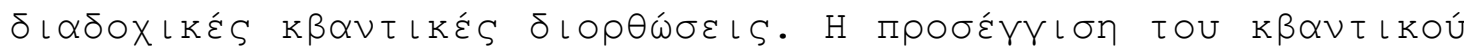

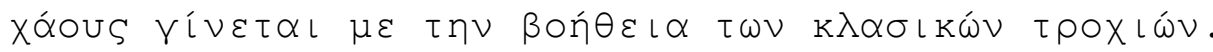

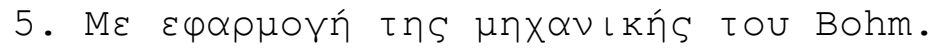

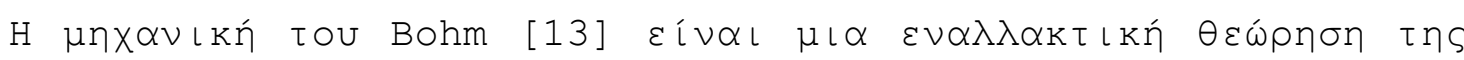

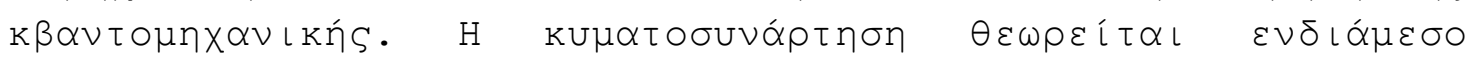

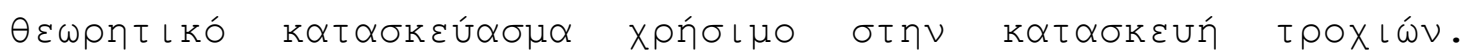

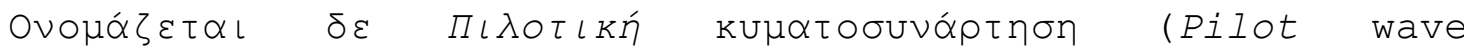

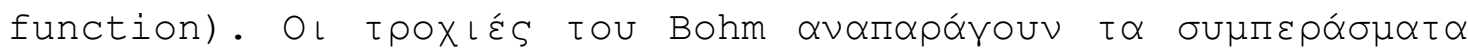

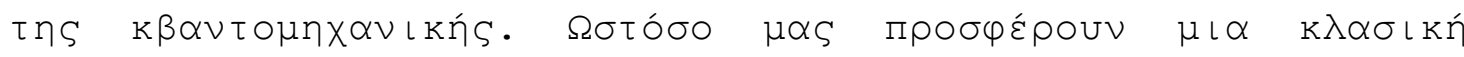

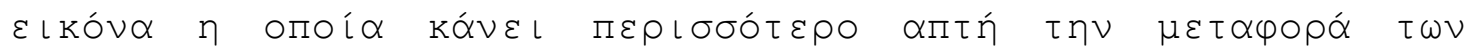

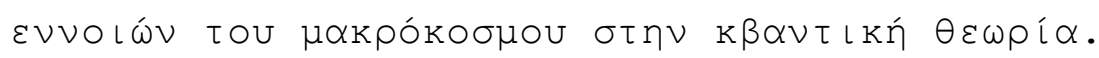

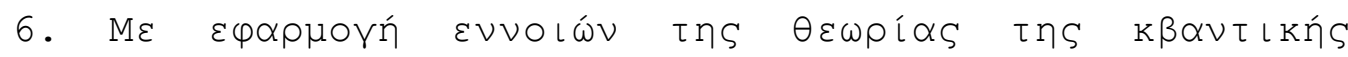

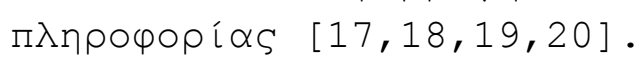

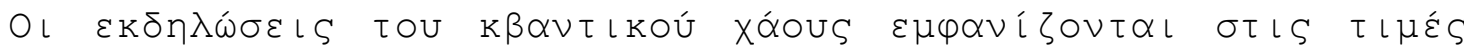

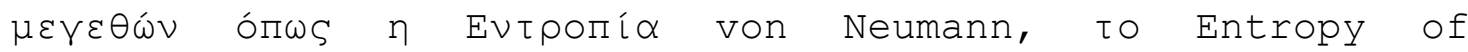

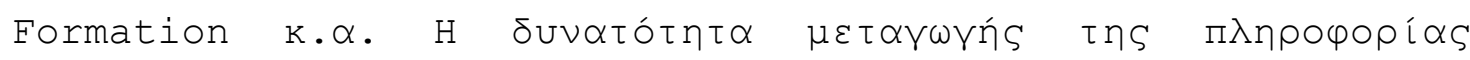

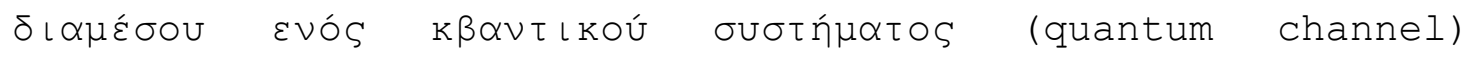

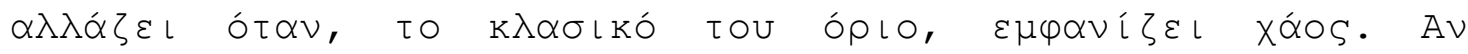

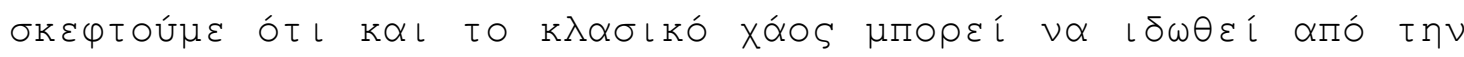

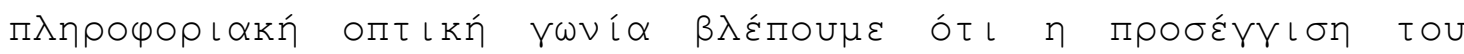

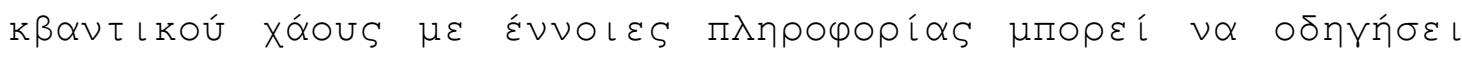

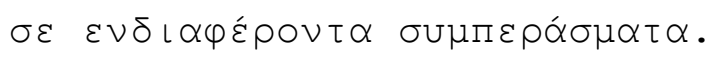

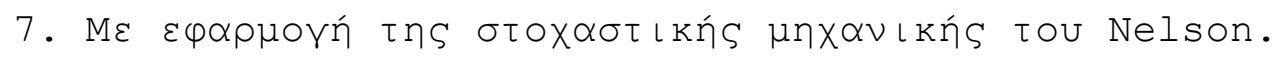

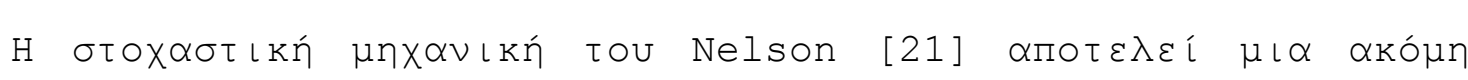

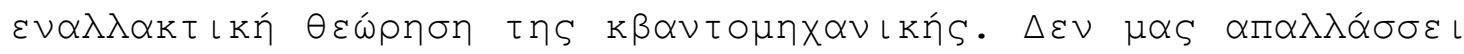

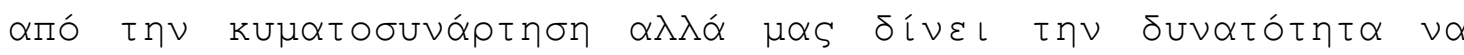

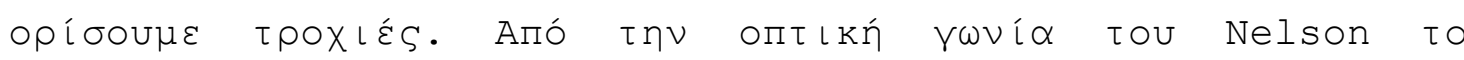

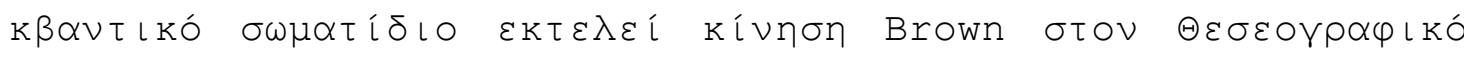
xẃpo.

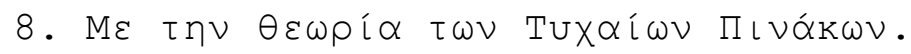

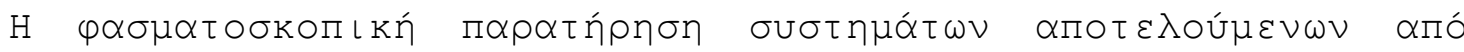

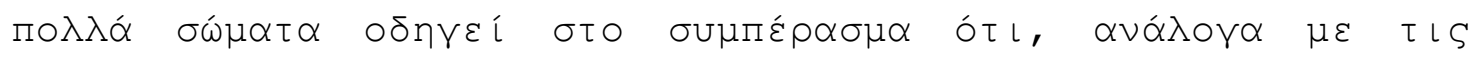




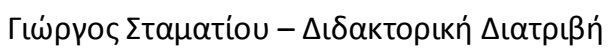

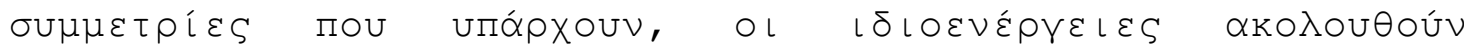

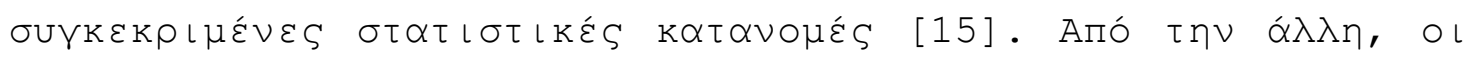

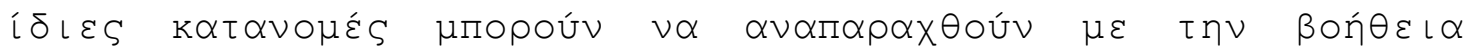

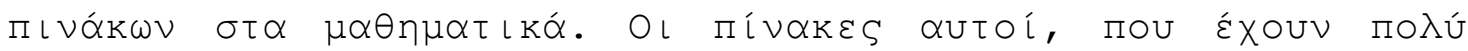

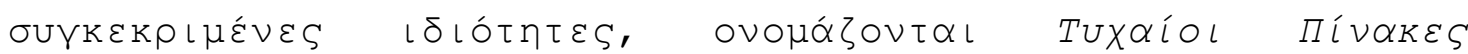

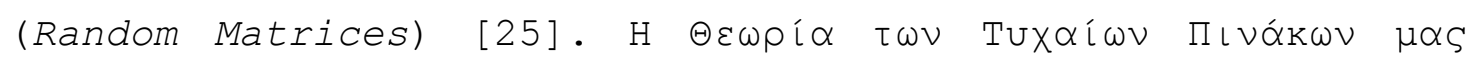

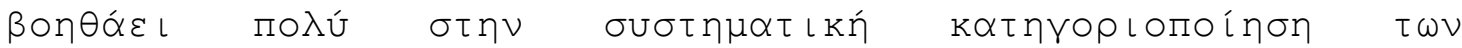

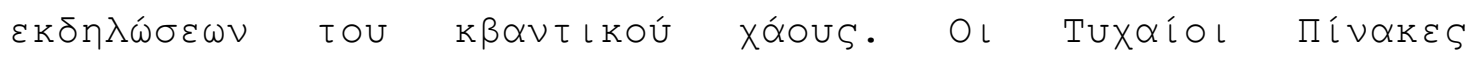

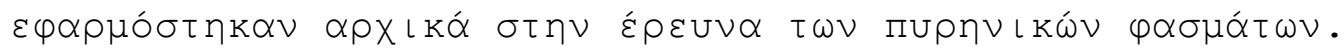

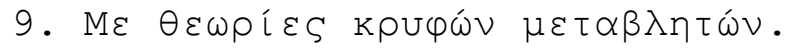

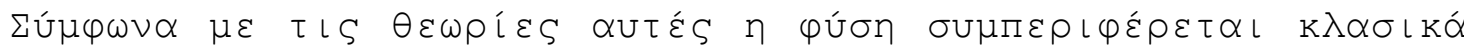

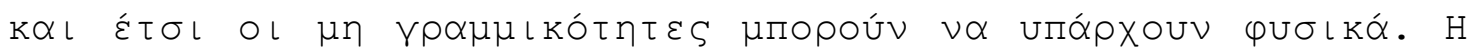

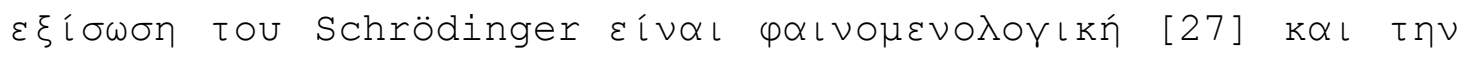

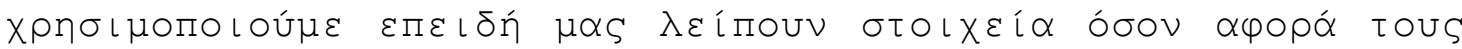

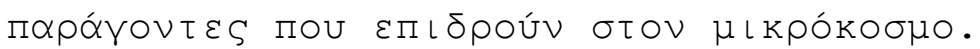

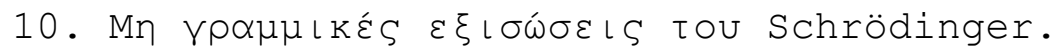

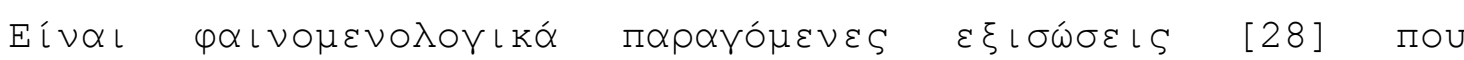

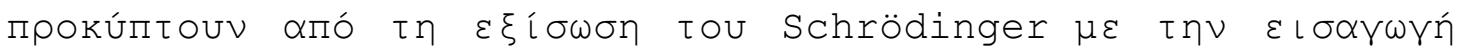

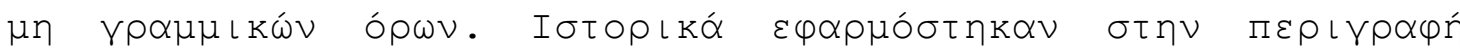

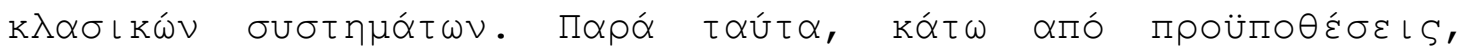

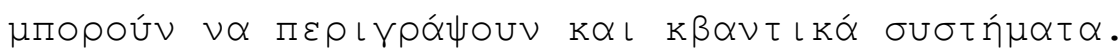

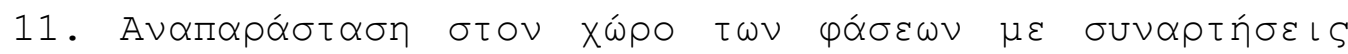
$\psi \varepsilon \cup \delta \circ-\Pi \iota \theta \alpha \nu o ́ t \eta \tau \alpha_{S}[30,31]$.

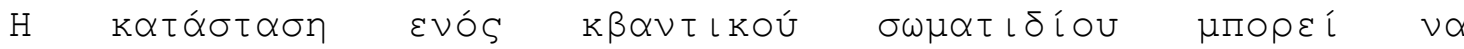

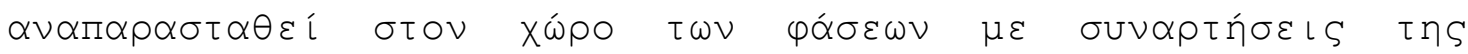

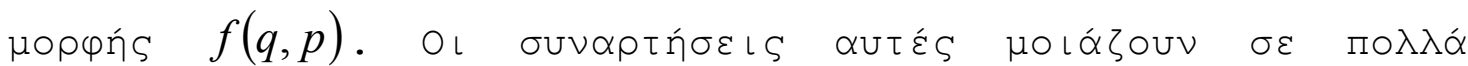

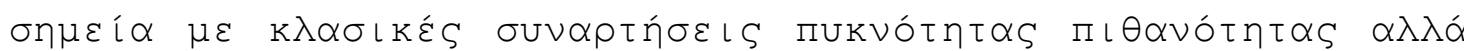

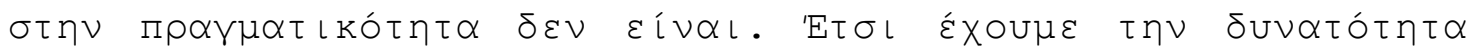

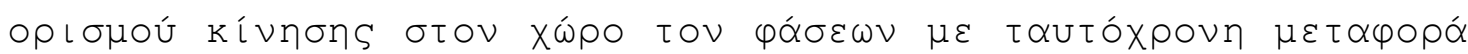

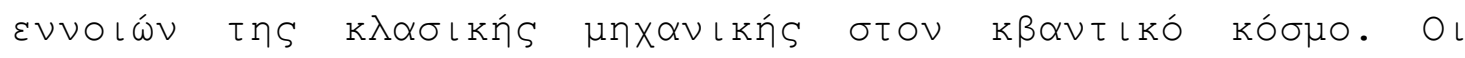

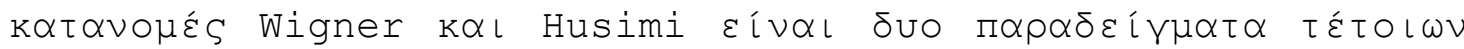

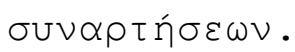

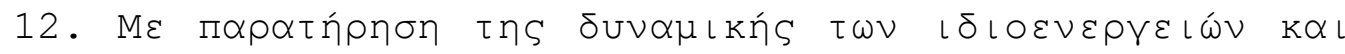
$\tau \omega \nu$ ı $\delta\llcorner O K \alpha \tau \alpha \sigma \tau \alpha ́ \alpha \varepsilon \omega \nu$. 


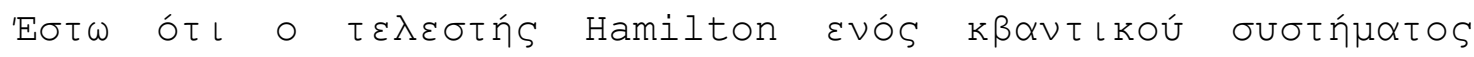

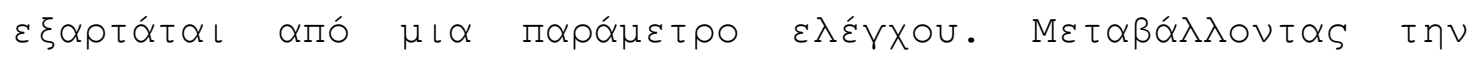

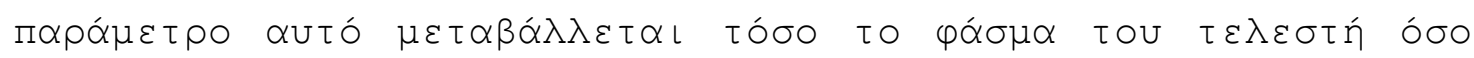

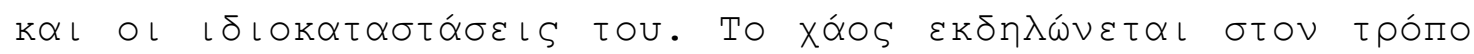

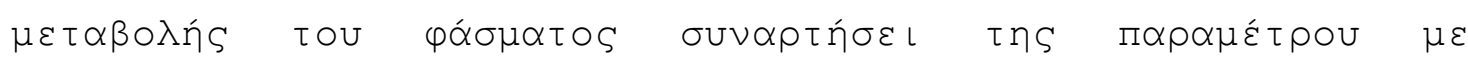

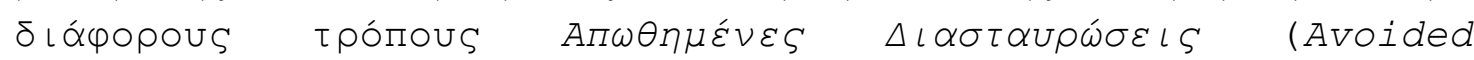

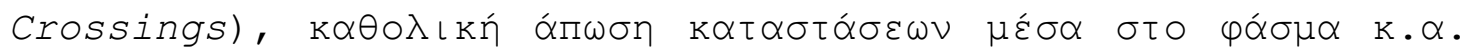

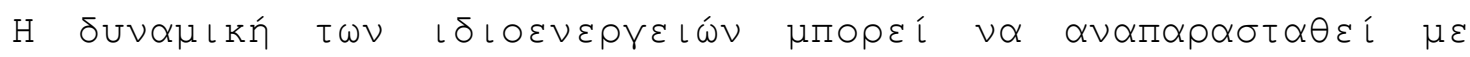

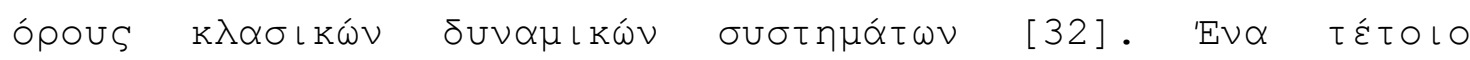

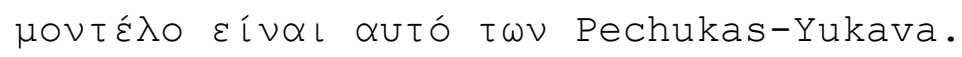

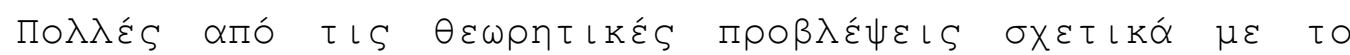

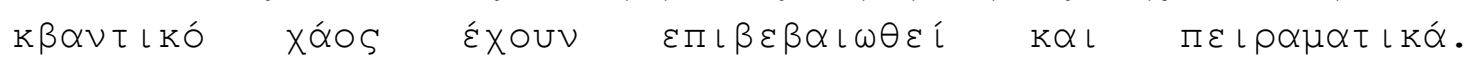

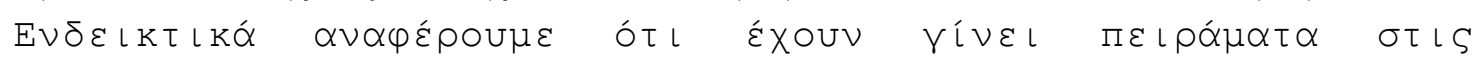

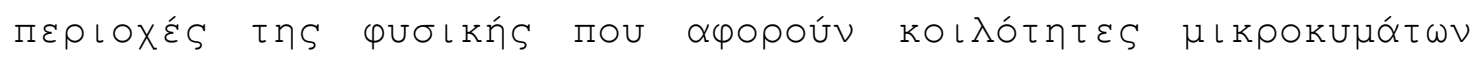

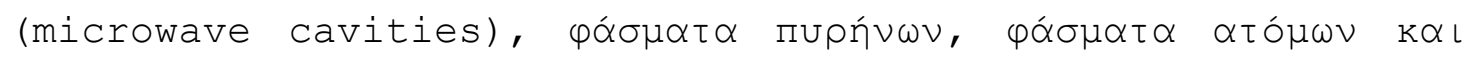

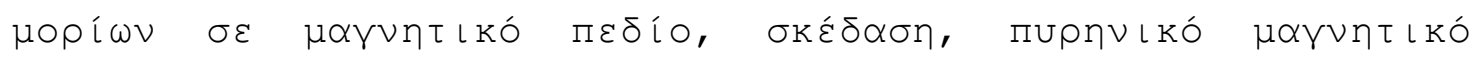

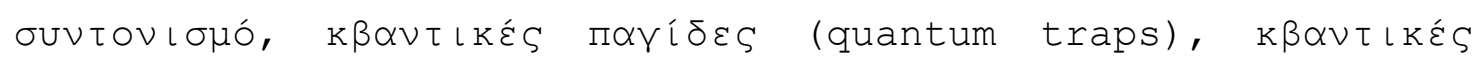

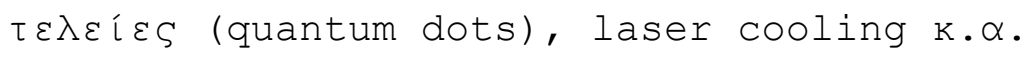

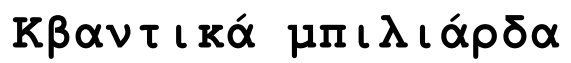

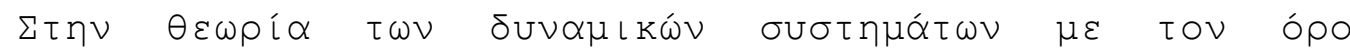

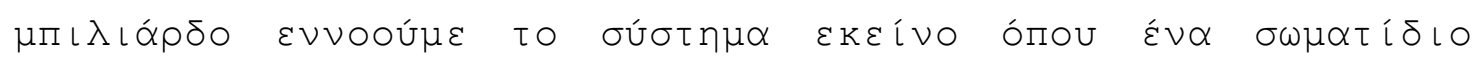

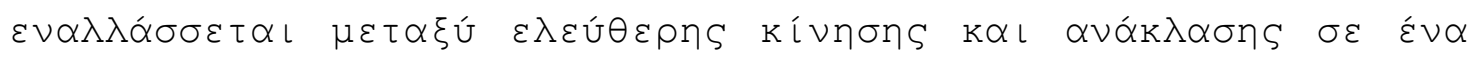

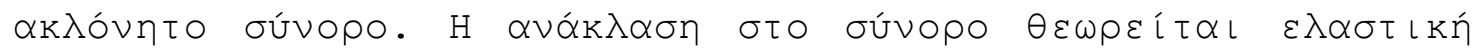

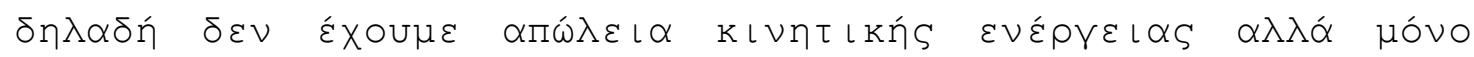

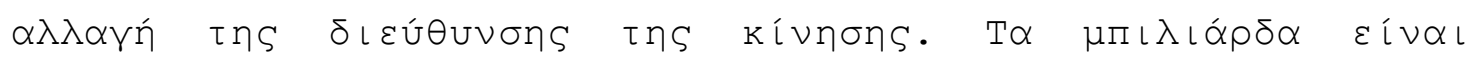

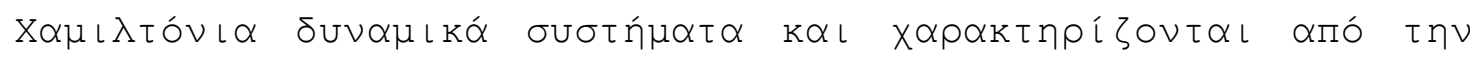

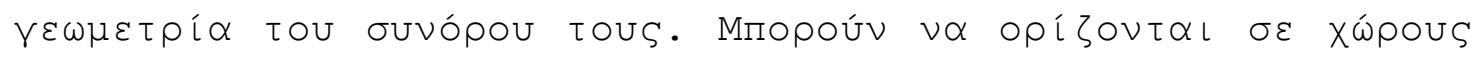

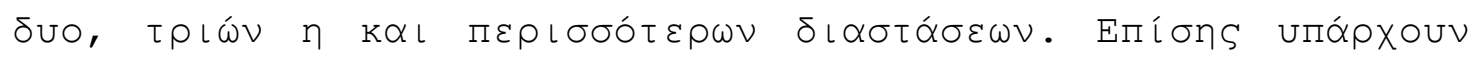

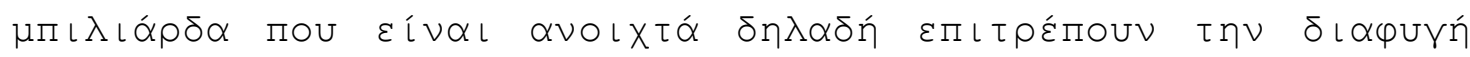

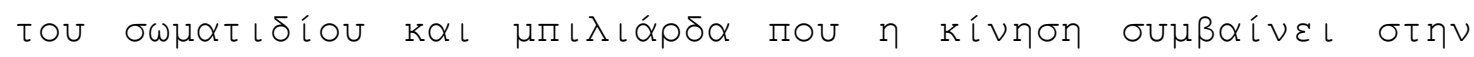

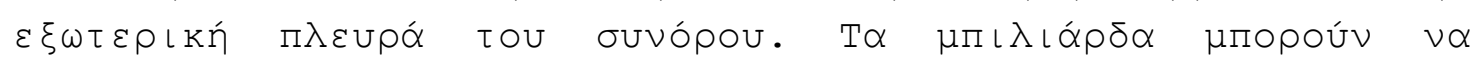

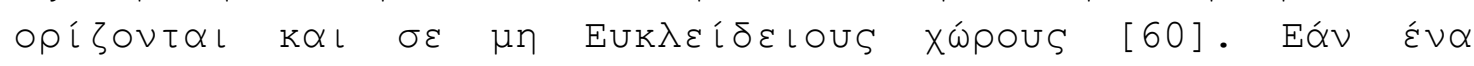

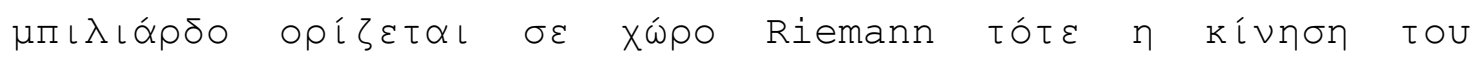

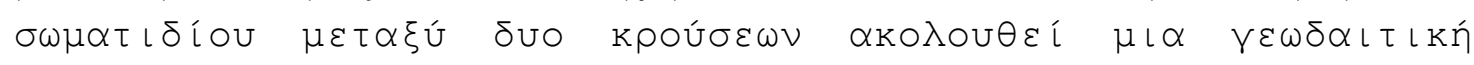

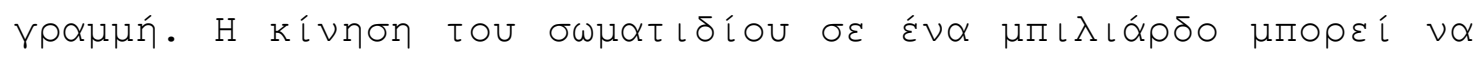

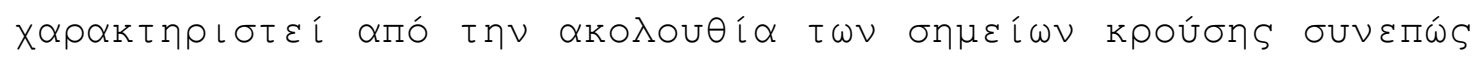

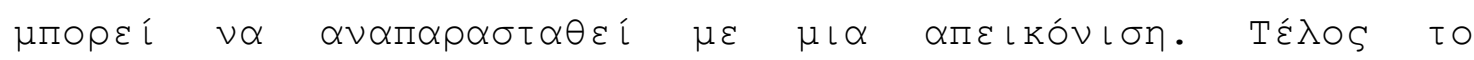




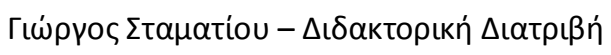

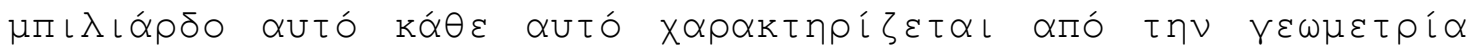

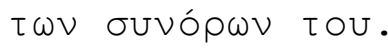

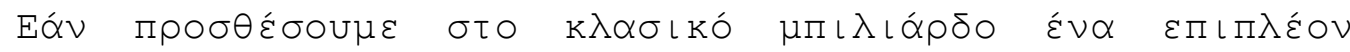

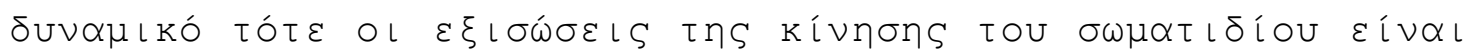
Ol $\varepsilon \xi n ́ S:$

$$
\begin{gathered}
H(p, q)=\frac{p^{2}}{2 m}+V(q) \\
\text { к ८ } V(q)=\infty \quad q \notin \Omega
\end{gathered}
$$

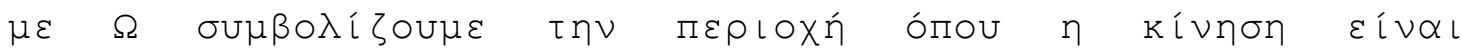
$\varepsilon \Pi \iota \tau \rho \varepsilon \Pi \tau \dot{\text {. }}$

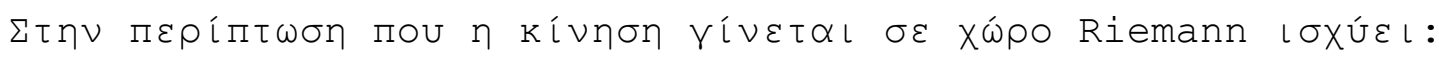

$$
H(p, q)=\frac{1}{2 m} \sum_{i j} p_{i} p_{j} g_{i j}(q)+V(q)
$$

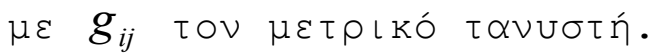

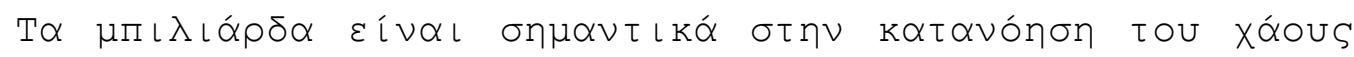

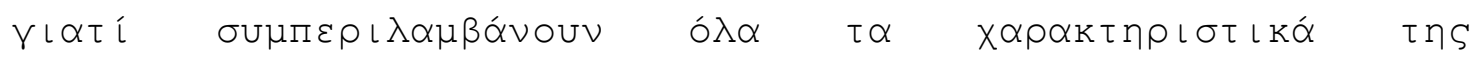

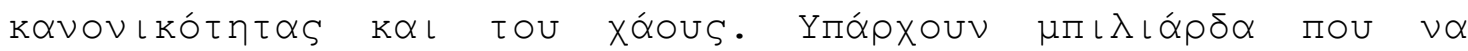

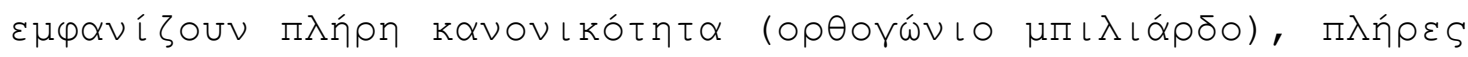

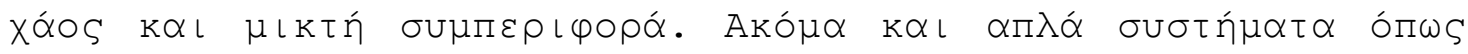

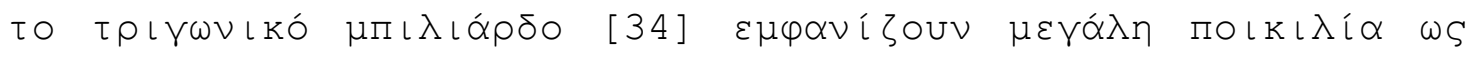

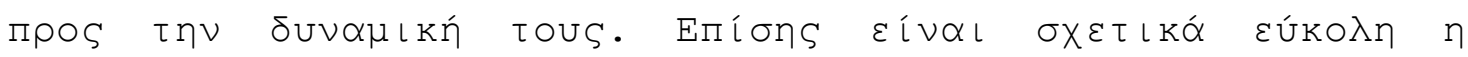

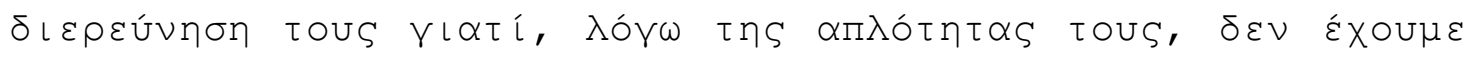

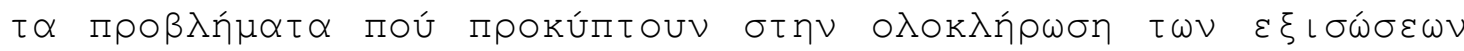

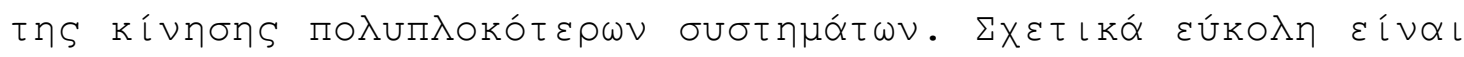

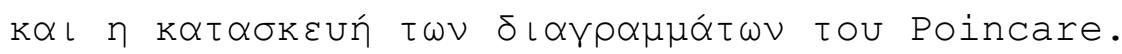




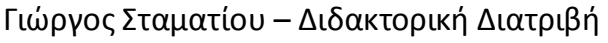

[http://commons.wikimedia.org/wiki/File:BunimovichStadium.png]

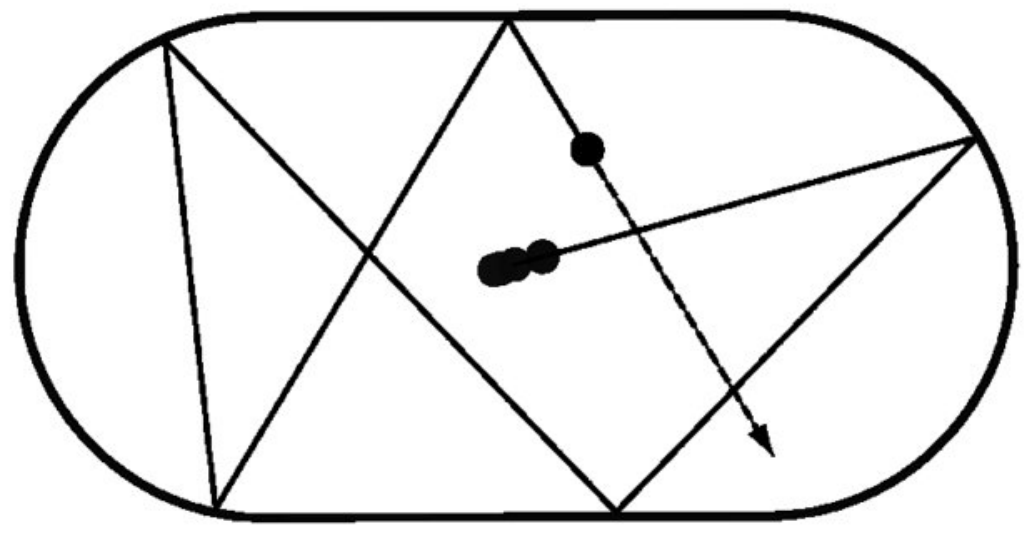

Bunimovich stadium $[\beta \lambda . \Pi \alpha \rho \alpha \rho \tau \eta \mu \alpha]$

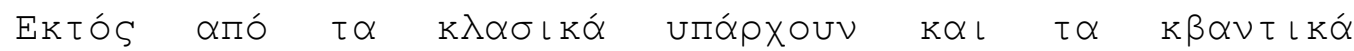

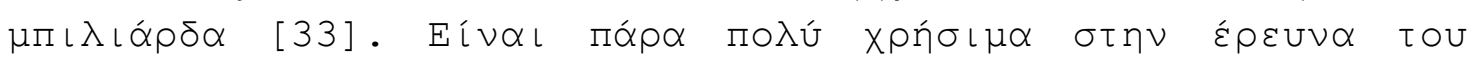

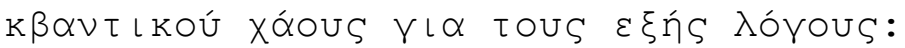

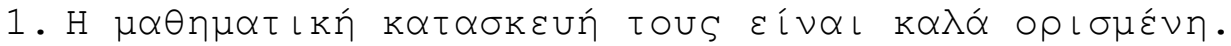

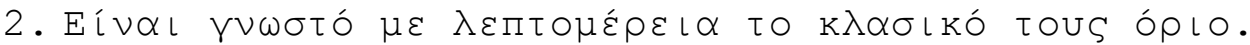

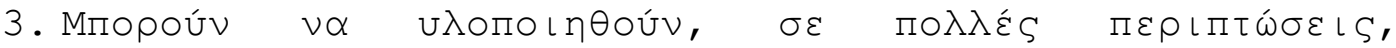

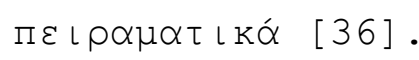

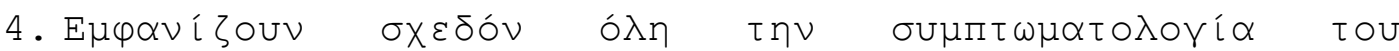

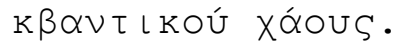

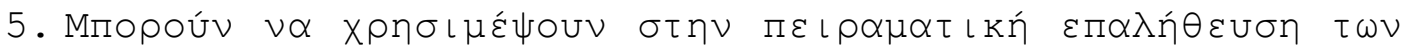

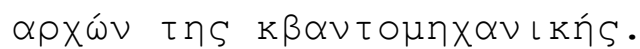

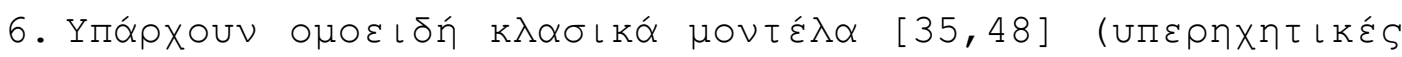

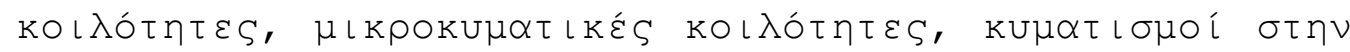

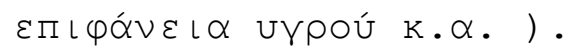




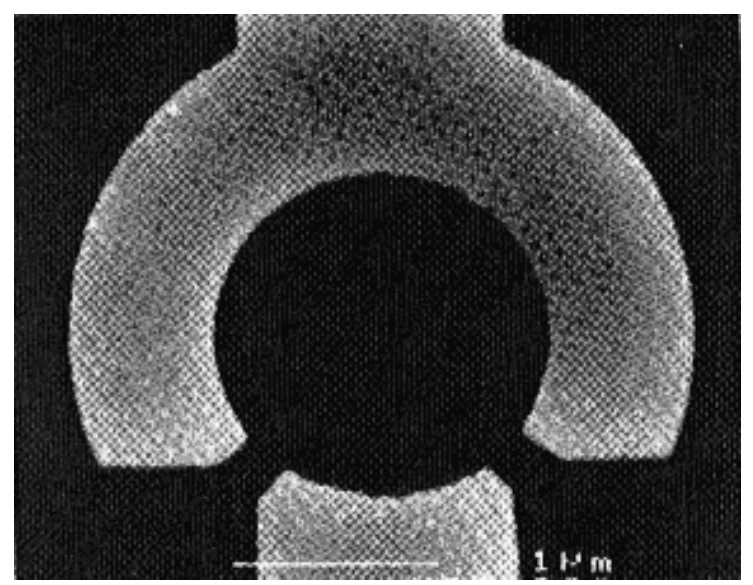

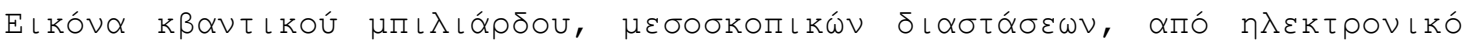

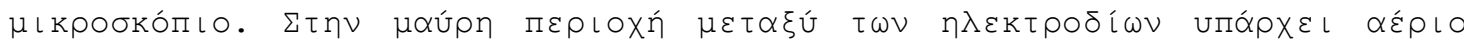

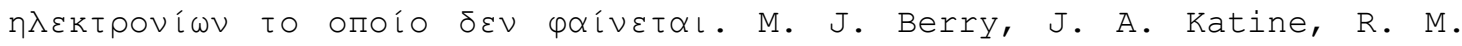
Westervelt, and A. C. Gossard, Phys. Rev. B 50, 17721 (1994).

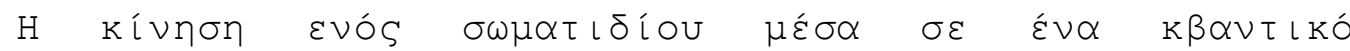

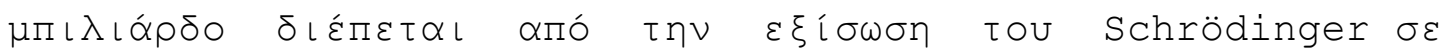

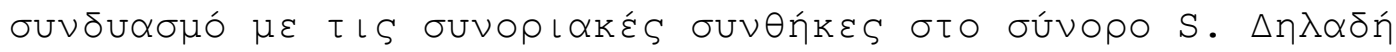

$$
\begin{aligned}
& -\frac{\hbar^{2}}{2 m} \nabla^{2} \psi=i \hbar \frac{\partial \psi}{\partial t}
\end{aligned}
$$

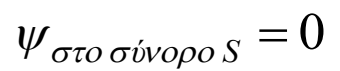

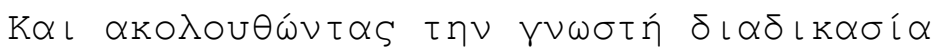

$$
\psi_{n}(x, t)=\psi_{n}(x) e^{i \omega_{n} t}
$$

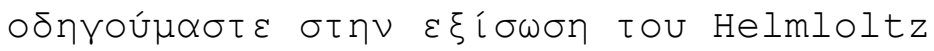

$$
\begin{gathered}
\left(\nabla^{2}+k_{n}^{2}\right) \psi_{n}(x)=0 \\
\omega_{n}=\frac{\hbar}{2 m} k_{n}^{2}
\end{gathered}
$$




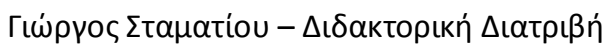

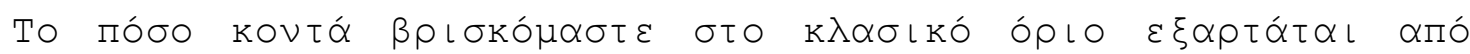

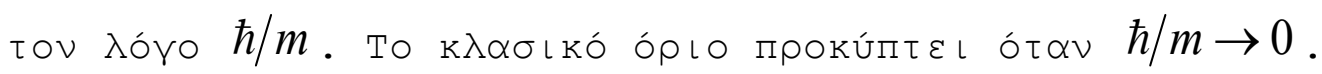

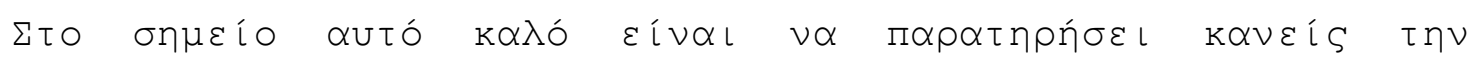

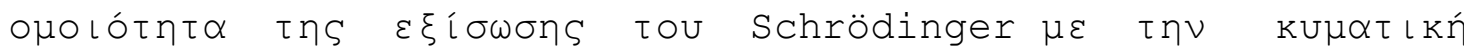
$\varepsilon \xi \hat{\imath} \sigma \omega \sigma \eta$

$$
\left(\nabla^{2}-\frac{1}{c^{2}} \frac{\partial^{2}}{\partial t^{2}}\right) \psi=0
$$

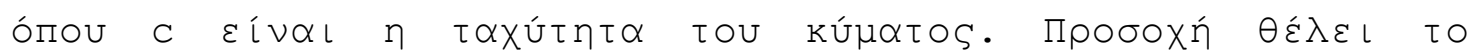

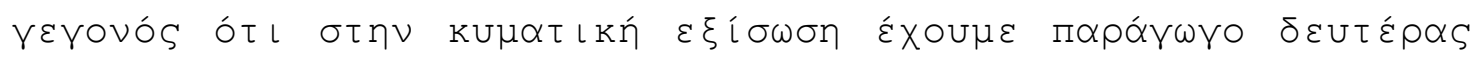

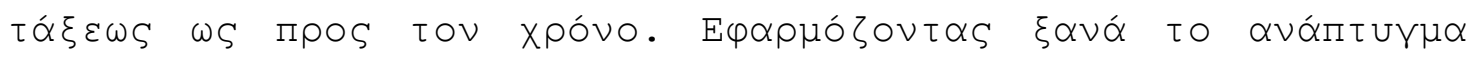

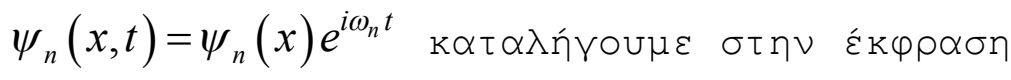

$$
\begin{gathered}
\left(\nabla^{2}+k_{n}^{2}\right) \psi_{n}(x)=0 \\
\omega_{n}=c k_{n}
\end{gathered}
$$

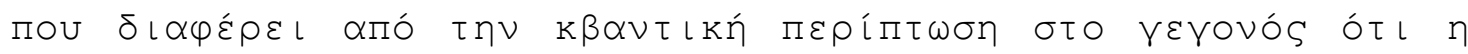

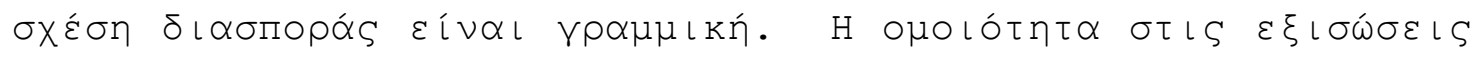

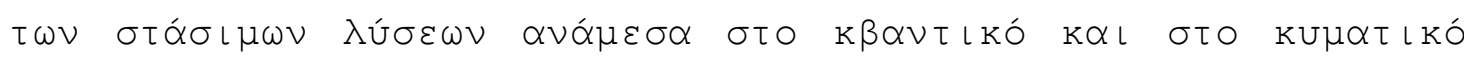

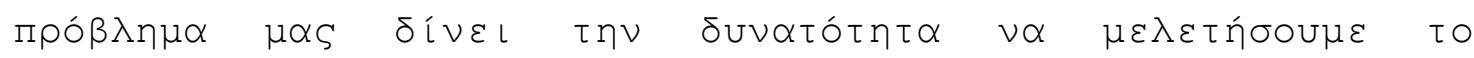

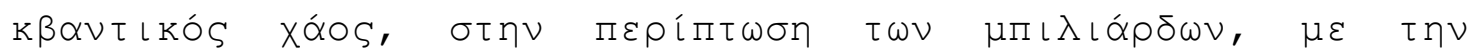

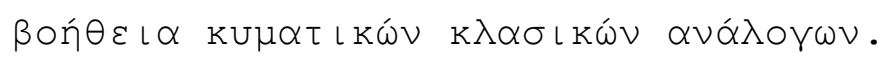




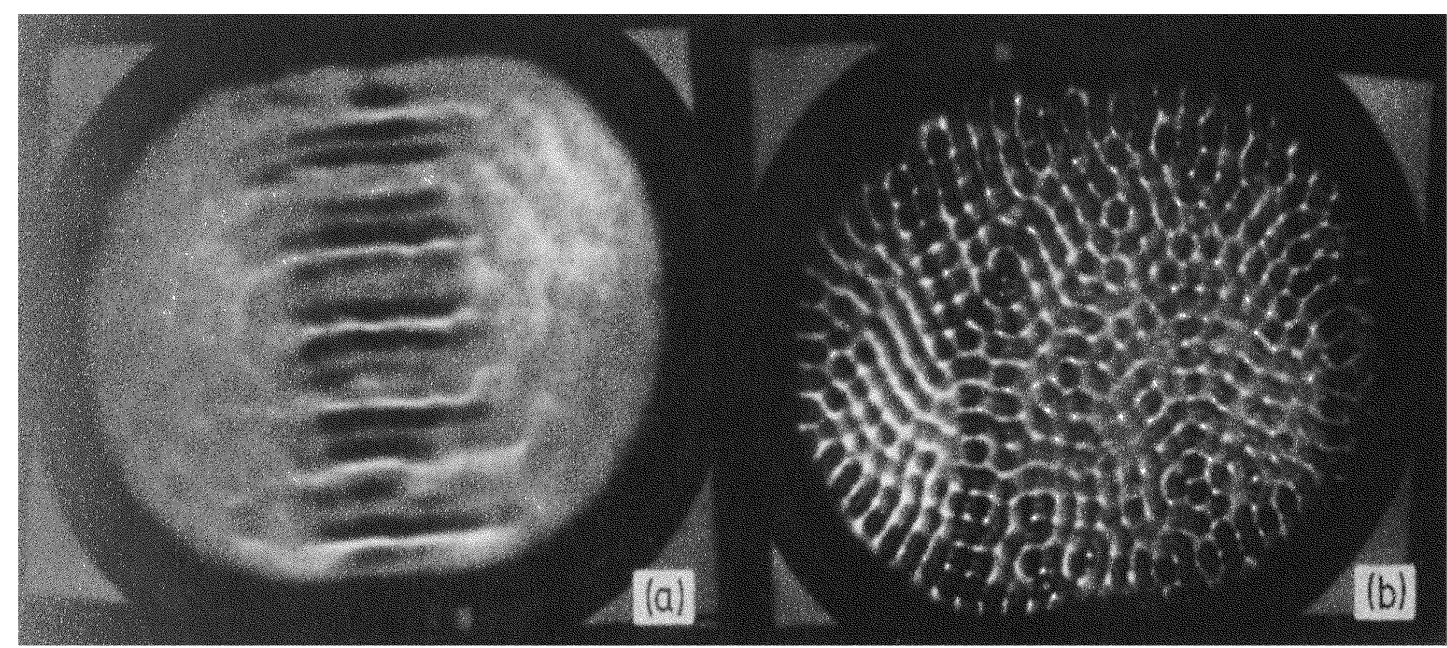

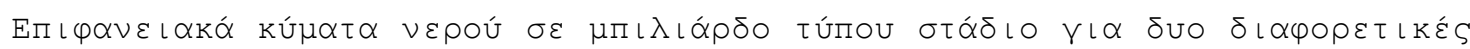

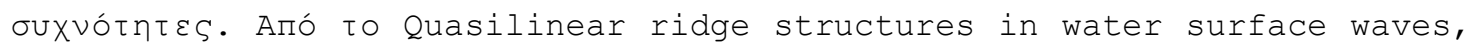
R. Blumel, I.H Davidson,.., Phys. Rev. A, vol. 45, 2641,1992

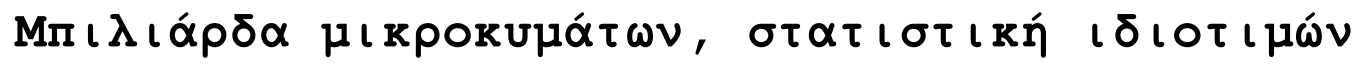

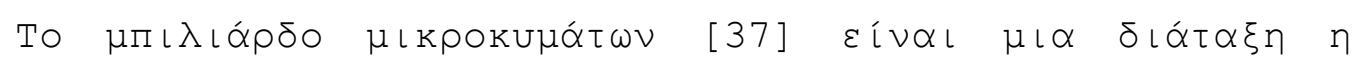

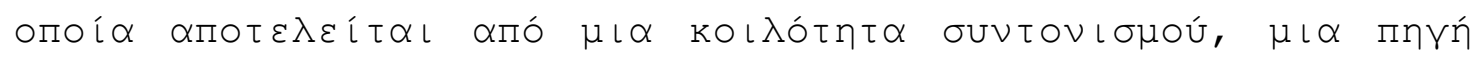

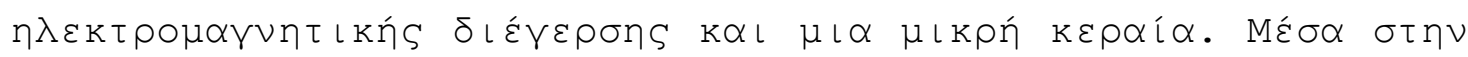

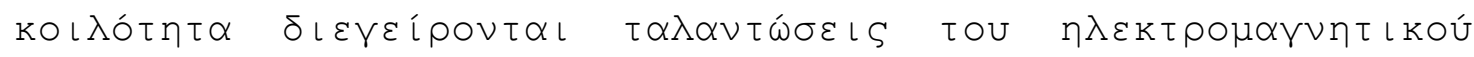

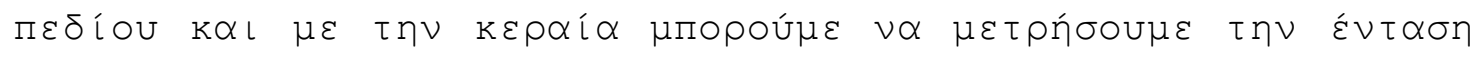

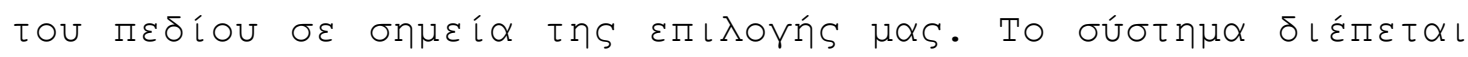

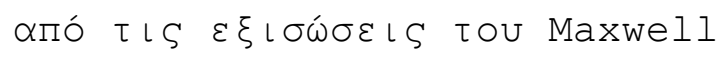

$$
\begin{gathered}
\nabla \times E=-\frac{\partial B}{\partial t} \\
\nabla \times H=\frac{\partial D}{\partial t} \\
\nabla \cdot D=0 \\
\nabla \cdot B=0
\end{gathered}
$$




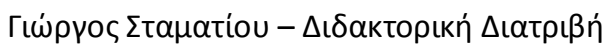

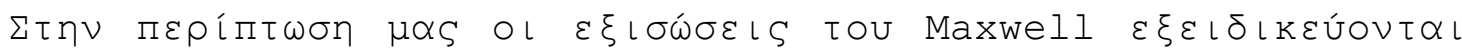
$\sigma \tau \iota \varsigma$

$$
\begin{aligned}
& \left(\Delta+k^{2}\right) E=0 \\
& \left(\Delta+k^{2}\right) B=0
\end{aligned}
$$

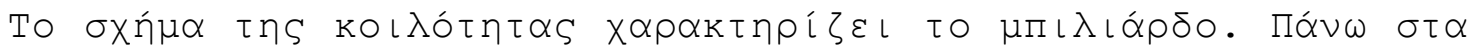

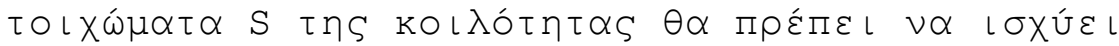

$$
E_{\text {parallel }}=0, \quad B_{\text {vertical }}=0
$$

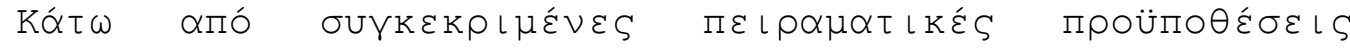

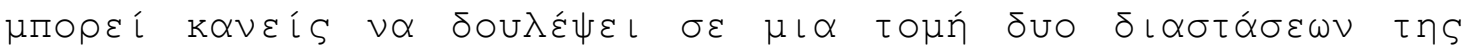
ко

$$
\left(\Delta+k^{2}\right) E=0
$$

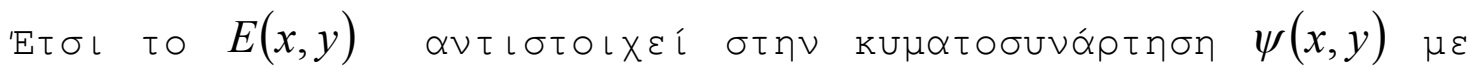

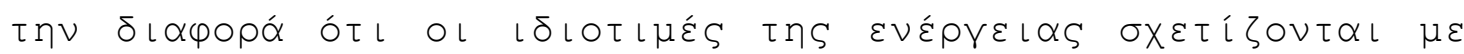
го $k^{2}$.

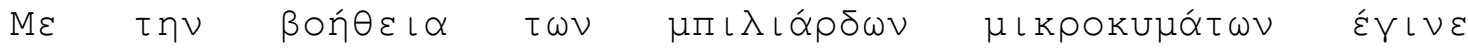

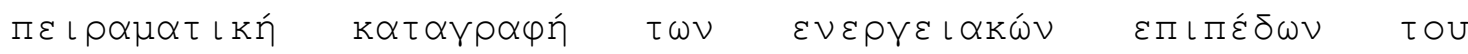

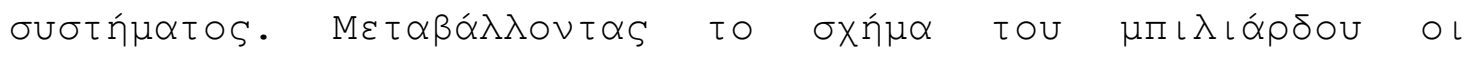

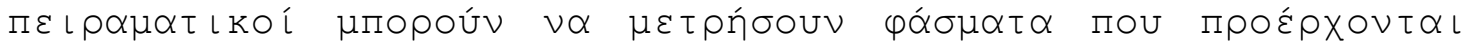

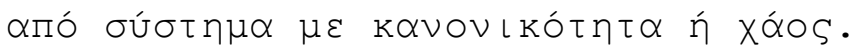

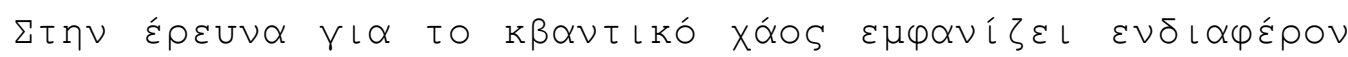

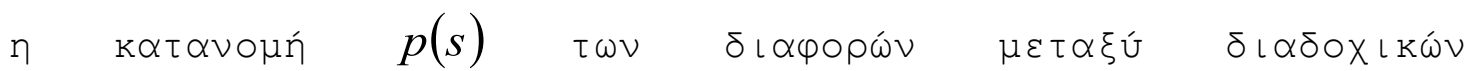

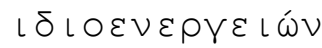

$$
\begin{aligned}
& s_{n}=E_{n}-E_{n-1}
\end{aligned}
$$

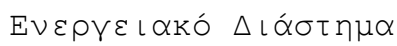

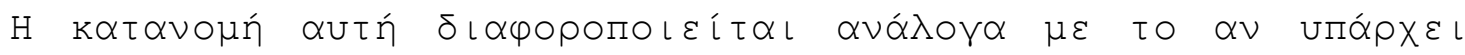

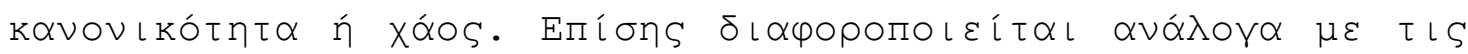

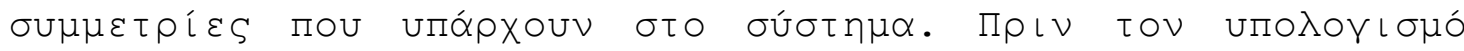




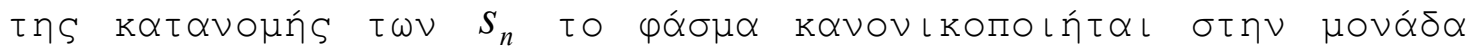

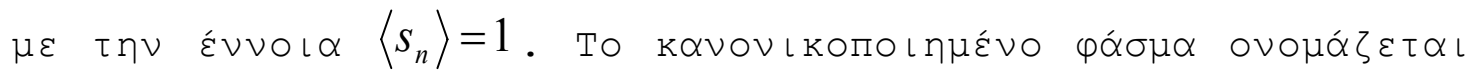
$\Xi \varepsilon \delta\left\llcorner\Pi \lambda \omega \mu \varepsilon^{\prime} \vee O\right.$ (unfolded spectrum).

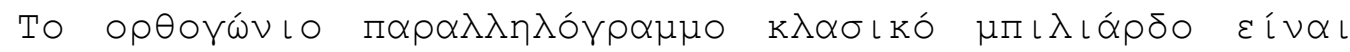

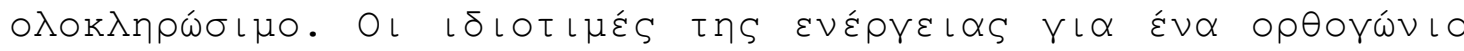

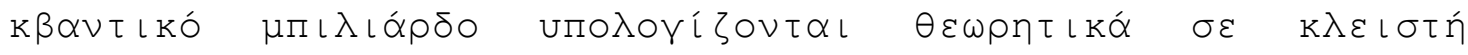

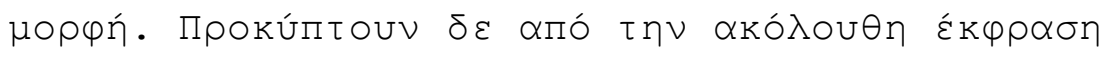

$$
\begin{gathered}
E=\frac{\hbar^{2} k^{2}}{2 m}=\frac{\hbar^{2}}{2 m}\left[\left(\frac{\pi n}{a}\right)^{2}+\left(\frac{\pi m}{b}\right)^{2}\right] \\
n, m=0,1,2, \ldots
\end{gathered}
$$

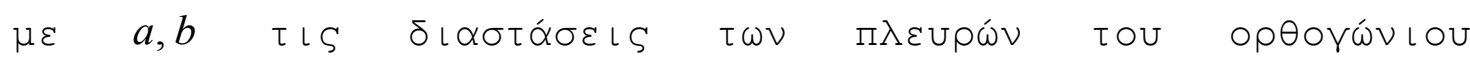

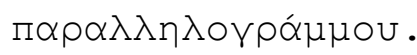

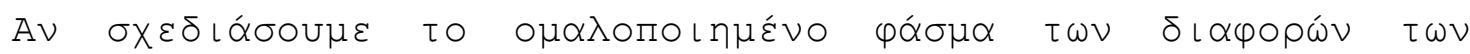

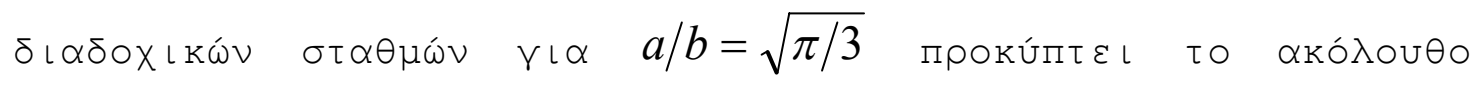
$\delta\llcorner\alpha ́ \gamma \rho \alpha \mu \mu \alpha$.

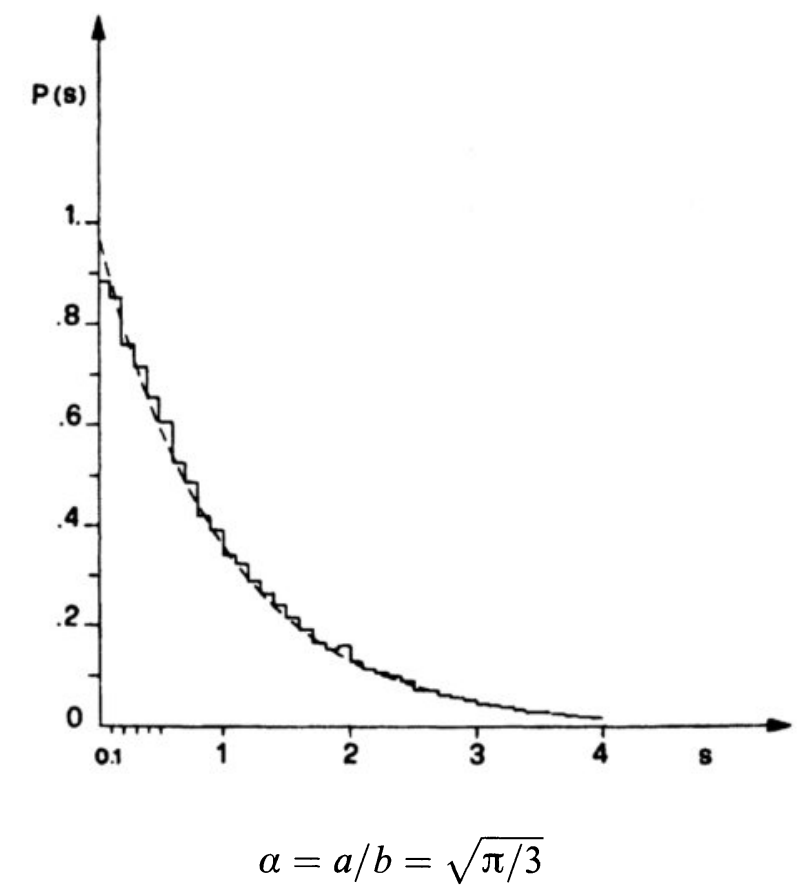




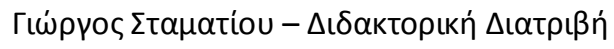

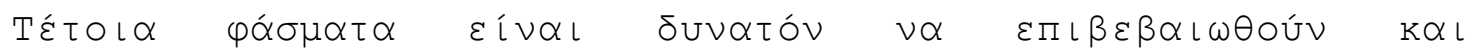

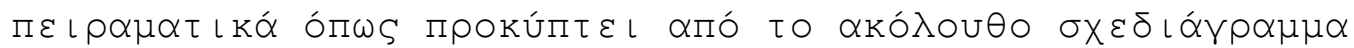

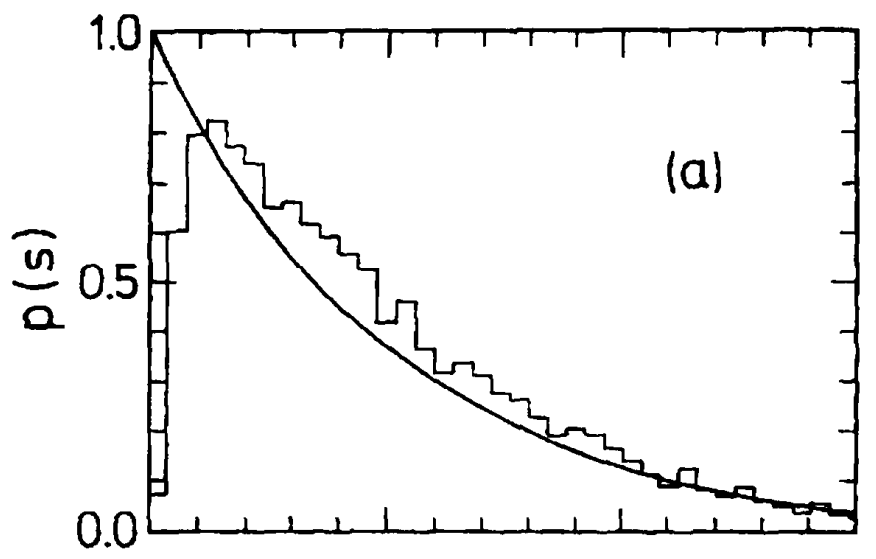

5

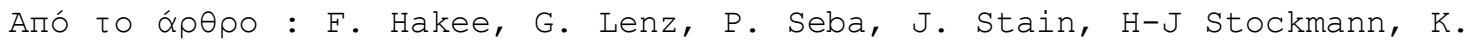
Zyczkowski. Manifestation of wave chaos..., Phys. Rev. A, vol. 44, R6161, (1991)

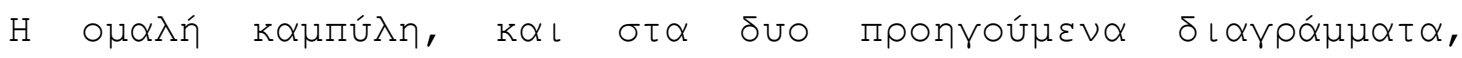

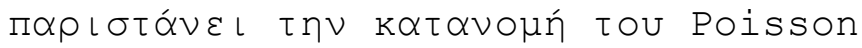

$$
P(s)=\exp (-s)
$$

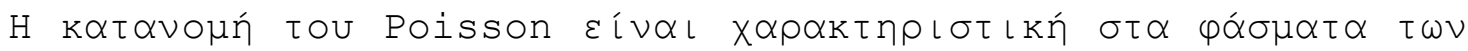

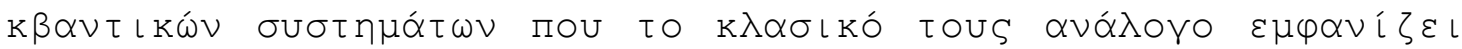

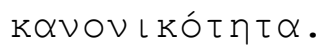

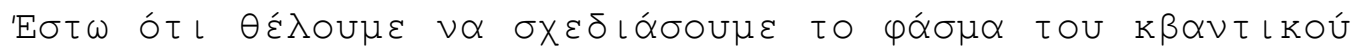

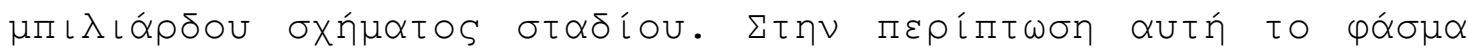

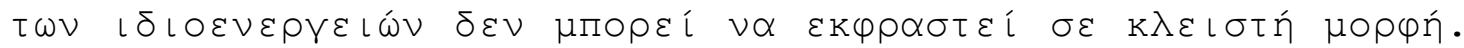

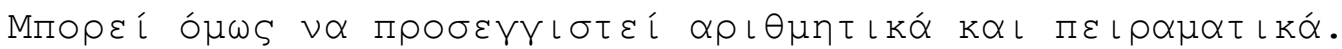



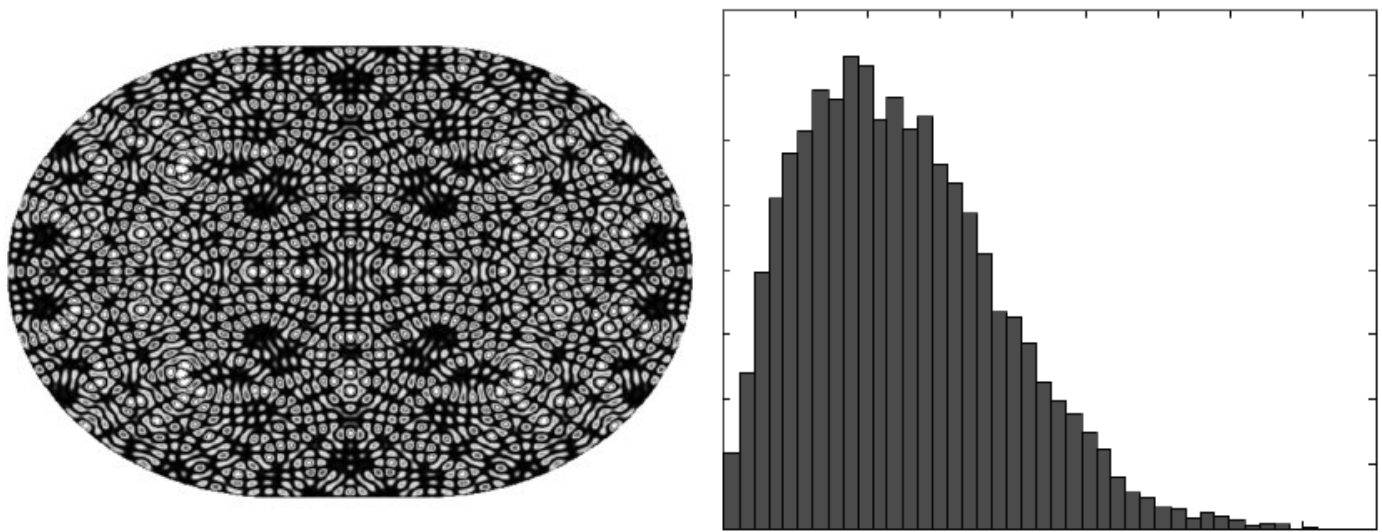

[from D.Stone's page http://www.eng.yale.edu/stonegroup/]

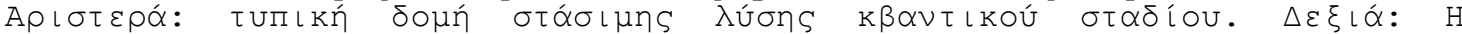

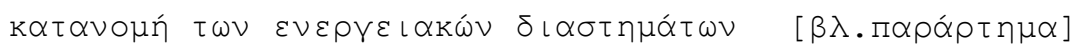

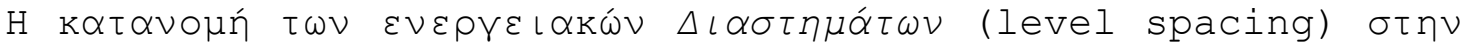

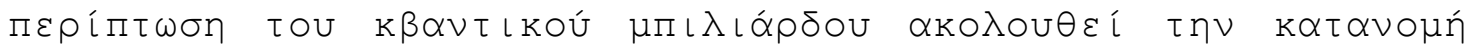
tou Wigner.

$$
P(s)=\frac{\pi}{2} s \exp \left(-\frac{\pi}{4} s^{2}\right)
$$

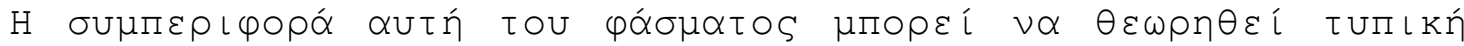

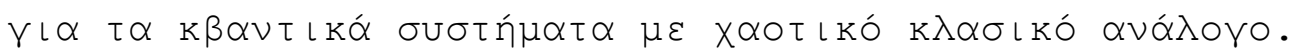
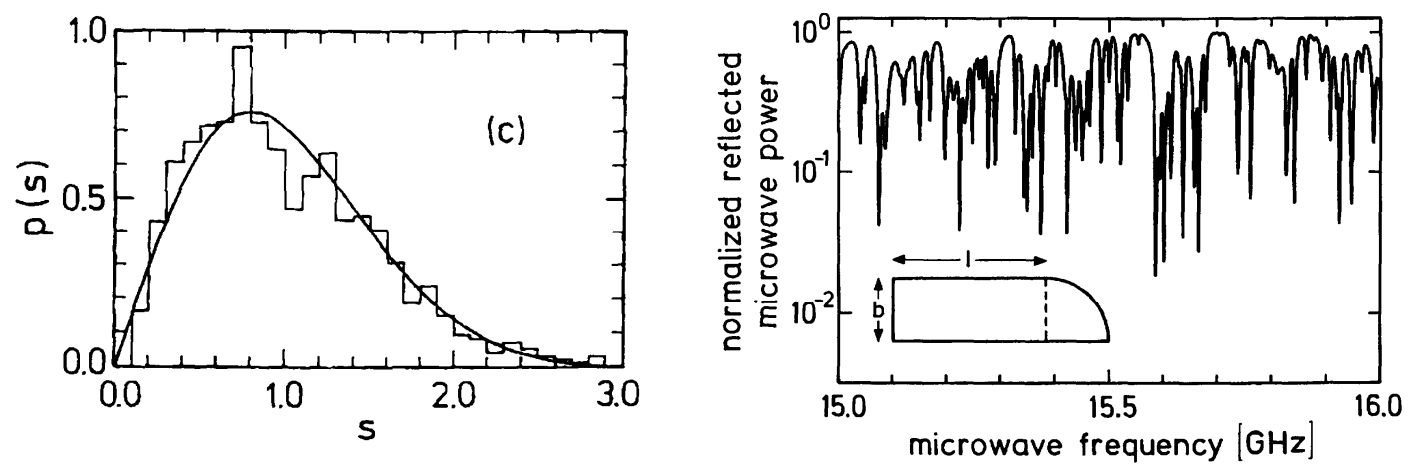

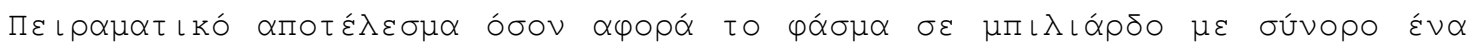

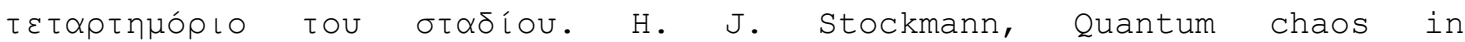
billiards..., Phys. Rev. Let. vol.64, 2215 (1990) 

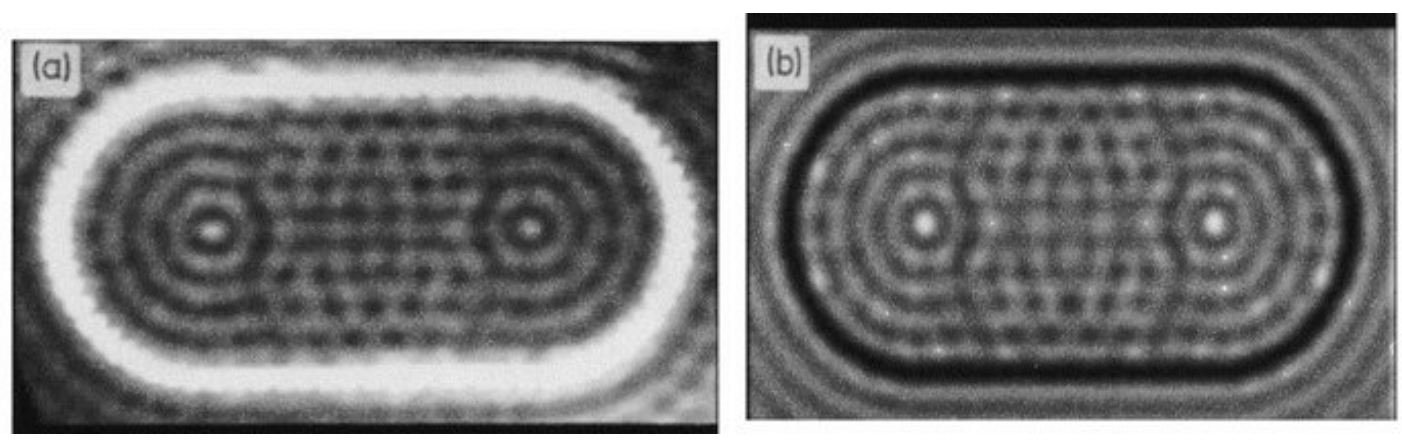

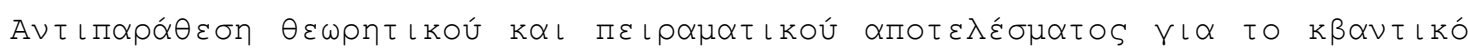

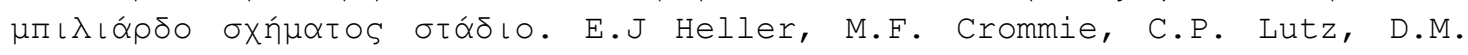
Eigler. Nature vol. 369, 464 (1994)

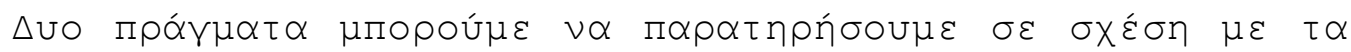

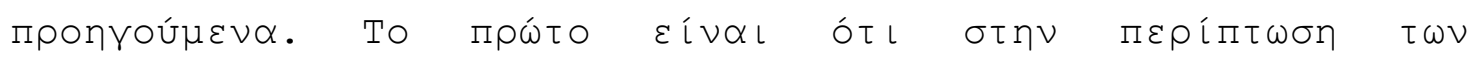

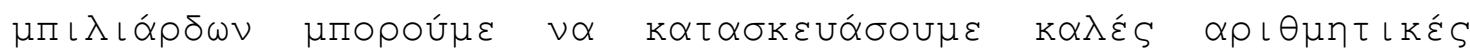

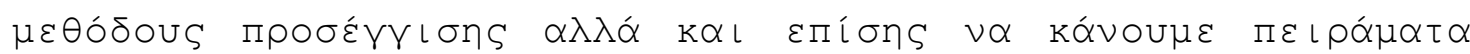

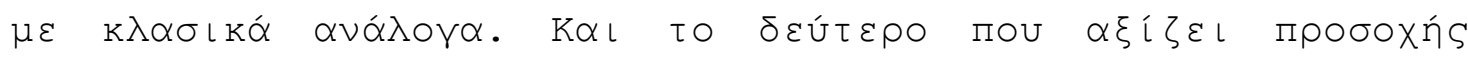

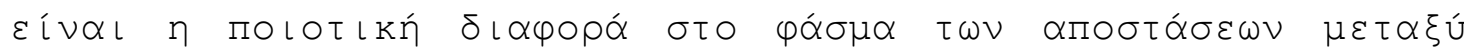

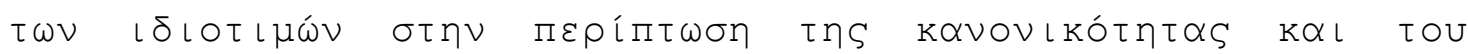

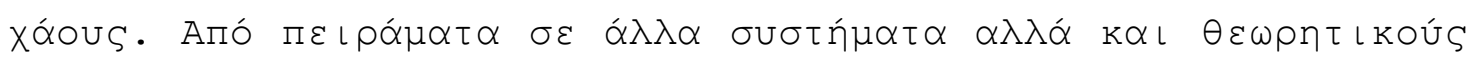

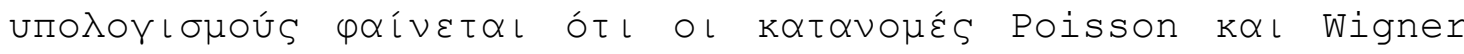

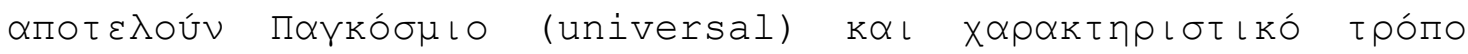

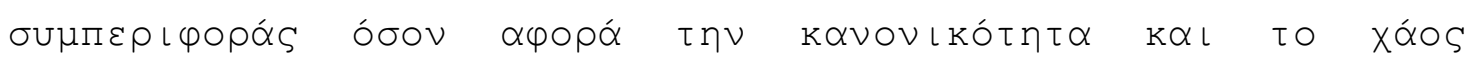
$\alpha \nu \tau i \sigma \tau \circ\llcorner\chi \alpha$.

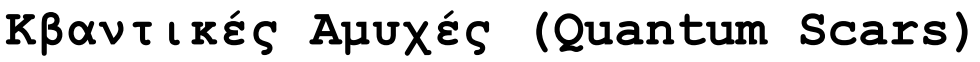

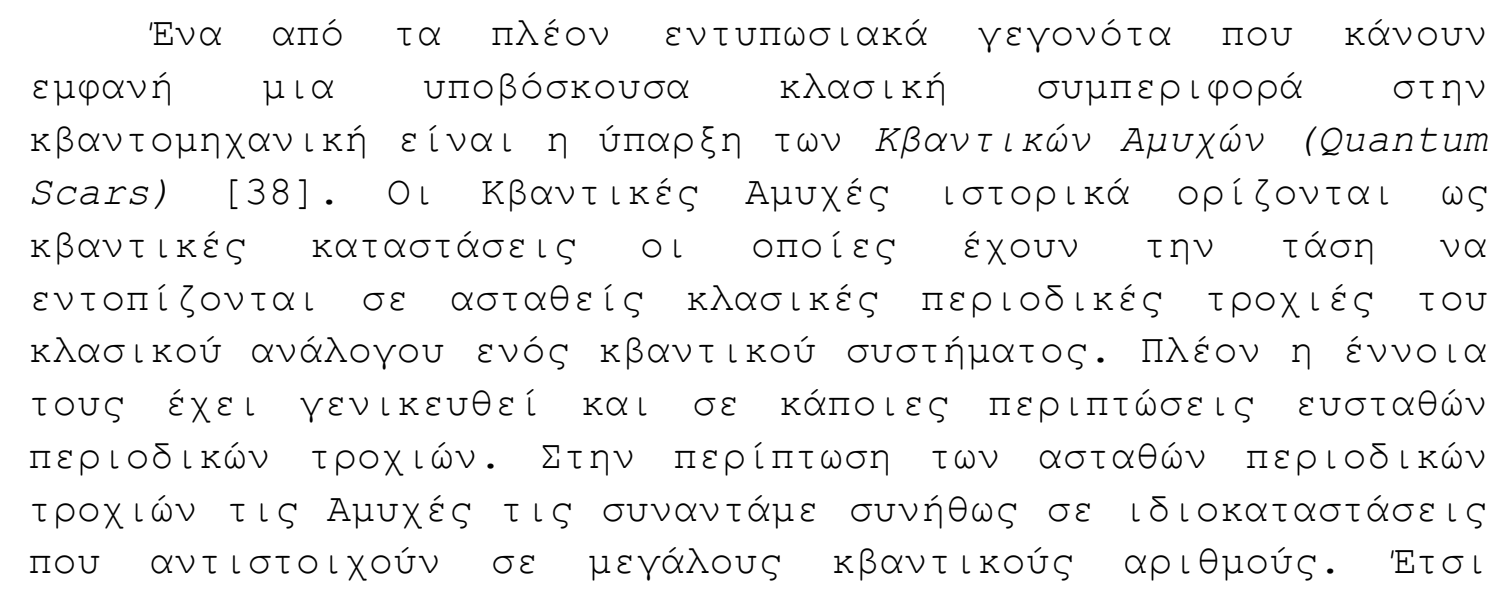




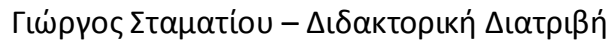

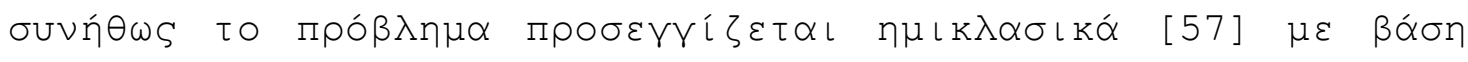

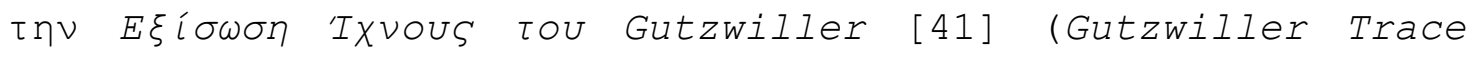

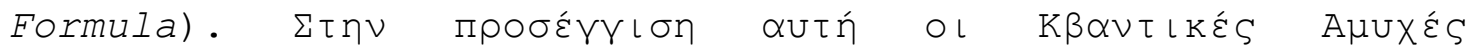

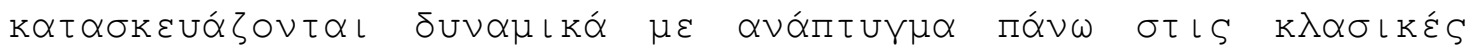

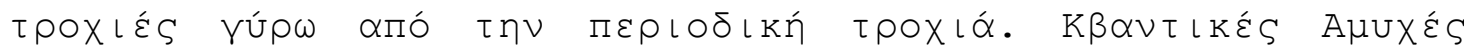

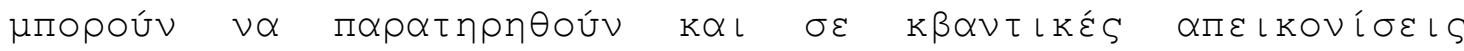

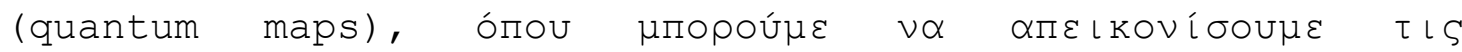

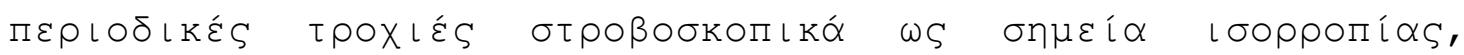

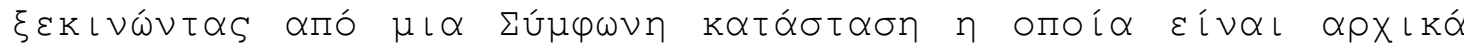

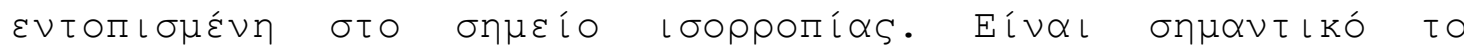

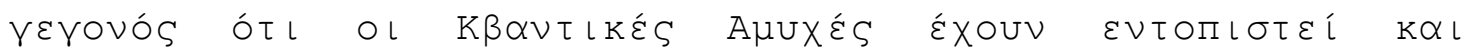

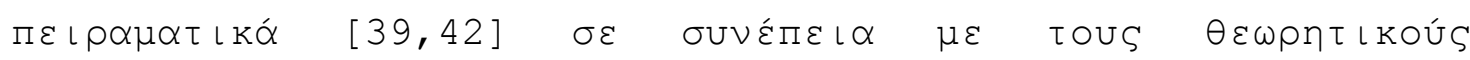
uпо入оY
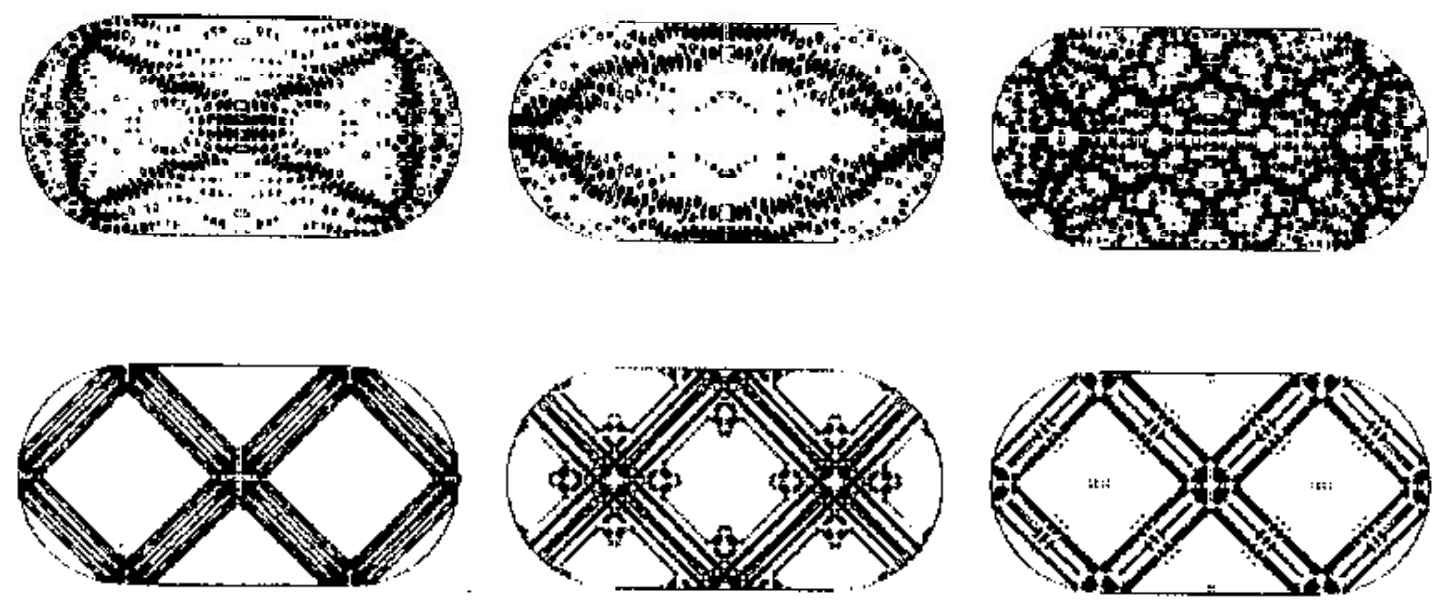

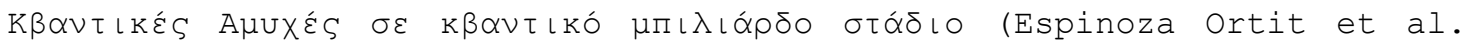
Journal of Statistical Physics, vol. 83, Nos. 1/2, 1996)

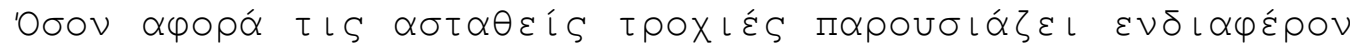

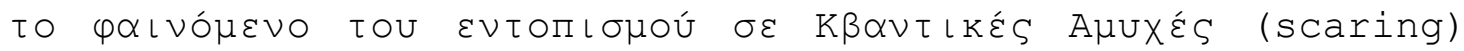

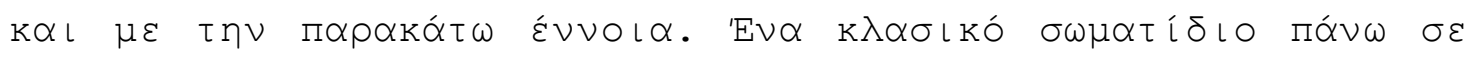
$\mu\llcorner\alpha \quad \alpha \sigma \tau \alpha \theta n$

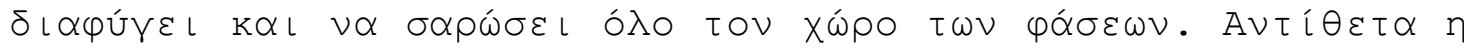

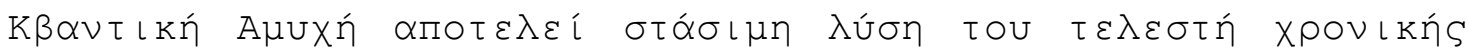

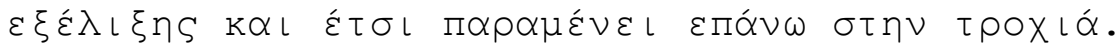




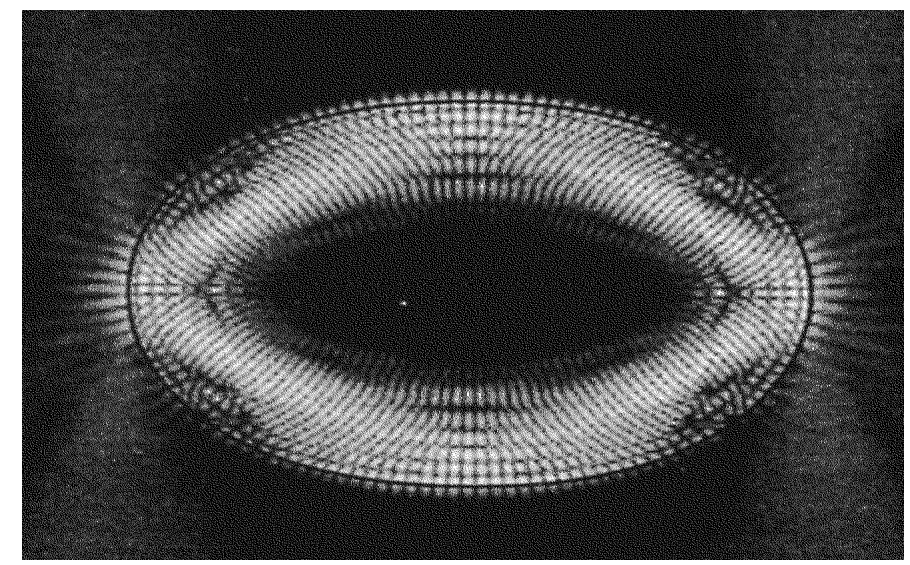

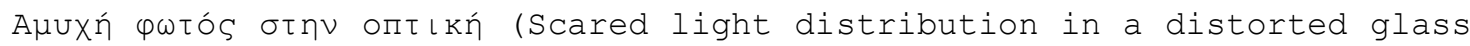
fibre cavity, J.U. Nockel and A.D. Stone. Ray and wave chaos in asymmetric resonant cavities. Nature 385, 45, 1997)

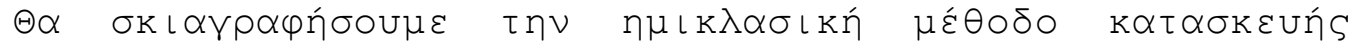

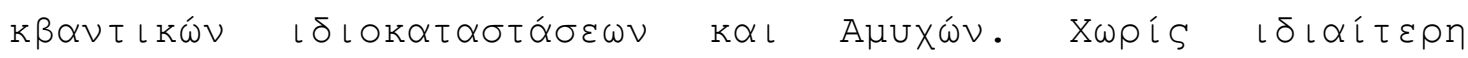

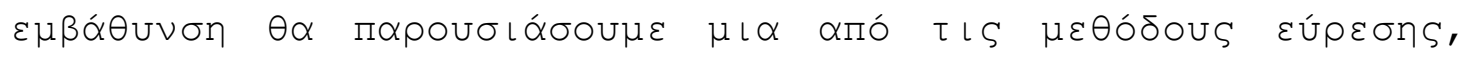

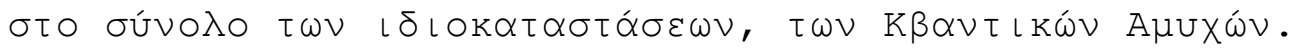

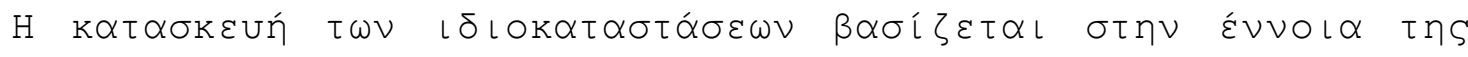
इuváptnons Green 1, 43

$$
\begin{gathered}
G\left(q, q^{\prime} ; E\right)=\left\langle q\left|\frac{1}{E-\hat{H}}\right| q^{\prime}\right\rangle=\sum_{j} \frac{\Psi_{j}(q) \Psi_{j}^{*}\left(q^{\prime}\right)}{E-E_{j}} \\
\text { इuvópinøn Green }
\end{gathered}
$$

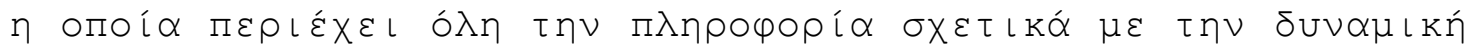

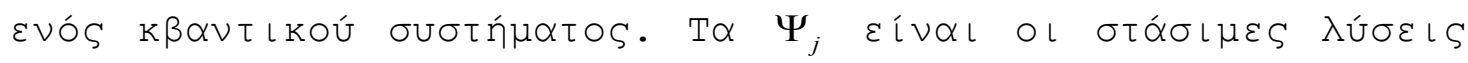

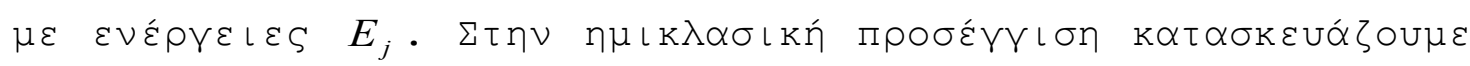

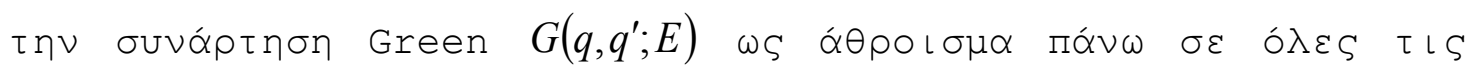

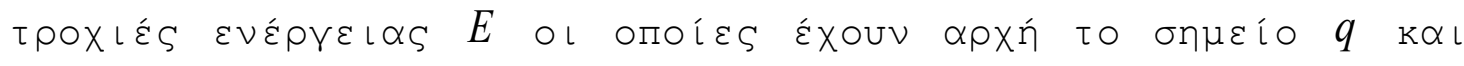

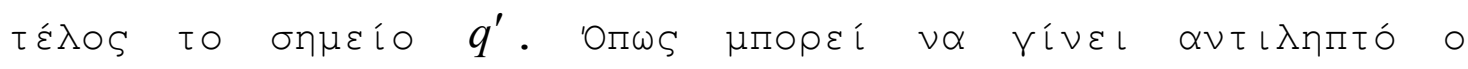

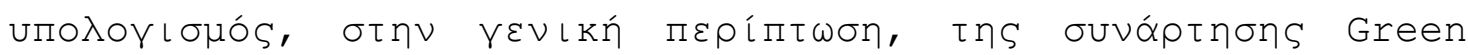

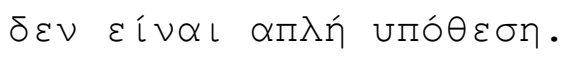




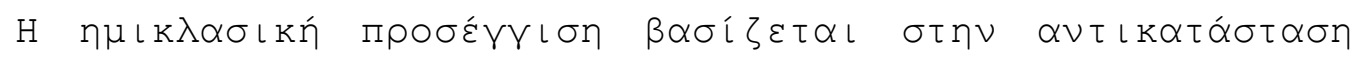

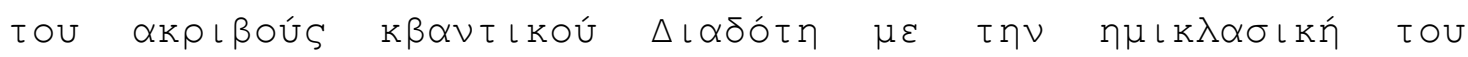
$\dot{\varepsilon} \kappa \delta \circ \sigma \eta$.

$$
G_{s c}\left(q, q^{\prime} ; t\right)=(2 \pi i \hbar)^{-d / 2} \sum_{j}\left|\operatorname{det}\left[\frac{\partial^{2} S_{j}\left(q, q^{\prime} ; t\right)}{\partial q \partial q^{\prime}}\right]\right|^{2} \exp \left[\frac{i S_{j}\left(q, q^{\prime} ; t\right)}{\hbar}-\frac{i \pi v_{j}}{2}\right]
$$

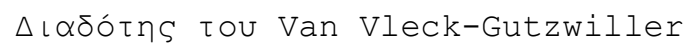

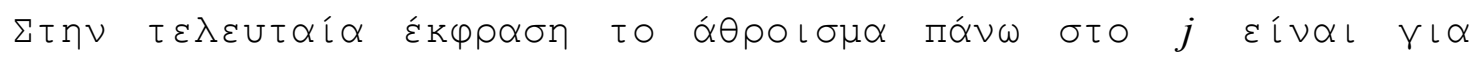

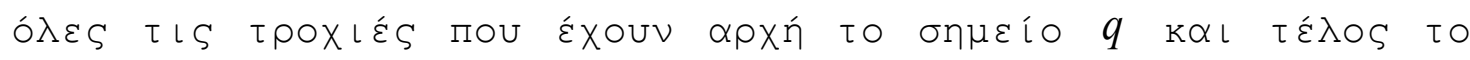

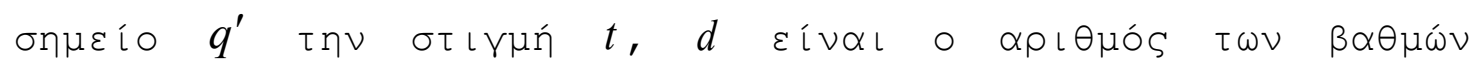

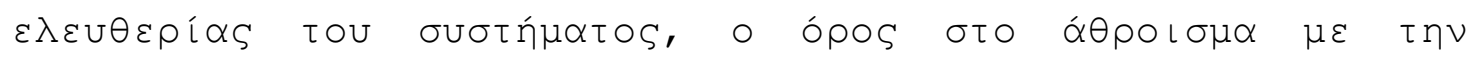

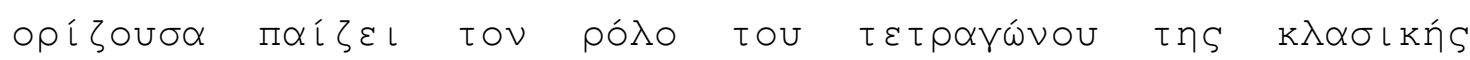

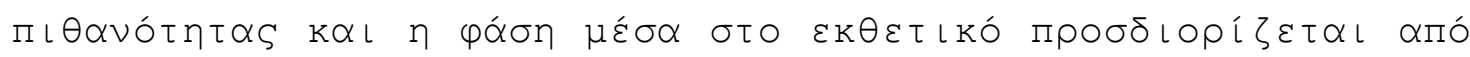

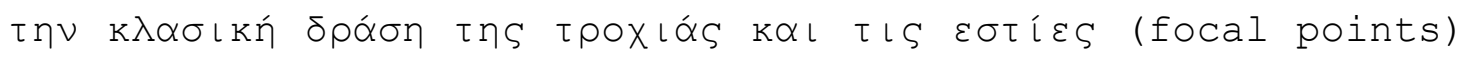

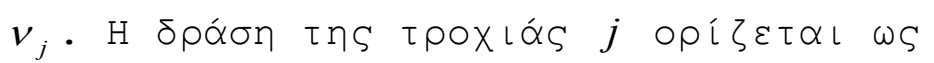

$$
S_{j}\left(q, q^{\prime} ; t\right)=\int_{0}^{t} d t^{\prime} \mathcal{L}=\int_{0}^{t} d t^{\prime}\left[p\left(t^{\prime}\right) \dot{q}\left(t^{\prime}\right)-H\left(p\left(t^{\prime}\right), q\left(t^{\prime}\right)\right)\right]
$$

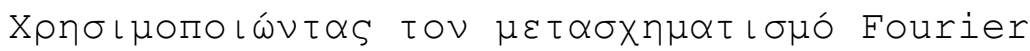

$$
\Psi_{j}(q)=\int_{-\infty}^{+\infty} d t \exp \left(i E_{j} t / \hbar\right) \Phi(q ; t)
$$

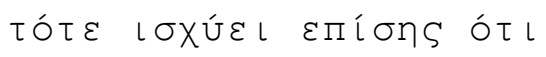

$$
\Phi(q ; t)=\int_{-\infty}^{+\infty} d q^{\prime} G\left(q, q^{\prime} ; t\right) \Phi\left(q^{\prime} ; 0\right)
$$

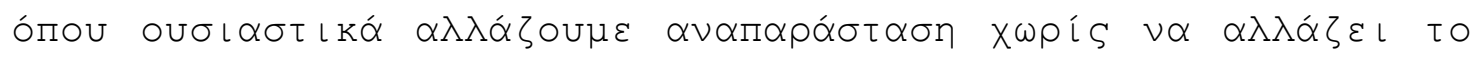

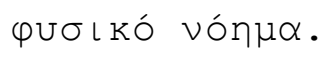

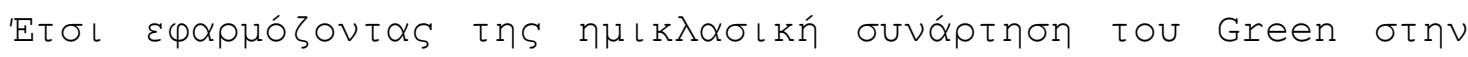

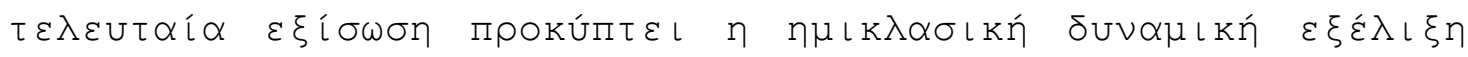

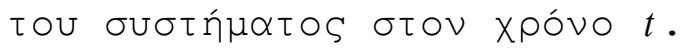




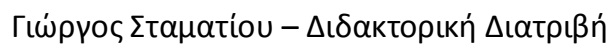

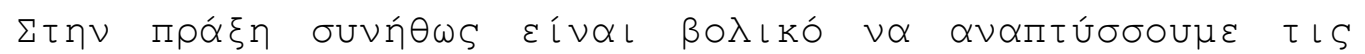

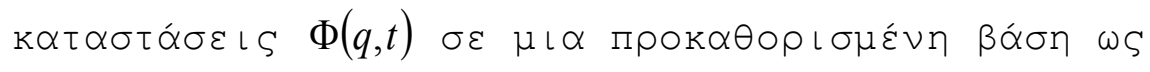

$$
\Phi(q ; t)=\sum_{k}\langle q \mid k\rangle\langle k \mid \Phi(t)\rangle
$$

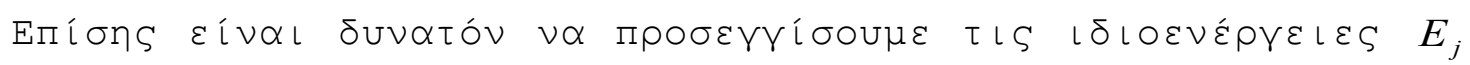

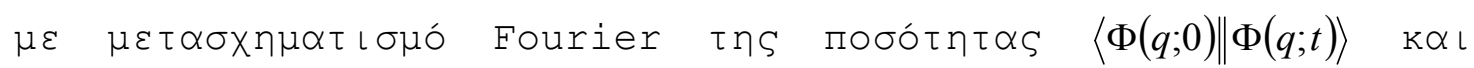

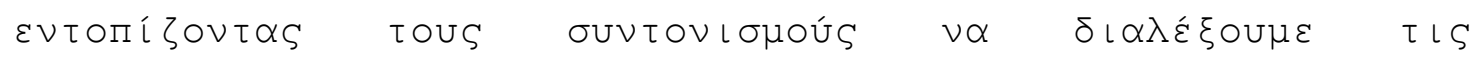

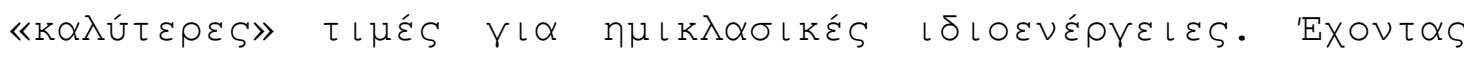

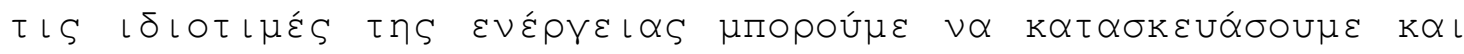

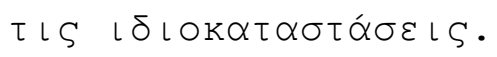

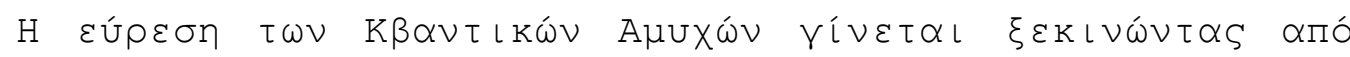

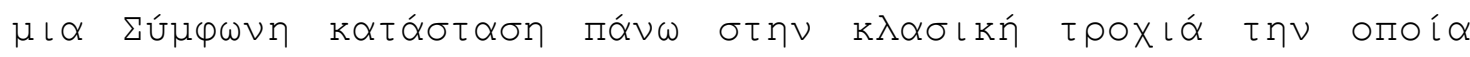

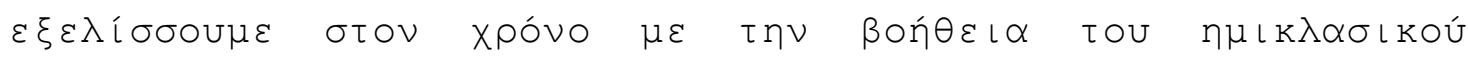

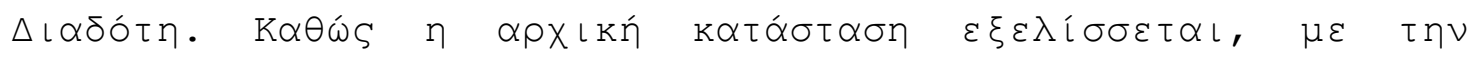

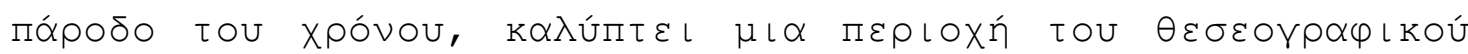

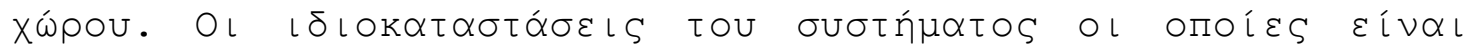

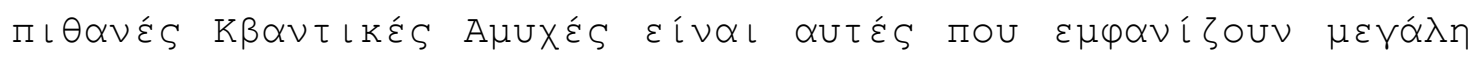

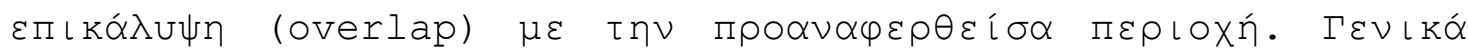

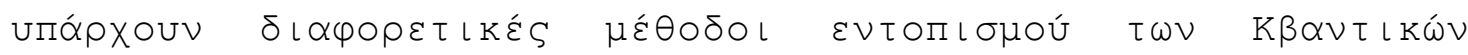

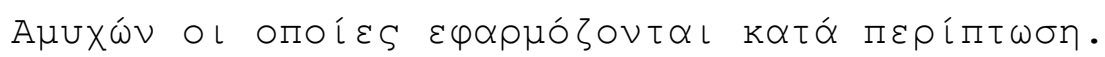

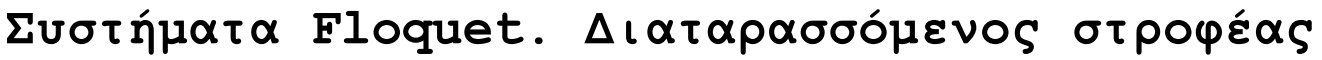 (Kicked Top). $\Sigma \tau \alpha \tau \iota \sigma \tau \iota$ кń}

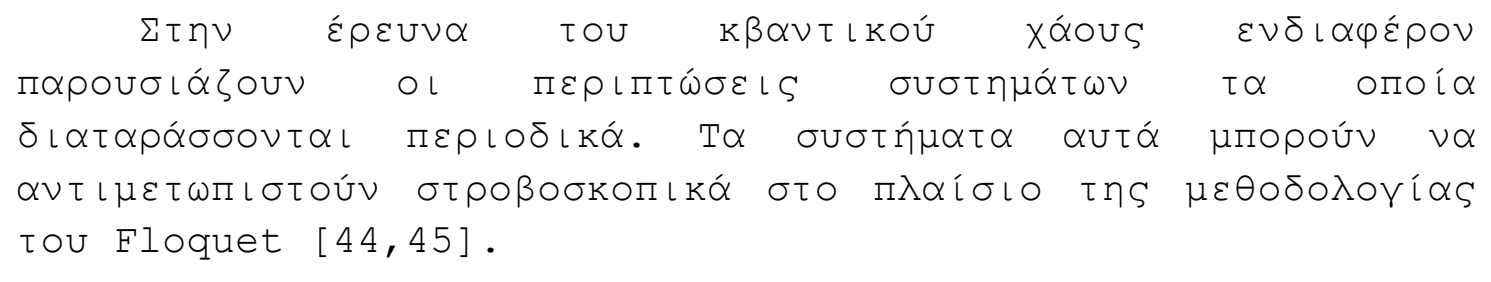




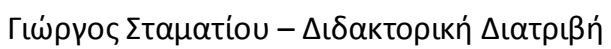

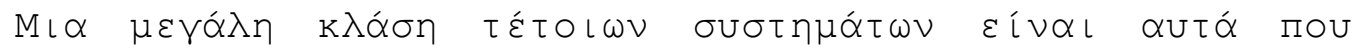

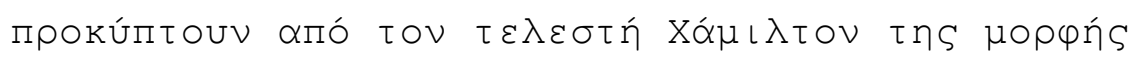

$$
H(t)=\left\{\begin{array}{cc}
H_{0} & n \tau<t<(n+1) \tau-\Delta \tau \\
H_{0}+\frac{1}{\Delta \tau} V_{0} & (n+1) \tau-\Delta \tau<t<(n+1) \tau
\end{array}\right.
$$

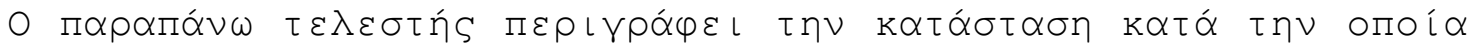

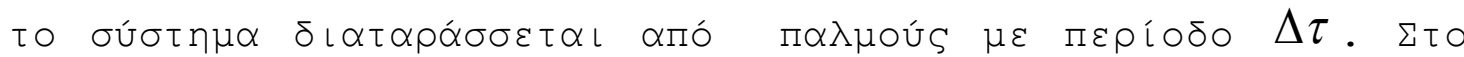

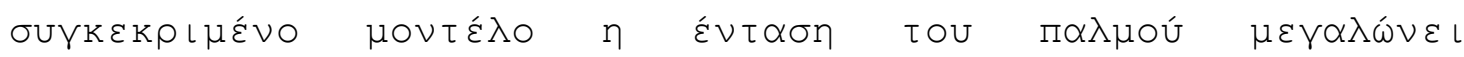

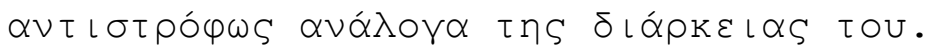

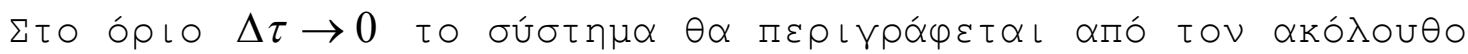
$\tau \varepsilon \lambda \varepsilon \sigma \tau \dot{n}$

$$
H(t)=H_{0}+V_{0} \sum_{n} \delta(t-n \tau)
$$

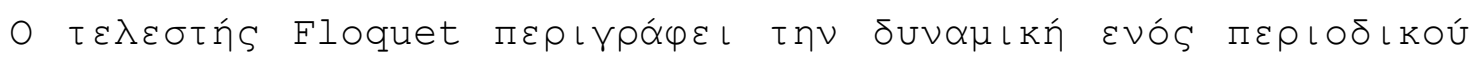

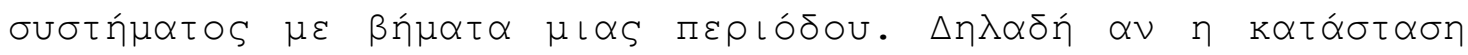

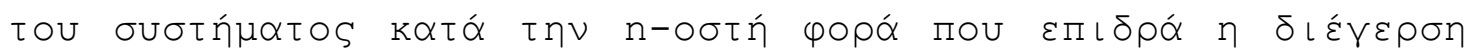

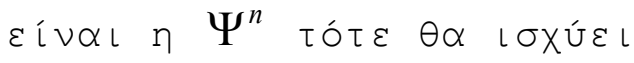

$$
\Psi^{n+1}=F \Psi^{n}
$$

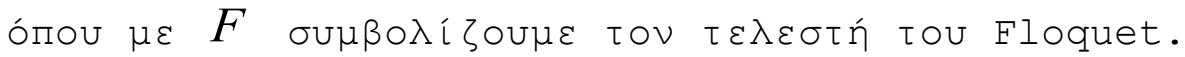

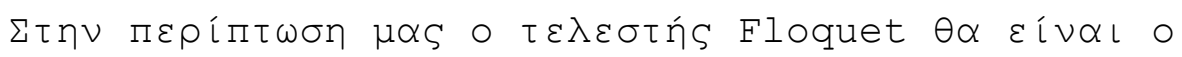

$$
F=\exp \left[-\frac{i}{\hbar}\left(H_{0}+\frac{1}{\Delta \tau} V_{0}\right) \Delta \tau\right] \exp \left(-\frac{i}{\hbar} H_{0}(\tau-\Delta \tau)\right)
$$

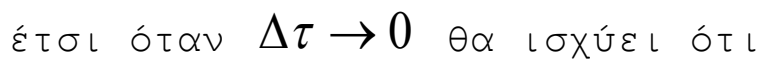

$$
F=\exp \left(-\frac{i}{\hbar} V_{0}\right) \exp \left(-\frac{i}{\hbar} H_{0} \tau\right)
$$




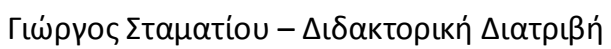

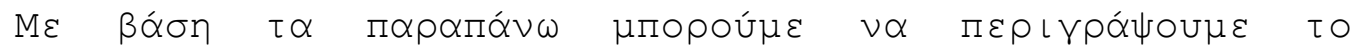

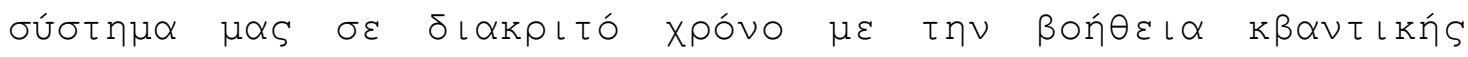
$\alpha \Pi \varepsilon\llcorner$ kóvıons (quantum map)

$$
\Psi^{n}=F^{n} \Psi^{0}
$$

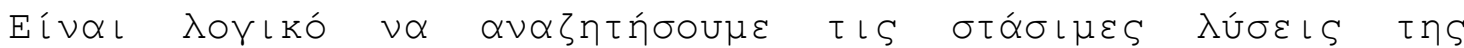

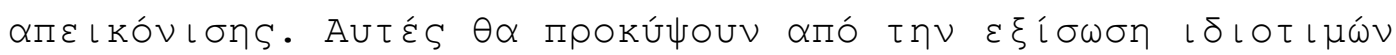

$$
F \psi_{k}=\exp \left(i \varepsilon_{k}\right) \psi_{k}
$$

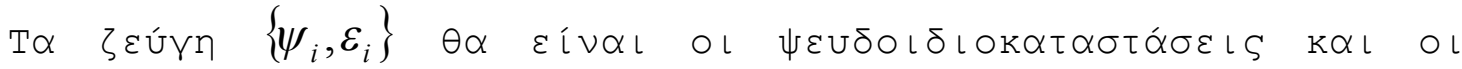

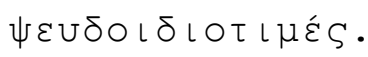

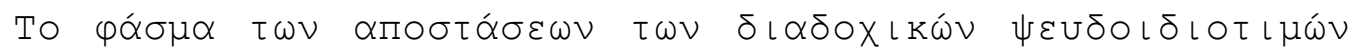

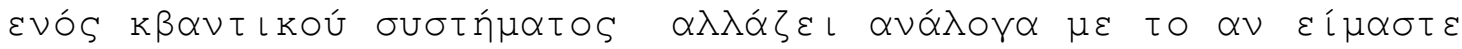

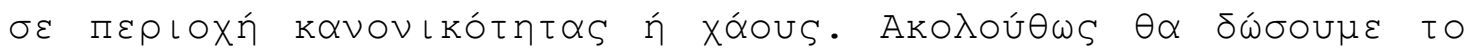

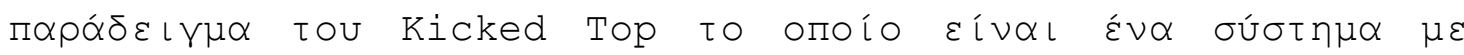

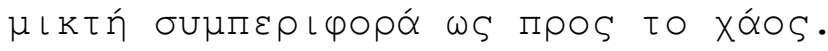

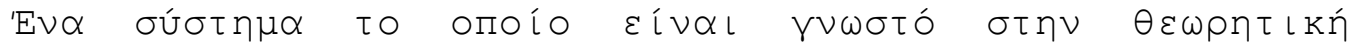

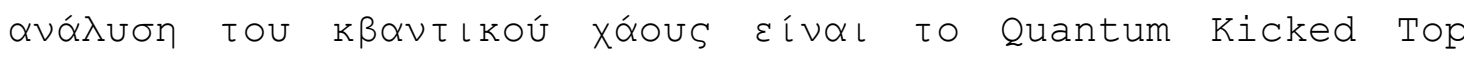

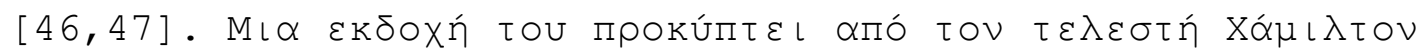

$$
H(t)=\frac{\hbar p}{\tau} I_{y}+\frac{\hbar k}{2 I} I_{z}^{2} \sum_{-\infty}^{\infty} \delta(t-n \tau)
$$

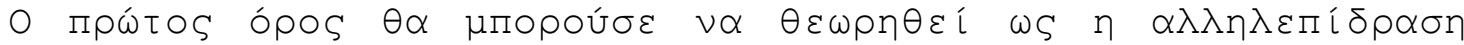

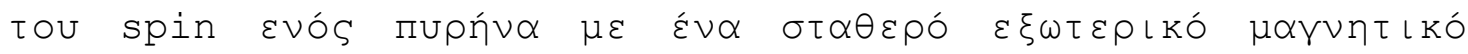

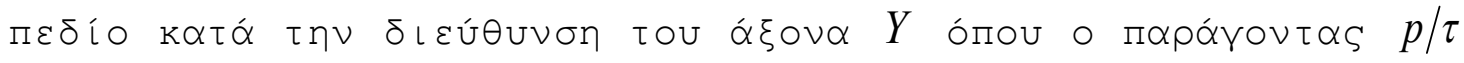

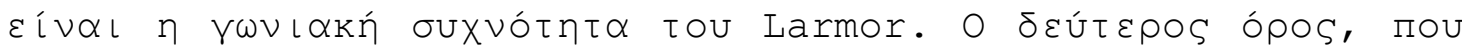

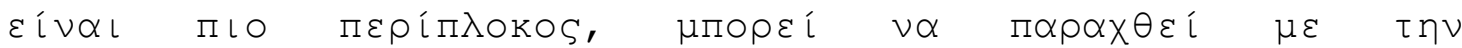

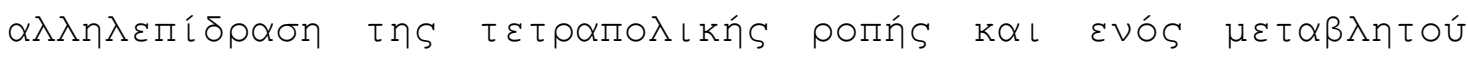

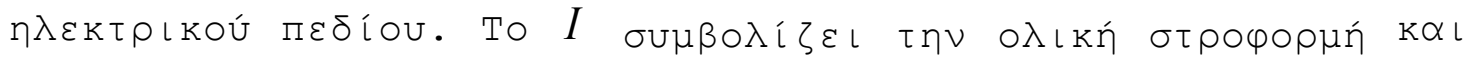

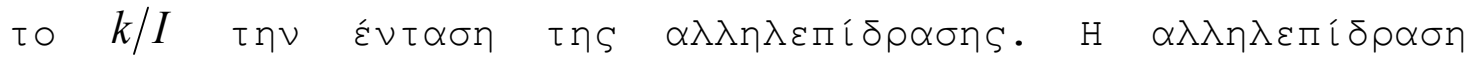

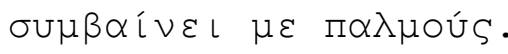




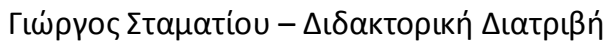

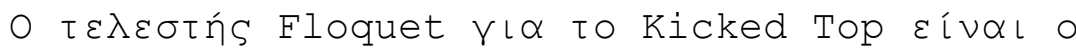

$$
F=\exp \left(-i \frac{k}{2 I} I_{z}^{2}\right) \exp \left(-i p I_{y}\right)
$$

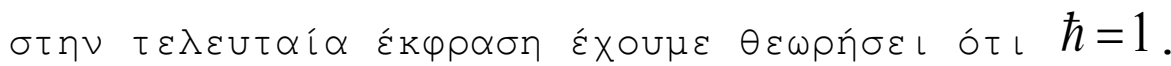

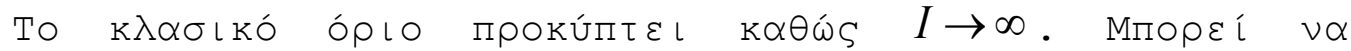

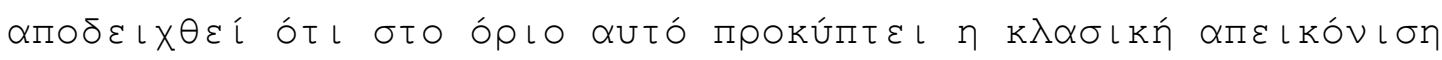

$$
\begin{aligned}
& X^{\prime}=\operatorname{Re}(X \cos p+X \sin p+i Y) e^{i k(Z \cos p-X \sin p)} \\
& Y^{\prime}=\operatorname{Im}(X \cos p+X \sin p+i Y) e^{i k(Z \cos p-X \sin p)}
\end{aligned}
$$

$$
Z^{\prime}=-X \sin p+Z \cos p
$$

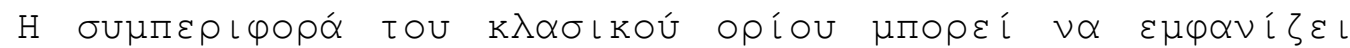

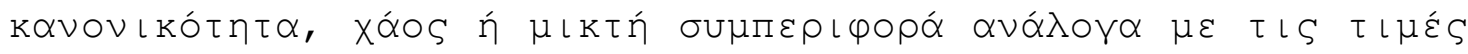

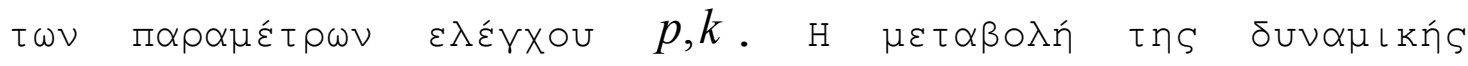

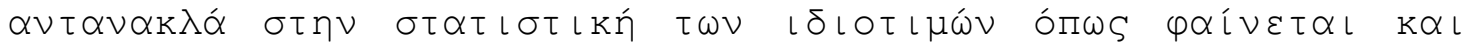

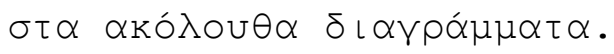

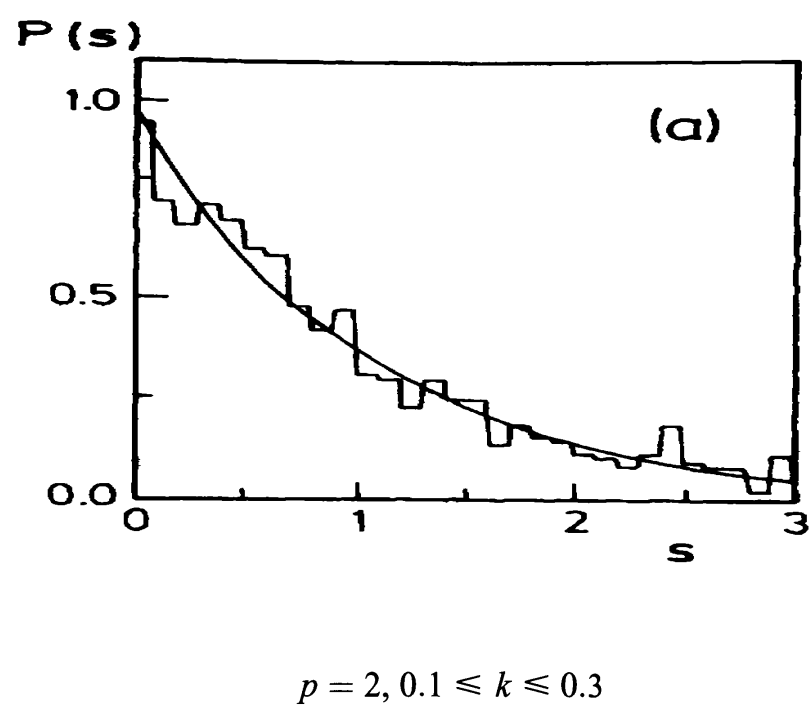




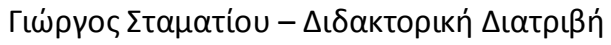

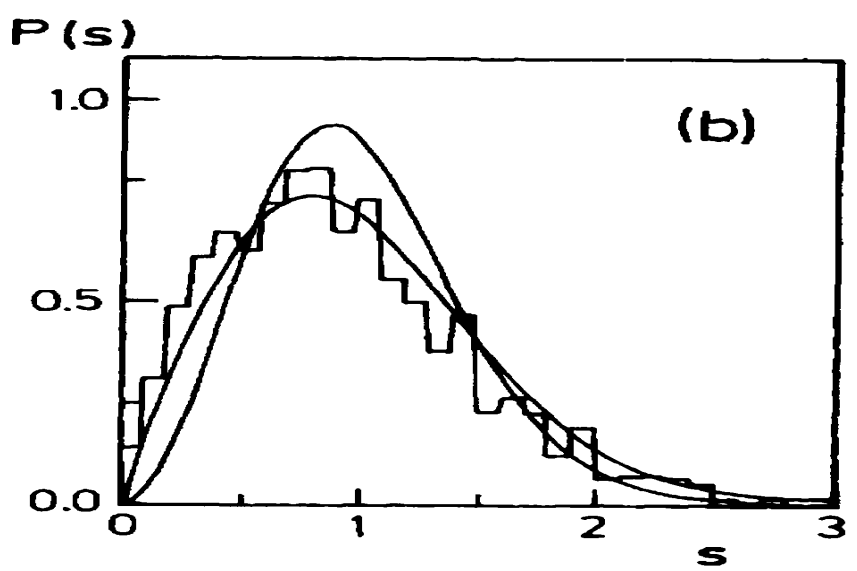

$$
p=1.7,10.0 \leqslant k \leqslant 10.5
$$

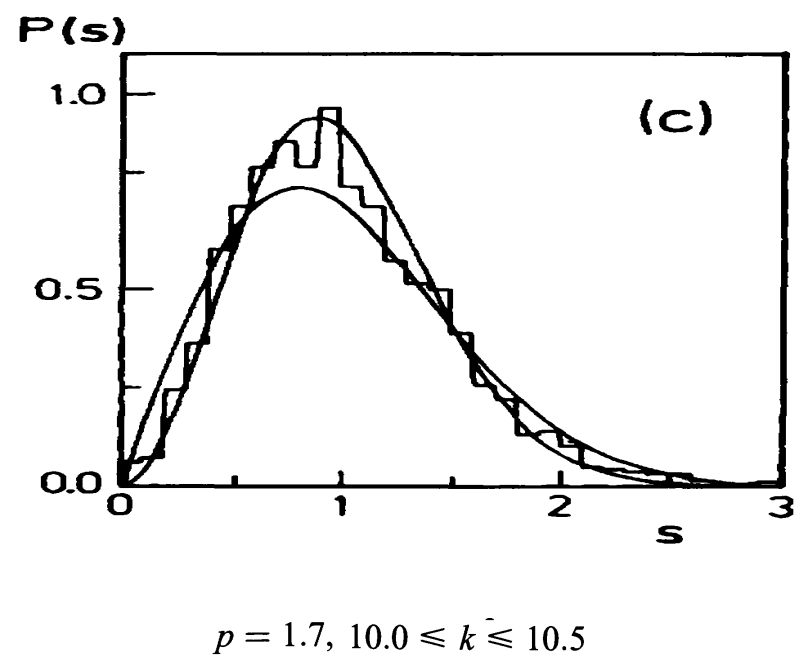

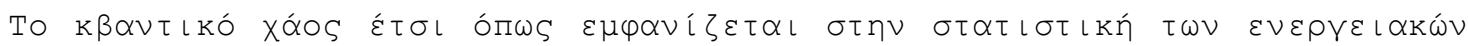

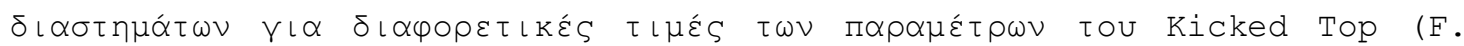
Haake, M. Kus and R.Scharf. Classical and quantum chaos for a kicked top. Z. Phys. B 65, 381, 1987)

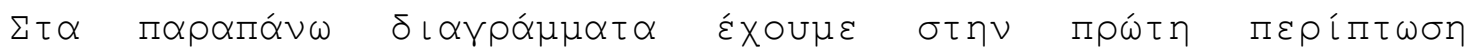

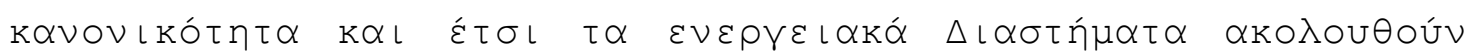

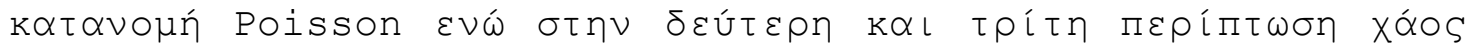

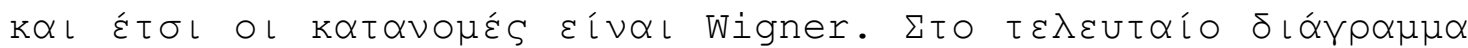

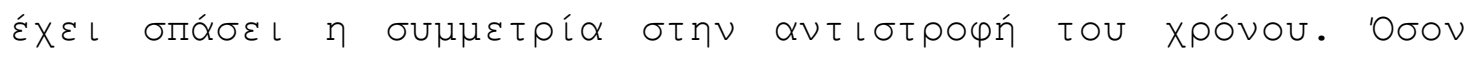

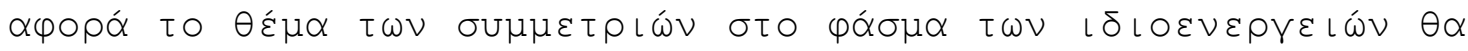

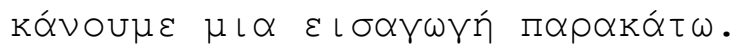




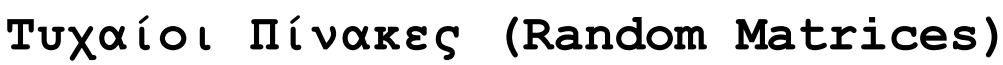

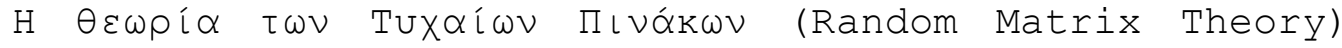

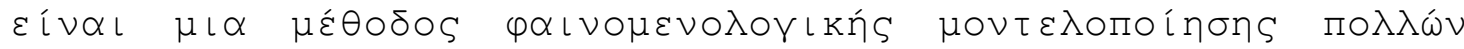

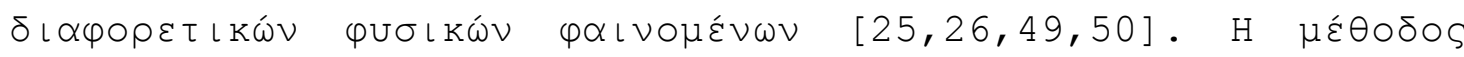

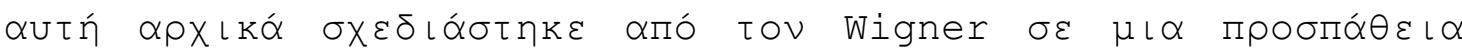

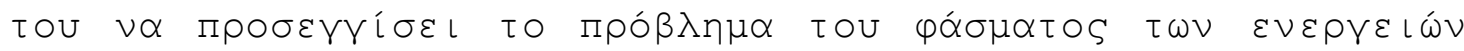

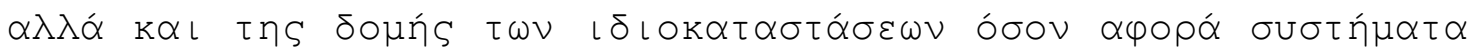

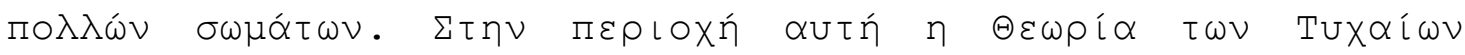

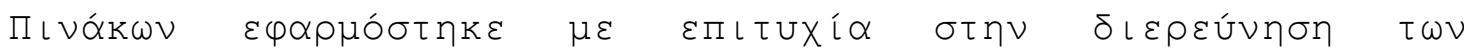

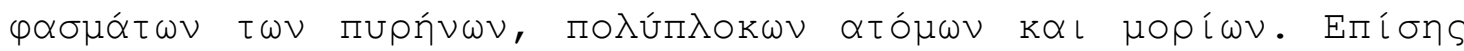

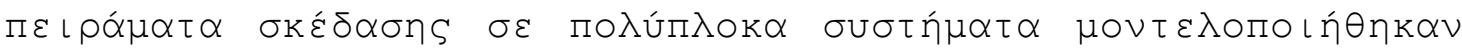

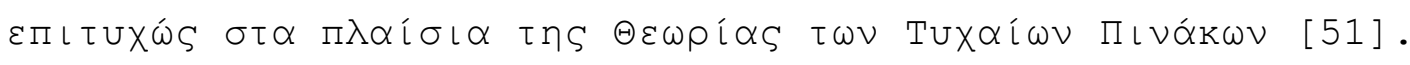

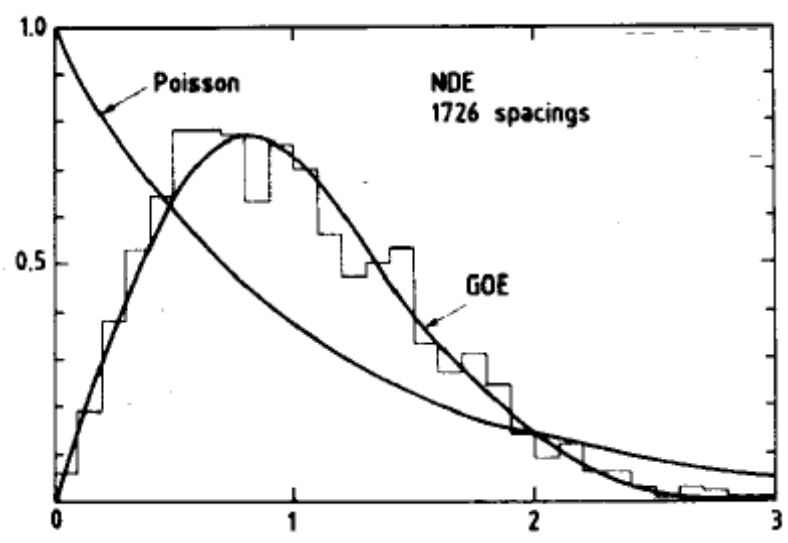

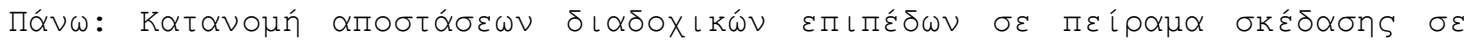
пUрńves(O. Bohigas, R.U. Haq and A. Pandey, in Nuclear Data for Science and Technology, K.H. Bochhoff (ed.), Reidel, Dordrecht (1983), p.809).

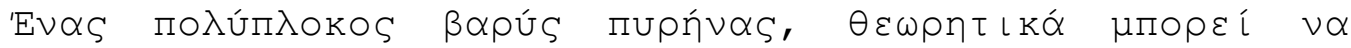

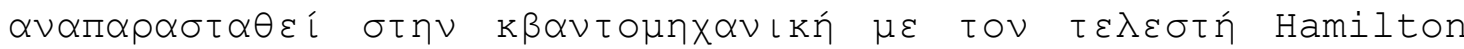

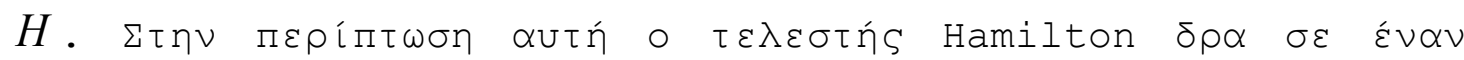

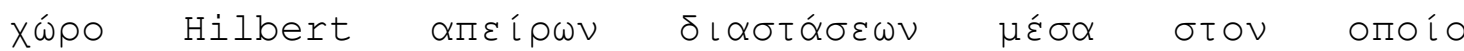

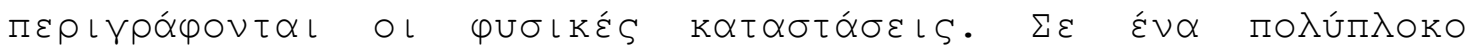

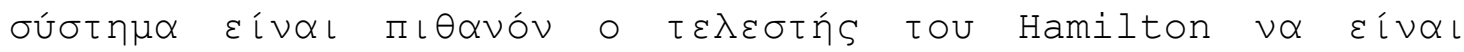

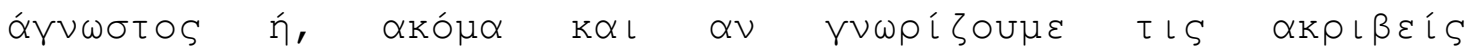

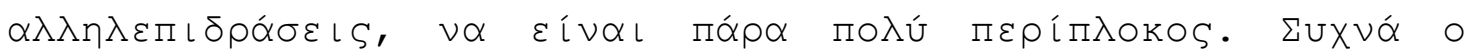

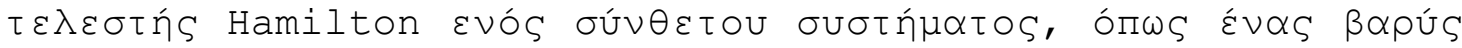




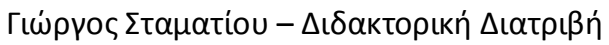

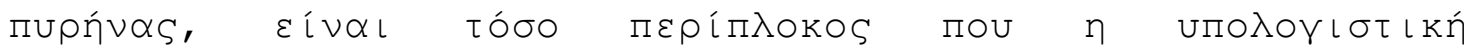

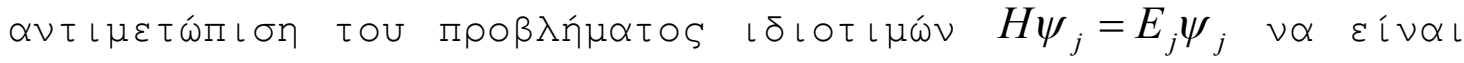

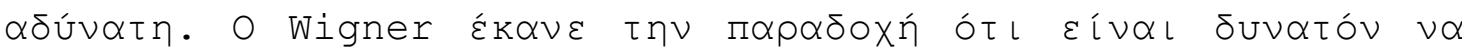

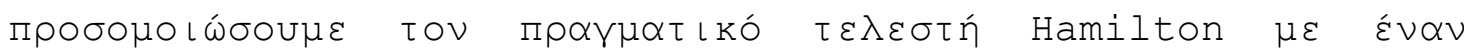

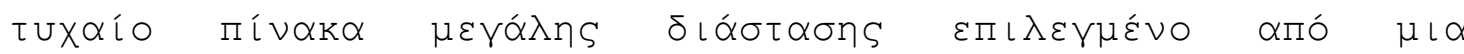

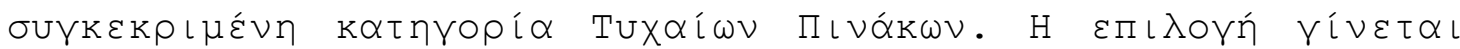

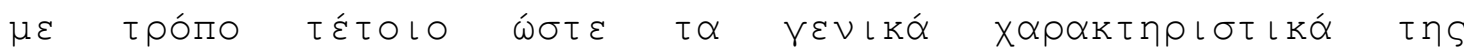

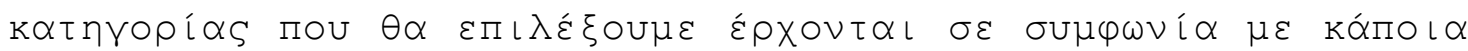

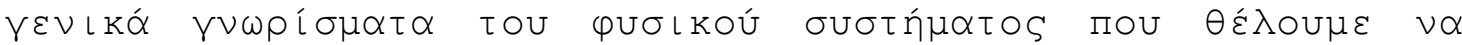

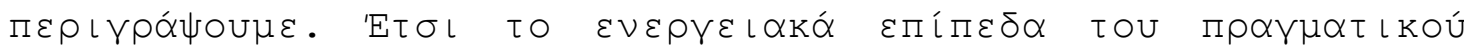

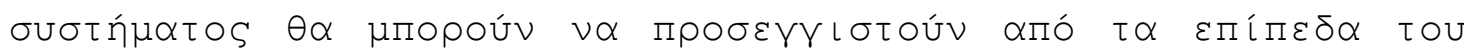

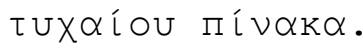

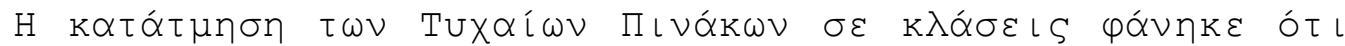

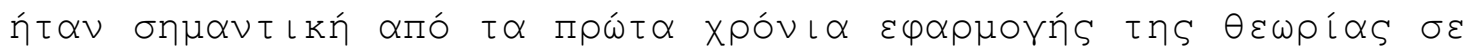

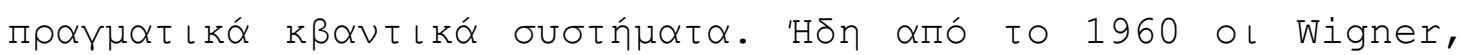

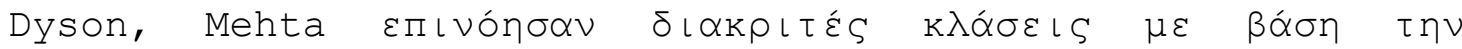

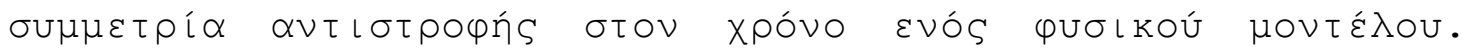

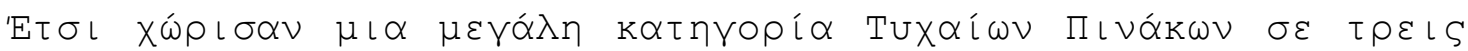

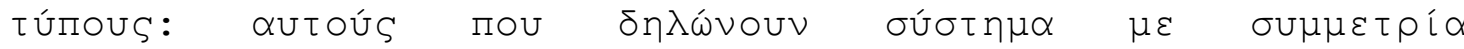

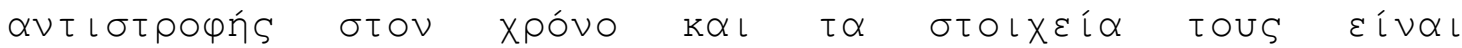

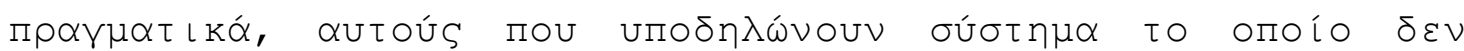

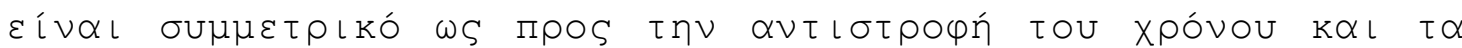

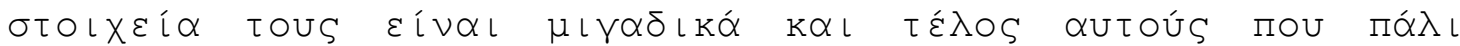

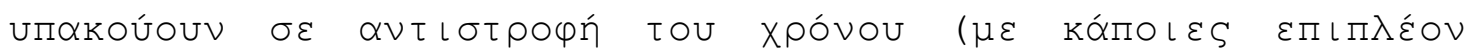

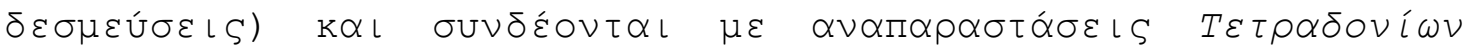
[52] (Quaternions).

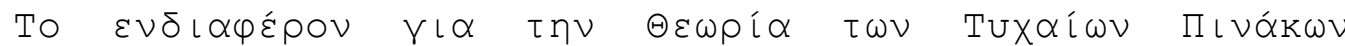

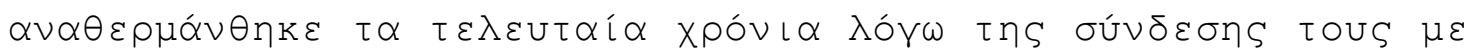

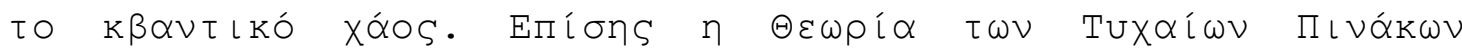

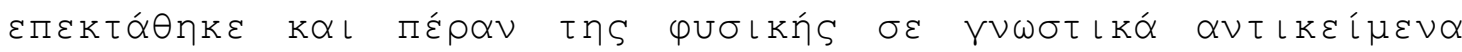

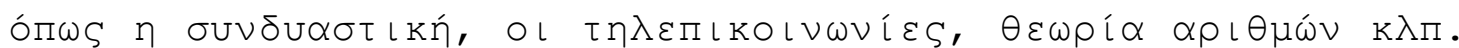

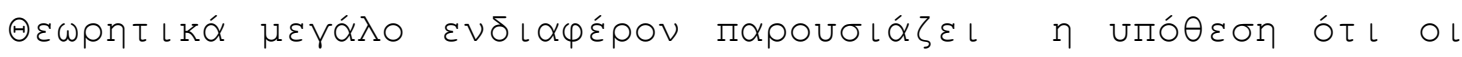

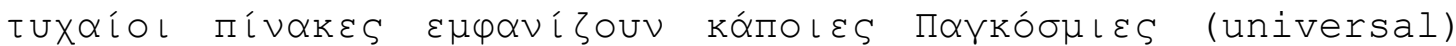

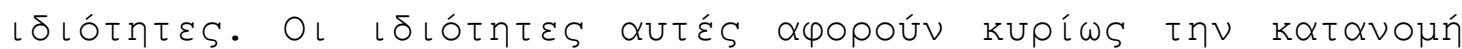

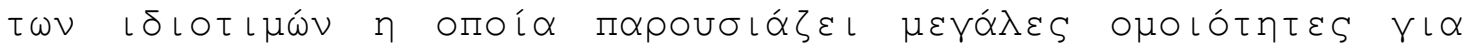

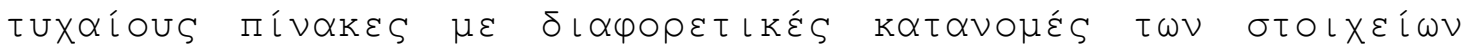

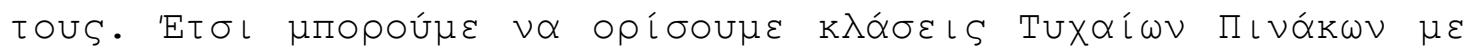

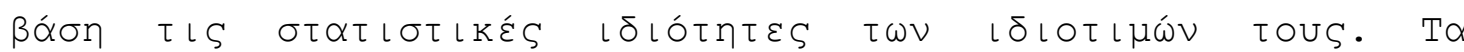

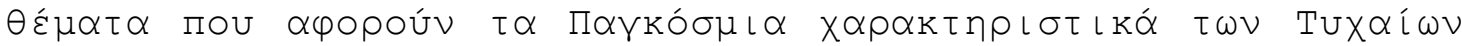




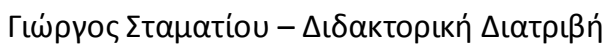

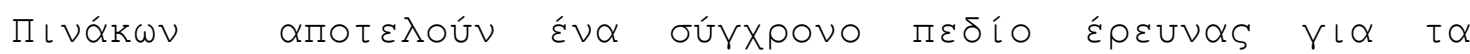

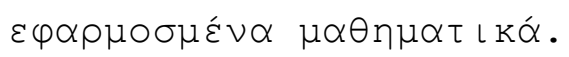

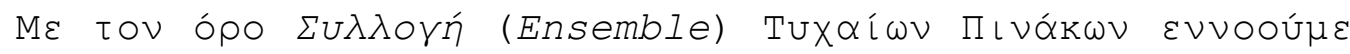

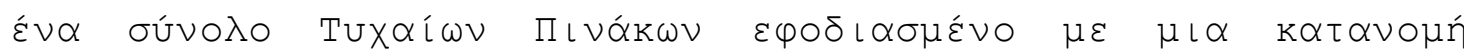

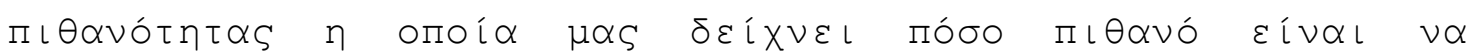

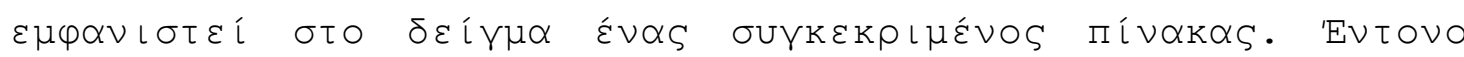

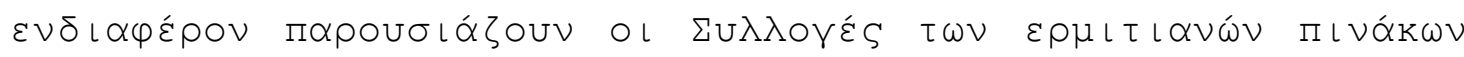

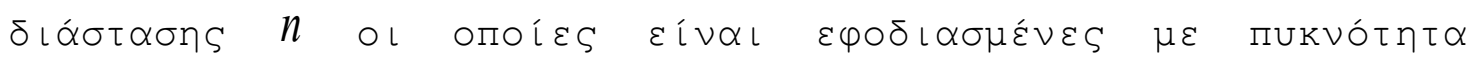

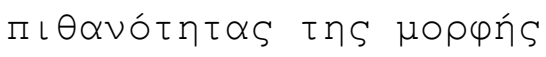

$$
p(H) \propto e^{-\beta \operatorname{Tr}[V(H)]}
$$

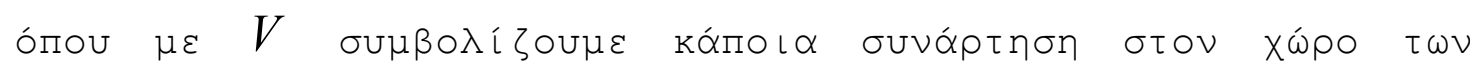

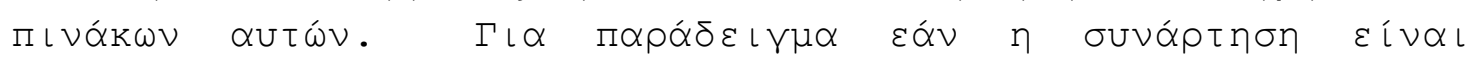
$V(H)=H^{2}$ тó c ₹ $\operatorname{Tr}[V(H)]=\sum_{i j}\left(H_{i j}\right)^{2}$.

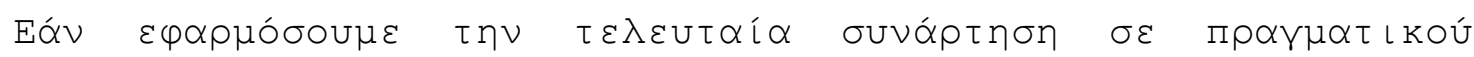

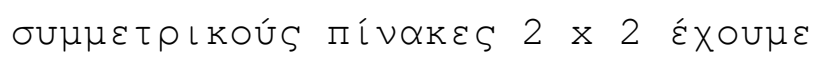

$$
H=\left(\begin{array}{ll}
H_{11} & H_{12} \\
H_{12} & H_{22}
\end{array}\right), H_{i j} \in R
$$

$K \propto l$

$$
\operatorname{Tr}\left(H^{2}\right)=H_{11}^{2}+H_{22}^{2}+2 H_{12}^{2}
$$

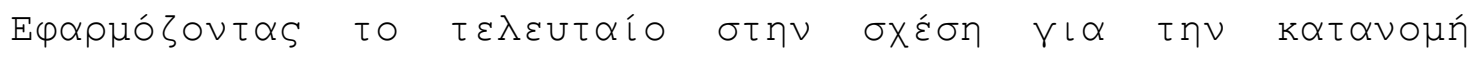

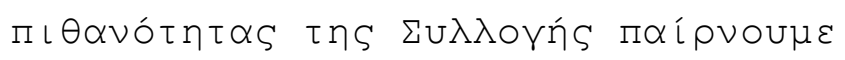

$$
p(H) \propto e^{-\beta\left(H_{11}^{2}+H_{22}^{2}+2 H_{12}^{2}\right)}
$$

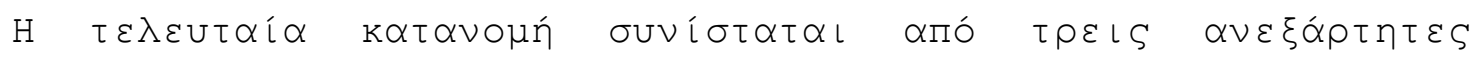

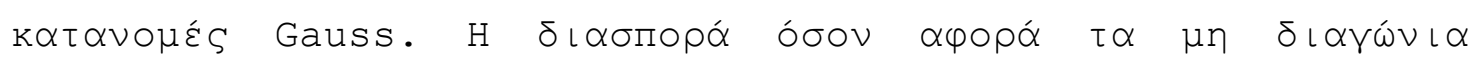

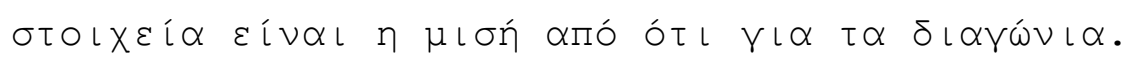

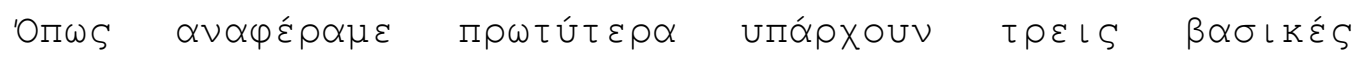

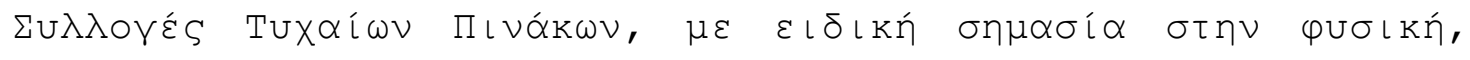




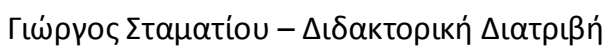

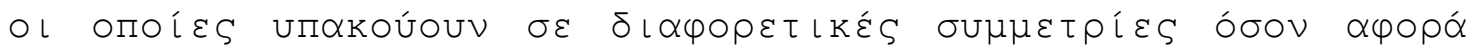

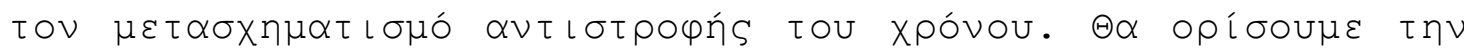

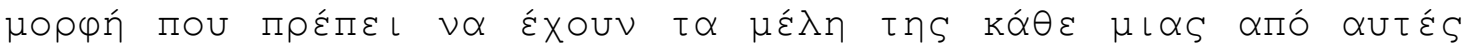

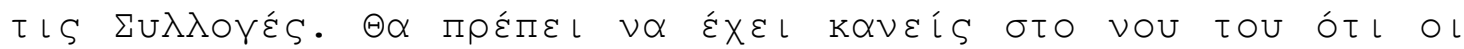

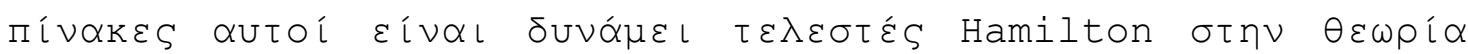

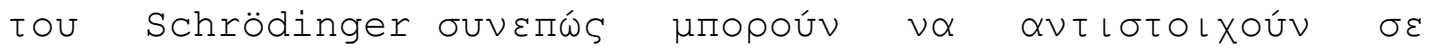

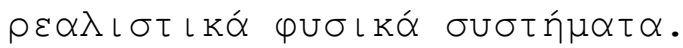

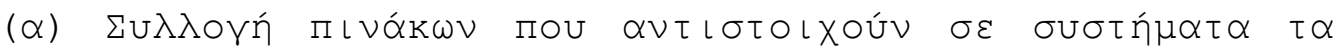

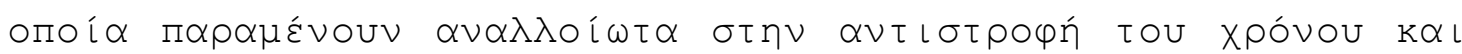

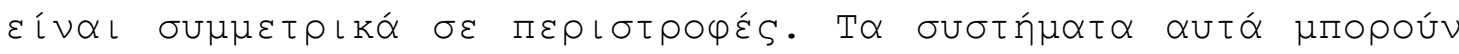

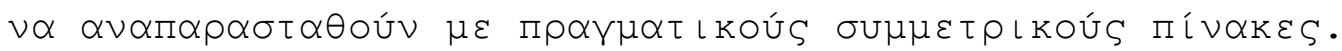

$$
H_{m n}=H_{n m}=H_{m n}^{*}
$$

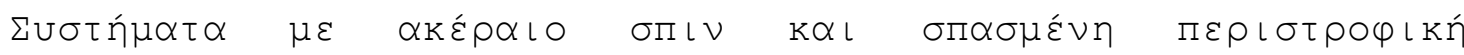

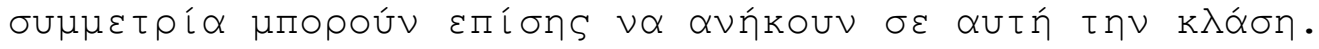

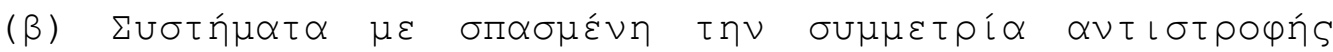

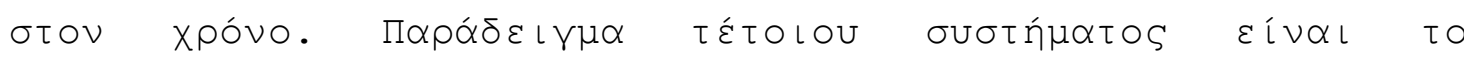

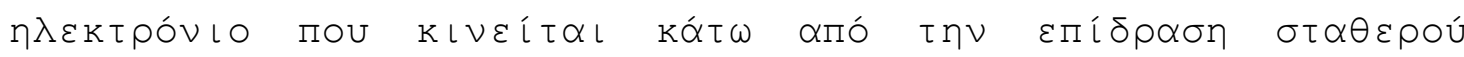

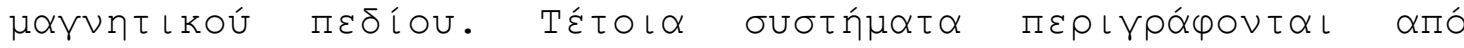

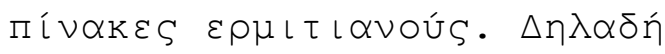

$$
H_{m n}=\left[H^{+}\right]_{n m}
$$

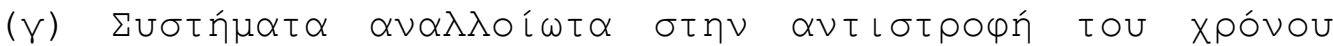

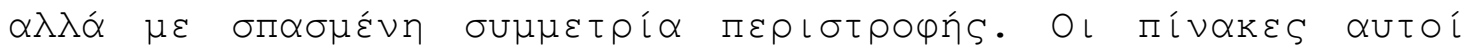

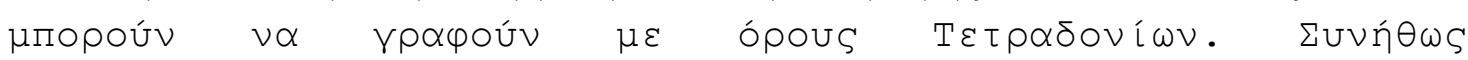

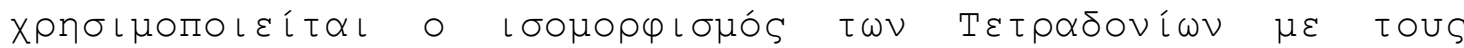

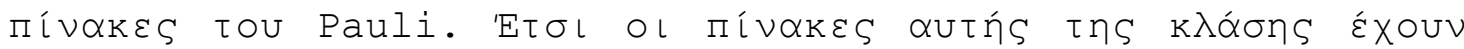

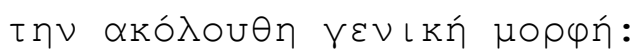

$$
H_{n m}^{(0)} 1_{2}-i \sum_{\gamma=1}^{3} H_{n m}^{(\gamma)} \sigma_{\gamma}
$$

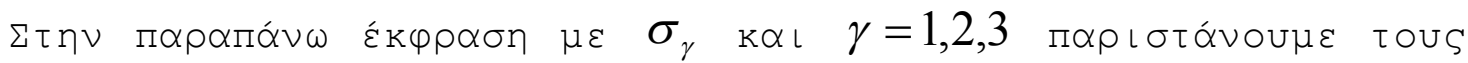

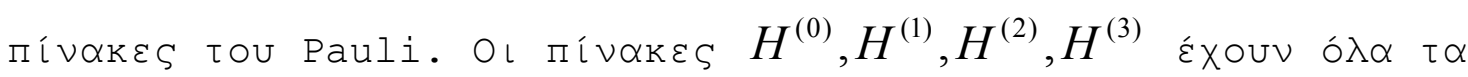




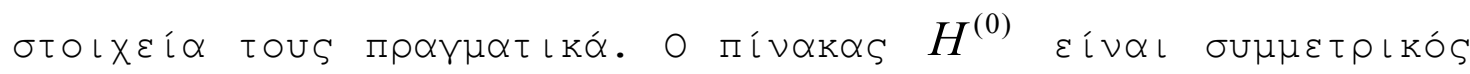

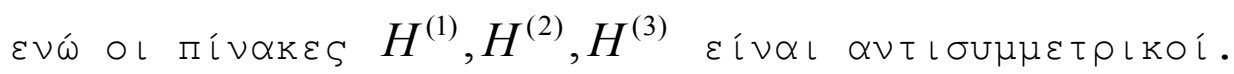

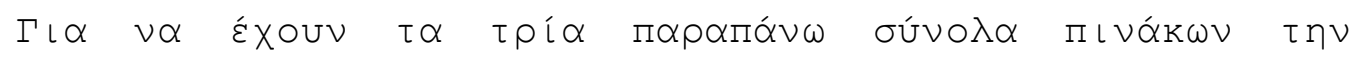

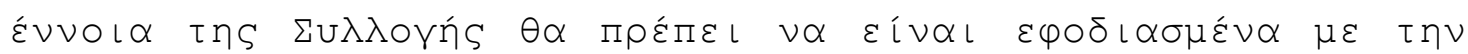

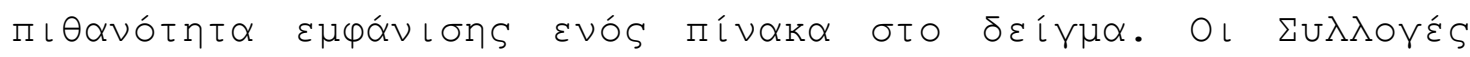

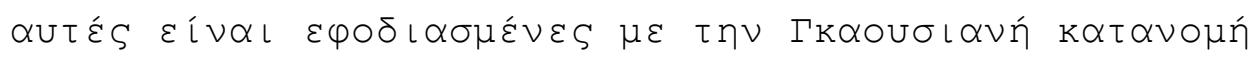

$$
P_{N \beta}(H) \propto \exp \left(-\frac{\beta N}{\lambda^{2}} t r H^{2}\right)
$$

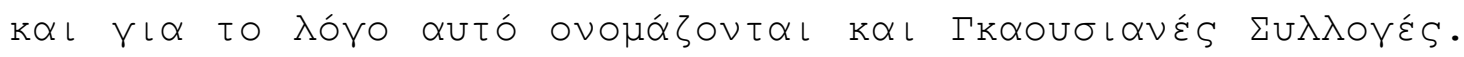

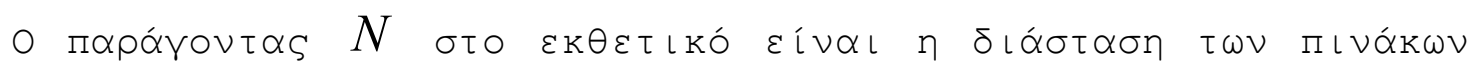

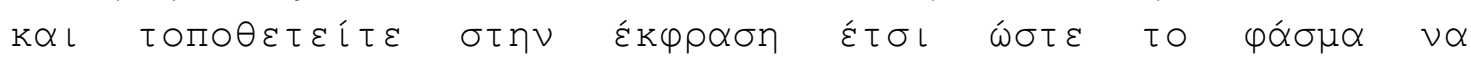

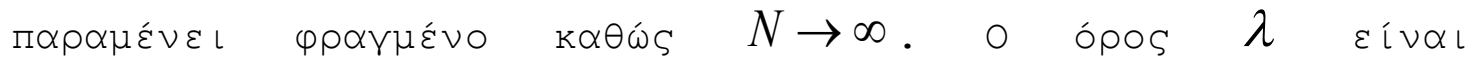

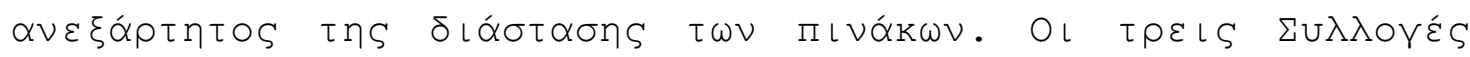

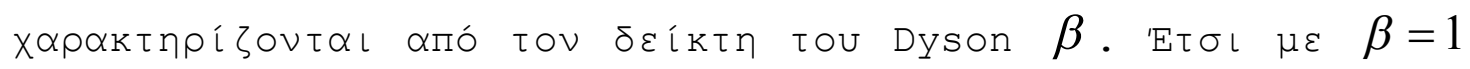

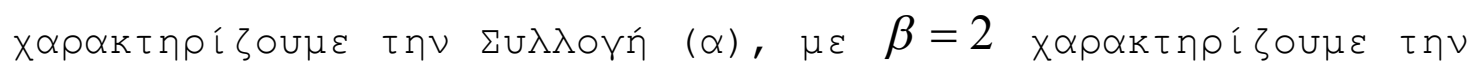

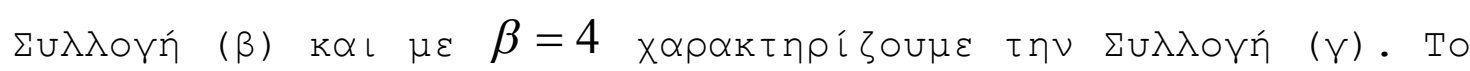

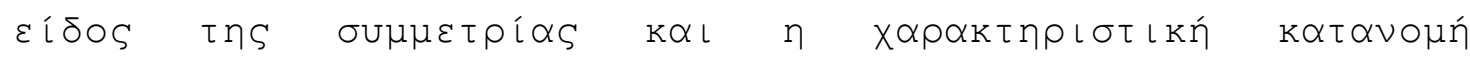

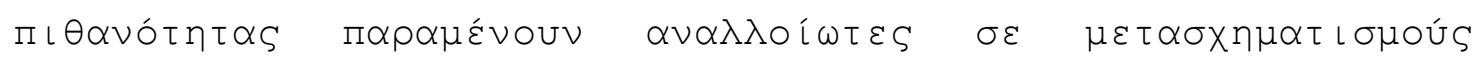

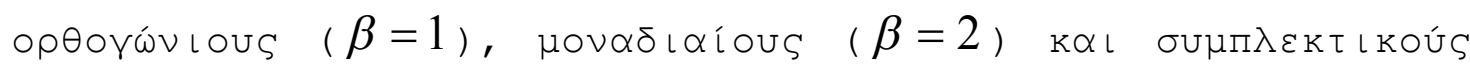

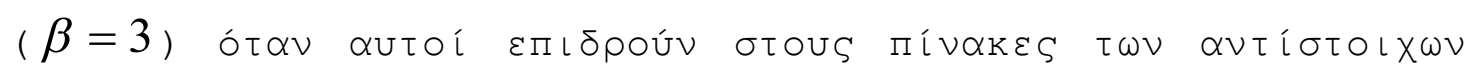

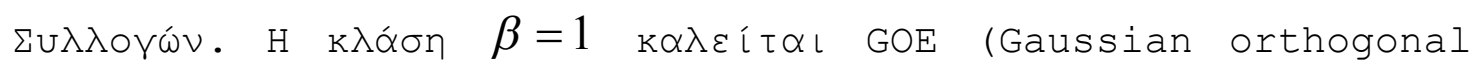

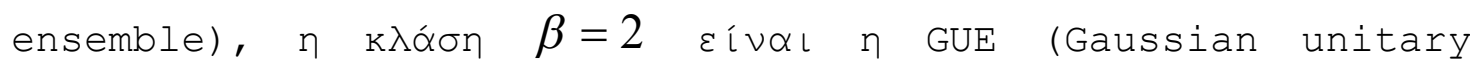

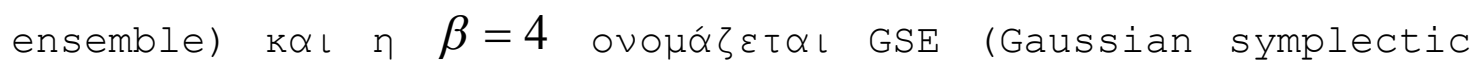

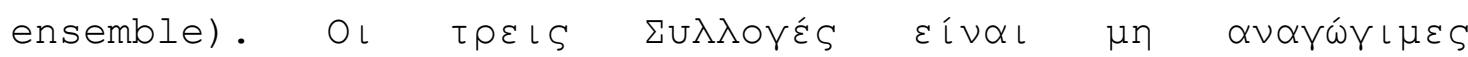

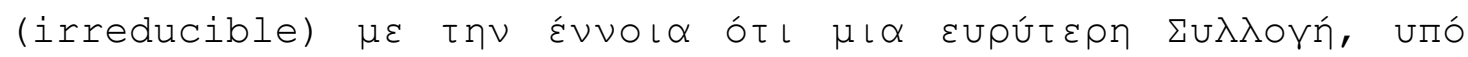

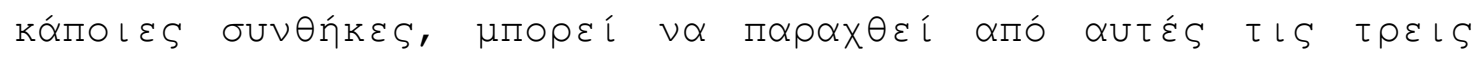
$\Sigma U \lambda \lambda \circ \gamma \tilde{\varepsilon} S$.

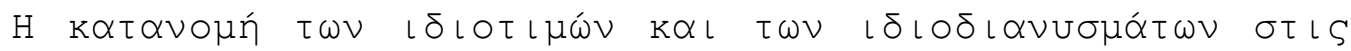

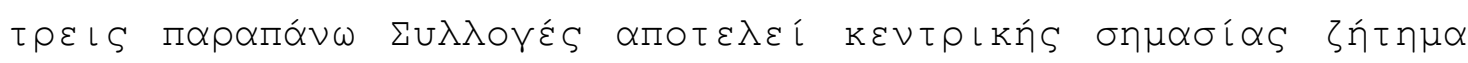

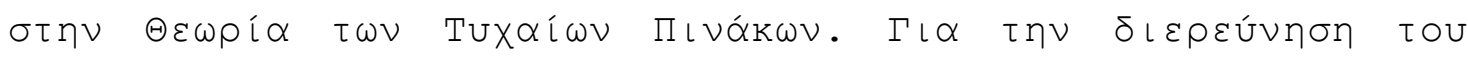

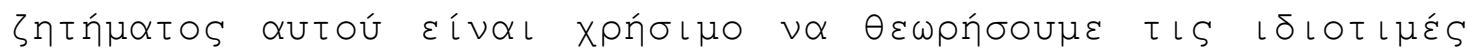

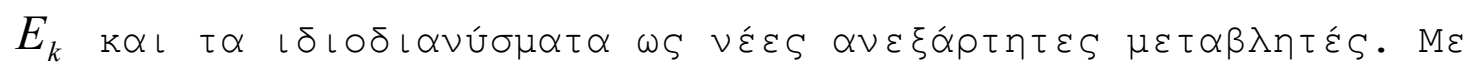




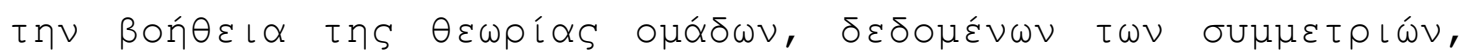

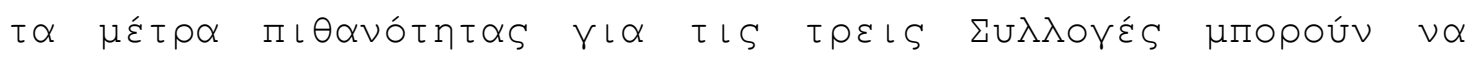

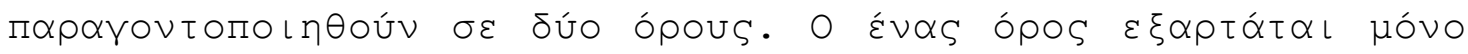

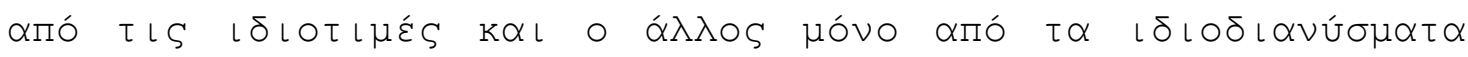

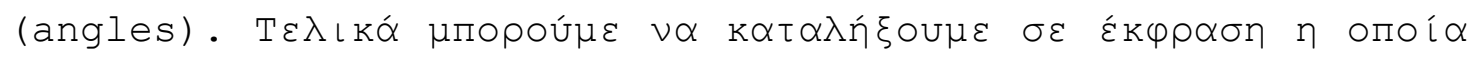

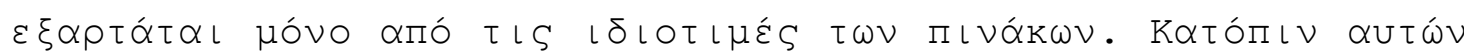

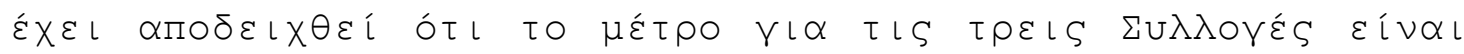
т०

$$
P_{N \beta}\left(E_{1}, \ldots, E_{N}\right) \prod_{m>n}\left|E_{m}-E_{n}\right|^{\beta} \prod_{i=1}^{N} d E_{i}
$$

$\mu \varepsilon \quad \beta=1,2,4$.

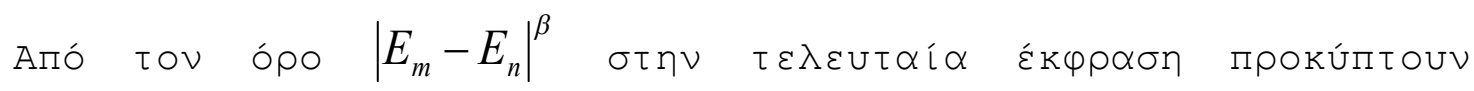

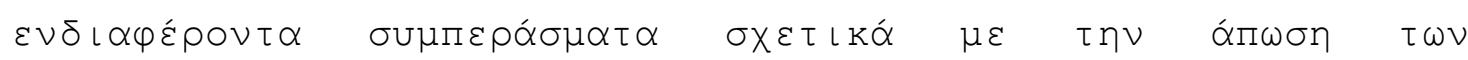

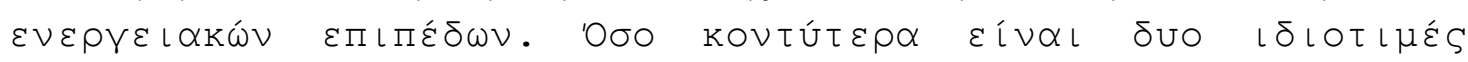

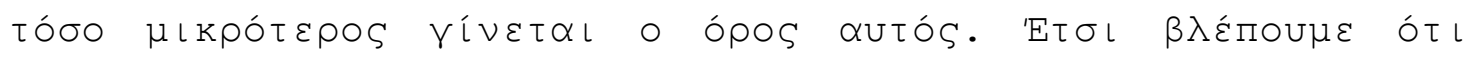

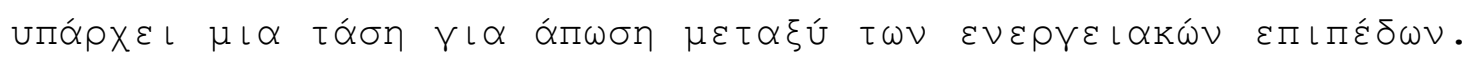

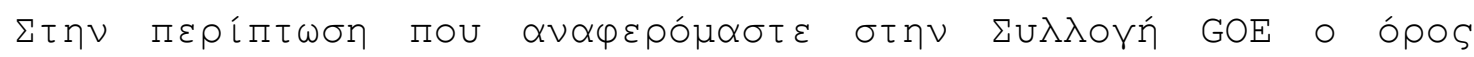

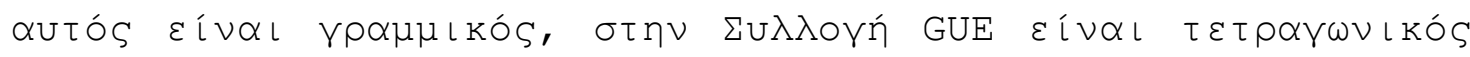

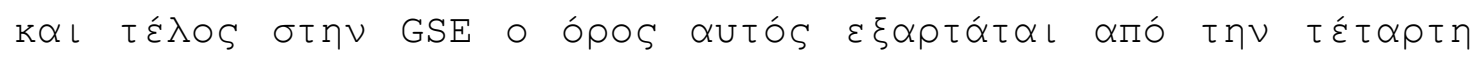

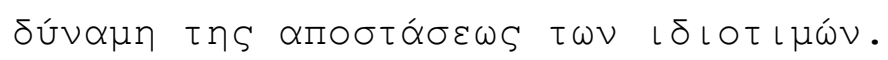

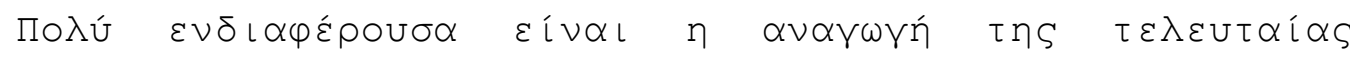

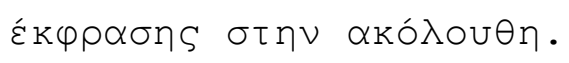

$$
\exp \left[-\beta N \sum_{n=1}^{N}\left(\frac{E_{n}}{\lambda}\right)^{2}+\frac{\beta}{2} \sum_{m>n} \ln \left(\frac{E_{m}-E_{n}}{\lambda}\right)^{2}\right] \prod_{i=1}^{N} d E_{i}
$$

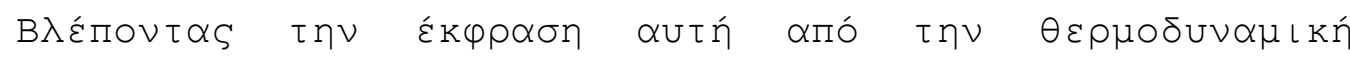

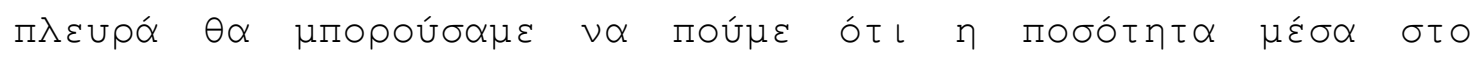

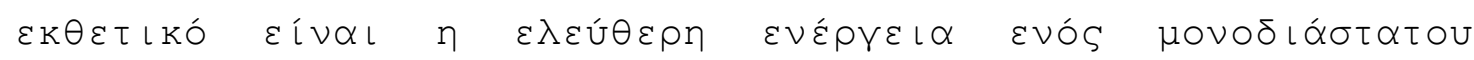

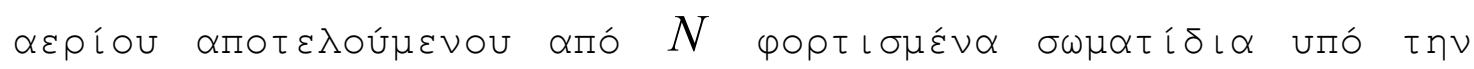

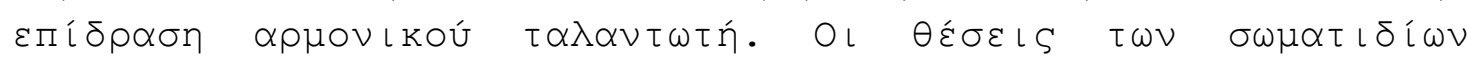

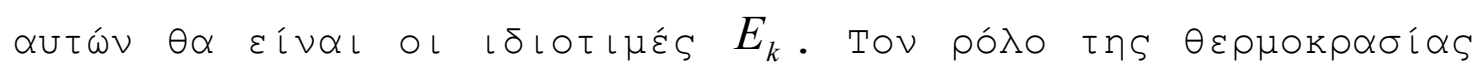

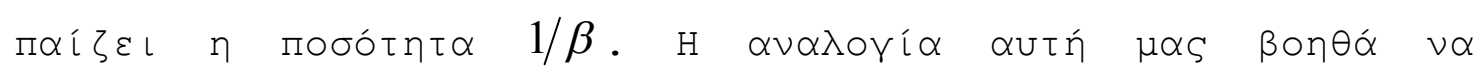

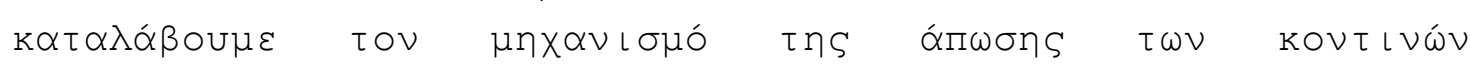




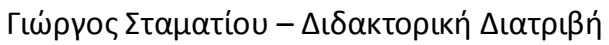

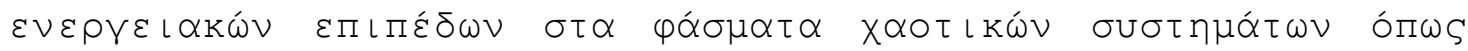

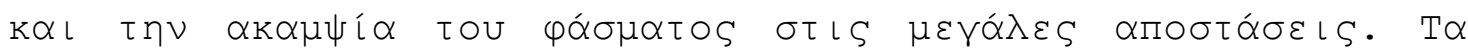

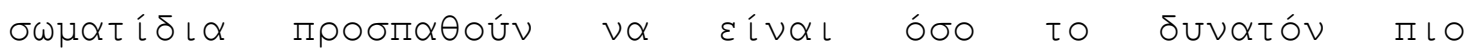

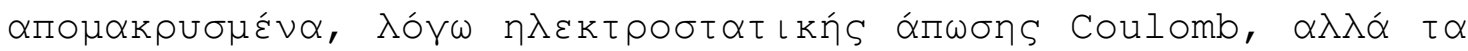

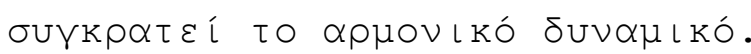

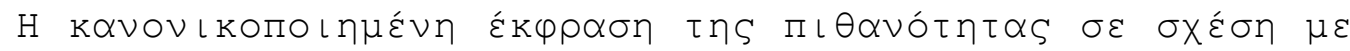

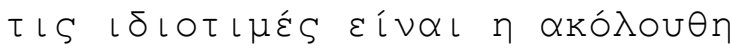

$$
\begin{gathered}
P_{N \beta}^{(E)}\left(x_{1}, \ldots, x_{N}\right)=C_{N \beta} \exp \left(-\frac{\beta}{2} \sum_{n=1}^{N} x_{n}^{2}\right)\left|\Delta_{N}(X)\right|^{\beta} \\
\Delta_{N}(x)=\prod_{n<m}\left(x_{n}-x_{m}\right)
\end{gathered}
$$

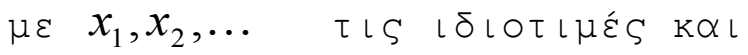

$$
C_{N \beta}=\frac{\beta^{N / 2+\beta N(N-1) / 4}}{(2 \pi)^{N / 2}} \frac{\Gamma^{N}(1+\beta / 2)}{\prod_{n=1}^{N} \Gamma(1+\beta n / 2)}
$$

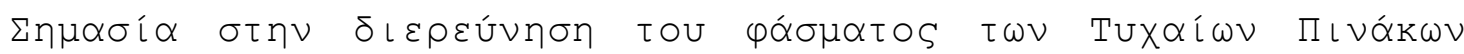

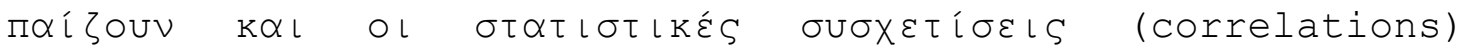

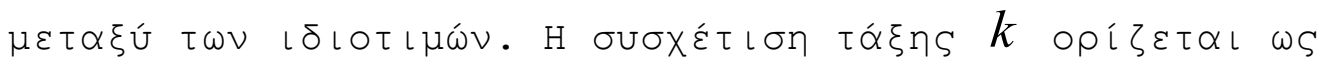

$$
R_{k}^{(\beta)}\left(x_{1}, \ldots, x_{k}\right)=\frac{N !}{(N-k) !} \int_{-\infty}^{+\infty} d x_{k+1} \ldots \int_{-\infty}^{+\infty} d x_{N}\left|\Delta_{N}(x)\right|^{\beta} P_{N}^{(\beta)}(x)
$$

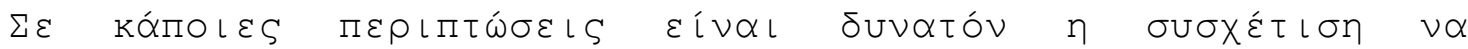

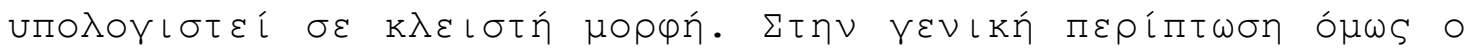

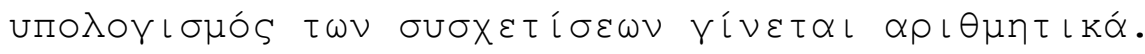

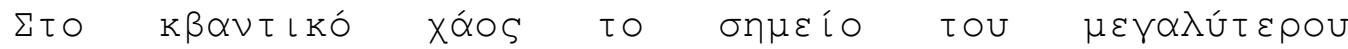

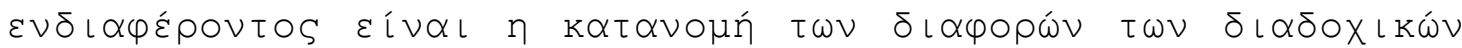

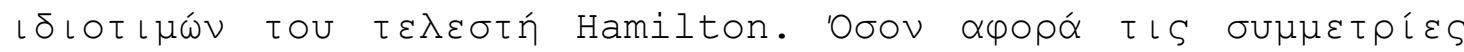

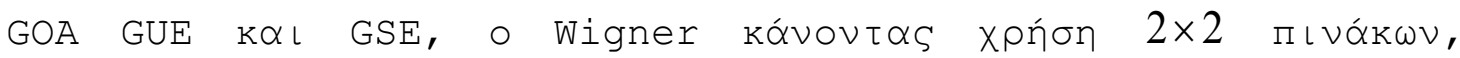

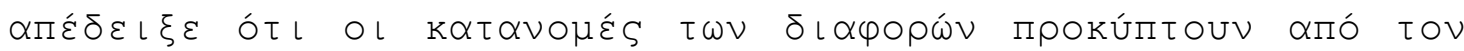

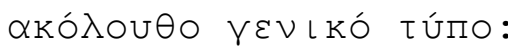




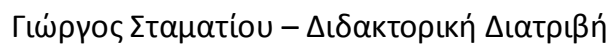

$$
p_{\beta}=a_{\beta} s^{\beta} \exp \left(-b_{\beta} s^{2}\right)
$$

ónоv

$$
a_{\beta}=2 \frac{\Gamma^{\beta+1}[(\beta+2) / 2]}{\Gamma^{\beta+2}[(\beta+1) / 2]} \quad \text { к } \alpha \iota \quad b_{\beta}=2 \frac{\Gamma^{2}[(\beta+2) / 2]}{\Gamma^{2}[(\beta+1) / 2]}
$$

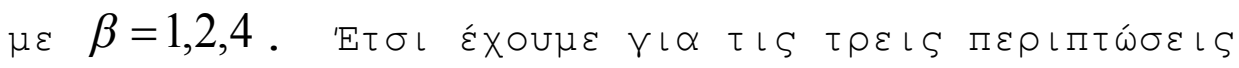

\begin{tabular}{|l|l|l|}
\hline GOE & $\alpha_{1}=\frac{\pi}{2}$ & $b_{1}=\frac{\pi}{4}$ \\
\hline GUE & $\alpha_{2}=\frac{32}{\pi^{2}}$ & $b_{2}=\frac{4}{\pi}$ \\
\hline GSE & $\alpha_{4}=\frac{262144}{729 \pi^{3}}$ & $b_{4}=\frac{64}{9 \pi}$ \\
\hline
\end{tabular}

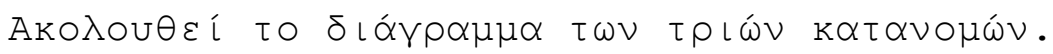

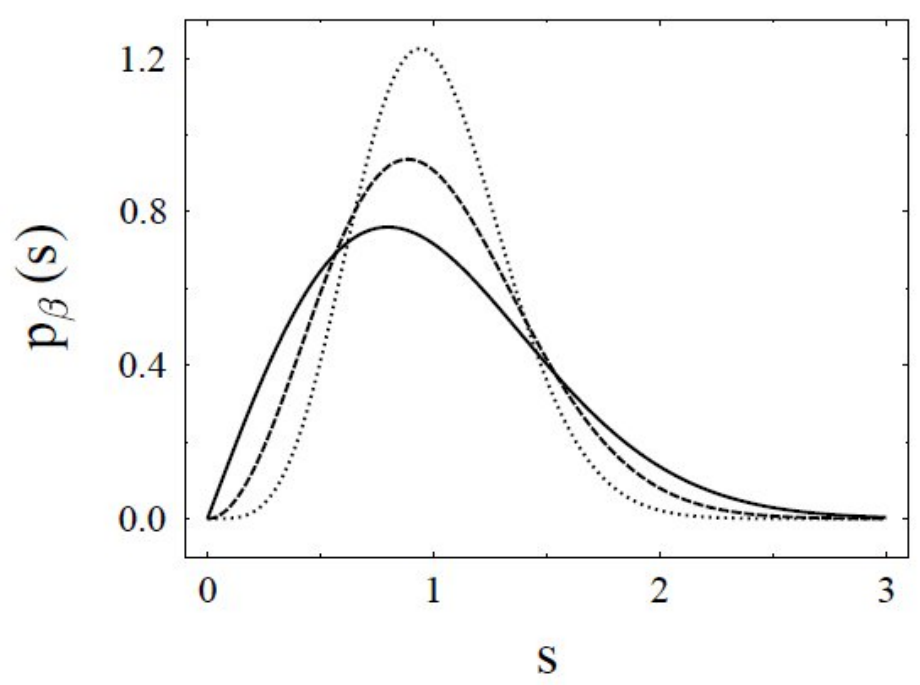

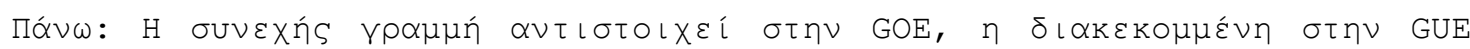

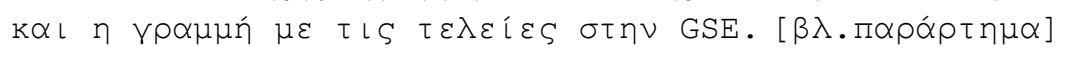




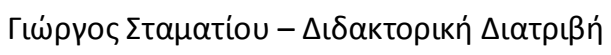

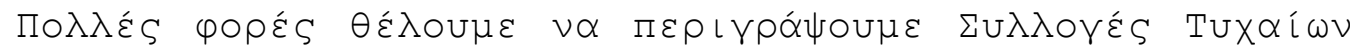

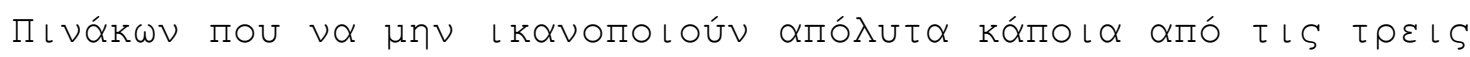

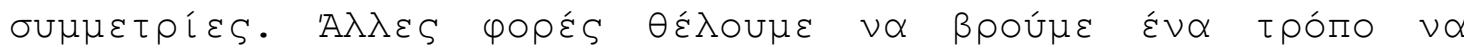

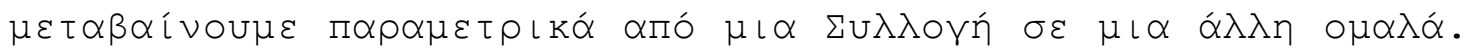

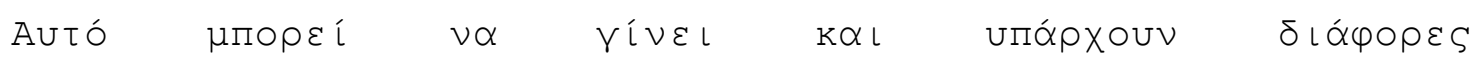

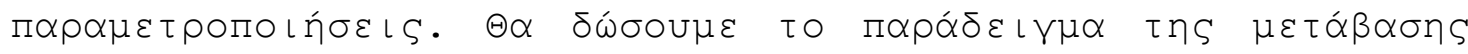

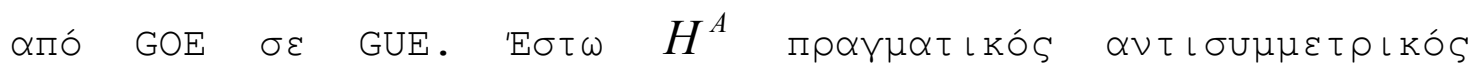

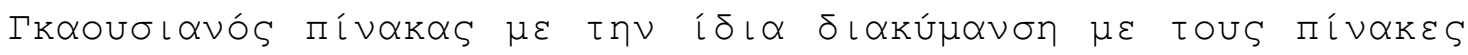

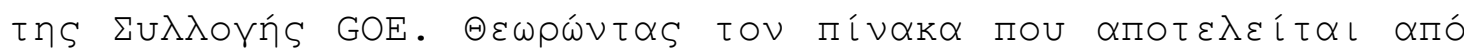

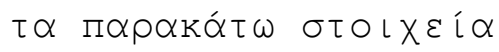

$$
H_{n m}=H_{n m}^{G O E}+i \sqrt{t / N} H_{n m}^{A}
$$

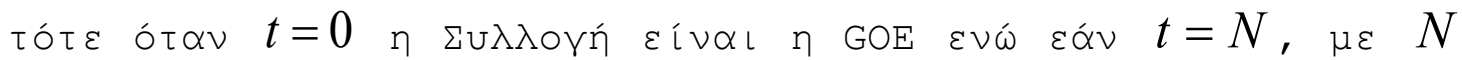

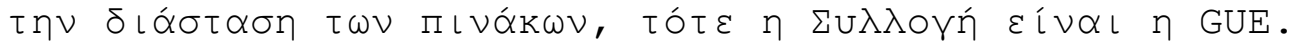

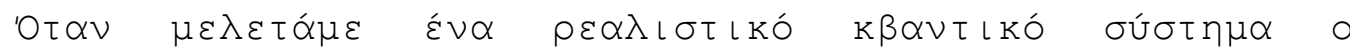

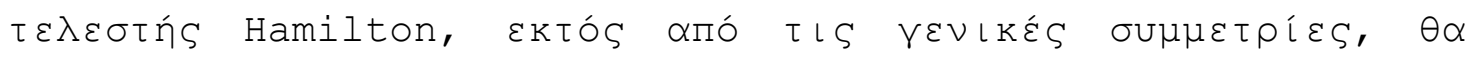

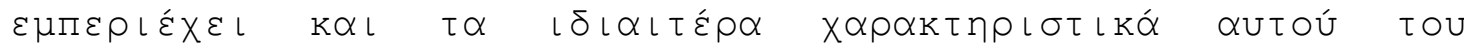

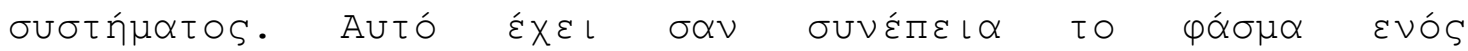

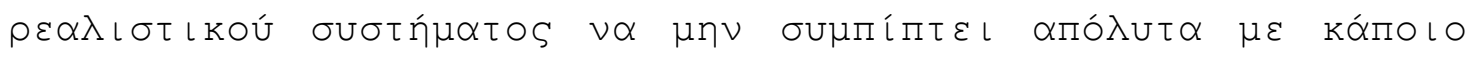

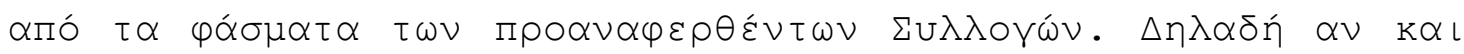

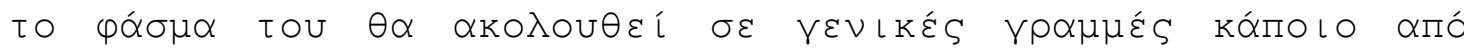

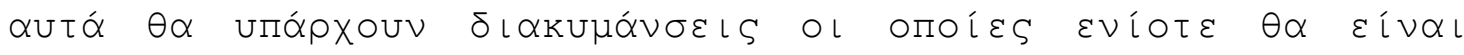

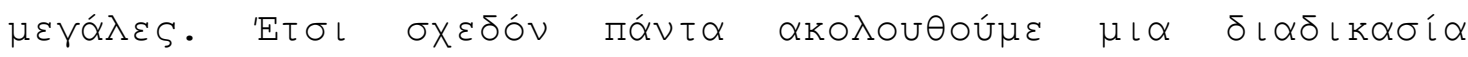

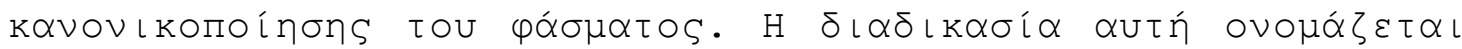

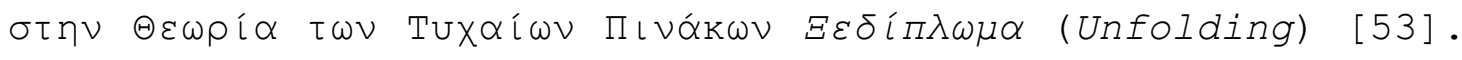

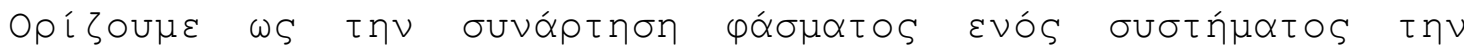
$\varepsilon \kappa \varphi \rho \alpha \sigma \eta$

$$
\rho(E)=\overline{\sum_{\mu} \delta\left(E-E_{\mu}\right)}
$$

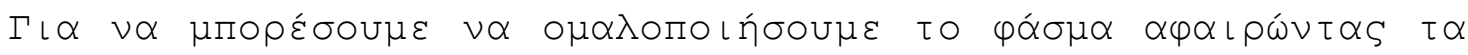

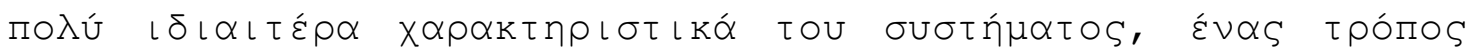

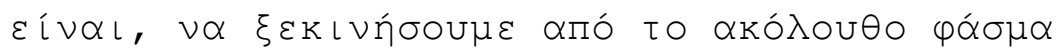

$$
\varepsilon_{\mu}=\int_{-\infty}^{E_{\mu}} d E \rho(E)
$$




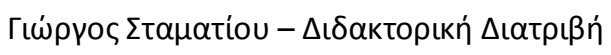

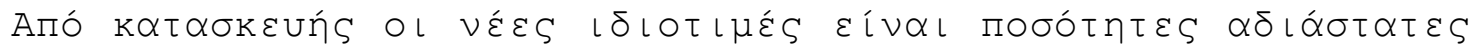

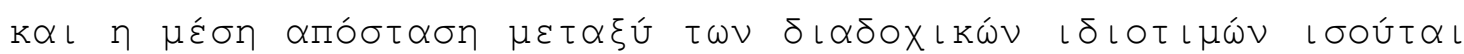

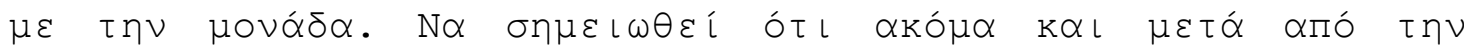

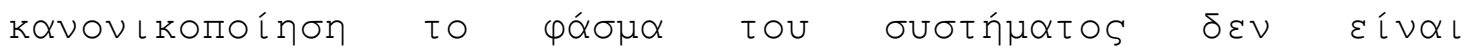

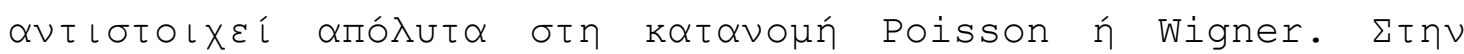

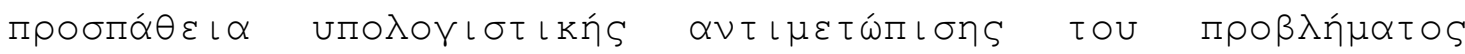

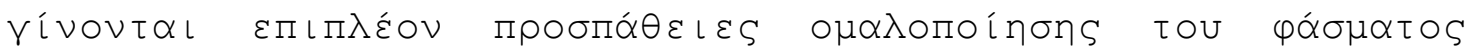

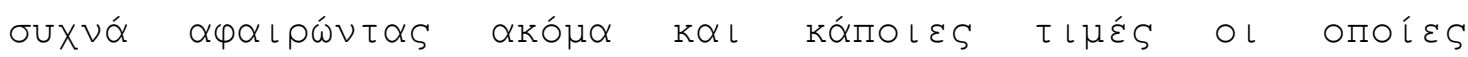

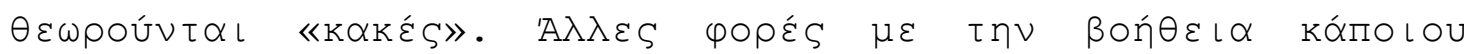

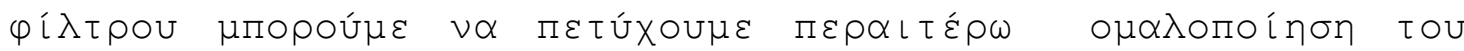

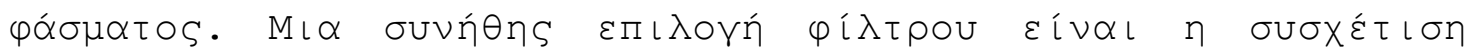

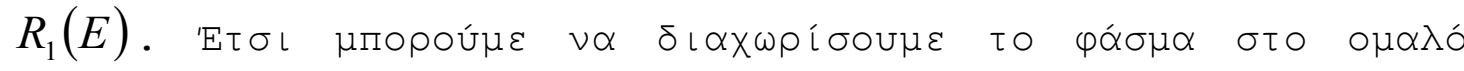

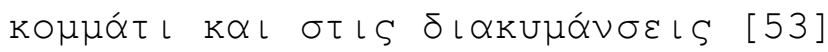

$$
n(\varepsilon)=n_{\text {smooth }}(\varepsilon)+n_{f l}(\varepsilon)
$$

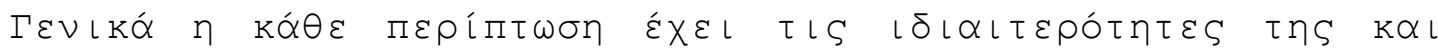

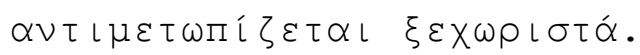

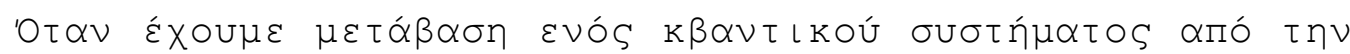

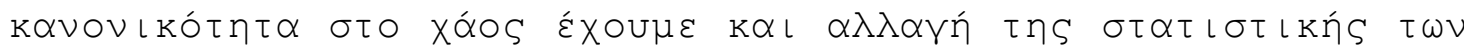

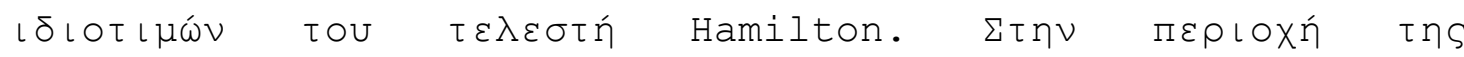

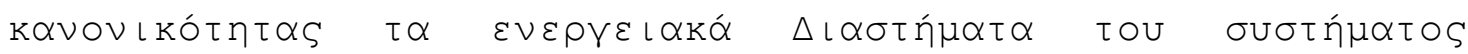

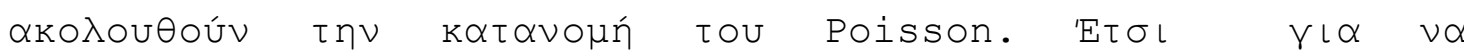

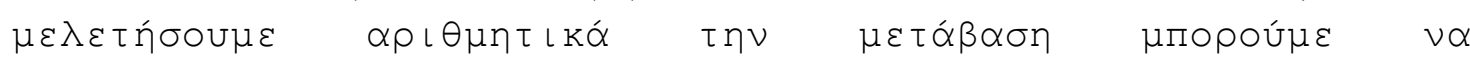

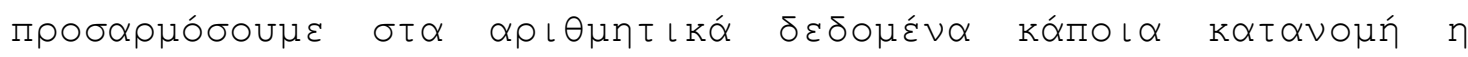

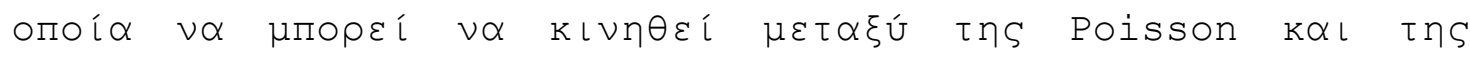

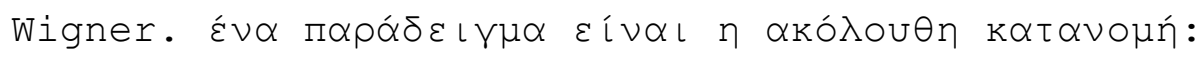

$$
p_{\beta_{e f f}}(s)=A_{\beta_{e f f}} s^{\beta_{e f f}} \exp \left[-\frac{\pi^{2} \beta_{e f f}}{16} s^{2}-\left(B_{\beta_{e f f}}-\frac{\pi \beta_{e f f}}{4}\right) s\right]
$$

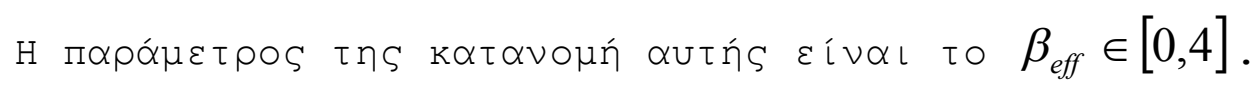

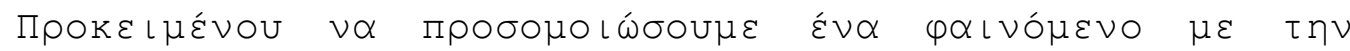

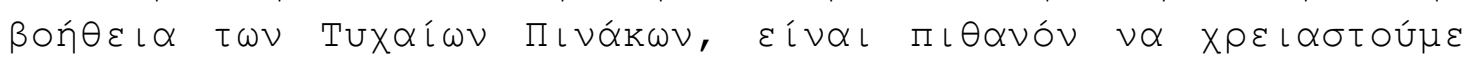

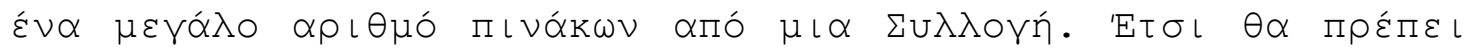

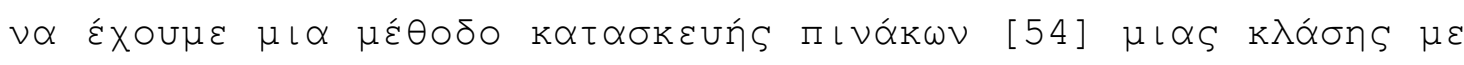

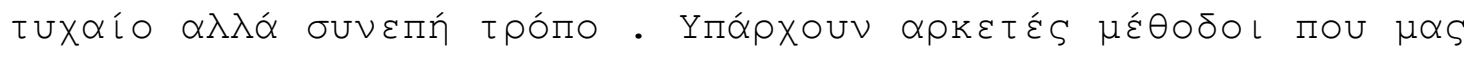




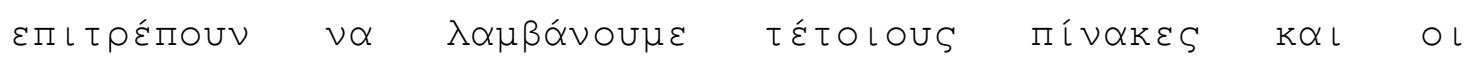

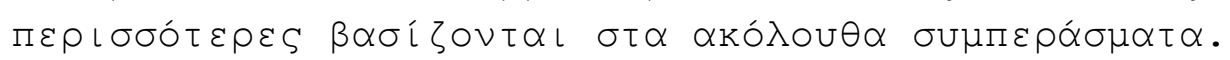

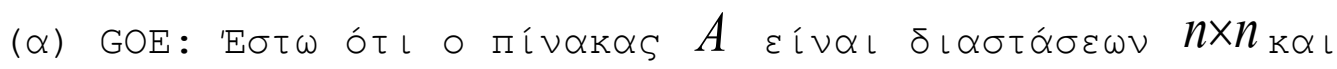

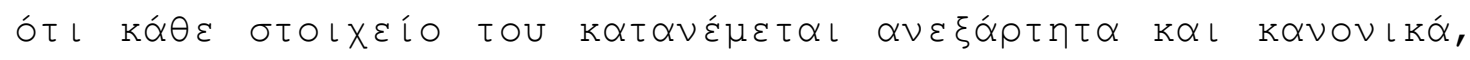

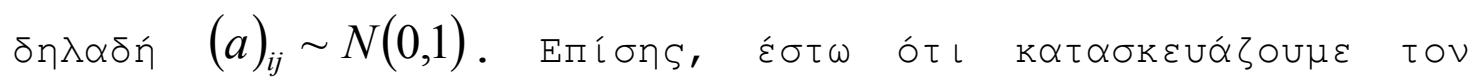

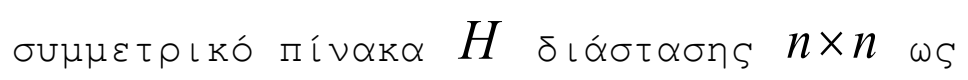

$$
H=\frac{A+A^{T}}{2}
$$

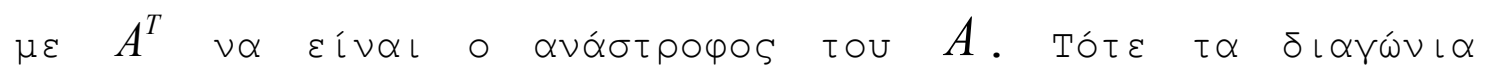

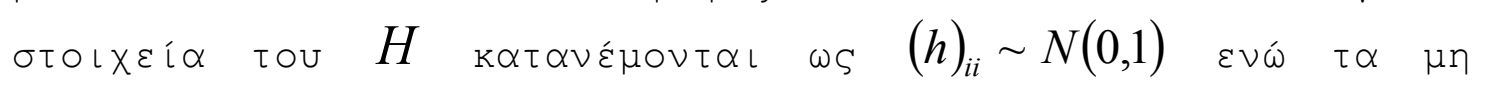

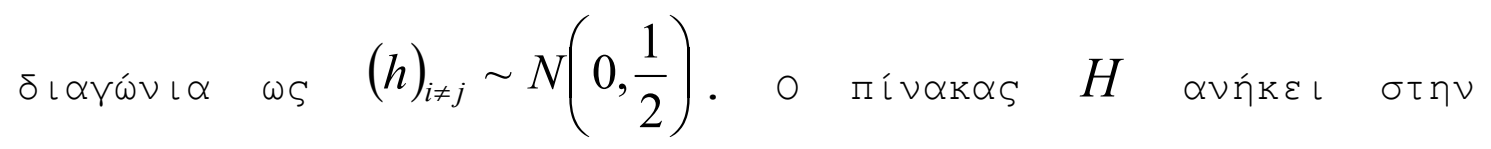

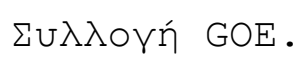

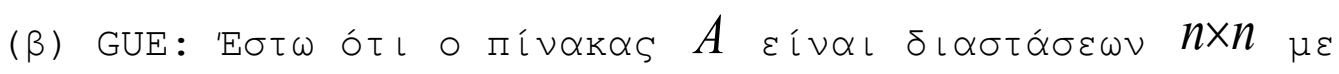

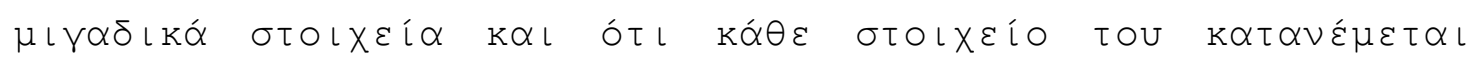

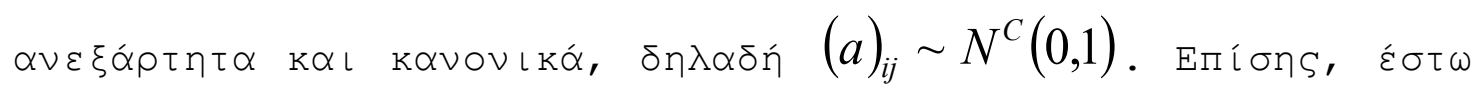

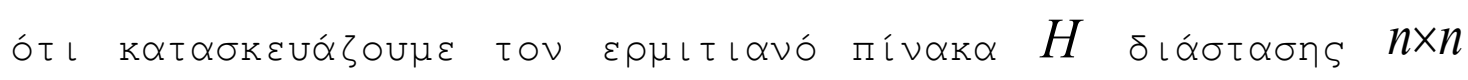
$\omega S$

$$
H=\frac{A+A^{*}}{2}
$$

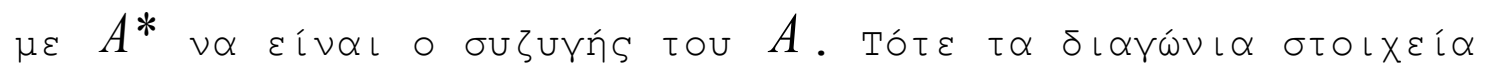

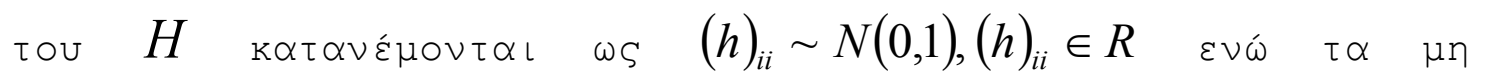

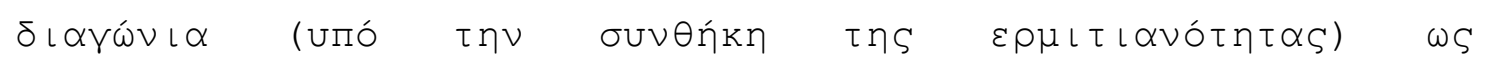

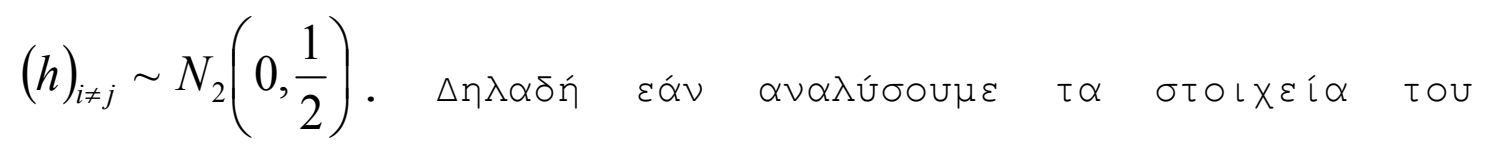

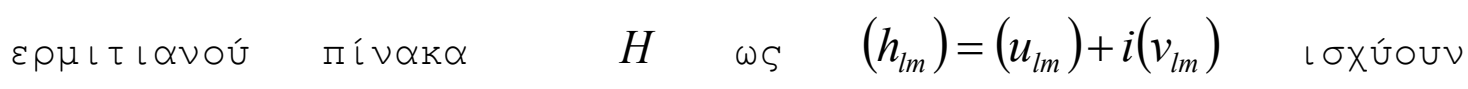




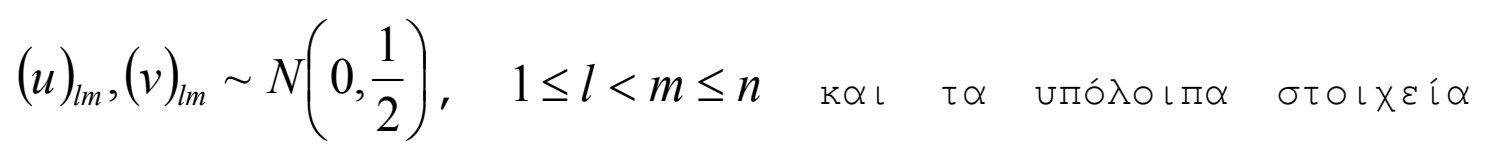

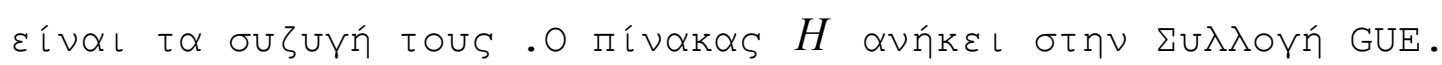

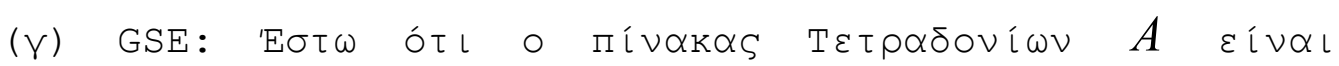

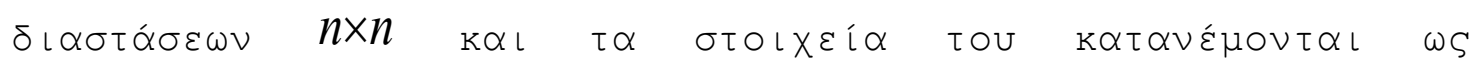

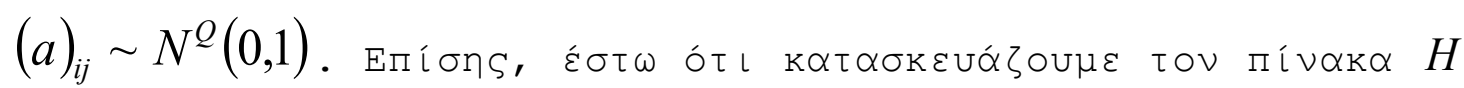
$\delta\llcorner\alpha ́ \sigma \tau \alpha \sigma \eta s \quad n \times n$ wS

$$
H=\frac{A+A^{D}}{2}
$$

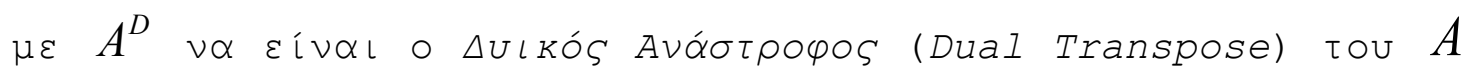

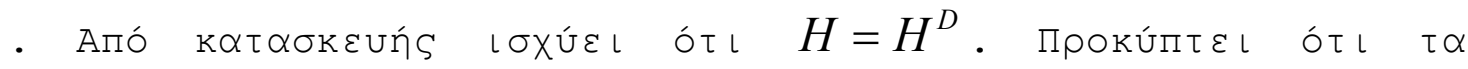

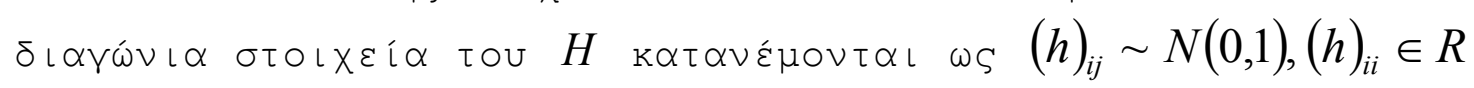

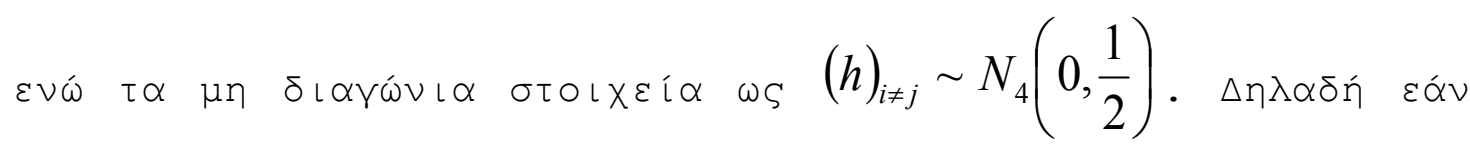

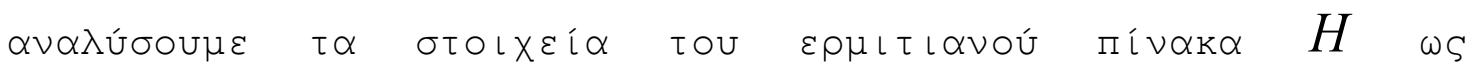
$\left(h_{l m}\right)=\left(u_{l m}\right)+i\left(v_{l m}\right)+j\left(w_{l m}\right)+k\left(z_{l m}\right)$

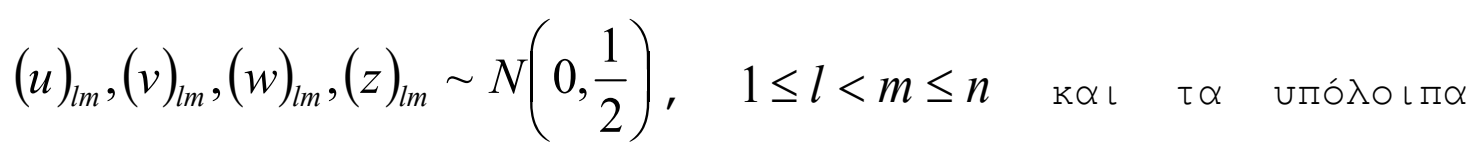

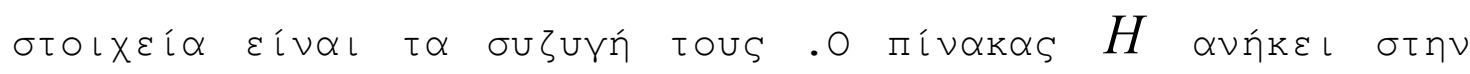

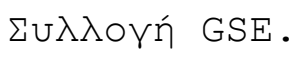

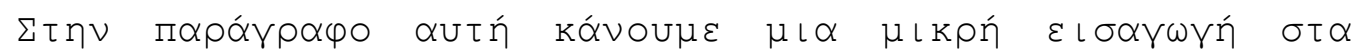

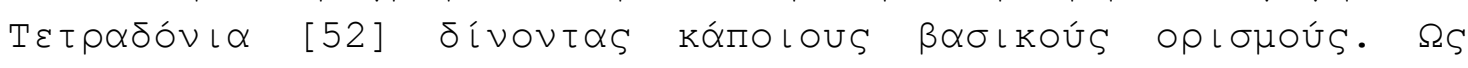

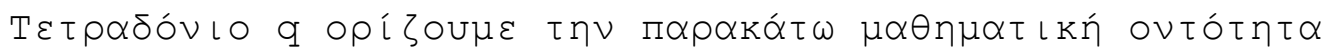

$$
a \cdot 1+b \cdot i_{1}+c \cdot i_{2}+d \cdot i_{3}, \quad a, b, c, d \in \mathbb{R}
$$

$\mu \varepsilon$

$$
i_{1}^{2}=i_{2}^{2}=i_{3}^{2}=i_{1} i_{2} i_{3}=-1
$$




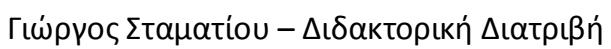

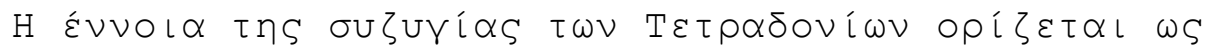

$$
\bar{q}=a \cdot 1-b i_{1}-c i_{2}-d i_{3}
$$

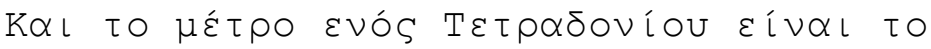

$$
\|q\|^{2}=q \bar{q}=\bar{q} q=a^{2}+b^{2}+c^{2}+d^{2}
$$

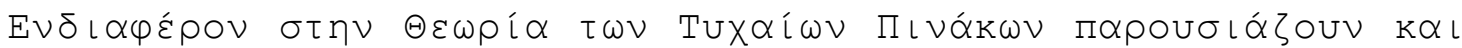

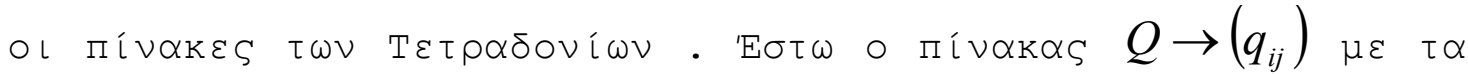

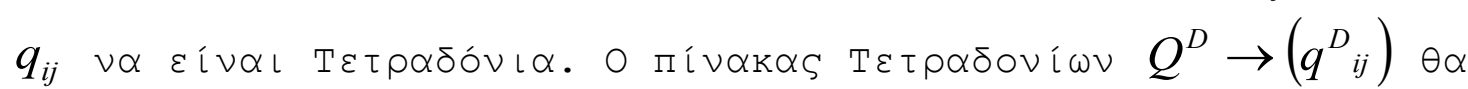

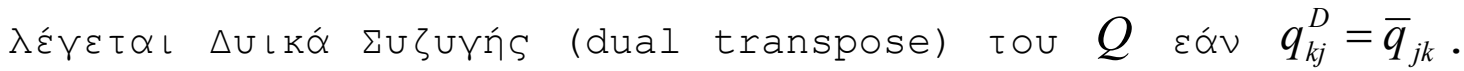

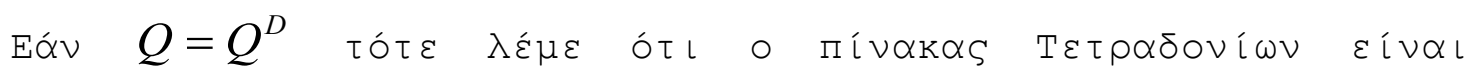
Autoduıkós (self dual).

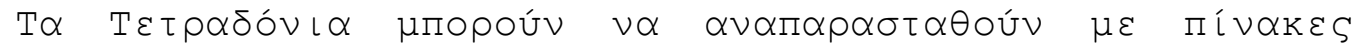

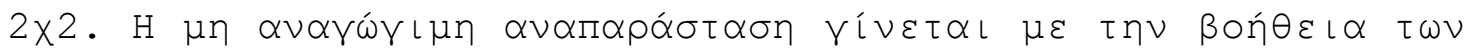

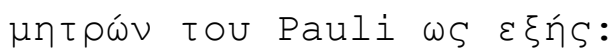

$$
1 \rightarrow\left(\begin{array}{ll}
1 & 0 \\
0 & 1
\end{array}\right), \quad i_{1} \rightarrow\left(\begin{array}{cc}
i & 0 \\
0 & -i
\end{array}\right), \quad i_{2} \rightarrow\left(\begin{array}{cc}
0 & 1 \\
-1 & 0
\end{array}\right), \quad i_{3} \rightarrow\left(\begin{array}{ll}
0 & i \\
i & 0
\end{array}\right)
$$

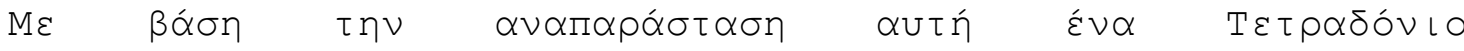

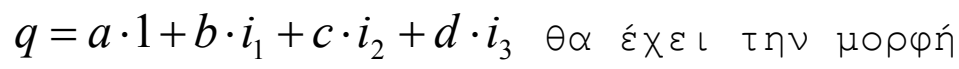

$$
A=\left(\begin{array}{cc}
z & w \\
-\bar{w} & \bar{z}
\end{array}\right)
$$

$K \propto l$

$$
\bar{q} \rightarrow A^{+}=\left(\begin{array}{cc}
\bar{z} & -w \\
\bar{w} & z
\end{array}\right)
$$

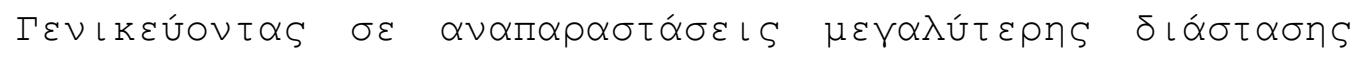

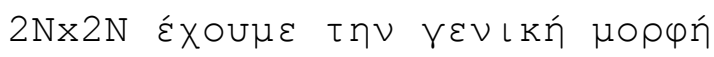




$$
Q=Q_{0} \otimes\left(\begin{array}{ll}
1 & 0 \\
0 & 1
\end{array}\right)+Q_{1} \otimes\left(\begin{array}{cc}
i & 0 \\
0 & -i
\end{array}\right)+Q_{2} \otimes\left(\begin{array}{cc}
0 & 1 \\
-1 & 0
\end{array}\right)+Q_{3} \otimes\left(\begin{array}{ll}
0 & i \\
i & 0
\end{array}\right)
$$

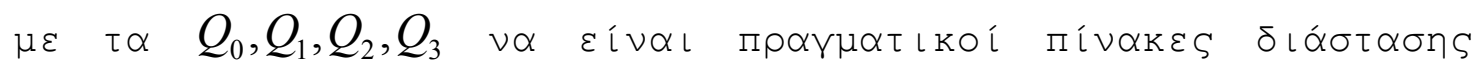

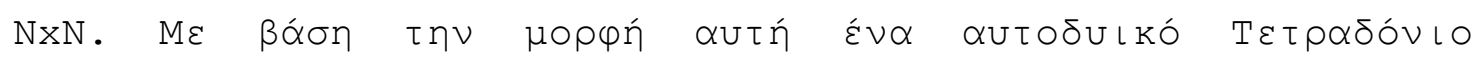

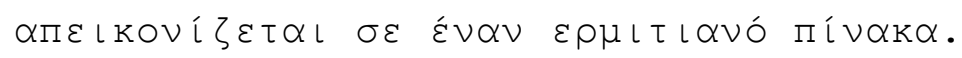

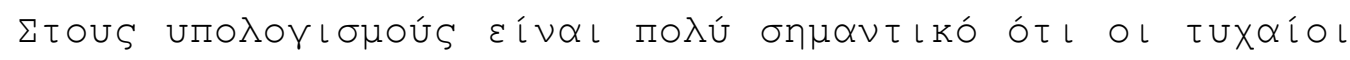

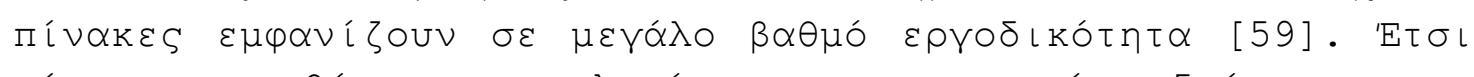

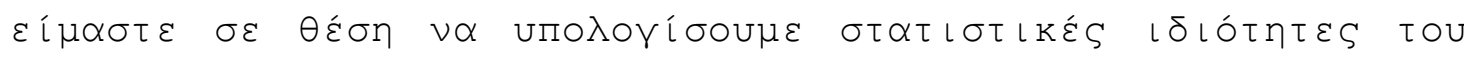

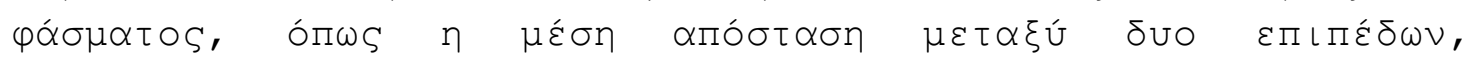

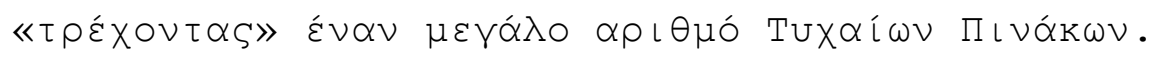

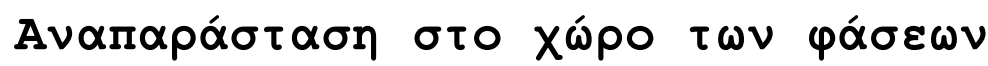

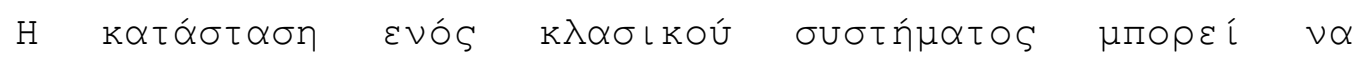

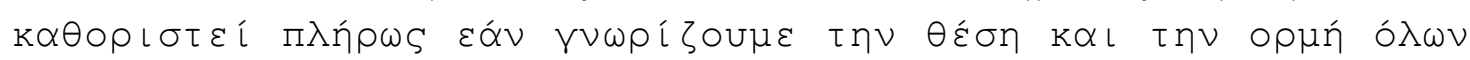

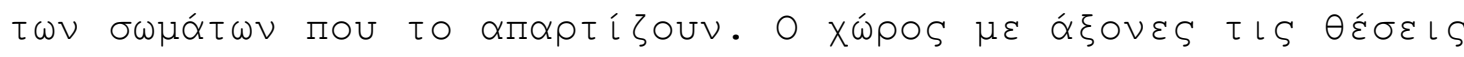

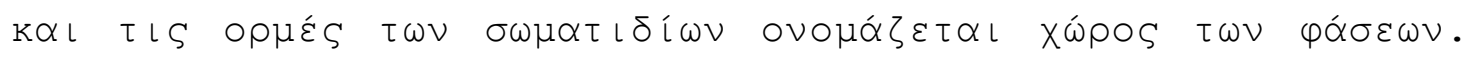

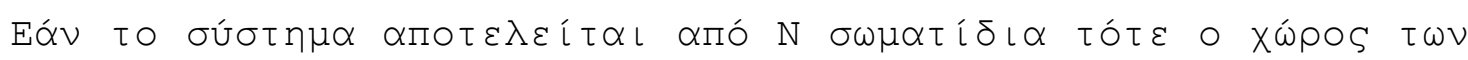

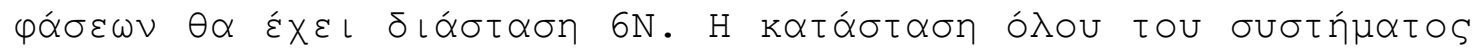

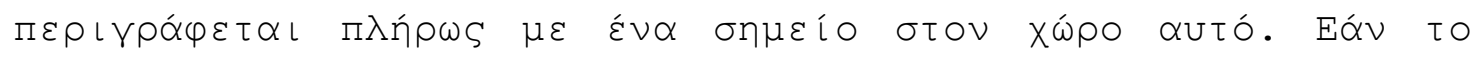

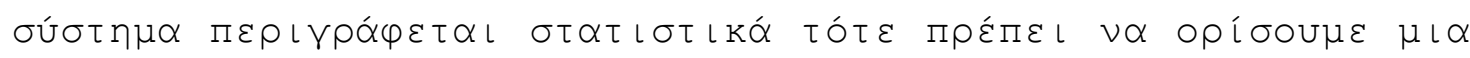

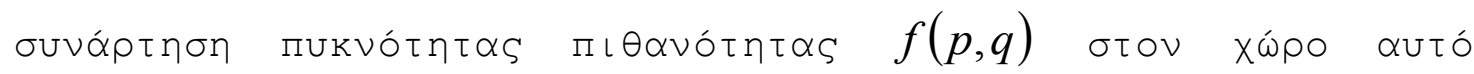

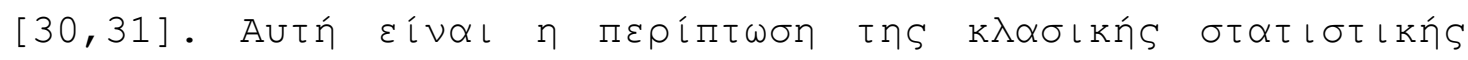

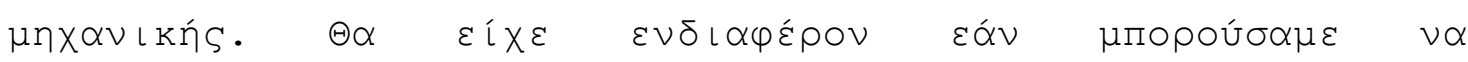

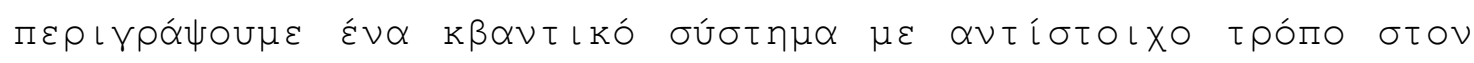

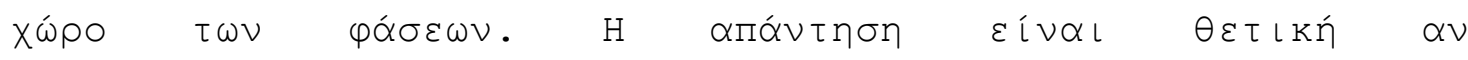

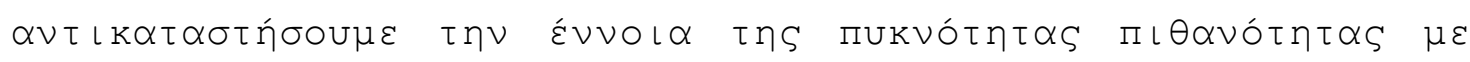

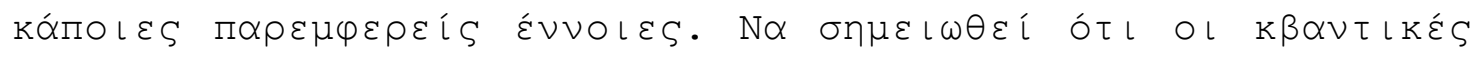

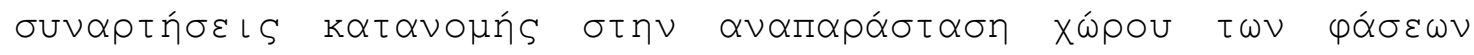

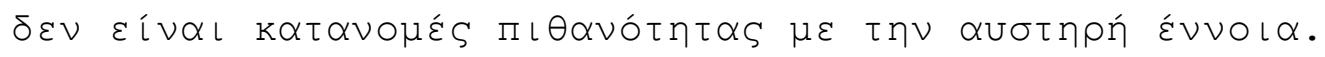

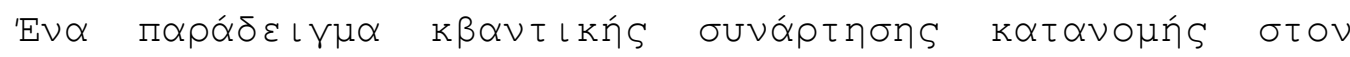

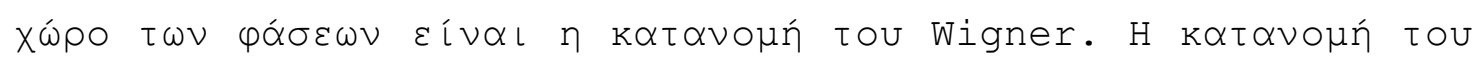

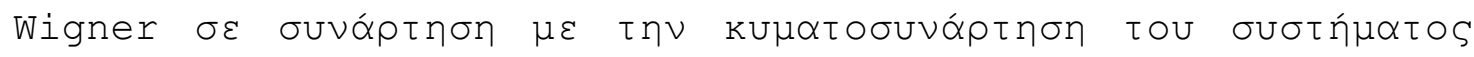

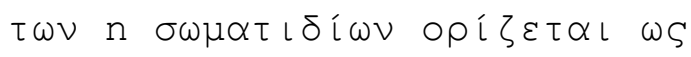




$$
P(q, p)=\left(\frac{1}{2 \hbar \pi}\right)^{n} \int d y \psi(q-y / 2) \psi(q+y / 2)^{*} e^{i p y / \hbar}
$$

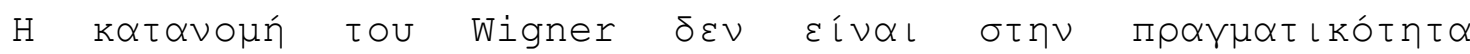

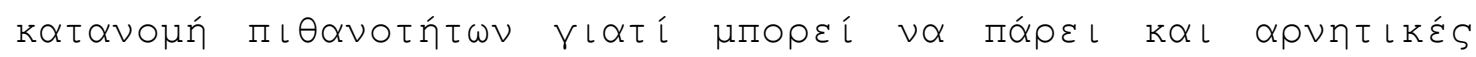

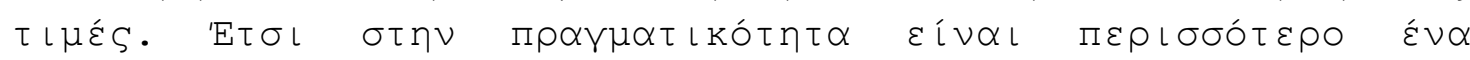

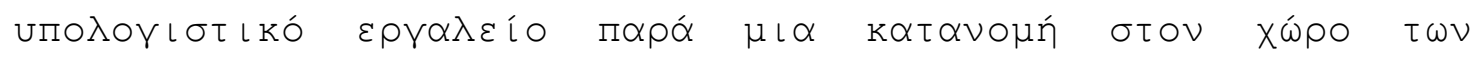

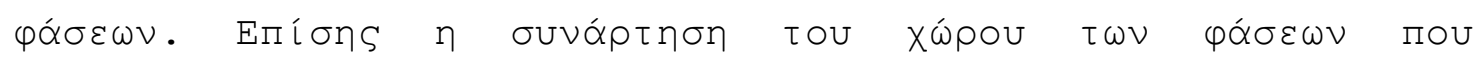

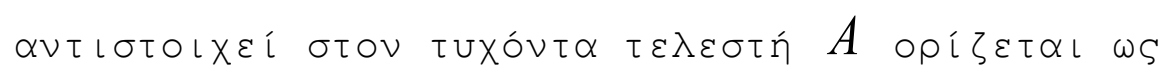

$$
A(q, p)=\int d y e^{i p y / \hbar}\left\langle q-\frac{y}{2}|A| q+\frac{y}{2}\right\rangle
$$

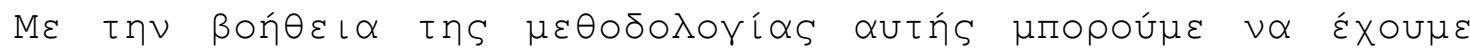

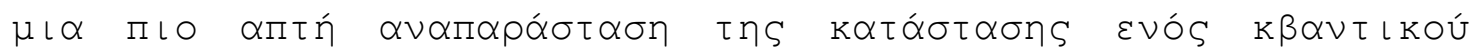

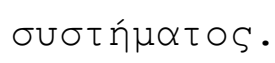

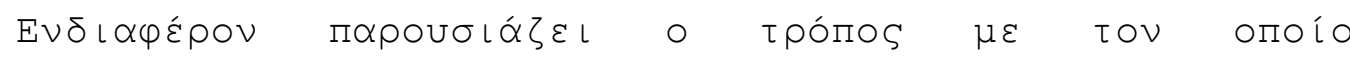

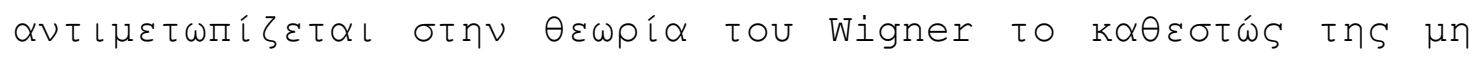

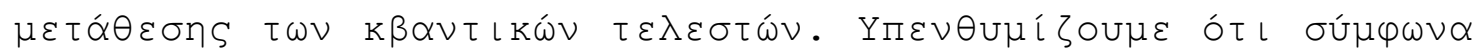

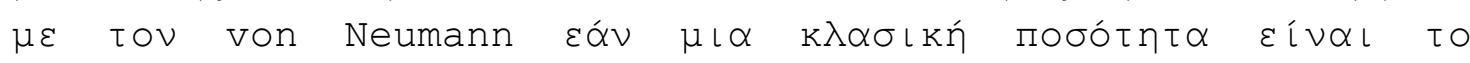

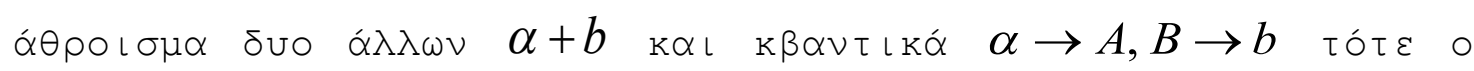

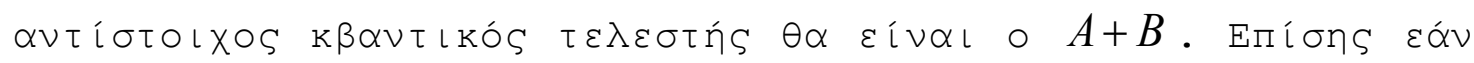

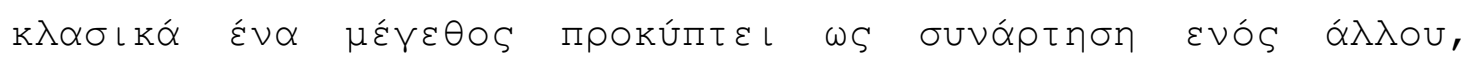

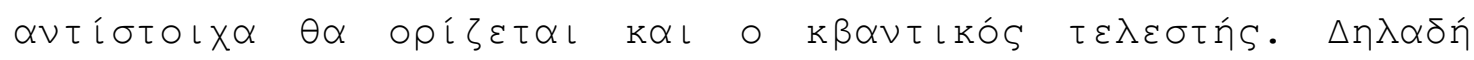

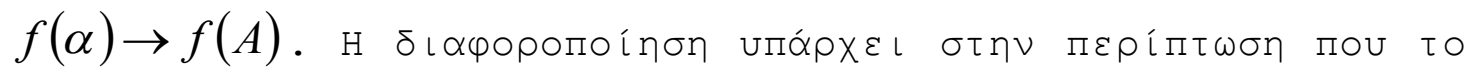

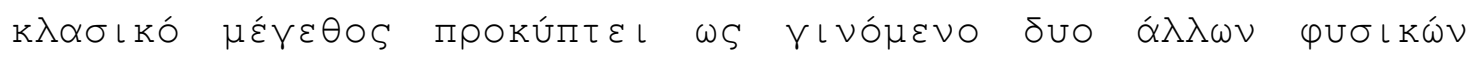

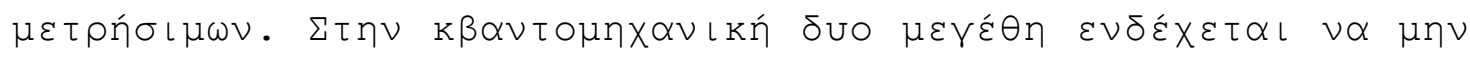

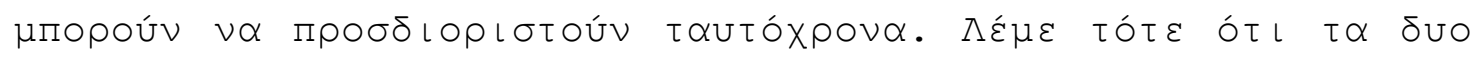

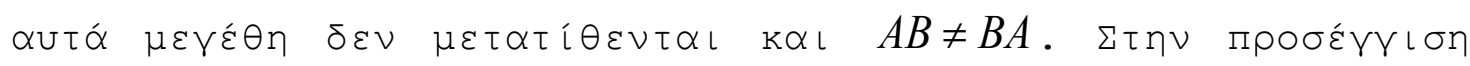

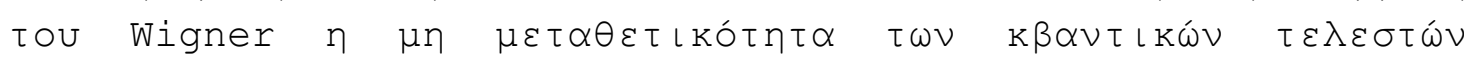

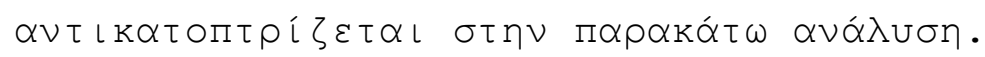

$' E \sigma \tau \omega$

$$
\hat{A} \hat{B}=\hat{F}
$$

$\tau o ́ \tau \varepsilon$ 


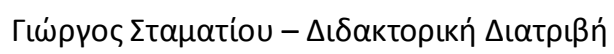

$$
\begin{aligned}
& F(q, p)=\int d z e^{i p z / \hbar}\langle q-z / 2|\hat{A} \hat{B}| q+z / 2\rangle \\
= & \int d z d q^{\prime} e^{i p z / \hbar}\left\langle q-z / 2|\hat{A}| q^{\prime}\right\rangle\left\langle q^{\prime}|\hat{B}| q+z / 2\right\rangle
\end{aligned}
$$

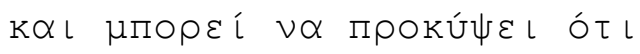

$$
F(q, p)=A(q, p) e^{\hbar \Lambda / 2 i} B(q, p)=B(q, p) e^{-\hbar \Lambda / 2 i} A(q, p)
$$

$\mu \varepsilon$

$$
\Lambda=\frac{\overleftarrow{\partial}}{\partial p} \frac{\vec{\partial}}{\partial q}-\frac{\grave{\partial}}{\partial q} \frac{\vec{\partial}}{\partial p}
$$

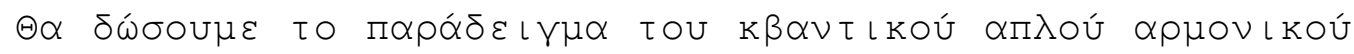

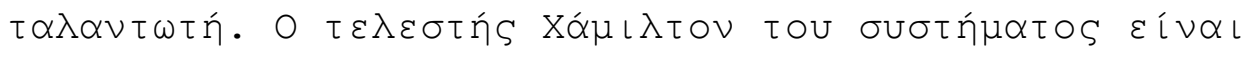

$$
\hat{H}_{0}=\frac{\hat{p}^{2}}{2 m}+\frac{m \omega^{2}}{2} \hat{x}^{2}
$$

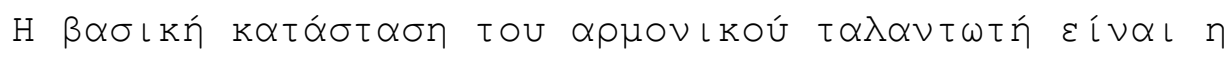

$$
\begin{gathered}
\psi(x)=\frac{1}{\sqrt{\sqrt{2 \pi} x_{0}}} \exp \left(-\frac{x^{2}}{4 a_{0}^{2}}\right) \\
a_{0}=\sqrt{\frac{\hbar}{2 m \omega}}
\end{gathered}
$$

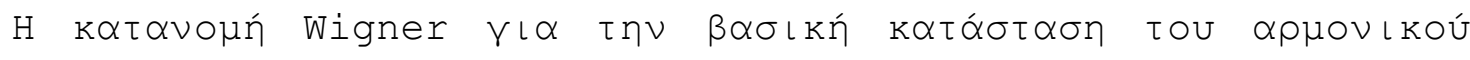

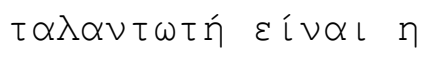

$$
\begin{gathered}
P(x, p)=\int d \xi \psi^{*}(x+\xi / 2) \psi(x-\xi / 2) e^{i p \xi / \hbar}=2 e^{\left(-\frac{x^{2}}{2 \alpha_{0}^{2}}-\frac{p^{2}}{2 q_{0}^{2}}\right)} \\
q_{0}=\frac{\hbar}{2 a_{0}}=\sqrt{\frac{m \omega}{2 \hbar}}
\end{gathered}
$$




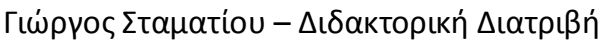

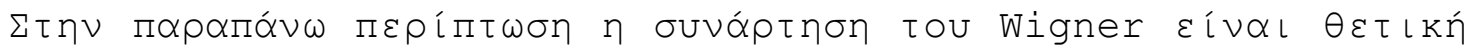

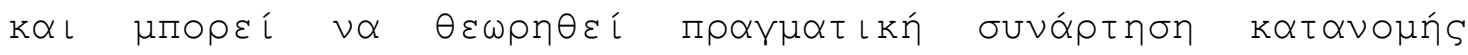

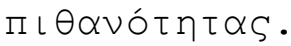

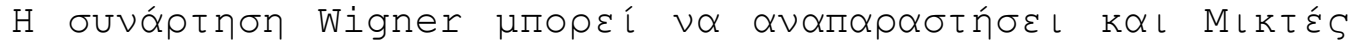

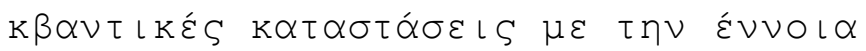

$$
P(q, p)=\sum_{i} w_{i} P_{i}(q, p)
$$

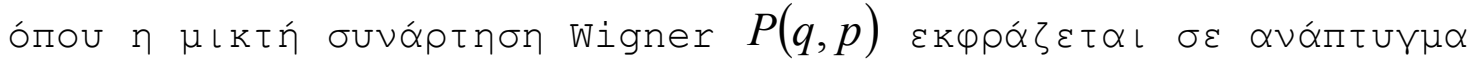

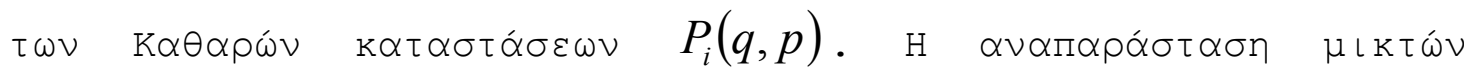

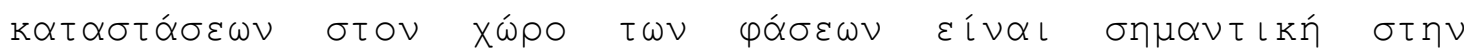

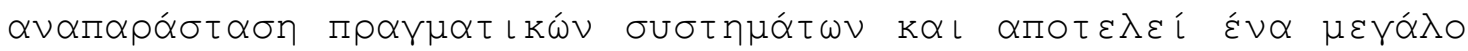

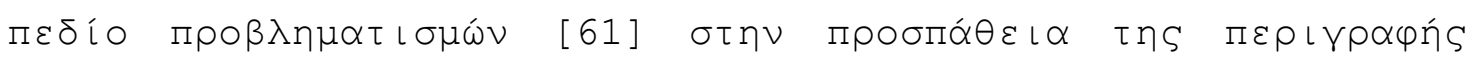

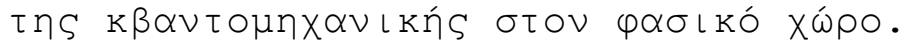

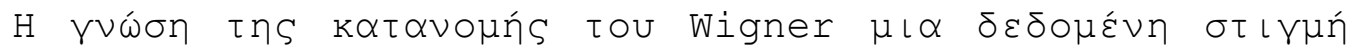

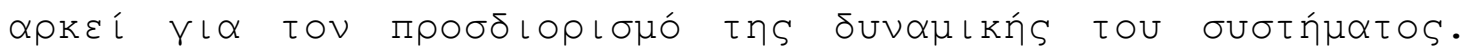

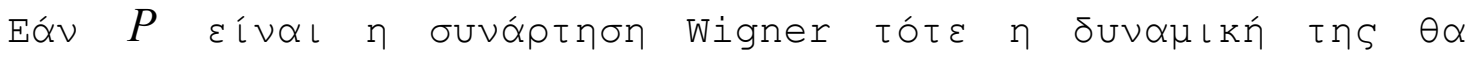

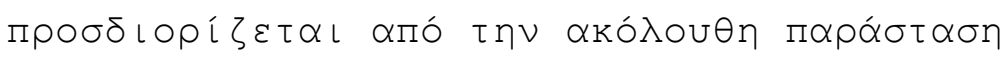

$$
\frac{\partial P(q, p)}{\partial t}=-\llbracket P(q, p), H(q, p) \rrbracket
$$

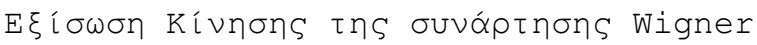

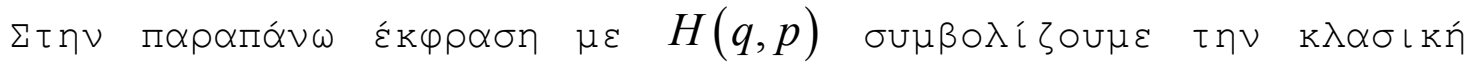

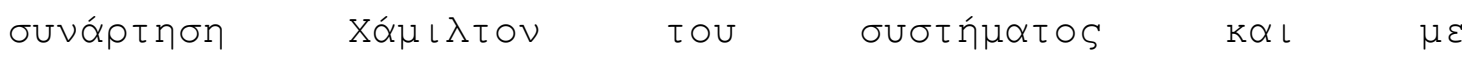

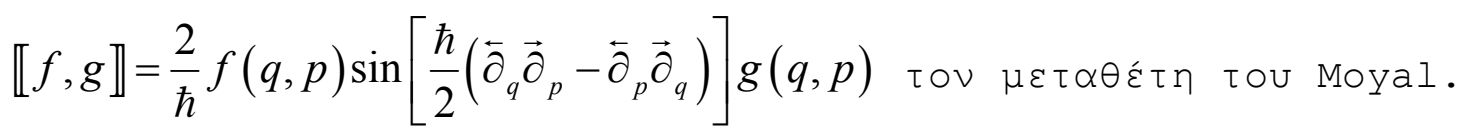

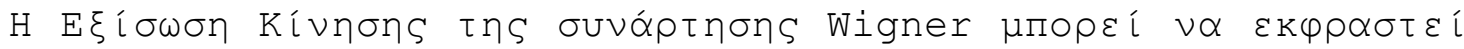

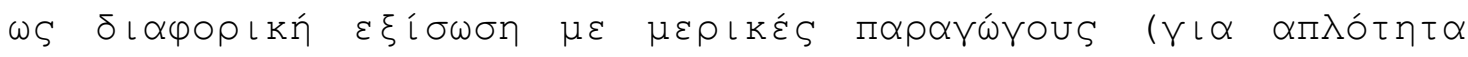

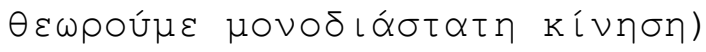

$$
\frac{\partial P(q, p)}{\partial t}=-\frac{p}{m} \frac{\partial P(q, p)}{\partial q}+\frac{\partial V}{\partial q} \frac{\partial P(q, p)}{\partial p}+\sum_{k=1 . . \infty} \frac{\left(-\hbar^{2} / 4\right)^{k}}{(2 k+1) !} \frac{\partial^{2 k+1} V}{\partial q^{2 k+1}} \frac{\partial^{2 k+1} P(q, p)}{\partial p^{2 k+1}}
$$




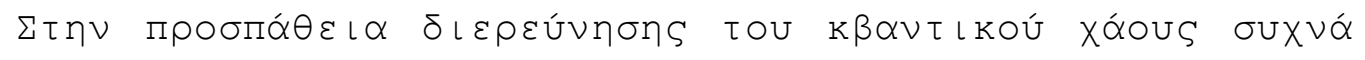

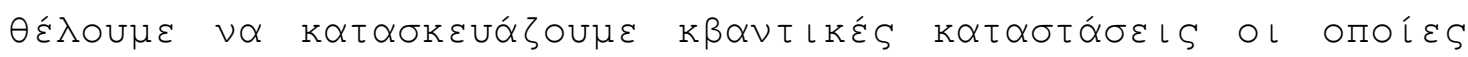

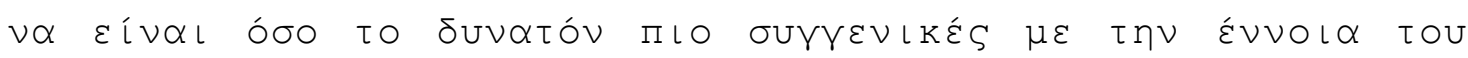

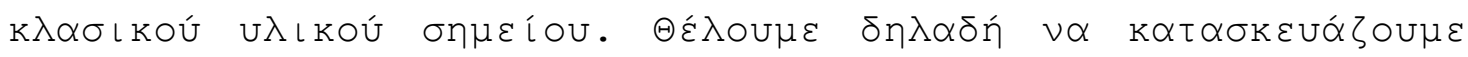

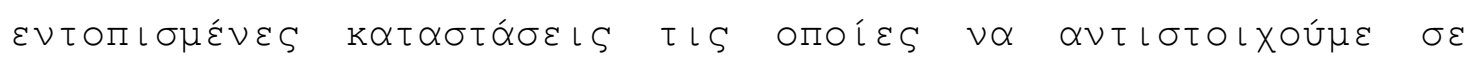

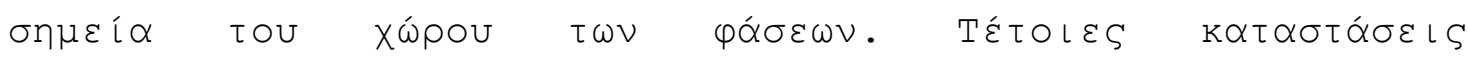

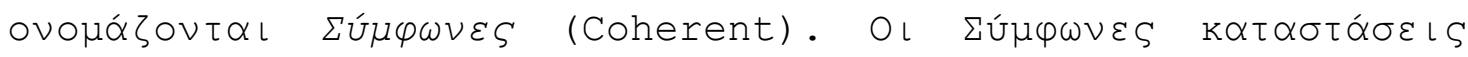

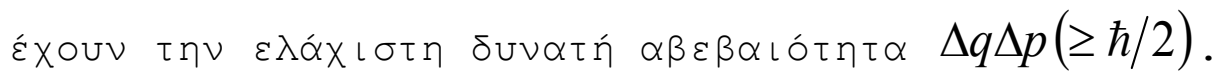

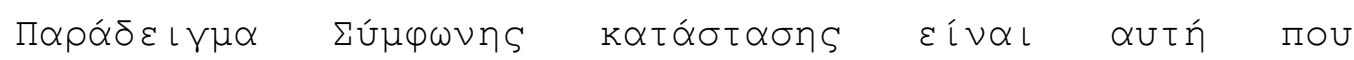

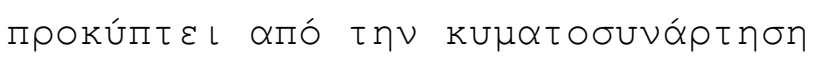

$$
\begin{gathered}
\psi_{0}(x)=C \exp \left[-\frac{(x-\langle x\rangle)^{2}}{4 b^{2}}+\frac{i\langle P\rangle x}{\hbar}\right] \\
\Delta x=b \\
\Delta p=\frac{\hbar}{2 b}
\end{gathered}
$$

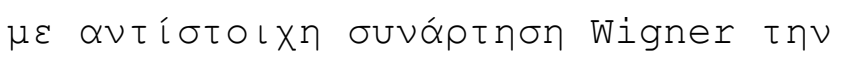

$$
W_{0}(R, P)=A \exp \left[-\frac{(R-\langle x\rangle)^{2}}{2 b^{2}}-\frac{4 b^{2}(P-\langle P\rangle)^{2}}{2 \hbar^{2}}\right]
$$

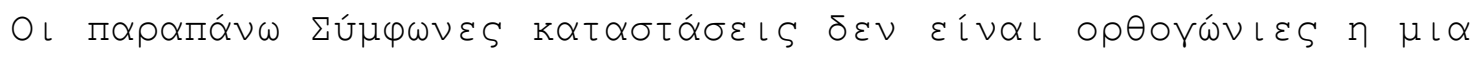

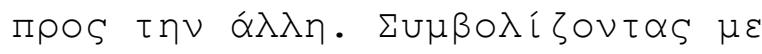

$$
\begin{gathered}
\langle x \mid q, p\rangle=\left(2 \pi s^{2}\right)^{-1 / 4} \exp \left[-\frac{(x-q)^{2}}{4 s^{2}}\right] \exp (i p x / \hbar) \\
\delta q=s, \quad \delta p=\frac{\hbar}{2 s}
\end{gathered}
$$




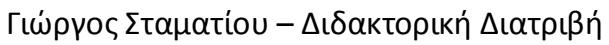

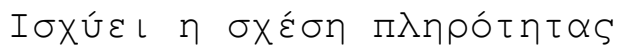

$$
\int|q, p\rangle\langle q, p| d q d p=2 \pi \hbar
$$

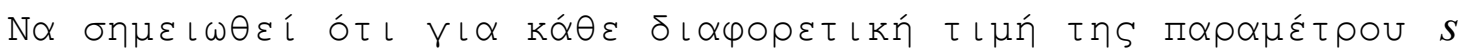

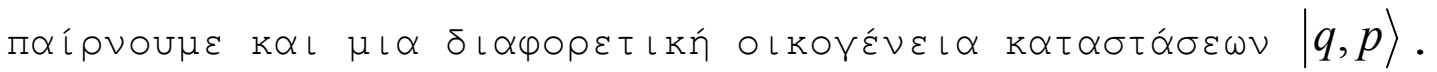

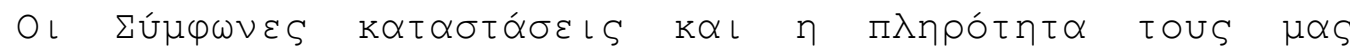

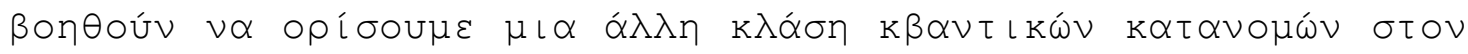

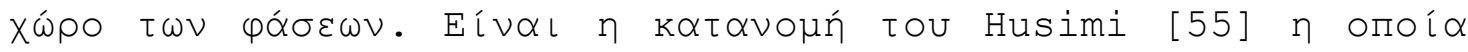

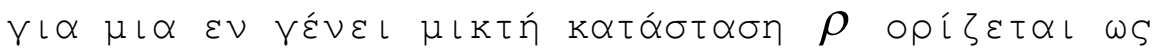

$$
\rho_{H}(q, p)=\frac{1}{2 \pi \hbar}\langle q, p|\rho| q, p\rangle
$$

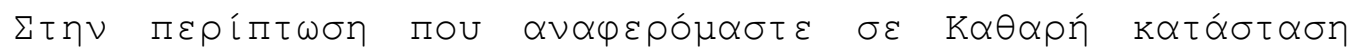

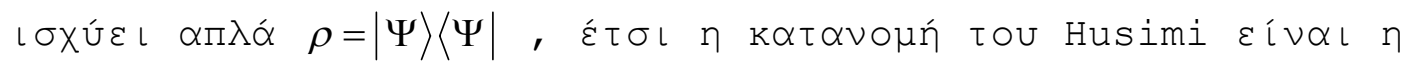

$$
\rho_{H}(q, p)=\frac{1}{2 \pi \hbar}|\langle q, p \mid \Psi\rangle|^{2}
$$

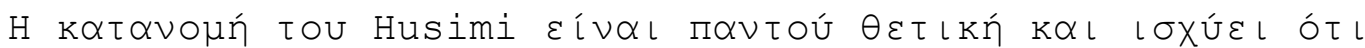

$$
\int \rho_{H}(q, p) d q d p=1
$$

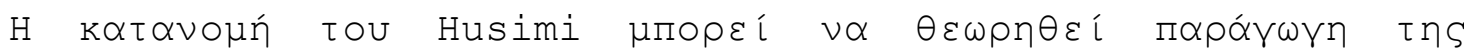

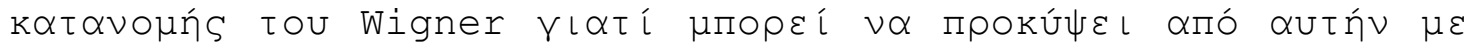

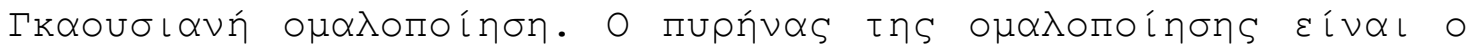

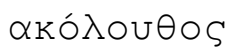

$$
\frac{1}{\pi \hbar} \exp \left[-\frac{\left(q^{\prime}-q\right)^{2}}{2 s^{2}}\right] \exp \left[-\left(p^{\prime}-p\right)^{2}\left(2 s^{2} / \hbar^{2}\right)\right]
$$

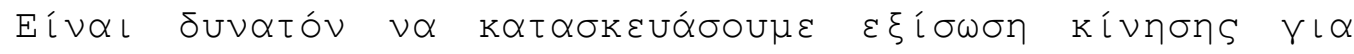

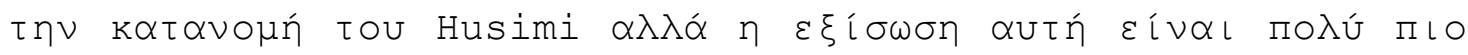

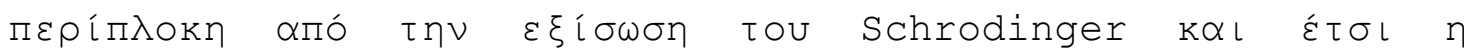

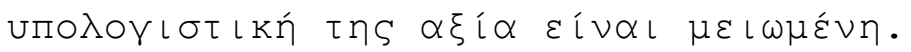




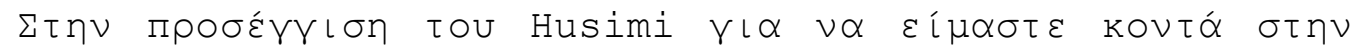

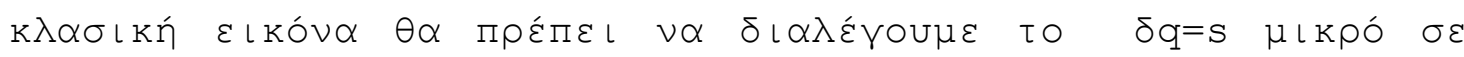
бX

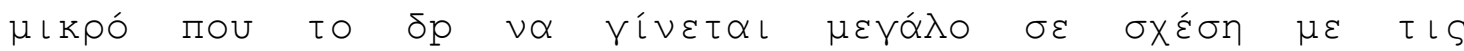

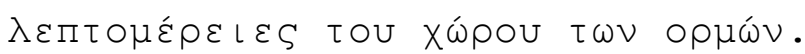

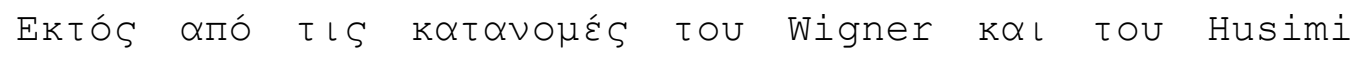

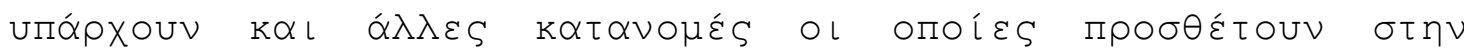

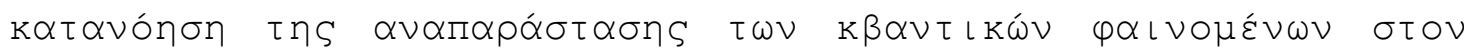

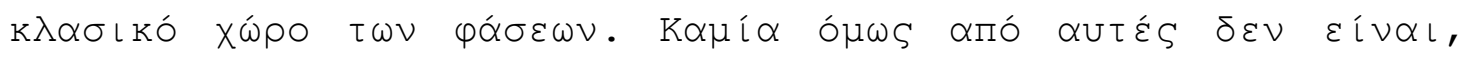

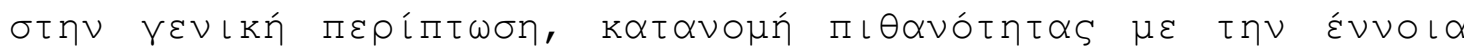

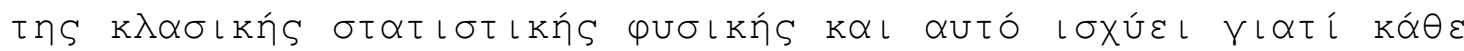

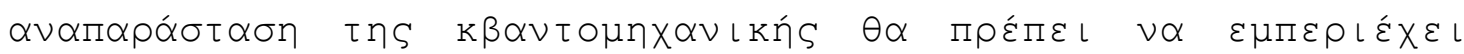

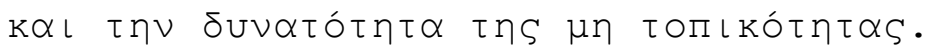

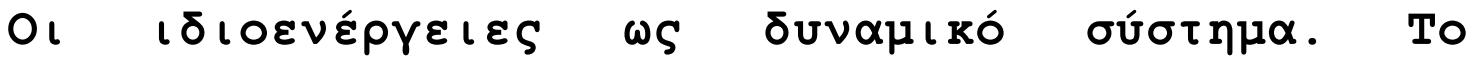

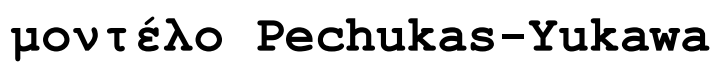

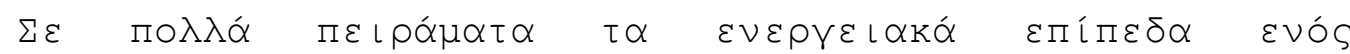

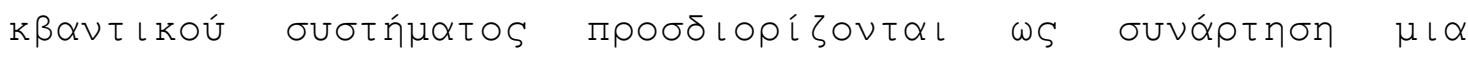

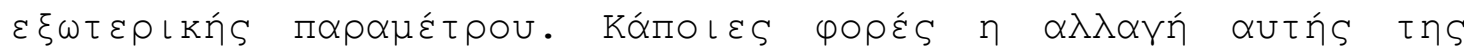

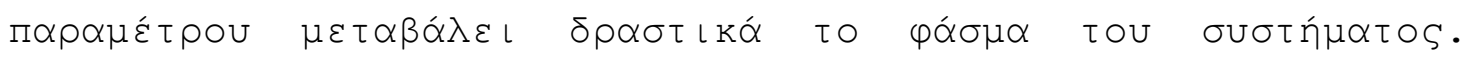

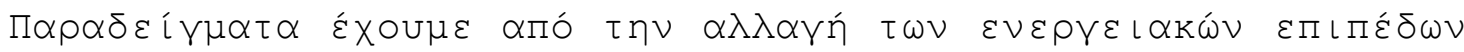

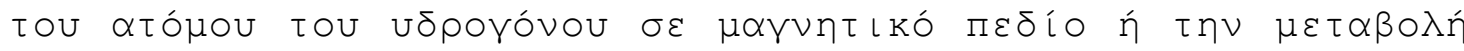

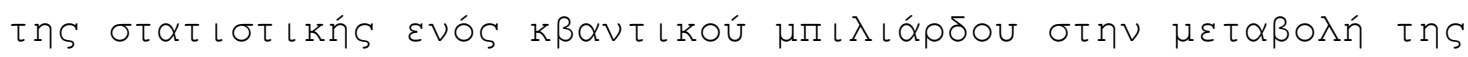

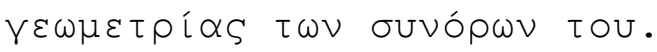

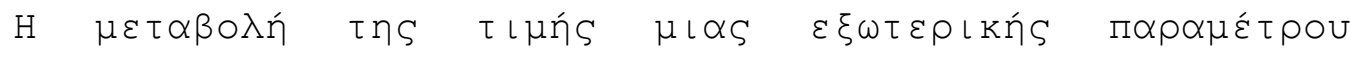

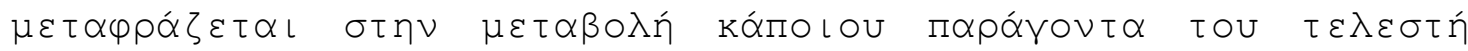

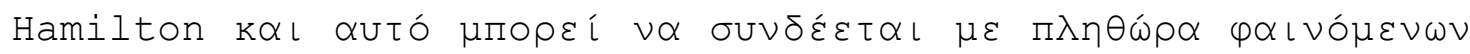

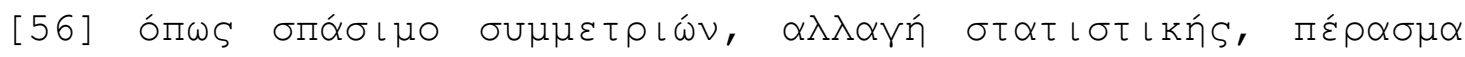

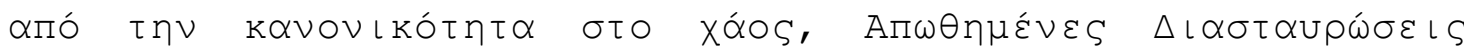

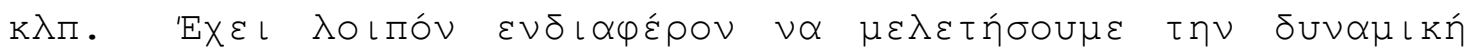

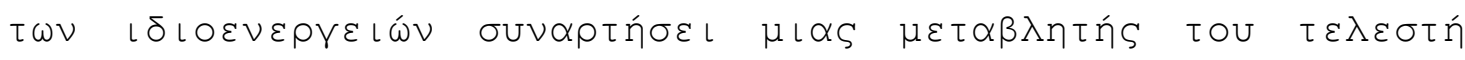

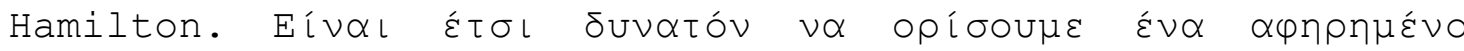

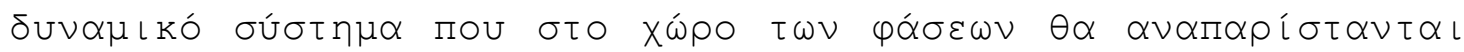

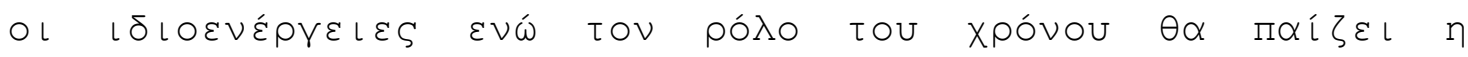

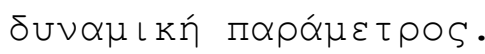




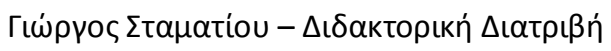

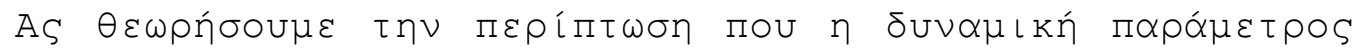

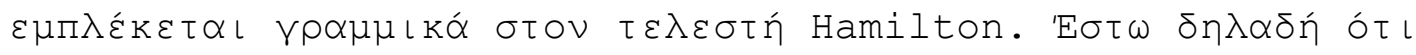

$$
H=H_{0}+t V
$$

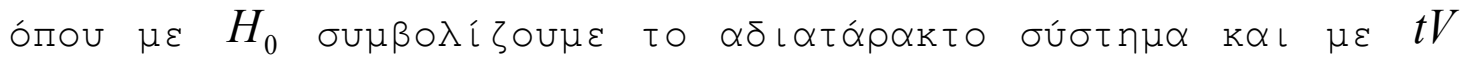

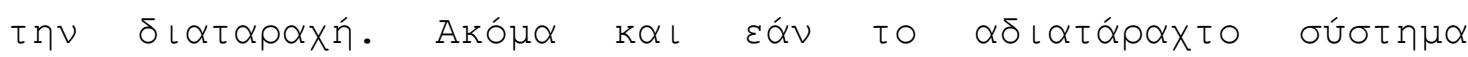

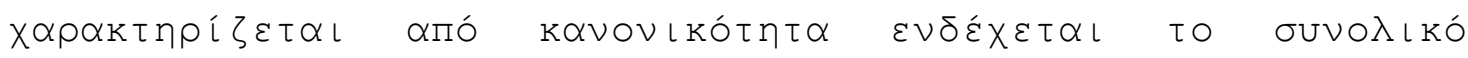

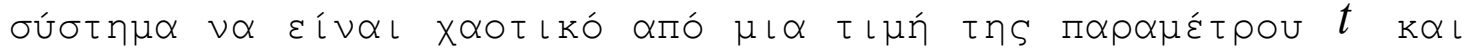

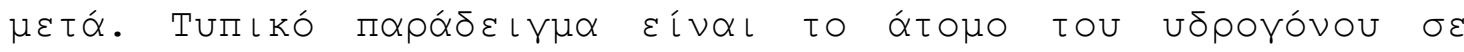

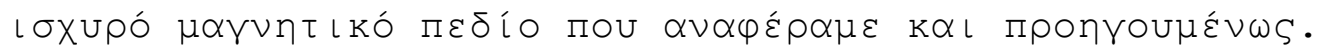

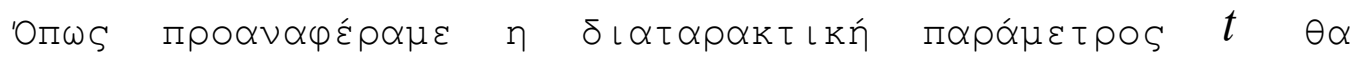

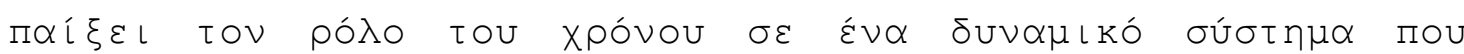

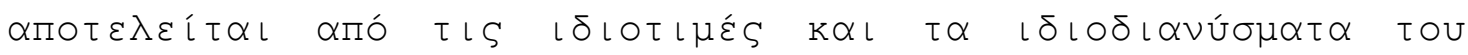

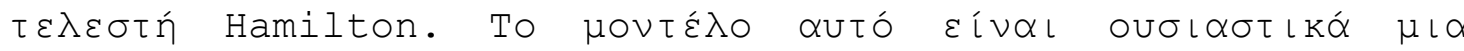

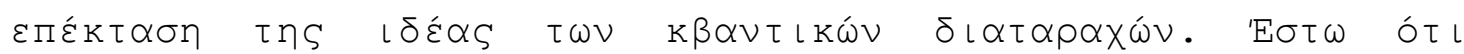

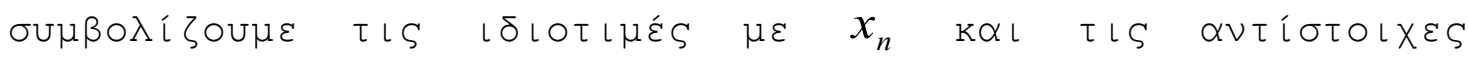

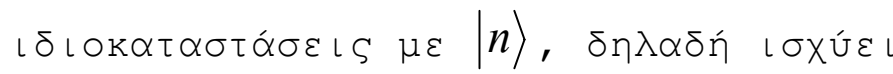

$$
H|n\rangle=x_{n}|n\rangle
$$

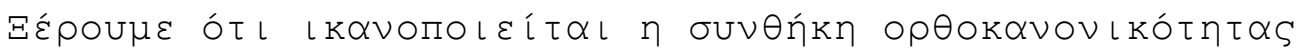

$$
\langle n \| m\rangle=\delta_{n m}
$$

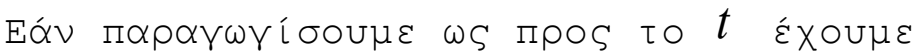

$$
\frac{\partial(\langle n|)}{\partial t}|m\rangle-\langle n| \frac{\partial(|m\rangle)}{\partial t}=0
$$

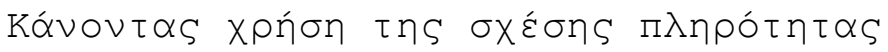

$$
\sum_{k}|k\rangle\langle k|=1
$$




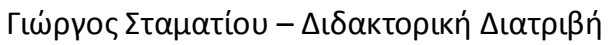

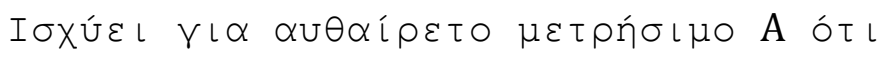

$$
\begin{gathered}
\frac{d}{d t}\langle n|A| m\rangle= \\
=\left(\frac{\partial}{\partial t}\langle n|\right) A|m\rangle+\left\langle n\left|\frac{\partial A}{\partial t}\right| m\right\rangle+\langle n| A\left(\frac{\partial}{\partial t}|m\rangle\right) \\
=\sum_{k}\left[\left(\frac{\partial}{\partial t}\langle n|\right)|k\rangle\langle k|A| m\rangle+\langle n|A| k\rangle\langle k|\left(\frac{\partial}{\partial t}|m\rangle\right)\right]+\left\langle n\left|\frac{\partial A}{\partial t}\right| m\right\rangle
\end{gathered}
$$

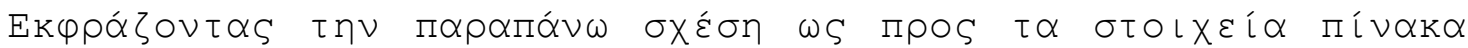

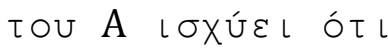

$$
\frac{d A_{n m}}{d t}=i \sum_{k}\left(H_{n k} A_{k m}-A_{n k} H_{k m}\right)+\left(\frac{\partial A}{\partial t}\right)_{n m}
$$

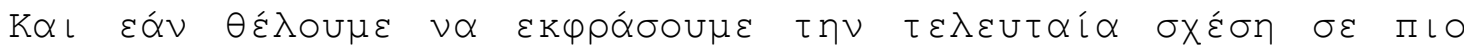

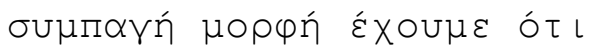

$$
\frac{d A}{d t}=i[H, A]+\frac{\partial A}{\partial t}
$$

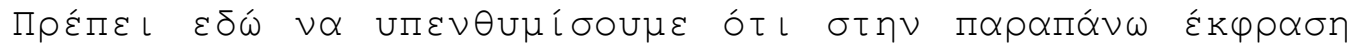

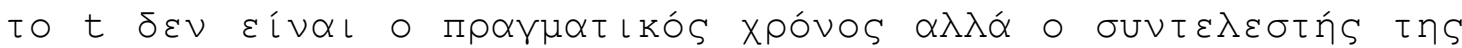

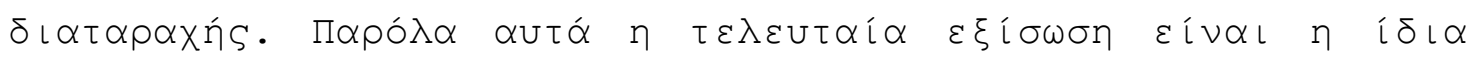

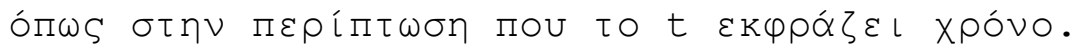

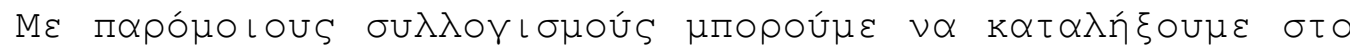

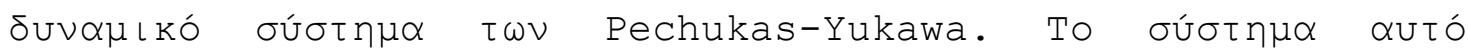

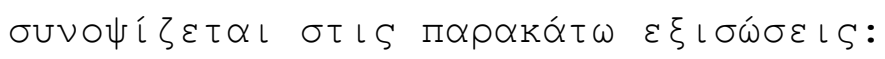

$$
\begin{gathered}
\dot{x}_{n}=p_{n} \\
\dot{p}_{n}=2 \sum_{k(\neq n)} \frac{\left|f_{n k}\right|^{2}}{\left(x_{n}-x_{k}\right)^{3}}
\end{gathered}
$$




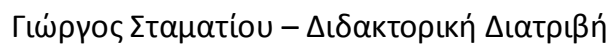

$$
\dot{f}_{n m}=\sum_{k(\neq n, m)} f_{n k} f_{k m}\left[\frac{1}{\left(x_{n}-x_{k}\right)^{2}}-\frac{1}{\left(x_{m}-x_{k}\right)^{2}}\right]
$$

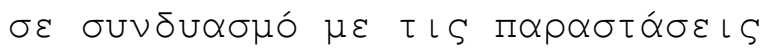

$$
\begin{gathered}
p_{n}=V_{n n} \\
f_{n m}=\left(x_{n}-x_{m}\right) V_{n m} \quad \mu \varepsilon \quad n \neq m
\end{gathered}
$$




\section{Av $\alpha \varphi \circ \rho \varepsilon ́ \varsigma$.}

[1]. Martin C. Gutzwiller, Chaos in Classical and Quantum Mechanics, (1990) Springer-Verlag, New York ISBN=0-38797173-4.

[2]. Roderick V. Jensen, Quantum chaos, Nature 355, 311 318 (23 January 1992); doi:10.1038/355311a0.

[3]. Hans-Jürgen Stöckmann (Author), Martin C. Gutzwiller (Reviewer), Quantum Chaos: An Introduction, American Journal of Physics, August 2000, Volume 68, Issue 8, pp. 777-778, http://dx.doi.org/10.1119/1.19544.

[4]. Quantum chaology, not quantum chaos, Michael Berry, 1989, Phys. Scr., 40, 335-336

[5]. Quantum Signatures of Chaos, Fritz Haake, Edition: 2, Springer, 2001, ISBN 3540677232, 9783540677239.

[6]. Jules Henri Poincaré (1890) "Sur le problème des trois corps et les équations de la dynamique. Divergence des séries de M. Lindstedt," Acta Mathematica, vol. 13, pages $1-270$.

[7]. Florin Diacu and Philip Holmes (1996) Celestial Encounters: The Origins of chaos and Stability, Princeton University Press.

[8]. Hadamard, Jacques (1898). "Les surfaces à courbures opposées et leurs lignes géodesiques". Journal de Mathématiques Pures et Appliquées 4: pp. 27-73.

[9]. George D. Birkhoff, Dynamical systems, vol. 9 of the American Mathematical Society Colloquium Publications (Providence, Rhode Island: American Mathematical Society, 1927)

[10]. P. Ehrenfest: Bemerkung über die angenäherte Gültigkeit der klassischen Mechanik innerhalb der Quantenmechanik Zeitschrift für Physik A Ausgabe 45, Nummern 7-8 / Juli, 1927, Seiten 455-457 


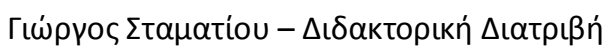

[11]. Kantz, H. und Schreiber, T.: Nonlinear Time Series Analysis. Cambridge University Press, Cambridge 2004, ISBN: $0-521-52902-6$

[12]. P. R. Holland, The quantum theory of motion Cambridge Univerity Press, Cambridge, 1993, ISBN: 0-521-48543-6

[13]. Bohmian Mechanics The Physics and Mathematics of Quantum Theory, Dürr, Detlef, Teufel, Stefan 2009, XII, 393 p. 41 illus., Hardcover, Springer ISBN: 978-3-540-89343-1

[14]. Bohr, N. (1920), "Über die Serienspektra der Element", Zeitschrift für Physik 2 (5): 423-478

[15]. O. Bohigas, M.-J. Giannoni and C. Schmit, Characterization of chaotic quantum spectra and universality of level fluctuation laws, Phys. Rev. Lett., 52: $1-4$ (1984).

[16]. Martin C. Gutzwiller (1971). "Periodic Orbits and Classical Quantization Conditions". Journal of Mathematical Physics 12: 343 .

[17].Vlatko Vedral: Introduction to quantum information science. Oxford Univ. Pr., Oxford 2006, ISBN 0-19-921570-7

[18].Dirk Bouwmeester: The physics of quantum information quantum cryptography, quantum teleportation, quantum computation. Springer, Berlin 2001, ISBN 3-540-66778-4

[19].Dieter Heiss: Fundamentals of quantum information quantum computation, communication, decoherence and all that. Springer, Berlin 2002, ISBN 3-540-43367-8

[20].Dagmar Bruss, Gerd Leuchs: Lectures on quantum information. Wiley-VCH, Weinheim 2007, ISBN 978-3-52740527-5

[21].E.Nelson, Phys. Rev., vol. 150, 1079 (1966)

[22].G.P. Berman and G.M. Zaslavsky, Physica 91A, 450 (1978). 


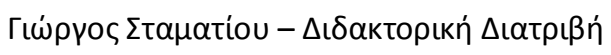

[23].F. Haake, M. Kus and R. Scharf, Z. Phys. B 65, 361 (1987).

[24].B.V. Chirikov, F.M. Israilev, and D.L. Shepelyansky, Physica D 33, 77 (1988).

[25]. Edelman, A. and Rao, N.R. (2005). Random matrix theory, Acta Numerica, 1-65.

[26]. Deift, P. (2006). Universality for mathematical and physical systems, arXiv:math-ph/0603038v2 19 May 2006.

[27]. Einstein, A., Podolsky, B. and Rosen, N. (1935) Can Quantum-Mechanical Description of Physical Reality Be Considered Complete?, Phys. Rev. 47, 777-780

[28]. On nonlinear Schrodinger equations, T Kato - Ann. Inst. H. Poincare Phys. Theor, 1987 - Springer

[29]. Quantum Chaotic Environments, the Butterfly Effect, and Decoherence, ZP Karkuszewski, C Jarzynski, WH Zurek Physical review letters, 2002, vol. 89, issue 17, 170405

[30]. Phase space picture of quantum mechanics: group theoretical approach, YS Kim, ME Noz - 1991, ISBN:981-02$0360-8$

[31]. Theory and application of the quantum phase-space distribution functions, HW Lee - Physics Reports, 1995, vol. 259, issue 3, 147-211

[32]. On the applicability of the energy level dynamics for the Hamiltonian systems in the ..., $\mathrm{H}$ Hasegawa, M Robnik EPL (Europhysics Letters), 1993, vol. 23, no. 3, 171

[33]. Classical and quantum billiards, integrable, nonintegrable, and pseudo- ..., $K$ Zyczkowski, I Pisyki, U Jagielloóski - Acta Physica Polonica B, 1992, vol. 23, no. $3,245-270$

[34]. Periodic orbits in triangular billiards, T Ruijgrok Acta Phys. Polon, 1991, vol. 22, no. 11-12, 955-981 


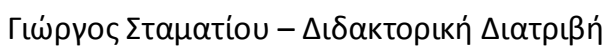

[35]. Signatures of chaos in quantum billiards: Microwave experiments, A Kudrolli, S Sridhar, A Pandey, R Ramaswamy Physical Review E, 1994, vol. 49, issue 1, R11-R14

[36]. Experimental studies of chaos and localization in quantum wave functions, A Kudrolli, V Kidambi, S Sridhar Physical review letters, 1995, vol. 75, issue 5, 822-825

[37]. Microwave studies of chaotic billiards and disordered systems, HJ Stöckmann, M Barth, U Dörr, U Kuhl, H ... Physica E: Low- ..., 2001, vol. 9, issue 3, 571-577

[38]. Quantum scars of classical closed orbits in phase space, MV Berry -Proceedings of the Royal Society of London. Series A, ..., 1989, vol. 423, 219-231

[39]. Lead-orientation-dependent wave function scarring in open quantum dots, JP Bird, R Akis, DK Ferry, D Vasileska, J Cooper, Y ... - Physical Review Letters, 1999, vol. 82, issue 23, 4691-4694

[40]. Wave function scarring effects in open stadium shaped quantum dots, R Akis, DK Ferry, JP Bird - Physical Review Letters, 1997, vol. 79, issue 1, 123-126

[41]. Semiclassical criterion for scars in wave functions of chaotic systems, O Agam, S Fishman - Physical review letters, 1994, vol. 73, issue 6, 806-809

[42]. Experimental observation of scarred eigenfunctions of chaotic microwave cavities, S Sridhar - Physical review letters, 1991, vol. 67, issue 7, 785-788

[43]. Green's functions in quantum physics, EN Economou 1979, Springer, ISBN: 3-540-28838-4

[44]. Quantum chaos with time-periodic Hamiltonians, G Casati, L Molinari - Prog. Theo. Phys., Suppl, 1989, vol. $98, \quad 287-322$

[45]. Floquet theory for partial differential equations, PA Kuchment - Russian Mathematical Surveys, 1982, vol. 37, no. 4,1 


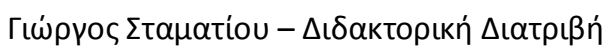

[46]. Classical and quantum chaos for a kicked top, F Haake, M Kuś, R Scharf - Zeitschrift für Physik B Condensed Matter, 1987, vol. 65, no.3, 381-395

[47]. Classical and quantum structures in the kicked-top model, GM D'Ariano, LR Evangelista, M Saraceno - Physical Review A, 1992, vol. 45, issue 6, 3646-3658

[48]. Vibrating soap films: An analog for quantum chaos on billiards, American Journal of Physics, , E Arcos, G Baez, PA Cuatlayol, MLH Prian, RA ,1998, vol. 66, issue 7, 601607

[49]. Random matrices in physics, EP Wigner - Siam Review, 1967, vol. 9, no. 1, 1-23

[50]. On the distribution of the roots of certain symmetric matrices, EP Wigner - The Annals of Mathematics, 1958, vol. 67 , no. 2, 325

[51]. Random matrices and their applications, JE Cohen, H Kesten, CM Newman, (Providence) ... - 1986, ISBN-10: 0-8218$5044-\mathrm{X}$

[52]. Rotations, quaternions, and double groups, SL Altmann, 1986, Clarendon Press

[53]. Chaotic motion and random matrix theories, O Bohigas, MJ Giannoni, Lecture notes in physics-Mathematical and computational ..., Springer, 1984, vol. 209, 1-99

[54]. Random unitary matrices, K Zyczkowski, M Kus Journal of Physics A: Mathematical and General, 1994, vol. 27 , no. 12,4235

[55]. Chaos and Husimi distribution function in quantum mechanics, K Takahashi, $N$ Saitô - Physical review letters, 1985, vol. 55, issue 7, 645-648

[56]. Quantum chaos: a new paradigm of nonlinear dynamics, K Nakamura, K Nakamura - 1993, Cambridge University Press, ISBN : $978-0-521-46746-9$ 


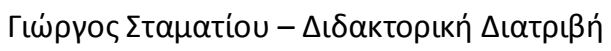

[57]. Quantum eigenvalues from classical periodic orbits, G Tanner, P Scherer, EB Bogomolny, B Eckhardt, D ... - Physical review letters, 1991, vol. 67, issue 18, 2410-2413

[58]. The transition to chaos, LE Reichl - 1992, Springer, ISBN: $0-387-98788-6$

[59]. Statistical properties of many-particle spectra. III. Ergodic behavior in random-matrix ensembles, A Pandey, Ann. Phys.(NY), 1979, vol. 119, issue 1, 170-191

[60]. Billiards in Finsler and Minkowski geometries, E Gutkin, S Tabachnikov - Journal of Geometry and Physics, 2002, vol. 40, issue 3-4, 277-301

[61]. Mixed states with positive Wigner functions, T Bröcker, RF Werner - Journal of mathematical physics, 1995, vol. 36, issue 1, 62

[62]. Quantum Chaos: Between Order and Disorder, Giulio Casati, Boris Chirikov - 2006, Cambridge University Press, ISBN-10: 0521031664

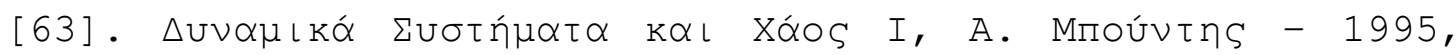

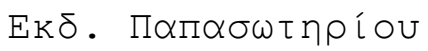

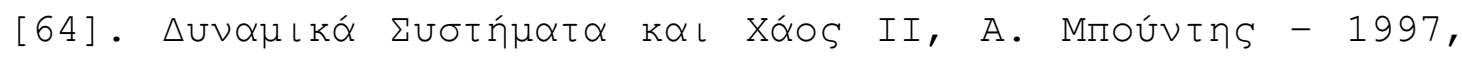

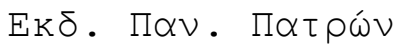

[65]. O Ө

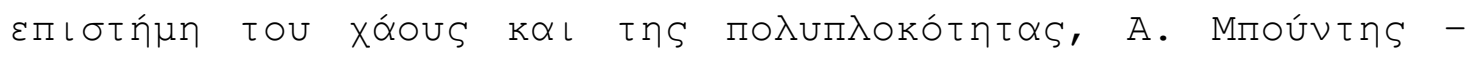
2004, Leader Books

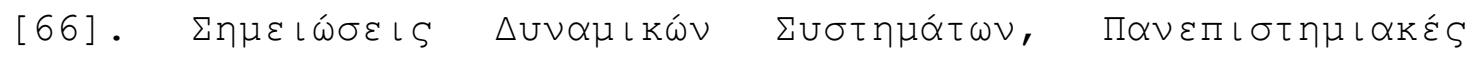

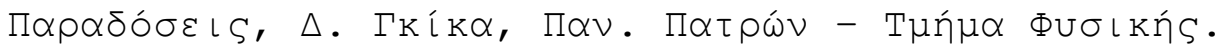




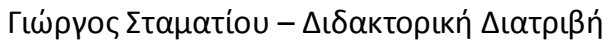

ENOTHTA $\Delta^{\prime}$

EEAPTHEH TOY ENTANGLEMENT

AПО THN КYРTOTHTA

TSN IDIOTIMQN TOY TEAEETH HAMILTON 



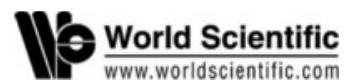

International Journal of Quantum

Information Vol. 5, No. 5 (2007)

685-704 (c) World Scientific

Publishing Company

A CURVATURE DEPENDENT BOUND FOR ENTANGLEMENT CHANGE IN CLASSICALLY CHAOTIC SYSTEMS

DEMETRIS P. K. GHIKAS and GEORGE STAMATIOU

Department of Physics, University of Patras

Patras 26500, Greece

ghikas@physics.upatras.gr

Received 5 July 2007

Using the Calogero-Moser model and the NakamuraLakshmanan equations of motion for eigenvalues and eigenfunctions associated with a multi-partite quantum system, we prove an inequality between the mean bipartite Entanglement rate of change under the variation of a critical parameter and the levelcurvature. This provides an upper bound for the rate of production or destruction of Entanglement induced dynamically. We then investigate the dependence of the upper bound on the degree of chaos of the system, which in turn, through the inequality, gives a measure of the stability of the entangled state. Our analytical results are supported by extensive numerical calculations.

Keywords: Entanglement; quantum chaos; Calogero-Moser Model; upper bounds.

PACS Number(s): 03.67.Mn, 05.45.Ac, 05.45.Pq 


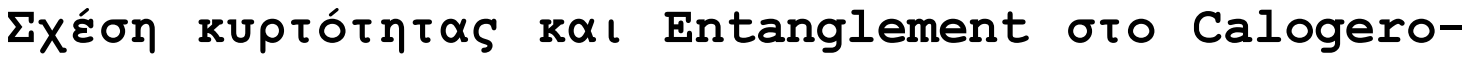 Moser System.}

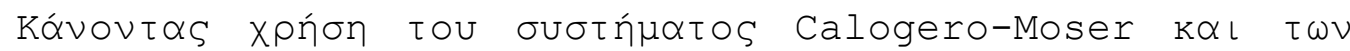

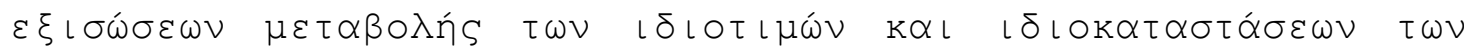

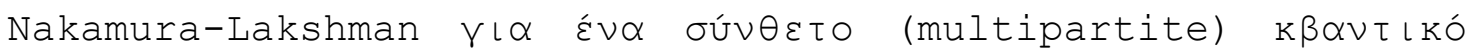

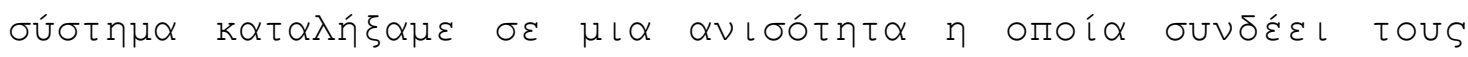

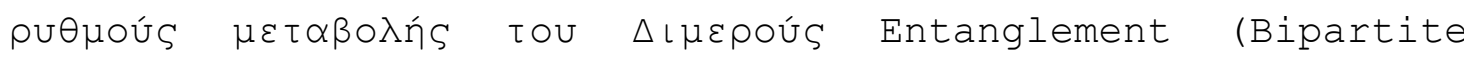

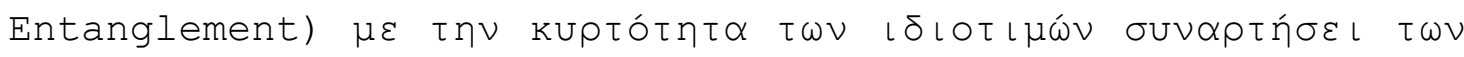

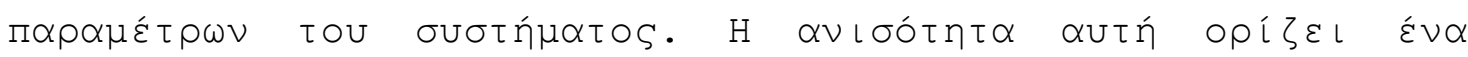

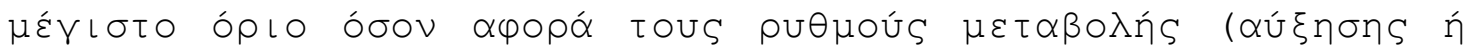

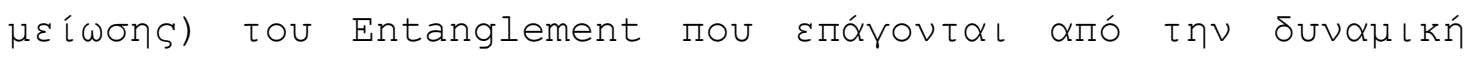

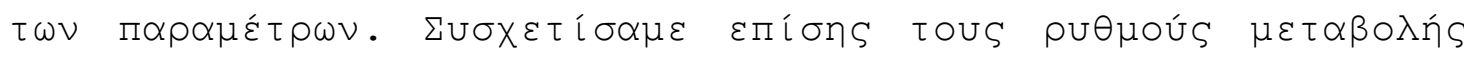

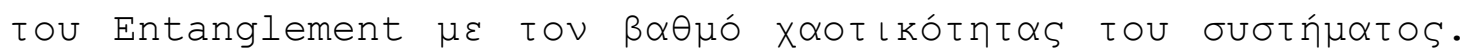

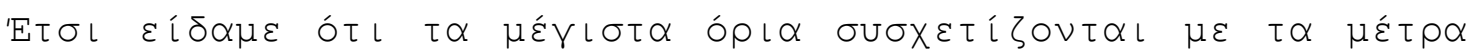

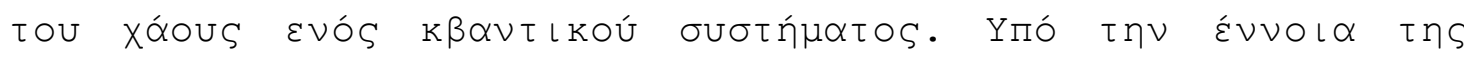

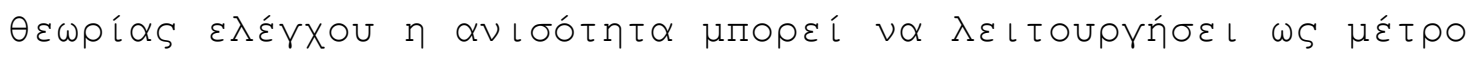

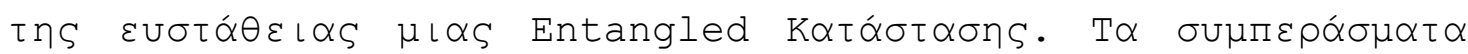

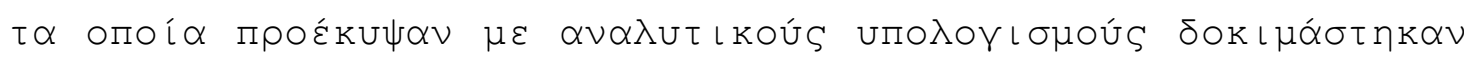

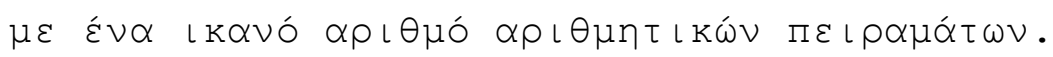

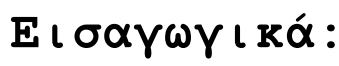

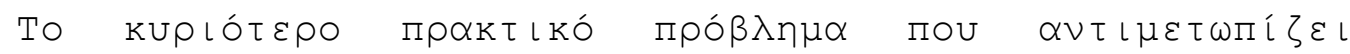
бń

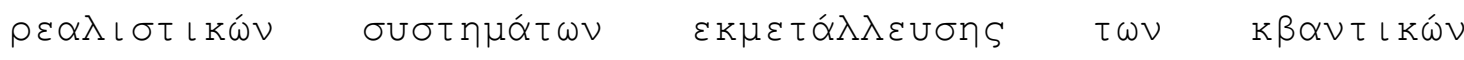

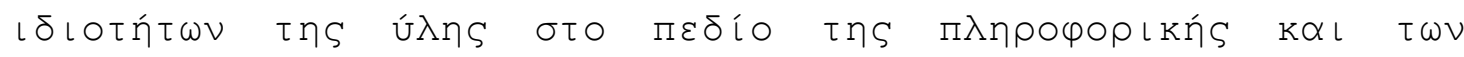

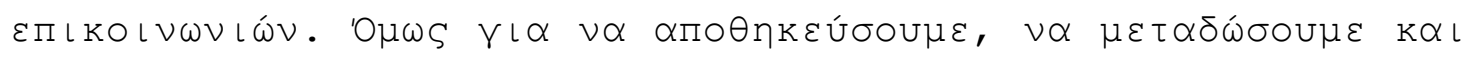

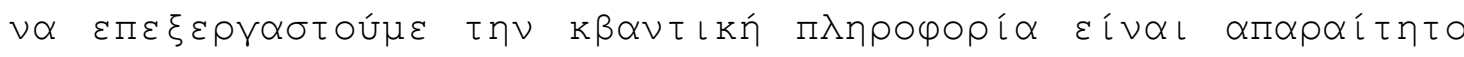

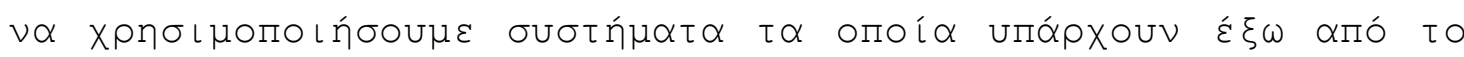

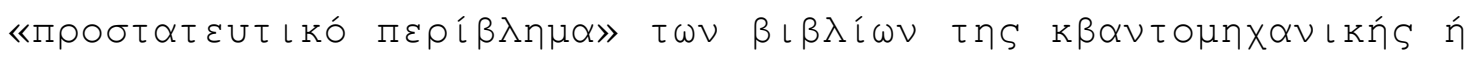

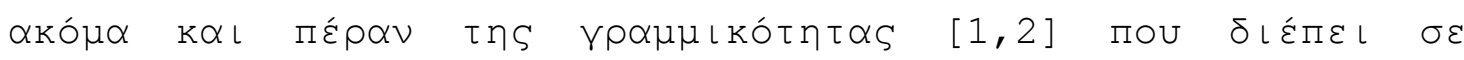

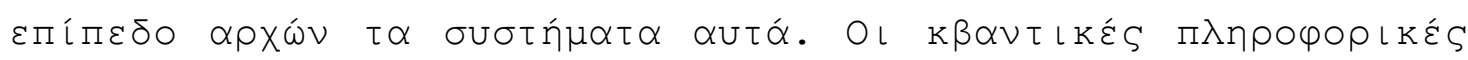

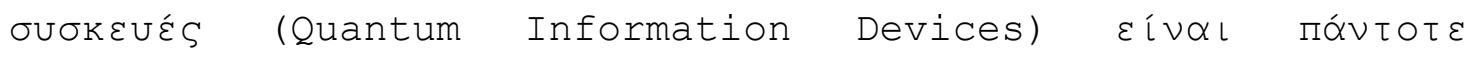

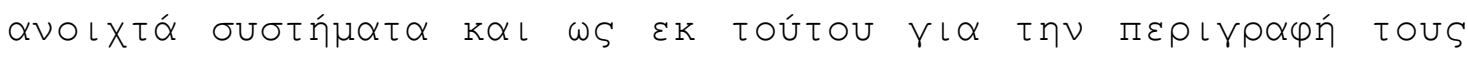

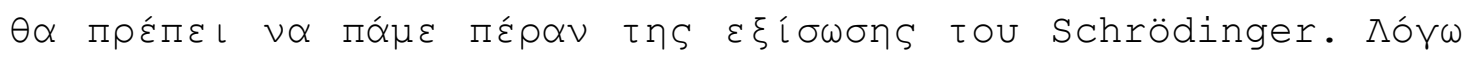

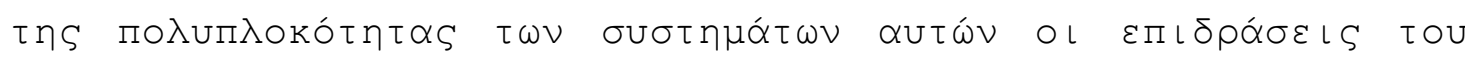

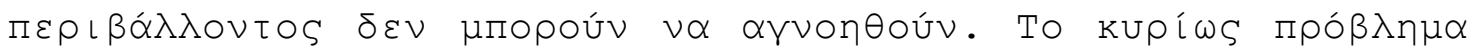

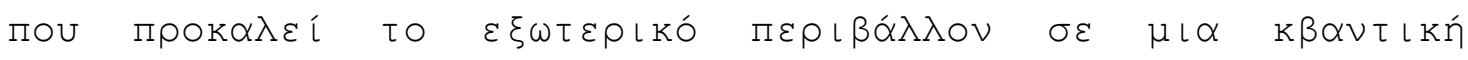




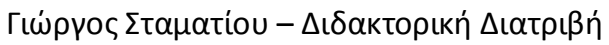

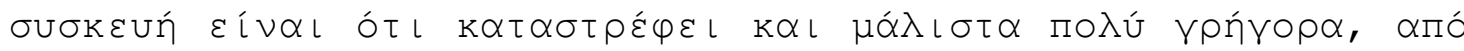

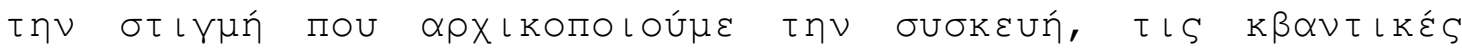

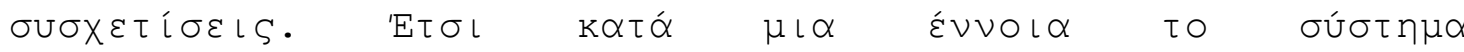

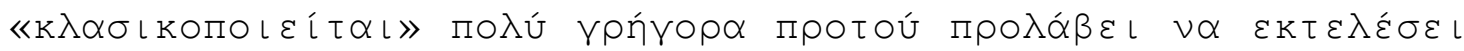

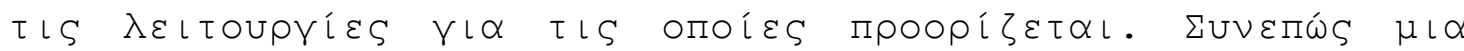

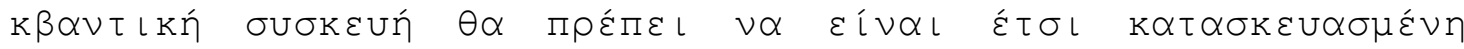

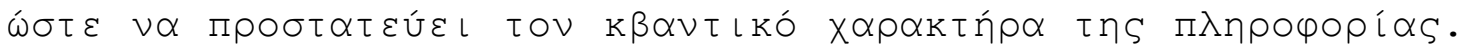

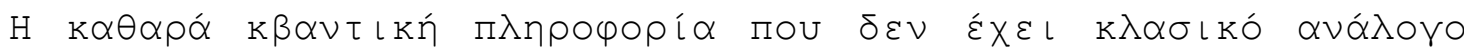

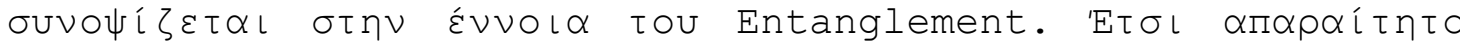

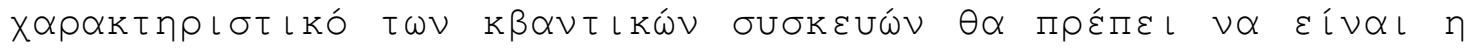

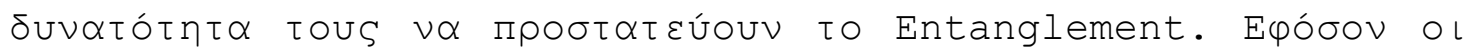

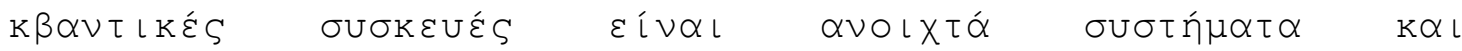

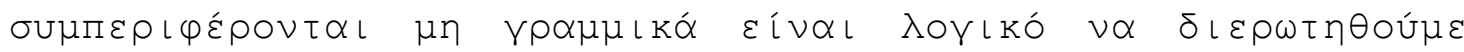

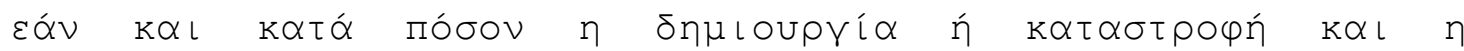

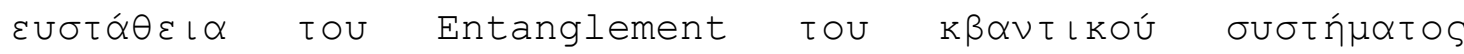

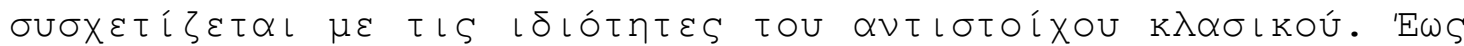

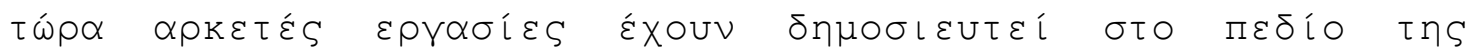

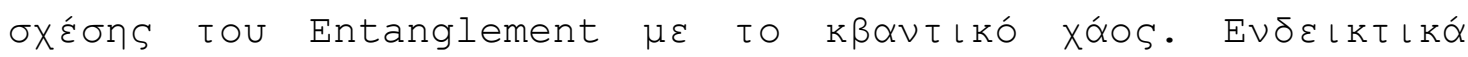

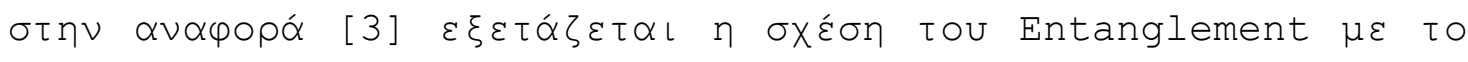

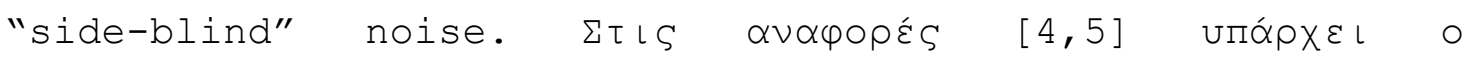

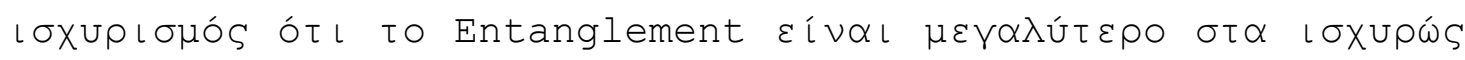

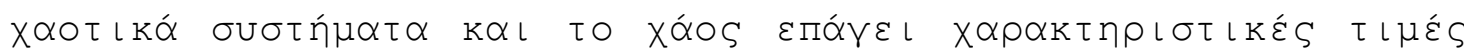

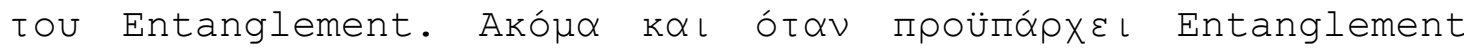

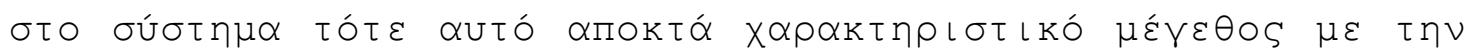

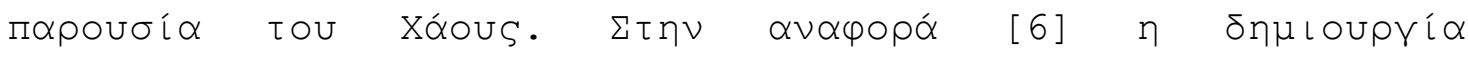

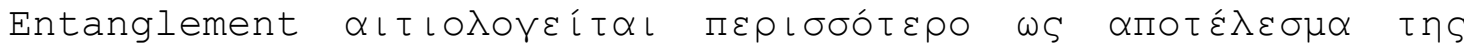

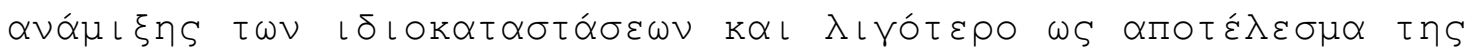

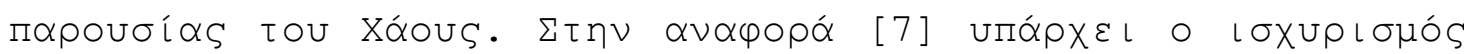

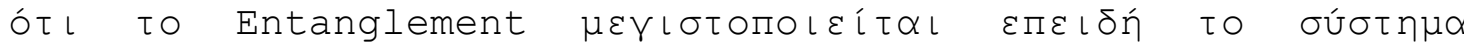

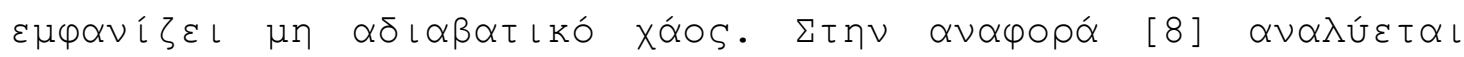

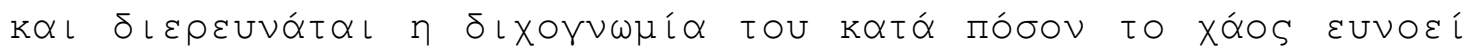

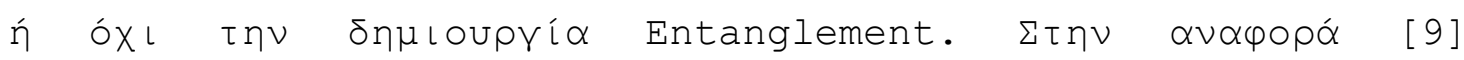

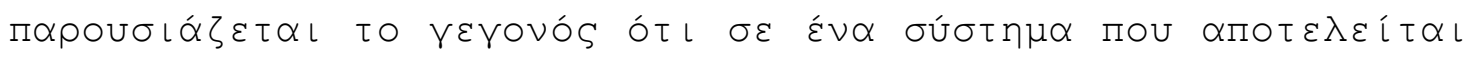

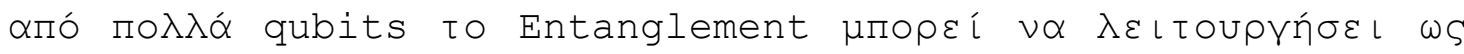

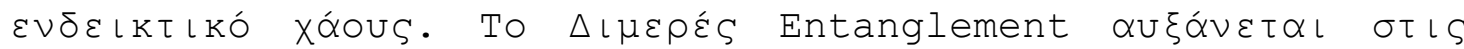

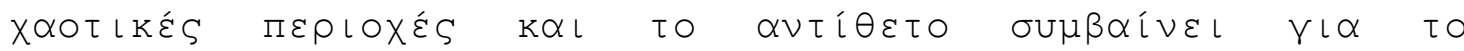

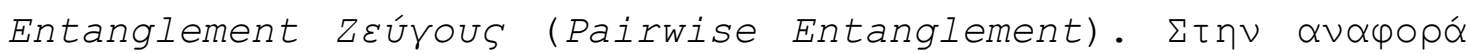

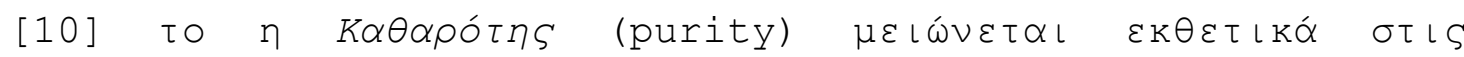

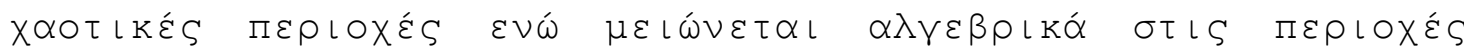

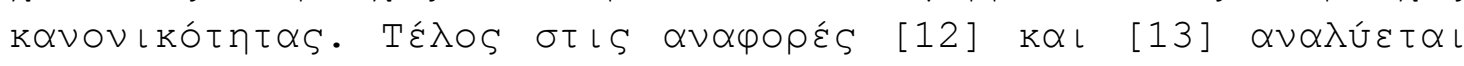

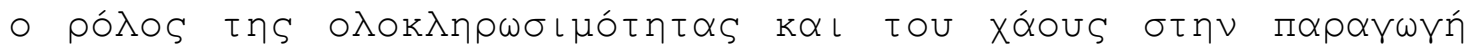




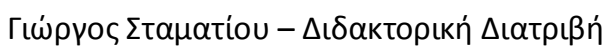

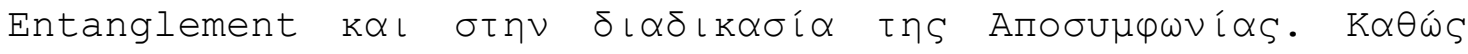

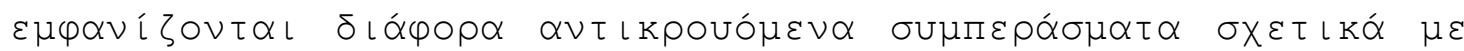

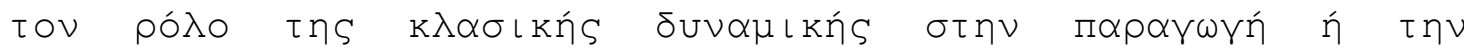

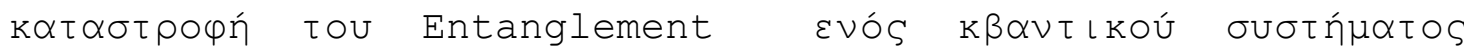

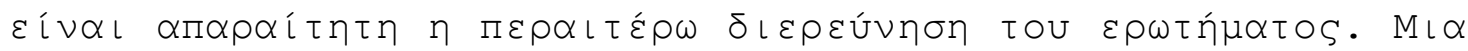

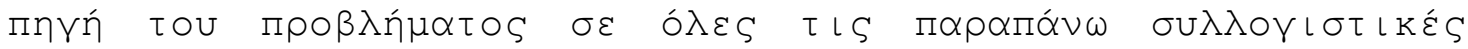

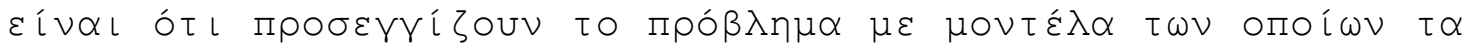

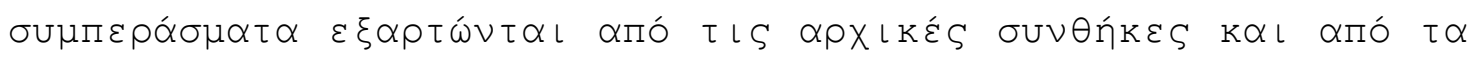

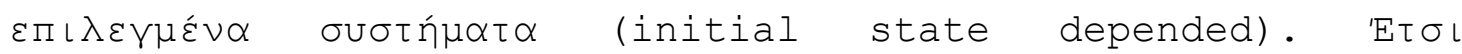

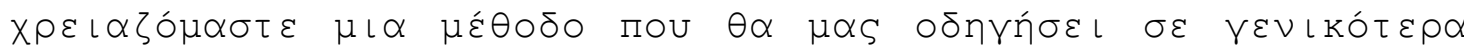

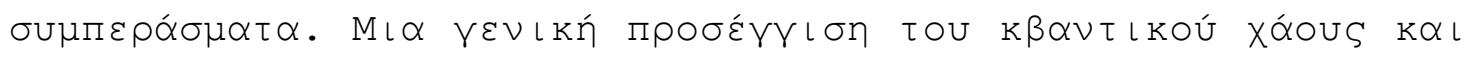

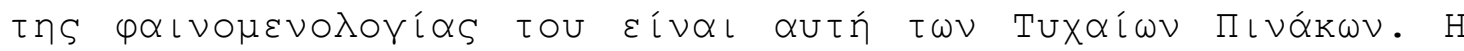

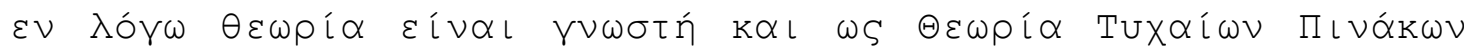

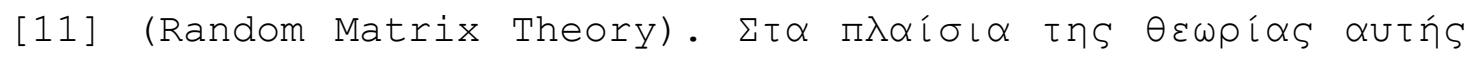

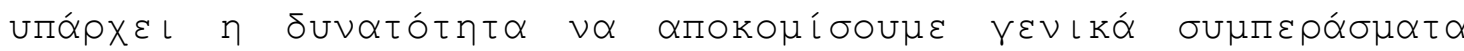

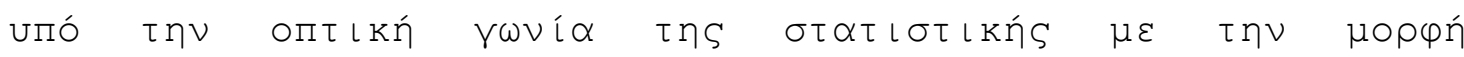

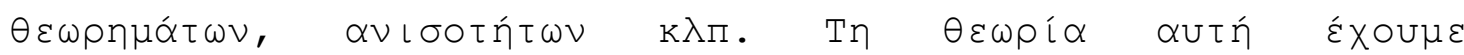

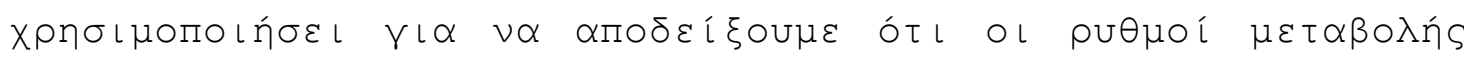

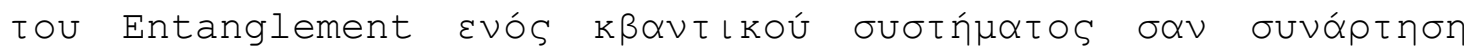

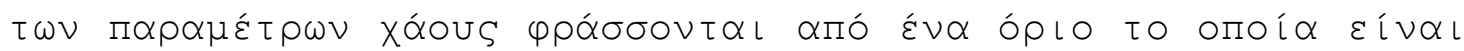

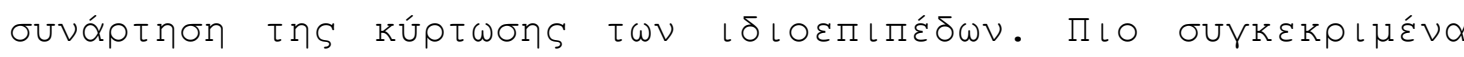

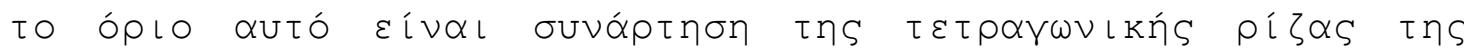

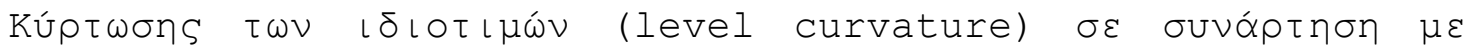

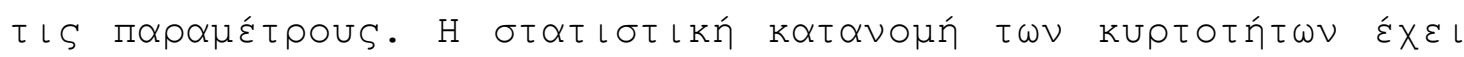

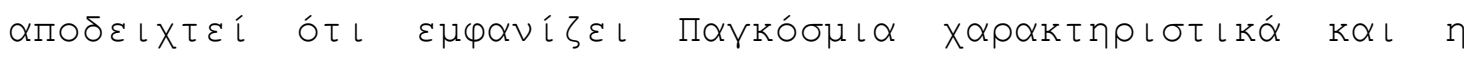

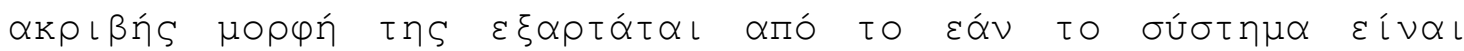

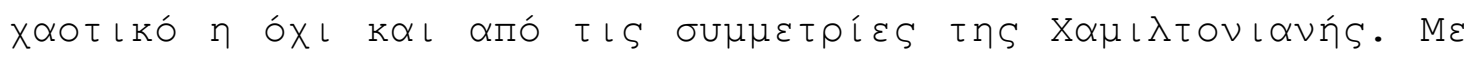

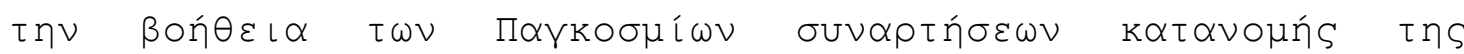

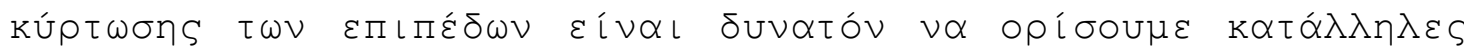

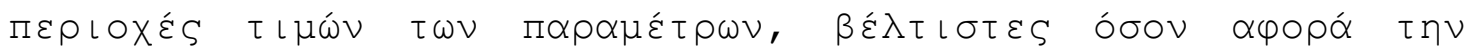

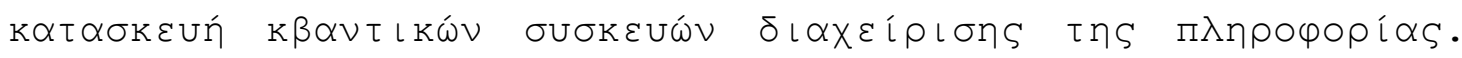

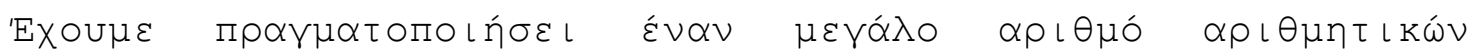

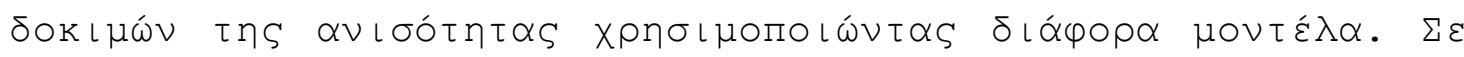

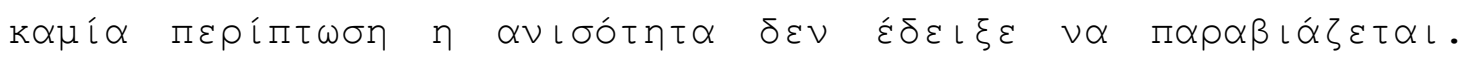

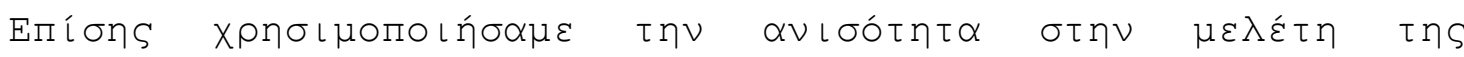

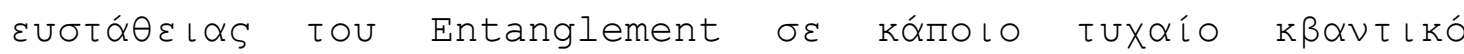

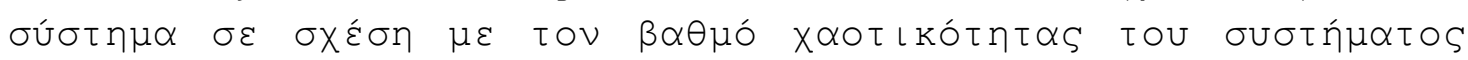

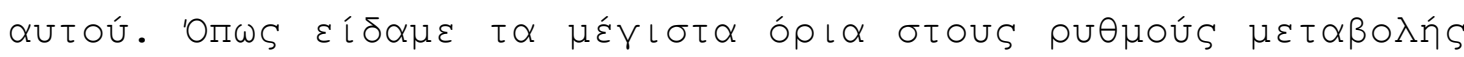

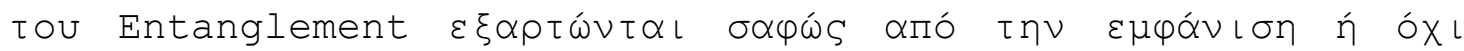

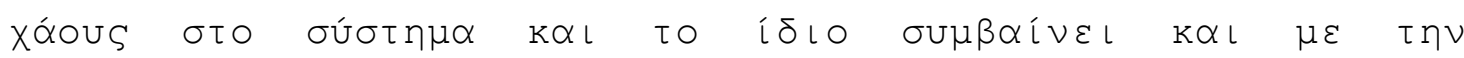

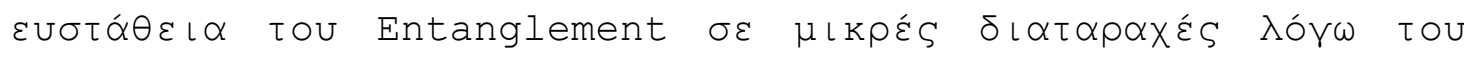




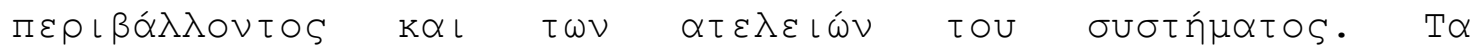

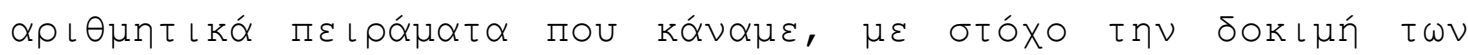
$\theta \varepsilon \omega \rho \eta \tau\llcorner$ k

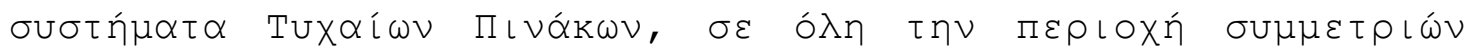

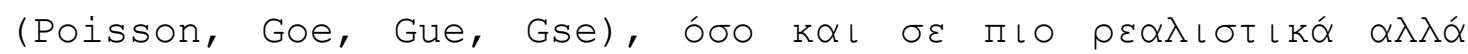

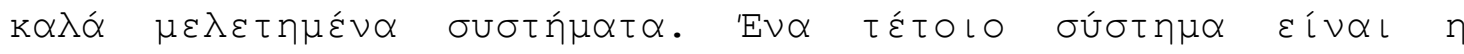

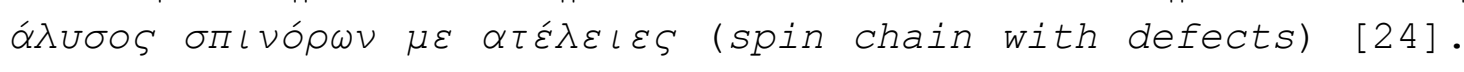

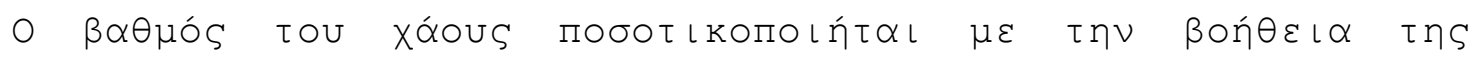

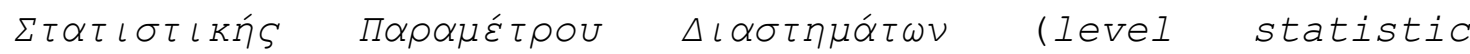

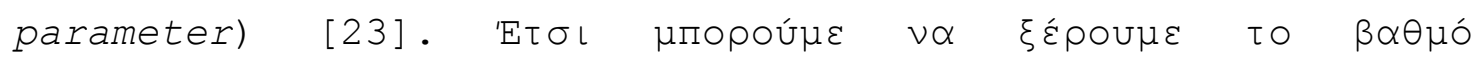

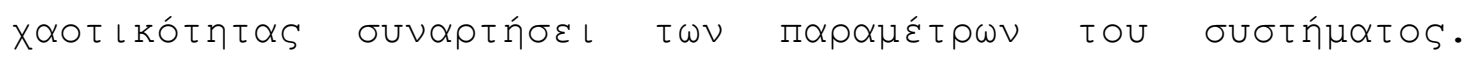

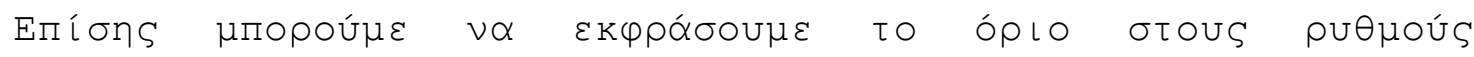

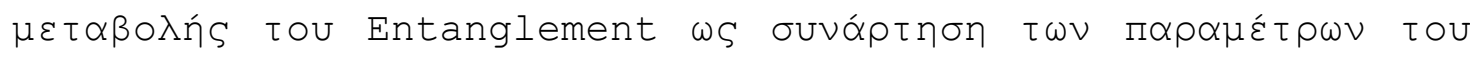

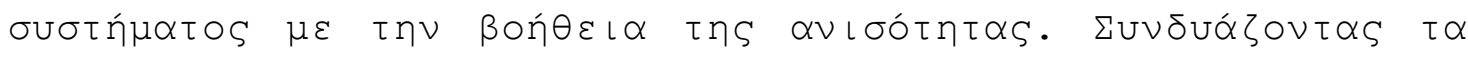

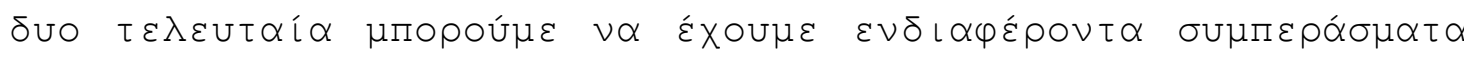

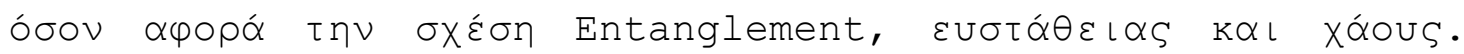

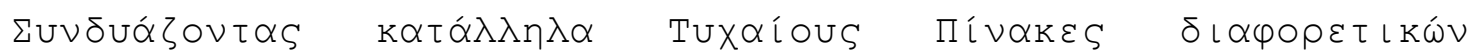

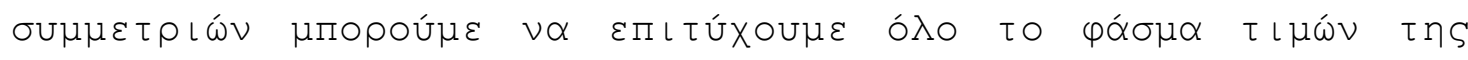

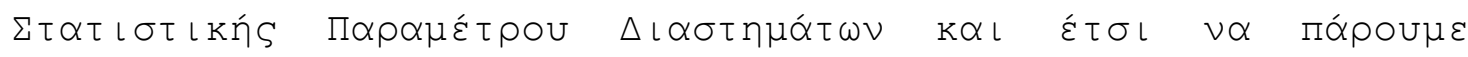

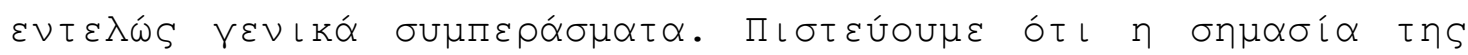

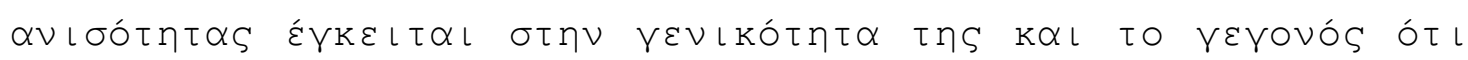

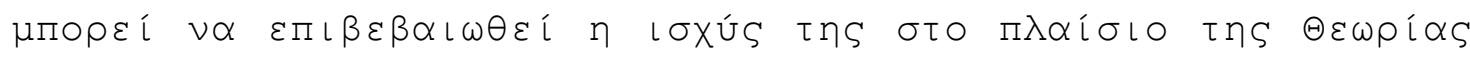

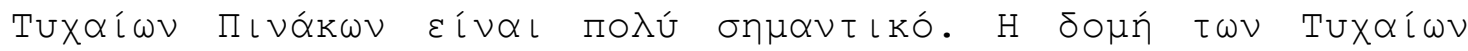

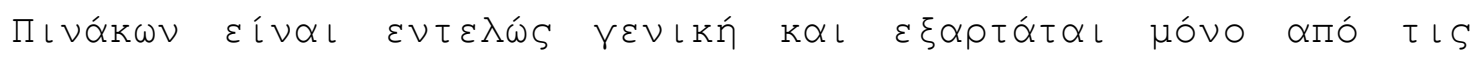

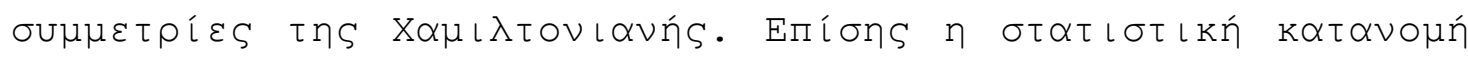

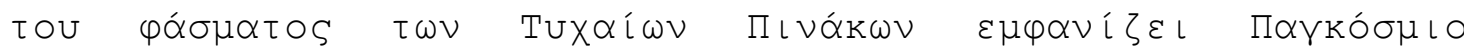

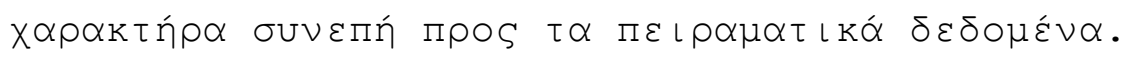

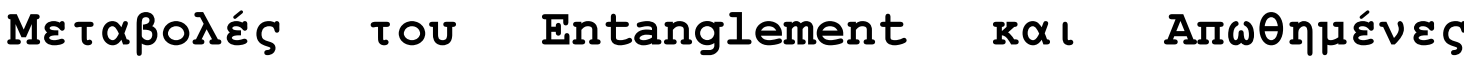 $\Delta \iota \alpha \sigma \tau \alpha u \rho \omega ́ \sigma \varepsilon เ \varsigma$.}

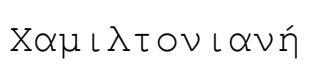

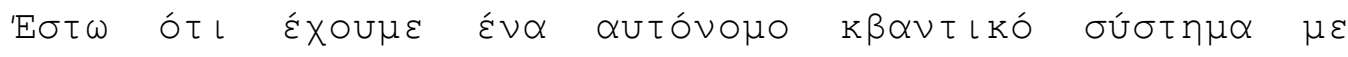

$$
H=H_{0}+\tau V
$$

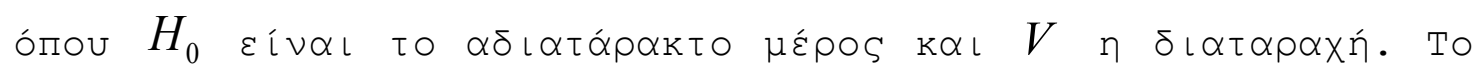

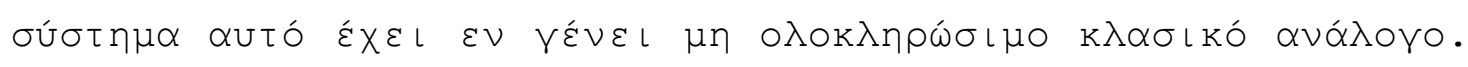

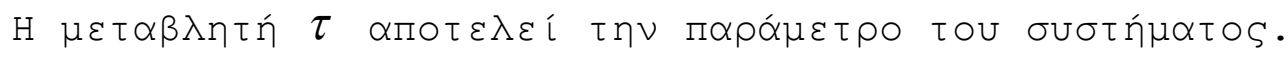




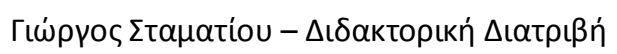

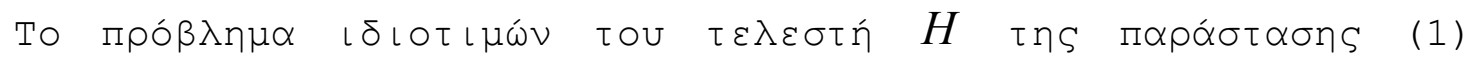
$\varepsilon \kappa \varphi \rho \alpha ́ \zeta \varepsilon \tau \alpha l \quad \omega S$

$$
H(\tau)|n(\tau)\rangle=\varepsilon_{n}(\tau)|n(\tau)\rangle
$$

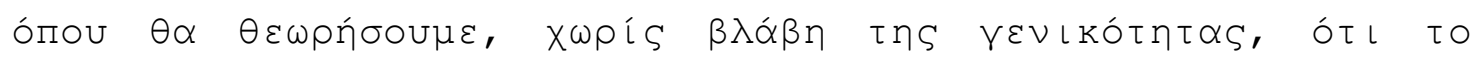

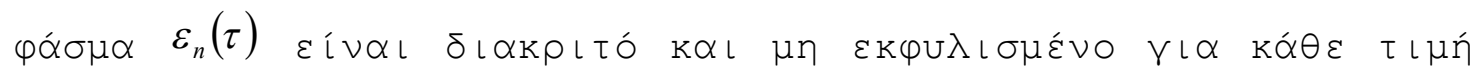

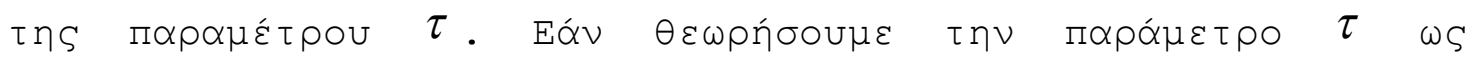

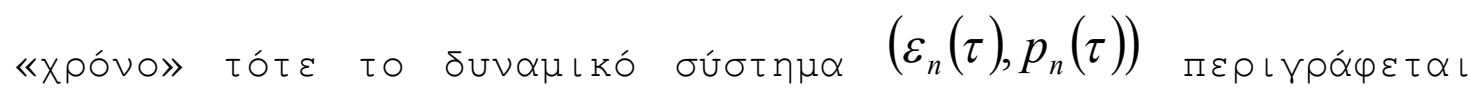

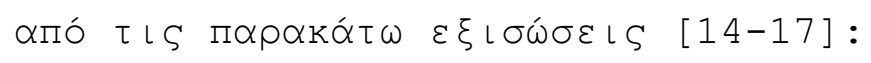

$$
\begin{gathered}
\frac{d \varepsilon_{n}(\tau)}{d \tau}=p_{n} \\
\frac{d p_{n}(\tau)}{d \tau}=2 \sum_{m(\neq n)} V_{n m}(\tau) \cdot V_{m n}(\tau)\left(\varepsilon_{n}(\tau)-\varepsilon_{m}(\tau)\right)^{-1} \\
\frac{d \varepsilon_{n}(\tau)}{d \tau}=p_{n} \\
\frac{d p_{n}(\tau)}{d \tau}=2 \sum_{m(\neq n)} V_{n m}(\tau) V_{m n}(\tau)\left(\varepsilon_{n}(\tau)-\varepsilon_{m}(\tau)\right)^{-1} \\
\frac{d|n(\tau)\rangle}{d \tau}=\sum_{m(\neq n)}|m(\tau)\rangle V_{m n}(\tau)\left(\varepsilon_{n}(\tau)-\varepsilon_{m}(\tau)\right)^{-1} \\
\frac{d\langle n(\tau)|}{d \tau}=\sum_{m(\neq n)}\langle m(\tau)| V_{n m}(\tau)\left(\varepsilon_{n}(\tau)-\varepsilon_{m}(\tau)\right)^{-1}
\end{gathered}
$$

о́поч

$$
p_{n}(\tau) \equiv V_{n n}=\langle n(\tau)|V| n(\tau)\rangle
$$

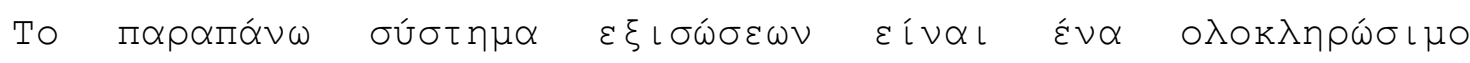

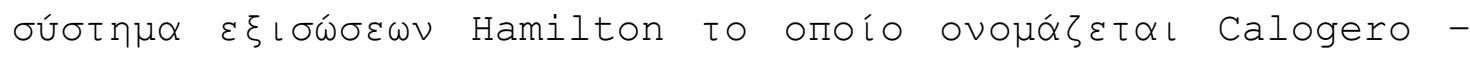




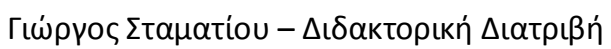

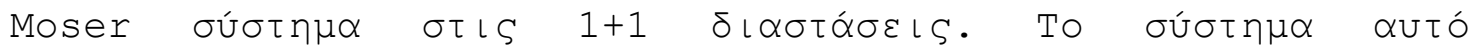

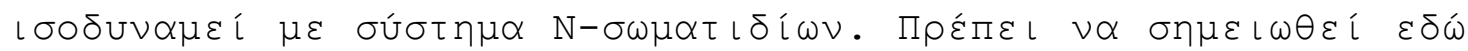

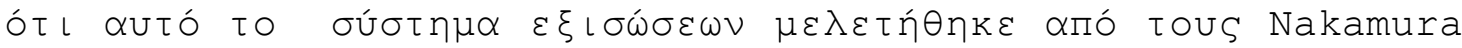

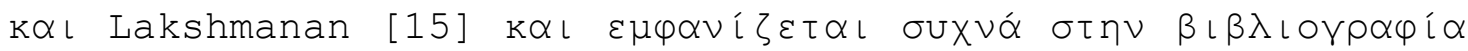

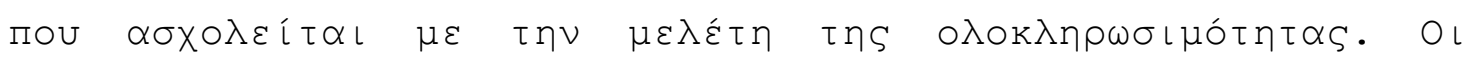

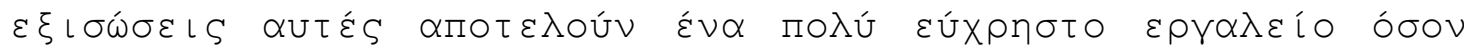

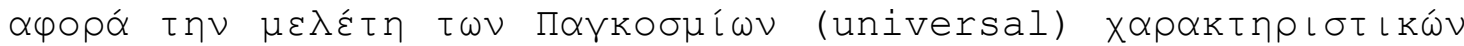

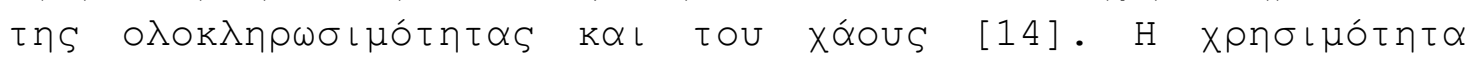

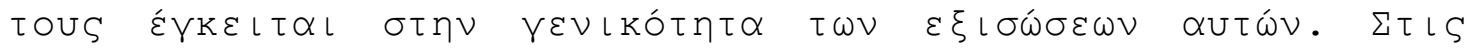

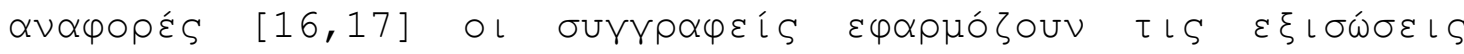

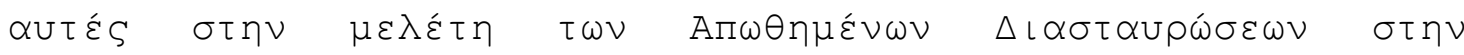

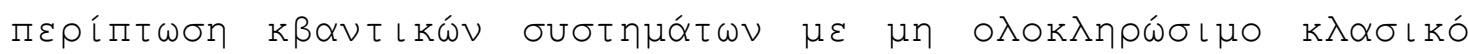

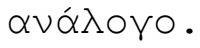

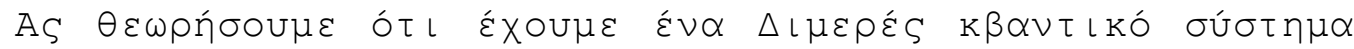

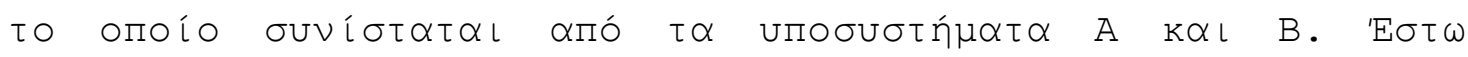

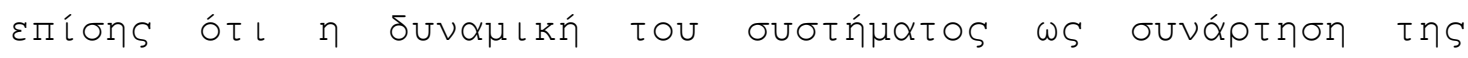

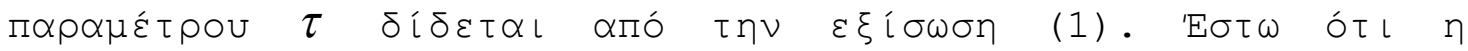

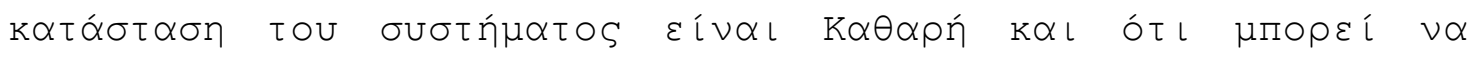

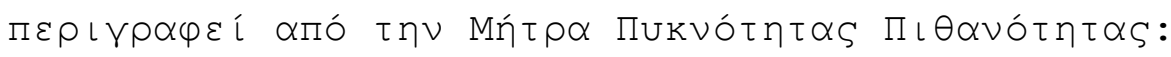

$$
\rho_{A B}^{n}(\tau)=|n(\tau)\rangle\langle n(\tau)|
$$

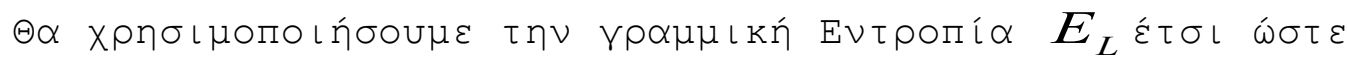

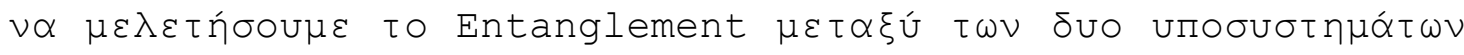

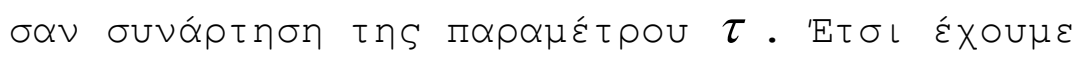

$$
E_{L}=1-\operatorname{tr}\left[\left(\rho_{A}^{n}\right)^{2}\right]
$$

о́поv

$$
\rho_{A}^{n}=\operatorname{tr}_{B}\left(\rho_{A B}^{n}\right)
$$

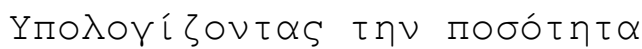

$$
d E_{L} / d \tau
$$




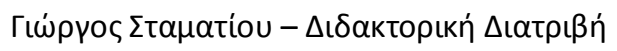

$\varepsilon \times \bigcirc u \varepsilon$

$$
\frac{\partial}{\partial \tau} \operatorname{tr}\left[\left(\rho_{A}^{n}(\tau)\right)^{2}\right]=2 \operatorname{tr}\left\{\operatorname{tr}_{B}\left(\rho_{A B}^{n}\right) \operatorname{tr}\left[\frac{\partial}{\partial \tau}\left(\rho_{A B}^{n}\right)\right]\right\}
$$

$\varepsilon \Pi i ́ \sigma \cap S$

$$
\begin{aligned}
&\left\langle k(\tau)\left|\frac{\partial}{\partial \tau} \rho_{A B}^{n}(\tau)\right| l(\tau)\right\rangle=\langle k(\tau) \mid\left(\frac{\partial}{\partial \tau}|n(\tau)\rangle\right)\langle n(\tau) \mid l(\tau)\rangle \\
&+\langle k(\tau) \mid n(\tau)\rangle\left(\frac{\partial}{\partial \tau}\langle n(\tau)|\right)|l(\tau)\rangle \\
&=\langle k(\tau)|\left(\frac{\partial}{\partial \tau}|n(\tau)\rangle\right) \delta_{n l}+\delta_{k n}\left(\frac{\partial}{\partial \tau}\langle n(\tau)|\right)|l(\tau)\rangle
\end{aligned}
$$

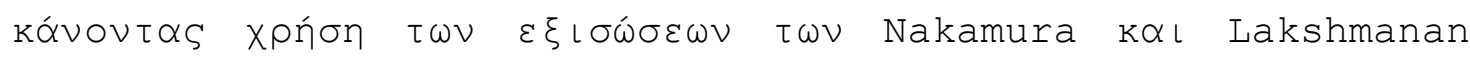

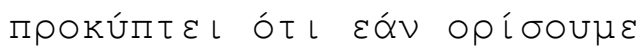

$$
\gamma_{k l}^{n}(\tau) \equiv \delta_{n l}\left(1-\delta_{n k}\right) \frac{V_{k n}}{\epsilon_{n}-\epsilon_{k}}+\delta_{k n}\left(1-\delta_{n l}\right) \frac{V_{n l}}{\epsilon_{n}-\epsilon_{l}}
$$

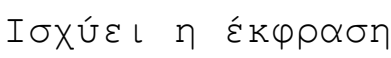

$$
\frac{\partial}{\partial \tau} \rho_{A B}^{n}(\tau)=\sum_{k l} \gamma_{k l}^{n}|k(\tau)\rangle\langle l(\tau)|
$$

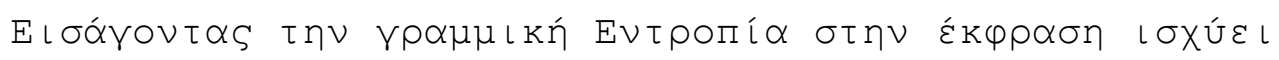

$$
\frac{\partial}{\partial \tau} E_{L}^{n}=-2 \sum_{k l} \gamma_{k l}^{n} \operatorname{tr}\left[\operatorname{tr}_{B}(|n(\tau)\rangle\langle n(\tau)|) \operatorname{tr}_{B}(|k(\tau)\rangle\langle l(\tau)|)\right]
$$

ń $\alpha \lambda \lambda \iota \dot{\omega} S$

$$
\frac{\partial}{\partial \tau} E_{L}^{n}=-2 \sum_{k l} \gamma_{k l}^{n} A_{k l}^{n}(\tau)
$$


о́поч

$$
A_{k l}^{n}(\tau)=\operatorname{tr}\left[\operatorname{tr}_{B}(|n(\tau)\rangle\langle n(\tau)|) \operatorname{tr}_{B}(|k(\tau)\rangle\langle l(\tau)|)\right]
$$

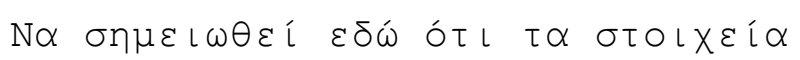

$$
A_{k l}^{n}(\tau)
$$

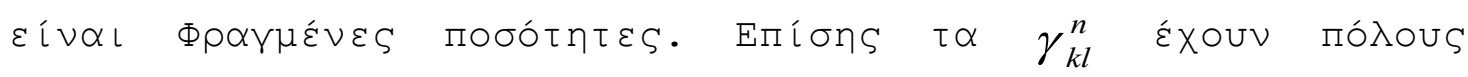

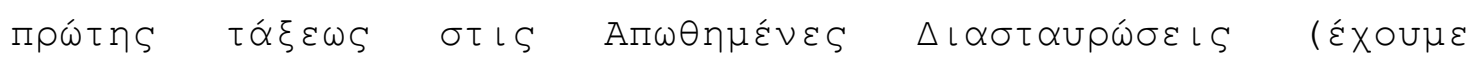

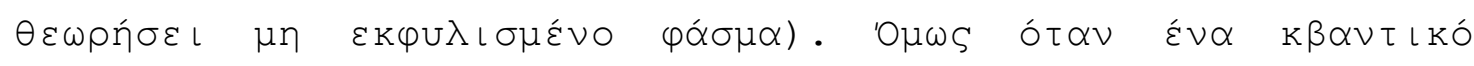

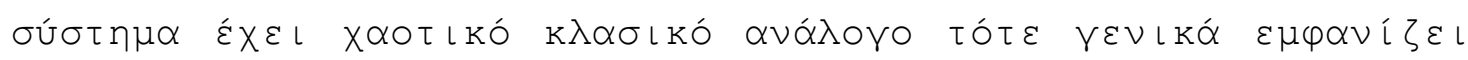

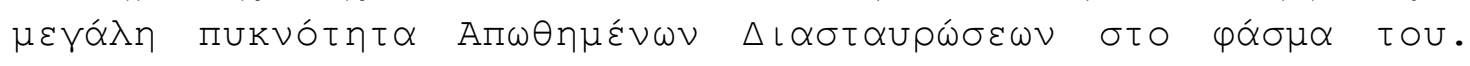

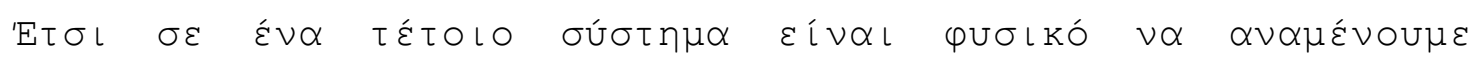

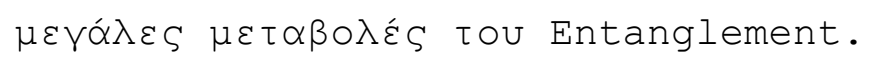

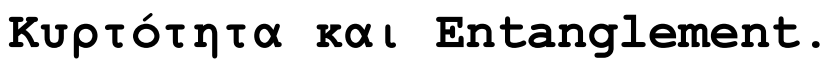

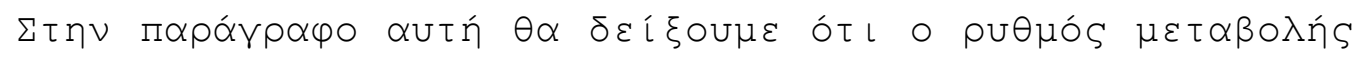

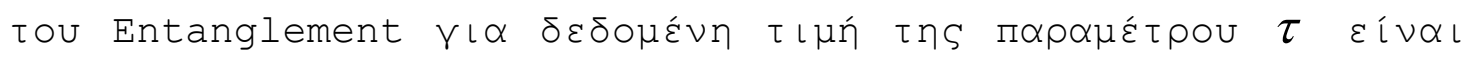

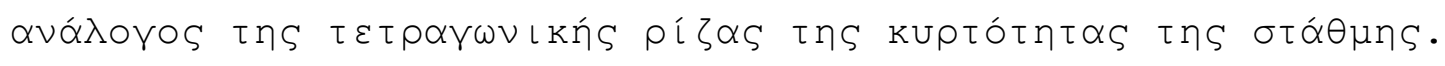

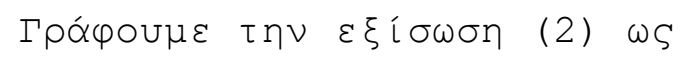

$$
\frac{\partial E_{L}^{n}}{\partial \tau}=-2 \sum_{k \neq n} \frac{V_{k n} A_{k n}^{n}+V_{n k} A_{n k}^{n}}{\epsilon_{n}-\epsilon_{k}}
$$

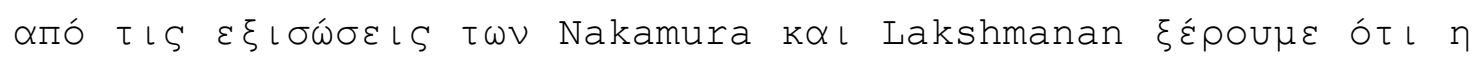

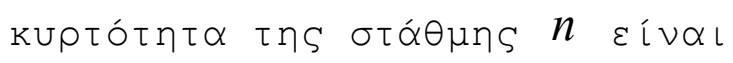

$$
K_{n} \equiv \frac{\partial^{2} \epsilon_{n}(\tau)}{\partial \tau^{2}}=2 \sum_{m \neq n} \frac{\left|V_{n m}\right|^{2}}{\epsilon_{n}-\epsilon_{m}}
$$

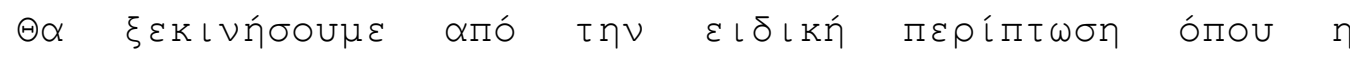

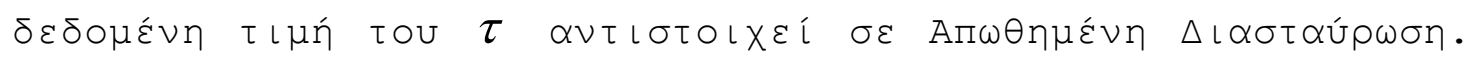

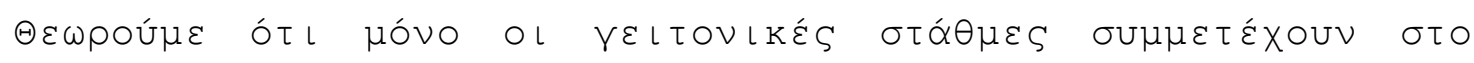




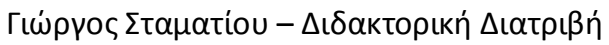

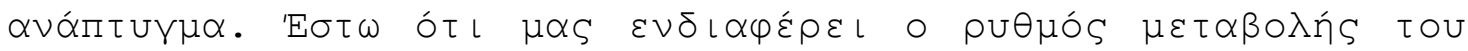

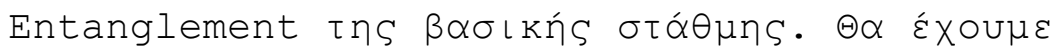

$$
\frac{\partial E_{L}^{0}}{\partial \tau} \approx-2 \frac{V_{10} A_{10}^{0}+V_{01} A_{01}^{0}}{\epsilon_{0}-\epsilon_{1}}
$$

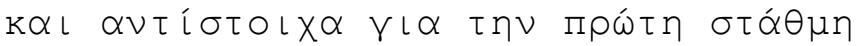

$$
\frac{\partial E_{L}^{1}}{\partial \tau} \approx-2 \frac{V_{01} A_{01}^{1}+V_{10} A_{10}^{1}}{\epsilon_{1}-\epsilon_{0}}
$$

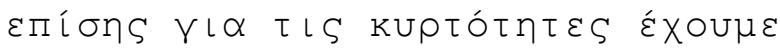

$$
K_{0}=2 \frac{\left|V_{01}\right|^{2}}{\epsilon_{0}-\epsilon_{1}}<0
$$

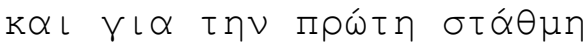

$$
K_{1}=2 \frac{\left|V_{01}\right|^{2}}{\epsilon_{1}-\epsilon_{0}}>0
$$

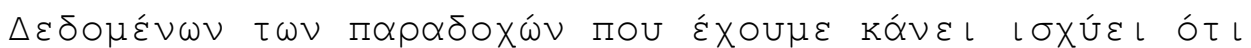

$$
K_{0}=-K_{1}
$$

$\dot{\varepsilon} \tau \sigma \iota$

$$
\frac{\partial E_{L}^{0}}{\partial \tau} \approx-\frac{V_{10} A_{10}^{0}+V_{01} A_{01}^{0}}{\left|V_{01}\right|^{2}} K_{0}
$$

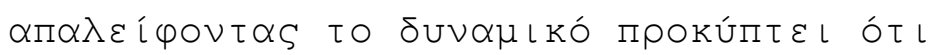

$$
\frac{\partial E_{L}^{0}}{\partial \tau} \approx \frac{2 \sqrt{2} \operatorname{Re}\left[A_{10}^{0} e^{i \phi}\right]}{\sqrt{\epsilon_{1}-\epsilon_{0}}} \sqrt{\left|K_{0}\right|}
$$

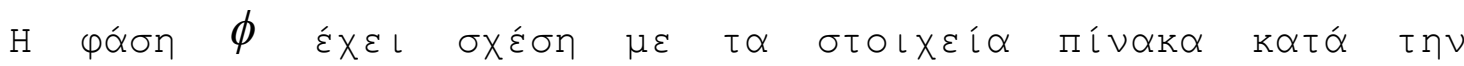

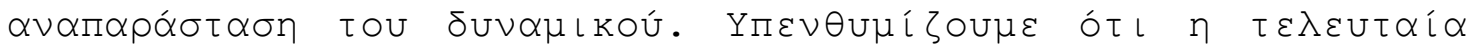

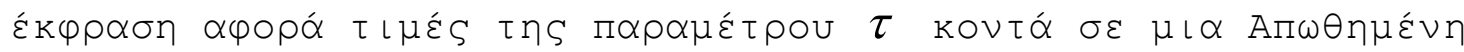




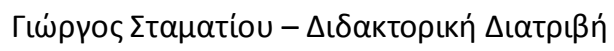

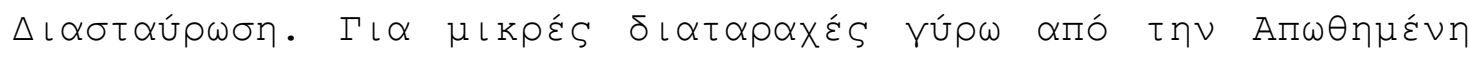

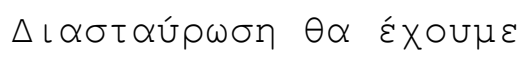

$$
\frac{\Delta E_{L}^{1}}{\Delta E_{L}^{0}} \approx \frac{\operatorname{Re}\left(A_{01}^{1} e^{i \phi}\right)}{\operatorname{Re}\left(A_{10}^{0} e^{i \phi}\right)}
$$

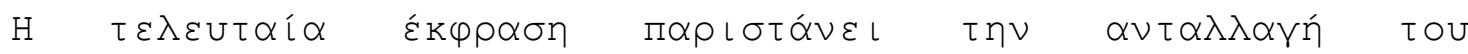

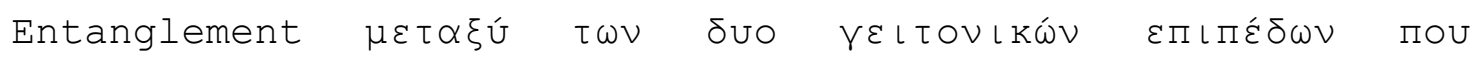

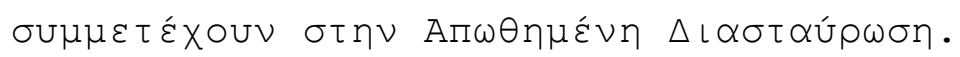

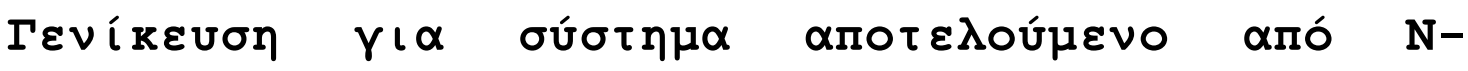 qubits.}

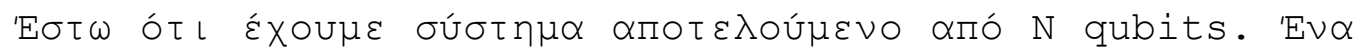

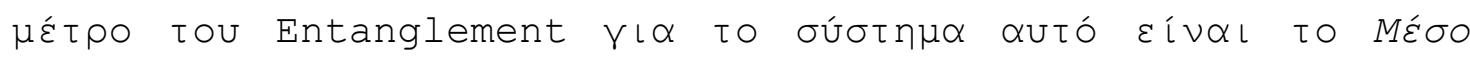
$\Delta \iota \mu \varepsilon \rho \varepsilon^{S}$ Entanglement (Mean Bipartite Entanglement) то опоі́

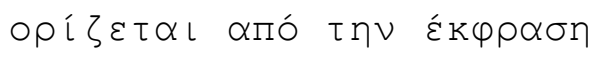

$$
Q^{n}=2-\frac{2}{N} \sum_{j=1}^{N} \operatorname{tr}\left[\rho_{j}^{2}\right]
$$

о́поч

$$
\rho_{j}=\operatorname{tr}_{A L L \neq j}(|n\rangle\langle n|)
$$

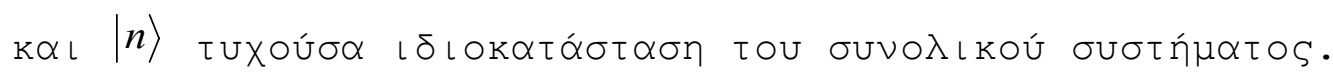

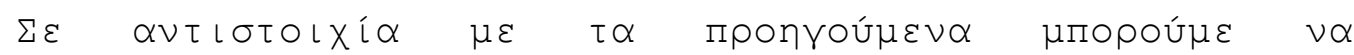

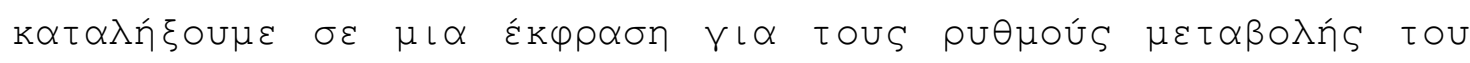

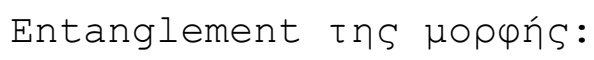

$$
\left|\frac{\partial Q^{0}}{\partial \tau}\right| \leq b \sqrt{\left|K_{0}\right|}
$$

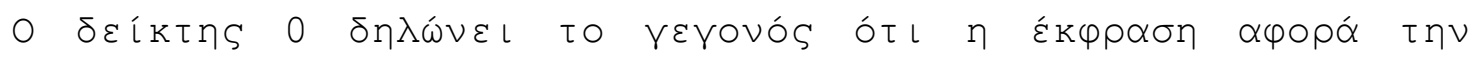

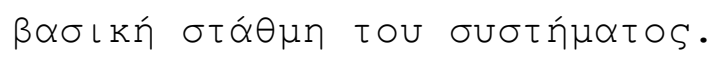




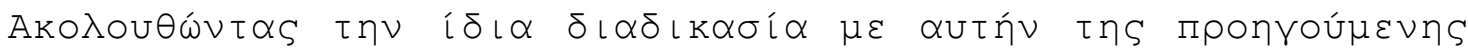

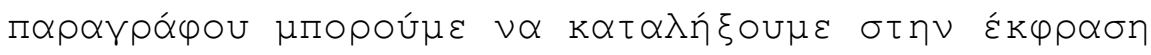

$$
\frac{\partial Q^{0}}{\partial \tau}=\frac{8}{N} \sum_{k=1}^{2^{N}-1} \frac{\operatorname{Re}\left[V_{0 k} A_{0 k}^{0}\right]}{\epsilon_{k}-\epsilon_{0}}
$$

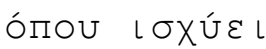

$$
-N \leq A_{k l}^{0} \leq+N
$$

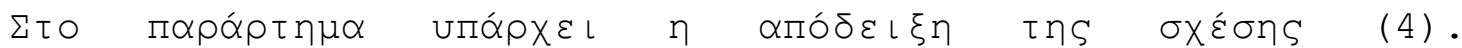

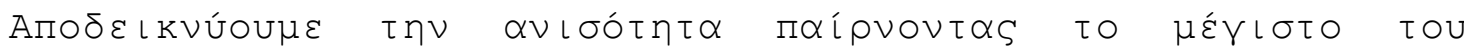

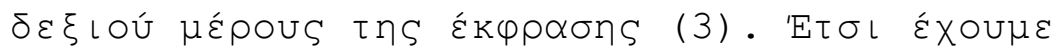

$$
\begin{aligned}
\max \frac{\partial Q^{0}}{\partial \tau} & =8 \sum_{k=1}^{2^{N}-1} \frac{\operatorname{Re}\left[V_{0 k}\right]}{\epsilon_{k}-\epsilon_{0}} \leq 8 \sqrt{\sum_{k=1}^{2^{N}-1} \frac{\operatorname{Re}^{2} V_{0 k}}{\epsilon_{k}-\epsilon_{0}}} \sqrt{\sum_{k=1}^{2^{N}-1} \frac{1}{\epsilon_{k}-\epsilon_{0}}} \\
& \leq 8 \sqrt{\sum_{k=1}^{2^{N}-1} \frac{\left|V_{0 k}\right|^{2}}{\epsilon_{k}-\epsilon_{0}}} \sqrt{\sum_{k=1}^{2^{N}-1} \frac{1}{\epsilon_{k}-\epsilon_{0}}}=b \sqrt{\left|K_{0}\right|}
\end{aligned}
$$

о́поч

$$
b=8 \sqrt{\sum_{k=1}^{2^{N}-1} \frac{1}{\epsilon_{k}-\epsilon_{0}}}
$$

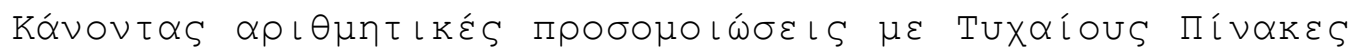

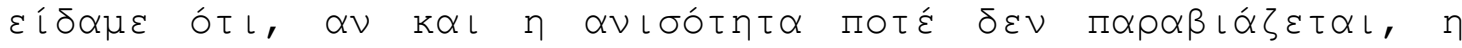

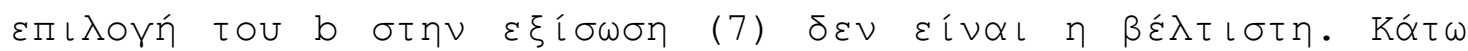

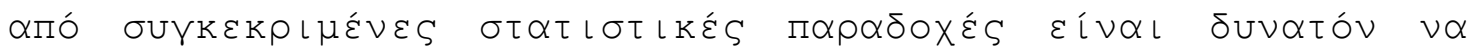

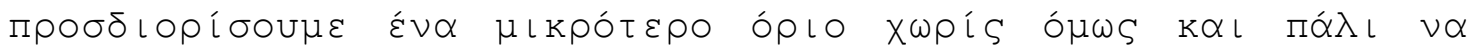

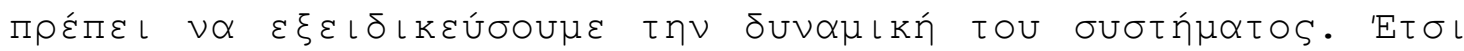

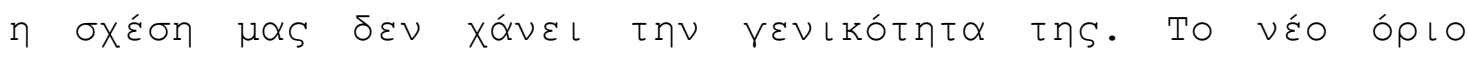

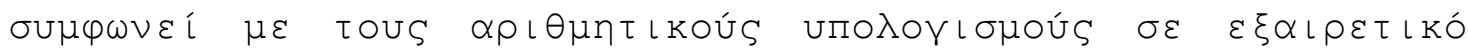




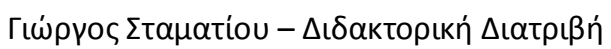

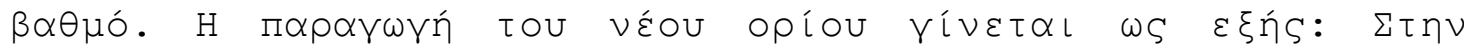

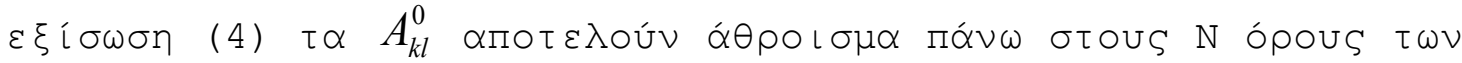
$\mathrm{N}$ qubits.

$$
A_{k l}^{0}=\sum_{j=1}^{N} A_{j ; k l}^{0}
$$

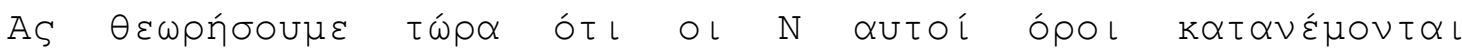

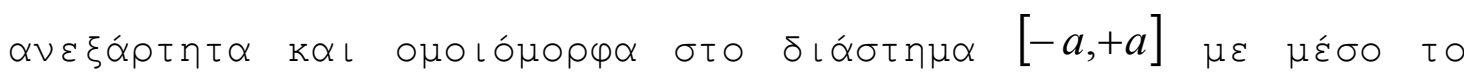

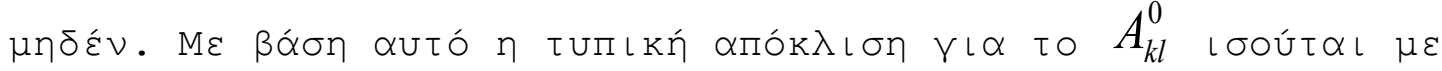

$$
\sigma\left(A_{k l}^{0}\right)=a \sqrt{N} / \sqrt{3}
$$

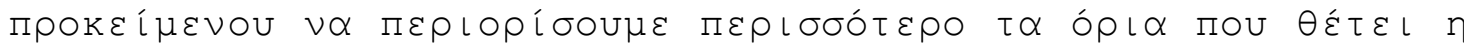

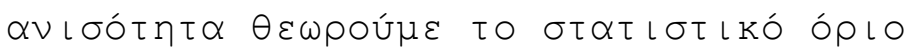

$$
-\sigma\left(A_{k l}^{0}\right) \leq A_{k l}^{0} \leq \sigma\left(A_{k l}^{0}\right)
$$

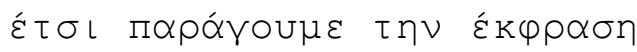

$$
b^{\prime}=\frac{8 a}{\sqrt{3 N}} \sqrt{\sum_{k=1}^{2^{N}-1} \frac{1}{\epsilon_{k}-\epsilon_{0}}}
$$

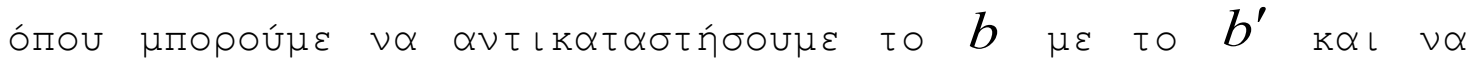

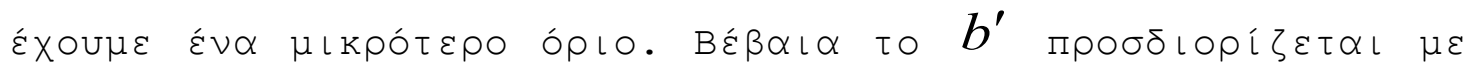

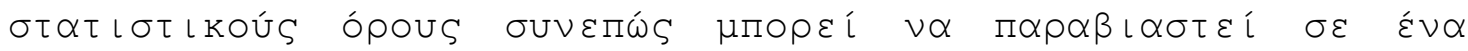

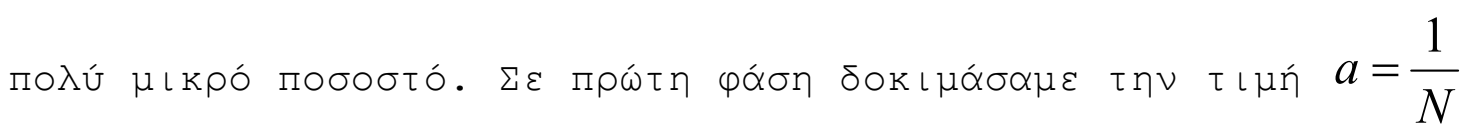

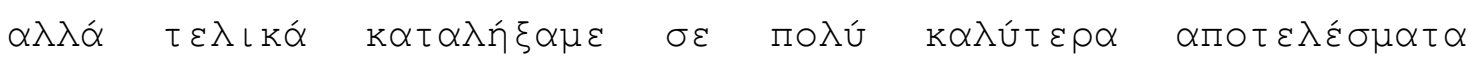

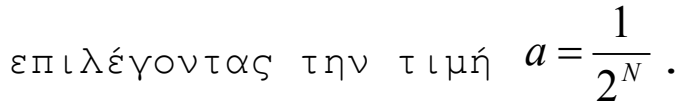




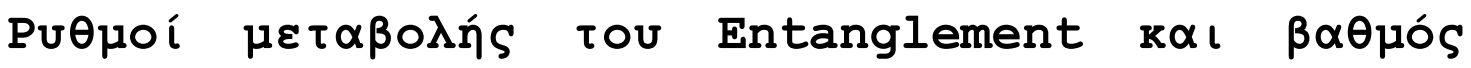 Xóous.}

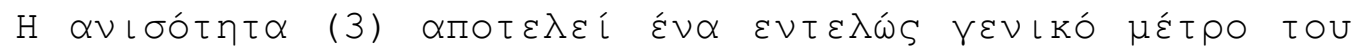

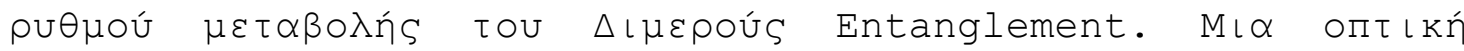

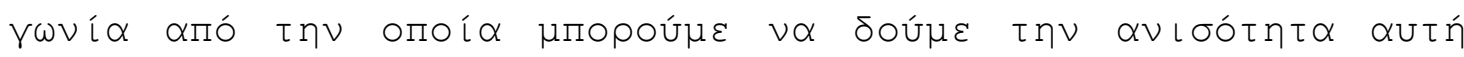

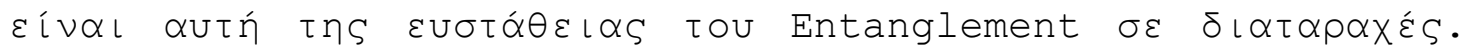

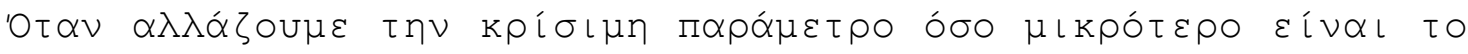

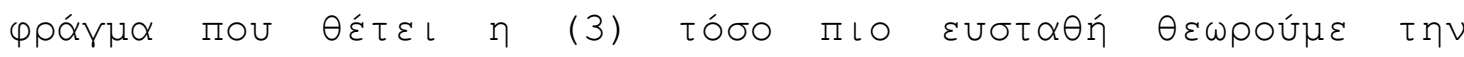

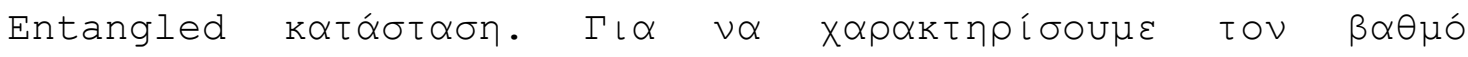

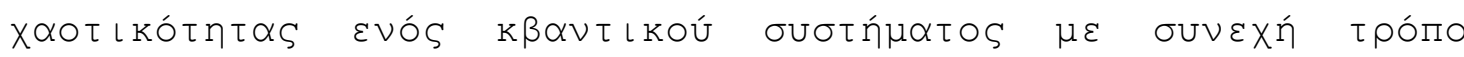

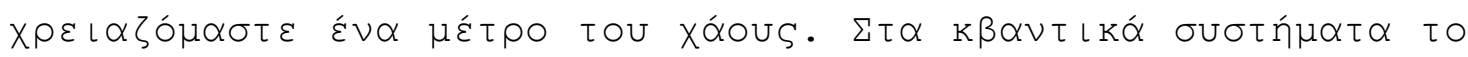

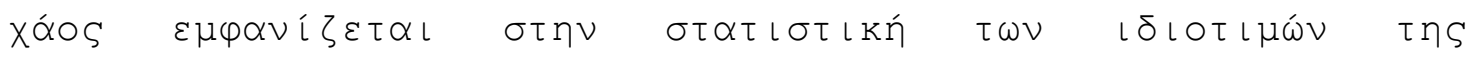

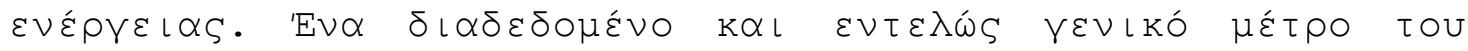

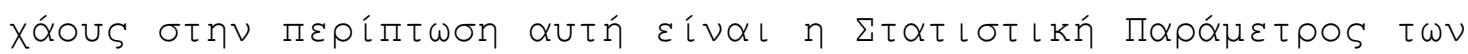
$\Delta \iota \alpha \sigma \tau \eta \mu \alpha ́ \tau \omega \nu[23]$.

$$
\gamma=\frac{\int_{0}^{s_{0}}\left[P(s)-P_{W D}\right] d s}{\int_{0}^{s_{0}}\left[P_{P}(s)-P_{W D}\right] d s}
$$

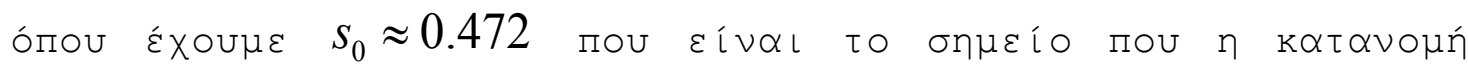
Poisson

$$
P_{P}(s)=\exp (-s)
$$

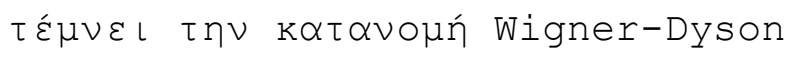

$$
P_{W D}(s)=\pi s / 2 \exp \left(-\pi s^{2} / 4\right)
$$

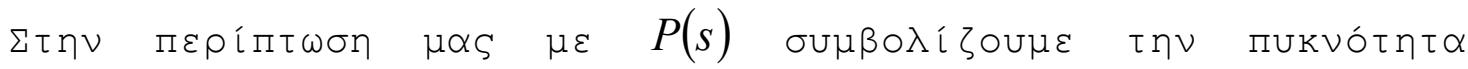

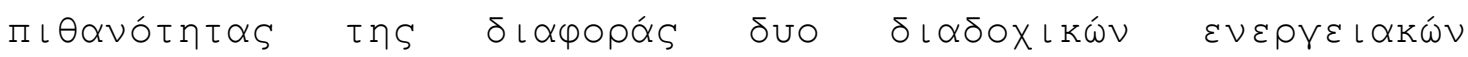

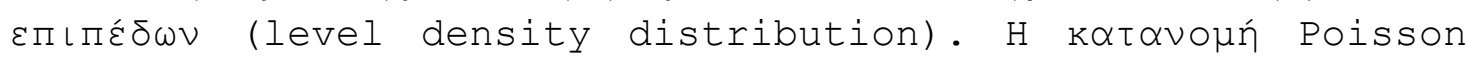

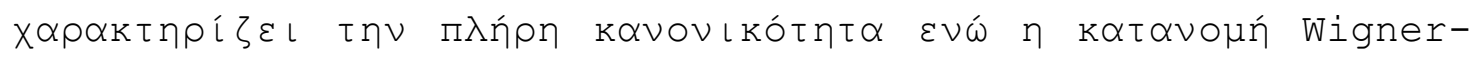

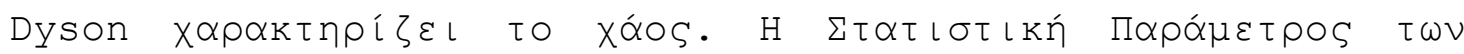

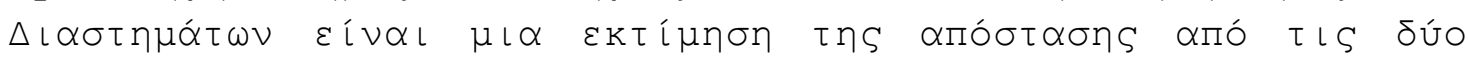

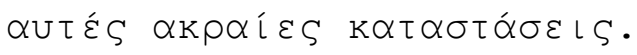




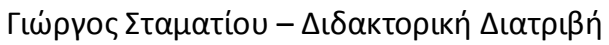

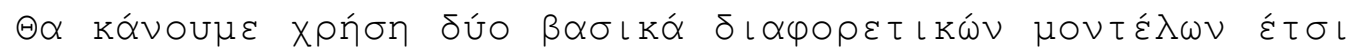

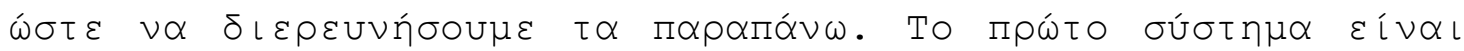

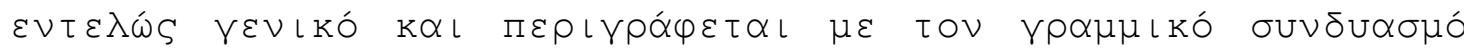

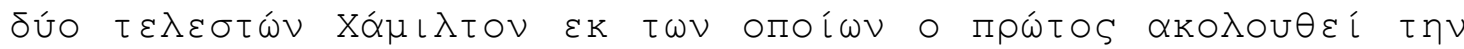

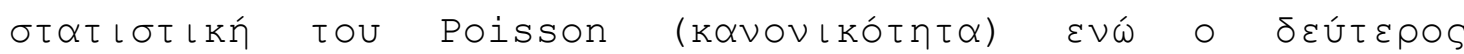

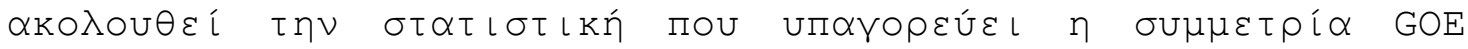

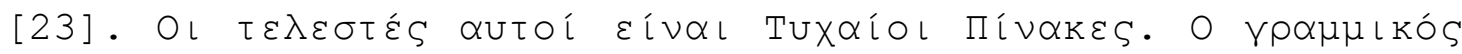

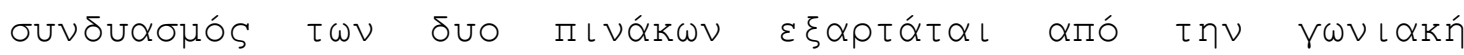

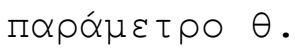

$$
H(\theta)=\cos (\theta) H_{P}+\sin (\theta) H_{W}
$$

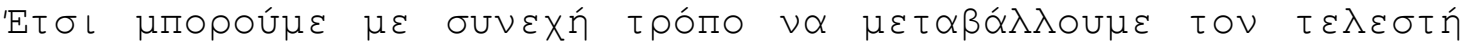

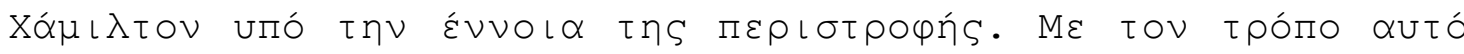

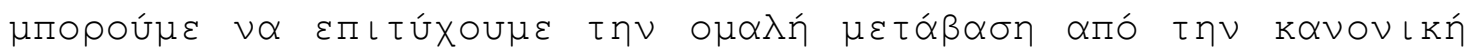

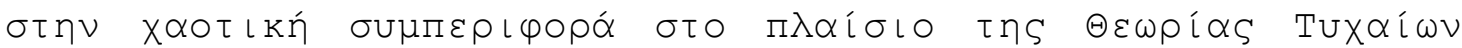

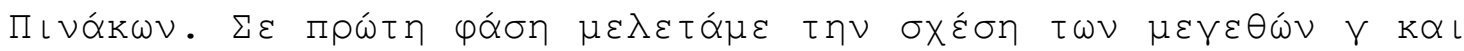

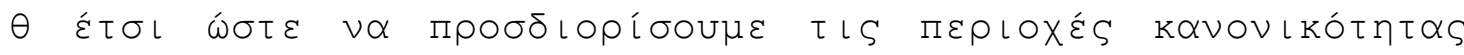

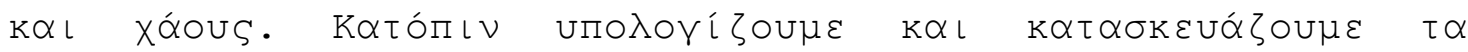

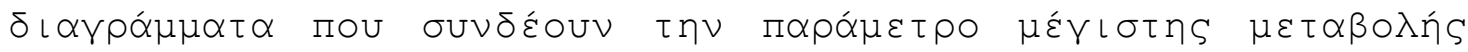

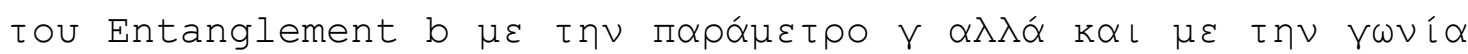

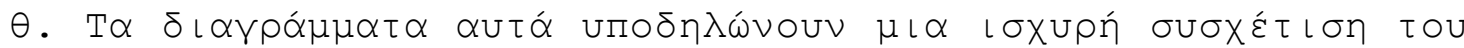

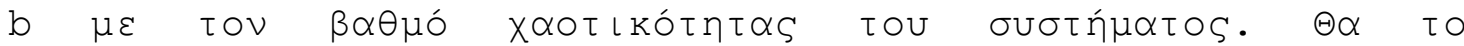

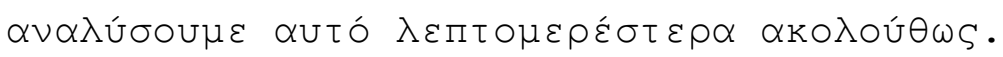

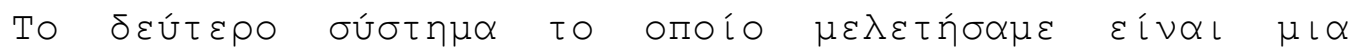

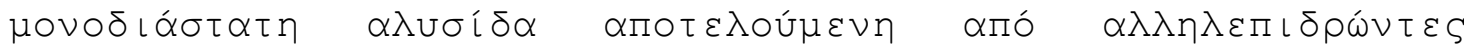

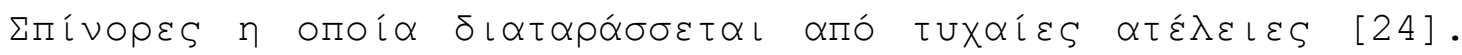

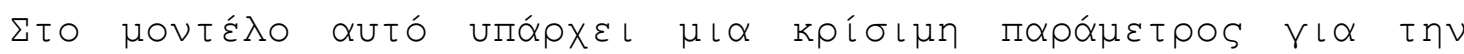

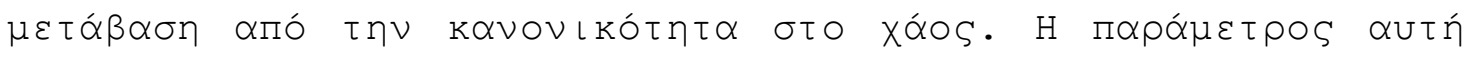

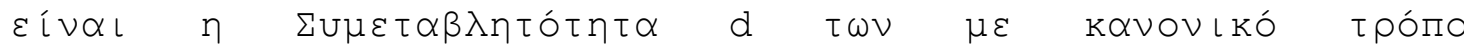

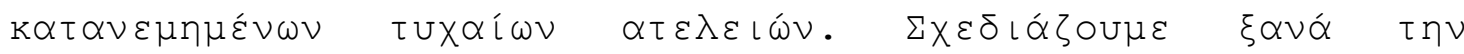

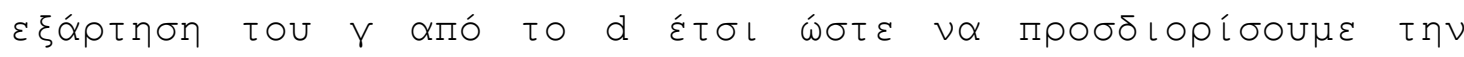

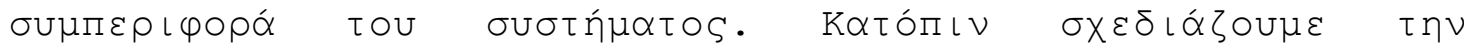

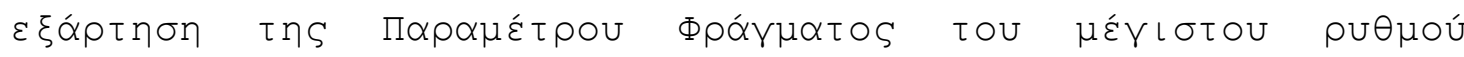

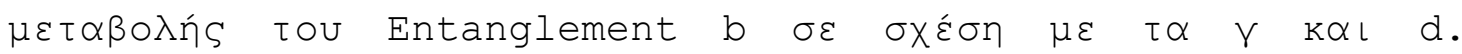

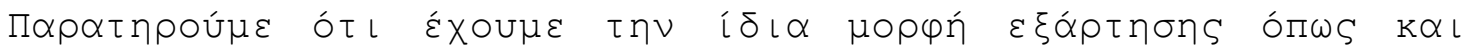

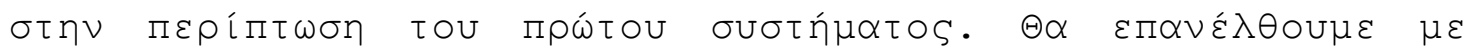

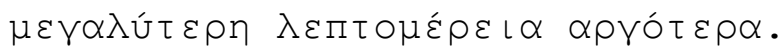




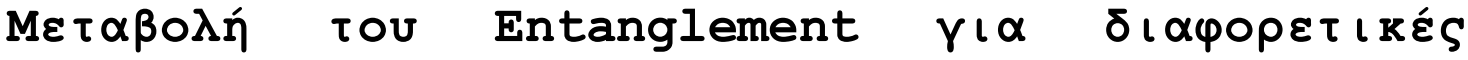

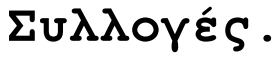

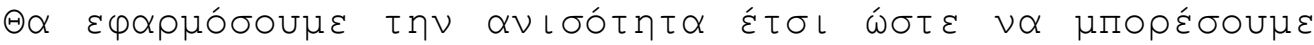

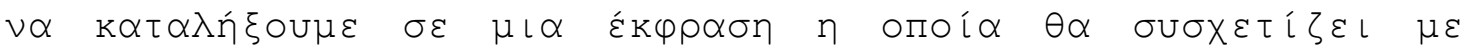

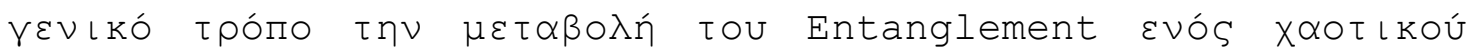

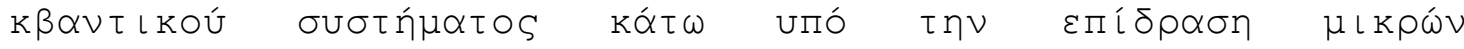

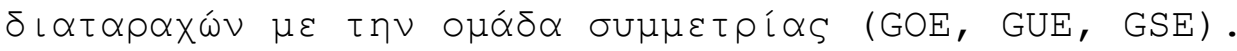

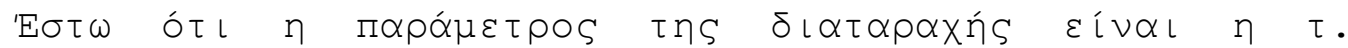

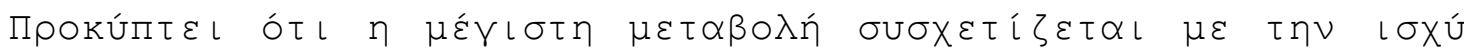

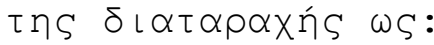

$$
\Delta Q=\left|\frac{\partial Q^{0}}{\partial \tau}\right| \Delta \tau=b \sqrt{K_{0}} \Delta \tau
$$

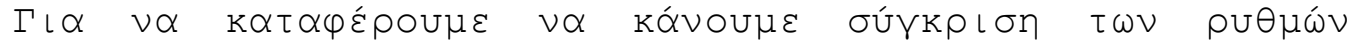

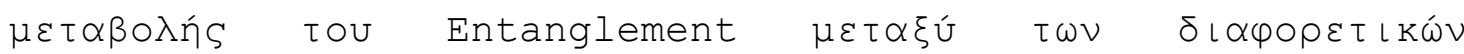

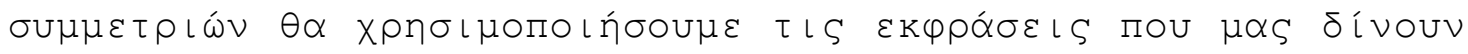

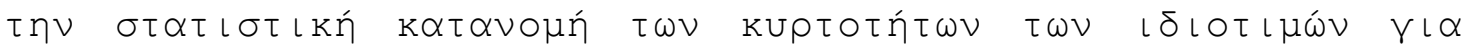

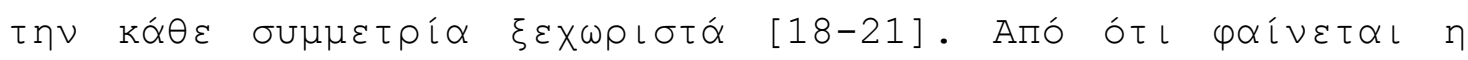

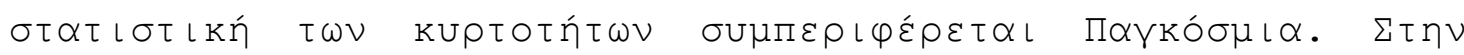

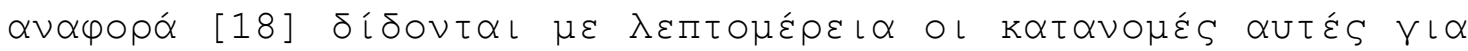

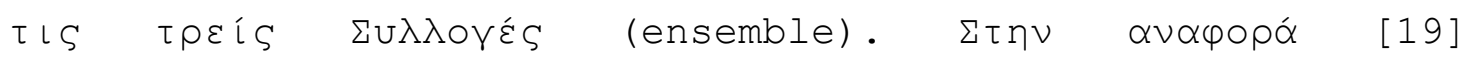

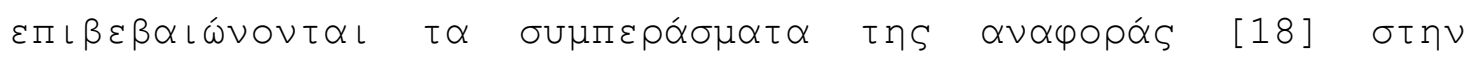

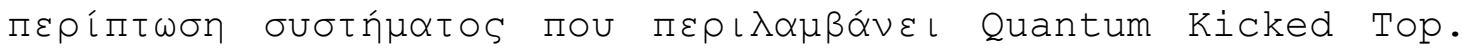

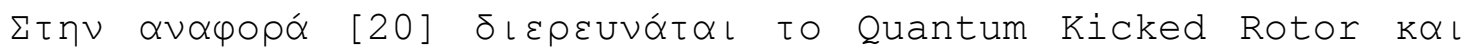

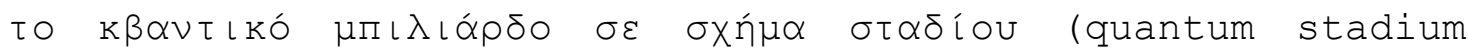

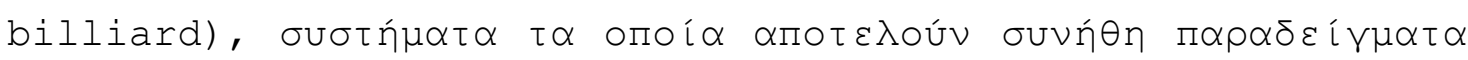

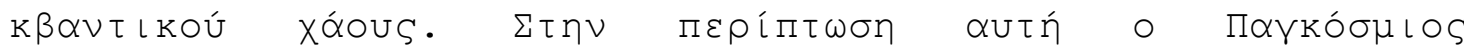

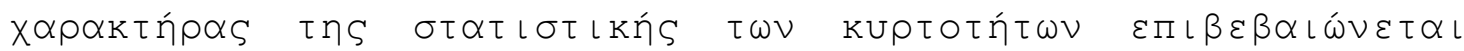

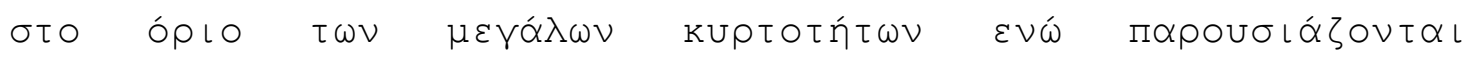

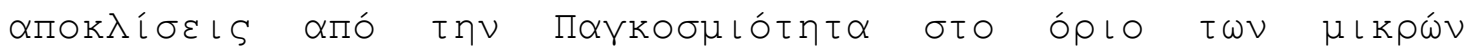

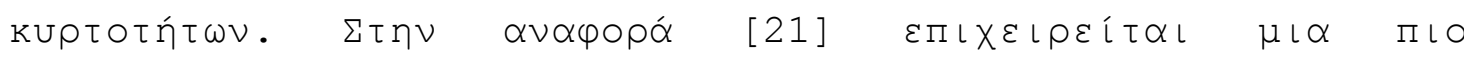

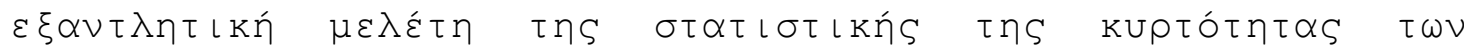

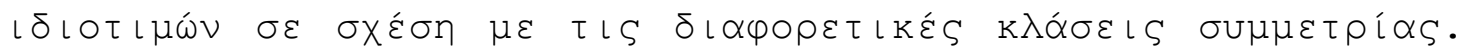

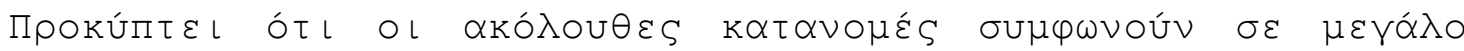

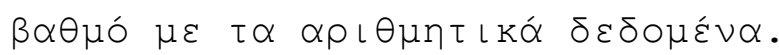




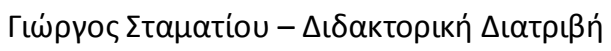

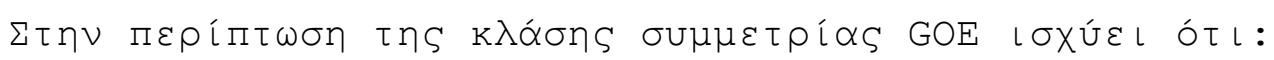

$$
P_{O}=\frac{1}{2} \frac{1}{\left(1+k^{2}\right)^{3 / 2}}
$$

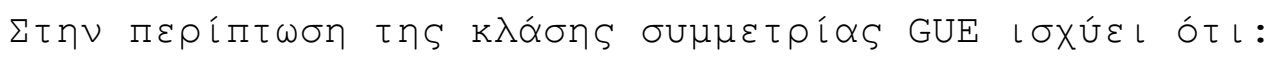

$$
P_{U}=\frac{2}{\pi} \frac{1}{\left(1+k^{2}\right)^{2}}
$$

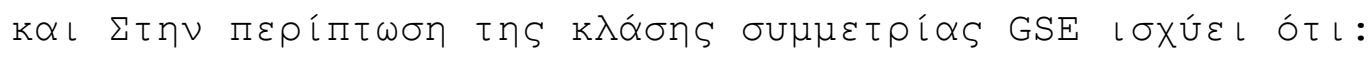

$$
P_{S}=\frac{8}{3 \pi} \frac{1}{\left(1+k^{2}\right)^{3}}
$$

о́по

$$
k=\frac{K}{\gamma_{\nu}}
$$

$k \alpha \iota$

$$
\gamma_{\nu}=\nu A
$$

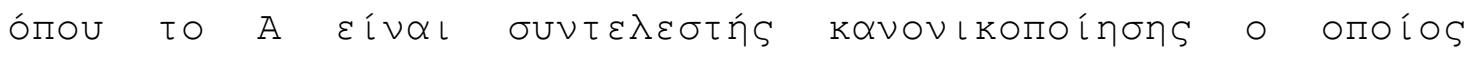

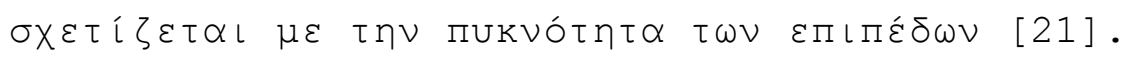

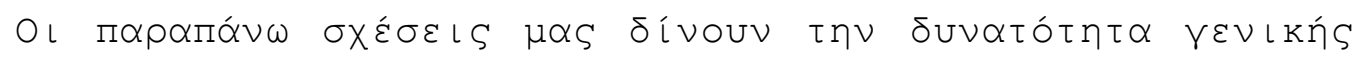

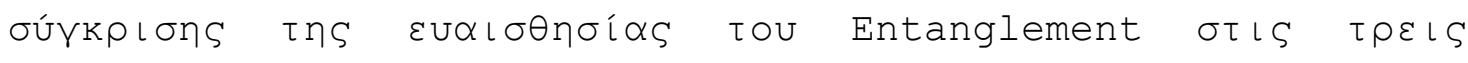

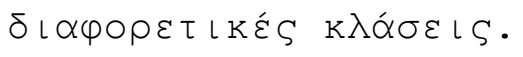

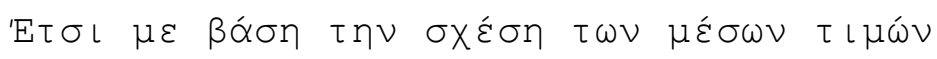

$$
\langle\Delta Q\rangle=b\left\langle\sqrt{K_{0}}\right\rangle \Delta
$$

$\dot{\varepsilon} \chi \circ \cup \mu \varepsilon$ 


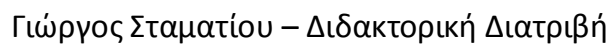

$$
\begin{aligned}
& \langle\sqrt{|K|}\rangle_{G O E} \approx \frac{1}{2} B\left(\frac{3}{4}, \frac{3}{4}\right) \sqrt{\gamma_{1}}=0.847 \sqrt{\gamma_{1}} \\
& \langle\sqrt{|K|}\rangle_{G U E} \approx \frac{2}{\pi} B\left(\frac{3}{4}, \frac{5}{4}\right) \sqrt{\gamma_{2}}=0.707 \sqrt{\gamma_{2}} \\
& \langle\sqrt{|K|}\rangle_{G S E} \approx \frac{8}{2 \pi} B\left(\frac{3}{4}, \frac{9}{4}\right) \sqrt{\gamma_{4}}=0.589 \sqrt{\gamma_{4}}
\end{aligned}
$$

о́поч ив В

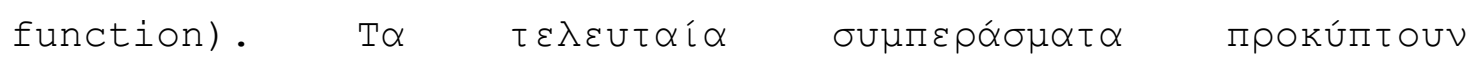

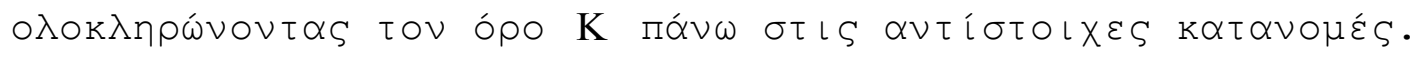

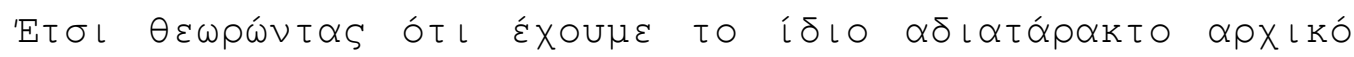

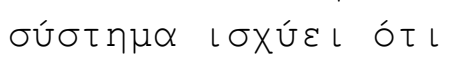

$$
\frac{\Delta Q_{G O E}}{\Delta Q_{G U E}} \approx 0.84, \quad \frac{\Delta Q_{G O E}}{\Delta Q_{G S E}} \approx 0.7
$$

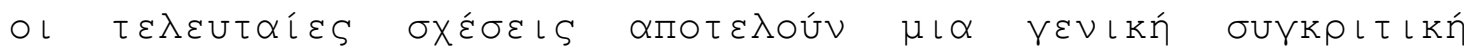

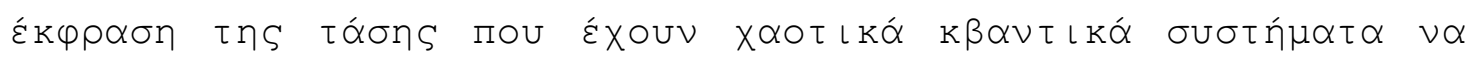

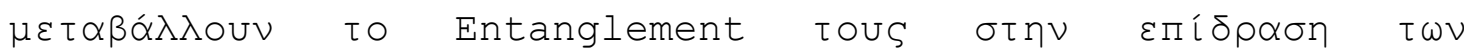

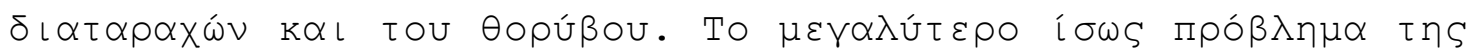

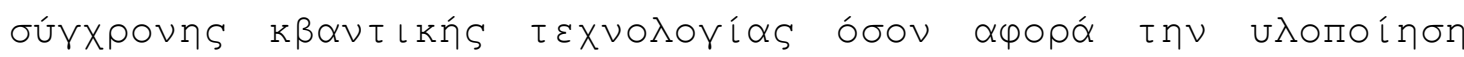

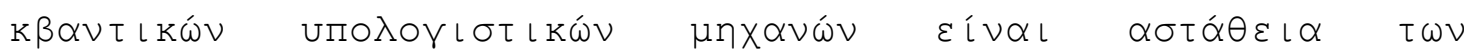

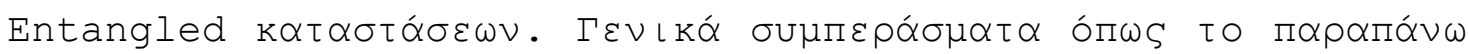

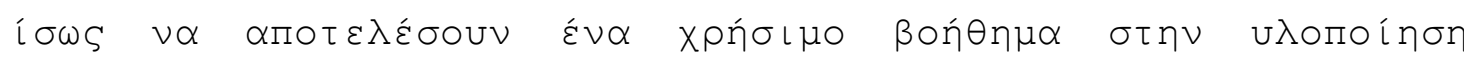

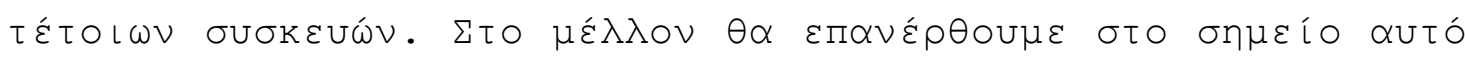

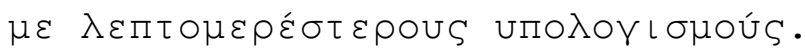

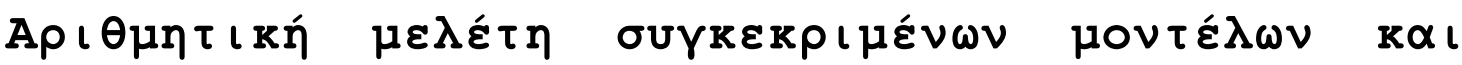

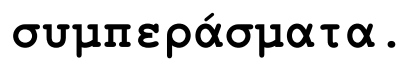

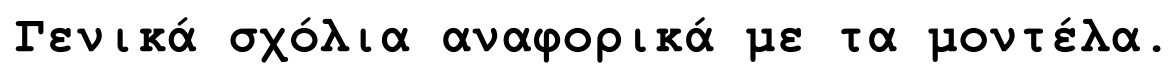

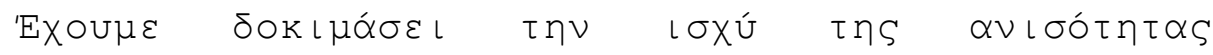

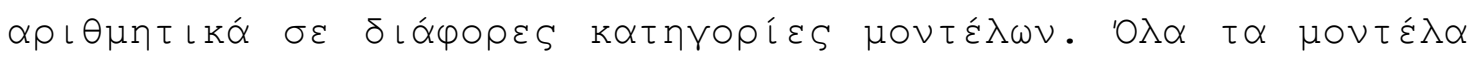

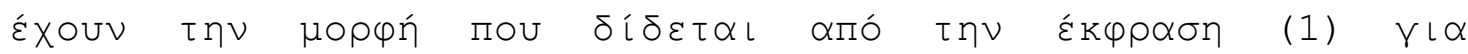

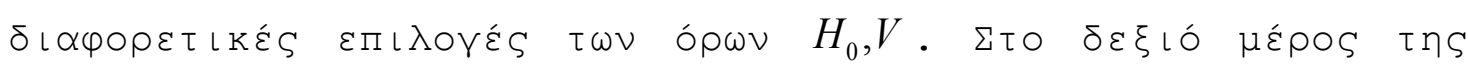




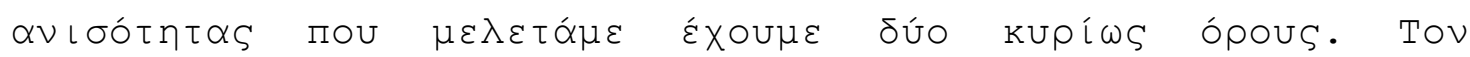

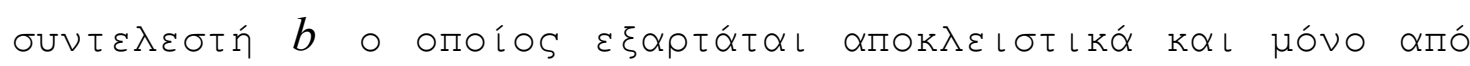

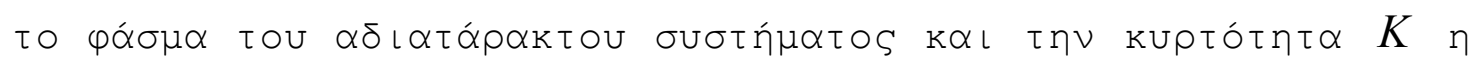

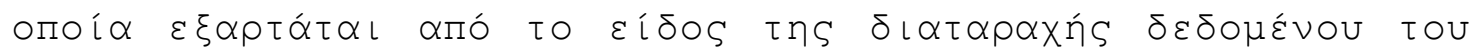

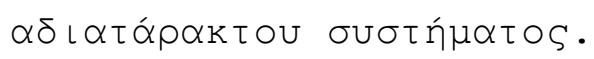

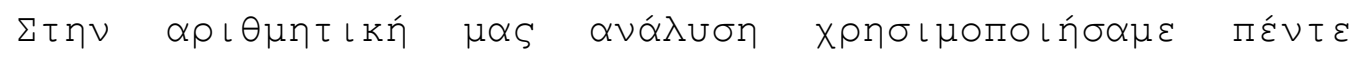

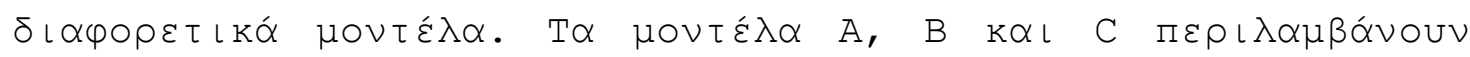

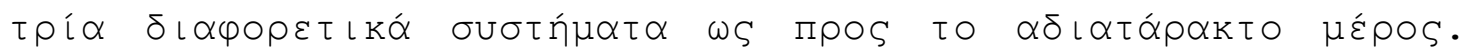

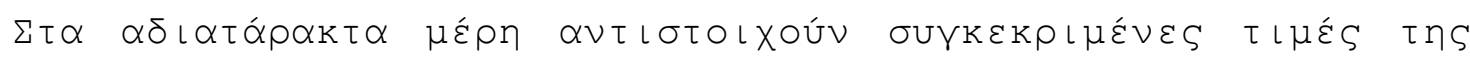

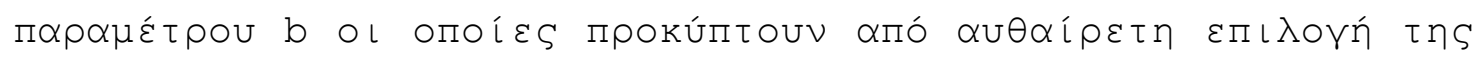

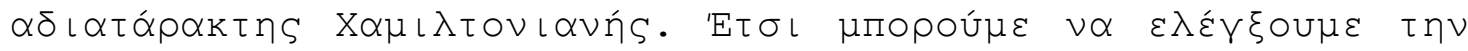

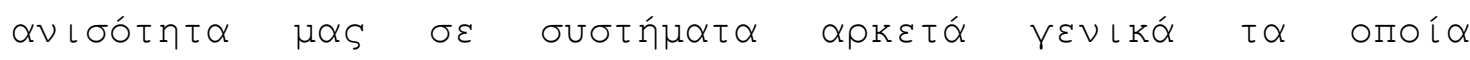

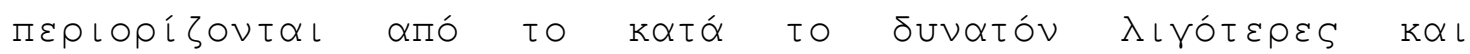

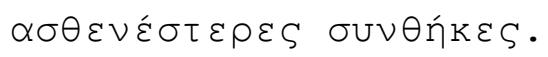

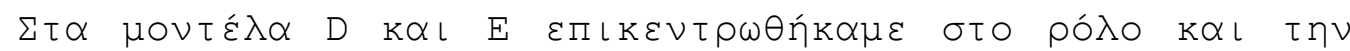

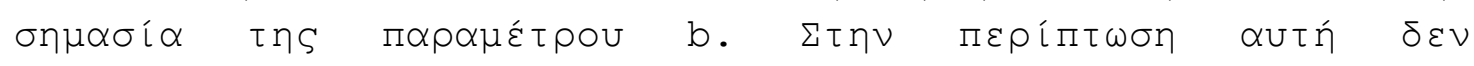

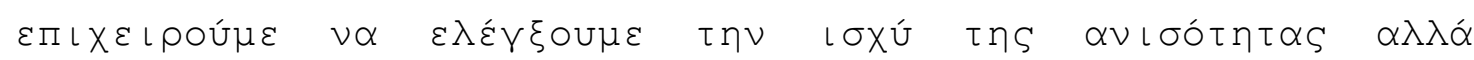

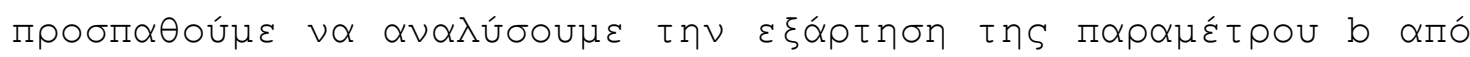

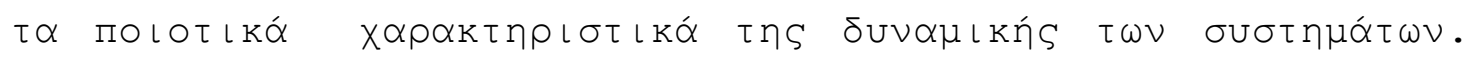

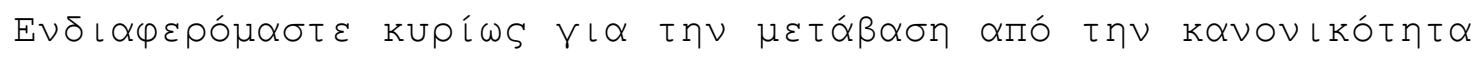

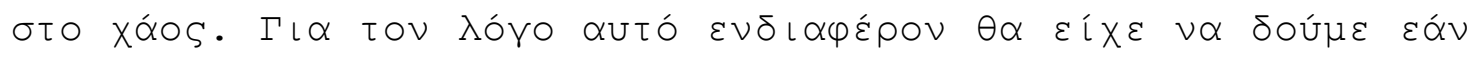

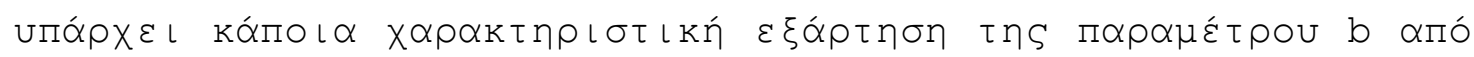

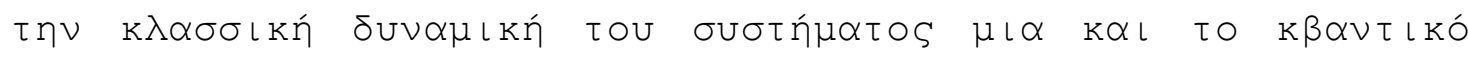

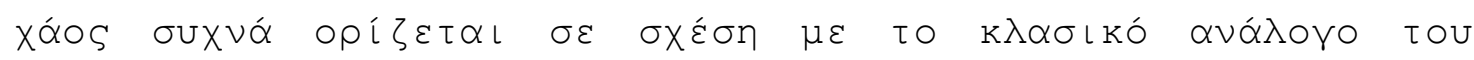

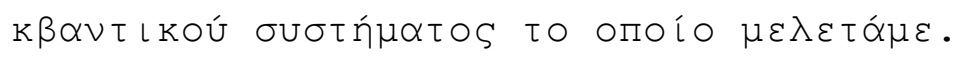

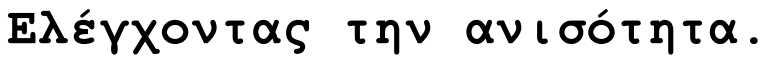

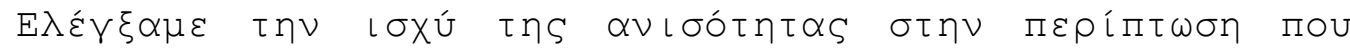

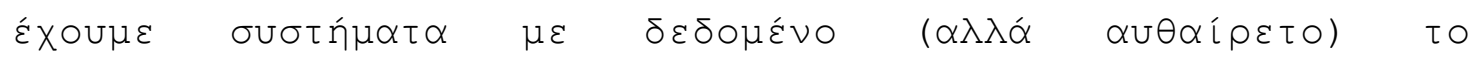

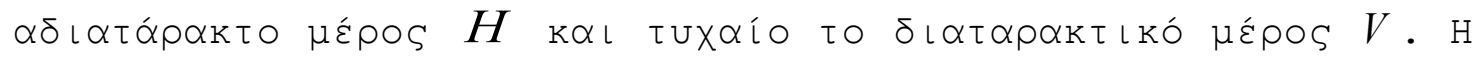

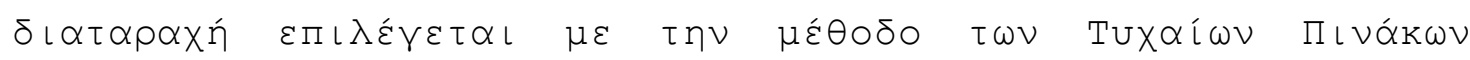

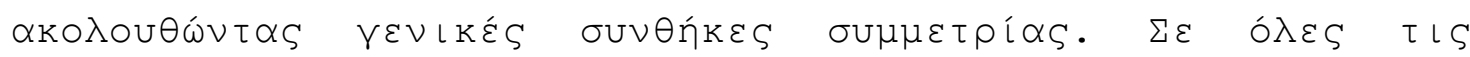

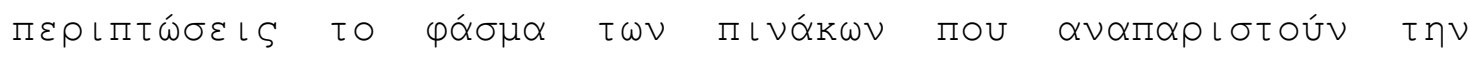

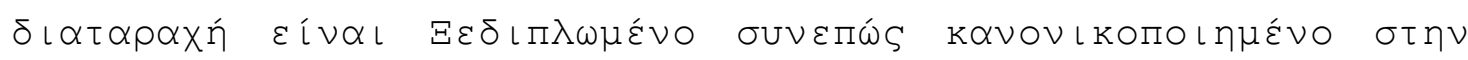
$\mu \circ \vee \alpha ́ \delta \alpha$.
} 


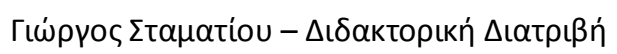

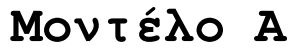

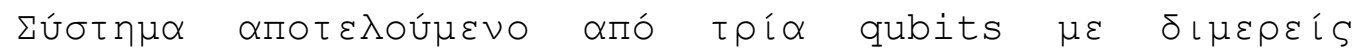

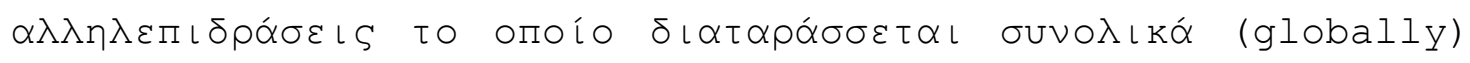

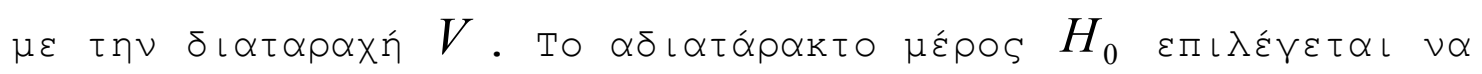

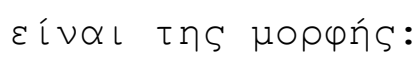

$$
H_{0}=\sum_{j=1}^{3} a_{j} \sigma_{z j}+\lambda \sum_{i<j} \overrightarrow{\sigma_{i}} \cdot \overrightarrow{\sigma_{j}}
$$

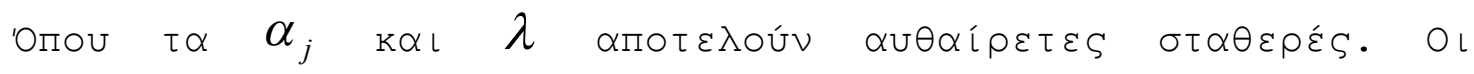

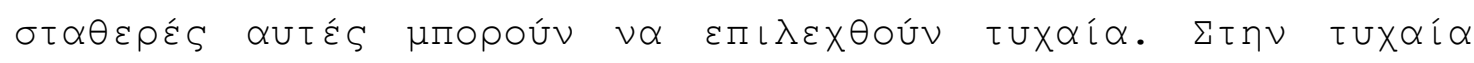

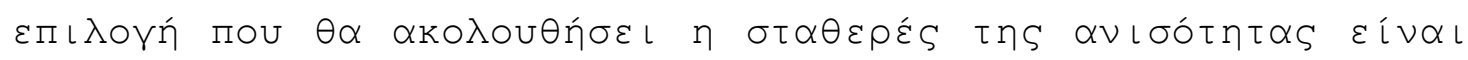
$b=9.849476$ каь $b^{\prime}=0.410395$.

н

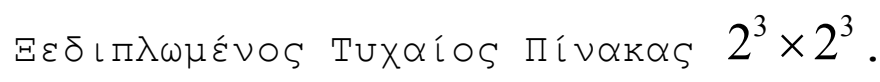

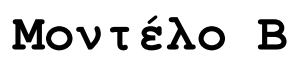

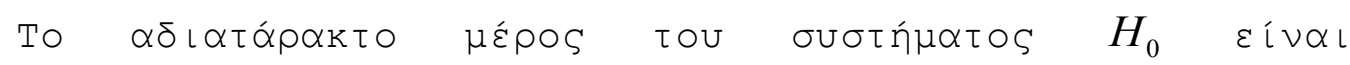

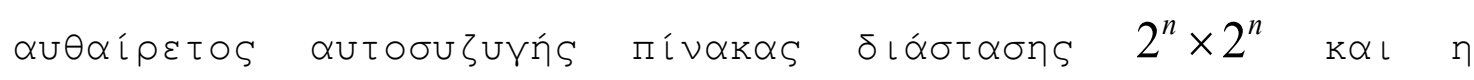

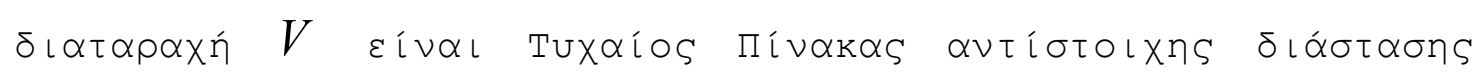

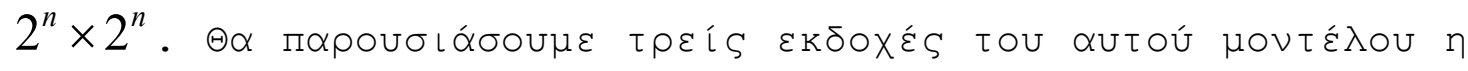

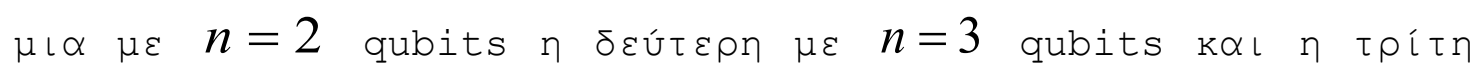

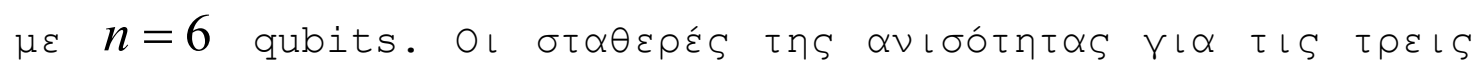

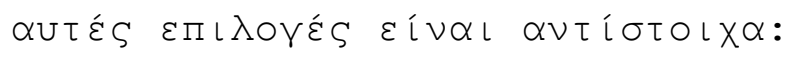

$$
\begin{aligned}
& b=11.66033, \quad b^{\prime}=1.190078 \\
& b=20.57092, b^{\prime}=0.8571215 \\
& b=38.85935, b^{\prime}=0.1431131
\end{aligned}
$$




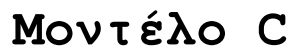

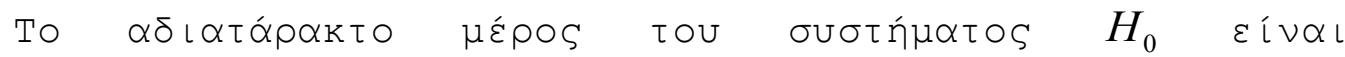

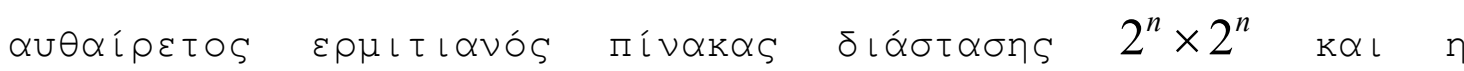

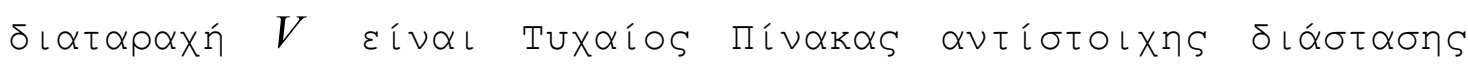

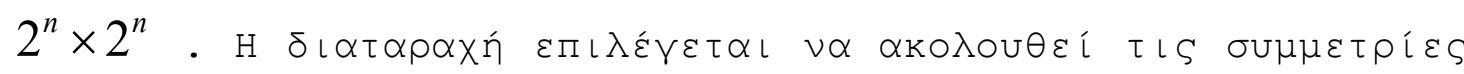

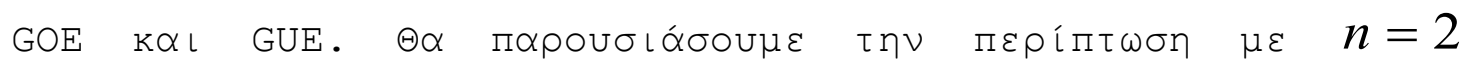
qubits.

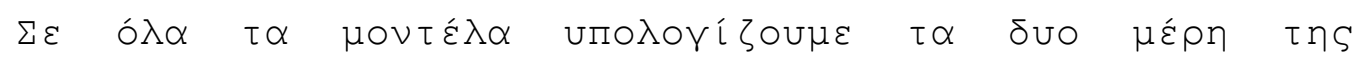

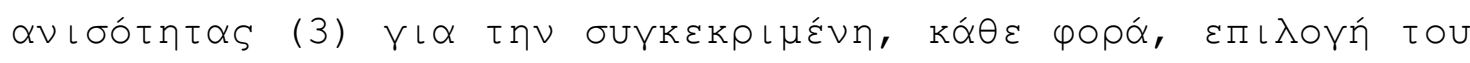

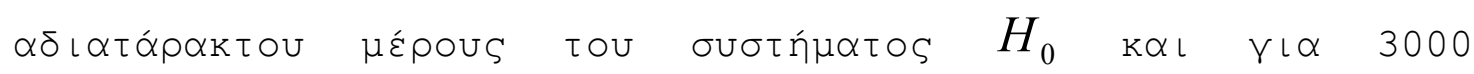

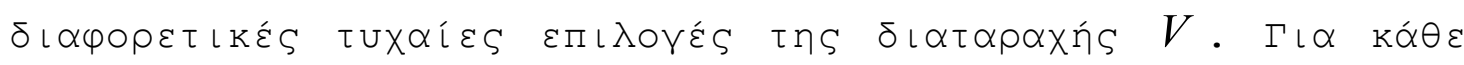

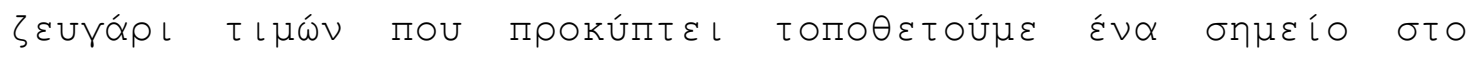

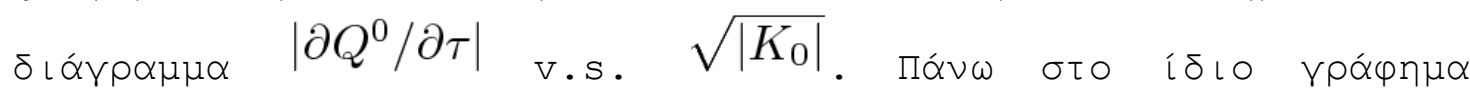

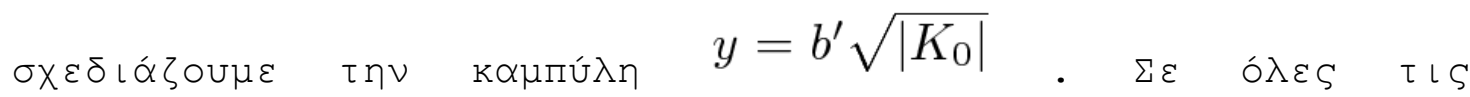

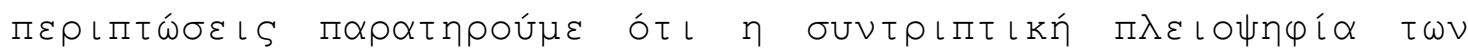

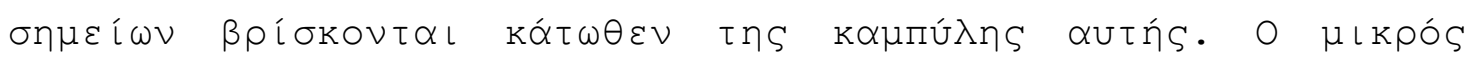

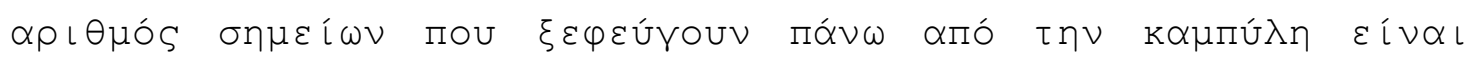

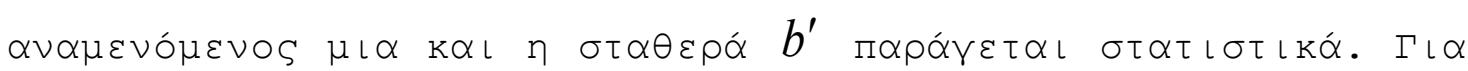

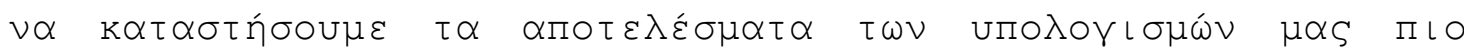

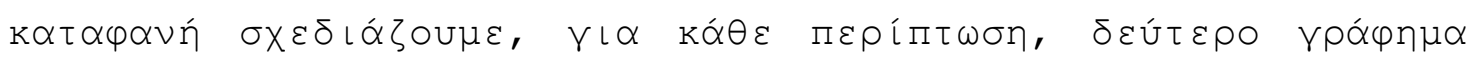

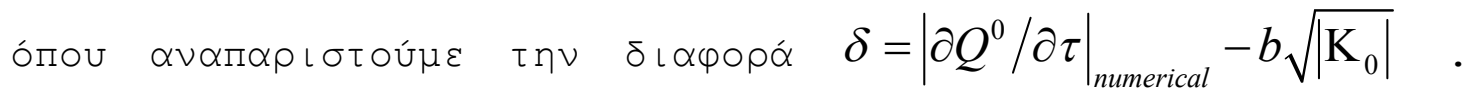

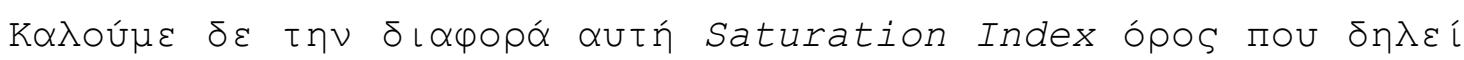

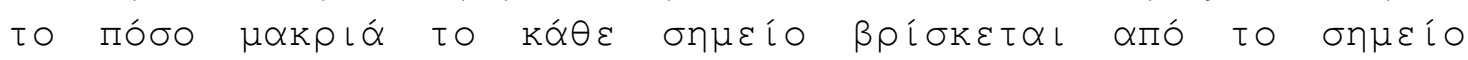

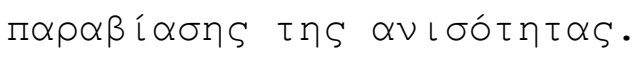

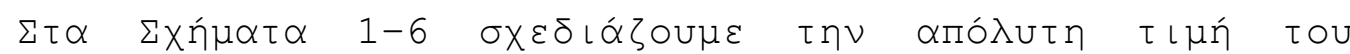

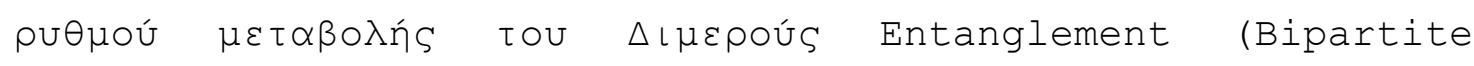

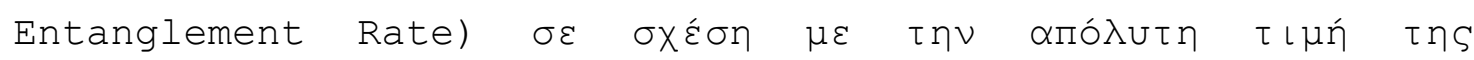

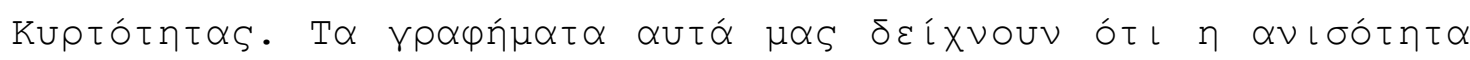

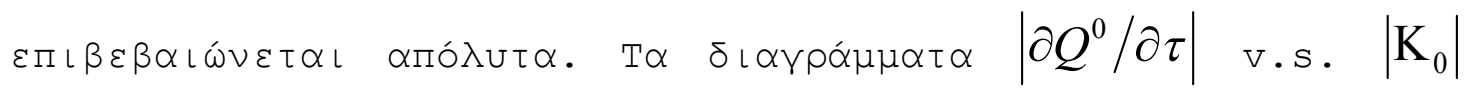




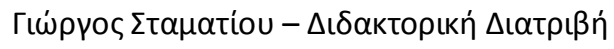

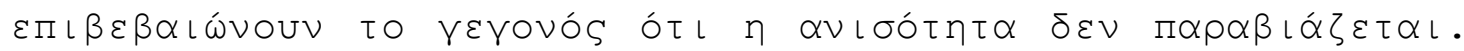

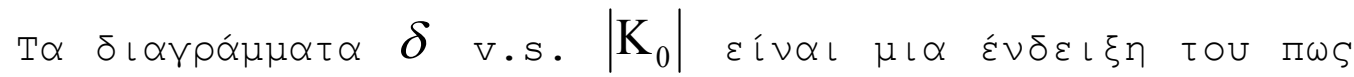

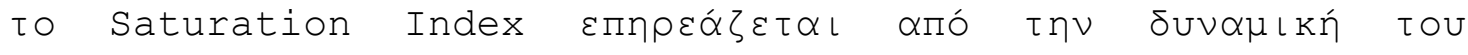

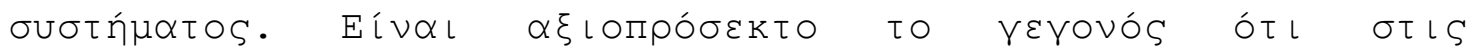

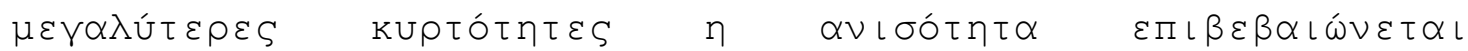
пв

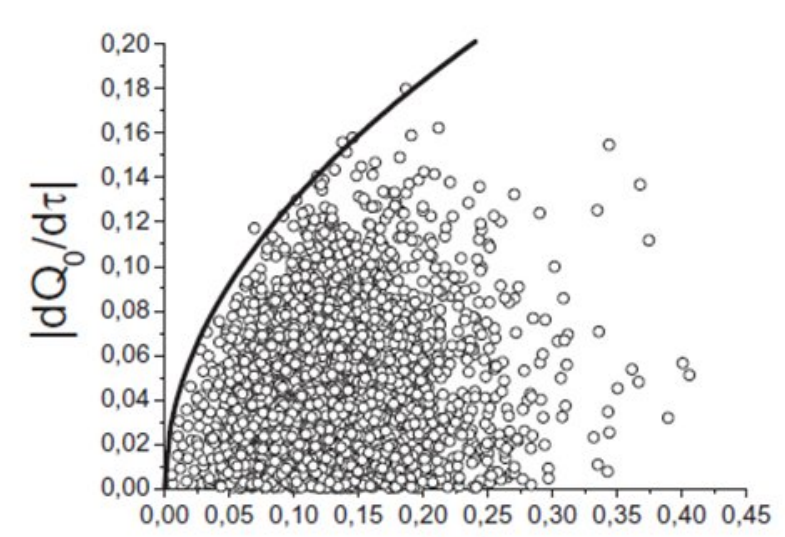

$\left|\mathrm{K}_{0}\right|$

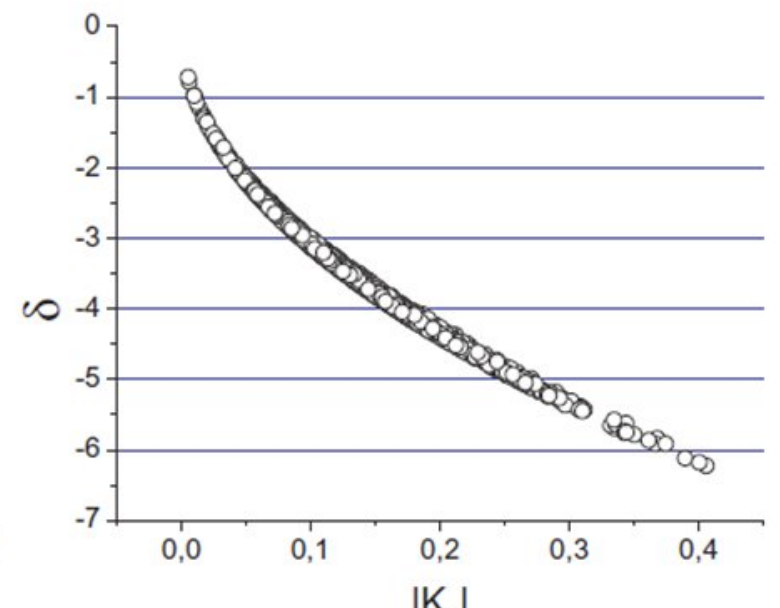

$\left|\mathrm{K}_{0}\right|$

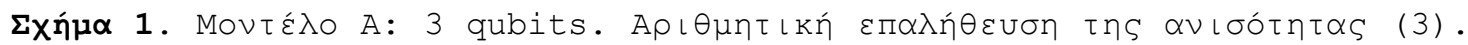

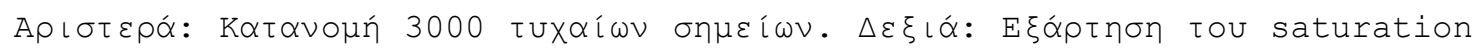

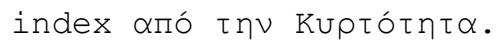



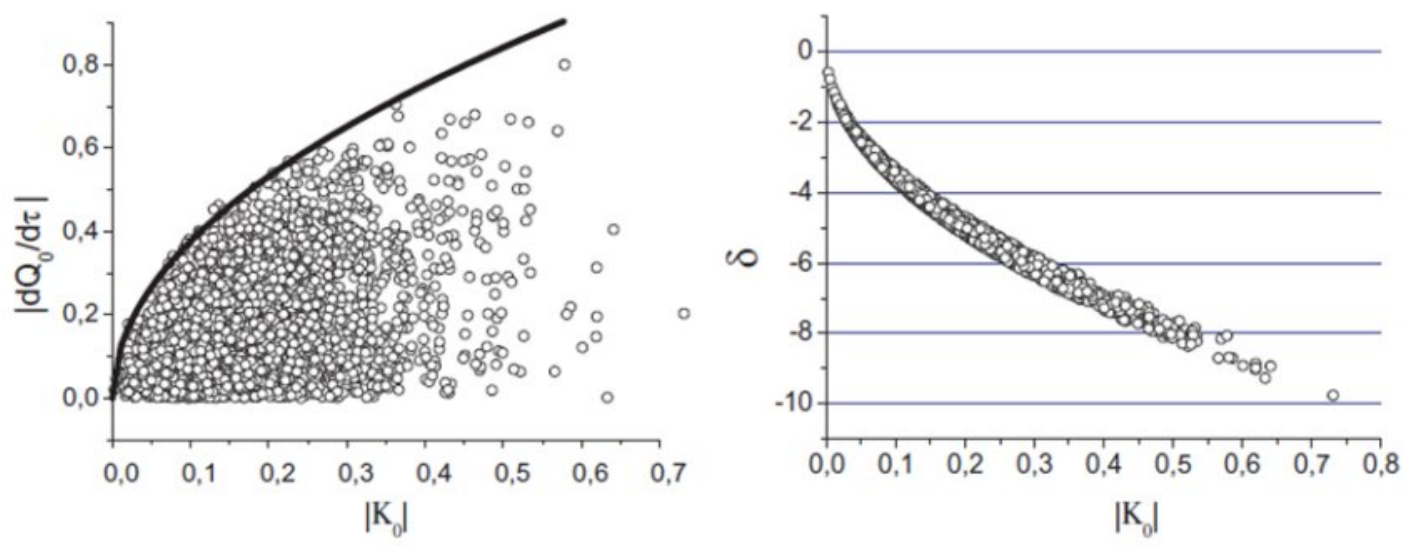

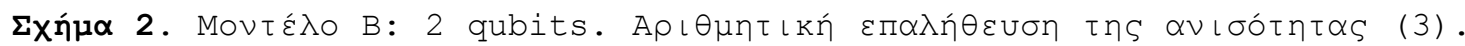

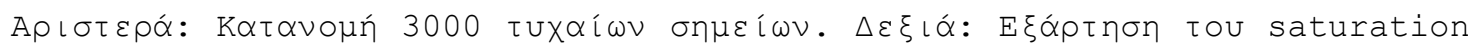

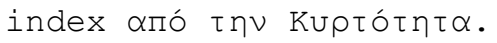
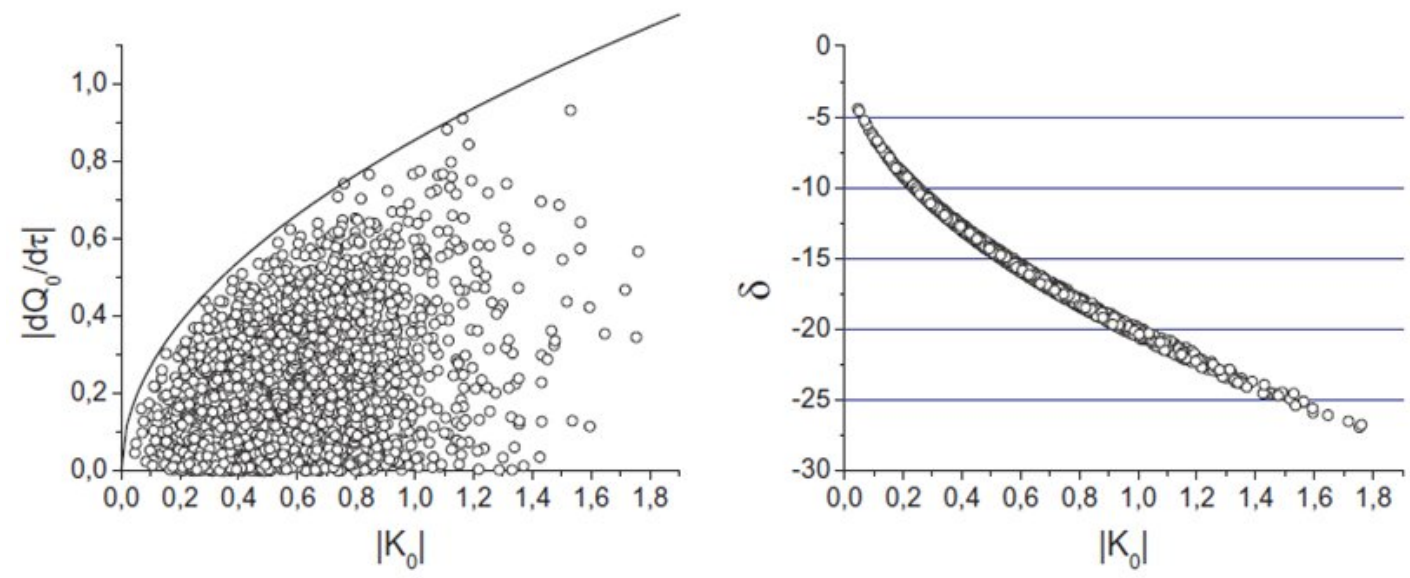

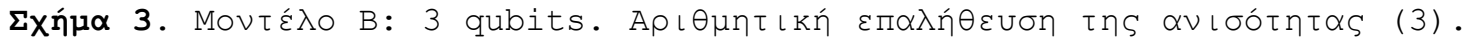

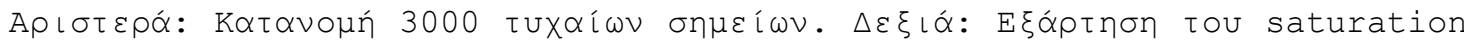

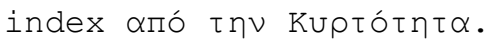



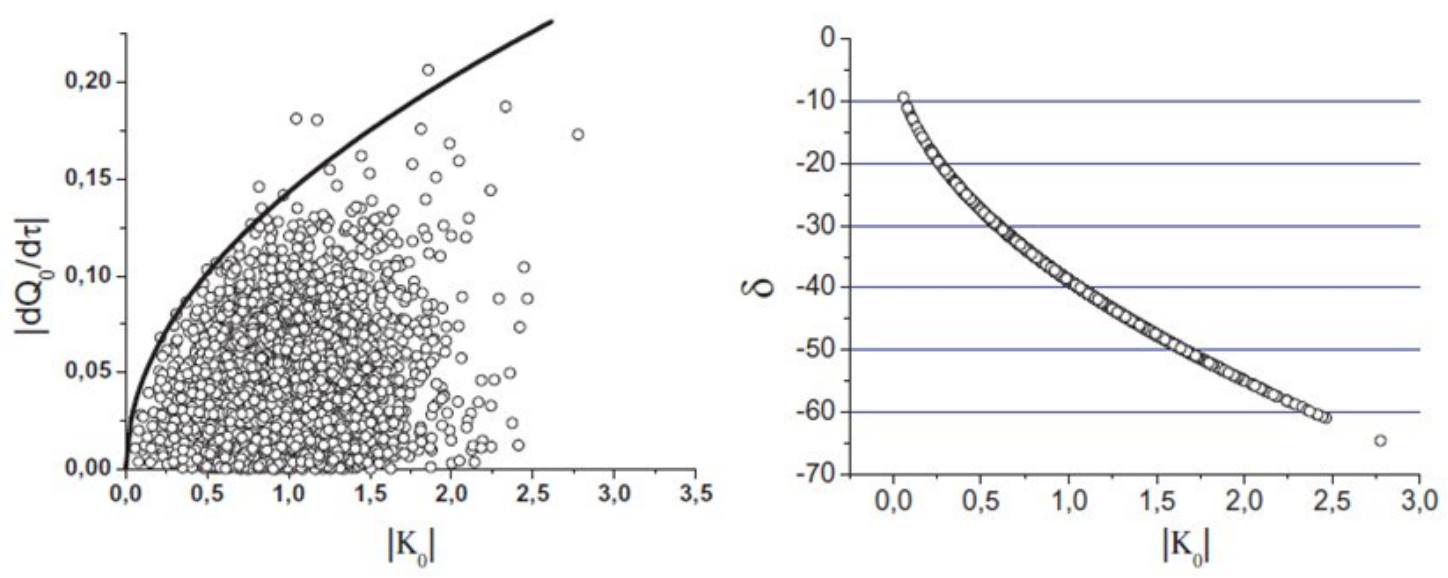

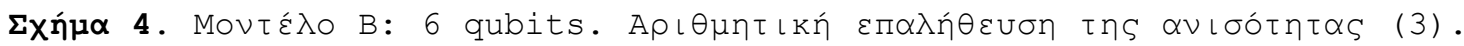

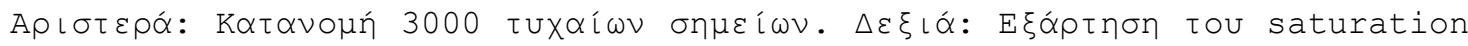

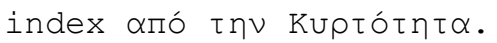
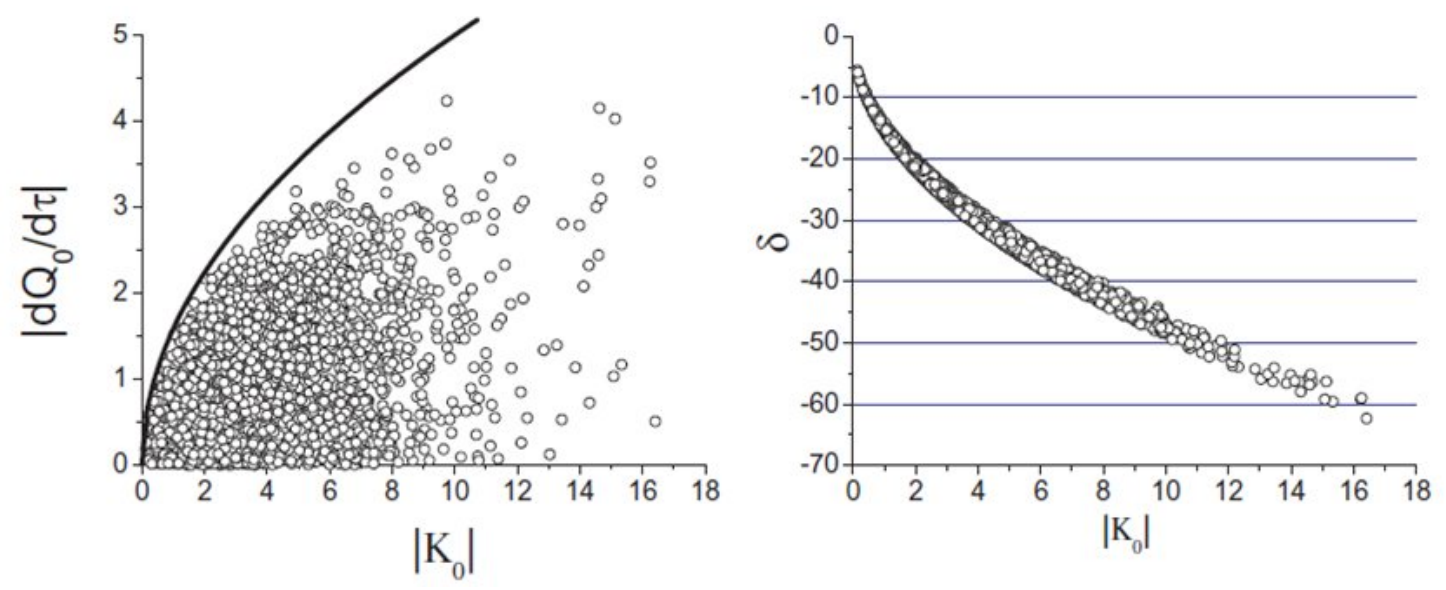

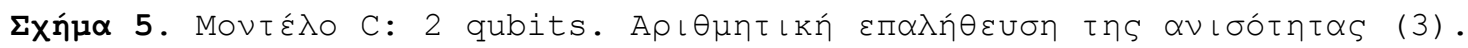

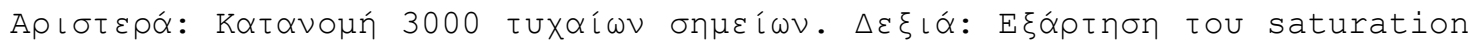

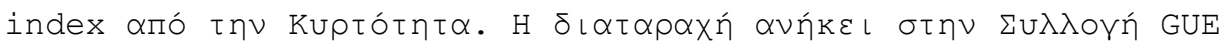



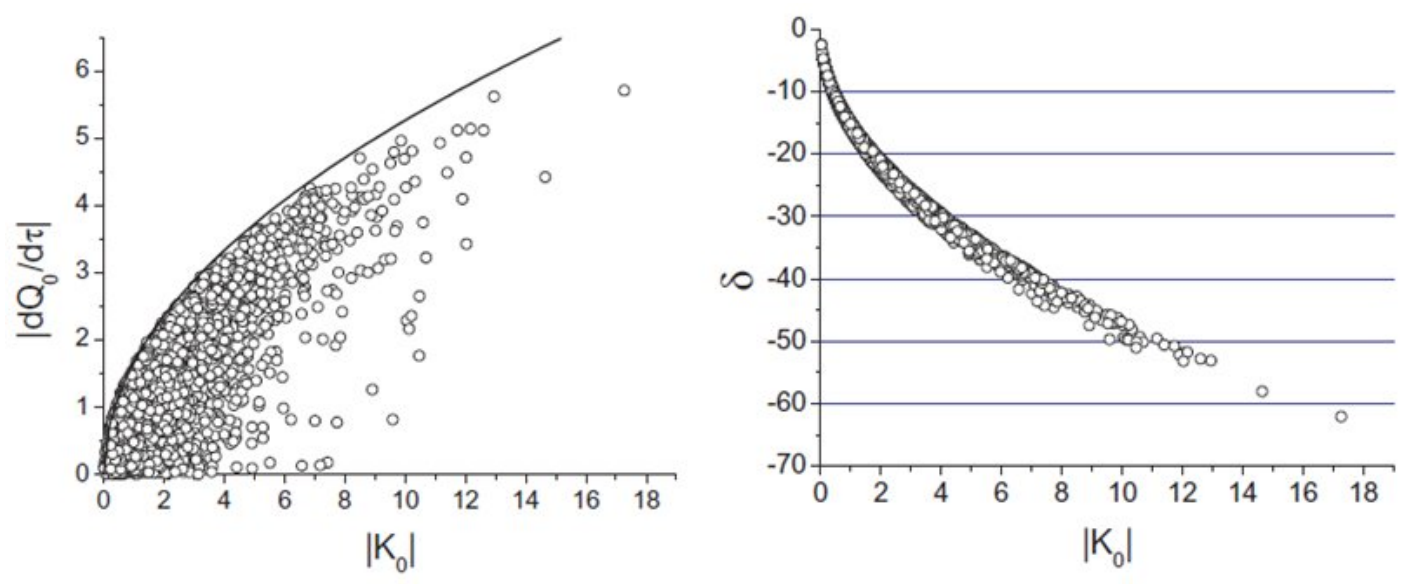

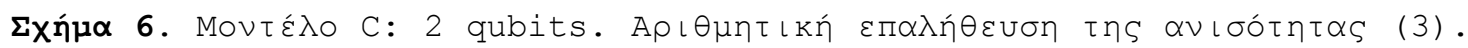

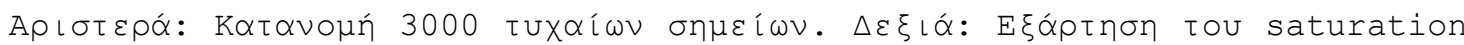

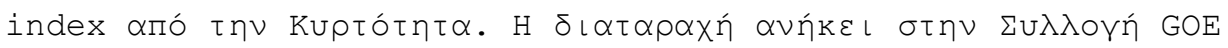

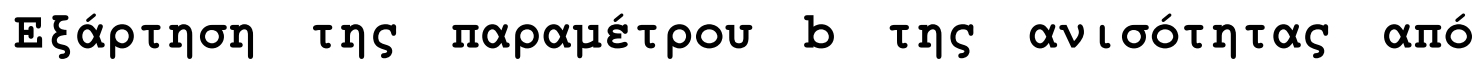

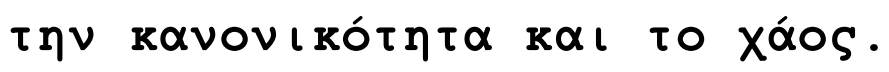

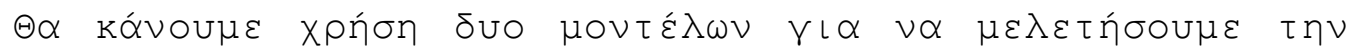

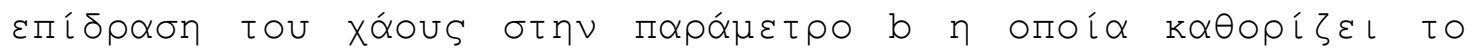

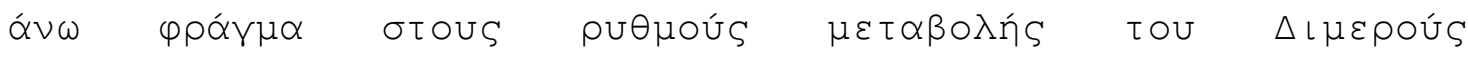

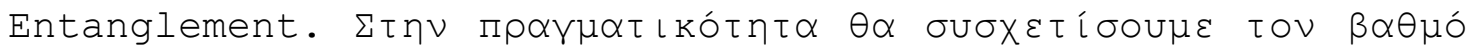

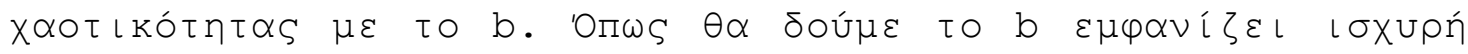

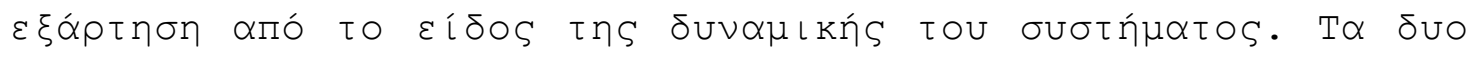

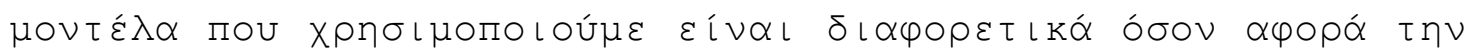

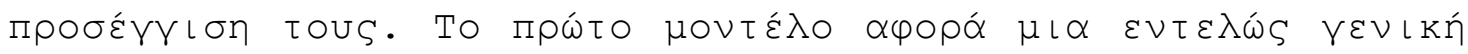

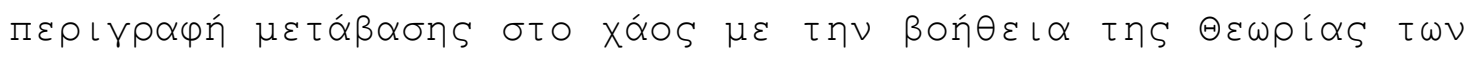

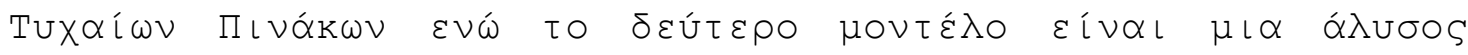

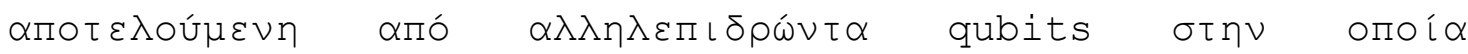

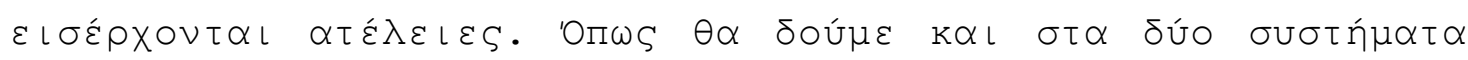

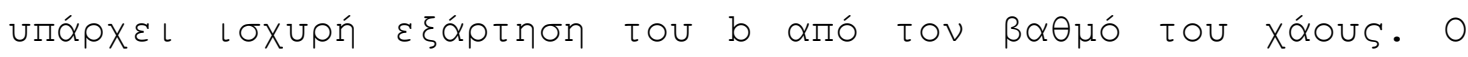

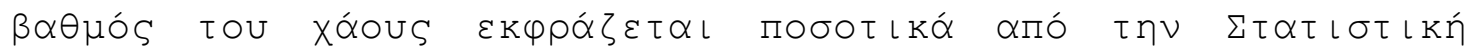

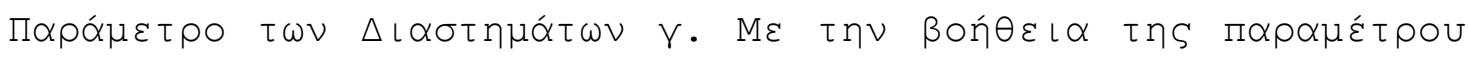

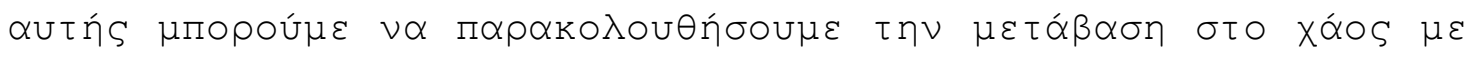
бUVEXń тро́по. 


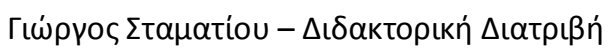

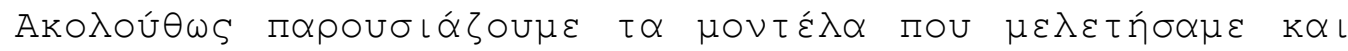

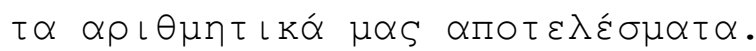

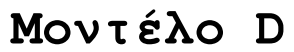

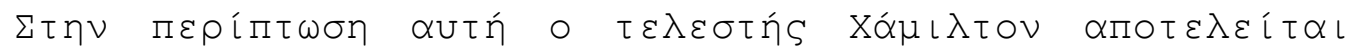

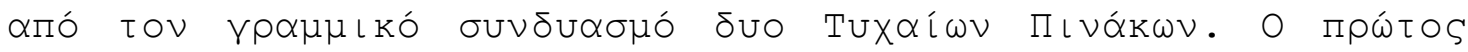

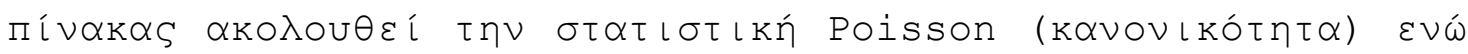

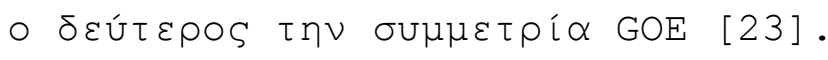

$$
H(\theta)=\cos (\theta) H_{P}+\sin (\theta) H_{W}
$$

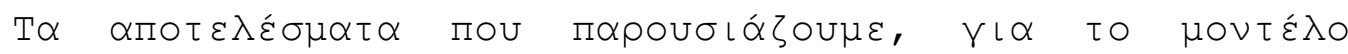

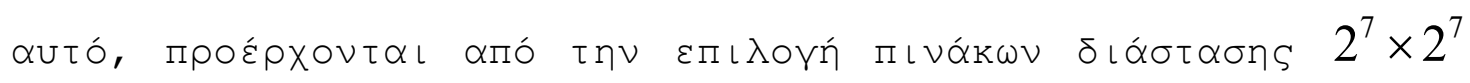

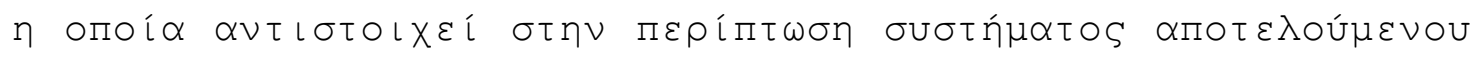

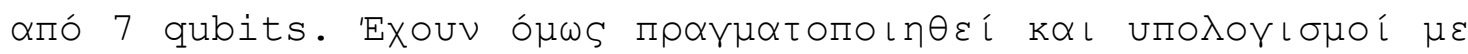

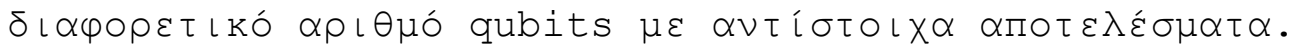

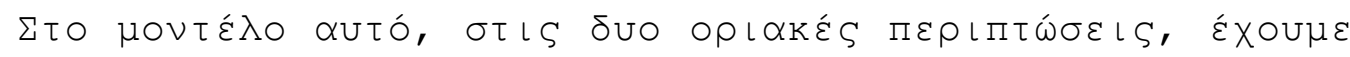

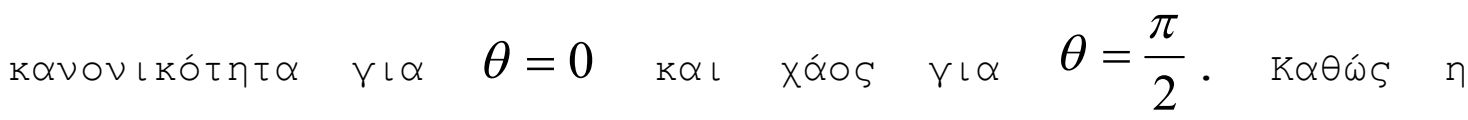

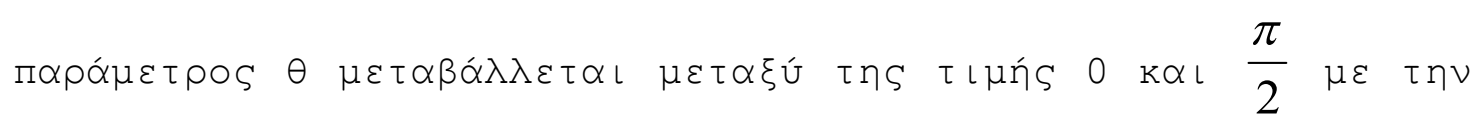

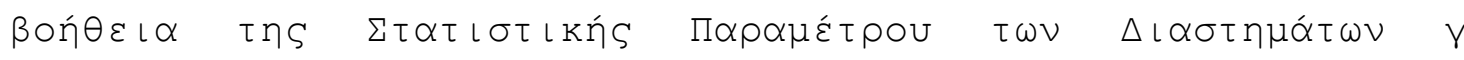

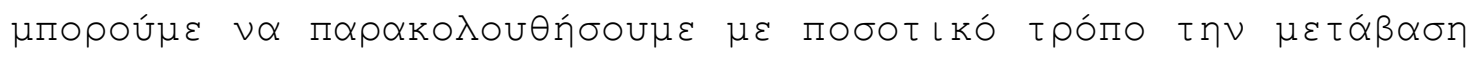

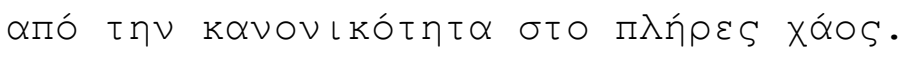

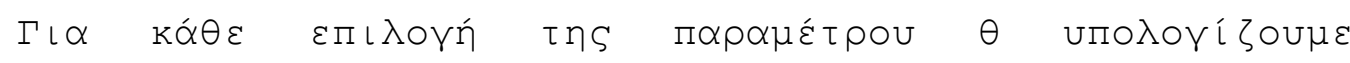

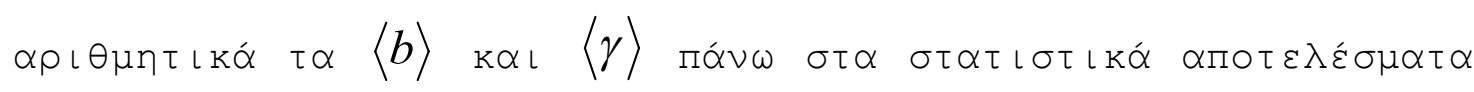

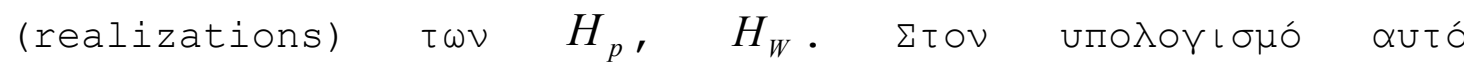

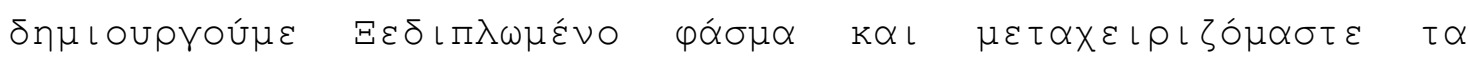

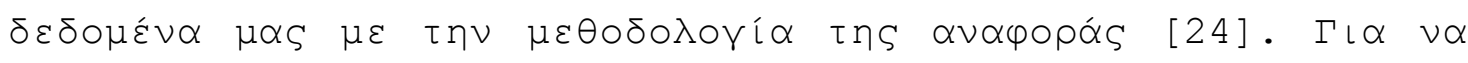

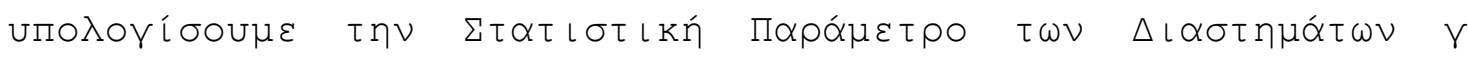

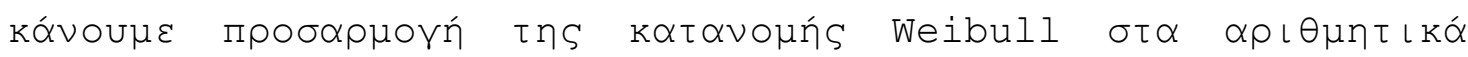
$\delta \varepsilon \delta \circ \mu \varepsilon \dot{\varepsilon} \vee \alpha$. 


$$
y_{a, c}(x)=a c x^{c-1} \exp \left(-a x^{c}\right), \quad x \in[0,+\infty]
$$

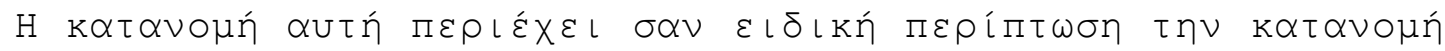

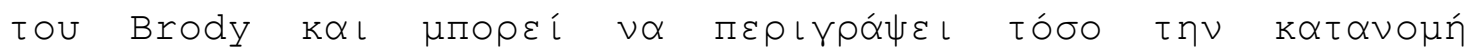

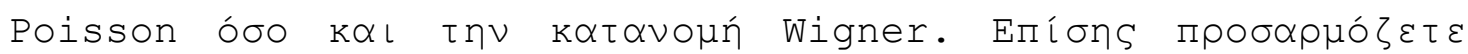

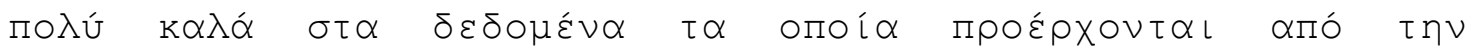

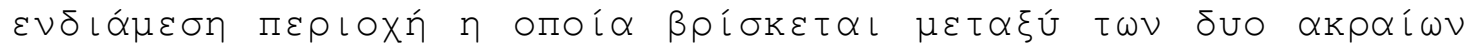

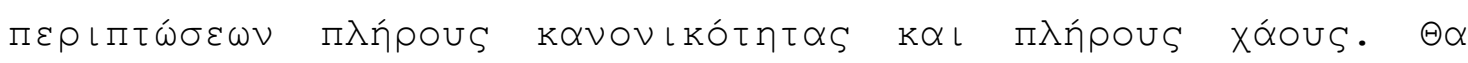

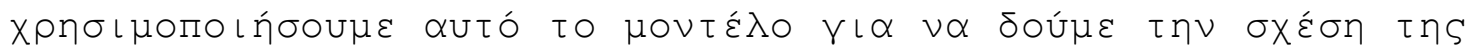

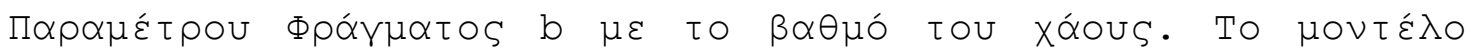

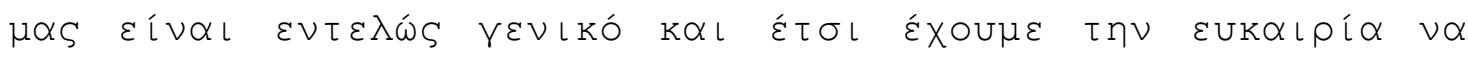

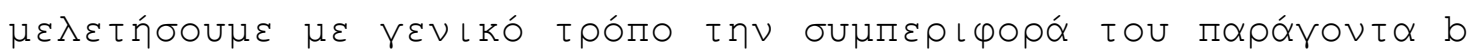

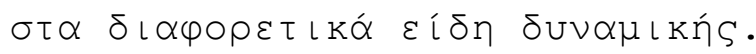

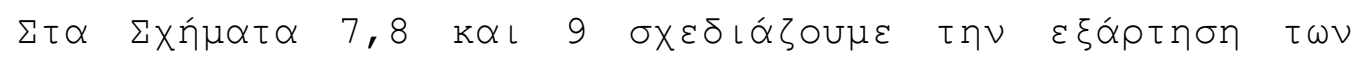

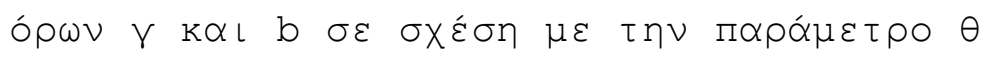

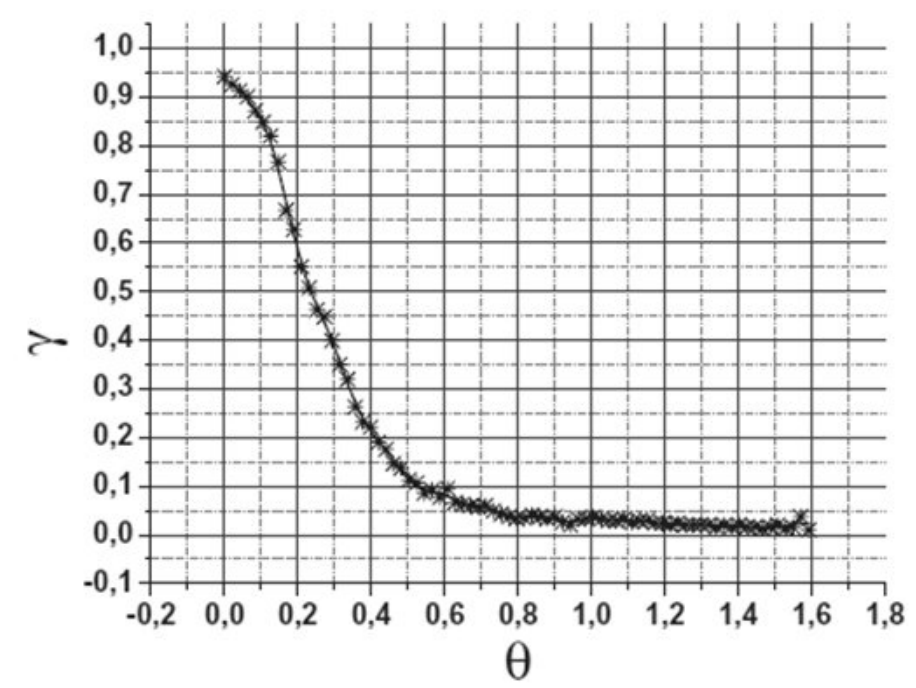

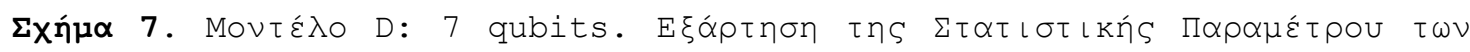

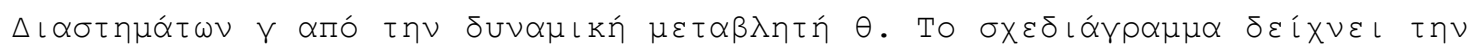

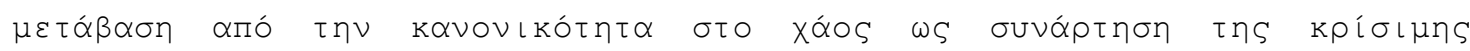

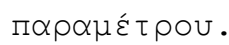




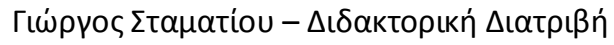

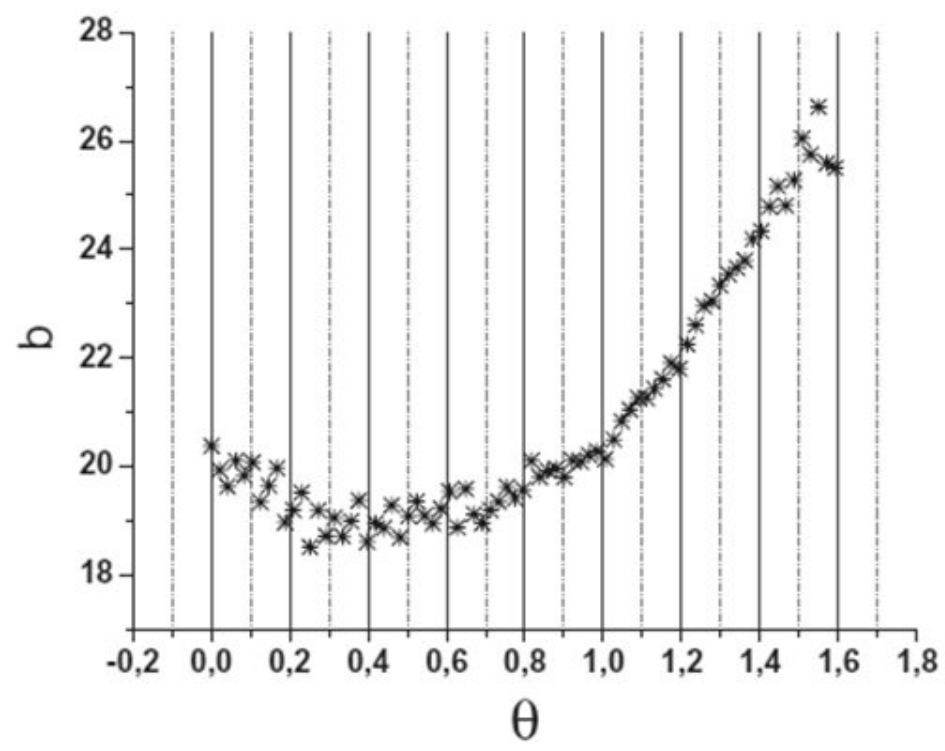

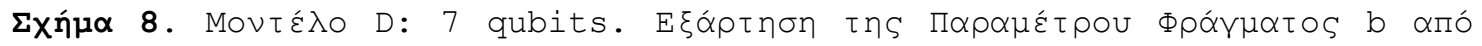

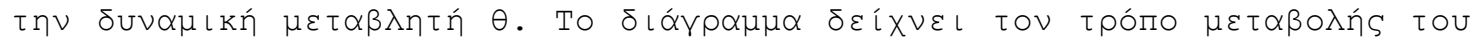

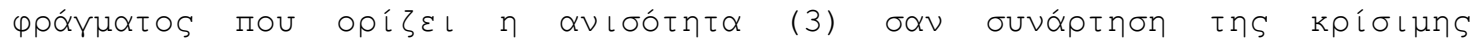
$\Pi \alpha \rho \alpha \mu \varepsilon \dot{\tau} \tau \circ \mathrm{U}$.

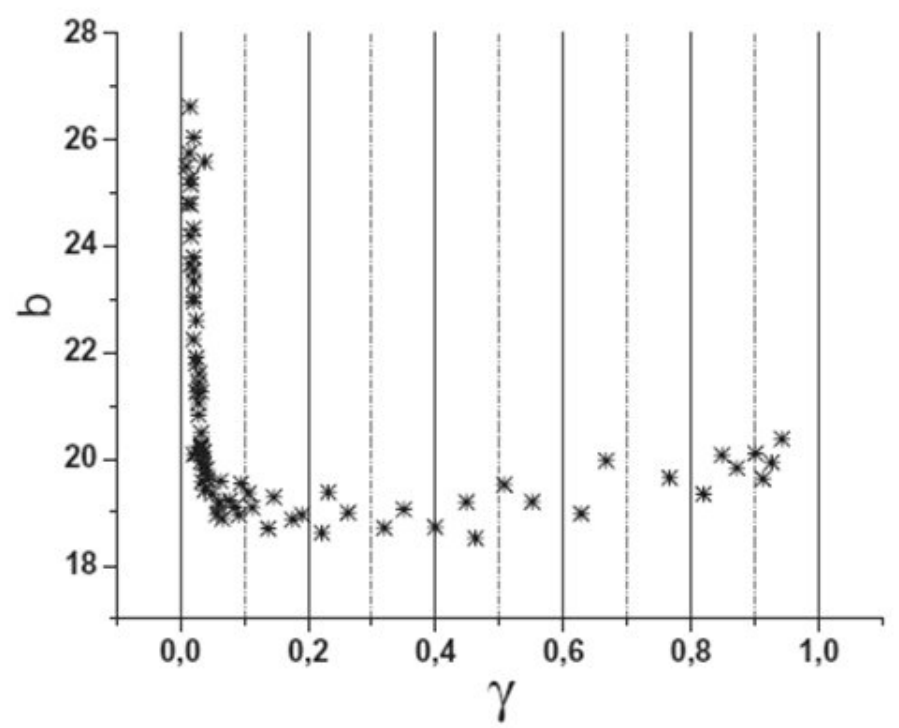

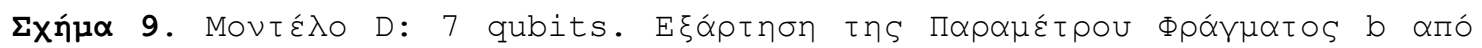

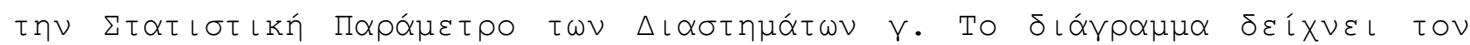

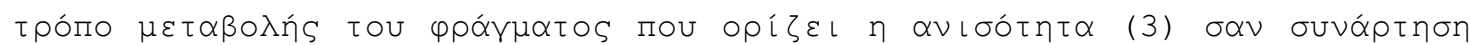

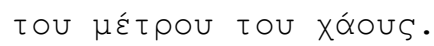




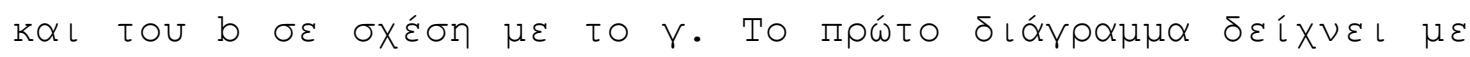

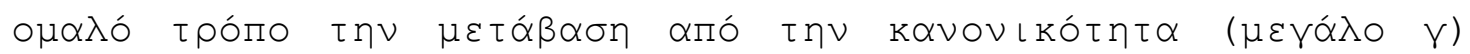

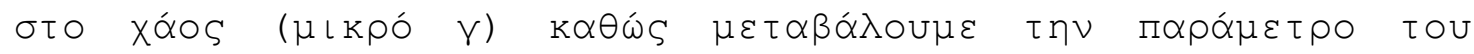

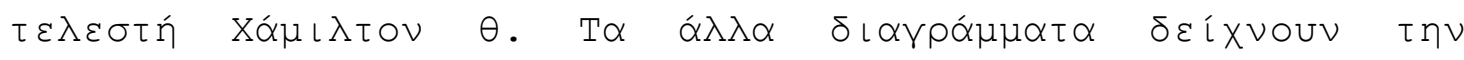

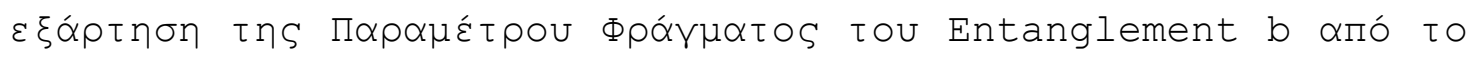

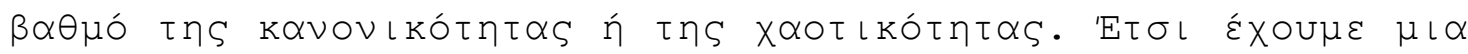

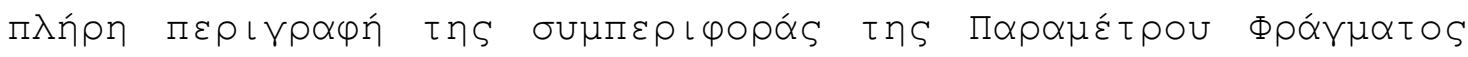

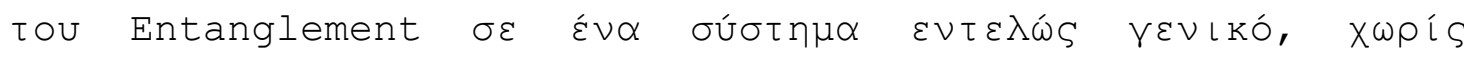

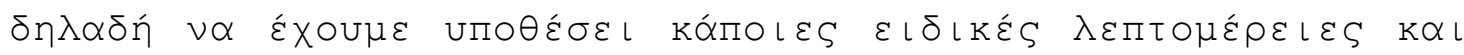

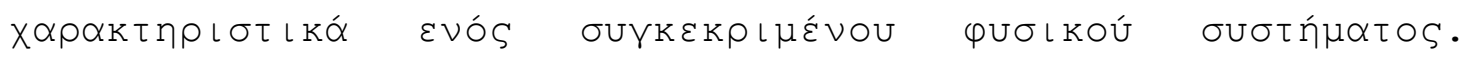

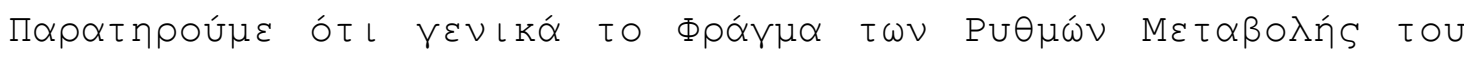

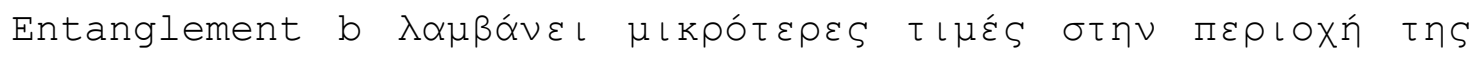

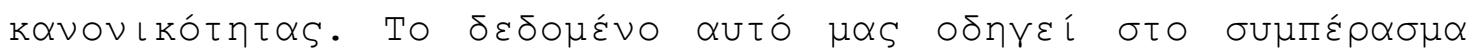

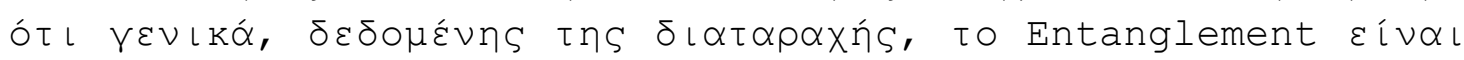

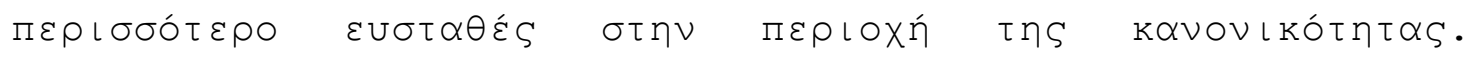

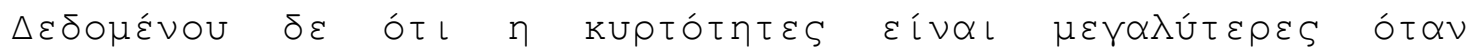

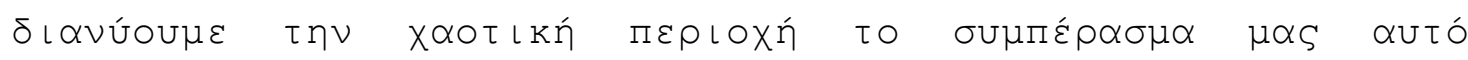
$\varepsilon \vee\llcorner\sigma X U ́ \varepsilon \tau \alpha \iota$.

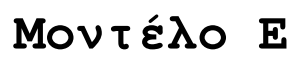

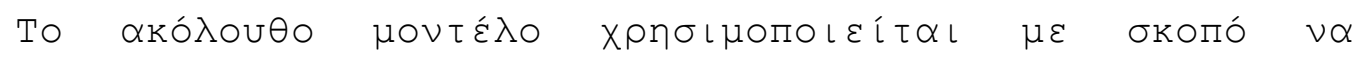

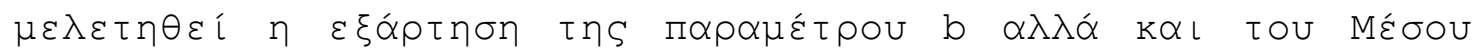

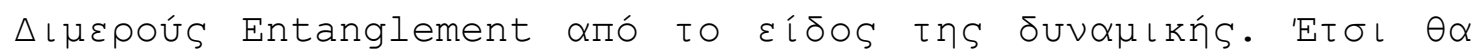

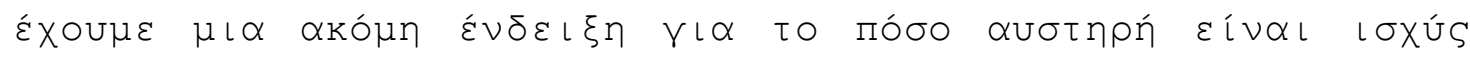

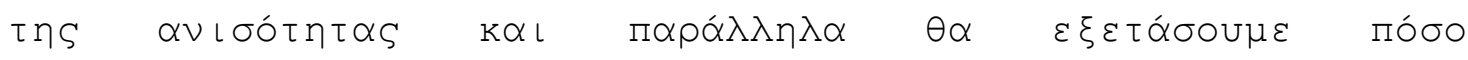

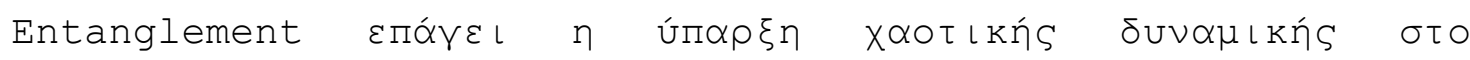
$\sigma \dot{\sigma} \sigma \tau \eta \mu \alpha$.

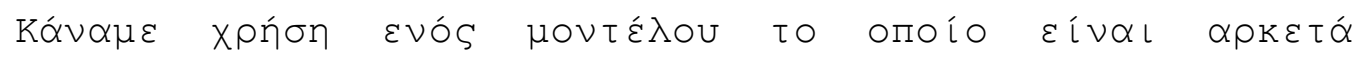

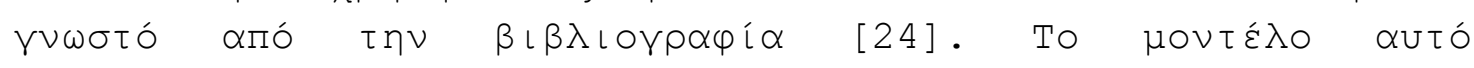

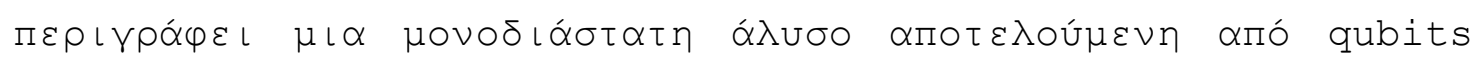

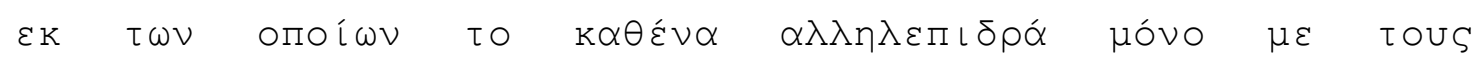

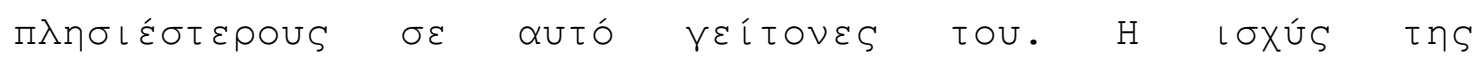

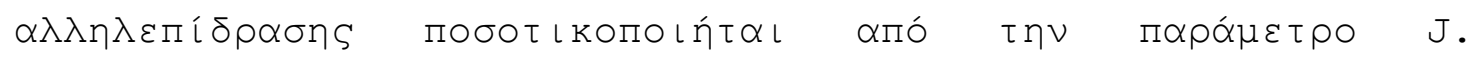

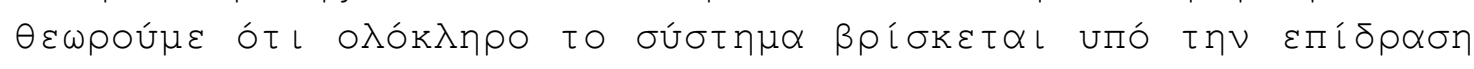




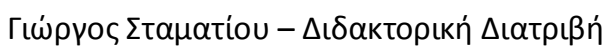

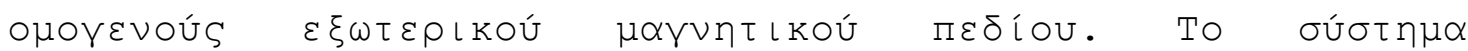

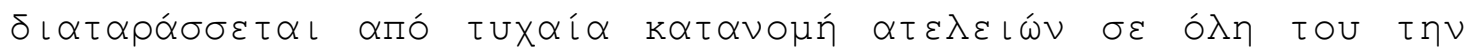

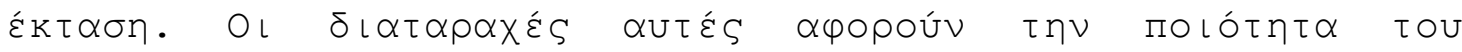

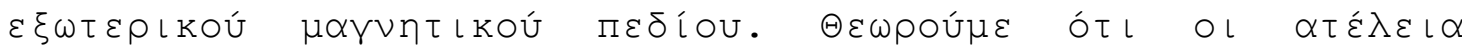

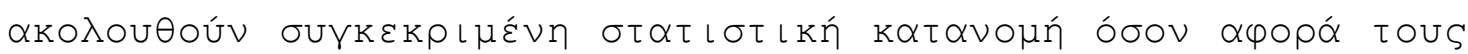

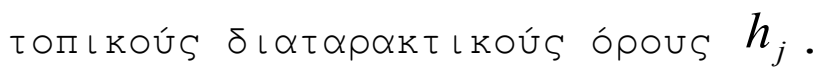

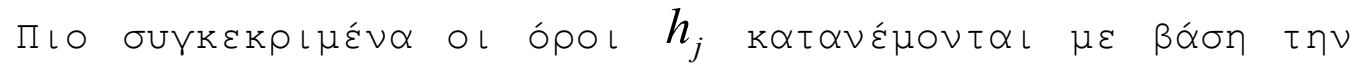

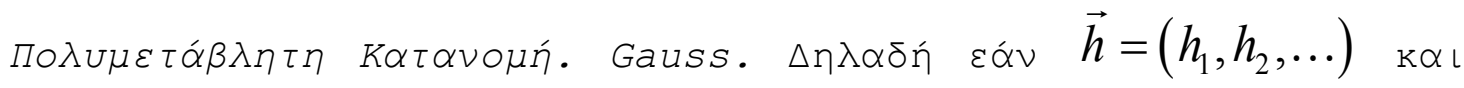

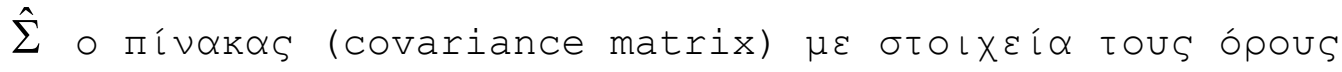

$$
\Sigma_{i j}=\operatorname{cov}\left(h_{i}, h_{j}\right)=\left\langle\left(h_{i}-\left\langle h_{i}\right\rangle\right)\left(h_{j}-\left\langle h_{j}\right\rangle\right)\right\rangle
$$

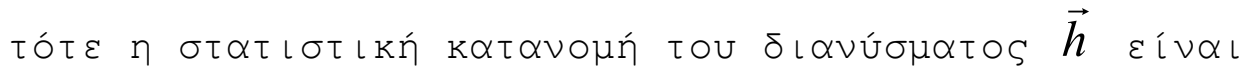

$$
\frac{1}{(2 \pi)^{k / 2} \sqrt{\operatorname{det}(\hat{\Sigma})}} \exp \left(-\frac{1}{2}(\vec{h}-\langle\vec{h}\rangle)^{\prime} \hat{\Sigma}^{-1}(\vec{h}-\langle\vec{h}\rangle)\right)
$$

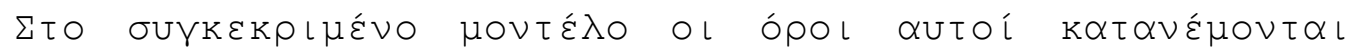

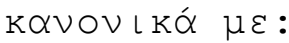

$$
\begin{gathered}
\left\langle h_{j}\right\rangle=0, \quad\left\langle h_{i} h_{j}\right\rangle=d^{2} \delta_{i j} \\
H=\sum_{j=1}^{N}\left(h+h_{j}\right) \sigma_{z j}+\frac{J}{4} \sum_{j=1}^{N-1} \overrightarrow{\sigma_{i}} \cdot \overrightarrow{\sigma_{j}}
\end{gathered}
$$

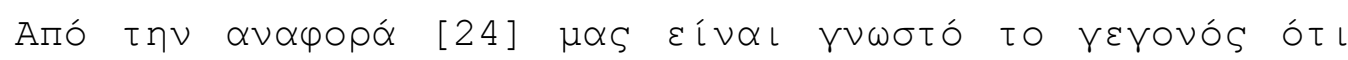

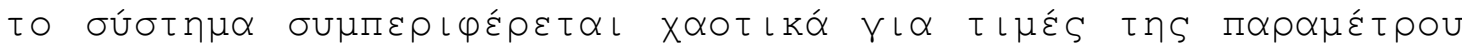

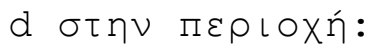

$$
0.1 \leq d \leq 0.5
$$




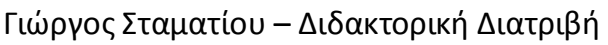

$\varepsilon \alpha ́ v$

$$
J=1
$$

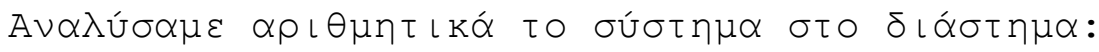

$$
0 \leq d \leq 2.5
$$

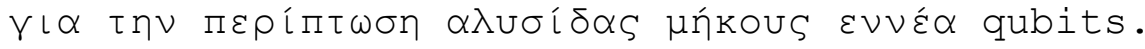

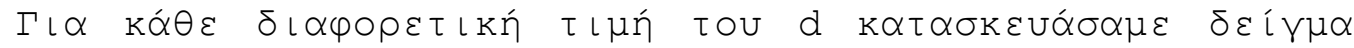

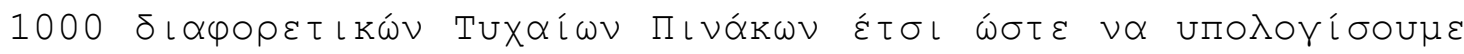

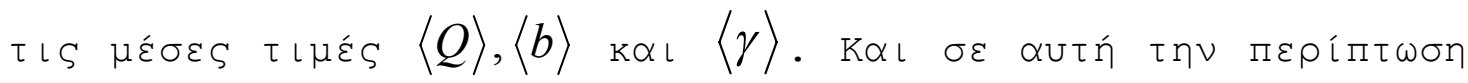

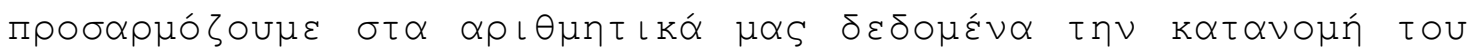

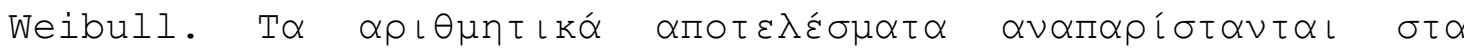

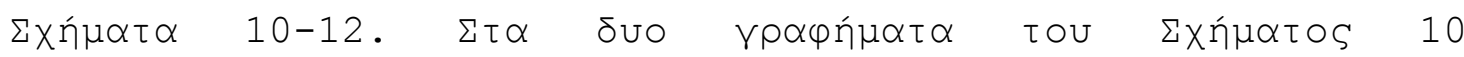

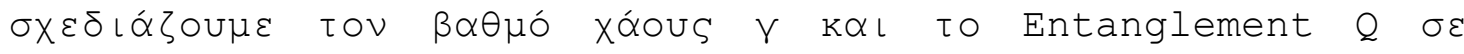

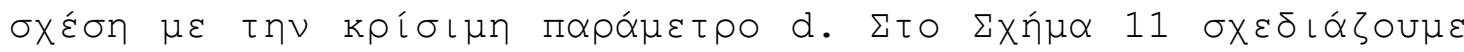

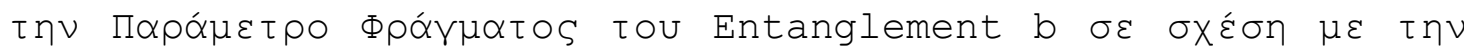

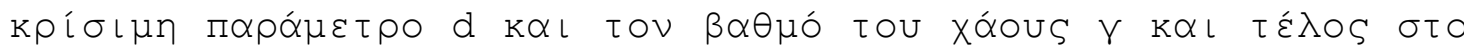

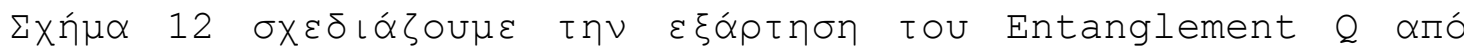

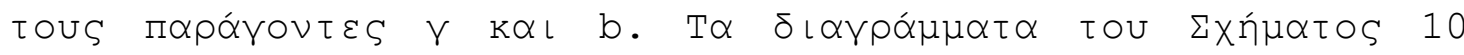

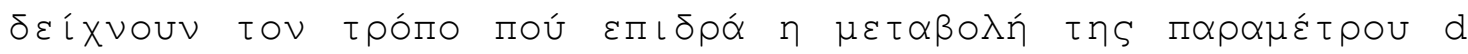

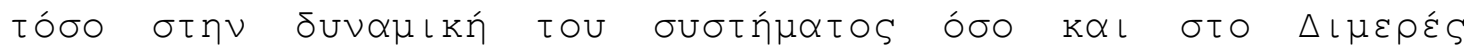

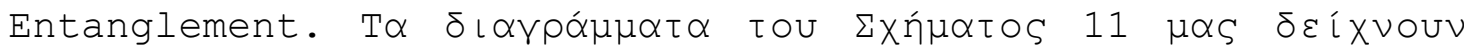

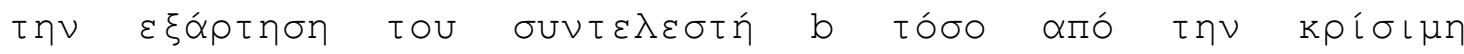

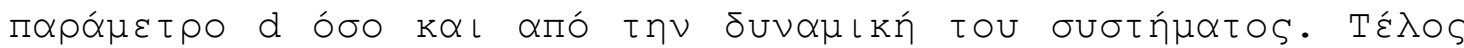

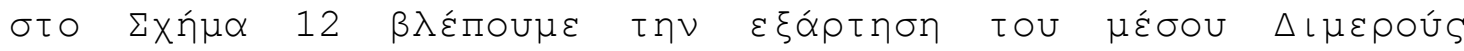

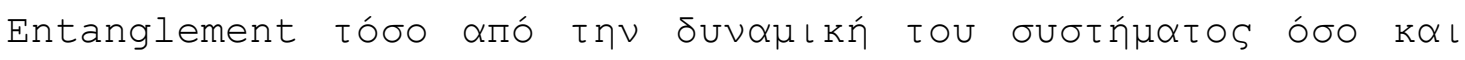

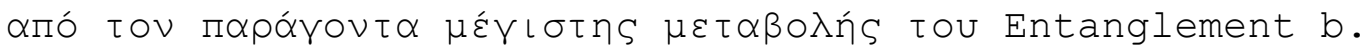

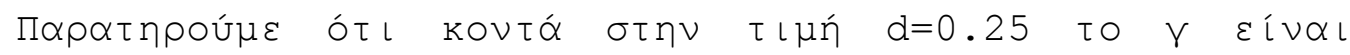

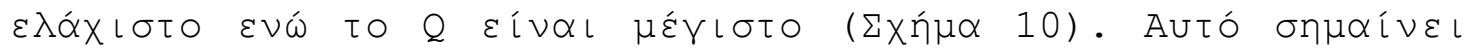
ót

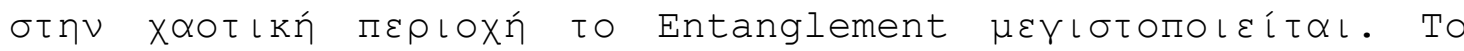

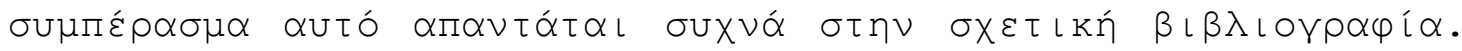

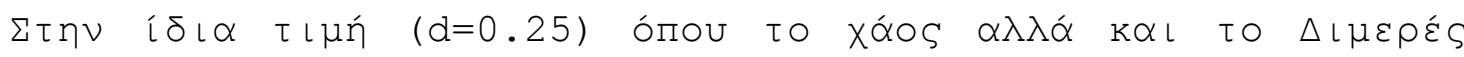

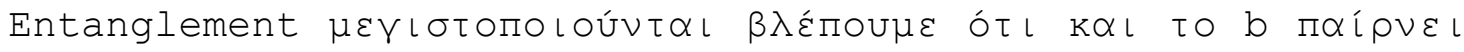

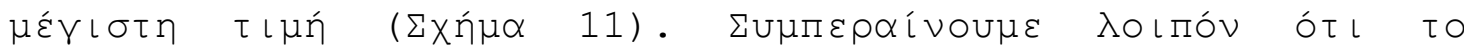

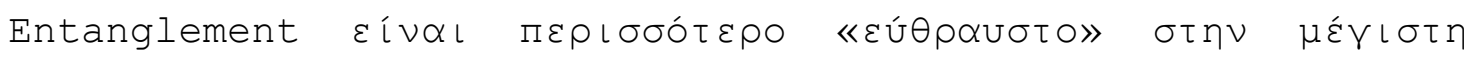

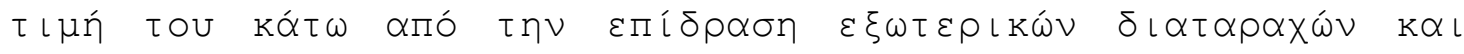




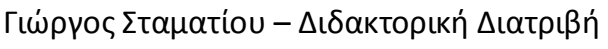

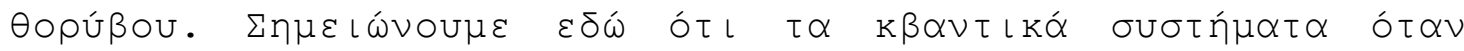

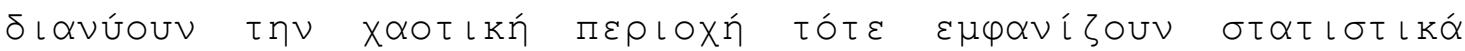

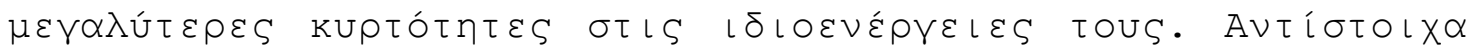

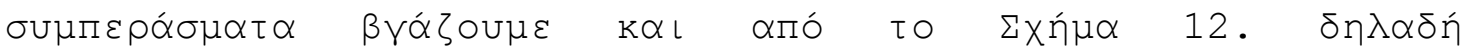

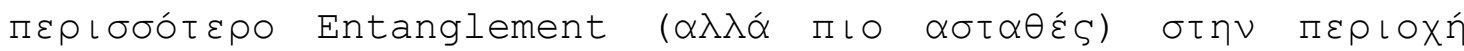
тOU XóOus.

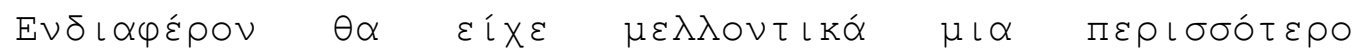

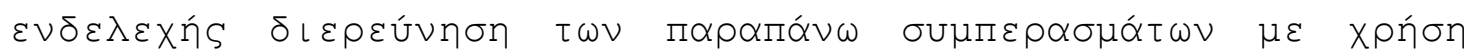

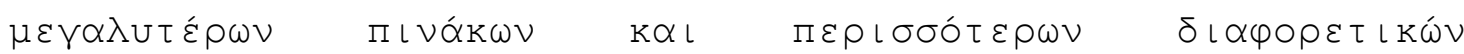

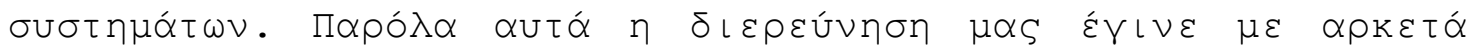

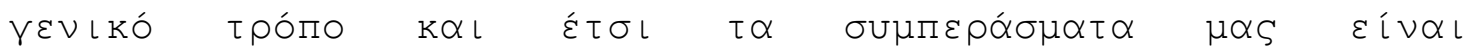

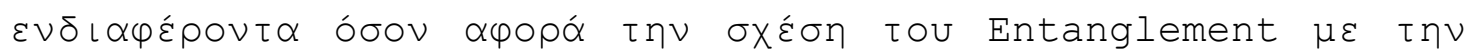

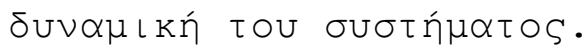
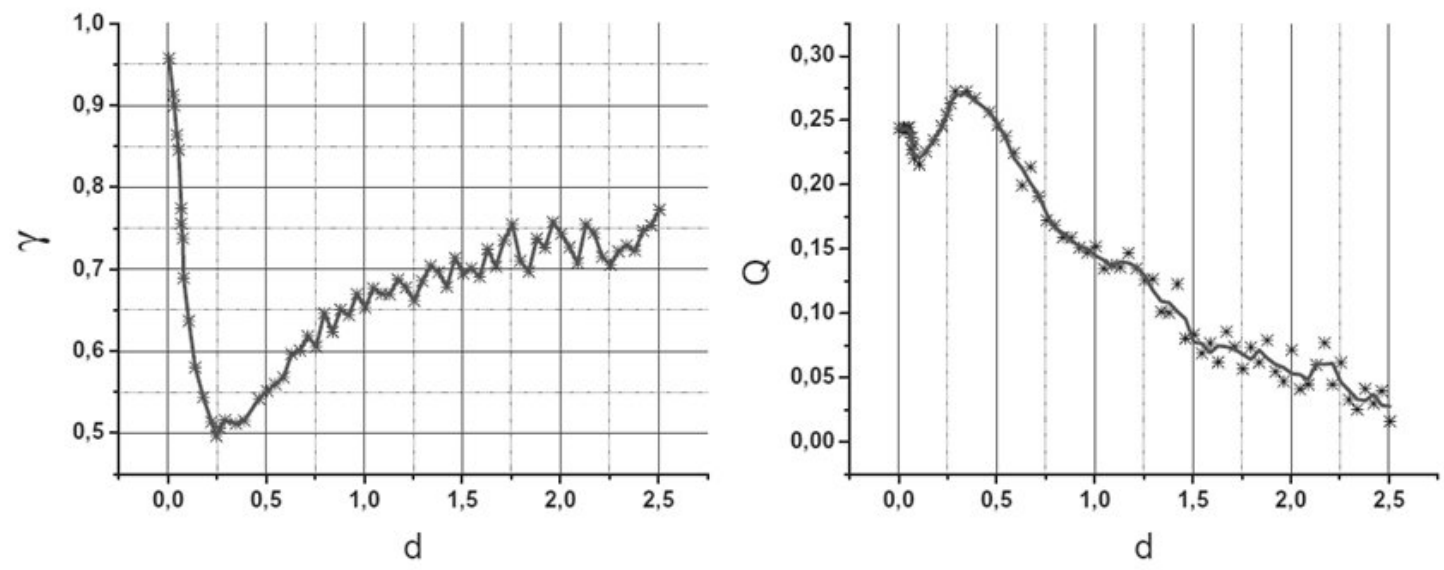

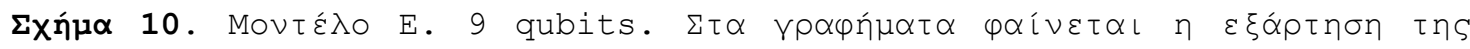

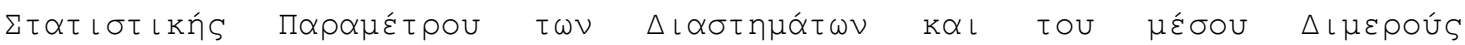

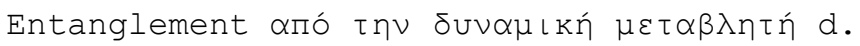



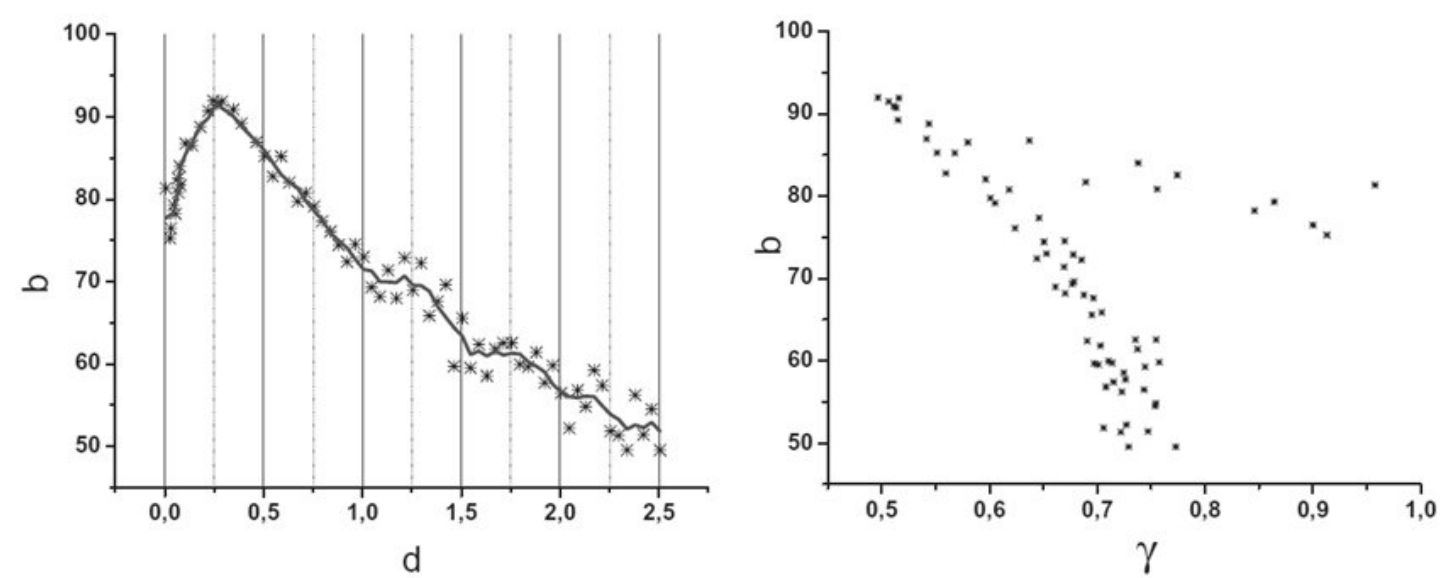

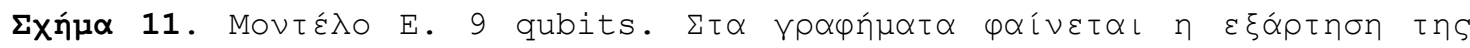

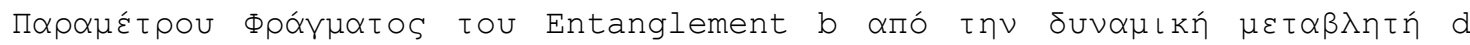

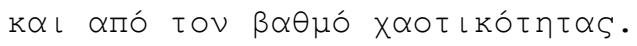
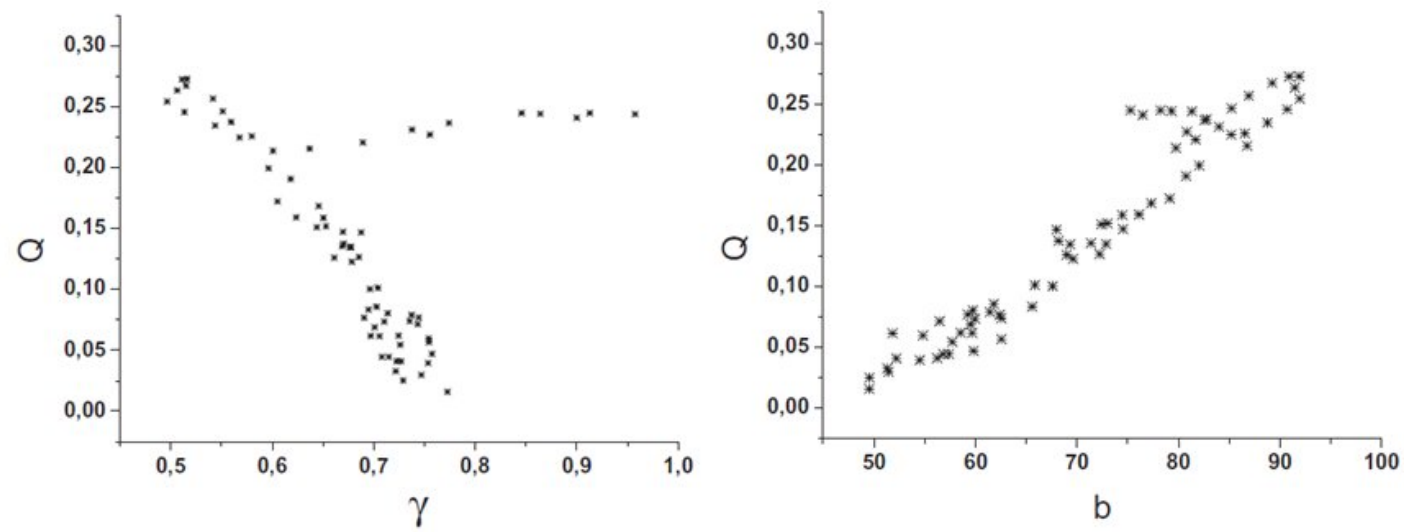

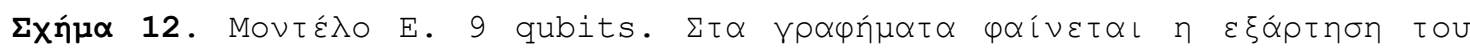

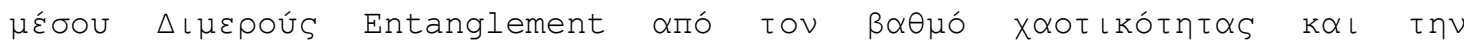

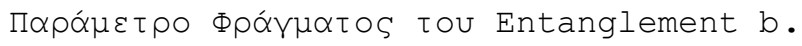

\section{$\Sigma \cup \mu \Pi \varepsilon \rho \alpha ́ \alpha \alpha \tau \alpha:$}

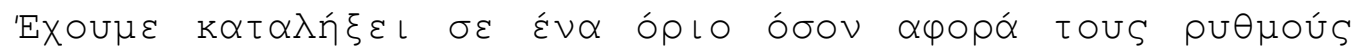

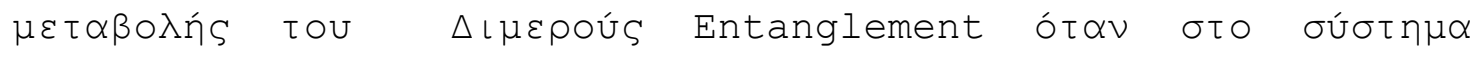

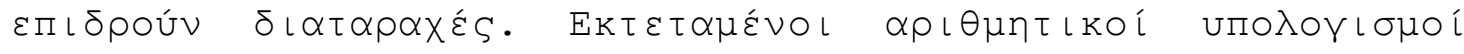




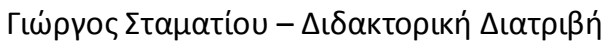

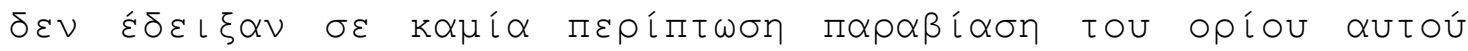

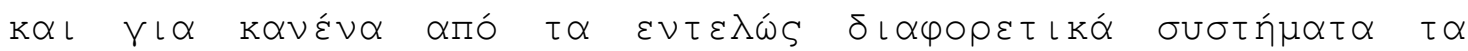

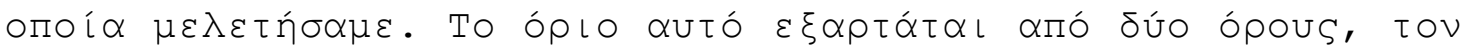

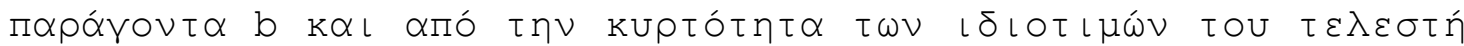

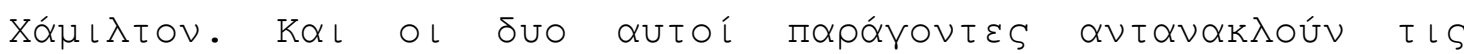

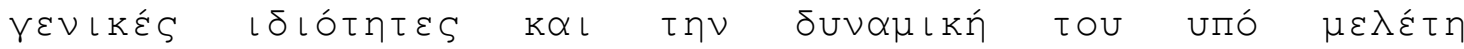

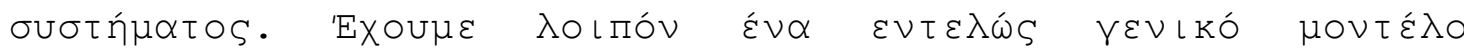

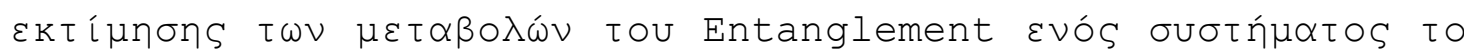

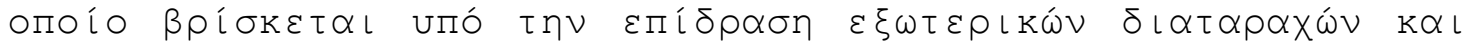

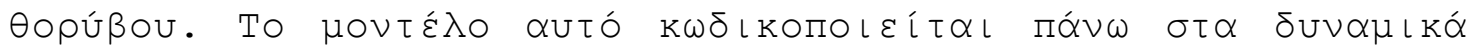

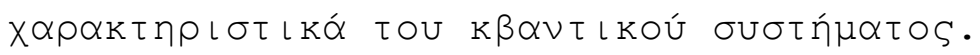

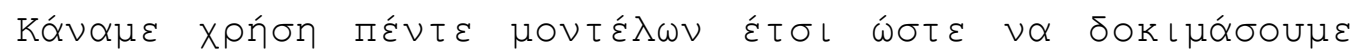

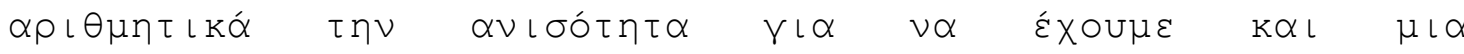

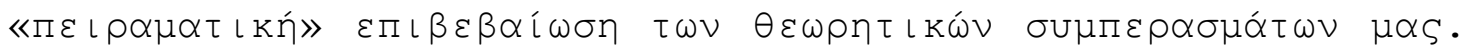

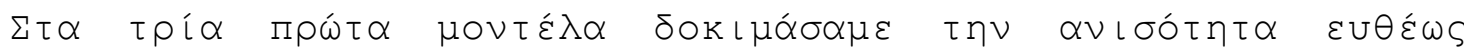

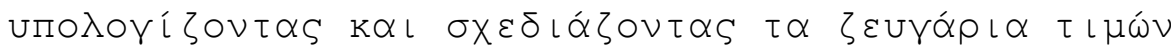

$$
\left|\partial Q^{0} / \partial \tau\right| \text { vs }\left|\mathrm{K}_{0}\right|
$$

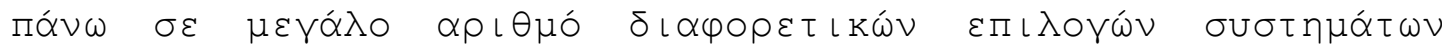

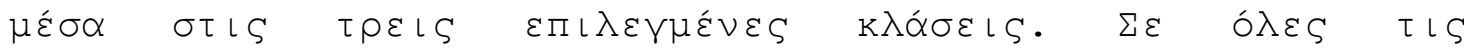

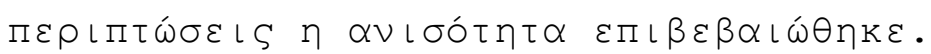

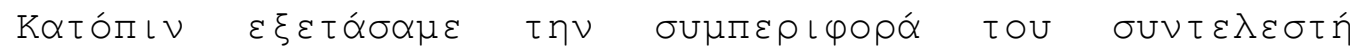

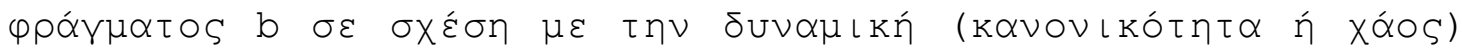

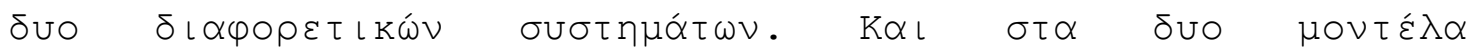

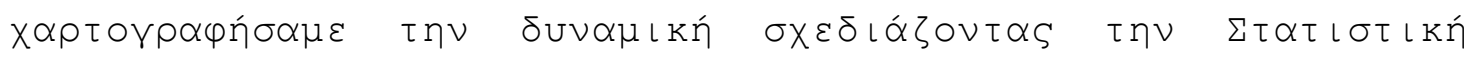

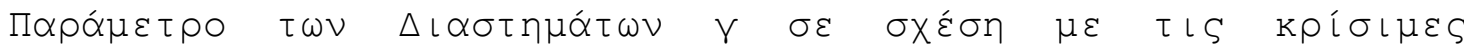

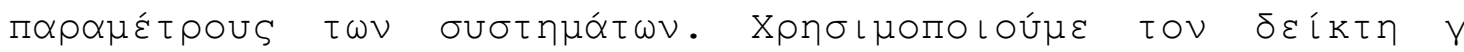

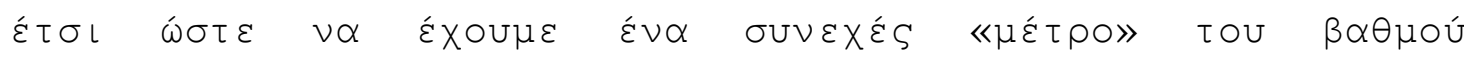

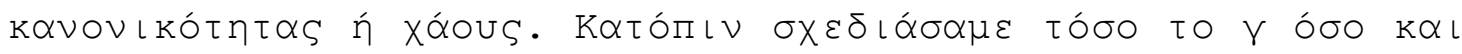

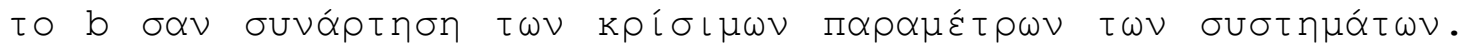

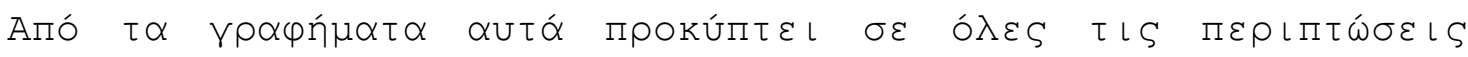

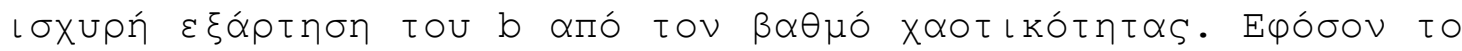

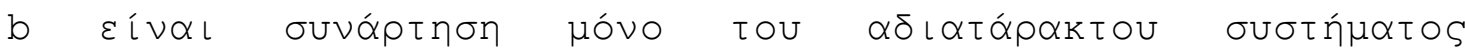

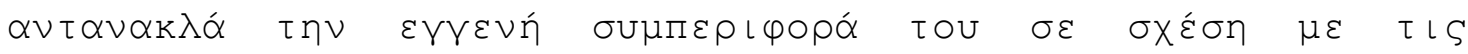

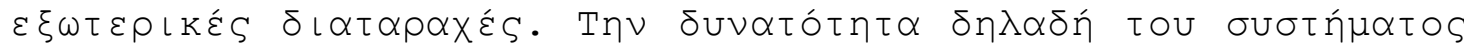

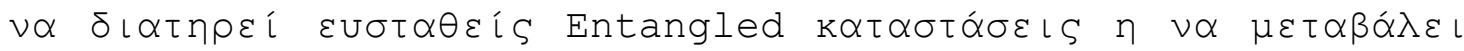

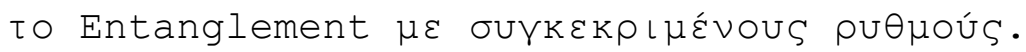




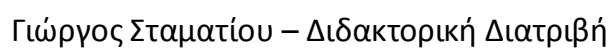

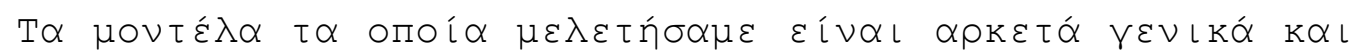

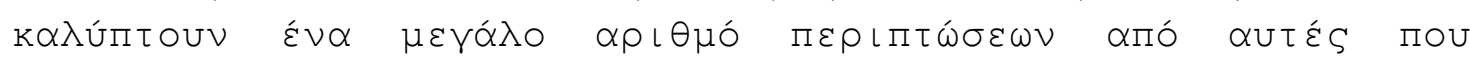

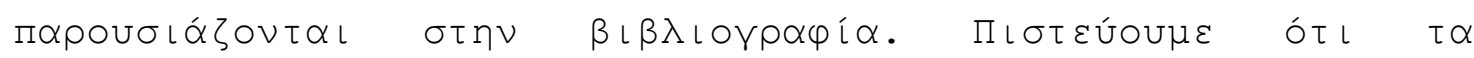

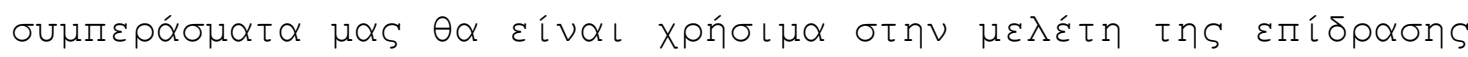

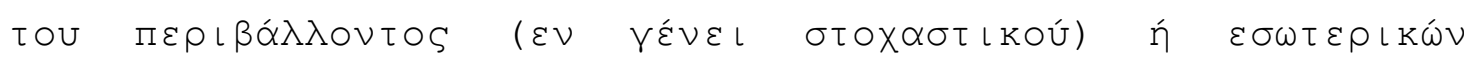

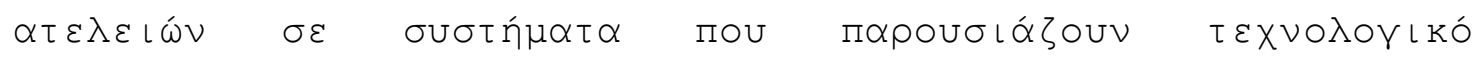
$\varepsilon \vee \delta\llcorner\alpha \varphi \varepsilon ́ \rho \circ \nu$.

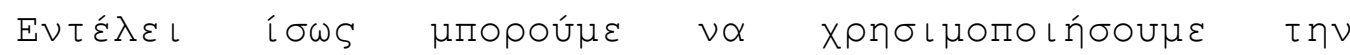

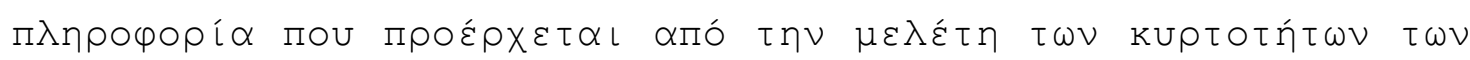

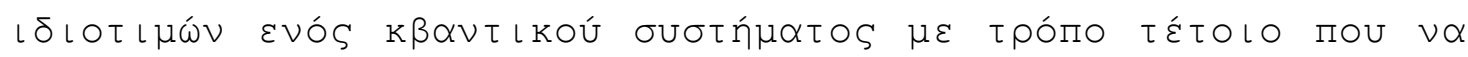

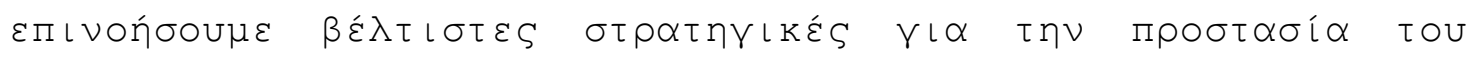

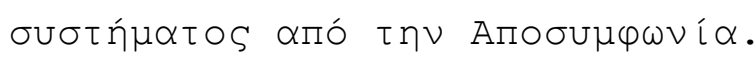

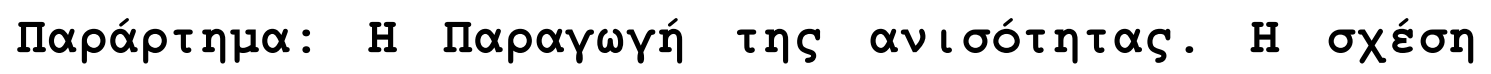
(4)

$' E \sigma \tau \omega$

$$
\rho_{\text {tot }}=|n(\tau)\rangle\langle n(\tau)|
$$

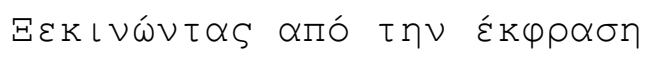

$$
Q^{n}=2-\frac{2}{N} \sum_{j=1}^{N} \operatorname{tr}\left(\rho_{j}^{2}\right)
$$

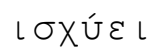

$$
\frac{\partial Q^{n}}{\partial \tau}=-\frac{2}{N} \sum_{j=1}^{N} \frac{\partial}{\partial \tau} \operatorname{tr}\left(\rho_{j}^{2}\right)
$$

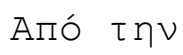

$$
\frac{\partial}{\partial \tau} \operatorname{tr}\left[\left(\rho_{j}\right)^{2}\right]=2 \operatorname{tr}\left[\operatorname{tr}_{A l l \neq j}\left(\rho_{\text {tot }}\right) \operatorname{tr}_{A l l \neq j}\left[\frac{\partial}{\partial \tau}\left(\rho_{\text {tot }}\right)\right]\right]
$$

$K \alpha \iota \quad \tau \eta \nu$ 


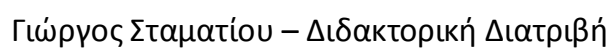

$$
\frac{\partial \rho_{\mathrm{tot}}}{\partial \tau}=\sum_{k \neq n} \frac{V_{k n}|k\rangle\langle n|+\text { c.c. }}{\epsilon_{n}-\epsilon_{k}}
$$

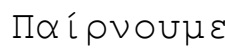

$$
\frac{\partial}{\partial \tau} \operatorname{tr}\left[\left(\rho_{j}\right)^{2}\right]=2 \sum_{k \neq n} \frac{V_{k n} A_{j ; k n}^{n}+V_{n k} A_{j ; n k}^{n}}{\epsilon_{n}-\epsilon_{k}}
$$

'Опоч

$$
A_{j ; k l}^{n}=\operatorname{tr}\left[\operatorname{tr}_{A l l \neq j}(|n\rangle\langle n|) \operatorname{tr}_{A l l \neq j}(|k\rangle\langle l|)\right]
$$

'E七 $\sigma$

$$
\frac{\partial Q^{n}}{\partial \tau}=-\frac{2}{N} \sum_{j=1}^{N} \frac{V_{k n} A_{k n}^{n}+\text { c.c. }}{\epsilon_{n}-\epsilon_{k}}
$$

$\mathrm{M \varepsilon}$

$$
A_{k l}^{n}=\sum_{j=1}^{N} A_{j ; k l}^{n}
$$

$K \propto l$

$$
-1 \leq A_{j ; k l}^{n} \leq+1
$$

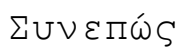

$$
-N \leq A_{k l}^{n} \leq+N
$$

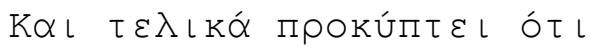

$$
\frac{\partial Q^{n}}{\partial \tau}=-\frac{8}{N} \sum_{j=1}^{N} \frac{\operatorname{Re}\left(V_{k n} A_{k n}^{n}\right)}{\epsilon_{n}-\epsilon_{k}}
$$




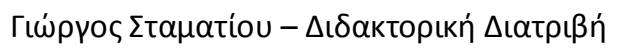

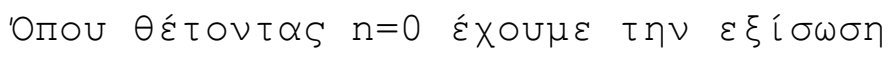

$$
\frac{\partial Q^{0}}{\partial \tau}=\frac{8}{N} \sum_{k=1}^{2^{N}-1} \frac{\operatorname{Re}\left[V_{0 k} A_{0 k}^{0}\right]}{\epsilon_{k}-\epsilon_{0}}
$$





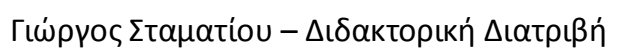

\section{Aข $\propto \varphi \circ \rho \varepsilon ́ \varsigma:$}

[1]. M. A. Nielsen and I. L. Chuang, Quantum Computation and Quantum Information, Cambridge University Press, Cambridge, UK, 2000, ISBN: 0-521-63503-9

[2]. Ph. Blanchard, D. Giulini, E. Joos, C. Kiefer and I.O. Stamatescu (eds.), in Proc. Decoherence, Theoretical, Experimental and Conceptual Problems, Bielefeld, Germany

1998 (Springer-Verlag, Germany, 2000).

[3]. N. F. Bell, R. F. Sawyer, R. R. Volkas and Y. Y. Y. Wong, Phys. Rev A 65 (2002) 042328.

[4]. J. N. Bandyopadhyay and A. Lakshminarayan, Phys. Rev. Lett. 89 (2002) 060402.

[5]. J. N. Bandyopadhyay and A. Lakshminarayan, Phys. Rev. E 69 (2004) 016201.

[6]. C. Mejia-Monasterio, G. Benenti, G. G. Carlo and G. Casati, Phys. Rev A 71 (2005) 062324.

[7]. H. Fujisaki, Phys. Rev A 70 (2004) 012313.

[8]. S. Ghose and B. C. Sanders, Phys. Rev A 70 (2004) 62315.

[9]. X.Wang, S. Ghose, B. C. Sanders and B. Hu, Entanglement as a signature of quantum chaos, e-print quant-ph/0312047.

[10]. Ph. Jacquod, Phys. Rev. Lett. 92 (2004) 150403.

[11]. T. Gorin and T. H. Seligman, Decoherence in chaotic and integrable systems: A random matrix approach, e-prints nlin.CD/0101018, quant-ph/0112030.

[12]. M. Znidaric and T. Prosen, Phys. Rev A 71 (2005) 32103 . 


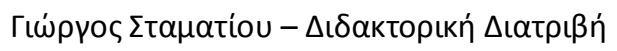

[13]. C. Pineda and T. H. Seligman, Evoluation of pairwise Entanglement in a coupled n-body system, e-print quantph/0503177.

[14]. K. Nakamura, Quantum Chaos (Cambridge University Press, Cambridge, UK, 1993).

[15]. K. Nakamura and M. Lakshmanan, Phys. Rev. Lett. 57 (1986) 1661 .

[16]. T. Takami, Phys. Rev. Lett. 68 (1992) 3371.

[17]. T. Takami, Phys. Rev. E 52 (1995) 2434.

[18]. P. Gaspard, S. A. Rice, H. J. Mikeska and K. Nakamura, Phys. Rev. A 42 (1990) 4015.

[19]. D. Saher, F. Haake and P. Gaspard, Phys. Rev. A 44 (1991) 7841 .

[20]. T. Takami and H. Hasegawa, Phys. Rev. Lett. 68 (1992) 419 .

[21]. J. Zakrzewski and D. Delande, Phys. Rev. E 47 (1993) 1650 .

[22]. Y. Fyodorov and H.-J. Sommers, Z. Phys. B 99 (1995) 123.

[23]. T. Guhr, A. Muller-Groeling and H. A. Weidenmuller, Phys. Rep. 299 (1998) 189.

[24]. L. F. Santos, J. Phys. A. Math. Gen. 37 (2004) 4723 


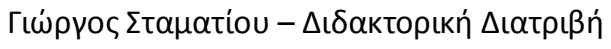

ENOTHTA $\mathbf{E}^{\prime}$

ENTANGLEMENT,

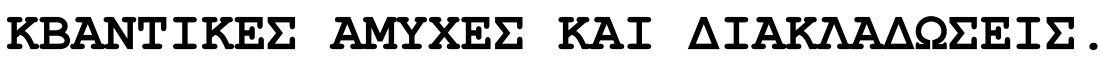

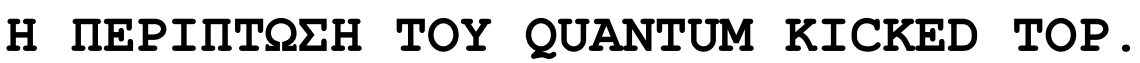





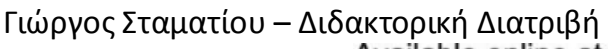

Available online at www.sciencedirect.com

ScienceDirect

Physics Letters A 368 (2007) 206-214

www.elsevier.com/locate/pla

\title{
Quantum Entanglement dependence on bifurcations and scars in non-autonomous systems. The case of Quantum Kicked Top
}

George Stamatiou, Demetris P.K. Ghikas *

Department of Physics University of Patras, Patras 26500, Greece

Received 2 August 2006; received in revised form 10 February 2007; accepted 2 April 2007 Available online 4 April 2007 Communicated by A.P. Fordy

\begin{abstract}
Properties related to Entanglement in quantum systems, are known to be associated with distinct properties of the corresponding classical systems, as for example stability,

integrability and chaos. This means that the detailed

topology, both local and global, of the classical phase space may reveal, or influence, the entangling power of the quantum system. As it has been shown in the literature, the bifurcation points, in autonomous dynamical systems, play a crucial role for the onset of Entanglement. Similarly, the existence of scars among the quantum states seems to be a factor in the dynamics of Entanglement. Here we study these issues for a non-autonomous system, the Quantum Kicked Top, as a collective model of a multi-qubit system. Using the bifurcation diagram of the corresponding classical limit

(the Classical Kicked Top), we analyzed the pairwise and the bipartite Entanglement of the qubits and their relation to scars, as a function of the critical parameter of the system. We found that the pairwise Entanglement and pairwise negativity show a strong maximum precisely at the bifurcation points, while the bipartite Entanglement
\end{abstract}




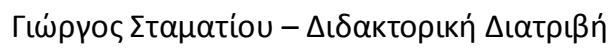

changes slope at these points. We have also investigated the connection between Entanglement and the fixed points on the branch of the bifurcation diagram between the two first

bifurcation points and we found that the Entanglement measures take their extreme values precisely on these points. We conjecture that our results on this behavior of

Entanglement is generic for many quantum systems with a nonlinear classical analogue. (c) 2007 Elsevier B.V. All rights reserved.

PACS: 03.67.Mn; 05.45.AC; 05.45.Pq; 05.45.Mt

Keywords: Entanglement; Quantum chaos; Bifurcations; Scars; Kicked-top model

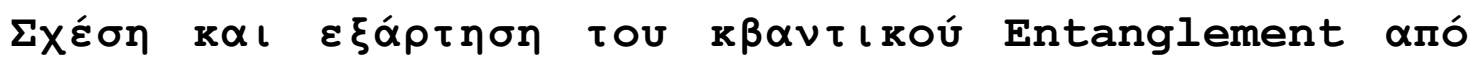

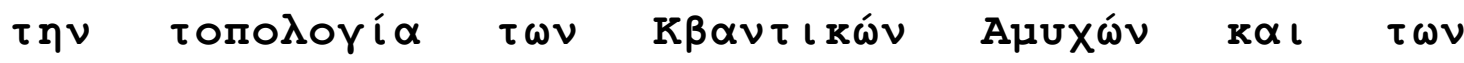

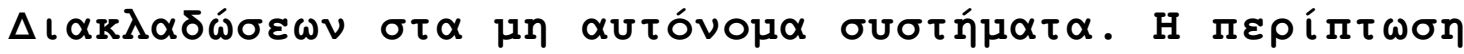
rou Quantum Kicked Top.

\section{$\Gamma \varepsilon \vee \iota k \alpha ́$ :}

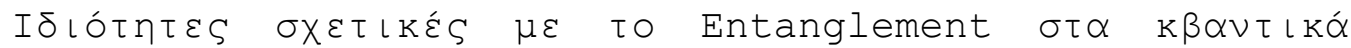

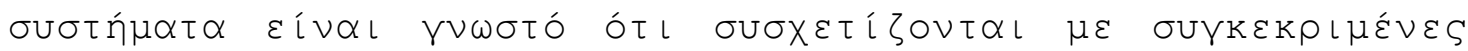

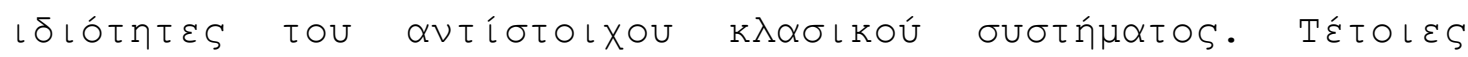

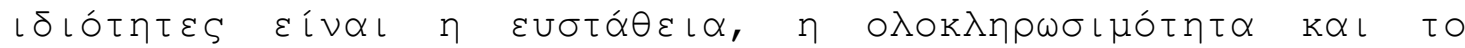

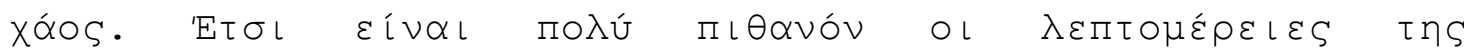

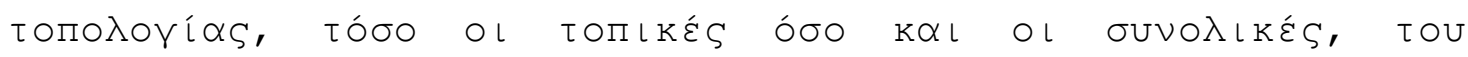

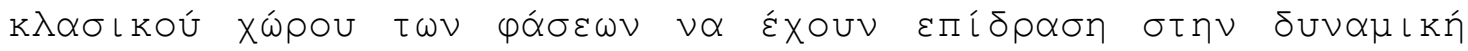

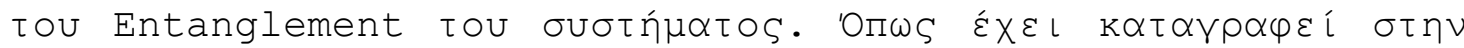

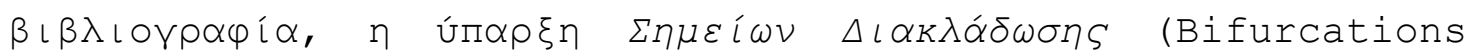

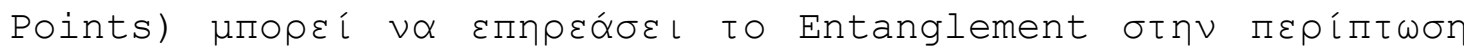

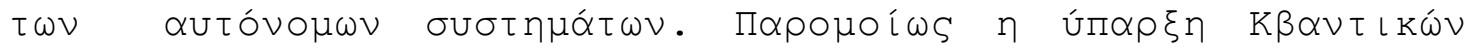

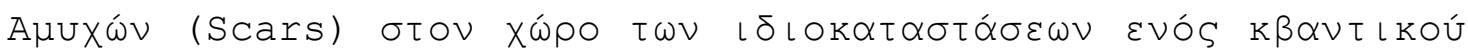

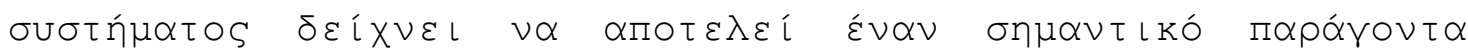

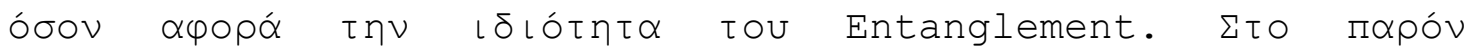




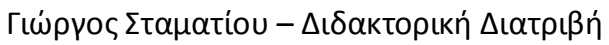

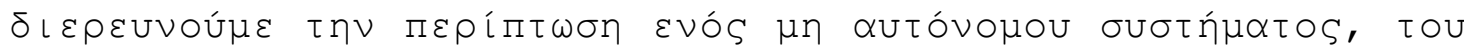

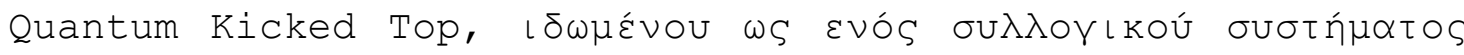

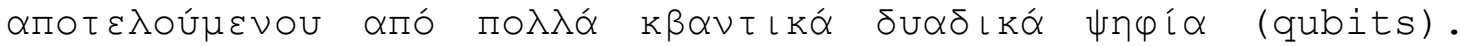

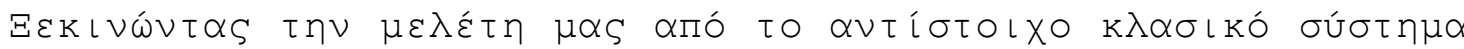

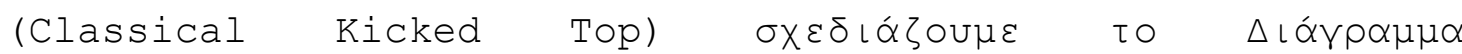

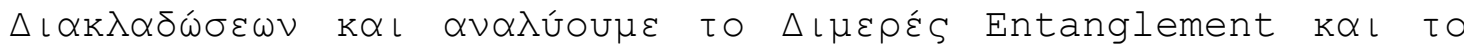

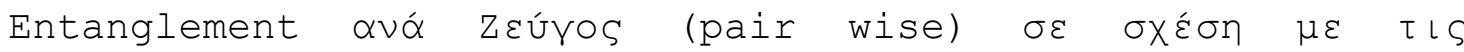

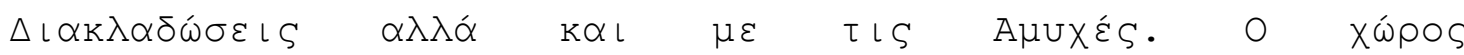

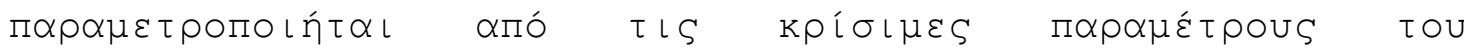

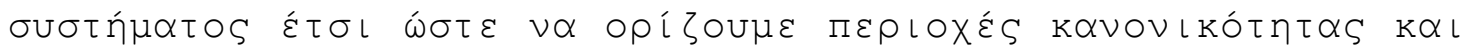
Xóous.

\section{Eı $\sigma \alpha \gamma \omega \gamma \eta \dot{~: ~}$}

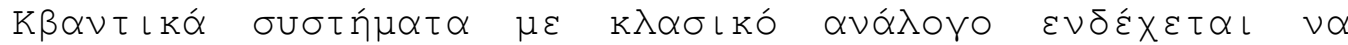

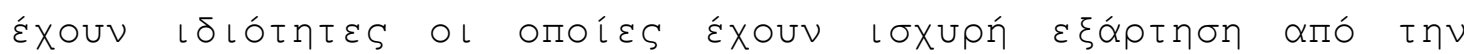

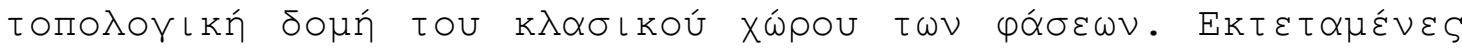

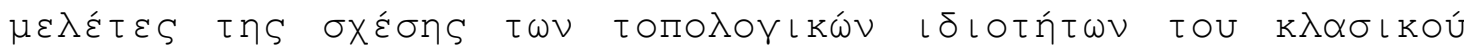

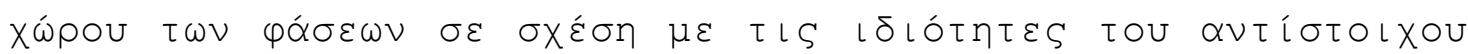

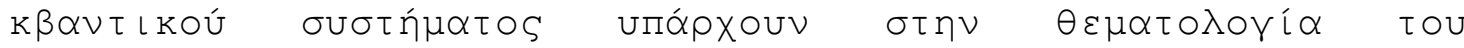

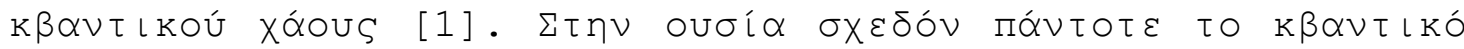

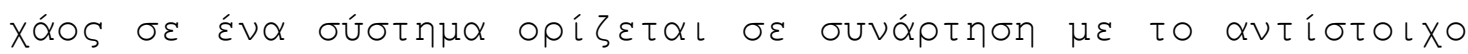

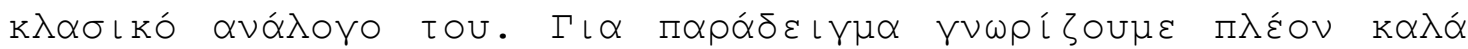

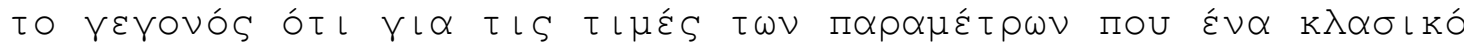

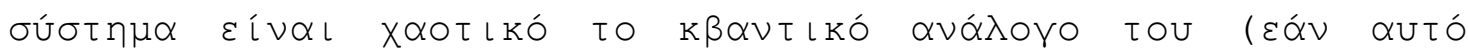

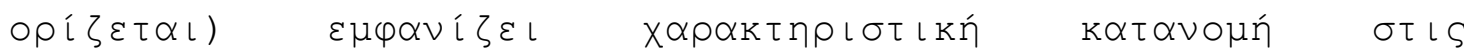

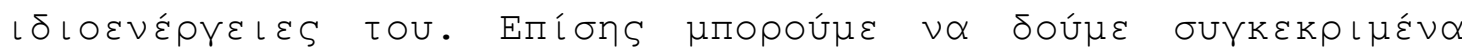

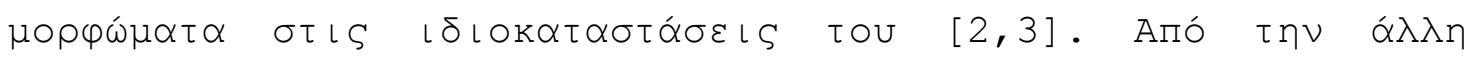

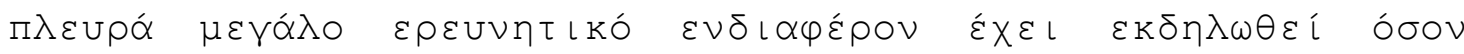

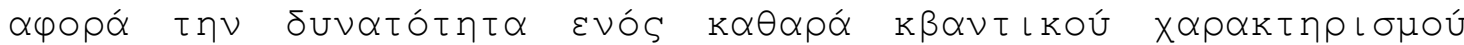

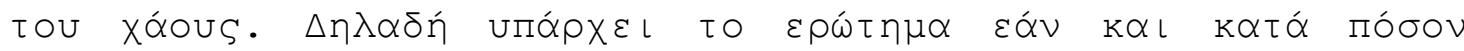

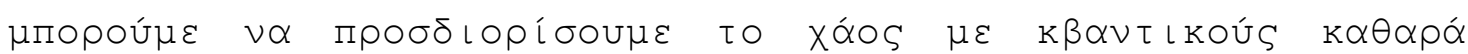

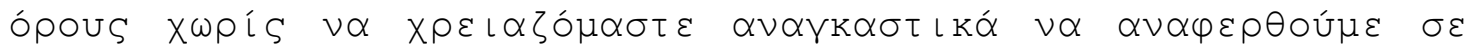

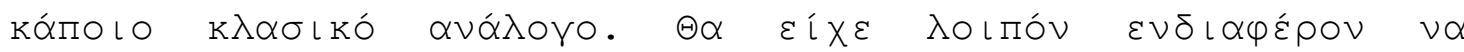

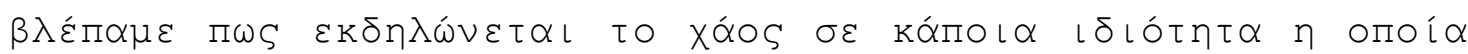

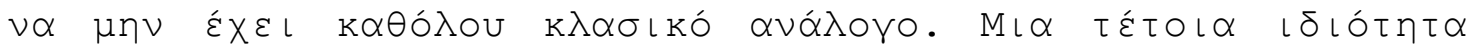

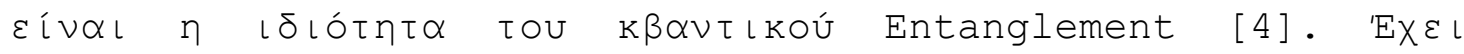

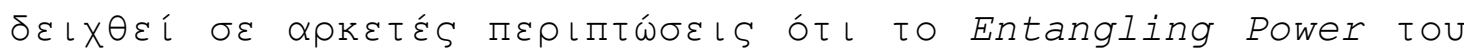

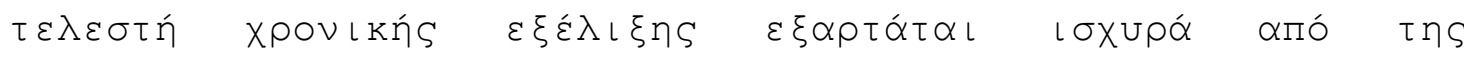

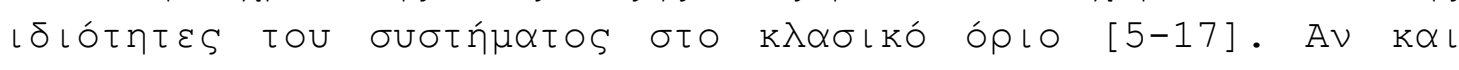




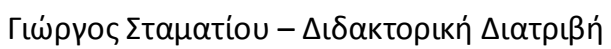

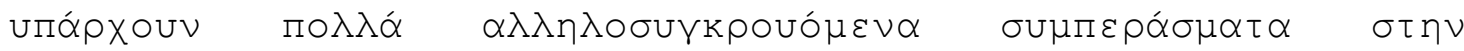

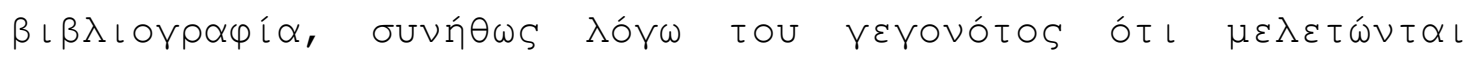

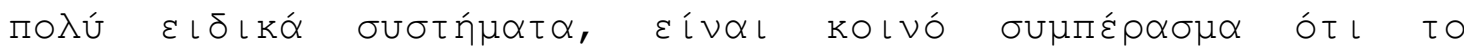

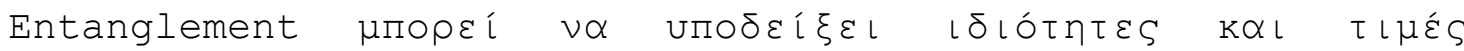

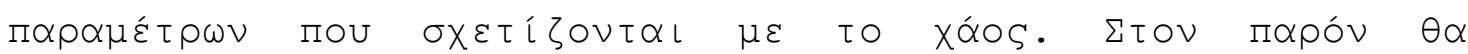

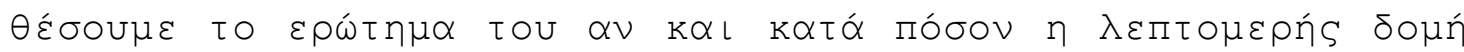

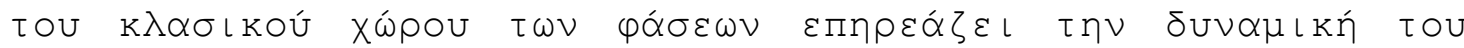

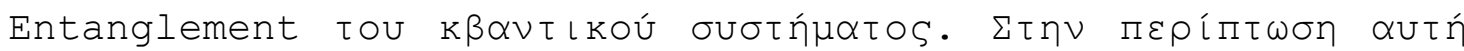

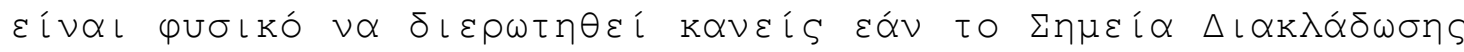

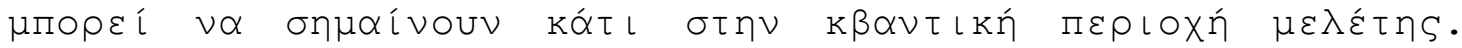

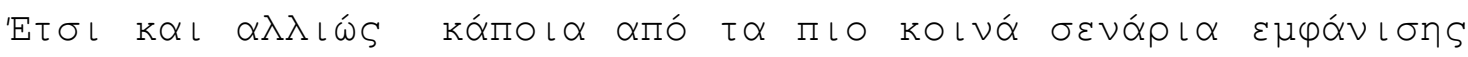

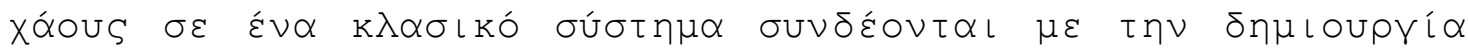

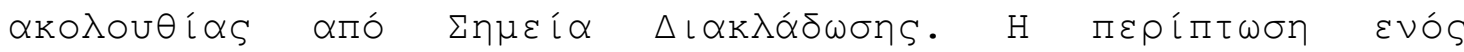

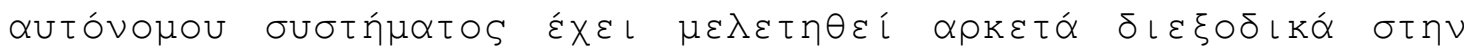

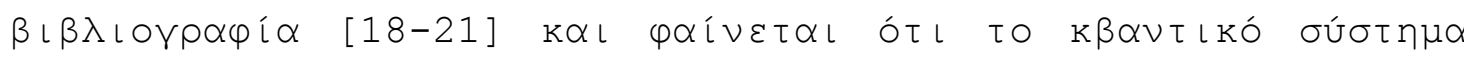

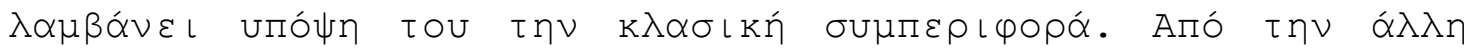

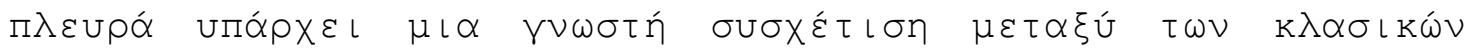

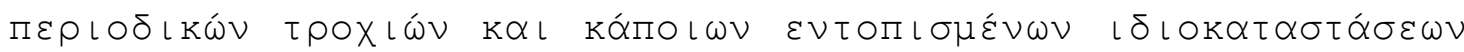

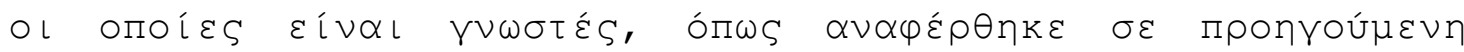

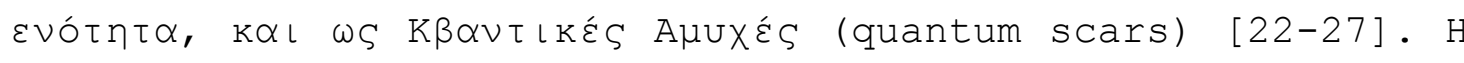

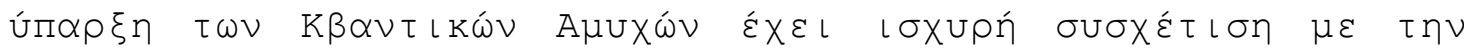

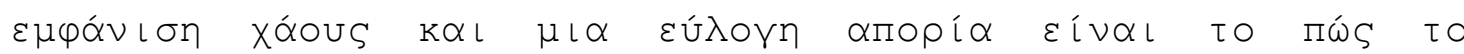

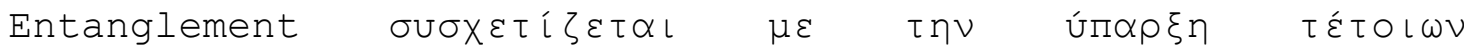

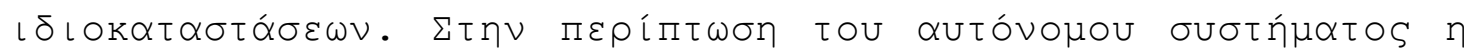

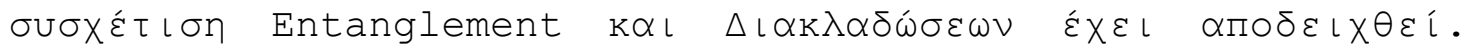

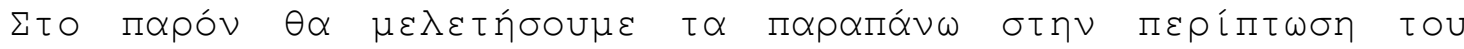

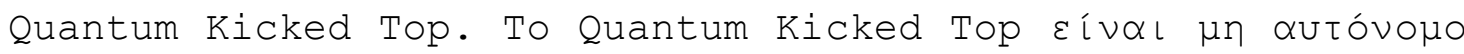

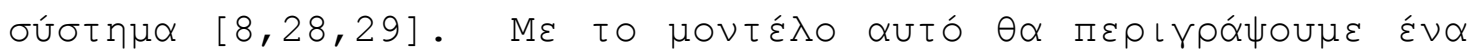

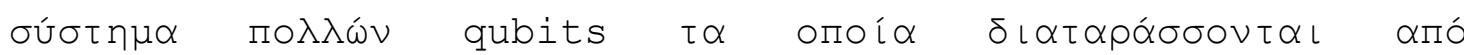

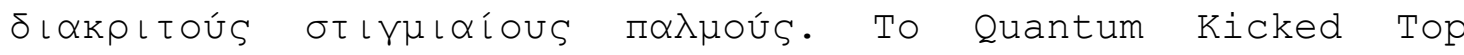

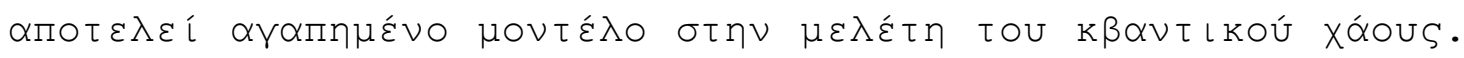

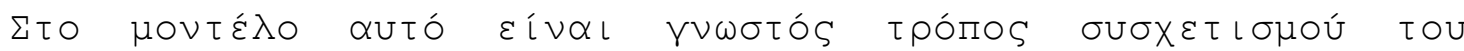

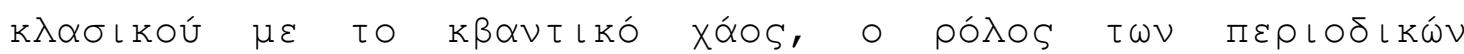

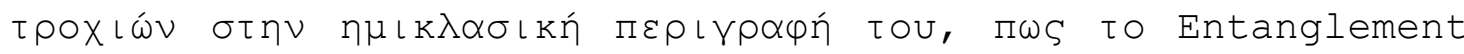

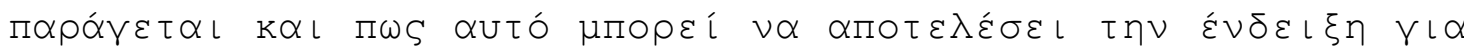

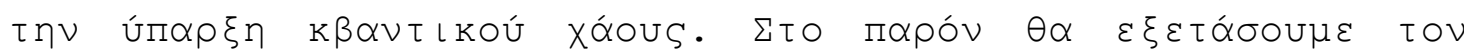

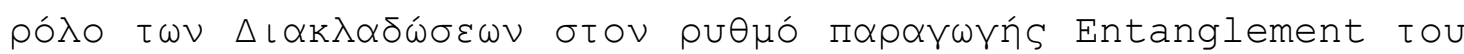

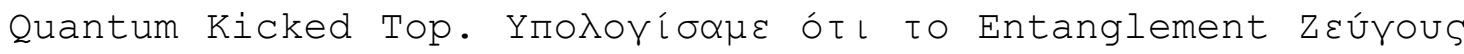

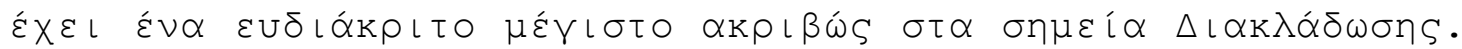

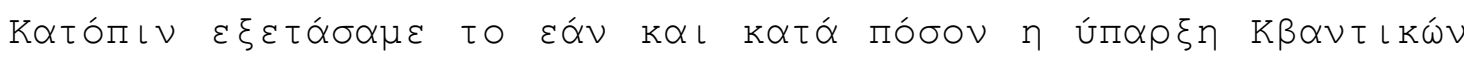

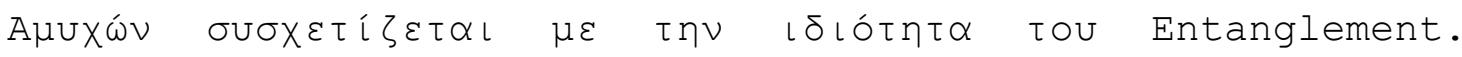




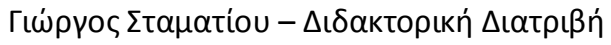

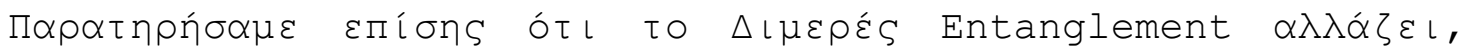

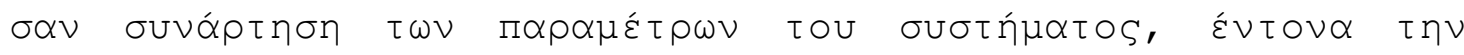

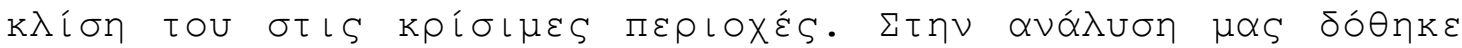

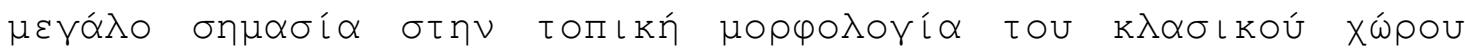

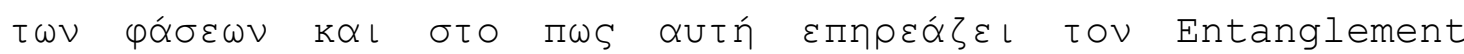

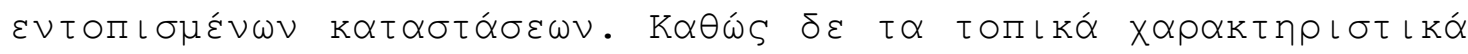

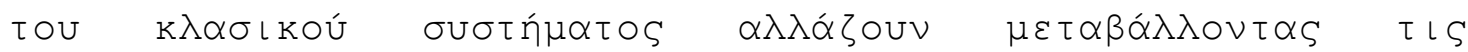

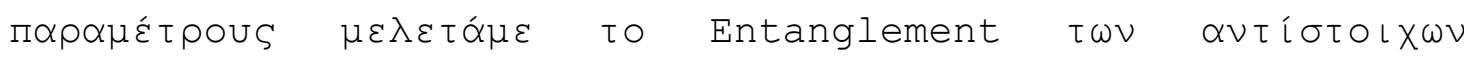

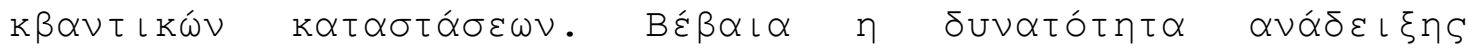

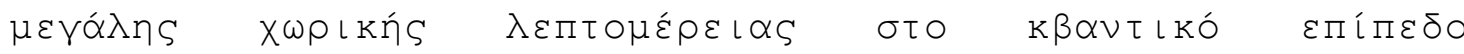

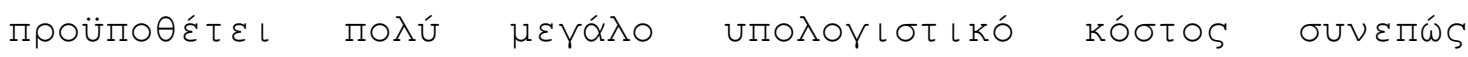

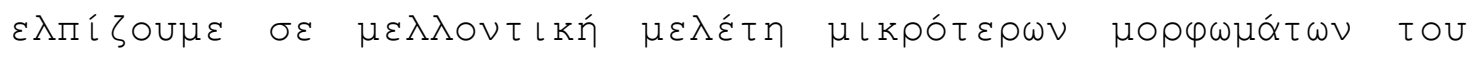

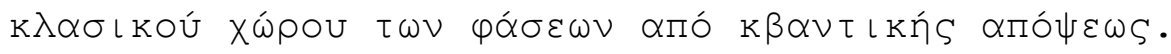

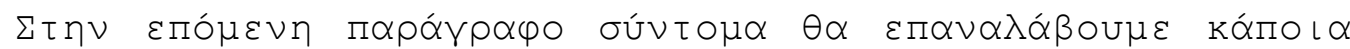

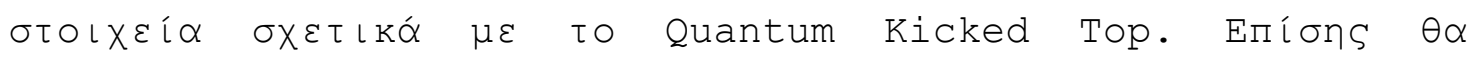

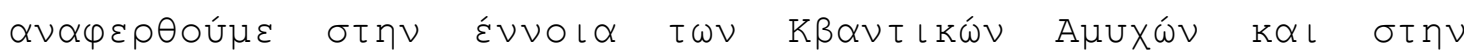
$\sigma \eta \mu \alpha \sigma i \alpha$ tous.

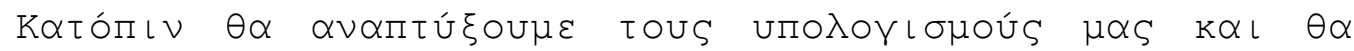

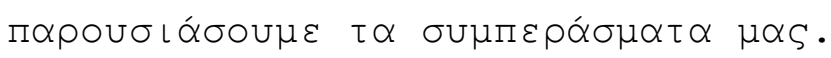

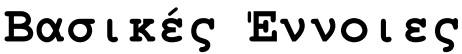

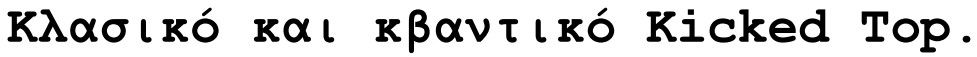

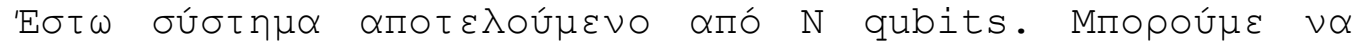

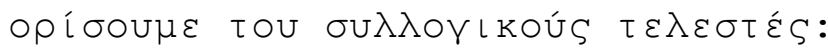

$$
\hat{J}_{a}=\sum_{i=1}^{N} \frac{\hat{\sigma}_{i a}}{2}
$$

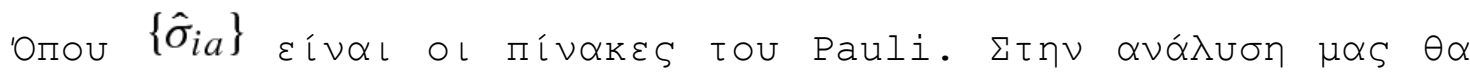

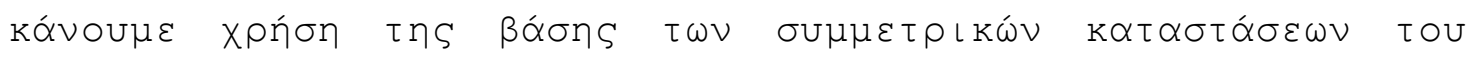
Diche [32]

$$
\{|j, m\rangle, m=-j, \ldots, j, j=N / 2\}
$$




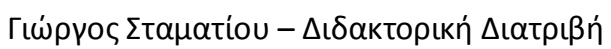

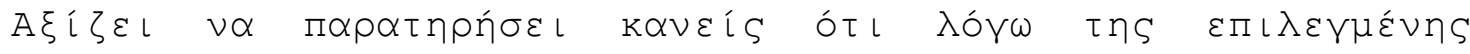

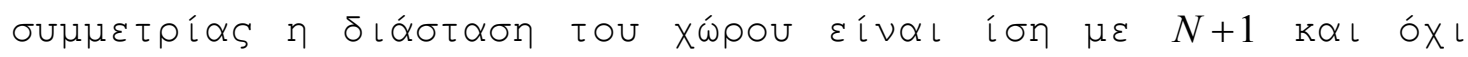

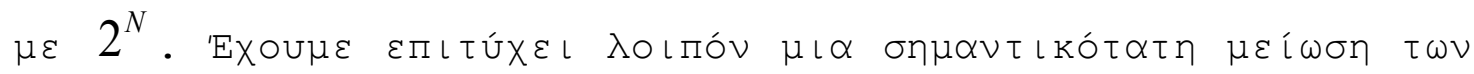

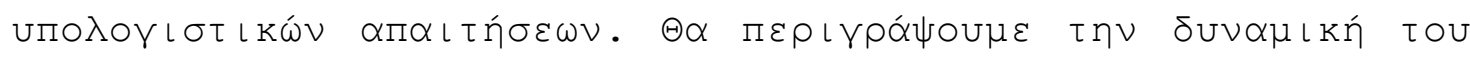

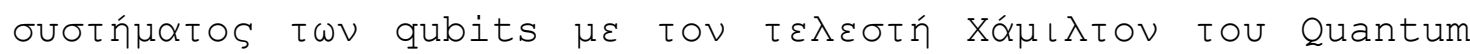

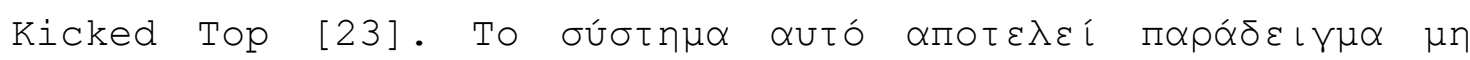

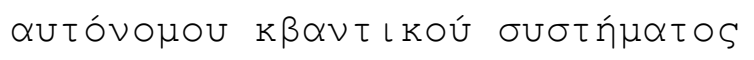

$$
\hat{H}(t)=(\hbar p / \tau) \hat{J}_{y}+(\hbar k / 2 j) \hat{J}_{z}^{2} \sum_{n=-\infty}^{+\infty} \delta(t-n \tau)
$$

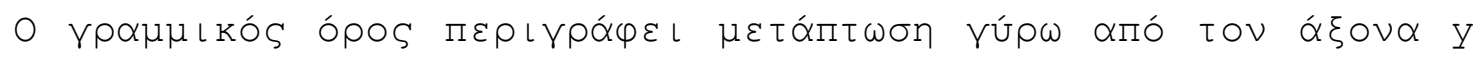

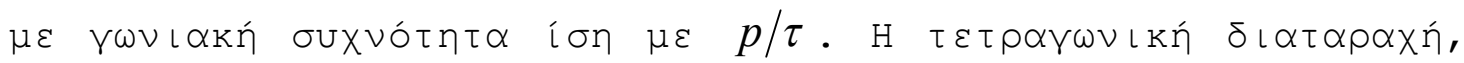

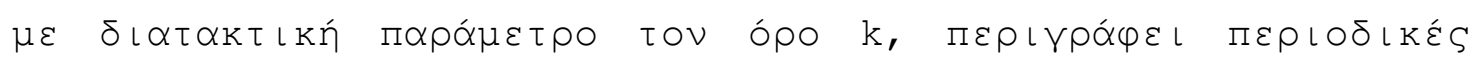

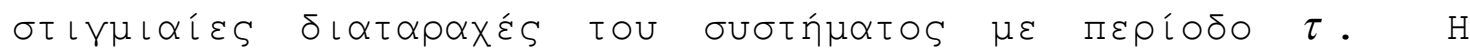

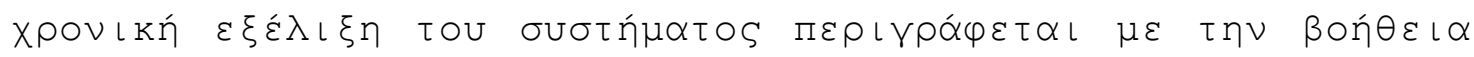

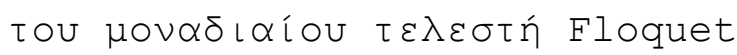

$$
\hat{F}=e^{-i(k / 2 j) \hat{J}_{z}^{2}} e^{-i p \hat{J}_{y}}
$$

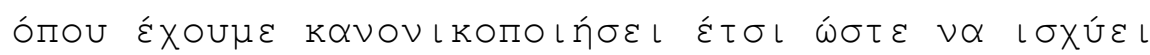

$$
\hbar=1, \tau=1
$$

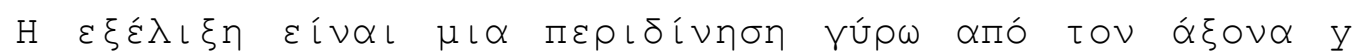

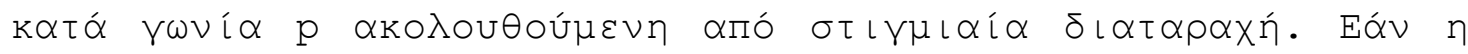

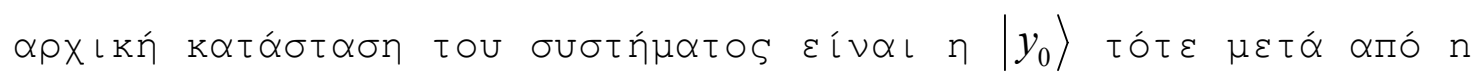

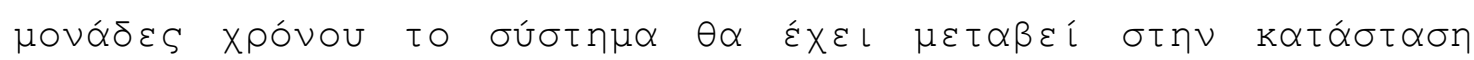
$\left|y_{n}\right\rangle=\hat{F}^{n}\left|y_{0}\right\rangle$

$\mathrm{H} \varepsilon \xi i \sigma \omega \sigma \eta$

$$
\hat{F}\left|\Phi_{m}\right\rangle=e^{i x_{m}}\left|\Phi_{m}\right\rangle
$$

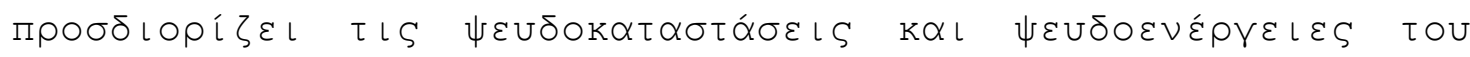

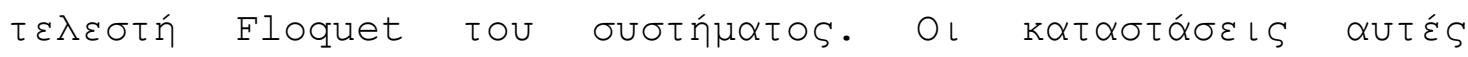

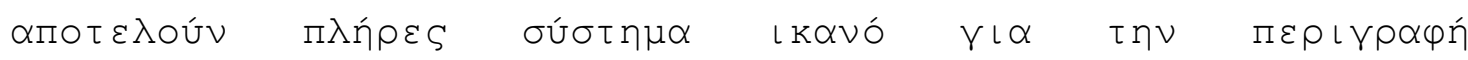

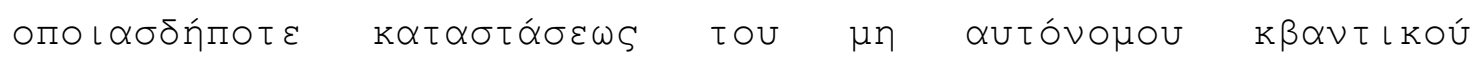




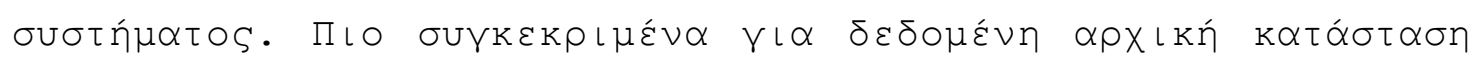

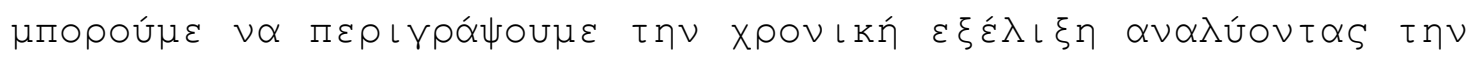

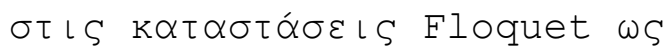

$$
\left|y_{n}\right\rangle=\sum_{m} e^{i n x_{m}}\left|\Phi_{m}\right\rangle\left\langle\Phi_{m} \mid y_{0}\right\rangle
$$

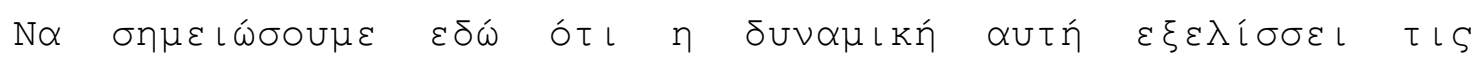

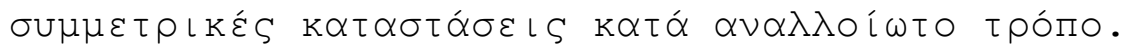

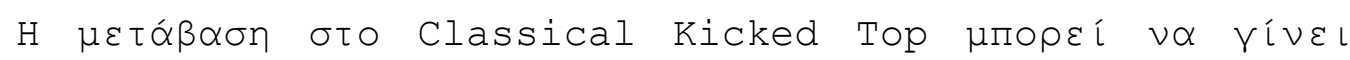

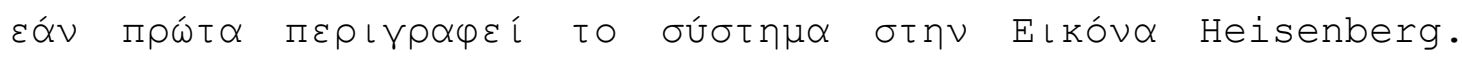

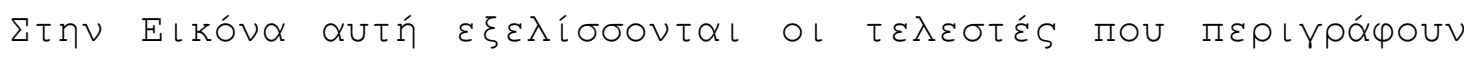

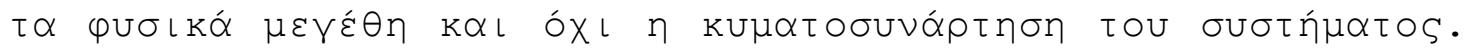

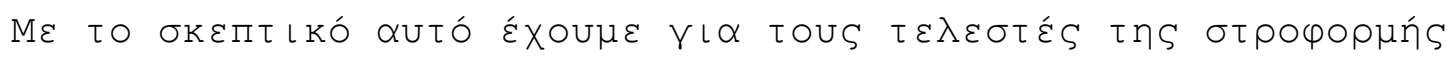

$$
\hat{J}_{i n}=\hat{F}^{\dagger n} \hat{J}_{0} \hat{F}^{n}
$$

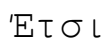

$$
\begin{gathered}
\hat{J}_{x}^{\prime}=\frac{1}{2}\left(\hat{J}_{x} \cos p+\hat{J}_{z} \sin p+i \hat{J}_{z}\right) e^{i \frac{k}{j}\left(\hat{J}_{z} \cos p-\hat{J}_{x} \sin p+\frac{1}{2}\right)}+\text { h.c. } \\
\hat{J}_{y}^{\prime}=\frac{1}{2 i}\left(\hat{J}_{x} \cos p+\hat{J}_{z} \sin p+i \hat{J}_{z}\right) e^{i \frac{k}{j}\left(\hat{J}_{z} \cos p-\hat{J}_{x} \sin p+\frac{1}{2}\right)}+\text { h.c. } \\
\hat{J}_{z}^{\prime}=\hat{J}_{z} \cos p-\hat{J}_{x} \sin p
\end{gathered}
$$

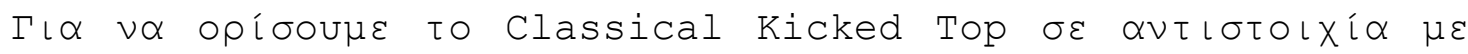

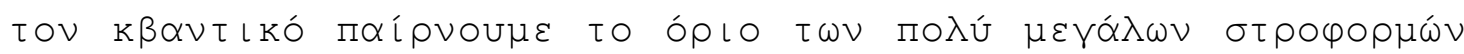

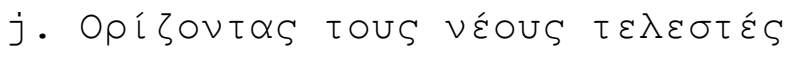

$$
\hat{T}_{a}=\hat{J}_{a} / j, a \in\{x, y, z\}
$$

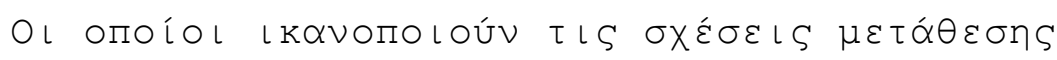

$$
\left[\hat{T}_{a}, \hat{T}_{b}\right]=\frac{i \epsilon_{a b c}}{j} \hat{T}_{c}
$$

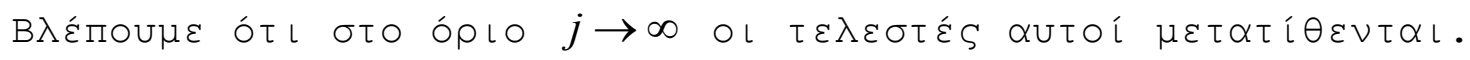

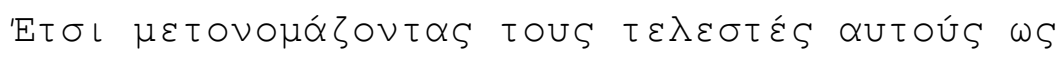




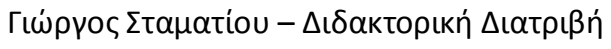

$$
X=T_{x}, Y=T_{y}, Z=T_{z}
$$

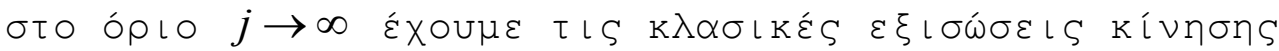

$$
\begin{aligned}
& X^{\prime}=\operatorname{Re}(X \cos p+X \sin p+i Y) e^{i k(Z \cos p-X \sin p)}, \\
& Y^{\prime}=\operatorname{Im}(X \cos p+X \sin p+i Y) e^{i k(Z \cos p-X \sin p),} \\
& Z^{\prime}=-X \sin p+Z \cos p .
\end{aligned}
$$

Eóv $\delta \varepsilon \quad \varepsilon \Pi \iota \lambda \varepsilon \dot{\xi} \circ u \mu \varepsilon \quad \tau \eta \nu \quad \tau \iota \mu n$

$$
p=\pi / 2
$$

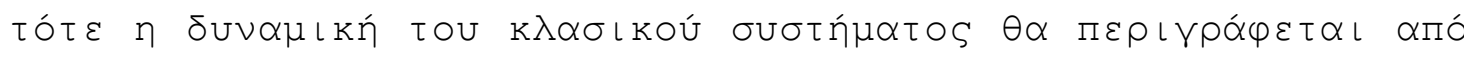

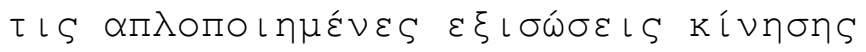

$$
\begin{aligned}
& X^{\prime}=Z \cos (k X)+Y \sin (k X), \\
& Y^{\prime}=-Z \sin (k X)+Y \cos (k X), \\
& Z^{\prime}=-X
\end{aligned}
$$

'Oחоu ı $\sigma x u ́ \varepsilon l$ ót l

$$
X^{\prime 2}+Y^{\prime 2}+Z^{\prime 2}=X^{2}+Y^{2}+Z^{2}=1
$$

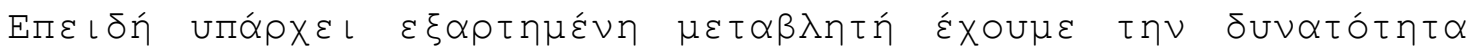

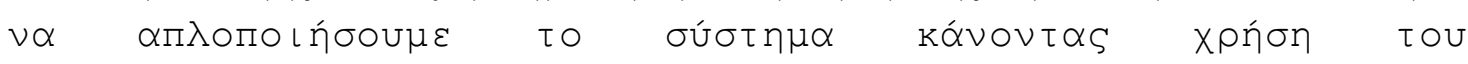
$\mu \varepsilon \tau \alpha \sigma \times \eta \mu \alpha \tau \iota \sigma \mu \circ u$

$$
\begin{gathered}
X=\sin \theta \cos \phi, Y=\sin \theta \sin \phi, Z=\cos \theta \\
\theta=\arccos (Z), \phi=\arctan \left(\frac{Y}{X}\right)
\end{gathered}
$$

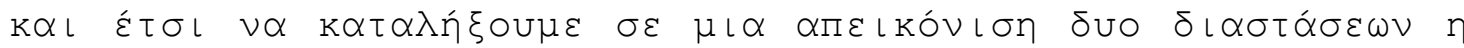

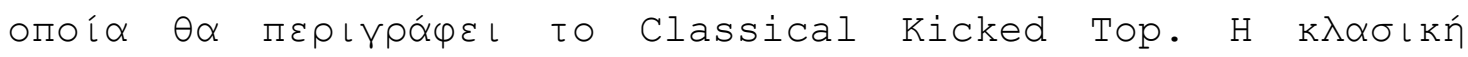

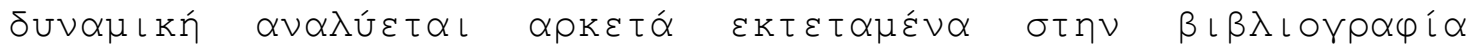

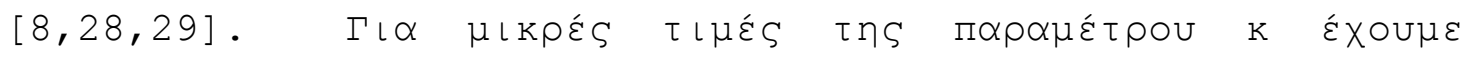

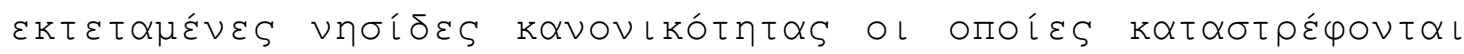

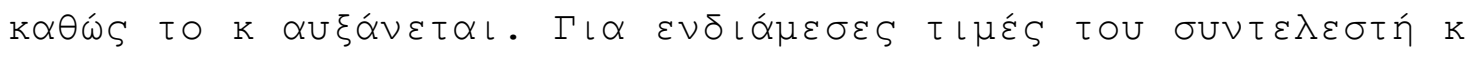

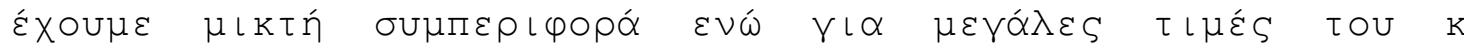




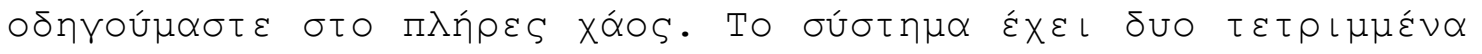

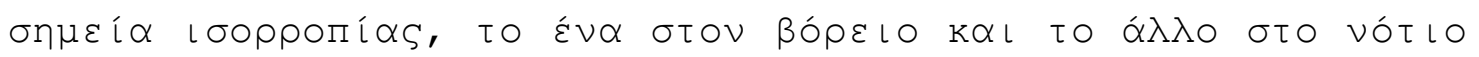

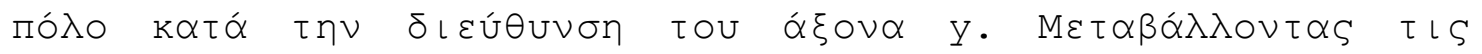

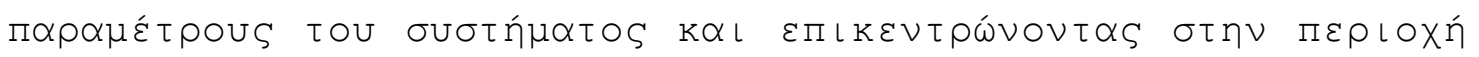

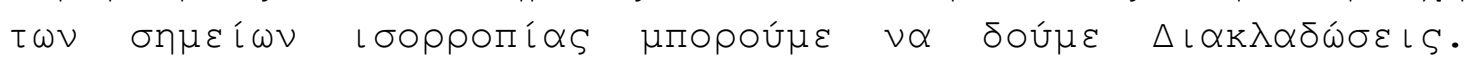

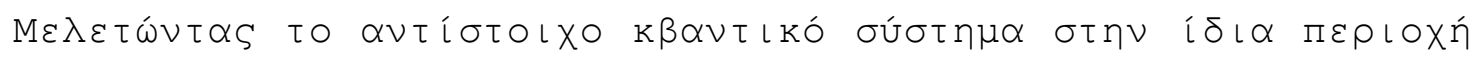

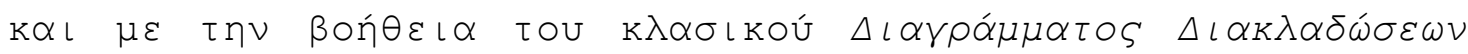

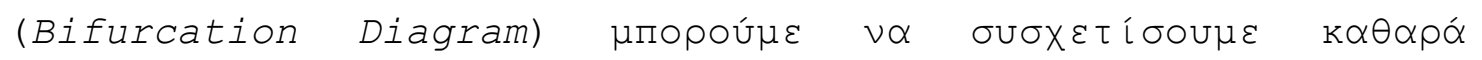

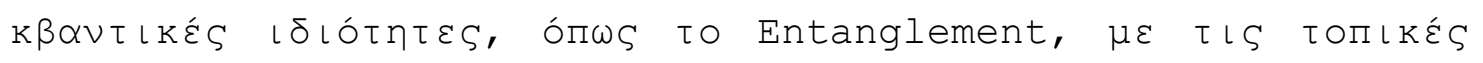

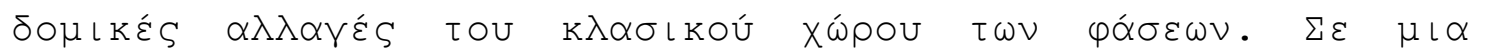

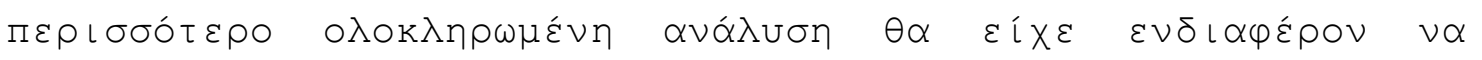

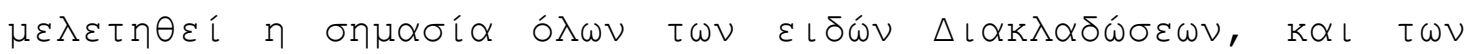

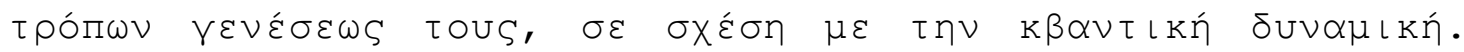

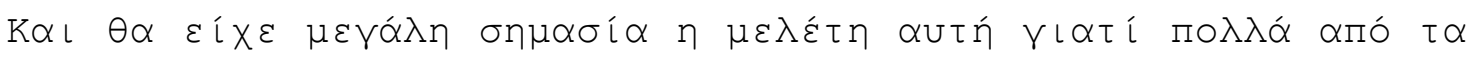

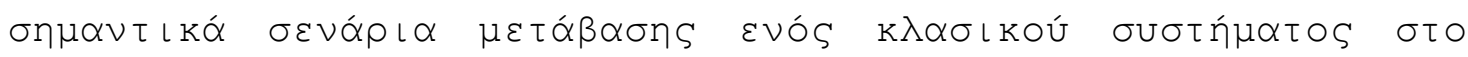

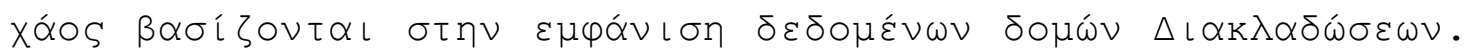

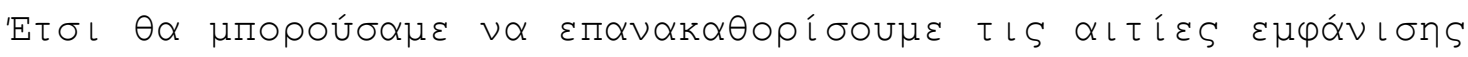

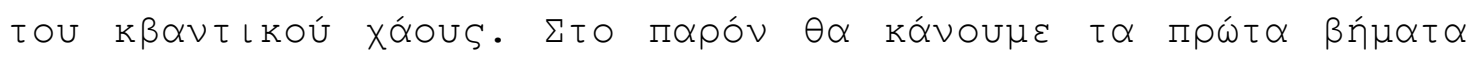

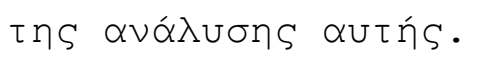

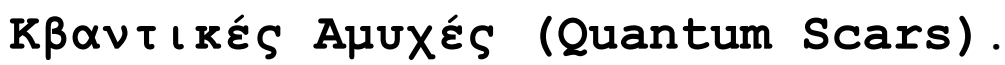

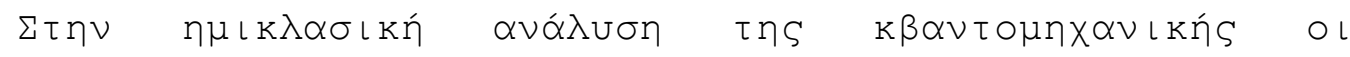

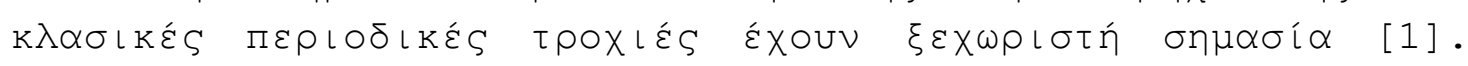
'ЕХદı

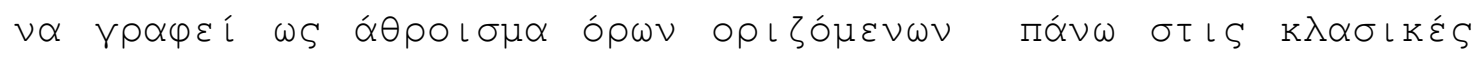

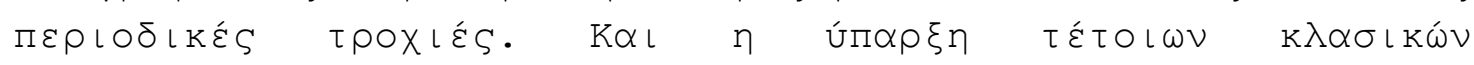

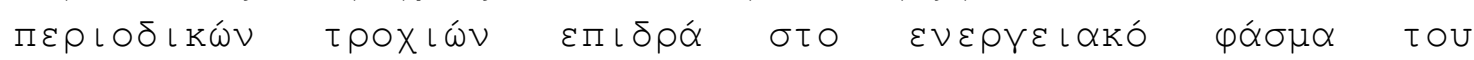

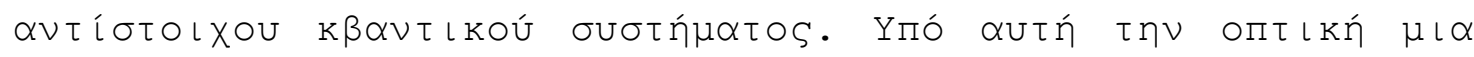

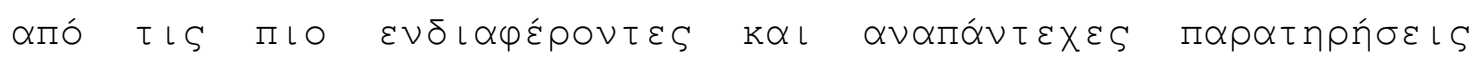

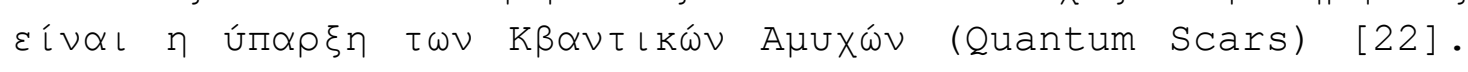

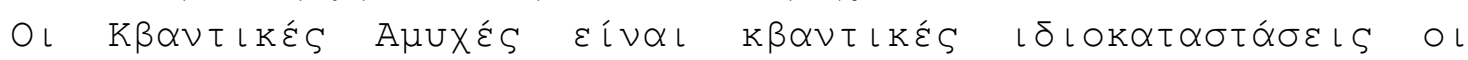

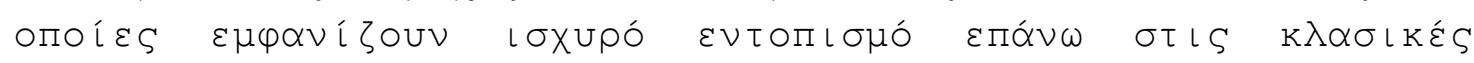

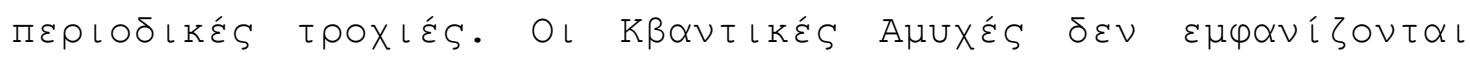

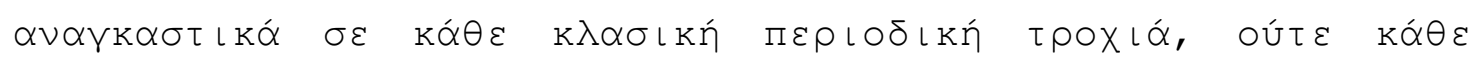

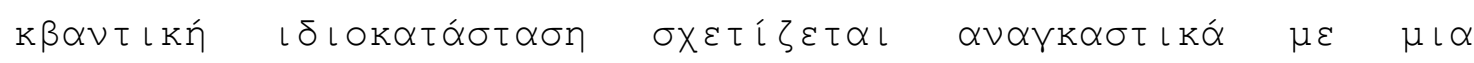

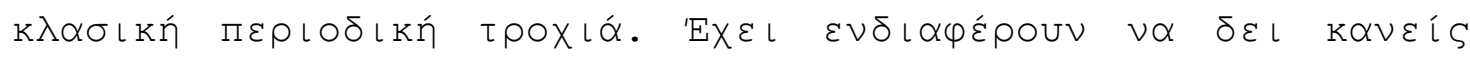

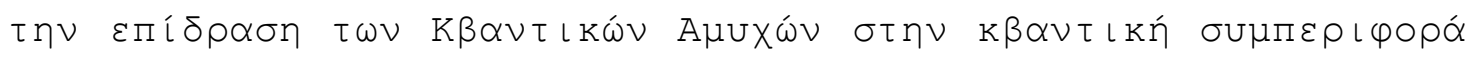

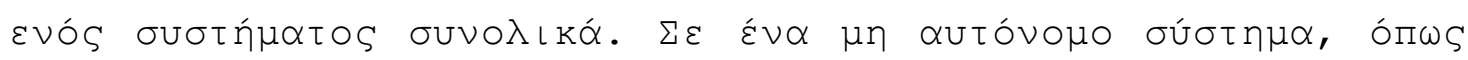

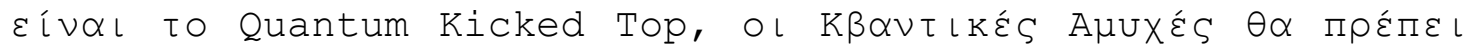




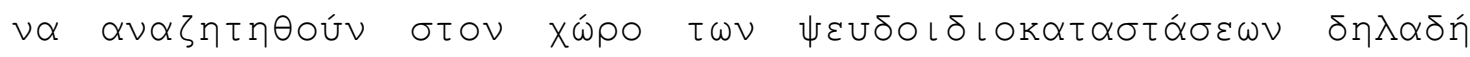

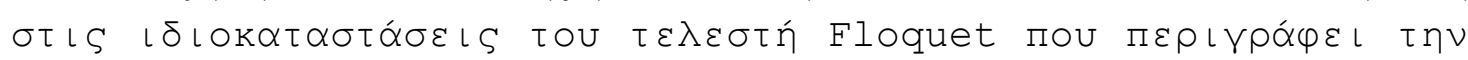

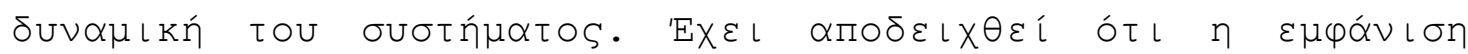

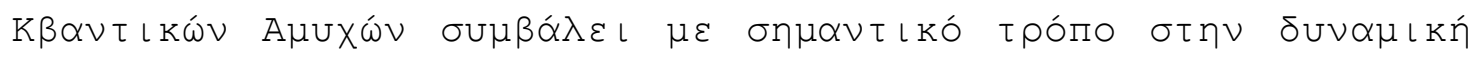

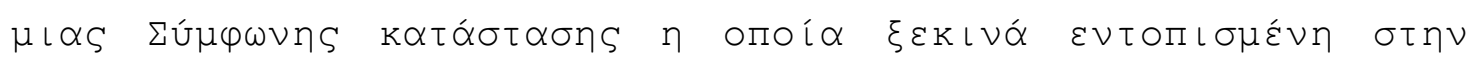

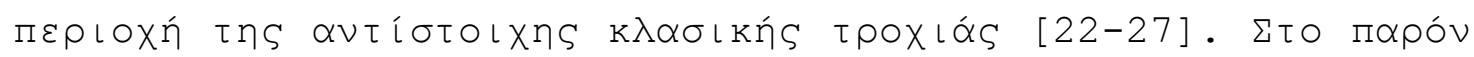

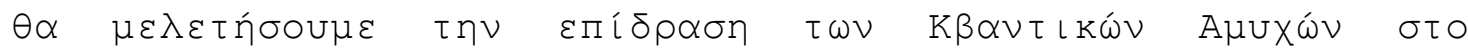

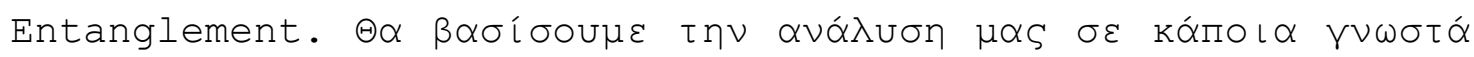
$\delta \varepsilon \delta \circ \mu \varepsilon \dot{\varepsilon} \vee \alpha$.

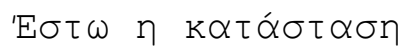

$$
|\gamma\rangle=|\theta, \phi\rangle
$$

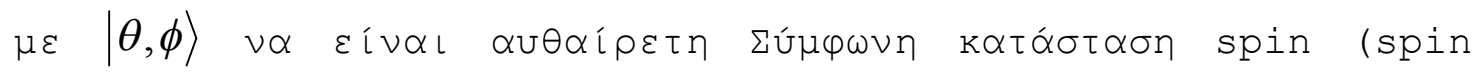

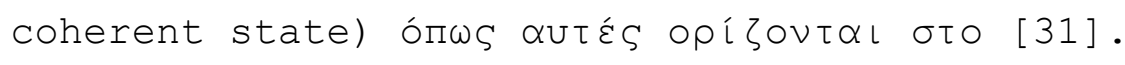

$$
\{|\theta, \phi\rangle=R(\theta, \phi)|j, j\rangle ;-\pi \leqslant \phi \leqslant \pi, 0 \leqslant \theta \leqslant \pi\}
$$

$\mu \varepsilon$

$$
R(\theta, \phi)=\exp \left\{i \theta\left[J_{x} \sin \phi-J_{y} \cos \phi\right]\right\}
$$

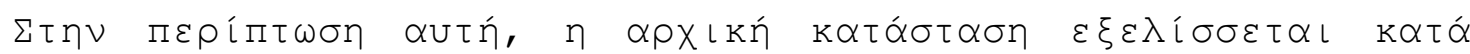

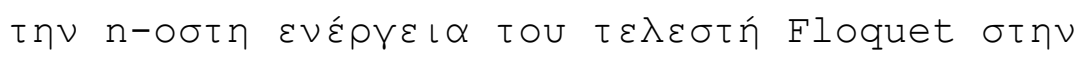

$$
\left|\gamma^{\prime}\right\rangle=\hat{F}^{n}|\gamma\rangle
$$

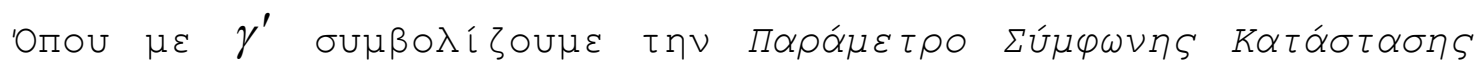

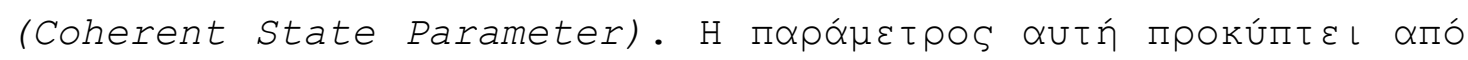

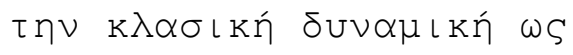

$$
\gamma^{\prime}=\underbrace{T(\cdots T(T(\gamma)))}_{n}
$$

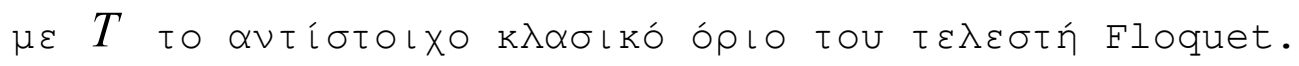

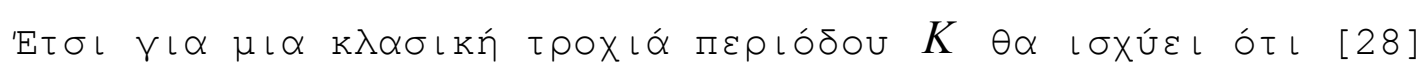




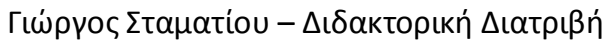

$$
\gamma_{0}=\underbrace{T\left(\cdots T\left(T\left(\gamma_{0}\right)\right)\right)}_{K}
$$

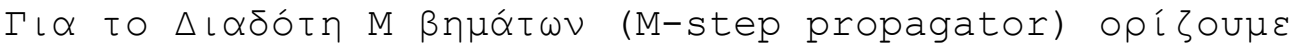

$$
f_{M}=\left\langle\gamma_{0}\left|\hat{F}^{M}\right| \gamma_{0}\right\rangle
$$

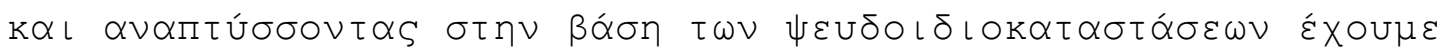

$$
f_{M}=\sum_{n=1}^{N} H_{n}\left(\gamma_{0}\right) e^{i M x_{n}}
$$

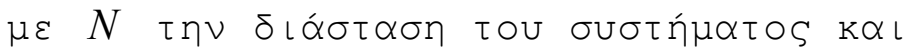

$$
H_{n}=\left|\left\langle\Phi_{n} \mid \gamma_{0}\right\rangle\right|^{2}
$$

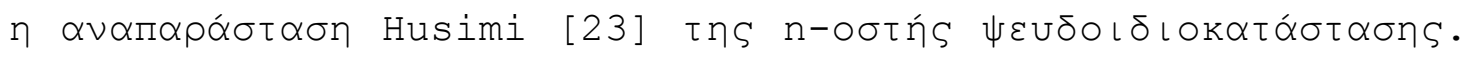

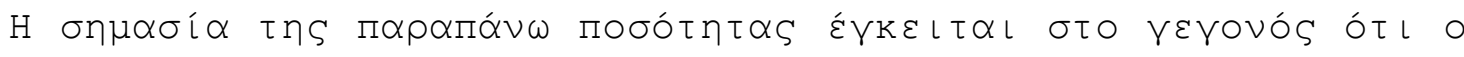
$\mu \varepsilon \tau \alpha \sigma \times \eta \mu \alpha \tau \iota \sigma \mu o ́ s$ Fourier

$$
\hat{f}_{\omega}=\sum_{M=-L}^{L} e^{-i \omega M} f_{M}=\sum_{n=1}^{N} H_{n}\left(\gamma_{0}\right) \sum_{M=-L}^{L} e^{i M\left(\phi_{n}-\omega\right)}
$$

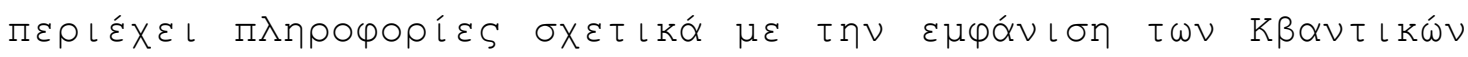

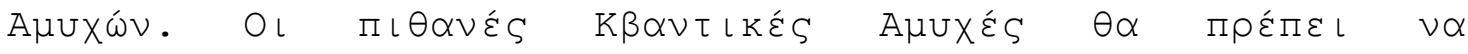

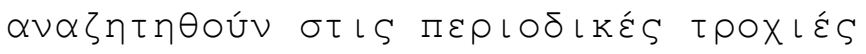

$$
\Gamma=\left(\gamma_{0}, \gamma_{1}, \ldots ., \gamma_{M-1}\right)
$$

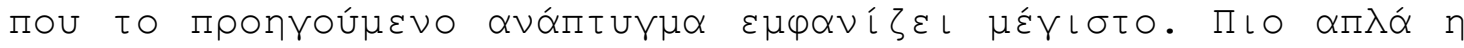

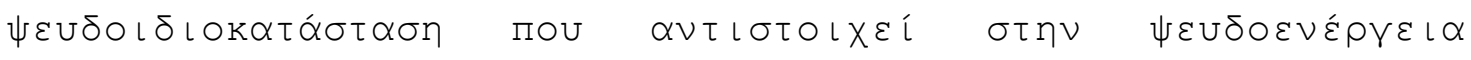

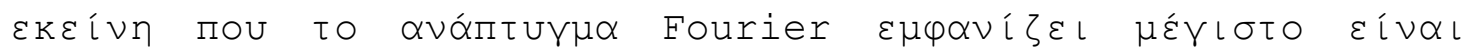

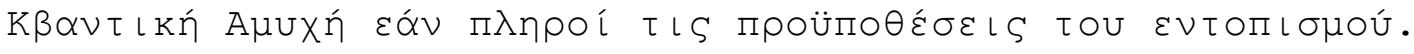

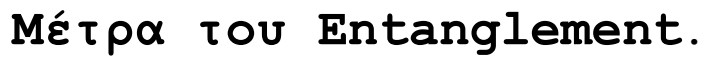




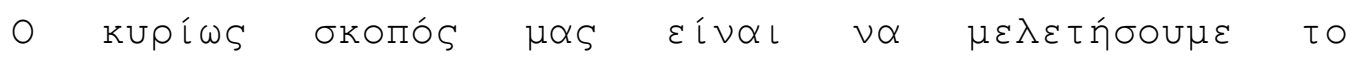

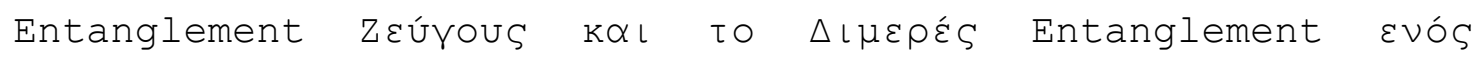

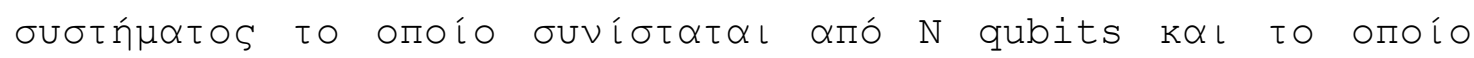

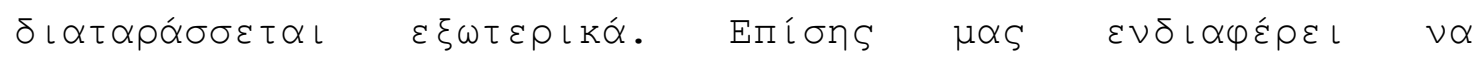

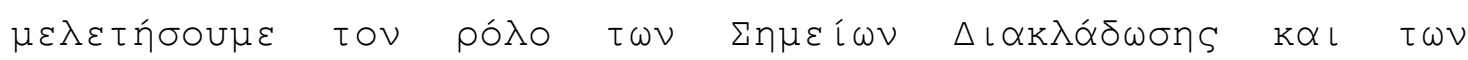

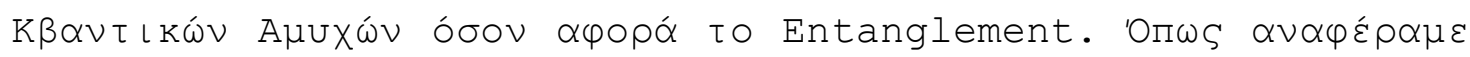

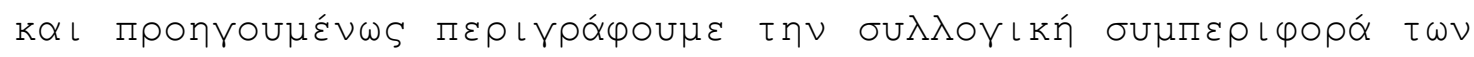

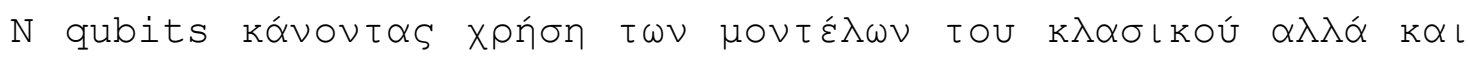

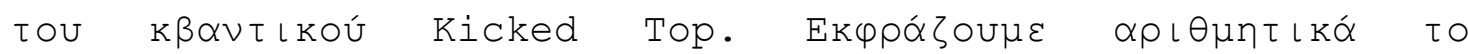

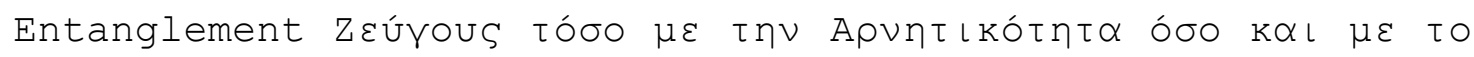

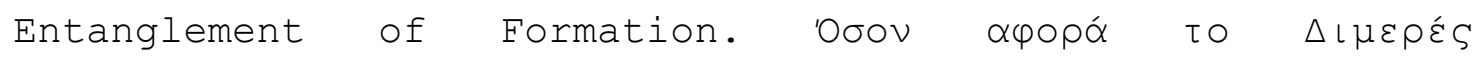

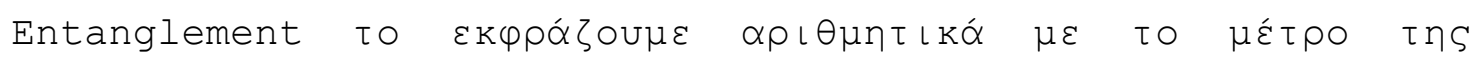

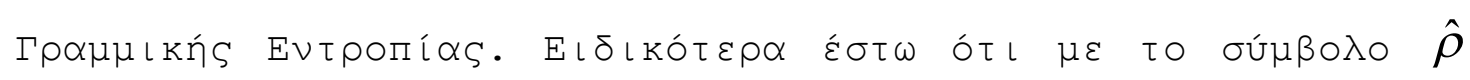

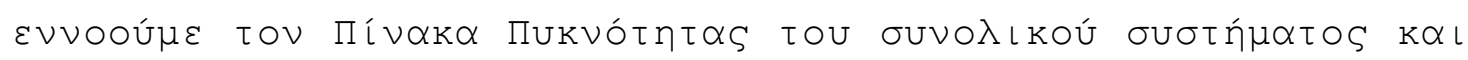

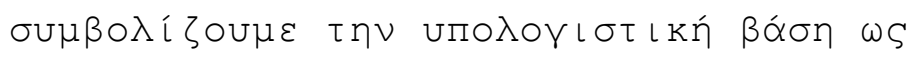

$$
\left|i_{1} i_{2} \cdots i_{N}\right\rangle i_{k} \in\{0,1\}
$$

Eníons

$$
\hat{\rho}_{k}=\operatorname{tr}_{A L L \neq k}(\hat{\rho})
$$

$K \propto l$

$$
\hat{\rho}_{k m}=\operatorname{tr}_{A L L \neq k, m}(\hat{\rho})
$$

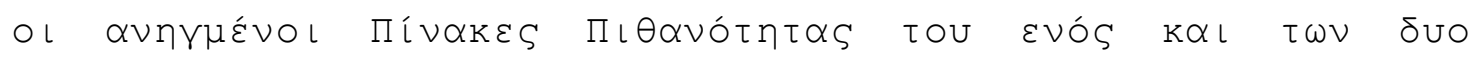

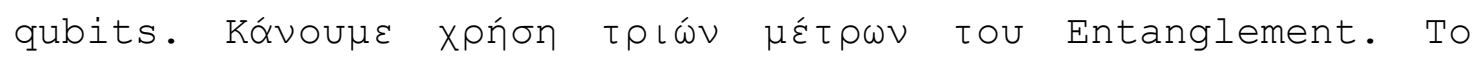

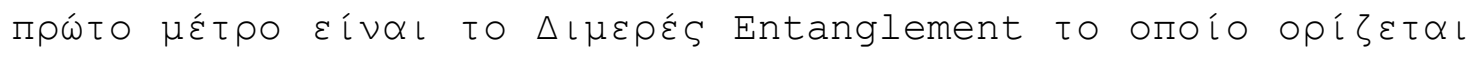
$\omega S$

$$
Q \equiv 2-\frac{2}{N} \sum_{i} \operatorname{tr}\left(\hat{\rho}_{i}^{2}\right)
$$

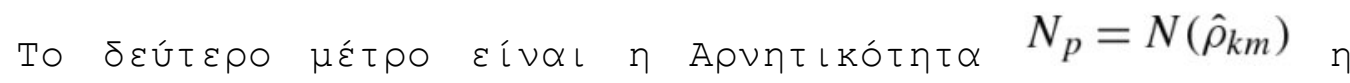

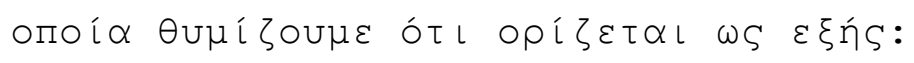

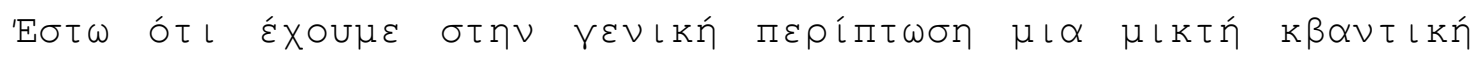

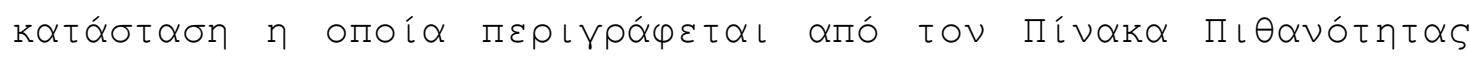

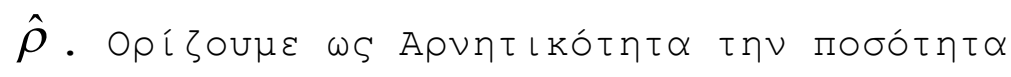




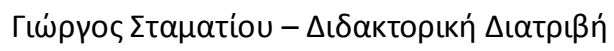

$$
N(\hat{\rho})=\sum_{j} \max \left\{0,-\mu_{j}\right\}
$$

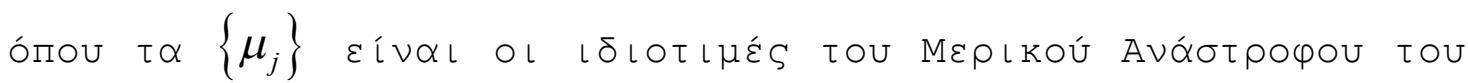
$\hat{\rho}$.

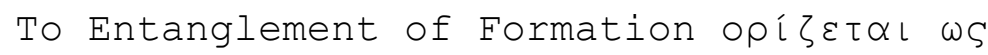

$$
E_{f}=H\left(\frac{1+\sqrt{1-C^{2}}}{2}\right)
$$

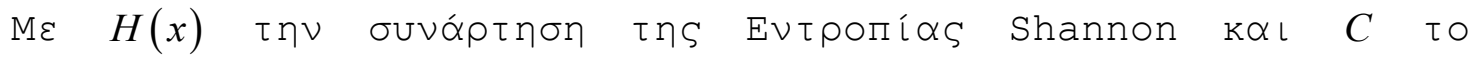

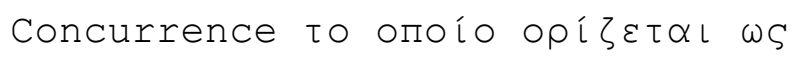

$$
C=\max \left\{0, \lambda_{1}-\lambda_{2}-\lambda_{3}-\lambda_{4}\right\}
$$

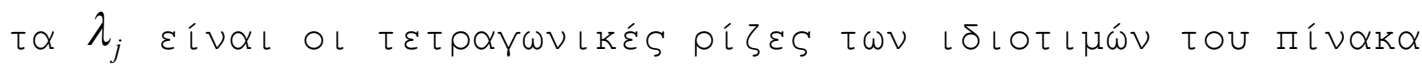

$$
\hat{\rho}_{12}\left(\sigma_{1 y} \otimes \sigma_{2 y}\right) \hat{\rho}_{12}^{*}\left(\sigma_{1 y} \otimes \sigma_{2 y}\right)
$$

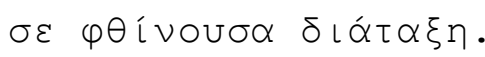

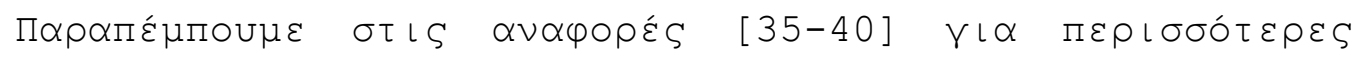

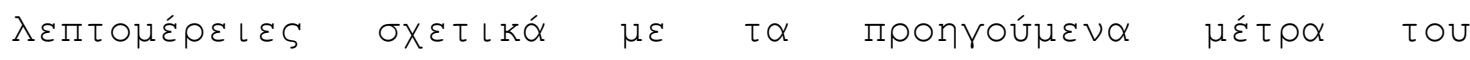
Entanglement.
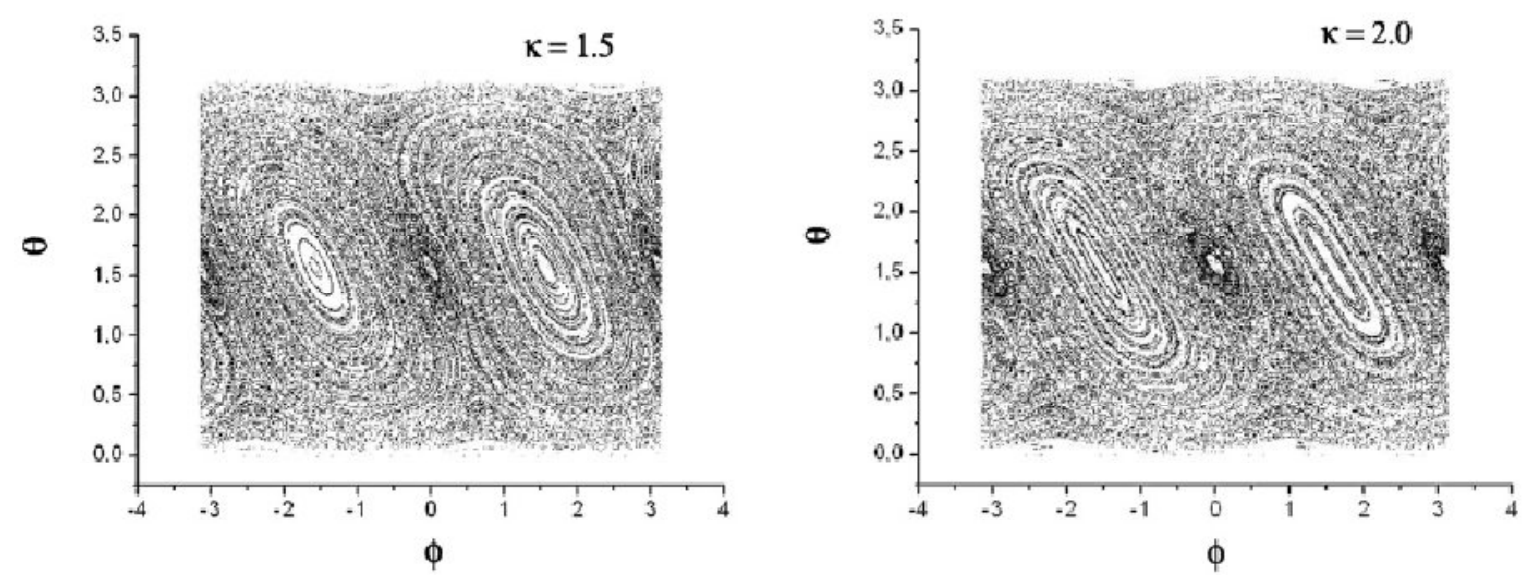

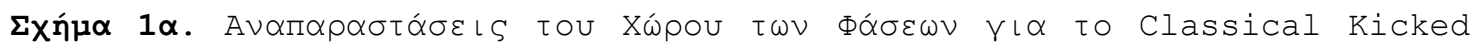

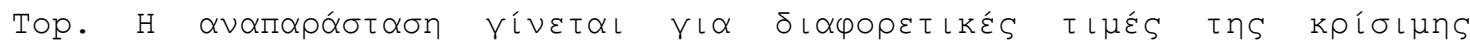




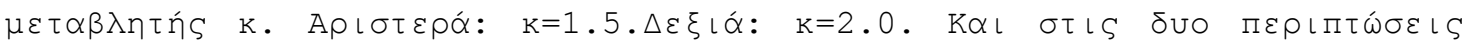

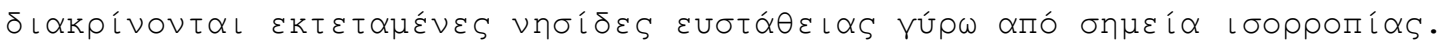
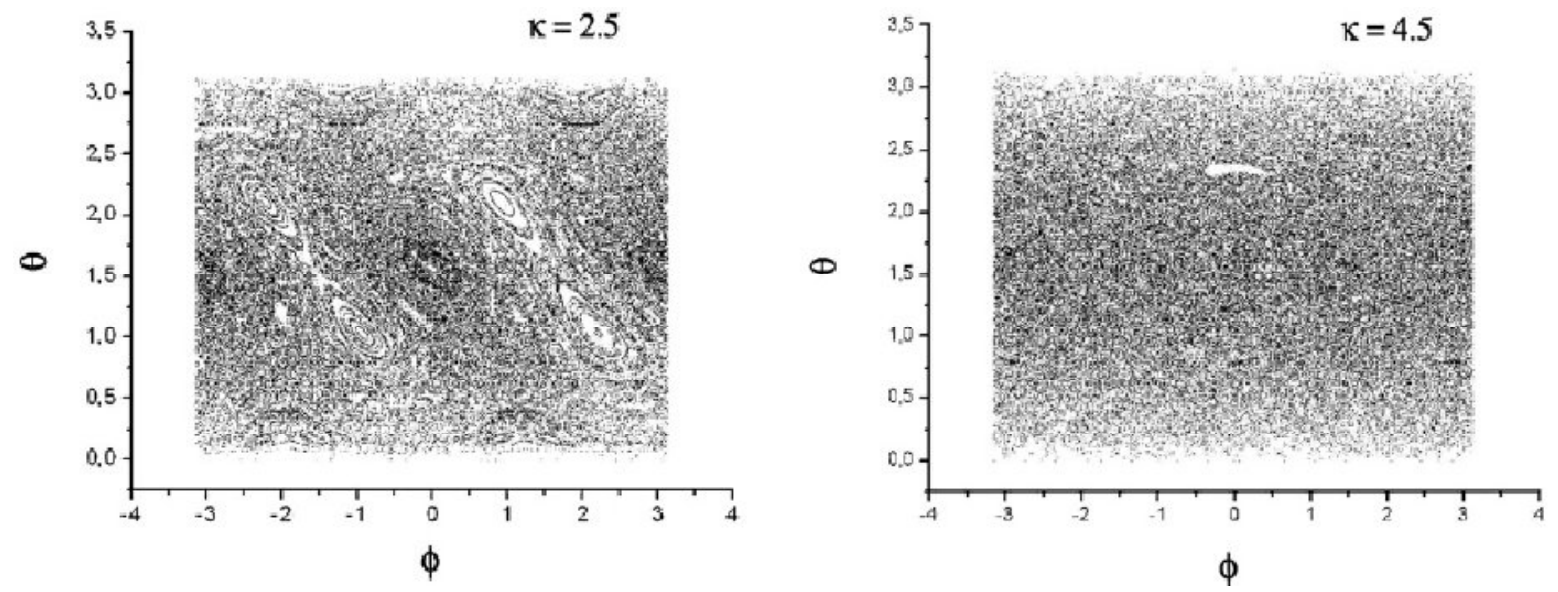

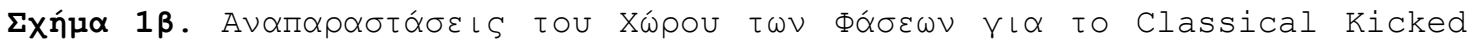

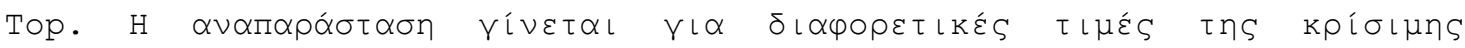

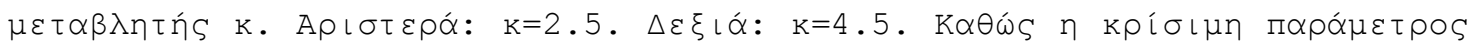

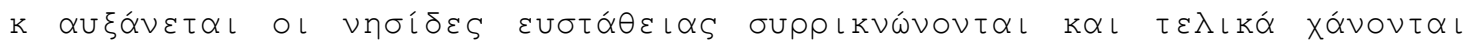

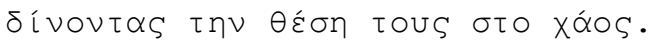

\section{Aпо $\varepsilon \lambda \varepsilon \dot{\varepsilon} \sigma \alpha \tau \alpha$.}

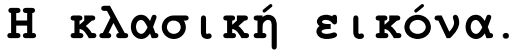

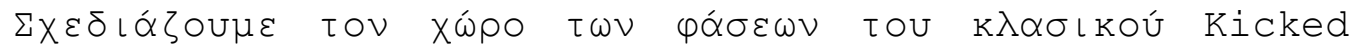

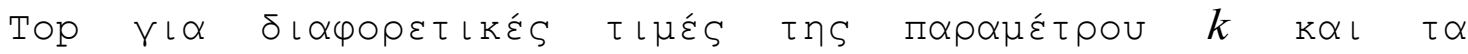

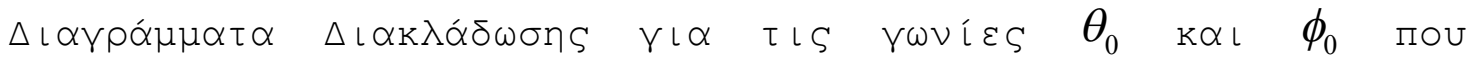

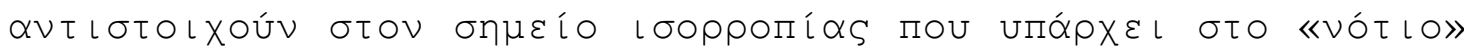

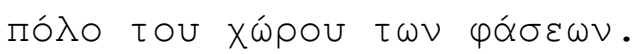

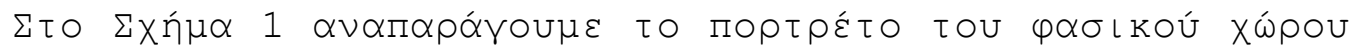

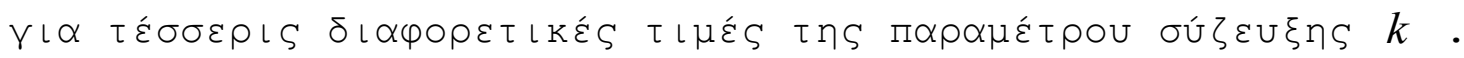

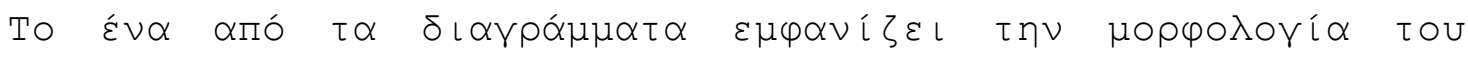

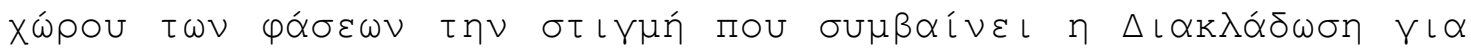

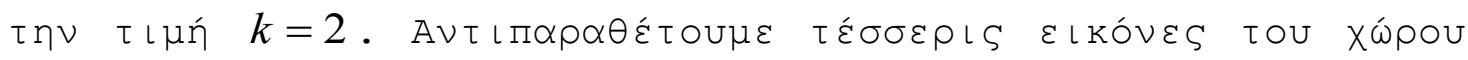

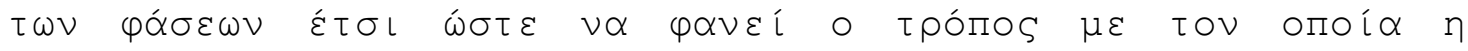

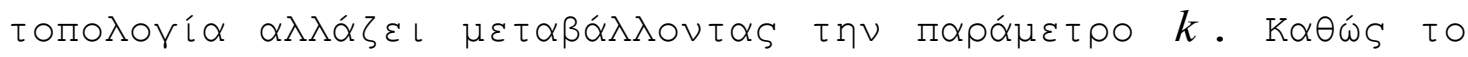

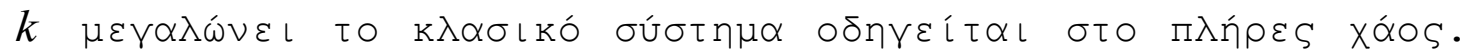




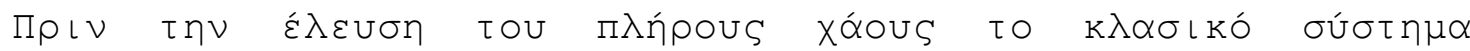

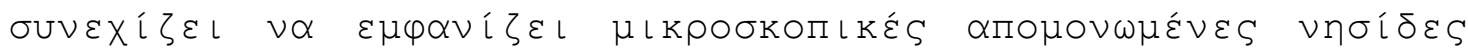

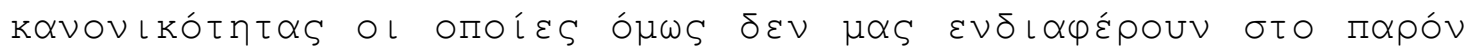

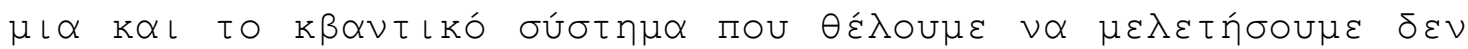

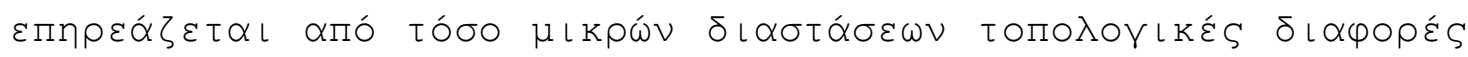

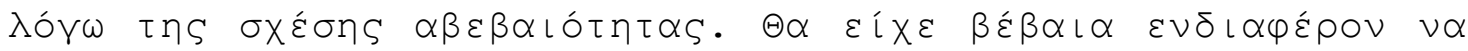

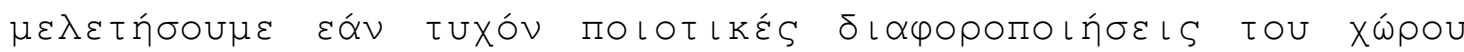

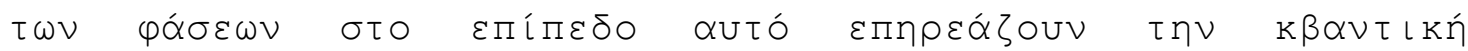

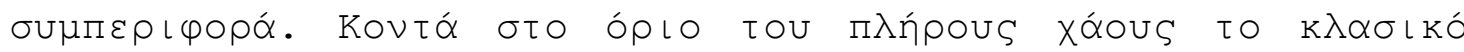

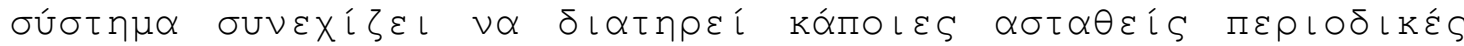

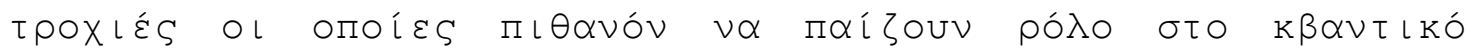

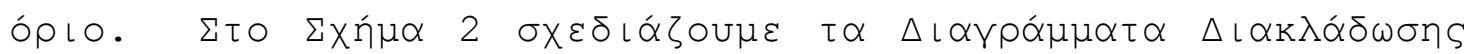

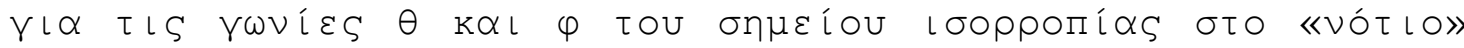

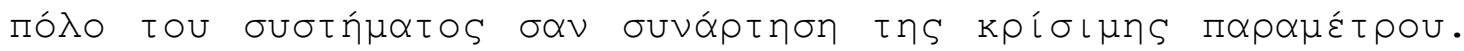

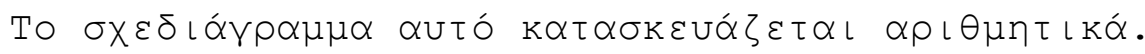

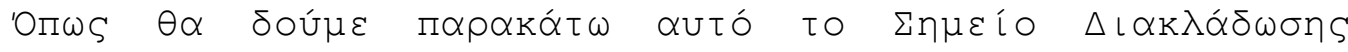

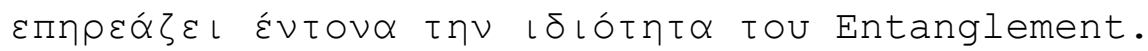

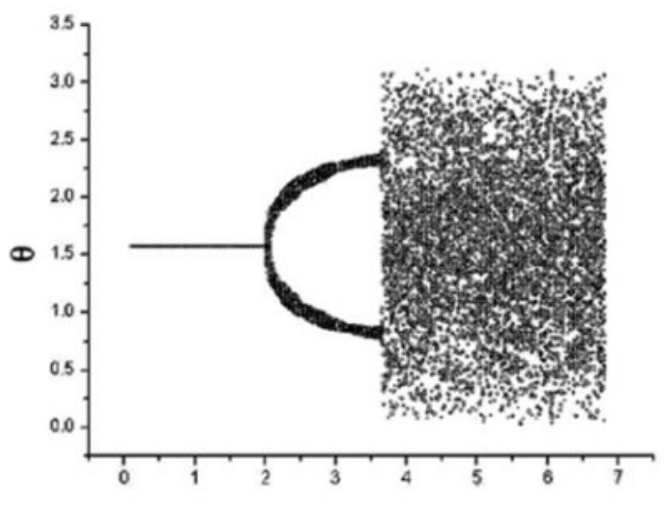

$\kappa$

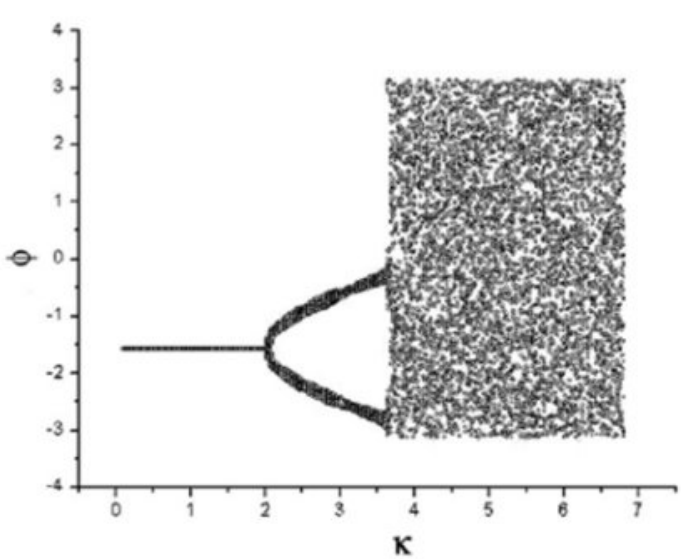

K

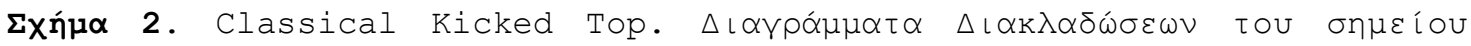

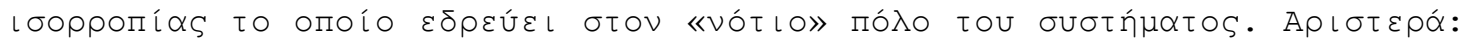

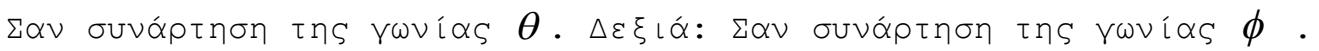




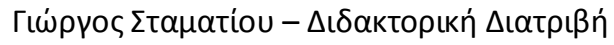
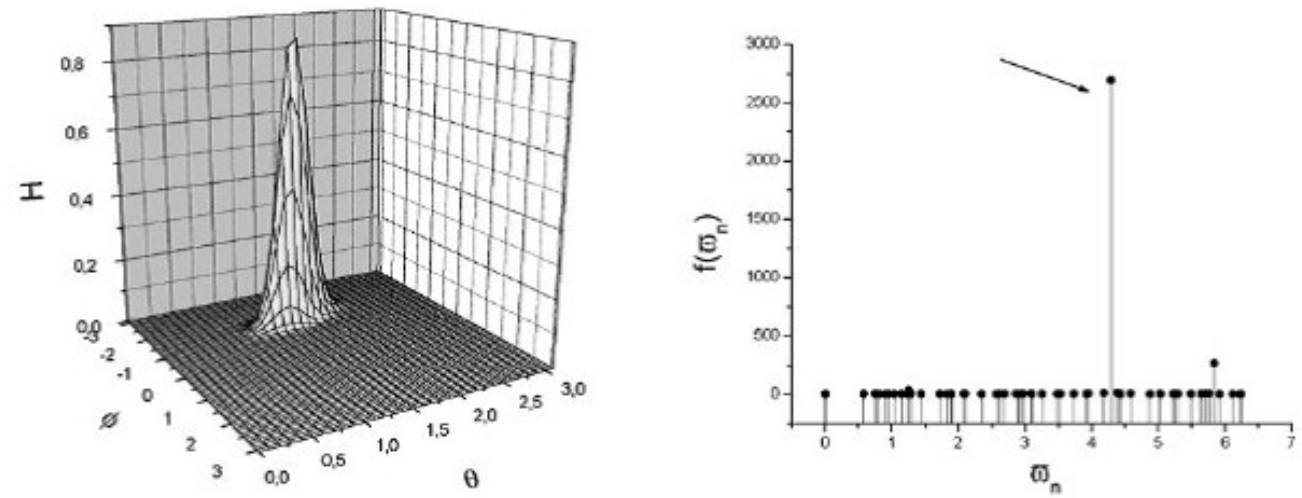

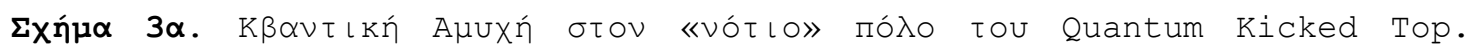

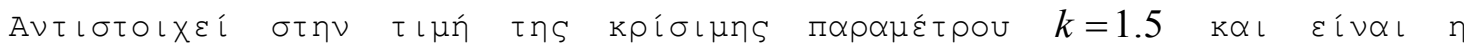

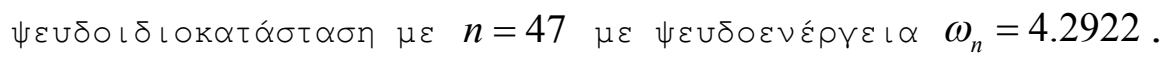
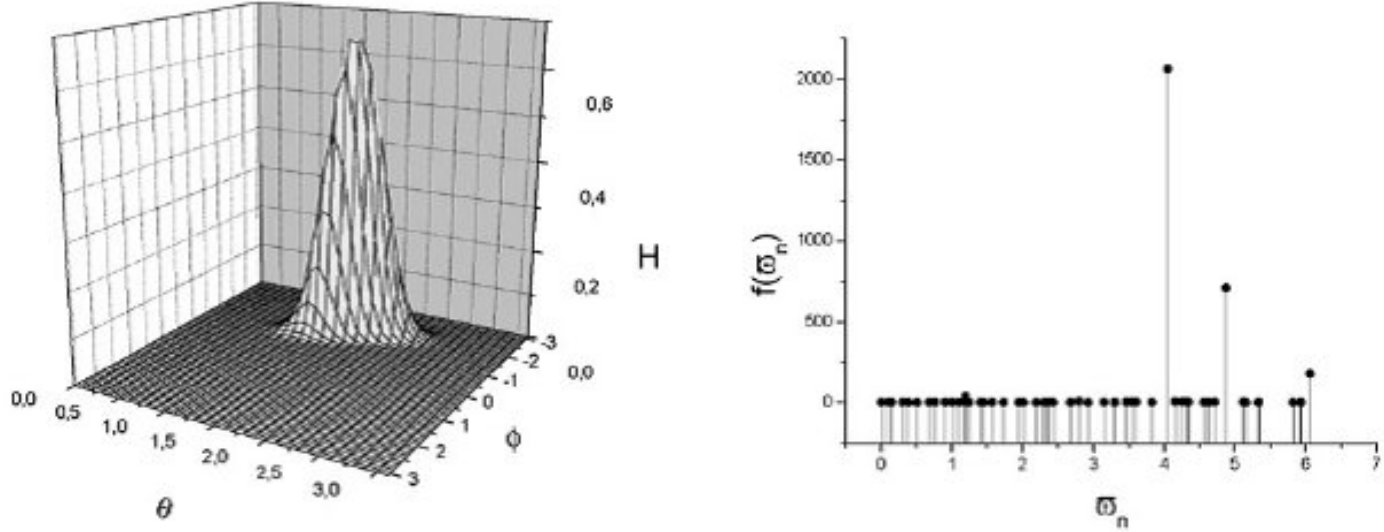

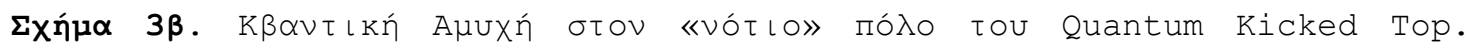

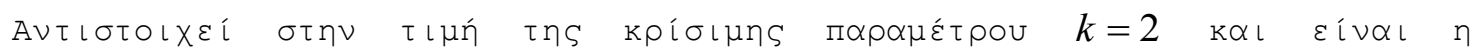

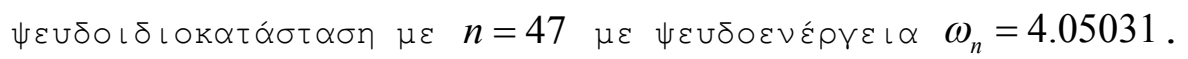



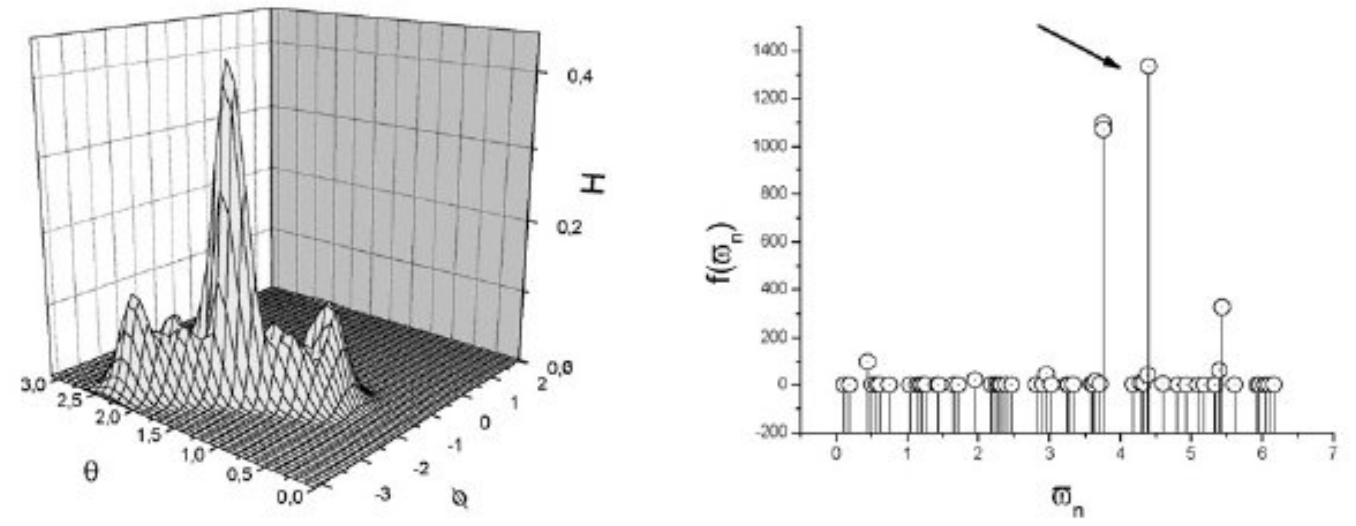

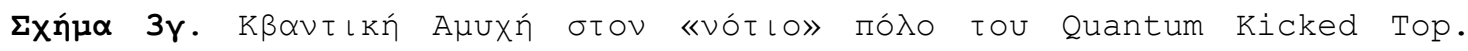

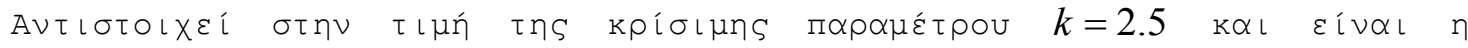

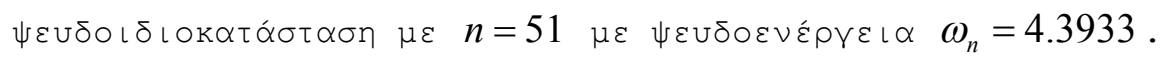
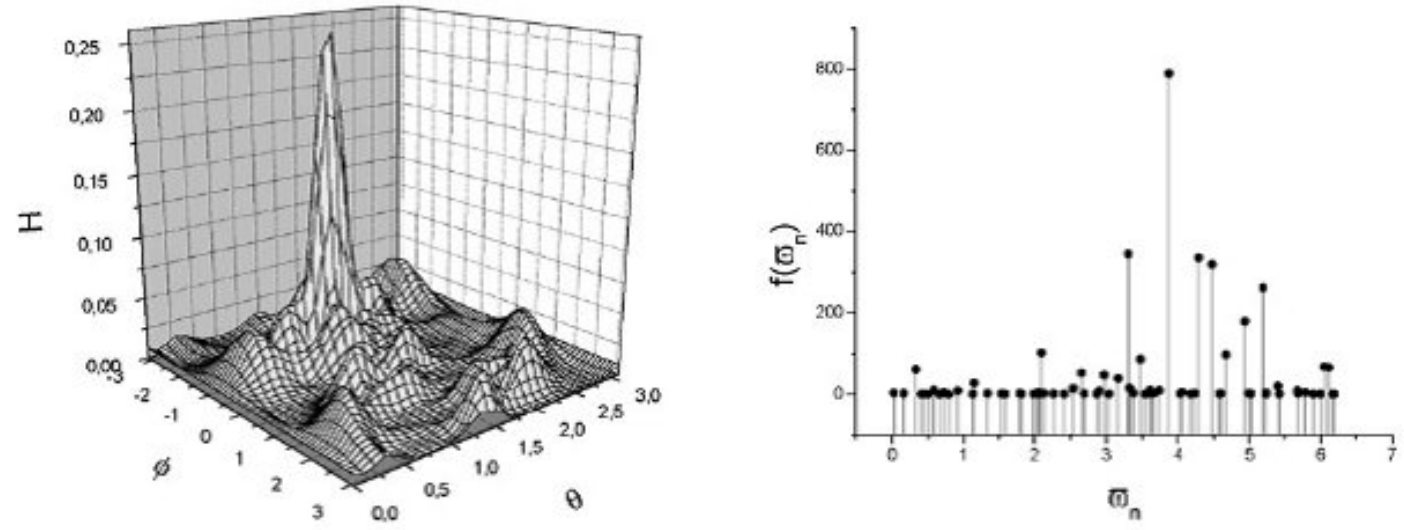

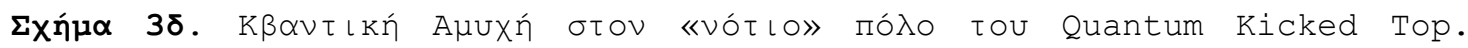

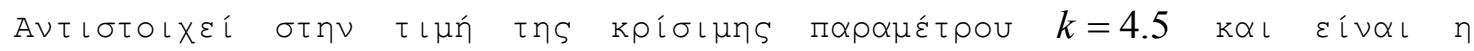
$\psi \varepsilon \cup \delta \circ\llcorner\delta\llcorner\circ \kappa \alpha \tau \alpha ́ \sigma \tau \alpha \sigma \eta \eta$ 


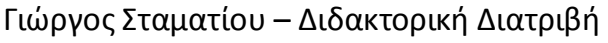
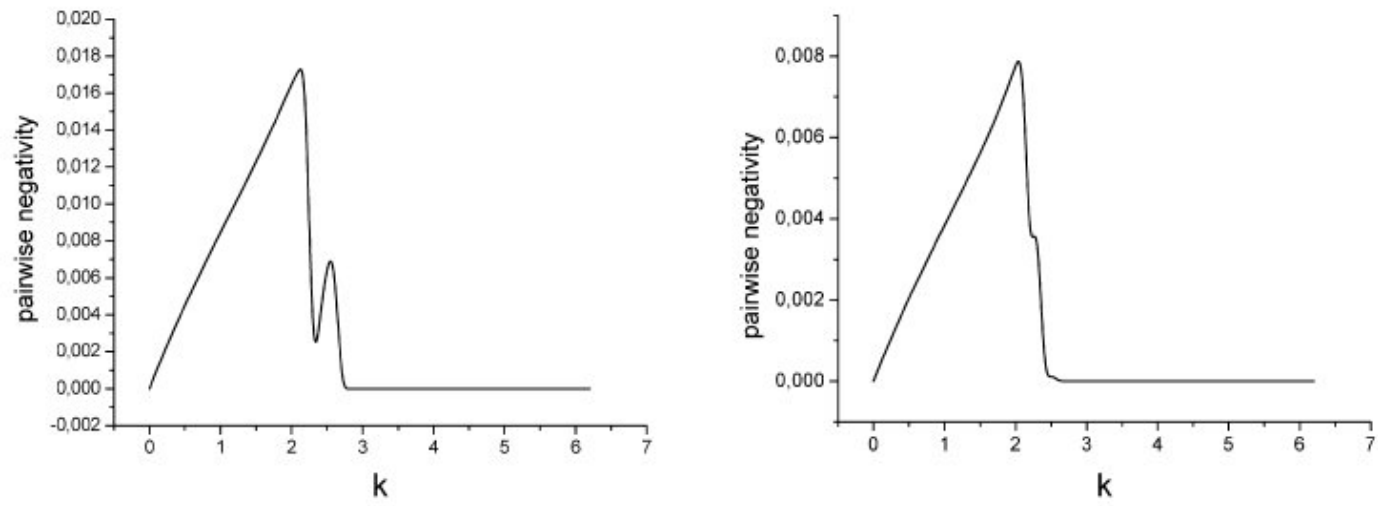

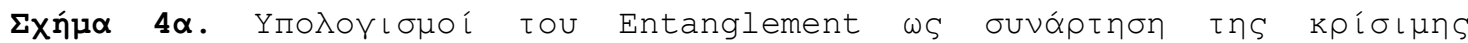

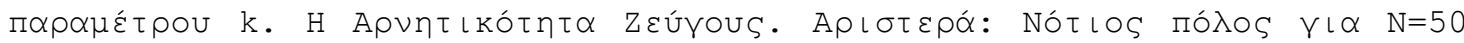

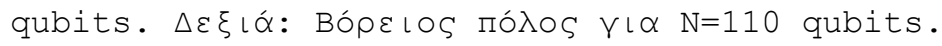
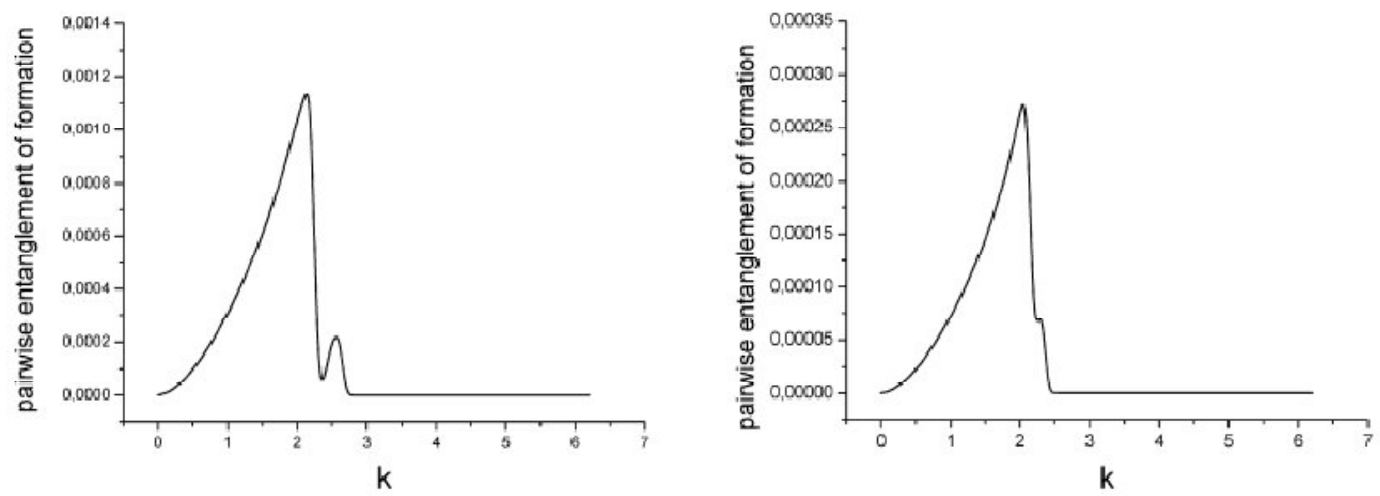

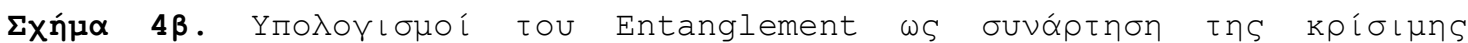

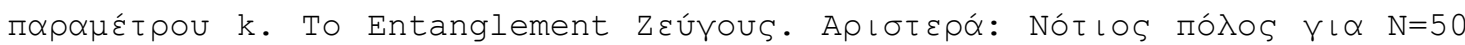

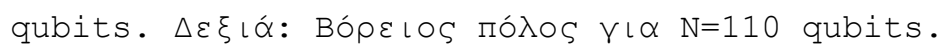



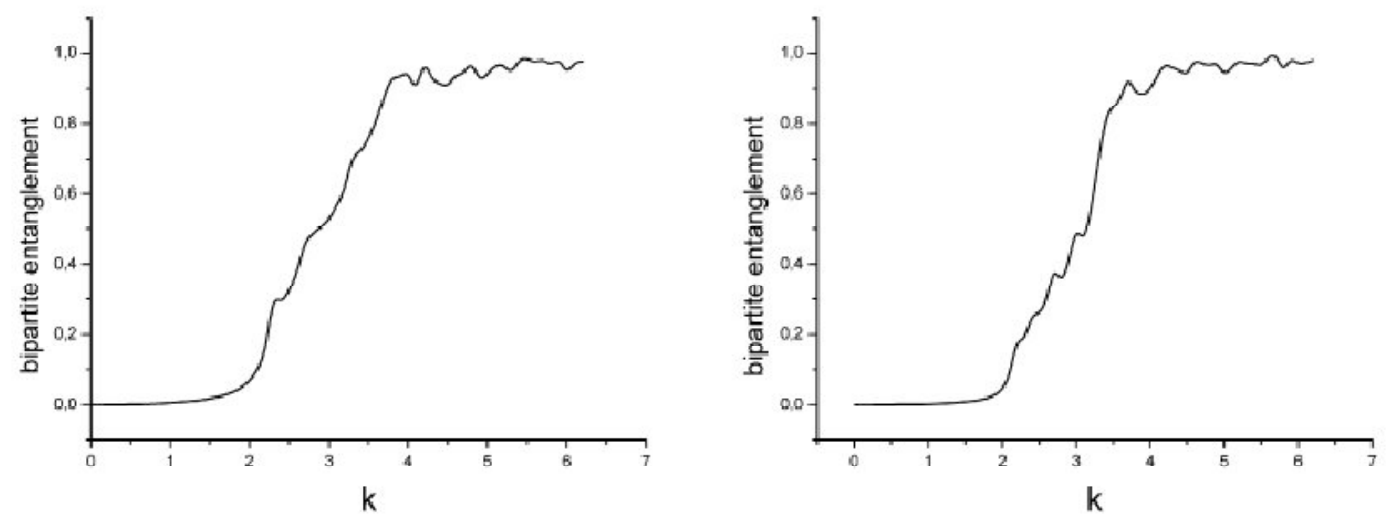

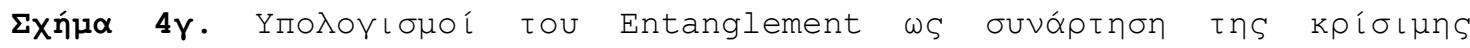

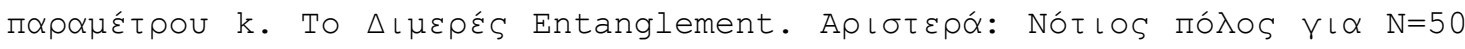

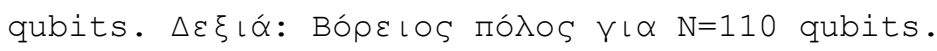

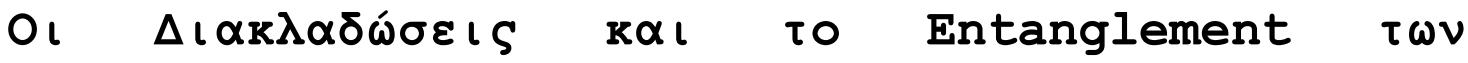

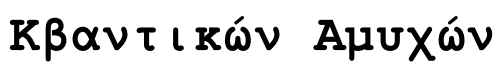

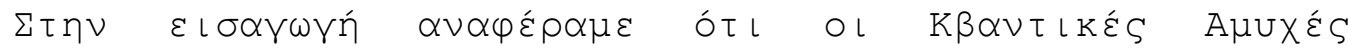

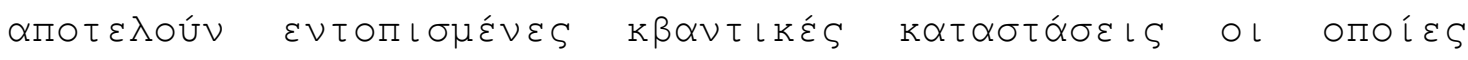

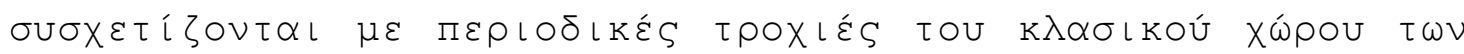

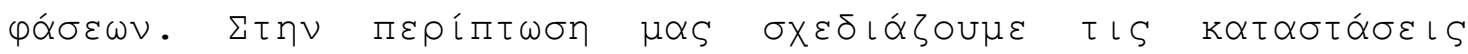

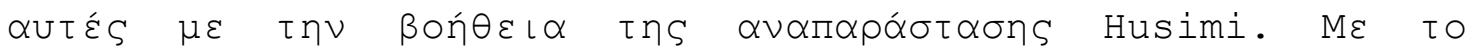

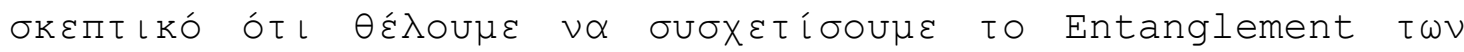

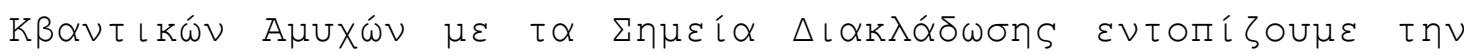

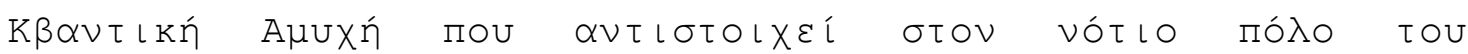

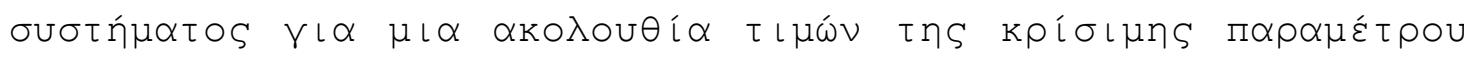

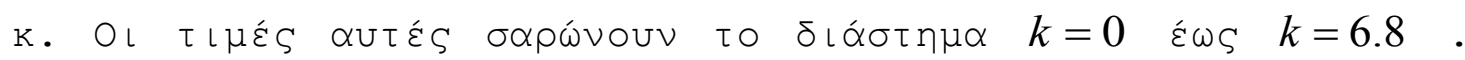

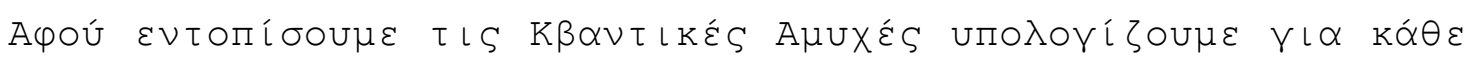

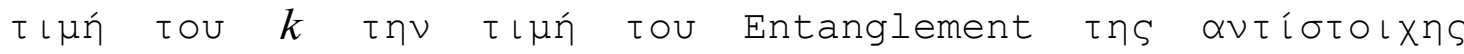

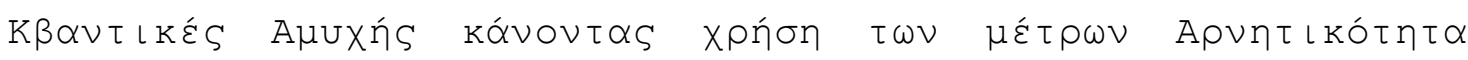
Zeúrous (Pairwise Negativity), Entanglement of Formation

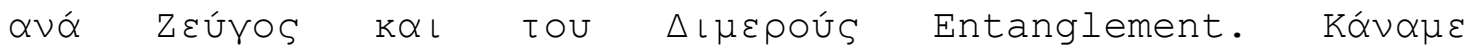

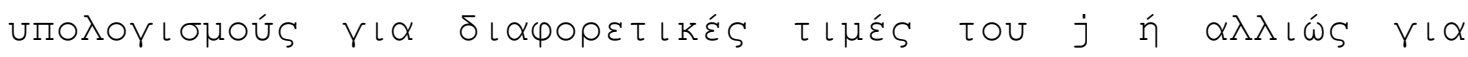

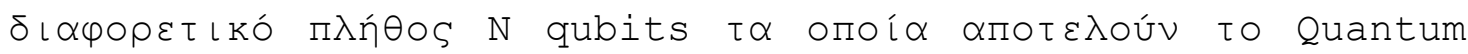
Kicked Top поч $\mu \varepsilon \lambda \varepsilon \tau \alpha ́ \alpha \varepsilon$.

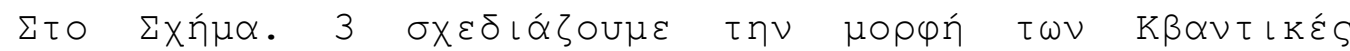

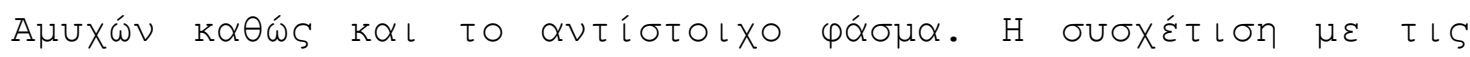

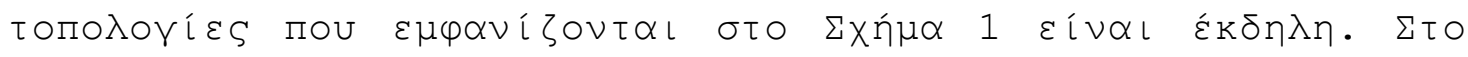




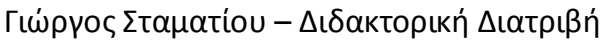

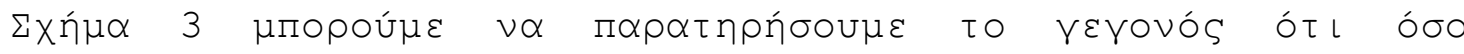

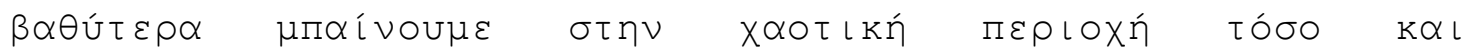

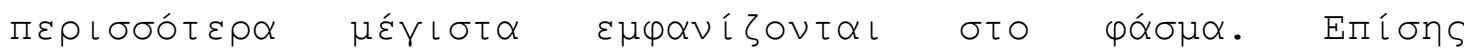

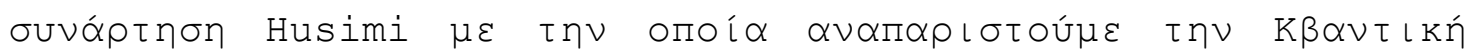

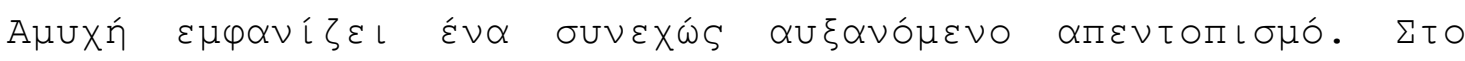

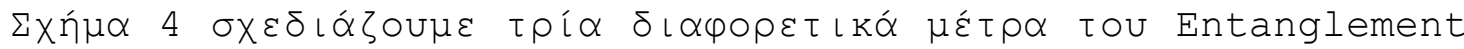

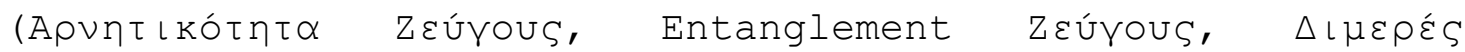

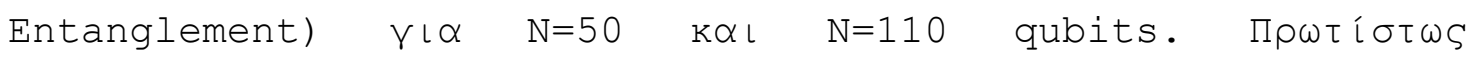

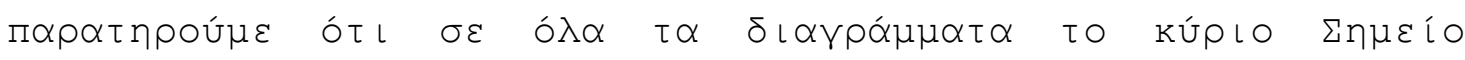

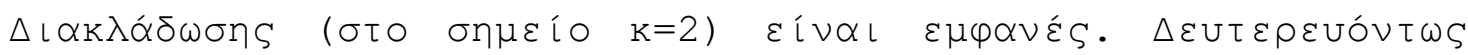

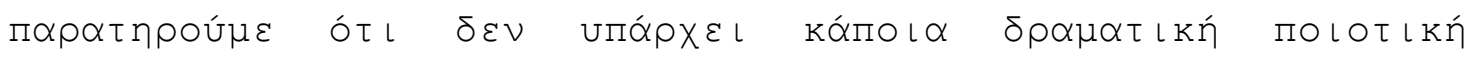

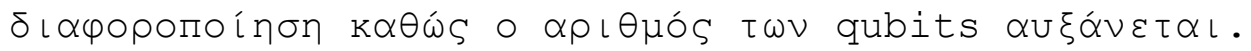
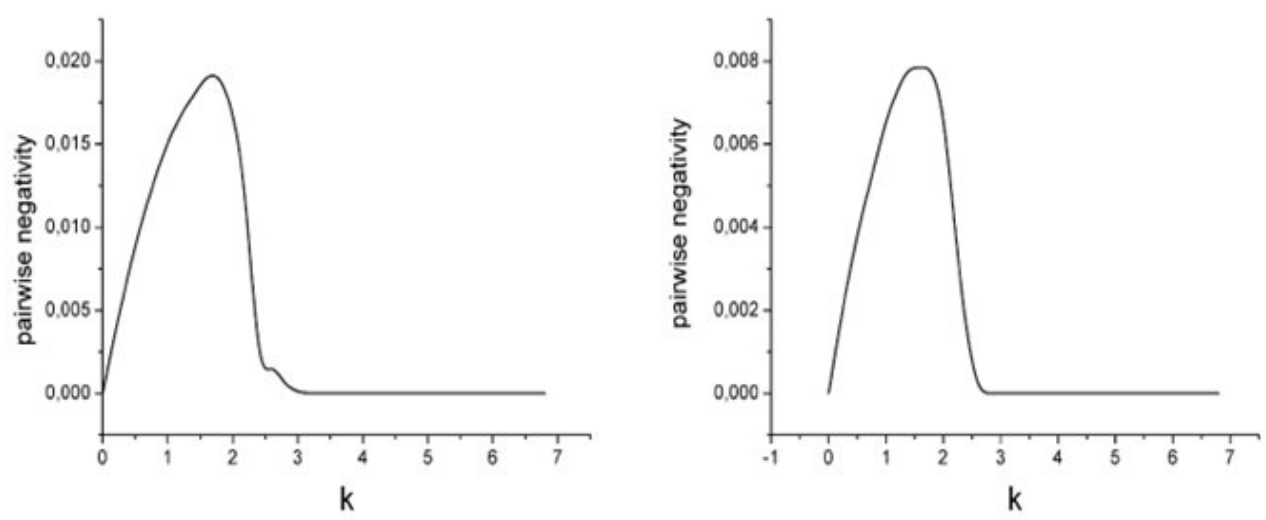

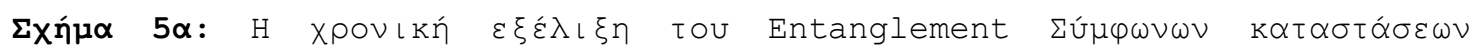

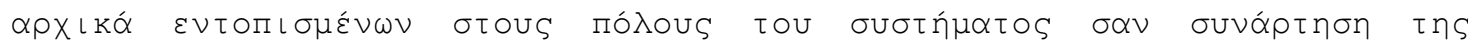

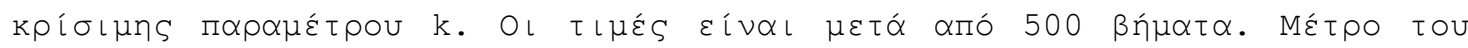

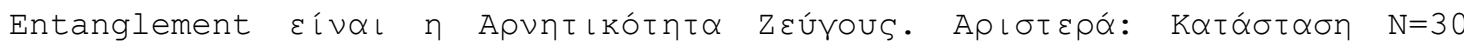

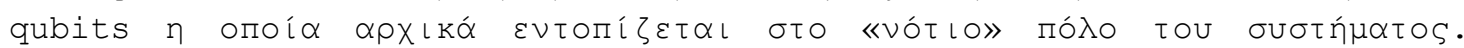

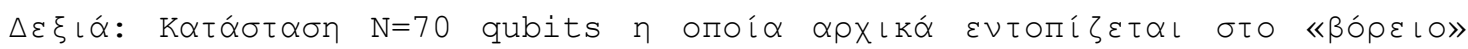

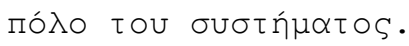



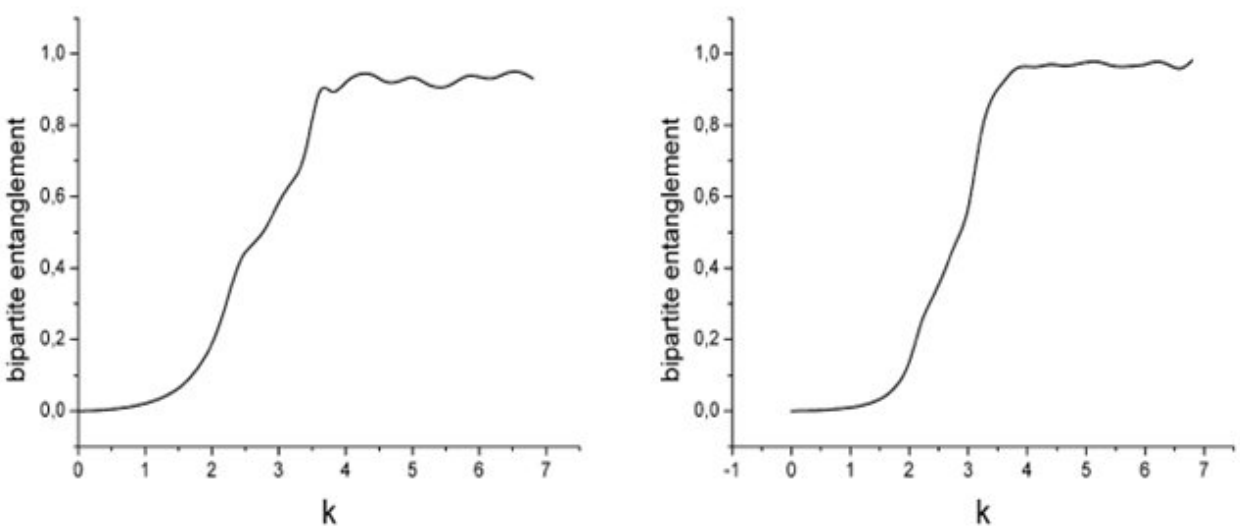

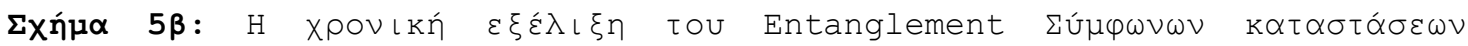

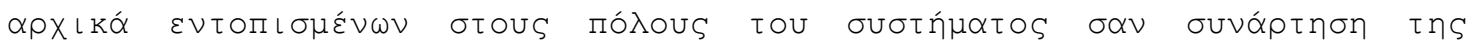

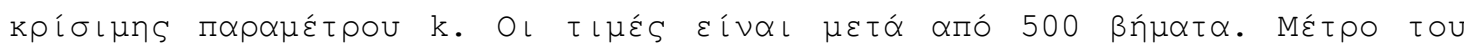

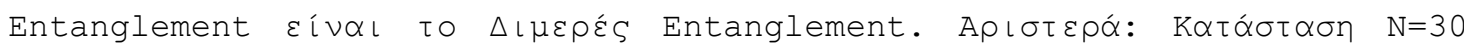

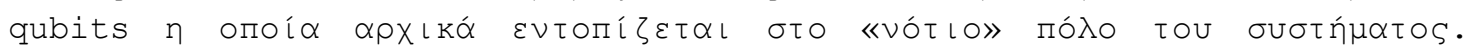

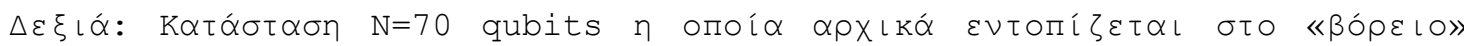

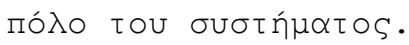

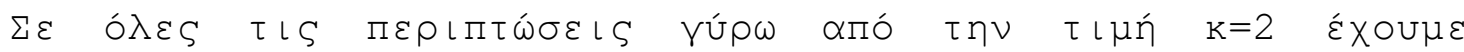

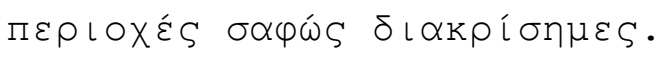

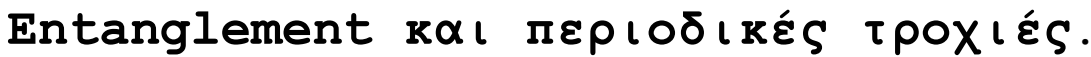

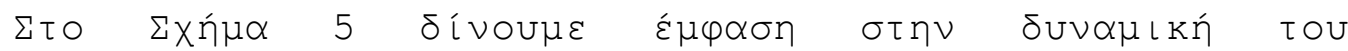

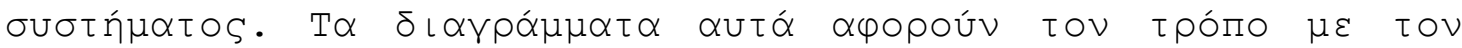

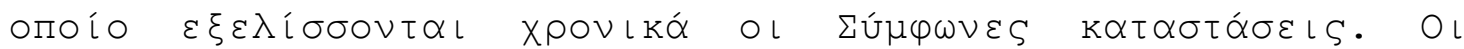

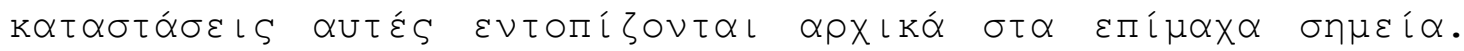

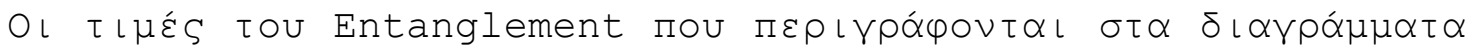

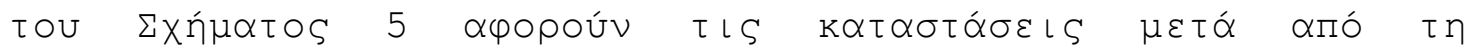

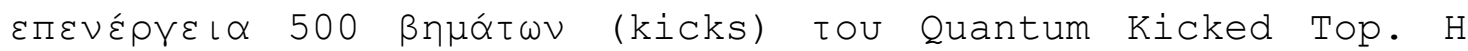

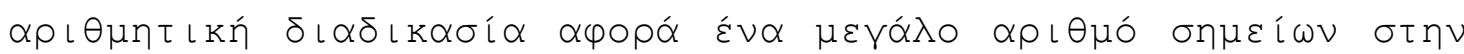

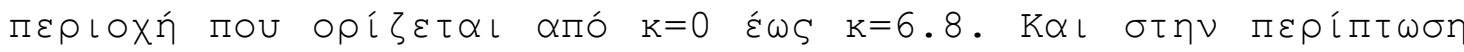

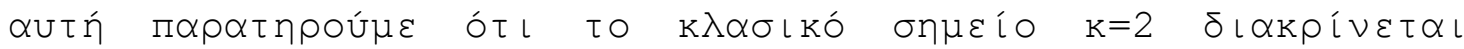

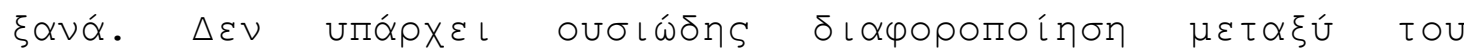

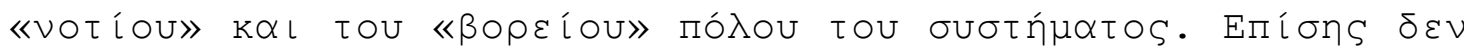

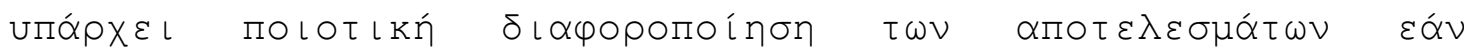

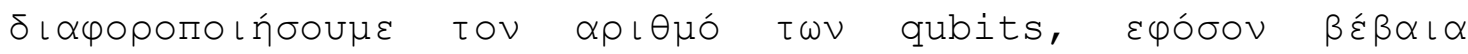

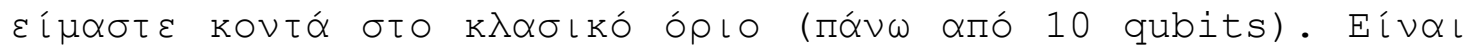




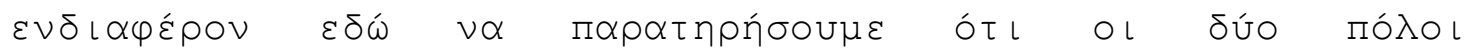

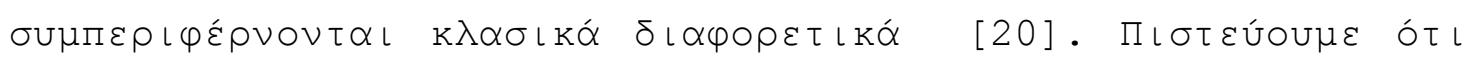

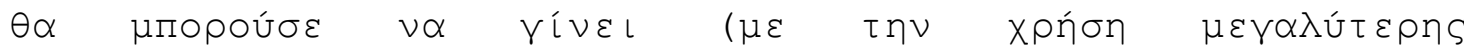

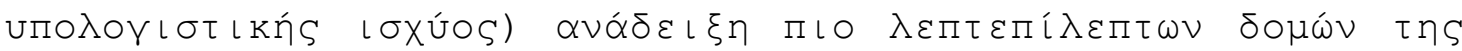

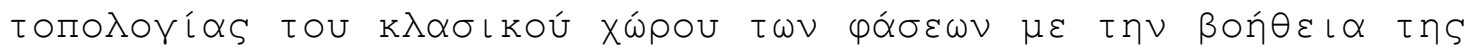

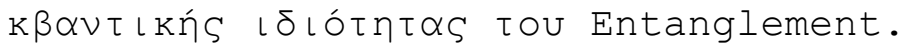

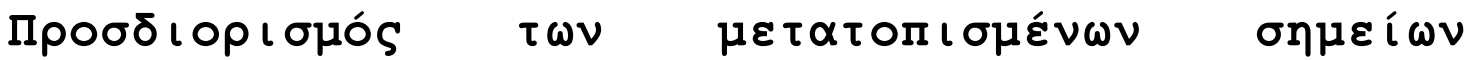

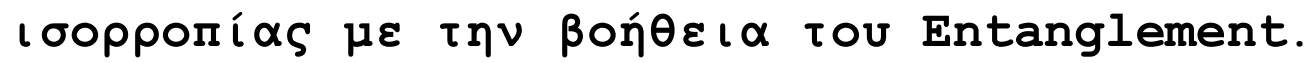

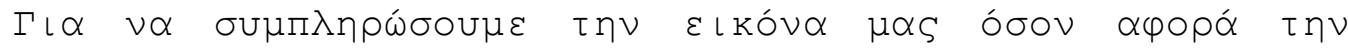

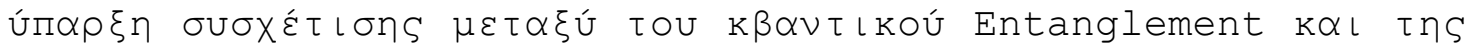

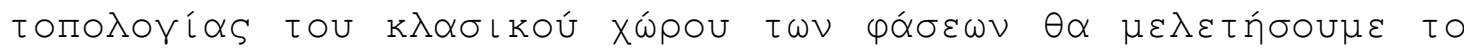

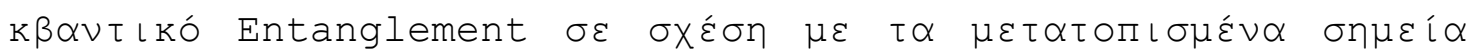

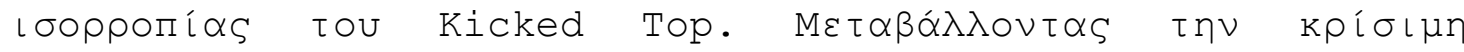

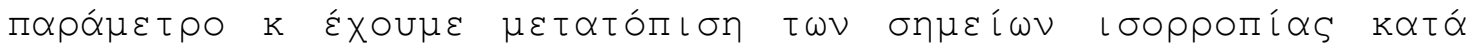

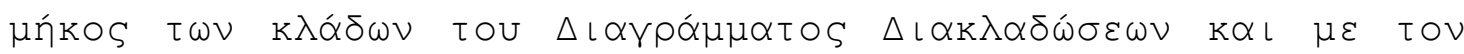

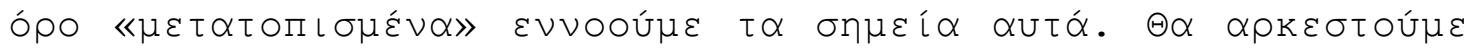

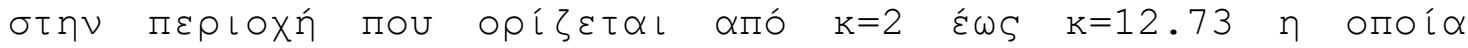

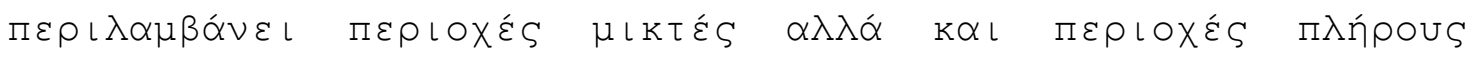

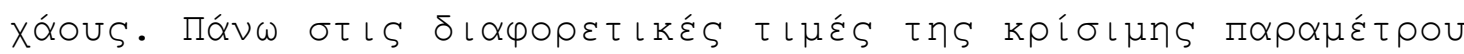

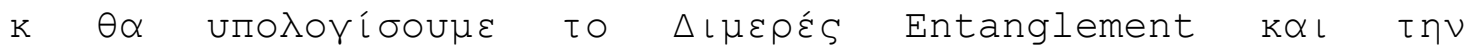

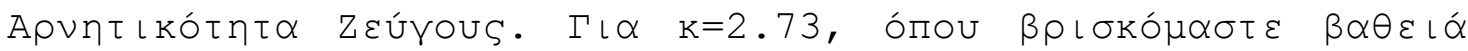

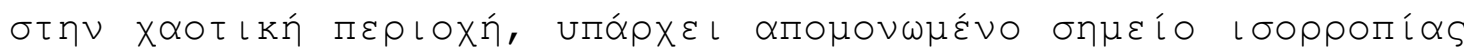

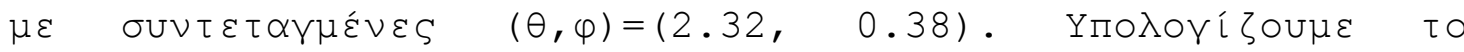

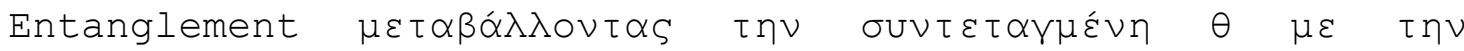

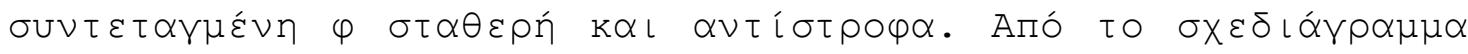

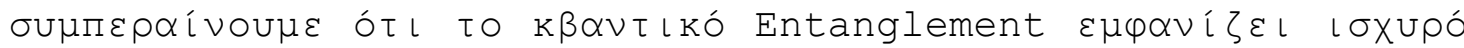

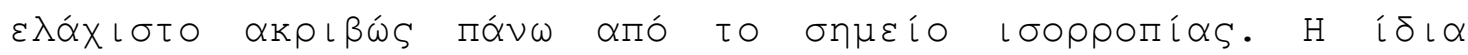

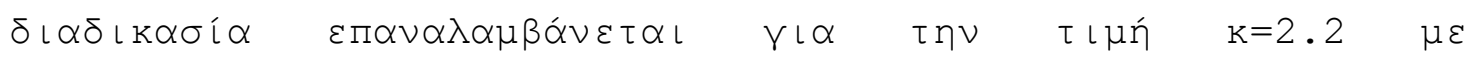

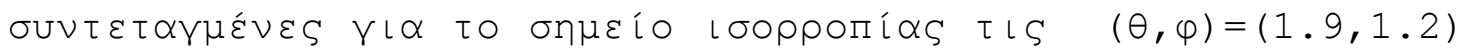

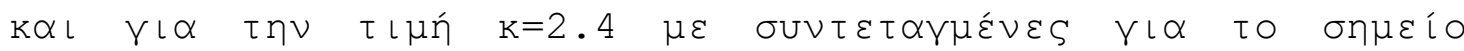

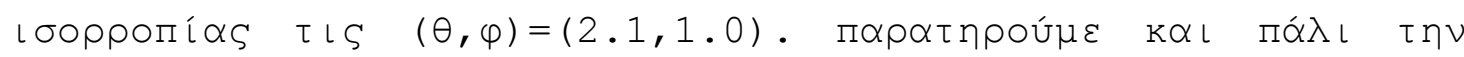

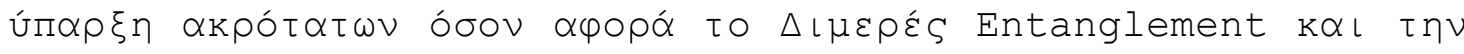

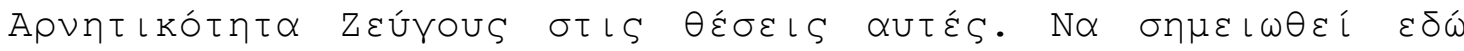

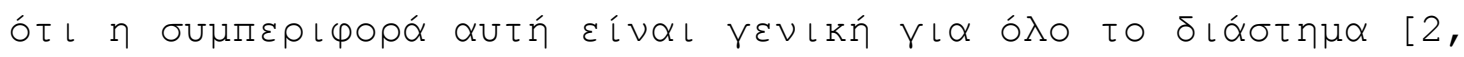

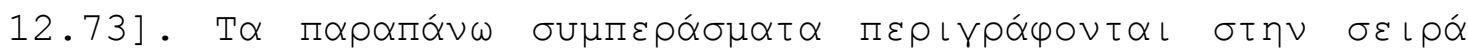

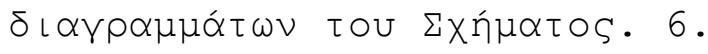




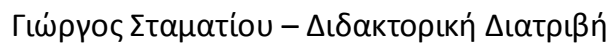
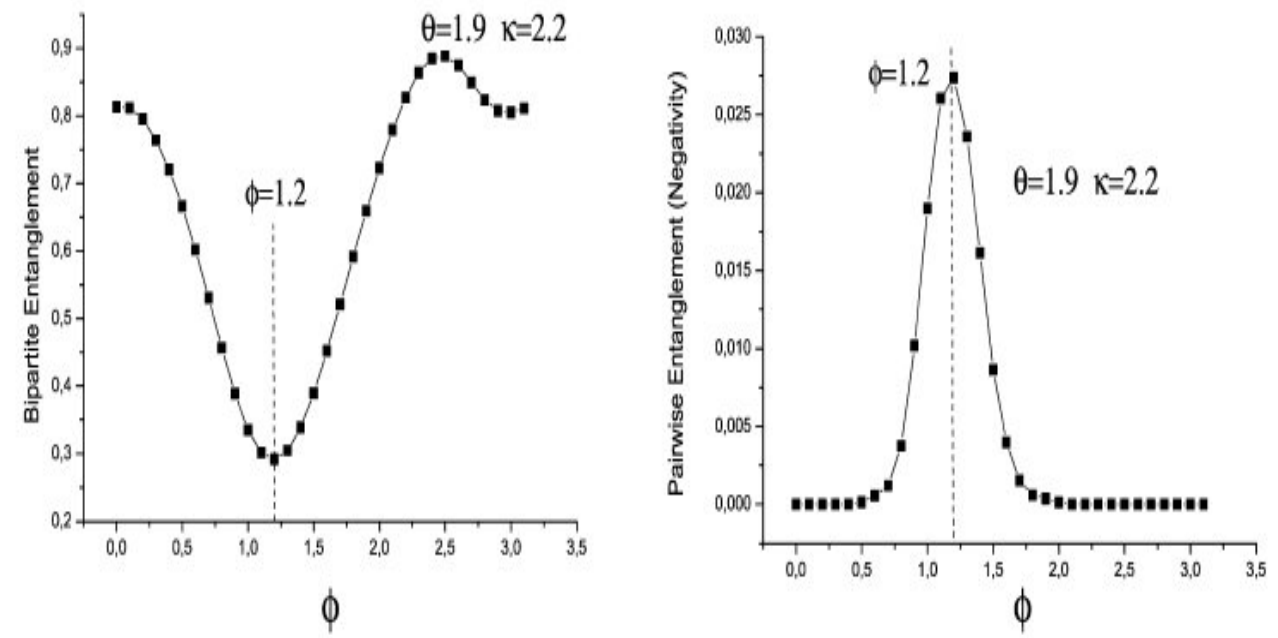

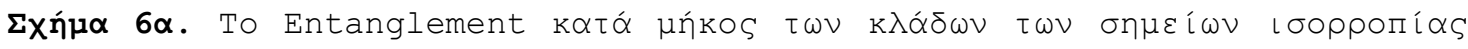

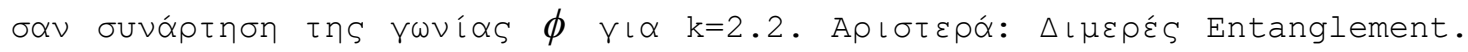

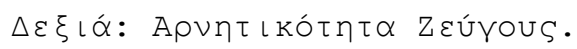
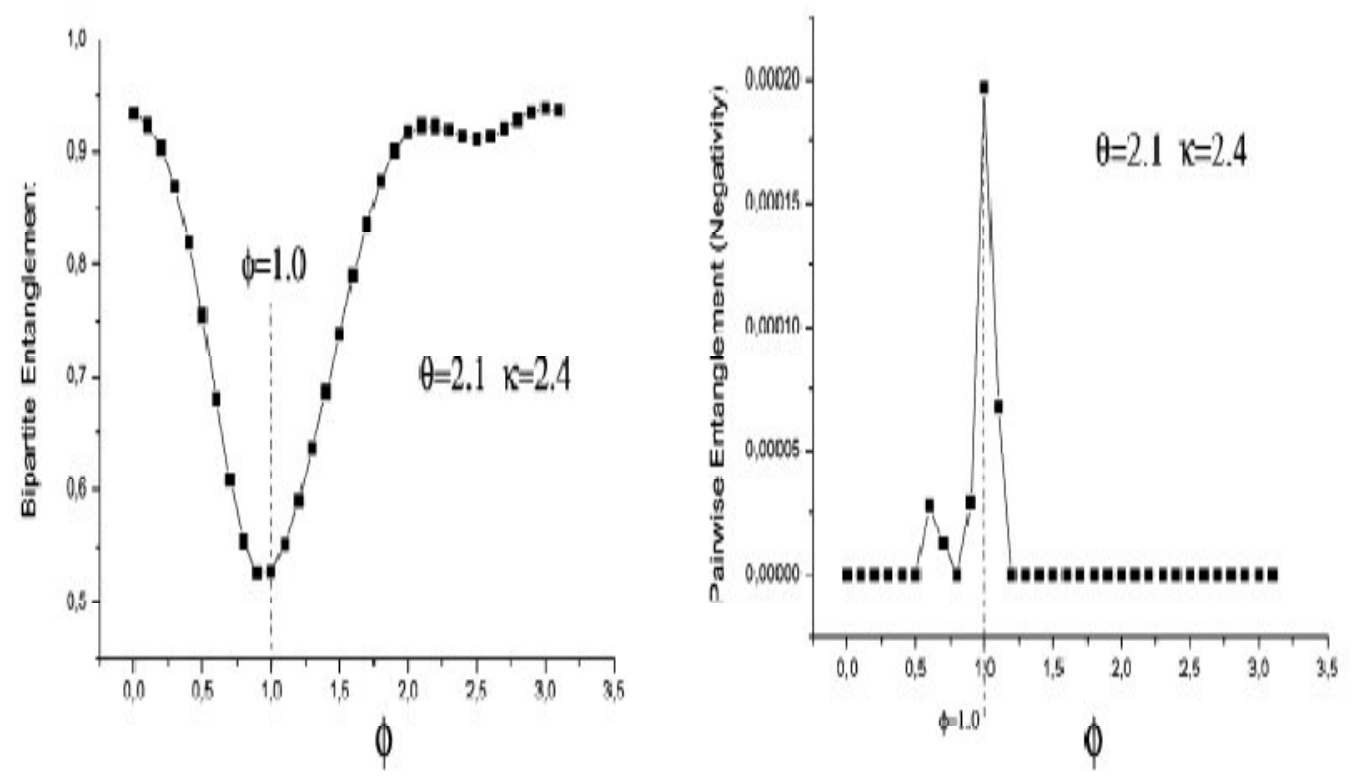

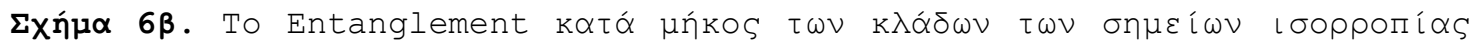

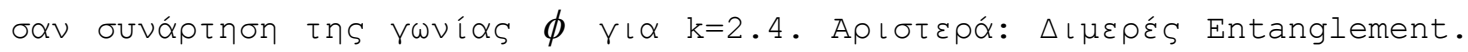

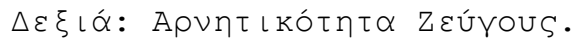



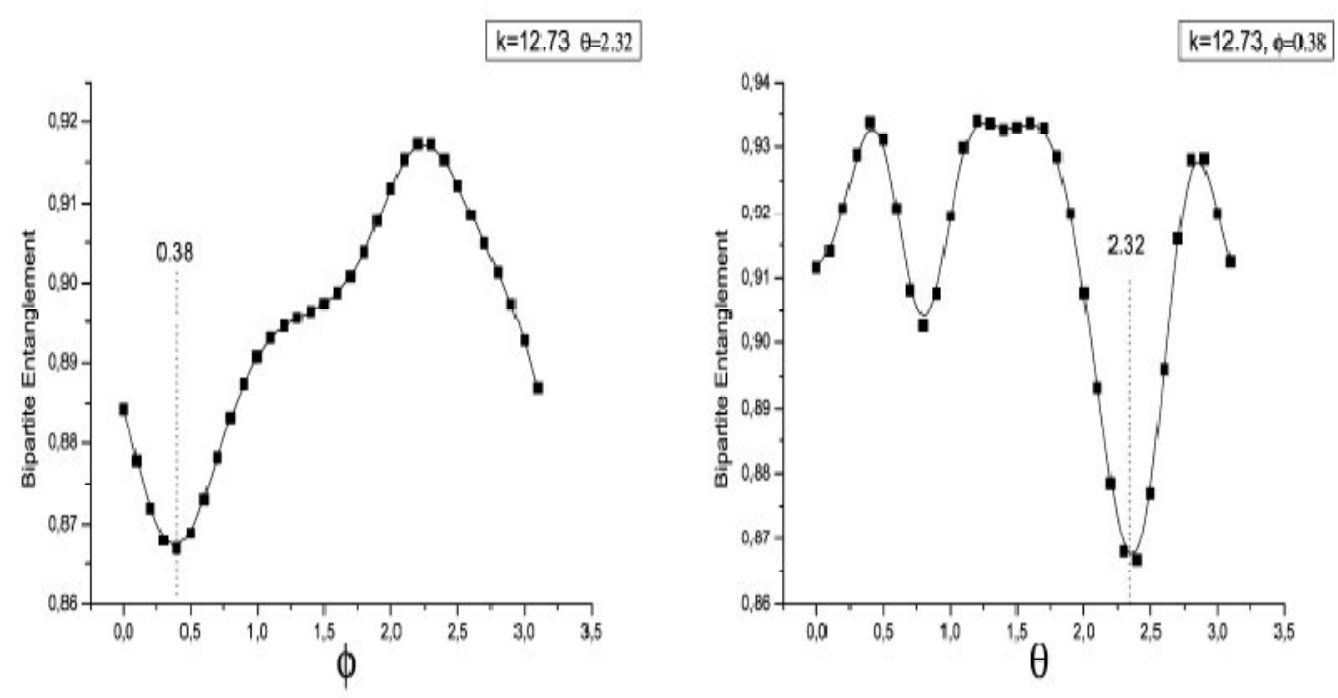

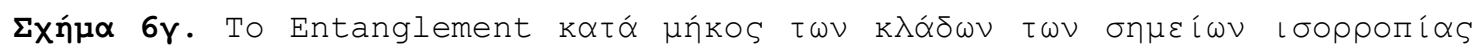

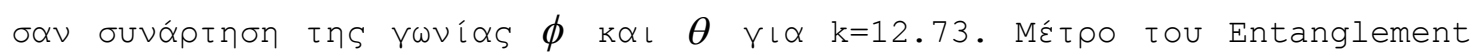

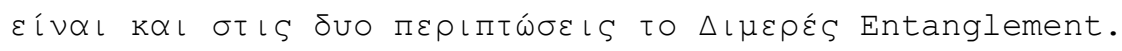

\section{Eníioyos}

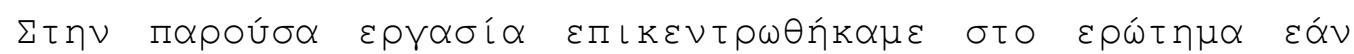

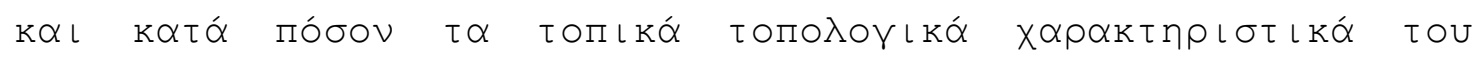

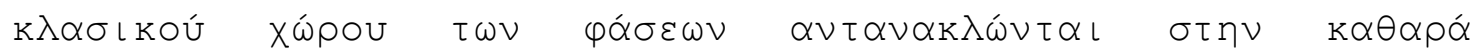

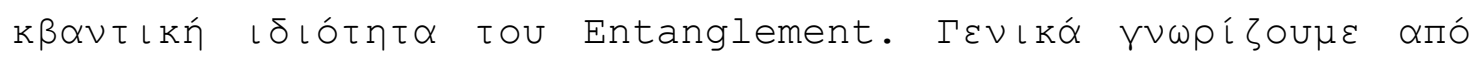

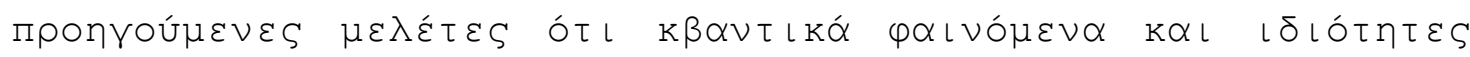

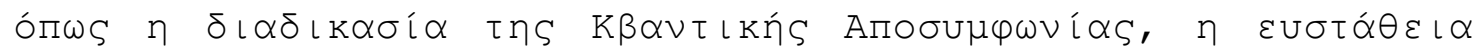

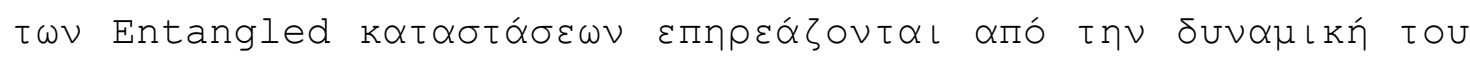

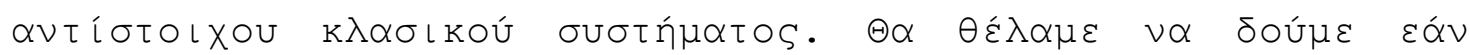

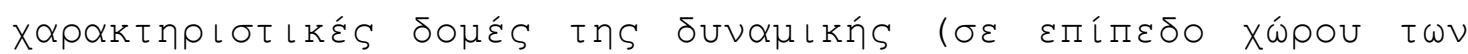

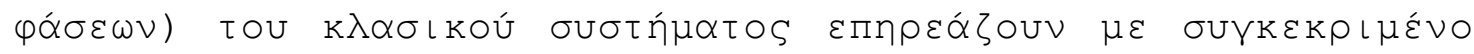

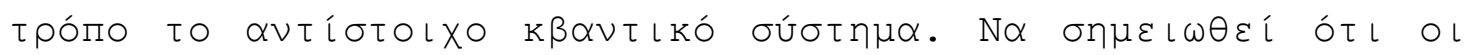

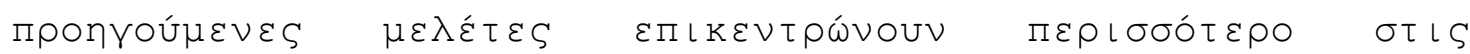

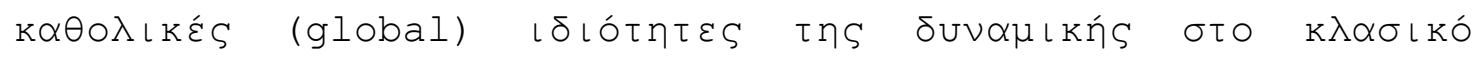

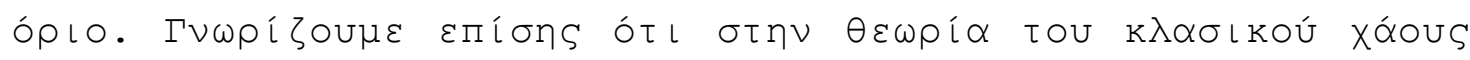

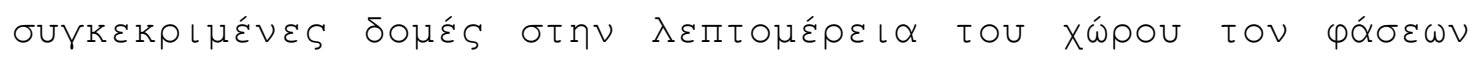

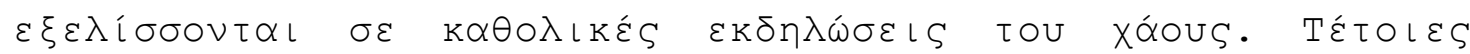

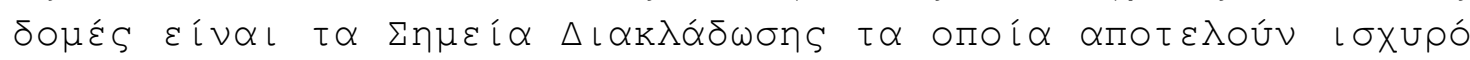




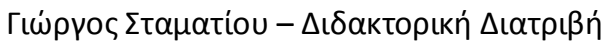

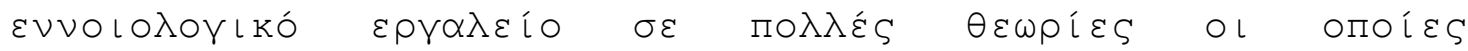

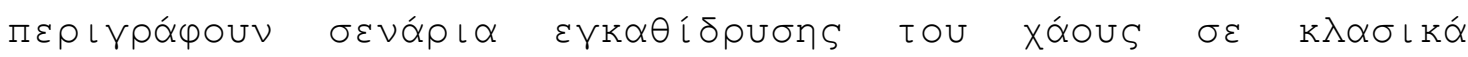

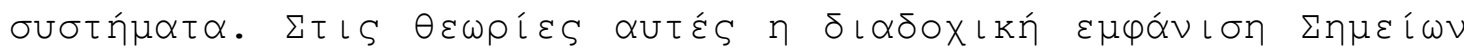

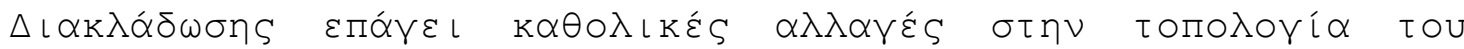

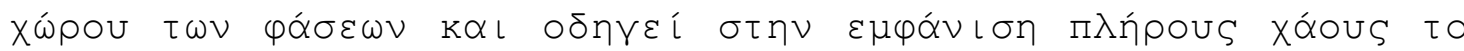

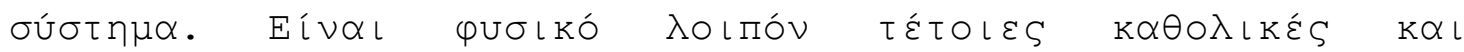

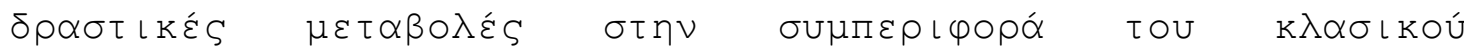

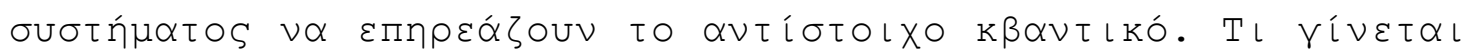

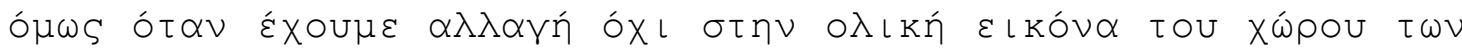

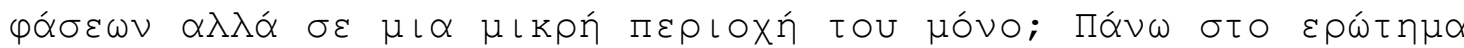

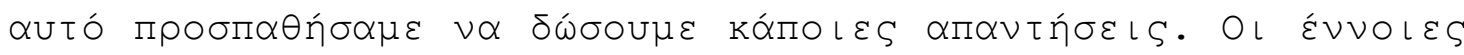

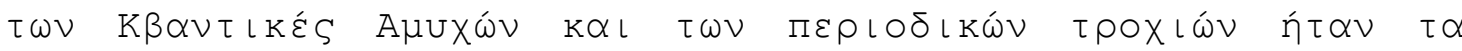

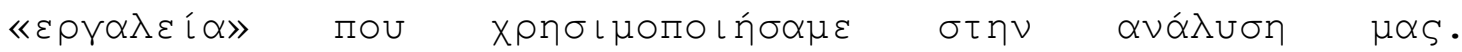

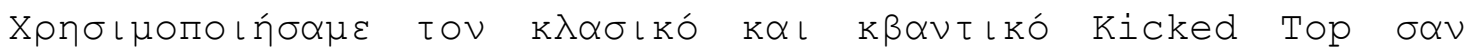

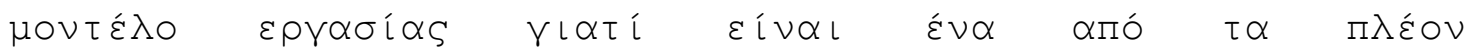

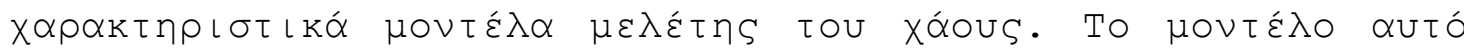

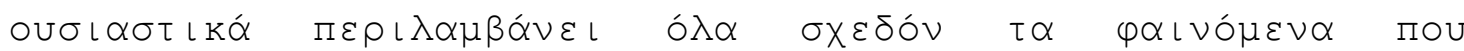

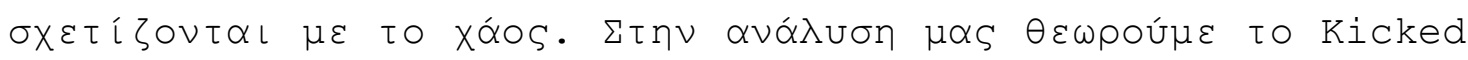

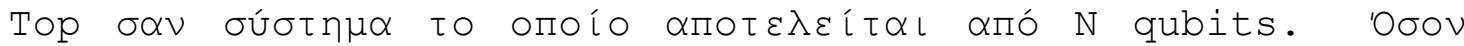

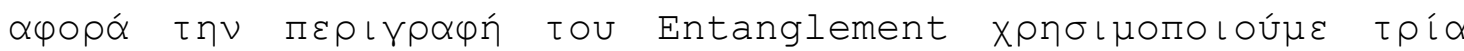

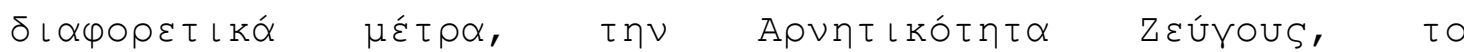

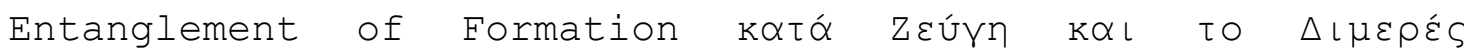

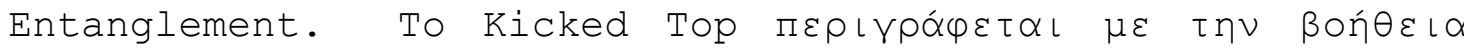

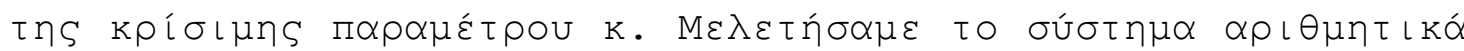

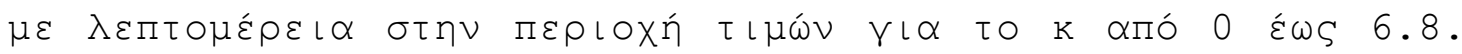

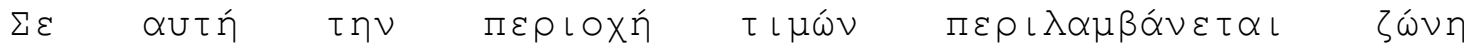

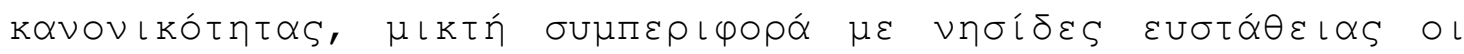

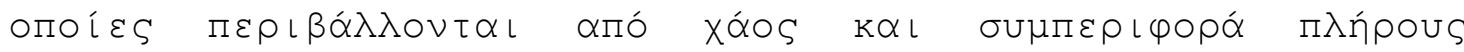

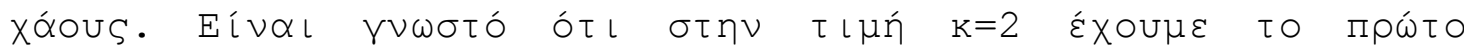

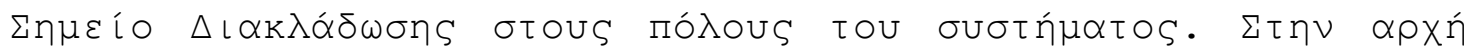

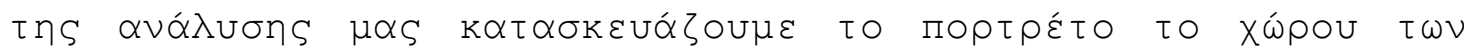

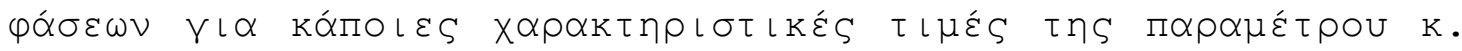

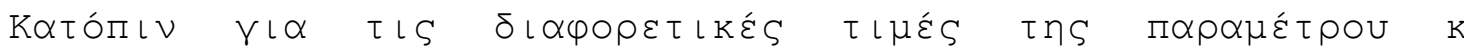

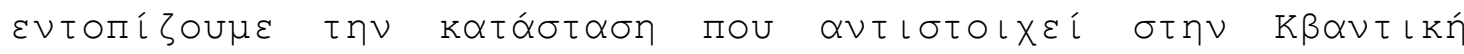

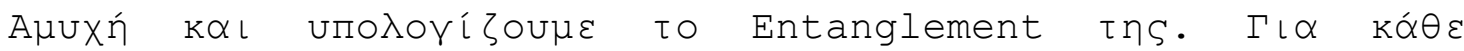

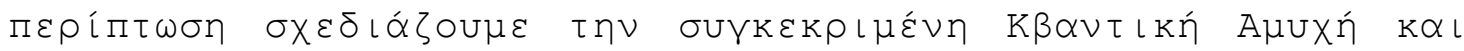

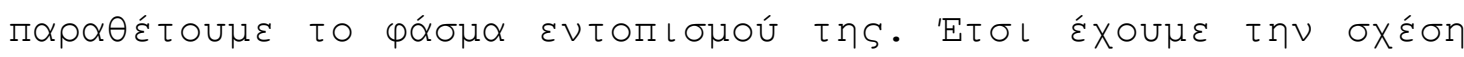

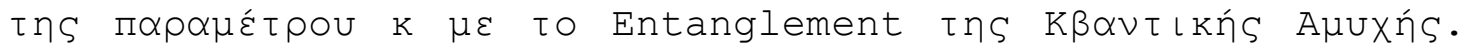

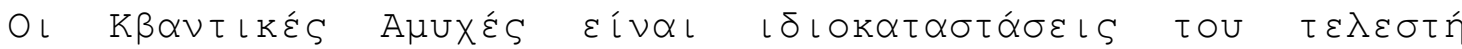

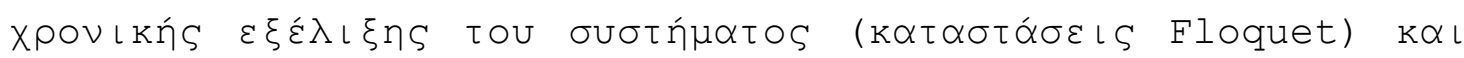

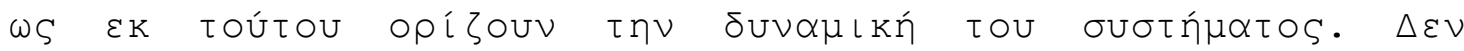




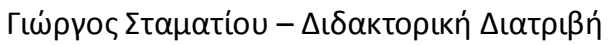

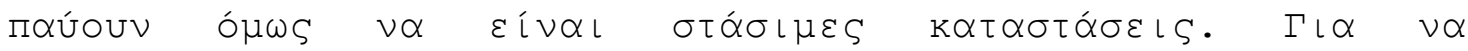

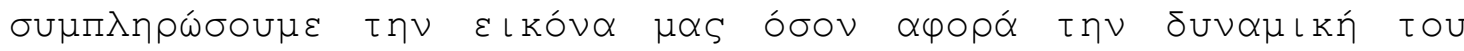

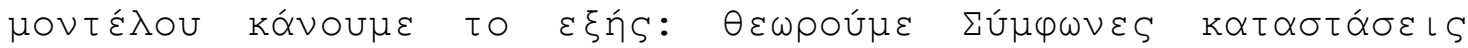

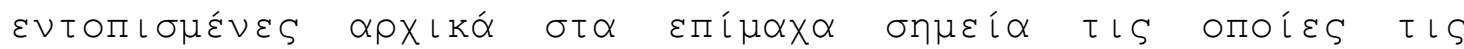

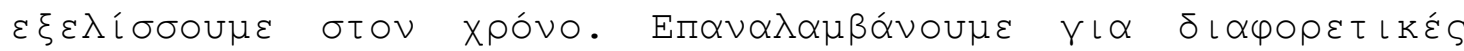

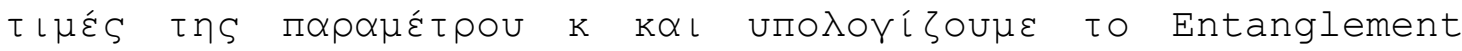

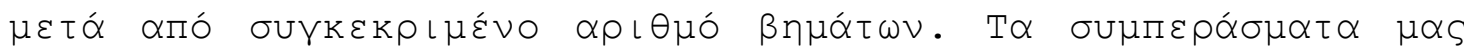

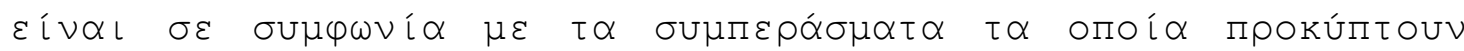

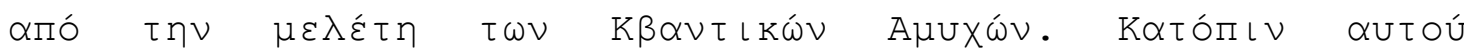

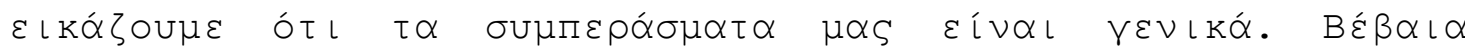

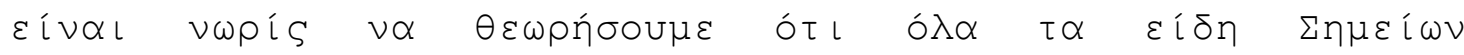

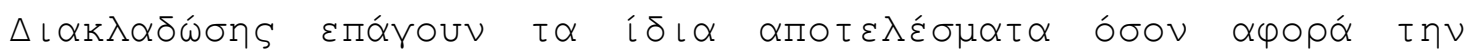

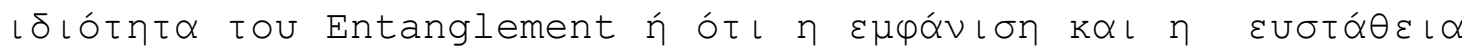

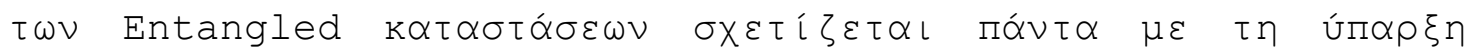

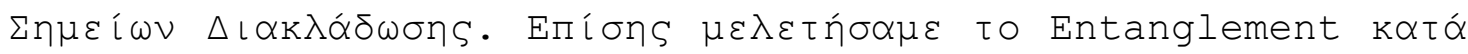

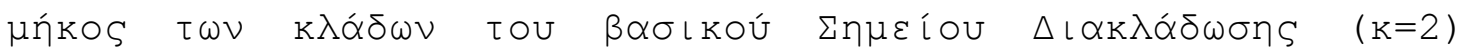

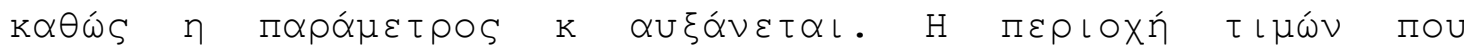

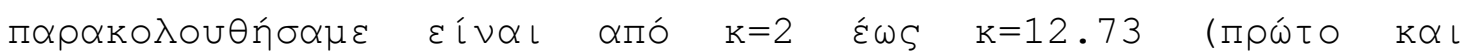

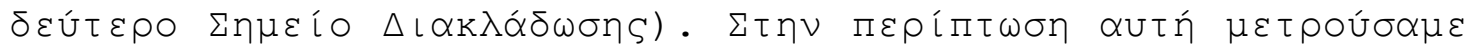

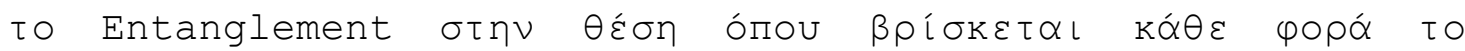

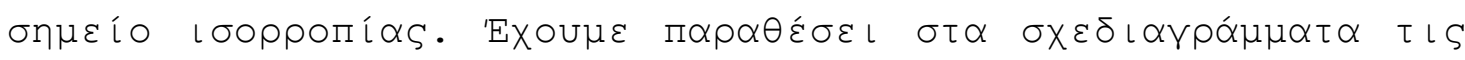

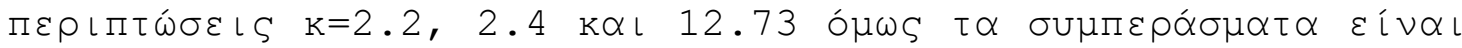

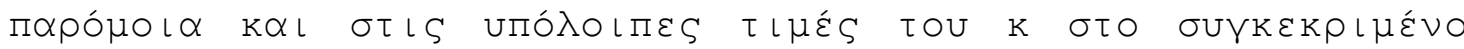
$\delta\llcorner\alpha ́ \sigma \tau \eta \mu \alpha$.

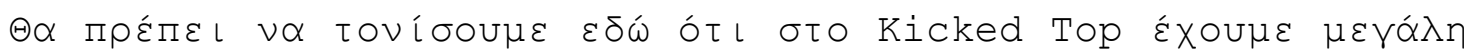

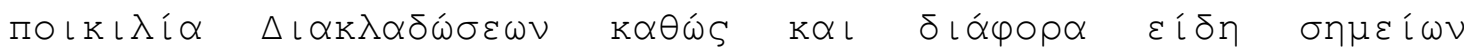

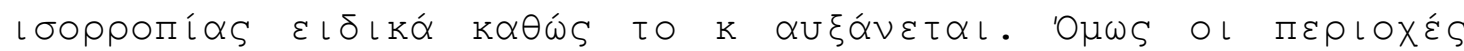

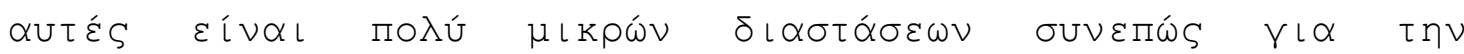

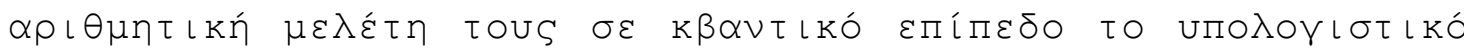

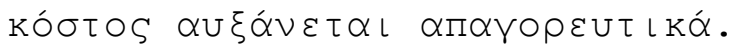

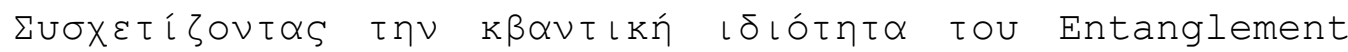

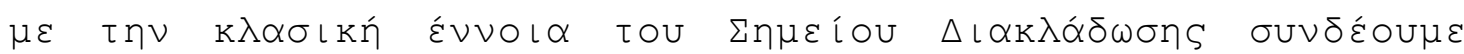

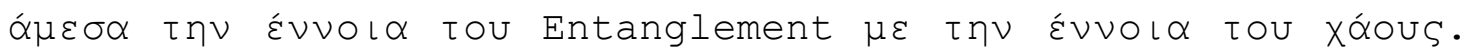

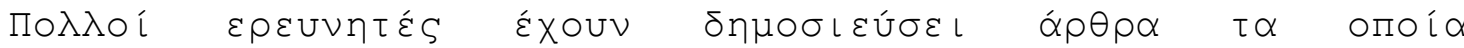

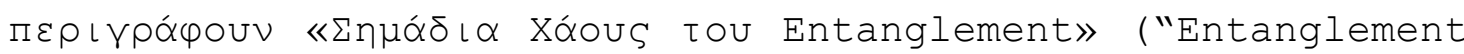

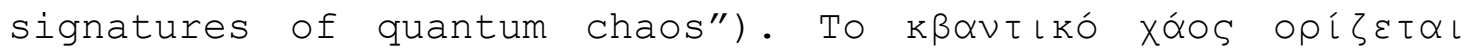

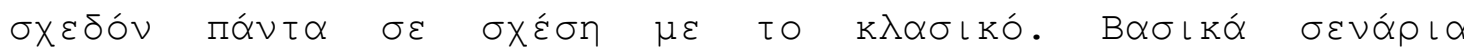

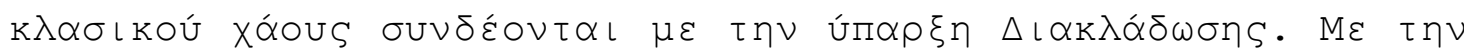

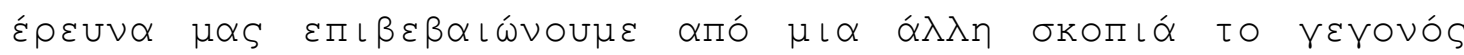

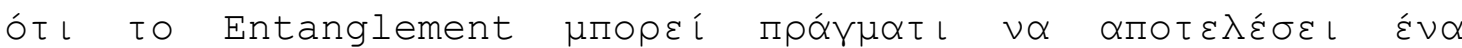




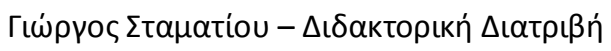

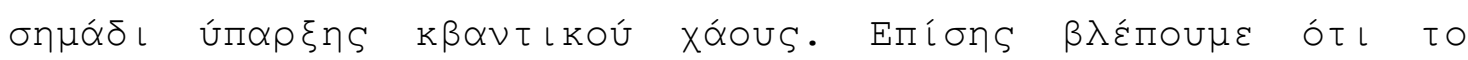

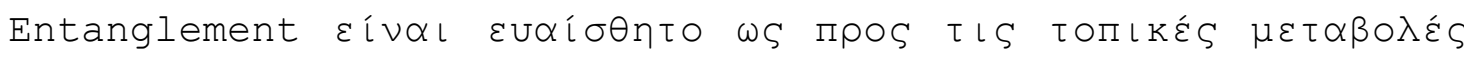

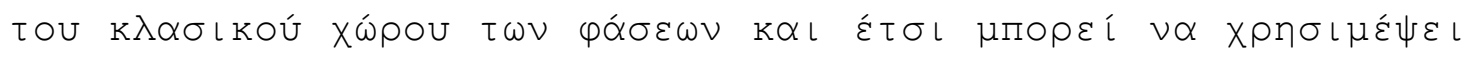

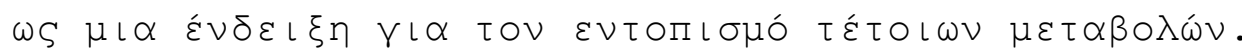

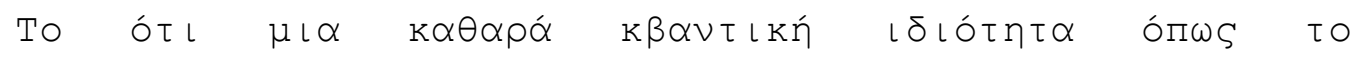

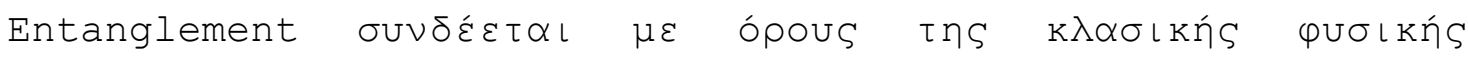

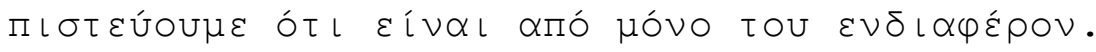





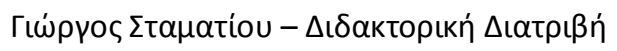

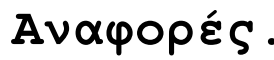

[1] M.C. Gutzwill, Chaos in Classical and Quantum Mechanics, Springer, New York, 1990.

[2] F. Haake, Quantum Signatures of Chaos, Springer, Berlin, 1991.

[3] L.E. Reichl, The Transition to Chaos, Conservative Classical Systems and Quantum Manifestations, Springer, New York, 2004.

[4] M.A. Nielsen, I.L. Chuang, Quantum Computation and Quantum Information, Cambridge Univ. Press, Cambridge, UK, 2000, ISBN: 0-521-63503-9

[5] K. Furuya, M.C. Nemes, G.Q. Pellegrino, Phys. Rev. Lett. 25 (1998) 5524.

[6] N.F. Bell, R.F. Sawyer, R.R. Volkas, Y.Y.Y.Wong, Phys. Rev. A 65 (2002) 042328.

[7] J.N. Bandyopadhyay, A. Lakshminarayan, Phys. Rev. Lett. 89 (2002) 060402.

[8] J.N. Bandyopadhyay, A. Lakshminarayan, Phys. Rev. E 69 (2004) 016201.

[9] C. Mejia-Monasterio, G. Benenti, G.G. Carlo, G. Casati, Phys. Rev. A 71

(2005) 062324 .

[10] H. Fujisaki, Phys. Rev. A 70 (2004) 012313.

[11] S. Ghose, B.C. Sanders, Phys. Rev. A 70 (2004) 062315.

[12] S. Ghosh, P.M. Alsing, I.E. Deutsch, Phys. Rev. E 64 (2001) 056119.

[13] X. Wang, S. Ghose, B.C. Sanders, B. Hu, quant$\mathrm{ph} / 0312047$.

[14] Ph. Jacquod, Phys. Rev. Lett. 92 (2004) 150403. 


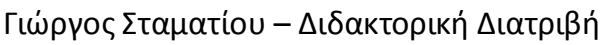

[15] M. Znidaric, T. Prosen, Phys. Rev. A 71 (2005) 032103.

[16] C. Pineda, T.H. Seligman, quant-ph/0503177.

[17] Y.S. Weinstein, L. Viola, quant-ph/0603071.

[18] C. Emary, N. Lambert, T. Brandes, Phys. Rev. A 71 (2005) 062302 .

[19] A.P. Hines, G.J. Milburn, R.H. McKentzie, quant$\mathrm{ph} / 0308165$.

[20] A.P. Hines, C.M. Dawson, R.H. McKenzie, G.J. Milburn, Phys. Rev. A 70 (2004) 022303.

[21] M.C. Nemes, K. Furuya, G.Q. Pellegrino, A.C. Oliveira, M. Reis, L. Sanz, quant-ph/0507026.

[22] E.J. Heller, Phys. Rev. Lett. 53 (1984) 1515.

[23] M. Kus, J. Zakrzewski, K. Zyczkowski, Phys. Rev. A 43 (1991) 4244 .

[24] G.G. de Polavieja, F. Borondo, R.M. Benito, Phys. Rev. Lett. 73 (1994)

1613.

[25] D.A. Wisniacki, F. Borondo, E. Vergini, R.M. Benito, Phys. Rev. E 62, (2000) R7583.

[26] D.A. Wisniacki, F. Borondo, E. Vergini, R.M. Benito, nlin.CD/0103031.

[27] S.-Y. Lee, S.C. Creagh, nlin.CD/0304018.

[28] F. Haake, M. Kus, R. Scharf, Z. Phys. B: Condens. Matter 65 (1987) 381.

[29] M. Kus, F. Haake, B. Eckhardt, Z. Phys. B: Condens. Matter 92 (1993), 221.

[30] A. Lakshminarayan, Phys. Rev. E 64 (2001) 036207. 


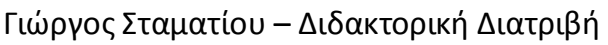

[31] F.T. Arrechi, E. Courtens, R. Gilmore, H. Thomas, Phys. Rev. A 36 (1987) 5543.

[32] R.H. Diche, Phys. Rev. 93 (1954) 99.

[33] H. Barnum, E. Knill, G. Ortiz, L. Viola, Phys. Rev. A 68 (2003) 032308 .

[34] H. Barnum, E. Knill, G. Ortiz, R. Somma, L. Viola, Phys. Rev. Lett. 92, (2004) 107902.

[35] C.H. Benett, D.P. DiVincenzo, J.A. Smolin, W.K. Wooters, Phys. Rev., A 54 (1996) 3824.

[36] W.K. Wooters, Phys. Rev. Lett. 80 (1998) 2245.

[37] A. Peres, Phys. Rev. Lett. 77 (1996) 1413.

[38] K. Zyczkowski, P. Horodecki, A. Sampera, M. Lewenstein, Phys. Rev., A 58 (1998) 883.

[39] G. Vidal, R.F. Werenr, Phys. Rev. A 65 (2002) 032314.

[40] M. Horodecki, P. Horodecki, R. Horodecki, Phys. Lett. A 223 (1996) 1 . 



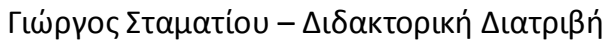

ENOTHTA $\Sigma T^{\prime}$

@EPMIKO ENTANGLEMENT

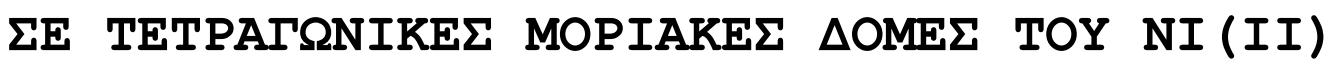





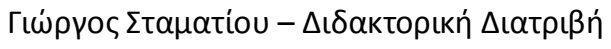

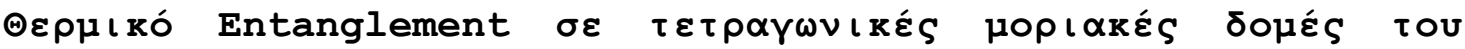 $\mathrm{Ni}(\mathrm{II})$}

\section{Eı $\sigma \alpha \gamma \omega \gamma \tilde{n}$}

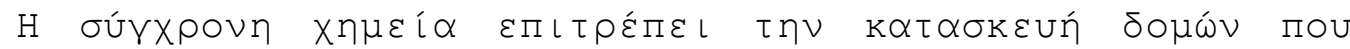

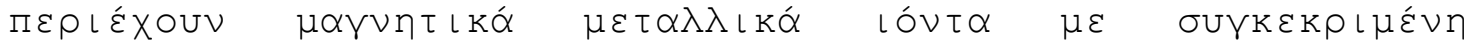

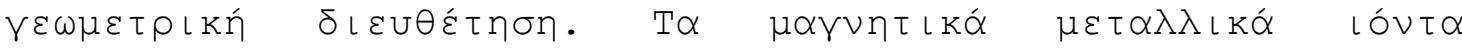

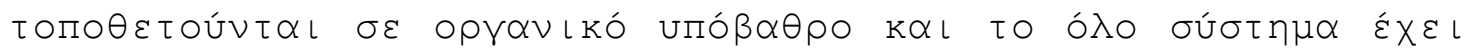

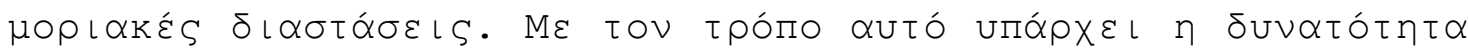

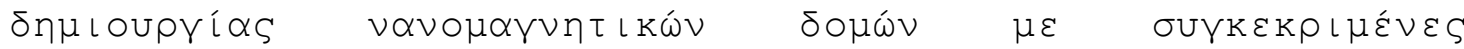

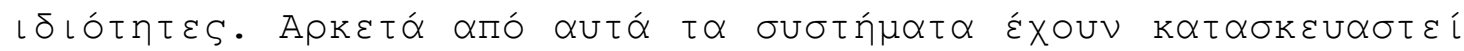

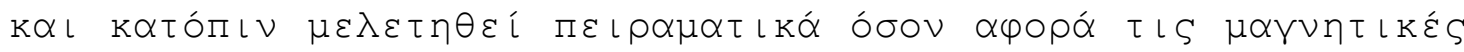

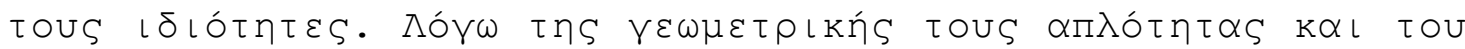

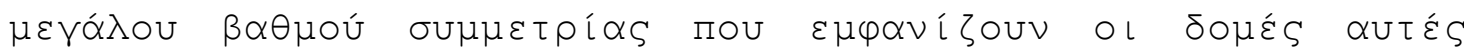

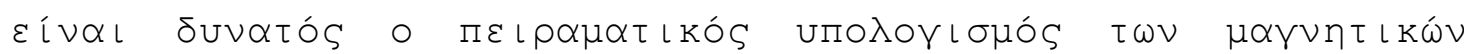

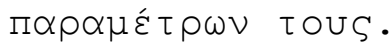

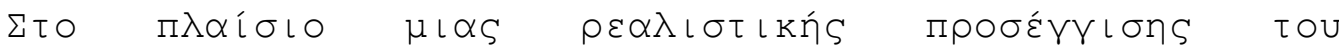

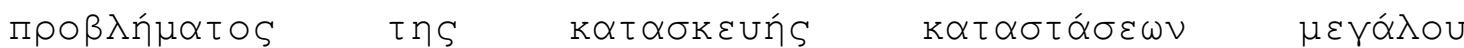

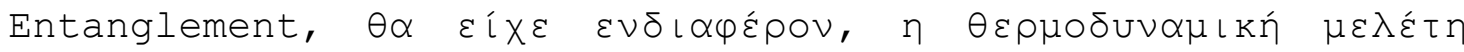

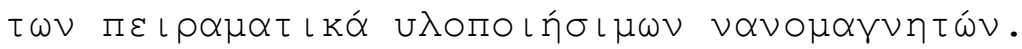

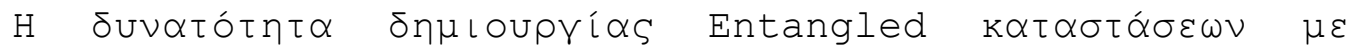

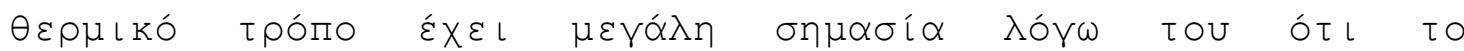

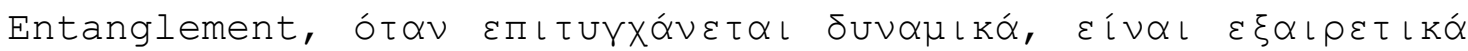
$\varepsilon u ́ \theta \rho \alpha u \sigma \tau$.

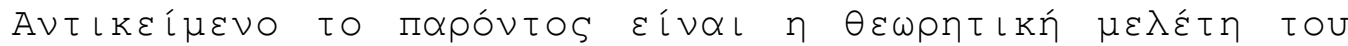

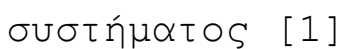

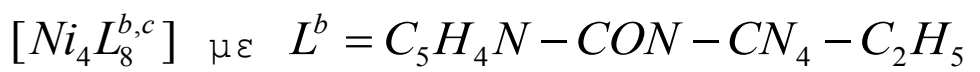

$$
\text { к } \propto\left\llcorner\quad L^{c}=\mathrm{C}_{5} \mathrm{H}_{4} \mathrm{~N}-\mathrm{CSN}-\mathrm{CN}_{4}-\mathrm{C}_{2} \mathrm{H}_{5}\right.
$$

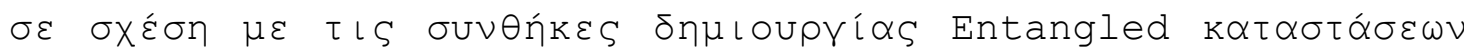

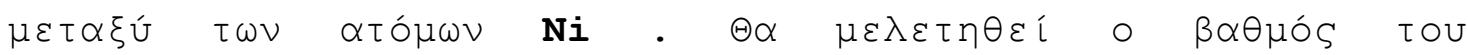

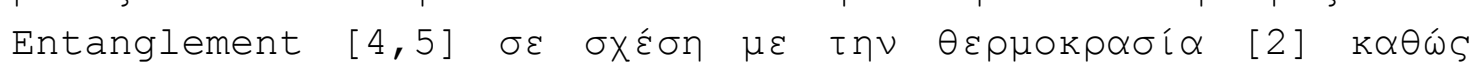

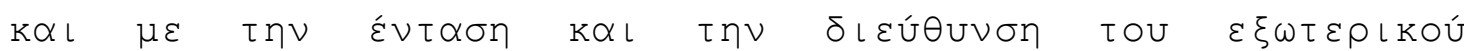

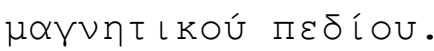




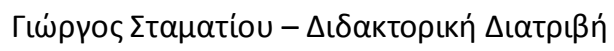

\section{To $\mu \circ \rho\llcorner\alpha$ Kó $\sigma u ́ \sigma \tau \eta \mu \alpha$}

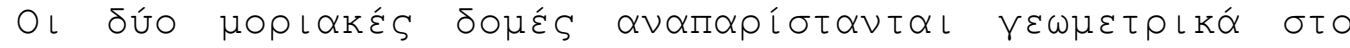

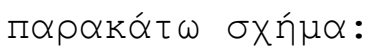

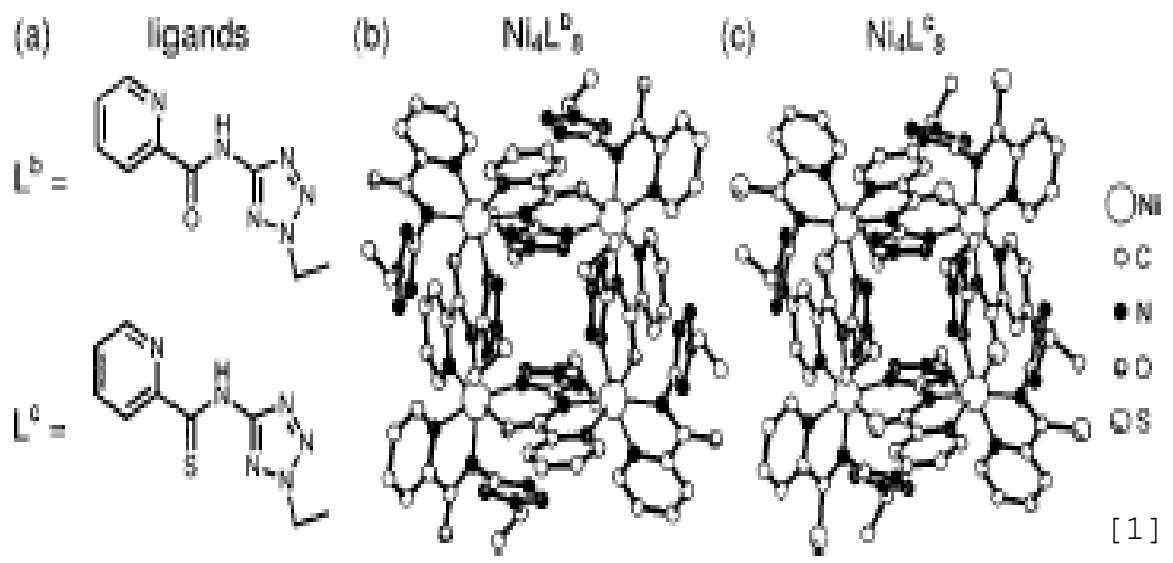

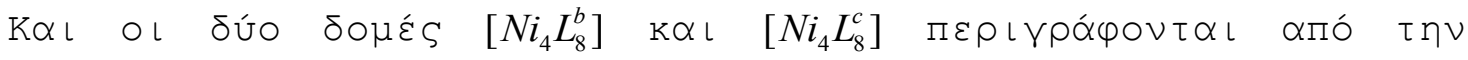

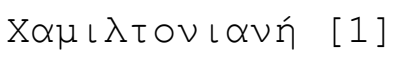

$$
\hat{H}-J \sum_{i<j} \hat{\vec{S}}_{i} \hat{\vec{S}}_{j}+D \sum_{i}\left(\hat{S}_{i, z}^{2}-\frac{2}{3}\right)+\mu_{B} g_{x y}\left(\hat{S}_{x} B_{x}+\hat{S}_{y} B_{y}\right)+\mu_{B} g_{z} \hat{S}_{z} B_{z}
$$

$\mu \varepsilon \quad S=1$.

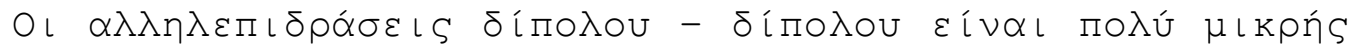

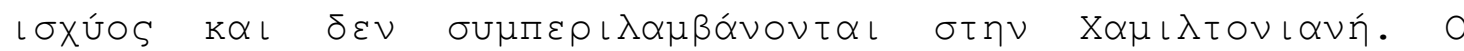

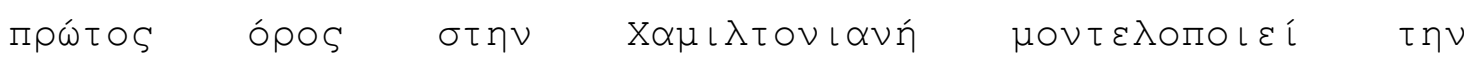

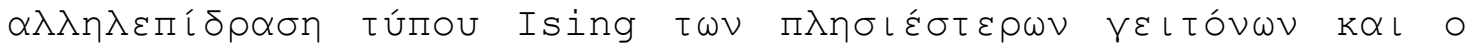

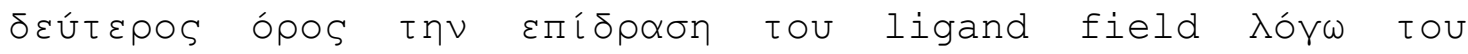

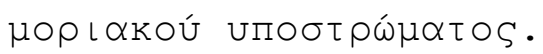

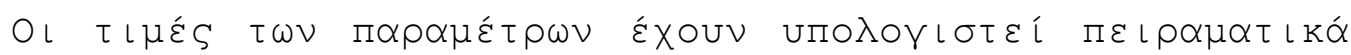

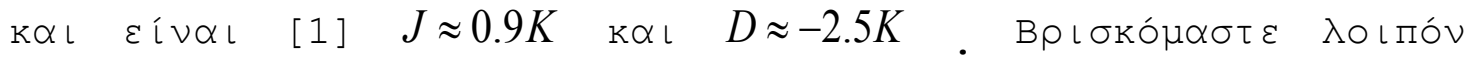




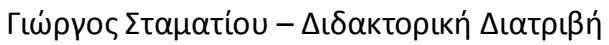

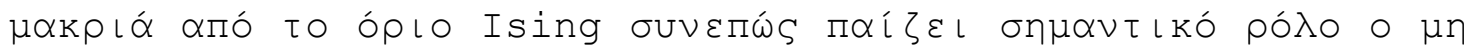
vранuи kós ópos $\hat{S}_{z}^{2}$

\section{н пробороі́}

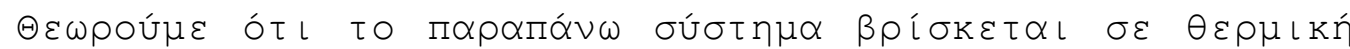

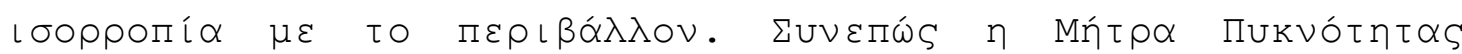

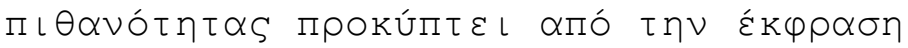

$$
\hat{\rho}(T, \vec{B})=\frac{e^{-\frac{\hat{H}(T, \vec{B})}{\kappa_{B} T}}}{z} \mu \varepsilon \quad z=\operatorname{tr}\left(e^{-\frac{\hat{H}(T, \vec{B})}{\kappa_{B} T}}\right)
$$

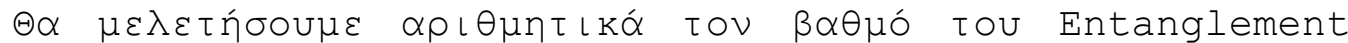

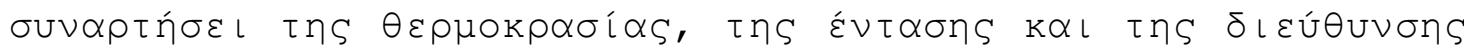

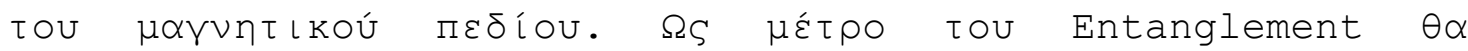

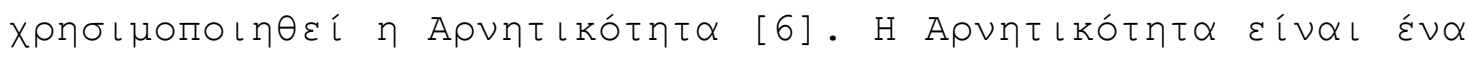

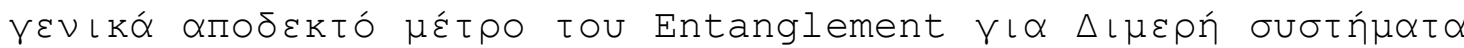

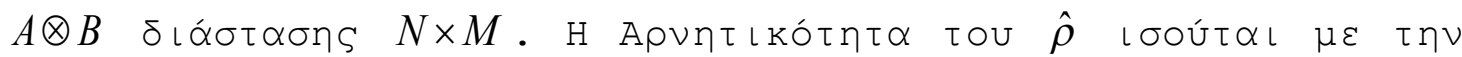

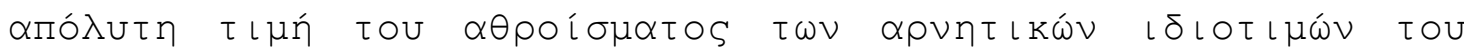

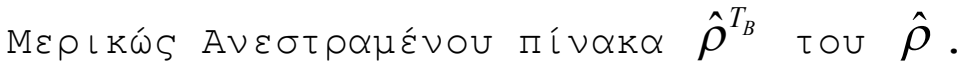

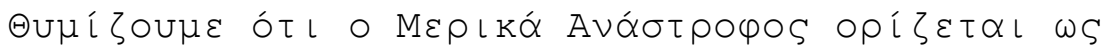

$$
\hat{\rho}^{T_{B}}=\sum_{i j}\left\langle i_{B}|\hat{\rho}| j_{B}\right\rangle \otimes\left|j_{B}\right\rangle\left\langle i_{B}\right|
$$

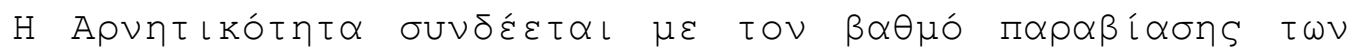

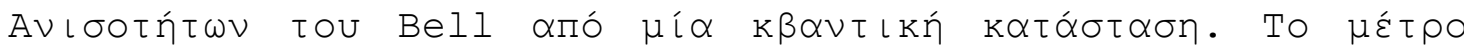

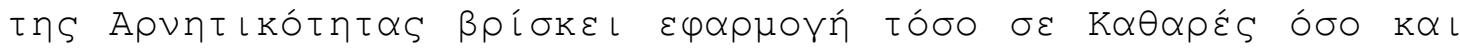

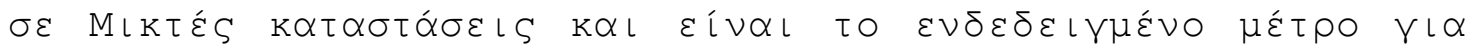

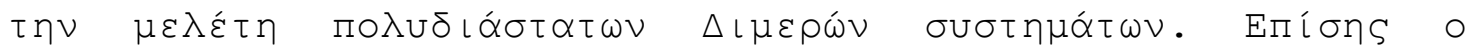

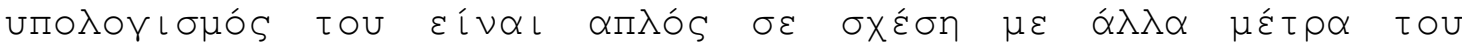
Entanglement.

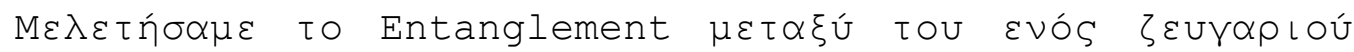

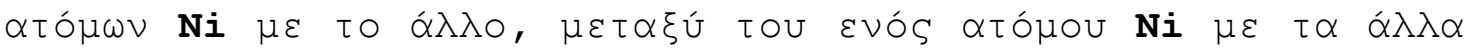

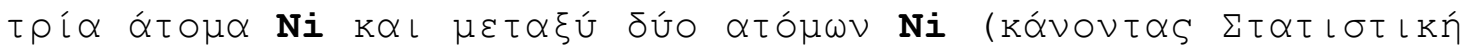




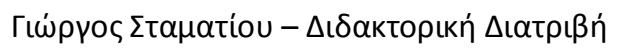

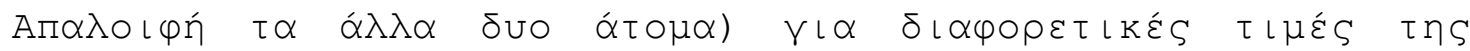

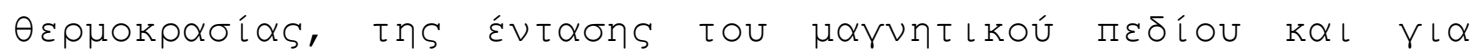

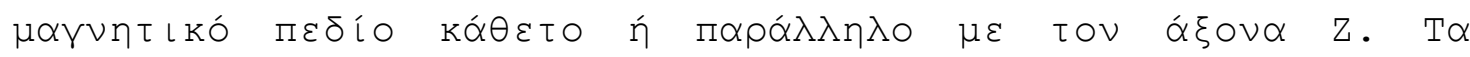

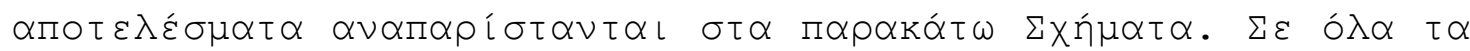

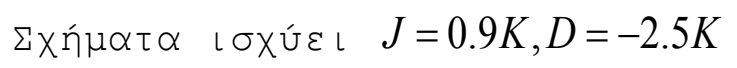

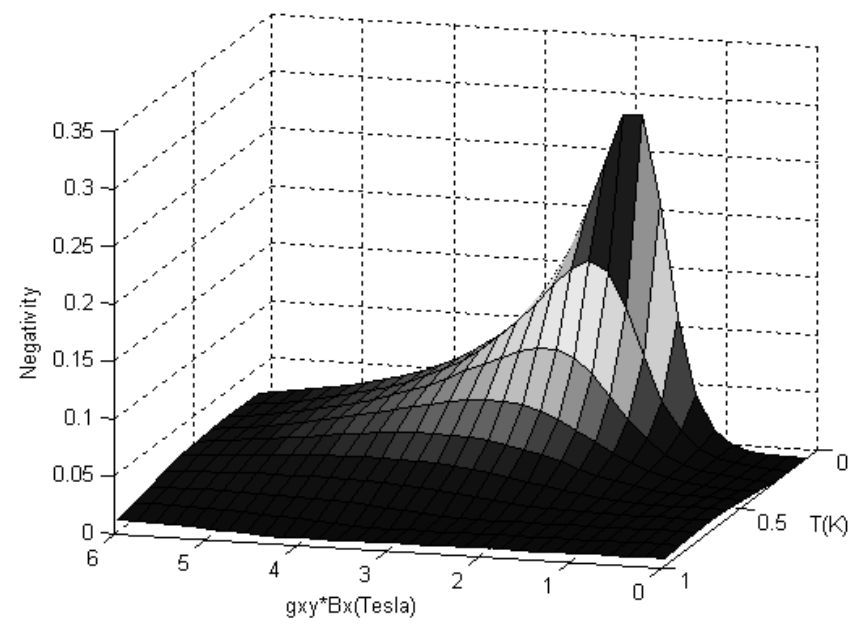

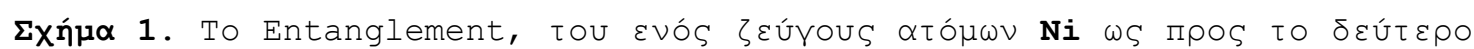

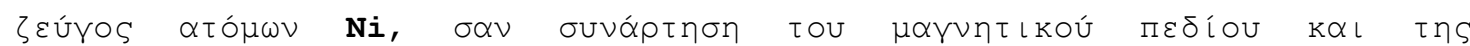

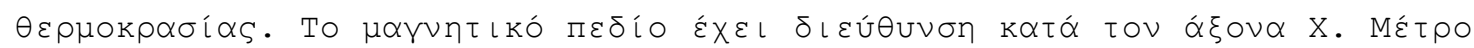

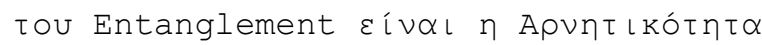




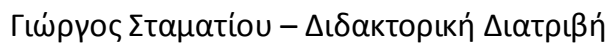

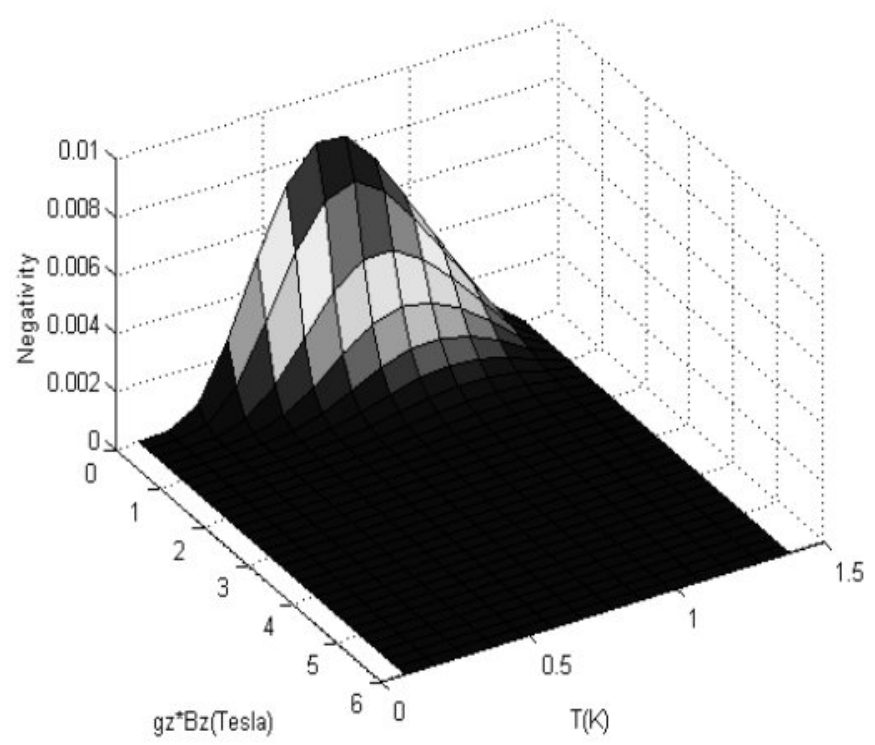

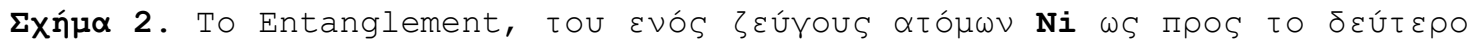

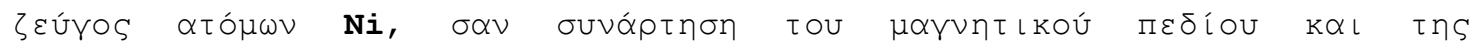

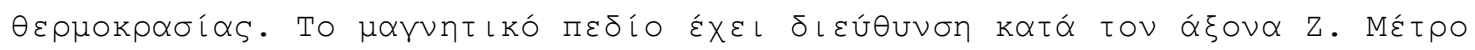

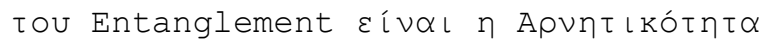

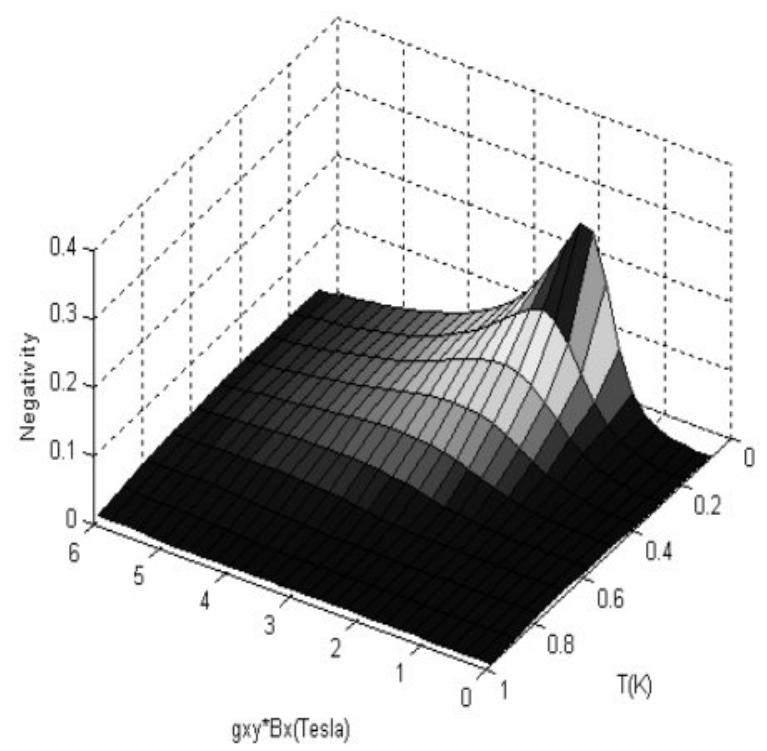

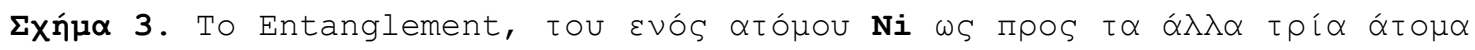

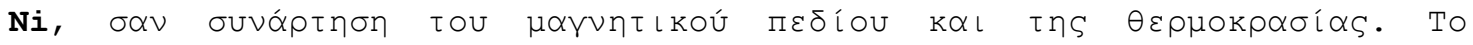

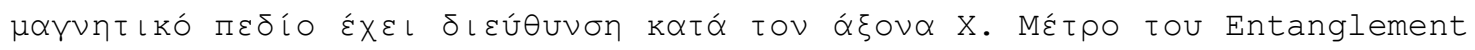

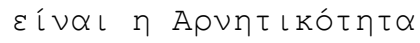

271 


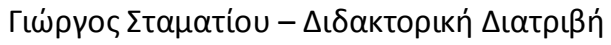

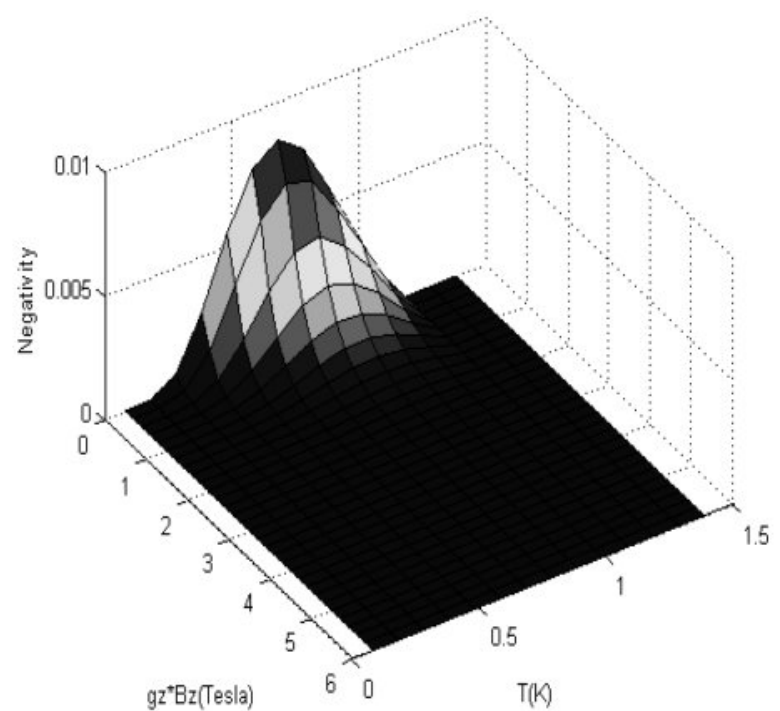

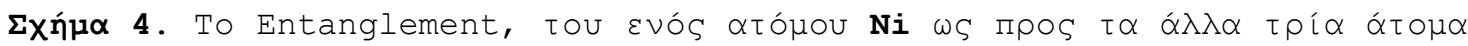

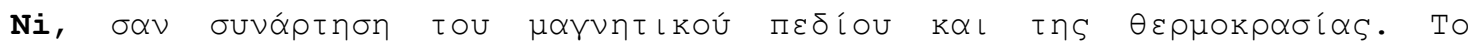

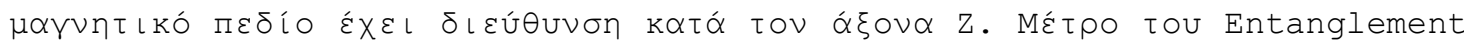

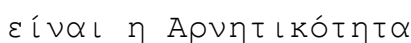

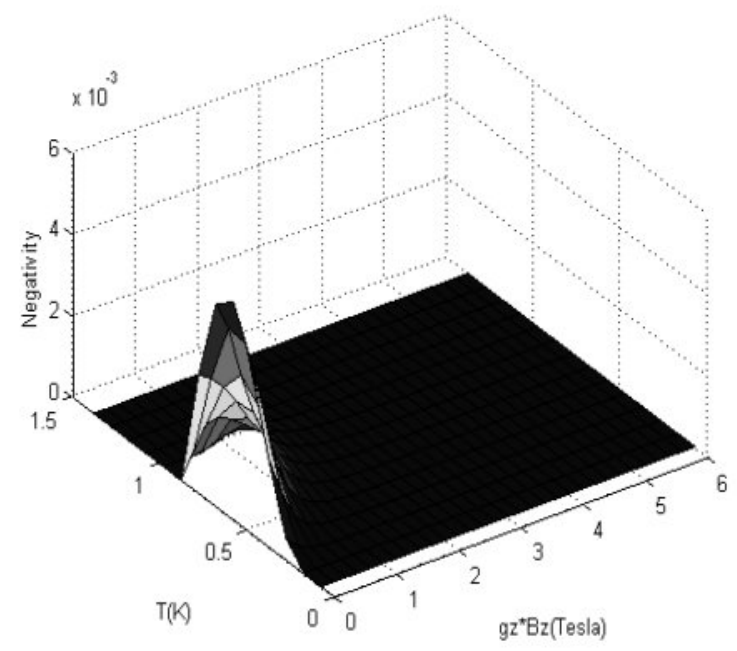

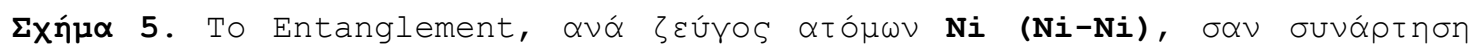

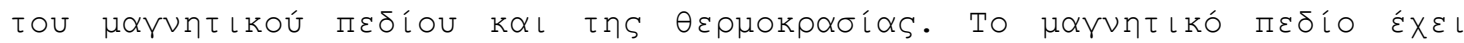

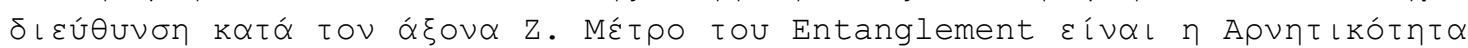
ZEúYOUS. 


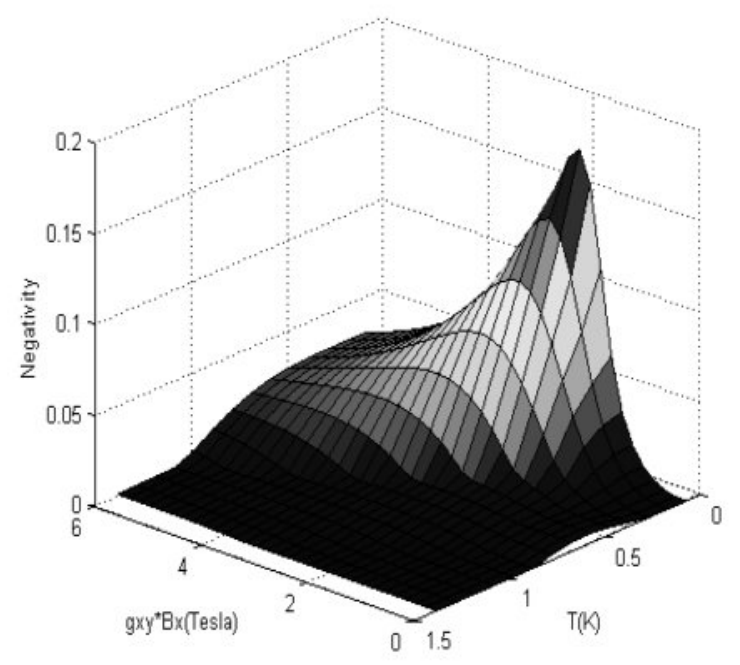

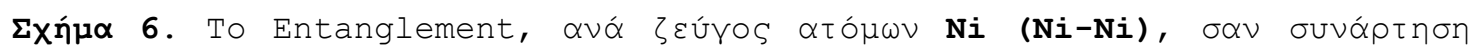

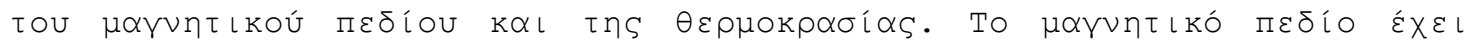

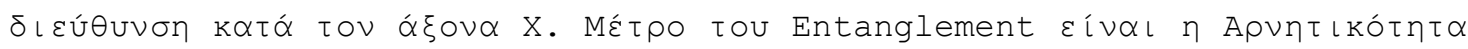
ZEúYOUS.

\section{$\Sigma \cup \mu \Pi \varepsilon \rho \alpha ́ \alpha \mu \alpha \tau \alpha$}

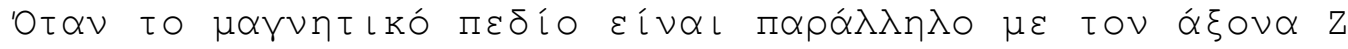

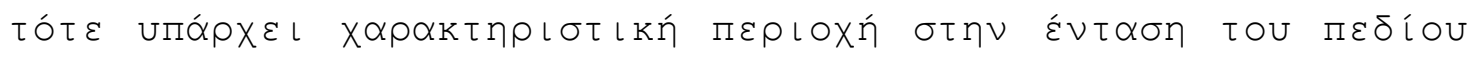

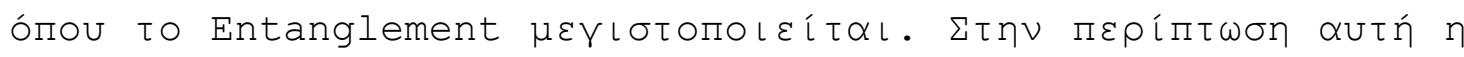

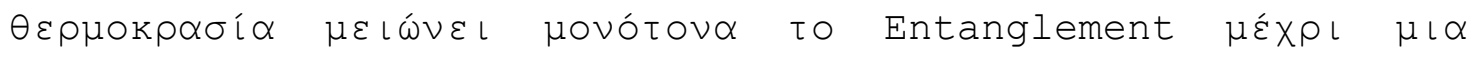

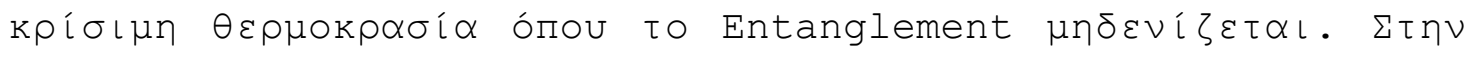

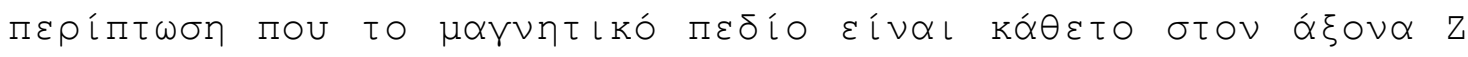

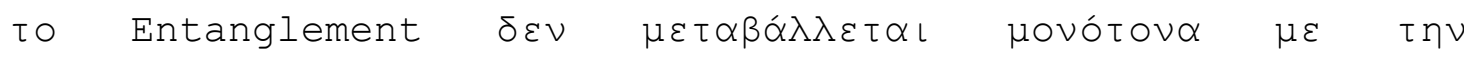

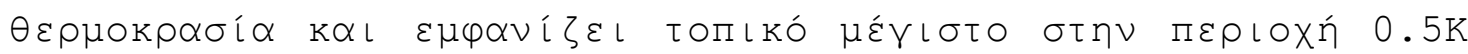

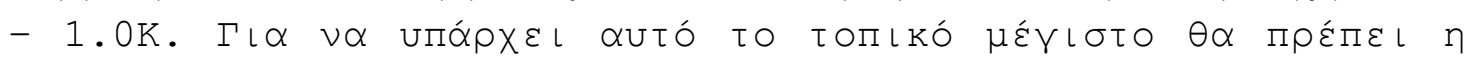

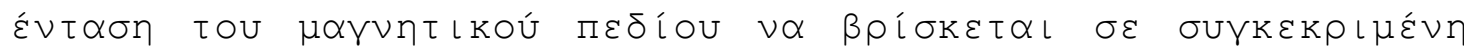

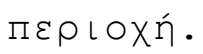

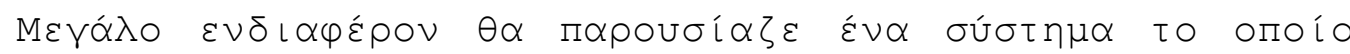

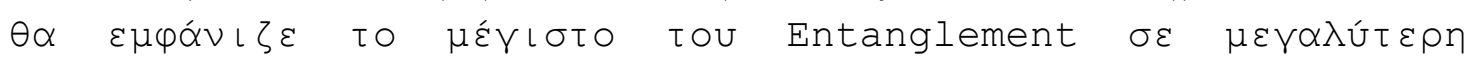
$\theta \varepsilon \rho \mu о к \rho \alpha \sigma i ́ \alpha$. 



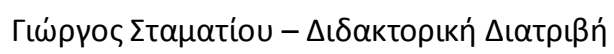

\section{Aข $\alpha \varphi \circ \rho \varepsilon ́ s$}

[1] Ferromagnetic coupling and magnetic anisotropy in molecular Ni(II) squares, R. Koch, O. Waldman, P. Muller, Ph. Rev. B, vol. 67, issue 9, 094407 (2003)

[2] Thermal Entanglement in the two-qubit Heisenberg XYZ model, G. Rigolin, Arxiv.org, quant-phys/0311185

[3] Thermal Entanglement of Bosonic atoms in an optical lattices with nonlinear couplings, L. Zhou, X. X. Yi, S. Song, Y. Q. Quo, Arxiv.org, quant-phys/0310169

[4]. Quantum information and computation, C. H. Bennett and D. P. DiVincenzo, 2000, Nature 404, 247

[5]. Quantum computation and quantum information, M. A. Nielsen and I. L. Chuang, Cambridge University press, Cambridge, 2000, ISBN: 0-521-63503-9

[6]. Logarithmic negativity: a full Entanglement monotone that is not convex, MB Plenio - Physical review letters, 2005, vol. 95, issue 9, 090503 



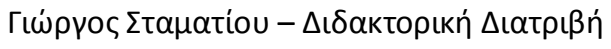

ENOTHTA $Z^{\prime}$

XAOE KANONIKOTHTA

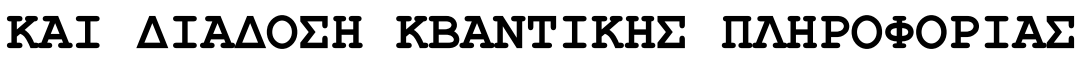

$\triangle I A M E \Sigma O Y$ KBANTIKOY KANAAIOY.

XSPHTIKOTHTA TOY KANAAIOY KAI XAOL.

$\triangle I A T H P H \Sigma H ~ T O Y ~ E N T A N G L E M E N T ~ \Sigma T O N ~ X P O N O$. 



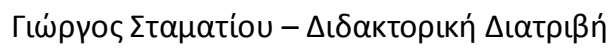

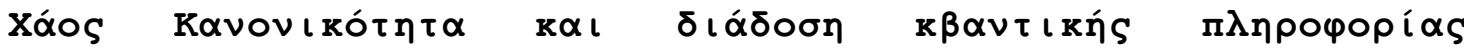

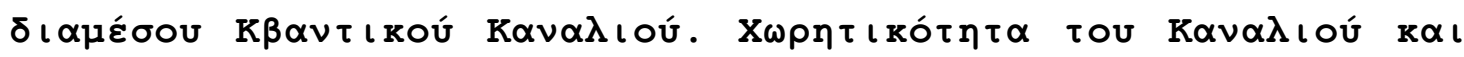

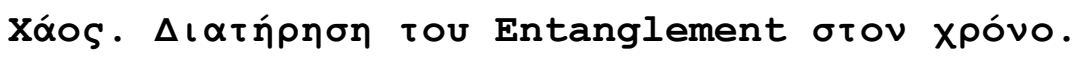

\section{Eı $\sigma \alpha \gamma \omega \gamma \eta ́$}

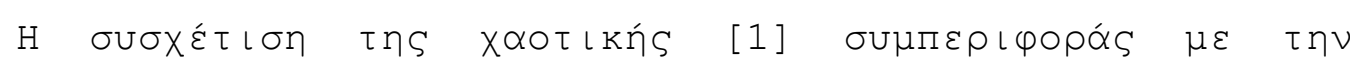

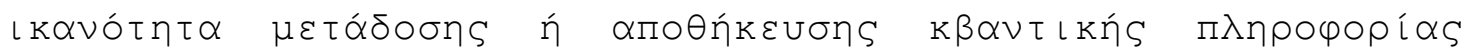

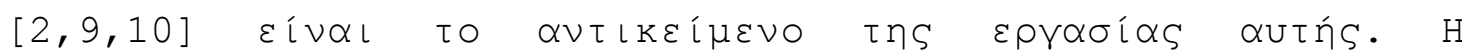

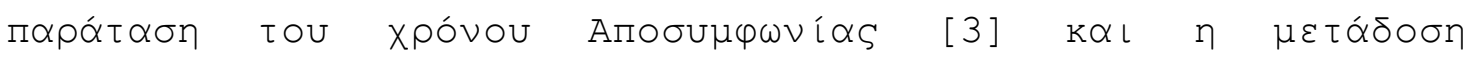

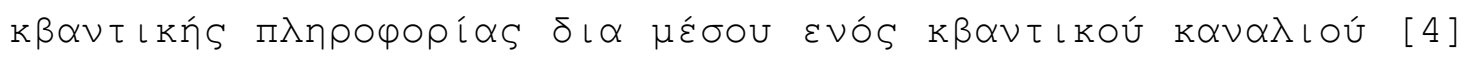

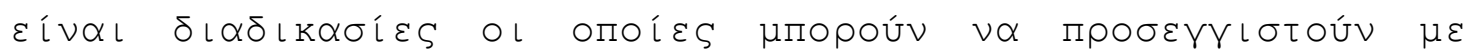

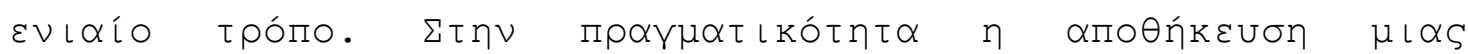

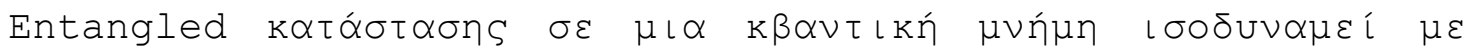

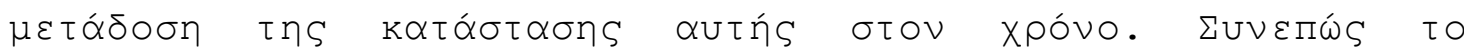

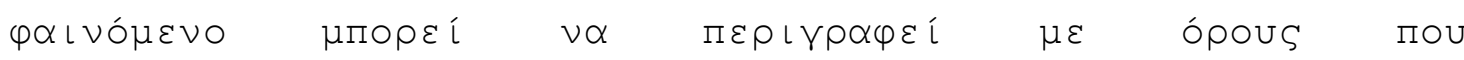

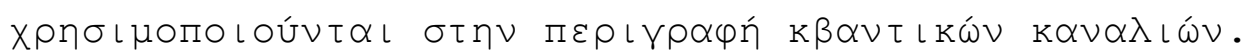

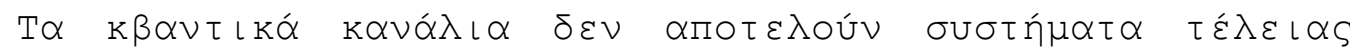

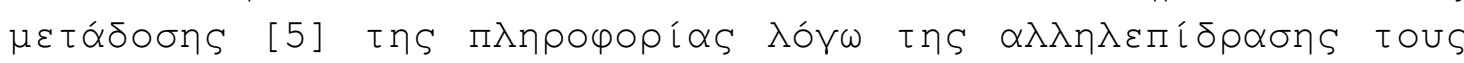

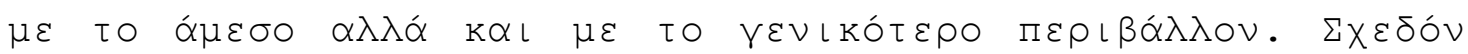

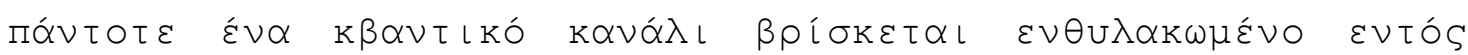

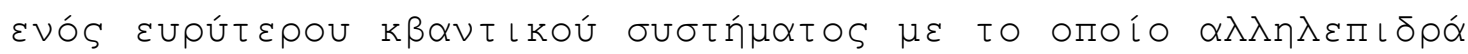

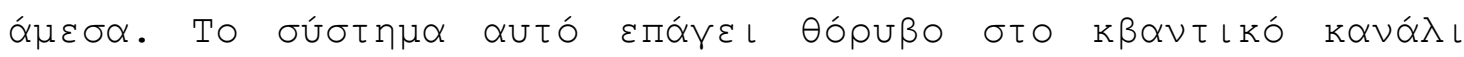

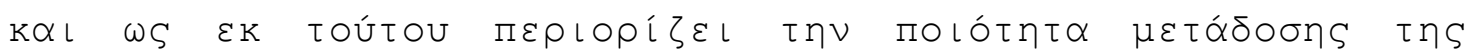

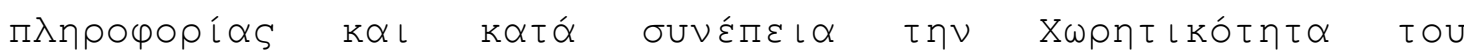

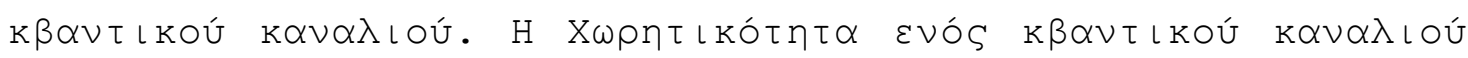

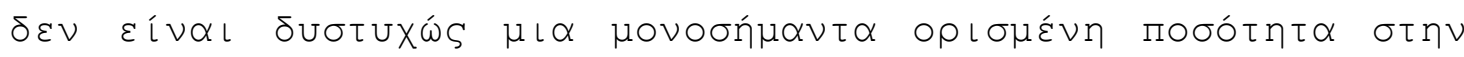

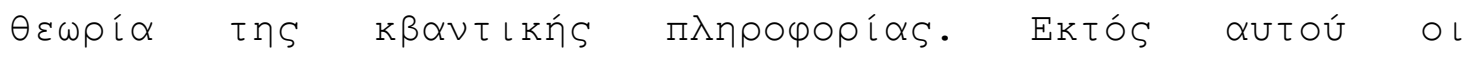

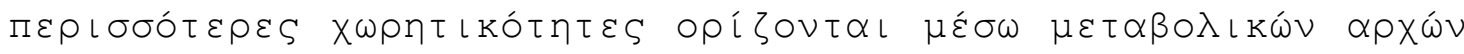

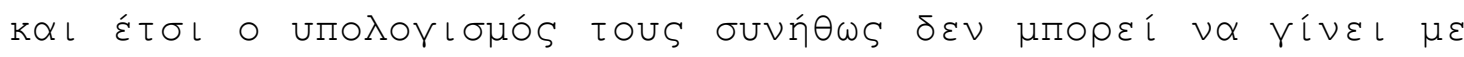
$\alpha \vee \alpha \lambda u \tau \iota k o ́$ тро́по.

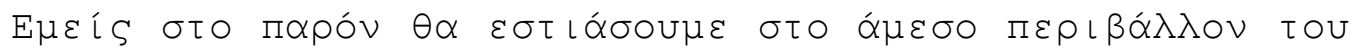

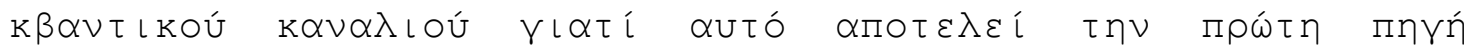

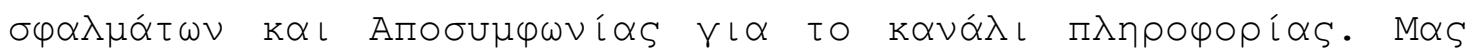

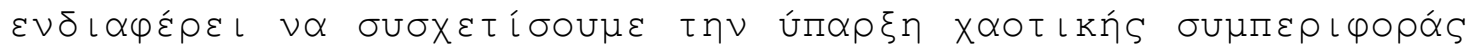

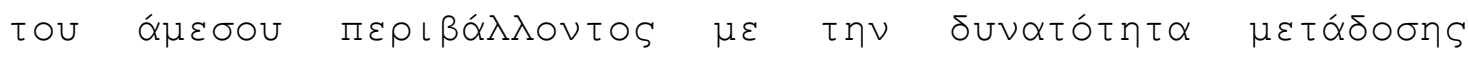

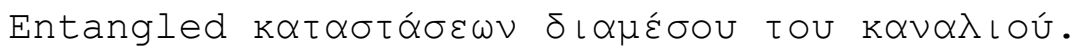

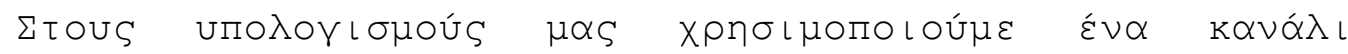

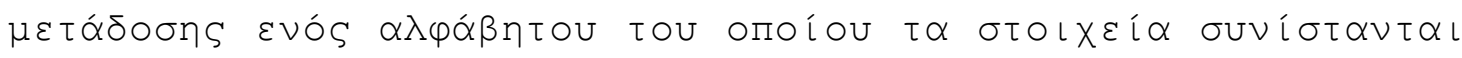




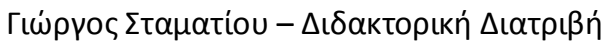

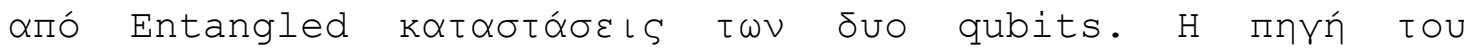

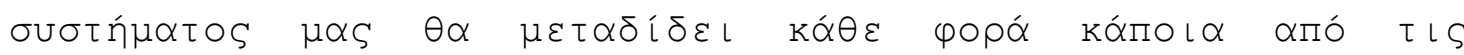

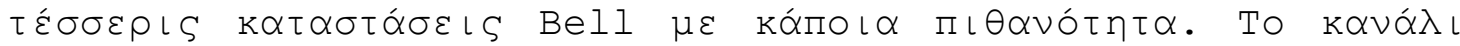

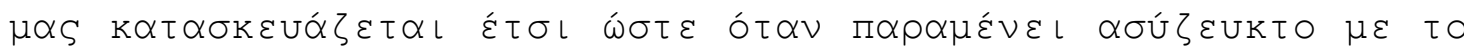

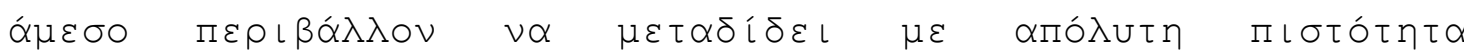

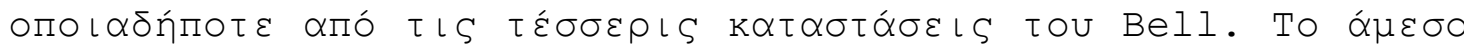

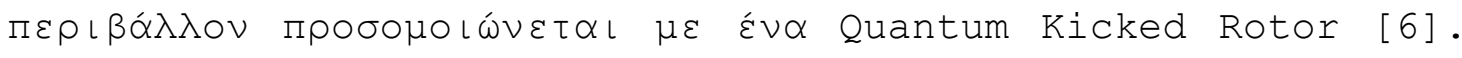

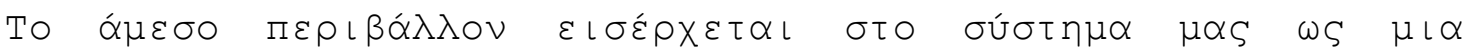

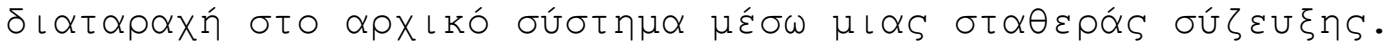

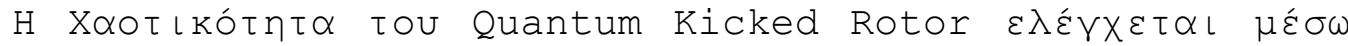

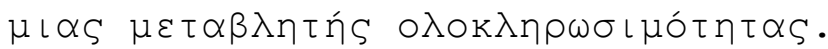

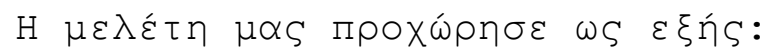

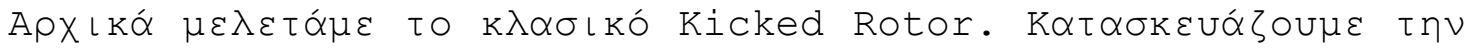

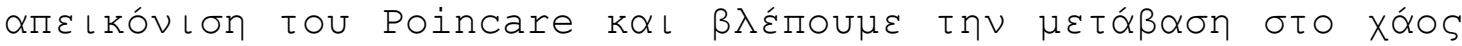

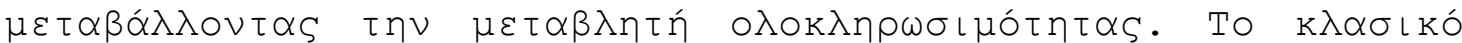

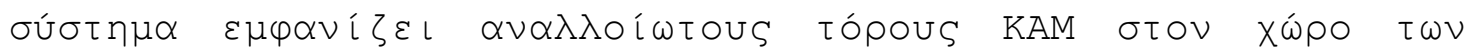

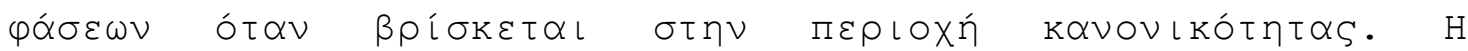

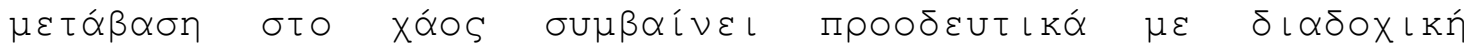

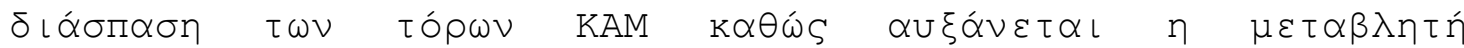

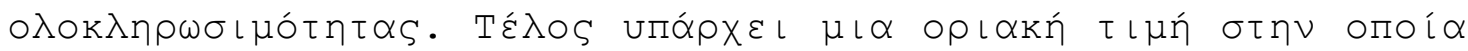

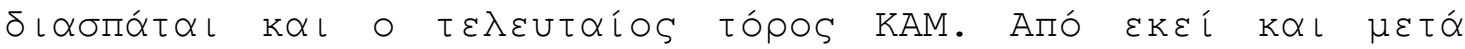

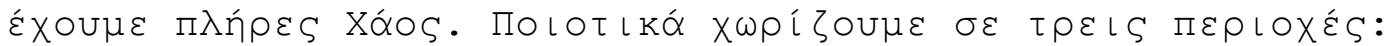

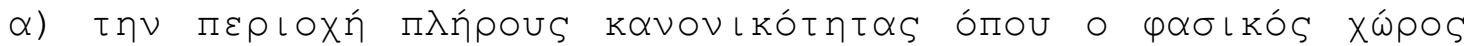

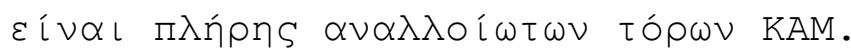

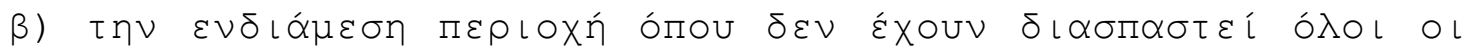

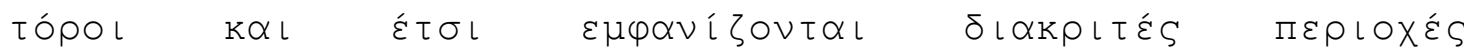

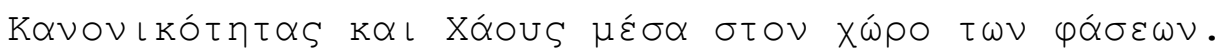

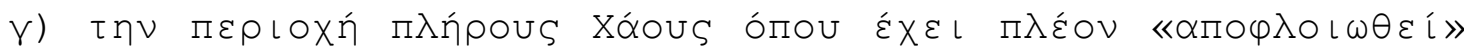

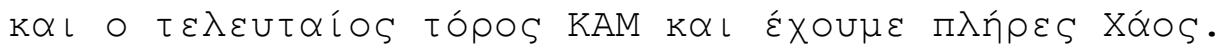

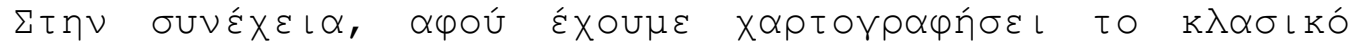

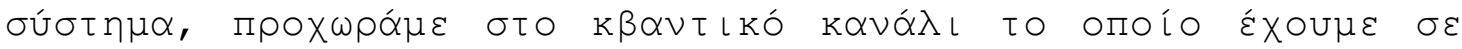

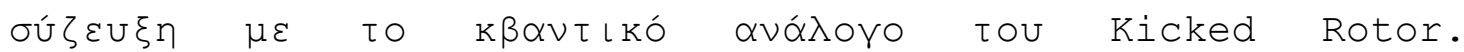
Е

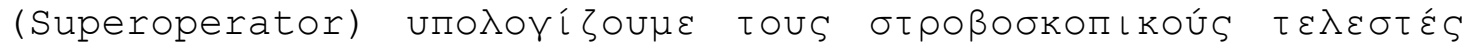

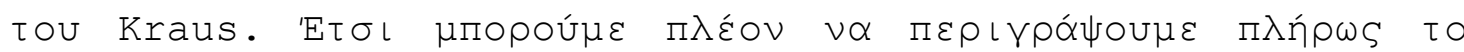

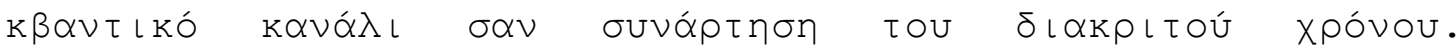




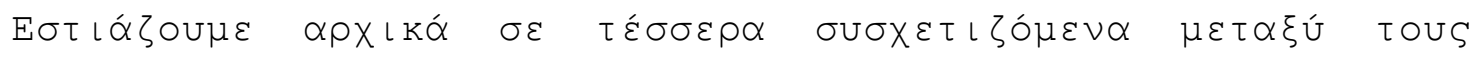

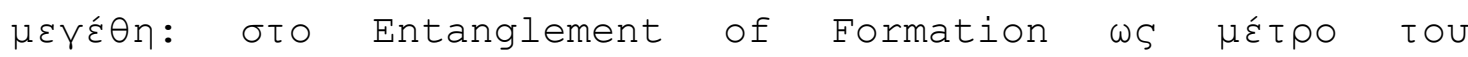

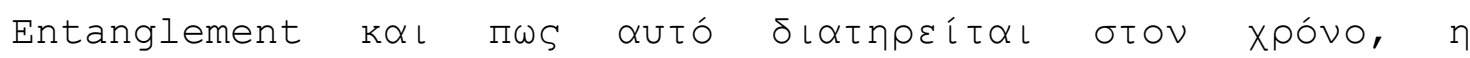

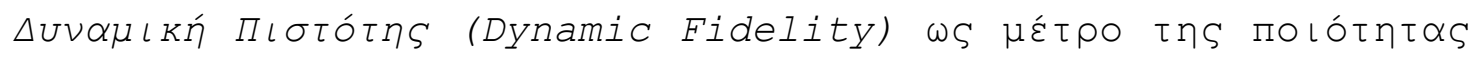

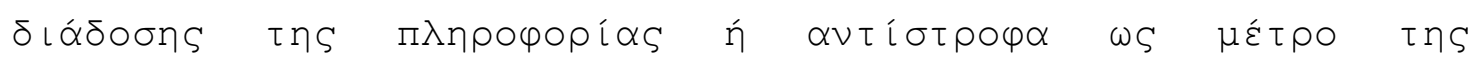

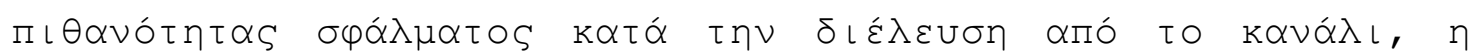

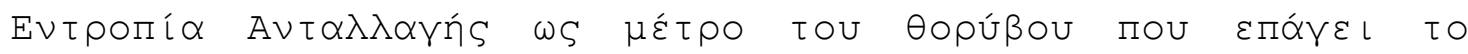

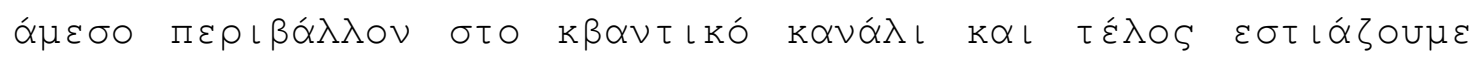

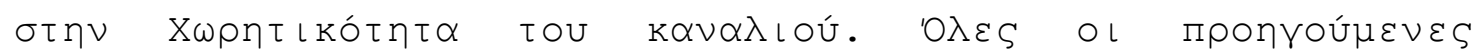

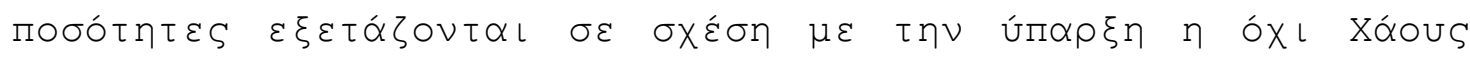

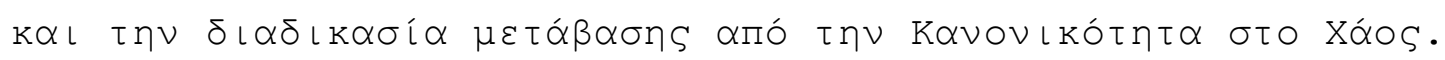

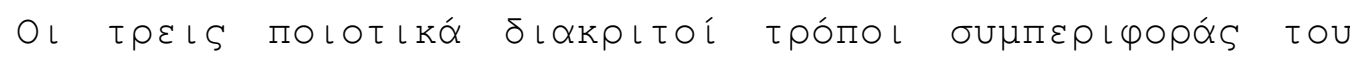

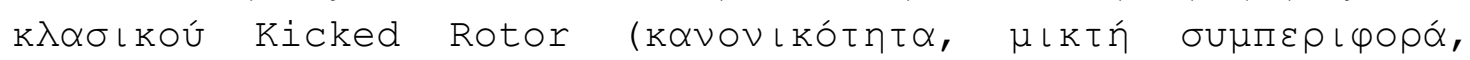

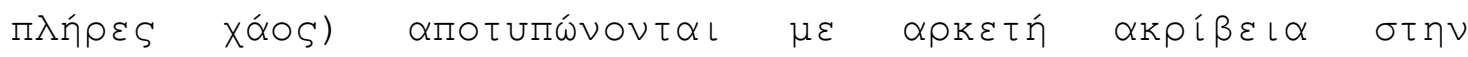

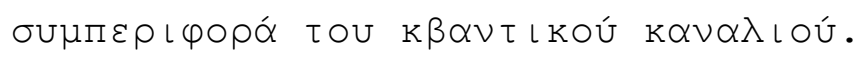

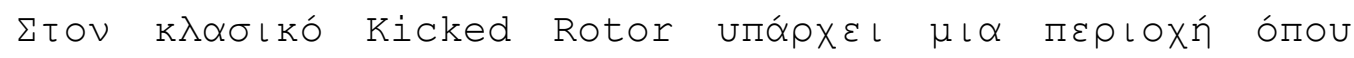

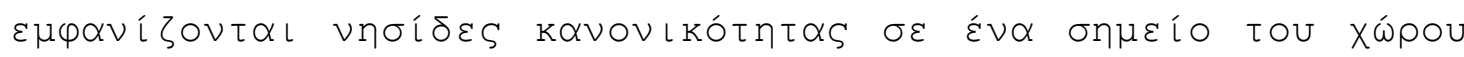

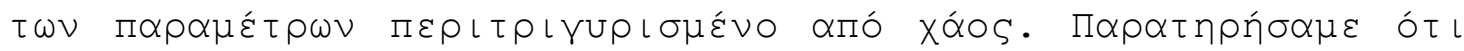

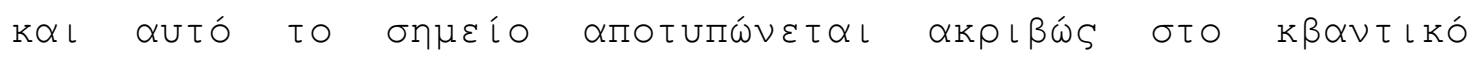

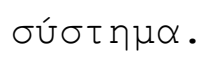

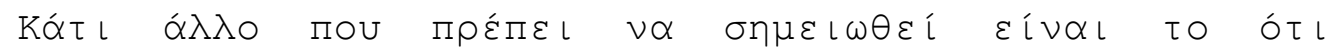

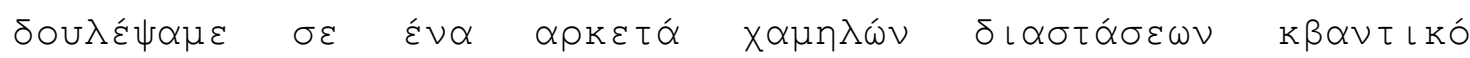

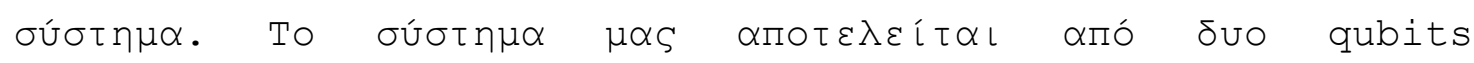

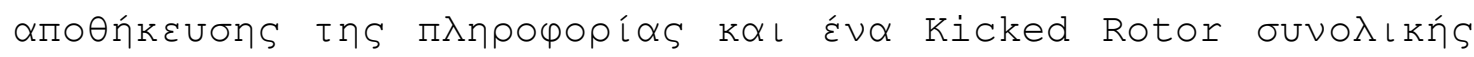

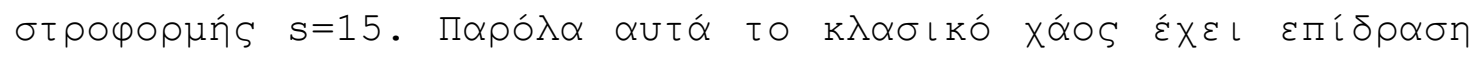

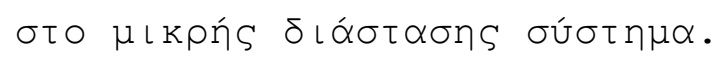

\section{Classical Kicked Rotor (CKR)}

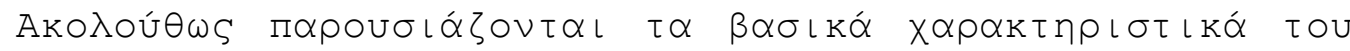

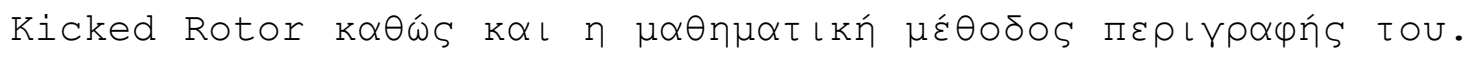

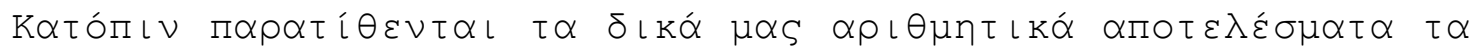

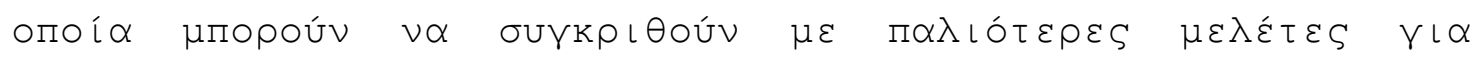

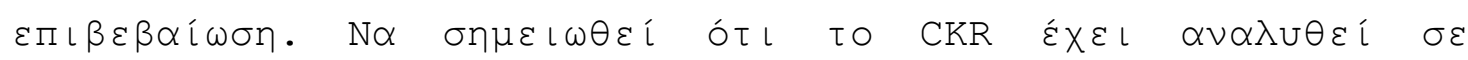

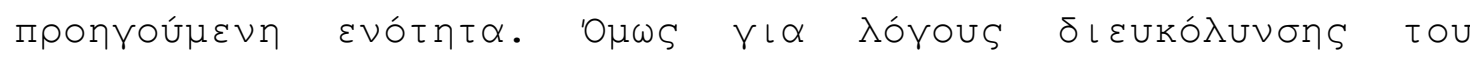

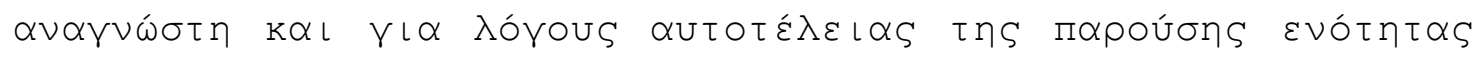

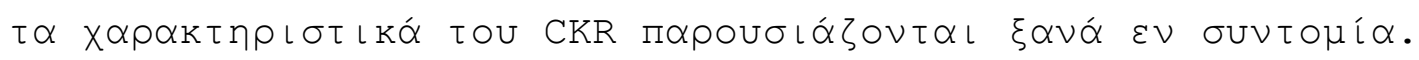




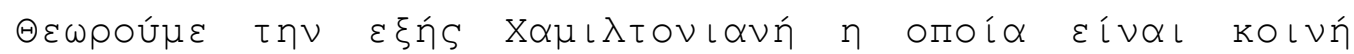

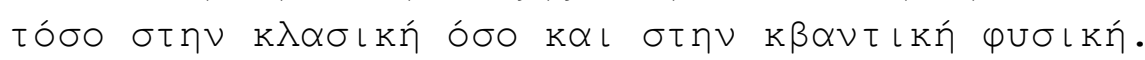

$$
H=A S_{z}^{2}-\mu B S_{x} \sum_{j=-\infty}^{+\infty} \delta(t-2 \pi j)
$$

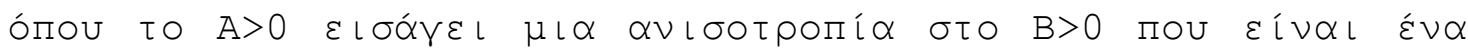

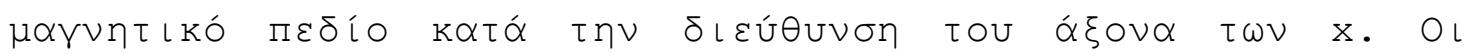

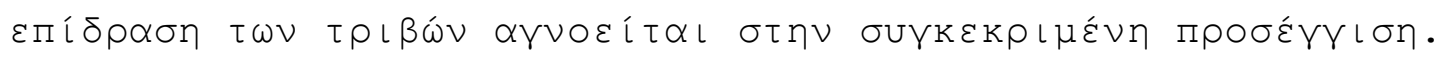

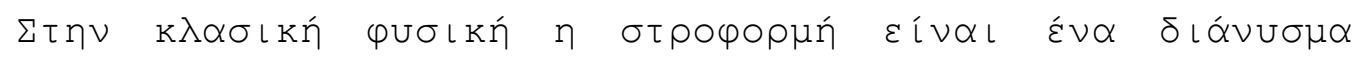

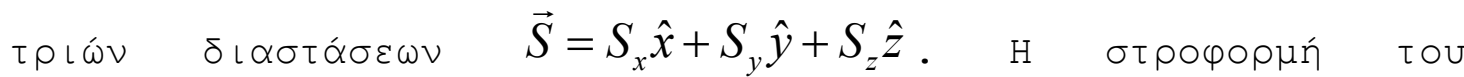

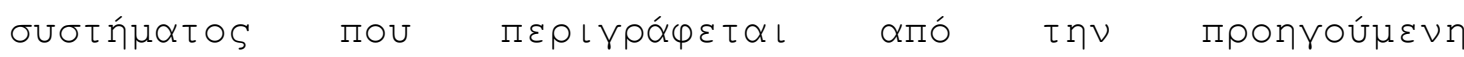

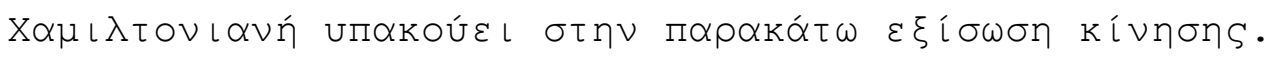

$$
\frac{d \vec{S}}{d t}=\vec{S} \times\left(-\frac{\delta H}{\delta \vec{S}}\right)=\vec{S} \times\left[-2 A S_{z} \hat{z}+\mu B \hat{x} \sum_{j=-\infty}^{+\infty} \delta(t-2 \pi j)\right]
$$

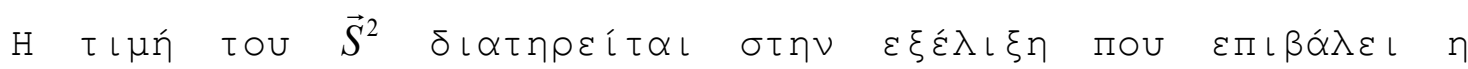

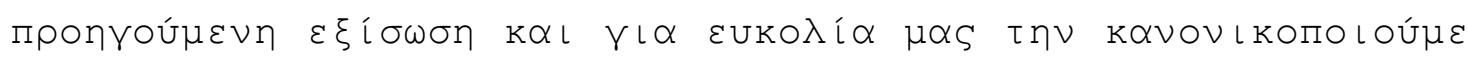

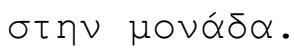

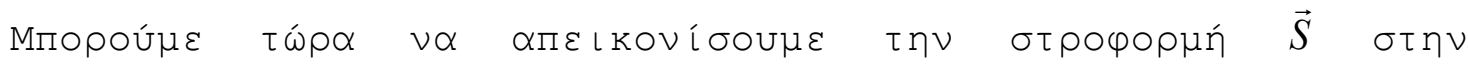

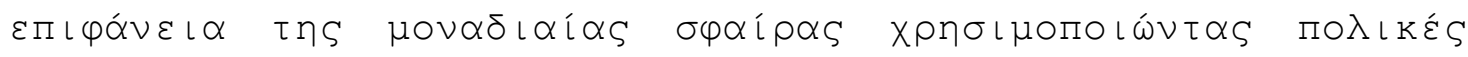

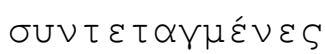

$$
\vec{S}=S_{x} \hat{x}+S_{y} \hat{y}+S_{z} \hat{z}=\sin \theta \cos \phi \hat{x}+\sin \vartheta \sin \phi \hat{y}+\cos \theta \hat{z}
$$

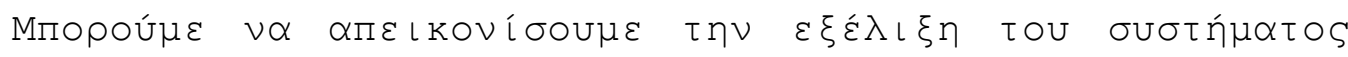

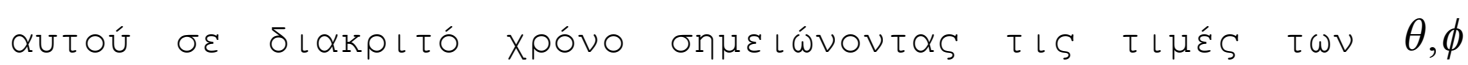

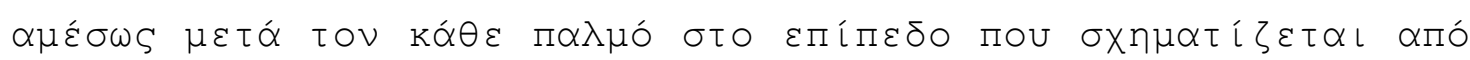

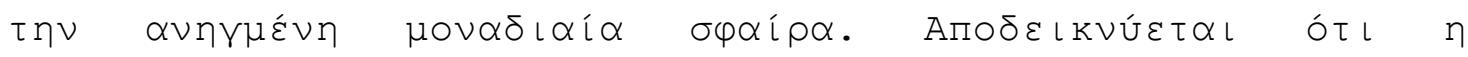

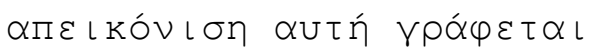

$$
\begin{aligned}
& S_{x}^{\prime}=\Gamma_{x} \\
& S_{y}^{\prime}=\Gamma_{y} \cos (\mu B)+\Gamma_{z} \sin (\mu B) \\
& S_{z}^{\prime}=-\Gamma_{y} \sin (\mu B)+\Gamma_{z} \cos (\mu B)
\end{aligned}
$$




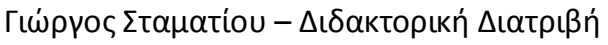

$\mu \varepsilon$

$$
\begin{aligned}
& \Gamma_{x}=S_{x} \cos \left(4 \pi A S_{z}\right)-S_{y} \sin \left(4 \pi A S_{z}\right) \\
& \Gamma_{x}=S_{x} \sin \left(4 \pi A S_{z}\right)+S_{y} \cos \left(4 \pi A S_{z}\right) \\
& \Gamma_{z}=S_{z}
\end{aligned}
$$

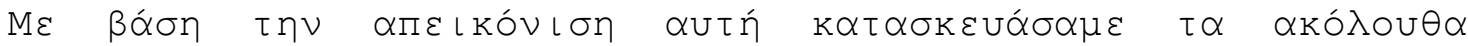
үрари́

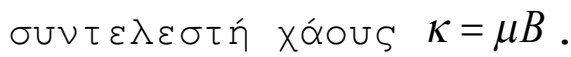

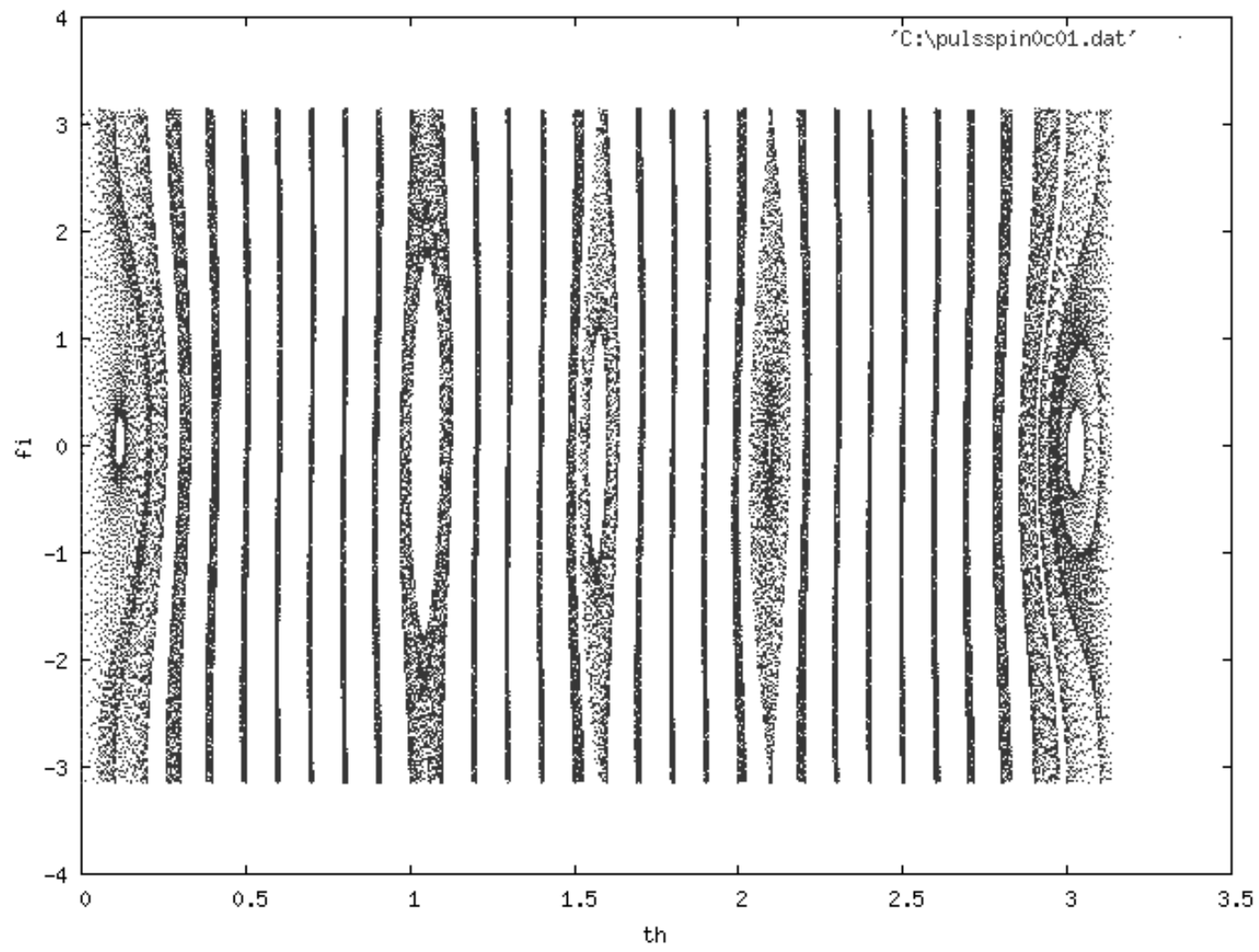

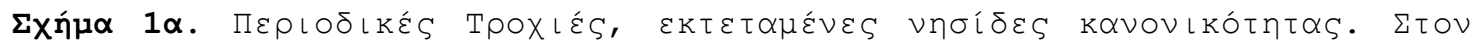

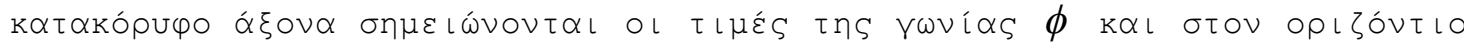

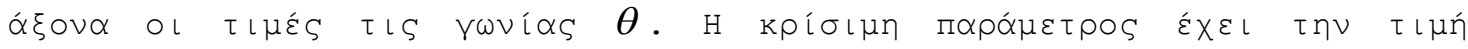

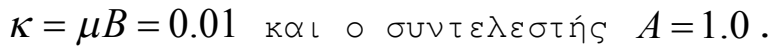




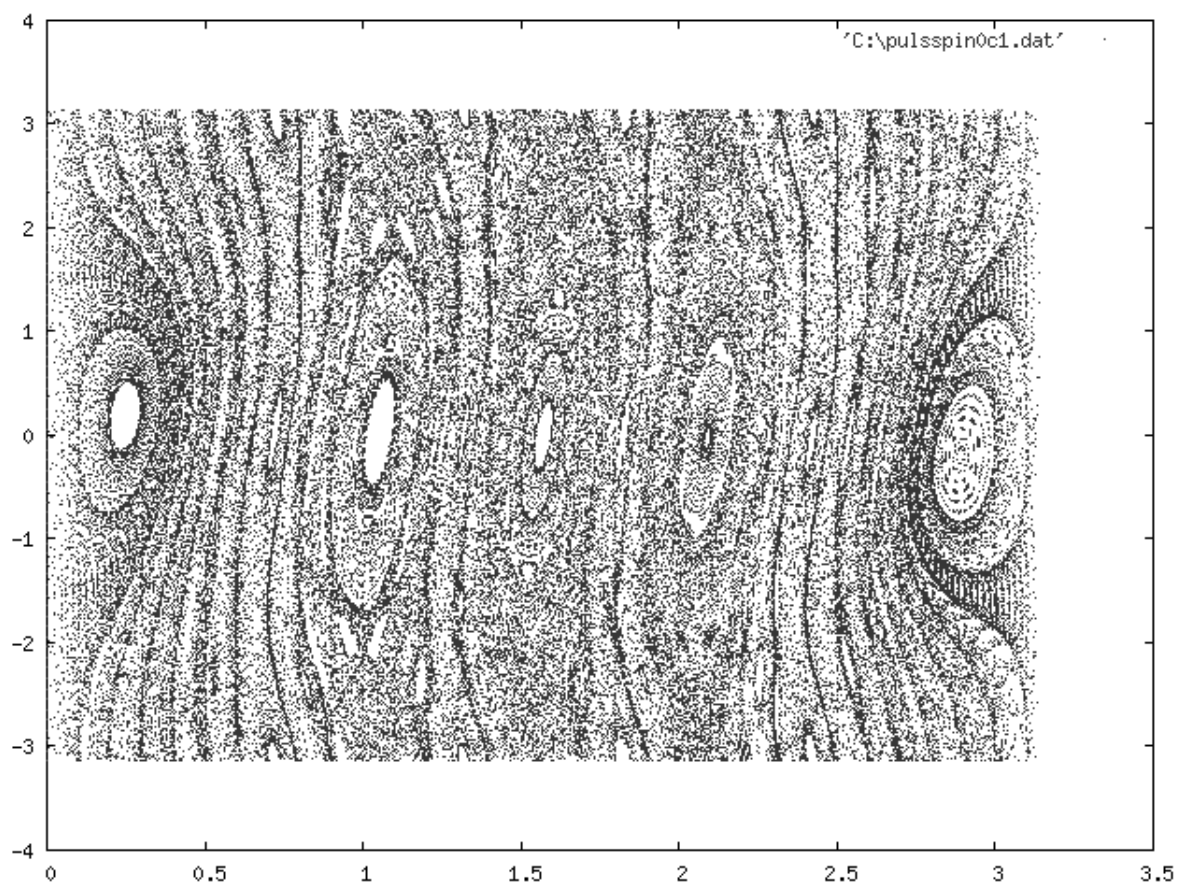

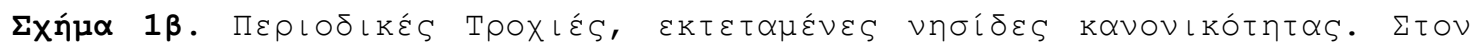

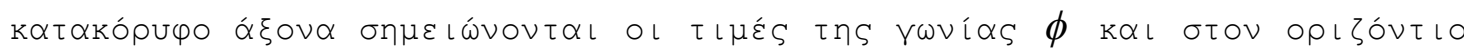

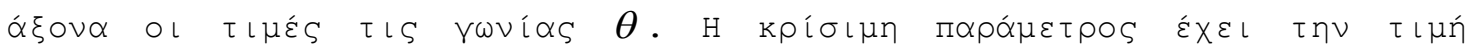

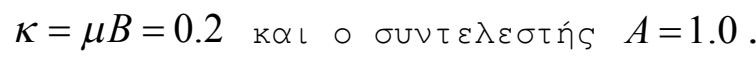




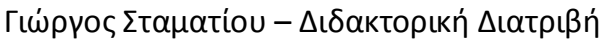

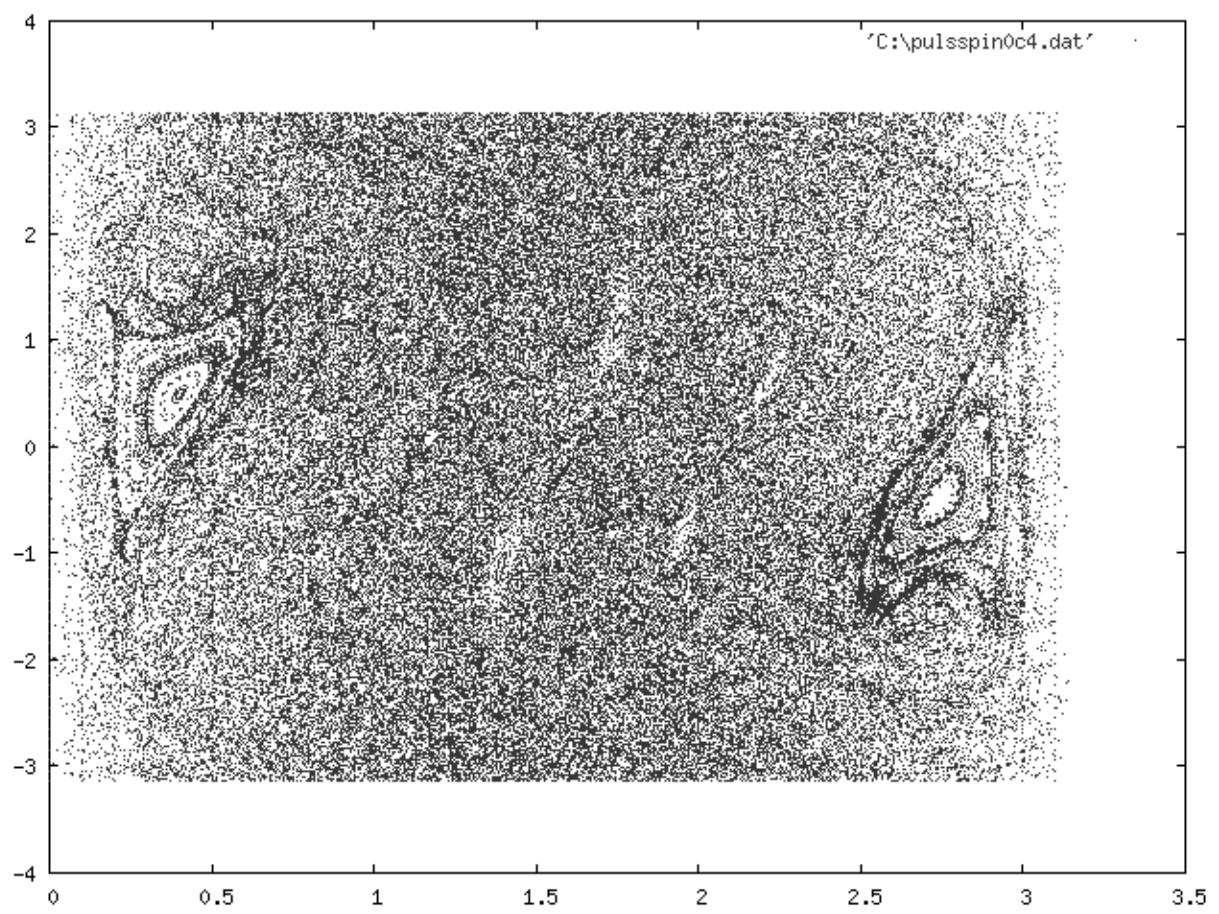

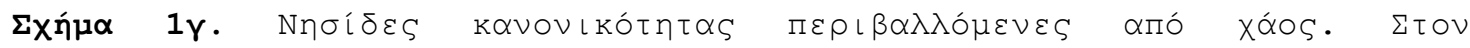

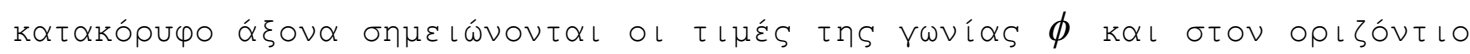

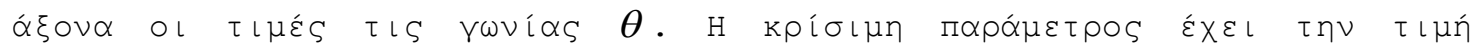

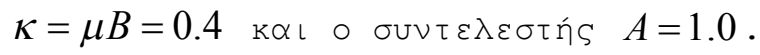




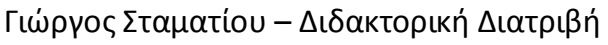

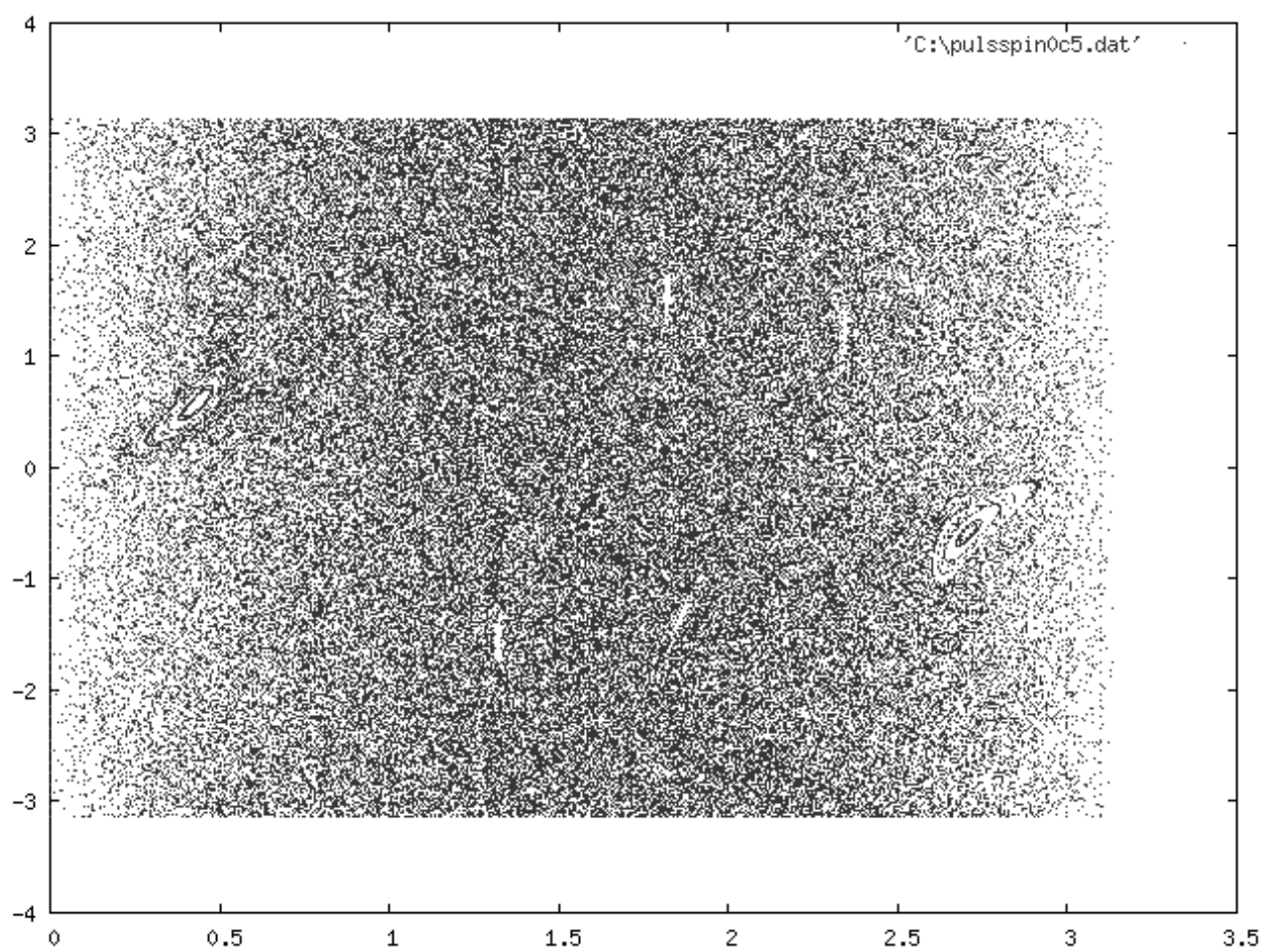

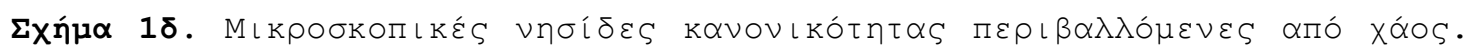

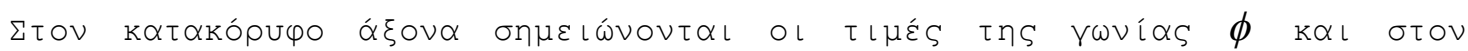

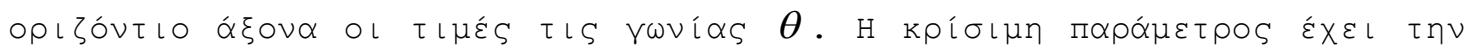

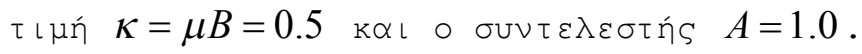




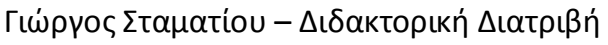

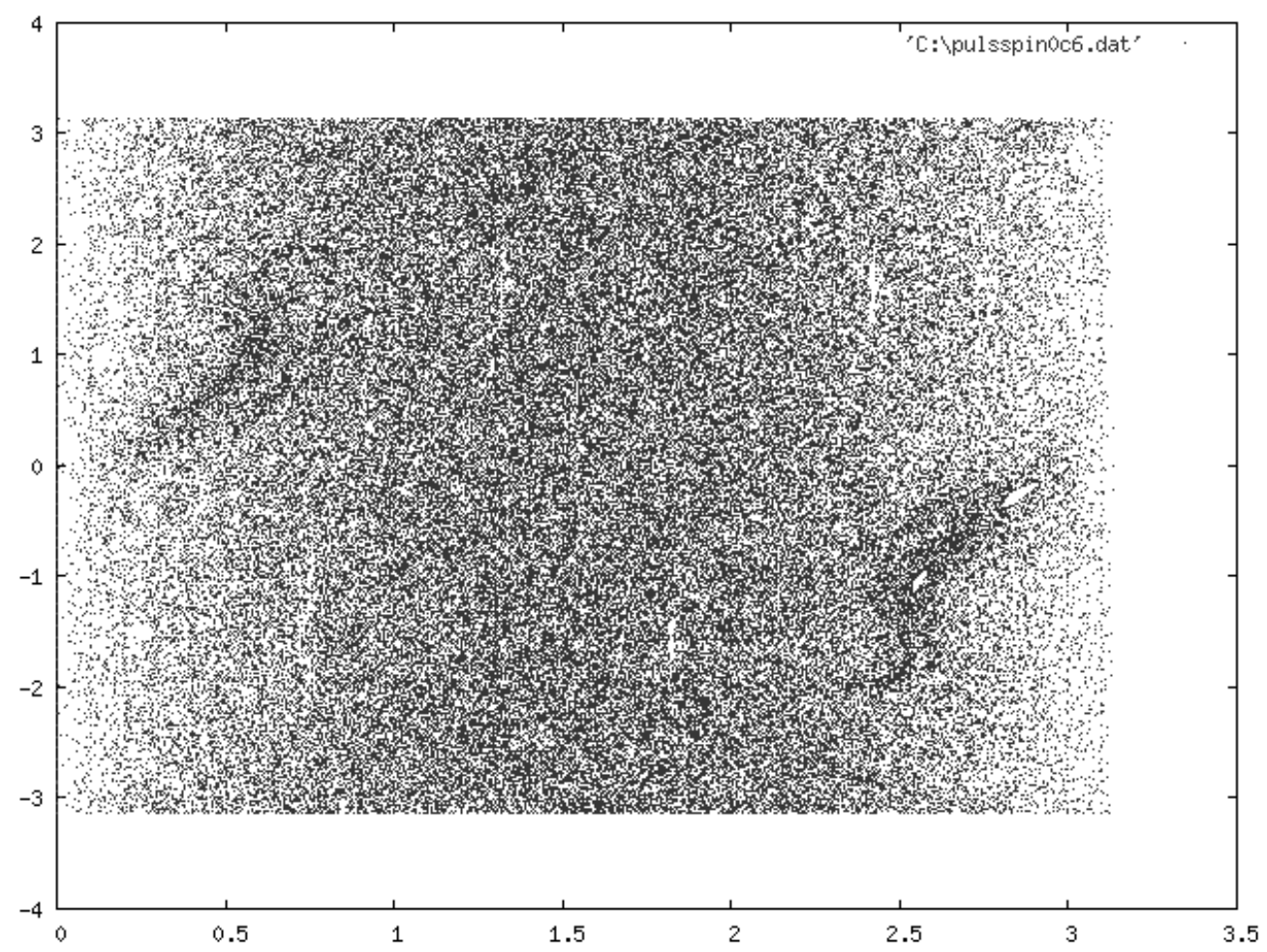

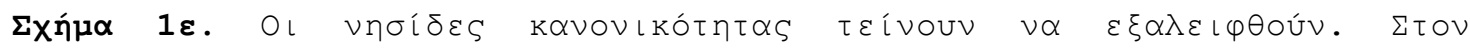

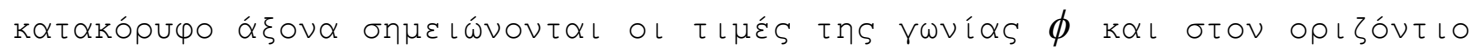

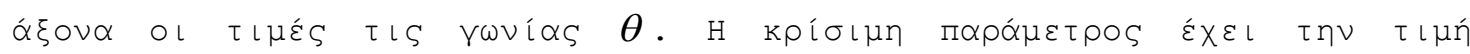

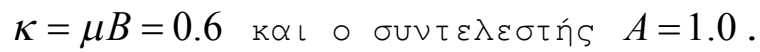




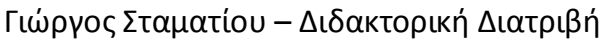

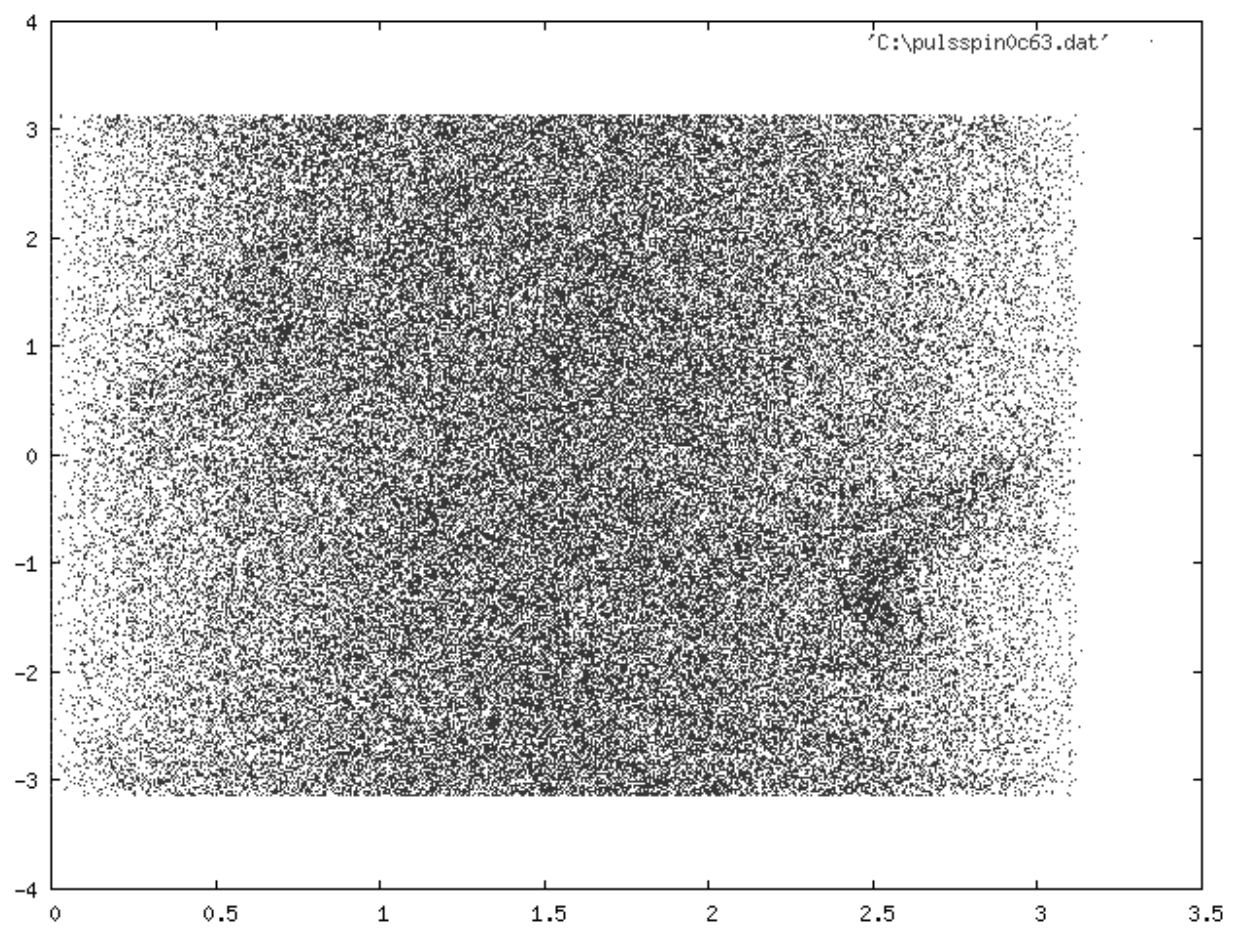

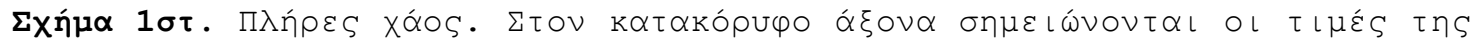

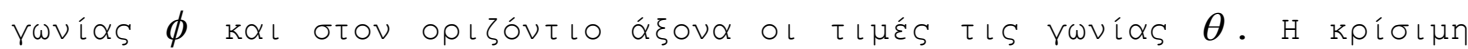

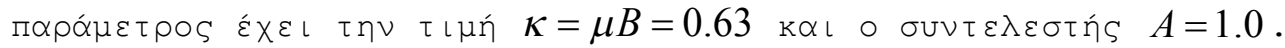




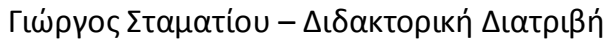

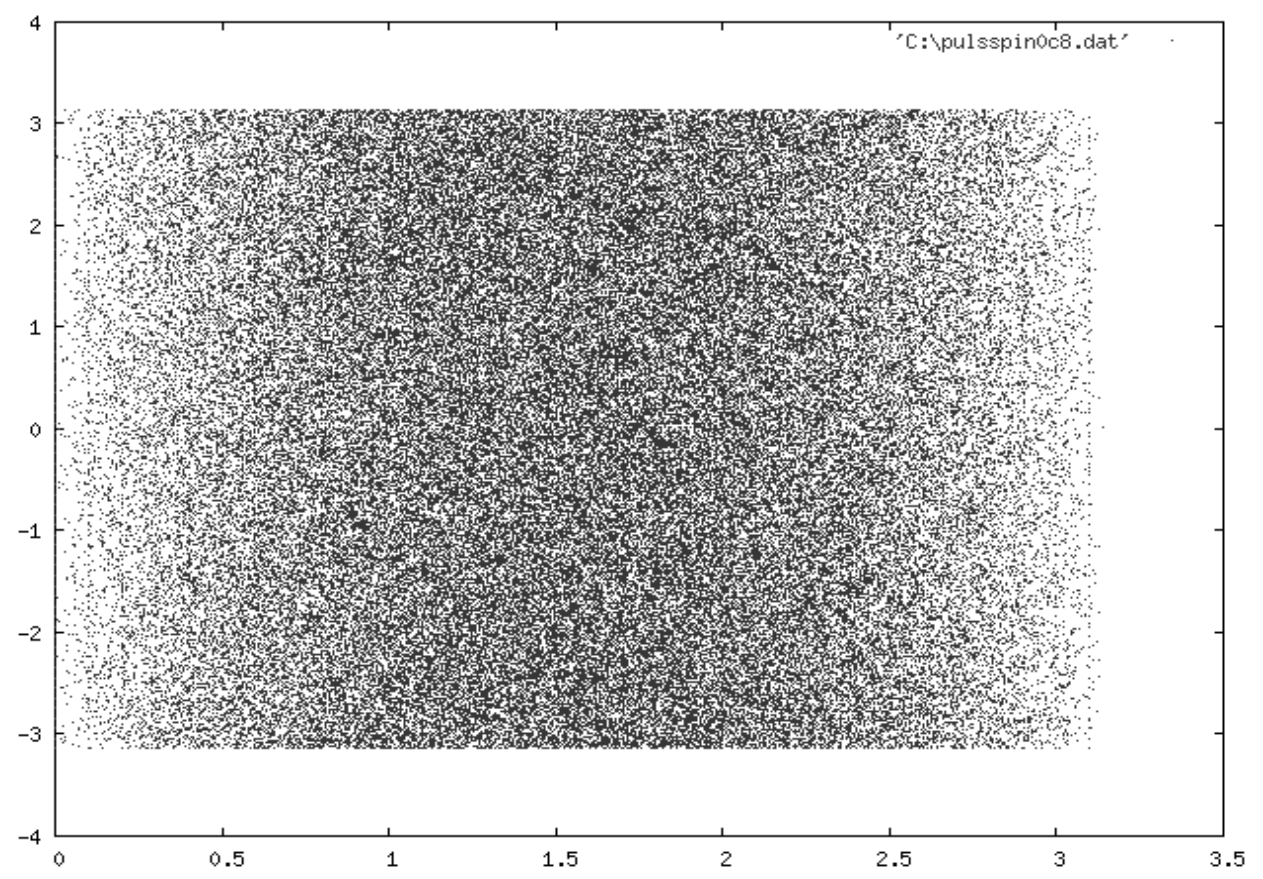

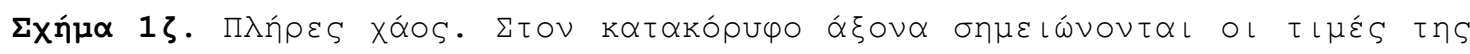

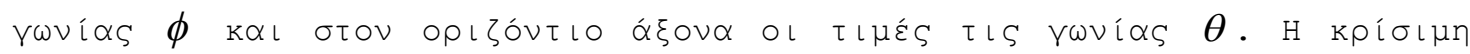

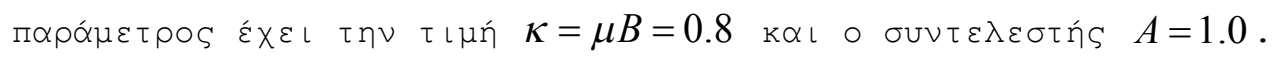

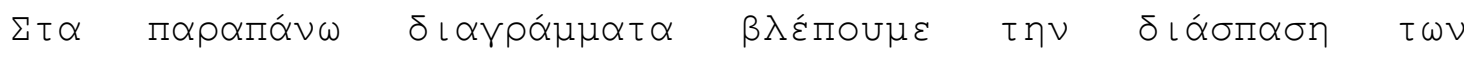

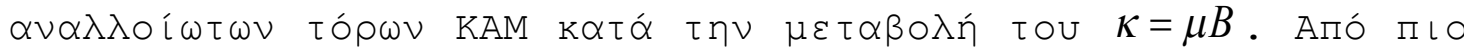

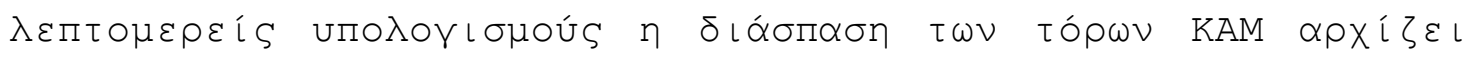

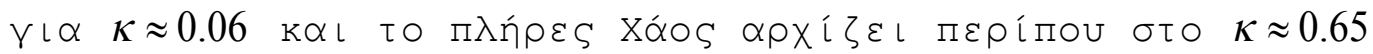

\section{Quantum Kicked Rotor (QKR)}

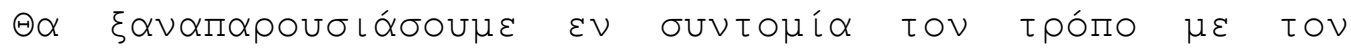

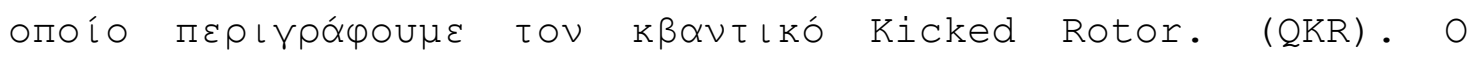

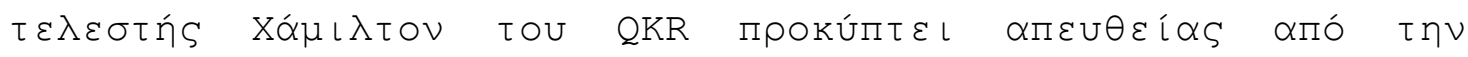

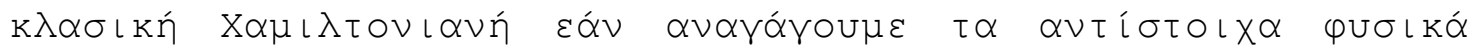

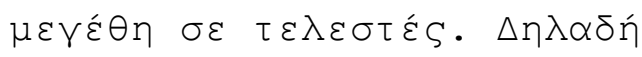

$$
\hat{H}=A \hat{S}_{z}^{2}-\mu B \hat{S}_{x} \sum_{j=-\infty}^{+\infty} \delta(t-2 \pi j)
$$




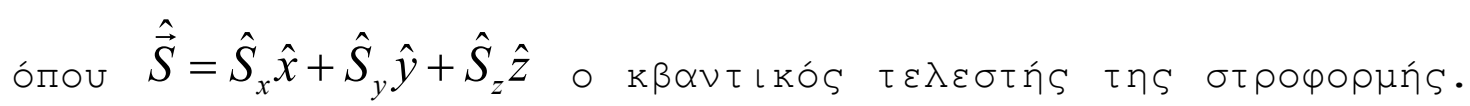

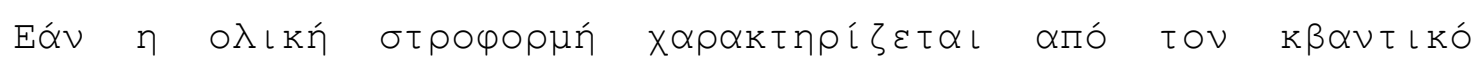

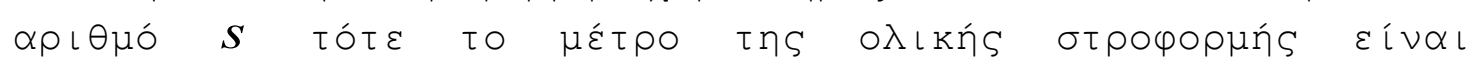

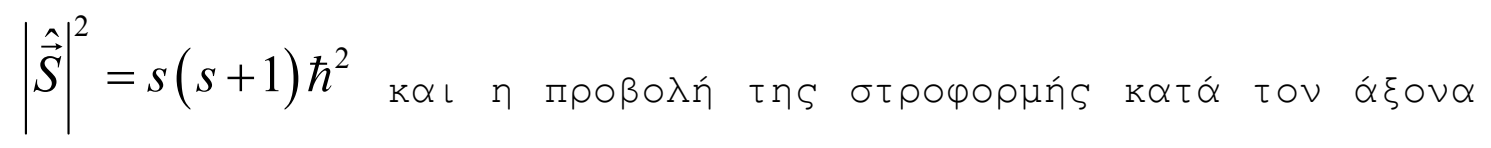

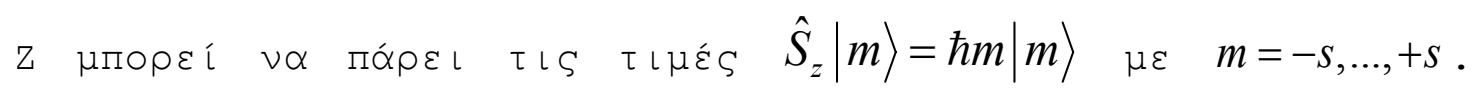

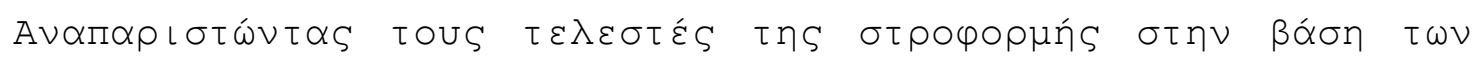

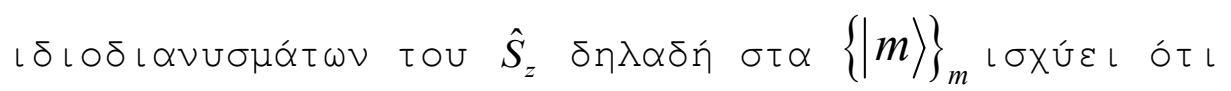

$$
\begin{gathered}
(\hat{S} z)_{m m^{\prime}}=\hbar m^{\prime} \delta_{m, m^{\prime}} \\
\left(\hat{S}_{ \pm}\right)_{m m^{\prime}}=\hbar \sqrt{\left(s \mp m^{\prime}\right)\left(s \pm m^{\prime}+1\right)} \delta_{m, m^{\prime} \pm 1} \\
\hat{S}_{x}=\frac{1}{2}\left(\hat{S}_{+}+\hat{S}_{-}\right) \\
\hat{S}_{y}=\frac{1}{2 i}\left(\hat{S}_{+}-\hat{S}_{-}\right)
\end{gathered}
$$

ónоu

$$
\hat{S}_{ \pm}|m\rangle=\hbar \sqrt{(s \mp m)(s \pm m+1)}|m \pm 1\rangle
$$

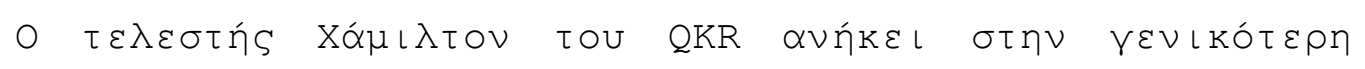

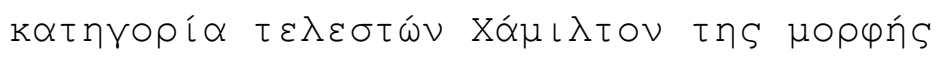

$$
\hat{H}(t)=\hat{H}_{o}+\hat{V} \sum_{j=-\infty}^{+\infty} \delta(t-2 \pi j)
$$

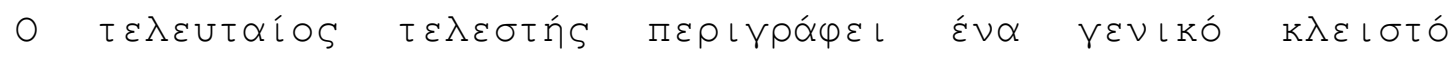

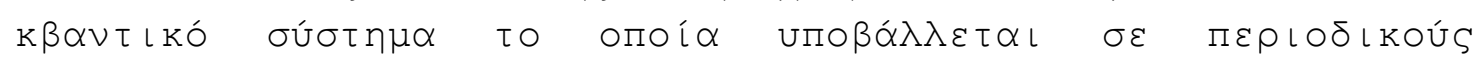

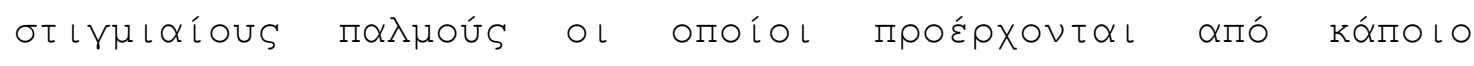

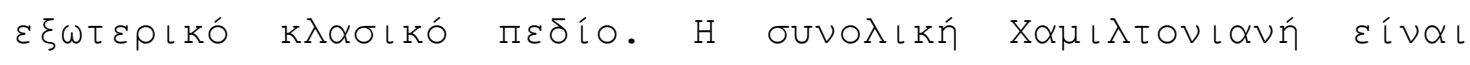

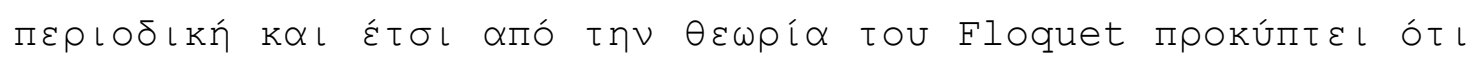




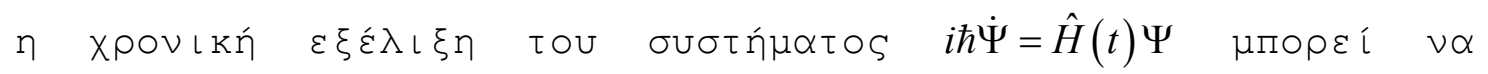

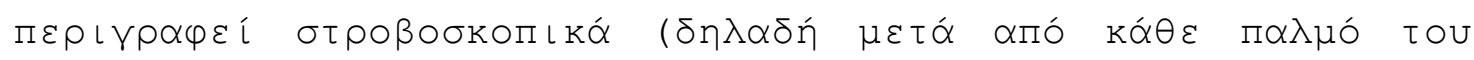

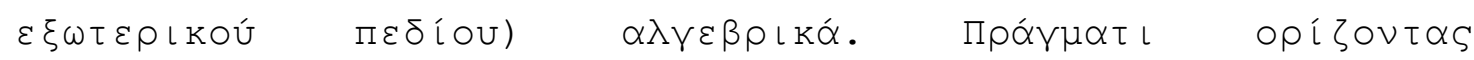

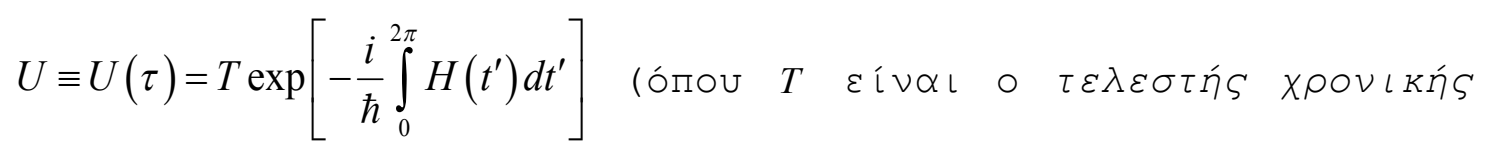

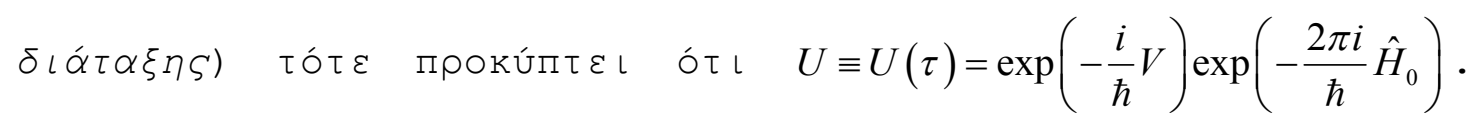

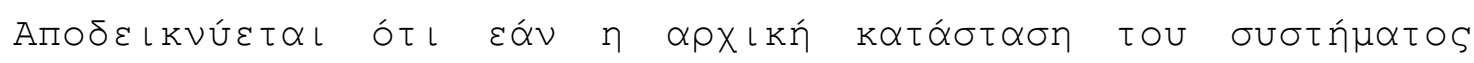

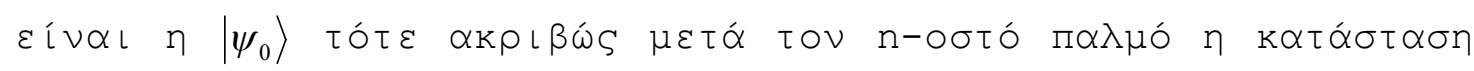

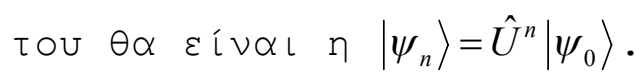

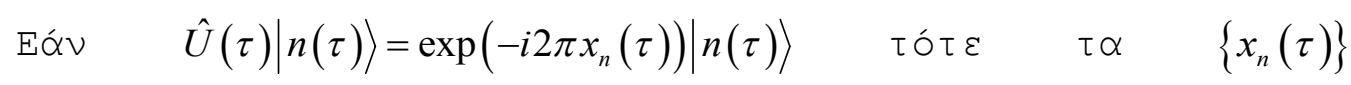

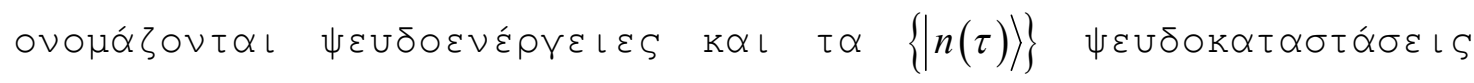

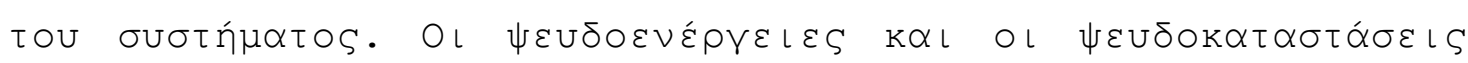

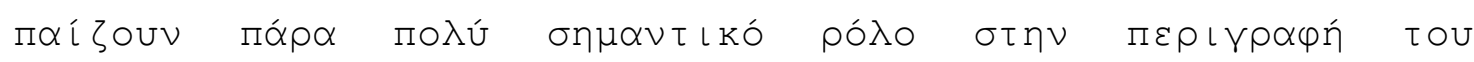

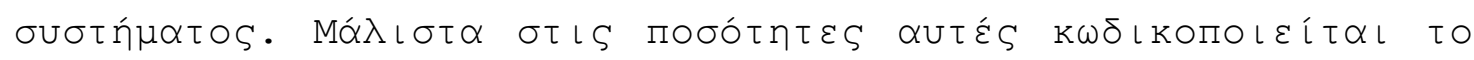

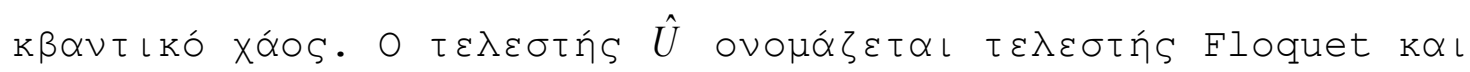

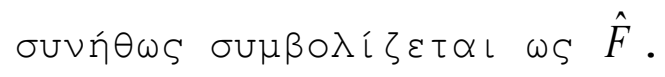

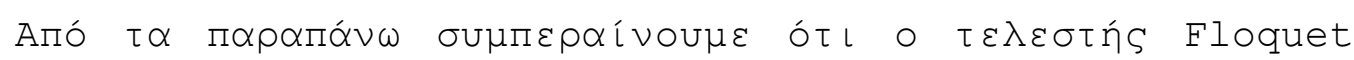

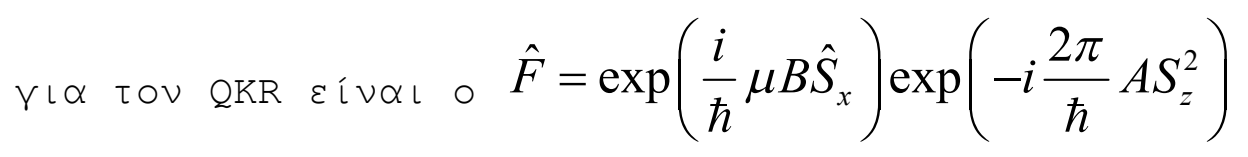

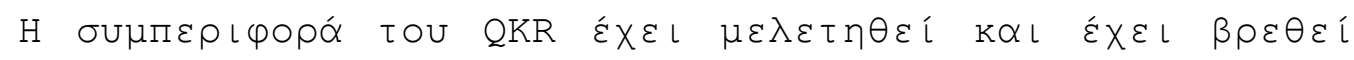

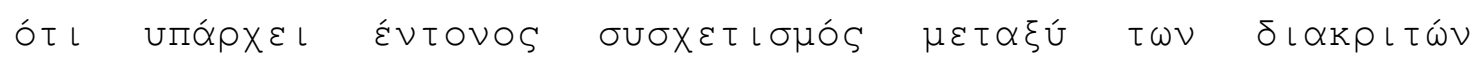

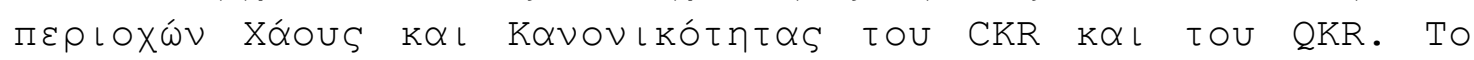

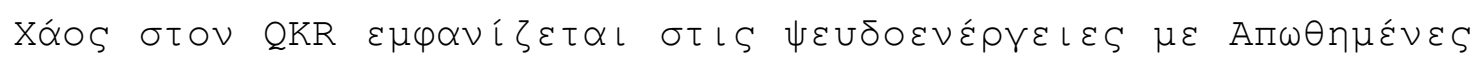

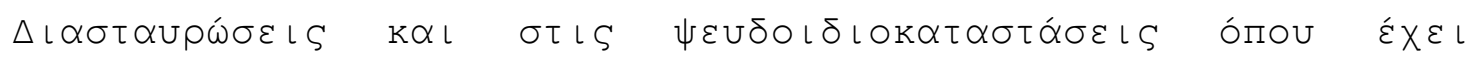

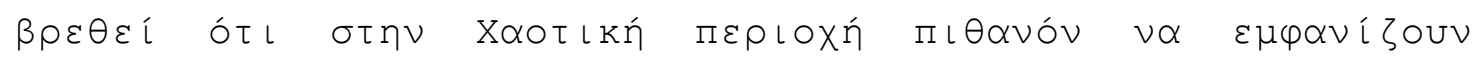

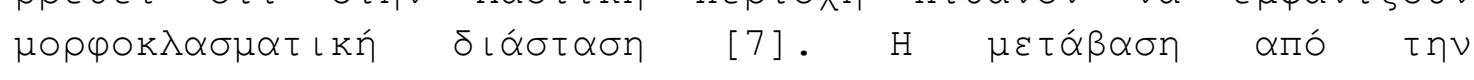

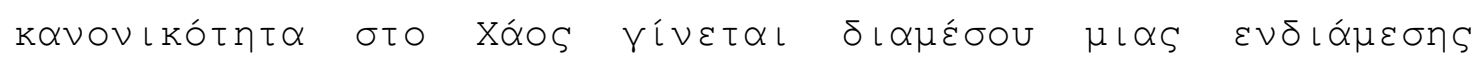

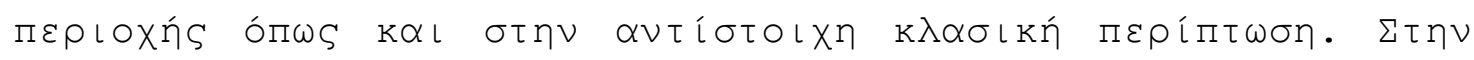

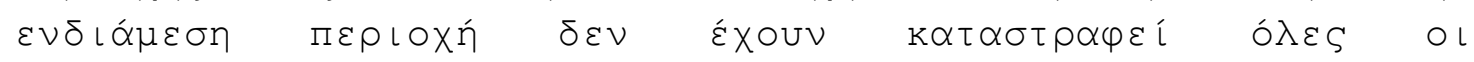

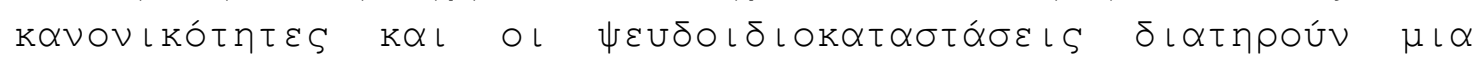

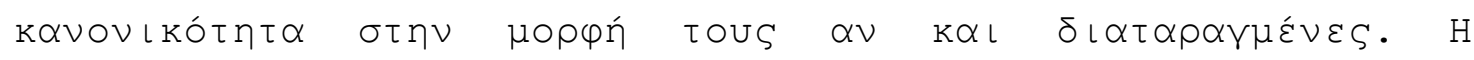

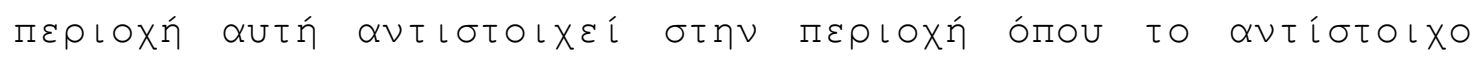

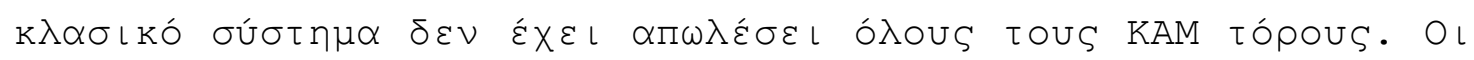




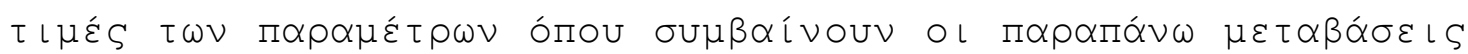

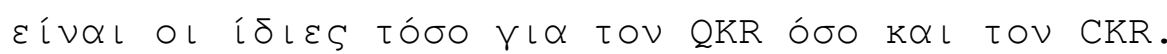

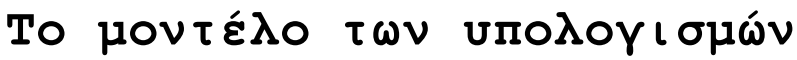

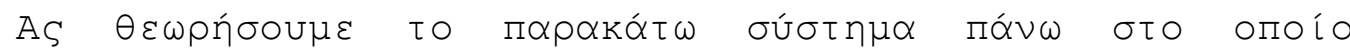

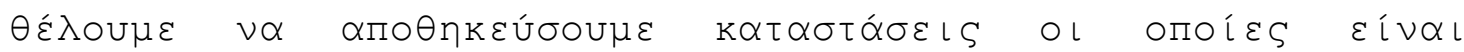

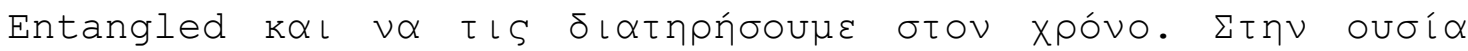

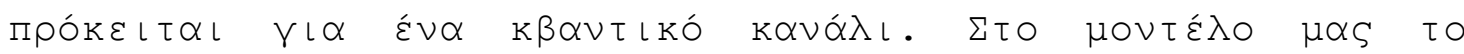

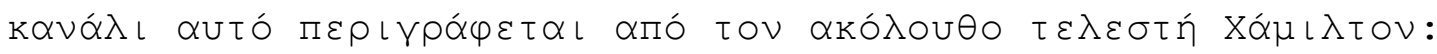

$$
\hat{H}=\hat{H}_{r e g}+\gamma \hat{H}_{c}+\hat{H}_{e}
$$

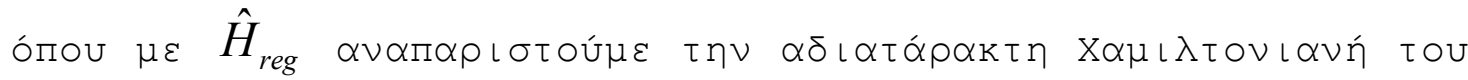

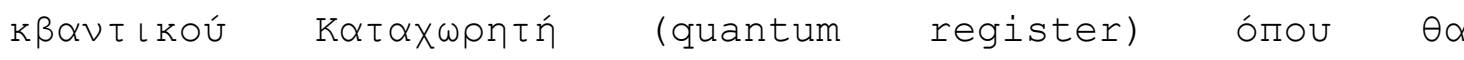

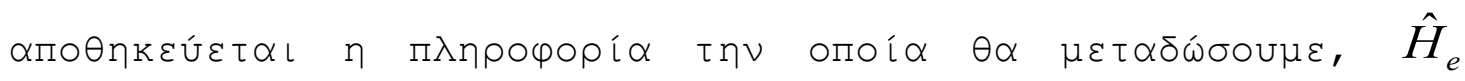

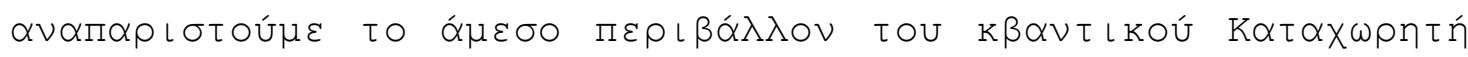

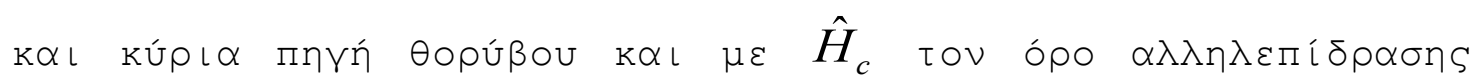

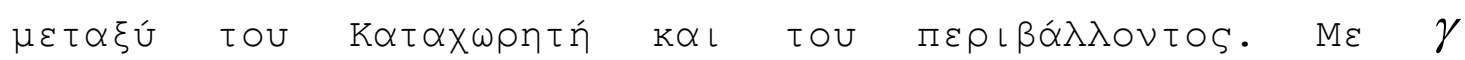

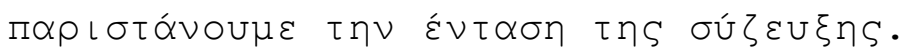

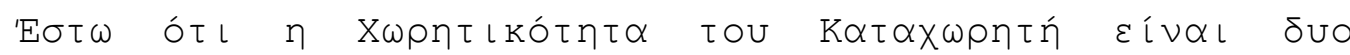

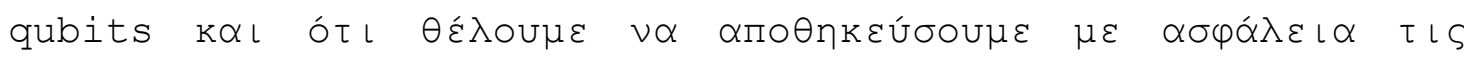

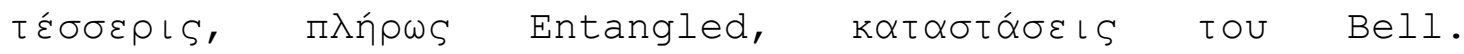

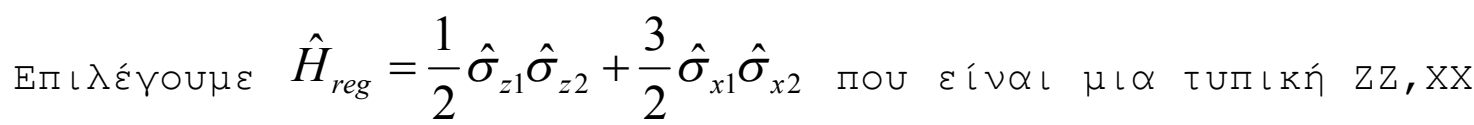

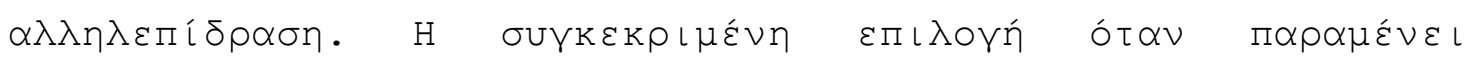

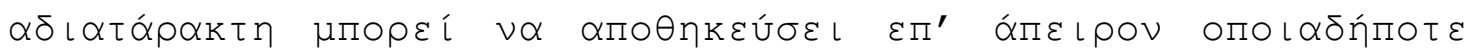

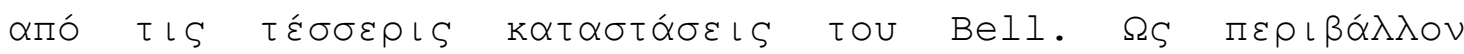

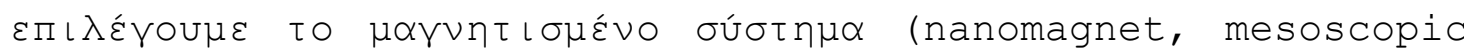

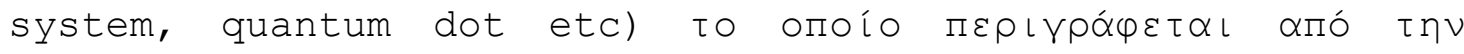

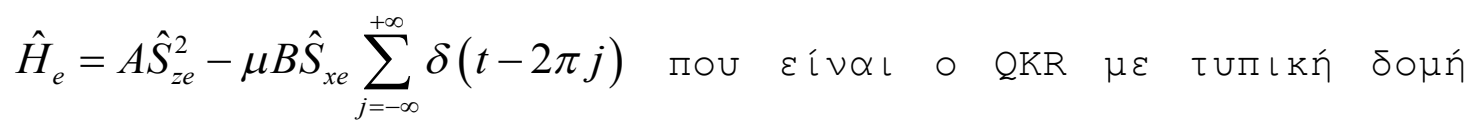

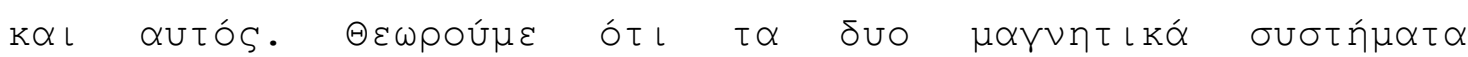

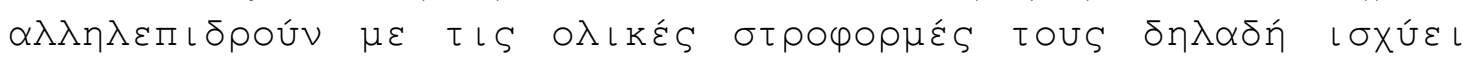




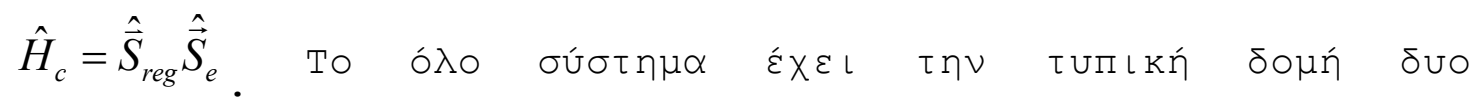

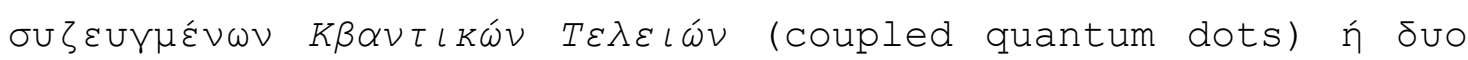

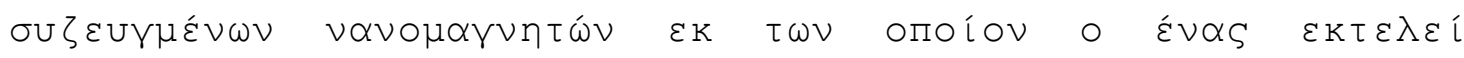

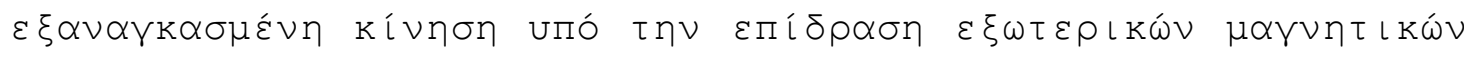

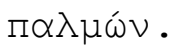

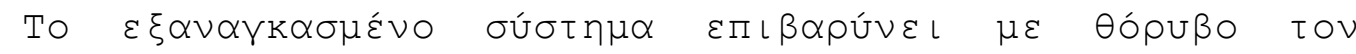

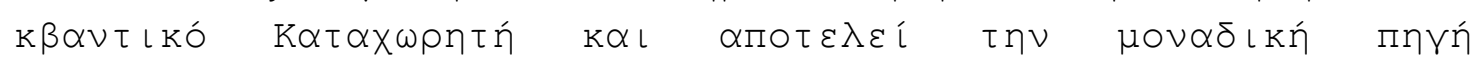

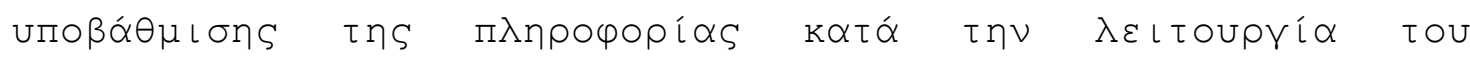

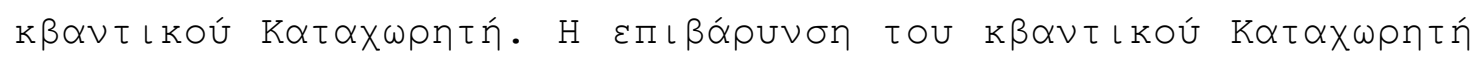

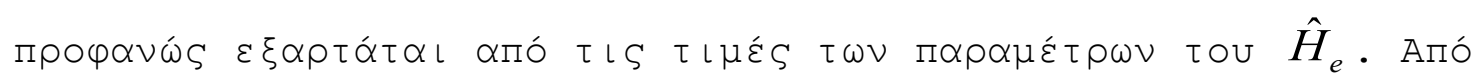

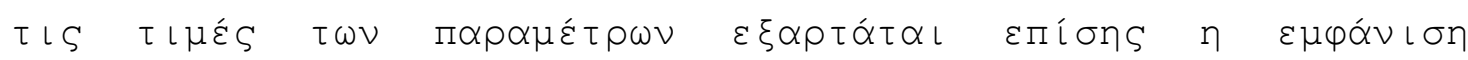

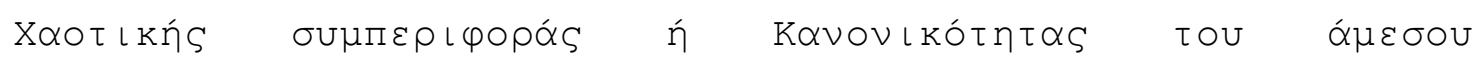

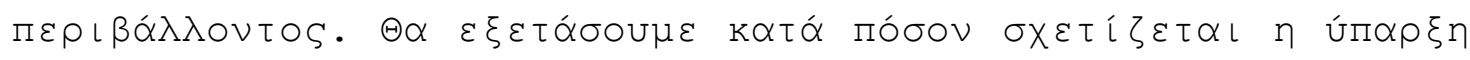

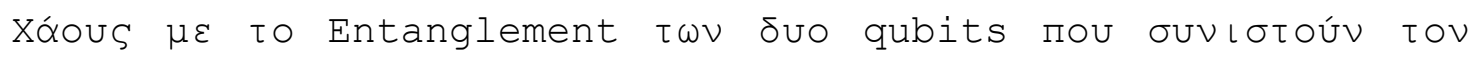

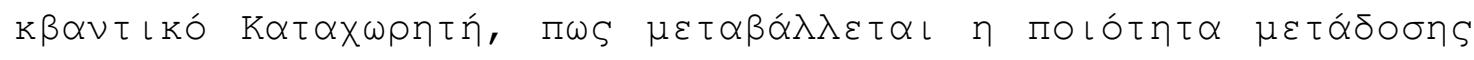

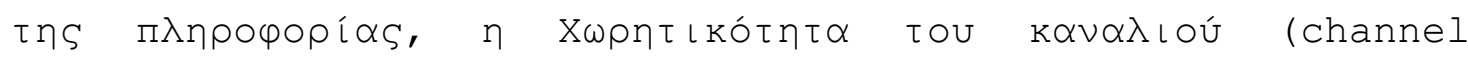

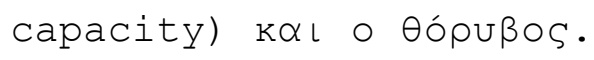

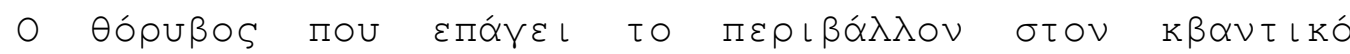

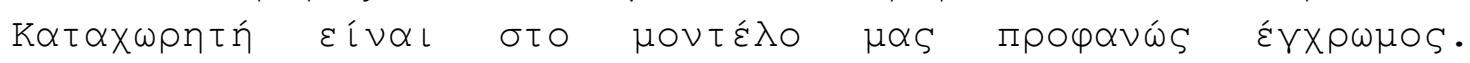

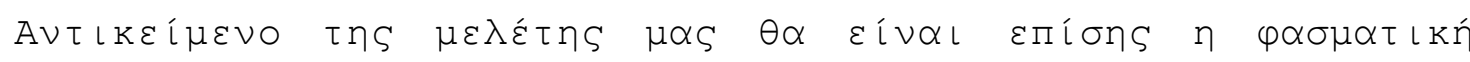

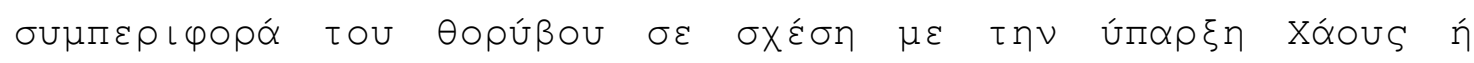

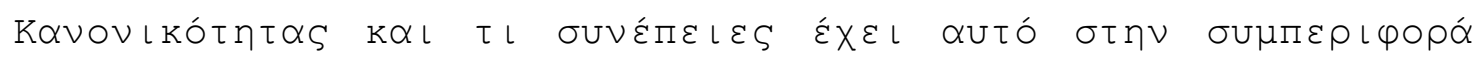

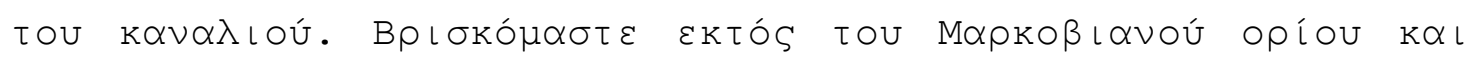

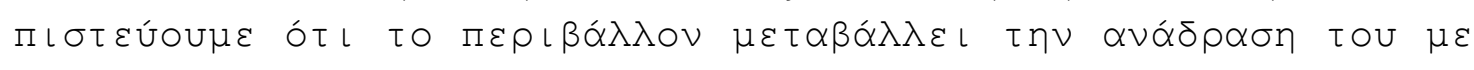

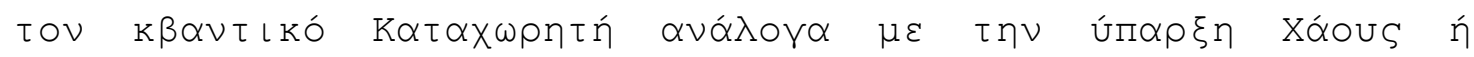

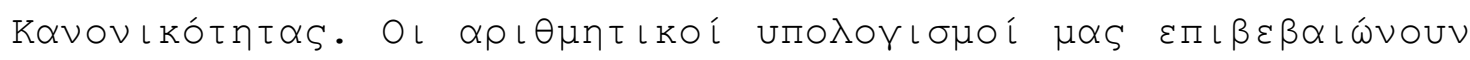

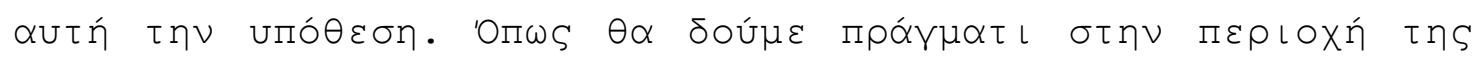

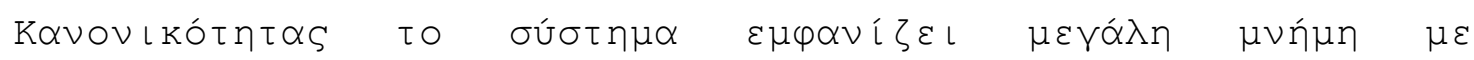

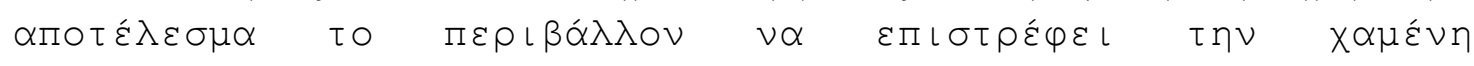

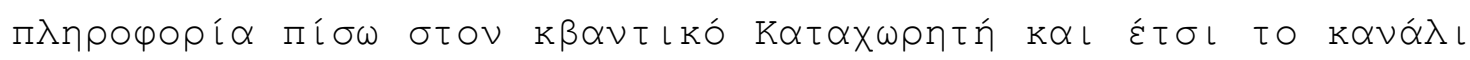

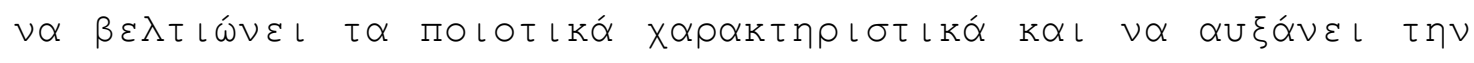

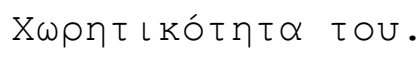

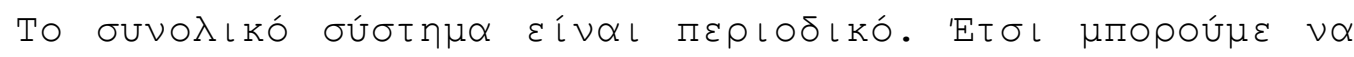

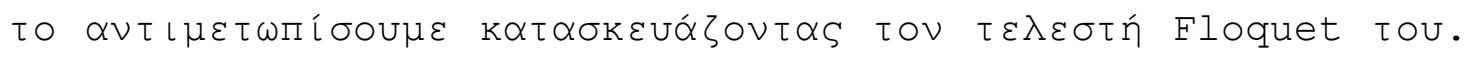

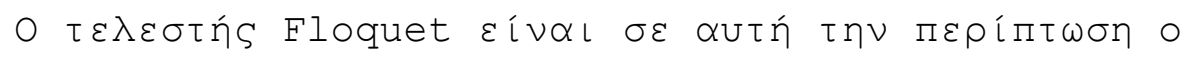




$$
\hat{F}=\exp \left(\frac{i}{\hbar} \mu B \hat{S}_{x e}\right) \exp \left[-\frac{2 \pi i}{\hbar}\left(\hat{H}_{r e g}+A \hat{S}_{z e}^{2}+\gamma \hat{H}_{c}\right)\right]
$$

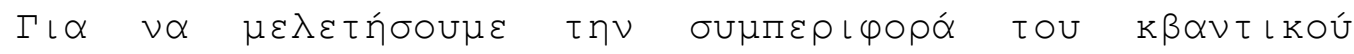

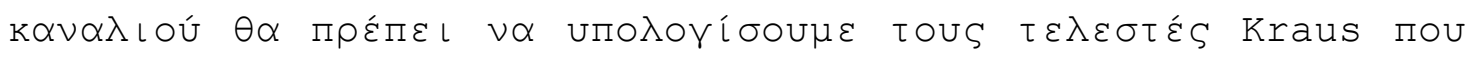

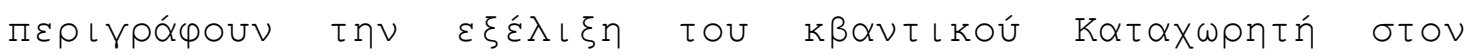

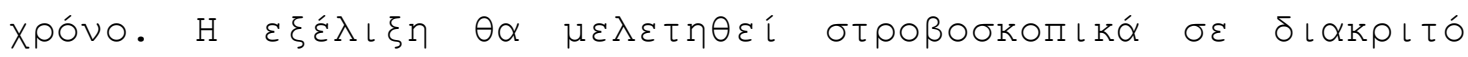

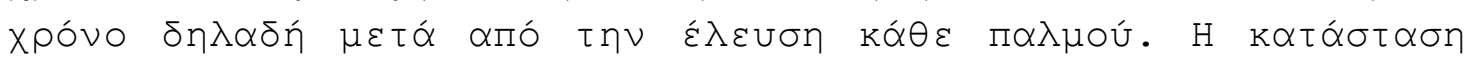

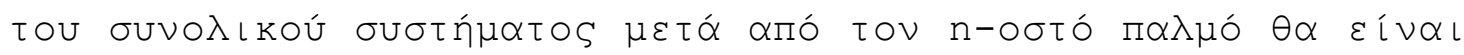

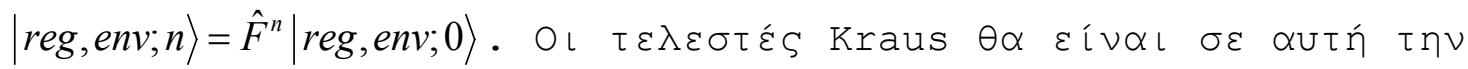

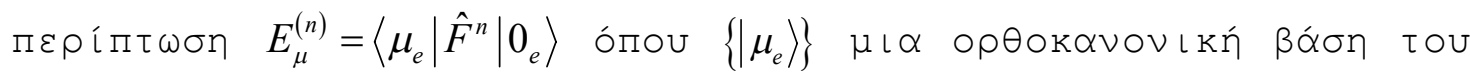

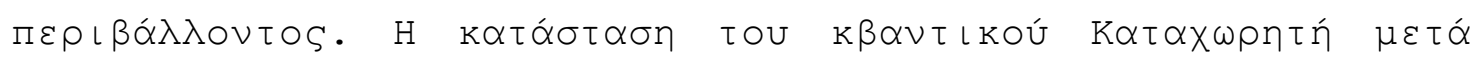

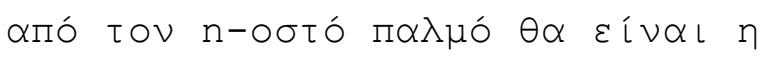

$$
\hat{\rho}_{r e g}^{(n)}=\sum_{\mu} E_{\mu}^{(n)} \hat{\rho}_{r e g}^{(0)} E_{\mu}^{(n)}
$$

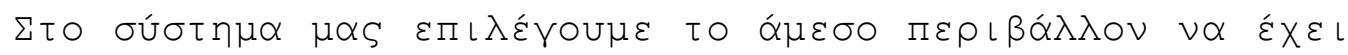

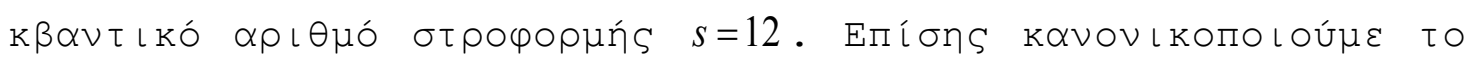

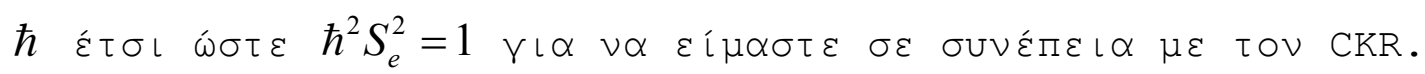




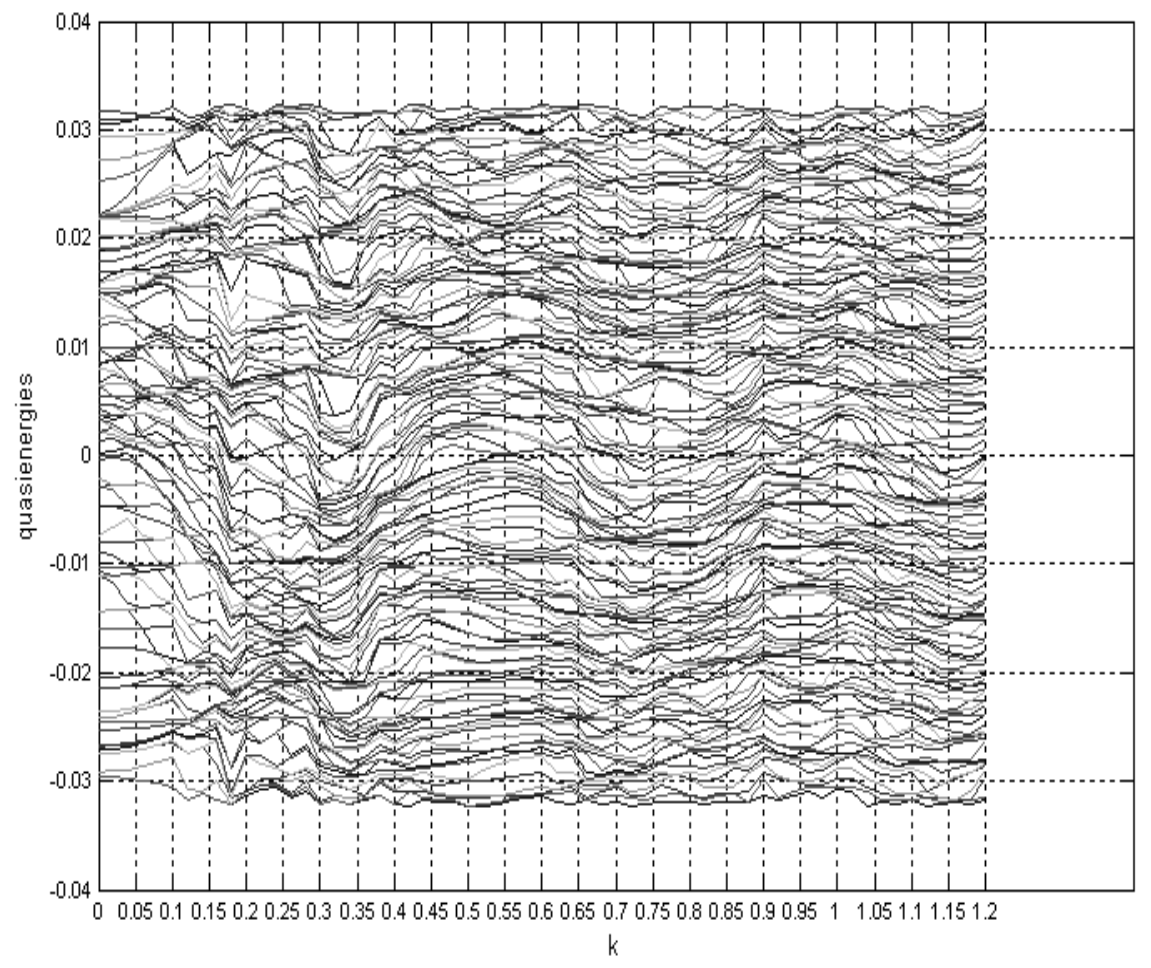

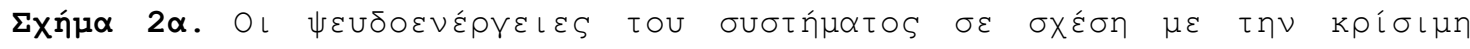

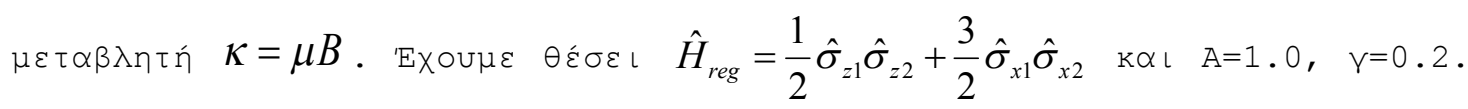

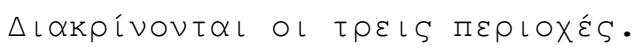




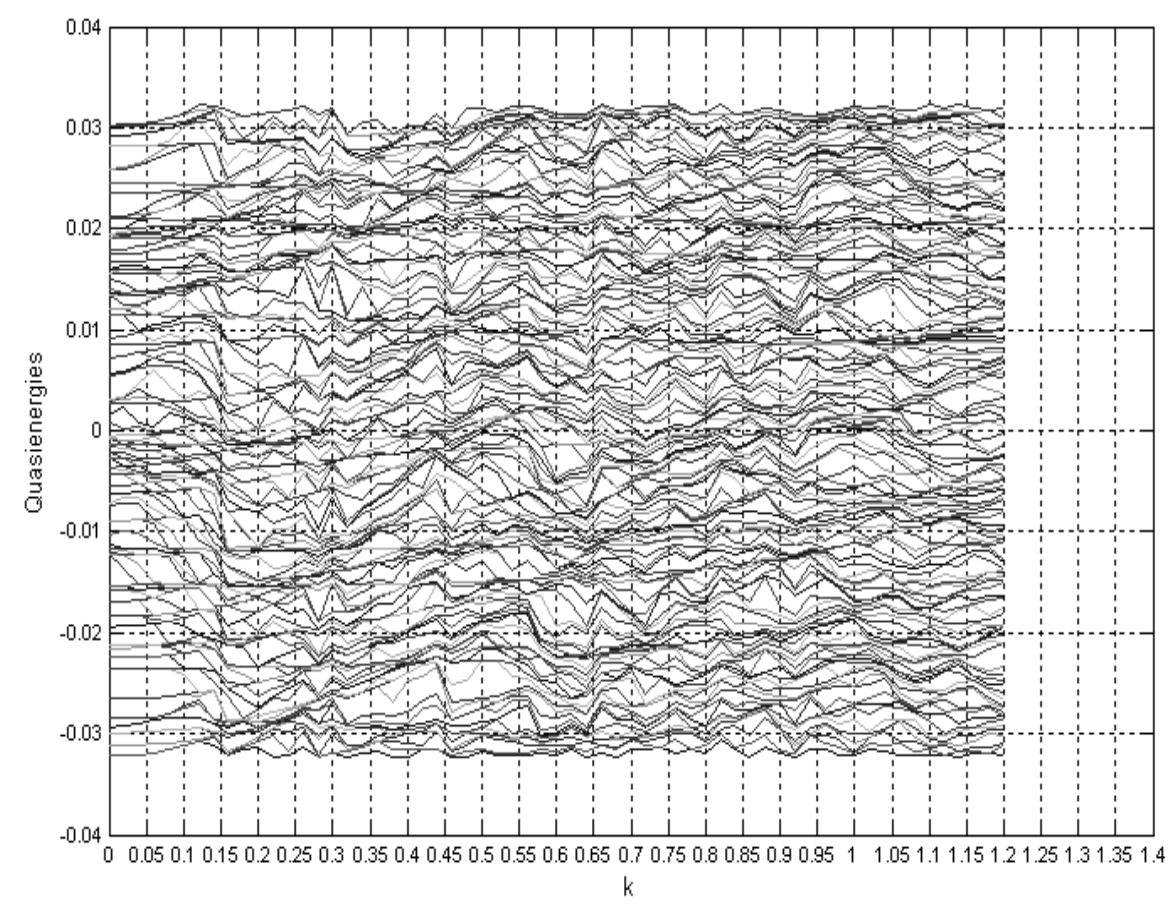

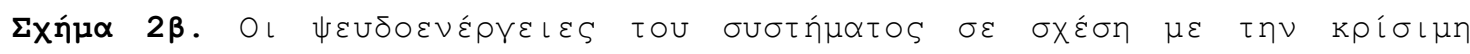

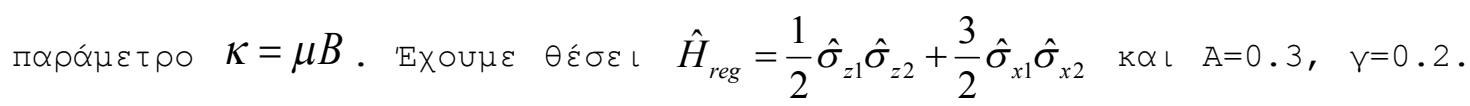

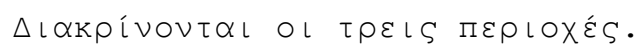

\section{Entanglement of Formation}

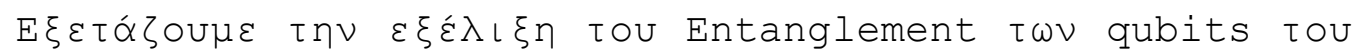

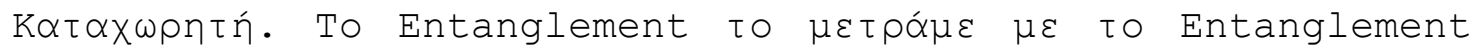

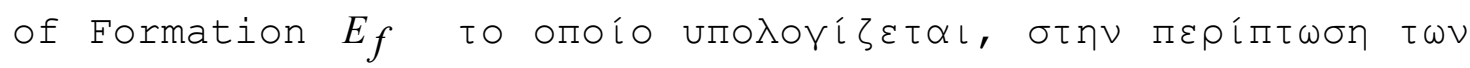

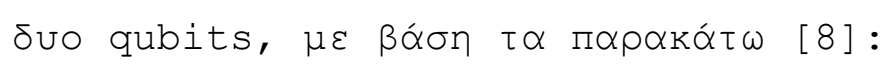

$$
E_{f}(\hat{\rho})=h\left[\frac{1}{2}\left(\sqrt{1-C(\hat{\rho})^{2}}\right)\right]
$$

$\mu \varepsilon$

$$
h(x)=-x \log _{2} x-(1-x) \log _{2}(1-x)
$$


$K \propto l$

$$
C(\hat{\rho})=\max \left(0, \sqrt{\lambda_{1}}-\sqrt{\lambda_{2}}-\sqrt{\lambda_{3}}-\sqrt{\lambda_{4}}\right)
$$

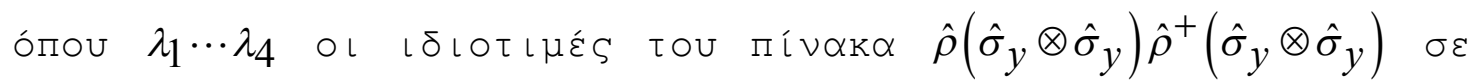
$\varphi \theta i ́ v o u \sigma \alpha \delta \iota \alpha ́ \tau \alpha \xi \eta$.

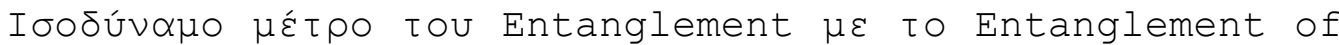

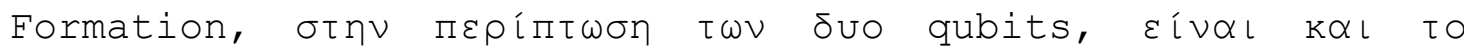

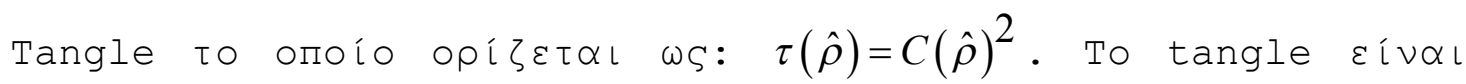

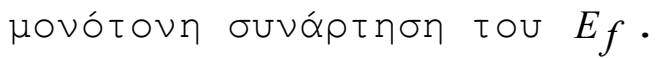

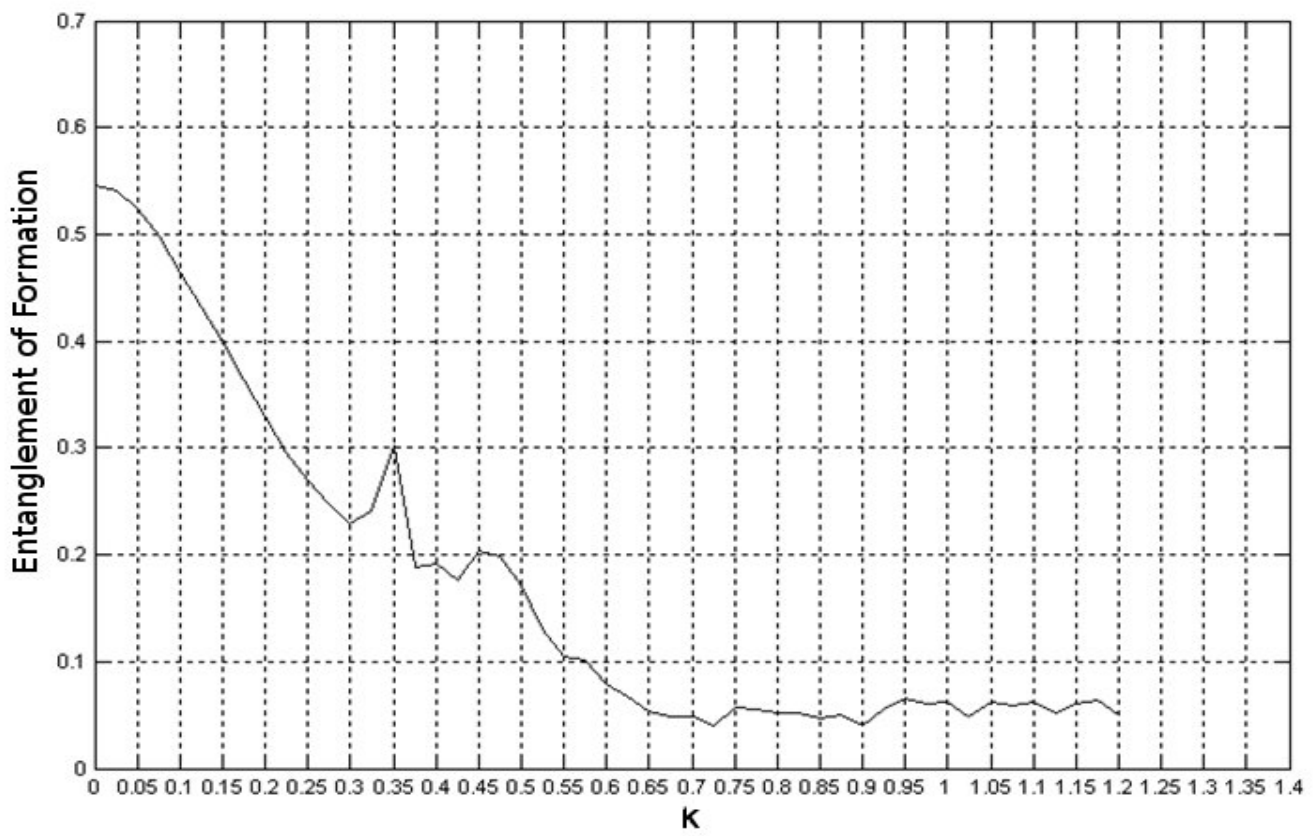

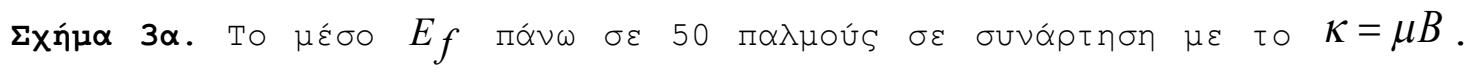

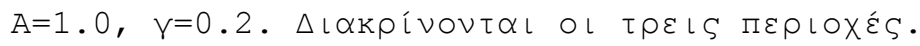




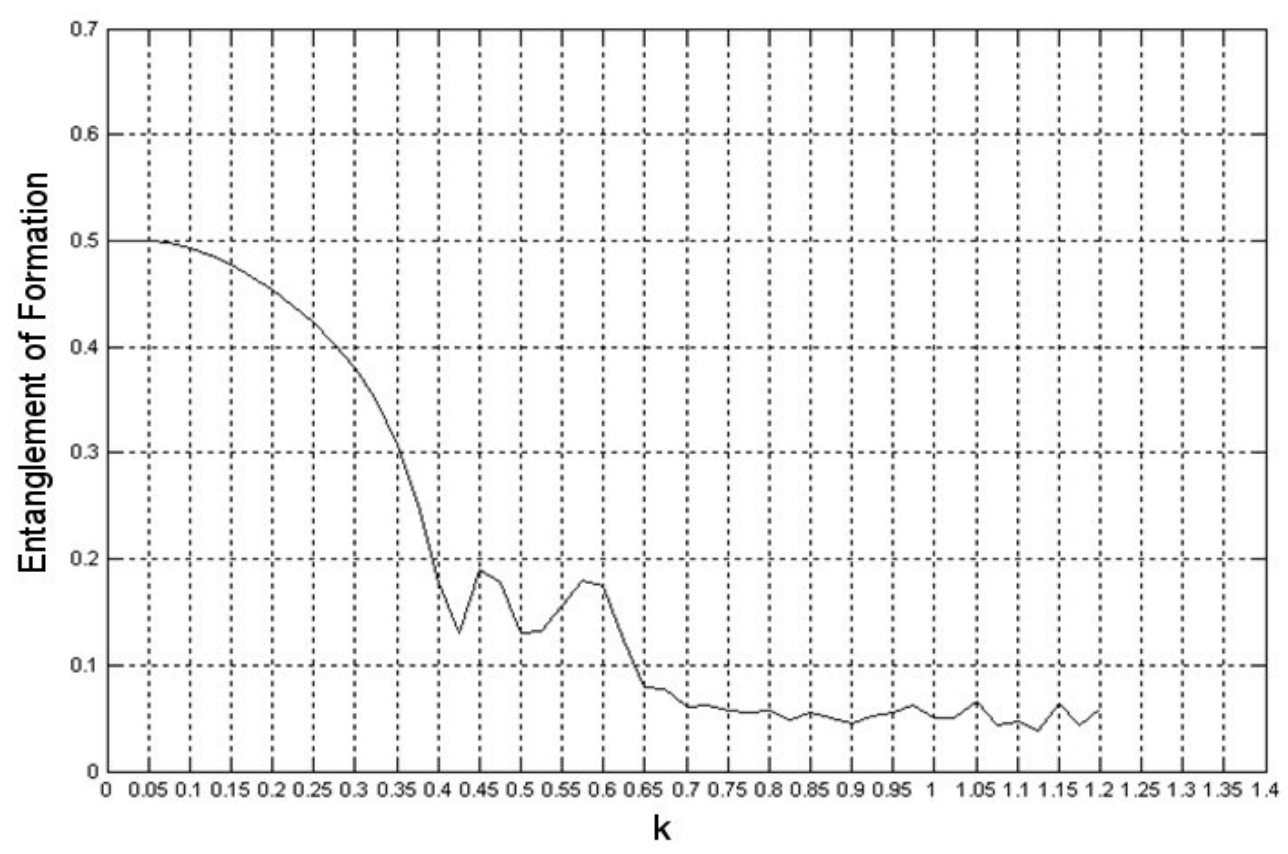

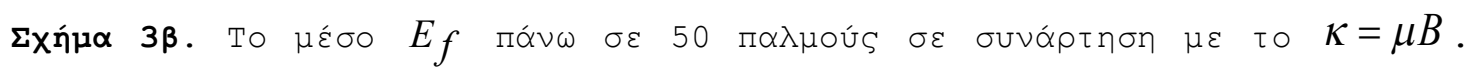

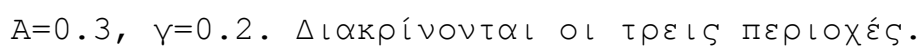

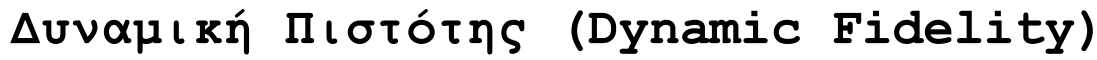

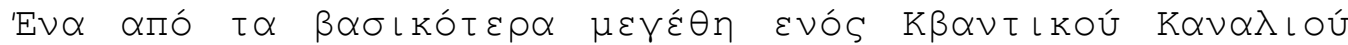

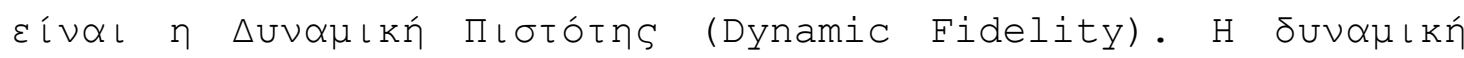

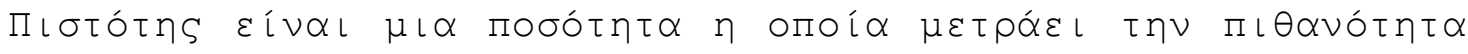

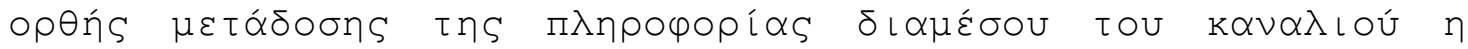

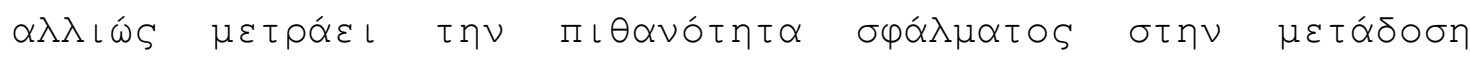

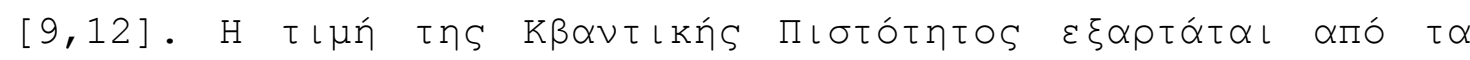

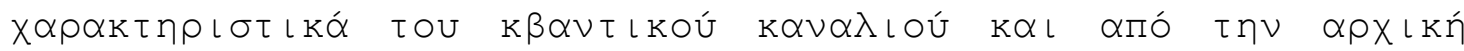

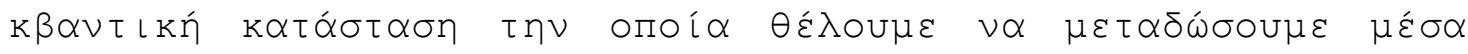

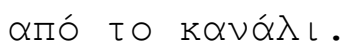

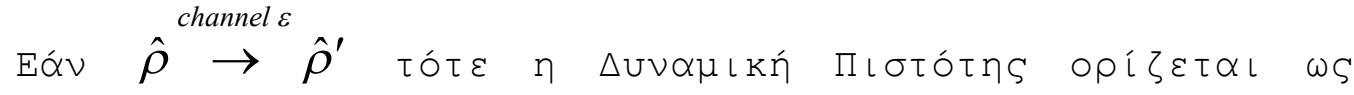

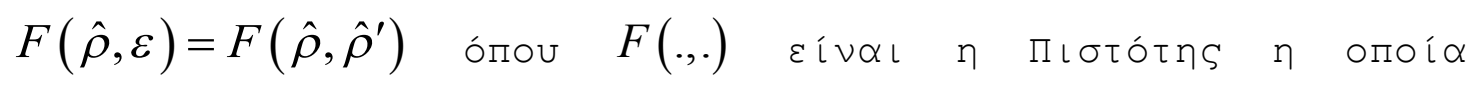

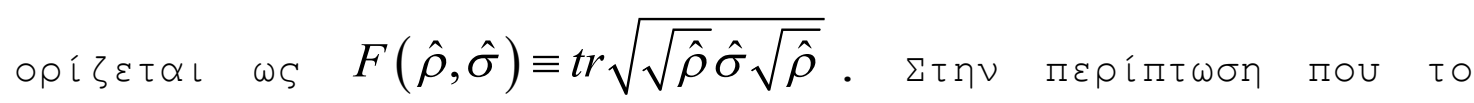

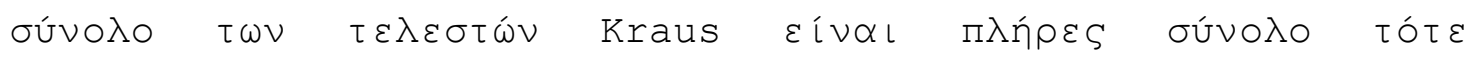

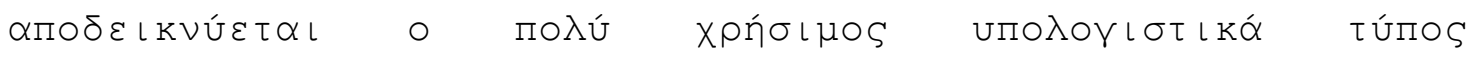




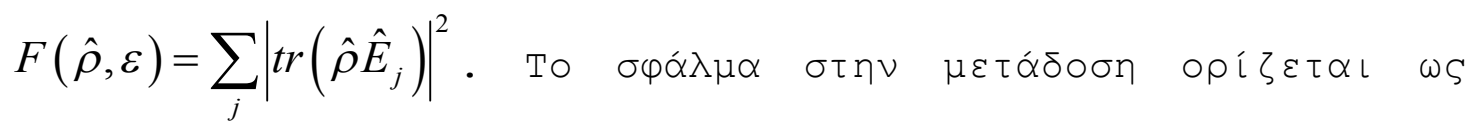

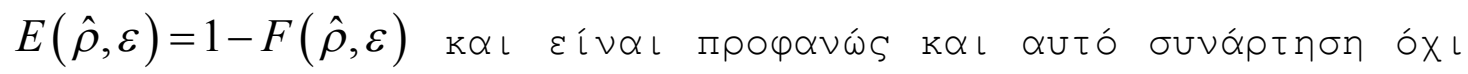

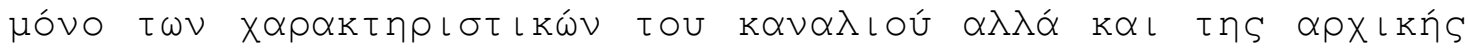

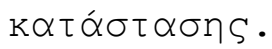

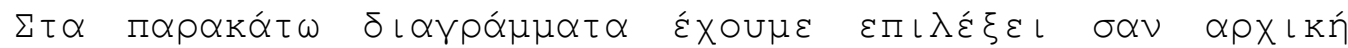

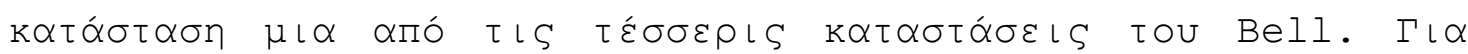

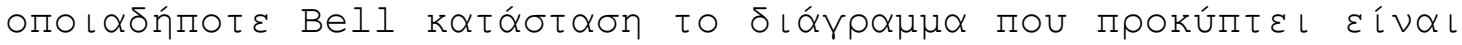
т०

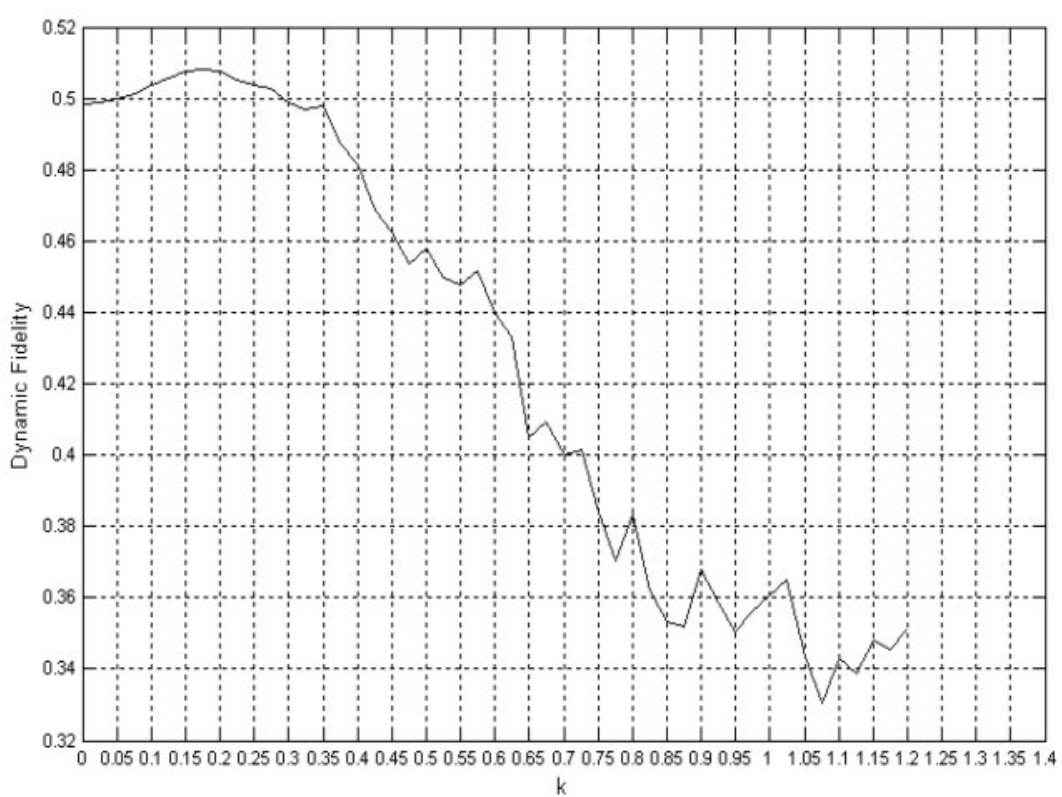

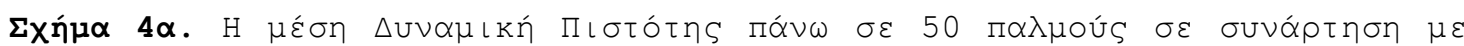

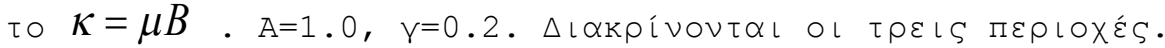




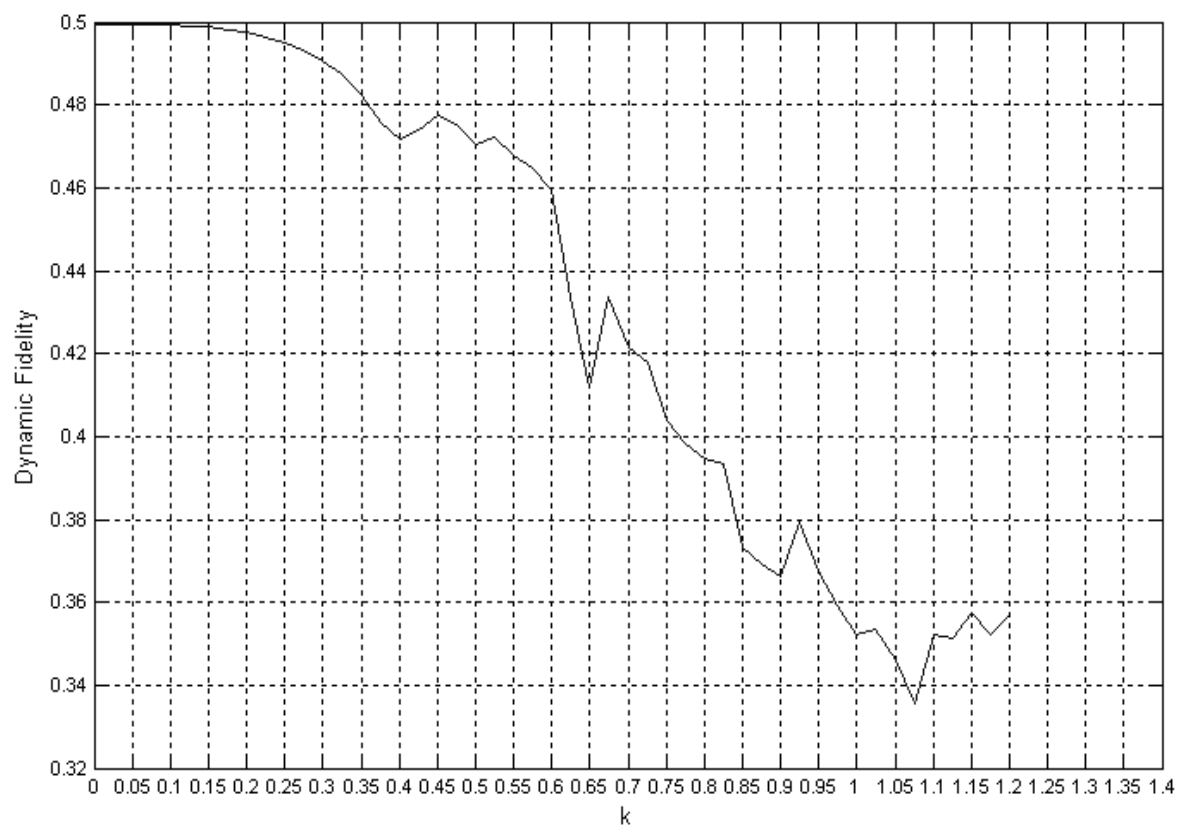

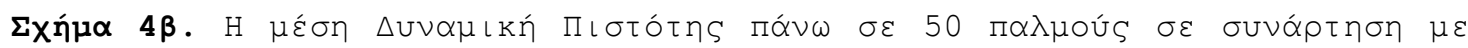

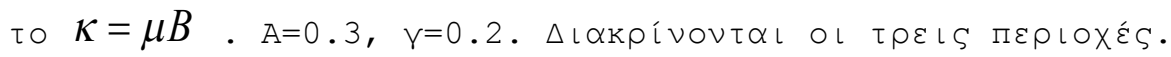

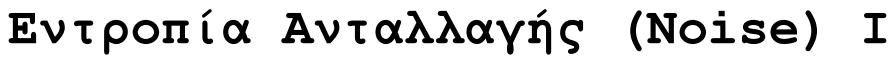

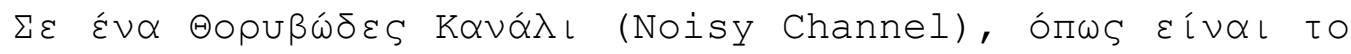

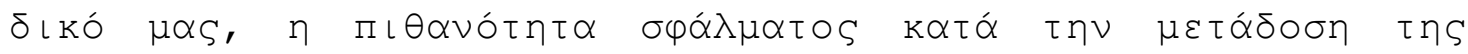

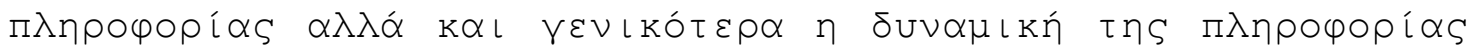

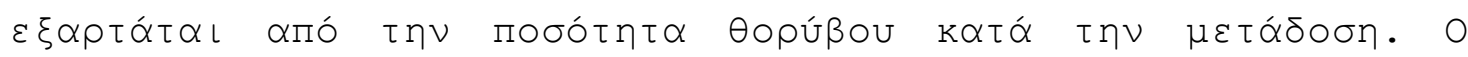

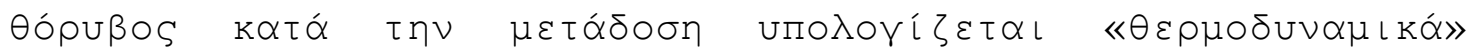

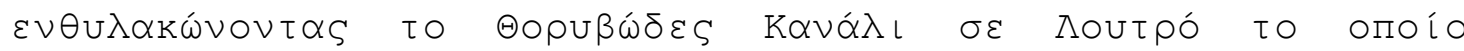

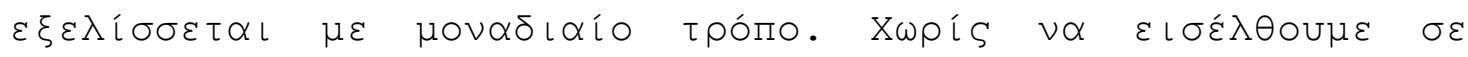

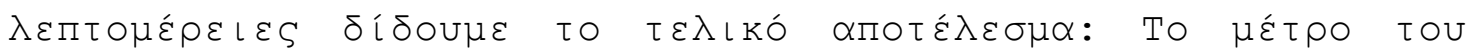

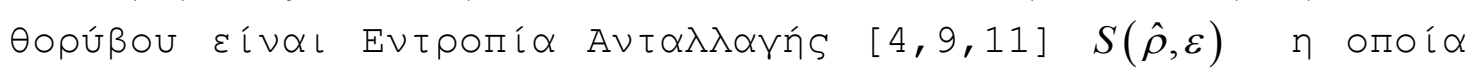

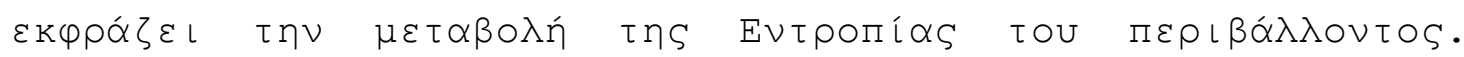

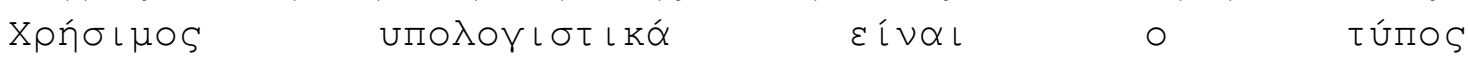

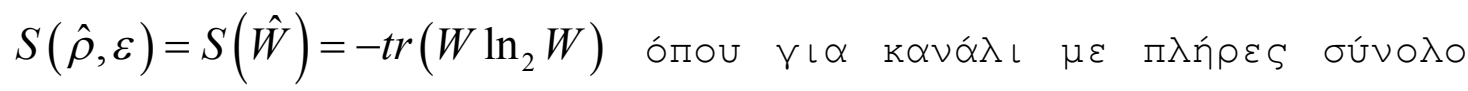

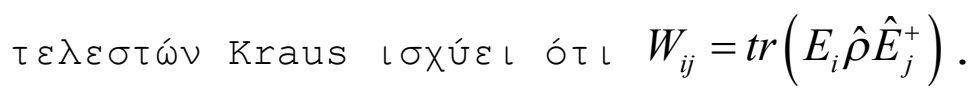




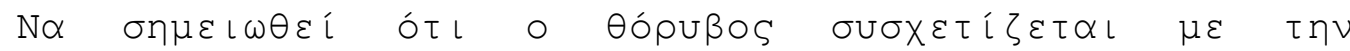

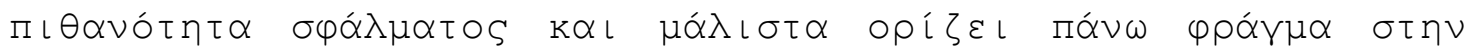

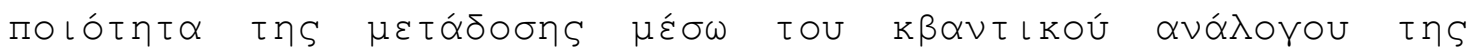

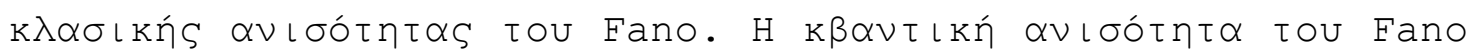

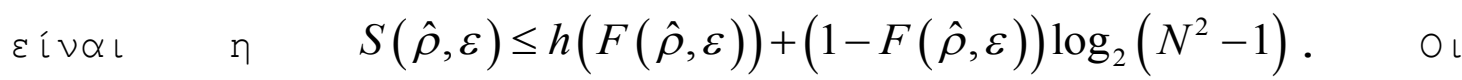

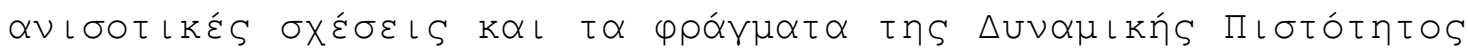

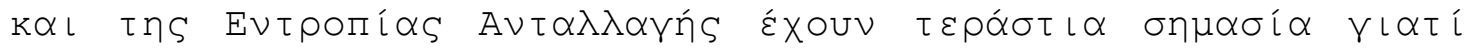

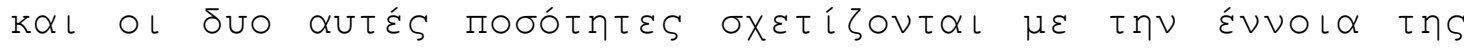

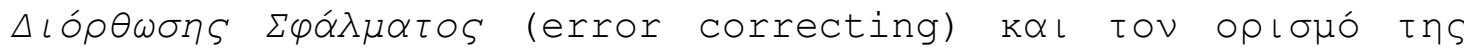

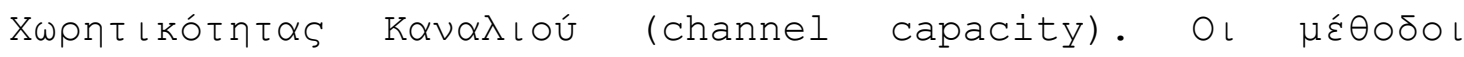

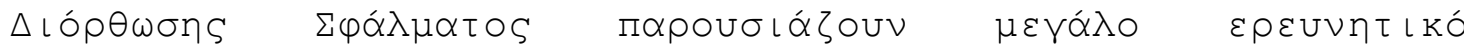

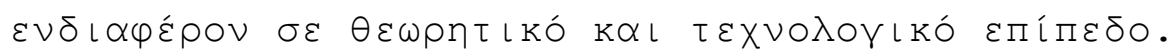

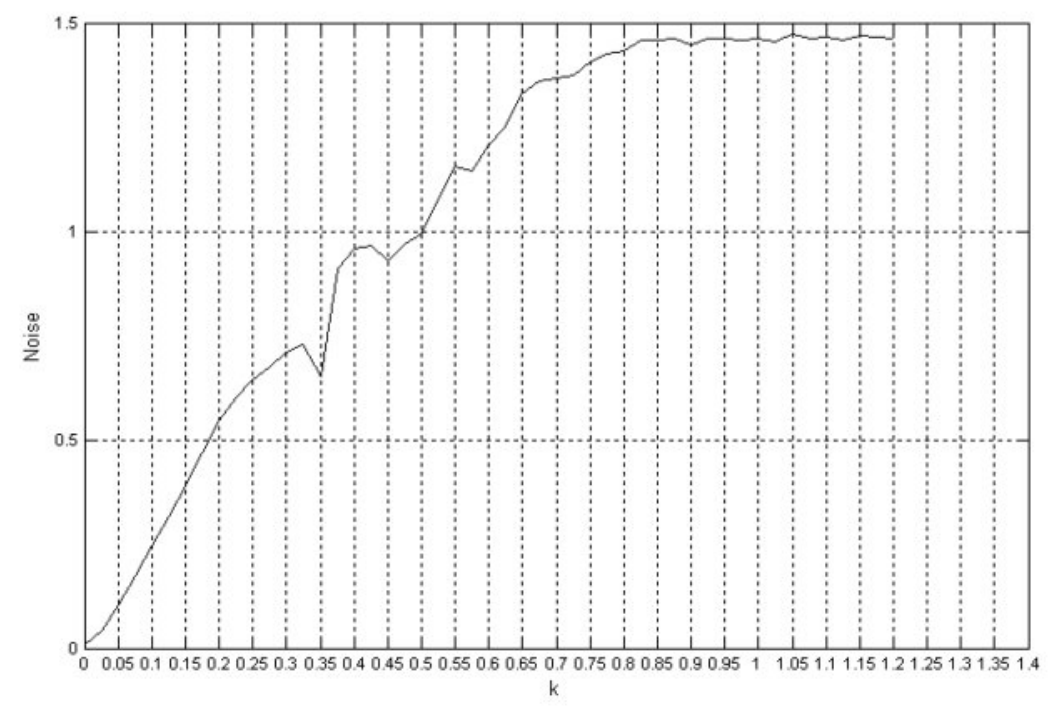

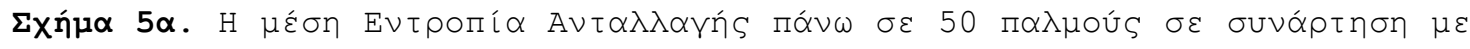

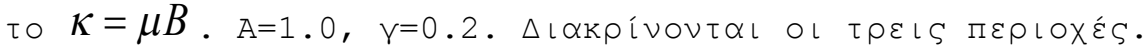




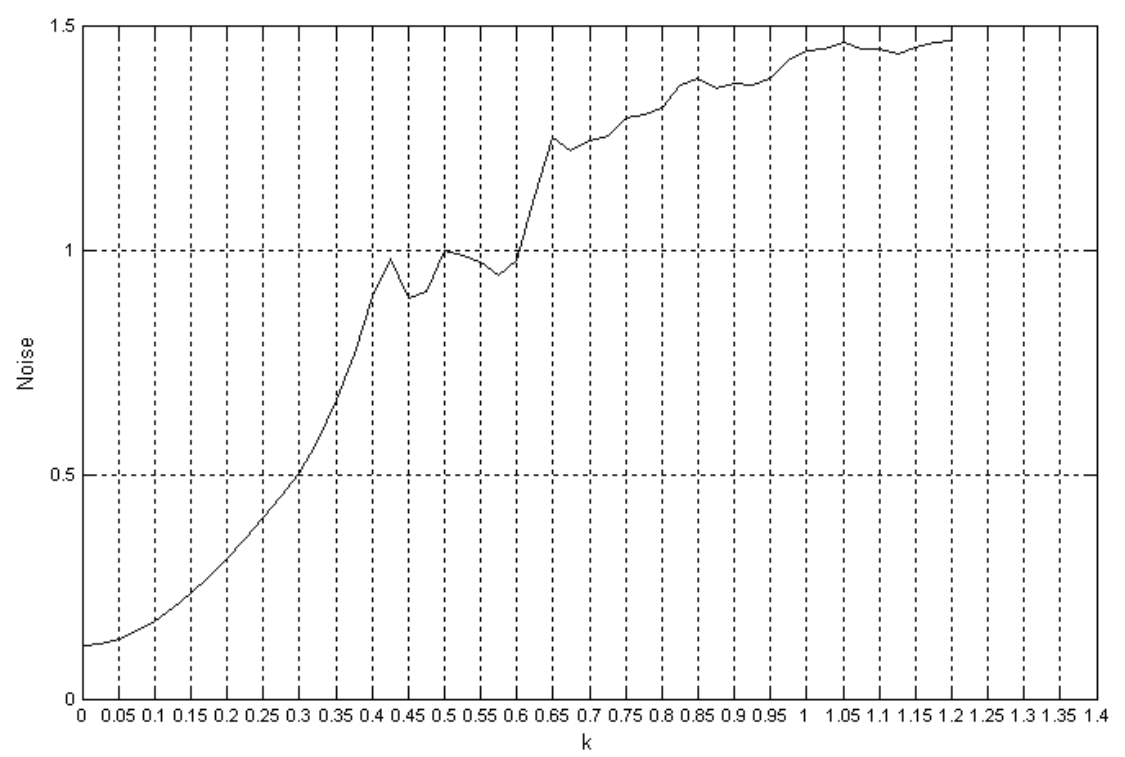

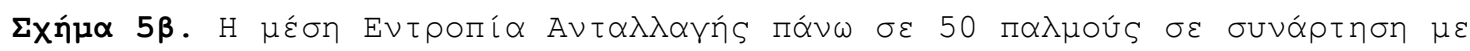

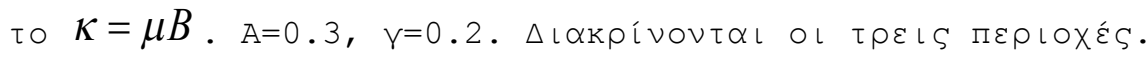

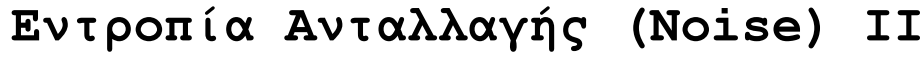

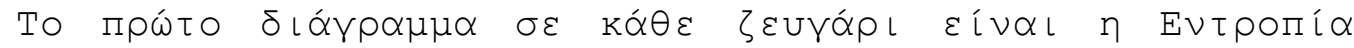

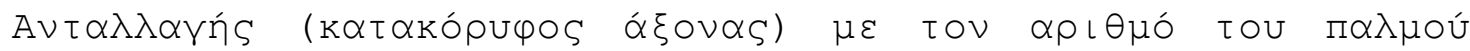

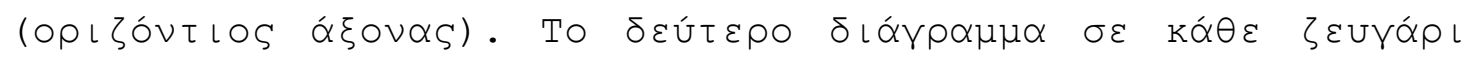

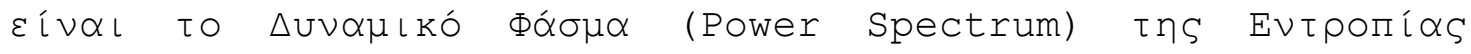

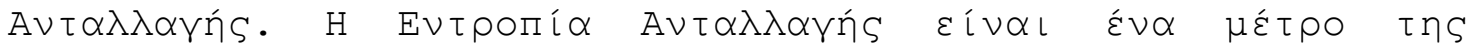
$\varepsilon \dot{\vee} \tau \alpha \sigma \cap S$ tou $\theta$ Opúßou. 

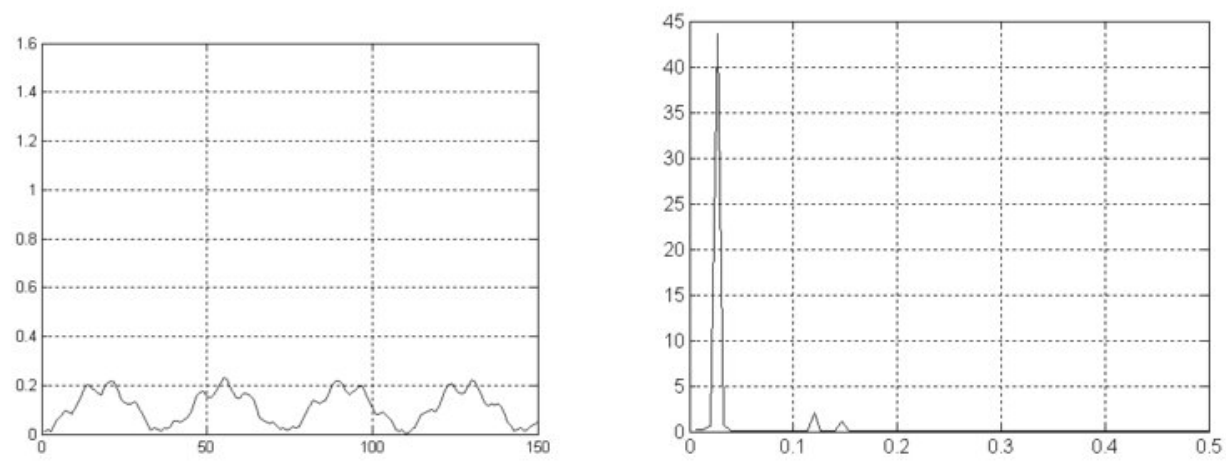

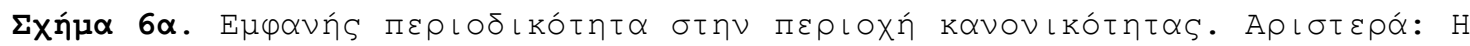

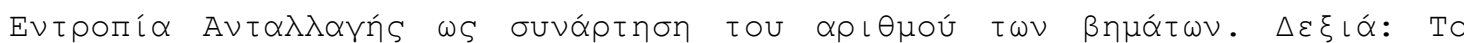

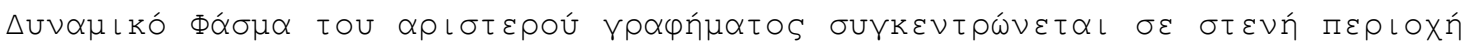

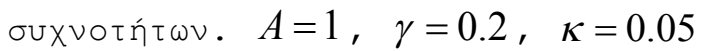
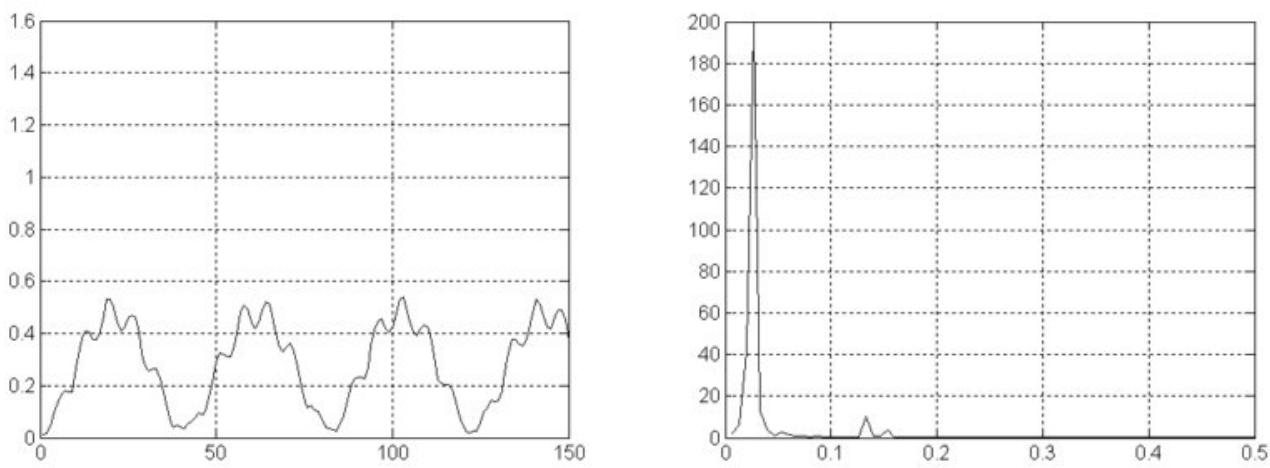

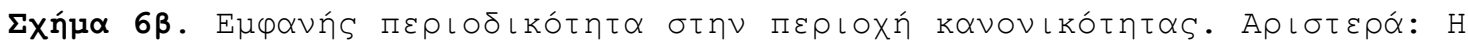

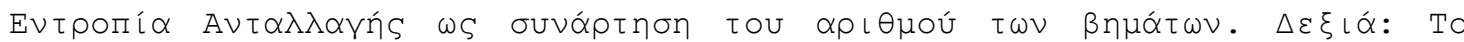

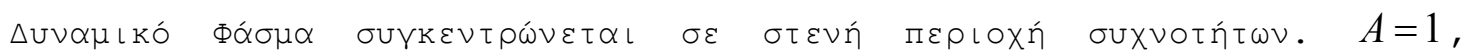
$\gamma=0.2, \kappa=0.1$ 

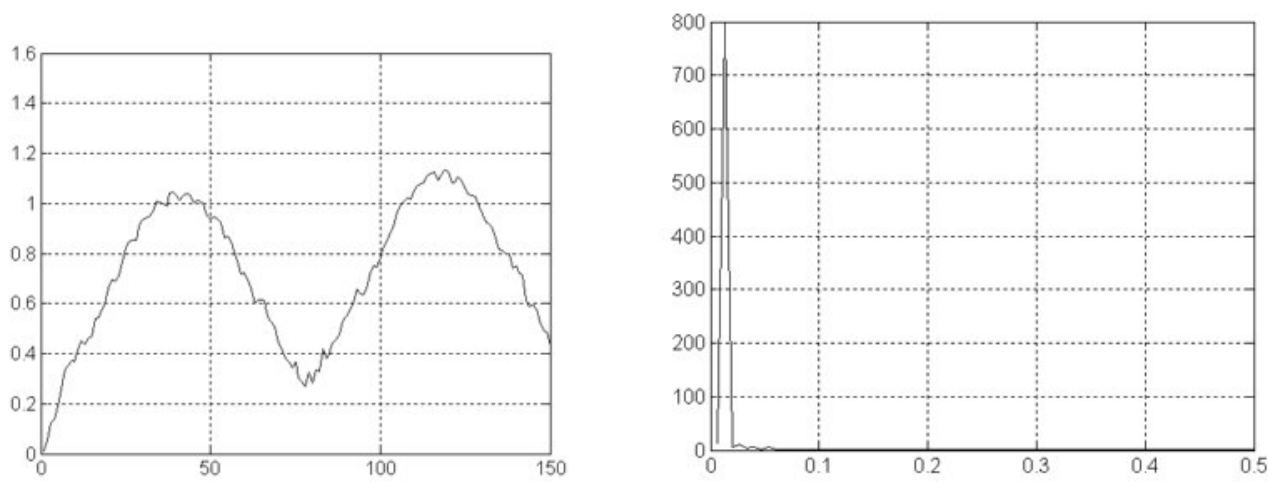

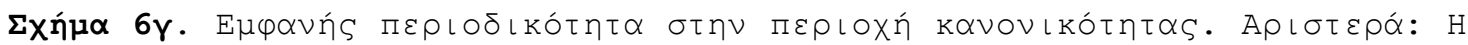

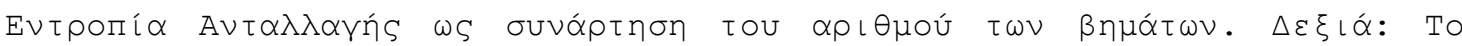

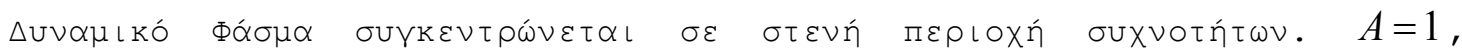
$\gamma=0.2, \kappa=0.3$
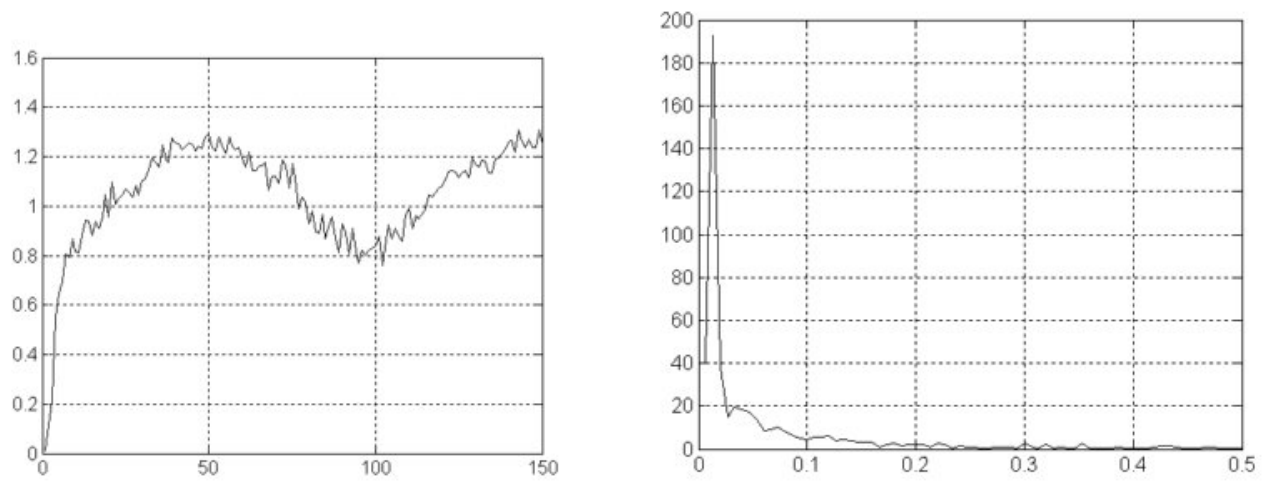

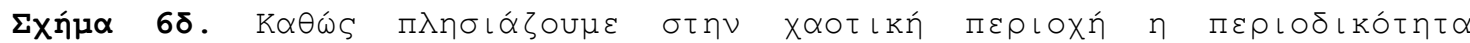

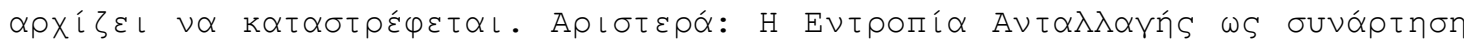

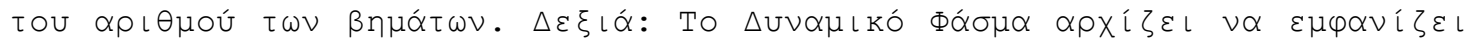

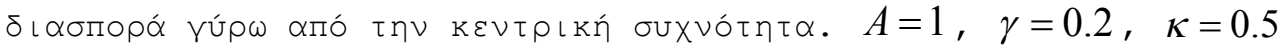



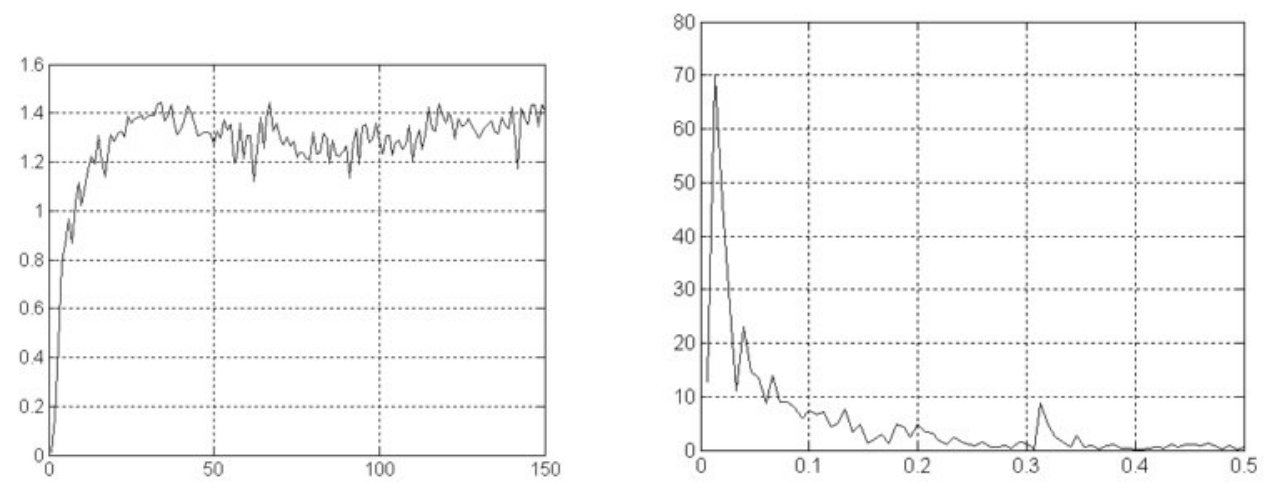

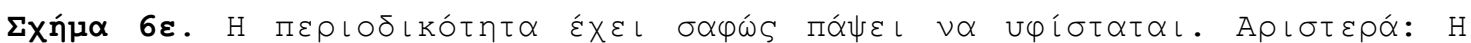

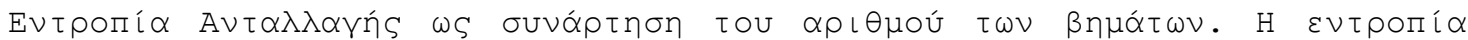

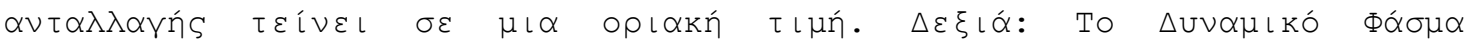

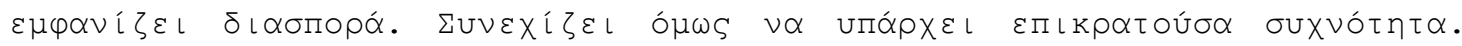
$A=1, \quad \gamma=0.2, \quad \kappa=0.6$
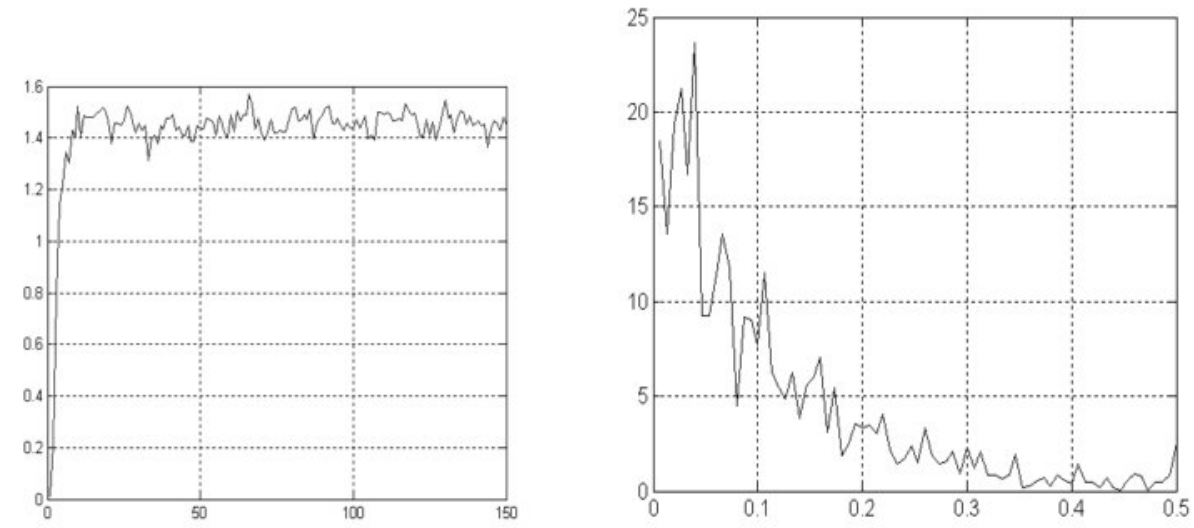

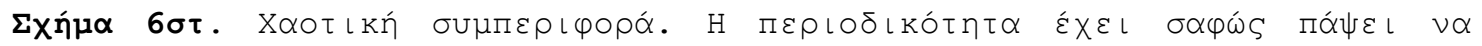

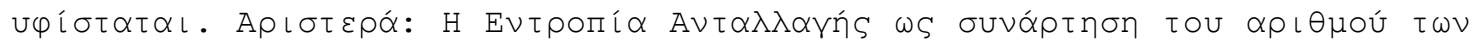

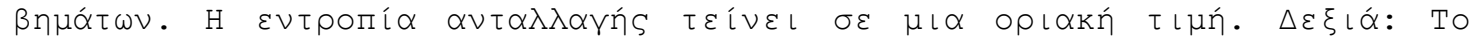

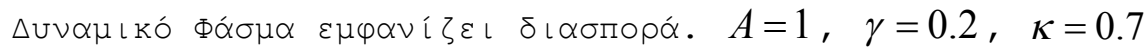



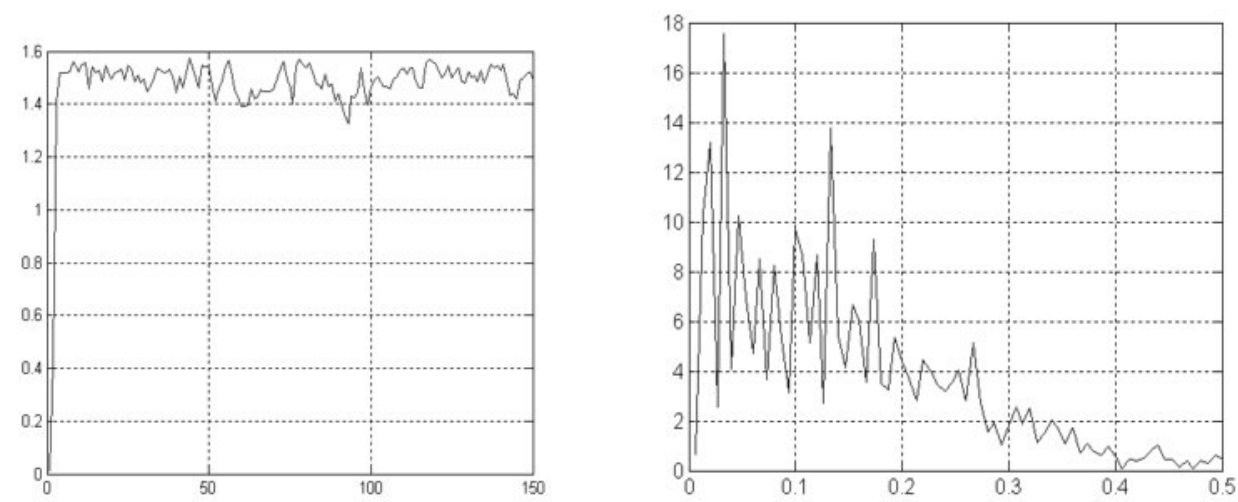

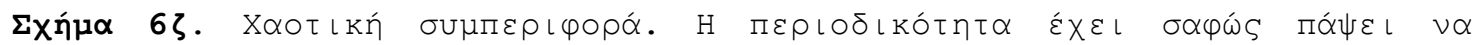

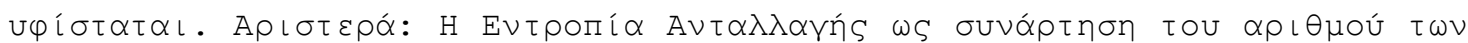

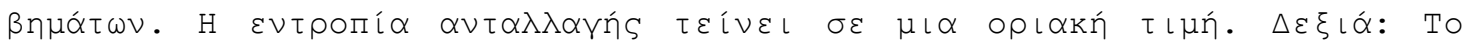

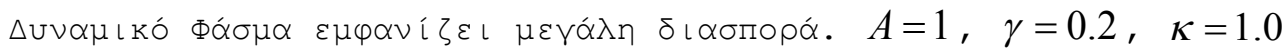
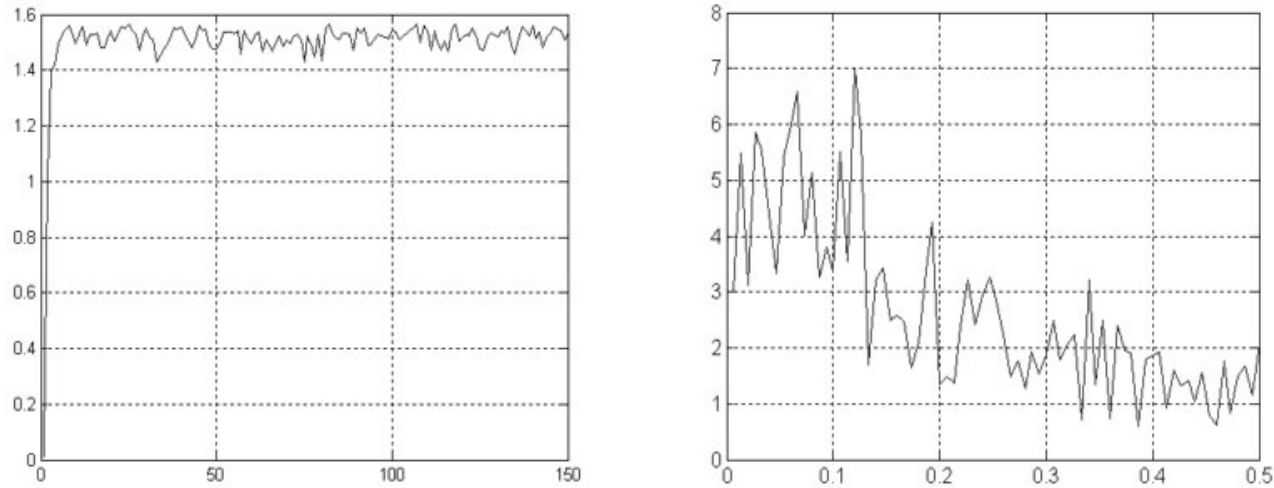

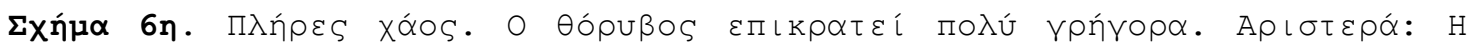

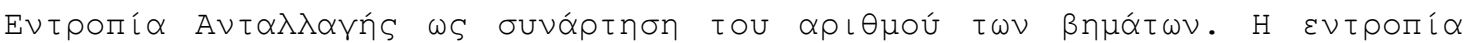

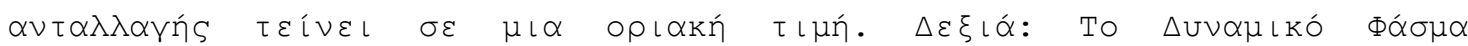

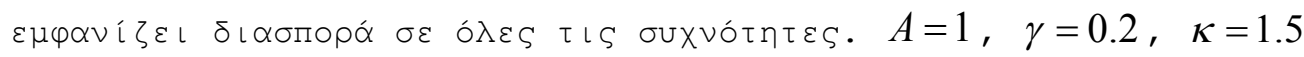

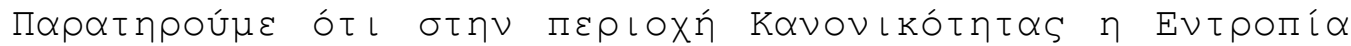

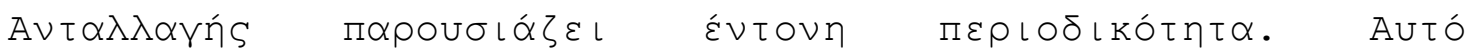

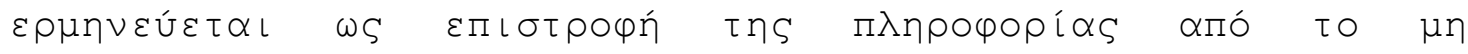

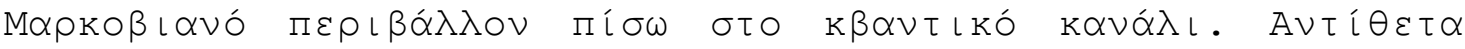

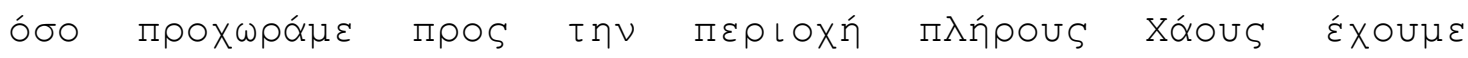

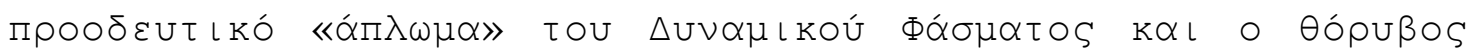




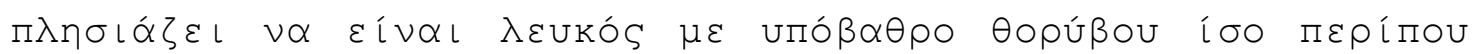
$\mu \varepsilon \quad 1.5$.

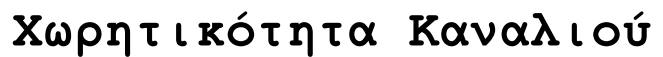

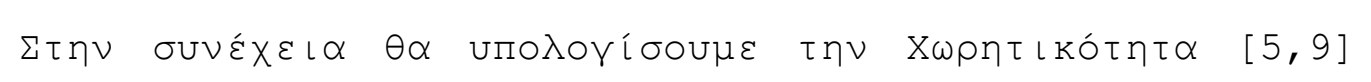

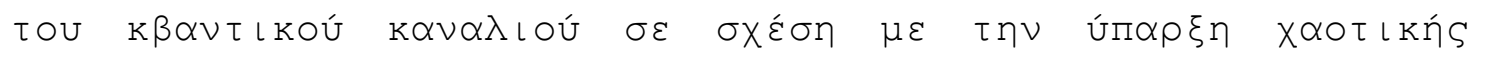

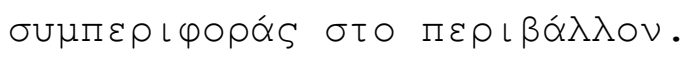

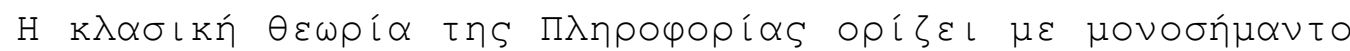

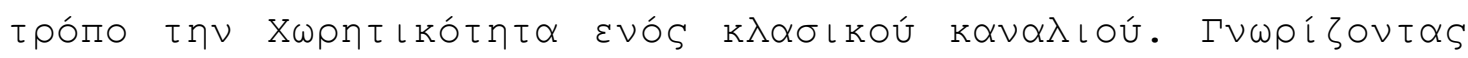

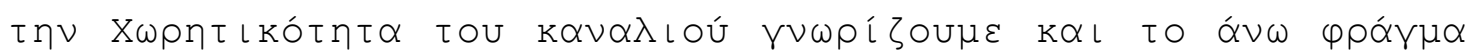

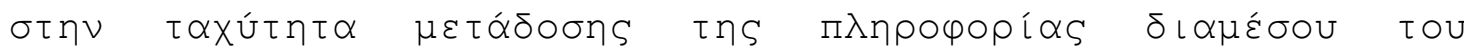

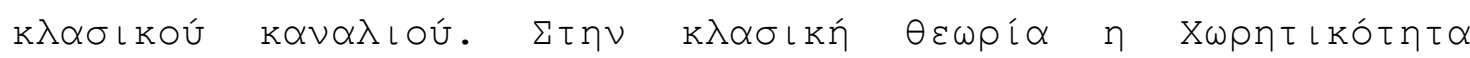

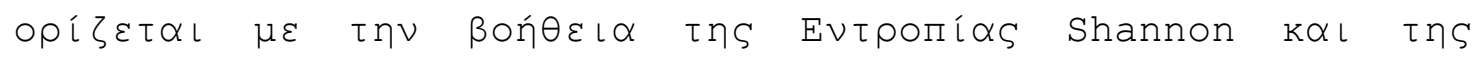

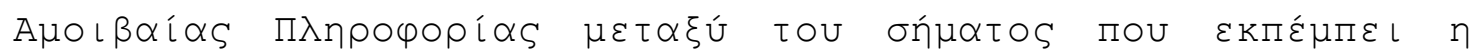

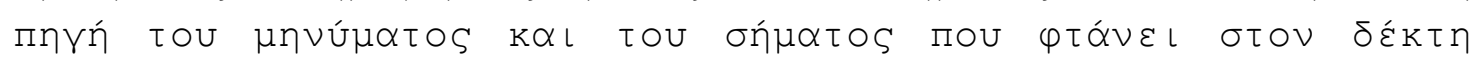

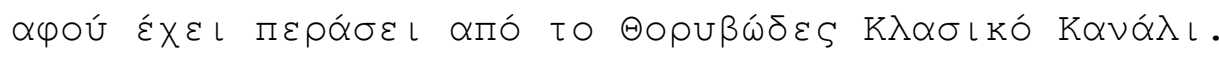

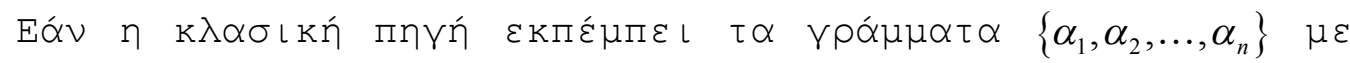

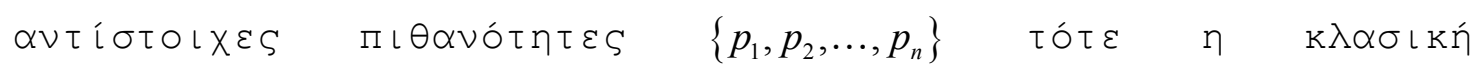

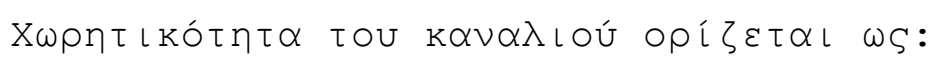

$$
C=\max _{\left\{p_{j}\right\}} I(X: N(X))
$$

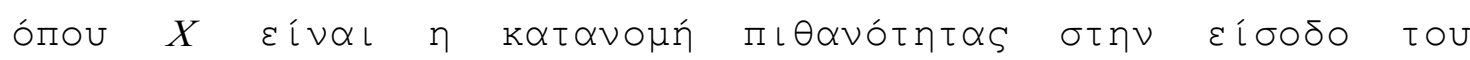

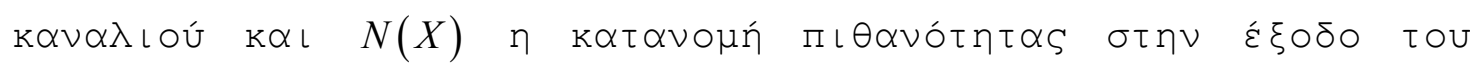

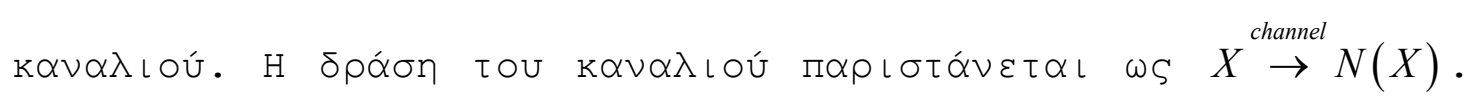

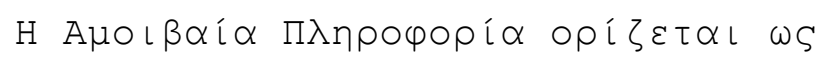

$$
I(X: Y)=H(Y)-H(Y \mid Y)=H(X)+H(Y)-H(X, Y)
$$

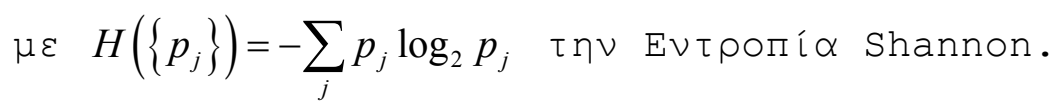

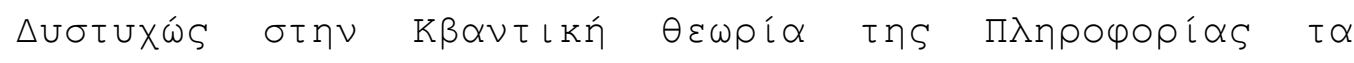

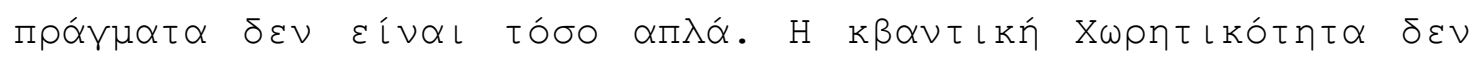

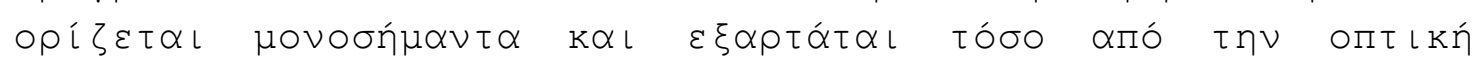




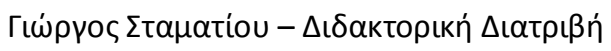

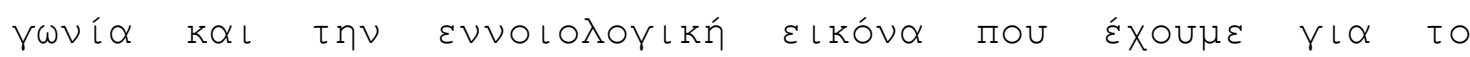

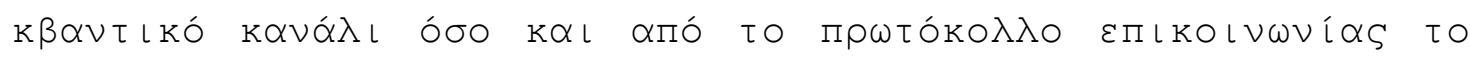

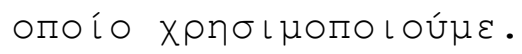

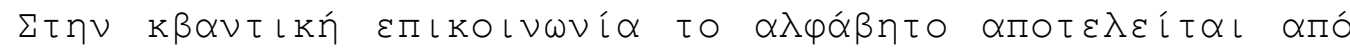

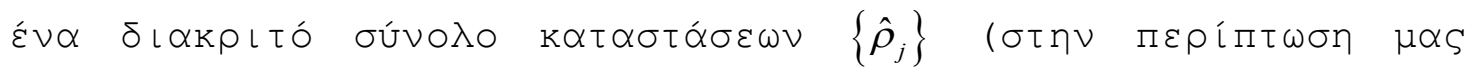

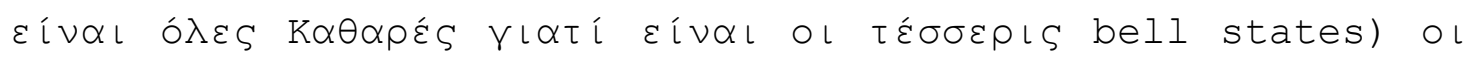

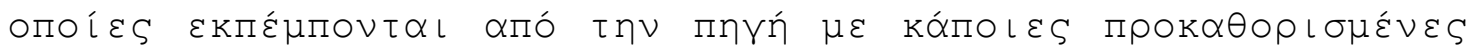

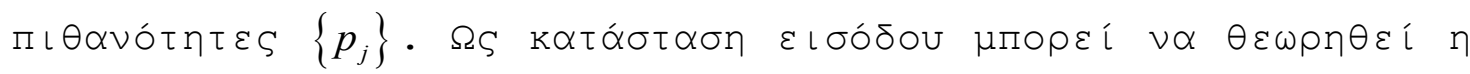

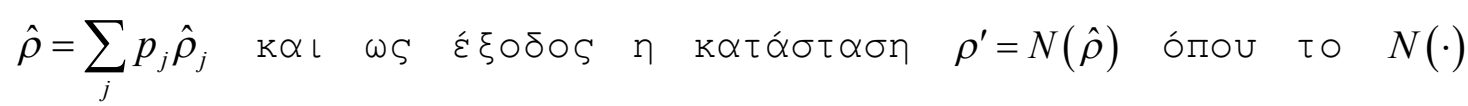

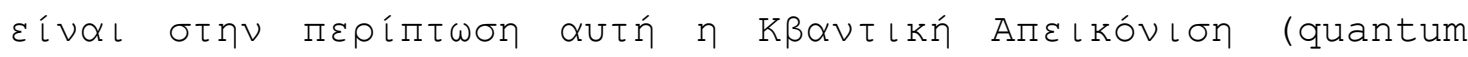

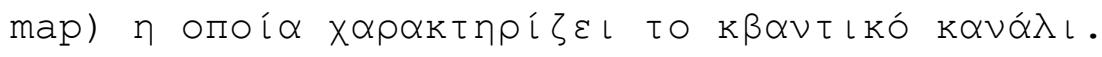

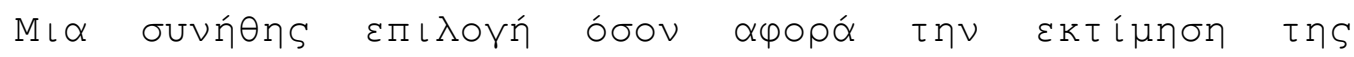

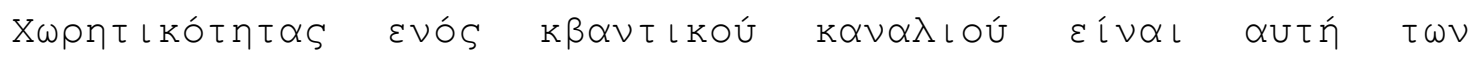
Hovelo, Schumacher, Westmoreland:

$$
C=\max _{\left.\left\{p_{j}, \mid v_{j}\right)\right\}}\left\{S\left[N\left(\sum_{j} p_{j}\left|v_{j}\right\rangle\left\langle v_{j}\right|\right)\right]-\sum_{j} p_{j} S\left[N\left(\left|v_{j}\right\rangle\left\langle v_{j}\right|\right)\right]\right\}
$$

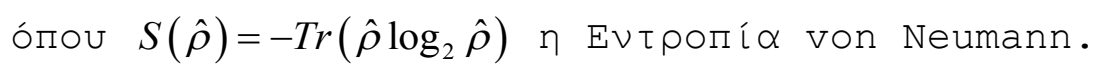

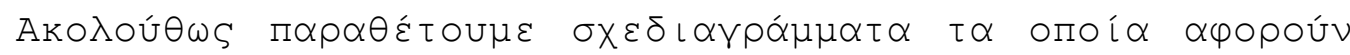

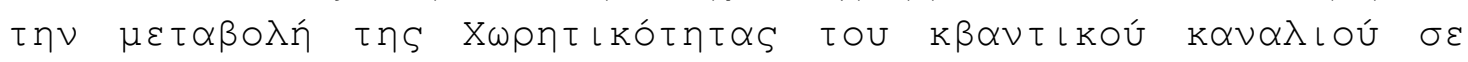

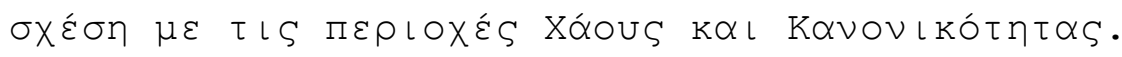



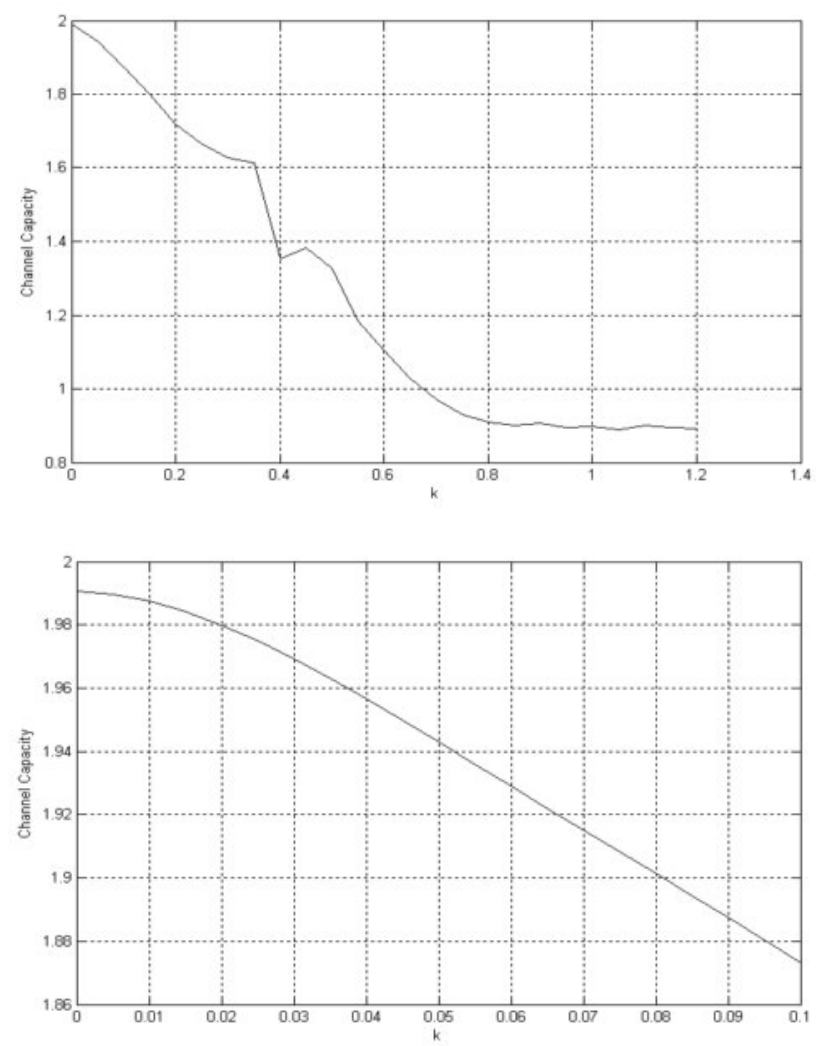

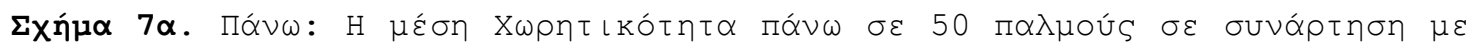

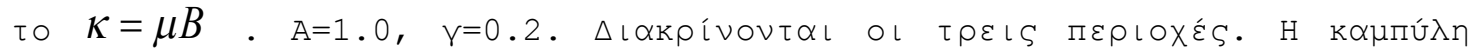

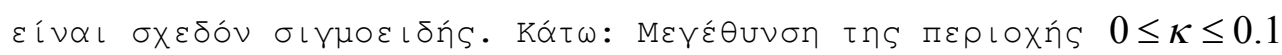




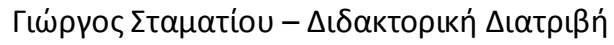

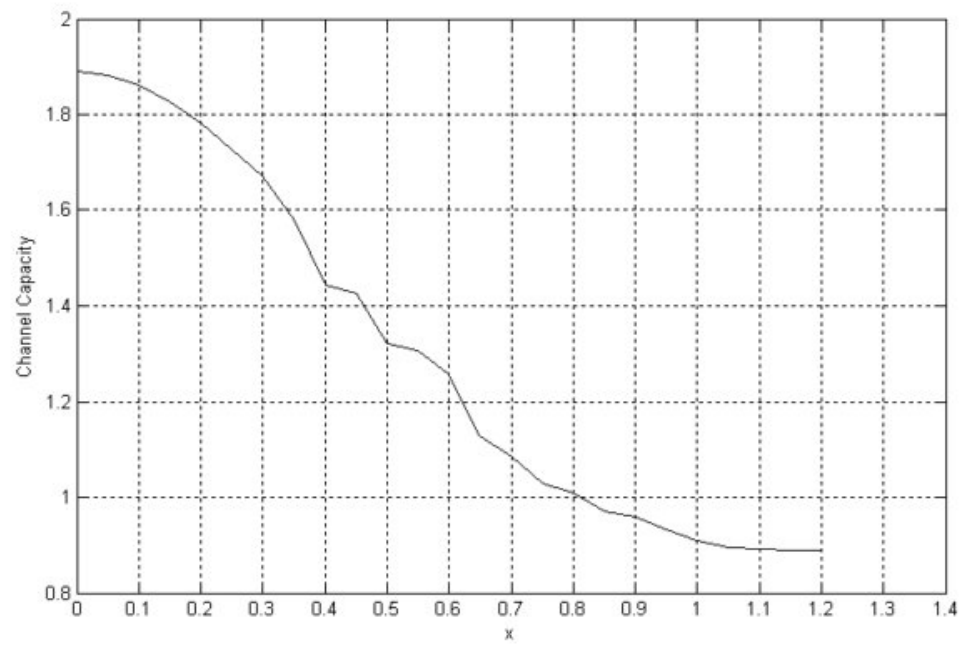

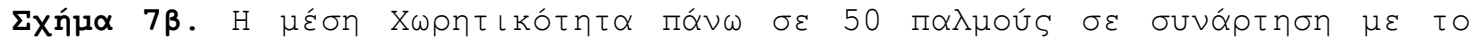

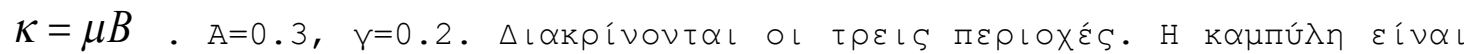

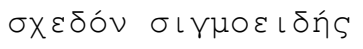



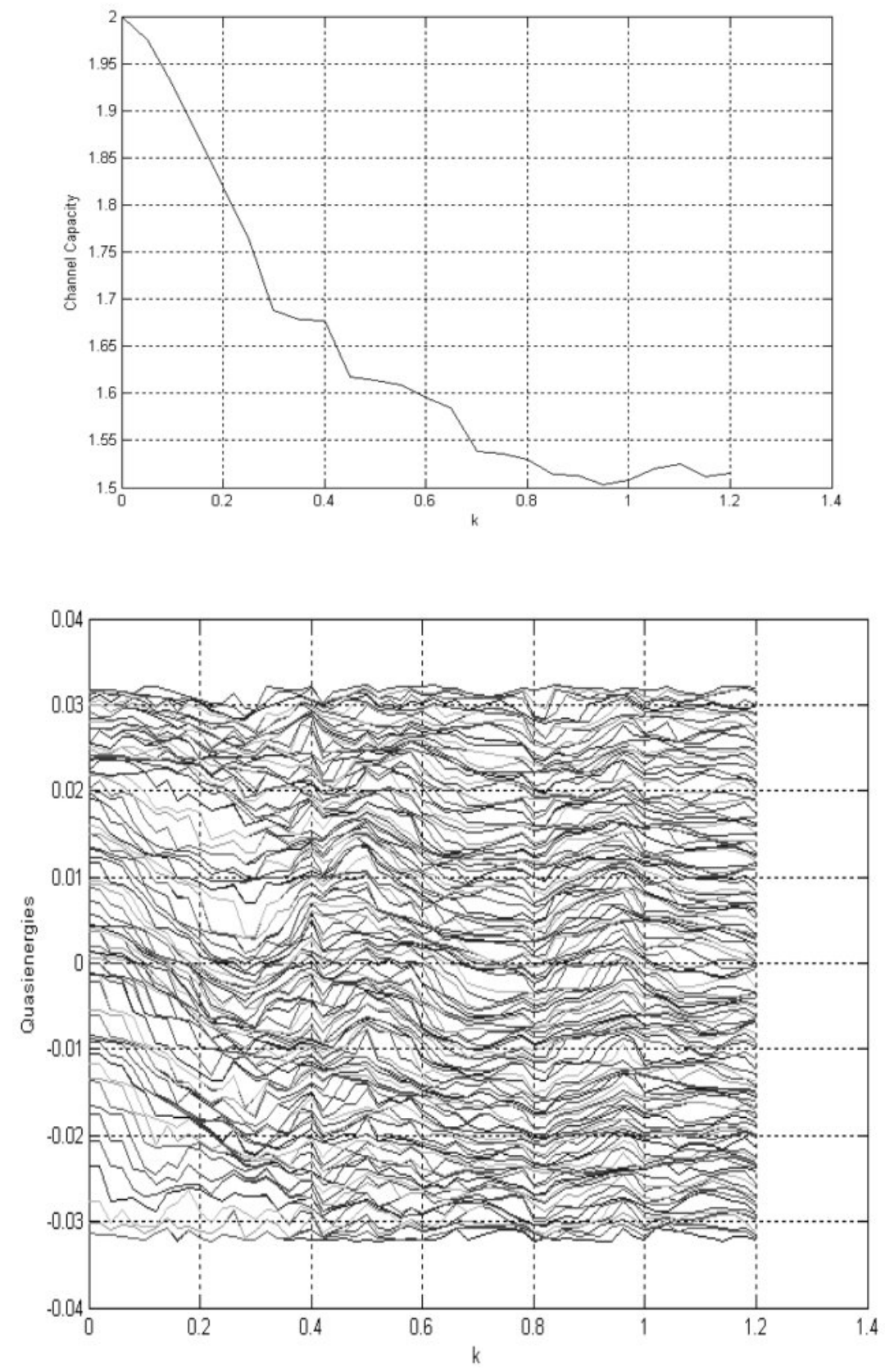

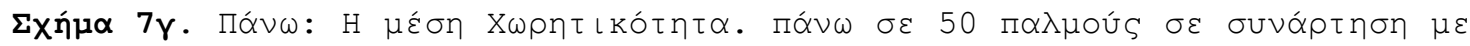

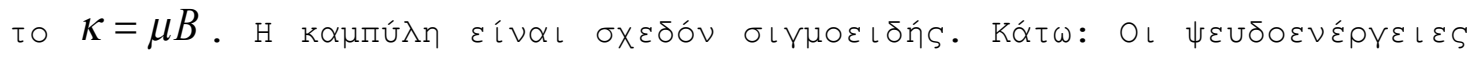

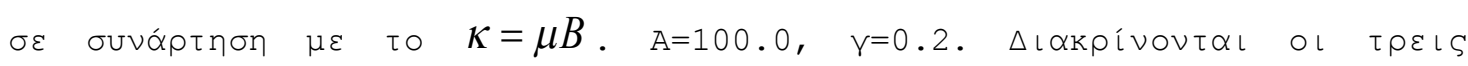
$\Pi \varepsilon \rho\llcorner O X \varepsilon \dot{\varepsilon}$ 


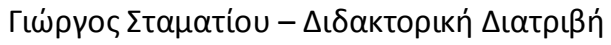

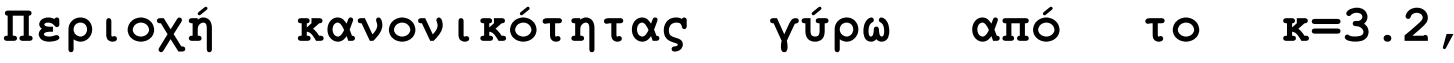 $A=1.0, \gamma=0.2$}

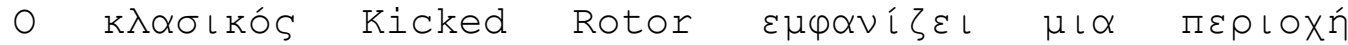

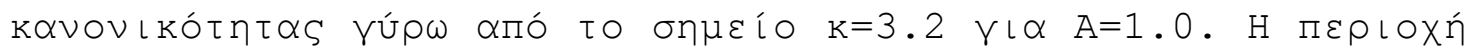

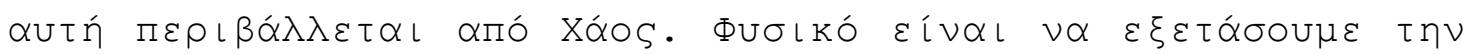

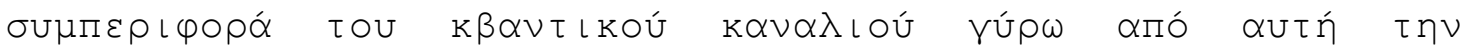

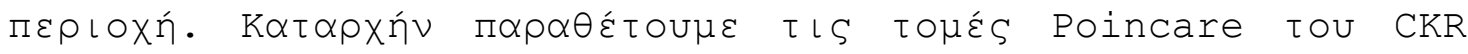

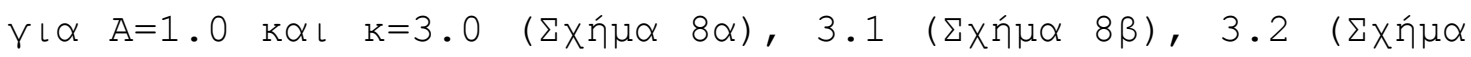

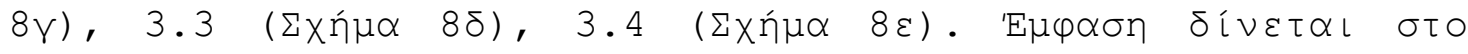

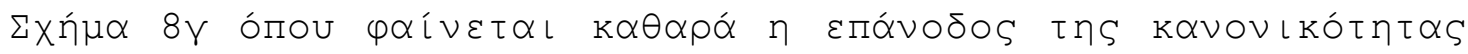

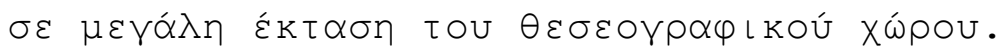

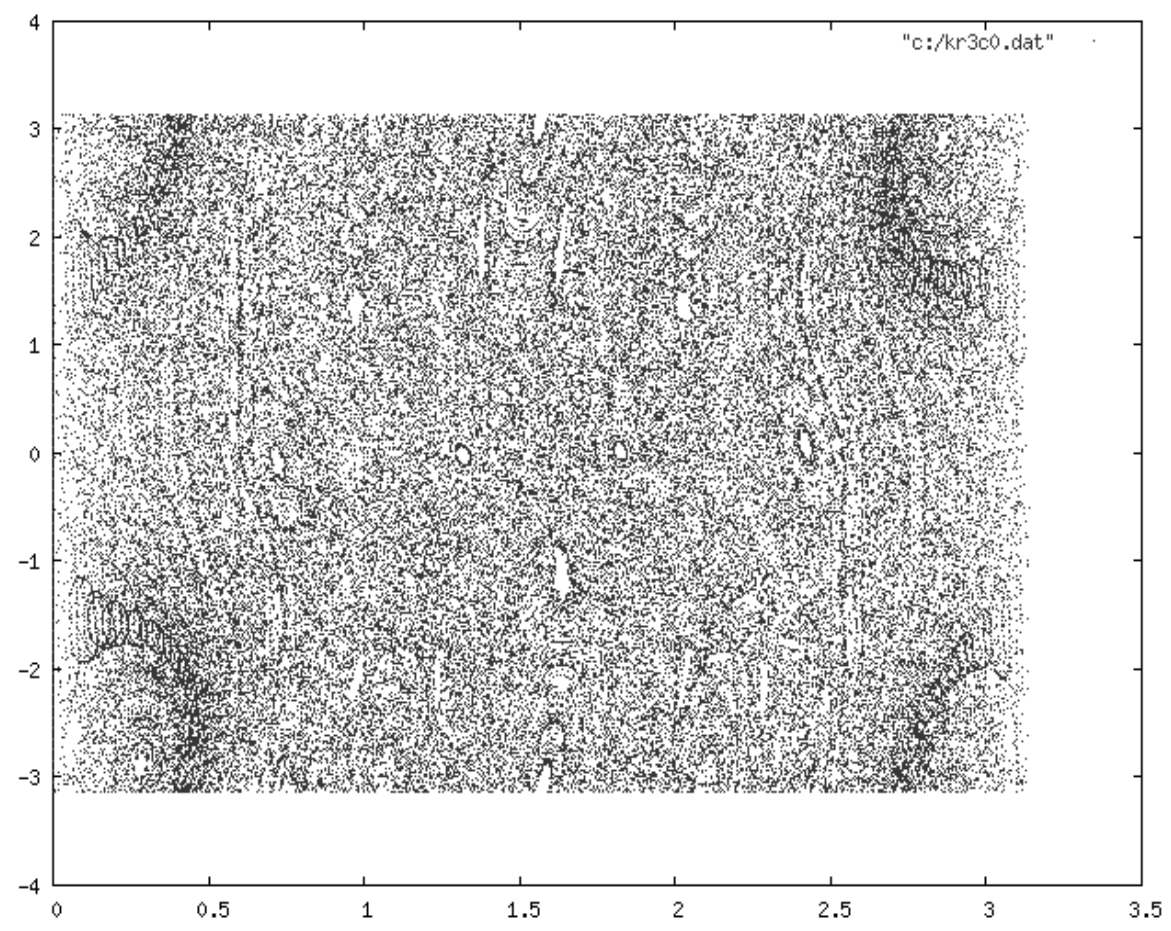

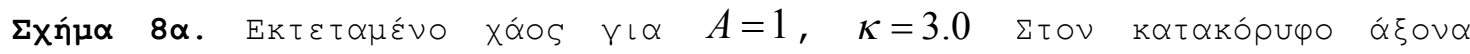

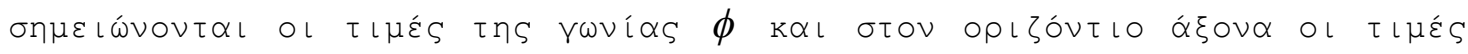

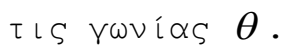




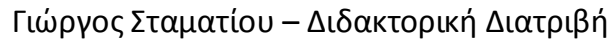

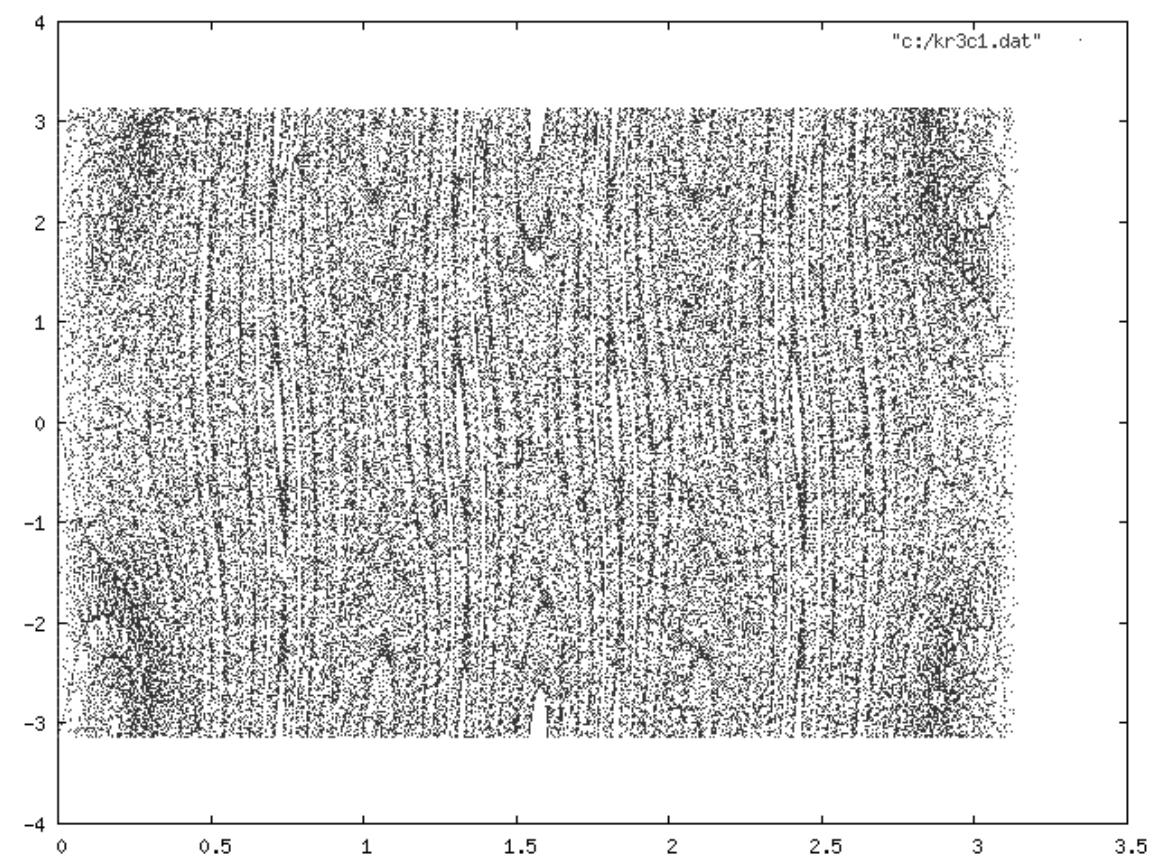

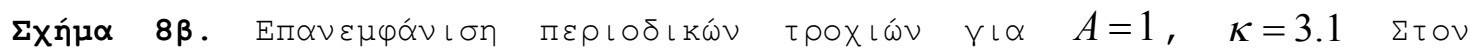

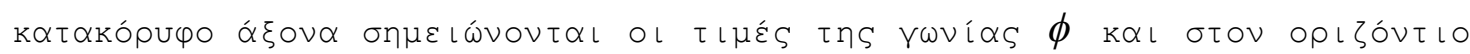

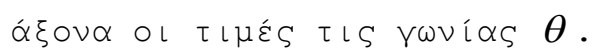




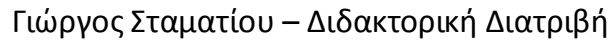

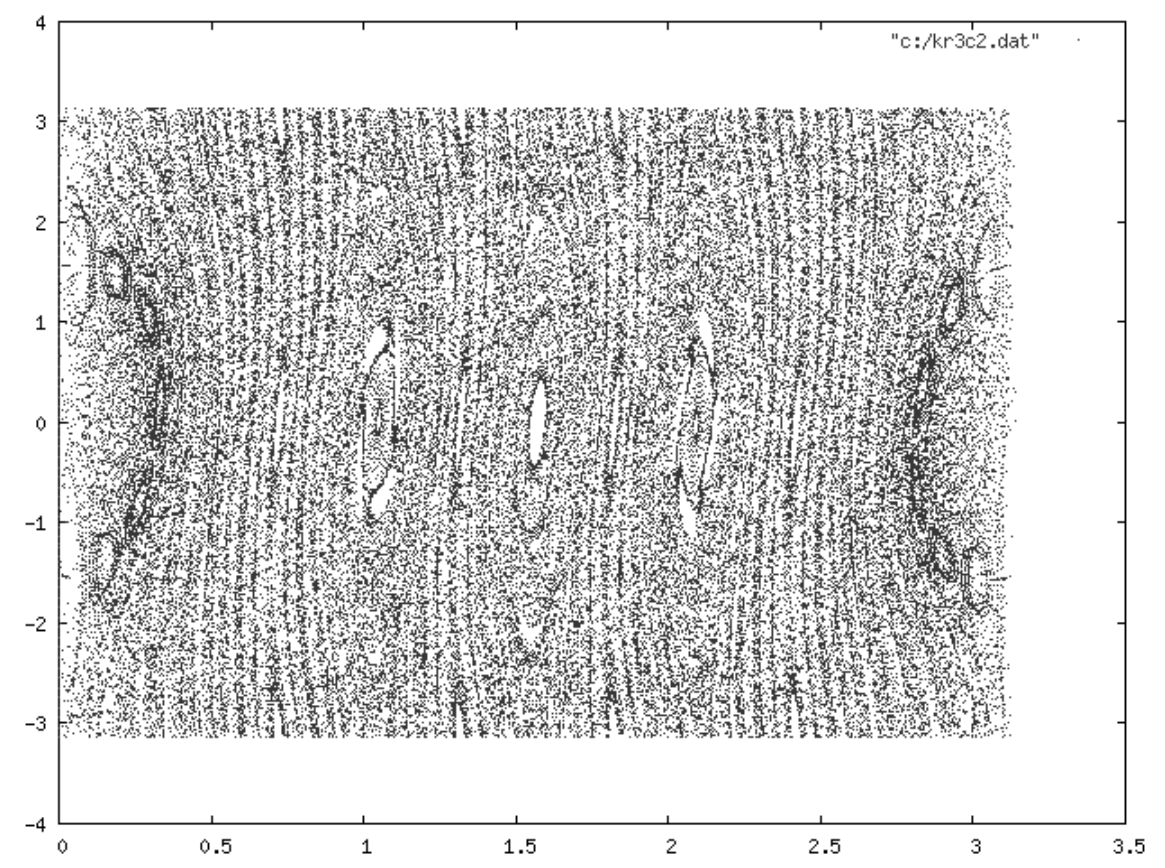

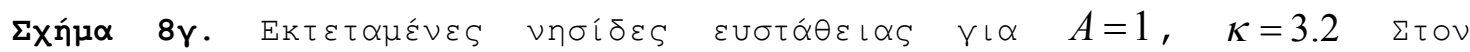

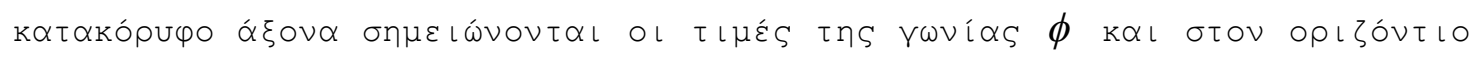

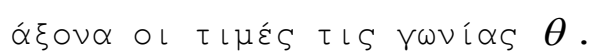




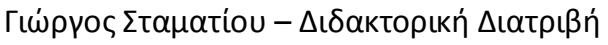

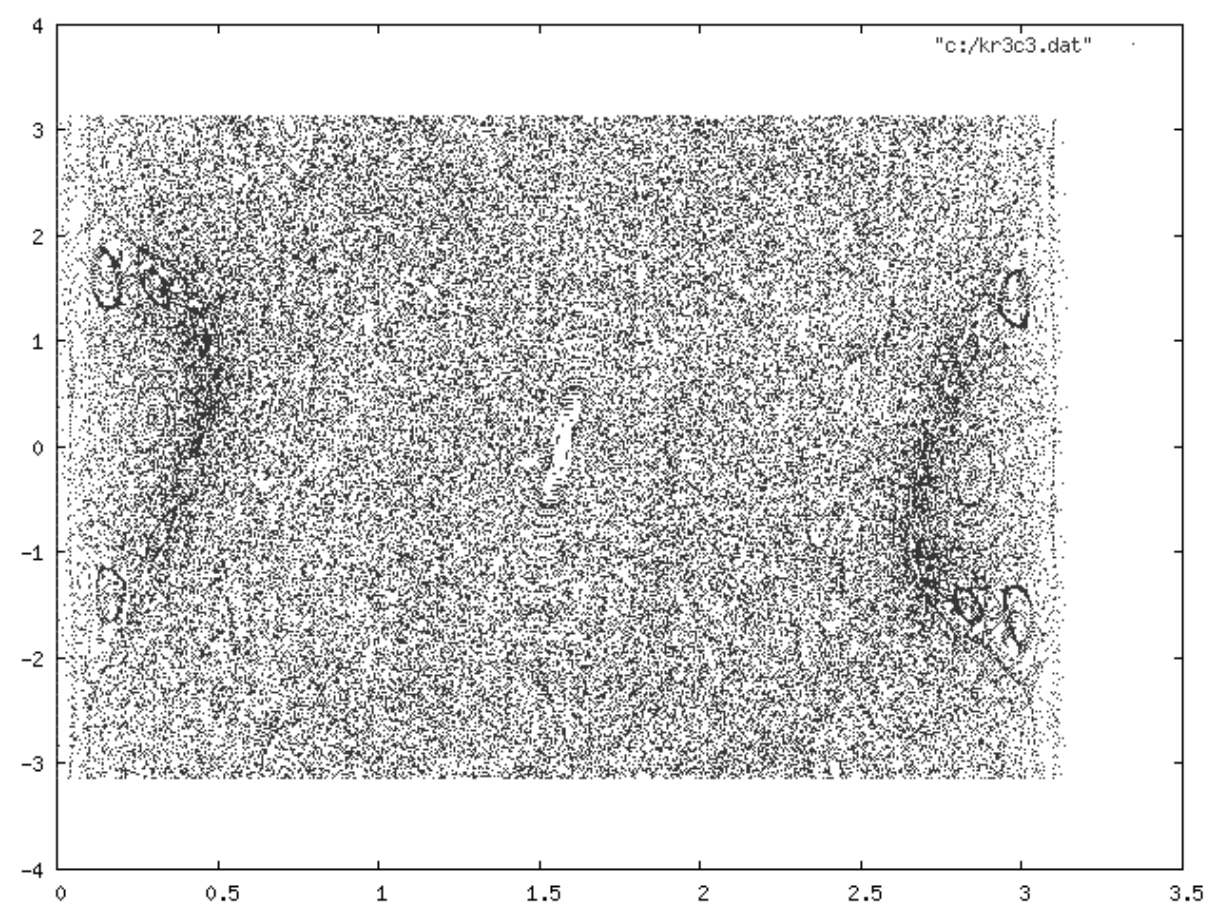

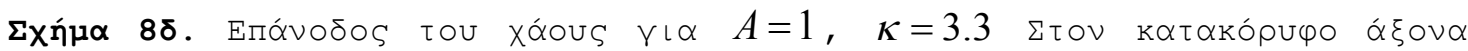

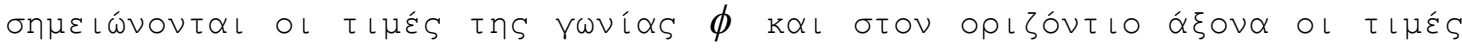

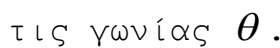




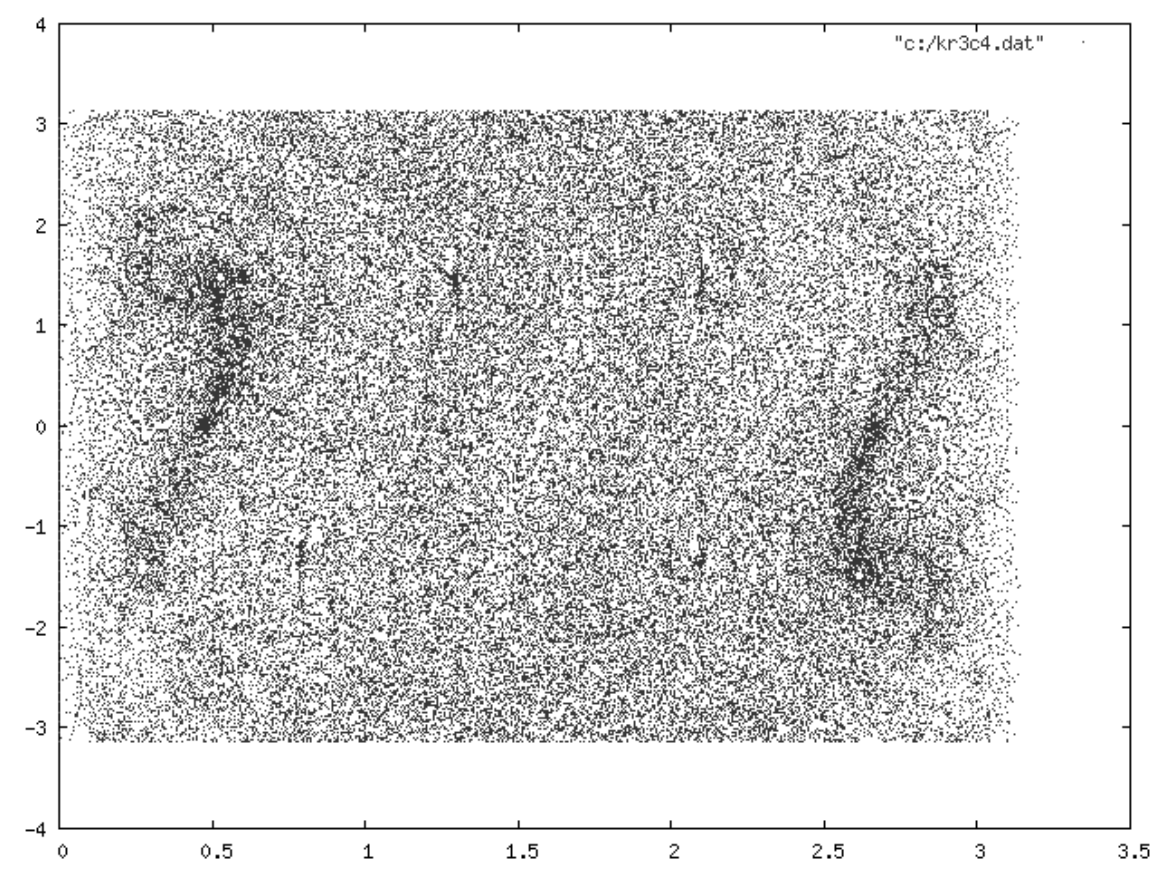

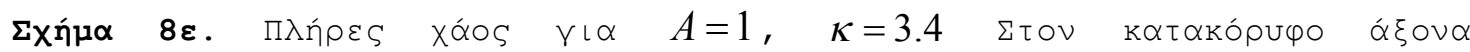

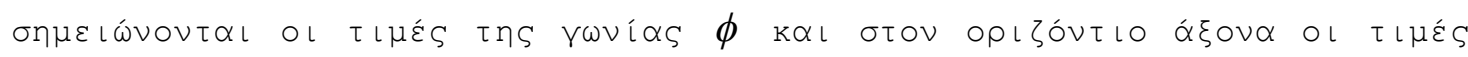

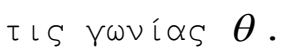

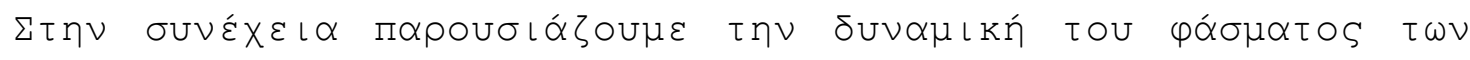

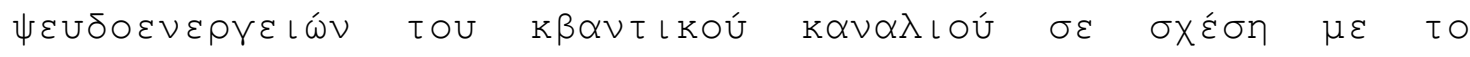

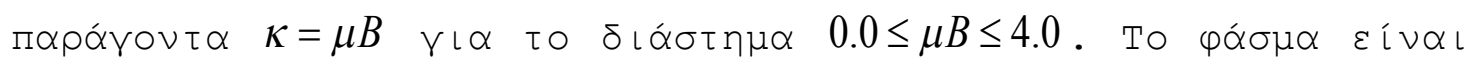

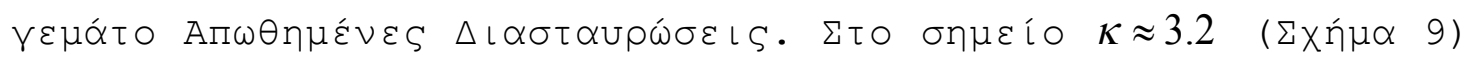

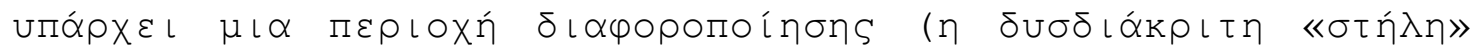

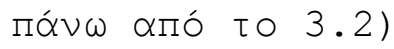




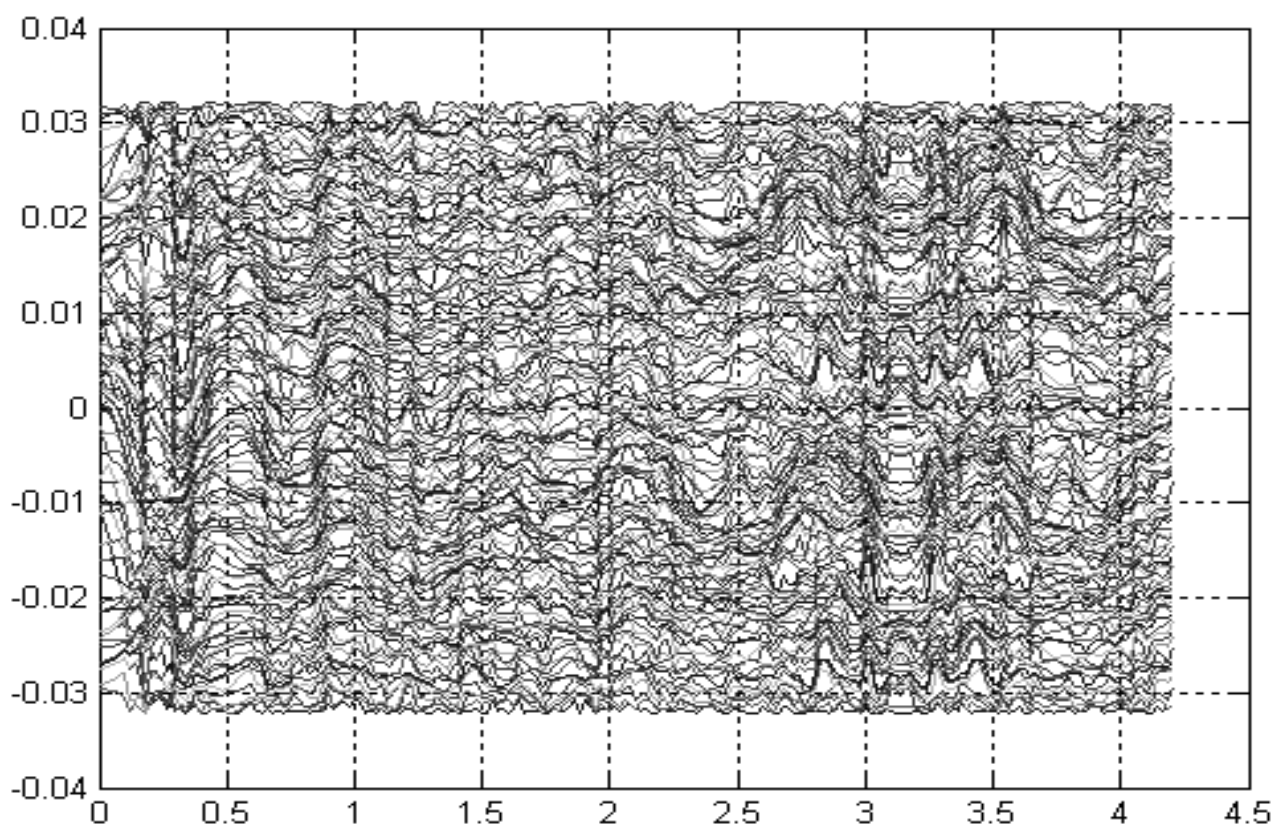

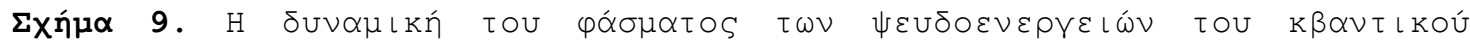

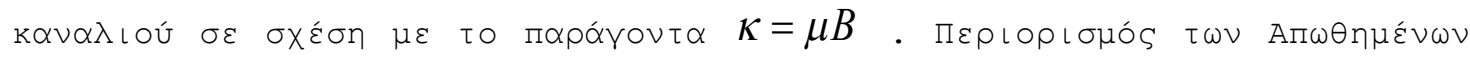

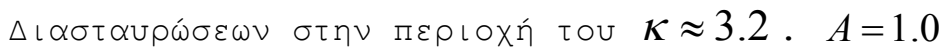

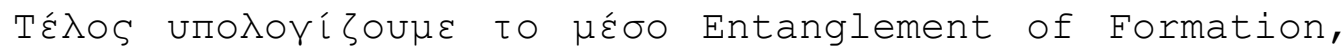

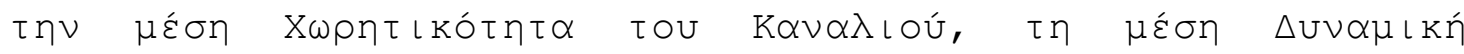

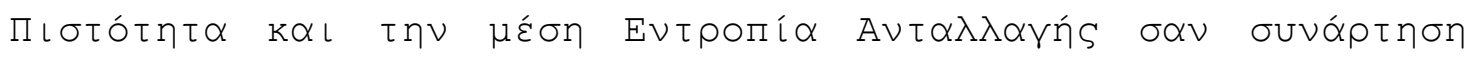

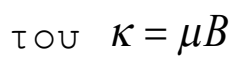

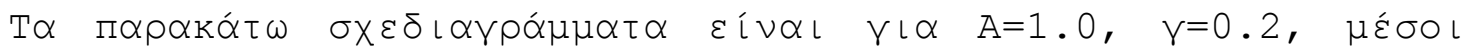

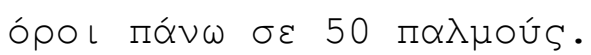



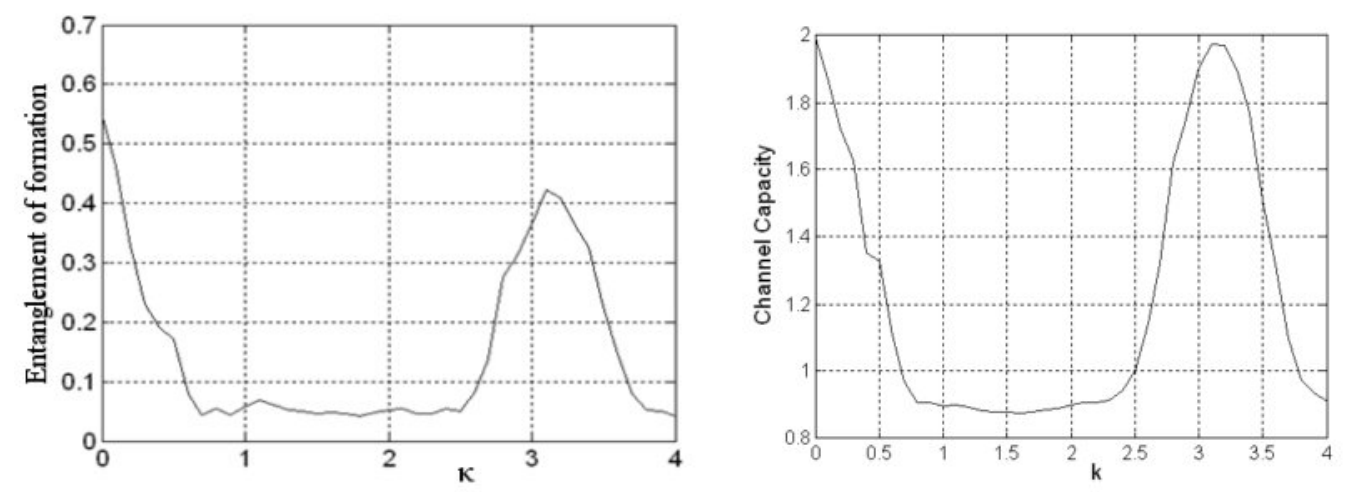

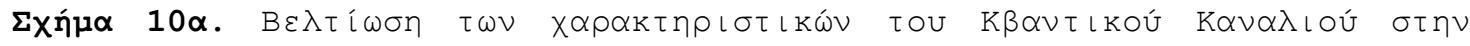

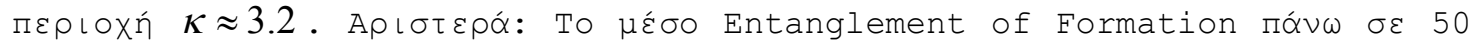

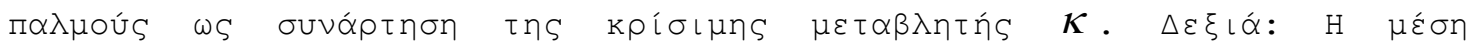

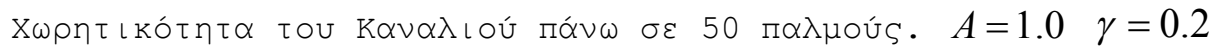
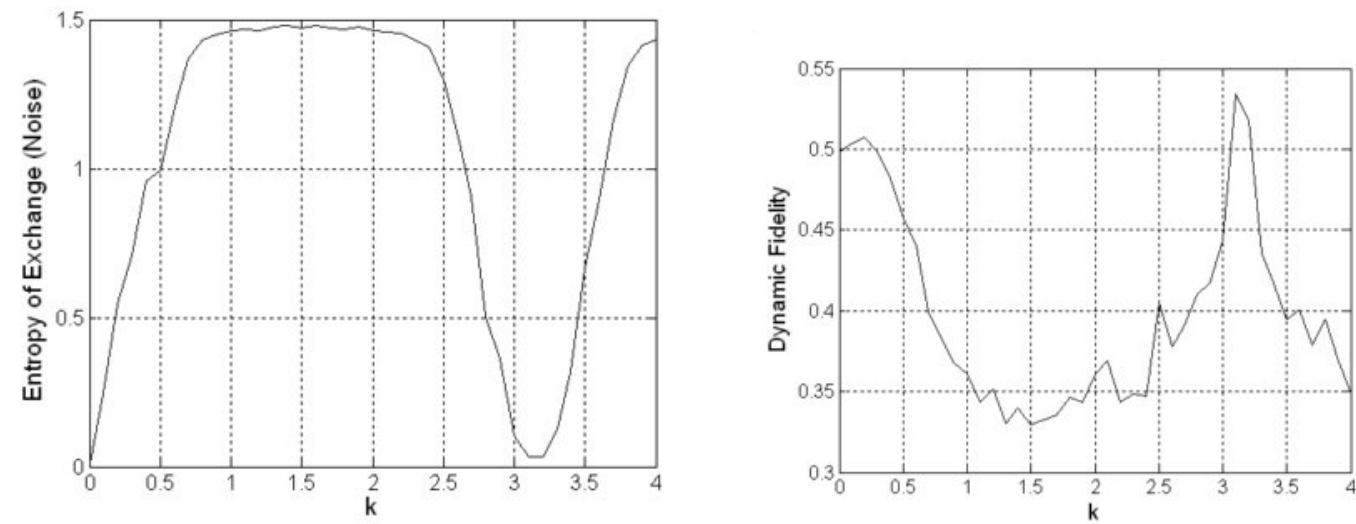

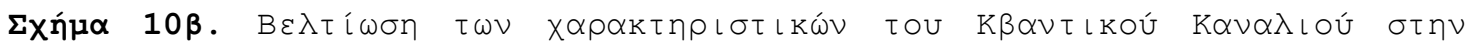

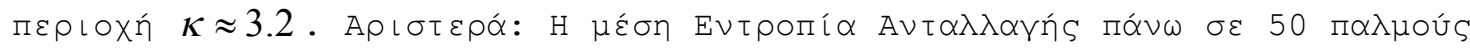

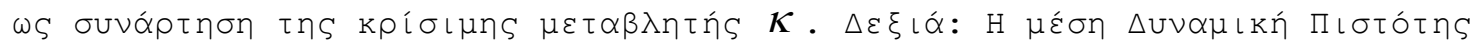

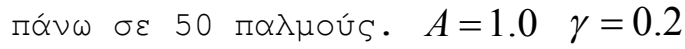

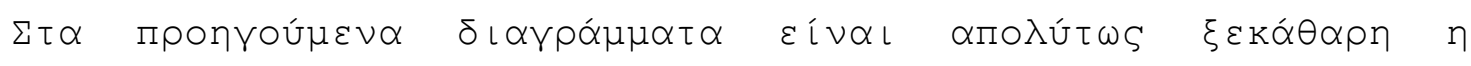

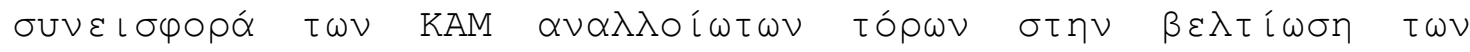

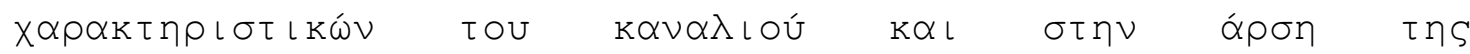

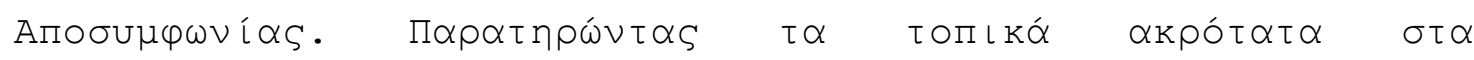

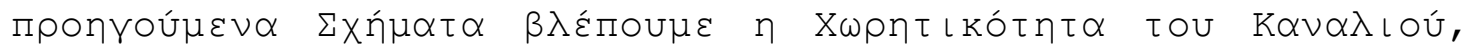

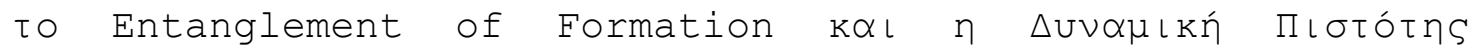

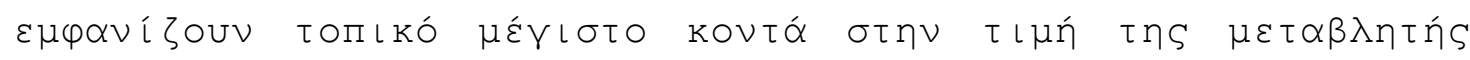




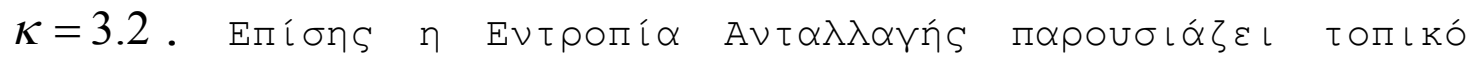

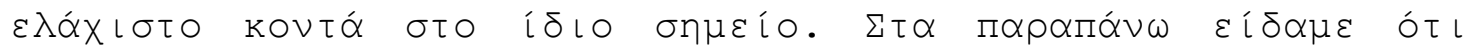

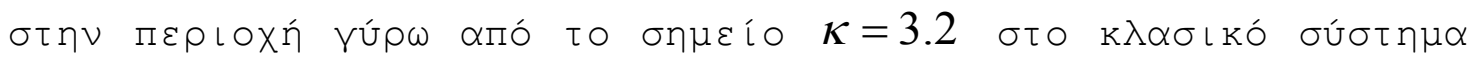

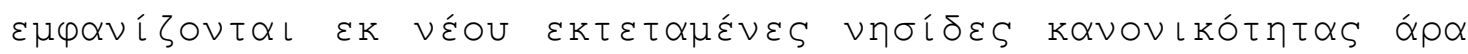

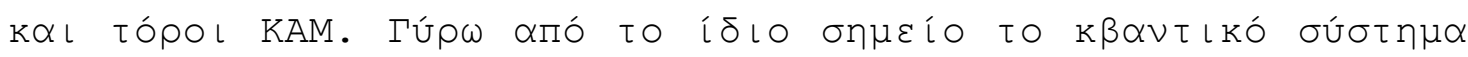

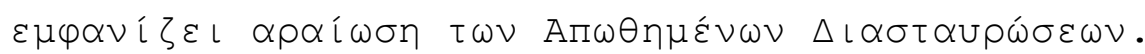

\section{$\Sigma \cup \mu \pi \varepsilon \rho \alpha ́ \alpha \mu \alpha \tau \alpha$}

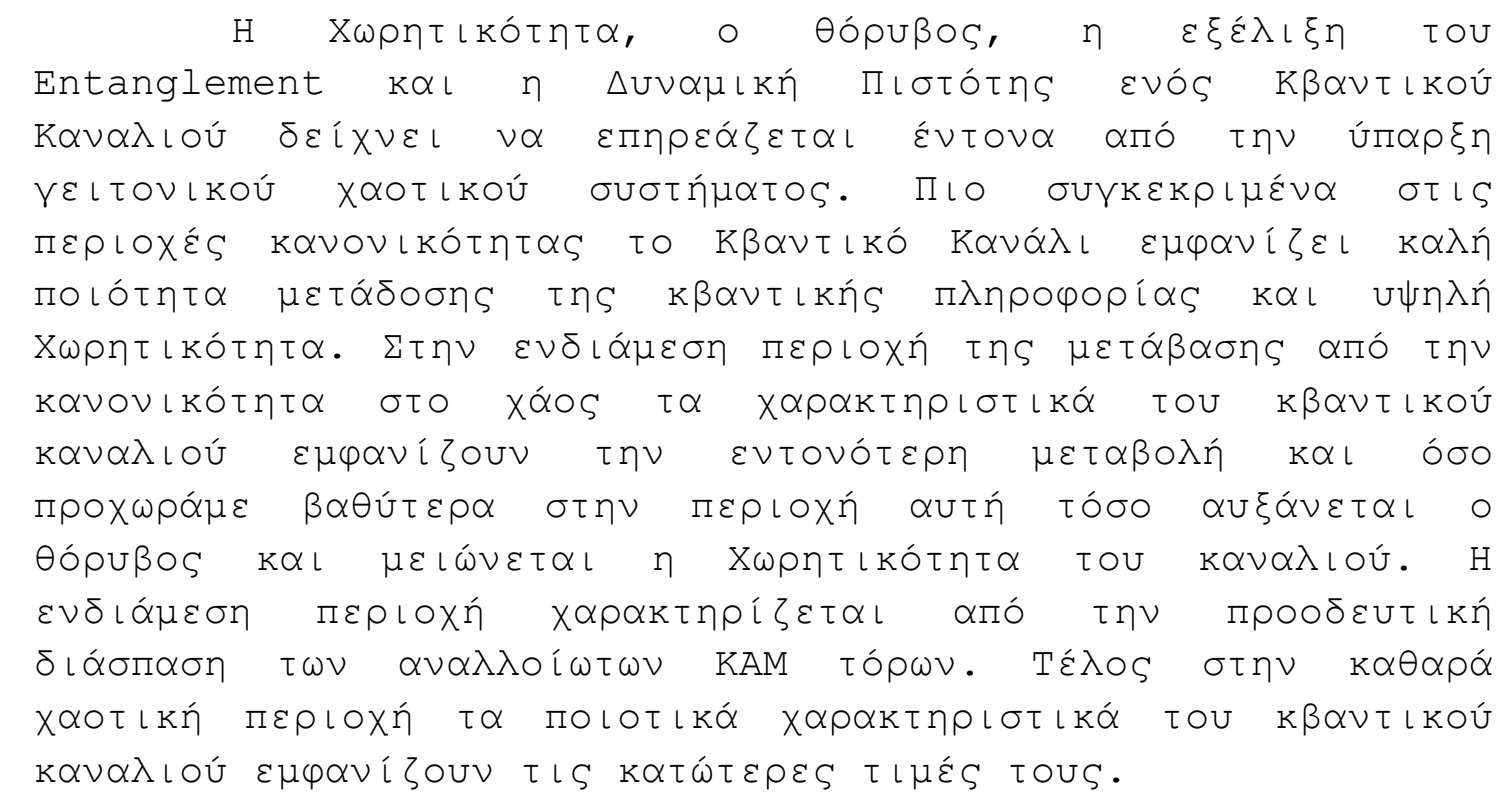

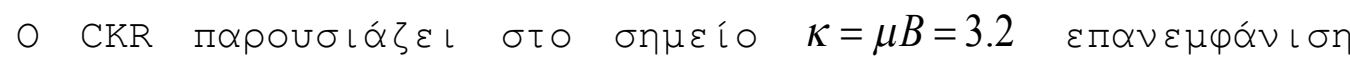

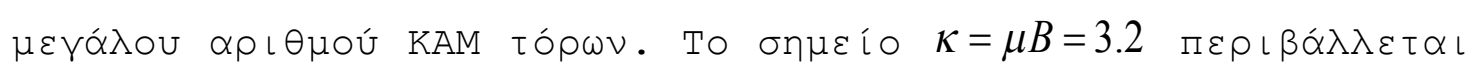

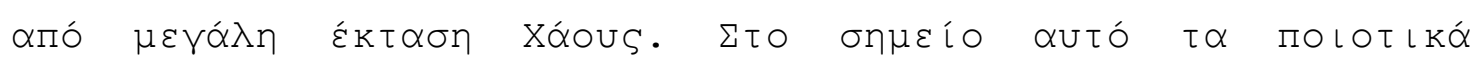

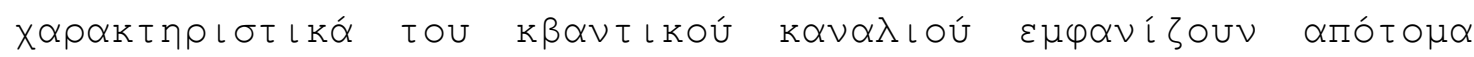

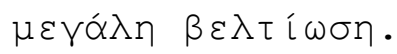





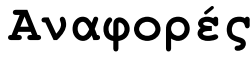

[1]. Martin C. Gutzwiller, Chaos in Classical and Quantum Mechanics, (1990) Springer-Verlag, New York ISBN=0-38797173-4.

[2]. Elements of information theory, TM Cover, JA Thomas 2006 - Wiley

[3]. Decoherence, chaos, and the second law, WH Zurek, JP Paz - Physical Review Letters, 1994

[4]. Sending Entanglement through noisy quantum channels, B Schumacher - Physical Review A, 1996

[5]. Capacity of the noisy quantum channel, $S$ Lloyd Physical Review A, 1997

[6]. G.Casati, B.V.Chirikov, J.Ford and F.M.Izrailev, Lect.NotesPhys.93, 334, 1979. F. M. Izrailev, Phys.Rep. $196,299,1990$

[7]. Quantum chaos: a new paradigm of nonlinear dynamics, K Nakamura, K Nakamura - 1993

[8]. Entanglement of formation of an arbitrary state of two qubits, WK Wootters - Physical Review Letters, 1998

[9]. Quantum Computation and Quantum Information, MA Nielsen, IL Chuang, Cambridge University Press, 2000, ISBN: $0-521-63503-9$

[10]. Lecture notes for Physics 229: Quantum information and computation, J Preskill - California Institute of Technology, 1998

[11]. Entropy exchange and Entanglement in the JaynesCummings model, E Boukobza, DJ Tannor - Physical Review A, 2005

[12]. Dynamics of Loschmidt echoes and fidelity decay, T Gorin, T Prosen, TH Seligman, M Žnidarič - Physics Reports, 2006 


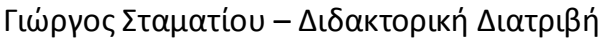




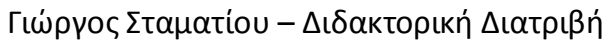

ENOTHTA H'

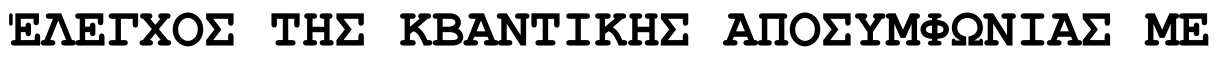

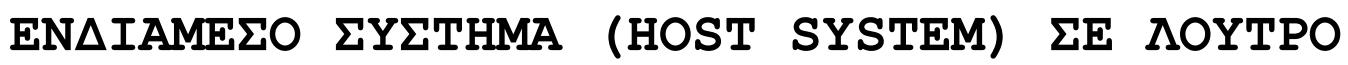

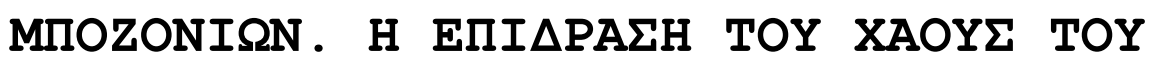

ENDIAME

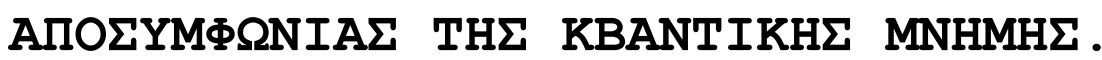





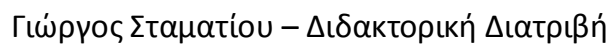

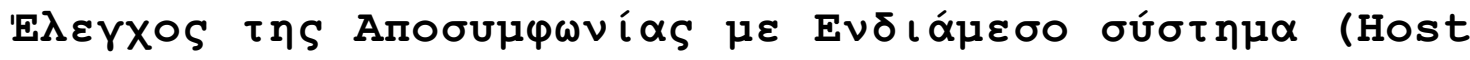

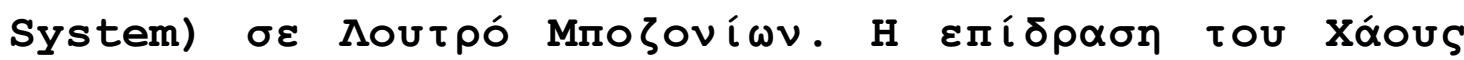

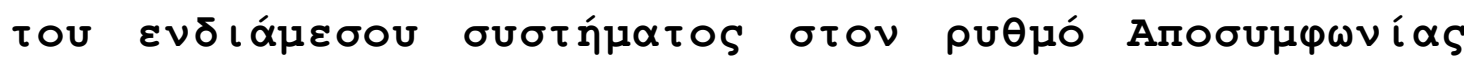

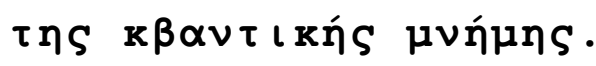

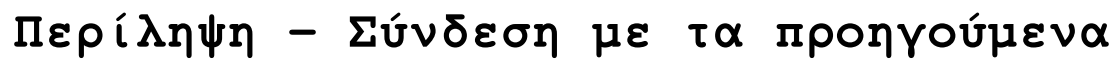

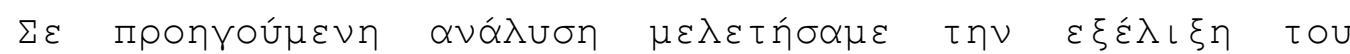

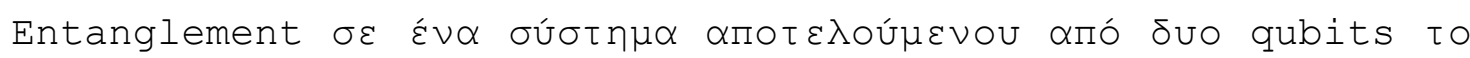

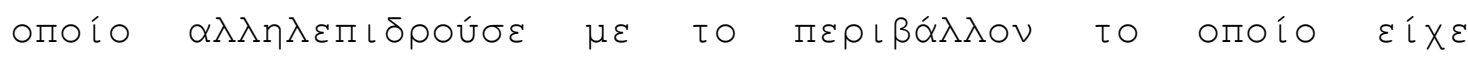

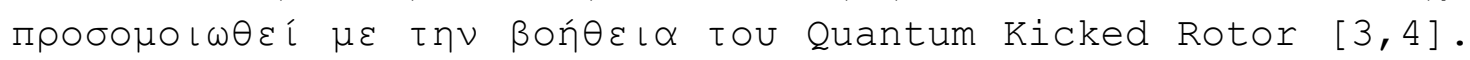

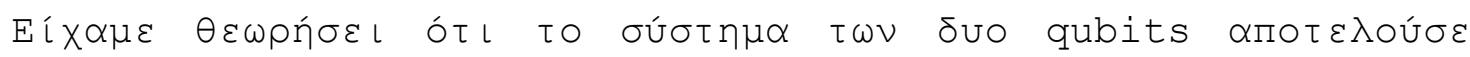

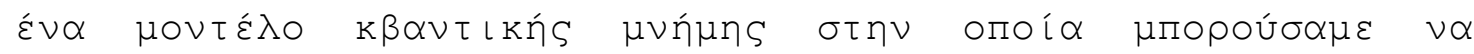

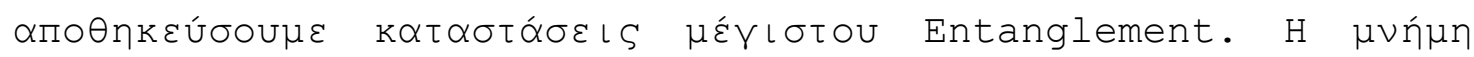

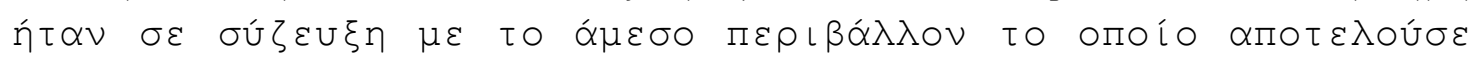

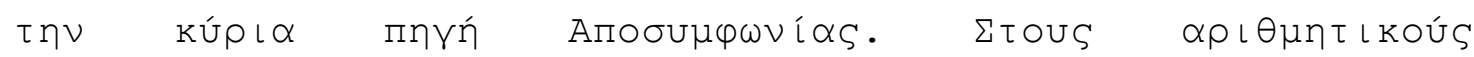

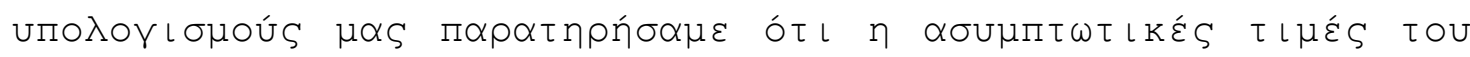

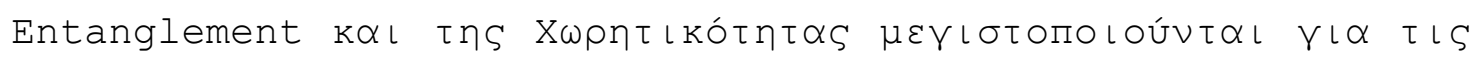

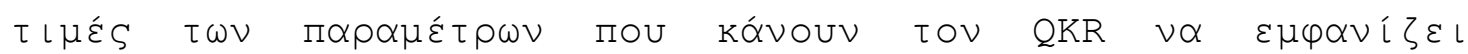

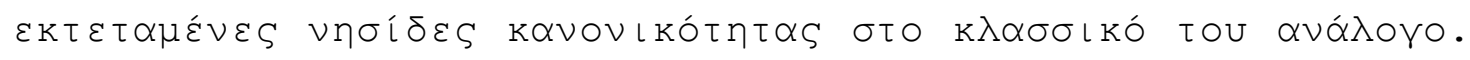

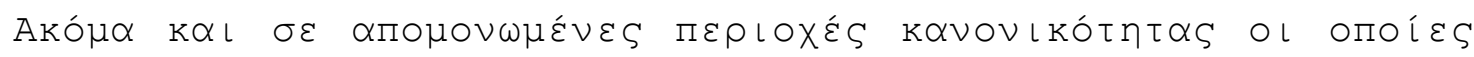

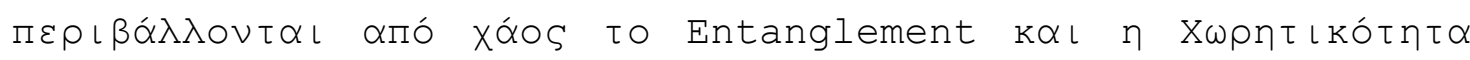

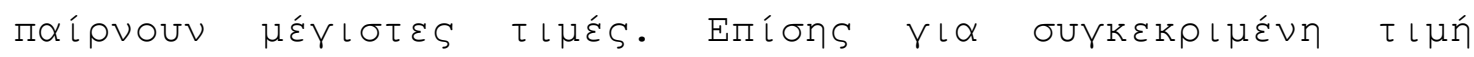

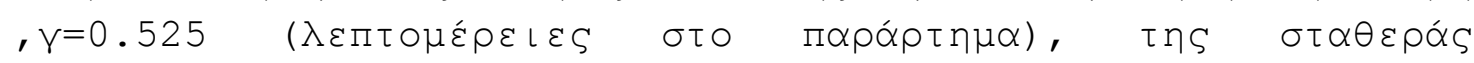

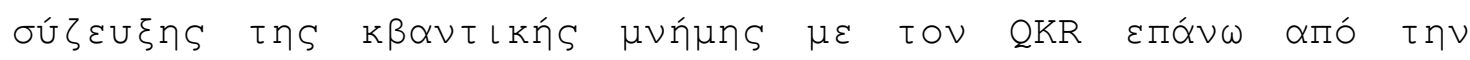

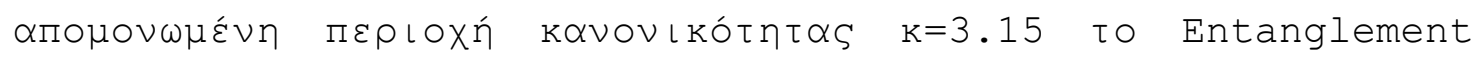

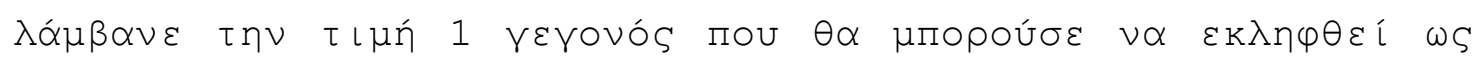

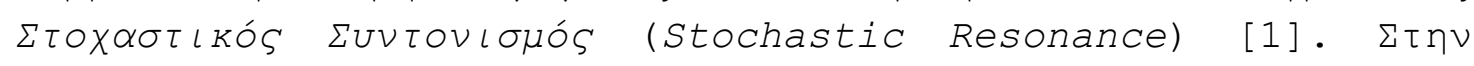

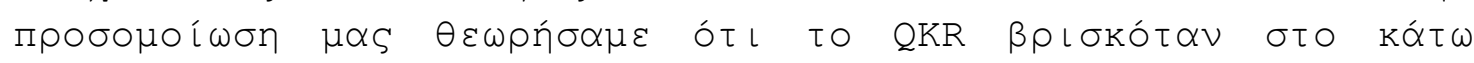

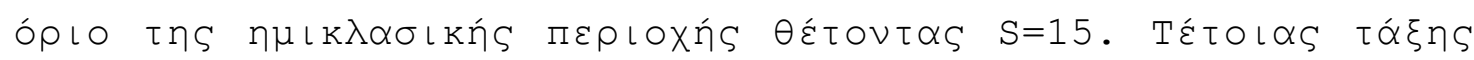

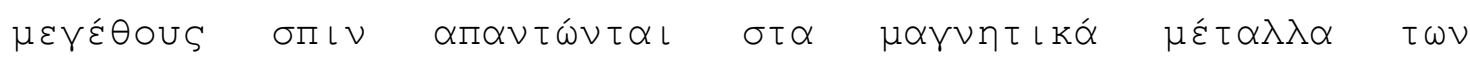

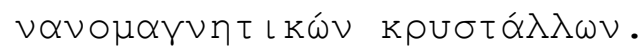

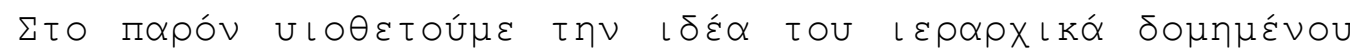

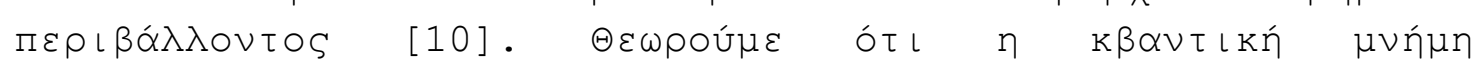

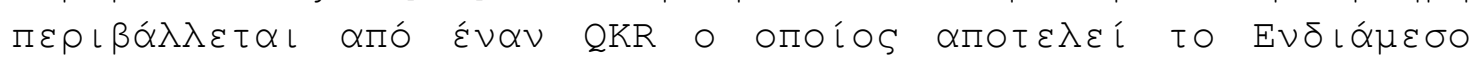

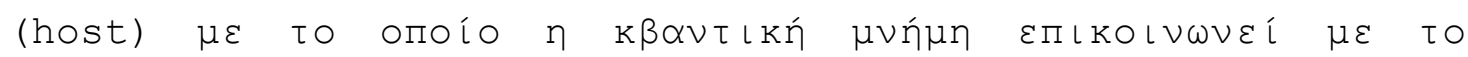

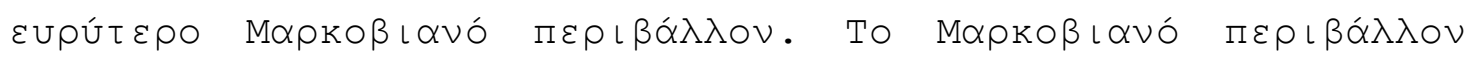

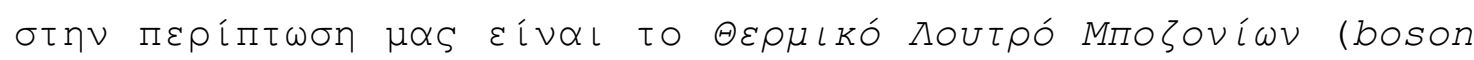




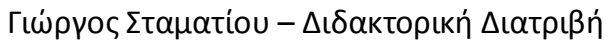

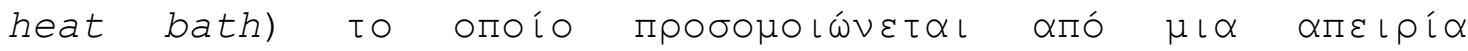

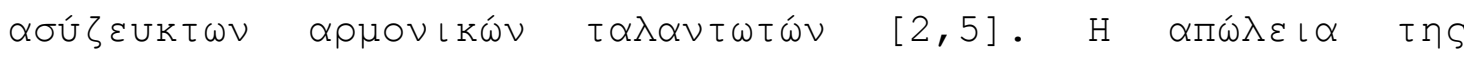

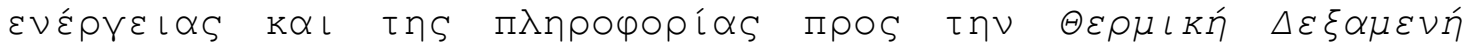

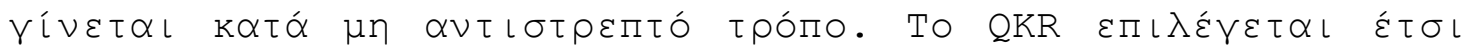

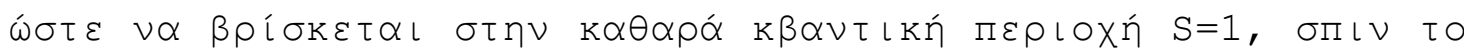

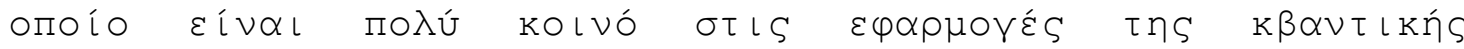

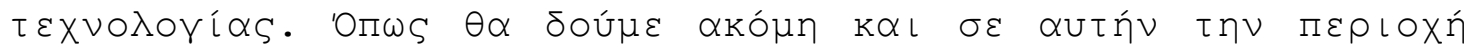

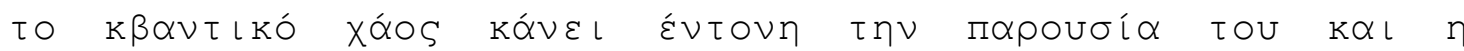

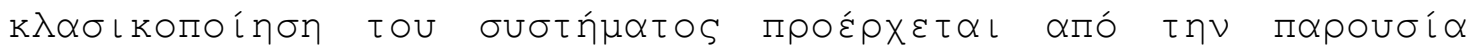

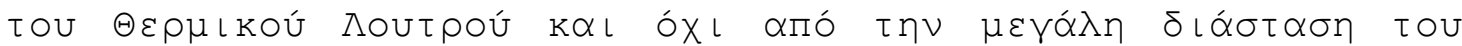

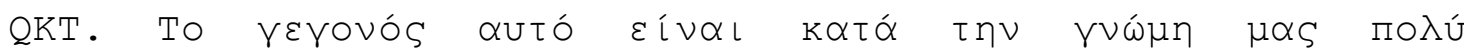

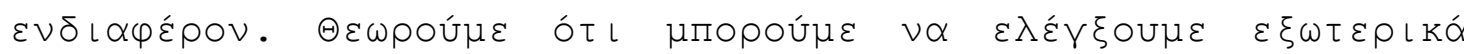

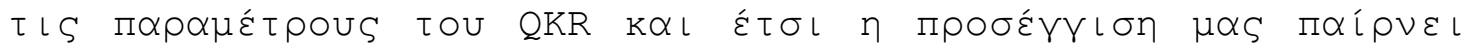

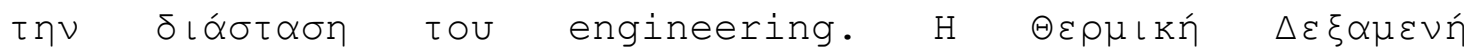

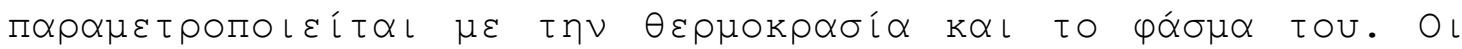

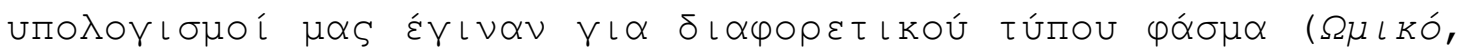

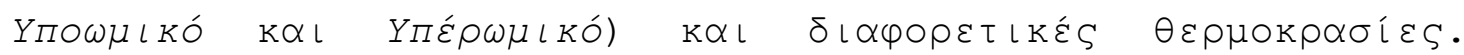

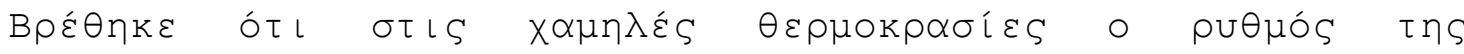

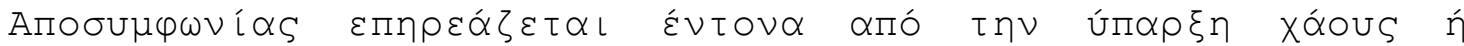

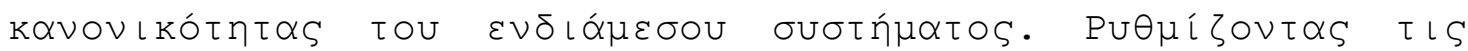

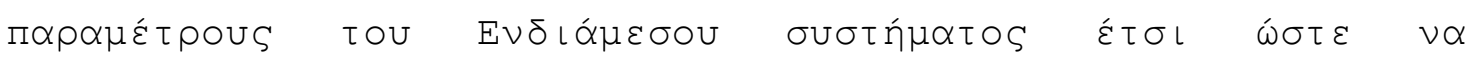

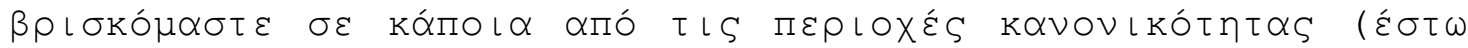

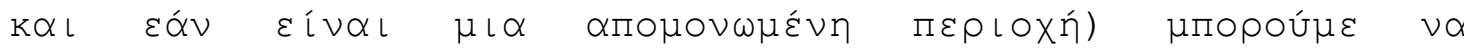

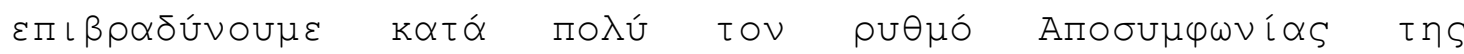

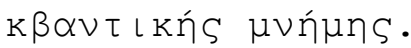

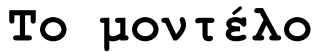

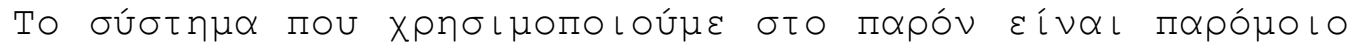

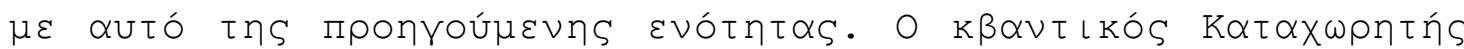

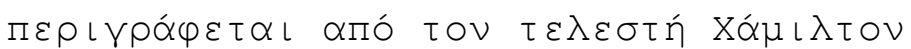

$$
\hat{H}_{r e g}=\frac{1}{2} \hat{\sigma}_{z 1} \hat{\sigma}_{z 2}+\frac{3}{2} \hat{\sigma}_{x 1} \hat{\sigma}_{x 2} .
$$

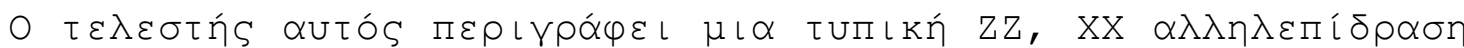

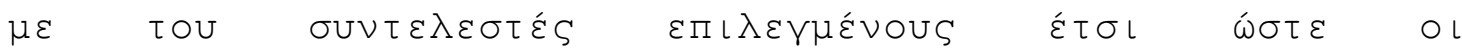

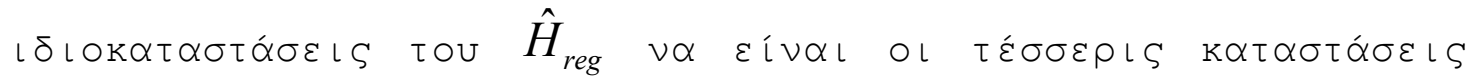




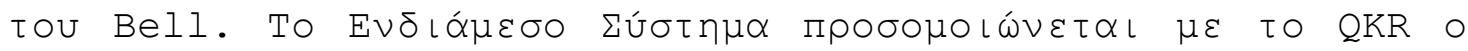

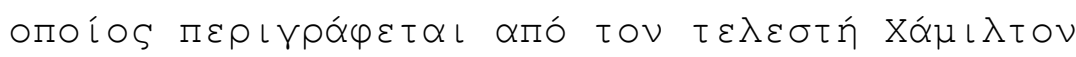

$$
\hat{H}_{h}=A \hat{S}_{z h}^{2}-\mu B \hat{S}_{x h} \sum_{j=-\infty}^{+\infty} \delta(t-2 \pi j) .
$$

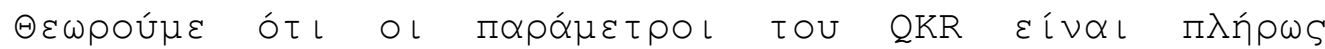

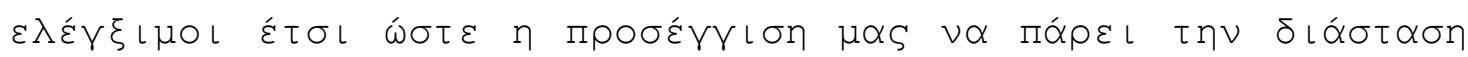

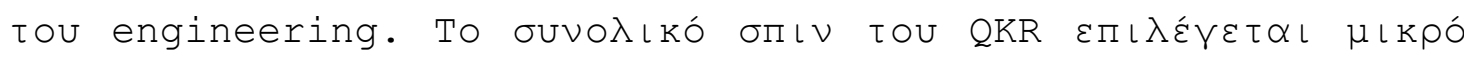

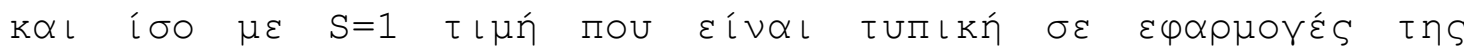

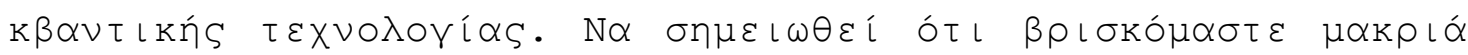

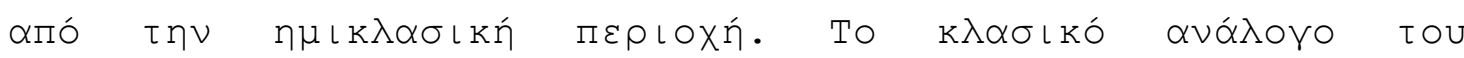

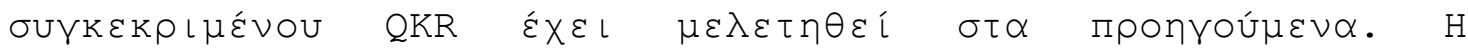

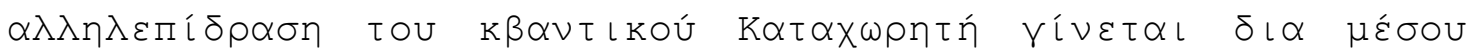

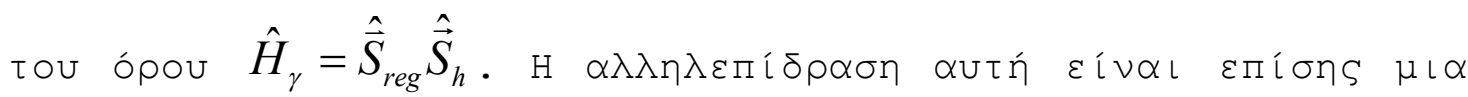

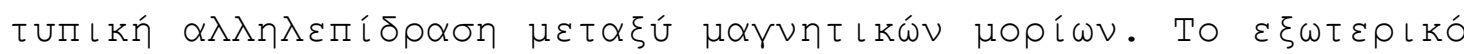

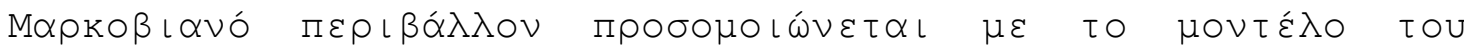

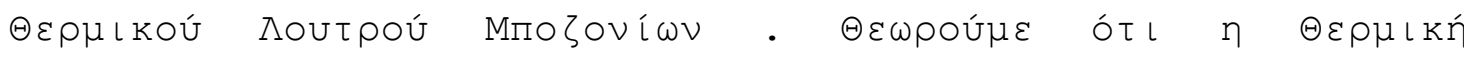

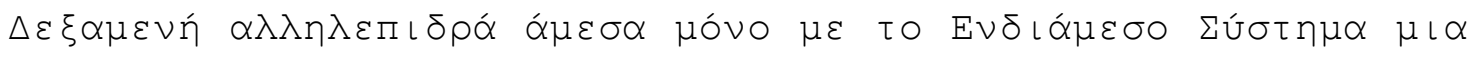

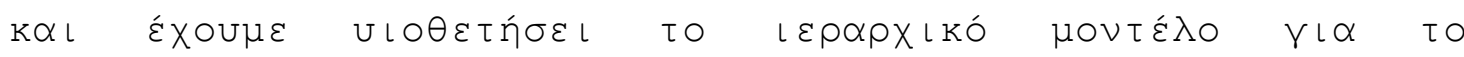

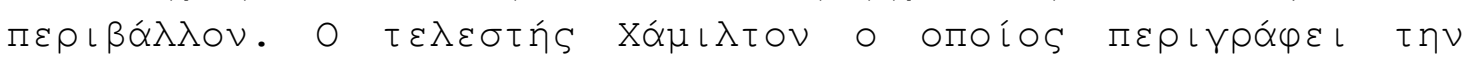

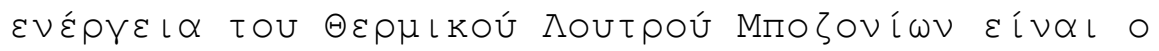

$$
\hat{H}_{R}=\sum_{\alpha} \hbar \omega_{\alpha}\left(b_{\alpha}^{+} b_{\alpha}+\frac{1}{2}\right)
$$

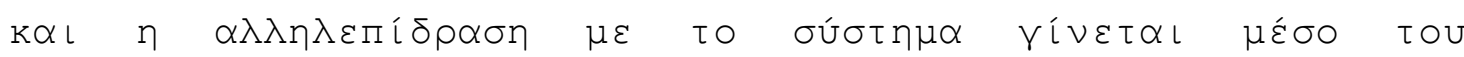
ópou $[2,5]$

$$
\hat{H}_{\mathrm{int}}=\lambda \sqrt{\hbar} \sum_{a} \gamma_{a}\left(b_{a}^{+}+b_{a}\right) \hat{X}+O\left(\hat{X}^{2}\right)
$$

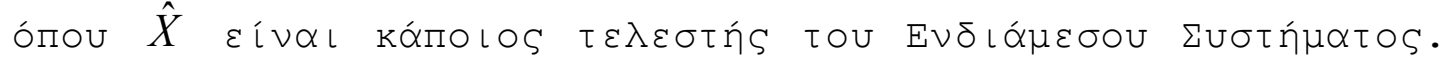

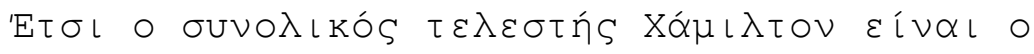

$$
\hat{H}=\hat{H}_{r e g}+\gamma \hat{H}_{\gamma}+\hat{H}_{h}+\hat{H}_{\mathrm{int}}+\hat{H}_{R}
$$




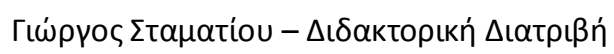

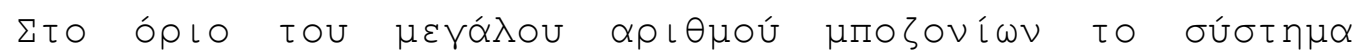

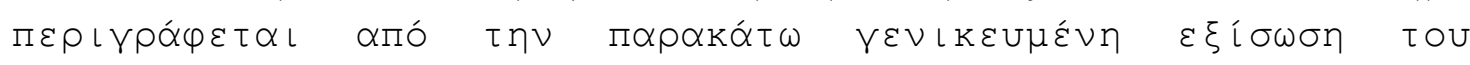
Lindblad:

$$
\dot{\hat{\rho}}_{S H}=-\frac{i}{\hbar}\left[\hat{H}_{S H}, \hat{\rho}_{S H}\right]-\frac{\pi \lambda^{2}}{\hbar}\left(\left[\hat{X}, \hat{R} \hat{\rho}_{S H}\right]+\left[\hat{X}, \hat{R} \hat{\rho}_{S H}\right]^{+}\right)
$$

ónоv

$$
\begin{aligned}
& \hat{H}_{S H}=\hat{H}_{r e g}+\gamma \hat{H}_{\gamma}+\hat{H}_{h} \quad \mathrm{k} \alpha \iota \quad\langle l|\hat{R}| m\rangle=\frac{1}{\hbar} X_{l m} \frac{I\left(\omega_{l m}\right)-I\left(-\omega_{l m}\right)}{\exp \left(\beta \hbar \omega_{l m}\right)-1} \\
& \mu \varepsilon \hbar \omega_{l m}=E_{l}-E_{m} \text { о́поч } \hat{H}_{o}|m\rangle=E_{m}|m\rangle \\
& \mu \varepsilon \quad \hat{H}_{o}=\hat{H}_{r e g}+\gamma \hat{H}_{\gamma}+A \hat{S}_{z h}^{2} \quad \text { к } \alpha \iota \quad \beta=\frac{1}{\kappa_{b} T} .
\end{aligned}
$$

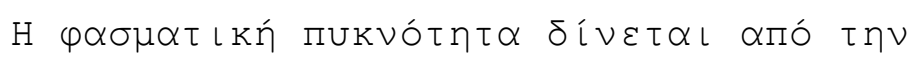

$$
\begin{aligned}
& I(\omega)=I_{o} \omega^{a} \theta(\omega) \quad \mu \varepsilon \quad \theta(\omega)=\left\{\begin{array}{l}
1, \omega \geq 0 \\
0, \omega<0
\end{array} \quad \vee \alpha \quad \varepsilon i \nu \alpha \iota \quad \eta \quad \kappa \lambda \iota \mu \alpha \kappa \omega \tau \dot{n}\right. \\
& \text { бuváptnon. }
\end{aligned}
$$

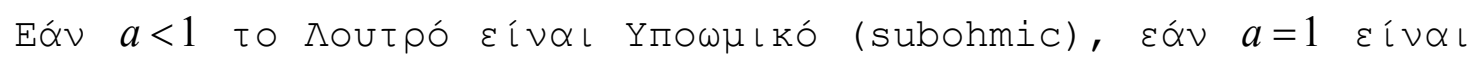

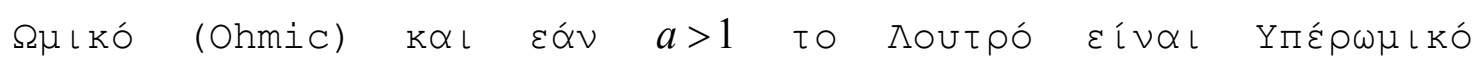
(Superohmic).

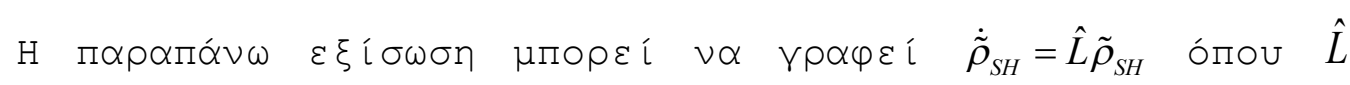

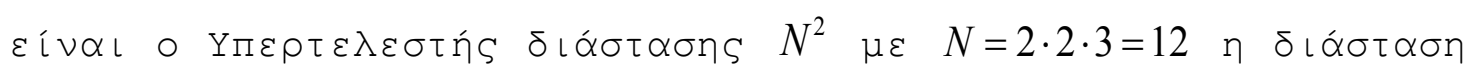

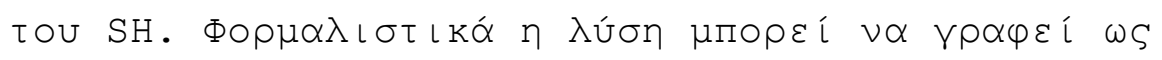

$$
\tilde{\rho}(t)=\hat{T} \exp \left(\int_{0}^{t} \hat{L} d t\right) \tilde{\rho}(0)
$$

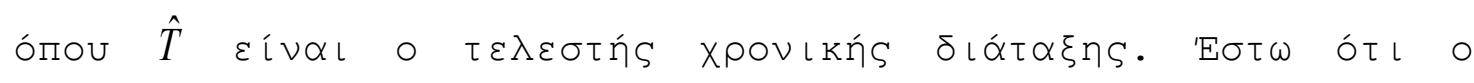

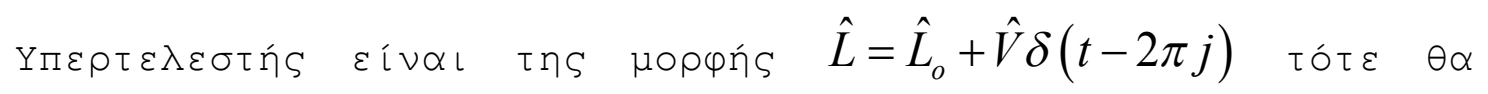




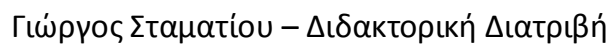

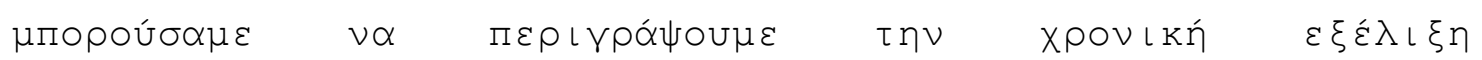

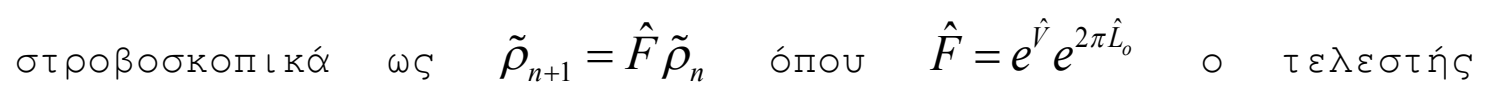

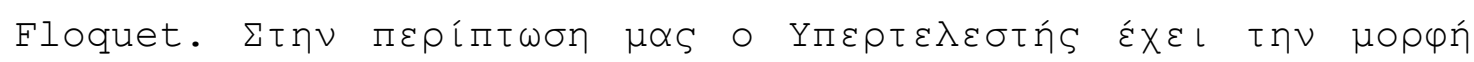

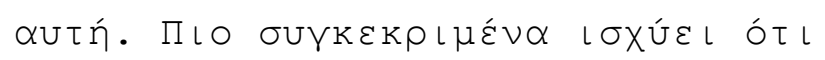

$$
\begin{gathered}
\hat{V} \leftrightarrow \frac{i}{\hbar} \mu B\left[\hat{S}_{x h}, \bullet\right] \\
\text { к } \propto\llcorner \\
\hat{L}_{o} \leftrightarrow-\frac{i}{\hbar}\left[\hat{H}_{o}, \bullet\right]-\frac{\pi \lambda^{2}}{\hbar}\left([\hat{X}, \hat{R} \bullet]+[\hat{X}, \hat{R} \bullet]^{+}\right)
\end{gathered}
$$

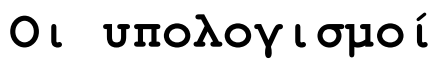

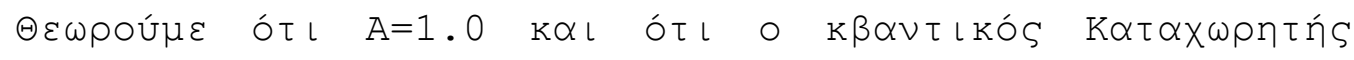

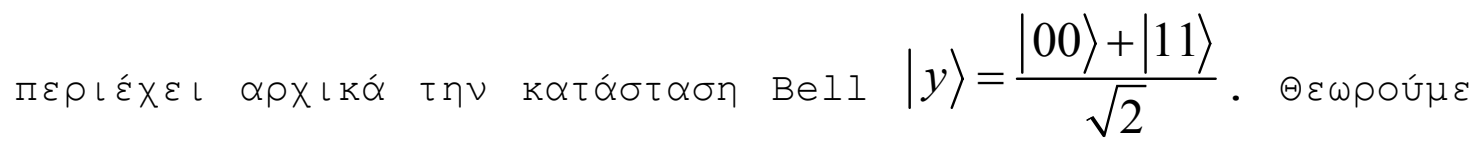

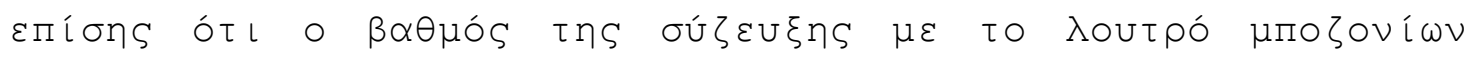

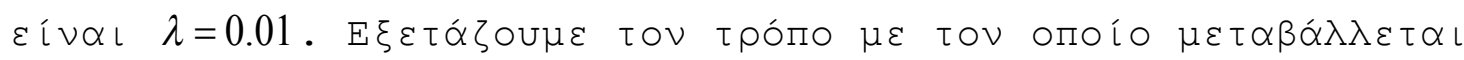

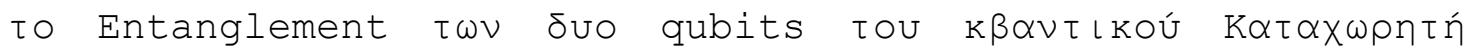

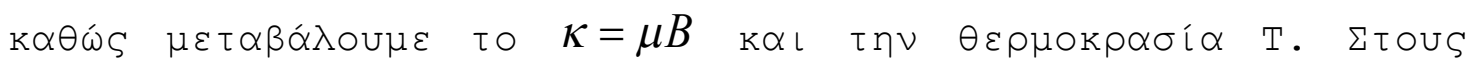

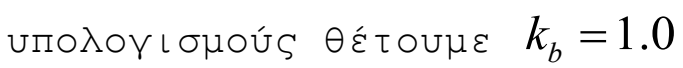

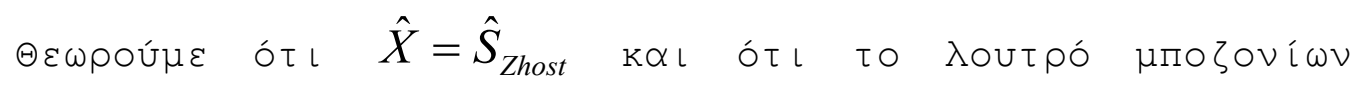
Eíval $\Omega \mu \iota k o ́$.

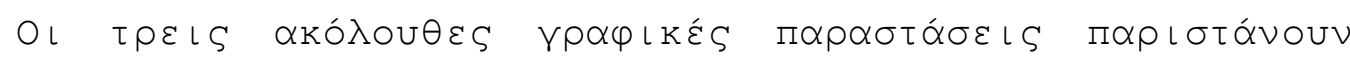

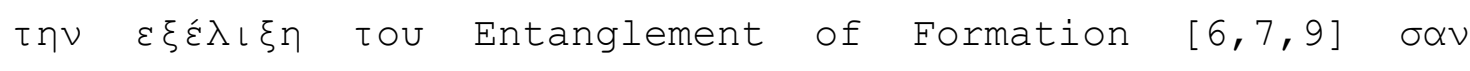

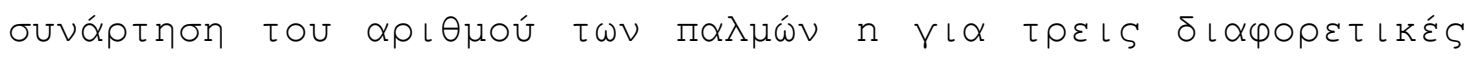

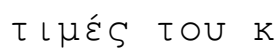




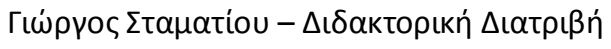

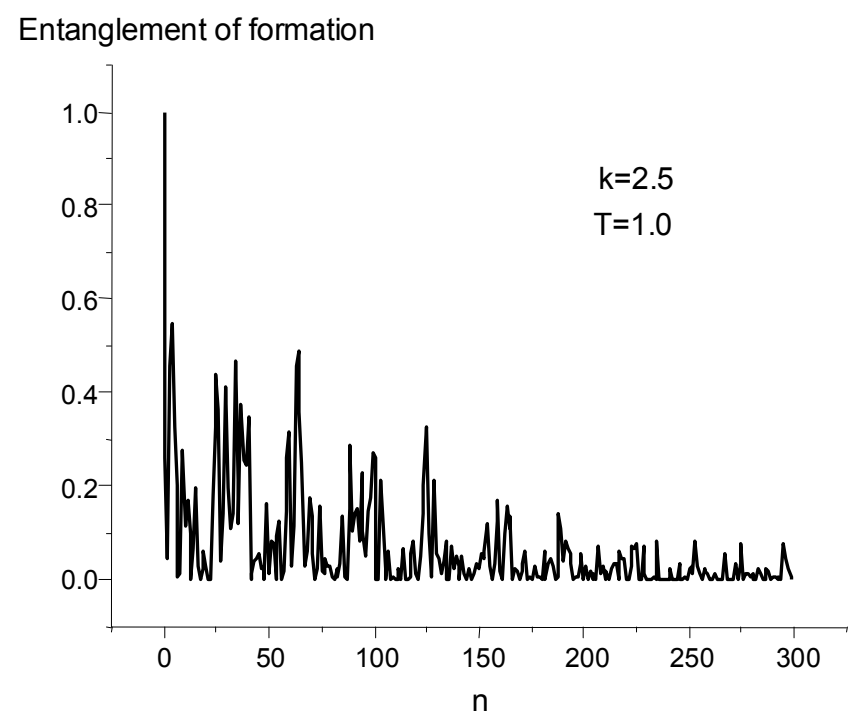

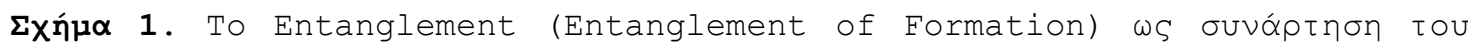

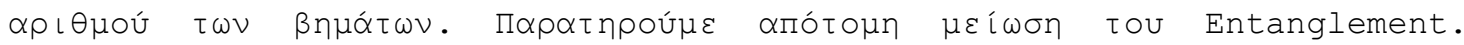
$\kappa=\mu B=2.5, T=1.0, \hat{X}=S_{\text {ZHost }}, A=1.0, \lambda=0.01$, Ohmic Bath.

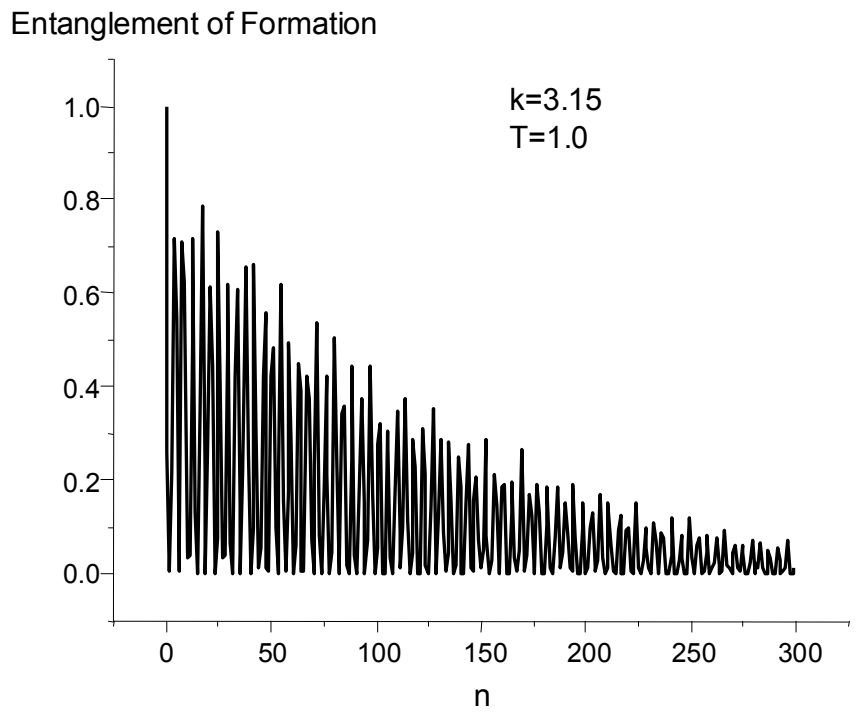

$\boldsymbol{\Sigma}$ X́n $\boldsymbol{\alpha}$ 2. To Entanglement (Entanglement of Formation) wS ouváptnon tou

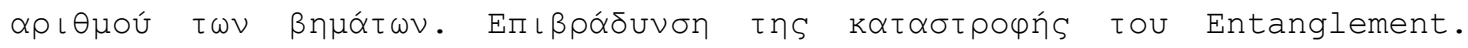
$\kappa=\mu B=3.15, T=1.0, \hat{X}=S_{\text {ZHost }}, A=1.0, \lambda=0.01$, Ohmic Bath. 


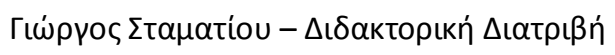

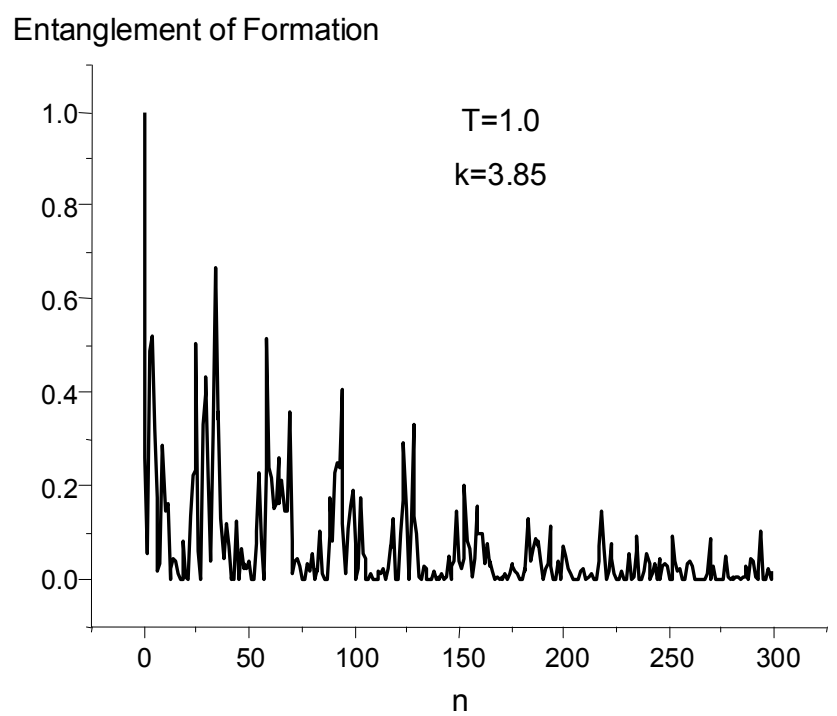

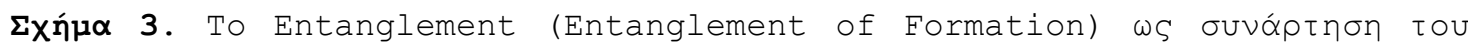

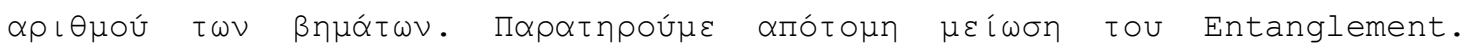
$\kappa=\mu B=3.85, T=1.0, \hat{X}=S_{\text {ZHost }}, A=1.0, \lambda=0.01$, Ohmic Bath.

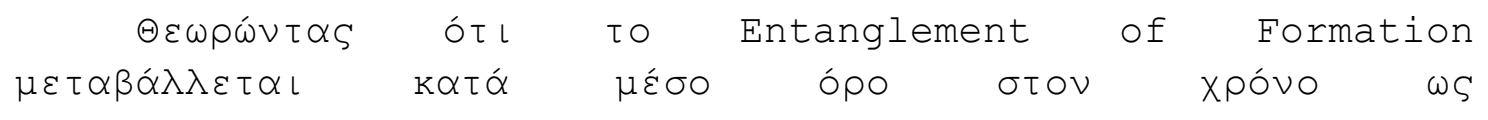

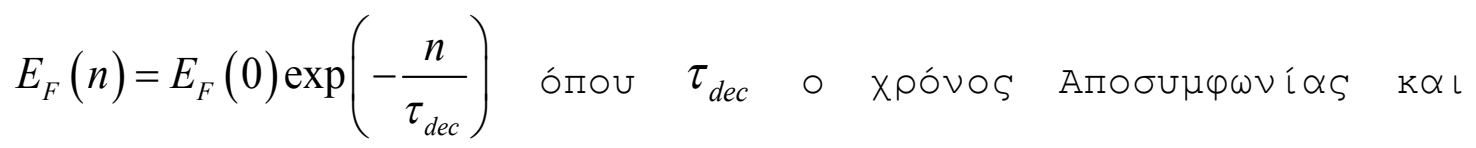

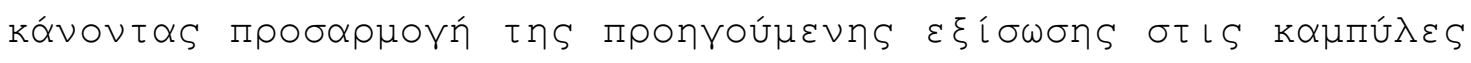

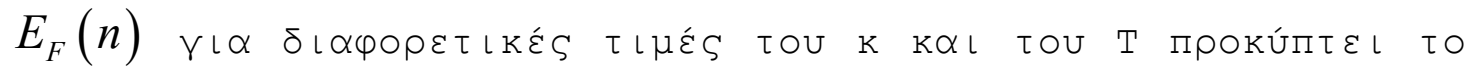

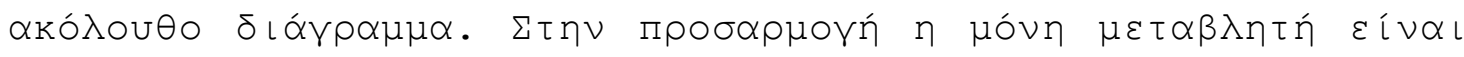

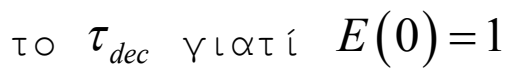




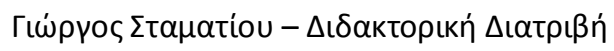

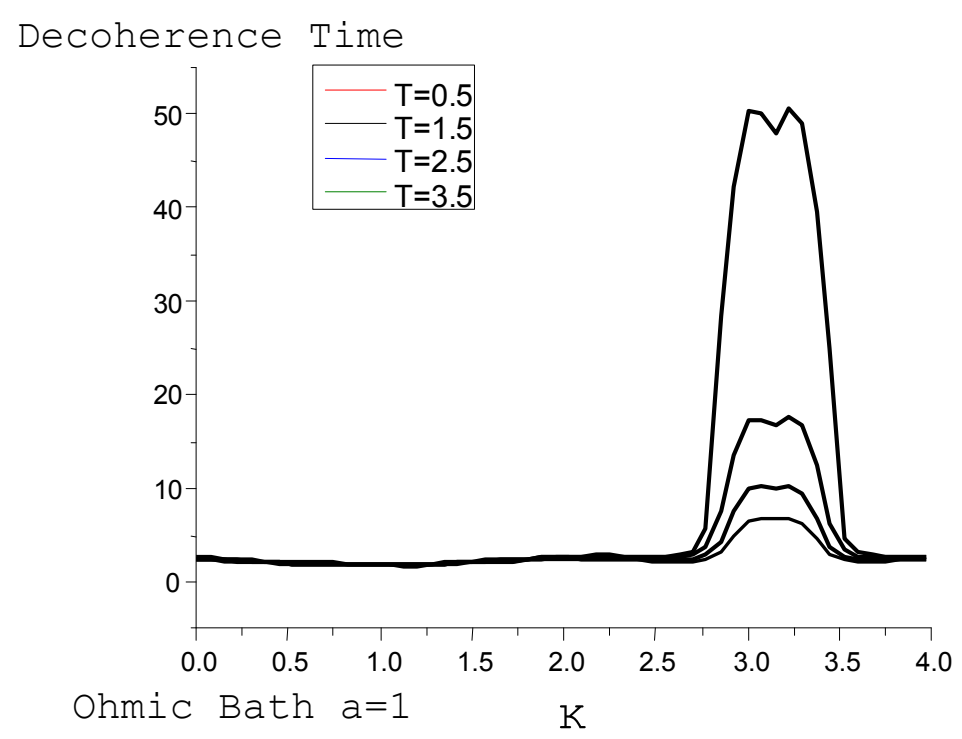

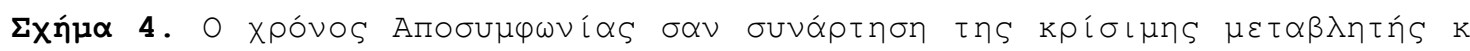

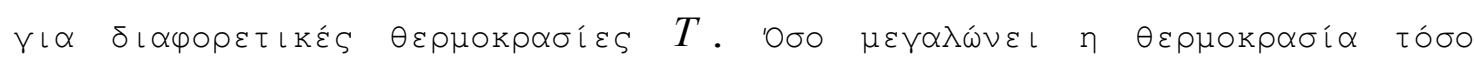

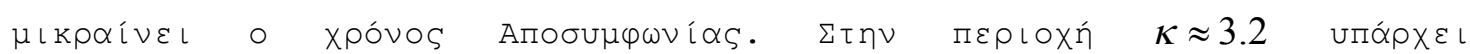

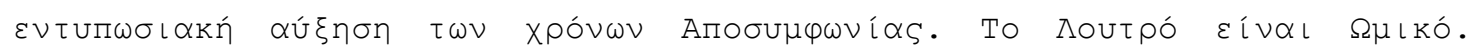
$\hat{X}=S_{\text {ZHost }}, \quad A=1.0, \lambda=0.01, \quad \gamma=0.525$

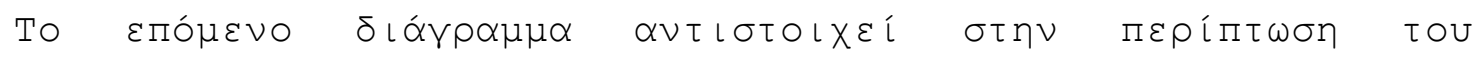

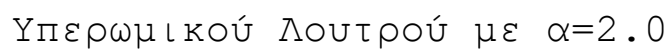




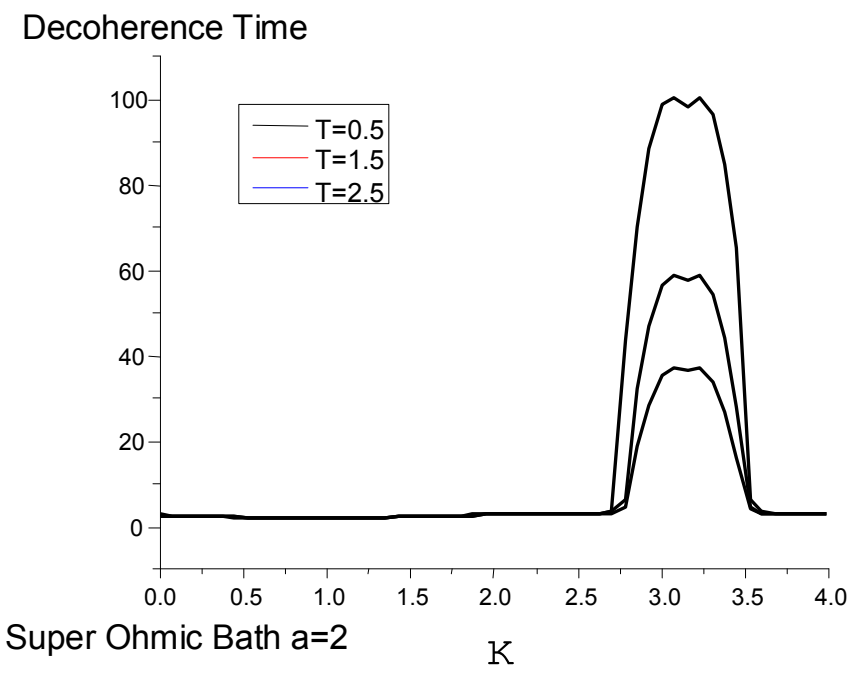

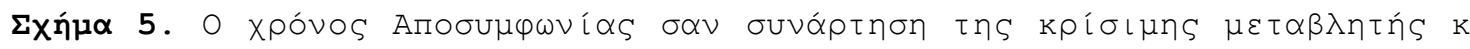

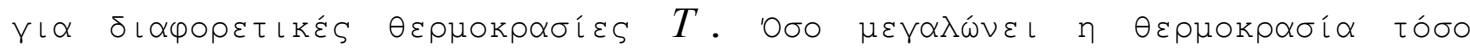

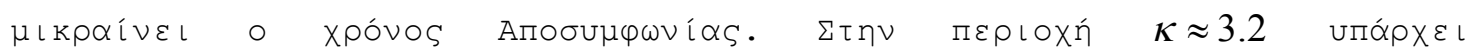

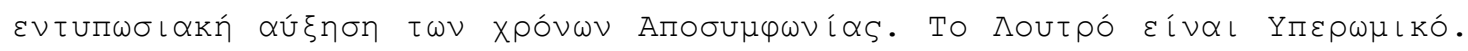
$\hat{X}=S_{\text {ZHost }}, \quad A=1.0, \lambda=0.01$

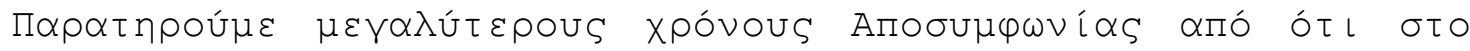

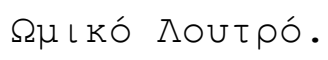

\section{$\Sigma \cup \mu \Pi \varepsilon \rho \alpha ́ \alpha \mu \alpha \tau \alpha$}

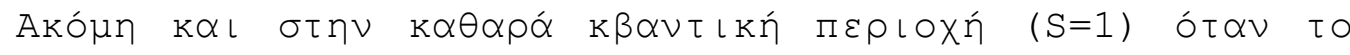

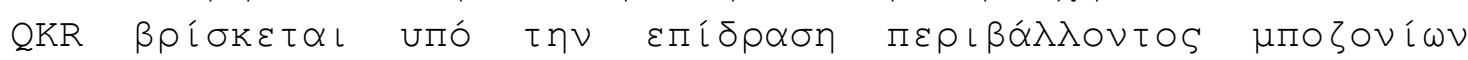

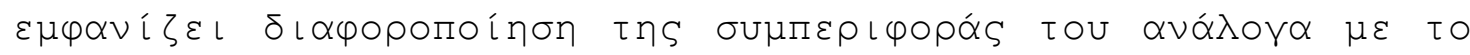

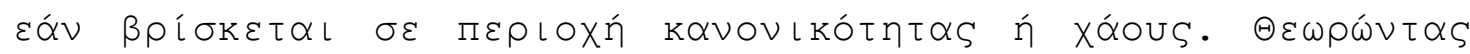

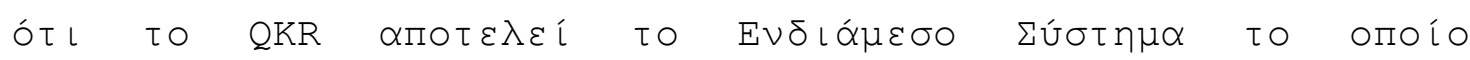

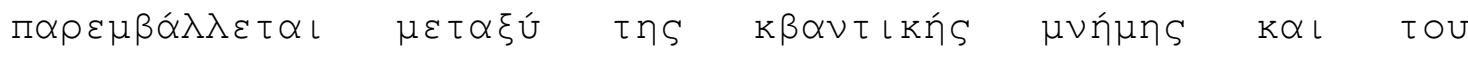

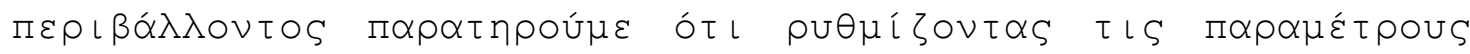

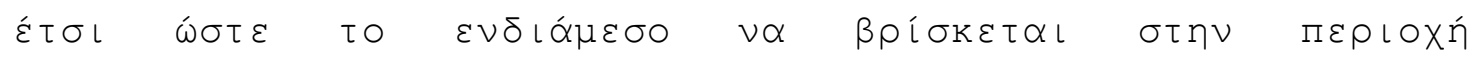

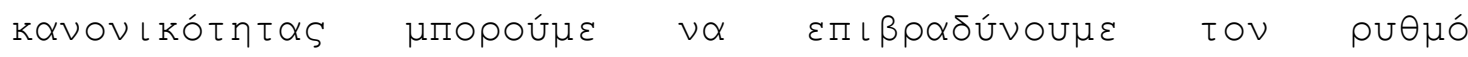

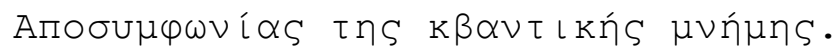




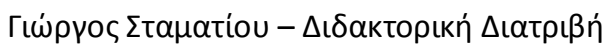

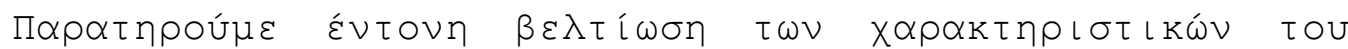

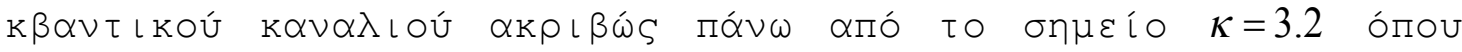

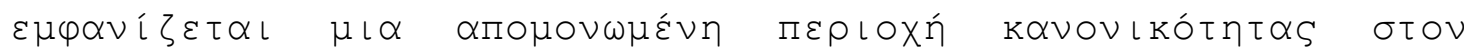

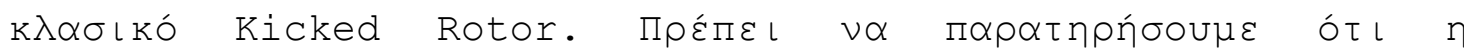

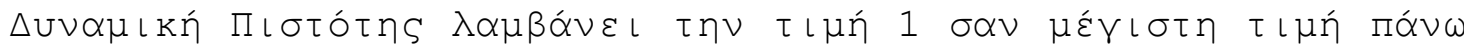

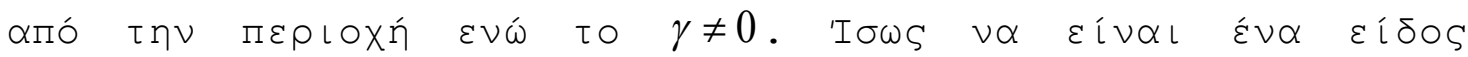

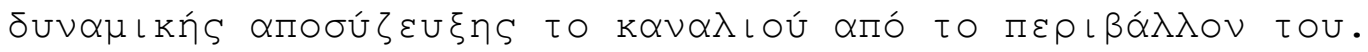

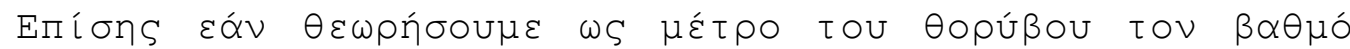

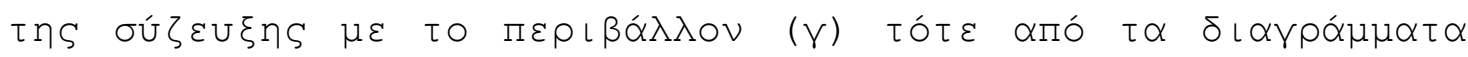

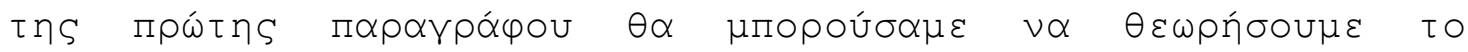

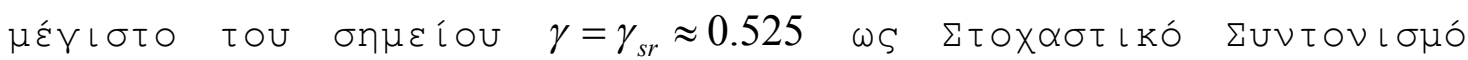
$[1]$.

\section{$\Pi \alpha \rho \alpha ́ \rho \tau \eta \mu \alpha$.}

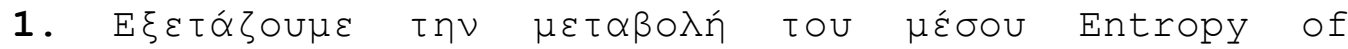

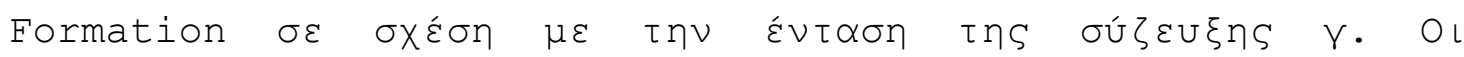

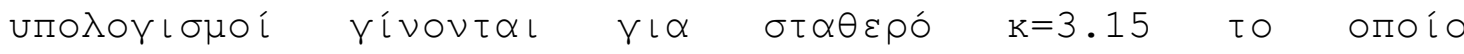

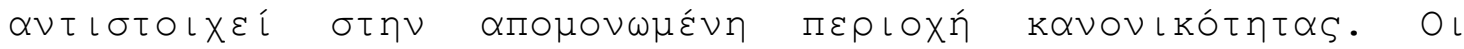

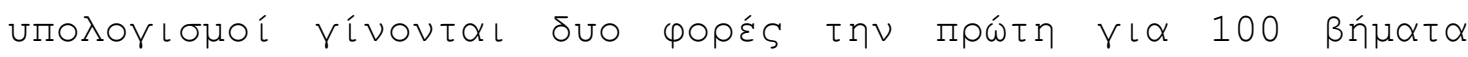

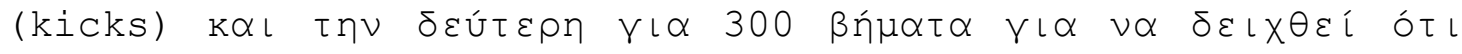

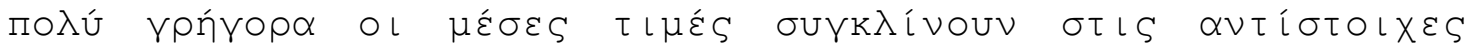

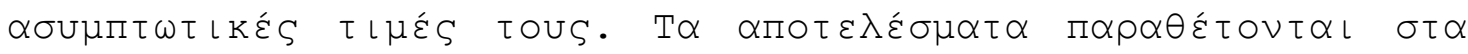

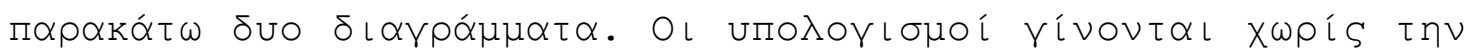

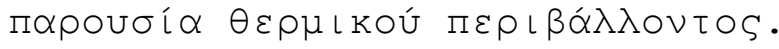




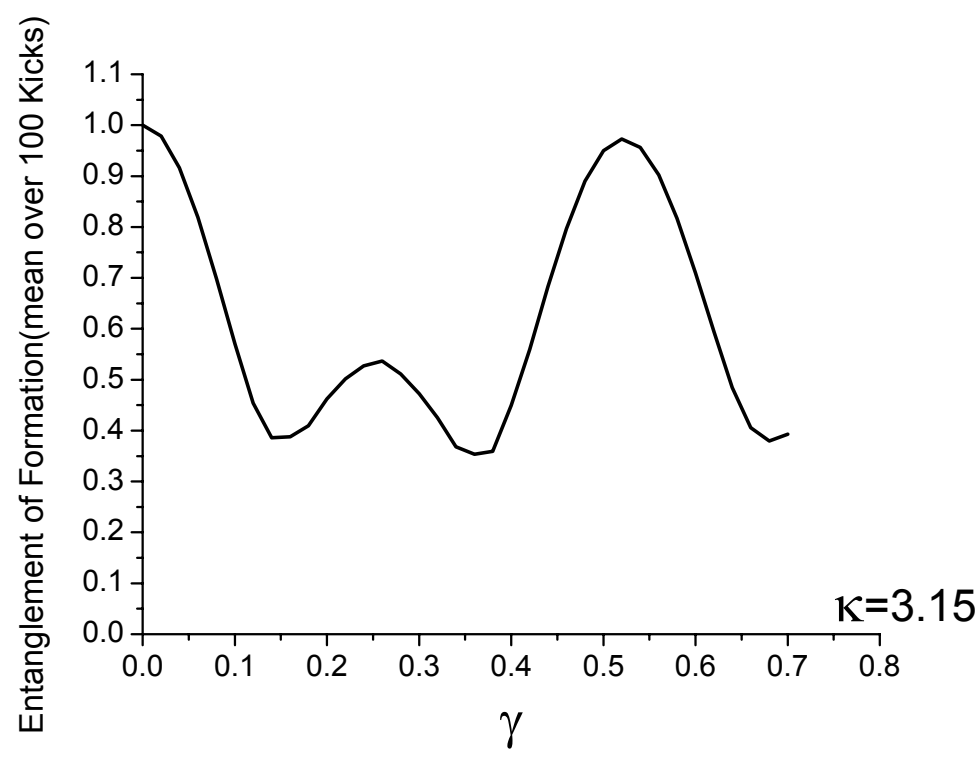

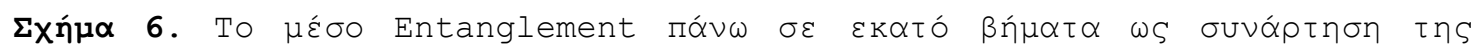

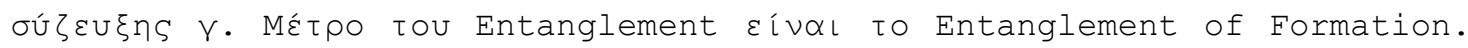
$\kappa=3.15$

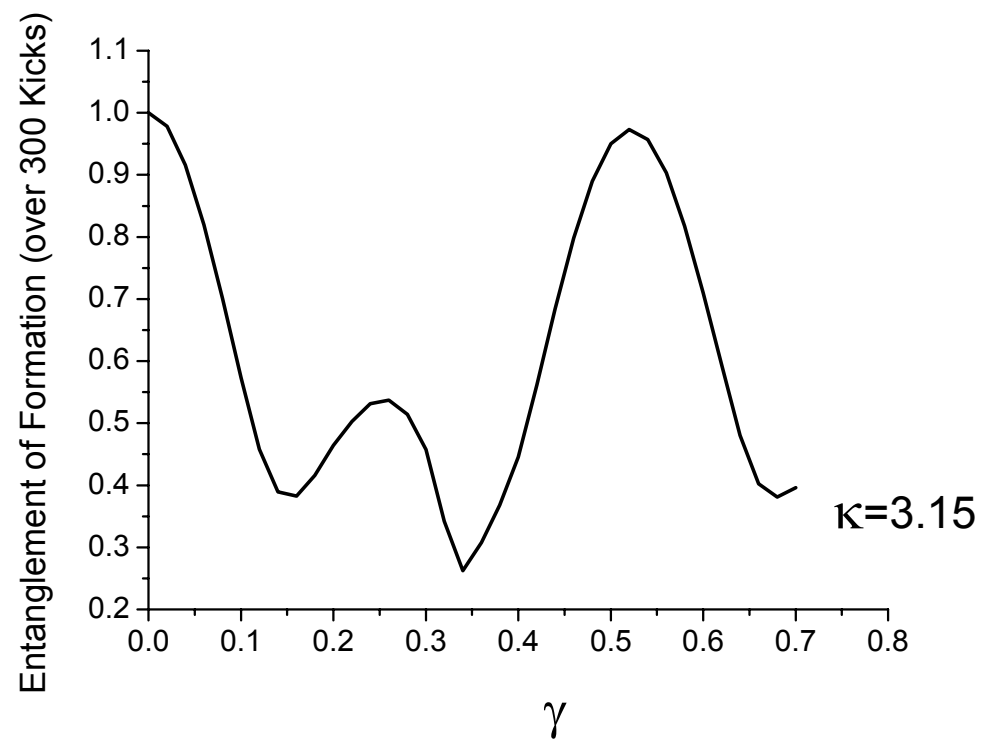

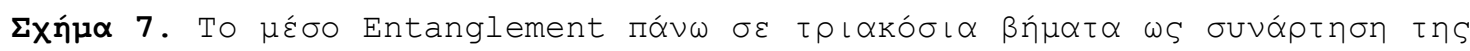

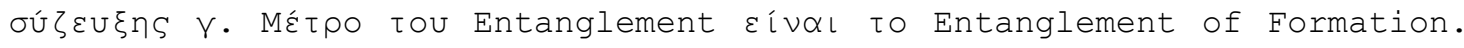
$\kappa=3.15$ 


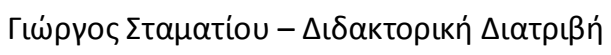

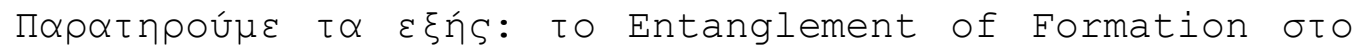

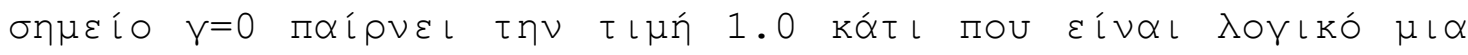

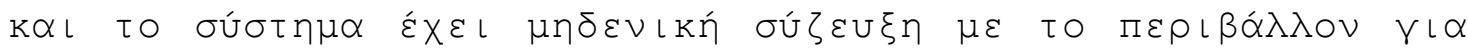

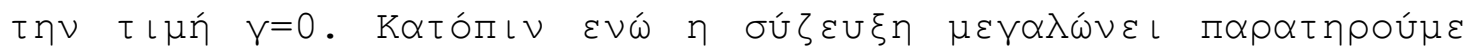

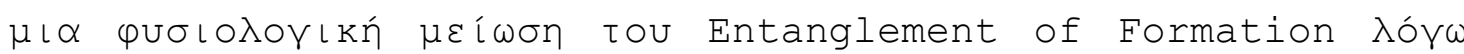

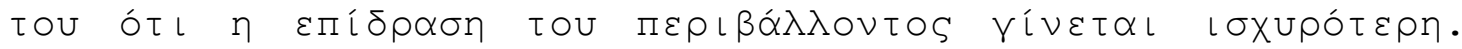

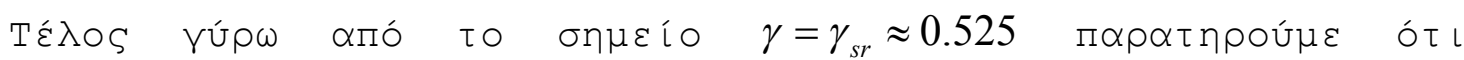

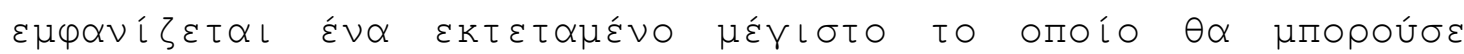

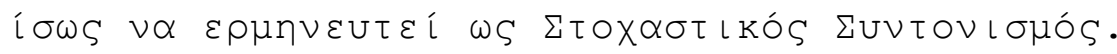

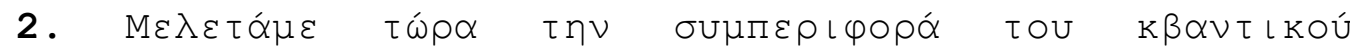

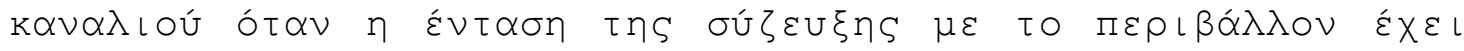

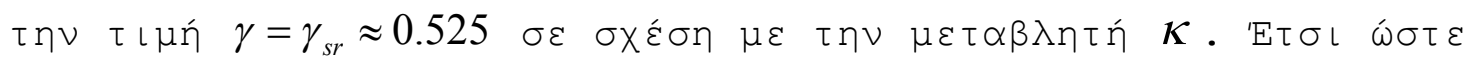

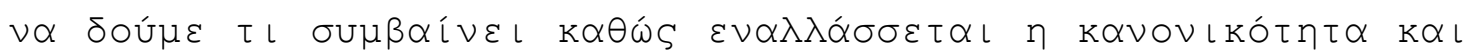

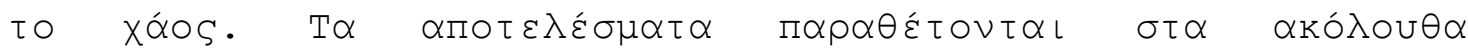

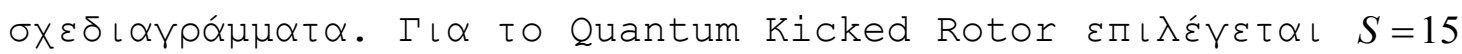

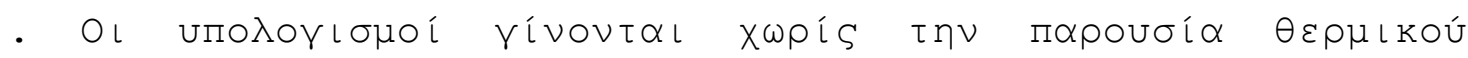

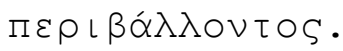

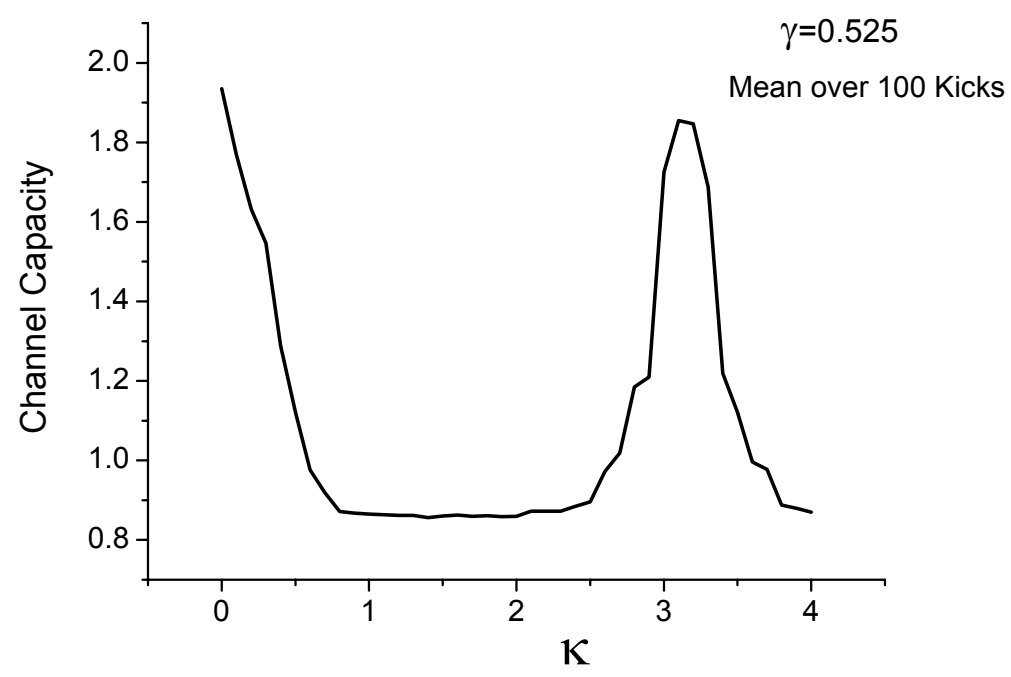

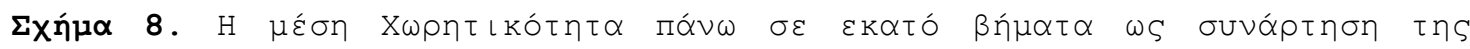

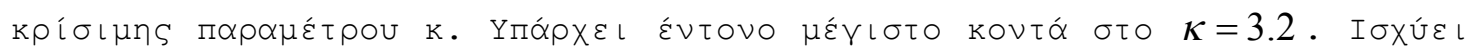
$\gamma=0.525$ 


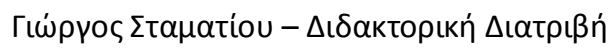

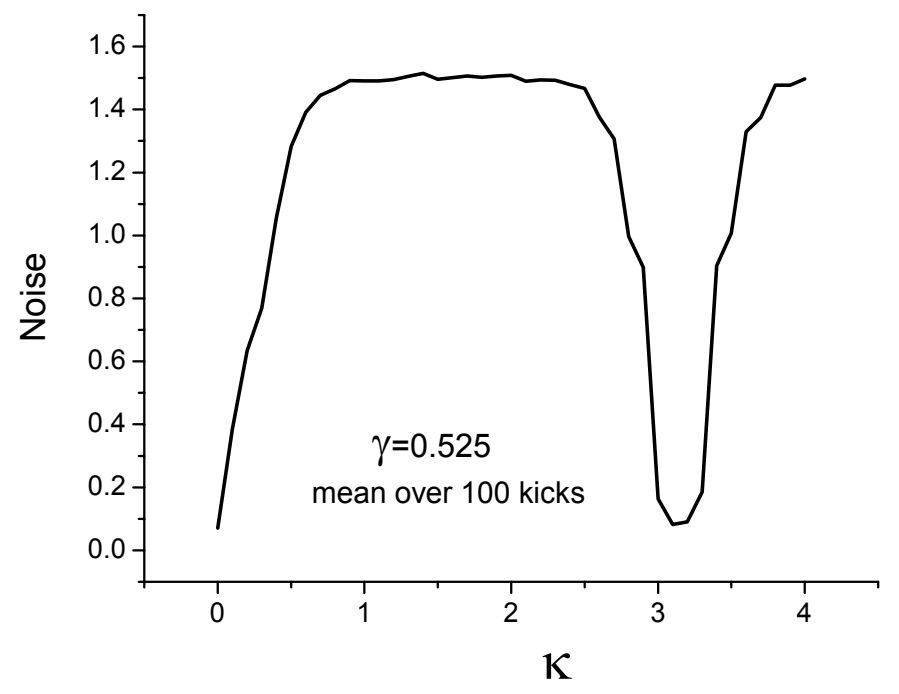

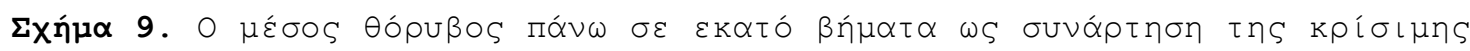

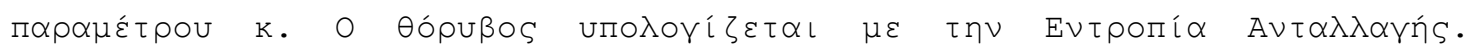

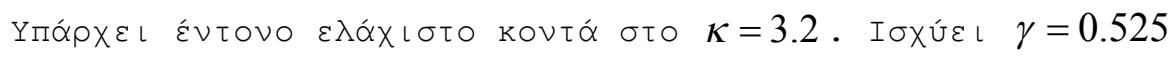

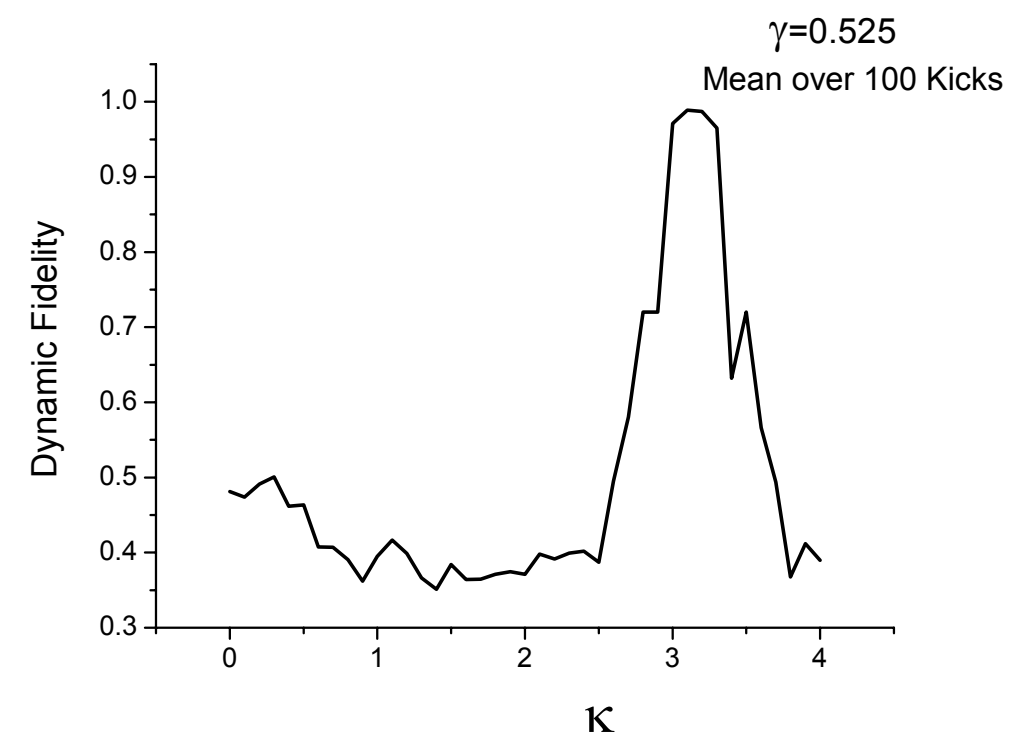

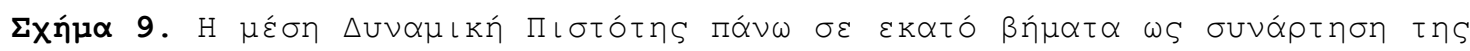

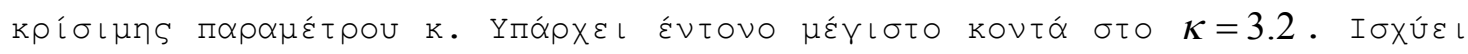
$\gamma=0.525$ 


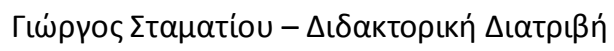

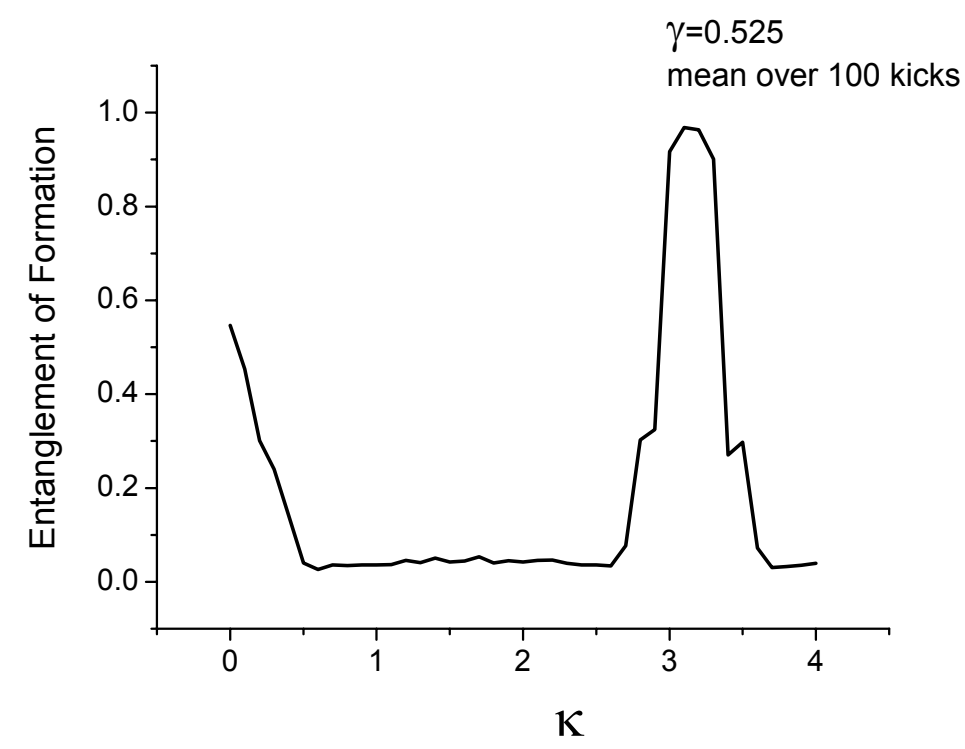

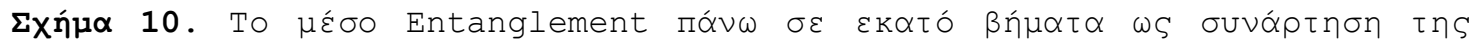

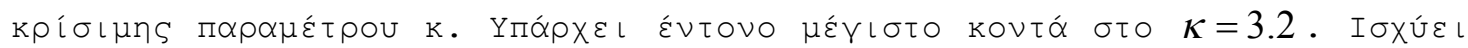
$\gamma=0.525$ 


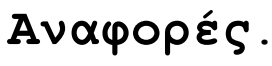

[1]. Stochastic resonance, L Gammaitoni, P Hänggi, P Jung, F Marchesoni - Reviews of Modern Physics, 1998, vol. 70, issue $1,223-287$

[2]. Energy transport in the integrable system in contact with various types of phonon ..., K Saito, $S$ Takesue, $S$ Miyashita - Physical Review E, 2000, vol. 61, issue 3, 2397-2409

[3]. G.Casati, B.V.Chirikov, J.Ford and F.M.Izrailev, Lect.NotesPhys.93, 334, 1979. F. M. Izrailev, Phys.Rep. $196,299,1990$

[4]. Martin C. Gutzwiller, Chaos in Classical and Quantum Mechanics, (1990) Springer-Verlag, New York ISBN: 0-38797173-4.

[5]. Effects of the environment on nonadiabatic magnetization process in uniaxial ..., K Saito, S Miyashita, H De Raedt - Physical Review B, 1999, vol. 60, issue 21, $14553-14556$

[6]. Entanglement of Formation, WK Wootters - Quantum Communication, Computing, and Measurement 2-Springer, 2000, ISBN : 978-0-306-46307-5, 69-73

[7]. Entanglement of formation of an arbitrary state of two qubits, WK Wootters - Physical Review Letters, 1998

[8]. Capacity of the noisy quantum channel, S Lloyd Physical Review A, 1997, vol. 55, issue 3, 1613-1622

[9]. Quantum Computation and Quantum Information, MA Nielsen, IL Chuang, Cambridge University Press, 2000, ISBN: $0-521-63503-9$

[10]. Method for modeling decoherence on a quantuminformation processor, G Teklemariam, EM Fortunato, CC Lopez, J Emerson, ... - Physical Review A, 2003, vol. 67, issue 6, 062316 


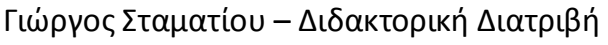




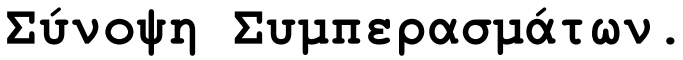

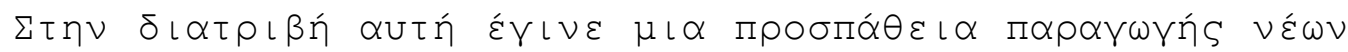

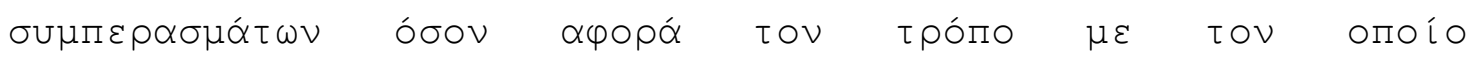

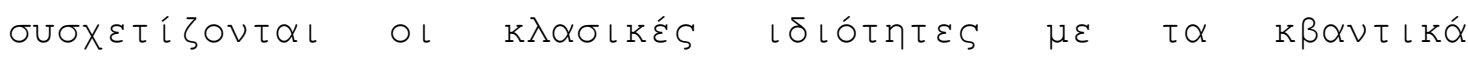

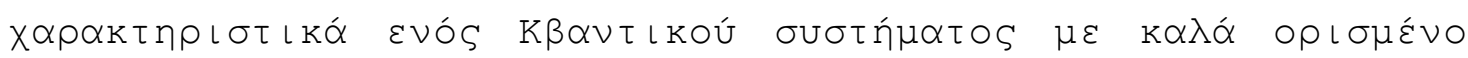

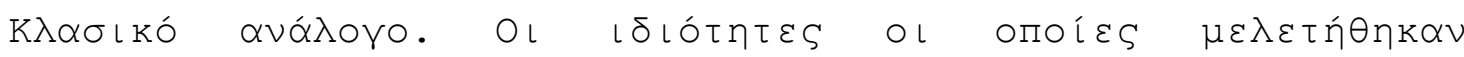

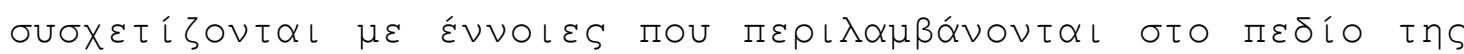

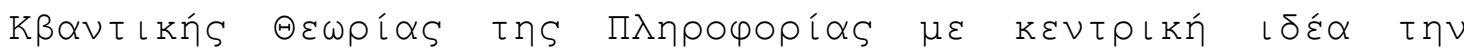

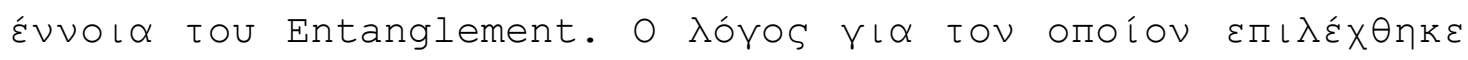

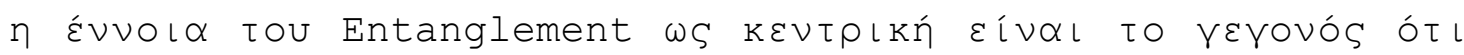

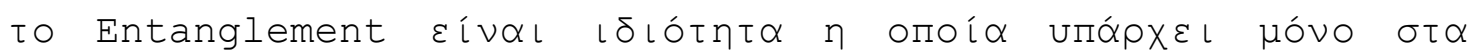

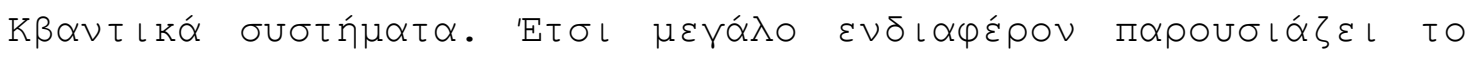

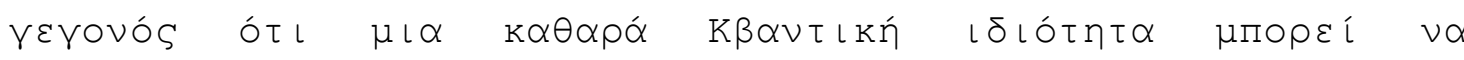

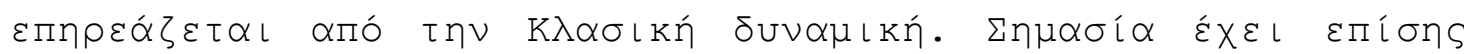

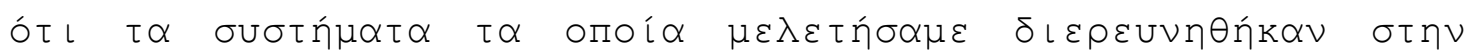

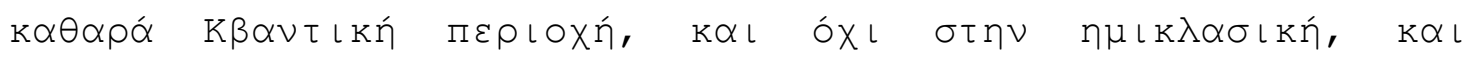

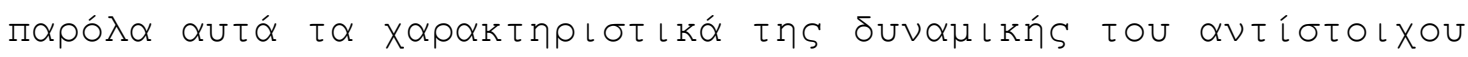

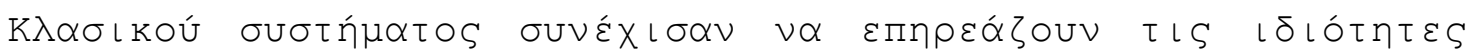

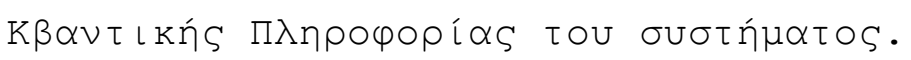

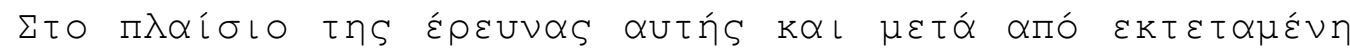

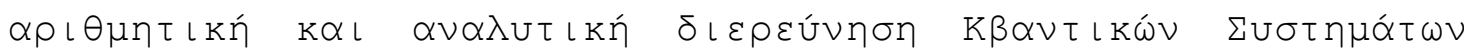

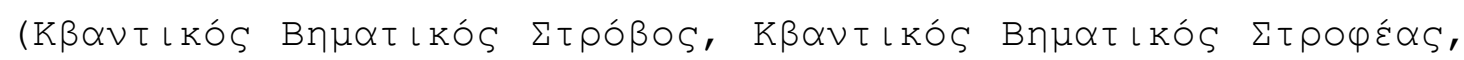

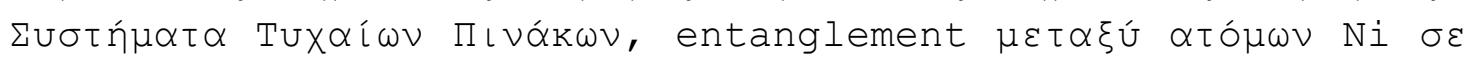

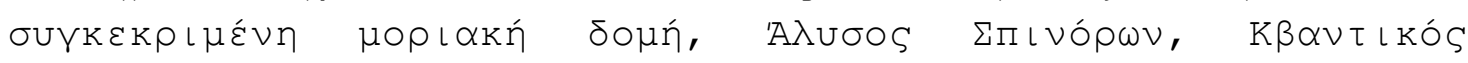

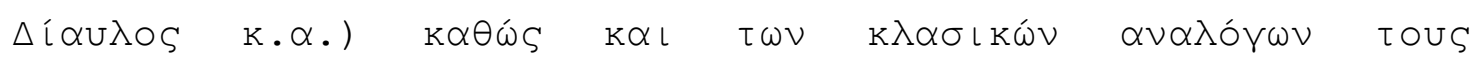

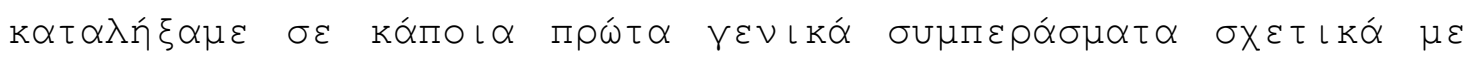

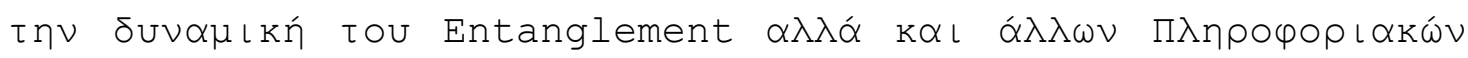

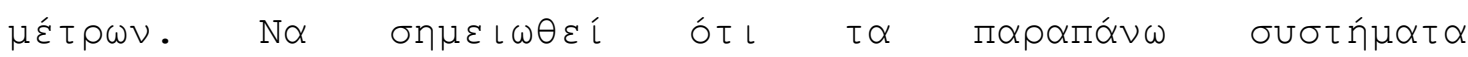

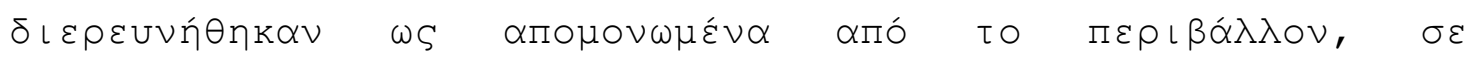

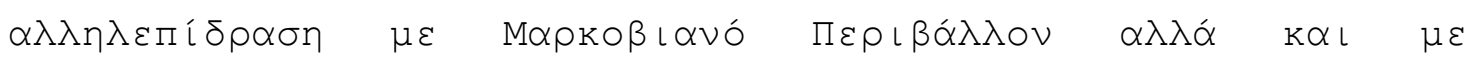

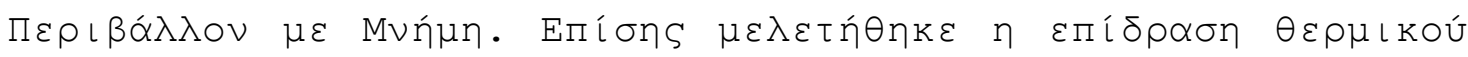

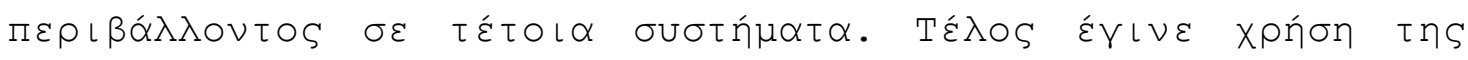

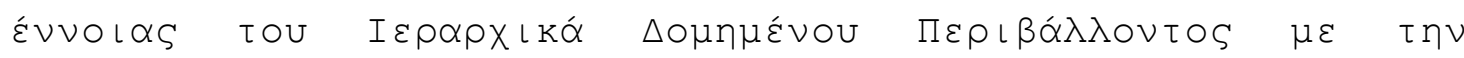

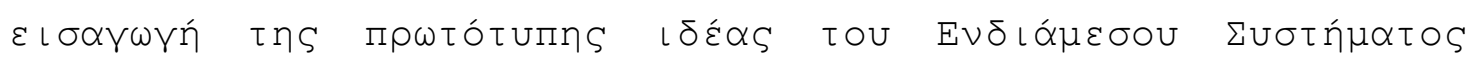
(Host System). 


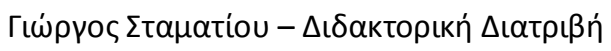

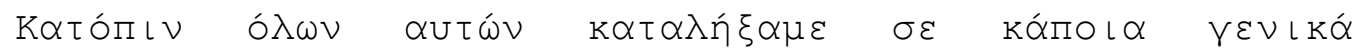

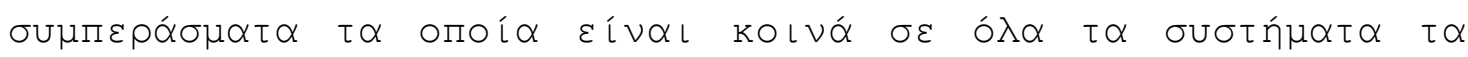

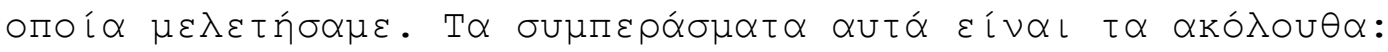

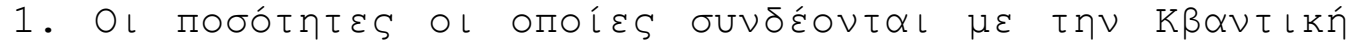

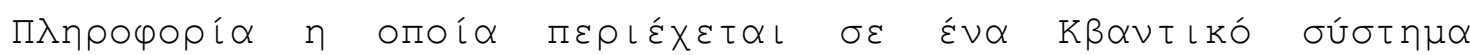

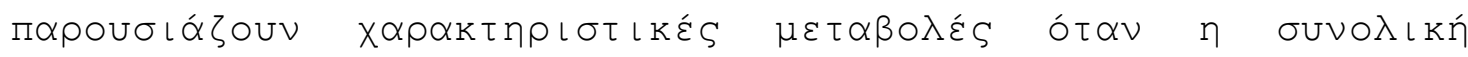

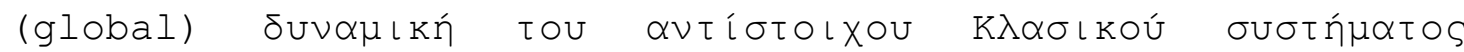

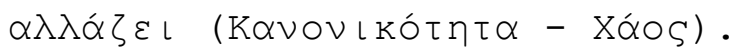

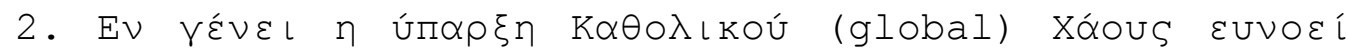

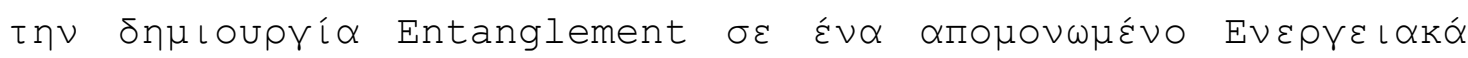

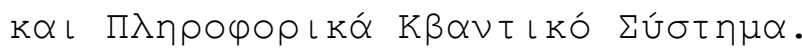

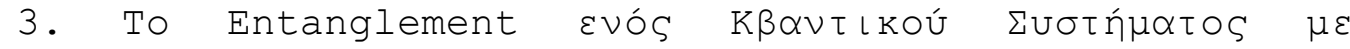

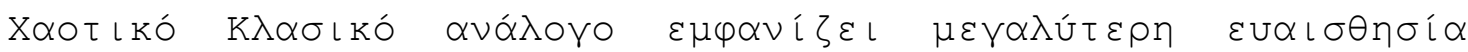

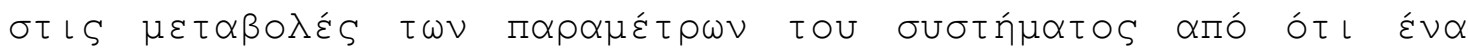

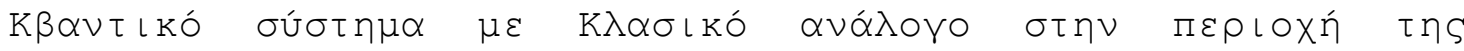

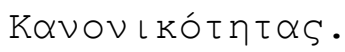

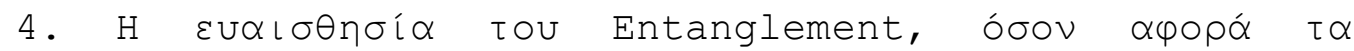

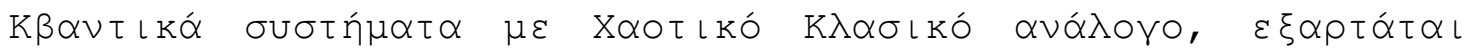

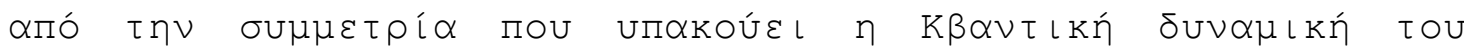

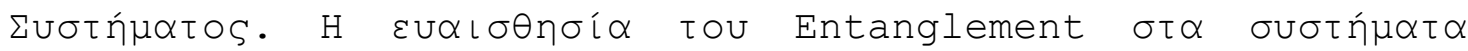

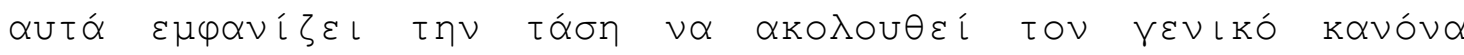

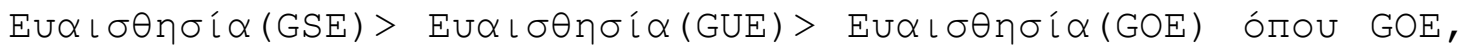

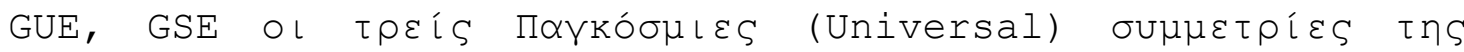

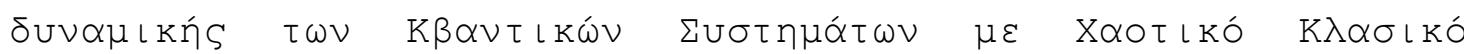

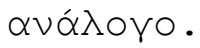

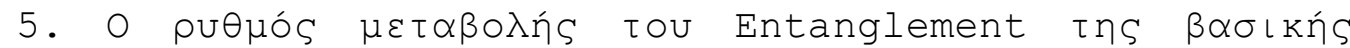

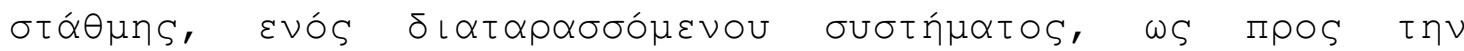

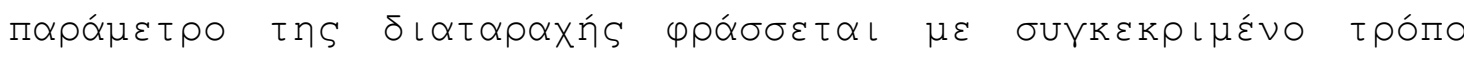

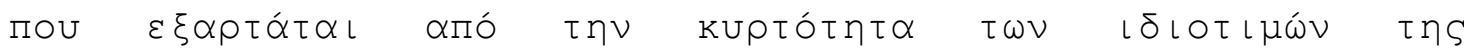
$\varepsilon \vee \varepsilon ́ \rho \gamma \varepsilon\llcorner\alpha \varsigma$.

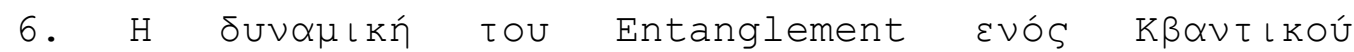

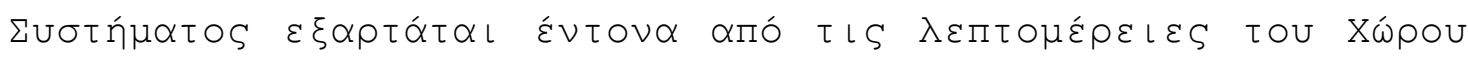

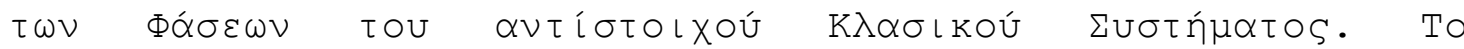

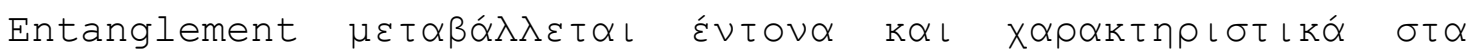

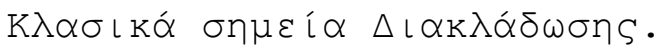




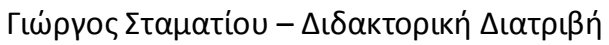

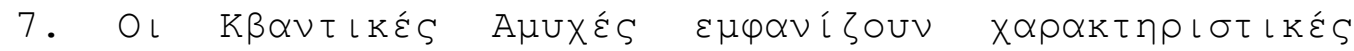

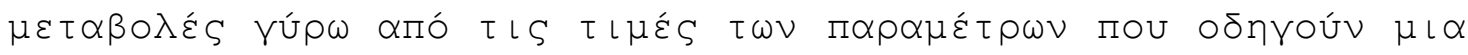

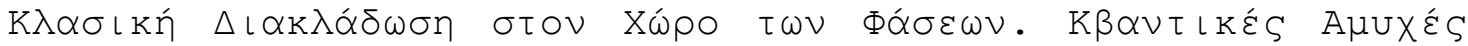

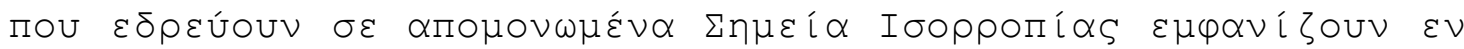

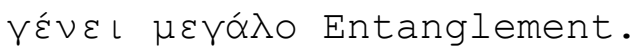

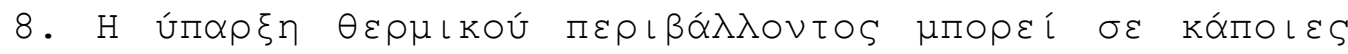

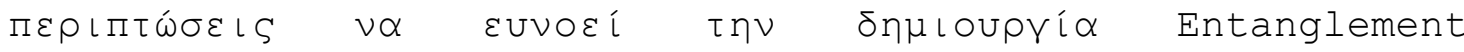

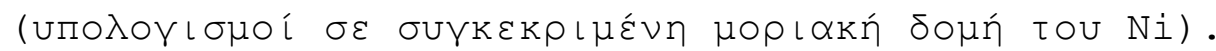

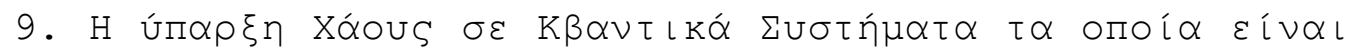

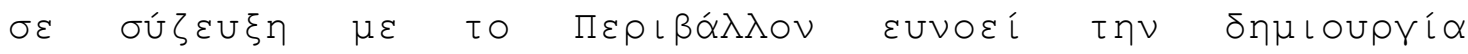

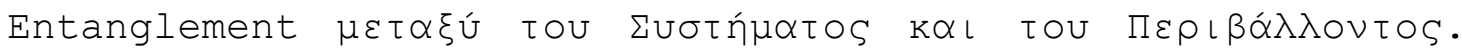

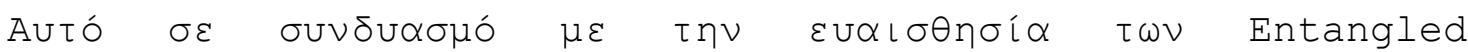

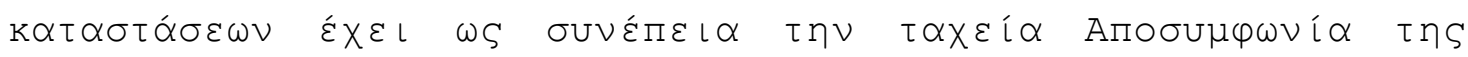

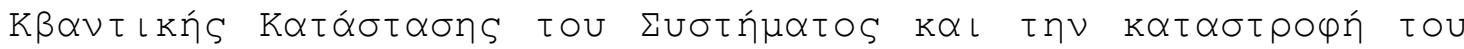

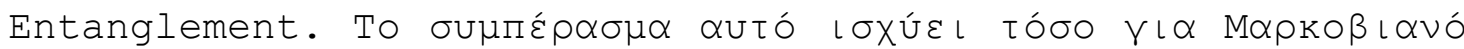

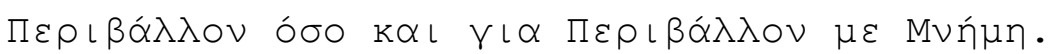

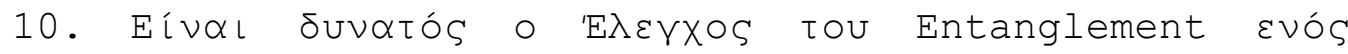

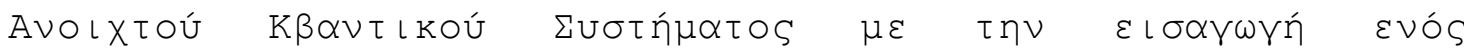

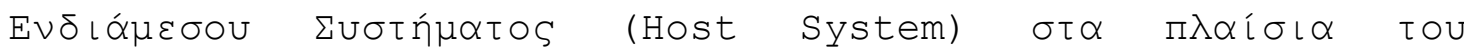

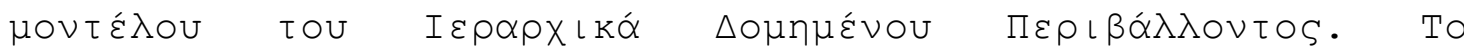

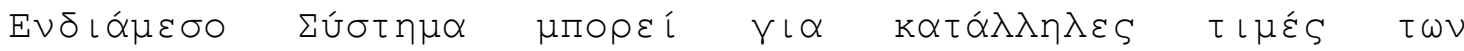

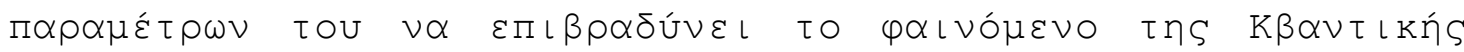

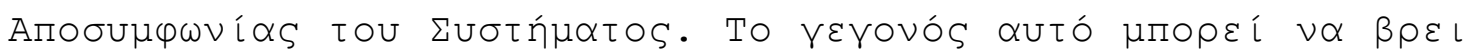

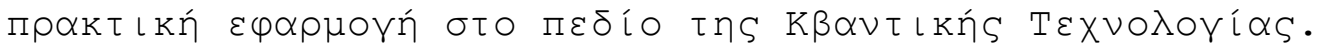

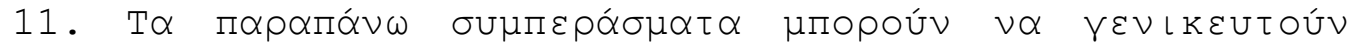

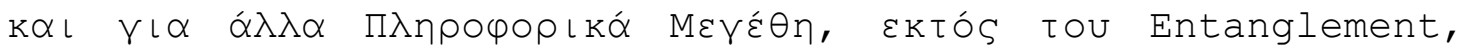

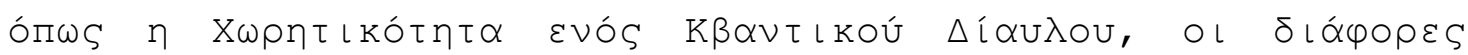

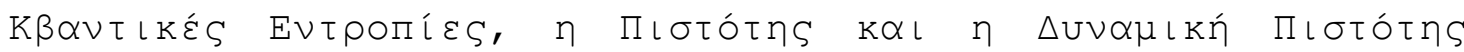

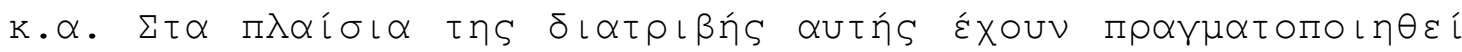

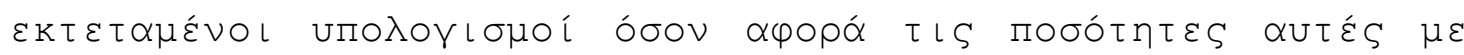

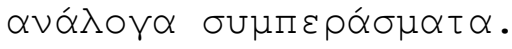

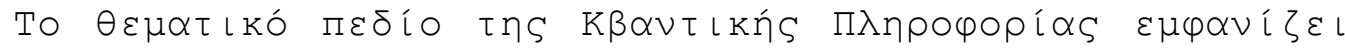

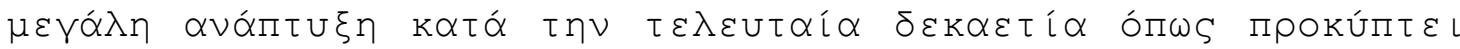

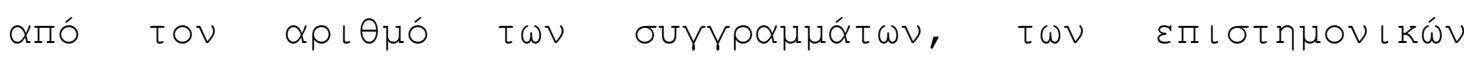

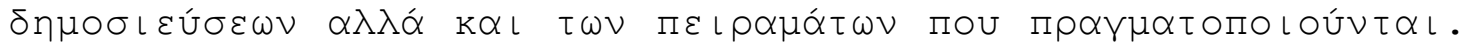

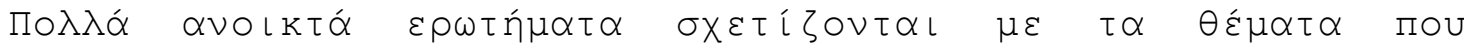

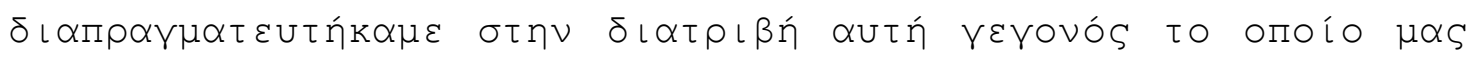




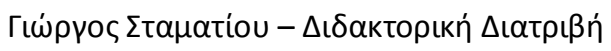

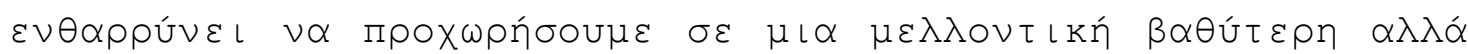

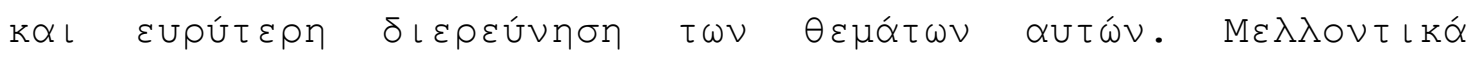

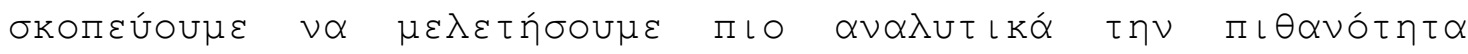

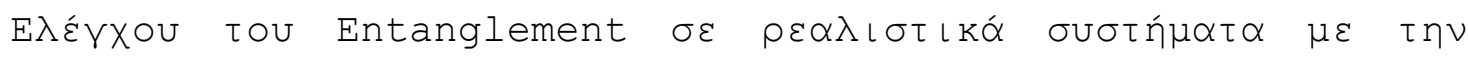

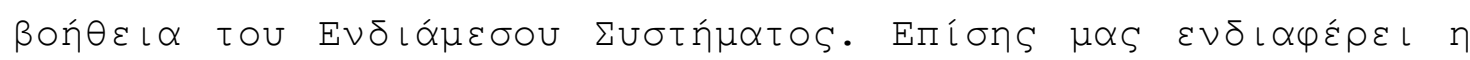

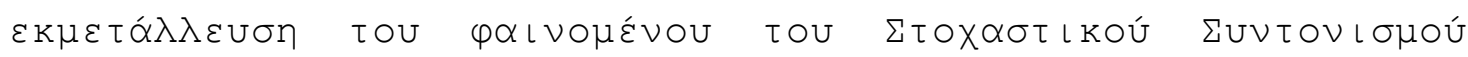

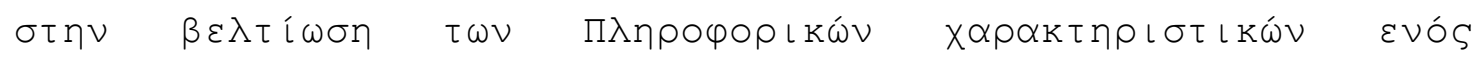

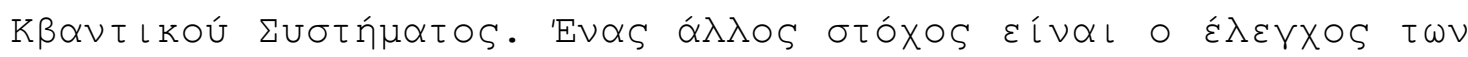

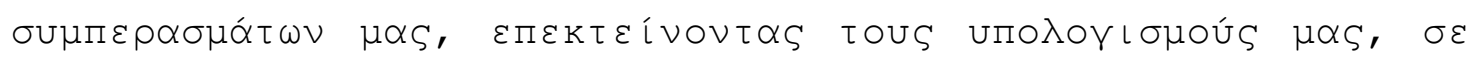

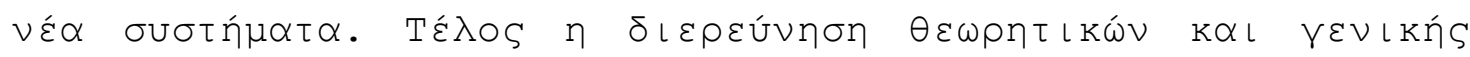

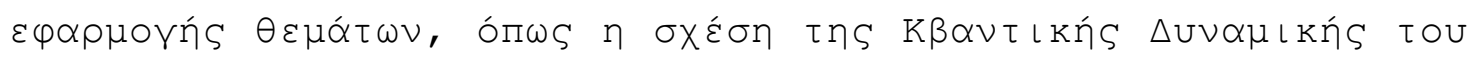
Entanglement $\mu \varepsilon$ to

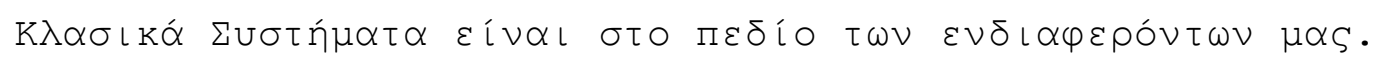

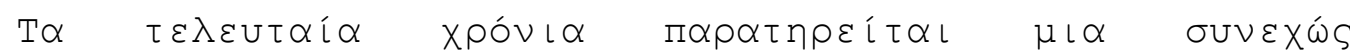

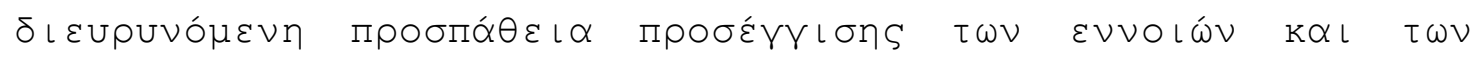

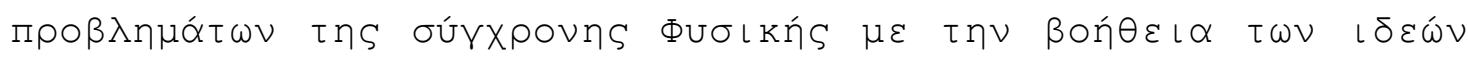

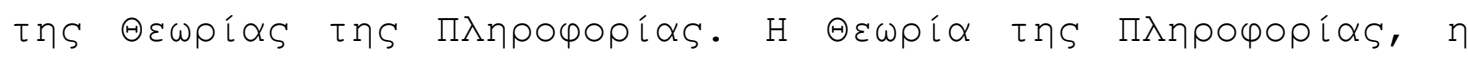

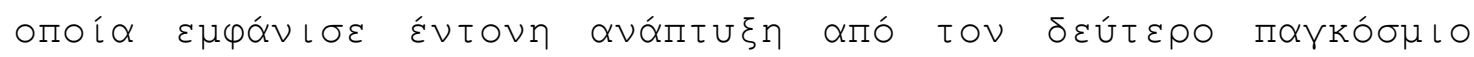

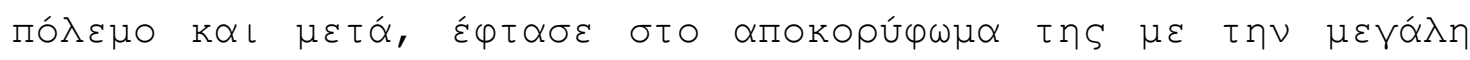

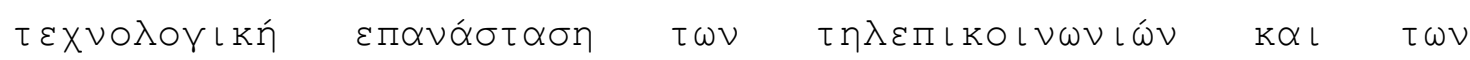

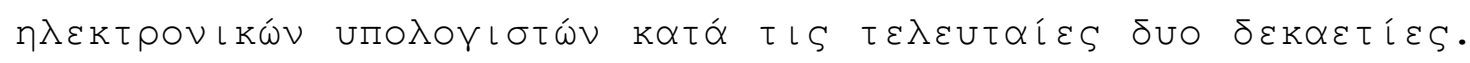

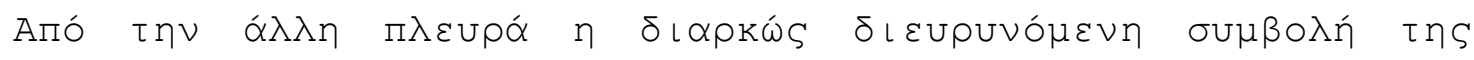

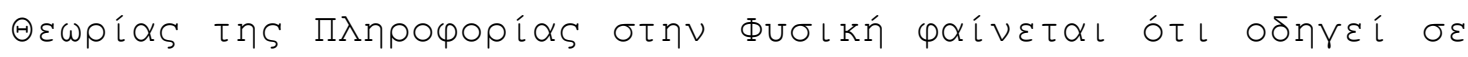

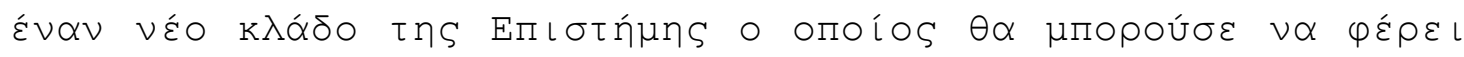

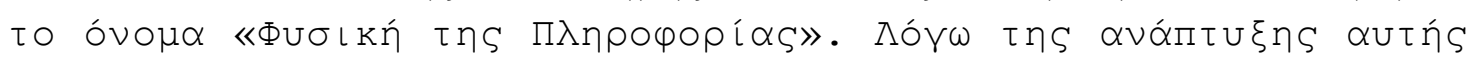

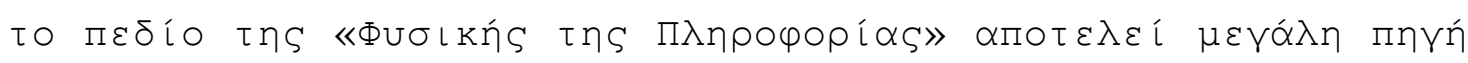

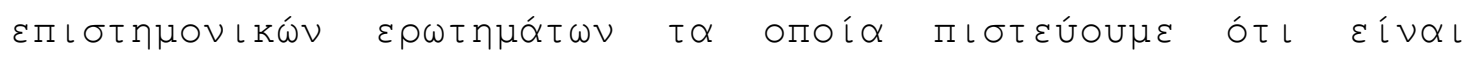

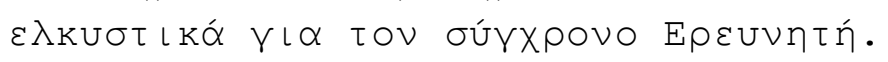




\title{
ПАРАРТНMA - ФЕМАТIKH ВIВАIOГРАФІA,

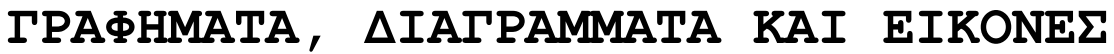

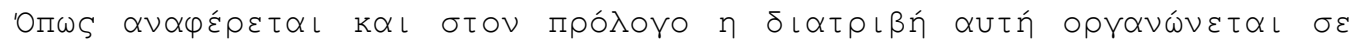

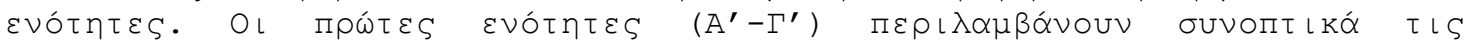

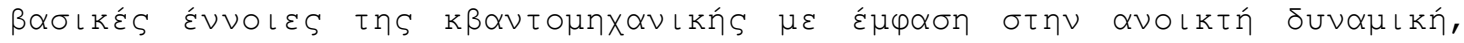

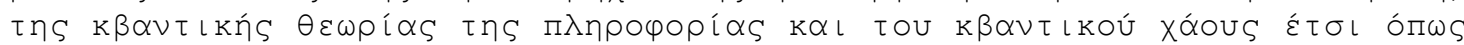

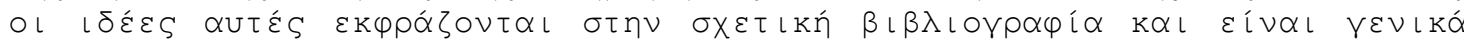

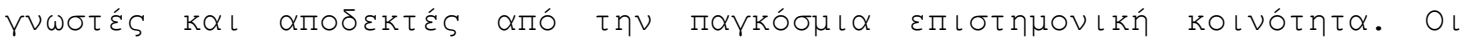

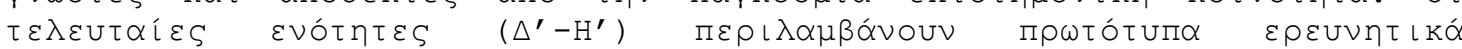

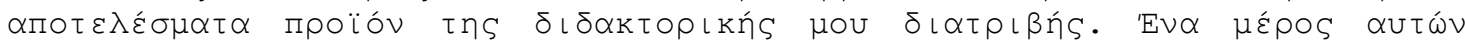

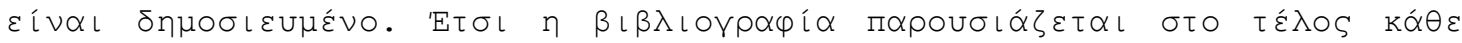
$\varepsilon \vee o ́ t \eta \tau \alpha s$.
\end{abstract}

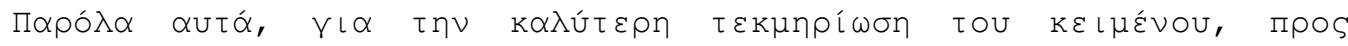

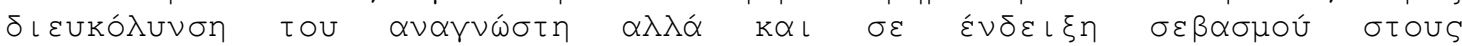

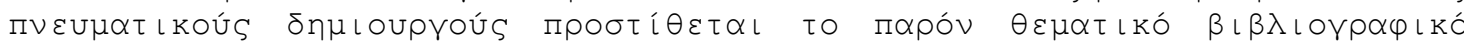

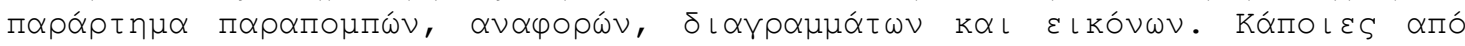

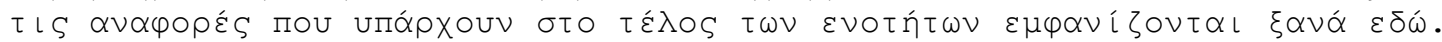

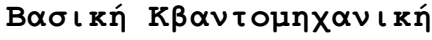

-Quantum theory, D.Bohm, Prentice-Hall, 1951

-The principles of quantum mechanics, PAM Dirac, Oxford University

Press, 1981

-Mathematical foundations of quantum mechanics, J Von Neumann,

Princeton University Press, 1996

-Quantum Mechanics Non-Relativistic Theory, L. D. Landau, L. M. Lifshitz, Butterworth-Heinemann, 1981

-Understanding quantum mechanics, R Omnès, Princeton University Press, 1999

-Quantum Mechanics, C Cohen-Tannoudji, B Diu, F Laloë, S Hemley, Wiley Interscience, 1977

-Quantum Mechanics, Albert Messiah, North-Holland, 1961

-Quantum Mechanics, L. I. Schiff, Mcgraw-Hill College, 1968

-A Modern Approach to Quantum Mechanics, Townsend, John S. 2nd ed..

Sausalito, CA: University Science Books, 2000

-Quantum Theory: Concepts and Methods, A. Peres, Kluwer Academic

Publishers, 1993

-Quantum Mechanics, Franz Mandl, Wiley, 1992

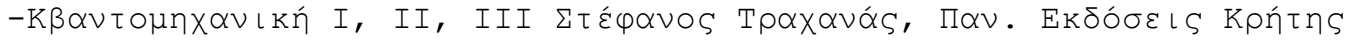

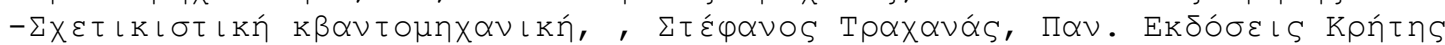

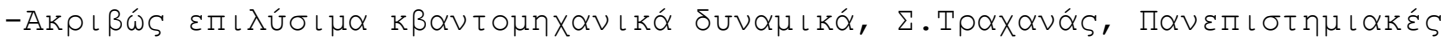

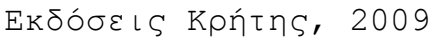

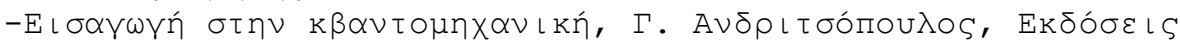

$\Pi \alpha \Pi \alpha \sigma \omega \tau \eta \rho$ íou.

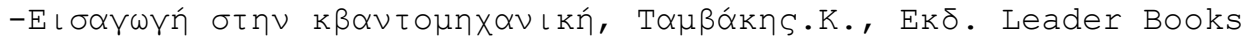

-Quantum Mechanics in Phase Space: An Overview with Selected Papers, Cosmas K. Zachos (Editor), David B. Fairlie (Editor), Thomas L.

Curtright (Editor), World Scientific,2005

-Phase space representation of quantum dynamics, Anatoli Polkovnikov, http://arxiv.org/abs/0905.3384v2, 2010

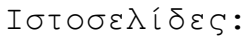

-http://en.wikipedia.org/wiki/Introduction_to_quantum_mechanics

-http://www. quantiki.org/

-http://en.wikipedia.org/wiki/Quantum_mechanics 
-Phase space QM

http://en.wikipedia.org/wiki/Wigner_quasi-probability_distribution

http://en.wikipedia.org/wiki/Husimi_Q_representation

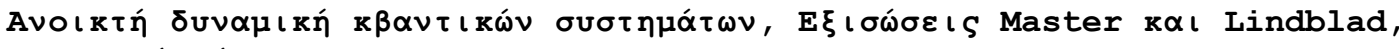

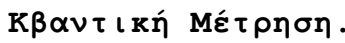

- Irreversible Quantum Dynamics (Lecture Notes in Physics), Fabio Benatti (Editor), Roberto Floreanini (Editor), Springer, 2003

-Quantum Dynamical Semigroups and Applications, Alicki R., Lendi K. Berlin: Springer Verlag, 1987.

- The Theory of Open Quantum Systems, Breuer, Heinz-Peter F.

Petruccione, Oxford University Press 2007

-Density matrix theory and applications, K Blum, Springer, 1996

-States, Effects, and Operations, K.Kraus, Lecture Notes in Physics 190, Springer, 1983.

-Open Quantum Systems I-III, Stéphane Attal, Alain Joye, Claude-Alain Pillet, Lecture Notes in Mathematics, Springer

-Quantum Computation and Quantum Information, M. Nielsen and I. Chuang, Cambridge University Press, 2000

-Quantum Theory and Its Stochastic Limit., Accardi L., Lu Y.G., Volovich I.V., New York: Springer Verlag, 2002.

-Quantum Mechanics of Non-Hamiltonian and Dissipative Systems, Tarasov V.E., Amsterdam, Boston, London, New York: Elsevier Science, 2008. -Quantum Dissipative Systems, Weiss U. Singapore, World Scientific, 1993

-Quantum state diffusion, I. Persival, Cambridge University Press, 1999 -Quantum Noise, C. W. Gardiner, Springer,2004

-Handbook of Stochastic Methods: for Physics, Chemistry and the Natural Sciences, C.Gardiner, Springer-Verlag, 1983

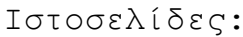

-http://en.wikipedia.org/wiki/Lindblad equation

-http://en.wikipedia.org/wiki/Quantum_operation

-http://en.wikipedia.org/wiki/Open_quāntum system

-http://en.wikipedia.org/wiki/Quantum_dissipation

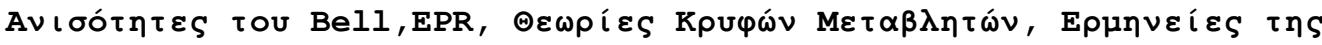

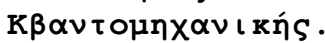

-J.S. Bell, Physics 1, 195-200, 1964

- Experimental Tests of Realistic Local Theories via Bell's Theorem, Aspect, 1981: A. Aspect et al., Phys. Rev. Lett. 47, 460, 1981 -Can Quantum-Mechanical Description of Physical Reality Be Considered Complete?, Einstein A., Podolsky B., Rosen, N., Physical Review 47 (10): 777-780,1935

-The Undivided Universe:An Ontological Interpretation of Quantum Theory, David Bohm, Basil J. Hiley, publisher: Routledge, 1993 -Derivation of the Schrödinger Equation from Newtonian Mechanics, Edward Nelson, Physical Review 150: 1079-1085, 1966

-Mathematical and Physical Aspects of Stochastic Mechanics, Ph. Blanchard, $\mathrm{Ph}$. Combe, W. Zheng, Lecture notes in physics, Springer, 1987 -Entanglement, Information, and the Interpretation of Quantum Mechanics, Jaeger, Gregg, Springer, 2009

-Bell's inequalities and density matrices. Revealing hidden nonlocality, Sandu Popescu, http://xxx.lanl.gov/abs/quant-ph/9502005, 1995

-Correlations, Bell Inequality Violation \& Quantum Entanglement, YeongCherng Liang, http://arxiv.org/abs/0810.5400,2008 


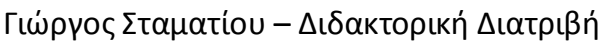

-Research on Hidden Variable Theories: a review of recent progresses, Marco Genovese, http://arxiv.org/abs/quant-ph/0701071,2007

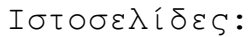

-http://en.wikipedia.org/wiki/Bell_test_experiments

-http://en.wikipedia.org/wiki/Hidden_variable_theory

-http://en.wikipedia.org/wiki/User:Caroline Th̆ompson/Bell's theorem

-http://en.wikipedia.org/wiki/Interpretations_of_quantum_mechanics

-http://en.wikipedia.org/wiki/CHSH_inequality

-http://en.wikipedia.org/wiki/De_Br̄oglie-Bohm_theory

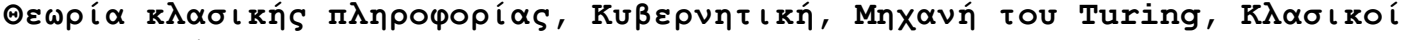

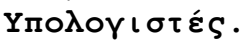

-Elements of information theory, TM Cover, JA Thomas, Wiley, 2006

-Information Science, David G. Luenberger, Princeton University Press, 2006

-An Introduction to Information Theory, Fazlollah M. Reza, Dover Books -Information Theory, Robert B. Ash, Dover books on mathematics

-The Mathematical Theory of Communication, Claude E Shannon, Warren Weaver, ISBN-10: 0252725484, 1971

-Cybernetics, Second Edition: or the Control and Communication in the Animal and the Machine, Norbert Wiener, The MIT Press, 1965

-An Introduction to Information Theory: Symbols, Signals and Noise, John R. Pierce, Dover Publications, 1980

-Mathematical Foundations of Information Theory, A. Ya. Khinchin, Dover, 1957

-Entropy and Information Theory, Robert M. Gray, 1990

-Information Theory and Statistics, Solomon Kullback, Dover, 1997

-The Universal Computer: The Road from Leibniz to Turing, Martin Davis, Publisher: W. W. Norton \& Company, 2000

-The Universal Turing Machine: A Half-Century Survey, editor: Rolf Herken, Springer, 1995

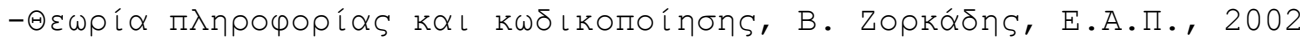

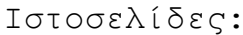

-http://en.wikipedia.org/wiki/Theory of computation

-http://en.wikipedia.org/wiki/Information theory

-http://en.wikipedia.org/wiki/Turing machine

-http://en.wikipedia.org/wiki/Logic_gate

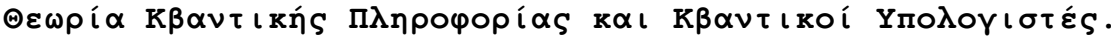

-Quantum Computation and Quantum Information, M.Nielsen and I. Chuang, Cambridge University Press, 2000

-Einstein, Bohr and the Quantum Dilemma: From Quantum Theory to Quantum Information, Andrew Whitaker, Cambridge University, 2006

-Quantum information and computation, Lecture notes for Physics 229, J.Preskill, California Institute of Technology, 1998

-Quantum Information: An Introduction to Basic Theoretical Concepts and Experiments, G. Alber et al., Springer, 2001.

-Introduction to Quantum Computation and Information, H.K. Lo, T. Spiller, S.Popescu, World Scientific, 1998

-Quantum information theory, M. A. Nielsen, PhD Dissertation, The University of New Mexico, 1998

-Lectures on Quantum Information, Dagmar Bruß and Gerd Leuchs(editors), Wiley-VCH Verlag, 2007

-Principles of Quantum Computation and Information, Vol.1: Basic Concepts, Giuliano Benenti, Giulio Casati, Giuliano Strini, World Scientific Pub Co Inc, 2004 


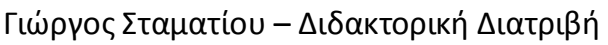

-Principles of Quantum Computation And Information: Basic Tools And Special Topics, Giuliano Benenti, Giulio Casati, Giuliano Strini, World Scientific Publishing Company, 2007

-Elementary gates for quantum computation, A. Barenco, C. H. Bennett , R. Cleve, D. P. DiVincenzo, N. Margolus, P. Shor, T. Sleator, J. Smolin,H. Weinfurter, http://xxx.lanl.gov/abs/quant-ph/9503016, 1995 -Quantum Information Theory and Quantum Statistics, Dénes Petz, Springer, Berlin Heidelberg,2008

-Quantum Computing, Andrew Steane, http://xxx.lanl.gov/abs/quant$\mathrm{ph} / 9708022,1997$

-Entanglement Measures and Purification Procedures, V. Vedral, M.B. Plenio, http://xxx.lanl.gov/abs/quant-ph/9707035, 1997

-Quantum Computer Science, N. D. Mermin, Cambridge, 2007

-An Introduction to Quantum Computing, Phillip Kaye, Raymond Laflamme, Michele Mosca, oxford university press, 2007

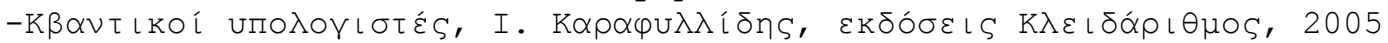

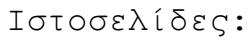

-http://en.wikipedia.org/wiki/Quantum information

-M. A. Nielsen's Phd: http://arxiv.org/abs/quant-ph/0011036

-http://en.wikipedia.org/wiki/Quantum_computer

-http://en.wikipedia.org/wiki/Qubit

-http://en.wikipedia.org/wiki/Quantum_gate

-http://en.wikipedia.org/wiki/Quantum_teleportation

-http://en.wikipedia.org/wiki/Quantum_channel

-http://en.wikipedia.org/wiki/Quantum error correction

-J.Preskill's page, http://www.theory.caltech.edu/ preskill/

-J.Prskill's lecture notes, problems and solutions

-http://www. theory.caltech.edu/people/preskill/ph229/\#lecture

-http://en.wikipedia.org/wiki/Quantum_entanglement

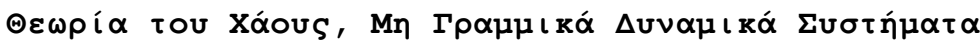

-Differential Equations and Dynamical Systems, Lawrence Perko, Springer, 2001

-Hamiltonian Chaos and Fractional Dynamics, George M. Zaslavsky, Oxford University Press, USA, 2008

-Chaos in Classical and Quantum Mechanics, Martin C.

Gutzwiller, Springer-Verlag, New York, 1990

-Classical and Quantum Chaos, Predrag Cvitanovic, Roberto Artuso, Freddy Christiansen, Per Dahlqvist, Ronnie Mainieri, Hans Henrik Rugh, Gregor Tanner, G'abor Vattay, Niall Whelan, Andreas Wirzba, version 7.0.1, www.nbi.dk/ChaosBook/, 2000

-Nonlinear Oscillations, Dynamical Systems, and Bifurcations of Vector Fields, John Guckenheimer, Philip Holmes, SPRINGER-VERLAG NEW YORK INC, 2002

-Introduction to the Modern Theory of Dynamical Systems, Anatole Katok, Boris Hasselblatt, Cambridge University Press, 1996

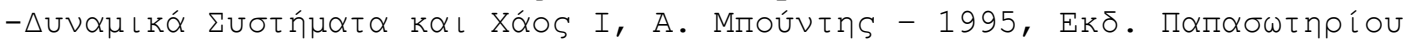

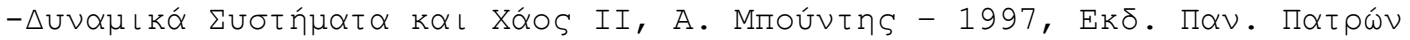

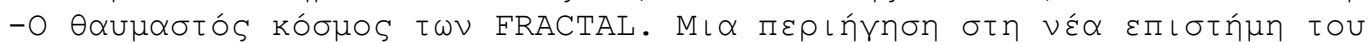

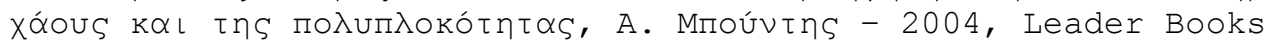

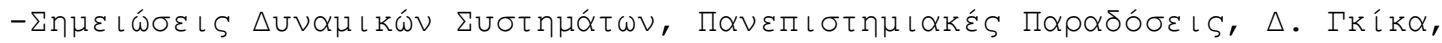

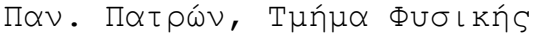

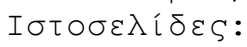

-http://en.wikipedia.org/wiki/Chaos_theory

-http://chaosbook.org/

-http://en.wikipedia.org/wiki/Dynamical_system

-http://en.wikipedia.org/wiki/Bifurcation_theory 


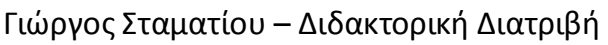

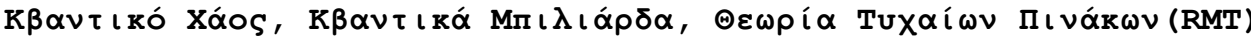

-Chaos in Classical and Quantum Mechanics, Martin C. Gutzwiller, Springer-Verlag, New York, 1990

-Quantum Signatures of Chaos, Fritz Haake, Edition: 2, Springer, 2001 -Quantum versus Chaos: Questions Emerging from Mesoscopic Cosmos, K. Nakamura, Springer, 1997

-Quantum Chaos and Mesoscopic Systems: Mathematical Methods in the Quantum Signatures of Chaos, N.E. Hurt, Springer, 1997

-Quantum Chaos: An Introduction, Stöckmann Hans-Jürgen, Cambridge University Press, 1999

-Dissipative Quantum Chaos and Decoherence, Daniel Braun, Springer, 2001

-Quantum chaos: a new paradigm of nonlinear dynamics, K.Nakamura, Cambridge University Press, 1993

-Quantum Chaos: Between Order and Disorder, Giulio Casati, Boris Chirikov, Cambridge University Press, 2006

-The Transition to Chaos: Conservative Classical Systems and Quantum Manifestations, Linda E Reichl, Springer, 2004

-Random Matrix Theories in Quantum Physics: Common Concepts, Thomas Guhr, Axel Muller-Groeling, Hans A. Weidenmuller, http://lanl.arxiv.org/abs/cond-mat/9707301v1,1997

-Introduction to random matrices. Geometric and quantum aspects of integrable systems, Tracy C. and Widom H.,103-130, Lecture Notes in Phys., 424, Springer, Berlin, 1993

-An Introduction to Random Matrices, Greg W. Anderson, Alice Guionnet, Ofer Zeitouni, Cambridge University Press, 2009

-Applications of Random Matrices in Physics, Édouard Brezin, Vladimir Kazakov, Didina Serban, Paul Wiegmann, Anton Zabrodin, Springer, 2006 -Quantum Dynamics with Trajectories: Introduction to Quantum Hydrodynamics, Springer, Robert E. Wyatt (Author), Corey J. Trahan (Contributor), 2005

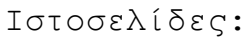

-http://en.wikipedia.org/wiki/Quantum_chaos

-M. Gutzwiller,

http://www.scientificamerican.com/article.cfm?id=quantum-chaossubatomic-worlds

-http://www.scholarpedia.org/article/Category: Quantum Chaos

-http://chaosbook.org/

-http://en.wikipedia.org/wiki/Dynamical_billiards

-http://en.wikipedia.org/wiki/Random maťrix

-http://www.scholarpedia.org/article/Random_matrix_theory

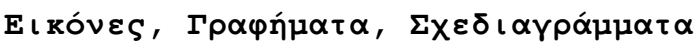

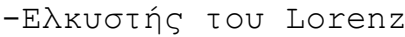

http://commons.wikimedia.org/wiki/File:Lorenz_attractor_yb.svg

http://commons.wikimedia.org/wiki/File:TwoLorénzorbits.jpg

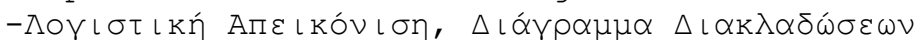

http://commons.wikimedia.org/wiki/File:LogisticMap_BifurcationDiagram.p ng

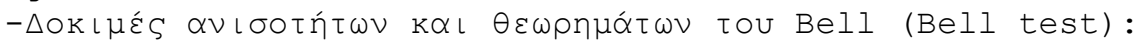

by C. Thompson:

http://commons.wikimedia.org/wiki/File:Two channel.png

http://en.wikipedia.org/wiki/File:Single-channel_Bell_test.png

human detectors:

http://arxivblog.com/wp-content/uploads/2008/03/human-entanglement.jpg

photon analyzer:

http://commons.wikimedia.org/wiki/File:Bell-test-photon-analyer.png 


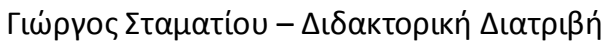

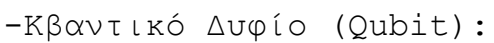

http://commons.wikimedia.org/wiki/File:Bloch_sphere.svg

http://commons.wikimedia.org/wiki/File:Blochsphere.svg

http://commons.wikimedia.org/wiki/File:Quantum_computer.svg

-Mnxavń tou Turing:

http://en.wikipedia.org/wiki/File:Turing machine 2a.svg

http://commons.wikimedia.org/wiki/File:Türing_machine_2b.svg

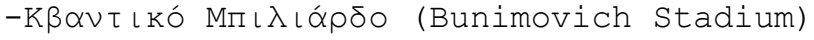

http://commons.wikimedia.org/wiki/File:Bunimovichstadium.png

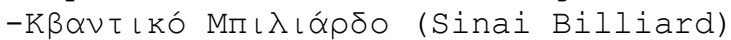

http://commons.wikimedia.org/wiki/File:SinaiBilliard.png

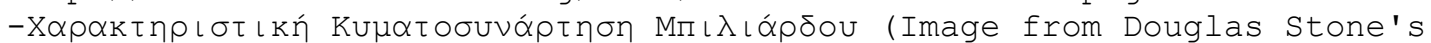
website)

http://www.aimath.org/news/que/typicalstadium.gif

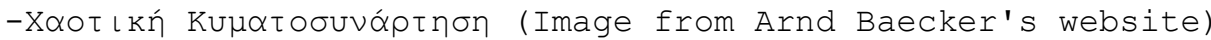

http://www.aimath.org/news/que/stadium scarring.jpeg

Exploring Quantum, Classical and Semiclassical Chaos in the Stadium Billiard, Chris King http://www.dhushara.com/DarkHeart/QStad/QStad.htm

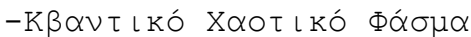

http://en.wikipedia.org/wiki/File:Qdfels.jpg

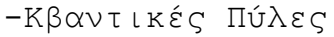

Cnot, http://en.wikipedia.org/wiki/File:Cnot-compared-to-xor.svg

Cnot, http://en.wikipedia.org/wiki/File:Cnotf.PNG

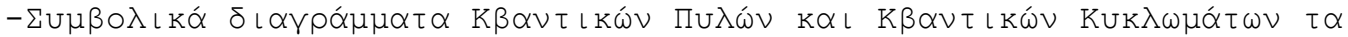

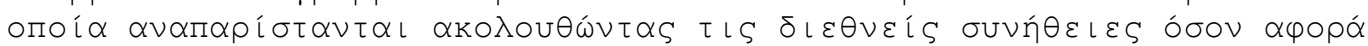

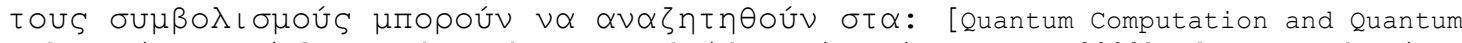
Information, M.Nielsen and I. Chuang, Cambridge University Press, 2000], [An Introduction to Quantum Computing, Phillip Kaye, Raymond Laflamme, Michele Mosca, oxford university press, 2007], [Quantum information and computation, Lecture notes for Physics 229, J.Preskill, California Institute of Technology, 1998]

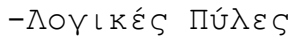

And, http://commons.wikimedia.org/wiki/File:AND_ANSI.svg

Or, http://commons.wikimedia.org/wiki/File:OR Ā̄SI.svg

Not, http://commons.wikimedia.org/wiki/File:NOT_ANSI.svg

Nand, http://commons.wikimedia.org/wiki/File:NĀ̄D ANSI.svg

Nor, http://commons.wikimedia.org/wiki/File:NOR ĀNSI.svg

Xor, http://commons.wikimedia.org/wiki/File:XOR_ANSI.svg

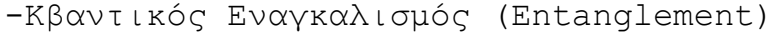

Art by Jim Flora

http://www.jimflora.com/fineartprints/entangled-couple.html

Sculpture by Ruth Bloch

https://picasaweb.google.com/106118540018705150389

Entangled light By Lisa Zyga (physorg.com)

http://cdn.physorg.com/newman/gfx/news/hires/2009/BECentangledlight.jpg

Art by Brose Partington

http://en.wikipedia.org/wiki/File:Entangled_front.JPG

Draws by Nick Harvey

http: //art-by-nick-harvey.blogspot.com/

http: / / quantumartandpoetry.blogspot.com/

Artwork by Pamela Ott, "Entanglement", acrylic, 1999

http://www.hottr6.com/ott/images/Entanglement.jpg 